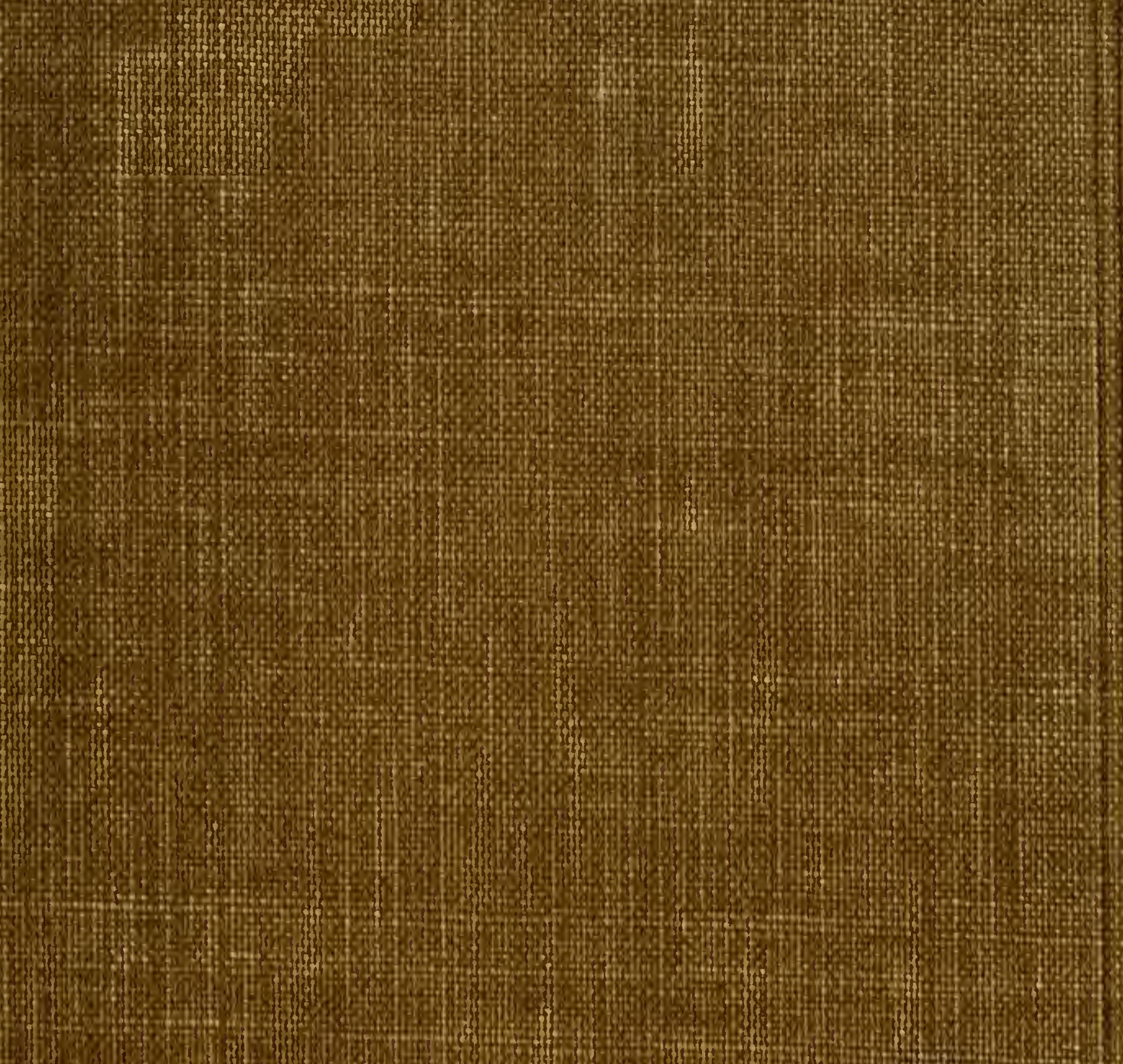

13. 
N. L. Noecker

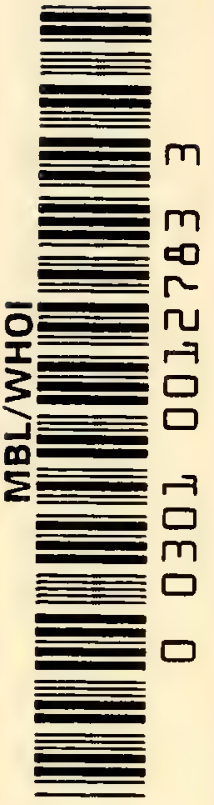



MCGRAW-HILL PUBLICATIONS IN THE BOTANICAL SCIENCES EDMUND W. SINNOTT, Consulting Editor 


\section{Selected Titles From \\ McGRAW-HILL PUBLICATIONS IN THE BOTANICAL SCIENCES}

Edmund W. Sinnotr, Consulting Editor

Babcock and Clausen-Genetics

Belling-The Use of the Microscope

Boysen Jensen-Growth Hormones in Plants

Braun-Blanquet and Fuller and Conard-Plant Sociology

Curtis-The Translocation of Solutes in Plants

Eames-Morphology of Vascular Plants

Eames and MacDaniels-Plant Anatomy

Fitzpatrick-The Lower Fungi

Gäumann and Dodge-Comparative Morphology of Fungi

Hill-Economic Botany

Hill, Overholts, and Popp-Botany

Loomis and Shull-Methods in Plant Physiology
Lutman-Microbiology

Maximov-Plant Physiology

Miller-Plant Physiology

Pool-Flowers and Plants

Seifriz-Protoplasm

Sharp-Cytology

Sinnott-Botany

Sinnott and Dunn-Genetics

Smith-Fresh-water Algae

Swingle-Systematic Botany

Weaver-Root Developnent of Field Crops

Weaver and Bruner-Root Development of Vegetable Crops

Weaver and Clements-Plant Ecology

Wodehouse-Pollen Grains

There are also the related series of McGraw-Hill Publications in the Zoological Sciences, of which A. Franklin Shull is Consulting Editor, and in the Agricultural Sciences, of which Leon J. Cole is Consulting Editor. 


\section{IN'TRODUC'TION TO}

C Y T O L O G Y

BY

LESTER W. SHARP

Cornell University

"The most important discoveries of the laws, methods and progress of nature have nearly always sprung from the examination of the smallest objects which she contains, and from apparently the most insignificant enquiries."-Lamarck, Philosophie Zoologique.

ThIRD EdITION

Fourth IMpression

MCGRAW-HILL BOOK COMPANY, INC.

NEW YORK AND LONDON

1934 
Copyright, 1921, 1926, 1934, ву тhe

McGraw-Hill Book Company, Inc.

PRINTED IN THE UNITED STATES OF AMERICA

All rights reserved. This book, or parts thereof, may not be reproduced in any form without permission of the publishers. 
To the memory of my father

LESTER ALLEN SHARP 



\section{PREFACE TO THE THIRD EDITION}

An unfortunate though inevitable result of advance in any field of knowledge is the rapid obsolescence of textbooks. In cytology, the years just past have witnessed the accumulation of a wealth of new observations, including a number of significant discoveries calling for a decided revision of certain concepts and statements which seemed adequate when the second edition of this book was prepared. Many of the questions troublesome at that time have received answers or what is often fully as important, more precise formulation. New and equally perplexing problems have arisen, but on the whole the cytological picture, particularly those portions of interest to the geneticist, appear notably clearer than they did eight years ago.

Next to the desire to bring the book into better accord with the present state of the subject in both text and illustration, the chief thought in preparing this edition has been that of improving its adaptability to the needs of students with comparatively little experience in the special field of cytology, without, however, making it an elementary treatise. To this end much of the material has been rearranged, the treatment of many points has been simplified, and certain border-line topics have been omitted altogether. Most of the citations to literature have been transferred from the body of the text to footnotes, where they are still available to the advanced student but need not confuse the beginner. Instructors will often find it advisable to omit or rearrange certain sections of the book in their class work. Much of the special matter in the opening chapters, for example, might well be considered later in courses. In accordance with repeated recommendations from users of the book, most of the former bibliography has been retained in spite of its length.

This edition, like the earlier ones, treats chiefly of the structural and genetical aspects of cytology. An especially valuable complemental work is J. Gray's A Textbook of Experimental Cytology, which deals at length with the results of physical and chemical researches on the cell. Among other recent general works of value to the student should be mentioned Special Cytology, edited by E. V. Cowdry, K. Bělař's Die Cytologischen Grundlagen der Vererbung, C. D. Darlington's Recent Advances in Cytology, and Traité de Cytologie Végétale by A. Guilliermond, G. Mangenot, and L. Plantefol. Of special interest to readers of Japanese are G. Yamaha's Saibô and Y. Sinotô's Japana Historio Citologia. 
The author takes pleasure in acknowledging his indebtedness to those who have aided him in many ways during the course of the work. He is particularly grateful to Dr. Barbara McClintock, Dr. L. F. Randolph, Dr. Lillian Hollingshead Hill, Dr. Harriet Creighton, and Dr. Marcus M. Rhoades for their valued criticisms of a number of the chapters and for generous contributions of new material.

L. W. S.

ITHACA, New York,

November, 1933. 


\section{PREFACE TO THE FIRST EDITION}

This book has been prepared for students of the biological sciences who desire a means of becoming more readily acquainted with the literature and problems of cytology. It does not pretend to be an exhaustive treatise for the use of experienced cytologists, though it is hoped that to them also some of its features may be of service.

For a number of years students of biology, especially those working along botanical lines, have been faced with the task of searching through a widely scattered literature for information on various cytological subjects. It is the purpose of this book not to render the consultation of that literature unnecessary, but only to make it easier; the student can scarcely be too strongly urged to derive his information from original sources wherever possible. The author does not presume to replace, but rather aims to supplement, Professor Wilson's well-known book, The Cell in Development and Inheritance, which, though written twenty years ago and with the emphasis primarily on the zoölogical side, will remain invaluable to all workers for many years to come. The more recent works of Gurwitsch (Morphologie und Biologie der Zelle), Heidenhain (Plasma und Zelle), and Buchner (Prakticum der Zellenlehre) are of importance, especially to the zoölogist.

The living cell, or protoplast, which represents an organized protoplasmic unit of structure and function, obviously cannot receive complete description in structural terms. Until a comparatively recent period cytological researches dealt primarily with cell structure, including particularly the conspicuous changes undergone by this structure in connection with the reproduction of the cell (cell-division) and of the multicellular organism (maturation and fertilization). A gradual shifting of emphasis has since led to the opening of fruitful fields in other directions, and the important results already achieved have shown with increasing clearness the need for a closer acquaintance with the physiological aspects of cell activity, not only in metabolism and growth, but also in the reproductive phases of the life cycle. The present work, though dealing mainly with the structural aspects of the subject, may aid indirectly in fulfilling the above need by making the prerequisite data of cell morphology more readily available.

Throughout the book, which in many of its chapters treats chiefly of the plant cell, attention is focused upon the protoplast; the cell wall is given only brief consideration, since it plays a relatively minor rôle in the processes of particular interest to the cytologist at the present time. 
Because of their fundamental importance in connection with the problems confronting the geneticist, the phenomena of nuclear division, chromosome reduction, and fertilization are described with considerable fullness, and their relation to the problems of heredity is taken up in five special chapters. With regard to many of the subjects treated, it has not been found possible to formulate final conclusions, since in many cases nothing more than tentative general statements are warranted by the facts in our possession. In some chapters little more than catalogs of conflicting opinions can be given, but in such a form the state of certain questions is not inaccurately represented. The student entering upon the field of cytology will be impressed by the large number of special points which remain undetermined and general questions which await adequate answers. If he can look upon cytology as a developing science, and if he has reached the stage at which he no longer demands categorical answers to all his questions, this book will be of interest to him as much for the problems it raises as for those it helps to solve. Not the least of its functions is to indicate lines of research along which he can hope to make contributions to the subject.

In compiling his materials the author has not hesitated to draw very freely upon the writings of others. In many cases where direct quotation is not made, the language of the originals has been closely followed in order to lessen the likelihood of misrepresentation. His great debt to Professor Wilson's book will be apparent to all those familiar with that admirable work. The majority of the diagrams and a number of the other figures are new. Most of the latter, however, have been redrawn from works cited in the text, not only that the value of the book may be enhanced by the presence of authoritative illustrations, but also that the student may be encouraged to become more familiar with the original papers. The general systematic positions of organisms indicated in the text by their scientific names only may be ascertained by referring to the generic names in the index.

The illustrations are largely the work of Miss Mildred Stratton, in whose skill and spirit of coöperation the author has had invaluable assistance. The criticisms of the text kindly given by Professor C. J. Chamberlain of the University of Chicago and Professor R. A. Emerson of Cornell University have been very highly appreciated. Acknowledgments are also made to the author's other colleagues for their advice and continued encouragement. Further criticisms looking toward the improvement of future editions will be welcomed.

L. W. S.

Iтhaca, New York, September 8, 1920. 


\section{CONTENTS}

PAGE

Preface to the Third Edition ................... vii

Preface to the First Entrion. . . . . . . . . . . . . . . . . . ix

\section{CHAPTER I}

Celis and Tissues.

Description of the cell-Living and fixed cells-Development-Differentiation-Correlation-Cell theory and organismal theory.

\section{CHAPTER II}

Protoplasis

Chemical nature-Physical nature-Colloidal state of matter-Colloidal nature of protoplasm-Ectoplasm and plasma membrane-Vacuole membrane-Ectoplast of Protista-Kinoplasm-Protoplasm and metaplasmProtoplasm and life.

\section{CHAPTER III}

The Nucleds.

General features-Structure-Nueleolus-Functions.

\section{CHAPTER IV}

Plastids.

Occurrence and general characters-Chromoplasts-Photosynthesis-Elaioplasts-Eyespot-Structure of chloroplast-Origin and multiplicationConclusions.

\section{CHAPTER V}

The Golg1 Material. ......................

Occurrence and general characters-Behavior in cell-division-Rôle in secretion-Comparison with plant cells.

\section{CHAPTER VI}

Chondriosones ........

Occurrence and general charaeters-Physical and chemical nature-Origin and multiplication-Function-Chondriosomes and plastids-Conclusion.

\section{CHAPTER VII}

Ergastic Substances.

Vacuoles-Cell sap-Carbohydrates-Proteins-Fats and allied substances -Crystals-Recapitulation: structure of the protoplast.

\section{CHAPTER VIII}

Somatic Cell-division

Outline of somatic mitosis-Cytokinesis-Cell-division in animals-Duration and periodicity of cell-division-Summary and conclusions. 
The Morphology of the Chromosonies

Size and general form-Spindle-attachment region-Nueleolus-forming region-Other specialized regions-The chromosome complement-Arrangement of chromosomes in the mitotie figure-Chromosome complements in related organisms.

\section{CHAPTER X}

The Structure of the Chromosomes

Chromonema theory-The mitotie cycle - Slender and small chromosomesChromonemata in sporocytes-Alveolation theory-Chromomere theoryChromomeres in the chromonema-Chromosome structure and the gene theory-Continuity of the chromosome.

\section{CHAPTER XI}

The Achronatic Figure.

Anastral figures-Amphiastral figures-Mechanism of mitosis.

\section{CHAPTER XII}

Cytokinesis and the Cell Wall

Furrowing in plants-Furrowing in animals-Cytokinesis by cell-platesCell wall-Composition of the cell wall-Ultramicroscopic strueture of wall-Walls of spores-Cell membranes of animals.

\section{CHAPTER XIII}

Atypical Mitosis and Other Nuclear Phenomena . . . . . . . . . . . . 183 Amitosis-Indueed aberrations of mitosis-Karyosome nuelei-Blue-green algæ-Bacteria-Chromidia.

\section{CHAPTER XIV}

Gametogenesis and Sporogenesis.

Alga-Fungi-Bryophytes-Pteridophytes-Gymmosperms-Angiosperms -Oögenesis in animals - Spermatogenesis in animals-Structure of other ciliated cells.

\section{CHAPTER XV}

Syngamy. . . . . . . . . . . . . . . . . .

Plants: Alga-Fungi-Bryophytes and Pteridophytes-GymnospermsAngiosperms. Animals: Nueleus-Centrosome-Cytoplasm and ehondriosomes-Conjugation and endomixis-Physiology of fertilization-Conelusion.

\section{CHAP'TER XVI}

Meiosis.

Stage of life cycle at which meiosis oceurs-Preliminary sketeh-Detailed deseription-Disjunetional and equational division-The ehiasma-The synaptic reaction-Meiosis in lower organisms-Conclusion.

\section{CHAPTER XVII}

Chromosomes and Mendelian Heredty.

Development and heredity-Rôle of the nueleus-Examples of Mendelian heredity-Terminology-Cytologieal basis of Mendelian heredity-Linkage 
and crossing-over-Assignment of linkage groups to chromosomes-Location of genes in the ehromosome-Chromatid exchange as the mechanism of recombination-Mendelism in monoploid plants-The gene theorySummary.

\section{CHAPTER XVIII}

Fragmentation and Translocation.

Fragmentation of ehromosomes-Behavior of free fragments-InversionTransloeation-Bearing on the ehromosome map-Discussion.

\section{CHAPTER XIX}

Reciprocal Translocation.

Origin of the theory (Datura)-Reciproeal translocation in Zea-CEnothera.

\section{CHAPTER XX}

Heteroploidy .

Terminology-Heteroploidy in related species-Chromosome numbers in related genera-Heteroploidy within species and individuals-How differences in number arise-Autoheteroploidy and alloheteroploidy.

\section{CHAPTER XXI}

The Cytogenetics of Autoheteroploid Plants.

Tetraploids and tetrasomies-Triploids and trisomies-Other heteroploidsMonoploid sporophytes-Other features of autoheteroploids.

\section{CHAPTER XXII}

The Cytogenetics of Hybrids.

Diploid hybrids with good synapsis and fertility-Partial asynapsis and its results-Synapsis in relation to polyploidy in hybrids-Analysis of alloheteroploid types-Amphidiploid hybrids-Parental characters in polyploid hybrids-Segregation of parental chromosome sets-A fertile tetraploid obtained through a sterile triploid-Conclusions.

\section{CHAPTER XXIII}

Chromosomes and Sex

Sex-chromosomes in animals-Bryophytes-Algæ and Fungi-Angiosperms-Sex-linkage-Heteroploidy and sex-Modification of sex-Conclusion.

\section{CHAPTER XXIV}

Apomixis and Related Phenomena . . . . . . . . . . . . . . . . . . .

Plants: Apomixis-Parthenogenesis-Apogany-Sporophytic buddingApospory-False hybrids-Pseudogamy-Androgenesis-M e r o g o n yCauses of apomixis. Animals: Parthenogenesis-Parthenogamy-False hybrids-Gynogenesis-Androgenesis-Merogony-Parthenogenesis and heteroploidy.

\section{CHAPTER XXV}

Cytoplasmic Heredity

Chlorophyll inheritance-Theories of chlorophyll inheritance-MossesOther cases-Conclusions. 
Historical SKetch.

Discovery of the cellular organization of plants and animals-Preformation and epigenesis-Renewal of study of organic structure-Cell theory-Cell multiplication-Protoplasm doctrine-Organismal theory-Syngany and embryogeny-Mitosis and meiosis-Cytology and heredity - The twentieth century.

BiBLIOGRAPHY

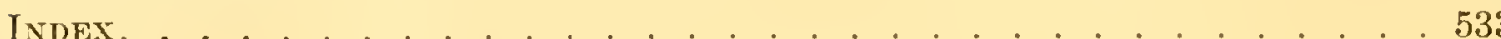




\title{
INTRODUCTION TO CYTOLOGY
}

\author{
CHAPTER I
}

\section{CELLS AND TISSUES}

Every living organism, plant or animal, is a protoplasmic system interacting with its environment. Although it is probable that the protoplasm of every kind of living thing, even every individual, differs physico-chemically from that of every other, it is nevertheless true that in certain major features of its organization protoplasm tends to be strikingly the same throughout almost the entire organic world. With the possible exception of certain lowly types of life, protoplasm everywhere is differentiated into two principal constituents, cytoplasm and nucleus. Furthermore, in most plants and animals protoplasm is organized in the form of cells, each cell consisting typically and essentially of a nucleus, a mass of cytoplasm, and a limiting membrane.

Cytology derives its name from the cell: kíros = hollow place (a cell). "Cytology, the study of cells" is a convenient definition, but in certain respects it is scarcely adequate. There are many tissues and many fairly complex organisms which are really large masses of nucleated protoplasm with actual cellular subdivisions partially or wholly absent, yet they carry on the same vital activities and confront the biologist with nearly all of the same fundamental problems as do the typically cellular forms. Hence it seems better to say that cytology is that branch of biology which deals directly with the structural and functional organization of protoplasm, usually in the form of cells, and with the relation of this organization to such phenomena as metabolism, ontogenetic differentiation, heredity, and phylogeny.

Throughout most of its historical development cytology concerned itself chiefly with the structural aspects of protoplasmic organization, its principal tool being the microscope. Since the nature of light and the structure of the human eye impose arbitrary limitations upon the applicability of microscopical methods, structural cytology came to be rather sharply set apart from the study of physiological function, in which a different set of techniques had to be employed. The artificial character of this distinction should be obvious. Every structure has its functional meaning, while every physiological process involves a structural alteration of some order in the protoplasmic system. Modern cytology is making 
increasing use of the methods and results of the physiologist, the physicist, the chemist, the geneticist, and the taxonomist, as well as those of the microscopist. The continued advance of this branch of biology will depend largely upon the measure in which these various investigators recognize the truly complementary nature of their tasks, and upon the rigor with which their guiding theories are checked by sound observation.

Description of the Cell.-The term cell was introduced in the seventeenth century by Robert Hooke and other microscopists, who applied it to the small cavities in the honeycomb-like structure which they discovered in plant tissues. Today the term denotes primarily the protoplasmic "cell contents," to which, strangely enough, the early workers attached little importance. The term protoplast, proposed by Hanstein (1880), is more appropriate and has come into fairly general use, but long usage and brevity have insured the permanence of the older term. The structural features of ordinary cells will now be sketched in their barest outlines by way of introduction to the detailed descriptions and discussions to follow in subsequent chapters.

The three mosi constant constituents of the typical cell are the cytoplasm, in which other cell organs and inclusions are imbedded, the nucleus, bounded by its own membrane, and the plasma membrane differentiated at the outer boundary of the cytoplasm. ${ }^{1}$ The cytoplasmic portion of the cell may be referred to as the cytosome.

The nucleus consists of a ground mass of hyaline fluid known as karyolymph ("nuclear sap"), an imbedded reticulum composed of karyotin, which is usually highly stainable, one or more true nucleoli, or plasmosomes, and a limiting nuclear membrane. Frequently masses of karyotin known as chromocenters are present at certain points in the reticulum (see Figs. 23, 24, and 73, 20).

The cytoplasm, a more or less transparent, viscous fluid, may, with its formed components and inclusions, occupy practically the entire volume of the cell. This is generally true of animal cells. In most plant cells a considerable amount of cell sap is present in one or more vacuoles. Often it far exceeds the cytoplasm in volume, the cytoplasm constituting a thin layer about it and lining the cell wall. In many cases the cytoplasm forms a system of strands that often show active streaming. Where it meets an enclosed vacuole it is limited by a vacuole membrane, or tonoplast. Externally the cytoplasm is bounded by a specialized layer of ultramicroscopic thinness, the plasma membrane. In some cells, for example certain amœbæ, a comparatively thick outer region known as the

${ }^{1}$ According to an older usage only the extra-nuclear portion of the protoplast was called "protoplasm," which was unfortunate because of the fact that the nucleus also is composed of protoplasm, or living substance in the broader sense. It is now the general custom to avoid this ambiguity by employing Strasburger's terms cytoplasm and nucleoplasm (karyoplasm, Flemming). The older usage has not, however, been entircly superseded. 
ectoplast may be distinguished; in some cases the plasma membrane appears to be the most superficial layer of the ectoplast (see Figs. 1, 48, and 49).

In plant cells plastids of one or more types are nearly always present in the cytoplasm. The most conspicuous among these are green chloroplasts, which are concerned in the fundamental process of carbohydrate synthesis (see Figs. 25 to 29).

A characteristic constituent of animal cells is the Golgi material, which may appear in the form of small bodies or more or less extensive networks

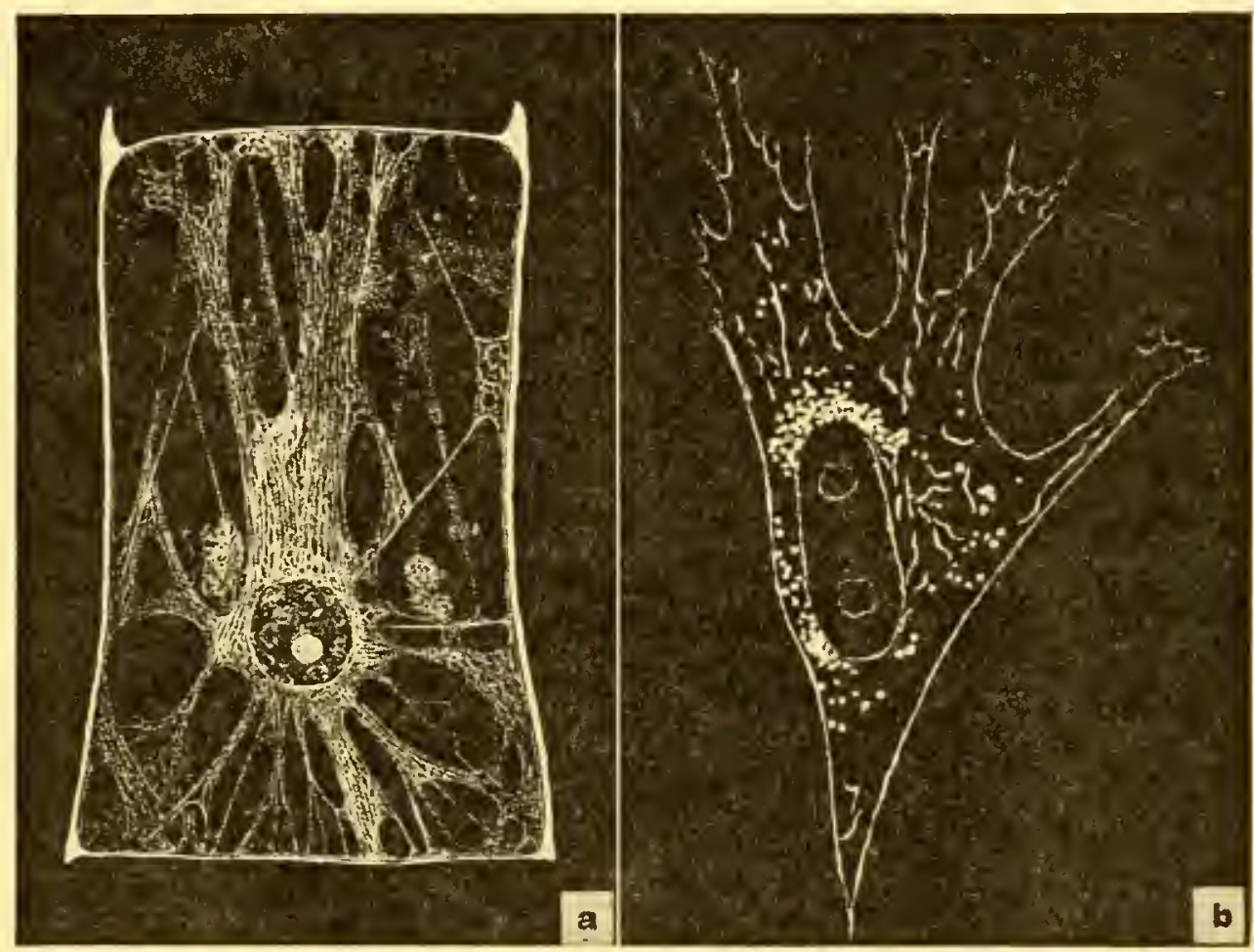

FIG. 1.-Living cells as they appear under dark-field illumination. $a$, cell from hair of squash. The cytoplasm lines the cell and extends in strands through the vacuolar matter; nucleus in lower center. (After Heidenhain, 1897.) b, cell from chick embryo in tissue culture. The cell is adhering to the underside of a cover slip. Near the nucleus are numerous fat globules; note also filamentous chondriosomes. (After Strangeways and Canti, 1927.)

in the cytoplasm. It is therefore often called the "internal reticular apparatus." It evidently plays a special rôle in secretion (see Figs. 35 and 37 ).

A centrosome is found in the majority of animal cells and in those of certain lower plants. It is typically made up of a central granule, or centriole, imbedded in a mass of hyaline or alveolar material known as the centrosphere, but either of these elements may be present alone. During stages of nuclear division the centrosome is surrounded by a conspicuous system of radiating astral rays, collectively called the aster (see Figs. 85 and 86).

Chondriosomes have been demonstrated in the cytoplasm of nearly all plant and animal groups. These are minute bodies having the form 
of granules, rods, or threads and constitute a group of materials of uncertain function (see Figs. 43 to 46).

Ergastic substances are accumulations of nutritive materials and other products of metabolic activity. These non-protoplasmic substances, which occur chiefly in the cytoplasm and vacuoles, may exist in the form of visible granules, droplets, or crystals. Such substances may also occur in the dissolved state.

The cell wall of plants, as at present understood, is usually regarded not as a part of the cell proper, or protoplast, but rather as a secretion of the latter. It is often absent, as in motile spores and gametes, which have only delicate limiting membranes. The intercellular substance of many animal tissues also bears a somewhat problematic relation to the protoplast.

Even in such a brief description as this, one is struck by the general similarity of plant and animal cells. This is obviously associated with the fact that all organisms, be they plants or animals, must carry on certain basic vital activities in common. Differences in cell structure may be taken to indicate differences in the vital processes habitually performed. The plastids, for instance, are to be viewed as structural and functional modifications by the aid of which plants are able to manufacture their own food out of simpler compounds derived from the environment, an act which animals cannot accomplish. The prevalence of large sap vacuoles in plant cells is also associated with peculiarities in metabolism. The elaborate cell walls of plants furnish support for large bodies, a problem which animals have solved in quite different ways. These are probably the three most conspicuous structural differences between the cells of the two kingdoms. Other minor differences will appear in subsequent chapters, but we shall continue to be impressed by the remarkable cytological unity of both branches of the organic world.

It is scarcely necessary to point out that the cell should not be thought of as a static thing with a permanent physical structure. It is rather a dynamic system in a constantly changing state of molecular flux, its constitution at any given moment being dependent upon antecedent states and upon environmental conditions. In the words of Harper (1919), it is a colloidal system in which the various processes have become progressively localized in certain regions, with the resulting formation of organs, which, with the increasing constancy of the processes involved, have come to possess a permanence and individuality of their own. The characteristic organization of the cell is a result of the differentiation of protoplasm, and this organization is of such a nature that it plays in turn an important part in determining the development of a higher degree of differentiation. Its efficiency in this respect is indicated by its almost universal occurrence in organisms of so many types. 
Living and Fixed Cells.-The majority of cytological descriptions have been based on material which has been prepared for observation by complicated processes involving (1) fixation, whereby the cell structures are rendered firm and capable of enduring the subsequent treatments without undue distortion, (2) dehydration, (3) imbedding in paraffin, (4) sectioning into thin slices, (5) staining with various dyes to bring out contrasts, and (6) mounting in a medium, usually Canada balsam, having a refractive index equal to that of the slide and cover-glass between which the material is supported. Consequently cytologists have had to meet the criticism that they are studying a series of artificial appearances rather than the natural structures of cells. It is well known that the

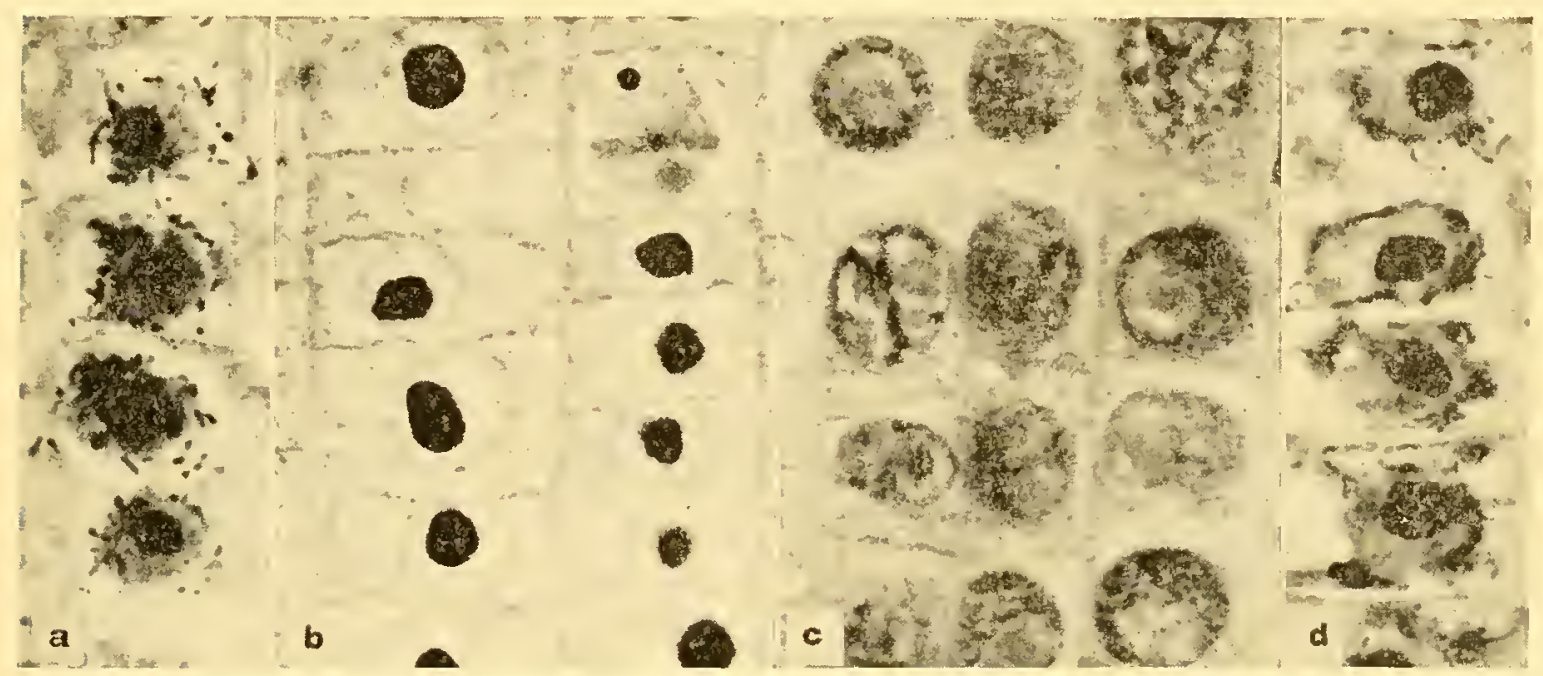

FIG. 2.- Root-tip cells stained with iron alum-hxmatoxylin after fixation in various aqueous solutions: $a$, ammonium and potassium bichromates, copper sulphate and pyridine; $b$, chromic anhydride, butyric acid, and nickel hydroxide; $c$, formic acid, chromic anhydride, and acetaldehyde; $d$, trichloracetic acid, formaldehyde, and nickel hydroxide. $a, b, d$ are from Zea; $c$ from Allium. Fluids with a $p \mathrm{H}$ below about 4.2 to 4.6 tend to give the "acid fixation image" (c) desired in studies of chromosomes, while those with a higher $p \mathrm{H}$ tend to give the "basic fixation image" $(d)$ showing the chondriosomes. (Photographs contributed by $C$. Zirkle.)

coagulative action of a fixing fluid may produce a visible structure not previously present in the protoplasm, and that some fluids swell certain cell components while others shrink them. Moreover, it is known that a given fluid may preserve the nucleus well and the cytoplasm poorly, or vice versa, and that the fixation images may differ characteristically according to the $p \mathrm{H}$ and other chemical features of the fluids used (Fig. 2). ${ }^{2}$ Hence careful cytologists are attempting to check their observations on fixed and stained cells against living material as far as possible and are constantly devising and refining methods for the study of untreated protoplasm.

Nearly all of the cell components mentioned on the foregoing pages may be observed to good advantage in living cells. For such study various kinds of cells can be kept alive for long periods if mounted in

${ }^{2}$ See Yamaha (1926 et seq.) and Zirkle (1928ab, 1929a). 
Ringer's solution or paraffin oil, the streaming of the protoplasm often continuing actively for hours or days. In the remarkable researches of R. G. Harrison and others ${ }^{3}$ it has been shown that cells from various tissues of vertebrate animals can be isolated and kept actively growing and multiplying in culture dishes for a length of time of ten far exceeding the normal life period of such tissues in the body. Under such conditions the cells appear pale and colorless, but by careful adjustment of the light the other components may be made to stand out with considerable clearness. The cytoplasm usually appears as an optically homogeneous fluid carrying various sorts of small inclusions which may show Brownian movement if the viscosity of the cytoplasm is not too high. Chondriosomes appear as dull granules or threads somewhat difficult to make out in many cells, while oil droplets stand out as highly refractive bodies. Centrosomes and centrioles may often be seen, especially if identified by surrounding astral rays. The nucleus may appear optically homogeneous except for the nucleolus, which is usually seen without difficulty, or it may present a finely mottled ("granular") appearance. In favorable material the reticulate structure is evident. The cell sap of plant vacuoles appears as a clear space, with or without inclusions. When properly illuminated in a dark field, many living cells are strikingly beautiful objects, the chondriosomes and other small bodies appearing as glistening specks in the pale protoplasm.

The visibility of certain components of living cells may be enhanced by the use of dyes which enter the cell without killing it. Examples of such "vital dyes" are neutral red and Janus green, which stain vacuoles and chondriosomes respectively. The vital staining of the nucleus with several dyes is also reported, ${ }^{4}$ but in several such cases it has been questioned whether or not the stained nuclei were actually uninjured and wholly normal.

Particularly noteworthy are the results obtained by a number of workers $^{5}$ through the use of micromanipulative methods. Apparatus and technique have been developed whereby it is possible to dissect, inject, and otherwise operate upon living cells under very high powers of the microscope. Many cells are highly tolerant of such treatment. A microneedle may be pushed carefully into the cell and even into the nucleus of a Tradescantia stamen hair without causing visible injury or cessation of activity, but a lowering of the internal pressure due to escape of material from the cell when the needle is withdrawn causes a series of alterations from which the cell may later recover (Martens and Chambers). In such ways it has been possible to learn much about the natural

${ }^{3}$ Carrel, Leo Loel, Burrows, H. V. Wilson, W. H. and M. R. Lewis, Drew.

${ }^{4}$ Küster (1918b, 1926), Gicklhorn (1927), Paltauf (1928), P. Dangeard (1923a).

${ }^{5}$ Kite (1913), Chambers (1914 et seq.), Seifriz (1918 et seq.), C. V. Taylor (1920 et seq.), Péterfi (1923), Martens and Chambers (1932). 
physical and chemical states of protoplasm and its differentiations in cells, as well as to correct many misconceptions due to inference from the aspects presented by fixed material. Of great interest also is the recent application of motion-picture photography to cytological problems. Films taken slowly of cells in tissue cultures and then projected at high speed have served to furnish information, especially with respect to time relations, which it would be difficult or impossible to obtain by any other means. Moreover, they have placed desirable emphasis on the conception of the cell as a dynamic system. As a consequence of the renewed study of living cells, it has been possible to evaluate anew the results obtained by the standard methods of fixation and staining and to improve upon such procedures. Critical studies have been made upon cells

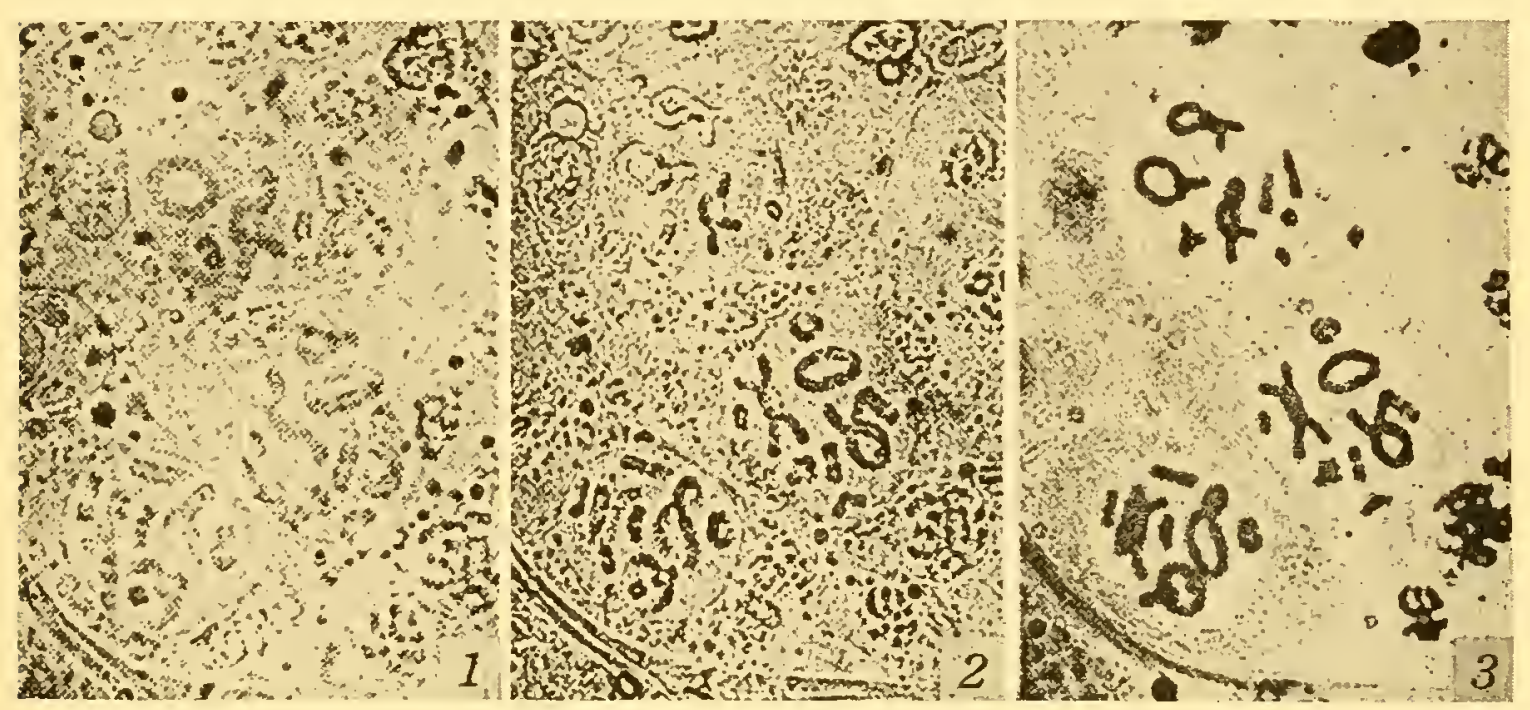

FIG. 3.-Spermatocytes of Stenobothrus (Chorthippus) lineatus: (1) living; (2) after treatment with $\mathrm{OsO}_{4}$ vapor and Flemming's fixing fluid; (3) after staining and mounting. (After Bèlaŕ, 1928b.)

before, during, and after fixation and staining ${ }^{6}$ (Fig. 3); and as a result it appears that, although the cytologist must be on his guard in interpreting the finer details even in his best fixed preparations, the general picture presented in such material is on the whole strikingly true to nature. Moreover, it is abundantly evident that no single set of methods will suffice to solve all of cytology's problems.

Development.-In studying the development of any small, relatively undifferentiated mass of nucleated protoplasm into a mature organism and in interpreting the rôle of cells and tissues in this process, there are certain general phenomena which may be considered before taking up the more special data of cytology. These are growth, morphallaxis, differentiation, and correlation.

By growth is meant primarily the synthesis of new protoplasm through the activity of the old, and secondarily the increase in body size which usually results. The synthetic processes involve an extensive interchange

${ }^{6}$ See Martens (1927bc) and Bělař (1929a). 
of materials between nucleus and cytoplasm, so that a certain proportion of nuclear and cytoplasmic substances (the "nucleoplasmic ratio") must be maintained if these processes are to continue. The part which any single spherical nucleus can play is strictly limited by the simple fact that its surface, through which the interchanges occur, does not increase at the same rate as does its volume during growth. The further growth of the protoplasmic mass therefore requires a relative increase in the nuclear surface. This is sometimes accomplished by a change in the shape of the nucleus, but the almost universal method is by nuclear division, whereby the nuclear surface is increased without a corresponding change in volume. This permits further growth up to the point at which

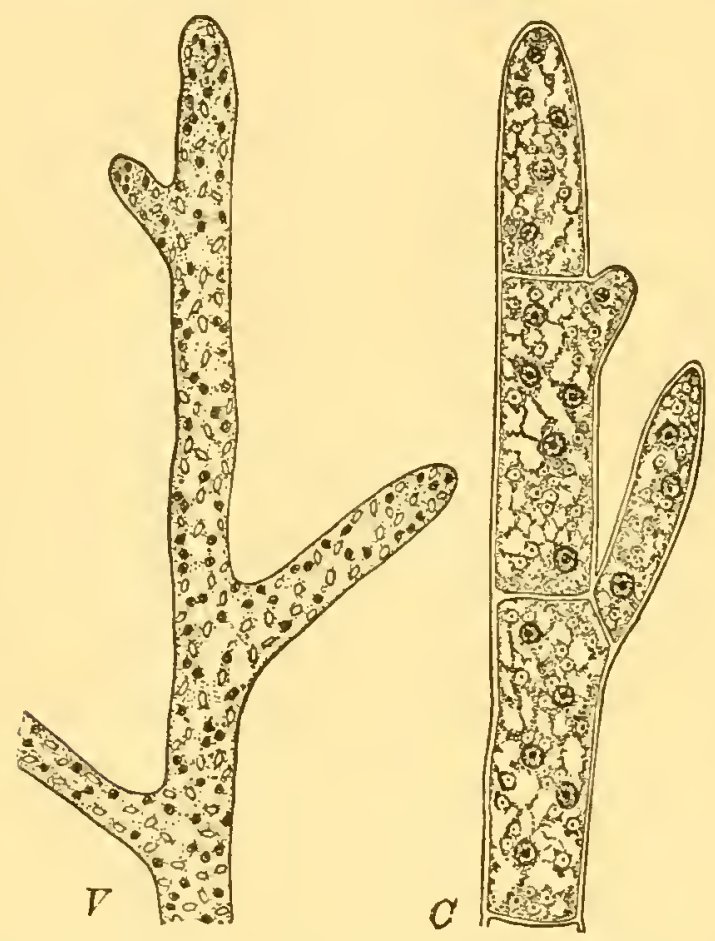

FIG. 4.- $V$, portion of coenocytic body of Vaucheria; nuclei dark and plastids in outline. $C$, portion of semi-coenocytic body of Cladophora.

and Protophyta). A second method is by change of shape. This is well exemplified in conocytic plants, such as Vaucheria (Fig. 4), Mucor, and the myxomycetes, in which large masses of protoplasm expose an extensive surface by assuming a flat, spreading form (myxomycetes) or by dereloping filamentous branching bodies (cœnocytic algæ and phycomycetes). At the same time the nucleoplasmic ratio is maintained by repeated nuclear division, the numerous nuclei being either relatively fixed in position (Caulerpa) or free to move about with the flowing cytoplasm (Bryopsis, phycomycetes, myxomycetes).

The formation of partitions and the division of the nuclei show various degrees of correlation in different organisms. The two processes may be quite independent, in which case the compartments (cells) contain vary- 
ing numbers of nuclei (e.g., Cladophora; Fig. 4). In many cases, however, they are so intimately related that they are like one process, and the result is a regularly uninucleate condition of the cells. Here growth may appear to be a matter of cell multiplication, but a consideration of protoplasmic masses not showing such a correlation between nuclear division and cytoplasmic septation suggests that the regularly uninucleate cellular condition is the result of a special refinement in mode of growth and differentiation. The great importance of this refinement in connection with the evolution of organisms is indicated by its prevalence. In certain organisms the number and arrangement of the cells are constant in a given organ or even in the whole body. ${ }^{7}$

By morphallaxis is meant the development of a particular form otherwise than through synthesis or enlargement directly. The specific form of the body is due in part to the fact that growth at certain periods is not equal in all directions, but in addition to this the rearrangement of materials already present may play a prominent rôle. A striking illustration of morphallaxis is afforded by small pieces of $H y d r a$ which under proper conditions will, without further growth, remold themselves into complete, though dwarfed, individuals.

As the young organism grows, it differentiates. Differentiation, in the words of Conklin, is "transformation from a more general and homogeneous to a more special and heterogeneous condition." It involves the development of unlike functions and structures, and the localization of these in different regions of the organism. Physiological division of labor and morphological division of substance constitute one inseparable process, and this process in its last analysis is the result of physical and chemical changes in protoplasm caused by the combined action of intrinsic and extrinsic factors. The next section will be devoted to the subject of differentiation.

One of the most remarkable features of a living organism is the perfect correlation which is normally maintained between the activities of its many diverse organs; without such correlation development could never occur at all. Differentiation and integration are, in fact, two aspects of one thing, namely, organization (Conklin). It is to be emphasized that correlation is not something which comes into being as the differentiation of functionally distinct regions occurs: the organism behaves as a consistent, correlated whole from the beginning of its development onward. The correlation existing between the activities of the parts of the cell with which development begins gradually becomes the more extensive correlation of the highly differentiated mature organism. Correlation is retained from the first, although new means for its maintenance are elaborated. What these means are will be pointed out below.

${ }^{7}$ See the review by Van Cleave (1932). For a review of the subject of cell form, see Gray (1931). 
Differentiation.-Differentiation occurs in uninucleate cells, multinucleate plasmodia, and multicellular masses. In the bodies of certain Protozoa (Fig. 5) one sees within a single cell a very elaborate regional differentiation in structure, certain functions being localized in definitely constituted organs. There are distinct locomotor, digestive, and excre-

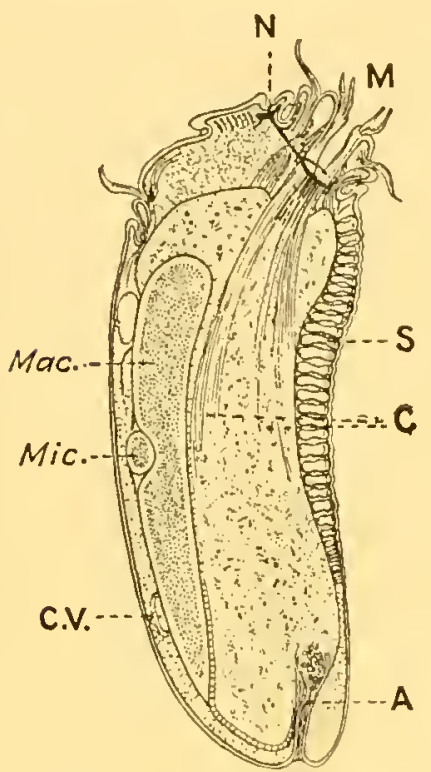

Fig. 5.-Diplodinium ecaudatum. MI, mouth; $N$, neuromotor apparatus; Mic, micronucleus; Mac, macronucleus; C.V., one of the contractile vacuoles; $A$, anal canal; $C$, contractile region; $S$, skeletal plates. (After R. G. Sharp, 1914.) tory systems, as well as a "neuromotor apparatus" which functions much as does the nervous system of larger animals. ${ }^{8}$ These are probably the most complex cells known. ${ }^{9}$

Differentiation in multinucleate masses of protoplasm may be seen in the plasmodia of myxomycetes, in the cœnocytic algæ and fungi, and in the embryos of certain higher animals and plants. In the myxomycete the protoplasm heaps up locally and develops into a highly characteristic fruit body with well-differentiated stalk, capsule wall, and supporting capillitium filaments, but there is no subdivision into cells until the spores are finally delimited. In the cœnocy tic algæ, such as Vaucheria and Caulerpa, the body develops a definite form and structure without cellular subdivisions. In Caulerpa (Fig. 6) there are well-formed "roots," "stems," and "leaves," yet the whole vegetative body is one continuous mass of cytoplasm with thousands of nuclei scattered through it. The nuclei may be relatively fixed in position, or they may move about freely with the streaming cytoplasm. In a number of cœnocytic plants, moreover, the spores and gametes themselves are cœnocytic ("cœnogametes" of Mucor and Albugo bliti; zoöspores of Vaucheria).

${ }^{8}$ R. G. Sharp (1914), Yocum (1918), C. V. Taylor (1920), Rees (1921, 1922b, 1931), Me Donald (1922), Koofoid and Swezy $(1922,1923)$.

${ }^{9}$ Certain writers, notably Dobell (1911a), hold that the protozoan body is "noncellular" rather than "unicellular," and restriet the term "cell" to the integral parts into which a "multicellular" organism is subdivided. Whatever may be thought of the practieability of this use of terms, its theoretical implications are worthy of attention. Although agreeing with Dobell that the protozoan body and the ordinary tissue cell are not homologous, we have ehosen to use "cell" more loosely as a term of convenience for both of them, as well as for other units for which morphological equivalence is not claimed.

It is also frequently urged that "organelle" rather than "organ" should be used for intracellular differentiations such as nuclei, plastids, and the neuromotor apparatus, and that the latter term should be applied only to multicellular structures. We have used "organ" in the more general sense in order to emphasize the fact that in all cases the structures indicated are regional differentiations of protoplasm associated with ecrtain functions, to which the presence or absenee of cell partitions is a subordinate feature. "Organelle" is, however, a useful term. 
The bodies of such organisms may be surprisingly elaborate, but the degree of complexity which they attain is nevertheless limited. The functional differentiation of regions in continuous large masses of protoplasm, which shows itself in visible structural differentiations, may be considerable; but the degree of differentiation reached by the higher classes of plants and animals seems to have been very largely conditioned by the development of partitions between the various centers of activity

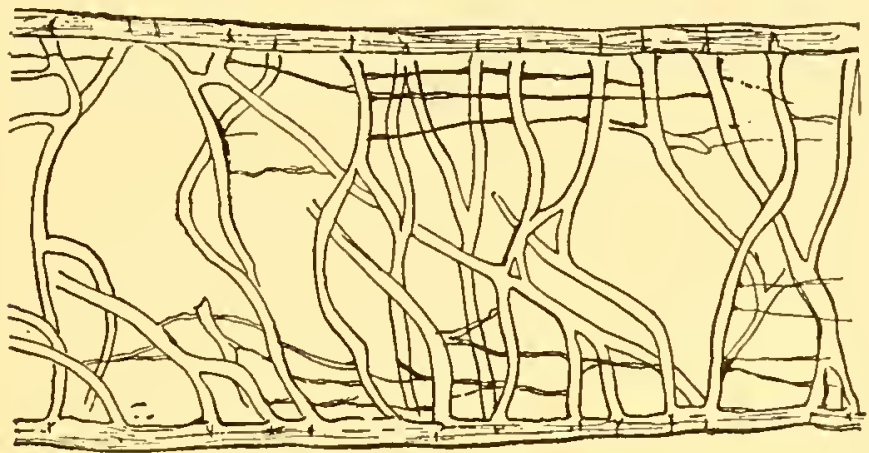

B

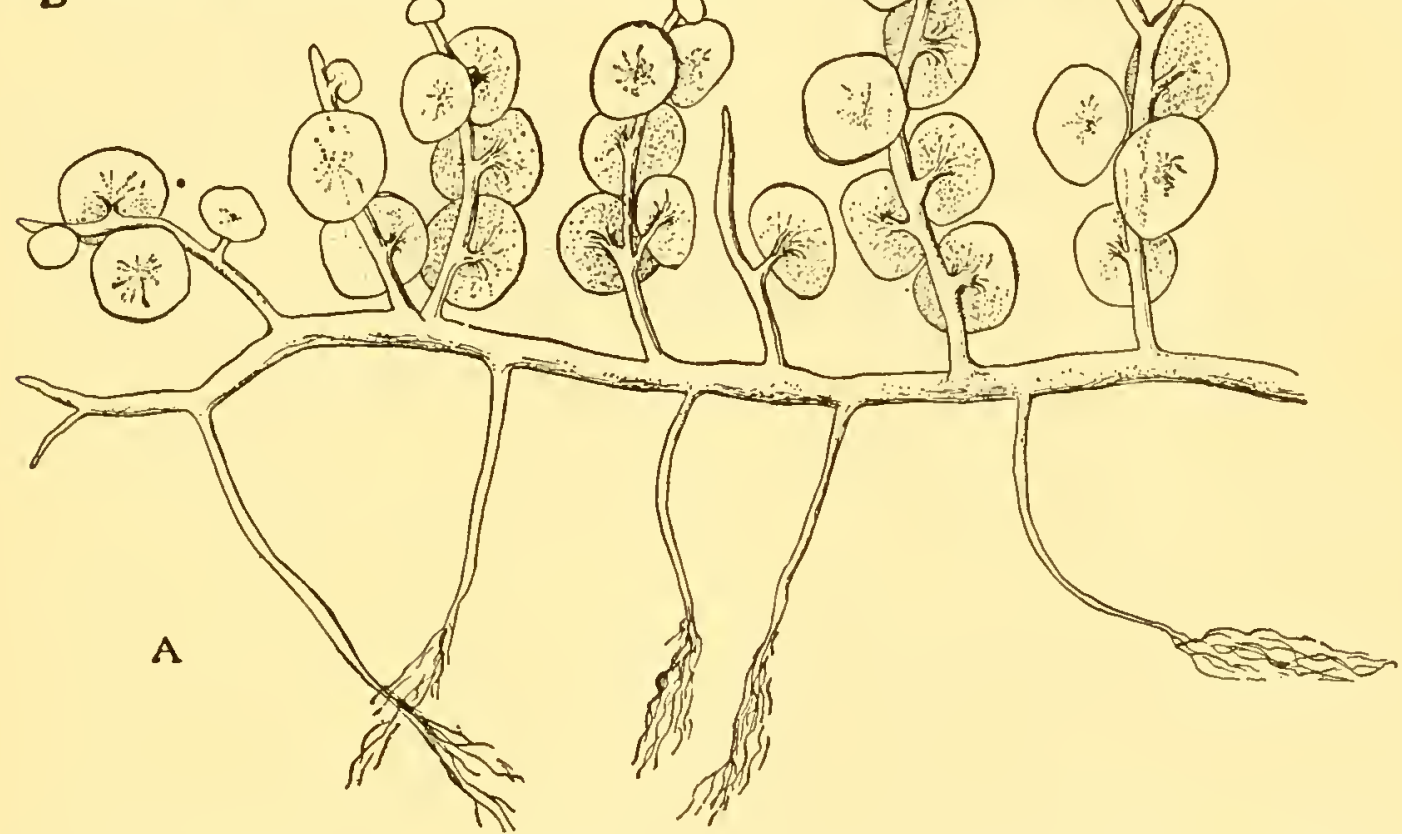

FIG. 6.-A, Caulerpa macrodisca, a cœnocytic plant. B, section of leaf of Caulerpa prolifera, showing supporting trabeculæ.

(After Oltmanns.)

(nuclei) and functionally differentiating regions, the regions thus set apart then becoming more fully specialized than would have been possible in a continuous aqueous colloidal medium (see R. S. Lillie, 1923). The protoplasmic body thus attains a multicellular organization.

Differentiation in multicellular masses is well exemplified in the growing points of vascular plants (Fig. 7). In the stem tip and root tip there are actively growing regions known as meristems, in which the cells are in a relatively undifferentiated "meristematic" or "embryonic" condition. ${ }^{10}$ In many plants there is another extensive region of such ${ }^{10}$ For an extensive account of meristems, see Schüepp (1926). 
cells in the cambium. As a rule, meristematic cells contain no conspicuous ergastic inclusions aside from vacuoles and are separated by very delicate walls with no intercellular spaces. As growth proceeds in such tissues, every nuclear division is accompanied by a division of the cytoplasm; hence growth results in the multiplication of the uninucleate cells. In regions farther from the point of greatest meristematic activity the cells gradually become visibly diversified in structure in connection with

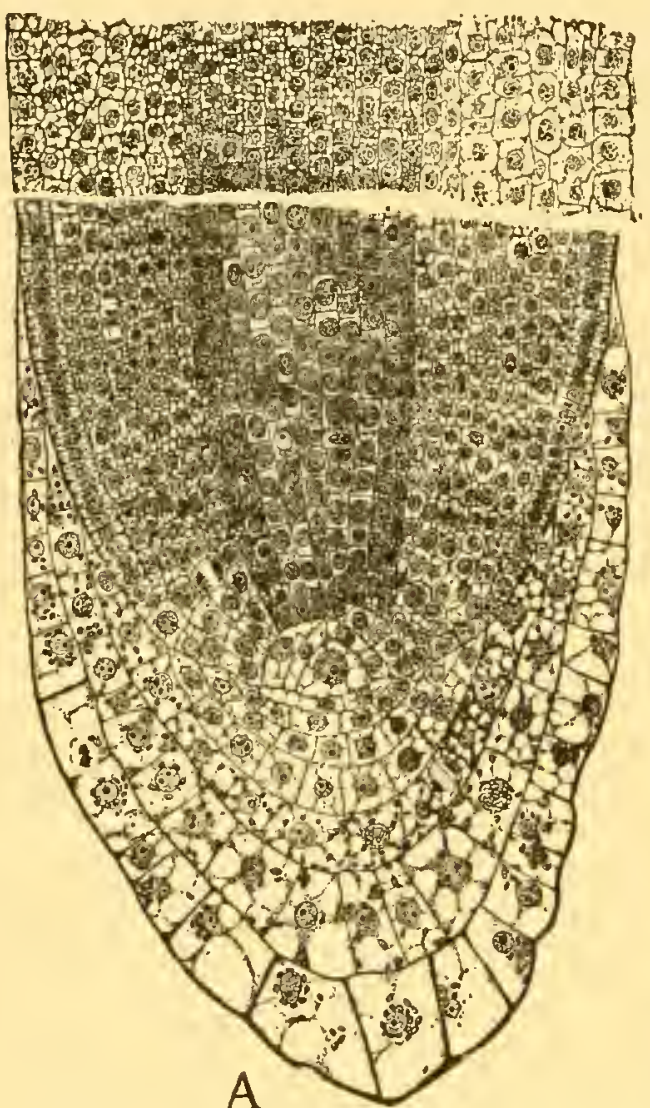
their increasing specialization in function. Throughout the active life of the plant the homogeneous meristematic cell mass thus continues to grow distally and differentiate proximally into tissues with very diverse histological characters (see Eames and MacDaniels, 1925).

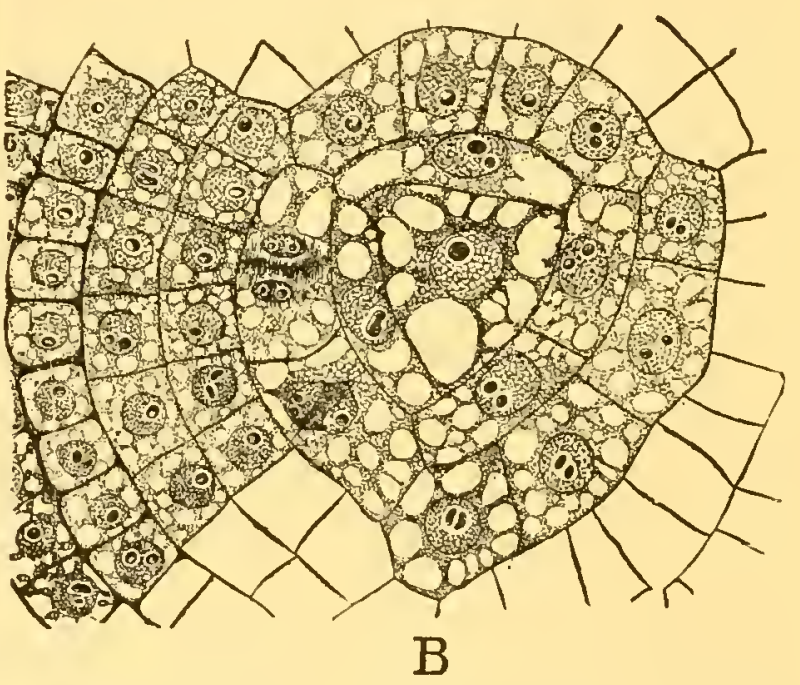

FIG. 7.-Longitudinal and transverse sections of apical meristem in root of Pteris, showing triangular pyramidal apical cell. The segments cut from the distal face of the cell go to form the root cap, while those from the three lateral faces develop in to the tissues of the root proper. In seed plants the meristematic activity does not center in a single distinguishable cell. (After Hof, 1898.)

Most of the visible characters which ordinarily serve to distinguish the various kinds of differentiated cells of the vascular plant are found in the cell wall rather than in the protoplast itself. Thus, besides meristematic and slightly modified parenchymatous cells, there are many other types, such as tracheids, vessels, wood fibers, phloëm fibers, and sieve tubes (Fig. 8), all of which are characterized by the peculiar ways in which their walls become modified through secondary and tertiary thickenings and by the form and arrangement assumed by the pits (see p. 175). The protoplasts may finally disappear completely from wood cells, leaving a tissue or framework composed of lifeless cell walls. All functional differences are accompanied by chemical or physical differences of some sort in the protoplasm, but it is mainly in the non-protoplasmic 
elements (including the wall) rather than in any conspicuous structural changes in the protoplasm itself that cell differentiation is rendered visible in the case of plants. Apart from differences in shape, amount of vacuolar material, accumulated food, and other products of differentiation, protoplasts performing widely different functions may appear much alike.

Structural differentiation in connection with division of labor is very striking in the protoplasm of animal cells, ${ }^{11}$ which are destitute of such walls as plant cells possess. The muscle cell shows fine longitudinal

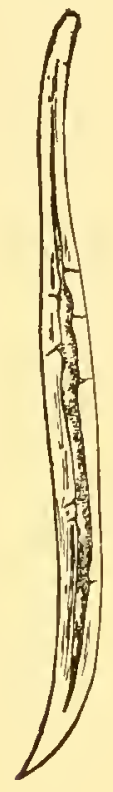

A

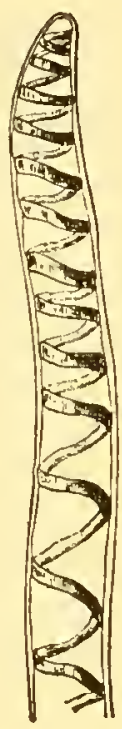

$B$

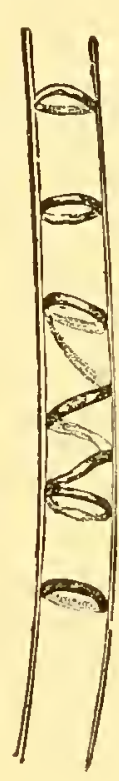

$C$
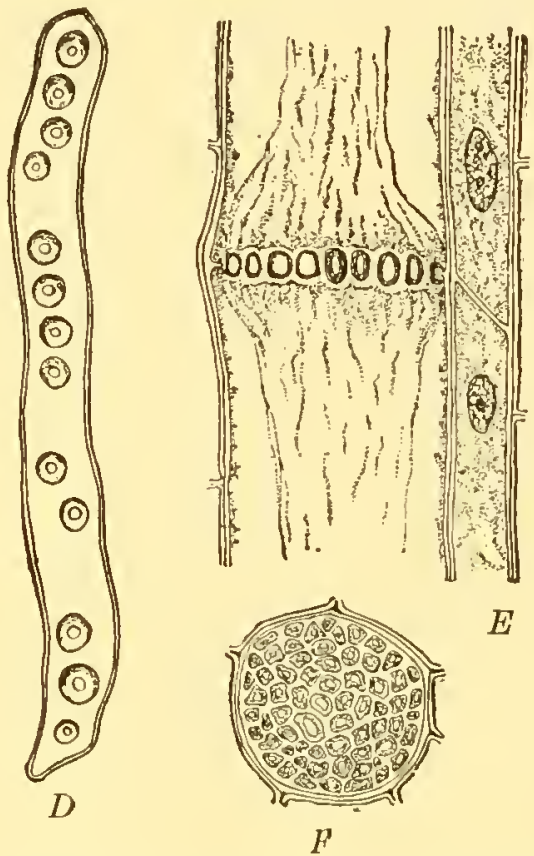

Fig. 8.-Differentiated cells from vascular plants. $A$, wood fiber with thiekened wall. $B, C$, portions of tracheids with spiral and annular thickenings. $D$, pitted tracheid. $E$, portion of sieve tube with adjacent companion cells. $F$, face view of sieve plate shown in section in $E$.

fibrils, which are in some way concerned with the cell's power of contractility. In certain muscles these fibrils show transverse membranes at regular intervals, and these so correspond in adjacent fibrils that the muscle has a transversely striped appearance.

The nerve cell (Fig. 9) typically possesses a single unbranched prolongation (axon) and one or more others (dendrites) which often become very elaborately branched, especially in the ganglion cells of the spinal cord and brain. In fixed preparations the cytoplasm of the nerve cell contains fine "neurofibrils"12 and also granules of chromatic "Nissl substance."

I1 For detailed descriptions of the cytological features of animal cells of many types, see Special Cytology, ed. by Cowdry (1932); see also Heidenhain (1907, 1911).

12 It has been reported that in healthy living eells the neurofibrils cannot be detected and probably represent coagulation artifacts (Matsumoto, 1920; Lewis and Lewis, 1924; de Moulin, 1923), but other investigators (Cowdry, 1914, 1928; Bozler, 1927; Boeke, 1926; Parker, 1929ab) are convineed that they eorrespond to real differentiations in the cell. 
Cells specialized in connection with motility, such as spermatozoa and the cells of certain epithelial tissues, show complex structural modifications not only in the flagella, cilia, and cirri which they bear but also in the other cell organs with which the activities of these motile structures are closely connected (Figs. 134, 136, 137). Secretory cells are often distinguishable by the accumulations of secretion products in their cytoplasm, or by the peculiar form assumed by their nuclei (Fig. 22).

In connective tissues (Fig. 10) the protoplasm is subdivided into cells with various degrees of distinctness depending on the relative amount of supporting substance they produce during their differentiation.
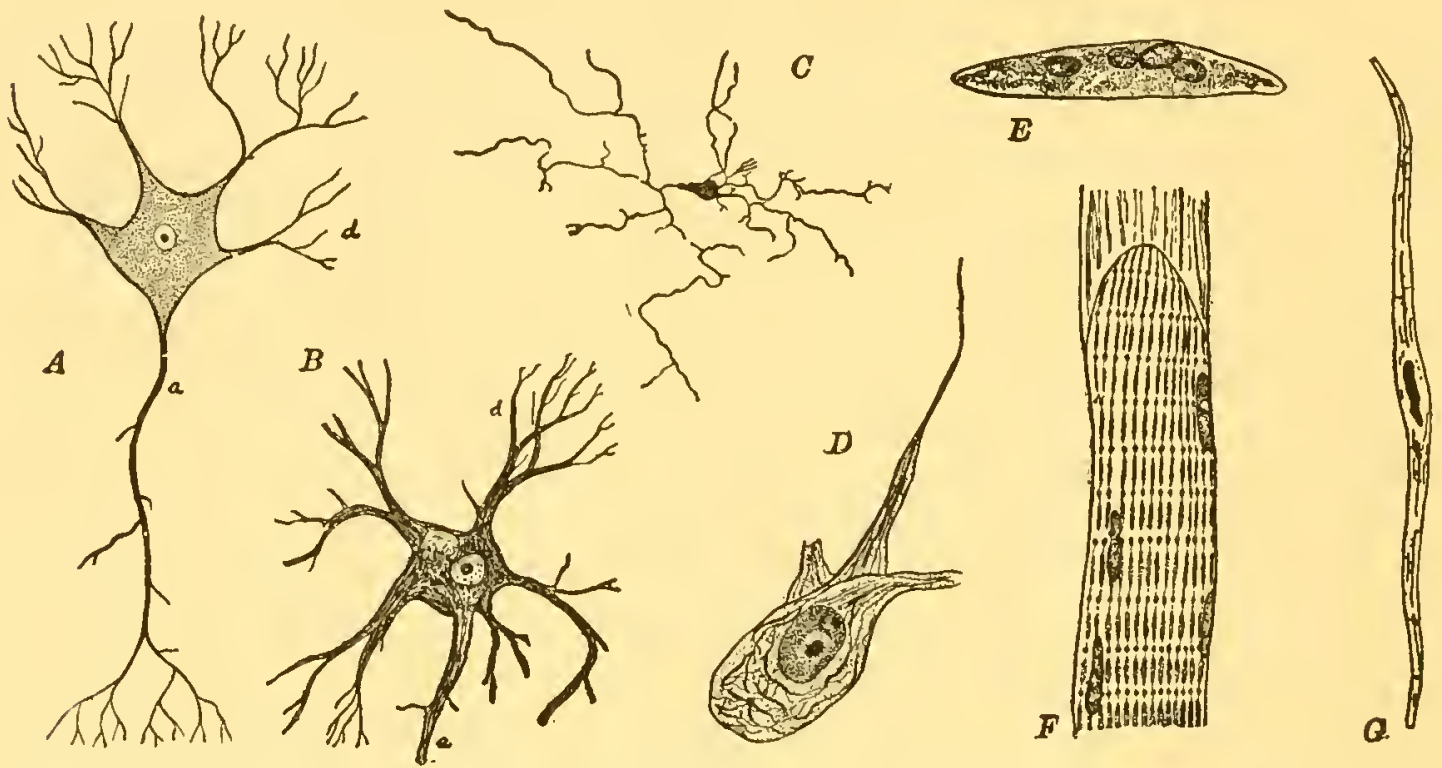

F1G. 9.- Nerve and muscle cells of animals. $A$, diagram of a typical neuron: $a$, axis cylinder process, or axon, ending in arborescent system; $d$, dendrites. $B$, cell from human spinal cord. ( $A$ and $B$ after Obersteiner and Hill.) $C$, nerve cell from eye. (After Lenhossék.) $D$, nerve cell from earthworm. (After Kowalski.) $E$, young voluntary muscle cell. $F$, portion of mature voluntary muscle cell, showing striations. $G$, involuntary muscle cell from intestine. ( $E-G$ after Piersol.)

Cartilage and bone cells are likewise imbedded in such substances, which are here produced in relatively enormous amounts and later form the main supporting framework of the body. Blood, which is sometimes spoken of as a "fluid tissue," consists of a plasma carrying cells of a variety of types.

Differentiation in a multicellular mass involves the gradual setting apart of special regions with modified structural and functional characters as in the body of a protozoön, but with the important difference that these regions include many cells, each of which retains in some degree the fundamental type of protoplasmic organization (nucleus, cytoplasm, semipermeable membranes) possessed by the cell which began the development of the differentiating mass. One therefore expects a greater capacity for independent action in these cellular components of the body than in the subcellular components of the protozoan body. This expec- 
tation is fulfilled in the results of experiments which have shown that many tissues and cells of higher organisms may, if given structural independence and a proper environment, continue to live or even in many cases to grow into a complete new body. The ability of such an
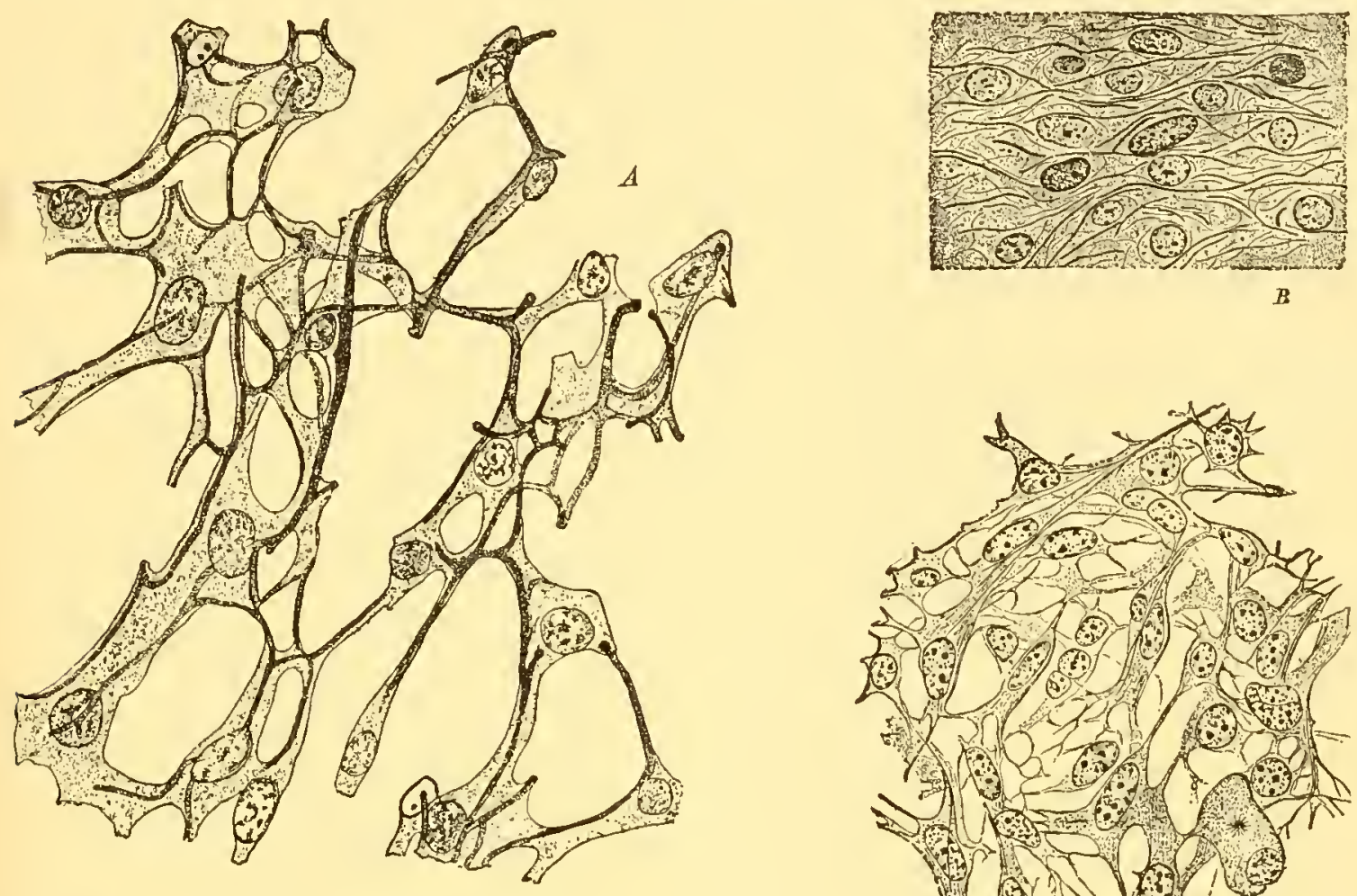

$\boldsymbol{B}$
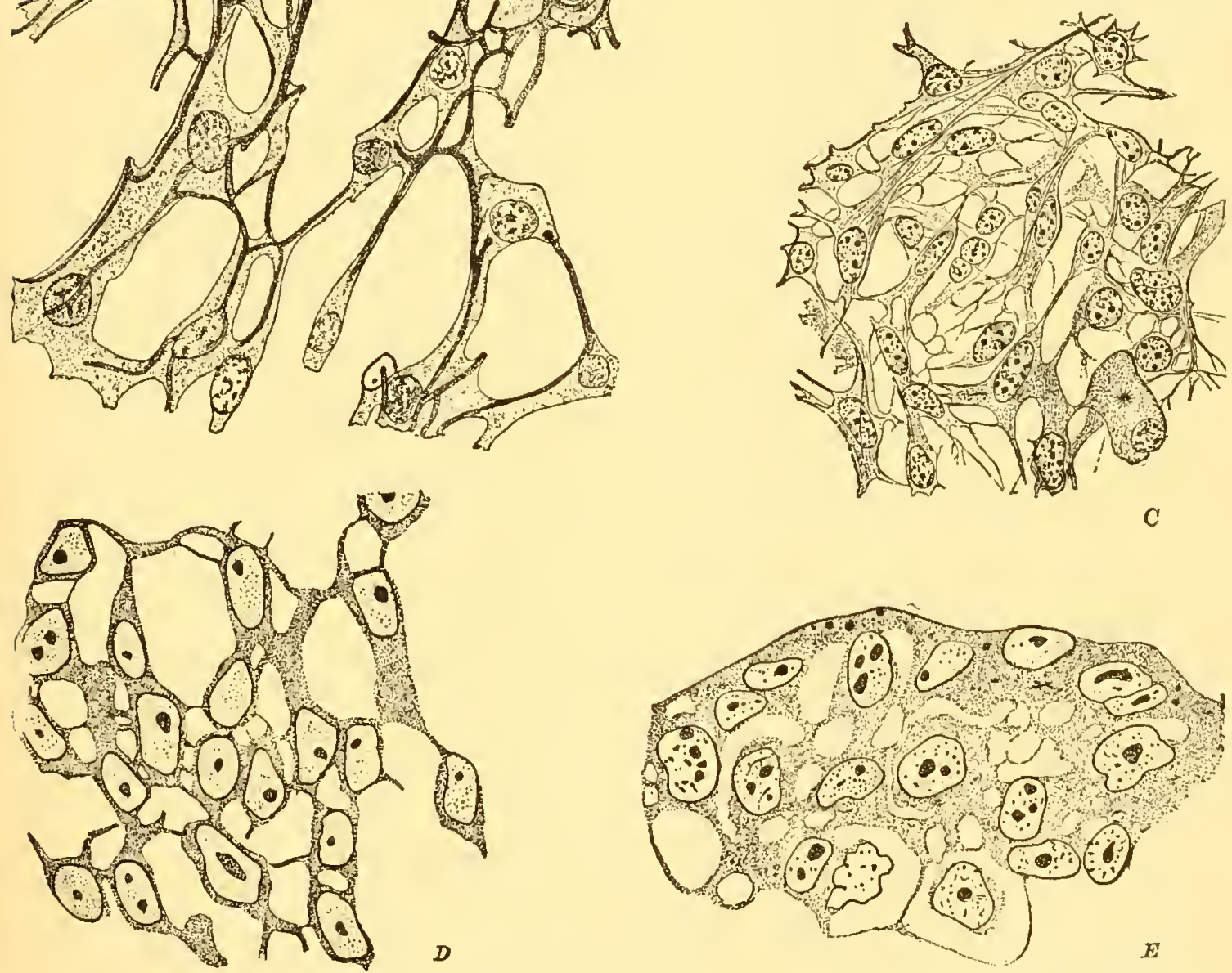

C

Fig. 10.-A, reticular connective tissue from lymph gland of cat. (After Heidenhain.) $B$, young heart muscle of dog embryo, showing myofibrils developing, but no subdivision into cclls. $C$, later stage of same. ( $B$ and $C$ after Godlewski.) $D$, development of cells in plasmodium by vacuole formation in human embryonic epithelium. (After Marchand.) $E$, development of cartilage tissue from plasmodium in Lophius. (After Studnicka.)

isolated part to reconstitute a whole depends upon the measure in which it has retained what is fundamental in the physico-chemical constitution of protoplasts through the period of its differentiation.

Correlation.- No such degree of differentiation and specialization of tissues as we see in organisms could be attained, and no such complex mechanism could continue to act as a unit or individual, were it not for 
adequate means of keeping the activities of the various parts fully coördinated. Among the means by which this correlation of the parts is maintained, we may consider protoplasmic continuity, the differentiation of specialized tracts, hormones, and physiological gradients.

The delicate intercellular strands in the tissues of multicellular plants have been described in a large number of papers. ${ }^{13}$ Two general types of connection are usually distinguished. In the red algæ and certain other thallophytes adjacent cells are connected at one relatively large region where membrane materials are deposited. The question of actual protoplasmic continuity through this region is at present a debated one (Jungers, 1933). Large protoplasmic strands are found between the egg and surrounding cells in cycads and in certain other tissues, but these seem to be due either to the enlargement of smaller pores present at an early stage or to actual solution of the intervening wall. Strands of the second general type, which are known as plasmodesms (Fig. 11), are of exceedingly small diameter, special methods being required for their demonstration. They may be distributed rather uniformly over the wall, or they may be aggregated in small groups, which are often located in pits or thin spots; frequently they are branched.

Very little is accurately known regarding the origin and development of plasmodesms. It can scarcely be doubted that the pores through which they pass are often present from the time the cell partitions are first formed, no wall substance being deposited at these points. There is also considerable evidence in support of the view that plasmodesms are secondarily developed structures. Strasburger (1901) stated that extensions from adjacent cells come into contact as the intervening wall begins to thicken but do not form continuous strands. The fact that in separating cells the break occurs through the thickened median portion of the strands lends support to this view (Hume, 1913). That the strands are actually continuous was emphasized by Meyer (1896 et seq.), who held that they are due both to retention and to new formation. He observed a secondary formation of connections in Volvox and in fungus hypha which came in contact.

Light on this question has been sought in parasites and graft hybrids, where cells of different species come together. Kienitz-Gerloff, Kuhla,

${ }^{13}$ Among these may be mentioned the works of Wille (1883) and Borzi (1886) on the Cyanophycex; Fohl (1891), E. Overton (1889), and Meyer (1896) on the Chlorophyces; Hick (1885) and Doubt (1928) on the Fucicex; Hick (1883), Massee (1884), Rosenvinge (1888), and Mangenot (1926) on Florideæ; Koht (1897) on mosses; and, on vascular plants, those of Tangl (1879), Russow (1882), Strasburger (1852, 1901a), Goroschankin (1883), Terletski (1884), Wortmann (1887, 1889), Haberlandt (1890), Kienitz-Gerloff (1891), Jönsson (1892), Kulıla (1900), Poirault (1893), Gardiner $(1884,1897,1900)$, Hill (1900, 1901), Gardiner and Hill (1901), Kolıl (1900, 1902), and Quisumbing (1925). For general accounts of protoplasmic eonnections, see Davis (1905a), Meyer (1920), Lundegardh (1922), and Jungers (1930). 
and Strasburger found no plasmodesms between the cells of Viscum and Cuscuta and their hosts, but in the case of graft hybrids both Buder (1911) and Hume (1913) report their presence in the walls separating cells which are supposed to be genetically unrelated. This seems to show that connections may arise secondarily, although uncertainty regarding the exact behavior of the protoplasts in the wounded region leaves an element of doubt. They arise secondarily in the abutting walls of tyloses, according to Molisch (1888).

At present the probabilities are in favor of the view that intercellular strands are both primary and secondary in origin, some of them representing regions in which the continuity of the protoplasm has never been broken and others being subsequently developed through the intervening

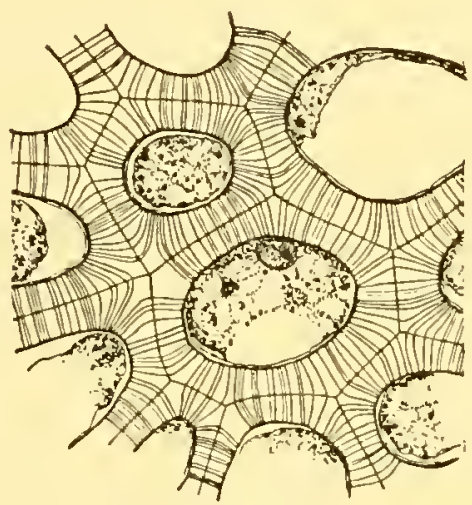

FIG. 11.-Endosperm of Diospyros, showing plasmodesms traversing the thickened cell walls. (After Quisumbing, 1925.)

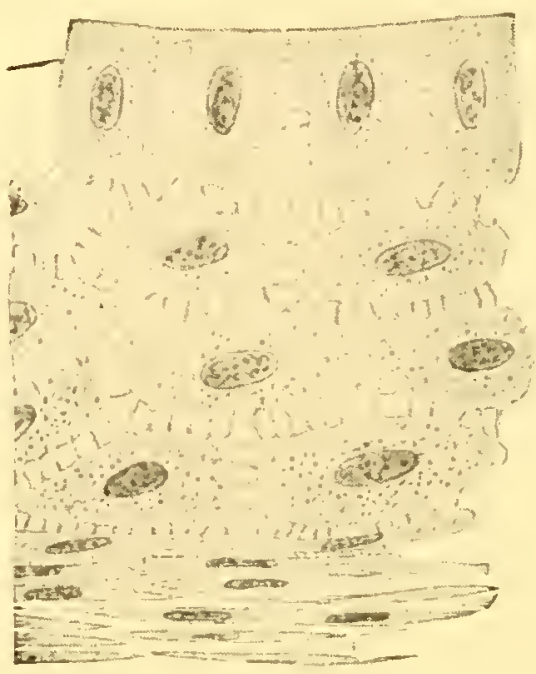

Fig. 12.-Protoplasmic continuity in human mesoderm tissue. (After Maurer.)

cell membranes. The very fact that a given area of cell wall becomes enormously extended during the growth of the tissues indicates that many of the pores seen at maturity must have been formed anew. It is also to be remembered that at the time when pore formation would necessarily occur the cell membranes are very thin and semifluid and would offer little resistance to dissolution or penetration by the protoplasts. Hence the fact that cells may glide or roll over one another during the early stages of development does not prove the impossibility of protoplasmic continuity between them. The exceeding fineness of many known plasmodesms, moreover, indicates that failure to find such connections in certain tissues does not necessarily prove their absence. On the other hand, proof that plasmodesms are continuous through the entire wall is not so complete as desired. Jungers (1930) has recently called into question the evidence for the view that plasmodesms in 
endosperm, sieve tubes, and thickened parenchyma are actually protoplasmic in nature. Their resistance to potassium hypochlorite and their arrangement in growing walls suggest to him that they are rather structural constituents of the wall itself.

Protoplasmic connections have been rather widely described in animals, and are best known in epithelial, muscle, and connective tissues (Fig. 12). In connective tissue they may be broad cytoplasmic extensions, giving the tissue the character of a protoplasmic network (Fig. 10), or they may be very fine threads. As in plants, they are the result either of an incomplete division or of a fusion of cell outgrowths. The fact that cells in animals are mostly divided by a process of furrowing indicates that connections, if present, must be for the most part secondarily developed. In his study of living tissues Chambers (1924) finds in the majority of cases no direct evidence of protoplasmic bridges and is inclined to agree with certain other workers in interpreting many reported connections as either fixation artifacts or fibrous differentiations in the intercellular substance, rather than actual protoplasmic strands. Such strands are clearly present, however, in squamous epithelium (Chambers and Rényi, 1925). Some investigators, as will be pointed out in a subsequent chapter, regard the intercellular substance itself as living matter of a special kind. The establishment of protoplasmic connections between blastomeres has been described. ${ }^{14}$

The chief significance of protoplasmic connections of all types probably lies in their coördinating function. That plasmodesms serve to transmit stimuli from one cell to another is indicated by their presence in tissues of plant parts known to be particularly responsive to external stimuli. $^{15}$ The effects of mechanical injury appear to be transmitted through epithelial tissue by way of such intercellular bridges according to Chambers and Rényi (1925). Their extensive development in storage tissues, such as the endosperm of seeds (Tangl, 1879; Gardiner, 1897), also suggests that they are in part responsible for the readiness with which nutritive materials are translocated in such specialized tissues.

The protoplasm of the entire individual is more or less continuous from the beginning of the ontogeny onward. It should not be thought, however, that without such connections there can be no correlation. Mere contact is sufficient for the passage of electrical stimuli, which, as will be indicated below, are recognized as important factors in the development and operation of the body. Cells separated by delicate colloidal membranes with no actual protoplasmic continuity are still able to interact and influence each other's behavior so that the entire

${ }^{14}$ G. F. Andrews (1897), E. A. Andrews (1898), Shearer (1906), Whong (1931).

${ }^{15}$ The leaves of Mimosa (Gardiner, 1884) and Dioncea (Gardiner, 1884; Macfarlane, 1892); the stamens of Berberis (Gardiner, 1884); the sensitive labellum of the orchid Masdevallia muscosa (Oliver, 1888). 
group tends to act as a unit. Thus without protoplasmic continuity the cells may still have a physiological continuity; it is the possession of both that conditions the coördinated action of most tissues. In any case the field of force which pervades the whole organism and which shapes its development is not interrupted by cell partitions (Thompson, 1917).

The differentiation of specialized tracts with a correlating function is exemplified not only in the nervous system of higher animals but also in the neuromotor apparatus of certain Protozoa. It has been found that after the delicate strands leading to the several groups of locomotor organelles in Euplotes are cut, the action of the groups is no longer properly coördinated (C. V. Taylor, 1920, 1929). It has been contended that the vascular bundle regions of higher plants serve to conduct correlating stimuli, but the evidence for this is as yet inconclusive.

In the vertebrates correlation is maintained in part through the agency of hormones. These are chemical substances which are elaborated in the endocrine glands and carried by the blood or lymph to the various parts of the body, where they exercise profound effects upon growth and other activities of the organs and tissues. The discovery of these endocrine secretions and the application of knowledge concerning them to the treatment of certain human disorders constitute one of the most significant developments in modern medical biology. ${ }^{16}$ The question of the possible rôle of hormones in plants is still a disputed one, although Went (1931) and others have adduced evidence indicating the presence of one, called "auxin," in growing points, while Haberlandt (1921 et seq.) attributes the healing of wounds to a hormone.

One of the most important contributions to our understanding of correlation is the conception of physiological gradients developed by Child (1911 et seq.) and his associates. It has been shown in a number of animals and plants that along each of the axes of symmetry there exists a physiological gradient (also called "metabolic gradient" and "axial gradient"), the rate of the physiological processes being highest at one end of the axis and diminishing progressively toward the other end. The anterior end of a planarian, for example, exceeds the posterior end in its rate of oxygen consumption and carbon dioxide output and in its susceptibility to poisons. Furthermore, the portions having a higher rate exercise a "dominating" influence over the development of those portions having a lower rate, with the result that the individual maintains a definite physiological correlation of anterior and posterior parts. Similarly in individuals with more than one axis of symmetry there may be a corresponding dorsal-ventral, as well as an axial-marginal, correlation. The gradient arises in the first place, according to Child, as a response to differential factors in the environment; and, although

${ }^{16}$ For a convenient account of hormones and their effects, see Guyer (1931). 
the types of organs developed depend upon the hereditary constitution of the organism, their arrangement and mutual behavior are due in large measure to the gradient. This is indicated by the fact that experimental alterations in the metabolic rate along the axis are followed by the expected abnormalities in structural development.

As to the means by which different regions along an axis influence one another, Child (1915, p. 224) adduces evidence in support of the theory that the fundamental relations of polarity "depend primarily upon impulses or changes of some sort transmitted from the dominant region, rather than upon the transportation of chemical substances." Very significant in this connection is the fact that there is a definite relation between physiological gradients and electrical polarity. The dominant and less active regions are respectively the negative and positive poles of the living system. Where the current enters the system from the exterior (negative pole), anabolic processes are promoted through increased oxidation; and where it leaves (positive pole), catabolic proccsses are furthered. ${ }^{17}$ Alterations in electrical polarity are accompanied by alterations in the mode of growth. ${ }^{18}$ Thus, in the opinion of Lillie (1923), "bioelectric currents exert a controlling and coördinating influence in normal growth processes as well as in normal stimulation."

The Cell Theory and the Organismal Theory.-Although not of the first importance to the student beginning work in cytology, the following discussion is added because of its theoretical interest.

The fact that the body of a higher organism comprises a vast number of specialized parts, the cells, led many years ago to the formulation of two general theories which differed in their interpretation of the relation existing between the two individualities: the organism as a whole and the cell. These theories are known as the cell theory and the organismal theory.

The cell theory set forth a century ago by Dutrochet, Schleiden, and Schwann exerted a dominating influence upon biology throughout the nineteenth century (see Chapter XXVI). The principal propositions involved in the theory are summarized by Heidenhain (1907, p. 29) essentially as follows: All living substance is concentrated in cells; the cells of the body are all individuals of the same morphological rank; the tissue cell is morphologically and physiologically an elementary individual, the unit of structure and function; the body is an aggregate of cells, which are its "building stones"; the action of the body is the sum of the many special actions performed by collaborating cells of many kinds. According to this theory, therefore, the cell is the fundamentally important individual-the "primary agent of organization." In ontogeny the multiplying elementary organisms, or cells, coöperate to

${ }^{17}$ Hering (1888), Mathews (1903), R. S. Lillie (1919, 1922, 1923).

${ }_{18}$ Bose (1918), Ingvar (1920), Lund (1921). See also Beutner (1933). 
build up an individual of a higher order, the multicellular organism. Such an organism is thus a "cell state," or "cell republic," secondarily formed by the aggregation of a vast number of elementary individuals.

The phylogenetic aspeet of the eell theory developed when it was diseovered that many minute organisms are single uninueleated masses of protoplasm much like the constituent eells of the multicellular forms. It was coneluded that sueh "unicellular elementary organisms" have in the course of time formed loose colonies, either by direet aggregation or by an aequired failure to separate after a period of multiplieation; and that the individual units have become inereasingly interdependent and knit together until individuals of a higher grade, multicellular organisms, have resulted. As a consequence of this interpretation, the individual protozoön has been homologized with a single cell of the human body. The dominanee of the cell theory, moreover, has resulted in the tendeney to deseribe everything of a biologieal nature so far as possible in terms of cells.

It has always been very difficult to make any plausible interpretation of plasmodial or coenocytic organisms in terms of the cell theory. Such organisms are multinueleate and non-septate masses of protoplasm, which nevertheless build up bodies of definite form and with a considerable degree of differentiation. The nuelei are centers of action whose reactions influence the eytoplasm to different distanees, especially in those forms in which their position is being constantly changed by protoplasmie streaming. Some have taken the standpoint that in sueh cœnoeytic bodies each nucleus with the portion of the cytoplasm it influences "represents" a cell, while others hold that the entire multinueleate body is one huge eell. Both of these interpretations contain elements of truth, but they also appear like attempts to save a theory based on a limited group of observations.

The early dissatisfaetion with the coneeption of the cell as the primaly and universal agent of organization led to the formulation of the organismal theory, in which the emphasis was plaeed on the living mass as a whole rather than on the eonstituent cells. ${ }^{19}$ According to this general interpretation, ontogenesis is a function primarily of the organism as a whole and consists in the growth and progressive internal differentiation of a single protoplasmic indiridual, this differentiation often, but not always, involving the septation of the living mass into subordinate semiindependent parts, the cells. Since the septation is rarely complete, all parts remain in eonnection and the whole continues to act as a unit. Hence development is not primarily the establishment of an association of multiplying elementary units to form a new whole but rather the

${ }^{19}$ For a list of biologists who developed and supported this theory, see p. 436. See also Whitman (1893), Sedgwick (1894), Dobell (1911a), Ritter (1919), Ritter and Bailey (1928), and Russell (1930). 
resolution of one persistent whole into newly formed metabolic units: it should be thought of not as primarily a multiplication and coöperation of cells but rather as the differentiation of growing protoplasm.

The real unity is that of the entire organism and, as long as its cells remain in continuity, they are to be regarded not as morphological individuals but as specialized centers of action into which the living body resolves itself and by means of which the physiological division of labor is effected (Wilson, 1893).

The phylogenetic implication of the organismal theory is that if multicellular organisms have arisen from unicellular forms, the process may have been the same as that seen in ontogeny: the differentiation and subdivision of a continuous growing mass of nucleated protoplasm into a system of uninucleate cells. If the septation coincided with nuclear division from the first, there was a direct transition to forms with several cells. If the two processes did not so coincide, cœnocytic or "plasmodial" types arose, some of which persisted as our cœnocytic organisms while others developed internal walls and became our multicellular forms. The ability of isolated tissue cells to live independently does not prove that independent cells have combined to form the multicellular body but only that such parts can still carry on essential protoplasmic functions. The phenomenon could as well be taken to prove the derived nature of Protista. The body is not an aggregation of elementary organisms, but a single organism which has evolved a multicellular structure. The cell colonies in certain green algæ ${ }^{20}$ and the remarkable polyp colonies in the Siphonophora (see J. S. Huxley, 1912) indicate the dependence of the part upon the whole even in a group formed by aggregation, and they show the possibility of an evolution by the combination of individuals; but it is not at all clear that they afford the key to the evolution of organisms in general. The protozoön is more properly compared with the whole man; both are organisms which have differentiated a series of specialized internal regions or organs, the one without cellular subdivision or increase in size and the other with them.

Some of the cytological facts underlying the organismal theory may be briefly cited as follows.

Many algæe and other organisms, as already noted, develop bodies of considerable size and with definitely differentiated form and structure without any cellular subdivision of the multinucleate protoplasm. In some of these (e.g., Bryopsis; Noll, 1903) the tips of the plant grow steadily forward as wholes, in spite of the fact that the cytoplasm and the nuclei it carries are constantly being changed by protoplasmic streaming. Interpreting the entire plant as a huge cell accomplishing the development is here equivalent to admission that it is the whole which dominates the "centers of activity" about the nuclei.

${ }^{20}$ Bock (1926) describes plasmodesms in Pandorina, Eudorina, and Gonium. 
The same general result may be achieved with or without cell-formation, as in the bodies of Vaucheria, Cladophora, and Stigeoclonium, which show essentially the same degree of elaboration although the first is cœnocytic, the second semicœnocytic, and the third strictly cellular. Eggs of Chatopterus have been observed to differentiate into swimming larvæ even when the usual cytoplasmic cleavages are suppressed by adding some $\mathrm{KCl}$ to the sea water (F. R. Lillie, 1902, 1906). It has been shown in a number of cases that alterations in the position of successive cleavage furrows do not disturb the normal course of development and differentiation. In the frog, for example, "normal development does not depend upon a specific number and succession of cleavages in definite positions but rather upon an egg pattern which may be cut up by the cleavage furrows in various ways without destroying the pattern or the normal results of development" (Conklin, 1924). Spemann (1918 et seq.) has shown that in very young amphibian embryos groups of cells can be cut out and reversed in position or transplanted into other regions, whereupon they develop as parts of the organs normally differentiating in those regions and not as those of the regions from which they came. Thus, as F. R. Lillie (1902) concluded for Chatopterus, "the process of cell-division, as such, is necessary neither to growth, differentiation, nor the earliest correlations; but it is accessory, in Metazoa, to all three as a localizing factor, often from the earliest stages."

It was to the same conclusion that W. Hofmeister, Sachs, de Bary, and other botanists were led many years ago through detailed studies of cellformation in the growing regions of plants. They found the growth of the organ as a whole to be the primary matter, the position of the cell walls within it being secondarily determined by the physical forces acting within the growing mass. "The formation of new cells in the vegetative point is accordingly a function of the general growth, not its cause" (Hofmeister, 1867). This view has been upheld by many researches on the mechanics of growth and form (see Thompson, 1917) and by the recent studies of Sinnott (1930) on the relation of the dimensions of cells to the size and form of the organ they constitute.

Many organisms which are cellular throughout the greater portion of the life cycle pass through a cœnocytic phase, often at a critical stage in the cycle, and in this phase as elsewhere growth and differentiation continue. In the young embryos of Agathis (Fig. 13) and other gymnosperms the characteristic mode of development is indicated by the positions taken up by the nuclei during the cœnocytic stage, the position of the subsequently formed cell partitions being determined by differentiations occurring in this stage. Similarly, there are among animals cases in which the embryo first passes through a free-nucleate stage, subdivision into cells occurring after differentiation, particularly that of the germ region, is well on its way. That differentiation in such cases is a function of the protoplasmic mass as a whole is indicated by the fact that any 
nuclei which happen to enter the germinal region become germinal nuclei, the rest becoming somatic nuclei (Huettner, 1923; on Drosophila). In many such eggs the cleavage is at first only superficial, the walls forming without special reference to the nuclei or their division, and the resulting compartments remaining for a time open into the common underlying mass of protoplasm. Many of the special tissues of the animal body may begin their development as plasmodial masses in which cellular differentiation occurs later. In some tissues the non-cellular condition may remain until maturity, as in certain "syncytial" types of connective tissue. The internal structures characterizing many tissue cells, such as neurofibrils and elastic fibrils, may arise before the subdivision into cells (see Rohde, $1908,1923)$.

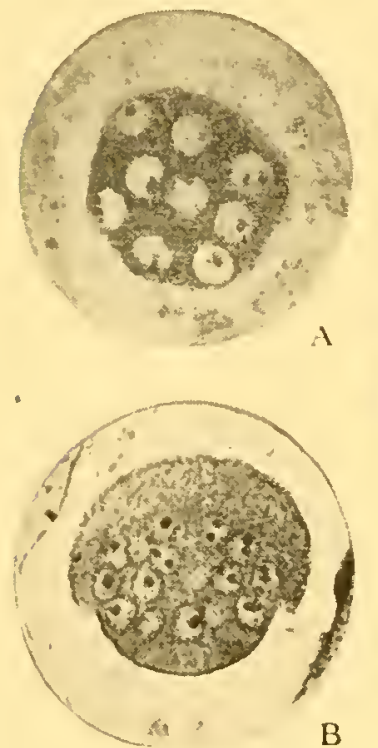

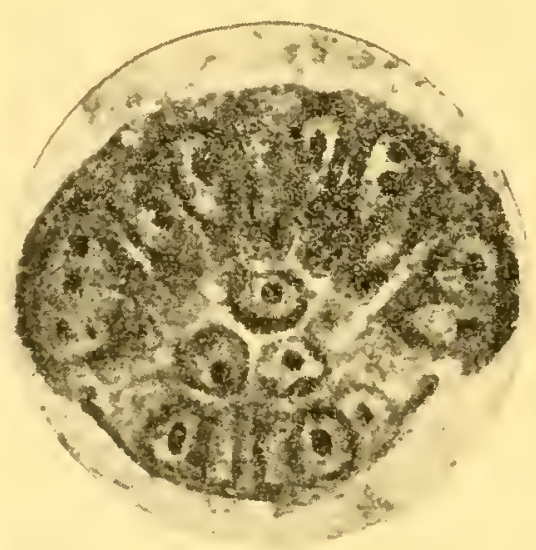

C

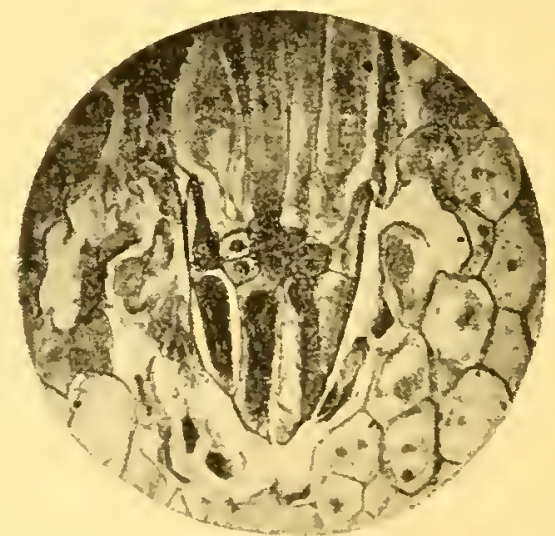

$\mathrm{D}$

FIg. 13.-Early stages in the embryogeny of Agathis australis. A, conocytic stage. $B, 32$-nucleate stage with cytoplasmic suspensor cap differentiated. $C$, proembryo subdivided into cells. Three regions distinguishable: suspensor initials above, embryo proper in middle, and embryonic cap below. $D$, later stage; cap well developed and suspensor elongating. (After Eames, 1913.)

All of this is not to be interpreted as a denial of the importance of cells in multicellar organisms but only as a recognition of the fact that the principle of functional protoplasmic differentiation is more general and fundamental than that of cells as units (Heidenhain, 1907). Although we thus look upon cellular organization as initially a result of differentiation, it is true that it has, in turn, conditioned differentiation of a higher degree. The presence of cell partitions allows a more effective segregation of functionally specialized regions and a fuller play to those important physico-chemical processes which depend on surfaces and thin films for their action. Furthermore, it permits the development of larger plant bodies by furnishing an ideal basis for the more effective operation of turgor and for the deposition of supporting materials. The evolution of higher organisms has unquestionably been very largely conditioned by the multicellular state, but we should think of such organisms primarily as highly differentiated protoplasmic individuals rather than cell republics. 


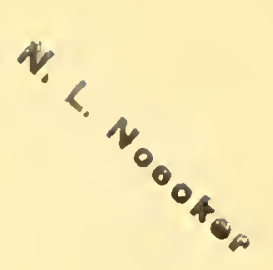

\section{CHAPTER II}

\section{PROTOPLASM}

In spite of the enormous amount of work which has been done upon protoplasm during a period of many years, our knowledge of its constitution and behavior must still be regarded as very superficial. Some have inclined to the view that a given kind of protoplasm is a single complex chemical compound, but at present it seems more probable that it represents a somewhat looser combination of substances, many of which are themselves very elaborate in composition. It furthermore seems probable that these substances differ from those which may exist apart from protoplasm, not so much in their fundamental chemical nature as in the degree of their complexity, their energy content or reactivity (Mathews, 1924), and especially in their mutual organization. Protoplasm is made up of proteins, fats, salts, water, carbohydrates, and other compounds, but it is not a mere mixture of these materials: it is an intricately organized system of substances of many types, and only by rirtue of this specific physico-chemical organization does it serve as the material substratum for those peculiar orderly activities characterizing the organism, namely, synthetic metabolism, irritability, reproduction, and adaptive response. Living protoplasm should always be thought of as a system in dynamic equilibrium; it is continuously maintaining itself through a balance of constructive and destructive processes. The "ability to transform environmental material into its own specifically organized and active substance is the distinctive criterion of living as distinguished from non-living matter" (R. S. Lillie, 1923).

The Chemical Nature of Protoplasm. '- The operation of any system, living or lifeless, depends upon the materials of which it is made up (its chemical composition), the arrangement of these materials (its physical organization), and the set of surrounding conditions under which it acts (its environment).

The chemical composition of protoplasm has been determined approximately in a number of instances. For this purpose the plasmodia

${ }^{1}$ See on this subject A. Zimmermann (1896), Hammarsten (1909), Zacharias (1910), Czapek (1913, 1920), Wells (1914), Bayliss (1915), Mathews (1916, 1924), Palladin (1923), Meyer (1920), T. B. Robertson (1920), B. Moore (1921), R. W. Thatcher (1921), Walter (1921), Pratje (1920), Tischler (1921-1922, Chap. II), Lundegårdh (1922, Pt. I, Chap. XI, B), Grafe (1922, Chap. IV), Onslow (1923), Trier (1924), Heilbrunn (1928), Gortner (1929), and Kiesel (1930). 
of myxomycetes ${ }^{2}$ and the spermatozoa of fish have been most frequently employed, the former because of the large mass of protoplasm readily available, and the latter because they yield large amounts of nuclear material in a fairly pure state. By the use of stains Unna and others have been able to determine the position in the cell occupied by certain organic constituents. Microchemical methods have been employed to some extent in the study of the inorganic constituents, but they are in need of further development. The results of Kiesel's analysis of the myxomycete Reticularia lycoperdon are as follows:

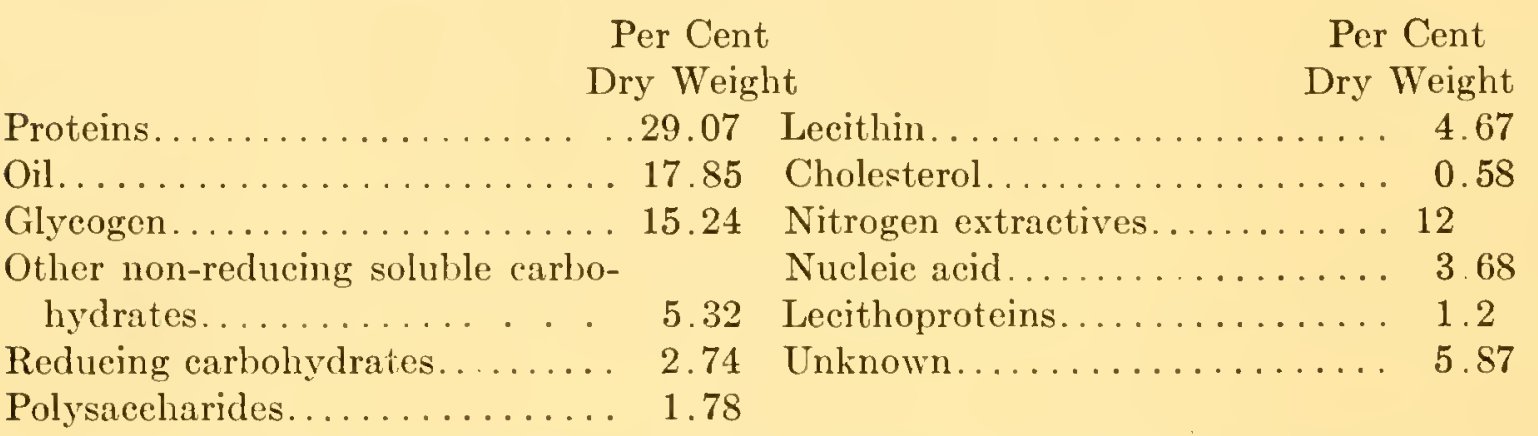

The protein matter of protoplasm exists in relatively complex forms. "The chief mass of the protein substances of the cells does not consist of proteids in the ordinary sense, but consists of more complex phosphorized bodies. ..." (Hammarsten). Such "phosphorized bodies" are the nucleo-proteins, which are "probably the most important constituents of the cell, both in quantity and in relation to cell activity" (Wells). A long series of chemical investigations ${ }^{3}$ has shown that these nucleo-proteins are essentially combinations of nucleic acid with proteins.

The nucleus, as a rule, contains little or no uncombined carbohydrate, fat, or salt, but is characterized rather by the abundance of a substance called "nuclein," isolated in 1871 by Miescher, who gave it the formula $\mathrm{C}_{22} \mathrm{H}_{49} \mathrm{~N}_{9} \mathrm{P}_{3} \mathrm{O}_{22}$. It was shown by Altmann (1889) that nuclein, like other nucleo-proteins, is a combination of two substances: nucleic acid and a simpler form of protein, the two existing in chemical combination much like an ordinary salt. Nucleic acid, which is, in turn, a combination of phosphoric acid with certain carbohydrates and bases, seems to be very much the same in all types of protoplasm. It is well known in two general forms, differing chiefly in the carbohydrate constituent of the molecule; in one form this is a pentose and in the other a hexose. The former is known to occur in yeast, wheat, and certain animals, while the latter has been found in thymus and lymph glands, blood corpuscles, and spermatozoa. The strong affinity of nucleic acid for organic bases is responsible for the staining reactions of the chromatic nuclear substance: a fixed and stained chromosome is a salt of nucleic acid with a

${ }^{2}$ Reinke and Rodewald (1881) and Lepesehkin (1923) on Fuligo varians (Aithalium septicum); Kiesel $(1925,1927)$ on Reticularia and Lycogala.

${ }^{3}$ By Miescher, Hoppe-Seyler, Reinke, Kossel, Lilienfeld, and later workers. 
basic dye. The basic constituent of this substance in a living nucleus may be a very complex protein, a simpler and more basic histon (blood, thymus, echinoderm sperm), or a still simpler and more strongly basic protamine (fish sperm). The following scheme will help to make clear the relationships of these substances:

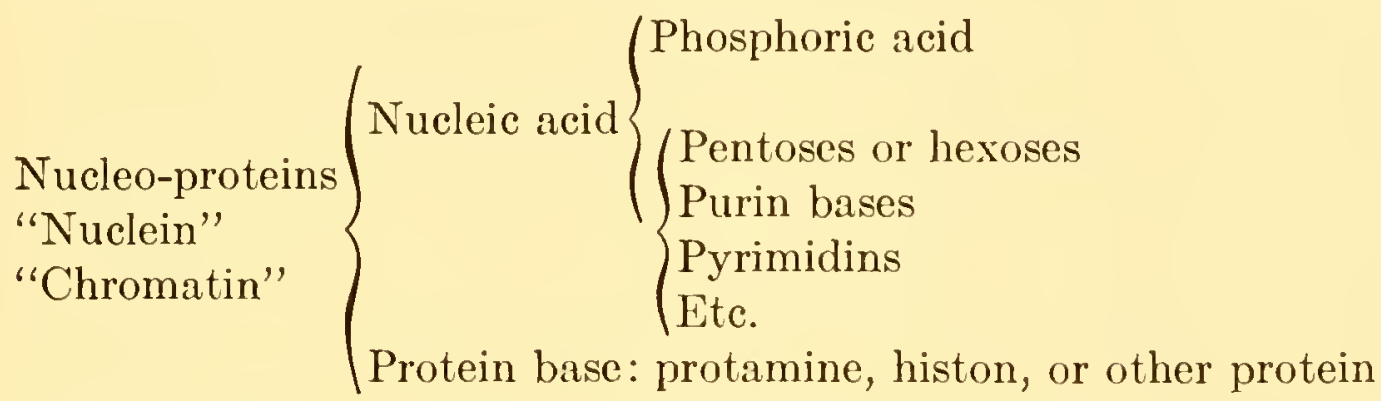

In the cytoplasm, in contrast to the nucleus, the proportion of protein constituents is relatively high. The cytoplasm normally has no "nuclein," but it is rich in nucleo-albumins, albumins, globulins, and peptones; these, unlike nuclein, contain little or no phosphorus. According to Hammarsten, "the globulins and albumins are to be considered as nutritive materials for the cell or as destructive products in the chemical transformation of the protoplasm." Volutin, a compound which resists strong acids and shows an affinity for basic dyes, ${ }^{4}$ is frequently present in the form of globules in the cytoplasm, notably in lower organisms. It is usually regarded as a reserve substance. The importance of proteins in protoplasmic activity is partly due to their amphoteric properties; they may act either as acids or as bases under proper conditions. In general, most of them act during life as acids.

The fatty constituents of protoplasm comprise the true fats and a number of more complex derivatives containing nitrogen and phosphorus, such as the phospholipides (phosphatides). Among the fatty substances which appear to have a peculiar importance in the organism are cholesterol, ergosterol, and lecithin. Under the influence of certain light rays ergosterol may be transformed into vitamin D. ${ }^{5}$ The staining reactions of fixed cytoplasm apparently depend not only upon its chemical composition, but also upon the degree of physical dispersion induced by the reagents used (see Yamaha, 1932).

The carbohydrates found in protoplasm are chiefly pentoses (plants) and hexoses (animals), which are, as a rule, combined with proteins and with lipides. Glycogen exists free in many tissues and serves as a source of energy. The important rôle played by pentoses and pentosans in the activity of the plant cell has been strongly emphasized; in fact, protoplasm has been called "an intermeshed pentosan-protein colloid." 6

${ }^{4}$ Reichenow (1909), van Herwerden (1917), Doflein (1918).

${ }^{5}$ See Gortner (1929, Chaps. XXXI-XXXII, XXXV).

${ }^{6}$ Spoehr (1917, 1919), MacDougal (1920). 
Inorganic salts are present in considerable variety, as shown by the presence of the following elements in the ash of Fuligo protoplasm: chlorine, sulphur, phosphorus, potassium, magnesium, sodium, calcium, and iron.

Enzymes, or organic catalysts, are of great importance in protoplasm because of their power of initiating and altering the rate of reactions. Their chemical nature is a matter of considerable dispute. Urease, isolated from jack-bean meal, is a globulin, ${ }^{7}$ and there is evidence that a number of other enzymes also are proteins. The high specificity characterizing the action of enzymes indicates the importance of their chemical composition, and it is also evident that such action depends largely upon the peculiar energy relations existing at the surfaces they occupy or present. ${ }^{8}$

The amount of water in protoplasm varies greatly under different conditions, but usually it is present in large proportions. It makes up from 85 to 95 per cent of the weight of actively streaming protoplasm such as is seen in Tradescantia, and in actively functioning cells it rarely drops below 70 per cent. In dry spores, however, it may be reduced to 10 or 15 per cent, the protoplasm then becoming very viscous. The body of a jellyfish may consist of more than 99 per cent by weight of water. The approximate composition of the human body is as follows: water, 65 per cent; protein, 15 per cent; fat, 14 per cent; salts, 5 per cent; other organic compounds, 1 per cent. A considerable portion of the water in the cell or body is physically "bound" in the colloidal structure of the protoplasm and must be considered an integral part of the living system.

No single substance is of greater significance in the life of the organism than water. In the words of Henderson (1913):

... the physiologist has found that water is invariably the principal constituent of living organisms. Water is ingested in greater amounts than all other substances combined, and it is no less the ehief excretion. It is the vehicle of the principal foods and exeretion products, for most of these are dissolved as they enter or leave the body [across the wall of the intestine and across the epithelia of kidneys, lungs, and sweat glands]. Indeed, as clearer ideas of the physico-ehemical organization of protoplasm have developed, it has become evident that the organism itself is essentially an aqueous solution in which are spread out colloidal substanees of great complexity [Bechhold, 1912]. As a result of these conditions there is hardly a physiological process in which water is not of fundamental importance.

It is not only by virtue of its great solvent power that water is so effective in promoting chemical interaction. Its exceptional ionizing power is especially noteworthy in this connection, while its high surface tension has a notable influence on reactions involving adsorption. There

${ }^{7}$ Sumner (1926), Sumner and Hand (1928).

${ }^{8}$ See Gortner (1929; Chap. XXXV). 
is also evidence that water is necessary to that most fundamental of vital processes, oxidation. The importance of these and other characteristics of water is discussed at length by Henderson in his inquiry into the biological significance of the properties of water.

Another highly important chemical feature of protoplasm is its hydrogen-ion concentration. Through the use of color indicators and the microinjection method Chambers and his associates ${ }^{9}$ have determined the $p H$ of a number of animal cells with considerable accuracy. They find the nucleus to be slightly alkaline $(p H$ about 7.5 to 7.6$)$ and the cytoplasm slightly acid ( $p \mathrm{H}$ about 6.7 to 6.9$).{ }^{10}$ Injury causes the cytoplasm to become much more acid ( $p \mathrm{H} 5.2$ to 5.5). If the $p \mathrm{H}$ remains altered, the cell dies. The experiments show the protoplasm to be well "buffered," or protected against sudden or radical alterations in $p \mathrm{H}$, by certain substances in solution. The principal buffers in organisms are carbonates, bicarbonates, and phosphates. It is a very significant fact that the vital reactions occur in a medium near the point of neutrality, and that protoplasm has a means of preserving its $p \mathrm{H}$ at the proper level under a considerable range of environmental conditions.

The $p H$ values for plant protoplasm are less well known, since the analyses have included considerable amounts of cell sap, which in mature cells is usually decidedly acid in reaction. As a result, the published values $^{11}$ in nearly all cases fall well on the acid side of neutrality. Moreover, in determinations made on expressed saps an increase of acidity due to injury is suspected.

Active acidity, due to the concentration of free hydrogen ions, is to be distinguished from potential acidity, which is determined by the quantity of hydrogen ions which can be made to combine with a base. The nucleus, although slightly alkaline to indicators, has a large amount of undissociated nucleic acid which reacts strongly with basic dyes in the usual staining procedures; hence the common statement that the nucleus reacts as an acid. In general the cytoplasm has a high potential alkalinity and a slight active acidity.

Protoplasm, because of the many combinations possible among its numerous constituents, is a substance which may exist in a vast number of different forms. When it is further recalled that many of the constituents

${ }^{9}$ Chambers (1928, 1930d, 1932), Chambers and Pollack (1927), Chambers, Pollack and Hiller (1927), Chambers, Pollack and Cohen (1929), Reznikoff and Pollack (1928), Pollack (1928), Chambers and Cameron (1932), Chambers and Kerr (1932).

${ }^{10}$ The reactions of solutions ranging from 0.1 normal in terms of hydrogen ions to $10^{-14}$ normal are expressed in 14 steps from $p \mathrm{H} 1$ to $p \mathrm{H} 14$. The neutral point is at $p \mathbf{H} 7$; a solution with a lower $p H$ is acid, while one with a higher $p \mathbf{H}$ is alkaline. The scale is a logarithmic one, a decrease of $1 \mathrm{in} \mathrm{pH}$ corresponding to a tenfold increase in hydrogen-ion concentration. See Gortner (1929, p. 92).

${ }^{11}$ Eichhorn (1927) and Small (1929) review the data for plant tissues. See the papers of Pfeiffer $(1930,1931,1932)$ on the isoelectric point. 
exhibit singly the phenomenon of isomerism, this number is seen to be incalculable. For example, it was shown by Miescher that an albumin molecule with 40 carbon atoms could have about 1,000,000,000 isomers, and some albumins probably have more than 700 carbon atoms. Albumin, moreover, is only one of many complex substances present in protoplasm. Hence, the statement that all living cells are composed of the same substance, protoplasm, is true only in a general sense. Although they are made up of the same categories of substances existing in the same general type of organization, the protoplasms of different organisms vary widely in the relative amounts of these leading constituents. For example, the fats and lipides are relatively more abundant in the protoplasm of animals than in that of plants. The carbohydrate-protein ratio also shows notable differences in the two kingdoms, carbohydrates being relatively more abundant in plant protoplasm. ${ }^{12}$ Qualitative differences are no less significant. The carbohydrates, which in plants are chiefly pentoses and in animals chiefly hexoses, may be cited. Analogous differences also exist between the smaller plant and animal groups, and with these differences in chemical constitution are associated many characteristic diversities in metabolic activity. Thus it is not simply with protoplasm, but with protoplasms, that the working biologist has to deal.

Special emphasis has been placed by Kossel, E. T. Reichert, and a number of other writers upon the relation of this great diversity in the constitution of protoplasms to the amazing variety observed among living organisms. Reichert and Brown (1909) have shown that each animal species examined has its own characteristic type of hæmoglobin, and that relationships may be indicated by degrees of similarity in the crystals of this substance. The same situation is found in the case of starch in plants (Reichert, 1913, 1919). That the specificity of organisms is largely a matter of protein diversity is strongly indicated by the phenomena of immunology. ${ }^{13}$ At the same time the importance of structural diversity must not be forgotten.

In concluding this section, the general significance of the results of analyses of protoplasm by chemical methods should be considered. It cannot be maintained that anything like an adequate chemical picture of living protoplasm has been obtained. As A. Meyer (1920) and others have pointed out, such analyses include ergastic reserves and by-products, which differ in amount and kind at different stages of the metabolic cycle. Furthermore, in such a complex and delicately balanced system as protoplasm the analytical methods employed can scarcely fail to induce

${ }^{12}$ E.g., compare the analyses of Sabellaria eggs (Fauré-Fremiet, 1921) and Zea pollen (Andersen and Kulp, 1922, 1923).

${ }^{13}$ See Reichert (1914) and R. S. Lillie (1923; Chap. II I and literature there cited), also the discussion by G. Reed (1923). 
reactions which result in the formation of compounds not present during life. In spite of their shortcomings, however, chemical analyses do yield valuable information concerning the types of substance present in protoplasm and the relative proportions in which they may occur. Such information, inadequate as it may be, is obviously prerequisite to any understanding of protoplasmic activity.

The chief point to be borne in mind is that chemical analyses do not reveal a most significant characteristic of protoplasm, namely, its peculiar organization. Just as in the case of any other operating system, such as a watch, the activities of protoplasm can be understood only if the structural relations of the materials composing it are known. It is now abundantly clear that the essential synthetic chemical reactions of protoplasm depend upon an essential type of structure present only during life (see R. S. Lillie, 1923, 1924).

The Physical Nature of Protoplasm. ${ }^{14}$ - Under the ordinary microscope living cytoplasm appears as a colorless, optically homogeneous fluid, called hyaloplasm, in which there are usually imbedded granules and globules of varying size, shape, and number. It is commonly observed to be in a state of active streaming, notably in highly vacuolate cells of plants. In the leaf cells of Elodea, for instance, the cytoplasm is in almost continuous rotation, while the stamen hairs of Tradescantia afford a beautiful example of a more complex type of circulation. Various theories, involving electricity, contractility, surface tension, imbibition, and other phenomena have been propounded to account for such protoplasmic movement. ${ }^{15}$ The specific gravity of protoplasm is very slightly above that of water.

Noteworthy among the general physical characteristics of living protoplasm are its elasticity and viscosity. These are properties which it is difficult to estimate quantitatively. Much has been learned about them through the use of microdissection apparatus, ${ }^{16}$ the centrifuge, ${ }^{17}$ and electromagnets acting on inserted iron or nickel particles. ${ }^{18}$ The viscosity varies greatly in different tissues, in different portions or organs of a cell, and at different stages of cell-division and differentiation. In general, it seems that animal protoplasm is on the average more viscous than that of plants. In nerve cells and non-dividing epithelial cells no Brownian movement is visible, even with dark-ficld illumination, but in plant cells, as well as in eggs and many tissue-culture cells of animals,

${ }^{14}$ See Chambers (1924), Lundegårdh (1922, Pt. I, Chap. XI; Pt. II, Chaps. I-III), Meyer (1921), Schaeffer (1920), Harper (1919), Wilson (1923, 1926), Heilbrunn (1927, 1928), Seifriz (1929a). Weber (1926a) and Spek (1926) give bibliographies.

${ }^{15}$ Meyer (1921), Beikirch (1925), S. Nichols (1925), Fitting (1927), Spek (1926), Unirath (1930).

${ }^{16}$ Chambers, Seifriz, C. V. Taylor.

${ }^{17}$ Lyon, Heilbrunn, Weber, W. Zimmermann.

${ }^{18}$ Heilbronn, Freundlich, Seifriz. 
it may readily be observed (see Chambers, 1924). Seifriz (1924a) states that the viscosity of protoplasm is on the average about like that of glycerine and seldom below that of machine oil. According to Heilbrunn (1926c), the viscosity of the granule-free cytoplasm of the Arbacia egg is 0.02 and that of the Cumingia egg 0.04 (water $=0.01$ ). With the granules included the values are 2 or 3 times as high as this. Myxomycete protoplasm is found to be 15 or 20 times as viscous as water (Heilbronn, 1922). The internal protoplasm of Paramocium is said to be 8,000 times as viscous as water (Fetter, 1926), although this may be due in part to fibrillar structures present. The effects of a variety of agencies on viscosity have been ascertained. ${ }^{19}$ The viscosity changes occurring during meiosis, syngamy, and cell-division will be referred to in a later chapter.

As already stated, cytoplasm nearly always contains visible globules and other particles. These represent vacuolar material, chondriosomes, and more or less transitory nutritive substances of various types. Some cells show no such elements or only chondriosomes, but this condition is rather exceptional. The smallest of the other visible granules, the "microsomes," are almost universally present, according to Chambers $(1917,1924)$. In the echinoderm egg there are, in addition, many larger "macrosomes" (called "alveolar spheres" by Wilson, 1899), which measure 3 or $4 \mu$ in diameter and seem to represent nutritive matter. Other bodies, such as fat and oil globules, are also present. All of these may be so abundant that the ground substance, or hyaloplasm, is almost invisible. Likewise, in plants especially, minute vacuoles may be so numerous that the protoplasm has an alveolar or foamy appearance. Fibrillar differentiations may further complicate the picture. It will be readily recognized that the optical appearance of a given sample of protoplasm depends very largely upon the kinds of inclusions present, their size and arrangement, and the degree to which they are crowded together.

A fact which is both striking and very significant is that cytoplasm may be deprived of all the above inclusions without losing the power of carrying on certain of its characteristic activities. Hyaline pseudopodia amputated from granular amobæ are irritable and move in a typical amoboid manner. Centrifuged sea-urchin eggs can be cut into two portions, one with all the risible granules (except oil globules) and the other with none, after which both portions may be inseminated and undergo cleavage (see Chambers, 1924). This means that the visible inclusions, although they should be regarded as a part of the living system to the degree in which they are active in protoplasmic reactions (see p. 46), are not an indispensable part of that system; and that "it is in the appar-

${ }^{19}$ Heilbrunn (1920c, 1924, 1925f, 1929ab), Heilbrunn and Young (1930), Weber (1922, 1923, 1927), Scarth (1924), and others. 
ently structureless hyaloplasm that the real problem of cytoplasmic organization lies" (Wilson, 1923).

With the aid of a Spierer objective, which has a portion of the rear surface of the front lens silvered, Seifriz (1931) has been able to see in the hyaloplasm of onion cells a structure not hitherto reported. In the optically empty ground substance ("cryptoplasm") there are dispersed innumerable droplets of a grayish substance ("phaneroplasm"). The cytoplasm thus has a finely mottled appearance. As the cytoplasm streams, the phaneroplasm takes the form of parallel rods or continuous strands 0.3 to $0.4 \mu$ thick and 0.2 to $0.3 \mu$ apart. It is the cryptoplasm which appears to be the actively streaming component.

The Colloidal State of Matter.-It is evident that many phenomena in protoplasm are in some way dependent upon its ultramicroscopic structure. It is here that cytology has received a notable contribution from the field of physical chemistry in the form of facts and hypotheses concerning the colloidal state of matter. ${ }^{20}$

Matter is said to be in the colloidal state when it is subdivided into ultramicroscopic particles. Matter in which the particles are large enough to be microscopically visible may show some of the properties of colloids, but such properties are exhibited most characteristically when the size of the particles lies between $0.1 \mu$ and $1 \mathrm{~m} \mu$. Such particles, or "micelles," are generally groups of molecules, although it seems that they may sometimes be single large molecules (e.g., in egg albumin). Although they cannot be seen with the ordinary microscope, which reaches the limit of its power with particles between $0.2 \mu$ and $0.1 \mu$ in diameter, their presence may be indicated by the Tyndall phenomenon or by the ultramicroscope, under which they appear as bright points against a dark field. Wolfgang Ostwald (1917) gives the following table of diameters:

\begin{tabular}{|c|c|}
\hline d blood corp & $7,500 \mathrm{~m} \mu(=7.5 \mu)$ \\
\hline Staphylococcus.. & 800 \\
\hline Particles in fine mastic suspension.. & $500-1,000$ \\
\hline Casein particles in milk. & $130-170$ \\
\hline Colloidal gold particles. & $2-15$ \\
\hline Molecule of soluble starch. . & 5 \\
\hline Molecule of hæmoglobin.... & 2.5 \\
\hline Molecule of grape sugar. . . . . . . . . . . & 0.7 \\
\hline Molecule of hydrogen ............. & 0.1 \\
\hline
\end{tabular}

The colloidal substance is, of course, enveloped in some other medium (gas, liquid, or solid), the two together constituting what may be termed a colloidal system. The subdivided substance is known as the "disperse phase," and the enveloping medium as the "dispersions medium."

${ }^{20}$ See Bancroft (1921), Bayliss (1915, 1923), Bechhold (1919), Czapek (1911b), Hatschek (1916), Lundegårdh (1922; Pt. I, Chap. XI), Meyer (1920; Chap. IV), T. B. Robertson (1920), Lepeschkin (1924), Svedberg (1928), Freundlich (1928), Gortner (1929), and Gray (1931). 
Each of the physically homogeneous constituents, or phases, may be chemically complex; an aqueous phase, for example, may contain salts and other compounds in solution. The different chemical substances, including the solvent, which make up a phase, are called components. Furthermore, more than two phases may be present; there may be "polyphase systems"; but it will be sufficient for our purpose to deal only with those having two phases.

Colloidal systems differ widely in general properties according to the liquid, solid, or gaseous nature of the phases. The following list of familiar substances will help to give a picture of colloidal structure, although some of them, because of the large size of their particles, are only large-scale models of such structure.

Liquid in liquid: mayonnaise Solid in liquid: muddy water Gas in liquid: foam Liquid in solid: pearl
Solid in solid: true ruby glass Gas in solid: bread Liquid in gas: fog Solid in gas: smoke

It is with the liquid colloidal systems that biolggy is chiefly concerned. Typically these are uncrystallizable, considerably more viscous than water, readily coagulable, only slightly or not at all osmotic, and poor as electrical conductors. In all of these features they differ markedly from true molecular solutions. It was formerly customary to classify them as "suspensoids," in which the suspended matter is solid, and "emulsoids," in which it is supposedly liquid. More adequate is the classification into lyophobic and lyophilic systems. A lyophobic system is one in which neither phase will dissolve in the other, whereas in a lyophilic system the disperse phase and the dispersions medium are more or less soluble one in the other (Martin Fischer). Gelatin in water is a lyophilic system; gelatin in alcohol is a lyophobic system. It is uncertain to what extent the dispersions medium actually dissolves in the micelles in the solvation of a lyophilic colloid (as when gelatin swells in water, a case of hydration) or only becomes closely "bound" in layers about them (Gortner, 1929, p. 212.)

Colloidal systems are referred to as sols if they flow readily and as gels if they do not. Gels include jellies (true gels) formed by lyophilic colloids, and coagula formed by precipitation in lyophobic ones. A sol may be made to become a gel (gelation) or a gel to become a sol (peptization) under certain conditions. In many cases such alterations are reversible. What structural change occurs during gelation is not well known, but there is evidence which indicates that an agglomeration or a partial coalescence of the suspended particles occurs, with the resulting formation of a reticulum or a spongework of threads. The consistency of a colloidal system obviously may vary according to the relative volumes of its phases, the closeness with which the dispersed particles are packed 
in the dispersions medium (Fig. 14), and the measure in which the dispersions medium is bound by the micelles.

The stability of a liquid colloidal system with a solid disperse phase depends largely upon the similar electric charge carried by the suspended particles. The addition of an electrolyte neutralizing the charges brings about a precipitation of the particles. In a liquid-in-

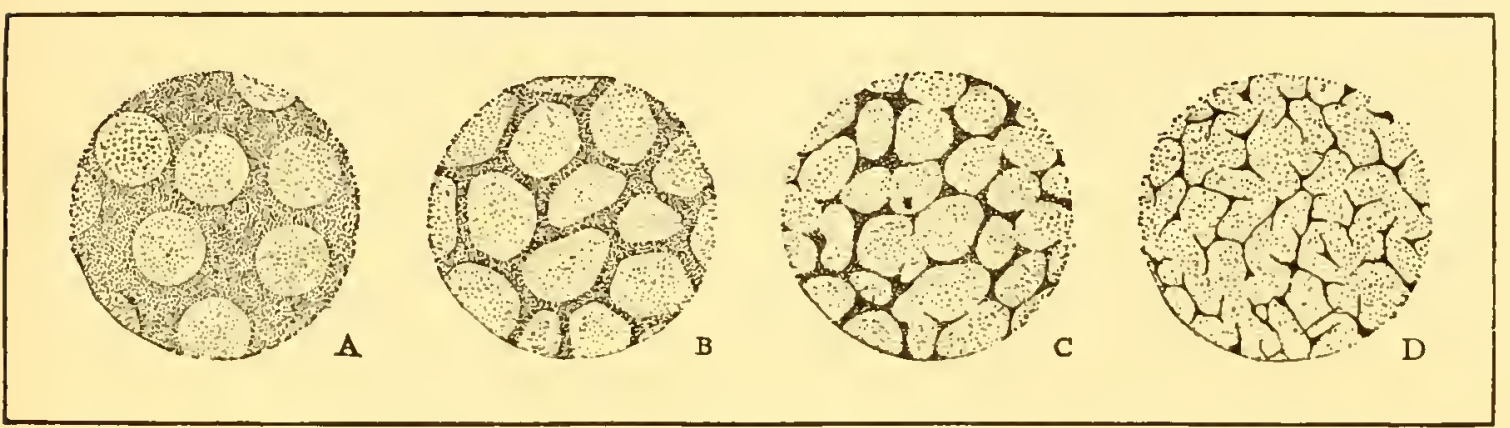

FIG. 14.-Diagram to show the various appearances observable in a two-phase colloidal emulsion. $A$, suspended droplets well separated, giving an alveolar appearance. $B$, droplets crowded, giving continuous phase a reticular appearance. $C, D$, aspects due to a proportionally smaller amount of the originally continuous phase and a coalescence of the droplets, both phases becoming continuous.

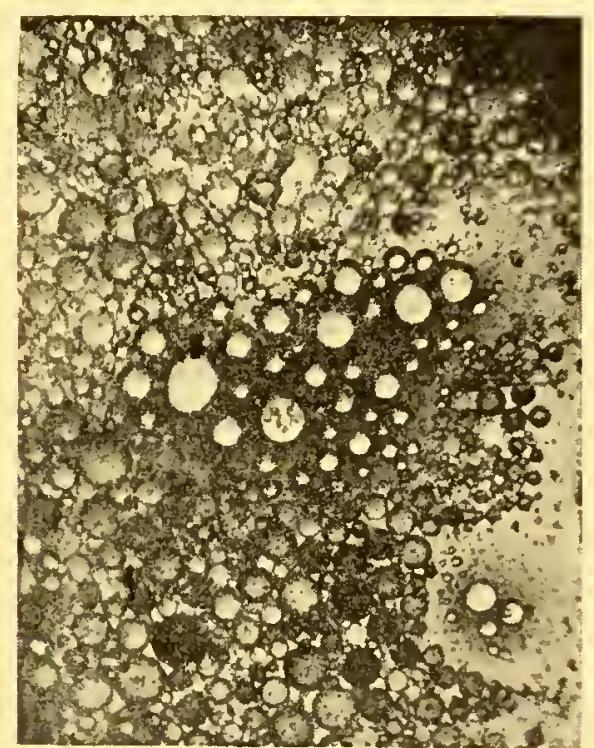

Fig. 15.-Photograph of an oil-water emulsion. (After Seifriz, 1930a.)

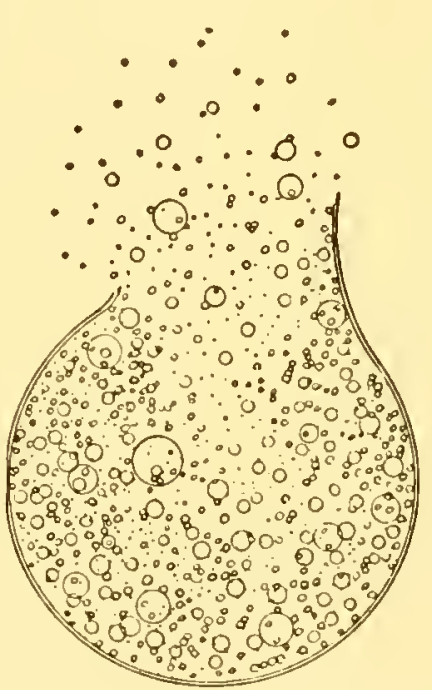

FIG. 16.-A torn Fucus egg. Compare with Fig. 15. (After Seifriz, 1930a.)

liquid system the matter is more complicated. A relatively coarse oil-water emulsion will serve to illustrate some of the principal facts. When olive oil and water are beaten up together, the oil forms droplets suspended in the water. If the mixture is allowed to stand, the two constituents quickly separate. If, however, one includes in the original mixture a third substance having a certain effect upon surface tension (an "emulsifier," or "stabilizer"), the resulting emulsion is stable, since the emulsifier enters the interface between the oil and the water and prevents the coalescence of the droplets (Fig. 15). Mayonnaise dressing is an 
emulsion of oil in an aqueous medium (vinegar) with egg protein as an emulsifier. Milk also is stabilized largely by proteins. When watersoluble sodium oleate is formed as the emulsifier in an olive oil-water mixture by adding $\mathrm{NaCl}$, the oil disperses in the water; this occurs even if the water constitutes only 1 per cent of the whole, the water forming a series of thin films separating the large oil droplets. When oil-soluble calcium oleate is formed by including $\mathrm{CaCl}_{2}$, the water disperses in the oil. By employing both sodium and calcium salts, varying their ratio, the important change known as phase reversal may be produced at will in either direction (Fig. 17). At a certain critical ratio there is a very delicate balance between the two conditions, and it is a biologically significant fact that the ratio at which colloidal proteins and lipides form

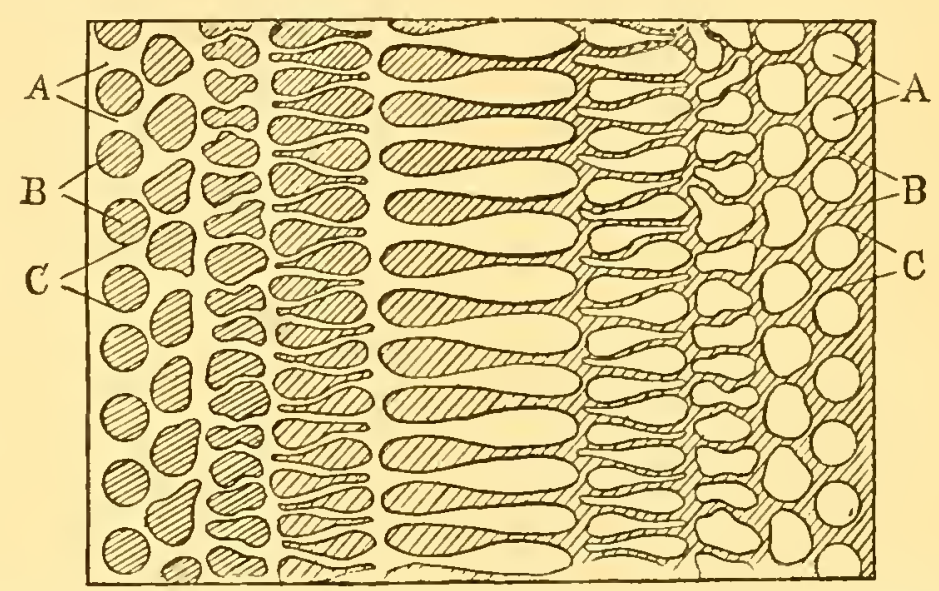

Fig. 17. -Diagram illustrating phase reversal in a colloidal emulsion. $A$, aqueous phase. $B$, oil or other non-aqueous phase. $C$, interfacial film formed by emulsifying agent. (After Clowes, 1916.)

such balanced emulsions is about the same as that in which the two classes of salts occur in both blood and sea water.

In many colloidal systems the finely divided substance is in the form of filaments or thin films rather than globules or small granules. Furthermore, these may extend throughout the mass as a network or platework, neither phase being wholly discontinuous. The structure of such an "interlacing system" is illustrated on a large scale by bread or a rubber bath sponge. What is essential to all types of systems is the enormous extent of the interface, and to obtain this condition it is therefore only necessary to make at least one dimension of the dispersed matter small. It can readily be calculated that a cube of matter 1 centimeter in each dimension and exposing 6 square centimeters of surface would expose 60 square centimeters if cut into 1 -millimeter cubes, 6 square meters if cut into $1-\mu$ cubes, and 6,000 square meters if cut into $1-\mathrm{m} \mu$ cubes.

The Colloidal Nature of Protoplasm. - The work of the past few years has made it abundantly clear that protoplasm is a colloidal system. This is manifest in its general physical properties. Its viscosity, surfacetension phenomena, power of adsorption, and high electrical resistance 
are like those of other known colloids. Its semipermeable properties are typically those of colloidal systems; a semipermeable region is probably present wherever protoplasm comes in contact with other substances, such as water. Protoplasm shows most strikingly its colloidal character in the alterations of physical state, involving imperfectly understood structural changes, which it undergoes as a result of variations in external conditions and internal reactions. Local temporary alterations of this nature are known to accompany a number of important life processes. Protoplasm, like many organic and inorganic colloids, is irreversibly coagulated by too high temperatures and a variety of chemical substances. The "fixation" of protoplasm by the reagents employed in cytological technique is primarily the transformation of a lyophilic system into an irreversible lyophobic coagulum.

Protoplasm is colloidal, but the question of the particular type or types of colloidal structure it possesses is one to which no satisfactory answer can yet be given. The most widely prevalent theory is that the emulsion type of structure, often visible with the microscope, is continuous with an ultramicroscopic colloidal structure of the same sort, with discontinuous phases of lipides, proteins, and other liquid and solid substances suspended in an aqueous medium. It has been thought by some that high electrical resistance indicates a discontinuity of the water phase (M. Fischer, 1923), some non-conducting material constituting the medium of dispersion. The suddenness of many permeability changes has been thought to favor the riew that protoplasm has an emulsion structure, at least in plasma membranes (Clowes, 1916). R. S. Lillie (1923) sees further evidence in a variety of phenomena, including the autolytic action of injured cells wherein enzyme and substrate are allowed to interact by the breakdown of films normally separating them.

The view that the submicroscopic colloidal constitution of protoplasm is primarily like that of an emulsion has been brought into question by Seifriz (1924ab, 1926abe, 1929b). He points out that living protoplasm, although it may show such a structure within the power of the microscope (Fig. 16), differs markedly from emulsions in its noticeable degree of elasticity, its power of imbibition, and its characteristic behavior in forming a granular irreversible coagulum at death. Comparisons with certain inorganic systems suggest that it has a structure in which the units are arranged in linear chains which may form a three-dimensional network or "brush heap."

It is not to be concluded that investigators insist that all protoplasm must have this or that particular type of colloidal structure. It is probable that the minute structure varies within wide limits in different tissues and in different regions of any differentiated mass of protoplasm. It may be that emulsions, interlacing systems, and molecular bridgeworks all actually exist and pass one into another according to general and local 
conditions. Only future research will permit an evaluation of the many conflicting views on this subject. Of special interest will be the results of studies to determine to what extent protoplasm may possess a micellar structure comparable to that of many of its products (p. 179). The point to be borne in mind at present is that the structure in any event is indisputably colloidal, and that our attempts to explain protoplasmic behavior must be based on this fundamental fact. We shall proceed, then, with the general conception of protoplasm as a complex system of many substances dispersed in the form of granules, globules, filaments, networks, and plates, which thus expose an enormous area of reacting surface in proportion to their volume. The system includes an extensive series of thin films in the interfaces between the continuous and dispersed phases, between the differentiated protoplasmic regions or organs, and around the mass as a whole.

Some of the activities of protoplasm which seem clearly to be conditioned by its polyphase, film-partitioned organization may now be briefly enumerated. ${ }^{21}$

A great many chemical substances coëxist in protoplasm without interacting until certain conditions prevail, whereupon interaction occurs suddenly and extensively. This is thought often to involve films separating the different phases of the system. Under appropriate circumstances the properties of these films are rapidly altered, allowing the substances on either side to interact, and the volume and the velocity of the reaction are due in large measure to the enormous area of reacting surface. The films separating various regions within a protoplasmic mass permit the localization of very diverse types of chemical activity within a small space and the consequent differentiation of organs in which these activities are then more efficiently carried on. A particularly important case of the control of reactions through changes in colloidal films is seen in general and local alterations in the permeability of the plasma membrane bounding any unit mass of protoplasm, a matter to be discussed in the next section.

The effect of colloidal structure upon processes involving adsorption is noteworthy. Substances are peculiarly subject to chemical change when adsorbed at surfaces because of the special energy conditions present there. Adsorption is frequently a necessary preliminary to chemical reaction. In protoplasm there exists an ideal structural basis for such "adsorption catalysis"; in fact, it seems that "the determination and control of chemical reactions by adsorption are universal in living protoplasm" (R. S. Lillie, 1923). It seems clear that a part of the catalytic activity of enzymes is due to their colloidal state; indeed, it appears that respiration itself is a surface reaction conditioned in this way (Warburg; sce, further, Beutner, 1933). These facts suggest why it is that certain ${ }^{21}$ See Bayliss (1923) and R. S. Lillie (1923). 
reactions, such as rapid oxidation, ordinarily occurring only at high temperatures, may take place at relatively low temperatures in the organism.

Finally, in the film-partitioned structure of protoplasm there is a basis for further electrical phenomena, a subject fully discussed by Lillie. Electric currents have been shown to accompany a variety of vital processes and to have a fundamentally important bearing upon problems pertaining to the reception and conduction of stimuli, automatic or reflex activity, muscular contraction, the movement of plant parts, correlation, polarity, growth, and other biological phenomena. "They appear, in fact, to be as essential a feature of protoplasmic action as the consumption of oxygen or the evolution of $\mathrm{CO}_{2}$."

Ectoplasm and the Plasma Membrane.--It has long been known that there is at the normal surface of any mass of protoplasm a layer whose physical properties differ somewhat from those of the substance within the mass. Sometimes such a differentiation can be easily seen: in amœbæ and myxomycetes a layer of hyaline ectoplasm surrounds the granular endoplasm within (Fig. 18). Frequently the boundary between these two is not at all sharp, and it seems evident that they may be readily converted one into the other. Among Protista, as will be shown farther on, the ectoplast is often elaborately differentiated and may be accompanied by additional envelopes.

There is a large body of evidence for the view that an exceedingly thin plasma membrane, or plasmalemma, is always present at the surface of

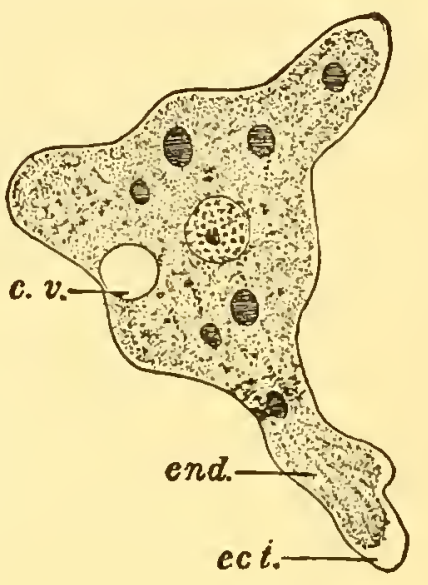

Fig. 18.- An amœba, showing ectoplasm, endoplasm, and contractile vacuole. the protoplast, whether any special ectoplasmic layer can be directly observed or not. In case such a layer is present, the plasma membrane represents its external surface film. Much has been learned about the plasma membranes of amœbæ and other cells by observing the behavior of particles adhering to it (Schaeffer, 1920) and by the use of the micromanipulator. ${ }^{22}$ In myxomycetes Seifriz finds the outer membrane to be distinctly more elastic and tenacious than the hyaline substance immediately beneath. Although it is very thin, it is a definite morphological structure which may be removed after death with dissecting needles. Moreover, it is capable of constant repair. Such a capacity to form a surface membrane immiscible with water, provided the injury has not been too sudden and extensive, seems to be generally present in healthy protoplasm. The development of the membrane is in many respects like that of the formation of superficial films by other colloidal systems, although in the case of protoplasm certain metabolic syntheses seem to be

${ }^{22}$ Kite (1913), Chambers (1917 et seq.), Seifriz (1918, 1921), Chambers and Höfler (1931), Plowe (1931a). 
involved. In the Arbacia egg there is a reaction of calcium with a substance visible in the cytoplasm, according to Heilbrunn (1928, 1930a).

Because of the very different surface conditions prevailing in tissue cells, the differentiations can scarcely be expected to be wholly the same as in "naked" masses of protoplasm. Nevertheless, there is reason to believe that here, too, specialized osmotic membranes are responsible for certain physiological phenomena, notably selective permeability, as was claimed long ago by Pfeffer (1890). Plowe (1931ab) has been able to show both by microdissection and by permeability studies that the plasma membrane in the familiar cell of the onion-bulb scale is a real structure. That surface membranes have properties not present in the endoplasm is shown by the fact that certain substances, eosin for example, to which the membrane is impermeable, will diffuse readily through the endoplasm if artificially injected through the membrane (Chambers). Such semipermeable membranes (membranes permeable to solvent but not to solute) are also present about nuclei and sap racuoles (see below). All such membranes of the protoplast appear to be lyophilic gels. In the case of a vacuolate cell, the permeability of the whole is determined by the permeabilities of all the regions traversed - plasma membrane, endoplasm and vacuole membrane- but it is due chiefly to the membranes.

Because of the great importance of the cell membrane in physiology many studies have been made with the hope of determining its exact physico-chemical constitution. ${ }^{23}$ One of the most influential theories has been that developed by E. Overton (1895 et seq.), who concluded that the membrane is composed primarily of lipides. This theory was founded chiefly on the high correlation found to exist between the lipide solubility of many organic compounds, notably dyes, and their ability to pass through cell membranes. Moreover, lipides would be expected to accumulate at free surfaces, and their presence would help to explain the immiscibility of the membrane with water. This theory has been much criticized, not only because it has been thought not to account satisfactorily for the high permeability of the membrane to water, but also on the basis of further studies on permeability and leaching. ${ }^{24}$

The probable importance of proteins in the cell membrane has been emphasized by a number of investigators ${ }^{25}$ partly because of the colloidal nature of these substances and the readiness with which they form pre-

${ }^{23}$ For discussions and literature pertaining to the eonstitution and physiological behavior of plasma membranes, see Schaeffer (1920), Bayliss (1921), Lundegårdh (1922), Höber (1922), R. S. Lillie (1923), Chambers (1924), Jacobs (1924), Stiles (1924), Weber (1926b), Gellhom (1929), Steward (1929), Gray (1931), and Beutner (1933).

${ }^{24}$ Ruhland (1908 et seq.), Höber (1909, 1922), Steward (1928ab, 1929), Bailey and Zirkle (1931). See, further, Gray (1931).

${ }^{25}$ Pfeffer (1900), Ramsden (1904), Osterhout (1911), Loeb (1911), T. B. Robertson (1908), Lepeschkin $(1910,1911)$. 
cipitation membranes. The high permeability of such membranes to strong electrolytes is, however, against the assumption that they can be primarily responsible for the selective properties of the living cell's surface (Gray); moreover, protein membranes do not exhibit the necessary osmotic properties.

As a result of such difficulties, physiologists have been forced to the conclusion that the membrane is characterized by a peculiar physical and chemical complexity. That both lipide and protein constituents are present is strongly indicated, but what other constituents may accompany them, and how they are all physically arranged, is not at all clear. According to Gortner (1929), it is possible to picture the membrane provisionally as consisting of a "protein gel, probably in the form of a fibrillar structure with fats, soaps, and lipides immeshed in the protein network. The transfer of lipide-soluble materials would be through the fat-soaplipide portion of the structure, whereas the passage of water and such water-soluble materials as actually do pass in and out of the cells would be through the hydrated filaments of the protein network."

Various conjectures have been made regarding the possible alterations in the structure of the colloidal cell membrane as it undergoes observed changes in permeability. Clowes $(1916,1918)$, who studied the relation existing between balanced antagonistic salts and phase reversal in olive oil-water emulsions, believed that similar reversals of lipide and aqueous phases might account for permeability changes in cell membranes, the substance originally forming a continuous path becoming discontinuous, and vice versa. Many membranes maintain their normal state of semipermeability only when the ratio of such antagonistic salts is held constant within narrow limits. Since colloidal systems are known in which the two phases differ chiefly in the relative proportion of water they contain, it has also been suggested that changes in permeability may be due to redistributions of water between such phases in the membrane (Lloyd, 1915; Free, 1918). Such changes would affect the size of dispersed droplets and the closeness with which they are packed and hence the action of the membrane as a whole. Such a hypothesis is thought to account more readily for very gradual changes in permeability than does the reversal hypothesis. Both gradual and sudden changes oceur, and it is highly probable that they involve structural alterations of more than one type.

In his discussion of this general subject Gray (1931) lays emphasis upon the fact that "the living cell is bounded by a surface or a membrane which is capable of generating energy or of transforming energy which is supplied to it in an appropriate form," and upon the further fact that no inanimate system known at present has the electrical properties manifested by the cell membrane. "In other words the plasma membrane is part of a dynamic system whose machinery is of a type not yet demonstrated outside the living cell." It remains true, however, that the 
results obtained with artificial systems, if rightly construed, cannot fail to contribute to our understanding of cell membranes and their action (see Beutner, 1933).

Vacuole Membranes. - The character of the tonoplast, or membrane bounding the ordinary sap vacuole, has been the subject of many investigations. In 1885 de Vries found that the large central vacuoles of Allium and Spirogyra could be isolated by placing the cells in hypertonic solutions of a potassium salt, and that the semipermeability of their membranes was retained in a measure for some time. Such observations on onion cells have been repeated and extended by Chambers and Höfler (1931), who have isolated the vacuole with a micromanipulator. They find the tonoplast membrane ${ }^{26}$ to be "a highly cohesive and extensible fluid film of unappreciable thickness." It resembles the plasma membrane in being immiscible with water when normal, in not wetting a clean glass micro-

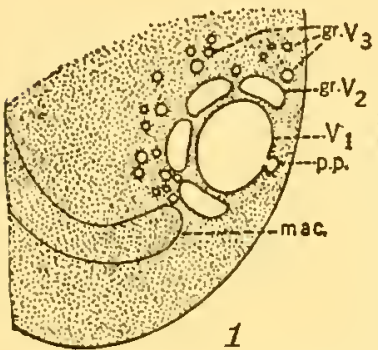

Fic. 19-Discharge of the contractil

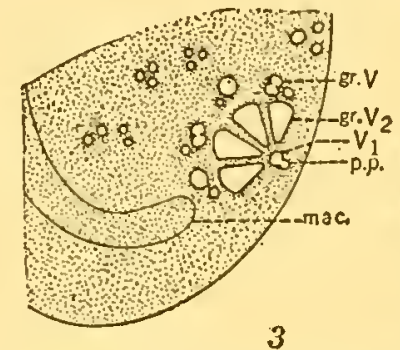

3

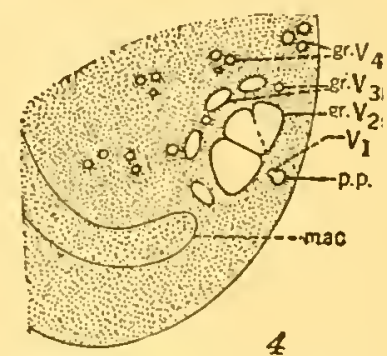

4

actile vacuole $\left(V_{1}\right)$ and the accompanying behavior of subsidiary groups of vacuoles $\left(g r . V_{2}, g r . V_{3}\right)$ in Euplotes. p.p., papilla pulsatoria. mac. macronucleus. (After C.V. Taylor, 1923.)

needle, and in being wetted by oil and chloroform. A further resemblance is seen in the failure of acid dyes to pass through either membrane when injected into the cytoplasm (Plowe, 1931b).

The osmotic properties of vacuoles are important not only in connection with metabolic processes but also in supporting many plant structures through turgor. It is also by virtue of osmotic action that vacuoles may function in subdividing large masses of protoplasm in the sporangia of certain fungi (p. 165), in pollen tube formation (Wóycicki, 1926), and doubtless in many other processes.

The contractile vacuoles of certain lower animals and plants have similar limiting membranes. Such vacuoles periodically absorb water and solutes from the protoplast and then expel them to the exterior. In some cases, as in Paramœcium trichium (Wenrich, 1926), there is evidence that the membrane is a permanent structure which contracts rhythmically to expel fluid received from subsidiary vacuoles. In fixed material the membrane shows a characteristic fibrous structure. In Euplotes, C. V. Taylor (1923a) finds that the primary contractile vacuole, which is

${ }^{26}$ Chambers and Höfler extend the term "tonoplast" to the vacuole as a whole with its membrane. De Vries applied it to the membrane alone. See also Plowe (1931a). 
accompanied by two adjacent series of smaller vacuoles, discharges its contents through a definitely localized but temporary ectoplasmic pore, whereupon it disappears completely. Its place is then taken by another formed by the union of secondary adjacent vacuoles (Fig. 19). These latter vacuoles arise in turn from still smaller ones, which seem either to be derived ultimately from vacuoles containing granules that dissolve, or to arise de novo as fluid centers causing the protoplasm to form a membrane by gelation. These observations have an interesting bearing on the problem of the origin of ordinary sap vacuoles (p.94) In amœbæ the membrane of the contractile vacuole appears to be formed anew periodically by gelation (Day, 1927) and shows certain resemblances to the plasma membrane in its permeability (Morita and Chambers, 1929).

Contractile vacuoles have usually been regarded as excretory organs, but there is evidence that they are mainly concerned in the regulation of hydrostatic pressure. ${ }^{27}$ In Spirogyra, Lloyd (1925, 192Sab) has shown that the action of contractile vacuoles is concerned in the movement of the gametes at the time of conjugation.

The Ectoplast of Protista.-The ectoplast shows its most elaborate structural differentiations in Protista, where it clearly has several functions-protective, motor, excretory, and sensory (see Minchin, 1912, Chapter V). In many forms there is a relatively tough outer envelope, or "pellicle," in addition to the more fluid hyaline ectoplasm. The origin and the degree of development of this envelope, however, are not the same in all cases. Commonly, it seems to arise as a modification of the outer region of the ectoplast. In Amoba proteus, Mast (1926) finds the internal protoplasm ("plasmasol") to be surrounded by a fairly rigid granular layer ("plasmagel") and this in turn by a tough "plasmalemma" about $0.25 \mu$ thick. The "periplast" of flagellates seems to represent the entire ectoplast modified. In still other forms the resistant envelope is formed indirectly by secretion.

Among the ectoplasmic structures with a motor function the simplest are the pseudopodia; in the larger ones there is a core of endoplasm, but the more delicate "filose" ones consist entirely of ectoplasm. Minute suspended particles can be detected in hyaline pseudopodia by the use of dark-field illumination, their Brownian movement affording an index of the fluidity of the ectoplasm (Bayliss, 1920). The cilia on the velum in certain molluscan larvæ have the form of thin blades, and each of these is composed of a number of long plates (Fig. 20, C). In Spirillum long contractile filaments are separable, but in Chromatium they are firmly united (Metzner, 1920). Cilia, which are short and numerous and show rhythmic pulsation, ${ }^{28}$ cirri, which are formed of tufts of cilia, membranelloe,

${ }^{27}$ See Calkins (1926); also Weatherby (1927) and Day (1930).

${ }^{28}$ For discussions of the structure and meehanics of flagella and cilia, see Heidenhain (1911), Lundegårdh (1922), Metzner (1920), Gray (1928), and Petersen (1929). 
representing fused rows of cilia, and undulating membranes, which are mainly sheet-like extensions of the ectoplasm (Fig. 20, $A$ ), are all essentially ectoplasmic organs. In Blepharisma, Chambers and Dawson find that when touched with a needle the undulating membrane breaks up into cilia, which may reunite. A further motor differentiation is seen in the minute contractile fibrils known as myonemes (Fig. 20, B), which are analogous to a system of muscle fibers. In ciliated forms they run beneath the rows of cilia.

Contractile or pulsating vacuoles sometimes appear to originate in the ectoplasm, although they may later lie much deeper. In certain cases definite actively protective organs, the trichocysts, are differentiated in the ectoplasm. A sensory function may be performed by the "eyespot,"

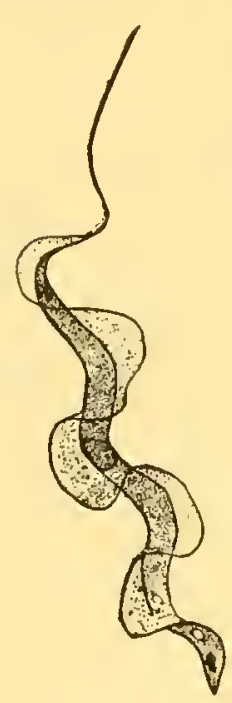

A

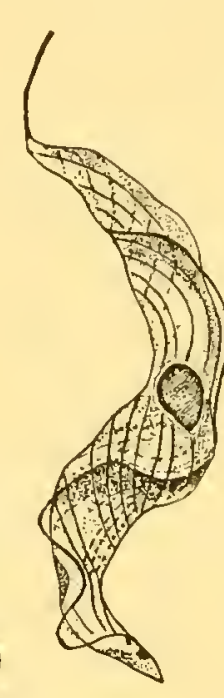

$B$

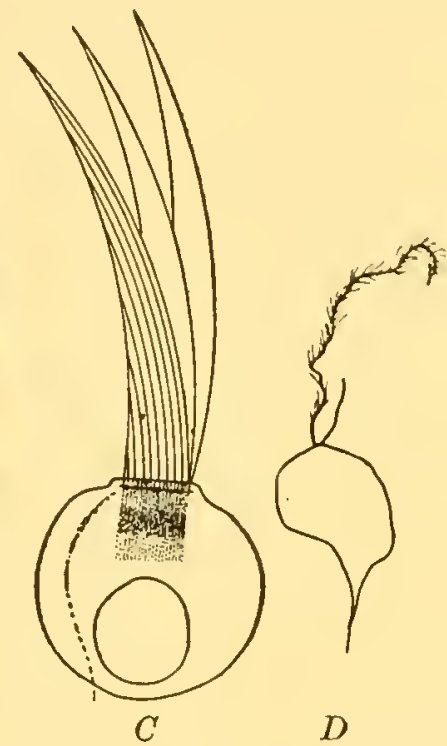

$D$
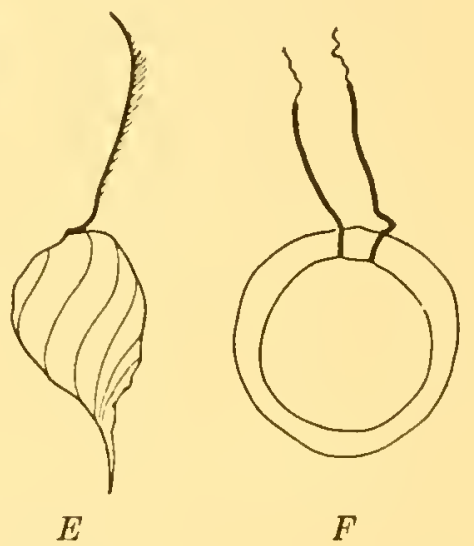

FIG. 20.-Types of motile apparatus. A, Trypanosoma tince, showing undulating membrane. $B$, Trypanosoma perce, showing myonemes. $C$, velar cilia of Asolis. $D$, Uroglena volvox. $E$, Phacus pyrum. F, Hxmatococcus pluvialis. (A and B after Minchin; $C$ after G. Carter (1926); D F after J. B. Pctersen, 1929.)

which in some cases appears to be ectoplasmic in origin (see p. 67), and also by flagella and cilia, which are often receptors of tactile stimuli.

Kinoplasm.- In his descriptions of the plant protoplast Strasburger (1892, 1897a, 1898) made a distinction between the nutritive alveolar trophoplasm and a specialized active kinoplasm, the latter constituting the plasma membrane, fibrils of the mitotic figure, centrosomes, and the contractile substance of cilia and allied structures. ${ }^{29}$ Although the theoretical aspects of such a distinction were overemphasized by certain writers, the terms have remained useful for descriptive purposes.

The importance of kinoplasm has been emplasized anew by Lloyd and Scarth ${ }^{30}$ in studies of living cells of Spirogyra, Symphoricarpos, Tradescantia, and Elodea. According to these workers, the kinoplasm

${ }^{29}$ Allied to this were conceptions involving archoplasm and ergastoplasm (see Wilson, 1925, p. 723).

${ }^{30}$ Scarth (1927), Searth and Lloyd (1927), Lloyd (1926), Lloyd and Scarth (1926). 
not only constitutes differentiated films about the cell, the nucleus and the plastids, but may also develop filamentous prolongations extending from these films into the cytoplasm and from the tonoplast into the vacuole. In extreme cases it may form a spongy framework throughout the cytoplasm and a "medusoid" growth over the surface of the vacuole. It appears to be the kinoplasm that is responsible for protoplasmic streaming; it flows through the relatively stationary trophoplasm, carrying with it the plastids and chondriosomes. ${ }^{31}$ There appears to be an inverse relationship between the abundance of chondriosomes and differentiated kinoplasm; moreover, the active kinoplasm does not stain, but as it metamorphoses into the tonoplast it colors faintly with Janus green. These and other facts suggest that lecithin enters into the composition of the kinoplasm and that the chondriosomes may represent a substance used in its elaboration.

Lloyd has shown that the contractile vacuole of the Spirogyra gamete (see p. 228) arises by a swelling of kinoplasmic globules in the cytoplasm. The cleavage of the protoplasm of many sporangia to form spores is also regarded as an activity of extending kinoplasm. Such observations raise a number of important cytological problems and emphasize the need for further work along this line.

Protoplasm and Metaplasm.-Because of the place they occupy in cytological discussions we may outline several classifications of the substances composing organisms which certain writers have found useful.

J. Hanstein (1S6S):

1. Protoplasm: the living substance.

2. Metaplasm: non-living substances in or on protoplasm.

M. Heidenhain $(1902,1907)$ :

1. Protoplasm: the principal form of living substance.

2. Metaplasm: a less active form of living substance, formed by a process of differentiation in protoplasm in connection with special functions, and capable of growth, response to certain stimuli, and further differentiation. It is represented chiefly by the intercellular substance of animals; also by certain other structures, such as elastic and connective tissue fibrils.

3. Non-living substances.

E. Rohde (1908, 1923):

1. Protoplasm: the principal form of living substance.

2. Metaplasm: a less active form of living substance; essentially the same as Heidenhain's metaplasm but including also contractile and nerve fibers, which Heidenhain regarded as protoplasmic. Metaplasm, according to Rohde, cannot again become protoplasm and does not continue from generation to generation.

3. Non-living substances.

A. Meyer $(1896,1920)$ :

1. Protoplasm: the principal form of living substance.

2. Alloplasm: a less active form of living substance, essentially the same as the metaplasm of Heidenhain and Rohde. Cilia, flagella, and cytoplasmic fibrils are "alloplasmatic organs." Organs of this class arise by direct transformation of all

${ }^{31}$ Seifriz's cryptoplasm and phaneroplasm (p. 33) would both be included in the kinoplasm. 
or part of a protoplasmatic organ, usually the cytoplasm. They may dissolve but do not become protoplasn again.

3. Ergastic substances: non-living substances arising anew in or on the protoplast; these correspond to the metaplasm of Hanstein. The intercellular substance, regarded as living by Heidenhain, is held to be ergastic by Meyer.

Protoplasm and Life---Huxley, in his famous essay of 1868, very aptly termed protoplasm "the physical basis of life." Since the true significance of protoplasm was first recognized, many suggestions have been ventured regarding the nature of the relation existing between life and its physical basis. The modern conception of protoplasm as a living system was preceded by a number of speculative "micromeric theories," or "atomic theories of biology," according to which the principle of life was held to reside in ultimate vital particles. ${ }^{32}$ Such fundamental particles were supposed to be for the most part of ultramicroscopic size, capable of growth and reproduction by division, and associated like members of a vast colony in protoplasm. Attempts were also made to account for the activities of protoplasm on the basis of the known chemical reactions of certain of its constituents, notably the proteins. It is not surprising that the peculiar properties of these compounds, which are certainly very significant, should have led to the belief that life is primarily a series of changes in special labile protein molecules, or "biogens" (Verworn).

The fundamental fallacy involved in all such speculation lies in attributing the properties of a system to some one of its constituent elements and consequently in attempting to draw a sharp line between "living" and "lifeless" components. Sachs $(1892,1895)$ and many others have urged that the various elements should be referred to as active and passive rather than living and lifeless. It cannot be emphasized too strongly that protoplasm is a living system of components which by themselves are non-living-a system composed of all the substances that are participating in essential protoplasmic reactions at a given moment ( $c f$. Wilson, 1923).

The various constituents of protoplasm share in all degrees in determining the activity of the system of which they are integral parts. It is probable that protoplasm always contains visible or invisible materials which might be removed without terminating the life processes. This does not prove, however, that these materials had no share in the processes while they were a part of the system, but shows only that the system which remains after their removal still has an organization permitting it to continue in the living state. It is an altered system and operates in a somewhat altered manner. Furthermore, the same chemical compound may

${ }^{32}$ For summaries of these theories, see Delage (1903), Heidenhain (1907), Kellogg (1907), and Meyer (1920). Some regard the gene theory as a modern development of the same kind. 
be active at one moment and relatively inactive at another, depending upon its physical state (e.g., whether in solution or not) and the presence of other substances with which to react. It is not a certain chemical composition, but activity, that marks a substance as a part of the living system. The system remaining after the supposed removal of all dispensable materials would comprise many classes of substances, some of which, notably water, carbon, and proteins, are probably essential in all forms of protoplasm because of their peculiar properties. The fact that these forms of protoplasm are almost innumerable suggests that the components of the fundamental system must vary quantitatively, qualitatively, and in their type of structural organization within considerable limits.

In any case it should be evident, in spite of the special importance of certain forms of matter, that it is not this or that component but the organized system as a whole which lives. Steel is not a time-keeping material, but it may be an important constituent of a time-keeping system, such as a watch. Proteins are not living compounds, but they are important constituents of living systems. That which is distinctively biological inheres not merely in the several components but in their unique integration and the consequent peculiar action of the organized system as a whole. The action of the whole can be said to be a sum of the action of the parts only when it is remembered that the parts act as they do because of their position in the whole. Hence a true conception of the organism can be approached only when analysis into physico-chemical components is followed by resynthesis into a biological whole. ${ }^{33}$

We may go a step farther and point out that the organism is not to be sharply set apart from the environment. The two are so inseparably interlocked that they must be conceived as a single integrated system whose orderly operation is necessary to life. ${ }^{34}$ Thus life is largely a relation or adjustment between the properties of the organism and those of the environment (Brooks, 1889), or, as Herbert Spencer put it, a "continuous adjustment of internal relations to external relations."

${ }^{33}$ See J. A. Thomson (1920), C. Lloyd Morgan (1923), Jennings (1927), Russell (1930), and Gray (1931). (1931).

${ }^{34}$ For discussions of this point, see Jennings (1924), Sharp (1925), and Carrel 


\section{CHAPTER III}

\section{THE NUCLEUS}

It is now a century since the nucleus was first recognized as a normal and characteristic element of cells, and half a century since a new era in cytology was ushered in by a series of researches revealing the remarkable behavior of the nucleus during the critical stages of the life cycle. Because of the peculiarly intimate relation which this behavior has been shown to bear to many outstanding biological problems, including that of Mendelian heredity, it is largely in nuclear phenomena that cytological interest has continued to center up to the present day. The most striking of these phenomena form the subjects of several subsequent chapters; at this point the nucleus will be considered only as it appears in the "metabolic" condition, i.e., when not undergoing division. ${ }^{1}$

General Features of Nuclei.- Whether or not one should say that all protoplasm or all cells are nucleated depends upon what is meant by the term "nucleus." If the chromatic substance, no matter whether distributed throughout the cell in the form of small particles or aggregated in a well-defined organ, be regarded as constituting a nucleus, then it follows that all complete plant and animal cells normally have nuclei. If, however, the term be employed only with reference to a distinctly delimited organ, we must regard those Protista with only scattered chromatic material as devoid of nuclei, although they may contain matter which performs at least certain nutritive functions of a nucleus. The question of the presence of nuclei in bacteria is discussed in Chapter XIII. In nearly all known organisms there are nuclei with roughly the same general type of organization, which indicates that the functional and structural differentiation of protoplasm into cytoplasm and nucleus doubtless occurred at a very early period in the history of the organic kingdoms.

The number of nuclei present in any mass of protoplasm depends largely upon the bulk of the mass, since within limits a certain ratio of nuclear surface to cytoplasmic volume must be maintained for the proper action of the whole system. A small mass with but one or a very few nuclei may grow into an extensive conocytic body with thousands of

${ }^{1}$ For an exhaustive review of the literature on plant nuclei, see Tischler (19211922, especially Chaps. I-IV). Von Neuenstein (1914) gives a systematic account of alga nuclei; for recent literature, sec Smith (1933). Agar's (1920) book on cytology is chiefly an account of the structure and behavior of the metazoan nucleus. 
nuclei produced by repeated division, as in the Siphonales and Phycomycetes. In such a body a few partitions may be formed, giving cells with varying numbers of nuclei, as in Cladophora (Fig. 4, C). Again, the subdivision into cells may be very closely correlated with the division of the nuclei so that every cell has one nucleus; this is the condition in the majority of organisms. Frequently, cells with two or more nuclei occur regularly in certain tissues of plants with uninucleate cells elsewhere throughout the body, as in the internodal cells of Characex, tapetal cells, and a number of other instances in vascular plants. ${ }^{2}$ A peculiar condition is found in the red blood corpuscles of mammals. These cells are originally nucleated, and the later enucleate condition is usually attributed to an actual loss or degeneration of the nucleus. In amphibians the enucleate erythrocyte ("erythroplastid") arises by the division of a nucleated cell without an accompanying nuclear division. ${ }^{3}$ Enucleate leucocytic elements are also known. The nuclei of a protoplast, whether many or one, are called the nucleome by Dangeard.

In certain infusoria two unlike nuclei are present: a small micronucleus and a much larger macronucleus (Fig. 5). This is usually the condition in Paramœecium caudatum, but races with no micronucleus are known. ${ }^{4}$ Some species may have more than one micronucleus: in $P$. woodruffi there are usually three or four, although the number varies from none to eight (Wenrich, 1928a). The micronucleus divides mitotically and the macronucleus is usually said to divide amitotically, but at least in some cases the story is evidently less simple than this (MacDougall, 1925). The macronucleus appears to be primarily a special nutritive organ, showing decided alterations under changing environmental conditions (Stolte, 1922). At certain intervals it is absorbed completely and replaced by a new one derived by a process of division from the micronucleus. C. V. Taylor (1923a), working with Euplotes, observed that individuals from which the micronuclei had been artificially removed underwent only one or two more fissions and died in a few days.

The position of the nucleus is determined largely by physical causes, such as surface tension, the position of the vacuoles, and the relative density of the cytoplasm in different portions of the cell. In a nonvacuolate cell it ordinarily occupies the center of the cytoplasmic mass. In a cell with vacuoles it is imbedded in the cytoplasm even when the latter is reduced to a thin parietal layer; it never lies free in the vacuole. Its position is also related to the functions of the cell; generally speaking,

${ }^{2}$ Arber (1920). A historical review of known cases is given by Beer and Arber (1920). See also Tischler (1921-1922, pp. 212ff.)

\footnotetext{
${ }^{3}$ Beyer (1921), Emmel (1924, 1925).
}

${ }^{4}$ Landis (1920), Woodruff (1921). 
it lies in the region characterized by the most active metabolism. ${ }^{5}$ For example, in young, growing root hairs and pollen tubes it is commonly found a short distance from the elongating tip. Wendel (1918) observed that the root hairs of Sinapis alba seedlings grow first at the apex, then at the base, and sometimes at the apex once more, and that the position of the nucleus changes accordingly. In thickening epidermal cells (Fig. 21) it frequently, though not always, lies near the wall upon which the thickening material is being deposited.

In form the nucleus is typically spherical or ellipsoidal, its shape, like its position, being determined by a number of physical factors.

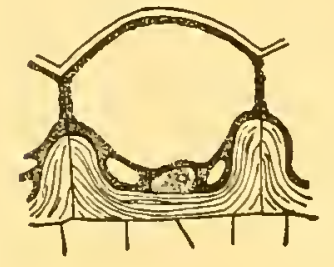

$A$

FIG. 21.-Position of nuclei in differentiating cells. $A$, thickening of inner wall of epidermal cell of Scopolia. B, origin of root hairs in Pisum. (After Haberlandt.)
Under comparatively uniform conditions, as where a small nucleus lies in a relatively large amount of nonvacuolate cytoplasm, a spherical shape is ordinarily assumed. Exceptions are often seen in cells with specialized functions. In the cells of the spinning glands of Pieris and Vanessa (butterflies) the physiological conditions lead to the assumption of very irregular forms whereby the nuclear surface is considerably increased (Fig. 22). Analogous changes occur in Lilium bulb cells (Goldstein, 1928). Nuclei seem rather commonly to undergo amøboid changes in shape, such active movement being directly observable in the nucleus of the living cycad spermatozoid. In the long, narrow cells of vascular bundles the nuclei, which are not free to grow in all directions, come to be correspondingly elongated. In Stentor and Spirostomum the nucleus has the form of a string of beads (Fig. 22, B). ${ }^{6}$

Nuclei show a wide variation in size, ranging in plants from extremely minute nuclei like those of $M u c o r, 1 \mu$ or less in diameter, to the relatively gigantic nucleus of the Dioön egg, with a diameter of $600 \mu$. A similar range is seen in animal nuclei. Although the nuclei of the fungi are characterized by small size, most of them being less than $5 \mu$ in diameter, they may grow to be very large at certain stages. The primary nucleus of Synchytrium, for instance, reaches a diameter of over $60 \mu$. The majority of nuclei, however, fall between 5 and $25 \mu$. In spite of the wide range in the size of nuclei of different organisms, it is generally uniform in a

5 This relation of position to funetion was emphasized in the works of Haberlandt (1887) and Gerassimow (1890, 1899, 1901).

${ }^{5}$ A discussion of the various factors influencing nuclear shape is given by Champy and Carleton (1921). Tischler (1921-1922) and Goldstein (1928) describe many unusual forms of nuclei. Tischler gives a long list of measurments of plant nuclei. 
given tissue, though it may vary considerably with the physiological activity (Maige, 1923).

The ratio of the volume of the nucleus to that of the cytoplasm in a cell is called the "nucleoplasmic ratio," or "karyoplasmic ratio." Many years ago it was held by Sachs and Strasburger that the size of a meristematic plant cell maintains a very definite relation to the size of the nucleus, owing to a supposed limitation of the sphere of influence of the nucleus. This latter conception has been emphasized anew by Winkler, and parallel views have been expressed by several zoölogists. ${ }^{7}$ In the case of certain terminal meristems of plants such a rule may well hold

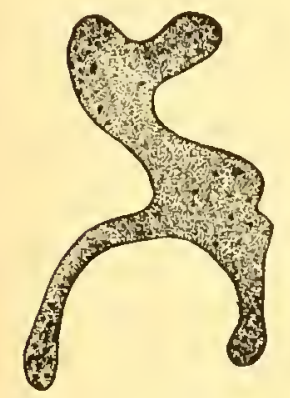

A
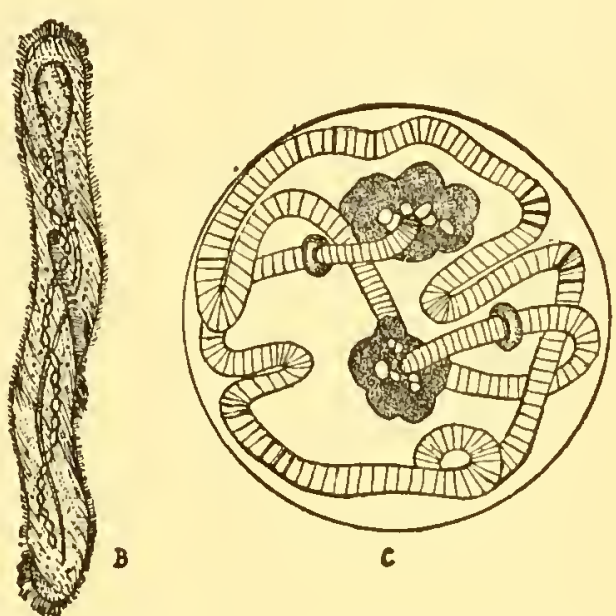

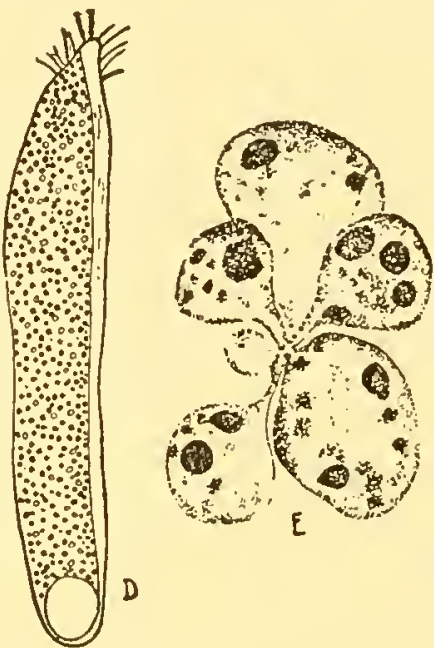

FIg. 22.-Peculiar nuclei. A, portion of nucleus from spinning gland of Vanessa urtice. (After Korschelt, 1896.) B, Spirostomum ambiguum, with moniliform nucleus. (After Stein.) C, nucleus from salivary gland of Chironomus; the chromatic material is in a convoluted thread which ends in two nucleoli. (After Balbiani, 1881. See also van Herwerden, 1910, 1911; Alverdes, 1912; Faussek, 1913; Tänzer, 1921.) D, Chæriu teres, with chromatic granules scattered throughout the body. (After Gruber, 1885.) $E$, lobed nucleus from yellowing lily leaf. (After Goldstein, 1928.)

true within limits, but the condition reported by Bailey (1920) in the lateral meristem (cambium) shows clearly that it cannot have universal application. The cambial initials may vary enormously in size with no corresponding variation in the size of their nuclei; two such initials, one of them having many hundreds of times the volume of the other, may possess nuclei of approximately equal size. Tischler (1924, 1925b) connects the ratio in pollen with the process of germination. The nucleoplasmic ratio has figured prominently in discussions of the problem of senescence and differentiation. ${ }^{8}$

In a given kind of cell there is a relation between the size of the nucleus and the number of chromosomes. In 1895 Boveri showed that the size

${ }^{7}$ Sachs (1892, 1893, 1895), Strasburger (1893), and Winkler (1916) on plant cells; Dolley (1913) on nerve cells; Hegner (1919) on Arcella; Hegner and Wu (1921) on Opalina; Dolley (1925) on pancreatic cells.

${ }^{8}$ R. Hertwig (1889, 1903, 1904, 1908), Minot (1891, 1908, 1913), Conklin (1912), Child (1915), Popoff (1907), Marcus (1908), Howard (1910), Howard and Schultz (1910). See Wilson (1925, p. 236); also Fauré-Fremiet (1925). 
of the nuclei in merogonic echinoderm larvæ is dependent upon the number of chromosomes each contains. In a more extended study (1905) he demonstrated that it is the surface of the nucleus that is proportional to the chromosome number, and also that the size of the cell is proportional to both. Gates (1909b), however, adduced evidence to show that this rule is by no means universal. This question has been investigated in connection with studies on polyploid plants with the general result that, although in the majority of cases a rise in the chromosome number of a species is seen to be accompanied by an increase in nuclear and cell size, there are cases in which such a change in related types seems to have been accompanied rather by a decrease in the size of the chromosomes. This indicates that it is volume of karyotin, and not mere number of chromosomes, that is of importance in this connection (see Tischler, 1921-1922, pp. 588ff.).

With respect to the physical nature of the nucleus as a whole, studies with the micromanipulator have shown ${ }^{9}$ that it ordinarily consists, at least in part, of an elastic gel having a higher viscosity than that of the cytoplasm, often being so firm that it can be handled without injury by the instrument. This obviously would be impossible were the nucleus merely a watery droplet or vesicle in the cytoplasm. The germinal vesicle (nucleus) of certain animal eggs Chambers finds to be a sol droplet with a gel membrane; when pinched in two by the instrument the two halves will reunite if they come in contact.

Studies on the electrical properties of cells have shown that the nucleus is apparently negative to the cytoplasm. Free nuclei and the heads of spermatozoa, which are composed almost entirely of nuclear material, pass to the anode in an isotonic cane-sugar solution; whereas, cells rich in cytoplasm, such as large leucocytes, pass to the cathode. ${ }^{10}$ The difference in electrical potential, on the two sides of the nuclear membrane may be of considerable significance in the vital processes.

The chemical nature of the nucleus has been touched upon in the preceding chapter (p. 26). The exact nature and distribution of the various constituents are not well known, but recent researches ${ }^{11}$ seem to show that the reticulum contains both nucleo-protein and lipide, the latter being in part combined with protein, while the karyolymph contains lipides but no nucleo-protein. The most nearly specific test so far devised for the chromatic nucleo-protein ("chromatin") of nuclei is the Feulgen reaction, in which the production of a purplish color in decolorized basic fuchsin indicates the presence of thymonucleic acid. ${ }^{12}$

${ }^{9}$ Kite (1913), Chambers (1914, 1917, 1921), Seifriz (1926c).

${ }^{10}$ R. S. Lillie (1903), Hardy (1913).

11 Němee (1909, 1910), Hansteen-Cranner (1919), Grafe (1925), Grafe and Magistris (1926), Wermel (1927), Gutstein (1927), Shinke and Shigenaga (1933).

12 Feulgen and Rossenbeck (1924), Feulgen (1926). See also MeClung's Microscopical Technique and Margolena (1932). 
The limitations of this method are not altogether clear, but it has already proved valuable in distinguishing chromosomal matter from other substances which stain like it with certain dyes. Most investigators have found that vital dyes do not stain the nucleus as long as it is living and healthy, but $\mathrm{P}$. Dangeard (1923a) reports that nuclei in certain cells with a somewhat retarded activity may be so stained with neutral red and methyl violet. Successful results are reported by several other workers. ${ }^{13}$

Structure of the Nucleus.-The typical nucleus is bounded by a nuclear membrane. The reality of this membrane has often been questioned, but researches on living cells ${ }^{14}$ leave no doubt that it is a definite structure. It is usually very thin; it has even been suggested that it may sometimes be but a single layer of molecules. In most cells under the micromanipulator it appears, however, to be thicker, remaining intact when the nucleus is pushed and pulled about or removed from the cytoplasm, and crumpling into folds when fluid is withdrawn from the nucleus with a pipette. Its origin with respect to the regions which it separates is obscure. The suggestion has been made that it is double, having distinct nuclear and cytoplasmic components. ${ }^{15}$

The larger portion of the volume of most nuclei is composed of a highly transparent ground substance known as karyolymph, or "nuclear sap." This usually appears optically homogeneous, with a few dispersed particles at most. The results of microdissection and the Brownian movement of suspended particles show that it may be in either the sol or the gel state. In old cells vacuole-like masses may appear within it (Kisser, 1922).

Associated with the karyolymph is another substance which appears in most preparations as a more or less continuous network of threads, the reticulum. The naturalness of this structure has also been a subject of debate. Many living nuclei, notably in animals, appear perfectly homogeneous (excepting the nucleolus) or at most very finely granular, a reticulum appearing gradually as the nuclei are injured experimentally or allowed to become moribund. ${ }^{16}$ The coarseness and the uniformity of the reticulum of ten vary considerably with the fixing agent employed; moreover, the coagulation of colloidal substances very commonly involves the formation of fibrous and reticulate structures. It has accordingly been inferred that the thready reticulum has no real existence in the normal living state but represents an artifact brought about by alterations in the nuclear colloids.

${ }^{13}$ Küster (1918b, 1926), Gicklhorn (1927), Paltauf (1928), Albach (1928). The reactions of plant and animal cells to vital dyes are compared by Benoist et al. (1929).

${ }^{14}$ Kite, Chambers, C. V. Taylor, Gross, Němec.

${ }^{15}$ Scarth (1927), Klingstedt (1928).

${ }^{16}$ Lewis and Lewis (1924), Chambers (1924), Schaede (1925, 1930), Strugger (1930). 
Opposed to the general validity of the foregoing interpretation are the results obtained by a number of investigators ${ }^{17}$ who have seen threads or a reticulum in apparently healthy nuclei under carefully controlled conditions of observation (Fig. 23). Martens, for example, working with the plumose stigmas of Arrhenatherum, stamen hairs of Tradescantia, and certain other tissues, watched the action of fixing fluids upon living nuclei

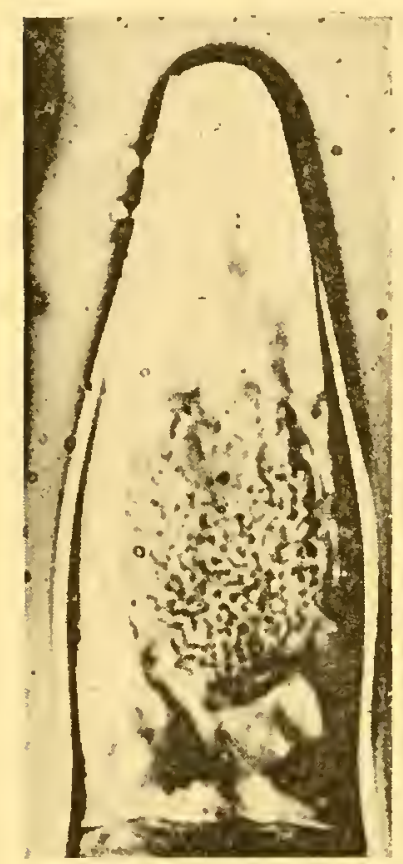

FIG. 23.-- Living nucleus in Tradescantia stamen hair mounted in paraffin oil. Note crooked chromonemata. (Photograph furnished by H. Tclezynski.) and found that the reticulate structure visible in healthy nuclei before fixation remained unchanged, except for a slight swelling of the threads and increased visibility due to alterations in refractive index. The same details appeared in material fixed for longer periods and stained with iron alumhæmatoxylin. These results, taken together with analogous findings of Chambers $(1924,1925)$ on the prophases in animal spermatocytes, indicate that the reticulum appearing in a well-fixed nucleus fairly represents a delicate thready structure actually present during life and rendered more distinctly visible by fixation. It is doubtless true that no fixer gives a wholly perfect rendition of all details of the living structure and that many fluids produce very great alterations, but it now seems evident that the reticulum cannot be explained away as merely a creation of technical procedures.

The reticulum is composed of threads. On the basis of evidence to be presented in Chapter $\mathrm{X}$, it may be said that these threads occur in a characteristic number and are derived each from the filamentous constituent (chromonema) of a chromosome. They appear to be connected laterally by fine strands (anastomoses) about whose origin and nature very little can be confidently affirmed (see p. 135). Since the main threads tend to be crooked and not appreciably thicker than their connecting strands, it is difficult or impossible to recognize them as individuals in the fully developed reticulum. They are characteristically chromatic: they usually stain well, particularly with certain basic dyes (basichromatism) and so offer a strong contrast with the achromatic karyolymph. In some nuclei the threads are rather evenly basichromatic throughout, the reticulum appearing as a system of uniform strands with only slight swellings here and there, especially at the nodes. In other cases the chromaticity may be more pronounced in the swellings, the other regions of the threads being relatively colorless or even oxychro-

${ }^{17}$ Lundegårdh (1912a), Kite (1913), de Litardière (1921b), Martens (1924, 1927abc, $1929 b$ ), Scarth (1927), Bělař (1929b, 1930), Guilliermond (1930a), Telezynski (1930), Belling (1933). 
matic (stainable with acid dyes). In such cases the appearance of the reticulum suggests a framework of one substance supporting differently stained particles of another substance

The interpretation of the above appearances has long been a major cytological problem, and no acceptable conclusion has yet been reached with regard to many points. The view was long ago put forward that the reticulum comprises two distinct elements: a supporting framework composed of relatively achromatic linin upon or in which are carried "granules" of a second, highly stainable substance, chromatin. The chromatin granules were held to be composed of "oxychromatin" or "basichromatin" according to their varying ratio of nucleic acid and protein. ${ }^{18}$ Thus the "chromatin" may be highly basichromatic (strongly acid) during nuclear division, but only weakly so or even oxychromatic (basic in reaction) in the metabolic nucleus where it is in a different functional state with its nucleic acid presumably combined with basic substances. At all stages it reacts positively to the Feulgen test (p. 52). Physical factors, such as degree of dispersion, as well as the order in which stains are applied, may also affect the results obtained in preparations. ${ }^{19}$

The evidence for such a structural distinctness of chromatin and achromatic supporting material in the reticulum has been questioned by many workers. ${ }^{20}$ It has been suggested that if there are two elements, chromatin and linin, they are not so distinct morphologically as the earlier workers supposed, the chromatin existing rather as a thin fluid impregnating the linin substratum. The chromatic lumps are often not sharply set off from the rest of the thread but taper off gradually. In such cases it has been found that purposes of cytological description are well served by the conception of a reticulum composed of a single complex substance which stains variously in different regions and at different stages of the nuclear cycle according to the size of the strands, their physico-chemical state, and the technical procedure employed. This one substance is loosely spoken of as chromatin, but because of the long application of this term to a supposedly distinct component of the reticulum it is advisable to use Lundegardh's (1910) term karyotin for the reticular substance as a whole. Only future research can decide whether karyotin ("chromatin" in the wide sense) is a true chemical compound

${ }^{18}$ Heidenhain (1890, 1907). As used by many writers, the term "oxychromatin" includes the linin, "chromatin" denoting only the "basichromatin." Heidenhain later ealled the chromatin granules "chromioles," a term introduced by Eisen (1899). See the discussions by Wilson (1925) and Stieve (1921). Oes (1908, 1910) and Němee $(1909,1910)$ reported that the two kinds of ehromatin differ in solubility.

${ }^{19}$ A. Fiseher (1899), Hardy (1899).

${ }^{20}$ Grégoire and Wygaerts (1903), Grégoire (1906), Sypkens (1904), MartinsMano (1904), Lundegårdh (1910, 1912), Malte (1910), Sharp (1913, 1920a), de Litardière (1921b), McClung (1924). 
or a looser combination of two or more constituents, only one of which is "chromatin" (in the narrow sense).

In nuclei with more sharply differentiated chromatic lumps and achromatic connecting strands the conception of two substances in the reticulum may seem more attractive. Studies on the chromosomal threads (chromonemata) during nuclear division have shown that the lumps (chromomeres) may be located in definite positions in the threads,
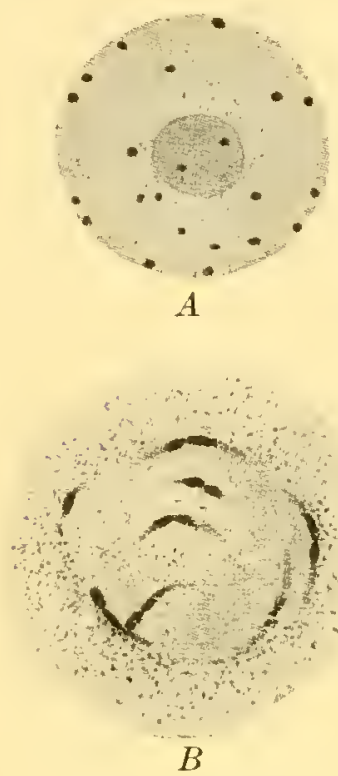

Fig. 24- $A$, nucleus of Phascolus, with chromocenters. (After Kuhn, 1929a.) B, nucleus of Impatiens in prophase, showing euchromocenters at spindle-attachment regions of developing chromosomes. (After Grégoire, 1932.) which in such a case have characteristic structural patterns (Fig. 79). Certain of the large lumps have been identified in the reticulum, and it is inferred that the small ones may also persist in some measure. Hence such chromomeres can scarcely be chance accumulations of chromatic matter; they are definite differentiations which there is much reason to believe are associated in some way with certain functional activities. They might be regarded as local swellings of the karyotin (one-substance theory) or as regions where "chromatin" is produced in characteristic amounts (two-substance theory). It may be that we shall come to look upon "chromatin" not as a substance existing primarily as definite units but rather as one whose presence indicates activities in other localized units or regions in the nuclear threads.

The above uncertainties do not prevent us from carrying forward the conception of the reticulum as a system of chromosomal threads connected by fine strands, or from speaking of the reticular substance in general as karyotin. As will be made evident in later chapters, the nucleus may be regarded as a group of closely associated chromosomes performing their metabolic functions together.

In many nuclei there are at certain stages one or more conspicuous masses of karyotin at several points in the reticulum (Fig. 24). Of the many terms applied to these the most suitable seems to be chromocenters. $^{21}$ After certain stains (iron alum-hæmatoxylin) they may at times closely resemble true nucleoli, but the distinctness of the two was long ago demonstrated with other stains (Rosen, 1892). In some cases it has been shown that they represent definite chromosomal regions which remain condensed and highly chromatic ("heteropyknotic")

${ }^{21}$ Bacearini (1908). They have been variously known as net knots (Flemming, 1882), karyosomes (Ogata, 1883), pseudonucleoli (Rosen, 1892), Nebennucleoli (Zacharias, 1895), and chromatin nucleoli. Karyosome is a widely adopted term, but the fact that it has been applied to other quite different elements has made the substitution of another term advisable. 
instead of forming portions of the reticulum. ${ }^{22}$ These euchromocenters appear to correspond in part to the "prochromosomes" which many writers have pointed out as regions at which prophasic chromosome condensation begins. In other cases chromatic accumulations may arise secondarily in the metabolic nucleus and disappear without showing any direct connection with chromosome development (Kuhn, 1929a).

The Nucleolus.-Nearly all metabolic nuclei contain one or more true nucleoli, or plasmosomes. ${ }^{23}$ In a young nucleus just formed by division there are often several small nucleoli which may unite to form two or one as the nucleus becomes fully developed. In the living nucleus the nucleolus appears as a dull, viscous droplet, usually round but frequently irregular in shape. Centrifuginger and the position it naturally assumes in certain eggs ${ }^{25}$ show it to be heavier than the rest of the nuclear matter. It may be homogeneous throughout, or it may contain vacuole-like masses and occasionally small granules. ${ }^{26}$ Chemically, it is composed mainly of proteins and lipides. ${ }^{27}$ It commonly shows an affinity for acid dyes, but in some procedures it takes the basic ones. Of greater interest is the fact that its chromaticity undergoes marked alterations during the nuclear division cycle, as will be pointed out in later chapters. Such alterations are thought to indicate interactions of some sort with the reticulum, with which the nucleolus is in contact at one or more points. The broad, clear zone often seen about the nucleolus in fixed material is, as a general rule, an artifact due to a contraction of the reticulum.

The functions of the nucleolus are obscure, but there is obviously some relation between its behavior and the cycle of alterations undergone by the other nuclear constituents. This topic is to be discussed in Chapter IX. It should, however, be pointed out here that the nucleolar matter arising in the young nucleus develops principally in close association with definitely localized regions of particular chromosomes, a fact which has only recently come to light (p. 118). The nucleolus or nucleoli tend to remain attached to such chromosomes and consequently to the metabolic reticulum which the latter form. As the reticulum again

${ }^{22}$ Strasburger (1904c), Miyake (1905a), Rosenberg (1907b, 1909ac), Overton (1905, 1909), Laibach (1907), Heitz (1929a), Grégoire (1932). These are called euchromocenters (true ehromocenters) by Grégoire.

${ }^{23}$ For general aceounts of the nucleolus and reviows of the literature pertaining to it, see Montgomery (1899), Wager (1904), Walker and Tozer (1909), M. Jörgensen (1913a), A. Meyer (1917a, 1920), Tischler (1921-1922), Ludford (1922a), and Guilliermond and Mangenot (1928).

${ }^{24}$ Mottier (1899), F. M. Andrews (1902), E. W. Schmidt (1914), Němec (1929f), Shimamura (1929).

${ }^{25}$ Gray (1927b) on Echinus, Wakayama (1929) on Pinus.

${ }^{26}$ Digby (1910), Reed (1914), Kuwada (1919), Carleton (1920), Saguchi (1920b).

${ }^{27}$ Zacharias (1885), A. Meyer (1920), Unna and Fein (1921), Fels (1926), Shinke and Shigenaga (1933). 
develops condensed chromosomes, the nucleolar matter diminishes in amount and commonly disappears completely. This long ago suggested that it is with the chromosomal changes especially that the nucleolus is concerned. ${ }^{28}$ Kossel suggested that the albuminous nucleolar material arises as one of the products formed when complex albumin-rich compounds are split to form albumin-poor "chromatin."

Less evident are certain other functions which have been ascribed to the nucleolus. It has often been regarded as a reserve material utilized in some unknown manner by the protoplast. ${ }^{29}$ To Strasburger (1895) its behavior suggested a connection with the development of the achromatic structures at the time of nuclear division (Chapter XI). Others have made observations suggesting a rôle in secretion ${ }^{30}$ or yolk formation ${ }^{31}$ in animals. The evidence in a number of such cases has been adversely criticized. ${ }^{32}$

The Functions of the Nucleus.-Many years ago Claude Bernard (1878) suggested that the nucleus is necessary for synthetic metabolism. Since that time various investigators ${ }^{33}$ have sought to test this hypothesis by experiments on plant cells artifically deprived of their nuclei by means of plasmolysis, centrifuging, direct section, cooling, or anesthesia. It was observed that in these cells such activities as the formation of cellulose membranes, assimilation, the utilization of reserves, growth, and the division of plastids ceased at once or continued for only a short time. The general conclusions were that these activities depend upon the presence of specific substances (building materials, enzymes, etc.) in the cytoplasm, that such substances are elaborated with the coöperation of the nucleus, and that the occurrence or non-occurrence of the activities in question in enucleated masses of cytoplasm depends upon the presence or absence of these substances at the time of the experiment.

Analogous results have been obtained in certain experiments on amœbæ, ${ }^{34}$ which can be cut into nucleate and enucleate pieces. The

${ }^{28}$ Flemming (1882), Strasburger (1884), F. M. Andrews (1901), Rhumbler (1893), R. Hertwig (1898), Lubosch (1902), Farmer (1907), Sheppard (1909), Maziarski (1910), Reed (1914), Schürhoff (1918), Digby (1919), de Litardière (1921b), Ludford (1922a), Cleland (1922, 1924), Lenoir (1923), Martens (1922), Van Camp (1924), Latter (1926, 1932), Gates and Latter (1927), Zirkle (1928b, 1931).

${ }^{29}$ M. Jörgensen (1913a), A. Meyer (1917, 1920), and many others.

${ }^{30}$ Macallum (1891), Maziarski (1911), Nakahara (1917, 1918a), Saguchi (1920), Kinney (1926), Beams and Wu (1929).

31 Macallum (1912), Lubosch (1902), Rohde (1903), Ludford (1921, 1922a), Koch (1925), Gardiner (1927), Nath and Mehta (1929), Nath and Mohan (1929).

${ }^{32}$ M. Jörgensen (1913a), A. Meyer $(1917,1920)$, de Winiwarter (1925).

${ }^{33}$ Kilebs (1887, 1888), Haberlandt (1887, 1919, 1921), Gerassimow (1890 et seq.), Townsend (1897), Palla (1889, 1890, 1906), Acqua (1891, 1910), van Wisselingh (1909), Bobilioff-Preisser (1917), Heitz (1922). See Tischler (1921-1922; pp. 142147).

${ }_{34}$ Štolc (1910), Lynch (1919), Phelps (1926), Becker (1926). 
enucleate pieces may move, respire, and respond to certain stimuliactivities which depend upon catabolic processes; but they do not undergo regeneration, growth through the formation of new albuminous material, or division-activities which involve organic synthesis.

Among the many reactions involved in metabolism the most important, according to modern physiology, is oxidation, for the energy utilized by the organism is derived immediately from the union of protoplasm or of its inclusions with oxygen. A relation between the nucleus and oxidation and reduction has therefore been sought.

Following the experiments of Spitzer (1897), who observed that nucleo-proteins extracted from certain animal tissues have the same oxidizing power as the tissues themselves, it was suggested by Loeb (1899) that the nucleus is the center of oxidation in the cell. Loeb pointed out that this would explain the inability of enucleated cellfragments to undergo regeneration. This conclusion was supported by R. S. Lillie (1903, 1913), who showed that rapid oxidation occurs both at the surface of the cell and at the surface of the nucleus. Osterhout (1917) found that "injury produces in the leaf-cells of the Indian Pipe (Monotropa uniflora) a darkening which is due to oxidation. The oxidation is much more rapid in the nucleus than in the cytoplasm and the facts indicate that this is also the case with the oxidation of the uninjured cell." Other investigators ${ }^{35}$ have opposed the oxidation theory. Further evidence in its support is brought forward by Chambers (1923a). In healthy cells (amobæ, ciliated cells, echinoderm eggs) Janus green does not enter the nucleus, but as the cells become moribund the dye enters and colors the reticulum red. This probably means that the normal resistance to the penetration of the dye is lost, whereupon the reducing ability of the nucleus becomes manifest in the change of Janus green to diethylsafranin. This reducing power is lost at death.

In interpreting the results of such experiments, particularly those in which the cell is enucleated by mechanical means, it has not always been sufficiently borne in mind that some of the effects upon metabolism may be due to the injury itself rather than to any sort of normal activity in the parts which remain. It is therefore very difficult to obtain by such direct means unequivocal evidence regarding the precise manner in which the nucleus functions during the normal life of the cell. Even when the results of enucleation seem clearly to indicate a dependence of certain functions on the nucleus, they do not limit to the nucleus the performance of those functions. Removal of the cytoplasm would also cause a cessation of such activities. Each function, though it may be peculiarly subject to the differential influence of some one organ, is, in reality, an act of the protoplasmic system as a whole. The nucleus may be said "to control" an activity only in the sense that ${ }^{35}$ Lynch (1919) on Amœba, Wherry (1913), W. Schultze (1913), Reed (1915). 
it contains something necessary to the performance of that activity, much as a chemical stockroom "controls" the activities carried on in the laboratory.

In a mature organism metabolic activity maintains the individual in the living condition. In a young and developing one it also results in growth and the differentiation of structural and functional characters. The manner in which the various constituents of the protoplast participate in metabolism and hence affect the course of development is studied by observing the effects of altering this or that constituent. In the case of the nucleus it has been found that differences in its constitution are associated with particularly clear-cut differences in certain of the characters developed. This leads to the subject of the rôle of the nucleus in development and heredity, which is to occupy our attention in later chapters of the book. 


\section{CHAPTER IV}

\section{PLASTIDS}

The most conspicuous cytoplasmic differentiations in plants are plastids. They may be looked upon as regions of the protoplast which have become structurally specialized mainly in connection with carbohydrate synthesis and transformation; hence they serve to call attention to one of the most profound metabolic differences between plants and animals. The total plastid outfit of the protoplast has been called the plastidome by Dangeard. ${ }^{1}$

Occurrence and General Characters.-Plastids are of almost universal occurrence in the tissues of plants, where they are found in one form or another in all groups with the possible exception of bacteria, blue-green algæ, myxomycetes, and certain fungi. Within a single cell there may regularly be but one plastid, as in many algæ, Anthoceros, and the meristematic cells of Selaginella; or two, as in Zygnema; or a higher number, as in the green tissues of most higher plants. They lie imbedded in the cytoplasm and are often closely associated with the nucleus; they are never found normally in the vacuole. The positions which they assume within the cell are frequently related in a definite manner to certain external conditions, as in palisade and stomatal cells of green leaves. ${ }^{2}$

Although they may vary considerably in size, Möbius (1920) found that 75 per cent of the plastids in 215 species of plants he examined had a long diameter lying between 4 and $6 \mu$. The size may be affected by temperature (O. Hartmann, 1919a). It seems that in some cases there is a relation between the size of the plastids and that of the cells in which they lie (Gates, 1923). Eyster (1929) observed characteristic size differences in certain genetic strains of maize.

Plastids are usually classified on the basis of the colors of their contained pigments. The arbitrary nature of such a classification is apparent when it is realized that two plastids of the same color, or with no color whatever, may be performing very different functions by virtue of unlike substances not visible to the eye; and, further, that the same plastid may be colorless, green, and yellow or red at different stages in its history. A separation on the basis of color is, however, very convenient and usually does correspond to functional diversity. This is shown by

${ }^{1}$ For general accounts of plastids, see A. Meyer (1883b), Schimper (1883, 1885), Senn (1908), and Schürhoff (1924).

2 Schimper (1885), Senn (1908), Haberlandt (1918), Weber (1925a). 
the interesting fact that the thallophyte groups with different predominating pigments are characterized by different principal products of metabolic activity. In the higher plants and the grass-green algæ this product is usually starch, in the yellow-green algæ and diatoms it is oil, in the brown algæ it is pentosan, in the red algæ (Florideæ) it is Floridean starch, and in the blue-green algæ it is glycogen.

Leucoplasts.-All colorless plastids, regardless of their size, function, or relation to other types of plastids, are known as leucoplasts (Fig. 25). They are found commonly in meristematic tissue and may be retained
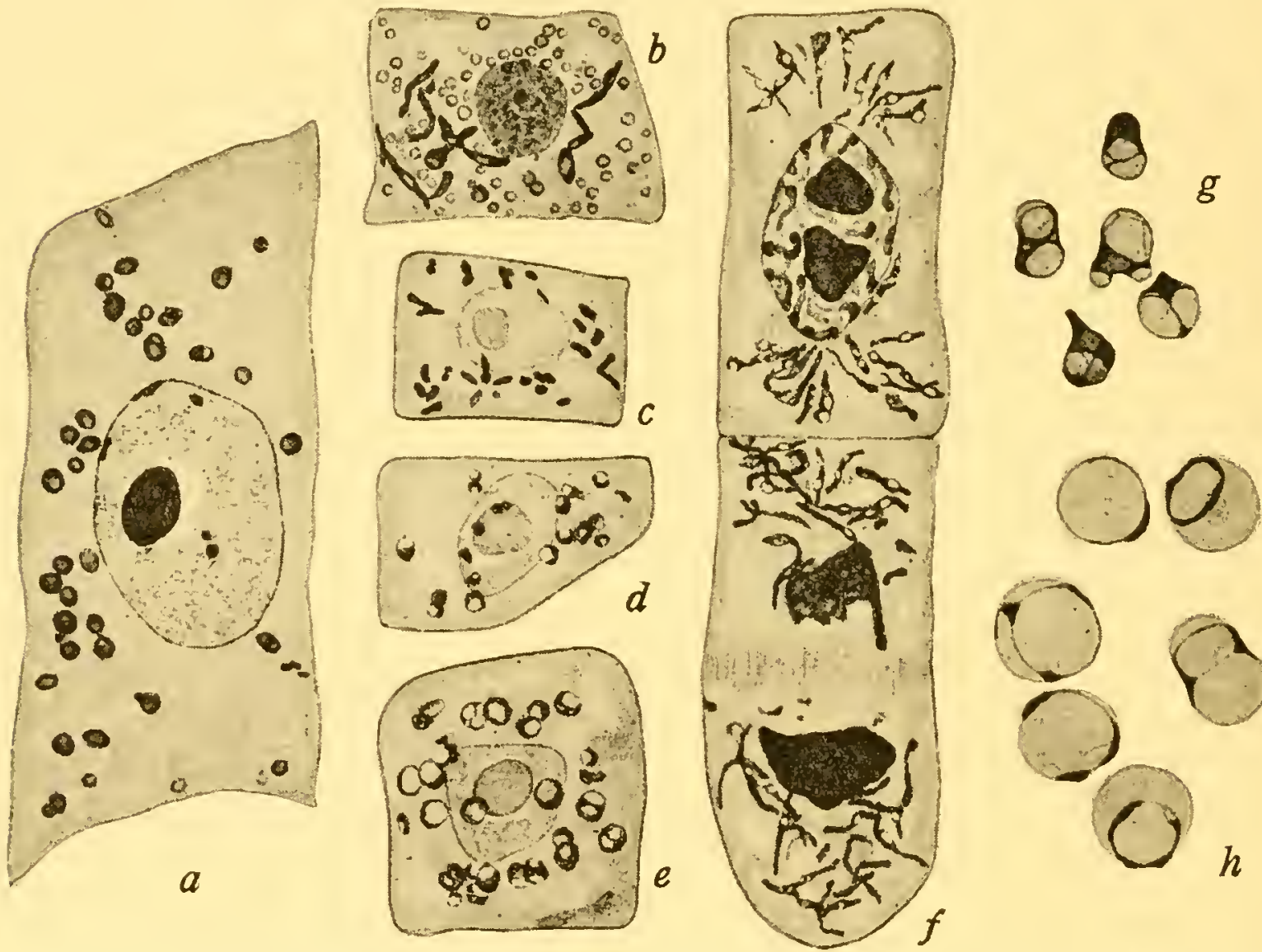

FIG. 25.-Leucoplasts in root cells. a-e, Vicia Faba; $f, g$, Pisum sativum; h, Hyacinthus orientalis. The smaller bodies in $b$ are chondriosomes. The light material in the enlarging leucoplasts is starch. (After Bowen, 1929a.)

in some kinds of differentiated cells, such as the glandular hairs of Pelargonium. The leucoplast in Orchis is of a very fluid consistency, undergoing amœboid changes of shape and multiplying by irregular fission (Küster, 1911). In Polytoma uvella it forms a reticulum or series of bands (Volkonsky, 1930). Many smaller leucoplasts represent juvenile stages in the development of plastids of more highly differentiated types, for under certain conditions they develop into the larger and more specialized leucoplasts known as amyloplasts (Fig. 28, C) and into the various kinds of chromoplasts mentioned below.

Chromoplasts.-Chromoplasts are plastids bearing one or more pigments. They are frequently called "chromatophores," but, since this 
term has been used with special reference to the plastids of algæ and also for certain pigmented cells in animals, chromoplast is preferable as a general term for all colored plastids. ${ }^{3}$

Of all chromoplasts the most familiar is the chloroplast, whose color is due to the presence of the green pigment chlorophyll. Chloroplasts are usually spherical, ovoid, or discoid in shape, but many bizarre forms are known particularly among the green algæ. For example, in Edo-

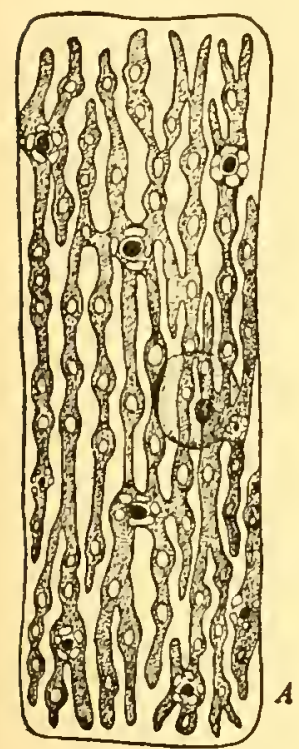

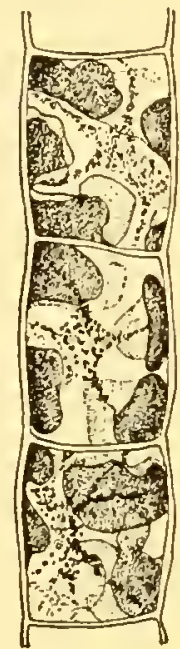

$\boldsymbol{B}$

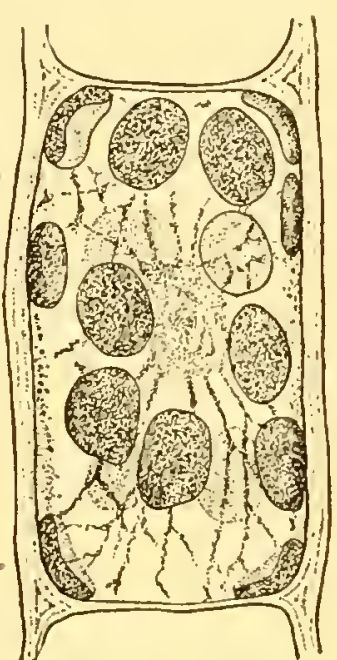

C

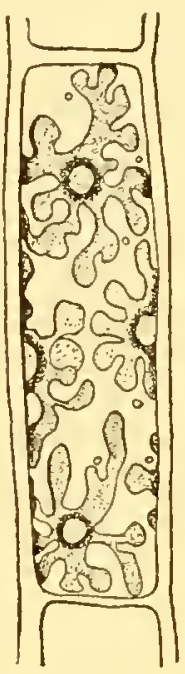

$D$

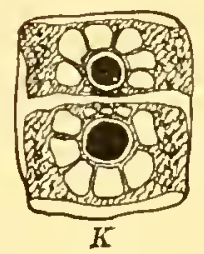

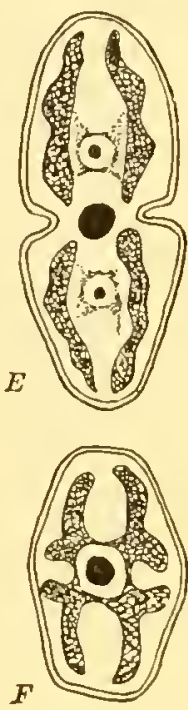

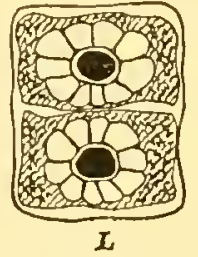

FIG. 26.-Various types of plastids. A, Edogonium, showing pyrenoids and stroma starch. B, Leptonema fasciculatum. C, Pilayella varia. D, Rhodochorton floridulum. $E, F$, Euastrum dubium, front and side views. $G-L$, Hyalotheca mucosa, showing division of plastid and pyrenoid with its surrounding starch masses. (From the works of Schmitz, Reinke, Kuckuck, and Carter.)

gonium the plastid has the form of an irregular net, while in Spirogyra it is a coiled ribbon and in desmids a series of radiating plates (Figs. 26, 27). The plastid of Anthoceros is spindle shaped and becomes chainlike in elongated columella cells (Scherrer, 1914). Chromoplasts are often sharply angular, owing in part to the presence of crystals.

It has been reported for several algæ that the chlorophyll is diffused throughout the cytoplasm, not being confined to definite plastids. In the case of Hydrodictyon, however, Lowe and Lloyd (1928) have shown that chloroplasts can be detected by the use of light lying in the region of the absorption bands of chlorophyll.

"A more common praetice is to limit the term "ehromoplast" to plastids with colors other than green, the chloroplast ("autoplast," Meyer) being placed in a class by itself. For accounts of ehromoplasts in this sense, see Rothert (1912) and A. Meyer (1926). 
The most important of all plastid pigments is chlorophyll, because of its peculiar relation to the elaboration of carbohydrates by chloroplasts. Chlorophyll ordinarily develops in the plastid only in the presence of light; apparent exceptions are found in certain woods and the embryo and endosperm of certain seeds. Other conditions necessary for the development of chlorophyll are a favorable temperature and the presence of iron, oxygen, and certain carbohydrates. Most young seedlings grown in the

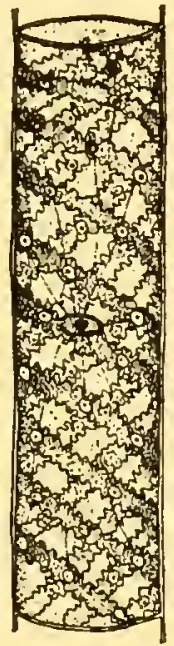

FI G. 27 .Cell of Spirogyra, with spiral chloroplasts. The nucleus is suspended in the middle of the cell by a series of cy toplasmic strands which meet the perip heral cytoplasm near the pyrenoids. (After Couch, 1931b.) is in chromoplasts, but red pigment in the same flower is held in solution in the cell sap. ${ }^{4}$

Photosynthesis.-Of all chromoplasts the chloroplasts stand first in importance, for by virtue of their chlorophyll they are able, in the presence of light, to recombine the elements of carbon dioxide and water in such a way as to form certain carbohydrates, oxygen being given off as a by-product. In this process the energy of sunlight is fixed, while in respiration this energy is again released for use by the organism. Repre-

${ }^{4}$ For accounts of plastid pigments, see the works of Palladin (1923), Onslow (1923), R. W. Thatcher (1921), Beauverie (1919), Willstätter and Stoll (1913), Jörgensen and Stiles (1917), Haas and Hill (1928), Rigg (1924), Oltmanns (1923), Palmer (1922), Fulton (1922), Lloyd (1924), Möbius (1927), and Gortner (1929). The distribution of carotin is discussed in an earlier paper by Tammes (1900). 
senting the energy involved in terms of calories, the reactions may be written as follows (Gortner):

Photosynthesis: $6 \mathrm{CO}_{2}+6 \mathrm{H}_{2} \mathrm{O}+677.2$ calories $=\mathrm{C}_{6} \mathrm{H}_{12} \mathrm{O}_{6}+6 \mathrm{O}_{2}$.

Respiration: $\quad \mathrm{C}_{6} \mathrm{H}_{12} \mathrm{O}_{6}+6 \mathrm{O}_{2}=6 \mathrm{CO}_{2}+6 \mathrm{H}_{2} \mathrm{O}+677.2$ calories.

The carbohydrate first elaborated is presumably in solution and therefore invisible. In many plants this product either accumulates in the dissolved state or is removed from the chloroplasts as fast as it is formed. Very commonly, however, there is a temporary excess, which is deposited as minute granules of starch in the chloroplasts. This "assimilation starch" is therefore the first visible product of photosynthesis in most green plants; in some species the product is evidently an oil. As Meyer originally showed, the starch is actually synthesized within the body of the chloroplast (Fig. 28, A). It is later transformed through the agency of enzymes into some soluble compound, usually a sugar, in which form it may be carried to growing regions where, after further changes, it is built into the structure of the plant, or it may pass to storage organs where it is transformed into "reserve" or "storage" starch. This deposition of reserve starch is brought about through the agency of amyloplasts, which are leucoplasts capable of changing already elaborated organic materials, such as glucose, into starch (Fig. 28, C).

The statement made by Schimper (1880) and Meyer $(1883,1895)$ that starch is always formed by plastids still holds good in its essential feature: so far as is certainly known, no primary product of photosynthesis is formed in the cytoplasm apart from plastids. The granules of "Floridean starch" in the red algæ lie in the cytoplasm, but they begin to form at the surface of the plastid as flat plates which become cup shaped or conical, with their flattened sides
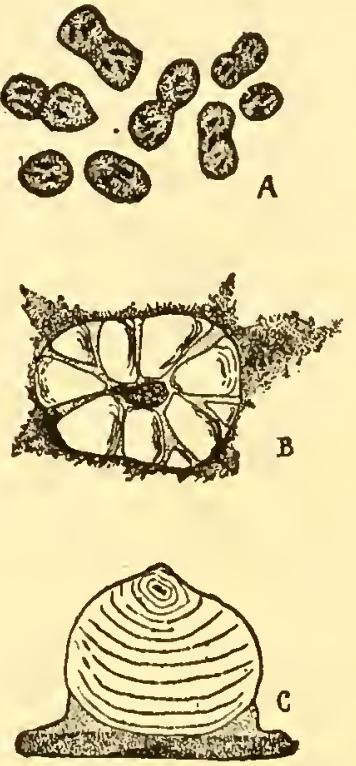

FIg. 28.-Formation of starch by plastids. $A$, dividing chloroplasts of Funaria, with grains of assimilation starch. (After Strasburger.) $B$, chloroplast of $Z y g-$ nema, with several large starch grains about a central pyrenoid. (After Bourquin, 1917.) $C$, leucoplast (amyloplast) in tuber of Phajus grandifolius, with grain of reserve starch. (After Strasburger.) remaining in contact with the plastid as long as they continue to grow. ${ }^{5}$ It is, however, reported that they are formed some distance away from the plastids in some species (Mangenot, 1923a). If such is actually the case, it is highly probable that they arise through the transformation of a non-visible product (sugar?) of the photosynthetic activity of the plastids, and are not immediately built up from water and carbon dioxide. A similar interpretation may be placed upon corresponding appearances reported in higher plants.

${ }^{5}$ Henckel (1901), Kylin (1913). 
Elaioplasts.-Special plastids concerned in the elaboration of oil are known in a number of angiosperms ${ }^{6}$ (Fig. 29). Küster and Harper have shown in the case of Ornithogalum that the plastid can be recognized before oil appears in it, and that the abundant oil which is present later may be extruded on its surface when the tissue is prepared for observation. Wóycicki has observed the fusion of small elaioplasts containing fat droplets to form a large "oleosome" with a central fat drop. In old cells the oleosomes degenerate. Since oil may also occur as droplets directly in the cytoplasm, Harper suggests that much of the current

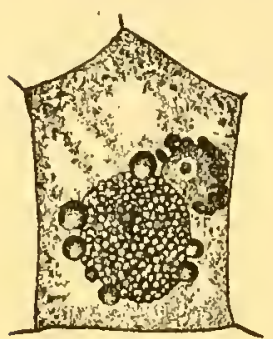

FI G. 29 .Elaioplast with oil droplets in epidermal cell of perianth of Polianthes tuberosa; nucleus with small plastids at right. (After Politis, 1914a.) difference of opinion regarding elaioplasts may be due to the fact that among these bodies there may be transitional forms between oily ergastic accumulations not causing any cytoplasmic differentiation and definite cytoplasmic organs in which the production of the oil is localized. Some reported "elaioplasts" seem to be only groups of disorganizing plastids with their products.

The Eyespot.-The so-called eyespot, or stigma, present in the flagellates and in the zoöspores and gametes of many algæ has certain characteristics in common with plastids and may therefore receive consideration here. This body, which nearly all workers agree is a light-sensitive organ, is an elongated or circular and flattened structure lying in the anterior region of the cell (flagellates) or near its lateral margin, usually in close association with the chromatophore and the plasma membrane ${ }^{7}$ (Fig. 30). With respect to its mode of origin, it has been variously reported to arise de novo in each newly formed zoöspore in several green algæ (Overton), to multiply by fission at the time of celldivision in flagellates (Klebs), to disperse as granules which reassemble in the daughter cells in Euglena (Jahn), to develop from a plastid in the antheridial cell in the case of the spermatozoid of $F^{r} u c u s,{ }^{8}$ and to arise as a differentiated portion of the plastid in the zoöspores and gametes of Zanardinia (Yamanouchi).

It is thought that the eyespot in many instances consists of a finely reticulate stroma in which an oily red pigment with many of the characteristics of hematochrome is held in the form of minute droplets or granules. $^{9} \quad$ The stroma may also bear one or more refractive inclusions, which in the Chlamydomonadaceæ and Volvocaceæ consist of starch and in the Euglenoidex of paramylum. These inclusions were thought by Franzé to

${ }^{6}$ Wakker (1888), A. Zimmermann (1893), Raciborski (1893), Küster (1894), Beer (1909a), Politis (1914a), Guilliermond (1922b), Harper (1929), Wóycicki (1929b).

${ }^{7}$ E. Overton (1889), Klebs (1883, 1892), L. N. Johnson (1893), Strasburger (1900a), Wollenweber (1907, 1908), Hall and Jahn (1929).

${ }^{8}$ Guignard (1889), Mangenot (1920e, 1921), Kylin (1920).

${ }^{9}$ Schilling (1891), Klebs (1883), Franzé (1893), Wager (1900a), Wollenweber (1907, 1908). 
increase the sensitivity of the eyespot by concentrating the light at certain points.

Of a different type is the eyespot in the zoöspore of Cladophora (Strasburger, 1900a), which appears to arise as a swelling of the ectoplast and to consist of an external pigmented layer with a lens-shaped mass of hyaline substance beneath it (Fig. 30, B). In Gonium and Eudorina (Mast, 1916) the lensshaped portion lies outside with the cupshaped opaque portion under it (Fig. 30, A), an arrangement suggesting the primitive eyes of certain animals. In neither portion has any finer structure been detected. Mast has shown that the orientation of the colony is brought about through changes in the intensity of the light falling upon the light-sensitive substance. As the unoriented swimming colony rotates on its axis, those zoöids turning away from the light have the hyaline portion of the eyespot shaded by the opaque cup; this sudden reduction in the amount of light energy received brings about an increase in the activity of the flagella of those zoöids, so that the colony as a whole turns more directly toward the source of light.

The Structure of the Chloroplast.- $\mathrm{No}_{\mathrm{O}}$ definite visible membrane has been demonstrated about the chloroplast, although it appears to be surrounded by a thin layer of modified cytoplasm. ${ }^{10}$ A prevalent view regarding the interior of the chloroplast is that it consists of a mass of somewhat denser cytoplasm, the stroma, in which there is a lipide carrying chlorophyll. Starch granules appear at one or more points in the stroma; hence
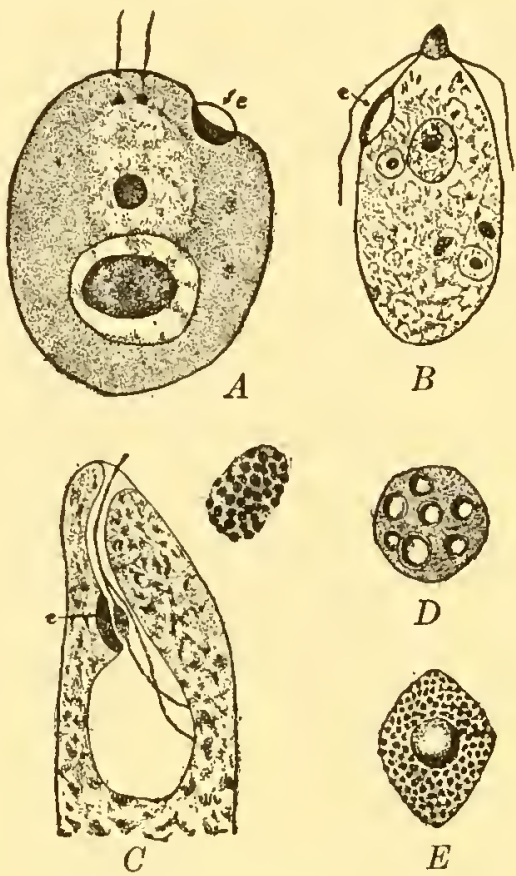

Fig. 30.-Eyespots of various types. A, zoöid of Eudorina; $e$, eyespot. (From Mast, after Grave.) B, zoöspore of Cladophora. (After Strasburger, 1900.) $C$, anterior end of Euglena viridis, showing eyespot at surface of cesophagus, and near it a swelling on one root of the flagellum; face view of eyespot at right, showing pigment granules. (After Wager, 1900.) D, eyespot of Euglena velata. $E$, eyespot of Trachelomonas volvocina, with pigment granules and crystalloid body. ( $D$ and $E$ after Franzé, 1893.) after fixation the chloroplast may appear like a hollow sphere, a honeycombed mass, or a series of threads or plates separating lighter regions (Fig. 31) in which starch may be demonstrated. It is claimed by Zirkle that one or more such regions, or "cavities," are present before starch appears in them and that they communicate with the exterior through fine pores (Fig. 32).

Because of the behavior of extruded chloroplasts in light, the reactions of green tissues to enzymes, organic solvents, and stains, and the absorp-

${ }^{10}$ Fitting (1909), A. Meyer (1920, 1922), Zirkle (1926). Zirkle reviews early views regarding plastid structure. 
tion spectra of chlorophyll mixtures it has been suggested that the chlorophyll may be in the colloidal state or adsorbed at the surface of colloidal particles in the stroma. ${ }^{11}$ K. Stern (1921), however, showed that chlorophyll exhibits its characteristic fluorescence only when molecularly dispersed, as in a true solution; moreover, he reported that chlorophyll mixtures are fluorescent only when lipide is present. ${ }^{12}$ Such facts indicate that chlorophyll, itself in the molecular state, exists in and perhaps on colloidal lipide droplets in the stroma.

A special structural feature of some plastids is the pyrenoid. Pyrenoids are small refractive masses of some proteinaceous substance lying in or on the chromatophores of many green algæ, red algæ, flagellates, and

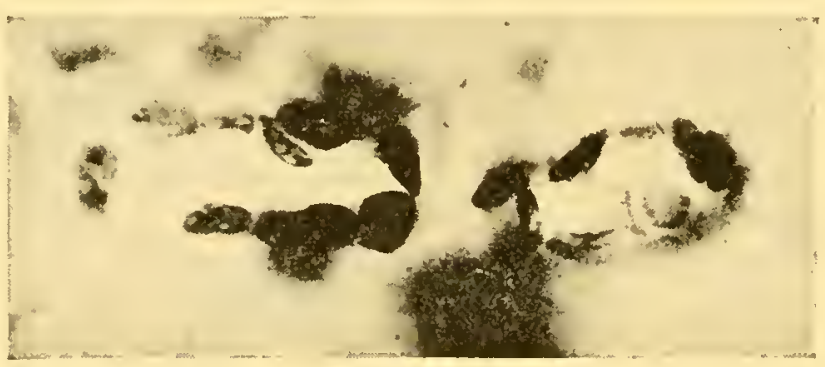

FIG. 31.

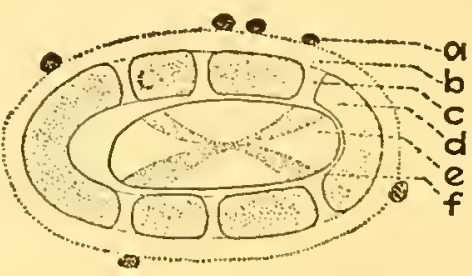

FIG. 32.

FIG. 31.-Chloroplasts in maize leaf, showing starch-containing regions. (Photograph by Weier.)

Fig. 32.-Diagram of cross section of chloroplast of Elodea as interpreted by Zirkle (1926). a, cytoplasmic granule adhering to the cytoplasmic sheath $(b) ; c$, pore in stroma; $d$, stroma; $e$, starch inclusion as it appears in polarized light; $f$, central cavity.

certain liverworts. ${ }^{13}$ They have been reported to originate de novo, ${ }^{14}$ by division, ${ }^{15}$ or by both methods. ${ }^{16}$ During the reproductive phases they may vanish and reappear. Pyrenoids may be simple (Protococcaceæ, probably), double (Ulothrix), or multiple (Conjugatæ, Anthoceros).

It seems clear that the pyrenoid is associated in some way with the synthetic processes carried on by plastids, although the precise nature of this association is by no means clear. When the product of synthesis is starch, as is commonly the case in Conjugatæ and Chlorophyceæ, it often appears as a series of grains about the pyrenoid (Fig. 26, $G$ ), but in other cases (e.g., certain Rhodophycex) the starch appears outside the plastid so that the pyrenoid is surrounded by no such "starch sheath." Some algæ with pyrenoids, for example certain diatoms and Chrysophyceæ,

1 Tswett (1906), Willstätter and Stoll (1913, 1918), Zirkle (1926).

12 See Lloyd (1924) on the fluorescent colors of plants.

${ }_{13}$ See the general account by Geitler (1926) and the paper by Czurda (1928).

${ }^{14}$ Schmitz $(1882,1884)$, Sehimper (1885), G. M. Smith $(1913,1914)$ on Tetradesmus and Scenedesmus, W. Zimmermann (1921) on Volvox, Hazen (1922) on Brachiomonas, Kater (1929a) on Chlamydomonas.

${ }^{15}$ M. Hartmann (1921) on Eudorina, Ishikawa (1921a) and Ikari (1923) on Porphyra, Bold (1931) on Chlorococcum.

${ }_{16}$ MeAllister (1914, 1927) on Anthoceros, Peterschilka (1922) on Mougeotia, N. Carter (1926) on Ulva, Czurda $(1928,1929)$ on Spirogyra, Geitler (1926). 
form no starch at all, the products of synthesis being compounds of other kinds (p. 62). In Anthoceros it is reported that the outer elements of the group of "pyrenoid bodies" develop starch while the inner ones multiply by division. In Selaginella also, the starch appears to develop from such proteinaceous bodies (R. Ma, 1930).

Origin and Multiplication.-The differentiated plastids seen in mature tissues may be traced back to plastid primordia, or proplastids, in the young cells of the meristem or embryo. ${ }^{17}$ These appear in the cytoplasm of living cells as minute bodies grading off to the limit of visibility. ${ }^{18}$ In the leaf they enlarge, sometimes becoming rod shaped, and develop chlorophyll (Fig. 33) when they are still no more than $0.5 \mu$ in diameter. Starch grains can be detected inside of them with polarized

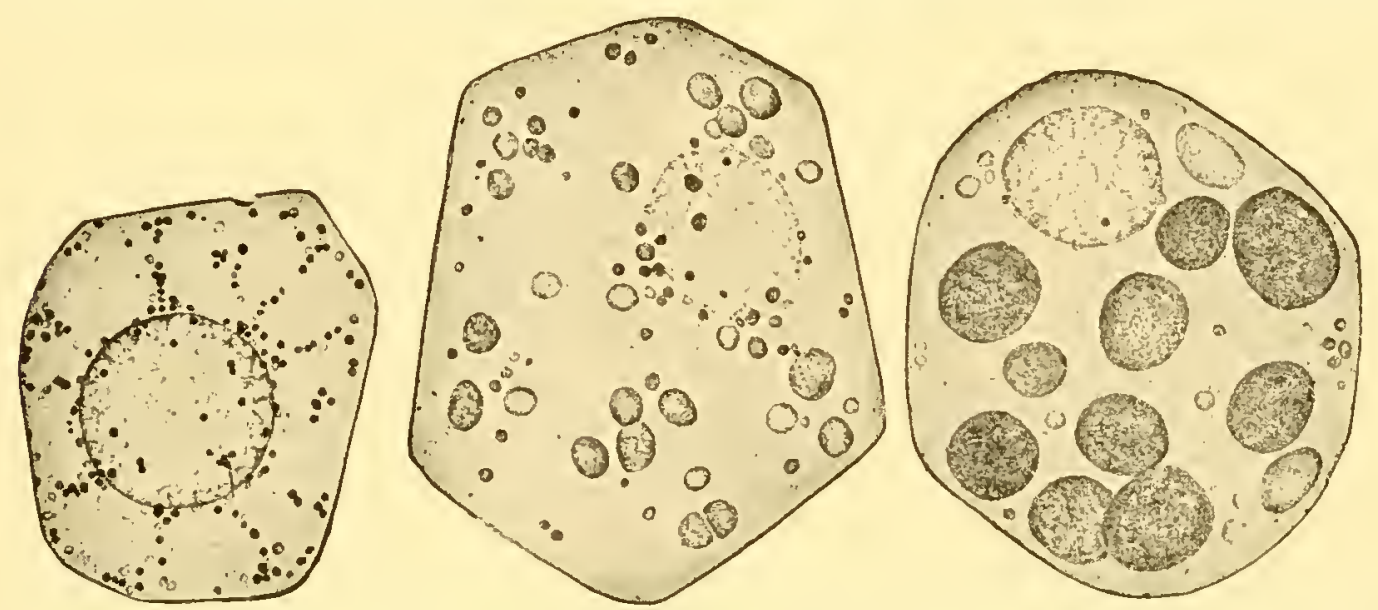

FIG. 33.-Development of chloroplasts from their primordia (proplastids) in living mesophyll of Zea Mays. (After Randolph, 1922.)

light (Zirkle, 1927a). They may be markedly altered by fixing reagents, ${ }^{19}$ fluids containing potassium bichromate often causing them to elongate and acetic acid destroying them altogether; later on they become more resistant to the latter reagent. After osmication or silver impregnation they may appear like hollow structures with a blackened periphery.

At all stages up to maturity chloroplasts can be seen to divide, although constriction often occurs without resulting in division (Kassmann). Division generally seems to occur in either of two ways: by simple constriction or through the formation of a hyaline cleavage zone. The process may occupy many hours or even several days. ${ }^{20}$ Few, if any, proplastids are left by the time the leaf-cell becomes fully differentiated. In the cells of the root tip the development of the leucoplasts is much the same, except for the absence of chlorophyll. Their division appears to be

${ }_{17}$ Pensa, Lewitsky, Guilliermond, Dangeard, Emberger, Noack, Friedrichs, Mottier, Randolph, Kassmann, Kiyohara, Bowen, Zirkle, Weier, Motte, and others.

${ }^{18}$ Randolph (1922) on Zea, Kassmann (1926) on Cabomba (chondriosomes ?, see p. 85 of this book). Bowen $(1929 a)$ disagrees for Vicia.

${ }^{19}$ Zirkle (1927a), Kiyohara (1927, 1930), Weier (1931a).

${ }^{20}$ Heitz (1922), Kiyohara (1926). 
independent of that of the nucleus and cell, although they may assume a characteristic polarized arrangement about the mitotic figure (Fig. 25, $f$ ).

It was believed by the early observers, notably Schimper (1883) and Meyer (1883), that plastids never originate de novo but always arise from preëxisting plastids by division. This view has the recent support of Bowen (1929a). Fully differentiated plastids, such as chloroplasts, can readily be seen multiplying in this manner in growing tissues with a frequency sufficient to account for the large number of plastids present in mature plant parts. However, since the individual plant arises from a zygote or a spore in which the plastids are usually in a relatively undifferentiated state, the problem of the individuality of the plastid is mainly one of determining whether these undifferentiated "plastid primordia," later developing into chloroplasts and other types, are continuous through the critical stages of the life cycle, multiplying only by division, or arise de novo as new differentiations of the cytoplasm.

Light on this question has been sought in a number of investigations on bryophytes. ${ }^{21}$ In Anthoceros each cell of the gametophyte contains a single plastid which divides with the nucleus at each cell-division. The egg likewise contains a plastid, but apparently the spermatozoid has none; the zygote and sporophyte cells which it later forms are, therefore, like the cells of the gametophyte, characterized by the presence of one plastid. Although it is difficult to demonstrate the plastid in the young sporogenous cells, every sporocyte shows one very clearly. The sporocyte plastid divides twice as the sporocyte begins to divide into a quartet of spores so that each spore receives one plastid. Upon germination the spore produces a gametophyte with one plastid in each cell, and the cycle is complete (Davis, Scherrer). According to Scherrer, the plastids remain as morphological individuals throughout the whole life cycle, multiplying exclusively by division. A similar claim is made for the plastids of mosses by Sapěhin.

More recent studies have added much to our knowledge of plastid behavior in the reproductive phases. In Polytrichum commune, Weier has followed the plastids through both sporogenesis and spermatogenesis. The cells of the young sporophyte contain several small plastids which multiply by division, and in each sporocyte there are usually two larger ones. In fixed preparations these show a deeply staining peripheral portion ("plastonema") and a lighter internal region ("plastosome") (Fig. 34). They pass through a temporary phase in which they appear loose and granular; they then divide to form four which are included in the four spores as the sporocyte divides. In the spore the plastid soon assumes a structure characteristic of the chloroplast. In the antherid-

21 Davis (1899), Scherrer (1914), Sapěhin (1915; see also 1911 and 1913, on pteridophytes), Senjaninova (1927b), Motte (1928), Weier (1930a, 1931ab), Chalaud (1929-1930). 
ium, also, the plastid shows a plastosome substance and a plastonema in the form of a network or series of plates. In the androcyte (spermatid), or cell which is to become a spermatozoid, the plastid becomes a large limosphere; this buds off a small portion which becomes an apical body at the anterior end of the spermatozoid (see Chapter XIV). In the sporocyte of Catherinox undulata the large plastid is said to be formed by the aggregation of several small ones present in earlier stages (Senjaninova). The plastid lying in each spore divides to several, each of which consists of a series of plate-like elements and clearer regions containing starch. ${ }^{22}$

In such cases as those just described the plastids in the spores divide and develop into those of the ensuing gametophyte generation. These in turn give rise to the plastids of the egg, in which, as a rule, they seem to be
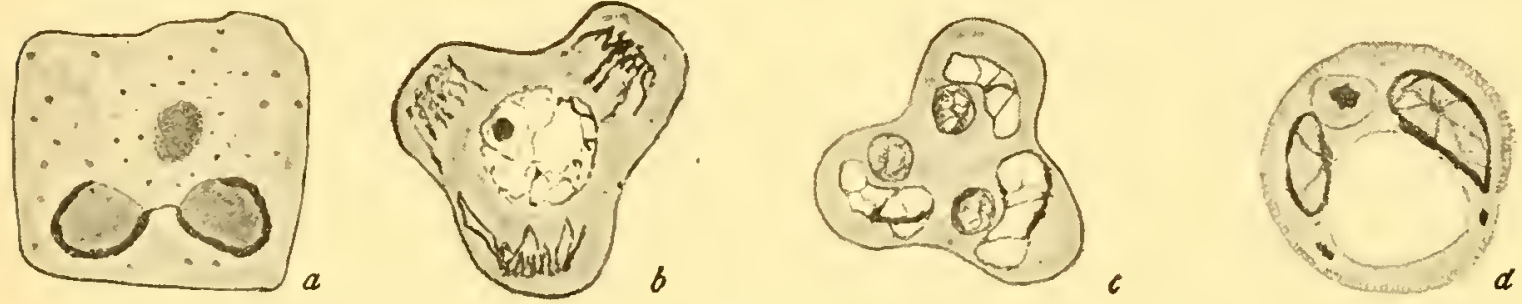

F1G. 34.-Behavior of plastids during sporogenesis in Polytrichum commune. a, division of plastid being completed in archesporial cell. $b$, reticulate stage of plastids in lobed sporocyte. $c$, quartet nuclei formed; each cell after cytokinesis will contain one plastid. $d$, young spore with two plastids formed by division. (After Weier, 1931a.)

reduced to primordia or small leucoplasts. The spermatozoid carries a plastid product (apical body) but apparently no true plastid; hence the plastidome of the sporophyte is derived from that of the egg.

Our knowledge of parallel phenomena in vascular plants is rather meager. In Ginkgo each microspore receives about one-fourth of the plastids of the sporocyte (Mann, 1924). A number of investigators ${ }^{23}$ have described a more or less equal distribution of numerous small bodies to the four spores as the sporocyte divides. Although these bodies have been referred to wholly or in part as chondriosomes, it is obvious that at least some of them are plastids or their primordia. Very little is known about plastids in angiosperm gametes. Guilliermond (1924) figures small plastids or chrondriosomes in the egg of Lilium. Ruhland and Wetzel (1924) find that the chloroplasts gradually lose their color and come to resemble chondriosomes in the generative cell in the pollen tube of Lupinus luteus, but their behavior in the uniting gametes

${ }^{22}$ In this and several other works in this field the minute bodies developing into or arising from plastids are referred to as "chondriosomes," and the thready or platelike elements of the plastid are regarded as "chondriosomal" in nature. This involves a puzzling question diseussed in Chap. VI.

${ }^{23}$ Nicolosi-Roncati (1910), Wóycicki (1912), Wagner (1915), Guilliermond (1920e, 1924), Suessenguth (1921), Devisé (1922), Lewitsky (1925), Senjaninova (1927c), Py (1929). See the review by Wagner (1927a). 
has not been reported. There is genetic evidence that plastid primordia are transmitted by both parents in some species and by the mother alone in others (Chapter XXV).

A single interesting case from the algæ may be cited. In Zygnema each vegetative cell contains one nucleus and two plastids, all of which divide at each vegetative cell-division. In sexual reproduction the entire protoplast, with its nucleus and two plastids, passes through the conjugating tube as a "male" gamete and unites with a similar complete protoplast ("female" gamete) of another filament. The two nuclei fuse, giving the primary nucleus of the new individual (zygospore nucleus), while the two plastids contributed by the male gamete degenerate, leaving the two furnished by the female gamete as the plastids of the new individual (Kurssanow, 1911). A similar process occurs in Spirogyra (Tröndle, 1911) (Fig. 162).

Conclusions. - With the possible exception of certain bacteria and some other protista, the entire organic world is dependent for its sustenance upon plastid activity, for it is by virtue of their chloroplasts that green plants are able to synthesize carbon-containing food necessary to the life of both plants and animals. Many theoretical questions, including that of the mechanism of heredity, involve the history of plastids as individuals. So far our knowledge of this history is incomplete. In some organisms plastids can be traced with fair confidence through the life cycle, but in most cases the situation is not clear. Not only is it difficult to obtain satisfactory evidence for the division of the smallest visible proplastids, but it is wholly impossible to determine by direct cytological observation what may be their history before they have passed above the limit of visibility. Hence "the question regarding the extent to which the plastids are to be considered permanent cell organs with an unbroken genetic continuity throughout the life cycle must remain an open one" (Randolph). The whole problem is complicated by the debated question of the relation of plastids to chondriosomes, a topic to be discussed in Chapter VI.

It may be remarked that cytologists have often been inclined to underestimate the capacity of protoplasm for epigenetic differentiation and so have been too averse to the idea of origin de novo. If plastids represent transformations of the cytoplasm resulting from the localization of certain processes, they may well be expected to differentiate anew as these processes begin, and to preserve varying degrees of permanence depending upon the processes carried on (Harper, Pensa). Their individual continuity through certain life cycles may accordingly be interpreted to mean that in such forms there is a persistence of certain types of physiological activity through all or nearly all the stages. In the next chapter it will be of interest to compare with plastids certain cytoplasmic differentiations in animals, whose metabolism differs in important respects from that of plants. 


\section{CHAPTER V}

\section{THE GOLGI MATERIAL}

A characteristic constituent of animal cells is the Golgi material. Interest in this substance has recently been stimulated anew by suggestions concerning its possible relation to certain components of plant cells. ${ }^{1}$

Occurrence and General Characters.-In 1898 Golgi, using a modification of a method devised by Cajal for the study of nervous tissue,
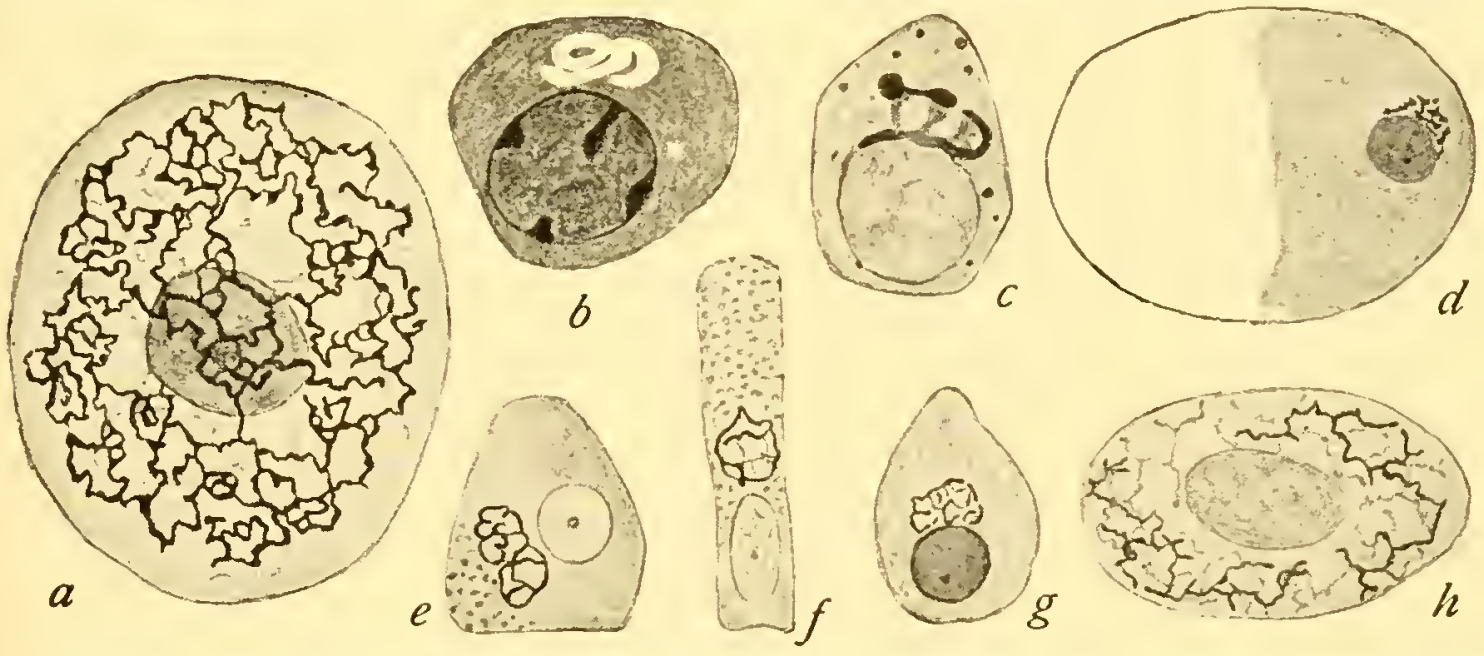

Fig. 35. - The Golgi material in animal cells of various types. $a$, spinal ganglion cell of rabbit. (After Golgi, 1899.) b, erythroblast of guinea pig, fixed in formalin and potassium bichromate. $c$, the same, fixed in osmic acid. (b and $c$ after $E$. V. Cowdry, 1921.) $d$, fat cell from human skin. (After Deineka, 1912.) e, cell from pancreas of cat. $f$, epithelial cell from prostate gland of dog. $g$, cartilage cell from cat. ( $e-g$ after von Bergen, 1904.) $h$, red blood corpuscle of frog, prepared by Golgi's silver impregnation method. (After Sinigaglia, 1910.)

found in the Purkinje cells of the barn owl's brain a structure which he called the "internal reticular apparatus." It consisted of a closed net of fine fibrils in a rather definite region of the cytoplasm between the nucleus and the cell surface. Subsequent investigation of a great variety of tissues from many animals showed the "Golgi apparatus" to be of general occurrence although varying considerably in form (Fig. 35). After the customary methods it commonly appears as a network

${ }^{1}$ For general accounts, see von Bergen (1904), Duesberg (1914ab), Cajal (1914), Pappenheimer (1916), E. V. Cowdry (1923b, 1924a), Nath (1926), Kopseh (1926) on human tissues, KKing (1927) on Protozoa, W. Jacobs (1927), and Bowen (1926cj, 1929d). For flagellates, see Hall (1929) and literature there cited; also later papers of Hall on Protozoa. For microtechnical methods, see Lee (1921), Pappenheimer (1916), and Bowen $(1928 b-g)$. Valuable literature lists are given by Duesberg (1914a), Cowdry (1924a), and Bowen (1929d). 
or group of fibrils lying at one side of the nucleus or entirely surrounding it. Often it consists of a few scattered vacuole-like "Golgi bodies," as in insect spermatocytes. In Protozoa the small, rather numerous vacuoles react like Golgi material (Hall et al.). Although the largest and most complex forms are found in vertebrates (spinal ganglion cells) while the simpler non-reticulate forms prevail in invertebrates, there is no constant correlation between form and taxonomic grouping. Furthermore, within some groups the form in different types of tissue is comparatively uniform, whereas in other groups it shows great diversity. The appearances presented are nevertheless rather characteristic in different cell types and in different stages of development: the "apparatus" is large in gland cells and small in muscle cells; it is well developed in active stages of differentiation and becomes less conspicuous as the cell ages. In pathological or physiologically abnormal tissues it often shows marked alterations, the significance of which is not understood. ${ }^{2}$

It has proved very difficult to investigate the Golgi material in living cells ${ }^{3}$ because of its low visibility. It is best seen in spermatocytes. The blackened images observed after impregnation with silver (Cajal, Golgi, Da Fano) or prolonged immersion in osmic acid (Kopsch, Kolatchev) create false impressions regarding the nature of the material in the living state; the clear canals appearing after other treatments (formalin and potassium bichromate) are somewhat better. In the living cell the Golgi material seems to be a fluid which is physically rather similar to the rest of the cytoplasm. Its solubility in alcohol and reactions to osmic acid and other reagents suggest the presence of some lipide. ${ }^{4}$ The fact that impregnated globules or strands of Golgi material often show chromophilic and chromophobic portions suggests that two main components are present, though not necessarily with the arrangement they show in the preparations. Different treatments often result in very different images according to the way in which the components are affected. This has led to the view that in the Golgi material we have to deal with a formless fluid rather than an individualized organ. However, we shall point out farther on that the material in question has come to be regarded by some workers as one component of a specialized cytoplasmic region where a definite function is being performed.

Behavior in Cell-division.-The Golgi material is apportioned with various degrees of regularity between the daughter cells at the time of cell-division. In the spermatocytes of a snail, Paludina vivipara, Perroncito $(1909,1910)$ observed the fragmentation of the Golgi network

${ }^{2}$ Cowdry (1924a) lists the alterations reported by many observers.

${ }^{3}$ Gatenby (1920), Nath and Nangia (1931), Murray (1898), Brambell and Rau (1925), Karpova (1925), Avel (1925). See Zweibaum and Elkner (1926) and Ludford (1927) on tissue cultures. Chambers does not detect it in his microdissection studies.

${ }^{4}$ Weigl (1910, 1912), Nussbaum (1913), Gatenby (1920), W. Ma (1928b), Ma and Chang (1928), Ma, Chang, and Liu (1929). 
into a number of elongated pieces, which formed a crown-like group and then passed to the daughter cells. He called the pieces dictyosomes and the entire process dictyokinesis. He reported a similar process in certain mammals. In opossum spermatocytes, according to Duesberg (1920), the process is much less regular; the network becomes a coiled thread, irregular fragments lie scattered between the two nuclei at anaphase, and the network is reconstructed in the daughter cells. In insects, on the other hand, Bowen (1920) finds that the distribution of the Golgi material is accomplished with much precision. In Euschistus, for example, it consists of scattered plate-like bodies (Fig. 129), and, as the nucleus prepares to divide, these bodies form a circular series at the equator of the cell and then fragment, after which they pass toward the poles in advance of the chromosomes. In some species they become arranged in two ring-like groups, the migrating chromosomes passing

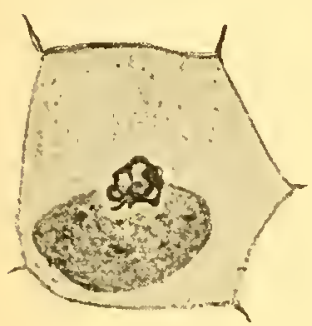

1

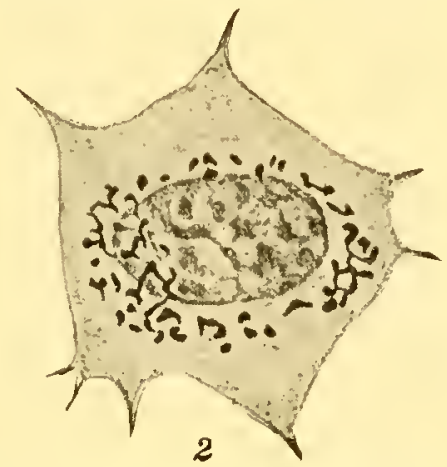

2

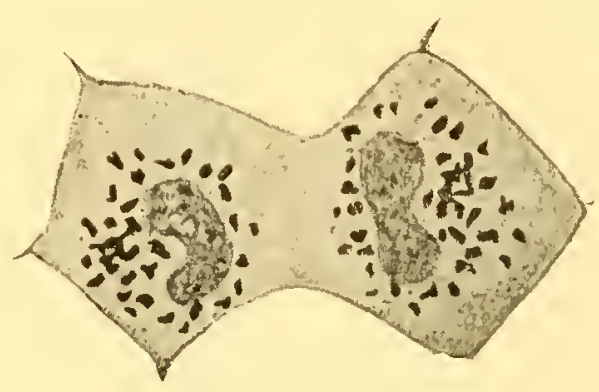

3

FIG. 36.-The division of the Golgi material (dictyokinesis) in epithelial cells of the cornea of the cat. (After Deineka, 1912)

through the rings. Eventually they lie scattered in the daughter cells and undergo further fragmentation.

The first clear account of dictyokinesis in somatic tissues was given by Deineka (1912). In the connective and epithelial tissues of the cornea in young dogs and cats he observed the fragmentation of the Golgi net into small pieces which were distributed more or less fortuitously as two groups to the daughter cells (Fig. 36). The process has been observed since in other tissues. In a protozoön, King and Gatenby (1923) find Golgi material in the form of rods which behave like those of a metazoön at the time of cell-division.

Rôle in Secretion.-Cytological phenomena in glandular tissues ${ }^{5}$ (Figs. 37 to 40 ) are of particular interest because of the evidence they furnish regarding the function of the Golgi material. Ordinarily the material lies between the nucleus and the side of the cell from which the secretion is delivered; such glandular cells have a definite physiological and morphological polarity. Cowdry (1922) and Reiss (1922) observed that a reversal in the secretory polarity is accompanied by a movement

${ }^{5}$ See the valuable review, with bibliography, by Bowen (1929d); also Special Cytology, ed. by Cowdry (1932), Secs. VI-X. 
of the Golgi material to the opposite end of the cell. The theory that the Golgi material is intimately concerned in the elaboration of secretions was placed on a firm foundation by the researches of Nassonow and of Bowen. It had long been known that the secretion in such cells appears in the form of small droplets or as small granules about which fluid accumulates (see Renaut, 1907, 1911). Parat (1928) found the fluid to be stainable with neutral red. It was clearly shown by Nassonow, Bowen, and others that these secretory droplets arise in the Golgi region. In intestinal goblet cells, for example, the secretion first appears in the form of minute granules or droplets along the strands of the basket-like

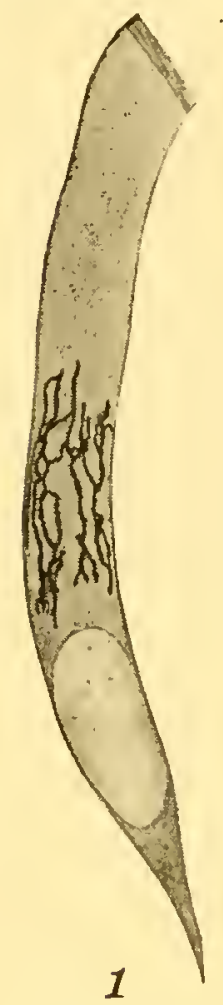

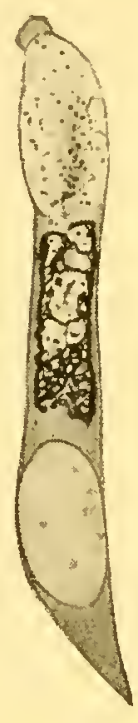

3
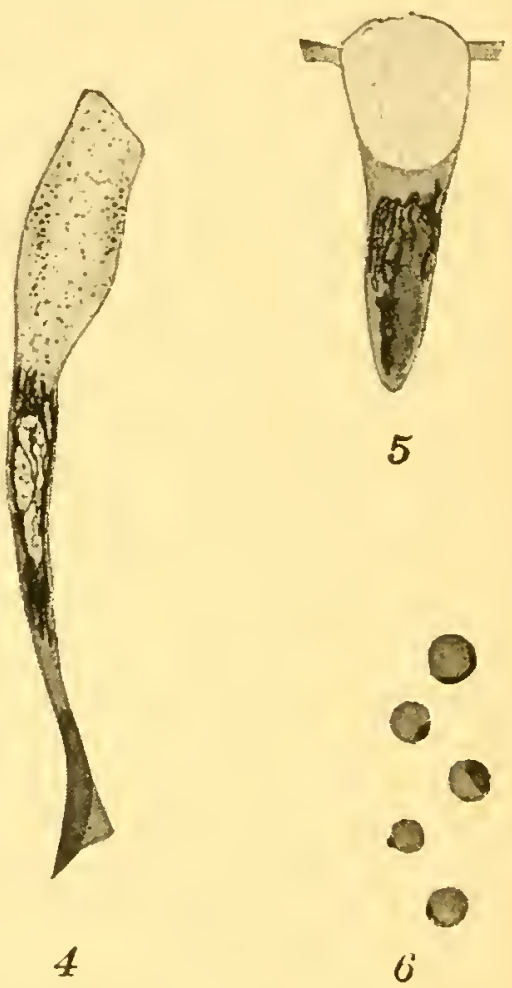

5

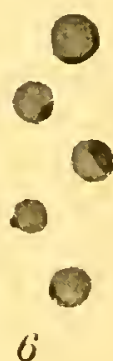

F1G 37.-The relation of the Golgi apparatus to secretion. 1-5, stages in the formation and accumulation of secretory droplets to form a typical "goblet cell" in the intestinal epithelium of Molge pyrrhogastra. 6, individual secretory granules with attached bits of Golgi material in the panereas of Cryptobranchus. (After Bowen, 1924a.)

Golgi network. The droplets pass toward the distal region of the cell, where they collect in large masses and complete their growth. As they move away from the net, each is seen to have a small chromophilic cap or girdle (Fig. 37, 6).

The principal interpretations which have been placed upon such observations may be outlined as follows. According to Nassonow and Bowen, the Golgi material in such cells is a definitely formed system with a reticulate structure like that illustrated in Fig. 37, and its special function is to produce the secretion. Whether it has a part in the actual synthesis of the secretion (Bowen) or only acts to concentrate a secretion elaborated elsewhere (Nassonow) is uncertain. The latter alternative would seem to be favored by the "secretion" of injected trypan blue, 
an acid dye, in the Golgi region of liver and kidney cells. ${ }^{6}$ The chromophilic girdle on the secretion droplet is regarded as a bit of the Golgi material. The gradual disappearance of this girdle as the droplet enlarges suggests an actual transformation of the Golgi substance into the secretion (Jacobs, 1928, 1929).

The view of Morelle (1923, 1926, 1927) is that the Golgi region, or "Golgi spot," in cells of the pancreas is a formless mass of substance
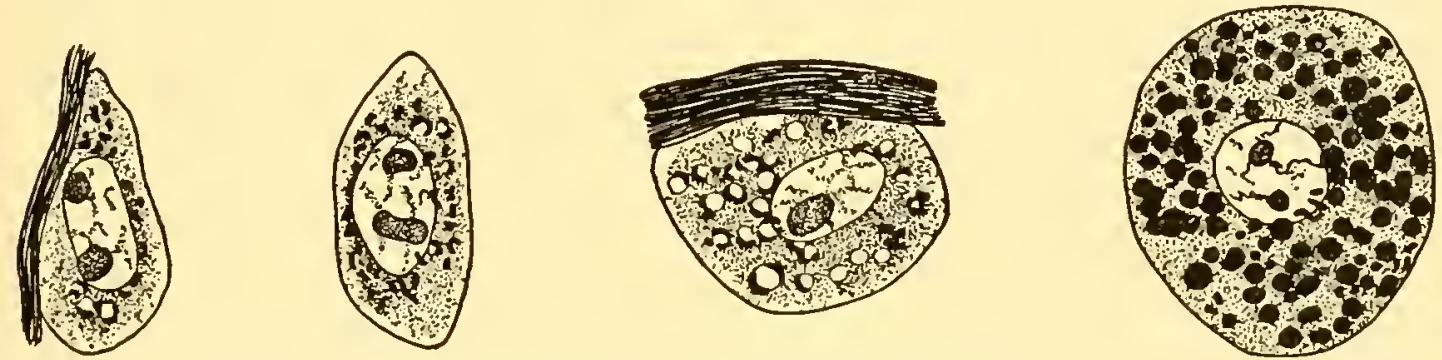

FIG. 38.-Formation of lipoidal secretion droplets by scattered Golgi bodies in sebaceous gland of mouse. (After Ludford, 1925.)

with fairly definite limits but without the organization shown in fixed preparations. The secretion droplets are formed within it, one of the materials for the synthesis being furnished by chondriosomes which appear to enter the Golgi region after being elaborated in the "basal protoplasm" (Fig. 39). The chromophilic girdle is attributed to unequal impregnation of the secretion droplet. Saguchi (1920) had previously described the formation of prozymogen granules from chondriosomes in this "secretogenous area" of the cell. Ludford (1928) suggests that a synthesis through enzyme activity occurs at the surface of the chondriosomes and that the product is concentrated in the form of droplets at the surface of the Golgi material.

Another interpretation is that of Parat and his collaborators. ${ }^{7}$ According to these workers, the cytoplasm of the animal cell includes only two constant formed elements: chondriosomes and vacuoles. In gland cells the "Golgi zone" consists of a group of secretory vacuoles, which stain with neutral red and lie in the midst of an ill-defined mass of cytoplasm containing "diffuse lipoids" and "active mitochondria"

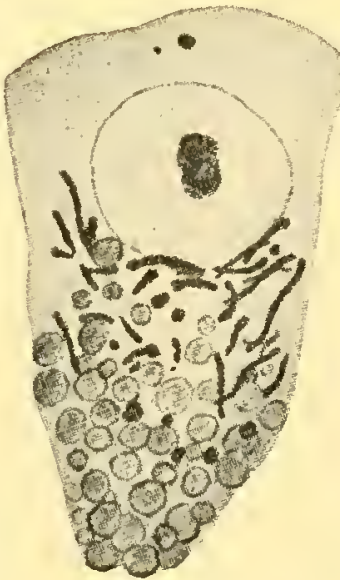

FIg. 39.-Cell of pancreas of frog. showing elongated chondriosomes in middle portion of the cell where the secretion is produced. (After Morelle, 1923.) Fig. $40, B$ ). It is not an organ with definite morphology, but a region in the cytoplasm where active secretory vacuoles accumulate and attract the other elements about them. The net-like aspects so often described

\footnotetext{
${ }^{6} J a s s w o i n ~(1925)$, Nassonow (1926), Ludford (1928).

${ }^{7}$ 1924-1930. See especially Parat (1928).
} 
are attributed to faulty preservation of the materials in and between the vacuoles. ${ }^{8}$

It will be observed that Parat holds the vacuoles to be the important
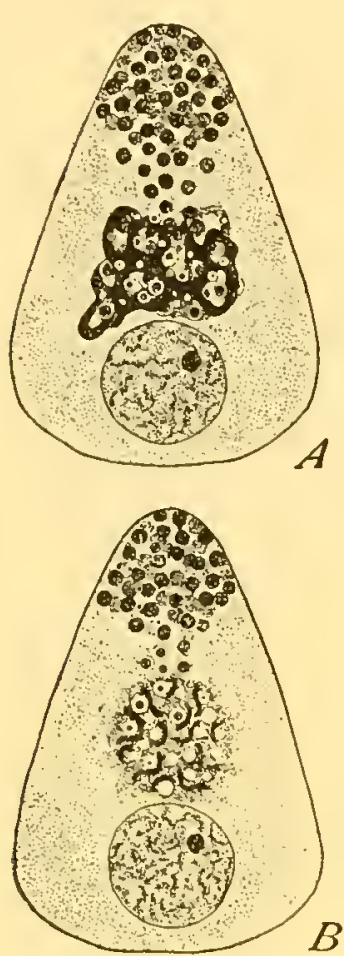

FIG. 40.-Schematized representation of two views of the architecture of the secretogenous region in a gland cell. $A$, the Golgi apparatus and its relation to the secretory droplets, according to Bowen and others. $B$, the secretory droplets (in their earlier phases, the vacuome) and their relation to the "diffuse lipoids" (more heavily stippled area) and "active chondriome" (black rods) of the secretogenous region, according to Parat, 1928 . ( After Bowen, 1929d.) active element of the Golgi region, whereas the other investigators regard them as results of an activity on the part of the material about them. Decision on the matter is scarcely warranted at present, but the fact remains clear that the Golgi region of many gland cells is intimately concerned in the function of secretion.

This general conclusion is supported by studies on oöcytes and spermatids of animals. A number of workers ${ }^{9}$ have associated the Golgi material with the production of fatty yolk in oöcytes. In the spermatid, or cell which is to transform into a spermatozoön, the Golgi material appears to form a definite body known as the acroblast; from this is differentiated and budded off an acrosome (apical body), which becomes in part the perforatorium of the spermatozoön. ${ }^{10}$ Bowen compares the development of the acrosome with the formation of secretion globules in goblet cells and inclines to the view that it, too, is a secretion of the Golgi material with a special rôle in fertilization. The topographical distinctness of the acroblast and chondriosomes is opposed to the view that the latter are incorporated in the secretion (Pollister, 1930).

The fact that Golgi material occurs in cells with no pronounced secretory activity indicates that it is not concerned solely with secretion in the ordinary sense (Cowdry, 1923b). We may adopt the provisional view that in general the Golgi material, whatever its exact form may be, characterizes a cytoplasmic region in which certain synthetic processes occur, and that in specialized gland cells we observe the results of a pronounced synthetic activity of a specific kind.

Comparison with Plant Cells.-The tendency to "homologize" structures in plants and animals has

${ }^{8}$ This interpretation of phenomena in gland eells is adversely criticized by Gatenby (1929, 1930), Beams (1930ab), and Beams and King (1932). Nath and Nangia (1931) distinguish Golgi bodies, vacuoles, and chondriosomes by their different colors in living oöcytes of Ophiocephalus and Rita. See also Payne (1932).

${ }^{9}$ Hirschler (1913), Cattaneo (1914), Gatenby and Woodger (1920), Voïnov (1925), King (1926), Parat (1927), Ikeda (1928), Kater (1928c), Hibbard (1928), Nath and collaborators (1928, 1929, 1931), Bhattacharya et al. $(1929,1930)$.

${ }^{10}$ Bowen $(1920,1922 b, 1923 b, 1924 a)$. See, further, Chap. XIV. 
found expression in three hypotheses regarding the relation of the Golgi material to components of plant cells.

In 1910 Bensley treated root tips of Allium, Iris, and Litium, and the tapetum of Lilium, with fixing reagents (composed chiefly of neutral formalin and potassium bichromate) which had been employed in the investigation of the Golgi region in animal tissues. In very young cells the vacuolar material had the form of a system of fine canals; in older cells these "canaliculi" enlarged and gradually coalesced to form the large vacuole (Fig. 41, $A, B$ ). Bensley succeeded in observing the various stages in living cells. In view of the striking resemblance between the vacuolar system and the Golgi canals of animal cells he ventured the suggestion that the two are morphologically and physiologically equivalent. This view has been supported, since, chiefly by Guilliermond. ${ }^{11}$ Guillier-

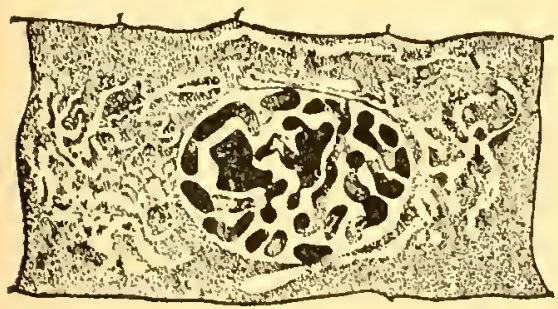

A

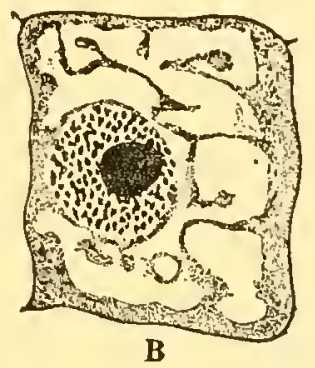

B

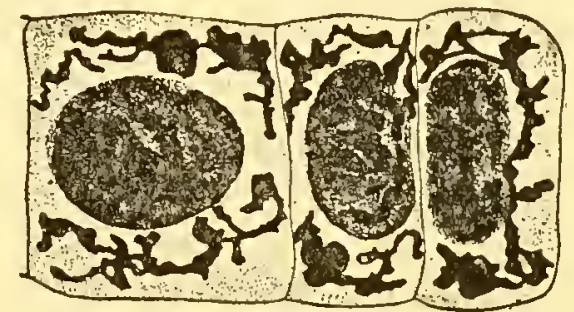

c

Fig. 41.- $A, B$, cells from the root tip of Allium, showing development of cytoplasmic canals into vacuoles. (After Bensley, 1910.) C, colls of the root of barley preserved by the method of Golgi. (After Guilliermond and Mangenot, 1922.)

mond and Mangenot (1922) treated barley roots with silver-impregnation methods and obtained blackened networks corresponding in form with the canaliculi observed by Bensley (Fig. 41, C). They, too, regarded these stages of the vacuolar system as homologous with the Golgi networks of animal cells. ${ }^{12}$ Further support for this view was seen by Guilliermond in the work of Parat, who considered the vacuole system (vacuome) to be a system common to both plants and animals. Zirkle (1932) observes the similarity between the tannin-filled vacuome of plant cells and the Golgi apparatus. Moreover, when such cells containing only traces of tannin are fixed with the salts of heavy metals, the insoluble tannates collect at the periphery of the vacuoles and give the impregnation pictures so commonly reported in the literature.

The second hypothesis is that proposed by Bowen, ${ }^{13}$ who found in plant cells subjected to osmic impregnation considerable numbers of "osmiophilic platelets." These were small flattened bodies in the cyto-

${ }^{11}$ Guilliermond (1926, 1927, 1928, 1929abe); see especially 1927 and 1929a. See also Drew (1920), Laburu (1916), Sanchez (1922), Scott (1929), and Bose (1927, 1931).

12 Long before this de Vries (1885) and Bokorny (1888) had blackened plant vacuoles with osmium and with silver. The reactions seem to be due to the presence of tannins; see Zirkle (1932).

${ }^{13}(1926 h, 1927 a b, 1928 a, 1929 a b c$.$) Gatenby (1928-1930) favors this hypothesis.$ 
plasm, and their blackened rim after osmic treatment strongly suggested the discrete Golgi bodies of insect spermatocytes. Bowen concluded that these elements, rather than the vacuoles, correspond to the Golgi material of animals, an interpretation which he thought was supported by phenomena in plant spermatids to be mentioned below. That the platelets are not a special class of elements but chondriosomes or young plastids showing a particular type of impregnation picture is claimed by several observers. ${ }^{14}$

The third hypothesis, namely, that it is the plastid in plants which most nearly corresponds to the Golgi material in animals, has been advocated chiefly by Weier (1930a, 1931, 1932), although it had been suggested by Bowen (1926c) only to be abandoned for the osmiophilic

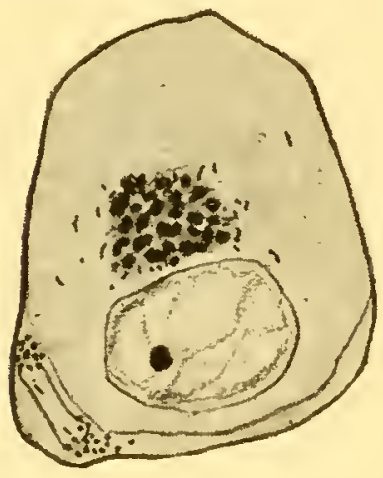

$a$

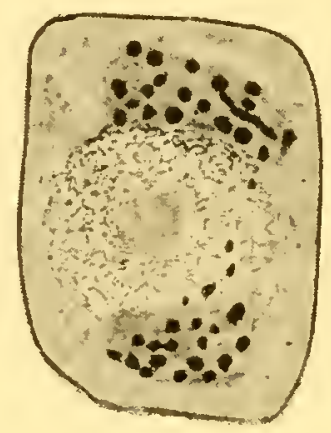

b

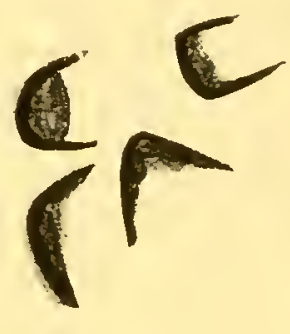

$c$

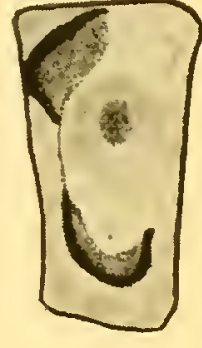

$d$

FIG. 42.-Comparison of Golgi zone and plastid. $a$, nerve cell of $B$ ufo tadpole; silver impregnation. $b$, spermatogenous cell of Polytrichum; osmic impregnation. Note similarity of plastids in $b$ and Golgi zone in $a . \quad c$, Golgi bodies in spermatocyte of Helix; osmic impregnation. $d$, plastid in sporogenous cell of Polytrichum; osmic fixation. (From Weier, 1932; $a$ and $c$ after Parat.)

platelet view. Weier has made a detailed comparison of the fixation images of the Golgi region in animal cells as described by Parat and other investigators with images obtained by similar methods (osmium and silver impregnation) in moss cells. He finds a striking similarity in the two series of cases. Before their characteristic activities begin, both Golgi region and plastid may appear relatively homogeneous, but after starch or the secretion product has accumulated each of them shows a blackened constituent in the form of strands or plates running through a mass of lighter substance or appears as a dense mass with one or more "cavities" inside it (Fig. 42). Starch occurs in these regions in the plastid. The dense substance and the cavities are compared respectively with the modified cytoplasm and "secretory vacuoles" of Parat's Golgi zone; the characteristic "network" or "platework" fixation image produced in both cases is attributed to a general similarity in constitution. It is concluded that the Golgi zone, like the plastid, should be considered as primarily a specialized region of the cytoplasm having to do with the ${ }^{14}$ Owens and Bensley (1929), Kiyohara (1930), Guilliermond (1928, 19296e). Weier (1931) argues for their distinctness in mosses. 
elaboration of certain compounds: carbohydrate in the moss and secretion droplets in the gland cell.

Since such comparisons are based so largely upon the results of microtechnical methods known to be somewhat capricious in their action, more cogent evidence has been sought in cells of other types. It was shown by Bowen that the Golgi material in the animal spermatid performs a curious but very definite morphogenetic function: as already noted, it becomes an acroblast, which in turn buds off or secretes the acrosome of the spermatozoön (Figs. 130, 133). It was also known that in the moss spermatid a limosphere gives rise by division to the apical body at the anterior end of the developing spermatozoid (Figs. 120, 121) (Allen, 1917a). Since it seemed reasonable to suppose that this very special function would be performed in the two cases by similar components of the cell, Bowen turned to the limosphere for evidence regarding the plant's analogue of the animal's Golgi material. In a preliminary study (1927b) he found the limosphere apparently developing in the midst of a group of "osmiophilic platelets"; in this he saw a confirmation of his view that the "platelets" in plants correspond to the Golgi material of animals. Weier, who has made a more extensive study of this matter, finds that the limosphere is derived from the plastids of the earlier spermatogenous cells; moreover, it appears that the osmiophilic platelets are in part small plastids modified in appearance by the methods of fixation (p. 69). The phenomena in sperm-forming cells, therefore, emphasize the similarity of Golgi region and plastid.

Such comparisons of plant and animal cells are of great interest, but they should not be pressed too far. Especially should it not be concluded that because two structures impregnate somewhat similarly with osmium or silver they are therefore homologous. Osmium tetroxide is reduced not only by unsaturated fats but also by certain proteins and phenolic compounds; it therefore may blacken structures which are neither homologous nor physiologically equivalent. The plant vacuole and the animal Golgi region are both so blackened; neutral red, however, colors the vacuole but only the secretion droplets (Parat's vacuome) in the Golgi region. Hall (1931a) warns against the use of impregnation methods as a criterion in identifying cell structures in flagellates and Protozoa, where they may often blacken contractile vacuoles, chromatopholes, eyespots, flagella, and other bodies, in addition to the sinall vacuoles which best meet the criteria for Golgi material in such organisms. The evidence becomes somewhat stronger when a definite morphogenetic function can be cited, as in the case of acrosome formation in spermatids. Perhaps the chief value of such comparisons at present lies in the fact that they lead to improvements in our technical procedures and foster a more critical attitude toward the results obtained through their use. 


\section{CHAPTER VI}

\section{CHONDRIOSOMES}

Chondriosomes, or mitochondria, are small globules, rods, and threads almost universally present in cytoplasm. They had no single discoverer; the elements described by many early observers correspond with them in some degree. Thus the "fila" of Flemming (1882), the "bioblasts" of Altmann (1890), and the "cytomicrosomes" of Strasburger (1882) and others are all recognizable among the chondriosomes of today. In 1897 and the following years Benda, through the use of newly devised technical methods, discovered chondriosomes in cells of many types, notably in the spermatogenous cells of animals, and applied to them the term "mitochondria." It was somewhat later, through the researches of Meves, Regaud, Fauré-Fremiet, Lewitsky, Guilliermond, and others, that they came into prominence. Since that time they have been very intensively studied, and a special literature of large volume has developed. Owing to the difficulties attending the observation of such minute objects and the determination of their relation to other cytoplasmic constituents, opinion regarding the origin, behavior, and biological significance of chondriosomes is still in a very unsettled state. ${ }^{1}$

Occurrence and General Characters.-Chondriosomes have now been reported in plants and animals belonging to nearly all of the larger natural groups. It is asserted by N. H. Cowdry (1917) that "in all forms of animals, from amoba to man, which have been investigated with adequate methods of technique, they occur without exception." Their presence has been reported, furthermore, in practically all tissues. In plants it is probable that they are no less universally present, although they have not yet been demonstrated with certainty in Cyanophyceæ and some Chlorophyceæ (Guilliermond). ${ }^{2}$

${ }^{1}$ Reviews of the subject are given by Fauré-Fremict (1910a), Duesberg (1911, 1919), E. Schmidt (1912), Cavers (1914), E. V. Cowdry (1916a, 1918, 1924a), Meves (1918a), Guilliermond (1919a, 1921i, 1923), Meyer (1920), Lundegårdh (1922), and Nath (1926). Giroud (1925) reviews researches on the physical and chemical nature of chondriosomes. For the effects of various reagents on chondriosomes, sce Kingsbury (1912), E. V. Cowdry (1914, 1918), N. H. Cowdry (1917), Meyer (1920), Ozawa (1927), and Milovidov (1928c, 1929). Findlay (1927) describes ehanges in injured cells. The valuable account by E. V. Cowdry (1918) has been drawn upon in the preparation of this chapter.

${ }^{2}$ They are abundant in myxomycetes (N. H. Cowdry, 1918; Lewitsky, 1924), Charales (Mirande, 1919; Riker, 1921), brown algæ (Mangenot, 1920abde), and red algæ (Nicolosi-Roncati, 1912). Alexieff $(1923,1925)$ reports their presence in bacteria. 
The chondriosomes have generally been regarded as essentially comparable in plants and animals, ${ }^{3}$ although their behavior may not be the same according to the view of some workers. N. H. Cowdry finds plant and animal chondriosomes to be practically identical in morphology, reaction to fixatives and dyes, and distribution in resting and dividing cells; any conspicuous differences in arrangement seem to be due to the more pronounced polarity of many animal cells.

Chondriosomes appear in living cytoplasm in the form of minute globules, vesicles, straight or curved rods, smooth threads, chains of granules, nets, and other more irregular bodies (Fig. 43). These forms
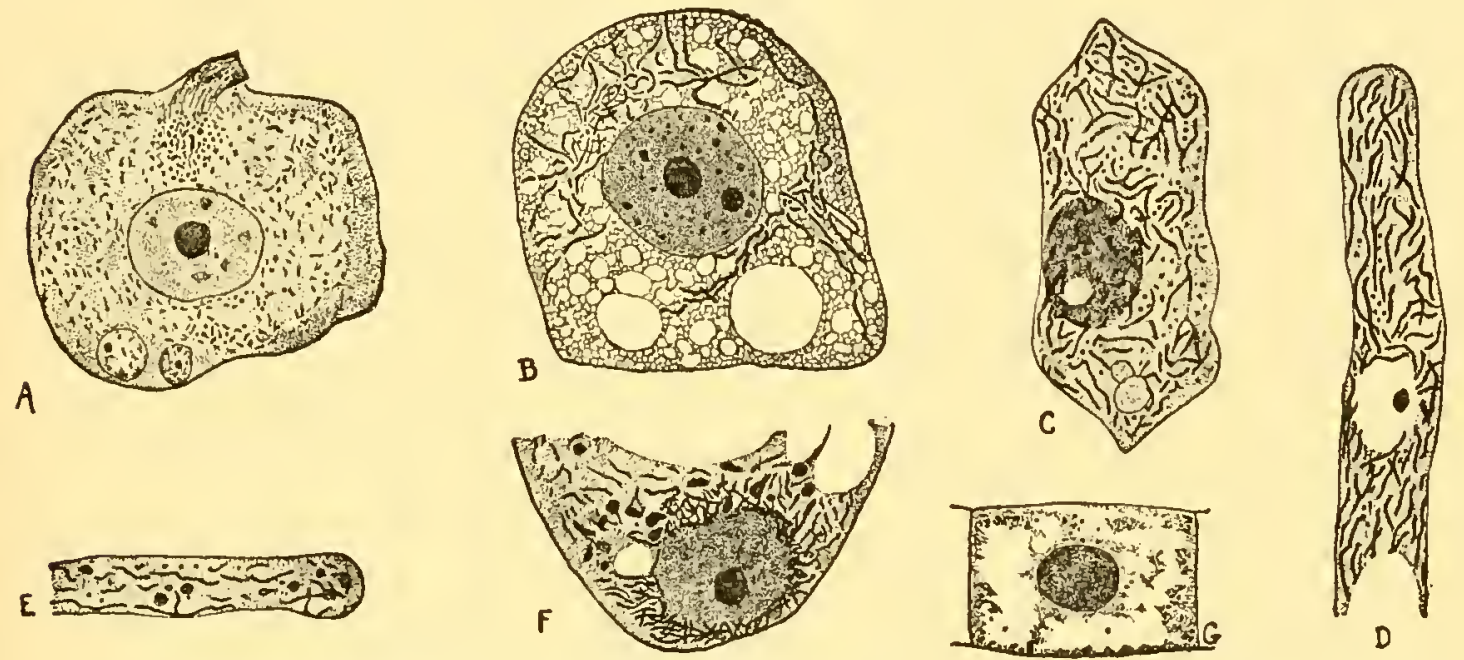

Fig. 43.-Chondriosomes in plant and animal cells. $A$, nerve cell of guinea pig. (After E. V. Cowdry, 1914b.) B, tapetal cell of Nymphoxa alba. (After Meves, 1904a.) $C$, living epidermal cell of tulip petal. $D$, ascus of Pustularia vesiculosa. E, hypha of Rhizopus nigricans. $F$, portion of embryo sac of Lilium; chondriosomes clustered about nucleus. $G$, cell of root tip of Allium. (C-F after Guilliermond, 1918.)

can be seen to change into one another, especially when the cultural conditions are altered. ${ }^{4}$ Such changes are in part the result of cytoplasmic streaming. The same variety of form is encountered in fixed tissues. Particular types tend to predominate in certain tissues. Although it may be convenient at times to designate rods or threads as "chondrioconts" and chains of granules as "chondriomites," it is to be remembered that the forms assumed by the chondriosomal material are of minor importance. For practically all purposes the now synonymous general terms chondriosomes and mitochondria are sufficient. The total chondriosomal content of the protoplast has been called the chondriome. ${ }^{5}$

${ }^{3}$ N. H. Cowdry (1917), Mangenot and Emberger (1920), Bouygues (1924).

${ }^{4}$ Lewis and Lewis (1915, 1924), Chambers (1915, 1924), Anitsehkow (1923), Guilliermond (1925a). N. H. Cowdry (1920) finds them to be more stable in Pisum roots.

${ }^{5}$ Meves, Guilliermond. P. A. Dangeard $(1924,1931)$ uses the terms cytosomes and cylome for these elements in plant cells. For an older and more prevalent use of the term "eytosome," see p. 2. 
The number of chondriosomes present varies greatly with the tissue and the degree of its differentiation. They are usually abundant in young or active cells and relatively few or even absent in fully differentiated ones. In living cells, moreover, they can be seen constantly appearing and disappearing. The arrangement of the chondriosomes within the cell varies; this is often clearly related to certain functional activities, as in certain polarized gland cells. ${ }^{6}$ In pathological animal tissues they show pronounced qualitative and quantitative changes, of which one of the most characteristic is the breaking up of filaments into granules (see E. V. Cowdry, 1924ab).

Physical and Chemical Nature.-Chondriosomes have a semifluid consistency. Under either bright- or dark-field illumination they appear homogeneous (Guilliermond, 1929c). It is reported that they are doubly refractive by polarized light (Giroud, 1928). When the cytoplasm is not too viscous they may be centrifuged out, which shows that their specific gravity exceeds that of the cytoplasm (Fauré-Fremiet, 1913). In both plants and animals they melt at a temperature of $48^{\circ}$ to $50^{\circ} \mathrm{C} .^{7}$ In hypotonic solutions they become swollen and vesicular, whereas in hypertonic media they shrink and become slender. In animal cells Rumjantzew (1926), studying the effect of variations in the $p H$ of the medium, observed that they remained unchanged between 6 and 8 , broke up or agglutinated between 5.8 and 3 , and formed networks and finally dissolved as the $p \mathrm{H}$ was raised to 10 . Lewis and Lewis (1924) observed filamentous ones become vesicular at 4.4 and shorten into granules at 9.6. As would be expected, many fixing reagents bring about decided alterations in their form, ${ }^{8}$ while those with much acetic acid destroy them altogether. They are well preserved by formalin and potassium bichromate, provided the solutions are not too acidic.

Chemical studies, though incomplete, indicate that chondriosomes are composed of phospholipides and albumins. ${ }^{9}$ Besides staining with hæmatoxylin and several other dyes commonly employed with fixed material, they show a characteristic affinity for certain intra-vitam stains, notably Janus green. According to E. V. Cowdry (1916), chondriosomes may be provisionally defined as "substances which occur in the form of granules, rods, and filaments in almost all living cells, which react positively to Janus green and which, by their solubilities and staining reactions, resemble phospholipins and, to a lesser extent, albumins."

Origin and Multiplication.-The question of the origin and multiplication of chondriosomes has been much debated. Evidence for their

${ }^{6}$ Bensley (1916), E. V. Cowdry (1918).

${ }^{7}$ Policard (1912), N. II. Cowdry (1917).

8 Schaxel (1911c), Kingery (1917).

${ }^{9}$ Regaud (1908), Fauré-Fremiet (1910a), Löwschin (1913), Lewitsky (1924), Giroud (1925ab, 1929), Milovidov (1928c). 
division in somatic cells has frequently been reported, and some investigators have even held this to be their sole mode of origin-that they arise only from preëxisting chondriosomes and are, therefore, permanent cytoplasmic organs. ${ }^{10}$ Others have questioned much of the evidence for division and are convinced that, whether or not division occurs,
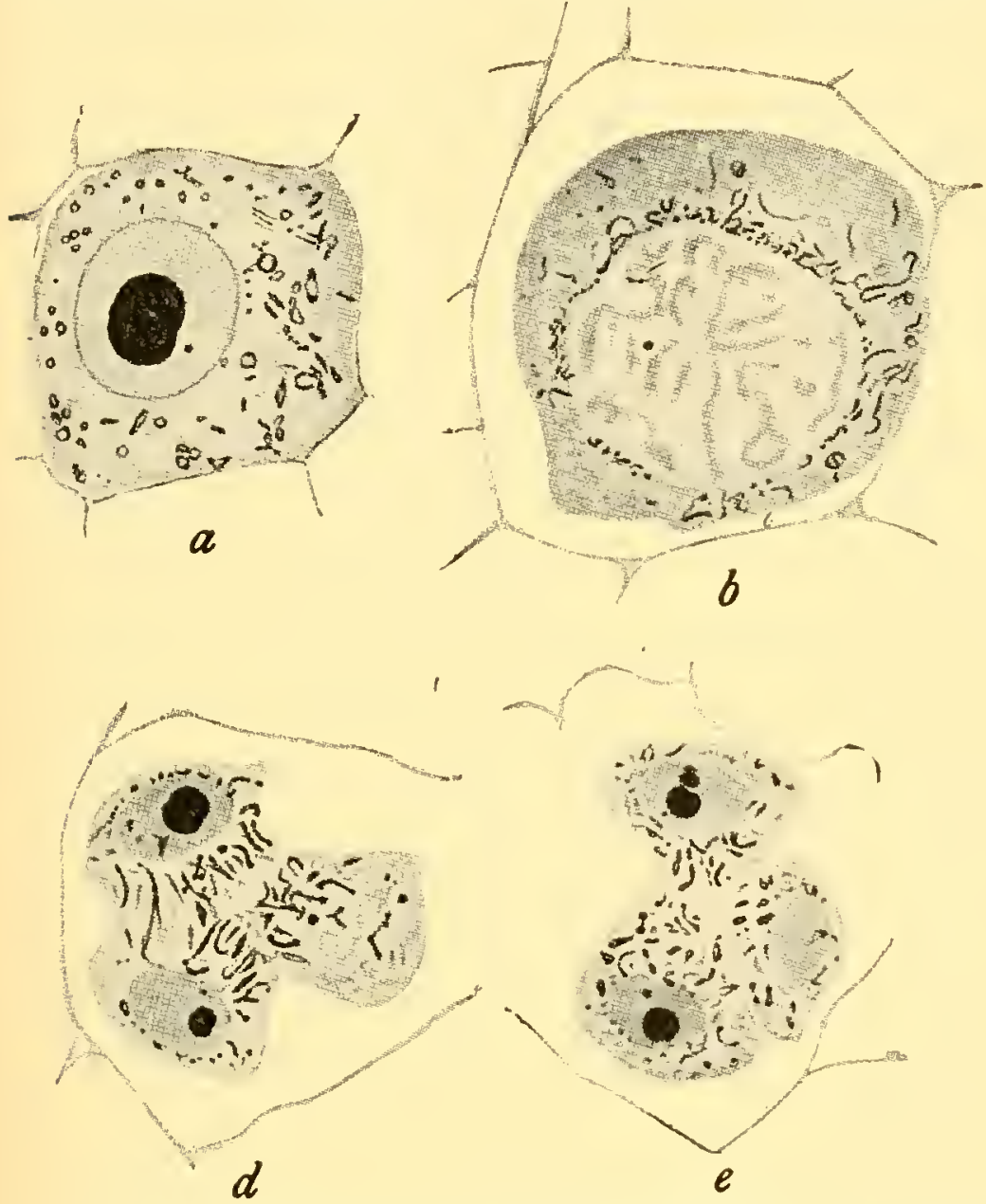
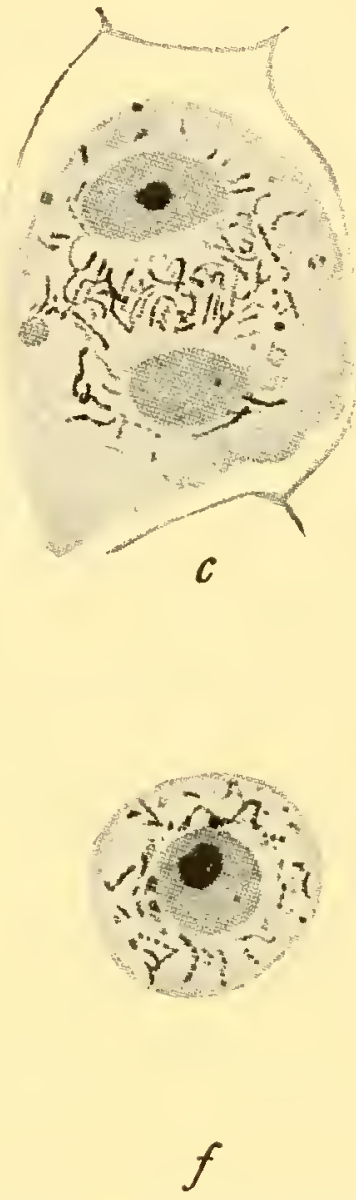

Fig. 44.-Behavior of "chondriosomes" during microsporogenesis in Helleborus. $a$, young sporocyte. $b$, prophase of first division. $c$, interkinesis. $d$, $e$, eytokinesis after second mitosis. $f$, young microspore. (After Wagner, 1927a.)

chondriosomes arise de novo in the cytoplasm. ${ }^{11}$ A third view, namely, that they arise from the nucleus, has not been well substantiated.

Researches on living material ${ }^{12}$ leave no doubt that chondriosomes may appear anew and disappear, probably with some relation to metabolic processes in the cytoplasm. They are often seen to divide, though the division appears to be passive. In the leaf-cells of Cabomba, Kassmann (1926) saw them rise from the limit of visibility, one or two days being

${ }^{10}$ Guilliermond (1912a), Terni (1914), Moreau (1914), Duesberg (1911), Arnold (1912), Friedrichs (1922), Horning (1926).

${ }^{11}$ Forenbacher (1911), Orman (1913), Löwschin (1913), Scherrer (1914), Beckwith (1914), Chambers (1915), Lewis and Lewis (1915), Twiss (1919), Meyer (1920), Morelle (1926), Kassmann (1926), Wagner (1927b).

12 Lewis and Lewis $(1914,1915,1924)$. 
required to reach the typical size; he also observed actual division several times. From his observations on myxomycetes (fixed material) Lewitsky (1924) concluded that the chondriosomes, which are capable of division, may become progressively smaller until they pass below the limit of visibility, and that by growth they may pass the limit in the reverse direction, giving the appearance of origin de novo. This places the problem of ultimate origin in the realm of the invisible.

The behavior of the chondriosomes at the time of nuclear division and cell-division varies widely in different cases. In somatic tissue cells their distribution is quite fortuitous, though approximately equal if the cytoplasm is equally divided. In microporocytes the chondriosomes tend to group about the nucleus and the mitotic figure and are apportioned

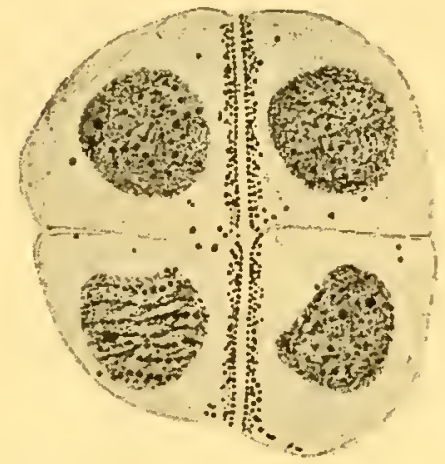

FIG. 45.-Young spore quartet of Nephrodium, showing cell partition formed in mitochondrial layer. (After Senjaninova,

1927c.) chondriosomal filaments lying across the equator of the cell are passively cut in two by the constriction furrow as the cell divides $^{15}$ (Fig. 129), but in Gryllotalpa borealis they appear to break and move apart before the furrow appears (Payne). In Gryllotalpa vulgaris, Voïnov $(1916,1925)$ states that the "mitochondria" fuse to form a thread which then segments into 60 or more "chondriosomes." These units are arranged in the spindle along with the chromosomes, which they may resemble, divide at both meiotic mitoses, and are thus equally distributed to the four resulting spermatids (Fig. 46).

In certain scorpions the chondriosomal material of the spermatocyte is distributed with remarkable precision (Wilson, 1916). In Centrurus it takes the form of a single ring-shaped body which lies by the side of the spindle figure. The ring divides accurately at both meiotic divisions along with the chromosomes, each of the four spermatids, and hence each spermatozoön, receiving a quarter of its substance. In Opisthacanthus

\footnotetext{
${ }^{13}$ Senjaninova (1927c). Cf. Yamanouchi (1908a). See also Lewitsky (1925) on Equisetum.

${ }^{14}$ Fauré-Fremiet (1910a), Korotneff (1909), Terni (1914), Payne (1916).

${ }^{15}$ Montgomery (1911), Bowen (1920).
} 
about 24 hollow spherical bodies are formed instead of a ring. These show no evidence of division but are separated into four approximately equal groups by the cell-divisions, each spermatid receiving six or occasionally five or seven. ${ }^{16}$ It is not improbable that this regularity of distribution in spermatocytes bears some relation to the definite function performed by the chondriosomal substance in the development of the spermatozoön (p. 218).

The Function of Chondriosomes.-Our knowledge of chondriosomes is far too incomplete to warrant categorical assertions concerning their function. The literature is not only complicated by conflicting statements regarding their observed behavior, but it is further encumbered with a variety of hypotheses, some of which rest on very narrow founda-

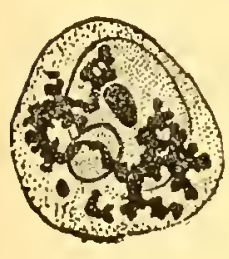

A

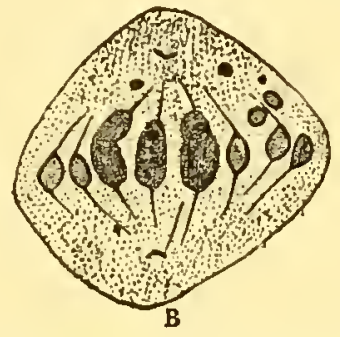

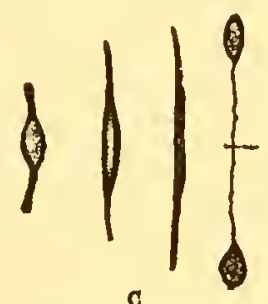

c

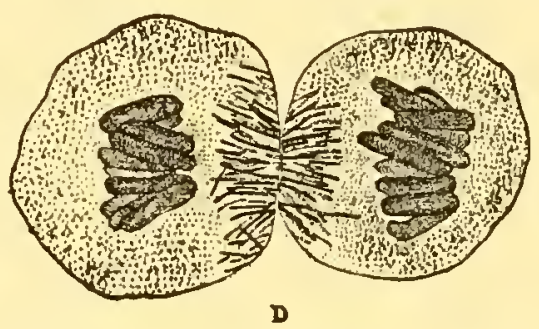

D

Fig. 46.--Examples of regular behavior of chondriosomes in cell-division. $A-C$ spermatocyte of Gryllotalpa vulgaris: $A$, chondriosomal material in cytoplasm; $B$, first meiotic mitosis, showing chondriosomes (at sides) occupying the achromatic figure with the chromosomes (at center); C, stages in the division of a chondriosome. (After Voünov, 1916.) $D$, dividing cell of Geotriton fuscus, showing division of chondriosomes as cell constricts at equator. (After Terni, 1914.)

tions. The more prominent views on the subject may be outlined as follows.

Meves in 1908 propounded a general theory according to which all the visible differentiations which develop in different types of cells during ontogenesis were regarded as modifications of the same elementary cytoplasmic constituents, namely, the chondriosomes. These were thought not only to be concerned in the formation of fibrils, plastids, and the like ${ }^{17}$ but also to play an important rôle in heredity. A close association was observed between chondriosomes and myofibrils, and it was claimed that the fibrils were actually transformed chondriosomes. ${ }^{18}$ A similar claim was made for neurofibrils ${ }^{19}$ and other such differentiations. This interpretation of the genesis of fibrils has been adversely criticized, ${ }^{20}$ and it now seems probable that, although the chondriosomes may possibly furnish material or coöperate in some other way in the formation

${ }^{16}$ Cf. Sokolow (1913) and Mark and Wyman (1922).

${ }^{17}$ See E. V. Cowdry (1918, p. 102).

${ }^{18}$ Benda (1899), Meves (1907ab, 1909), Duesberg (1909ab).

${ }^{19}$ Meves (1907a), Hoven (1910a), G. Arnold (1912).

${ }^{20}$ Heidenhain (1911), Levi (1911, 1916), Gurwitsch (1913), Gaudissart (1913), Lewis (1917), E. V. Cowdry (1914d, 1918), Laguesse (1926), Conklin (1931), and others. 
of fibrils of certain types, they do not actually transform into them. Probably the clearest case of the formation of a definite structure by chondriosomes in animal cells is furnished by the nebenkern, which is a chondriosomal body developing into an important constituent of the tail sheath of the spermatozoön (p. 219).

The observations of several investigators have suggested a connection between chondriosomes and the formation of secretion and storage products. In the pancreas, for example, it was thought ${ }^{21}$ that chondriosomes become zymogen granules, but Regaud (1911), who with Meves first emphasized the secretory theory in general, thought it more probable that the chondriosomes in some way synthesized the zymogen from materials selected from the cytoplasm (the "electosome theory"). Reference has already been made to the newer view that chondriosomes furnish a material for the synthesis of secretions in the Golgi region of gland cells (see p. 77). The development of the secretion product in glandular hairs of Salvia has been associated with chondriosomes. ${ }^{22}$ Chondriosomes have also been assigned a rôle in the elaboration of certain types of yolk in developing eggs ${ }^{23}$ and of fat in liver cells. ${ }^{24}$

The theory that chondriosomes are in any sense protoplasmic organs with special rôles in differentiation and secretion was strongly opposed by A. Meyer (1911, 1920), who contended that they are simply ergastic accumulations of an albuminous nature. Their ergastic nature, according to Meyer, is indicated by the fact that their distribution and behavior are just what would be expected of reserve substances necessary as sources of building material and energy (in meristematic cells, eggs, regenerating tissues, muscles, etc.). They do not transform into differentiations such as myofibrils, but they may lie near them and possibly furnish materials or energy for their upbuilding by the cytoplasm. They do not elaborate or secrete ergastic substances but are themselves such substances in process of formation by the cytoplasm. The general view that chondriosomes are products of metabolic activity rather than distinct protoplasmic organs has been held in one form or another by many workers and appears to be well supported by many observations on both living and fixed cells.

The almost universal occurrence of chondriosomes in protoplasm suggests a connection with some fundamental process common to all living matter. That this process may be oxidation, the chondriosomes being a "structural expression of the reducing substances concerned in cellular respiration," was suggested by Kingsbury (1912), who pointed

${ }^{21}$ Hoven (1910b), G. Arnold (1912).

22 Jaretzky and Triebel (1932).

${ }^{23}$ Loyez (1909), Dubreuil (1913), Van Durme (1914), Shaffer (1920), Gatenby and Woodger (1920), L. Harvey (1925), Konopacki (1927), Bhatia and Nath (1931). See Wilson (1925, p. 341).

${ }^{24}$ D. Smith (1931), Kater and Smith (1932). 
out that they are best fixed by oxidizing agents depending for their effect on reducing substances, probably the lipidal chondriosomes, in the cytoplasm. The chemical fitness of chondriosomes for processes involving oxidation and reduction was further emphasized by the work of Mayer, Rathery, and Schaeffer (1914), who also pointed out that various reagents which diminish respiratory oxidations attack lipides. Joyet-Lavergne (1928) reports that chondriosomes give a positive test for glutathione, which is thought to be of special importance in cell oxidations and reductions (probably because of a respiratory ferment associated with it, according to Warburg). The observations of E. V. Cowdry on the action of vital dyes are also in harmony with the respiration theory. Evidence regarded as unfavorable has been brought forward by Kropp and May (1924), who find that the chondriosomes in the white blood cells of animals made to inhale oxygen, carbon dioxide, and ether differ in no discernible way (Janus-green method) from those of normally respiring animals.

A theory that chondriosomes are the site of enzymatic syntheses has been put forward by Marston $(1923,1926)$, who finds evidence that they contain proteolytic enzymes and that protein synthesis in vitro is accelerated by colloidal lipides. Such synthesis would be greatly facilitated by adsorption in the extensive interface between the chondriosomes and the cytoplasm (Cowdry, 1926b). The enzyme theory is supported by Horning $(1926,1927 a b, 1928 a)$, who observes their adherence to food particles in the Protozoa and their disappearance as the food is digested.

One of the most striking views regarding chondriosomes is that they are microörganisms. Altmann (1890) thought many years ago that protoplasm was essentially a vast colony of living "bioblasts" in a non-living ground substance. Recently Portier $(1917,1918)$ and Wallin (1922-1927) have independently suggested that "mitochondria are, in reality, bacterial organisms, symbiotically combined with the tissues of higher organisms." Wallin finds evidence for this view in the fact that bacteria and chondriosomes show certain similarities in form, staining reaction, chemical composition, physical properties, and activity, and in peculiarities in the development of Bacillus radicicola in the cells of the clover nodule. Assuming that chondriosomes develop into plastids, Wallin suggests further that chloroplasts are not organs developed in the cytoplasm, but chlorophyll-forming organisms that have invaded protoplasm, with which they live in a state of complete symbiosis. ${ }^{25}$

This bacterial theory has been severely criticized. ${ }^{26}$ Cowdry has shown that chondriosomes and bacteria simultaneously present in the

${ }^{25}$ See in this connection Pascher (1929b) on endosymbiosis by blue-green algæ.

${ }^{26}$ Regaud (1919), Guilliermond (1919c), Cowdry and Olitsky (1922), Cowdry (1923a, 1924a), Nicholson (1923), Bowen (1923a), Horning (1927b), Milovidov (1928b). 
clover nodule show distinct differences in fixing and staining reactions. Similar results are obtained by Milovidov, using another method on lupine nodules. Horning reports that chondriosomes, but not bacteria, are stained with a new vital dye derived from Janus green. Bowen cites as further evidence against the bacterial interpretation the remarkable behavior of the nebenkern (chondriosomal material) in the animal spermatid. Wallin attributes the peculiar properties of chondriosomes to their long symbiotic history and is inclined to interpret the nebenkern as a stage in the bacterial cycle somewhat analogous to the "symplasm" in other bacterial forms (Löhnis). He reports further that experiments appear to indicate that chondriosomes can be cultivated independently on nutrient agar. The final evaluation of this evidence is awaited with the greatest interest.

Chondriosomes and Plastids.-The general theory of Meves that many intracellular differentiations are due to transformations of chondriosomes included the proposition that the plastids of plant. cells arise from these bodies. This view has been expressed in one form or another by a large number of workers. ${ }^{27}$ In Asparagus and Pisum, for example, Lewitsky concluded that chondriosomes become leucoplasts in the root and chloroplasts in the stem and leaf. Other investigators ${ }^{28}$ have maintained that plastids are surely or in all probability quite distinct from all other elements in the cell and that confusion has arisen because different kinds of minute bodies are so similar in appearance. No problem in the cytology of recent years has been more perplexing than this one. ${ }^{29}$

That some distinction must be made between the small bodies which develop into plastids and those which do not has become increasingly evident. Guilliermond, whose extensive studies ${ }^{30}$ are particularly noteworthy, came to recognize such a distinction, but he considered both types as chondriosomes essentially equivalent to those of animals: the "active" ones are elaborative elements developing into plastids, while the relatively "inactive" ones remain small and perform undetermined

${ }^{27}$ Lewitsky (1910, 1925), Forenbacher (1911), Guilliermond (1911b et seq.), Cavers (1914), Moreau (1914), Nassonow (1918), Emberger (1920, 1927), Alvarado (1923), Friedrichs (1922), Bouygues (1924), Mangenot (1925), Kirby (1928), Cowdry (1926b), Senjaninova (1927bc), Motte (1928), Cunha (1929), Zirkle (1929c), Loui (1930), and others.

${ }^{28}$ Meyer (1911), P. A. and P. Dangeard (1919 et seq.), Lundegårdh (1910), Rudolph (1912), Mottier (1918, 1921), Scherrer (1914), Noack (1921), Harper (1919), Löwschin (1913, 1914), Sapěhin (1915), Krupko (1926), Bowen (1927ab, 1929a), Bowen and Buck (1930). Kassmann (1926) expresses uneertainty.

${ }^{29}$ See the discussions by Bowen (1929a) and P. A. Dangeard (1931). The indiscriminate use of the terms "chondriosomes" and "mitochondria" for small bodies of many kinds must be borne in mind when reading the literature in this field.

${ }^{30}$ A elassified list of Guilliermond's researches up to 1921 is given by himself (1921i), together with a list of works by his associates. See also Guilliermond, Mangenot, and Plantefol (1933). 
functions. Others contended that the proplastids should not be included under the heading of chondriosomes nor homologized with anything in animal cells. Meyer's (1920) claim that plastids constitute a fundamentally distinct class even in their earliest stages of development has been supported by Bowen (1927ab, 1929a), who has employed a variety of methods little used by botanists. In root meristems and other plant tissues Bowen claims that the two classes of elements can be distinguished on the basis of general form, structure, reactions to stains, and behavior during nuclear division and cell-division. As a rule the chondriosomes ${ }^{31}$ in the root tips are round and vesicular, are best preserved and stained by the ChampyKull method, and show no regular arrangement in the cell during mitosis; whereas the proplastids are elongate and usually not vesicular, are best preserved by the MottierBenda method, and show a characteristic polarized arrangement during mitosis (Figs. 25,47 ). Bowen further finds, with others, ${ }^{32}$ that the two classes react somewhat differently in impregnation procedures and cites reports ${ }^{33}$ of unlike reactions to vital dyes and heat. A difference in appearance and behavior in tissue affected with mosaic diseases is described by Dufrenoy (1931). As a result of his studies on living cells, Pensa concludes that plastids may arise either by division or by a direct differentiation of the cytoplasm; in the latter case the chondriosomes group about the plastids and seem to furnish material for the latter as they grow
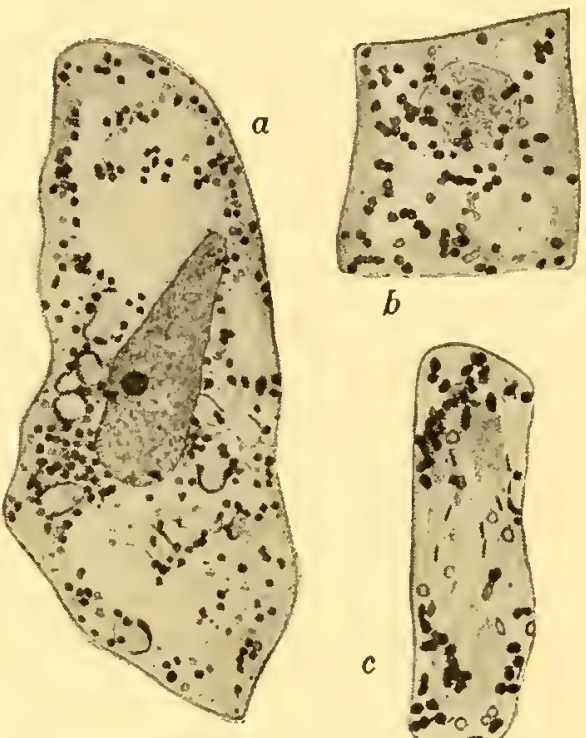

FIg. 47.-Plastids and chondriosomes. $a$, plastids (large) and chondriosomes (small) in cell of growing point of Equisetum. b, chondriosomes in root tip of Vicia; some are dividing. $c$, distribution of chondriosomes (dark bodies) during mitosis in Hyacinthus root. (After Bowen, 1929ab.)

or become green. Loui (1930), who has used the fluorescence microscope, can find but one kind of initial body which he regards as chondriosomal in nature.

It is plain that the confusion in this subject is in part a matter of terminology, since the words "chondriosome" and "mitochondrion" have been used in such different ways. Hence it has been suggested that the terms should be dropped altogether (Mottier, Dangeard). Zirkle $(1929 c)$, on the other hand, uses the term "mitochondria" for all small inclusions which are preserved by bichromates with a $p H$ higher than

${ }^{31}$ Not wishing to eommit himself on the question of the relation of these bodies to the chondriosomes of animals, Bowen employs for them the term "pseudochondriosomes." Dangeard calls them "eytosomes."

${ }^{32}$ Pensa (1925), Ruhland and Wetzel (1924).

${ }^{33}$ Guilliermond (1923a), Polieard and Mangenot (1922). 
4.2 to 5.2 and are destroyed by more acid fluids, and the term "plastids" only for those bodies containing starch or chlorophyll. We have here chosen somewhat arbitrarily to retain the term "chondriosome" but to restrict its application to those elements with a fairly characteristic composition which do not behave as the primordia of plastids. When the nature of a small element is unknown, such non-committal terms as "microsome" and "particle" are always available. It remains for future research to reveal more precisely the chemical and genetic relationship of proplastids and chondriosomes at the time of their first appearance in the cytoplasm and to evaluate the claim of Meyer and others that the chondriosomes are, like all other formed bodies in the cytoplasm except plastids, ergastic in nature.

Conclusion.- In the present state of our knowledge no final judgment can be rendered on the question of the nature and function of "chondriosomes." Bearing present probabilities in mind, we may adopt as a tentative working basis the view that in both plants and animals the chondriosomes, or mitochondria, are a special class of small bodies fairly distinguishable chemically from other materials of an ergastic nature, that they represent elaborated products which are somehow employed as sources of matter and energy for further growth and differentiation, and that they are best regarded as distinct from the plastids of plant cells. This provisional disposition of the matter will be convenient until wholly decisive evidence is obtained.

In spite of the fact that the study of chondriosomes has so far raised more problems than it has solved, it has proved of much value, for it has turned to the cytoplasm some of the attention so long directed almost exclusively to the nucleus. It has also been of great service in bringing about a closer scrutiny of the effects of fixation and a renewed emphasis upon the importance of the study of living protoplasm. 


\section{CHAPTER VII}

\section{ERGASTIC SUBSTANCES}

In Chapter II emphasis was laid on the conception of the protoplast as a living system of active components which by themselves would be nonliving. It is nevertheless convenient to deal separately with materials which, for the time at least, are relatively inactive. These, for the most part, are accumulations of the products of protoplasmic activity and represent by-products, supporting structures, and reserves which are later to be used in metabolism. They are thought of as non-living substances, but to whatever extent they affect the vital activities of protoplasm they must logically be regarded as a part of the living system.

The relatively inactive or "lifeless" substances in or on protoplasm were called metaplasm by Hanstein (1868). This term has been commonly employed in this sense, but unfortunately it has been widely used in another meaning (see p. 45). We have, therefore, adopted Meyer's (1896) expression, ergastic substances. ${ }^{1}$

Vacuoles. - Although the semipermeable membranes bounding vacuoles are definitely a part of the protoplasmic system (p. 42), it is convenient to treat vacuoles in this chapter because of the ergastic nature of their contents.

Any spaces within the protoplast containing ergastic liquid or (very rarely) gas may be regarded as vacuoles, but the term is ordinarily used with reference to those occupied by the aqueous mixture known as cell sap. Vacuoles of this general nature are small and comparatively inconspicuous in animals, but in plants they appear to be almost universally present and clearly play a prominent rôle in metabolism. In terminal meristems of vascular plants, as shown especially by the recent work of Zirkle (1932), they are usually rather numerous, small, and spherical, although they may be drawn out into other shapes by the streaming cytoplasm. In the apical cells of Osmunda (fern) and Lunu-

${ }^{1}$ For an extensive account of the ergastic materials in plants, sec A. Meyer (1920, 1926). For the chemistry of plant products, sce R. W. Thatcher (1921), Onslow (1923), Czapek (1913, 1920), Molisch (1913), Rigg (1924), Trier (1924), Haas and Hill (1928), Gortner (1929), and Wiesner et al. (1932). Ergastic materials are not to be confused with the ergastoplasm of Garnier, Bouin, Prenant, and others. This term was applied to a supposedly very active or "superior" type of protoplasm; see FauréFremiet (1910a) and Wilson (1925). For general accounts of vacuoles, see Meyer (1920-1921; Chap. VI, Sec. 6), Lundegårdh (1922; Pt. 2, Chap. VIII), P. Dangeard (1923a) and Guilliermond (1929a). 
laria (liverwort) they are very large. In the cambium of woody plants they are very abundant and exhibit a surprising variety of form (Fig. 48) (Bailey, 1930). As the meristematic cells multiply, the vacuoles are distributed, often after passive division, to the daughter cells. During the final growth and differentiation of the cells the vacuoles coalesce, commonly forming a single enormous vacuole which far exceeds the volume of the cytoplasm in which it lies. The vacuolar system of a cell or other protoplasmic mass, whether this system consists of one or more vacuoles, is called the vacuome (Dangeard).

Much of the difference of opinion regarding the origin and occurrence

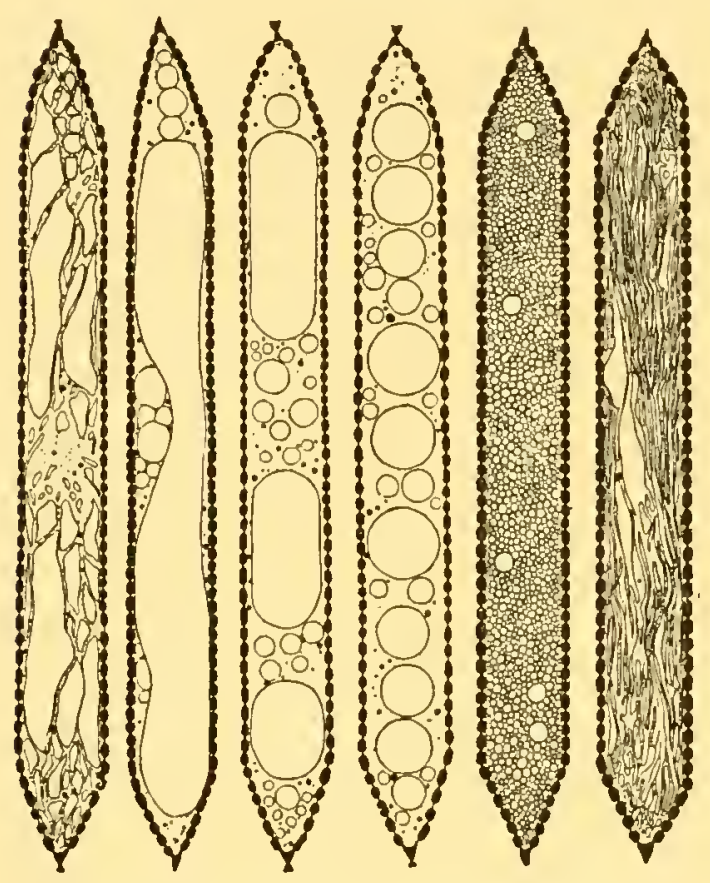

FIG. 48.--Various forms assumed by the vacuome in fusiform cambial initials of Robinia. (After Bailey, 1930.) of vacuoles has been due to the fact that many fixing fluids, particularly those containing acetic and chromic acids, may destroy the true vacuoles and greatly alter the appearance of the cytoplasm. Certain fluids containing iron or chromium salts preserve very well the size and form of the vacuoles but not their membranes (Zirkle). The only trustworthy studies on this subject, as in the case of so many others in the field of cytology, are those which are checked as far as possible by observations on living material.

As just intimated, the mode of origin of vacuoles has long been a debated question. An old and still prevalent view is that they simply arise de novo in the cytoplasm wherever water and certain dissolved substances become abundant enough to form visible droplets (von Mohl, Nägeli). Pfeffer (1890), who found that droplets with membranes were formed when granules of asparagin were introduced into protoplasm, favored this view. Strasburger (1898), who regarded protoplasm as mostly alveolar in structure, accounted for the origin of vacuoles by the enlargement and coalescence of alveoles, while Meyer (1912, 1920) classed them as accumulations of ergastic fluid around which membranes are secondarily formed.

Sharply opposed to this interpretation was that of de Vries (1885), who advanced the theory that vacuoles do not arise de novo but rather from individualized bodies which he called tonoplasts. As the tonoplast secretes cell sap within itself, it gradually enlarges and becomes the vacuolar membrane, which is still referred to as the tonoplast. Since the tonoplast bodies were supposed to multiply only by division, de Vries 
looked upon vacuoles as permanent constituents of protoplasm. This theory had the support of various other workers, ${ }^{2}$ but the general tendency for many years was to view it with skepticism.

A new period in the study of vacuoles began with the more recent researches of P. A. and P. Dangeard, ${ }^{3}$ who again advanced a theory that
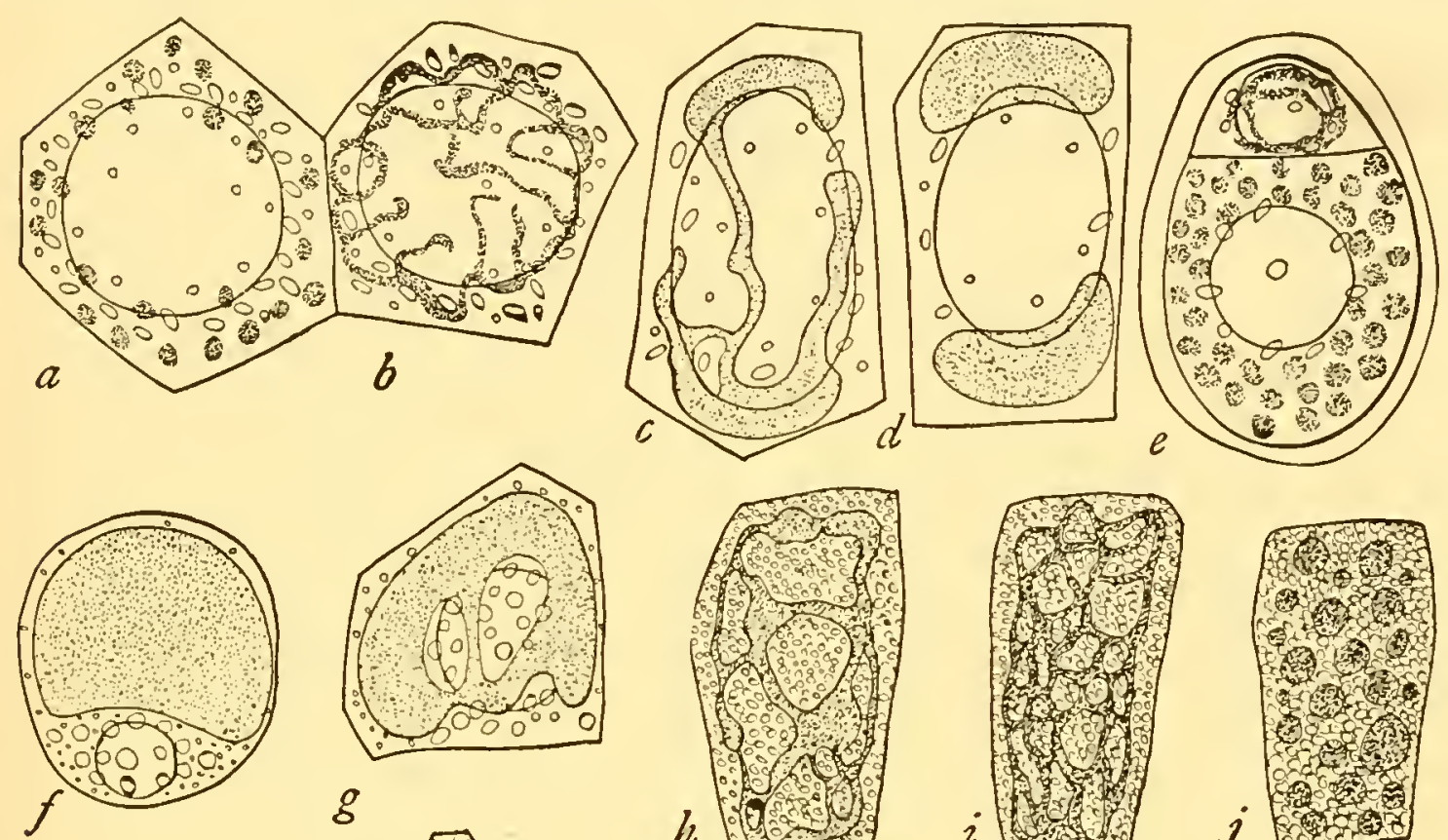

$g$
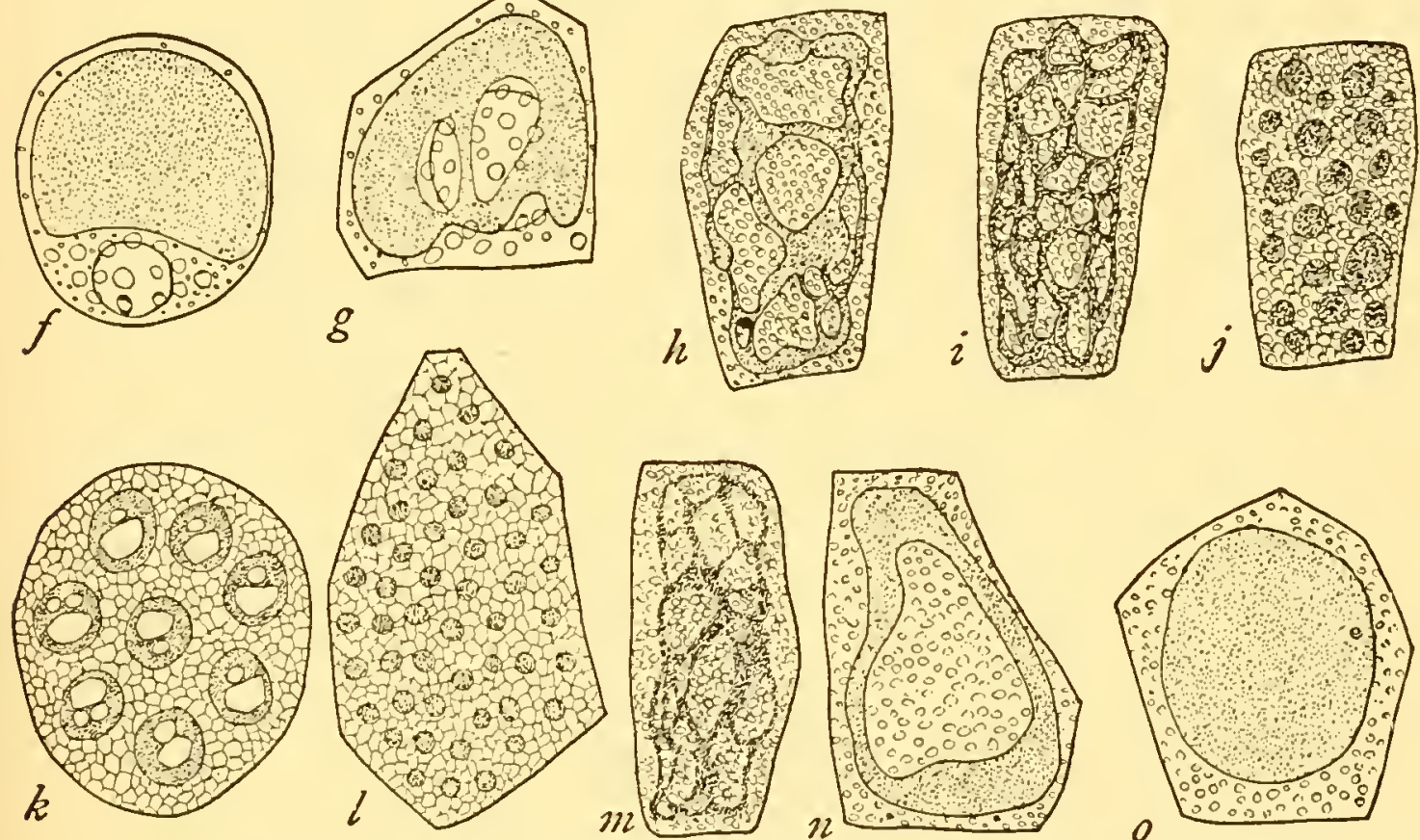

FIG. 49.-Behavior of vacuole system in certain plant cells. $a-d$, successive stages in bud of Abies. $e$, pollen grain of Cephalotaxus. $f-j$, formation of aleurone grains from vacuome in endosperm of Ricinus. $k$, deeply lying endosperm cell of Ricinus, with typical aleurone grains. $l-o$, peripheral cells of Ricinus endosperm, showing development of vacuole from simple aleurone grains during germination. (After P. Dangeard, 1923a.)

the vacuome is a permanent system arising not de novo but from small units in the cytoplasm. According to these observers, the vacuome exists at first in the form of minute "metachromes"; these absorb water, enlarge, develop into a system of canals, ${ }^{4}$ and finally become one or more large vacuoles (Fig. 49, $a$ to $d$ ). It was reported further that in

${ }^{2}$ E.g., Van Tieghem (1888) and Went (1888).

${ }^{3}$ P. A. Dangeard (1916 et seq.), P. Dangeard (1922, 1923, 1927c).

${ }^{4}$ Such a system of canals had been seen several years earlier in root tips by Bensley (1910), who, like Dangeard, found that they were not well preserved by ordinary methods of fixation (see p. 79). 
the maturing endosperm of Ricinus the protein-containing vacuole passes through a reticular stage and breaks up into smaller ones which, through dehydration, become aleurone grains (Fig. $49, f$ to $j$ ). At the time of germination the process is reversed: the grains absorb water and become small vacuoles, which then coalesce to form a network and finally a large vacuole (Fig. $49, l$ to $o$ ). It has accordingly been concluded by the Dangeards that the vacuome is an autonomous system present in all plant cells and multiplying by repeated division.

That vacuoles may arise de novo is maintained by Guilliermond, who has made intensive studies of fungi and terminal meristems in the living condition. In vitally stained young branches of Penicillium hyphæ and developing buds of yeast they are said to appear often without any connection with the vacuome of the main portion. The same is reported for Saprolegnia. Dangeard (1927c) contends that in untreated hyphæ and yeast the new vacuoles can be seen to originate from the old ones by budding or constriction and that the vital dyes used by Guilliermond cause certain alterations in the morphology of the young vacuome. No evidence for origin de novo is found in Erisiphe. ${ }^{5}$ The question of the origin of vacuoles in hyphæ therefore remains unsettled.

In their studies on terminal and lateral meristems of vascular plants Bailey and Zirkle found no evidence for the origin of vacuoles de novo, although, as Zirkle points out, such a mode of origin in a cell whose vacuoles are constantly fragmenting, changing their shape, and fusing would easily escape notice.

Cell Sap.-The fluid in the ordinary vacuoles of plants is somewhat viscous and usually homogeneous in appearance, and it quickly shows a marked increase in viscosity when exposed to the air. It consists of water with a variety of substances in molecular and colloidal solution. Analyses have shown that the following elements and classes of compounds occur in such saps: magnesium, aluminum, sodium, potassium, calcium, manganese, iron, nitrogen, phosphorus, sulphur, chlorine, iodine; organic acids (oxalic, malic, citric, acetic, tartaric, formic, etc.); alcohols; carbohydrates (dextrose, fructose, lactose, inulin, etc.); slimes, glucosides; anthocyanin and soluble yellow pigments, most of which exist in the form of glucosides; amides (glutamin, asparagin); alkaloids; albumins; certain enzymes; tannins; a variety of other aromatic compounds (see A. Meyer, 1920). The vacuole is thus a reservoir of nutritive materials and a depot for by-products.

Although the sap rather characteristically shows an alkaline reaction in meristematic cells, its behavior toward vital dyes indicates a change to a neutral or acid state with the appearance of organic acids and certain phenolic compounds, such as tannin (P. Dangeard, 1923a). In the

${ }^{5}$ Guilliermond (1925b) on Penicillium and yeast, Cassaigne (1931) on Saprolegnia, Baache-Wiig (1925) on Erisiphe. 
cambium of woody plants and other tissues Bailey and Zirkle ${ }^{6}$ find two kinds of vacuoles in the same cell: relatively alkaline ones staining reddish orange in neutral red (B-type), and markedly acid ones staining bluish magenta with the same dye (A-type). Those of the latter type contain phenolic compounds and tend to form copious precipitates. It is observed that vacuoles in the cambium of conifers and many dicotyledons are alkaline when stainable and become acid in the xylem and phloëm; whereas, in some dicotyledons they are acid and show changes to alkalinity and vice versa in the differentiating cells. Seasonal variations also occur. Although the accumulation of basic dyes appears to be correlated with the presence of specific substances in the vacuoles, these workers do not favor Dangeard's hy pothesis that a substance called "metachromatin" is chiefly responsible for their staining reactions and other activities. It is further found that colorimetric methods are very unreliable for determining the $p \mathrm{H}$ of B-type vacuoles. It is this type which occurs in Nitella and Valonia, which are so widely used in physiological studies.

Nearly all of the red, blue, and purple colors of flowers, fruits, and other plant parts are due to anthocyanin pigments in the cell sap. These pigments are usually reddish in an acid medium and bluish in a neutral or alkaline one, though these colors may be masked by other pigments in the sap or plastids. The yellow flavone and flavonol pigments are widely distributed in the cell sap of plants, but they occur in such dilute solution that they do not give a noticeable color to tissues except in rare cases (e.g., Antirrhinum). Yellow color in plants is usually due to plastid pigments. ${ }^{7}$

Frequently the cell sap contains visible solid or fluid particles in suspension; these may be so numerous as to give the sap a milky appearance. The latex found in special cells or vessel systems in certain plants is a sap which contains a great variety of substances in solution, together with suspended droplets or granules of oils, tannins, gum, starch, resin, caoutchouc, and other compounds. As familiar examples may be cited the juices of the rubber tree, dandelion, milkweed, and poppy. ${ }^{8}$

Under certain circumstances organic and inorganic substances dissolved in the cell sap may crystallize or precipitate. This may result from an increase in the concentration of such substances beyond the saturation point, often because of a decrease in the amount of solvent, as in drying seeds; it may also be due to the appearance of some other compound which precipitates them. As examples may be cited the formation

${ }^{6}$ Bailey (1930), Bailey and Zirkle (1931), Zirkle (1932a). See also Went (1888) and Mangenot $(1927,1929 b)$ on the presence of two vacuole types.

${ }^{7}$ For accounts of plant pigments, see Onslow (1923), Möbius (1927), Meyer (1926), Howard (1925), and Gortner (1929).

${ }^{8}$ For accounts of such saps, see Czapek (1913), Meyer (1920), and Went (1926). 
of aleurone grains in maturing endosperm (p. 96), the appearance of stained masses when certain vital dyes are used, and the formation of crystals. Very striking alterations in the form, number, and reactions of vacuoles have been observed in stimulated secretory and conducting cells of insectivorous plants ${ }^{9}$ and in diseased tissue. ${ }^{\text {I0 }}$.

Carbohydrates. - The most conspicuous visible carbohydrate materials in plants are starch and cellulose. In most green plants the excess carbohydrate elaborated by the process of photosynthesis (p. 65) is deposited in the form of starch granules. These range in size up to about $200 \mu$ and vary considerably in appearance in different plants.

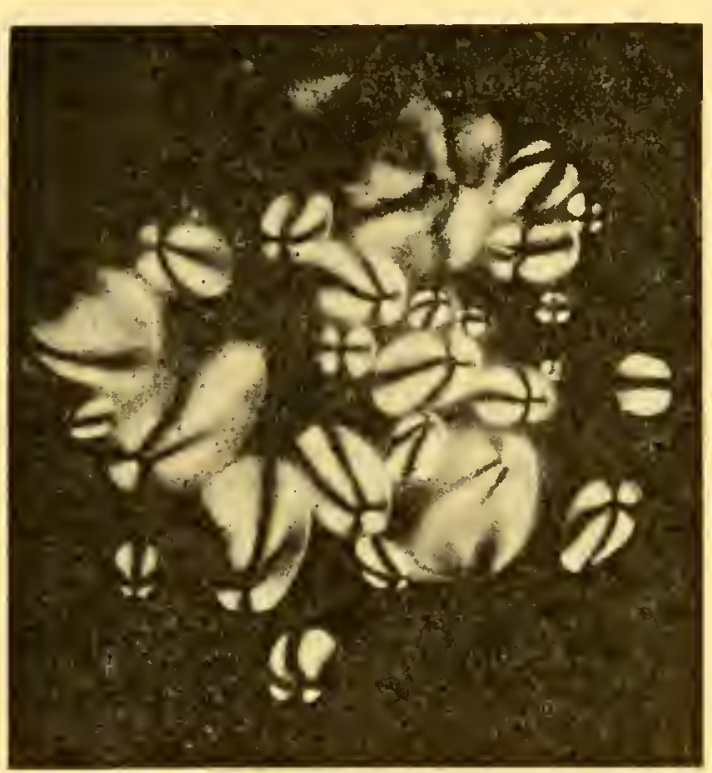

FIG. 50.-Potato-starch grains photographed by polarized light. Each granule is made up of a series of concentric layers successively deposited about a center, or "hilum." Meyer showed that in certain cases the stratification is correlated with the alternation of day and night and therefore with a periodic activity of the protoplast. Granules from wheat grown under constant conditions are reported not to show it (SandeBakhuyzen, 1926). In case the granule starts to form near the middle of an amyloplast it may develop symmetrically, but commonly it lies near the periphery and becomes very eccentric in form, owing to the unequal deposition of new material on its various sides. The amyloplast may become greatly distended as the granule grows, often reaching invisible thinness; in extreme cases it becomes ruptured and remains in contact with the granule only at one side, where all new material is thenceforth deposited. Several granules may start to develop simultaneously in a single amyloplast and later grow together to form a "compound granule" with more than one hilum. In case the parts making up the compound granule are enveloped in one or more common outer layers, the granule is said to be "half-compound." The successively deposited layers making up the granule differ mainly in water content, the innermost layers being richest and the outermost poorest in water. As a result of this non-uniformity the granule often splits radially when dehydrated. Viewed through a polarizing microscope with crossed Nicols the granule appears as a light body traversed by a dark cross (Fig. 50). By this means starch can often be detected in very minute plastids.

${ }^{9}$ Gardiner (1885), Dufrenoy (1927), Mangenot (1929ab), Homès (1929).

${ }^{10}$ Dufrenoy (1928bc, 1929bc, 1930c, 1931ac), Dufrenoy et al. (1929de). Küster (1929) describes pathologieal ehanges in protoplasm. 
As a result of his classic researches Nägeli $(1858,1881)$ developed the theory that the starch granule is made up of ultramicroscopic particles ("micellæ"), differing in size and in the thickness of their surrounding water films in the various layers of the granule. He finally decided that the micellæ are crystalline in nature, a conclusion supported by the work of Schimper (1881) with polarized light. This conception of the starch granule as a spherocrystal with radially arranged elements was adopted and elaborated by A. Meyer (1883, 1895), who attributed the stratification of the granule to differences in the length, thickness, closeness, and richness of branching of the constituent needle-shaped crystals, or "trichites." The more recent X-ray analysis by Sponsler $(1922,1923)$ indicates that the granule is made up of units regularly arranged in a space lattice; it is therefore crystalline in the modern sense of the term. Instead of having single atoms in planes as in an ordinary crystal, it consists of $\mathrm{C}_{6} \mathrm{H}_{10} \mathrm{O}_{5}$ units arranged in curved layers.
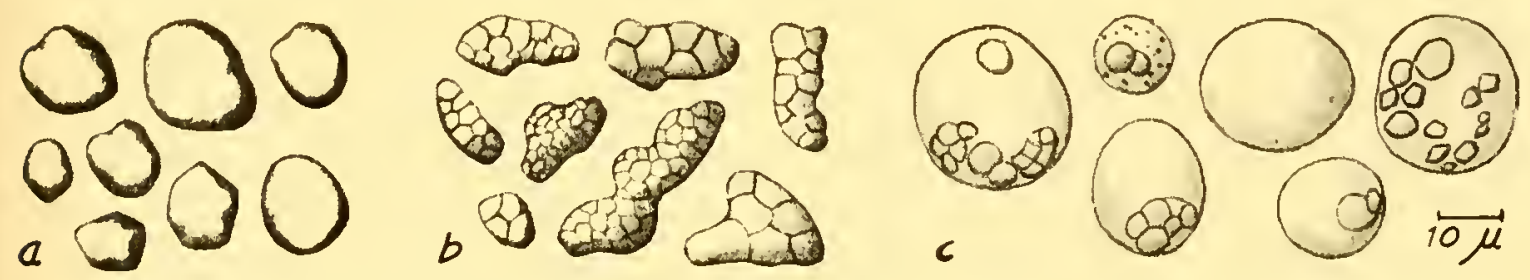

FIG. 51.-Carbohydrate reserves in endosperm of maize 50 days after pollination. $a$, simple grains of starch from starchy maize. $b$, compound grains of starch from sweet maize. $c$, globules of liquid dextrin from sweet maize; some of them contain simple and compound starch grains. (After Lampe, 1931. Figure redrawn and furnished by that author.)

Starches vary considerably in composition and in reaction to reagents. The principal constituents are $\alpha$-amylose and $\beta$-amylose, which usually become reddish and bluish, respectively, with iodine. In maize endosperm, according to Brink and Abegg (1926), the two amyloses occur in different proportions in waxy and non-waxy starches; furthermore, both turn red in the waxy starch and blue in the non-waxy. It may be that such differences are due to variations in the degree of colloidal dispersion (W. Harrison, 1911).

In the endosperm of maize the visible reserves of carbohydrate, which are deposited in plastids, are specific in form and composition in the four genetic types: starchy, waxy, sweet, and waxy-sweet. In the nonsweet maize simple grains of solid carbohydrate occur. They are composed of starch in starchy maize and of red-staining "starch" in waxy maize (Fig. 51,a). In the sweet types there are compound grains, which are composed of starch in sweet maize and of the red-staining "starch" in waxy-sweet maize (Fig. 51, b). In the sweet types globules of liquid dextrin also occur in the upper central portion of the endosperm. In sweet maize these globules may contain small simple and compound 
grains of starch, while in waxy-sweet maize they contain similar grains of the red-staining "starch" (Fig. 51, c). ${ }^{11}$

Cellulose is the chief constituent of the cell wall in most groups of plants. It is not often found in the pure state in the wall, other substances, notably lignin, ordinarily being present in physical or chemical combination with it (p. 176). The proportion of pure cellulose may be as high as 90 per cent in cotton fiber, but in beech and oak wood it is as low as 35 per cent. Although chiefly supporting in function, cellulose is sometimes used as a reserve product; the hemicelluloses more often function in this capacity. Closely allied to the celluloses are the pectins. It is a striking fact, as remarked by Sponsler (1923), that starch, cellulose, and pectic substances, which are about the only solid materials deposited directly and in quantity by plant protoplasm, have the same proportional formula $\mathrm{C}_{6} \mathrm{H}_{10} \mathrm{O}_{5}$.

Glycogen, a substance of great importance in animals, is found also in the Cyanophyceæ, myxomycetes, fungi, and bacteria. It may exist in the form of viscous or solid masses in the cytoplasm, or in colloidal solution in the vacuoles. In these plants it appears to function much as starch does in higher plants. The many mucilages and gums which occur so widely in plant tissues are composed chiefly of carbohydrate materials, the former being condensation products of various sugars and the latter these products together with complex acids (Onslow). Plant slimes may apparently arise in different cases as modifications of cell-wall substance, within the cytoplasm, or at the boundary between the cytoplasm and the vacuoles (Czapek; E. L. Smith, 1923). Sugars of several types are of special importance in the metabolism of plants and are frequently present as storage products.

Proteins.-Ergastic protein bodies are constantly being encountered in cytological study. These may be either crystalline or non-crystalline and may lie in the cytoplasm, plastids, nucleus, or vacuoles. In animal eggs the storage materials commonly occur in the form of yolk globules, or "deutoplasm spheres," which consist for the most part of relatively complex protein compounds; globules of fat or oil are usually associated

${ }^{11}$ Lampe and Meyers (1925), Lampe (1931). For the composition of starch, see Meyer (1895, 1913, 1920), Czapek (1913), Abderhalden (1923), Reichert (1913), and Brink and Abegg (1926). For the structure of the starch granule, see papers of Nägeli, Schimper, Meyer, Kabsch, Binz, Dodel, Salter, Kramer, and Sponsler. For the oceurrence of starch, see Meyer (1895) and Winkler (1898). Belzung (1887) and Eberdt (1891) deal with the origin of stareh in chloroplasts and amyloplasts, respectively. For accounts of "Floridean starch," see Schmitz (1882), Bruns (1894), O. Darbishire (1896), Henckel (1901), Kylin (1913), and Mangenot (1923a). The development of maize endosperm and its storage products is described by Lampe (1931). The relation of genes to the production of such products in endosperm or pollen is discussed by Brink and MacGillivray (1924), Demerec (1924), Longley (1925), Brink and Burnham (1927ab), Brink and Abegg (1926), and Brink (1927ce, 1928, $1929 a b)$. 
with them. The eggs of gymnosperms may contain large globules or shapeless masses of albuminous reserves. Refractive "metachromatic corpuscles," composed of metachromatin, a nucleic acid compound, may occur in the vacuoles and cytoplasm of certain algæ, fungi, and Protozoa (Guilliermond); these are what A. Meyer calls volutin globules. In Meyer's opinion both chondriosomes and nucleoli are ergastic protein masses.

Protein crystals occur widely in both plants and animals. They consist chiefly of albumins and globulins and may be found in the cytoplasm, plastids, nucleus, or vacuoles. ${ }^{12}$ The best known albumin crystals are those found in aleurone grains, which are ordinarily made up of both crystalline and amorphous protein elements. These grains occur in the endosperm, embryo, and perisperm of ripe seeds, being especially prevalent in such oily seeds as those of Ricinus, Juglans, and Bertholletia. In maize and wheat kernels they lie in the outermost layer of endosperm cells. Aleurone grains differ considerably in color, form, and structure. ${ }^{13}$ In many cases (e.g., Pisum) the grain consists only of an amorphous substance. In other cases this ground substance encloses a rounded "globoid" (in grasses), a crystal of calcium oxalate (in certain Umbelliferæ), or a large angular albuminous "crystalloid." The well-known aleurone grain of the

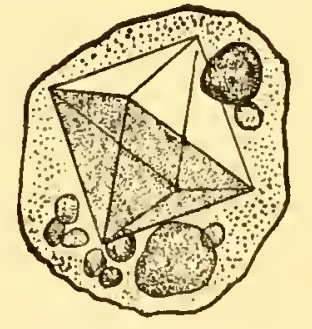

FIG. 52.-Aleurone grain from endosperm of Ricinus communis. (After A. Meyer, 1920.) deeply lying endosperm cells of Ricinus consists of a protein ground substance, a crystalloid, and a globoid composed of a double phosphate of calcium and magnesium together with certain organic constituents (Fig. 52).

The development of the aleurone grain has been repeatedly studied, especially in Ricinus. It now seems clear that the early investigators (Maschke, Gris, Wakker) were correct in their statement that the grains appear in vacuole-like cavities as the seed matures. They regarded these cavities as actual sap vacuoles, a view which was again emphasized by the Dangeards. ${ }^{14}$ According to this interpretation, the vacuolar material passes through a reticular stage and breaks up into a number of small vacuoles in which the gradually condensing constituents differentiate as crystalloid, globoid, and ground mass. According to Mottier (1921), aleurone formation involves the activity of permanent plastid primordia. These aggregate in large numbers in the vacuole-like cavities, where their combined products unite to form the aleurone grains. Vouk (1925)

12 Protein erystals are treated at length by Meyer (1920). For nuclear crystals, see also Tischler (1921-1922) and Wóycicki (1929b).

${ }^{13}$ Hartig (1856), Maschke (1859), Gris (1864), Pfeffer (1872), Tschirch (1887), Wakker (1888), Lüdtke (1890), Guilliermond (1907a).

${ }^{14}$ P. A. Dangeard (1919, 1920ac), P. Dangeard (1920, 1921abc, 1922a, 1923a). 
maintains that the vacuole-like cavities in Triticum are not true sap vacuoles but masses of an albuminous material which condenses about the plastids with the aid of certain enzymes, the plastids themselves eventually disappearing. An analogy with starch formation by leucoplasts is pointed out. This conflicts with the Dangeards' claim that aleurone grains represent a stage in the evolution of the vacuolar system.

Fats and Allied Substances.-Fats and oils are of widespread occurrence as reserve materials in plants as well as in animals. In plants they are found commonly in seeds, spores, embryos, and meristematic tissues, and occasionally in differentiated vegetative parts. They are chiefly neutral, free fatty acids seldom being present in any considerable amount. They occur emulsified with water and appear in the form of droplets in the cytoplasm, and occasionally in the nucleus also in animals. The assemblage of oily or osmiophilic bodies in the cell is called the ergastome by P. A. Dangeard.

It appears that materials of this class are ordinarily elaborated directly by the cytoplasm, although in certain plants elaioplasts are evidently concerned (p. 66). Mangenot (1923b) attributes oil formation to phæoplasts in certain brown algæ. The characteristic "oil bodies" in the cells of many liverworts appear to be formed by the fusion of small masses arising in the cytoplasm. ${ }^{15}$ Cytoplasmic origin of essential oils is reported by Popovici (1925) for glandular hairs, and Leeman (1928) describes the passage of such oil into special preformed droplets. It has long been the claim of Guilliermond and his associates that volatile oils have a chondriosomal origin (Guilliermond and Mangenot, 1923).

Waxes also bear a resemblance to fats in composition and form waterproof coatings on many fruits, stems, and leaves.

Crystals.-Crystals of many kinds occur in the differentiated tissues of plants. They may lie in the cytoplasm, vacuoles, and occasionally the nucleus. They may be attached to or imbedded in the cell wall; often the cells containing them are considerably modified in size and appearance. They are usually salts of calcium, the oxalate being especially prevalent, and are chiefly by-products. 'The bundles of needle-shaped crystals of calcium oxalate known as "raphides," which are found in many leaves, arise in the vacuole and come to be surrounded by a layer of mucilage ${ }^{16}$ (Fig. 53, L). Definite homogeneous regions in the cytoplasm are thought by Robyns to be concerned in their production. The spherical crystalline structures known as "druses" (Fig. $53, C)$ are also chiefly calcium oxalate, though they have a central mass of some more complex organic substance. The origin of the druse and its relation to the protoplast have been a subject of controversy,

15 Pfeffer (1874), Rivett (1918). Gargeanne (1903) held them to arise in sap vacuoles.

${ }^{16}$ E. L. Smith (1923), Robyns (1928). 
Jeffrey's (1922) view that the druse is formed as a casing around the protoplast being opposed by Lloyd (1923), who contends that druses, more than one of which may be present in one cell, arise within the protoplasm and may later pass into the vacuole. Gaiser (1923) finds that the stellate crystal of Anthurium arises in the vacuole, and that after it comes to occupy nearly the whole cell the cytoplasm with the nucleus can still be seen surrounding it.

The curious "cystoliths" in the Ficus leaf (Fig. 53, A) represent outgrowths of the cellulose wall heavily impregnated with calcium carbonate. In the cystoliths of certain Acanthacex the calcium may later disappėar (Linsbauer, 1921). Crystals of silica are very abundant
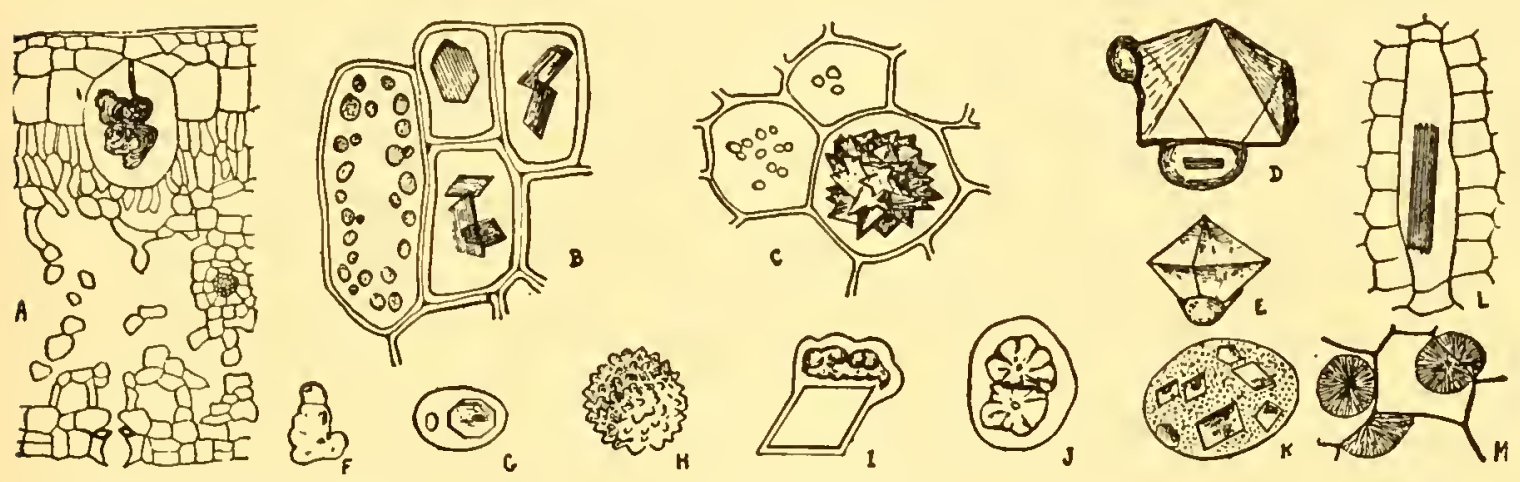

Fig. 53.-Crystalline and other ergastic materials in plant cells. A, cystolith in subepidermal cell of Ficus leaf. B, crystals in Arctostaphylos. C, druse in Rheum palmatum. $D-K$, aleurone grains: $D, E$, from Iyristica; $F$, from Datura; $G$, from Ricinus; $H$, from Amygdalus; $I$, from Bertholletia; $J$, from Foniculum; $K$, from Elois. $L$, raphides from Agave leaf. $M$, inulin crystals in preserved cells of artichoke. (B-K after Tschirch.)

in the thickened walls of wood cells and in many other tissues, such as the outer portion of the Equisetum stem. In an earlier section reference was made to the protein crystals of aleurone grains and to those frequently observed in the nucleus. Mirande (1923) states that the radiocrystals in the epidermis of white-lily-bulb scales consist of a phytosterol which he calls "liliostérine." The carbohydrate inulin, which often occurs in solution in the vacuole, appears as nodules of radiating crystals in tissues which have been preserved in alcohol (Fig. 53, $M$ ).

Recapitulation: Structure of the Protoplast.-In the preceding chapters the various structural elements of the protoplast have been passed in review. Before proceeding further it will be well to draw up a classification of these elements-a classification which in some points must be regarded as provisional only. From the point of view of convenience and present probabilities the differentiations and inclusions of protoplasts may be listed as follows:

$A$. The Nucleus, a highly specialized organ consisting of

1. Nuclear membrane, lying against the cytoplasm;

2. Reticulum, composed chiefly of chromonemata of chromosomes; 
3. Karyolymph, or nuclear sap;

4. Nucleolus or nucleoli; and

5. Occasional ergastic matter.

$B$. The Cytosome, the extra-nuclear region comprising

6. Cytoplasm, with differentiated

7. Membranes at its outer surface (plasma membrane) and bounding the sap vacuole (tonoplast);

8. Plastids, characteristic of plants, with a special rôle in the elaboration and storage of carbohydrates (chiefly);

9. Golgi material, or "Golgi zone," characteristic of animals, and concerned in the elaboration of secretions; regarded by some workers as ergastic;

10. Centrosomes, present in animals and some lower plants; and 11. Ergastic substances, non-protoplasmic constituents comprising

(a) Chondriosomes, small masses of a substance reacting as phospholipide and albumin, produced and used by the protoplast; regarded by many as cytoplasmic organs;

(b) Vacuolar materials, in particular the cell sap of plants;

(c) Other ergastic substances, chiefly reserves and byproducts.

C. The Cell Wall of plants, mainly ergastic in nature, but possibly incorporating protoplasm; or

THE INTERCELLULAR SUBSTANCE of animals.

Because of their prominence in the literature, the partial classifications employed by P. A. and P. Dangeard, Guilliermond, and Meyer for plant cells are presented here.

Dangeard (1923, 1929, 1931):

1. The nucleome, the nucleus or nuclei.

2. The vacuome, comprising all the vacuoles.

3. The plastidome, comprising all the plastids.

4. The cytome, or assemblage of cytosomes [plant chondriosomes].

5. The ergastome, including the oily or osmiophilic bodies called liposomes by Fauré-Fremiet.

Guilliermond (1919 et seq.):

1. The chondriome, comparable to that of animal cells and comprising chondriosomes of two kinds:

(a) Ordinary ones common to plants and animals;

(b) Active ones forming plastids in green plants.

2. The vacuome, or vacuole system.

3. The lipoid granulations, which are ergastic.

[4. The nucleus.]

Meyer (1896, 1920):

1. The nucleus.

2. The cytoplasm.

3. The plastids.

4. Ergastic substances. 


\section{CHAPTER VIII \\ SOMATIC CELL-DIVISION}

In most organisms growth involves a multiplication of cells by division. In unicellular forms this results in the multiplication of the organisms themselves. In multicellular forms the protoplast which is to undergo development into a new individual initiates a series of subdivisions which will eventuate in the many cells of the body, or soma. Ordinarily each subdivision involves both the division of the nucleus by a complicated process known as mitosis, or karyokinesis, and the division of the cytosome, called cytokinesis. These two processes may be correlated in various ways.

In the present chapter typical somatic cell-division will be described in its main outlines by way of preparation for detailed discussions of the more specific and problematic points in subsequent chapters. ${ }^{1}$

Outline of Somatic Mitosis.-The remarkable character of the process of mitosis finds its meaning in the peculiar organization of the nucleus. The nucleus contains a number of well-individualized units, the chromosomes, each of which in turn has a characteristic organization. In normal cell-division and differentiation it appears to be essential that each chromosome should be so divided that this organization, and hence the organization of the nucleus as a whole, will be reproduced in each of the two daughter nuclei.

In such a tissue as that of the root tip the nucleus in the metabolic condition consists of a bounding membrane, a mass of karyolymph, a reticulum composed chiefly of thread-like chromosomal elements (chromonemata or their persistent basis), and one or more nucleoli. As the prophase of mitosis begins (Fig. 54), the reticulum gradually separates into its constituent chromonemata through the disappearance of the small strands connecting them. At first the chromonemata are thin and crooked. They are also longitudinally double; in some cases this split condition is visible from the very beginning of the prophase.

The double chromonemata soon become straighter and thicker. As they do so their split condition becomes more obvious, so that they appear as double and somewhat twisted threads imbedded in the karyolymph. Their arrangement is often very irregular, but in rapidly multi-

${ }^{1}$ A useful list of works on mitosis in angiosperms is given by Picard (1913). Ruys (1925) lists the angiosperm genera in which nuelear studies have been made. A valuable review of researches on many chromosome problems is given by Reuter (1930). See also the works of Tischler (1921-1922) and Sehürhoff (1926). 
plying nuclei they usually tend to lie more or less parallel. During this middle portion of the prophase a second component of the chromosome,
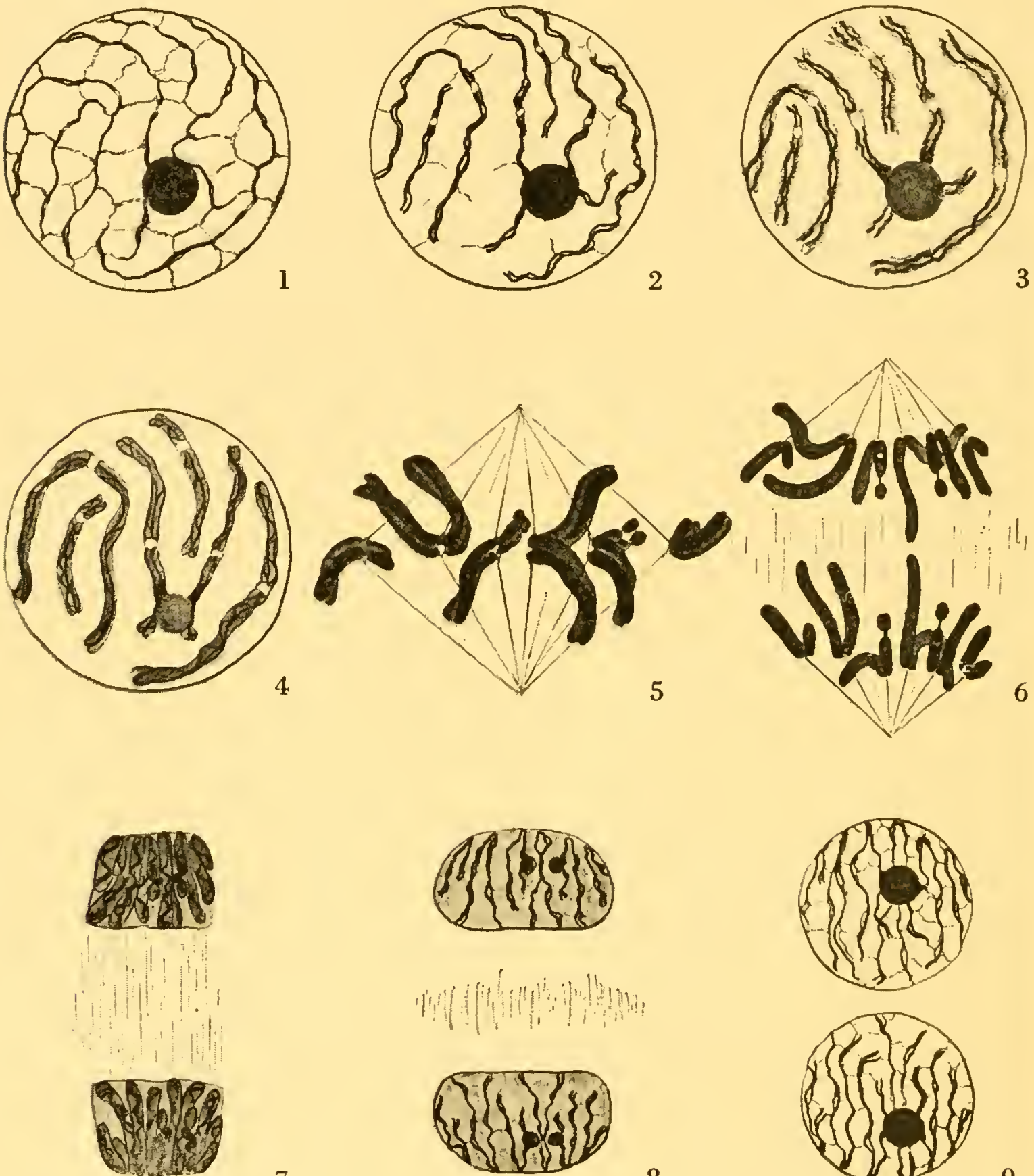

7
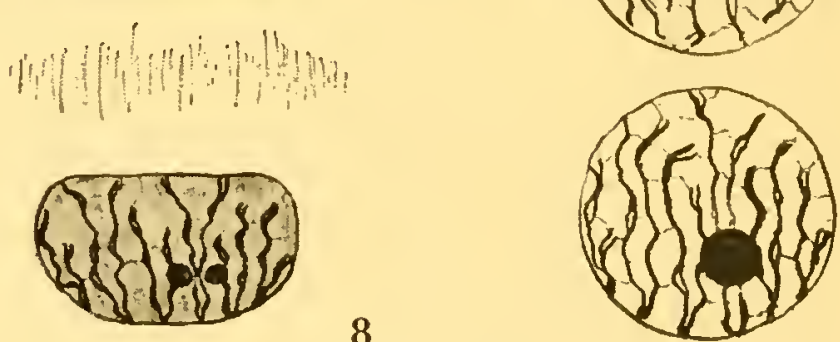

8

FIG. 54.-Semidiagrammatic representation of somatic mitosis based on studies of species with large chromosomes. 1, metabolic stage: the chromonemata with anastomoses form a reticulum. 2, early prophase: chromonemata individually distinet and show longitudinal doubleness. Note relation of certain chromosomes to nucleolus. $3, \mathrm{medium}$ prophase: matrix becoming more evident; spindle-attachment regions distinct. 4, later prophase: matrix becoming more chromatic, obseuring chromonemata. 5, metaphase: attachment regions all in equator of spindle formed by karyolymph. 6 , anaphase: divergence of the chromosomes. 7 , early telophase: matrix less chromatic; nucleoli developing in connection with certain chromosomes. 8, medium telophase: anastomoses forming between chromonemata; karyoly mph developing; spindle disappearing. 9, later telophase: nuclei larger; reticula more fully developed. For further explanation, see text.

namely, the matrix, becomes increasingly evident. This is a substance of uncertain history. It is probable that in some form and amount it 
accompanies the more stainable chromonema at all times. Although it may sometimes be distinguishable from the surrounding karyolymph rather early, it ordinarily becomes conspicuous only when the prophase is more advanced.

The chromosomes, each composed of two chromonemata and surrounding matrix, now become markedly thicker and of ten shorter. In many nuclei this involves a contortion of the chromonemata, which then appear to be crowded or coiled within the translucent matrix. At this period the matrix develops a strong affinity for the stains commonly employed, so that the whole chromosome may look like a solid body with no internal structure. As the prophase draws toward a close each chromosome tends gradually to assume its final form, which may differ characteristically from that of the other chromosomes of the group. At this time the nucleolus commonly disappears, although a portion of its substance may sometimes persist through the subsequent phases.

At the end of the prophase a marked change occurs in the other constituents of the nucleus. The nuclear membrane commonly disappears, and the clear karyolymph in which the chromosomes are lying develops a polarized arrangement which appears in fixed preparations as a series of striations or fibrils lying parallel to the longitudinal axis of the developing "mitotic figure." This mass of modified karyolymph rapidly assumes the form of a bipolar spindle and the double chromosomes group themselves in its middle region.

In the metaphase every chromosome lies at least in part in a welldefined equatorial plane through the spindle. If the chromosomes are very short, they may lie wholly in this plane; if they are long, all but a small portion of each one may lie with no regular arrangement in the figure. The portions lying in the equatorial plane are definitely determined: each chromosome has a specially differentiated and constantly located "attachment region" at which it manifests a peculiarly intimate connection with the spindle in the equatorial plane. Hence in the metaphase and immediately succeeding stage a given chromosome usually exhibits clearly its characteristic morphology; the attachment region is indicated by its relation to the spindle and by other features so that the relative length of the "arms" on either side of this region can be easily determined. At the attachment region the two longitudinal halves of each chromosome lie facing the two poles of the spindle; in other portions of the chromosome they often show no such orientation (Fig. 55). In fixed preparations the most strongly developed "spindle fibers" extend poleward from the attachment regions.

In the anaphase the two halves of each chromosome move apart and pass through the spindle toward its two poles, the movement beginning at the attachment region. The shapes assumed by the various chromosomes during this phase depend upon the location of the attachment region and 
the positions in which the remaining portions happened to lie during the metaphase. Long chromosomes, because of their trailing ends, may thus present a rather confused picture during these phases (Fig. 56). The attachment regions reach the poles in advance of the other portions, of ten before the trailing ends have separated at the equatorial plane in the case of very long chromosomes; such chromosomes may shorten considerably after their ends draw away from the equator. Eventually all the chromosomes lie more or less parallel in two fairly compact groups between which the spindle substance may be seen. In certain cases persistent nucleoli have been observed to pass poleward.

In the telophase each group of chromosomes reorganizes as a metabolic nucleus. This involves a series of changes which in many respects are the reverse of those seen in the prophase. During the anaphase the

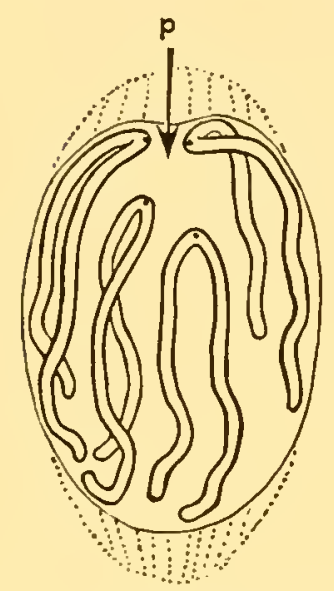

1

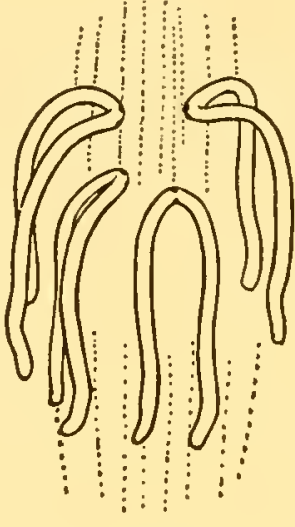

2

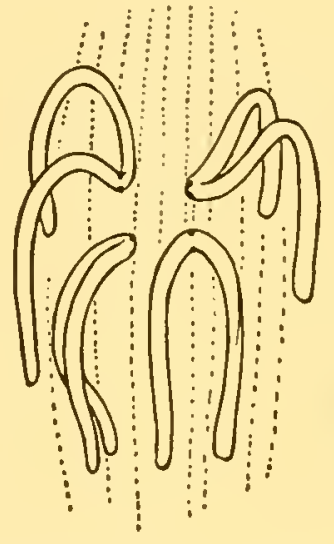

3

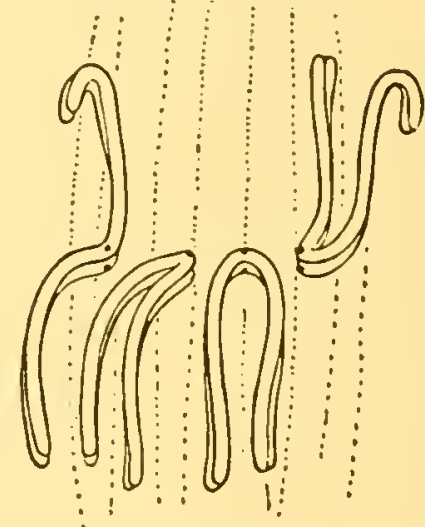

4

FIG. 55.-Development of metaphasic arrangement of chromosomes. Spindle-attachment regions indicated by dots. P, “pole field." (After Bèlar̆, 1929b.)

chromosome matrix is highly chromatic, but as the telophase begins this chromaticity decreases, so that the chromonemata again become evident. Furthermore, these chromonemata may be double as the result of a splitting which has been shown in some cases to have taken place shortly before the metaphase. Meanwhile the nuclear membrane, karyolymph, and nucleoli appear. As the two nuclei grow, the chromonemata tend to become less basichromatic and join to form the more or less continuous and uniform reticulum characterizing the metabolic stage.

In rapidly growing tissue with the mitoses occurring in quick succession, as in the meristem of the root tip, the telophasic changes often are not carried far enough to obscure the limits of the chromosomes in the reticulum before the changes of the ensuing prophase begin. In such nuclei it can often be seen that many or all of the attachment regions occupy a rather restricted area ("pole field") at the side away from the equator (Fig. 55), and that the parallel arrangement of the chromosomes assumed in the anaphase persists until the next prophase. The 
nucleus as a whole may, however, show a change in orientation. ${ }^{2}$ The period between two rapidly succeeding mitoses is referred to as the interphase.

As a result of the mitotic process, the organization characterizing the original nucleus is reproduced exactly in the two daughter nuclei. These
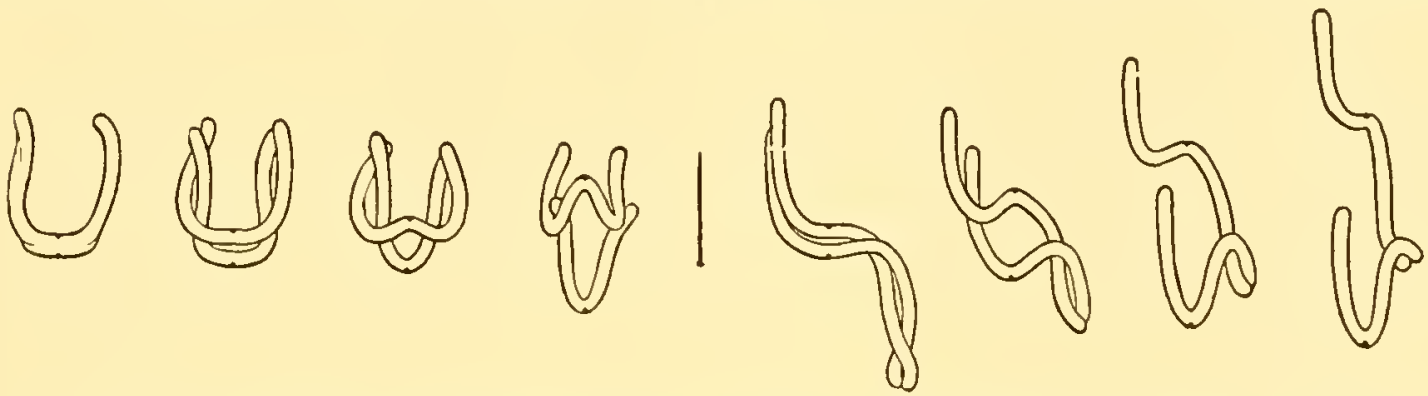

Fig. 56.-Configurations often assumed by long chromosomes in anaphase. Spindleattachment regions indicated by dots. (After Bëlaŕ, 1929b.)

nuclei are structurally alike and have the same functional capacities. When it is remembered that all of the nuclei of the body are produced by a succession of such mitoses, it should be obvious that the equational character of the process is of the greatest importance with respect to problems of metabolism, development, and heredity.
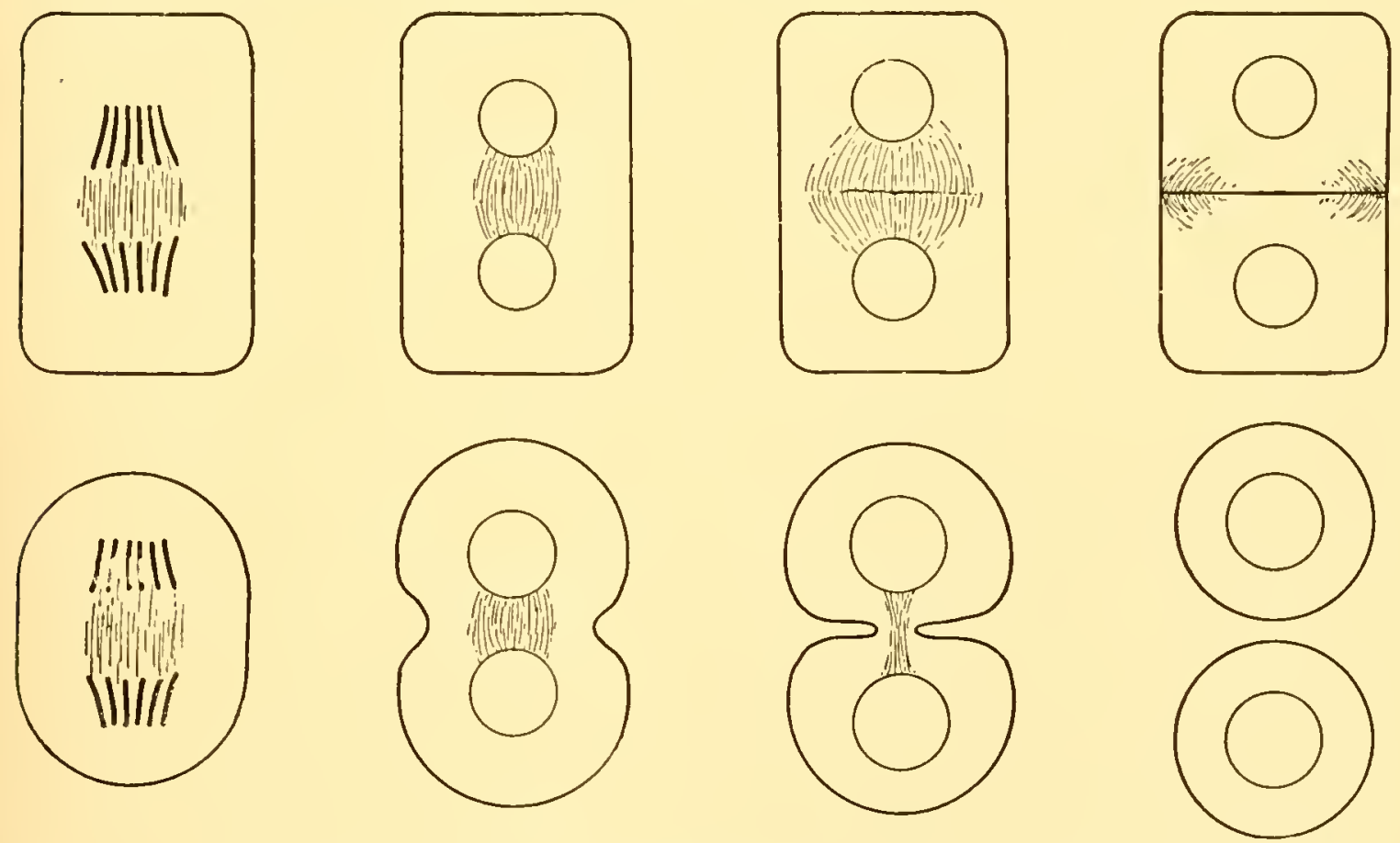

FIG. 57.-Diagram of cytokinesis by cell-plate formation (first row) and by furrowing (second row).

Cytokinesis.-The division of the cytosome is accomplished in a variety of ways in different organisms (Fig. 57). A furrow may form at the plasma membrane and gradually extend inward, cleaving the proto-

${ }^{2}$ See the discussion by Bělař (1929b). 
plast into two portions. In other cases a delicate membrane appears in the cytoplasm and develops into a partition between the two resulting cells. In the meristems of higher plants this membrane commonly forms before the spindle substance has disappeared from the equatorial region, so that the spindle seems to give rise to it.

In the last mentioned case the formation of a "cell-plate" appears to be a feature of the mitotic process, but it should be pointed out that this is probably because cytokinesis follows mitosis so closely. All degrees of correlation between these two processes are known. No correlation whatever is seen in certain plasmodial masses, cleavage furrows developing through the cytoplasm without any evident relation to the nuclei. In other cases the division of the cytosome is in some way

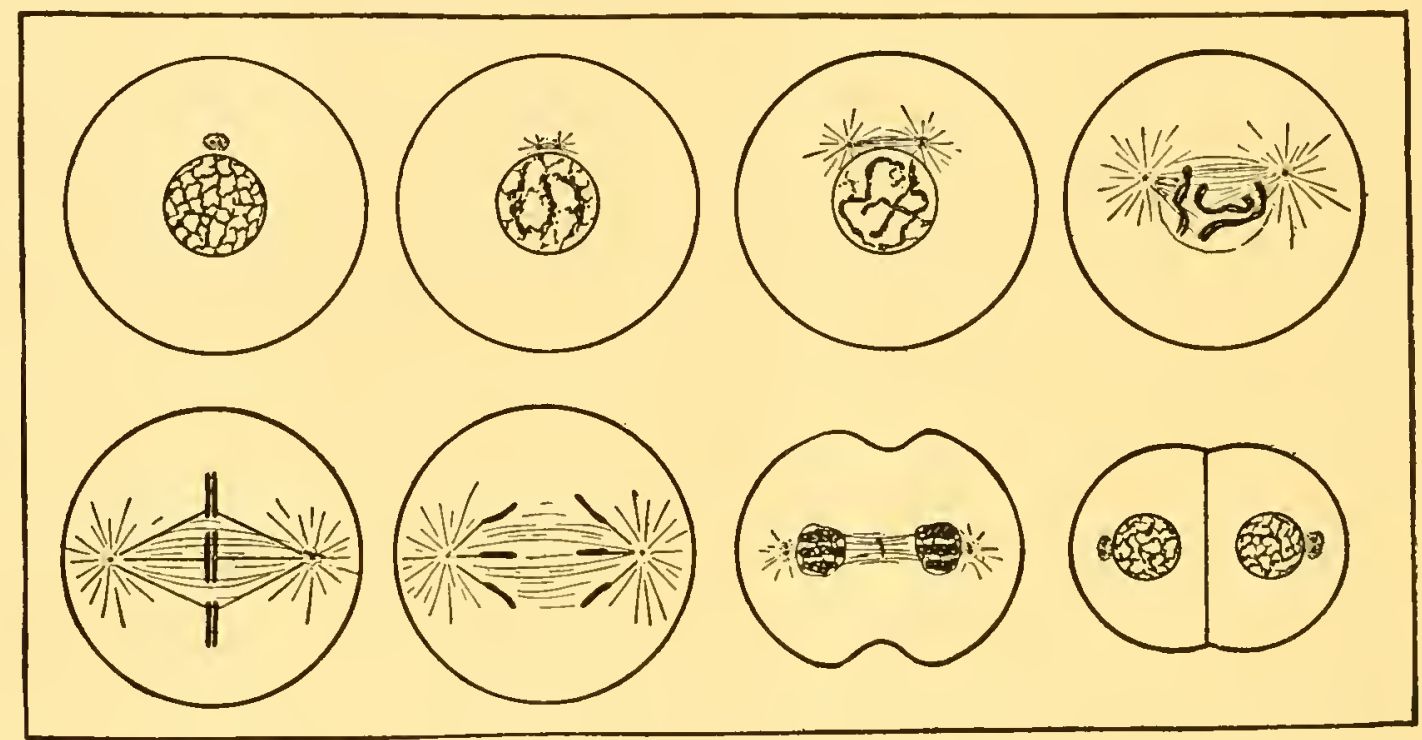

FIG. 58.-Diagram of a typical case of nuclear division and cell-division in animals.

related to nuclear influence but not to the mitotic process. Thus cleavage furrows or membranes may develop in positions clearly dependent upon the positions of the nuclei, the result being definitely uninucleate cells rather than the irregular multinucleate blocks seen in certain cases of plasmodial division. Frequently, as in the budding of yeast and the division of certain sporocytes, division of the cytosome follows so closely upon nuclear division that it seems in some way to depend upon it, without, however, involving the mitotic mechanism itself. Finally, cytokinesis may be so intimately connected with mitosis that the two are like a single process, the presence of the spindle between the nuclei causing a special series of changes in the plane of cytokinesis. In the interest of accuracy, however, the term "mitosis" should be applied only to nuclear division; it is not synonymous with "cell-division."

Cell-division in Animals.-Cell-division in animals is fundamentally similar to that in plants. Typically it differs, however, in the presence of centrosomes and in the mode of cytokinesis (Figs. 58, 59). As mitosis 
begins, the centrosome, if not already double, undergoes division, the daughter centrosomes then moving apart. Each of them occupies the center of a semisolid region, or aster, with conspicuous "astral rays," and between them is a central spindle with fine fibrils. The centrosomes, surrounded by their asters, reach opposite sides of the nucleus and remain at the poles of the mitotic figure through the metaphase, anaphase, and telophase. Cytokinesis is commonly brought about by a cleavage furrow which grows inward from the periphery of the protoplast, rather than by the differentiation of a plate-like wall as in the somatic tissues of higher plants.

Although cell-division in animals usually differs from that in plants in the above two respects, the distinction is by no means a sharp one. Centrosomes are regularly present in many algæ and fungi, and cytokinesis by furrowing also occurs in certain cells in both the lower and the higher plant groups. The point to be borne in mind is that the essential features of mitosis, the general results of cell-division, and the significance of these events in the life of the organism are the same in the two kingdoms.

Duration and Periodicity of Cell-division.-The duration of the process of cell-division, particularly that of the various phases of mitosis, has been determined by observing living material. ${ }^{3}$ In Spirogyra, de Wildeman (1891) found that mitosis was accomplished in 45 minutes at $12^{\circ} \mathrm{C}$., but that several hours were required when the temperature was a few degrees higher or lower. In the Tradescantia stamen hair mitosis was carried through in 30 minutes at $45^{\circ}$ (about the maximum temperature at which it would occur at all), in 75 minutes at $c a .25^{\circ}$, and in 135 minutes at $c a .10^{\circ} \mathrm{C}$. In stigma cells of Arrhenatherum at $19^{\circ} \mathrm{C}$. Martens (1927c) finds that the prophase occupies 36 to 45 minutes, the metaphase 7 to 10 , the anaphase 15 to 20 , and the telophase 20 to 35 ; total, exclusive of interphase, 78 to 110 minutes. In Sphacelaria fusca, a brown alga, growing at $17^{\circ}$ to $18^{\circ} \mathrm{C}$, the prophase occupies 10 minutes, the metaphase 7 , the anaphase 4 , and the telophase 9 ; total, 30 minutes (W. Zimmermann, 1923). W. and M. Lewis (1917) give the following figures for mesenchyme cells of the chick growing in tissue cultures at $39^{\circ} \mathrm{C}$.: prophase, 5 to 50 minutes, usually more than 30 ; metaphase, 1 to 15 , usually 2 to 10 ; anaphase 1 to 5 , usually 2 to 3 ; telophase up to cytokinesis, 2 to 13 , usually 3 to 6 ; telophasic reconstruction of daughter nuclei, 30 to 120 ; total, 70 to 180 minutes. In similar cultures of choroidal cells from the eyes of chick embryos and cartilage cells from adult fowls, Strangeways (1922) finds the process to be more rapid at the same temperature, complete division being accomplished in from 23 to 65 minutes, with the average at about 34 minutes. In a statistical study of mitosis in fixed onion root tips Laughlin (1919) observes that each stage shows a characteristic velocity reaction to temperature increments, and ${ }^{3}$ See Tischler (1921-1922), Martens (1927c), and Jaretzky (1930). 
that these approximate the expectations based on Van't Hoff's law for the velocity of chemical reactions.

When cytokinesis follows mitosis immediately, as it does in most tissues, it is carried through with considerable rapidity. In higher plants the cell-plate commonly appears during the closing phases of mitosis and by the time the daughter nuclei are fully reorganized the new cell membrane is well formed. In accelerated motion pictures of living fibroblasts and animal eggs the cells, after a series of interesting movements which escape the observer of fixed material, constrict and complete their division with dramatic suddenness.

In root tips, which are favorite objects for the study of somatic mitosis in plants, several investigators ${ }^{4}$ have found a periodicity in the occurrence of cell-division. Although their results are not in close
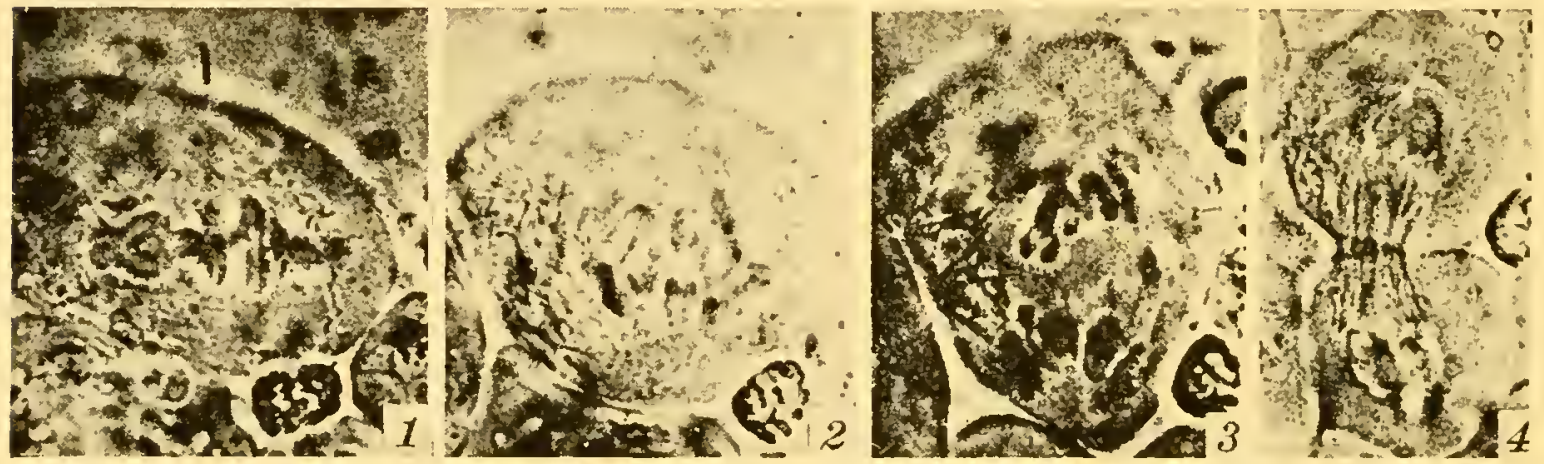

Fig. 59.-Mitosis in a living Chorthippus lineatus spermatocyte in $1 N$ Ringer solution. The change from 1 to 2 occupied 13 minutes; from 2 to 3,21 minutes; from 3 to 4,46 minutes. (After Bělařr, 1929a.)

agreement, they indicate that there are often about two division maxima during a 24-hour period, one of these falling near the middle of the day, and also that the rate of root elongation tends to be highest at the time of minimum cell-division. Many experiments have shown that while the periodicity in such cases may be altered by changing the environmental conditions, there is a noticeable tendency to retain the habitual rhythm. This indicates the action of both external and inherent factors, whose respective rôles in determining the observed results have not been fully analyzed.

Summary and Conclusions.-Typical somatic cell-division includes both mitosis and cytokinesis. In the prophase of mitosis the nuclear reticulum is resolved into a number of threads; these threads, or chromonemata, are the chromosomal elements which together formed the reticulum in the preceding telophase. They are double as the result of an accurate longitudinal splitting. A less highly stainable chromosomal matrix becomes evident about the chromonemata. In the metaphase

${ }^{4}$ Kellicott (1904), Karsten (1915, 1918), Friesner (1919, 1920), Stålfelt (1919, 1921), Fortuin (1926), Kojima (1928). 
the thickened double chromosomes are arranged with their attachment regions in the equator of the spindle, which is formed chiefly by the karyolymph. In the anaphase the longitudinal halves of each double chromosome pass toward opposite poles of the spindle. In the telophase the chromosomes of the two resulting groups reorganize two daughter nuclei, the chromonemata becoming joined to form the reticula while the matrix substance becomes less evident.

Anticipating evidence to be presented in following chapters, it may be stated further that in any particular kind of organism the chromosomes in the nucleus occur in a characteristic number and are differentiated among themselves in function and frequently in visible structure; they constitute a definitely organized system. Moreover, this organization is maintained by virtue of the genetic continuity of essential constituents of each chromosome through successive nuclear generations. This means that the chromosomes are not merely bodies which are temporarily formed by the nucleus but represent definite and persistent individuals reproducing by division and passing through a complicated series of visible changes in each nuclear cycle.

In view of these facts, it appears that the significant feature of somatic mitosis is this: each chromosome is longitudinally divided into equal halves which are distributed to the two daughter nuclei. These two nuclei are consequently similar to each other and to the original nucleus as regards their chromosome complements; in other words, somatic mitosis is equational. Furthermore, since all the nuclei of the body are normally derived through such mitoses from a single nucleus, each of these nuclei contains descendants of all the chromosomes present in the first nucleus of the series: the somatic nuclei are all alike in their chromosome complements, barring, of course, the effects of occasional aberrant chromosome behavior and other alterations to be described later. The great theoretical importance of these features of somatic nuclear behavior will be apparent when we take up the application of cytological phenomena to the problems of heredity and development.

Cytokinesis, or the division of the cytosome, follows mitosis quickly in most tissues and tends to separate the various elements-cytoplasm, plastids, chondriosomes, and vacuoles-more or less equally according to their arrangement in the protoplast. Occasionally some of these elements are passively divided. The daughter cells are initially similar in architecture and functional capacity. The course of their further differentiation depends largely upon their position in the developing whole. 


\section{CHAPTER IX}

\section{THE MORPHOLOGY OF THE CHROMOSOMES}

As they pass through a mitotic cycle, the chromosomes undergo a remarkable series of changes in form and structure. Nevertheless, in the metaphase and anaphase, when they are most condensed and clearly evident as individuals, they exhibit many characteristics of size and form which have proved useful not only in determining the functions with which they are associated, but also in comparing related species and hybrids. For the study of chromosome morphology in vascular plants the root tips and dividing microspores are particularly favorable. In animals the tissues of young embryos and the spermatogonia have been most frequently used. Different fixing reagents may produce a considerable variation in the appearance of chromosomes, but the studies of S. Nawaschin, Lewitsky, Taylor, and others have furnished us with methods which are very trustworthy when properly employed. ${ }^{1}$

Size and General Form.-As they appear in the metaphase or anaphase of somatic mitosis, the chromosomes of different organisms show a great range in size. In some cases they are extraordinarily minute, being less than $1 \mu$ long, while in others they may reach a length of $20 \mu$ or more; the breadth may vary likewise. Some natural groups, such as fungi and certain insect orders, have small chromosomes as a rule, while in others, notably amphibia, grasshoppers, and liliaceous plants, they are characteristically large. Within narrow circles of affinity, chromosome size sometimes affords evidence for probable taxonomic relationship. In this connection it should be noted that the size may be somewhat altered by cultural conditions and microtechnical treatments.

The chromosome is typically an elongated body. Sometimes very small chromosomes may appear practically spherical at metaphase or anaphase, but they are usually elongated in the prophase. Moreover, studies on larger chromosomes lead to the inference that even the smallest spherical chromosome contains an elongated chromonema. The essential point is that every chromosome adequately known possesses a longitudinally differentiated organization. This differentiation belongs primarily to the chromonema, but it is usually evident also in the entire metaphase chromosome with its abundant matrix.

${ }^{1}$ For an account of the development and present status of our knowledge of chromosome morphology, see Lewitsky (1931a); see also $1931 b$ for fixation. 
Noteworthy in this connection is the fact that the characteristic size differences shown by the several chromosomes of the same nucleus are almost wholly a matter of length; commonly the diameter of all of them is the same. This suggests that chromosomes are composed of smaller units arranged in a row, and that the longer chromosomes of a group have more units than the shorter ones. This interpretation may not be universally applicable, but genetical evidence to be presented in later chapters indicates its general validity.

Spindle-attachment Region.-The metaphase or anaphase chromosome usually shows at some point a differentiation which has commonly

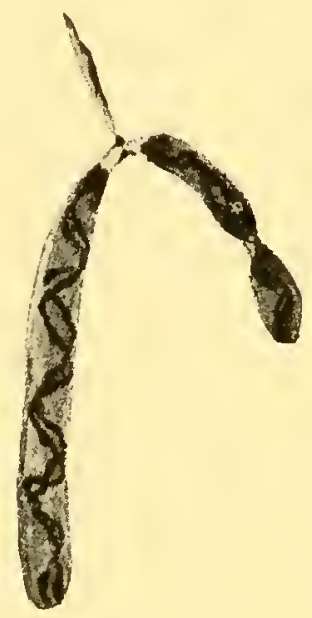

FIG. 60.

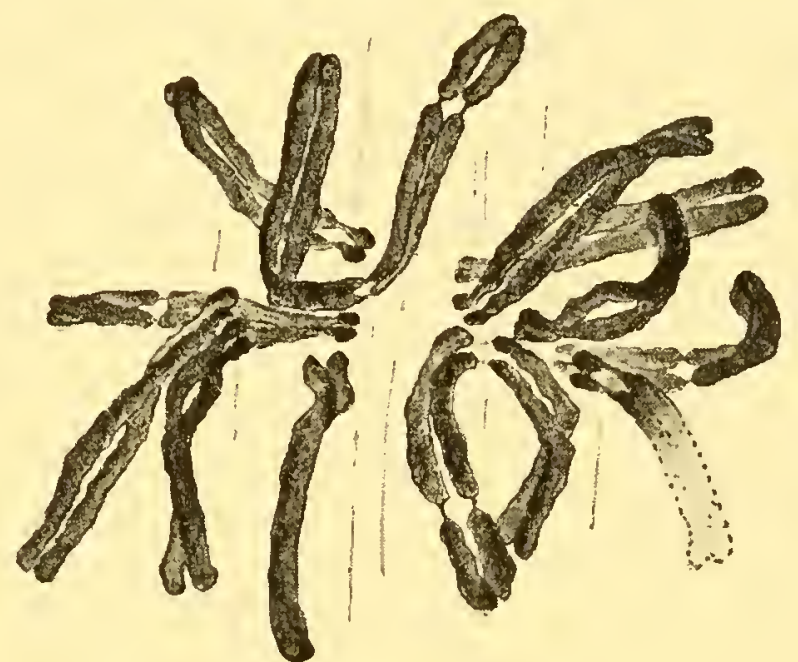

Fig. 61 .

Fig. 60.-A chromosome with submedian spindle-attachment region passing to the pole in anaphase. The shorter arm has a "secondary constriction."

Fig. 61.-Diploid chromosome complement at metaphase in root tip of Vicia faba. Spindle-attachment regions of all chromosomes near equator. Nucleolus-forming regions are by the "secondary constrictions" above and below. In this strain one pair of short chromosomes has conspicuous "constrictions" also (left and right). $\quad \times 1750$.

been referred to as a "constriction," since it appears as a relatively slender region in most preparations showing it clearly in these stages (Fig. 60). Careful studies have shown that the region in question differs from the rest of the chromosome in its structure and achromatic nature, and not merely in apparent diameter. In some preparations, especially of the prophase, it may appear as wide as or even wider than the adjacent portions; hence the term "constriction" is not truly descriptive. Some chromosomes have two or more achromatic regions. It is a striking fact that in a given chromosome these have definite and constant locations, ${ }^{2}$ although they may be very differently placed in different chromosomes.

${ }^{2}$ Exception should of course be made for individuals or races in which their positions differ because of translocation, inversion, or other alteration. See McClung (1917), Carothers (1921), King (1923), Helwig (1929); also McClintock (1931) on Zea. It was found by Sakamura (1920) that chloral hydrate shortens and thickens the chromosomes, thus accentuating the "constrictions." The effects of fixation have been studied by S. and M. Nawaschin, Lewitsky (see 1931b), and others. 
It was observed by Agar (1912) in a fish that it is at an achromatic "constriction" that the chromosome establishes connection with the spindle in the metaphase and separates first in the anaphase. This is now known to be true of chromosomes generally. Hence the region functioning in this manner has been variously called the "fiber-attachment point," "insertion region," "primary constriction," "kinetic constriction," "attachment constriction," and "Trennungstelle" (separation place). ${ }^{3}$ Chromosomes with apparently terminal spindle attachment are said to be telomitic; those with attachment elsewhere are atelomitic (Carothers, 1917).

As the result of the definite location of the spindle-attachment region, each atelomitic chromosome has two main segments, or "arms," with a characteristic length ratio. This is easily seen in side views of anaphase figures, also in polar views of metaphase ones if the chromosome is not too long. Such a chromosome passes poleward as a $V$ or $\downarrow$, the apex leading, whereas a telomitic one appears as an unbent rod. The same mitotic figure may include all types. It has been held by certain investigators ${ }^{4}$ that all chromosomes are probably two-armed, "telomitic" ones having an extremely minute second arm which easily escapes observation. Supposedly telomitic chromosomes have been shown in some instances to have their attachment region slightly back from the end, and in other cases a minute body seen at the end may represent the second arm; but that strictly one-armed chromosomes do not exist is at present improbable. What may have been the primitive location of the attachment, and how other types have arisen, are interesting subjects for speculation.

The structure of the spindle-attachment region has been the subject of some study. It may appear clearly in the prophase as a region in which highly stainable matter is lacking. It may be straight sided or somewhat swollen in some preparations (Fig. 152), and in certain instances it is known to have a characteristic length (Fig. 188). It appears to be homogeneous except for minute granules ${ }^{5}$ at the middle point (Figs. $62,63)$. The reaction which "attaches" the chromosome to the spindle seems to center in these "kinetic bodies," for it is from them that the strongly developed "spindle fibers" appear to extend in fixed preparations, and it is they which take the lead in the anaphasic movement. They have been observed at the extreme end of the attachment region

3 The convenient term kinetochore (= movement place) has been suggested to the author by J. A. Moore. The use of this term is recommended.

${ }^{4}$ S. Nawasehin (1916), Delaunay (1929), Heitz (1928a), Lewitsky (1930, 1931a). Chromosomes with apparently equal arms are termed isobrachial and unequal armed ones heterobrachial by Sorokin (1929). Lewitsky (1931a) ealls those with a small, rounded second arm "cephalobrachial," or "headed" chromosomes.

${ }^{5}$ Observed by S. Nawasehin (1913, 1916), Belling (1928b), Bělař (1929a), Trankowsky (1930a), Sharp (Fig. 63), and others. 
of "telomitic" chromosomes. In Trillium delicate strands extend out to the granules from the single arm of the telomitic chromosome and from both arms of atelomitic ones (Fig. 63). A comparison of these two types strongly suggests that in the former the attachment point is truly terminal. What relation the delicate strands may bear to the chromonemata is difficult to determine, especially since the chromosome tends to stain very deeply on either side of the attachment region.

The spindle-attachment region, then, is a well-differentiated feature of the chromosome: it is a region specialized with reference to the metaphasic arrangement and anaphasic separation of double chromosomes
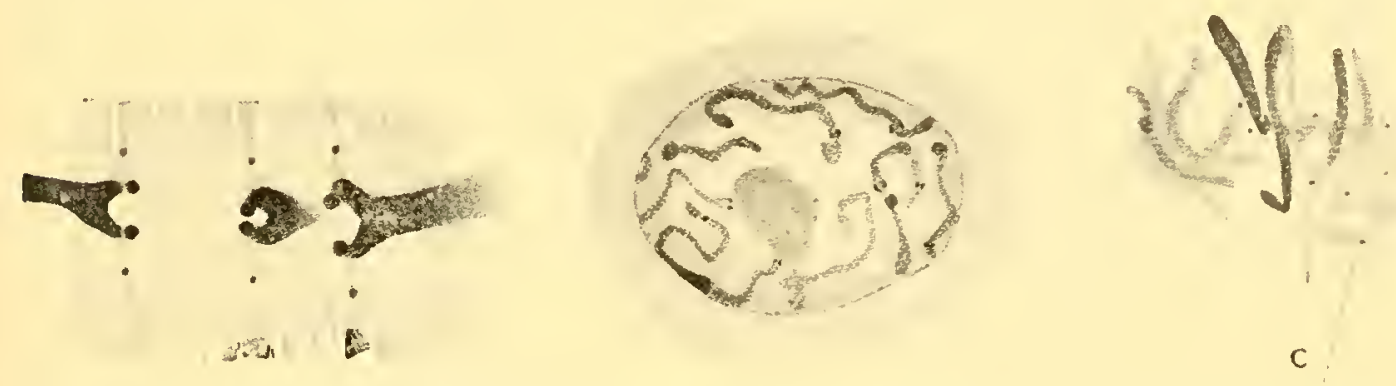

FIG. 62.-Kinetic bodies at spindle-attachment regions. A, in Galtonia mierospore. [After S. Nawaschin, 1927 (1913).] B, prophase in Najas major; C, anaphase in Crepis palestina. (After Trankowsky, 1930a.)
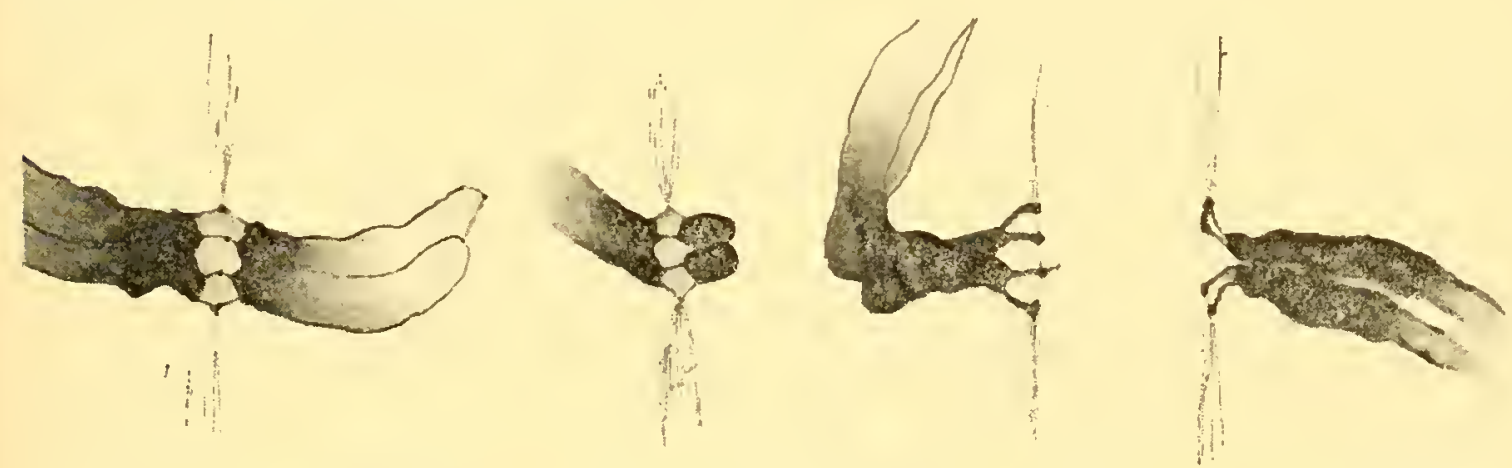

FIG. 63.-Submedian, subterminal, and apparently terminal spindle-attachment regions in Trillium grandiflorum.

(p. 107) and is not to be confused with other achromatic regions or "constrictions." That it persists through the interphase is indicated in Impatiens, where it can be seen at all stages (Fig. 24, B). What is known about the behavior of fragmented chromosomes (p. 317) suggests that spindle-attachment regions cannot be formed anew. In all probability the regular occurrence of one such region in every chromosome is due to the eventual loss of fragments without them because of their inability to interact normally with the spindle, and to the irregular behavior of any chromosome which happens to get an extra attachment region through translocation. Hence the constancy and the regular behavior of a group of chromosomes are directly dependent upon the attachment regions. Whether or not a chromosome may fragment 
directly through the attachment region, giving two chromosomes with terminal attachment regions, is not certainly known, but the possibility is strongly suggested by certain aberrations in Zea (p. 317).

Nucleolus-forming Region.-In addition to the spindle-attachment region the chromosome may exhibit one or more other differentiations affecting its general morphology. Among these perhaps the most interesting is the nucleolus-forming region, which seems as a rule to be conspicuously developed in but one chromosome of a set. Although it is reported to occupy the end of the chromosome in certain cases, it is more commonly situated in a non-terminal position. Moreover, its location is normally constant in a given race; like the spindle-attachment region, it is a definite "organ" of the chromosome.

The structure of this region is best known in sporocytes of Zea Mays, where it appears at certain stages as a broad and chromatic region of chromosome VI immediately adjacent to an achromatic region setting off a "satellite," or small portion of the chromosome arm (Figs. 65, 170). It is the chromatic region that is active in the accumulation of the nucleolar matter in the telophase. This is shown by the fact that in strains lacking the satellite, the achromatic region, and a portion of this chromatic region, the nucleolus is seen to arise from the remaining portion of the chromatic region. Normally, when chromosome VI is present in its entirety, the growth of the nucleolus causes the achromatic region to become extended as a slender filament lying over the nucleolar surface, so that the satellite may be situated at some distance from the remainder of the chromosome arm. In the ensuing prophase this filament may shorten more or less as the nucleolus decreases in size and disappears, but it remains visible as a conspicuous achromatic region, or "secondary constriction." 6

In most of the papers on this subject so far published it has been stated that the nucleolus is associated with the "secondary constriction" and satellite, the active chromatic region not having been recognized. Thus S. Nawaschin and his associates ${ }^{7}$ observed that during certain phases of mitosis, notably the late prophase, the satellites lie on the surface of the nucleolus (Fig. 64). In early prophases the satellite may appear like any other equal portion of the extended chromosome (Fig. $65)$, but during the condensation of the chromosome the satellite typically becomes a small spherical body (Figs. 64, 67, 72). In Vicia faba, investigated by Heitz (1931ab), the somatic chromosome complement (Fig. 61)

${ }^{6}$ Data in this paragraph from MeClintock (1931b and unpublished).

${ }^{7}$ S. Nawasehin $(1912,1915,1916$ et seq.), Sorokin (1924, 1929), Baranov (1926), Senjaninova (1926). Satellites were first described by S. Nawaschin (1912) for Galtonia candicans. Wenrich (1916) observed the attachment of the nucleolus to a certain portion of a chromosome in Phrynotettix. Kuhn (1928a) reviews the subject of satellites and lists the plants in which they have been reported. 
includes two long members with conspicuous achromatic regions setting off segments larger than those usually called satellites. Owing to the position assumed by the chromosomes in late anaphase these two regions lie at a certain level in each telophase nucleus, and a nucleolus develops near each of them. If the two chromosomes concerned lie far apart in the nucleus the two nucleoli remain separate, but if they lie near each other the nucleoli may come into contact and fuse. Hence some nuclei in the root show two nucleoli while others show one, and as a rule sister nuclei recently formed by division are mirror images of each other. This feature is represented in Fig. 54. In Zea roots the nucleolus is seen to be connected with the developing chromosomes at two points

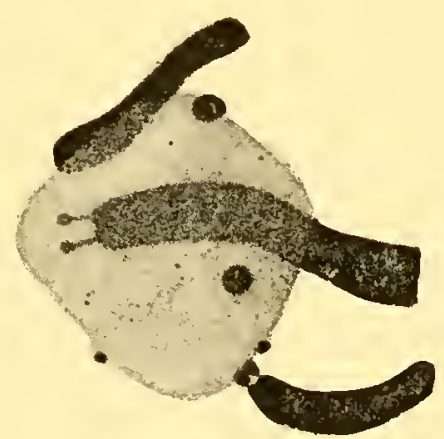

FIG. 64.

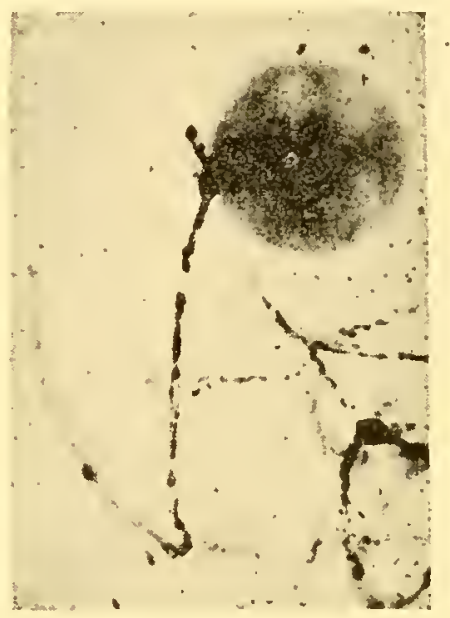

FIG. 65.

FIG. 64.- Satellited chromosomes attached to nucleolus in Galtonia. [After S. Nawaschin, 1927 (1913).]

F1G. 65.-Chromosome VI (synapsed pair) attached to nucleolus in microsporocyte of Zea Mays. The satellite projects upward from the chromatic swelling against the nucleolus. A short distance below the swelling is the spindle-attachment region, which here appears like a gap. Note chromatic knob toward left end. Compare Fig. 170. (After McClintock, 1931b, 1933.)

(Zirkle, 1928b); these are presumably the nucleolus-forming regions of the two chromosomes VI.

Because of the mode of their formation, it seems that nucleoli are as a rule connected with certain parts of certain chromosomes in the nuclear reticulum. At present too little is known to warrant anything more than suggestions regarding the significance of this phenomenon. It has long been thought by many observers that some nucleolar constituent passes into the chromosomes in the prophase and out of them in the telophase. This view is supported by the appearance of nucleoli as the chromosome matrix diminishes in amount and loses its chromaticity in the telophase, their disappearance as the chromaticity returns and the matrix becomes abundant in the prophase (Martens, 1922), and the results obtained with special fixing reagents (Zirkle, 1928b). Frequently it seems that only one of two principal nucleolar constituents functions in such a manner (Selim, 1930). If there is such a transfer 
of substance, microchemical tests indicate that it cannot be nucleic acid, "chromatin," or "karyotin." Heitz, who reports that in Vicia both nucleolus and secondary constriction react negatively in the Feulgen test for nucleic acid, ${ }^{8}$ inclines to the view that the nucleolus is formed by a substance existing throughout the nucleus and later accumulated near the constriction. In Zea sporocytes it is clear that the nucleolus bears a very close relation to the chromosome matrix (Fig. 65a). An

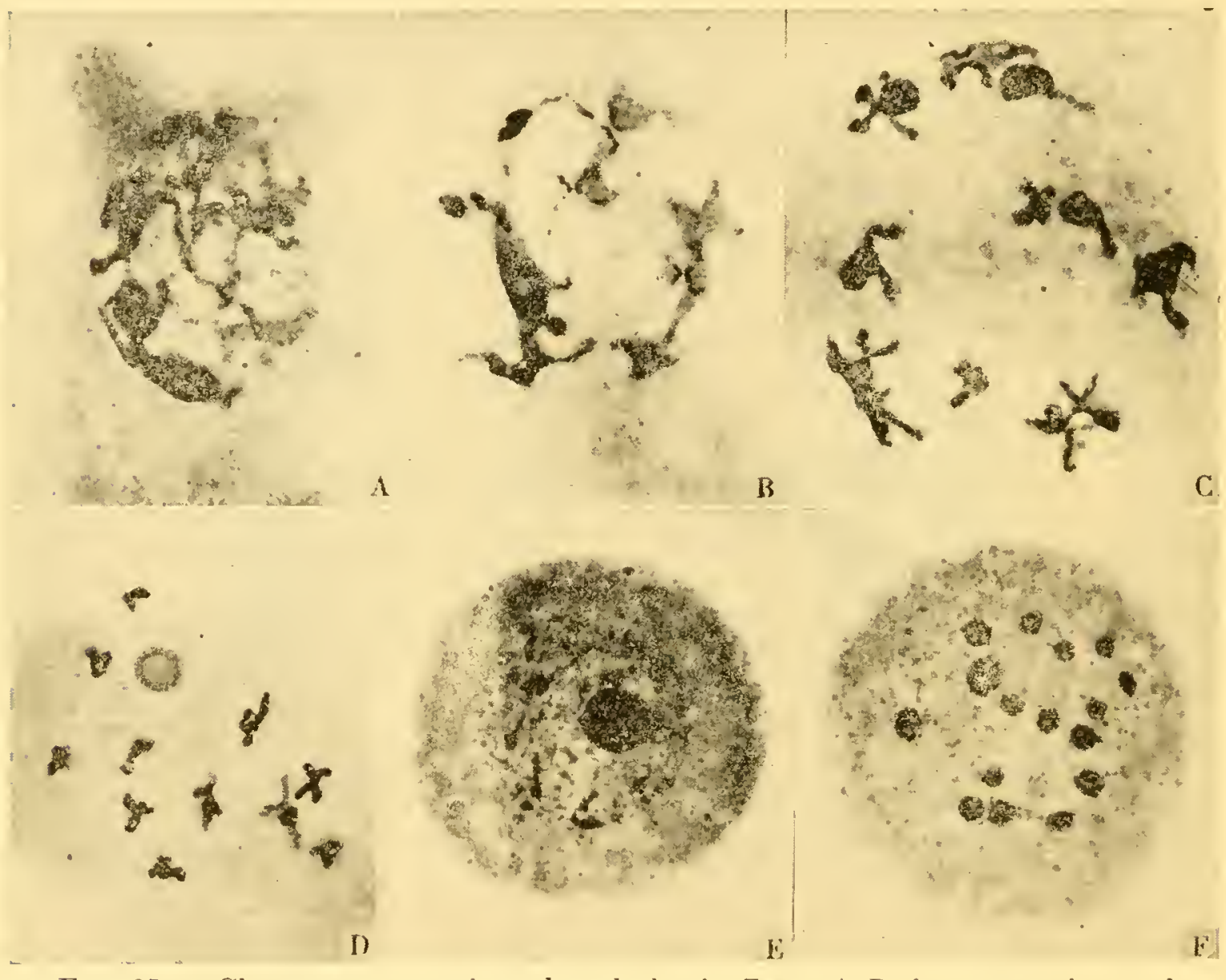

Fig. 65a.-Chromosome matrix and nucleolus in Zea. $A-D$, four stages in prophase of second meiotic division in microsporocyte. The nucleolus and the matrices of the several chromosomes are at frst eonfluent. The distinetion between matrix and chromonema shows well in $B$ and $C$. $E$, normal microspore with one principal nucleolus. $F$, microspore lacking the region of chromosome VI specially coneerned in nucleolus formation; many seattered nucleolus-like bodies present. (After McClintock, 1934.)

association of nucleolar behavior with the transfer of products of genic activity to the cytoplasm has been suggested by Zirkle (1928b) and Fikry (1930) (see p. 309).

The observations and hypotheses cited above indicate that certain chromosomes of the complement have regions specialized in connection with the transfer of material to and from them during the nuclear cycle (Haase-Bessell, 1928b). Accordingly, it might seem that every nucleus must have at least one such chromosome in order to pass through a

${ }^{8}$ Negative tests for the nucleolus are also reported by Wermel (1927), Yamaha (1932): Shinke and Shigenaga (1933), and others. 
normal cycle, this in turn suggesting why practically every chromosome set, well studied, shows at least one achromatic region, or "secondary constriction," which so often lies near a nucleolus-forming region. It is known, however, that nucleoli may develop at other points in the chromosome set under certain circumstances, as when the nucleolusforming region has been removed in the deletion of a portion of the chromosome. Thus when a strain of Zea Mays in which one member of chromosome pair VI had lost this region produced microspore quartets, it was found that two spores of each quartet had the region and a single large nucleolus, while the other two had no such region and a considerable number of small nucleolus-like bodies (Creighton) (Fig. 65a). These small masses of substance, which would normally contribute to the formation of a single nucleolus, may appear at one or more points on each chromosome. Heitz (1931b) reports that when a small extra nucleus is formed by a lagging chromosome in Vicia, it may contain a small nucleolus in addition to the two normal ones in each main nucleus. From such facts it may be concluded that all of the chromosomes give off a material during the telophase, and that under normal circumstances this material is incorporated in typical nucleoli formed in close association with specialized regions of certain chromosomes.

Other Specialized Regions.-Besides the spindle-attachment region and the nucleolus-forming region, other differentiations can frequently be observed in the chromosomes at metaphase and anaphase. These may sometimes appear as "constrictions" (Fig. 61), but very little is known about the functions they may perform or concerning the number of kinds of them which may be present in a chromosome set. It seems improbable that constrictions are necessarily places where fragments of chromosomes have formerly joined, ${ }^{9}$ although translocated pieces in Drosophila are sometimes set off by narrow regions.

The Chromosome Complement.-In any given kind of plant or animal each nucleus contains an outfit, or complement, of chromosomes composed of a certain number ${ }^{10}$ of members showing characteristic differences in form and function. As a general rule, the nucleus of an egg before fertilization contains a complement made up of one each of several kinds of chromosomes. Such a complement is called a set, or genom. Because each kind of element is represented but once, a nucleus (or organism) with such a chromosome outfit is said to be monoploid

${ }^{9}$ M. Nawaschin (1931c), Lewitsky and Araratian (1931).

${ }^{10}$ The known chromosome numbers in all plant groups are listed by Tischler (1927, 1931). Gaiser $(1926,1930)$ gives lists for angiosperms. See also Aase and Powers (1926) and Sisa (1929) for crop plants, Stolze (1926) for grains, Avdulow (1931) for Gramineæ, Kawakami (1930) for Leguminosæ, and Kihara, Yamamoto, and Hosono (1931) for plants cultivated in Japan. For animals, see Bresslau and Harnisch (1927) and Harvey (1916, 1920); also Oguma and Makino (1932) on vertebrates. Wilson (1925) gives a fairly extensive list for plants and animals. 
(or often haploid). The set is evidently a definitely differentiated group of elements all of which are usually necessary for normal development. In the male gamete there is a similar set of chromosomes. After the union of the gametes, therefore, the fusion nucleus of the zygote contains two sets of chromosomes. Since every kind of chromosome is present in duplicate, the nucleus is said to be diploid. All of the nuclei of the higher plant or animal body which develops from the zygote are derived by a series of equational mitotic divisions from the zygote nucleus; hence they are all diploid.

In the case of animals, gametes are formed when the individual becomes sexually mature; in this process the diploid zygotic number of chromosomes is reduced to the monoploid gametic number. This is done in such a manner that each gamete contains one complete set of elements, although this set may include elements from both of the original sets (Chapter XVI).

In vascular plants, bryophytes, and some thallophytes the situation is somewhat more complicated. The zygote and the sporophyte body into which it develops are typically diploid. A reduction to the monoploid number occurs when reproductive cells are formed, but in such plants these are spores rather than gametes. Each spore develops into a body of another type, a gametophyte with monoploid nuclei. Eventually the gametophyte produces gametes without further change in chromosome number, and these unite to form new zygotes. Hence such a plant life cycle differs from that of a higher animal in having two kinds of reproductive cells (spores in addition to gametes) and a monoploid body developing between sporogenesis and gametogenesis. It shows an "alternation of generations" which is usually, though not always, associated with an alternation of monoploidy and diploidy in the nuclei."1 Chromosome morphology in plants has been studied most in root tips, where two chromosomes of each kind are present, but it is often advantageous to use gametophyte tissue or spores because a monoploid group is easier to interpret, other things being equal.

Since the description of the chromosomes of Brachystola by Sutton in 1902, morphological differences in the members of the complement have been reported for a considerable number of animals belonging to various

11 The two nuclear phases, irrespective of their relation to the "generations," have been called gamophase and zygophase (Winkler). It is generally supposed that in the course of evolution the monoploid condition was first developed through a differentiation of the multiplying nuclear elements, the diploid condition arising as a result of fusion and necessitating a reducing process. The diploid generation is thought to have developed as the latter process became delayed in the cycle and to have hastened the evolution of diverse types by affording larger opportunity for the formation of new combinations through meiosis and syngamy (Svedelius, 1921). The diploid condition is also advantageous in affording a measure of protection against the detrimental effects of unfavorable recessive mutations. 
natural groups, especially the insects. ${ }^{12}$ In Drosophila melanogaster, so extensively used in genetical studies, the set is made up of four unlike members: one medium-sized chromosome with terminal spindle-attachment region (chromosome I), one large chromosome with median attachment (II), one slightly larger chromosome of the same type (III), and one very small chromosome (IV) (Fig. 71). In somatic cells and immature germ cells there are two such sets; as is characteristic of the Diptera, the corresponding chromosomes (the "homologues") actually lie near each other in pairs. ${ }^{13}$ The functional characteristics of these chromosomes will be discussed in a later chapter.

The morphology of the chromosome complement has been described in a large number of plants, particularly among the angiosperms. Several

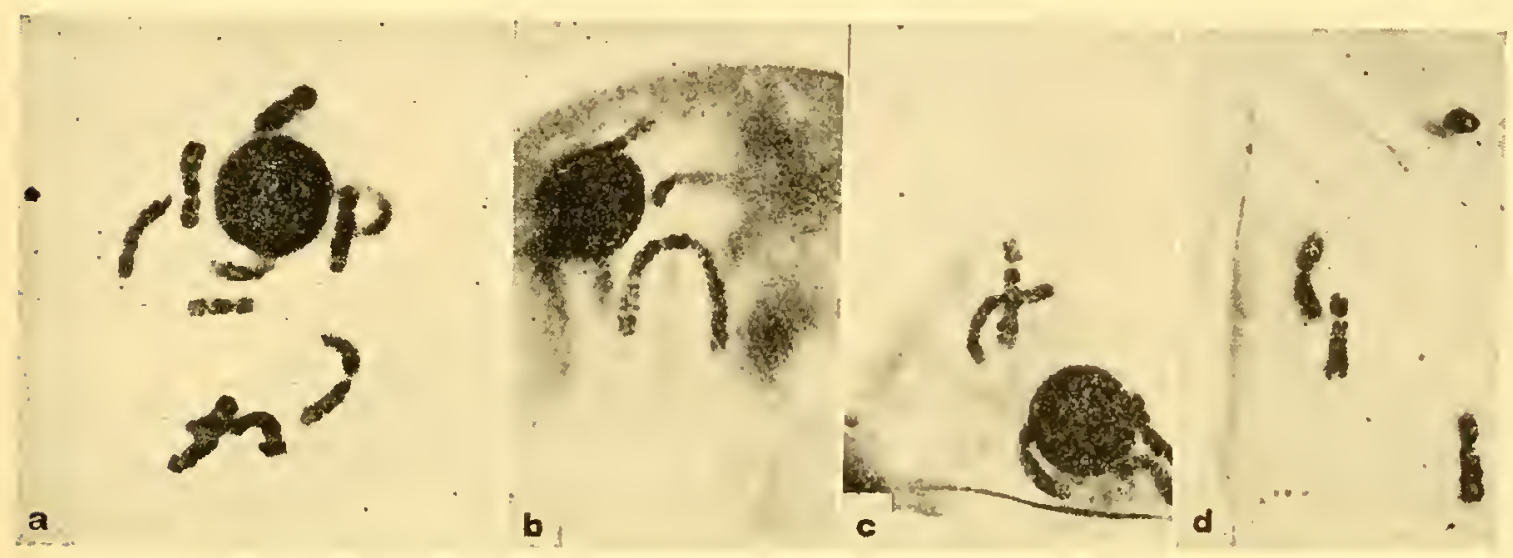

Fig. 66.-Photographs of Zea chromosomes in late prophase of mitosis in microspore. $a$, monoploid group; note spindle-attachment regions in chromosomes in good focus. $b$, chromosome I. c, chromosome IV, showing "knobs" near lower end. d, chromosome X. Compare Figs. 152 and 170. (Photographs by McClintock.)

well-known illustrative examples are the following. In Zea Mays ${ }^{14}$ the monoploid group comprises 10 chromosomes. These can be distinguished from one another on the basis of absolute length, the relative length of the two arms (each has a submedian spindle-attachment region), and other structural characteristics visible at certain stages (Figs. 66, 152, 170). One of them, number VI, has a conspicuous achromatic region setting off a satellite. In some races certain members of the set possess definitely located chromatic "knobs" which differ in character from large chromomeres; these aid further in identifying the various members. It is known that the chromosome sets of different races of maize vary in certain minor structural details, some such variations presumably being correlated with genic differences. Moreover, certain variations should also be expected in view of the observed fact of occasional translocations,

${ }^{12}$ McClung (1905 et seq.), Harman (1915, 1920), W. R. B. Robertson (1916 et seq.), Carothers (1917, 1921), Metz (1914 et seq.), and many others.

${ }^{13}$ Bridges, Morgan, Muller, Metz, Dobzhansky, Painter.

${ }^{14}$ Kuwada (1919), Fisk (1925, 1927), Longley (1927c), Randolph (1928 el seq.), McClintock (1929b et seq.). 
deletions, and inversions (Chapter XVIII). The same is doubtless also true of many other species in which a "typical" or "standard" chromosome set has been described.

In Datura stramonium there are 12 chromosomes in the set. These vary somewhat in different races. In "line $1 A$ " there are one very long, two long, five large medium, two small medium, one short, and one very short members. ${ }^{15}$ According to Lewitsky, all 12 may be distinguished on the basis of size, arm ratio, and the presence of small rounded segments. In Pisum sativum the seven pairs in the root cells are distinguishable $^{16}$ (Fig. 67, A). In Vicia faba there are five pairs with subter-

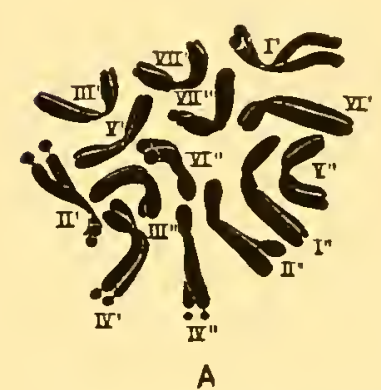

A
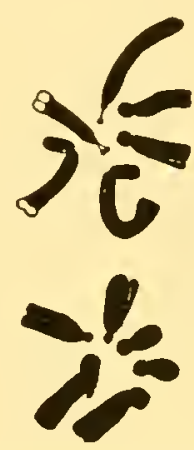

B

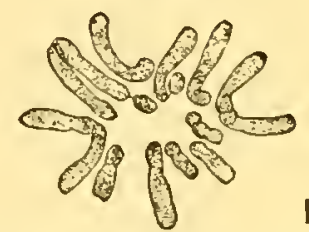

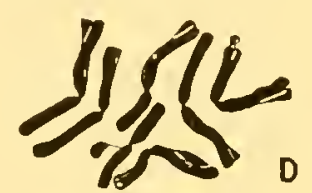

E

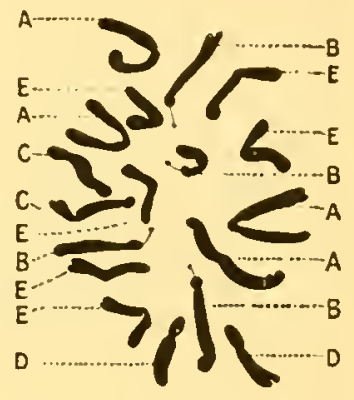

$\mathbf{F}$

FIg. 67.-Somatic chromosome complements in various higher plants. A, Pisum sativum. (After Lewitsky, 1930.) B,C, Crepis capillaris, after regular treatment and after shortening by cooling. (After Delaunay, 1930.) D, Allium allegheniense, with translocated fragment on one member. (After Levan, 1932.) E, Najas major. (After Tschernoyarow, 1914; see also Miuller, 1912, Winge, 1927a, and Takamine, 1927.) F, Nieotiana alata var. grandiflora. (After Avery, 1929.)

minal spindle-attachment regions and one much larger pair with a median attachment region and a conspicuous "secondary constriction" (Fig. 61). The chromosome set of Crepis capillaris (= virens) consists of a large chromosome with its attachment region between the middle and end, a medium-sized one with very unequal arms and a satellite, and a small one with unequal arms. Hence the somatic cells show three distinguishable pairs ${ }^{18}$ (Figs. $69 ; 72, B$ ). In Nicotiana alata var. grandiflora the nine pairs of chromosomes fall into five classes on the basis of their morphology (Fig. 67, F).

In a large number of plants and some animals the chromosome complements of gamete and zygote do not have merely one set and two sets of

${ }^{15}$ Belling and Blakeslee (1922 et seq.), Blakeslee, Belling, Farnham, and Bergner (1922), Lewitsky $(1930,1931 a)$. In much of the literature on Datura the term "set" is used rather for any group of homologous chromosomes, a diploid plant having 12 "sets" of two, a triploid 12 "sets" of three, and so on.

${ }^{16}$ Lewitsky (1930), Marshak (1931).

${ }_{17}$ Sharp (1913, 1914a), Sakamura $(1914,1915,1920)$, Mæda (1930b). For other species of Vicia, see Sweschnikowa $(1927 a b, 1928,1929)$.

${ }_{18}^{18}$ Rosenberg (1909a, 1918, 1920), de Smet (1914), Collins and Mann (1923), Mann (1925), M. Nawasehin (1925ab, 1926), W. R. Taylor (1925c), Hollingshead (1930c), Lewitsky and Araratian (1931). 
chromosomes respectively, as in the foregoing "diploid" types, but other numbers, such as two and four sets ("tetraploid" types), three and six sets ("hexaploid" types), and so on. Such individuals or species are said to be polyploid. This phenomenon and its significance are to receive detailed consideration in Chapters XX to XXII.

The Arrangement of the Chromosomes in the Mitotic Figure.As a general rule it seems that the various chromosomes of the complement have no constant relative position in the nucleus or mitotic figure. A comparsion of their positions in a number of metaphases in the same plant usually shows that the arrangement varies greatly. It frequently happens, however, that the arrangement is the same in figures which lie near each other, as in the same row of cells in a root tip, or in neighboring sporocytes. This is easily understood when it is remembered that the halves of the chromosomes pass rather directly to opposite poles from the equatorial plane and thus form similar patterns in the sister nuclei in the telophase. If the next mitosis follows soon, this pattern may be retained through another nuclear and cell generation. As the division rate becomes retarded and the cells compared come to lie farther apart, the arrangement is somehow altered. To what extent the chromosomes may change their relative position in interphasic and metabolic nuclei is not known, but irregularities in their grouping at the end of the anaphase seem to be in part responsible for the alteration observed.

What has been said above applies particularly to the elongated somatic chromosomes of plants whose morphology has been most studied. An examination of the chromosomes in certain sporocytes, where they are short and well separated from one another in the developing mitotic figure, shows that they tend strongly to assume arrangements like those of floating magnets of the same relative dimensions; they behave as if they were all acting under a mutual repulsion, especially of their spindleattachment regions. The most stable arrangement occurs most frequently, other less stable ones being due to such modifying factors as the viscosity of the medium and the time required to assume the arrangement. ${ }^{19}$

In animals also, particularly in the insects, cases are known in which the chromosomes show a more or less constant and characteristic arrangement in the metaphase. Often the smaller chromosomes lie in the middle of the figure with the larger ones around them (Fig. 215), or they may form a circle with certain members inside or outside it. ${ }^{20}$ Here again the phenomenon is best displayed where the size and spacing of the chromosomes are such as to permit their free movement as the metaphase stage develops. In the Diptera, as already mentioned, the chromosomes

${ }^{19}$ Kuwada and collaborators (1929). Cf. R. S. Lillie (1905), Cannon (1923), Müller (1912), and Heimans (1928).

${ }^{20}$ See Wilson (1925, various figures in Chap. XI; also 1932). 
of the diploid complement tend to show a very regular paired arrangement (see Metz, 1916). Similar but less distinct pairing is sometimes reported in plants. An especially interesting case is described by Robertson (1930) in Paratettix texanus, a grasshopper (Fig. 68). In individuals produced by the union of two gametes in the usual way the two parental chromosome sets tend to remain distinct in the developing tissues, whereas in individuals produced by parthenogenesis the two sets (evidently formed here by chromosome division in the incomplete maturation of the egg) have a distinctly paired arrangement. In both cases the
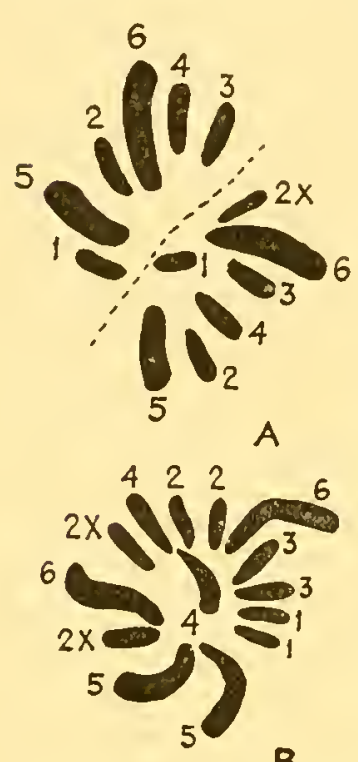

FIG. 68.-Chromosomes of Paratettix texanus. $A$, from biparentally produced individual; the dotted line passes between the parental sets. $B$, from parthenogenetically produeed individual. (After $W$. Robertson, 1930.) configuration in the egg as it begins development is maintained through the successive cell-divisions.

All of these facts suggest that there are always factors, some of them purely physical and some involving the chemical and genetic constitution of the chromosomes, which tend to give the members of a complement a certain arrangement, and that if these factors were always given unrestricted opportunity for action each type of complement might always exhibit about the same characteristic configuration.

Chromosome Complements of Related Organisms. The discovery of the relative uniformity of the number of chromosomes in an individual or a species led to the determination of the number in many organisms belonging to all of the major groups. A certain amount of speculation regarding the general phylogenetic significance of chromosome number has been indulged in, but students of the subject have reached the conclusion that the data at hand do not warrant many broad generalizations. So far as the large groups are concerned, there appears to be no correlation between taxonomic position and structural complexity, on the one hand, and the number, length, width, or volume of the chromosomes, on the other. It is, however, generally agreed that within restricted circles of affinity the number of chromosomes, and especially their size and form, may often afford important evidence in the determination of genetic relationships. ${ }^{21}$

The type of chromosomal complex characteristic of any individual or group of allied forms may be called its karyotype (Lewitsky, 1924). ${ }^{22}$

${ }^{21}$ MeClung (1908), Strasburger (1910b), Farmer and Digby (1914), Tischler (1916, 1921-1922), Winge (1917), Gates (1920b, 1924ab), Marchal (1920), Meek (1920), Heilborn (1924). See especially Lewitsky (1931c) for chromosome morphology. See also footnotes, pp. 23-28.

${ }^{22}$ The term was originally used in a more restricted sense by Delaunay (1922). See the discussion by Lewitsky (1931c). 
The diagrammatic representation of a karyotype, as in Fig. 70, is called an idiogram (S. Nawaschin, 1921). One of the most important lines of investigation in modern cytology is that in which the nuclear constitutions of related organisms are studied through comparisons of their idiograms. Such studies afford invaluable evidence supplementing that of the geneticist, taxonomist, and student of phylogeny.

In Crepis, which has come to be one of the most important genera for investigations in this field, ${ }^{23}$ there are many species with only three, four, or five pairs of chromosomes. These appear to be of about five distinguishable kinds as regards size and shape, each species having a characteristic complement composed of from three to five of these chromosome types (Fig. 69). It is observed that a chromosome of a given type

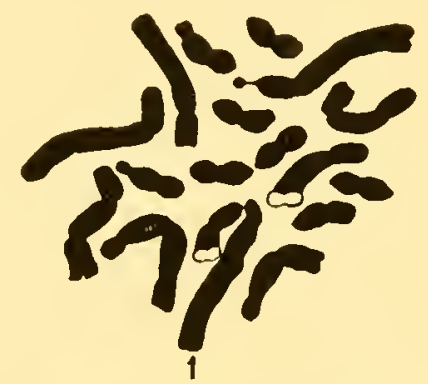

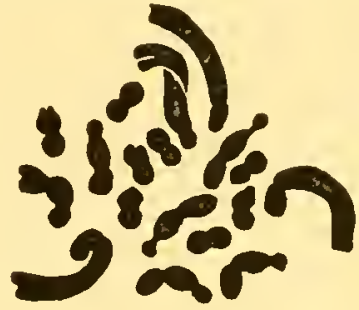

2

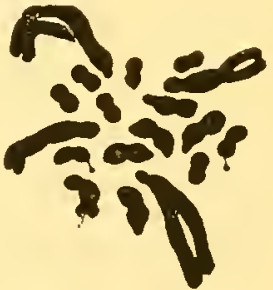

3

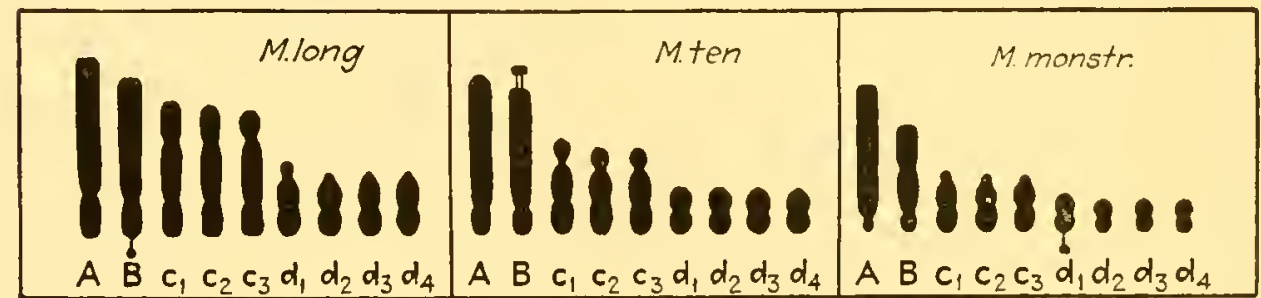

Fig. 70.-Somatic chromosome complements of three species of Muscari: longipes, tenuiflorum, and monstrosum. (After Delaunay, 1926.)

may vary considerably in length in the complements of the different species. The fact that the order of the species, when arranged in a series on the basis of a progressive lengthening or shortening of a given chromosome of the set, may differ from the order in a series similarly arranged for a different chromosome is taken to mean that the chromosome set is not a simple unit, but is rather a harmonious system of autonomous entities which are in some measure independent of one another in their evolutionary alteration.

In Muscari, Delaunay (1926) finds that the idiograms in three species differ chiefly in the absolute and relative length of the members (Fig. 70) and believes that in many such cases a gradual shortening of the chromosomes may have occurred during the evolution of the genus. Studies on the karyotypes of several groups, notably the Helleboreæ, ${ }^{24}$ have led

${ }^{23}$ See especially M. Nawaschin (1925a, 1926), Hollingshead and Babcock (1930), and Babcock and Nawaschin (1930).

${ }^{24}$ Lewitsky (1931a); also Senjaninova-Korezagina (1930, 1931) on Agilops ard Vicia, Avdulow (1931) on Agropyrum, and Lewitsky and Tron (1930) on Bellevalia and Muscari. 
Lewitsky to the view that in various tribes and genera there has been a progressive shortening of one arm in many of the chromosomes, primitive members of a group having mostly isobrachial chromosomes and derived members mostly heterobrachial ones. In Drosophila the idiograms in the various species bear a general resemblance to one another but show characteristic minor differences ${ }^{25}$ (Fig. 71). Even within a Linnæan species the idiograms may not be alike in all individuals. Thus in Rumex acetosa the plants fall into several classes on the basis of the

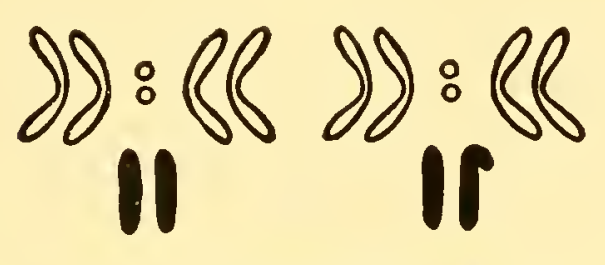

q D. melanogaster $\delta^{x}$

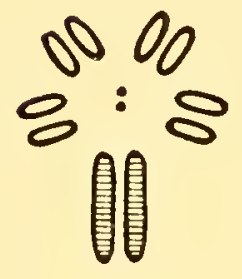

O.funebris

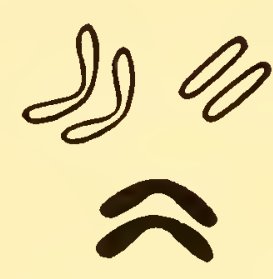

D. willistoni

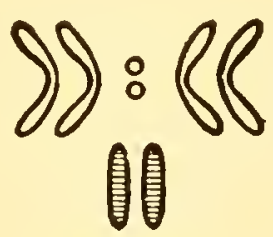

D.simulans

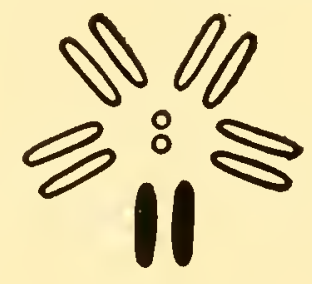

D. virilis

Fig. 71.-The somatic chromosome complements of several species of Drosophila. Pairs known to be sex-chromosomes shown in solid black; those presumed to be are marked with cross lines. (After Metz, Moses and Mason, 1923.)

karyotype. Such cytological studies have now been made on a considerable number of genera ${ }^{26}$ and are to receive further consideration in Chapter XX.

Groups of related genera, as well as the related species of a genus, often have the same general karyotype. For example, in Haworthia, Gasteria, and Aloë the complement consists of four long and three short pairs, with minor peculiarities in each genus ${ }^{27}$ (Fig. 72, $E$ to $G$ ). A notable example of similarity of chromosome complements throughout

${ }^{25}$ Metz (1914, 1916), Metz and Moses (1923), Metz, Moses, and Mason (1923), Frolowa (1926), Frolowa and Astaurow (1929).

${ }^{26}$ E.g., Vicia (Sweschnikowa, 1927ab, 1928, 1929; Heitz, 1931; SenjaninovaKorczagina, 1932); Triticum (Kagawa, 1927ab, 1929abc); Pellia (Heitz, 1928ab); Egilops (Senjaninova-Korczagina, 1930); Mecostethus (McClung, 1928ab); Rumex (Kihara and Yamamoto, 1931); Nigella, Delphinium, Aconitum, and other Helleboreæ (Lewitsky, 1931c).

${ }^{27}$ W. R. Taylor (1925b). See also Darlington (1926) on Scilleæ, Langlet (1928) on Berberidacex, Kachidze (1929) on Dipsacacex, Tschcchow (1930) and Tschechow and Kartaschowa (1932) on Leguminosa, and Saez (1928) on mammals. 
a large group is afforded by the Aerididæ, a family of grasshoppers. ${ }^{28}$ It is stated that the degree of relationship here is as clearly expressed in the chromosomes as in the externally visible characters, and that the evidence indicates a descent by variation from a common ancestral series of ehromosomes paralleled by corresponding variation in somatic structures (Robertson).

Very often among plants and oceasionally among animals the chromosome complements in a related group of individuals, races, or species differ in the number of sets present, i.e., they form a "polyploid series."
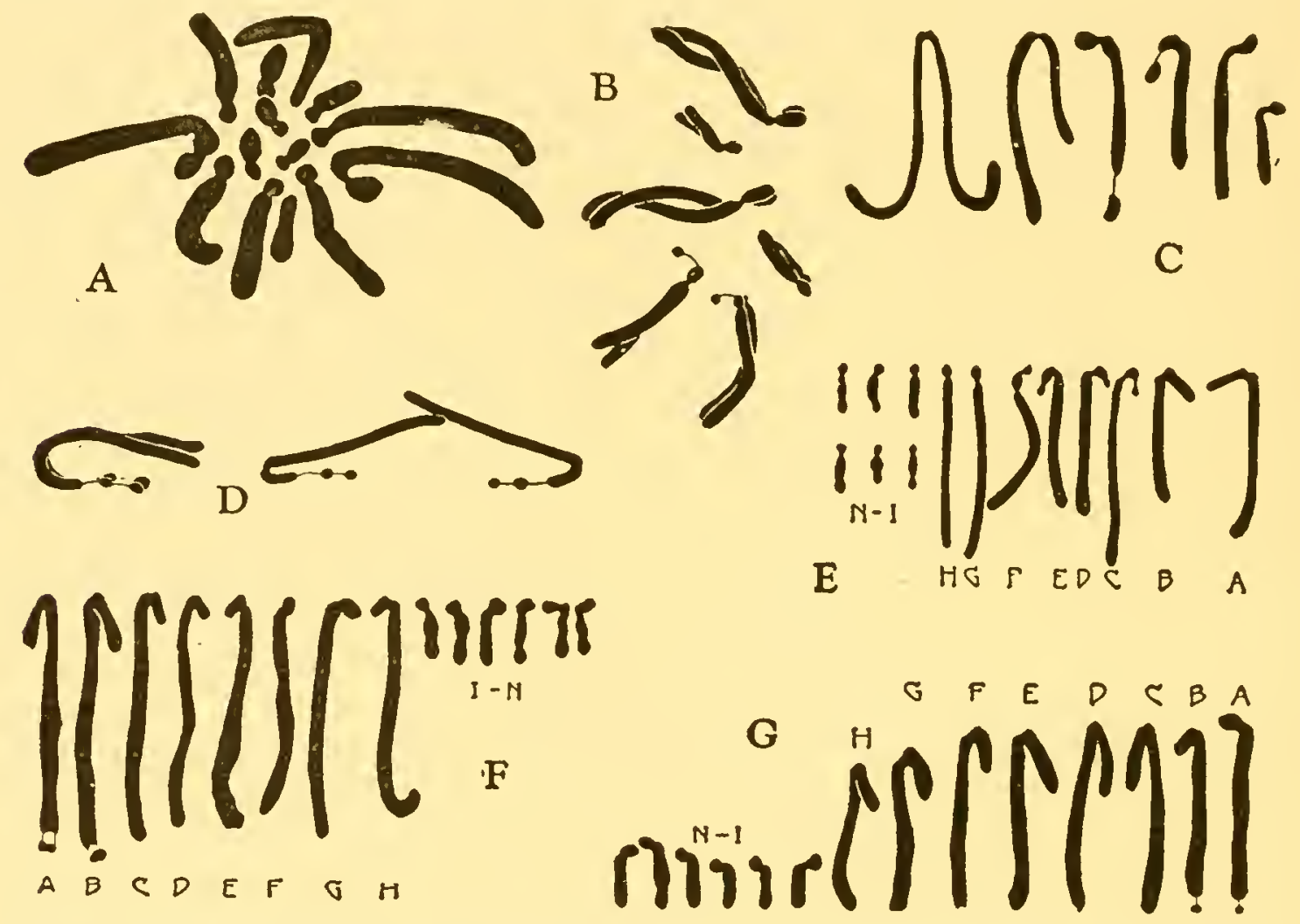

FIG. 72.- Morphology of somatic chromosomes in several angiosperms. A, Gasteria verrucosa. B, Crepis capillaris (virens). C, Cyrtanthus parviflorus: the six forms exhibited by the eight pairs of chromosomes. D, Allium cepa: chromosomes in metaphase and anaphase, showing two satellites in tandem; seen in one plant. E, Haworthia cymbiformis. $F$, Gasteria verrucosa. G, Aloë arborescens. Compare $E, F$, and $G$. (After W. R. Taylor, $1924,1925 a b$.)

Sometimes the chromosome numbers do not form a series of multiples. Consideration of the origin and consequences of these conditions must be postponed to later chapters.

It is not to be doubted that such similarities and differences in chromosome complements are of the greatest importance to students of genetic relationships among organisms. At the same time caution is necessary in the use of such evidence. It should not be assumed simply because two chromosomes in different organisms look alike that they correspond in function, nor that chromosomes with similar funetions should always show exact resemblance (see Fig. 71). Not only is it ${ }^{28}$ MeClung (1905 et seq., 1923, 1924), W. R. B. Robertson (1916), Saez (1930). 
known that the size and morphology of the chromosome may vary to some extent with cultural methods and mode of fixation, but in many cases it has been found that a chromosome may break into pieces, one of the pieces often becoming attached to another chromosome and so altering the idiogram (see Chapter XVIII). In this way the karyotypes of sister individuals or closely allied groups may come to show marked differences. Conversely, the same general karyotype may characterize species which are morphologically very unlike. Evidently the differentiation of genera, species, and varieties has involved genetic alterations not directly observable in the nuclear substance, as well as changes which modify the chromosome in a more obvious manner. The fact remains, however, that when these considerations are borne in mind, chromosomal characters as well as external features should be of great service in the solution of many taxonomic and phylogenetic problems. No student of such problems can afford any longer to ignore the data of cytology. 


\section{CHAPTER X}

\section{THE STRUCTURE OF THE CHROMOSOMES}

In most fixed and stained preparations the chromosomes in the metaphase and anaphase of mitosis appear as uniformly colored, more or less homogeneous bodies. Attention must now be given to the important question of the actual internal structure of such chromosomes and the alterations undergone by this structure during the various phases of the mitotic cycle. Students of this subject have investigated intensively somatic chromosomes, particularly those of root tips, but sporocytes and spermatocytes have come to be the favorite objects for such studies because of the size of their nuclei and the ease with which they may be examined without imbedding or sectioning.

In the development of our knowledge of chromosome structure three principal interpretations have been prominent. These are (1) the chromonema theory, according to which the fundamental structural element of the chromosome is a slender filament, or chromonema, present in some form throughout the mitotic cycle and associated with a second substance, the matrix; this interpretation was used as a basis for our preliminary description of mitosis in Chapter VIII; (2) the alveolation theory, according to which the filament is a temporary form assumed by the chromatic nuclear matter in the prophase as the result of an alveolation of the more or less homogeneous chromosome in the preceding telophase; (3) the chromomere theory, according to which small autonomous bodies, the chromomeres, are supported by relatively achromatic threads and reproduce regularly by division in mitosis. It will be seen in what follows that our present interpretation of chromosome structure incorporates elements of the chromonema and chromomere theories; that is, the typical chromosome in its condensed stages is now regarded as a mass of matrix enclosing one or more chromonemata in which there are small differentiations called "chromomeres."

The Chromonema Theory.-In 1880 Baranetzky examined fresh Tradescantia sporocytes in spring water and saw what appeared to be spiral filaments within the chromosomes. Janssens (1901) suggested that such spirals in the telophase were the same as those observed by himself and many others in the prophase. This conception was embodied in the chromonema hypothesis, developed especially by Bonnevie (1908, 1911) and Vejdovsky (1912). As a result of her studies on Ascaris, Allium, and other forms, Bonnevie concluded that each chromo- 
some in the telophase develops an endogenous spiral thread which persists through the interphase and emerges from the chromosome in the following prophase. The achromatic material is not continuous from one nuclear generation to the next but is differentiated anew in each telophase as the spiral develops. According to Vejdovský, who first applied the term "chromonema" to the spiral filament, the latter arises much earlier than stated by Bonnevie: it first develops in the prophase within the yet unsplit antecedent chromonema, and, as the latter splits, the new chromonema becomes broken up into irregular rings or strands which in some undetermined manner form a continuous spiral again in the telophase. The achromatic portion of the chromosome swells and becomes the karyolymph, at the periphery of which the nuclear membrane is formed. Such chromonemata have since been observed and discussed by many writers, one of the principal questions at issue being that of the permanence of such filaments through the nuclear cycle.

That the chromonema is a permanent constituent of the chromosome and not a temporary formation has been rendered evident by recent studies on both somatic cells and sporocytes. In root tips prepared by methods rendering the matrix less stainable the chromatic chromonema can be observed as a contorted or coiled filament lying mostly in the peripheral region of the metaphase or anaphase chromosome. After certain other methods the chromatic matter appears to be more uniformly distributed in the peripheral region of the chromosome, which thus has a tubular appearance. ${ }^{1}$ In the telophase the chromonema becomes a portion of a new nuclear reticulum, which resolves into its constituent chromonemata again in the next prophase. What is known about the morphology of the individual chromosomes and the characteristic structural patterns exhibited by certain chromonemata (see p. 141) indicates that the prophasic chromonemata are actually the same as those which formed the reticulum in the preceding telophase, although they have undergone temporary alterations in the meantime.

In large chromosomes it appears that the chromonema in the telophase, metabolic stage, and prophase is double as the result of splitting one mitotic cycle in advance of the anaphase in which the halves so formed are to separate. ${ }^{2}$ Whether or not this is also true of small chromosomes is unknown.

The Mitotic Cycle.-Large chromosomes like those just mentioned appear to pass through somatic mitosis essentially as follows (Figs. 73, 74).

${ }^{1}$ E.g., Bonnevie (1908), Zirkle (192S), and Blunt (1932).

2 K. Schneider (1910) on Salamandra; Dehorne (1911) on Salamandra and Allium; Kaufmann (1925, 1926ab, 1931a) on Tradescantia, Podophyllum, Allium, and Iris; Sharp (1929) on Trillium, Vicia, Podophyllum, Allium, and Tradescantia; Telezynski (1930ab, 1931ab) on Tradescantia and Hamanthus; Nebel (1932) on Tradescantia: Hedayetullah (1931) on Narcissus; Perry (1932) on Galanthus; F. H. Smith (1932) on Galtonia; Huskins and Huates (unpubl.) on Anthoxanthum. 

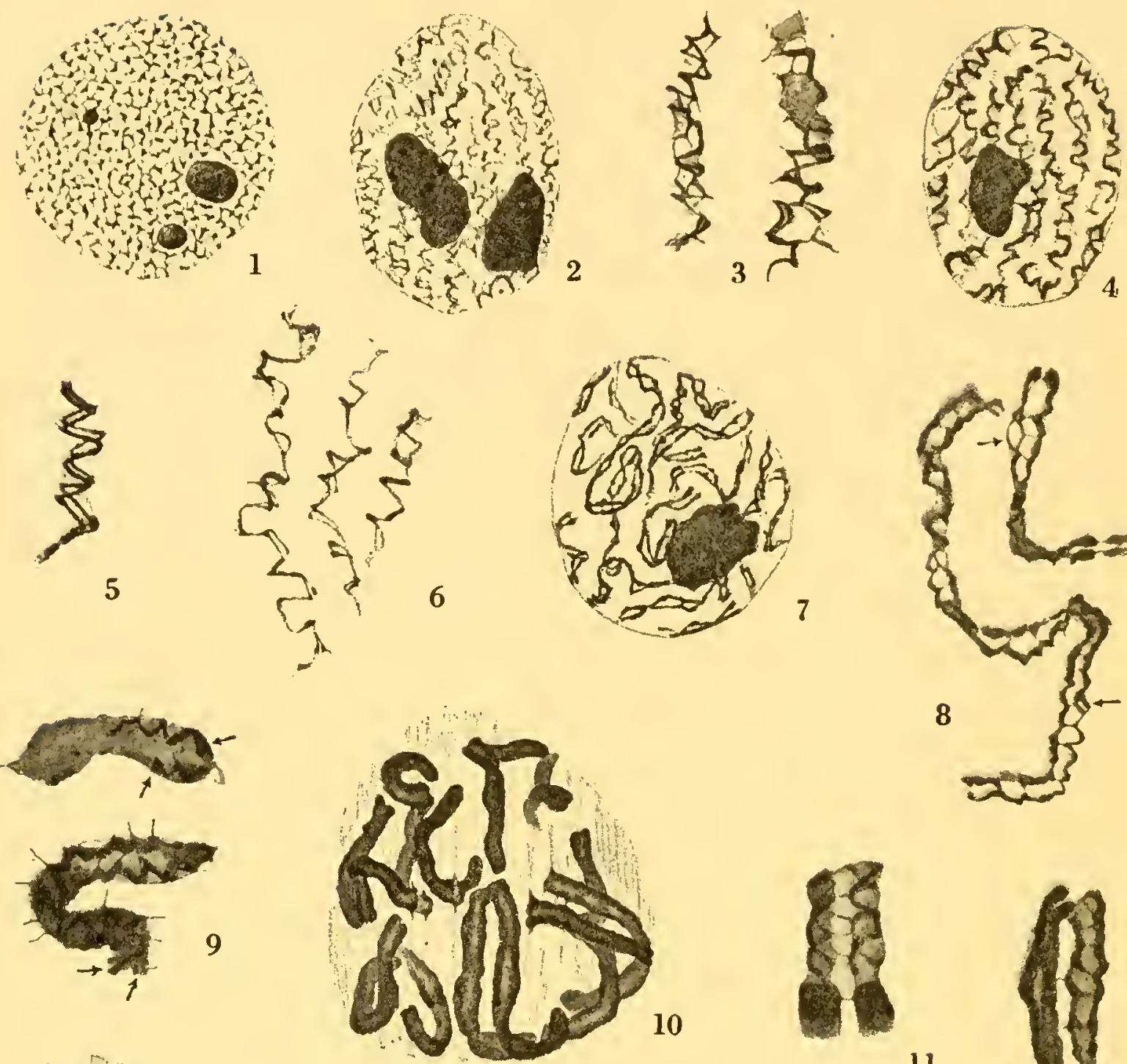

8
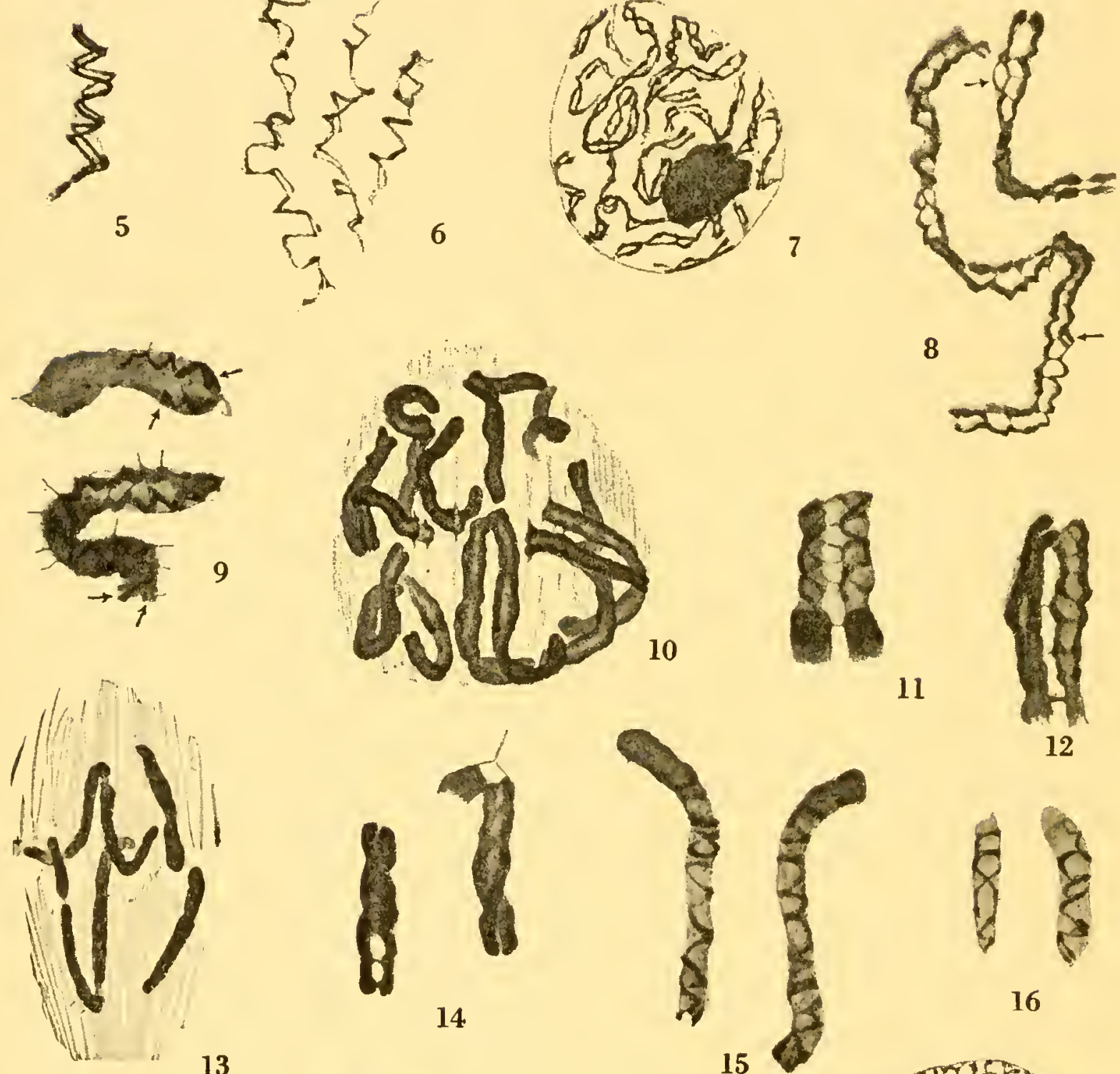

13
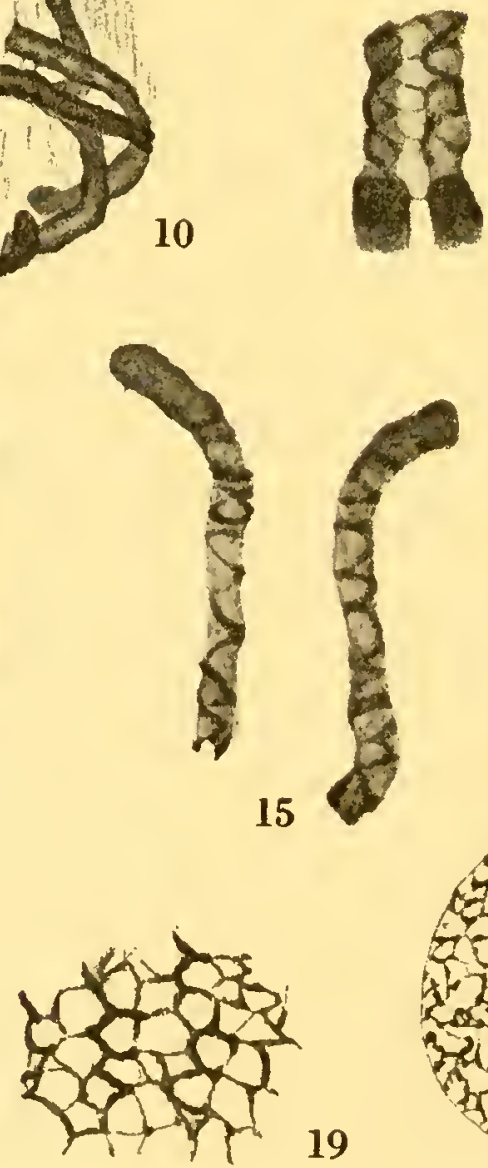

11

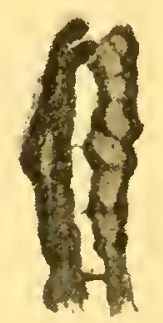

12

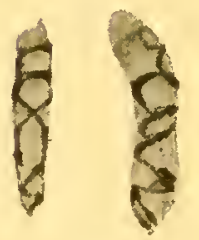

16
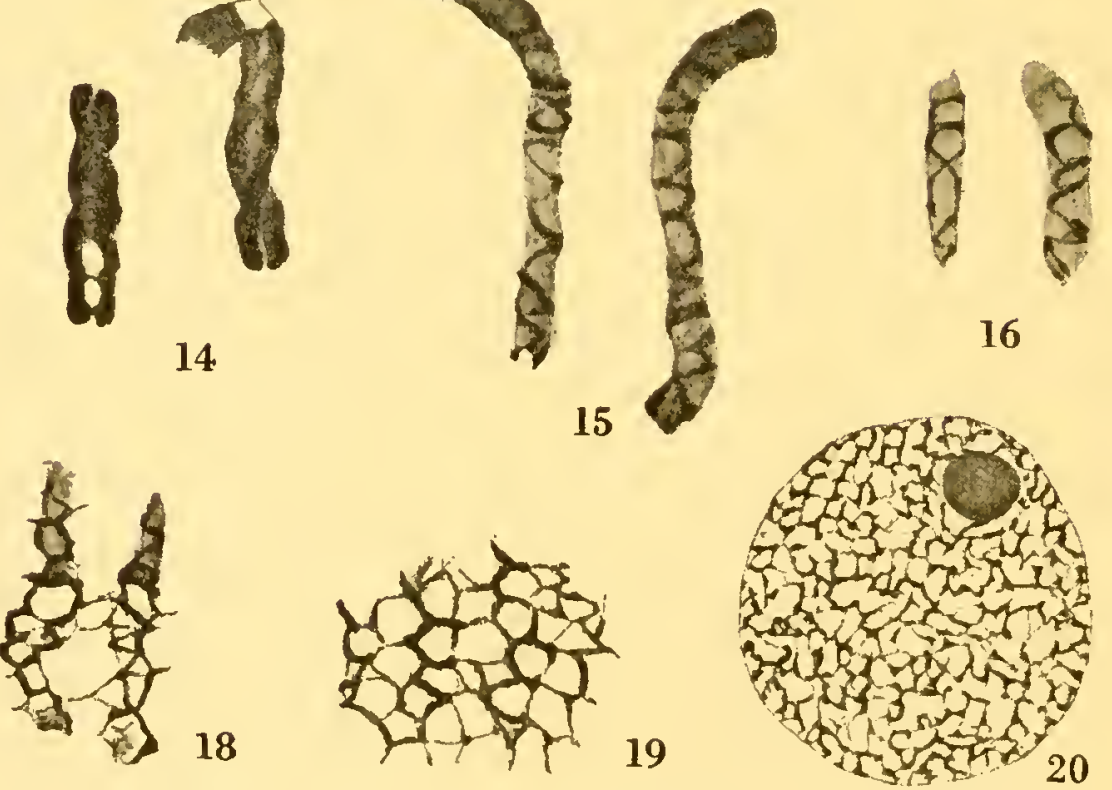

FIG. 73.-Stages in somatic mitosis as they appear in preparations of root tips of Vicia, Trillium, Podophyllum, and Allium. 1, metabolic stage. 2-9, prophase. 10, metaphase figure developing. 11, 12, metaphase. 18-16, anaphase. 17, 18, telophase. 19, 20, interphase. (After Sharp, 1929.) 
Beginning with the anaphase, it is observed that each chromosome consists of matrix and chromonema. The latter is double, i.e., there are in reality two chromonemata; these may lie very closely parallel to each other and so appear as a single thread, or they may be rather widely separated. The chromaticity of the matrix usually obscures this structure unless special methods are employed. At the end of the anaphase the chromosomes tend to form a fairly compact group at each pole (tassement polaire), a condition which may be accentuated by fixation. The chromonemata become somewhat more contorted when the chromosomes shorten.

As the telophase begins, the chromaticity of the matrix decreases markedly, so that the chromonemata are more easily observed. As each group of chromosomes enlarges and becomes a telophase nucleus, the

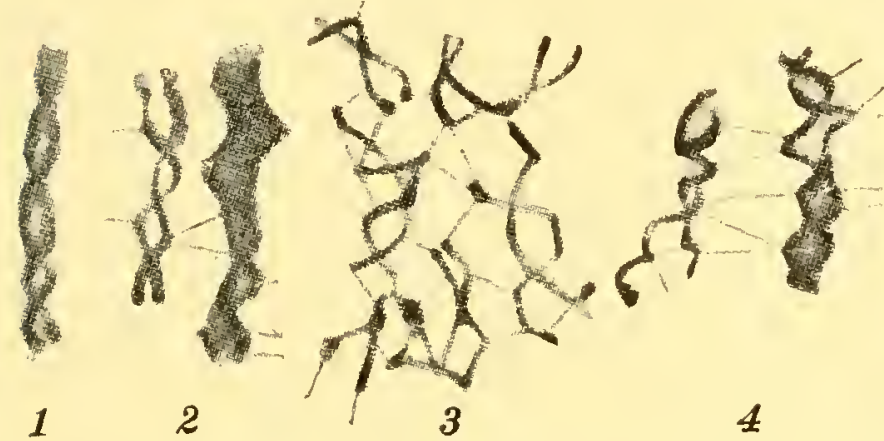

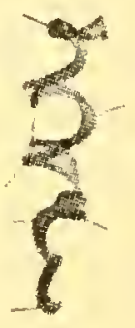

5

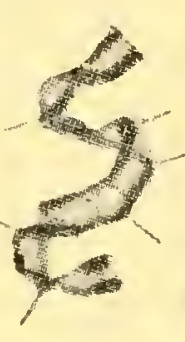

6

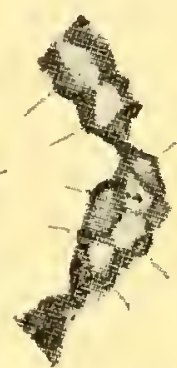

$\gamma$

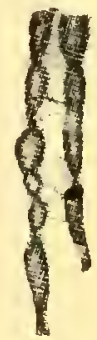

8

Fıg. 74.-The ehromonemata in somatic mitosis in Hamanthus. 1, anaphase; 2. early telophase; 3 , interphase; 4 , early prophase; 5 , prophase; 6 , mid-prophase; 7 , late prophase; 8 , metaphase. The magnification in $3,5,6$, and 7 is greater than that in $1,2,4$, and 8 . (After Telezynski, 1931b.)

chromonemata of the several chromosomes become joined together by anastomoses to form the reticulum. The origin and the nature of these connecting strands are uncertain. They have generally been regarded as short lateral extensions of some component of the chromonema, but in Zea microsporocytes they are clearly formed by the matrix (McClintock) (Fig. 65a). As the telophasic changes continue, the chromonemata of the various chromosomes become increasingly difficult to distinguish as individuals. In rapidly multiplying nuclei where the interphase is short, the next prophase may begin before they are lost to view; otherwise the condition of the interphase passes into that characteristic of the metabolic stage, when the reticulum is finer and relatively uniform in texture. Such fineness of texture is probably due in some measure to a tendency on the part of the two crooked chromonemata in each chromosome to become more independent during this stage.

Little is known about the fate of the matrix in the telophase and the origin of the achromatic constituents of the nucleus. Because of the large volume of the karyolymph in which the reticulum is imbedded at the end of the telophase, it is obvious that at least some of its material 
must have entered from outside during the telophasic enlargement of the nucleus. It is held by a number of investigators ${ }^{3}$ that this material is primarily the spindle substance which has functioned during the preceding stages. Although some have inclined to the view that the matrix and the entering fluid remain distinct, it has also been commonly thought that in such nuclei the two become intermingled to form karyolymph, new matrix differentiating about the chromonemata again in the ensuing prophase, when matrix and karyolymph are easily distinguishable. ${ }^{4}$ By some investigators ${ }^{5}$ this has been interpreted as an actual impregnation of the matrix by the other fluid, the metabolic nucleus thus consisting of chromosomes swollen and in contact or even fused so far as their matrix is concerned. Accordingly, in the prophase the matrix and the other fluid again separate, the former condensing about the chromonemata and

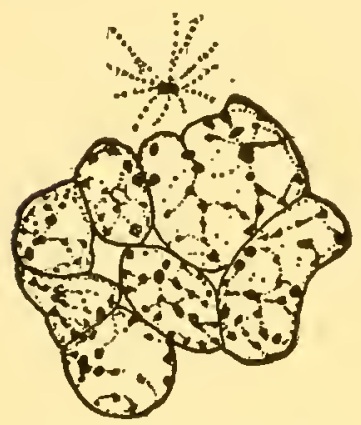

Fig. 75.-Chromosomal vesicles in embryo of Fundulus. (After Richards, 1917.) becoming chromatic as the latter accumulates between the chromosomes in the form of future spindle substance. On this hypothesis, therefore, the karyolymph of the metabolic nucleus is matrix plus spindle substance ("parachromosomic substance": Koerperich), while in the advanced prophase it is the latter substance alone. On the other hand, the conditions observed in Zea sporocytes (Fig. 65a) strongly suggest the possibility that in nuclei generally the matrix may maintain its identity from telophase to prophase in the form of anastomoses, nucleolar matter, and possibly thin sheaths about the chromonemata.

Of considerable interest are those nuclei in which every chromosome of the telophase group forms an individual vesicle, or karyomere. ${ }^{6}$ In some cases the karyomeres may eventually fuse partially or completely, but in others they remain separate although in contact, forming what is virtually a group of small nuclei containing one chromosome each (Fig. 75). It is thus possible to think of chromosomes as bodies coöperating with the cytoplasm somewhat after the manner of plastids, ${ }^{7}$ from which they differ, however, in acting in highly organized groups of differentiated individuals and undergoing a complicated series of changes during their reproductive cycle.

The nuclear membrane has usually been regarded as one which forms, perhaps in the manner of a precipitation membrane, ${ }^{8}$ where the

${ }^{3}$ E.g., de Litardière (1921b), Martens (1929b), and Koerperich (1930).

${ }^{4}$ Kaufmann (1926a), Sharp (1929).

${ }^{5}$ Martens (1929b), Koerperich (1930).

${ }^{6}$ Conklin (1901a) on Crepidula, Wenrich (1916) on Phrynotettix, Reuter (1909) on Pediculopsis, Richards (1917) on Fundulus, Pinney (1918) on fish hybrids, Eisentraut (1926) on Acridida, Heberer (1927) on Cyclops, B. G. Smith (1929) on Cryptobranchus.

7 Grégoire (1925, 1928).

${ }^{8}$ Gates (1909a), Tischler (1921-1922). 
telophasic karyolymph comes in contact with the cytoplasm. According to the interpretation of the metabolic nucleus as a group of chromosomes in contact, the membrane may be regarded as being made up of the surfaces of the outermost members of the group. In the prophase it is often observed that the formed chromosomes remain in contact with the nuclear boundary over a portion of their surface, particularly at their spindle-attachment regions. At this stage the membrane obviously must extend from these areas of contact over the mass of prophasic karyolymph, or separated spindle substance. This point requires further study.

As the prophase begins, the individual chromonemata composing the reticulum separate from one another through a disappearance of the anastomoses. It seems probable that the substance of the latter becomes a part of the matrix appearing about the chromonemata. At first the chromonemata stand out as contorted double threads, but they soon tend to straighten out. From this stage onward each chromosome, composed of a matrix with the halves of the split chromonema along its sides, undergoes a progressive thickening and often some shortening. Meanwhile the matrix becomes more abundant and highly chromatic, obscuring the contorted chromonemata within it. Toward the end of the prophase the matrix undergoes division, so that the chromosome as a whole, as well as its chromonema, is double.

The prophase now passes into the metaphase, each chromosome establishing connection with the spindle figure by its spindle-attachment region. In the large chromosomes here being considered each longitudinal half of the chromosome shows its chromonema to be double. ${ }^{9}$ Exactly when this subdivision of the chromonema takes place is not known. Certain appearances suggest that it first becomes visible some time during the prophase when the chromonemata are still more or less extended, although the multiplication of elements within the thread may well occur at some other stage of the mitotic cycle. The new chromonemata so formed remain together through the ensuing anaphase and separate in the anaphase of the next mitosis. In other words, the chromonema divides one mitotic cycle in advance of the anaphase in which the halves so formed are to separate.

Slender and Small Chromosomes.-To what extent the above interpretation of chromosome structure and the nuclear cycle can be applied to chromosomes of small dimensions is not known. It has been suggested that, if the division of the nuclear elements is related in some definite way to their growth and absolute mass, the stage at which the chromonema becomes visibly double may not be the same for all nuclei. On the other hand, small chromosomes may differ from larger ones only in the size or the compactness in arrangement of their constituent ele-

9 Kaufmann (1926a), Sharp (1929), Nebel (1932), Hedayetullah (1931.) 
ments. In any event the arrangement of the chromatic matter in the form of a long, slender, and relatively straight thread during the prophase strongly suggests that it is at this time that it becomes morphologically double, whatever the number of nuclear cycles in advance of the anaphasic separation. Very little can be said at present concerning how or at what stages any smaller elements within the chromonema undergo multiplication. Physiological considerations suggest that this occurs during the interphase or metabolic stage, but adequate evidence is not yet available.
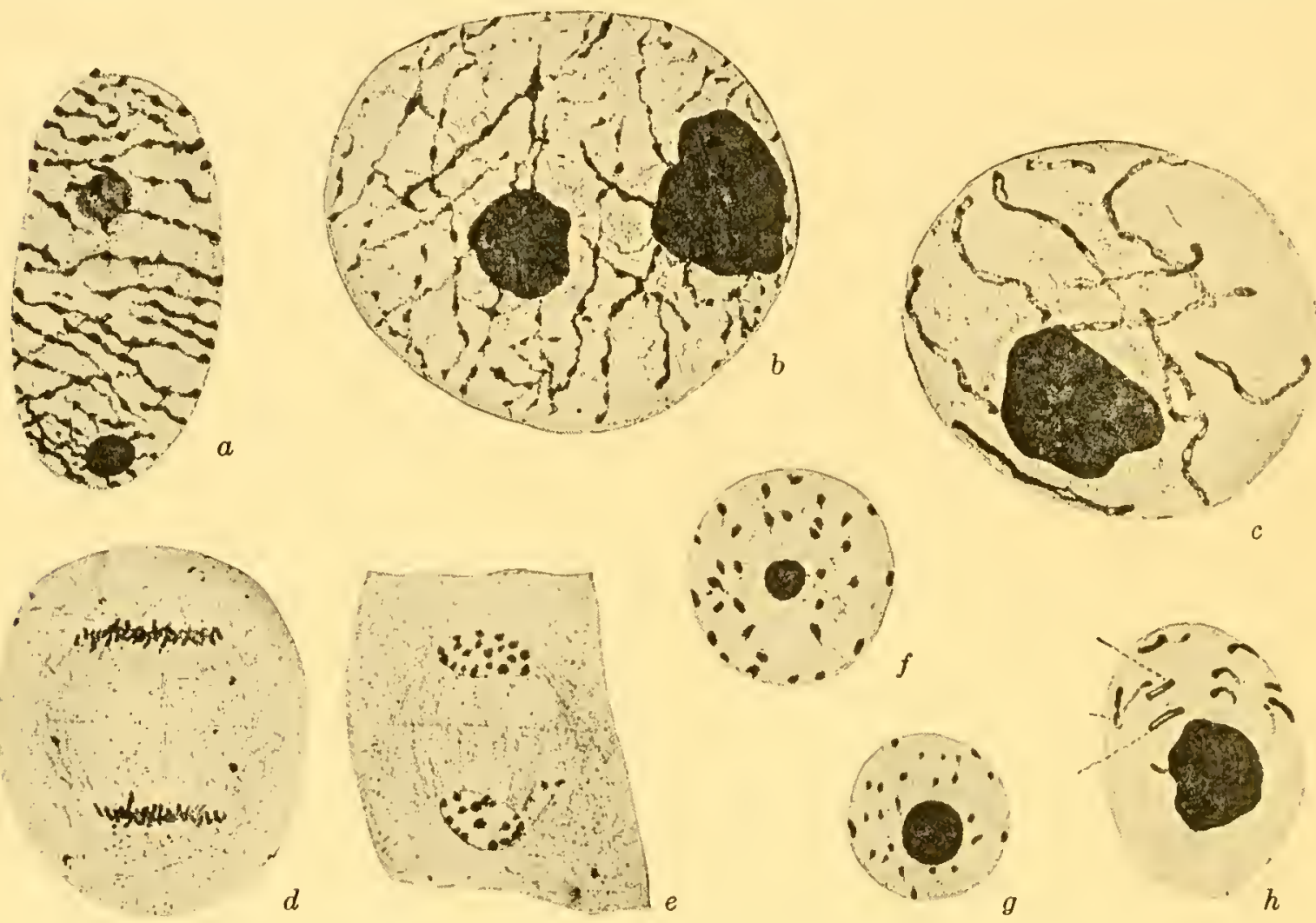

Fig. 76.-Somatic mitosis in plants with slender or small chromosomes. $a$, telophase in Ceratopteris. b, interphase in Pteris. $c$, prophase in Polypodium. $d-h$, anaphase, telophase, interphase, early prophase, and late prophase in Azolla. (After de Litardière, 1921b.)

As examples of the appearance of smaller chromosomes in some of the stages of the mitotic cycle may be cited certain cases in ferns described by de Litardière (1921b). In Pteris cretica and other forms with very slender chromosomes, the latter undergo no conspicuous changes in internal structure during the telophase but are drawn out into filaments connected by anastomoses to form the interphasic reticulum. In the prophase the anastomoses disappear and the chromatic substance is concentrated directly into slender threads which soon appear double and thicker (Fig. 76, a to c). In Azolla caroliniana the chromosomes are very small, ovoid bodies. They undergo no obvious change other than the development of anastomoses during the telophase and remain clearly visible through the interphase. In the prophase the anastomoses disappear, after which the chromosomes elongate somewhat, become double, 
and again shorten (Fig. 76, $d$ to $h$ ). These nuelei are of interest in eonnection with the problem of the origin of karyolymph, which is relatively abundant here. In the opinion of de Litardière it moves in between the chromosomes during the telophase, but its exact relation to chromosomal constituents is undetermined.

Chromonemata in Sporocytes.-The mierosporocytes of certain plants are favorite objects for the demonstration of ehromonemata because of the large size of the ehromosomes and the ease with which they may be treated with various reagents or examined in the living condition. ${ }^{10}$ The chromonemata stand out clearly at late prophase,
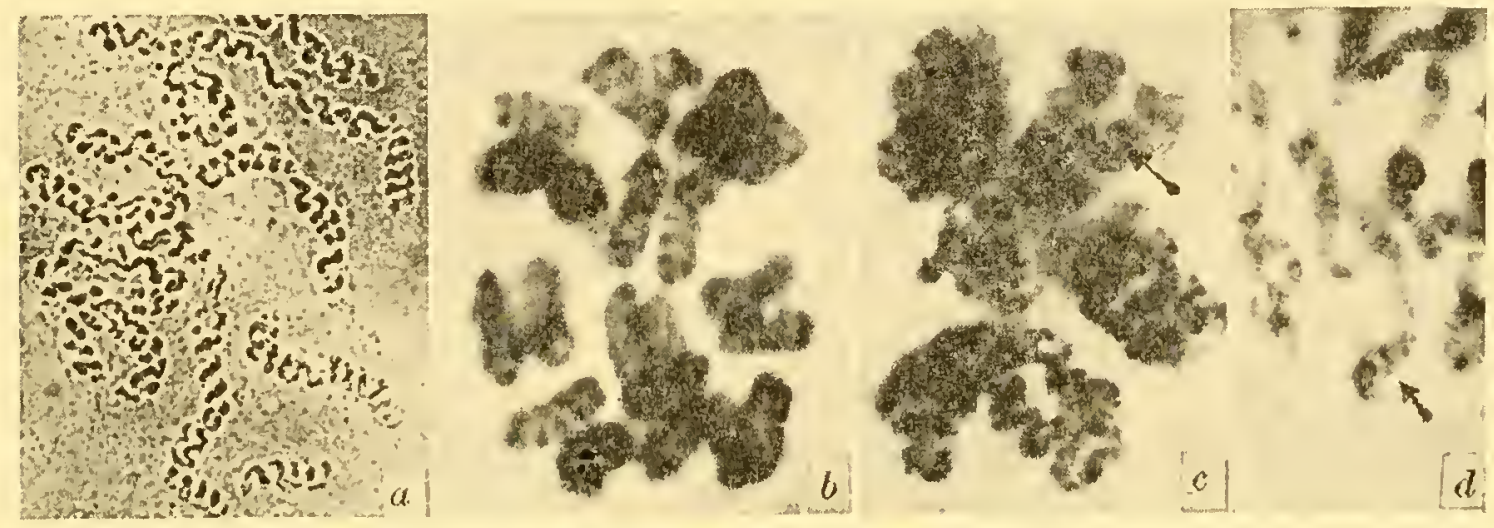

Frg. 77.-Photographs of chromonemata. a, sporocyte of Tradescantia virginica treated with hot water. (After Sakamura, 1927a.) b, sporocyte of Tradescantia reflexa, anaphase I. (After Nebel, 1932b.) c, sporocyte of Trillium erectum. (From C. L. Huskins and S. G. Smith, unpublished.) d, salivary gland of Drosophita. (From Kaufmann.)

metaphase, and anaphase in smear preparations fixed with chrom-osmicacetic mixtures and stained with hæmatoxylin (Kaufmann, Taylor) or fixed with chrom-aceto-formalin and stained with crystal violet ( $\mathrm{Sax}$ ) or brazilin (Belling); also in preparations in acetocarmine (Shinke), neutral violet (Kuwada), or ruthenium tetroxide (Nebel). They can be seen in Iiving cells mounted in Ringer's solution (Martens), sugar solution or olive oil (Sakamura) (Fig. 77). The matrix of the chromosome can be dissolved away with hot water, leaving the spiral chromonemata isolated (Sakamura). Prefixation treatments may increase their visibility (Kuwada, Nebel). For the suecessful observation of chromonemata in fresh sporocytes special attention must be given to the $p \mathrm{H}$ of the

10 For accounts of chromonemata in sporocytes, see Kaufmann (1926ab) on Tradescantia and Podophyllum; Kuwada and Sugimoto (1926), Kuwada (1927, $1932 a b$ ), Kuwada and Sakamura (1926), Sakamura (1927ab), Martens (1929a) and Nebel (1932) on Tradescantia; Belling (1928b) on Lilium; Maeda (1928, 1930a) on Lathyrus; Inariyama (1928) on Hosta; Babcock and J. Clausen (1929) on Crepis; Shinke (1930) on Tradescanlia, Lilium, Narcissus, and other genera; Sax (1930c) on Secale and Lilium; Taylor (1931) and Tuan (1931) on Gasleria; F. H. Smith (1932) on Galtonia. Binder (1927) describes them in the spermatocyte of the kangaroo. Lucas and Stark (1931) photographed them with ultra-violet light in living grasshopper spermatocytes. 
medium. ${ }^{11}$ Certain studies on the chemical composition of plant chromosomes suggest that the chromonemata consist of nucleo-protein and probably lipide, and the matrix of lipides free or combined with protein (Shinke and Shigenaga, 1933).

In many plants used in such investigations the chromonema appears in late prophase, metaphase, and anaphase as a rather smooth thread coiled with striking regularity within the matrix. The clearest preparations show it to be double. In other plants the chromosomes in the sporocytes do not show such regularly coiled and smooth chromonemata as those described above. In Zea Mays, for example, the shortened chromosome in these stages presents an appearance rather similar to that observed in the earlier phases. The chromonemata, which show their chromomeres clearly, seem to shorten with the chromosome as a whole and preserve much of their early structure without being thrown into very wide coils. This point will be considered further in a subsequent section. The behavior of the chromonemata through the mitoses in the sporocyte will be discussed in Chapter XVI.
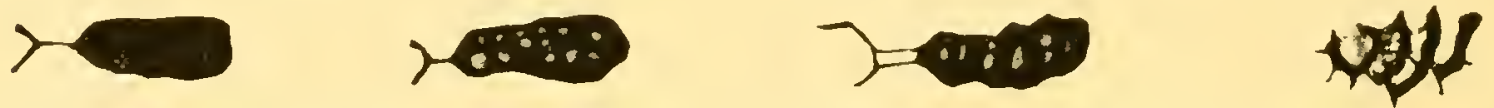

FIG. 78.-Chromosomes in metaphase of first meiotic mitosis, showing aspects observed after different fixations. (After Kaufmann, 1926a.)

The Alveolation Theory.-According to this theory of chromosome structure, which was founded chiefly on studies of somatic cells in plants, ${ }^{12}$ the more or less homogeneous chromosomes undergo a progressive "alveolation" during the telophase. Lightly staining regions appear in irregular positions in the chromosomes, whose chromatic matter assumes a spongy structure and forms the reticulum of the interphase and metabolic stage. In the ensuing prophase the reticulum again breaks up into its constituent "elementary nets," each of which gradually transforms into a slender filament. This filament splits, shortens, and thickens to become the double chromosome whose halves pass to the poles in the anaphase. The slender chromatic thread is accordingly looked upon as a temporary formation of the prophase related to splitting rather than a structure persisting throughout the nuclear cycle.

It is true that such figures as those published by the advocates of this theory represent rather accurately the aspects often observed in the telophase and early prophase; but it is now held by various workers, including some who formerly supported the alveolation theory, that they should be looked upon as inadequate images of the chromonemata and matrix during these stages (Fig. 78). The "alveolation" is therefore

${ }^{11}$ Kuwada and Sakamura (1926), Sakamura (1927a).

12 Grégoire and Wygaerts (1903), Grégoire (1906), Lundegårdh (1910, 1912), Sharp (1913, 1920a), de Litardière (1921b), J. B. Overton (1911, 1922), Koerperich (1930). 
interpreted as a series of aspects observable when the matrix swells and loses its chromaticity in the telophase. Certain cases of "telophase splitting by median alveolation" formerly reported are interpreted on similar grounds. The chromatic structure first appearing in the telophase chromosomes is not actually produced at that time by alveolation or any other process but is simply rendered visible by an alteration in the chromaticity of the matrix.

The Chromomere Theory.-Many years ago the theory was propounded that the small chromatic lumps often visible in the reticulum are arranged in a linear series in a slender thread during the prophase and by their division initiate its splitting. ${ }^{13}$ It was further supposed by some workers that these small bodies, or chromomeres, are composed of still smaller "chromioles," which are supported in the achromatic substance

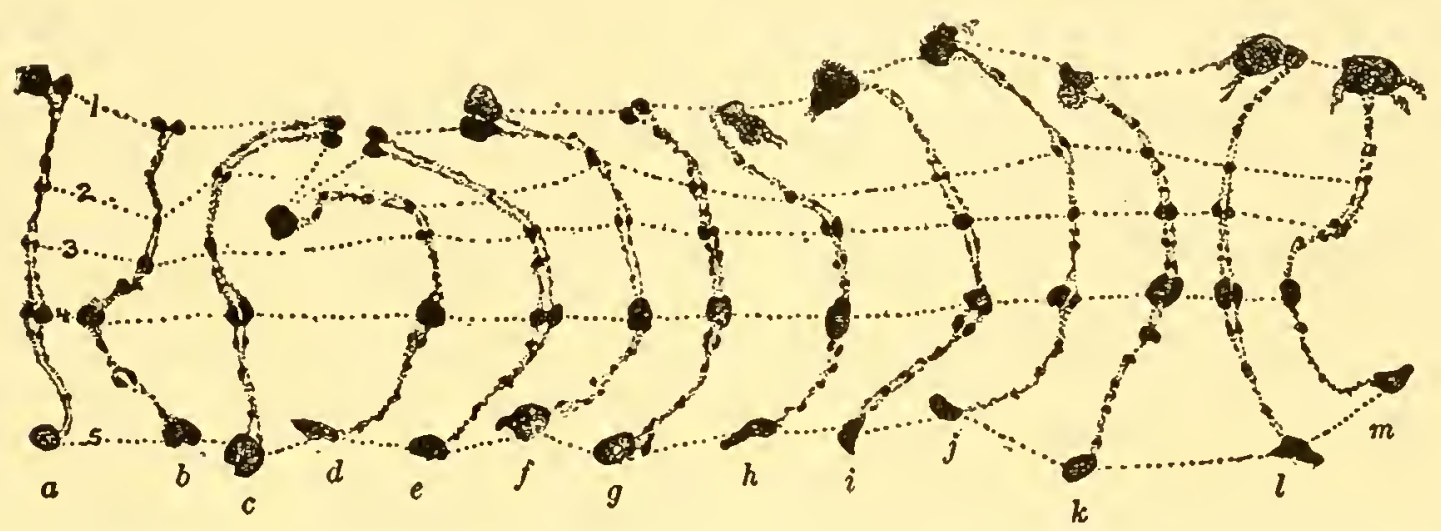

FIG. 79.-Chromosome $B$ (synapsed pair) from spermatocytes of 13 individuals of Phrynotettix magnus, showing constancy in size and arrangement of principal chromomeres. The same constancy is shown in different cells of a single individual. (After Wenrich, 1916.)

of the reticulum. The general opinion that such chromatic bodies are more or less persistent nuclear units multiplying regularly in mitotic division harmonized well with the theory of heredity propounded by Roux and Weismann, according to which the chromatic matter is qualitatively unlike in different regions of the nucleus, the arrangement of this matter in the form of a long thread prior to its splitting being a means whereby all the qualities, and hence hereditary potencies, are divided and distributed to the daughter nuclei.

Since 1900, chromomeres have been studied chiefly in animal spermatocytes and the microsporocytes of plants. In the spermatocytes of insects, especially grasshoppers, the chromomeres appear with great clearness. ${ }^{14}$ In some examples studied with particular care, it has been found that in the early prophase the chromomeres not only differ in size, as claimed long ago by van Beneden, but are arranged in a characteristic

${ }^{13}$ Balbiani (1876, 1881), Pfitzner (1881), van Beneden (1883).

${ }^{14}$ McClung (1905 et seq.), Carothers (1917), Pinney (1908), Robertson (1915, 1916), Wenrich (1916), Janssens (1924). 
and constant pattern in a given chromosome of the complement (Fig. 79). That such chromomeres are not primarily artifacts is indicated by their appearance in the same configurations after a variety of fixing reagents, as well as by the further fact that they may be seen in threads in unfixed nuclei, especially when stretched out by the micromanipulator (Chambers, 1924). Although their appearance may be modified in some measure by microtechnical treatment, they surely correspond to local differentiations of some kind in certain regions of the threads (Agar, 1923).

Very definite chromomeres have been described in the prophase of the first meiotic mitosis in many sporocytes, notably those of liliaceous plants. ${ }^{15}$ The significance of these bodies has been a much debated question among botanists, some maintaining that they are autonomous units, while opponents of such a view, especially those working on somatic tissues, have suggested other explanations for the appearances observed. ${ }^{16}$ It is true that their irregularity and indefiniteness of form in many preparations suggest that they are fortuitously thickened regions of a more or less homogeneous thread. On the other hand, their great distinctness and regularity in other cases, notably in smear preparations of Lilium and Zea, exclude so simple an interpretation. It now seems evident, for reasons which will appear below, that chromomeres should be regarded neither as elements wholly distinct from the rest of the thread nor as mere chance accumulations of chromatic matter, but as definitely localized differentiations of the chromonema, which in all probability indicate the sites of certain special reactions.

Chromomeres in the Chromonema.-In 1912 Vejdovský, working on the spermatocytes of Decticus, suggested that the chromonema is made up of chromomeres which have been brought into contact. This interpretation applies well to the many cases, particularly sporocytes, in which chromomeres in the early prophase are reported to give way to a comparatively uniform chromonema in later stages. Kuwada (1926a) likens the chromomeres to "balls embedded in a rubber thread," which, as it shortens, brings the balls into contact and then becomes contorted within the contracting matrix. Similarly, in the sporocytes of Crepis, Babcock and J. Clausen (1929) figure chromomeres in the middle prophase, but at the end of the prophase only a spiral chromonema whose coils may appear superficially like large chromomeres. In Lilium sporocytes, fixed in chrom-aceto- formalin and stained with iron-brazilin, Belling $(1928 b c g, 1931 a)$ finds the chromomeres as bead-like bodies about $0.23 \mu$ in diameter and separated in the thread by regions of about twice this

${ }^{15}$ E.g., by Strasburger (1884, 1905 et seq.), C. E. Allen (1905), Mottier (1907), Müller (1912), Belling (1928bcg), and W. R. Taylor (1931).

${ }^{16}$ Grégoire and Wygaerts (1903), Martins Mano (1904), Grégoire (1906 1907), Maréchal (1907), Bonnevie (1908), Stomps (1910), Lundegårdh (1912), Sharp (1913, 1920), Tischler (1908, 1921), de Litardière (1921b). 
length in the earlier portion of the prophase. ${ }^{17}$ At this stage all the threads taken together have a total length of about $1,500 \mu$, and as the prophase advances they become reduced to about one-tenth of this length. This brings the "ultimate chromomeres" into contact and causes coiling of the chromatic thread so formed. Aggregates of these chromomeres, together with additional chromatic matter, constitute the conspicuous "compound," or "secondary," chromomeres seen in the later portion of the prophase.

Although the chromosomal threads have chromomeres and probably always shorten in some measure during the prophase of mitosis in sporocytes, the appearance of the chromosome at the end of the prophase varies greatly in different plants. In Tradescantia and Secale rather uniform chromonemata lie coiled within the matrix (Fig. 77), but in Zea the large chromatic accumulations in the threads continue to be visible, the chromosome showing many of the characteristic structures of the early prophase in spite of its greatly reduced length (Figs. 66, 152). Hence such chromosomes, like those of grasshoppers (Fig. 79), are especially well suited to studies on the functions of their component parts. Vegetative nuclei (e.g., in root tips) commonly show rather smooth chromonemata from mid-prophase onward, though it is likely that improved methods will reveal more concerning the architecture of such threads, at least in their earlier stages.

A useful hypothesis covering the above observations is that the chromatic substance appearing in small masses, or chromomeres, at certain regions of the thread, together with any additional chromatic matter, varies in amount at different stages of the nuclear cycle and at a given stage in different organisms. When such chromatic substance is scanty the chromomeres are distinct, but when it is abundant they are more or less united and the thread appears more uniform. Hence the chromomeres should be expected to appear most plainly in the early prophase, when the masses of chromatic substance are small and well separated from one another because of the extended and straightened condition of the thread.

That the structural differentiation exhibited by the chromonemata determines in large part the general form of the metaphase chromosome seems evident. In Zea, for example, the general pattern formed by the spindle-attachment region, the nucleolus-forming region, larger chromomeres, and chromatic "knobs" in the early prophase is maintained as the chromosome shortens, but the positions of the smaller elements become difficult to make out (Fig. 66). It remains to be ascertained to

${ }_{17}$ Early pachynema stage. The threads are paired in synapsis, hence the chromomeres appear double. In the late pachynema stage each component thread shows its split, the chromomeres then appearing as groups of four "chromioles" (see Chapter $\mathrm{XVI})$. 
what extent the matrix shares in determining the form of the chromosome aside from giving it a smoother contour.

Chromosome Structure and the Gene Theory.-In later chapters it will be shown why the chromosomes are thought to contain a series of persistent units, or genes, which affect profoundly the course of development in successive generations and thus represent causal agents in heredity. At this point, those who are familiar with the gene theory may consider certain points of a hypothesis which attempts to relate the genes to the visible differentiations in the chromosome. ${ }^{18}$

As suggested by Alexander and Bridges, the fundamental constituent of the chromosome is the "gene string," a linear aggregate of genes held together primarily by their own attractive forces. The exact nature of the genes is unknown, but they may be thought of provisionally as minute bits of substances growing by autocatalysis and synthesizing a number of materials including basichromatin, the most conspicuous of such materials. In the early prophase each gene is surrounded by its own small mass of basichromatic matter; this is a chromomere. As the prophase advances, some chromomeres increase in size more rapidly than others because of a greater activity on the part of their genes, and they sooner or later fuse to form the coarser and more uniformly chromatic chromonema. Hence larger lumps in this thread indicate groups of more active genes. As the thread becomes thicker, the basichromatin just beneath its limiting membrane is transformed into oxychromatin; this is the chromosome matrix surrounding the still basichromatic chromonema with its gene string. Because of its liquid nature the oxychromatic matrix tends to round up and thus shorten the chromosome as a whole in the later prophase, the relatively firm chromonema then being thrown into coils. The matrix now becomes basichromatic again. In the telophase the basichromatin decreases in amount and may largely or completely disappear by the time the metabolic reticulum is fully developed; in the next prophase it is elaborated anew by the genes. This discontinuity of the basichromatin is taken to mean that "chromatin" is not the important "hereditary substance" but only a by-product of the autocatalytic or reproductive activity of the persistent genes. Belling calls the chromatic matter about each gene "gene chromatin" and the abundant substance present later "extra chromatin." In certain cases he observes at the center of each chromomere a minute dot which he regards as "either a bare gene, or close to one" (1931a, p. 156). ${ }^{19}$

${ }^{18}$ Alexander and Bridges (1928). See also Belling (1928bc, 1931a) and Reuter (1930).

${ }^{19}$ Certain provisional calculations of the possible size of genes place them well below the limit of visibility with the ordinary microscope and suggest that they are of the same order of magnitude as eertain large protein molecules (see Morgan, 1922, and Gowen and Gay, 1933). Recent evidence on the spacing of genes in the chromosome (p. 323) offers a basis for new speculations on this subject. 
On the basis of this hypothesis - and it is at present no more than a hypothesis - the chromomere is regarded not as a fundamental autonomous unit but rather as a product of such a unit, the gene. As already suggested, a way may be found between the view that the chromomere is a fundamental unit and the view that it is only a chance thickening of the thread, if we construe it as a mass of material definitely associated with some localized reaction and often becoming continuous with neighboring masses. The hypothesis summarized above goes a step further and attributes these localized reactions to genes. Similarly, the division of the chromonema is thought to result from the division of the genes. At present very little can be said concerning the time relation which may exist between gene-division (or other mode of multiplication) and the visible splitting of the chromonema. It is conceivable that a double chromonema might be more highly compound as regards its gene strings. Finally, it should be pointed out that although the chromosome may have a characteristic lengthwise differentiation visibly manifested by its chromomeres, as well as a functional differentiation manifested in the results of genic activity, there is as yet insufficient evidence to warrant a confident statement regarding the spatial and functional relationships of chromomeres and genes. Research in progress promises to yield evidence on this important point (see, further, p. 318).

The Continuity of the Chromosome.-In each succeeding prophase in the nuclei of a growing tissue there appears a group of chromosomes made up of a certain number of characteristically different individuals. Are these chromosomes in any real sense the same as those which developed the nucleus in the preceding telophase? That they do preserve their identity as individuals through the metabolic stage, arise only by division, and therefore maintain a genetic continuity throughout the life cycle, was held by many early observers. ${ }^{20}$ The question has been much debated, and, although many statements of the conception of chromosomes as persistent entities have been too crude, it remains true that the chromosomes "must at least be regarded as genetic homologues that are connected by some definite bond of individual continuity from generation to generation of cells" (Wilson, 1909c).

The evidences upon which this view is based are briefly as follows. With rare exceptions, which have been found not to militate against any proper conception of continuity, the chromosomes in successive mitoses are the same in number and form. When the total number is altered in any way, or when a number of the group is changed by loss, fragmentation, translocation, or the like, the altered group appears in the next mitosis; the original complement is in no way restored during the intervening metabolic stage. The limits of the several chromosomes remain visible through this stage in certain nuclei; in extreme cases the nucleus is ${ }^{20}$ E.g., van Beneden (1883), Rabl (1885), and Boveri (1887, 1888b, 1891). 
virtually a group of separate elementary nuclei, or karyomeres (Fig. 75). Each chromosome may even have its own elementary spindle at the time of mitosis; this is known as merokinesis (Reuter, 1909). It has also been observed that when the chromosomes lie in a certain relative position when the telophasic reticulum is formed, they tend to appear in the same position in the following prophase. ${ }^{21}$ It is therefore inferred that, even when the whole reticulum appears like a single unit under the microscope,

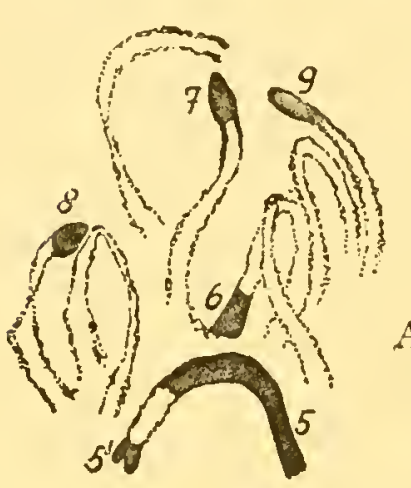
the integrity of the constituent chromonemata is nevertheless somehow preserved. This idea is supported by those cases in which certain portions of certain chromosomes remain dense and deeply chromatic ("heteropyknotic") and hence distinguishable while the remaining portions develop an otherwise uniform reticulum (Fig. 80). Furthermore, when two species with chromosomes of unlike size are crossed, the chromosomes of the two parents can be distinguished in the dividing

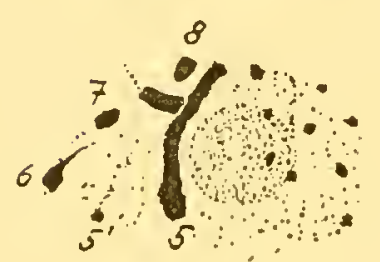
nuclei of the hybrid (Fig. 206). Often the parental groups do not intermingle but tend to remain rather distinct through several embryonal cell generations ${ }^{22}$ (Fig. 143).

Many years ago Boveri was led by the results

B. of his brilliant researches on echinoderm eggs to conclude that the number of chromosomes arising from the reticulum in the prophase is directly and exclusively dependent upon the number which built it in the preceding telophase; this, he contended, finds its most logical explanation in a

FiG. 80.-Heteropyknosis in chromosomes of Pellia epiphylla. $A$, prophase. $B$, late telophase. The heteropyknotic regions are numbered. Compare Fig. 220. (After Heitz, 19286.) genetic chromosomal continuity. These conclusions have been confirmed repeatedly by researches on the chromosomes of hybrids, by observations on cells subjected to the influence of various agencies causing aberrant chromosome behavior, and by critical analyses of the chromosome complements in organisms showing unexpected departures from the normal chromosome number and morphology.

The chromosome is not a body with unchanging form and composition. In the course of the nuclear cycle it passes through a complicated series of chemical and structural alterations, as does the organism itself during its individual life cycle. At certain stages, notably the metabolic stage

${ }^{21}$ E.g., in the segmenting egg of Ascaris, according to Boveri (1887a, 1891, 1909c) and Herla (1893). See also p. 125.

${ }^{22}$ Haecker (1895c), Rückert (1895), Conklin (1897, 1901a), Moenkhaus (1904), Tennent (1912), Morris (1914), Richards (1916), B. G. Smith (1919, 1929) 
and the growth period in animal oöcytes, the basichromatic character of the chromonema largely or completely disappears and the limits of the individual matrix, whether this is temporarily swollen or removed, become indistinguishable. Later on, a reverse series of changes takes place and the chromosome is again evident as an individual. This is not to be construed as an actual destruction and recreation of the chromosome but rather as a cyclic change undergone by a definite group of materials which must assume a structure suitable at one stage to the performance of metabolic functions and at another stage to individual division and distribution to daughter nuclei.

The above conclusion seems obvious enough when, as has been frequently observed, a single chromosome separated from its neighbors forms an individual metabolic nucleus and later passes through an essentially normal series of division stages. The formation of a mitotically dividing nucleus by a single isolated chromosome, taken together with what is known of karyomeres and merokinesis, serves to emphasize the compound nature of the ordinary nucleus and leads to the view that the usual mitotic figure is essentially a group of associated chromosomes undergoing division and separation in unison. That the same interpretation is to be placed upon the chromosome when it is a member of a group, whether as a karyomere (Fig. 75) or an indistinguishable portion of a metabolic nucleus, is now required by the evidence. That something essential in the characteristic structural and functional organization of each chromosome persists through the metabolic as well as other stages of the cycle is practically proved in recent investigations on the effects of X-ray treatments (Chapter XVIII). The results of treatments during the metabolic stage indicate that even at this time the peculiar linear organization of certain elements of the chromosome is normally maintained. In other words, the genes are somehow held to their characteristic linear order at all times. No other plausible explanation of the facts has been suggested. Only our limited vision prevents us from seeing directly that a given chromosome has as characteristic an organization in the metabolic stage as it has in other stages. This being the case, less direct but none the less cogent evidence must be relied upon. What persists as a chromosome through the nuclear cycle is evidently a group of substances held together in a characteristic pattern (in a chromonema or "chromonema axis") and accompanied at certain periods by additional materials, the whole system undergoing a recurrent cycle of physicochemical transformations in successive nuclear generations. To what extent the achromatic materials remain individualized is not known.

The chromosome, therefore, stands out as a persistent individual reproducing by division. It does not follow, however, that this individual necessarily remains rigidly fixed and unaltered in character in successive generations. It will be shown in subsequent chapters that a portion 
of a chromosome is occasionally lost or transferred to another chromosome. Under the proper conditions such altered chromosomes continue as individuals of new types. Since such alterations evidently have occurred with some frequency in the past, a given chromosome should be looked upon as a member of a long series of generations in which structural and functional alterations occur from time to time: it is an assemblage of elements which may remain relatively constant for a long period or perhaps through only a limited number of generations. It may be likened to a persisting society whose membership occasionally changes through losses, additions, and the alteration of personal action. Its persistence as an individual distinct from its neighbors in spite of all such alterations is insured by the spindle-attachment region, a definite "organ" with which the other chromosomal elements are associated. The precise nature of the chromosomal substances which persist and of the chemical transformations which they undergo remains for future investigators to determine. 


\section{CHAPTER XI}

\section{THE ACHROMATIC FIGURE}

The separation of the longitudinally divided chromosomes into two daughter groups involves the action of the achromatic figure. In many cells, notably those of vascular plants, this consists solely of the spindle, a bipolar structure formed chiefly by achromatic constituents of the nucleus. In other cases, including most animal cells and those of certain lower plants, the figure has in addition at each pole a system of cytoplasmic radiations known as the aster, often with a centrosome at its focus. Achromatic figures of the latter type are known as "amphiastral" figures, while those without asters are called "anastral" figures.

Anastral Figures.-All vascular plant cells, with the exception of certain spermatogenous cells of species with motile spermatozoids, have anastral achromatic figures. A typical example is seen in the root-tip cell, where the development occurs in the following manner.

According to an interpretation set forth in the preceding chapter, the metabolic nucleus contains within its membrane three easily distinguishable components: a reticulum made up of anastomosed chromonemata, a nucleolus (or nucleoli), and karyolymph. As the prophase of mitosis advances, each chromonema becomes more or less free from its neighbors and is seen to be surrounded by a distinct mass of matrix substance. The karyolymph, in which the developing chromosomes lie, is to form the spindle; hence it may be termed "spindle substance." In the root-tip cell (Fig. 81) the nucleus commonly enlarges during the prophase. Its membrane then gradually shrinks inward from the two poles, leaving behind two "polar caps" of spindle substance, which seems to have filtered through the membrane. As the membrane shrinks more closely about the crowded chromosomes, the caps become larger and gradually form a more definitely spindle-shaped figure. Eventually the membrane disappears and the double chromosomes become arranged with their spindle-attachment regions in the equator of the spindle. Through all these changes the appearance of the cytoplasm remains essentially unchanged (Robyns, 1924, 1929).

The spindle usually terminates in rather sharp points at its poles, but cases are known in which the polar regions are nearly or quite as wide as the equator. In the living condition the spindle is optically

${ }^{1}$ It is called "parachromosomic substance" by Koerperich (1930) and the "paragenoplast" by Bleier (1930c). 
homogeneous, but for reasons which will be given later it is evident that its substance has a definite longitudinal orientation of some kind. The fine "fibrils" or "lamellæ" seen running from pole to pole in fixed preparations are a visible modification of this oriented structure. In addition to these fine fibrils, there are coarser ones which begin to develop at the attachment regions of the chromosomes and extend poleward along the spindle; these have often been called "tractile fibers."

In the anaphase the halves of each split chromosome begin to move apart at the spindle-attachment region. The tractile fibers are very prominent (in fixed preparations) at this time, but when the chromosomes reach the poles they soon disappear. The spindle substance extending between the two chromosome groups still shows its characteristic fine

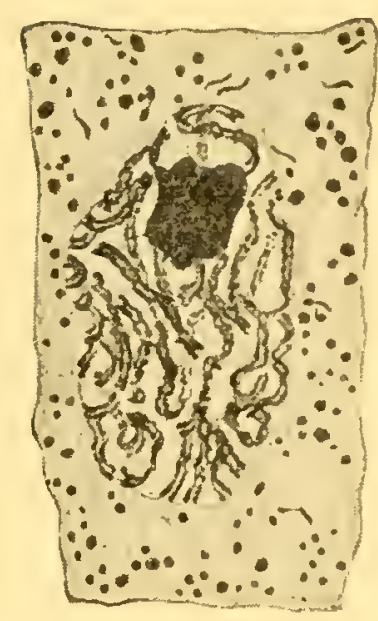

1

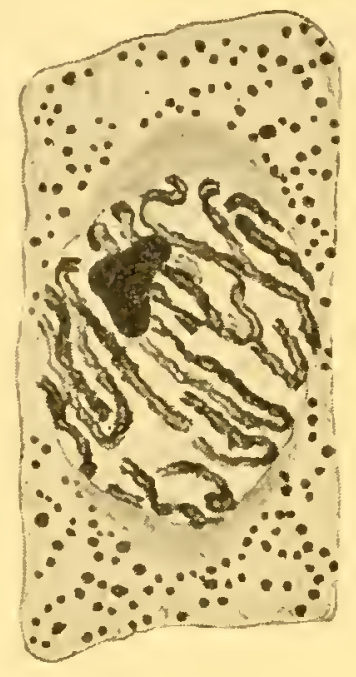

2

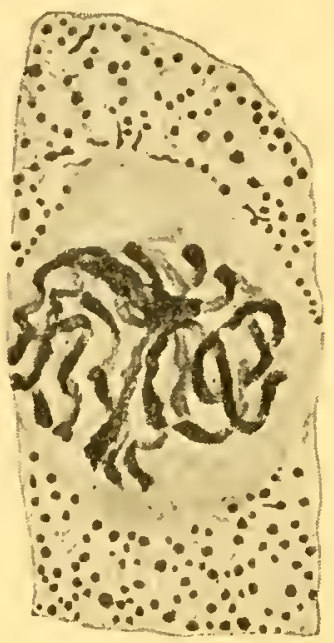

3

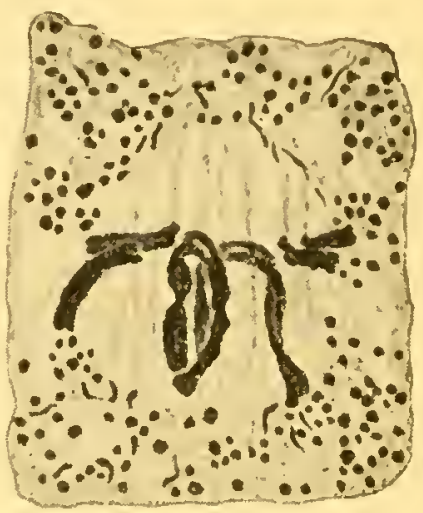

1

FIG. 81.-Development of achromatic figure in root tip of Hyacinthus orientalis. 1, nucleus after reaching its maximum size in prophase. 2, nuclear membrane shrunken, leaving polar caps. 3, membrane partly gone; caps about to become spindle cones. 4 , spindle established; metaphase. Note that chondriosomes do not invade the spindle. (After Robyns, 1924.)

striations. As the two groups develop into telophase nuclei, this mass of substance widens in the equatorial region to form a barrel-shaped phragmoplast, which usually continues to extend laterally until it comes in contact with the wall at one or more sides of the cell. It seems probable that a portion of the spindle substance or some constituent of it reënters the growing daughter nuclei while the remainder is functioning as the phragmoplast.

While the above changes are taking place, the partition which is to divide the original cell into two begins to develop. There is first formed a delicate membrane known as the cell-plate through the equator of the phragmoplast. The time at which it appears with reference to the nuclear changes varies considerably in different cases. Commonly it appears first in the middle region and extends laterally as the phragmoplast widens. The phragmoplast, or spindle substance, soon disappears 
near the nuclei but continues to be evident for some time at the margin of the growing cell-plate (Fig. 101). When a cell contains a large central vacuole the parietal cytoplasm may form a broad strand through it, the mitotic figure occupying this strand. In some cases the figure has been reported to move across a broad cell as the cell-plate develops from one side to the other. In all these examples, therefore, the achromatic figure is not only concerned with nuclear division, but it is also involved in the process of cytokinesis, which here follows very closely upon mitosis. Cytokinesis and the development of the cell wall about the cell-plate will be described in the next chapter.

In microsporocytes, which at the time of their division are usually suspended more or less free from one another in sporangial fluid, the development of the achromatic figure is essentially like that described above, but owing to the shape and polarity of such cells the figure ordinarily presents a somewhat different aspect in its earlier stages. In Larix, for example (Fig. 82), the cytoplasm when adequately fixed appears rather homogeneous and contains many rod-shaped chondriosomes. During the late prophase the chondriosomes move endwise toward the nucleus and lie parallel with its membrane, forming a dense "perinuclear chondriosomal mantle." Improper fixation here gives the "radial" and "felted" aspects so often described. The chondriosomal mantle remains intact throughout mitosis, its inner boundary marking the limit of the nuclear region. As the nuclear membrane shrinks and disappears, the chromosomes become grouped at the center of the nucleus, whose peripheral region is then occupied by the intranuclear spindle substance. The cytoplasm contributes no formed element to the figure. The "tractile fibers" appear first at the chromosomes and develop centrifugally until the completed spindle extends across the nuclear region with its poles at the chondriosomal mantle and the remaining intranuclear substance surrounding it on the sides. The figure is bipolar from the beginning; multipolar appearances and extra fibers surrounding the spindle proper are held to be due to inadequate fixation. In the telophase the terminal portions of the spindle and much of the remaining intranuclear substance become two masses of hyaline fluid in which the daughter nuclei are reconstituted; hence the hyaline substance of these nuclei is continuous with that of the original nucleus. The chondriosomes form mantles about the two nuclei (Devisé, 1914, 1922). In the peculiar fourlobed sporocytes of certain liverworts the developing spindle passes through a definitely quadripolar stage. ${ }^{2}$

Many variations of the processes described above are known in somatic cells and sporocytes. Among these the most instructive are certain cases in which there is little or no shrinkage of the nuclear membrane before the spindle differentiates. Frequently in sporocytes

${ }^{2}$ Farmer (1894, 1895), B. M. Davis $(1899,1901)$, A. C. Moore (1903). 
and even in root cells occasionally (Fig. 73, 10) the late prophasic karyolymph assumes the orientation characteristic of the spindle, and the attachment regions of the chromosomes move to the equator as the membrane disappears without shrinking inward. Cases are also known in which the partially shrunken membrane disappears when in some inter-
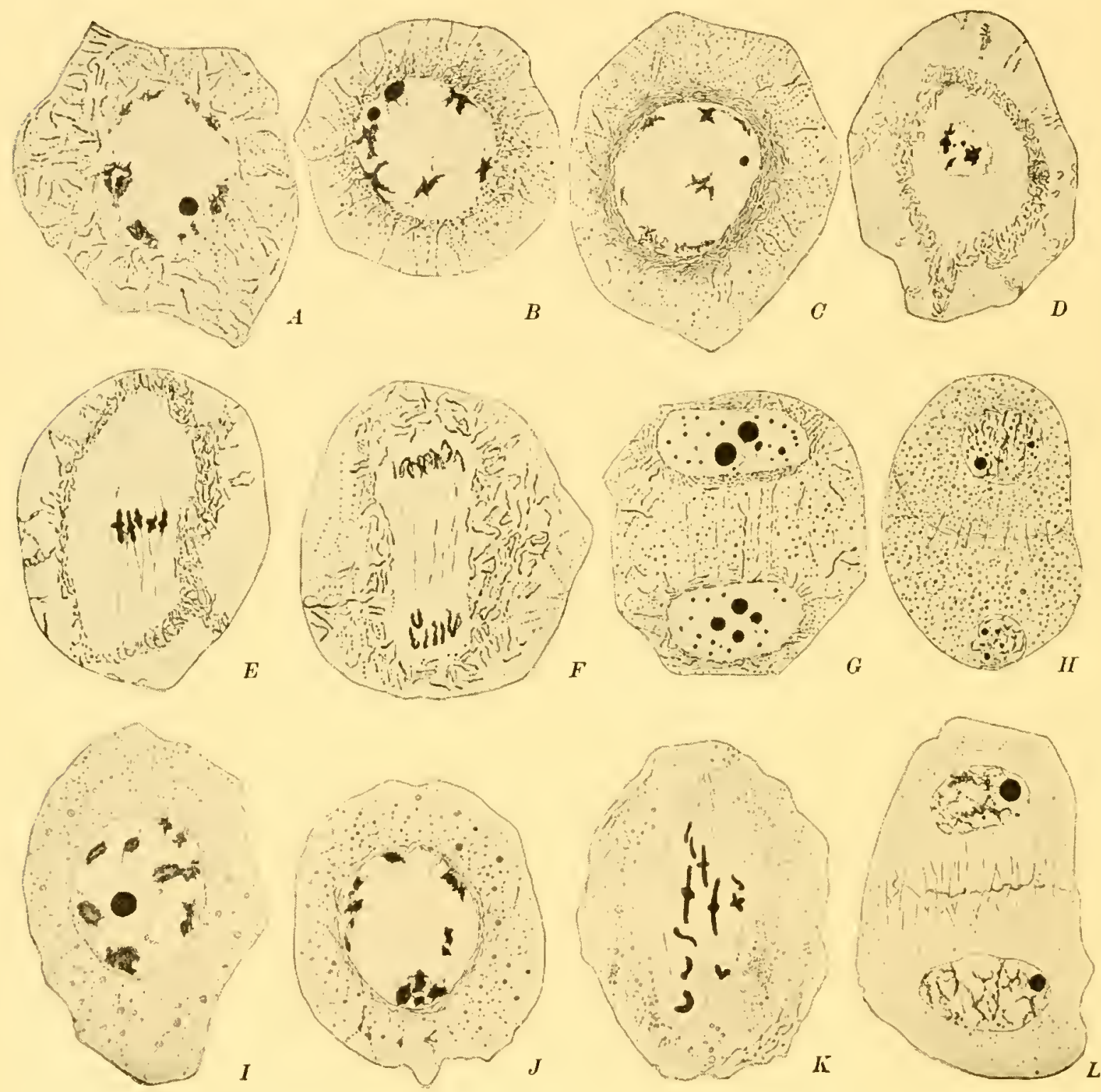

FIG. 82.-Development of achromatic figure in microsporocyte of Larix europcea. $A-H$, after fixation in Benda's fluid. $I-L$, after fixation in Flemming's fluid. $L$ and $H$ show two successive stages in cytokinesis. (After Devisé, 1922.)

mediate position. Hence it becomes easy to reconcile the many early accounts in which the spindle was variously stated to arise from the cytoplasm, from the nucleus, or from both. What was being observed in all these cases was a progressive transformation of the late prophasic karyolymph, or nuclear "spindle substance," into a polarized spindle, with or without a shrinkage of the nuclear membrane before its disappearance. The reactions involved in this transformation are unknown, but it has 
been thought probable that the karyolymph is allowed to react with some cytoplasmic constituent at this time by the disappearance of the nuclear membrane or by alterations in its permeability, the reaction occurring inside or outside the membrane in different cases. ${ }^{3}$

It seems proper, then, to regard the complete anastral mitotic figure in the metaphase simply as the nucleus in a stage of division, its several chromonemata being doubled and surrounded by condensed matrices and its prophasic karyolymph being organized as the spindle. The division is carried out in a cytoplasmic medium, but the structures concerned are primarily those of the nucleus. That mitosis in an ordinary nucleus is in a sense a coöperative undertaking on the part of its several chromosomes is suggested by cases in which each chromosome of the group begins by developing an individual spindle more or less independently of the others.

Amphiastral Figures.-Achromatic figures of the amphiastral type are characterized by the presence of a system of radiations known as the aster about each pole in the metaphase and a centrosome at the focus of each aster. Such figures are the rule in animals; relatively few cells, notably certain oöcytes, are devoid of centrosomes and asters during mitosis. They are also found in certain algæe and fungi, as well as in the spermatogenous cells of bryophytes and those vascular plants having motile male gametes. Since the asters are cytoplasmic, the amphiastral figure, unlike the anastral type, is not composed wholly of nuclear materials.

The centrosome varies widely in structure in different tissues. ${ }^{4}$ What may be called a "typical" centrosome lies in the cytoplasm (centrosomes rarely occupy the nucleus) and consists of a deeply staining granule known as the centriole together with a surrounding mass of substance called the centrosphere. Either of these elements may be present alone. The centriole may be single, but more commonly it is double as a result of division during the later phases of the previous mitosis. Occasionally there are several centrioles, constituting together a "microcentrum." The centrosphere substance is often fairly abundant in resting cells (Fig. 83).

In 1887 van Beneden and Boveri observed in Ascaris that the centrosome, prior to cell-division, divides to form two daughter centrosomes, which move apart to opposite sides of the cell and form the poles between which the mitotic figure is established; and further, that after celldivision is completed the centrosome included in each daughter cell does

${ }^{3}$ Nothnagel (1916), Tischler (1921-1922).

${ }^{4}$ An exhaustive account of centrosomal differentiations is given by Heidenhain (1907). The terminology of the subject has long been in a confused state (see Wilson, $1900,1925)$. In the present account we have chosen more or less arbitrarily a terminology which seems to have found favor with a number of writers. 
not disappear but remains visible in the cytoplasm through the ensuing metabolic stage. They concluded that the centrosome, like the nucleus, is a permanent organ maintaining its individuality throughout successive cell generations. This conclusion is supported by the conditions observable in many tissues, but it is evidently not valid for all. In certain instances it disappears at the close of cell-division, a new one appearing just before the next mitosis. Moreover, the formation of numerous asters with centrosomes can be induced in the cytoplasm of certain animal eggs, notably those of echinoderms, by treating them with solu-

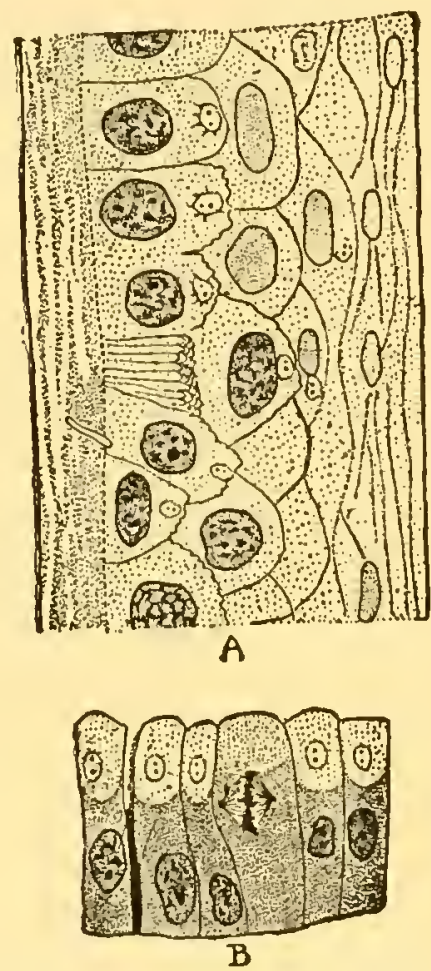

FIg. 83.-Centrosomes in epithelium of cornea of monkey $(A)$ and of human gastric gland $(B)$. (After A. Zimmermann.)

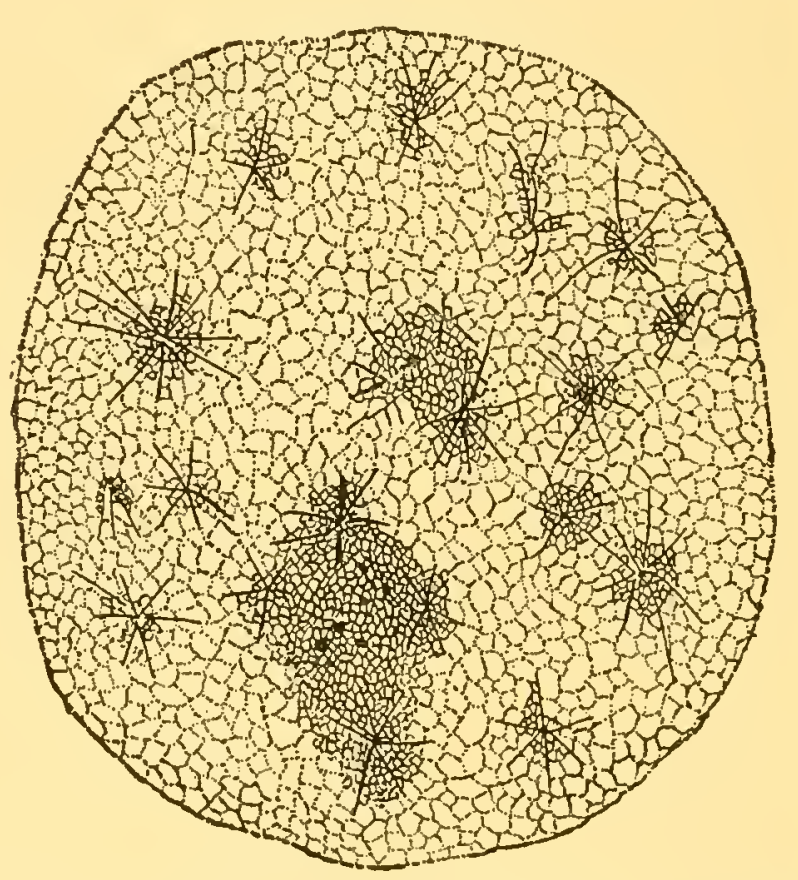

Fig. 84.-Artificial cytasters in egg of Arbacia. (After Morgan, 1899.)

tion of various chlorides (Fig. 84). Such "artificial cytasters" were thought by some to be quite distinct from normal ones in lacking the ability to function in mitosis, but it now seems clear that they do have this ability, and hence that functional centrosomes can be differentiated independently of preëxisting ones. ${ }^{6}$ Tharaldsen finds that, although they may arise anywhere in the cytoplasm in Asterias eggs, the region near the nucleus is "most susceptible," and that cytasters arising here are usually dominant in the mitotic activity which follows. This recalls Yatsu's observation that in Cerebratulus the cytasters do not appear unless the nuclear boundary has begun to break down in late prophase.

${ }^{5}$ Mead (1898), Morgan (1896, 1899), Wilson (1901), Yatsu (1905), and later workers. See Wilson (1925; p. 684).

6 Tharaldsen (1926), Bataillon (1929), Fankhauser (1929). 
Such facts, taken together with the frequent reports of the appearance of a normal centrosome only after the aster has become partly developed, suggest that the centrosome is a result of activity in this region rather than its cause. Moreover, cases are on record in which bodies described as centrosomes have turned out to be effects of microtechnical treatment. Such an interpretation of the phenomena at mitotic centers in cleaving animal eggs has recently been advanced by Fry $(1928,1929,1932)$,
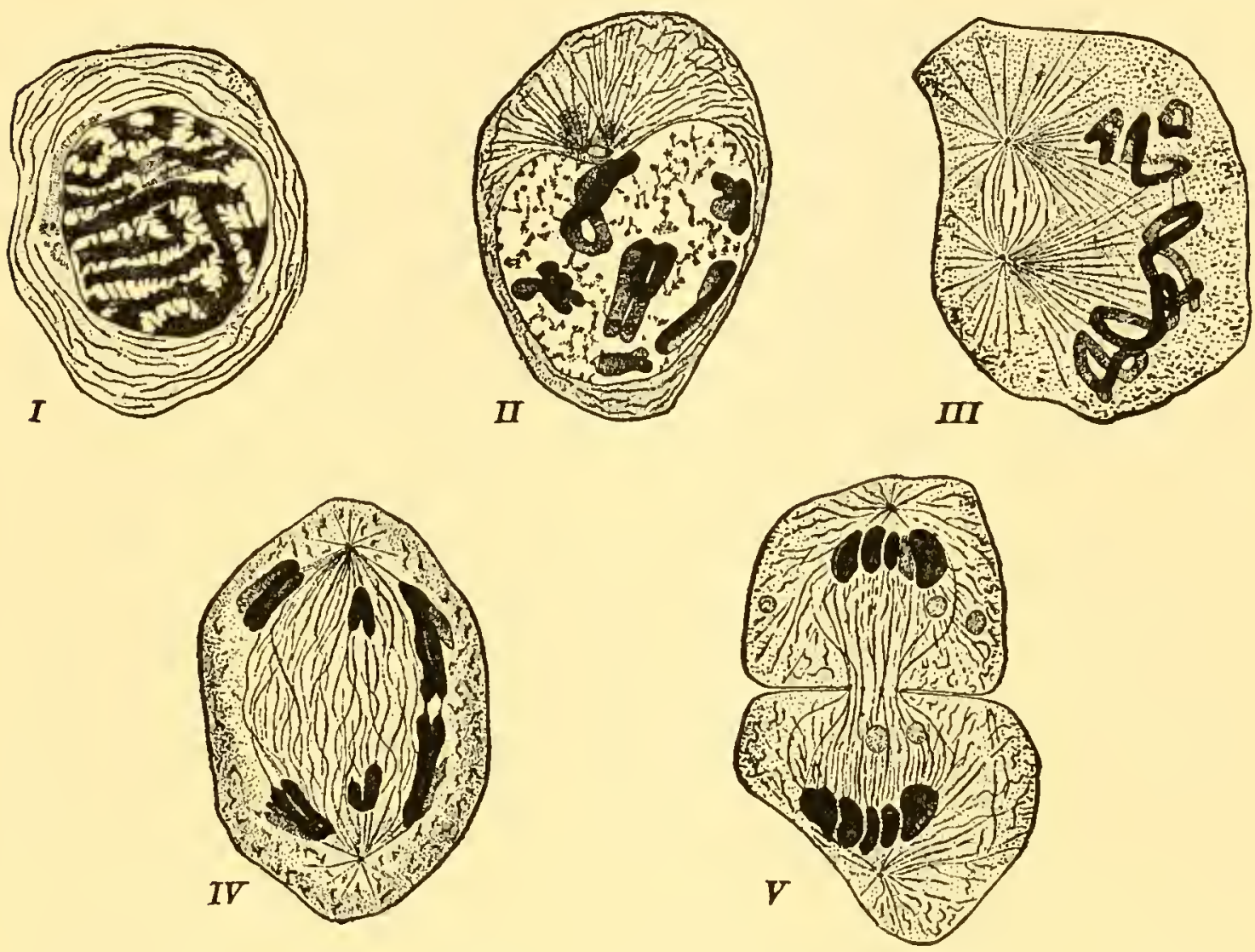

FIG. 85.-First meiotic mitosis in the spermatocyte of Salamandra. I, prophase: centrioles in centrosphere substance, which is spread out on nucleus. II, prophase: bivalent chromosomes formcd; centrioles beginning to diverge; central spindle and asters developed. III, late prophase: nuclear membrane gone; centrioles moving apart; "fibrils attaching to chromosomes." $I V$, anaphase: connecting fibers prominent. $V$, telophase cytokinesis by constriction nearly completed; mid-body forming at equator. (After Meves, 1897.)

but its general validity is strongly opposed by other observers. ${ }^{7}$ Centrosomes have been seen in living spermatocytes (Johnson, Pollister, Bělař).

In animal cells the amphiastral figure commonly develops essentially as follows. The centriole, with or without centrosphere substance, is usually double as the result of a division during the course of the preceding mitosis. As mitosis begins, the daughter centrioles begin to move apart, and as they do so they are seen to be connected by a small "central spindle." About each of them an aster develops (Fig. 85). The result-

7 Wilson (1930b), Pollister (1930, 1932), Bělař (1929c), Wilson and Huettner (1931), H. H. Johnson (1931). 
ing achromatic figure is known at this stage and later as an amphiaster. As the divergence of the centrioles continues, the asters and central

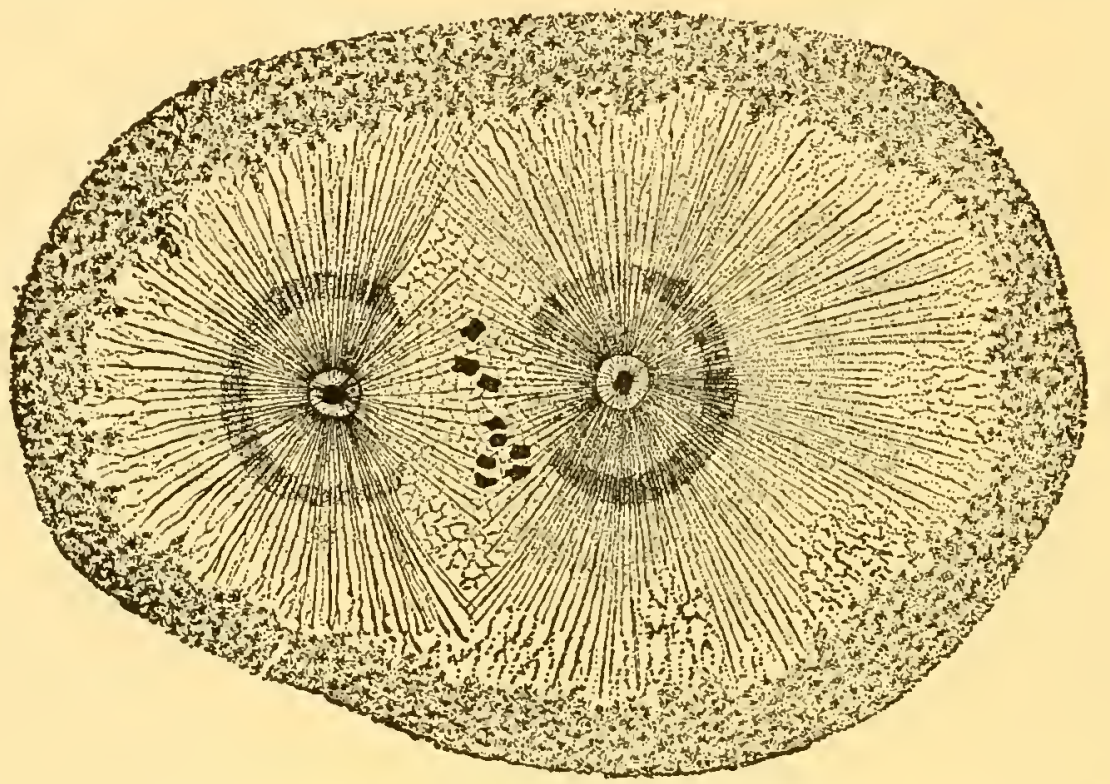

FIG. 86.-Achromatic figure with large asters in oöcyte of Pisciola. (After M. Jörgensen 1913.)
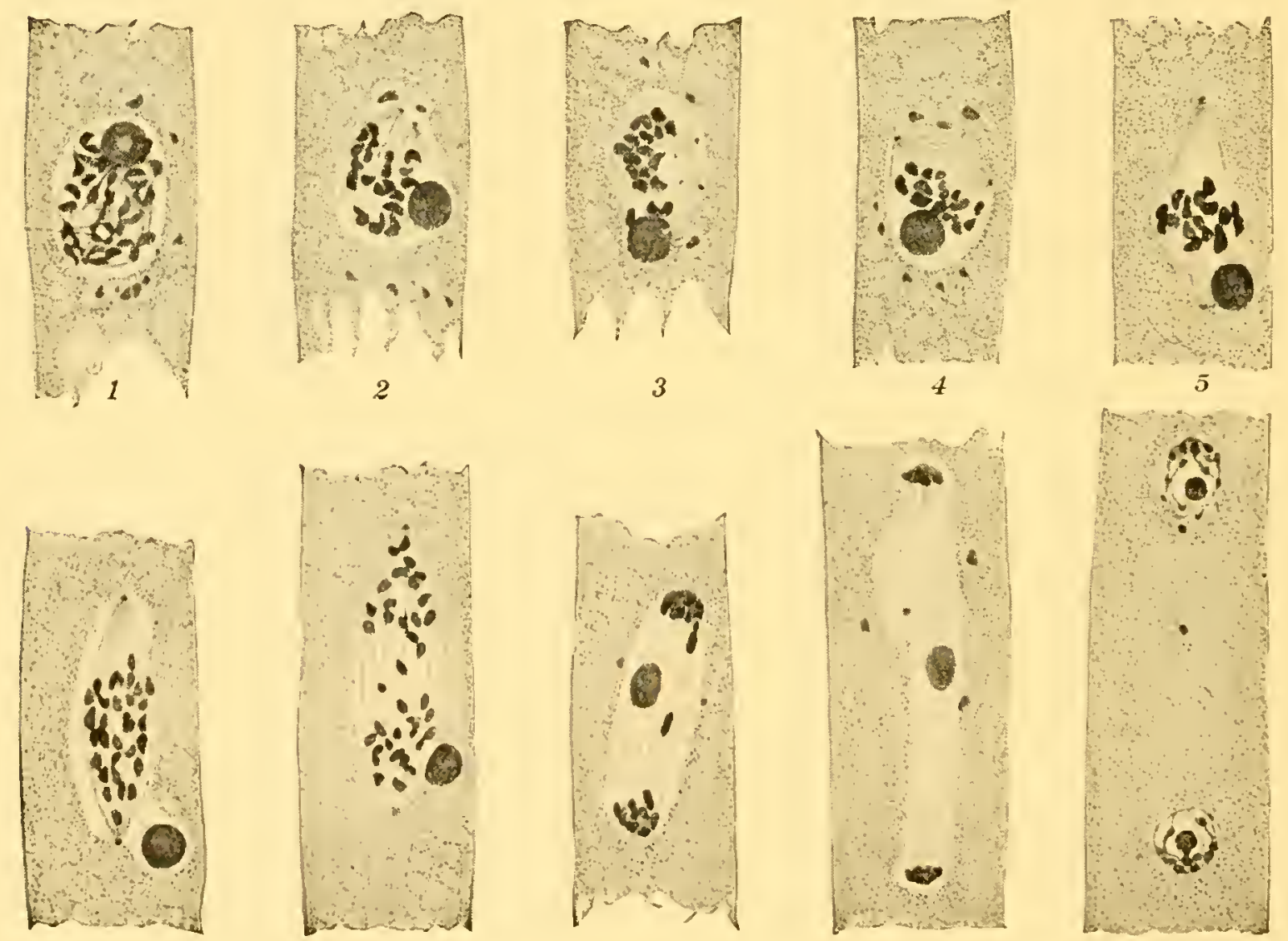

$\gamma$

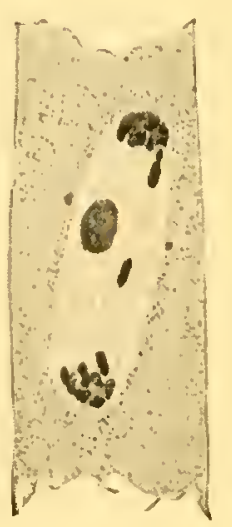

8

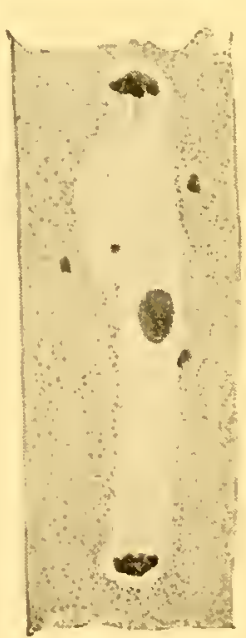

9

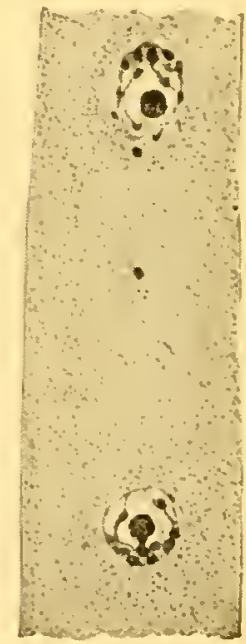

10

FIG. 87.-First meiotic mitosis in the ascus of Pustularia bolarioides. (After Bagchee, 1925.)

spindle increase in prominence. In some cases the nuclear membrane disappears while the centrioles are still diverging, while in others it 
persists until they have reached opposite sides of the cell. In either event it may fade out in its original position or first shrink inward before the enlarging asters. In the literature are many reports of "a growth of the astral rays into the nucleus" and "a lateral movement of the central spindle into the nucleus." To what extent this may involve a progressive alteration of extruded karyolymph beginning in the region near the amphiaster is not clear, but the spindle figure in such cases at least appears to incorporate structures not originally nuclear. ${ }^{8}$

With regard to the asters, it was shown by Chambers $(1921 b, 1924)$ that in living echinoderm eggs they are regions which are somewhat more
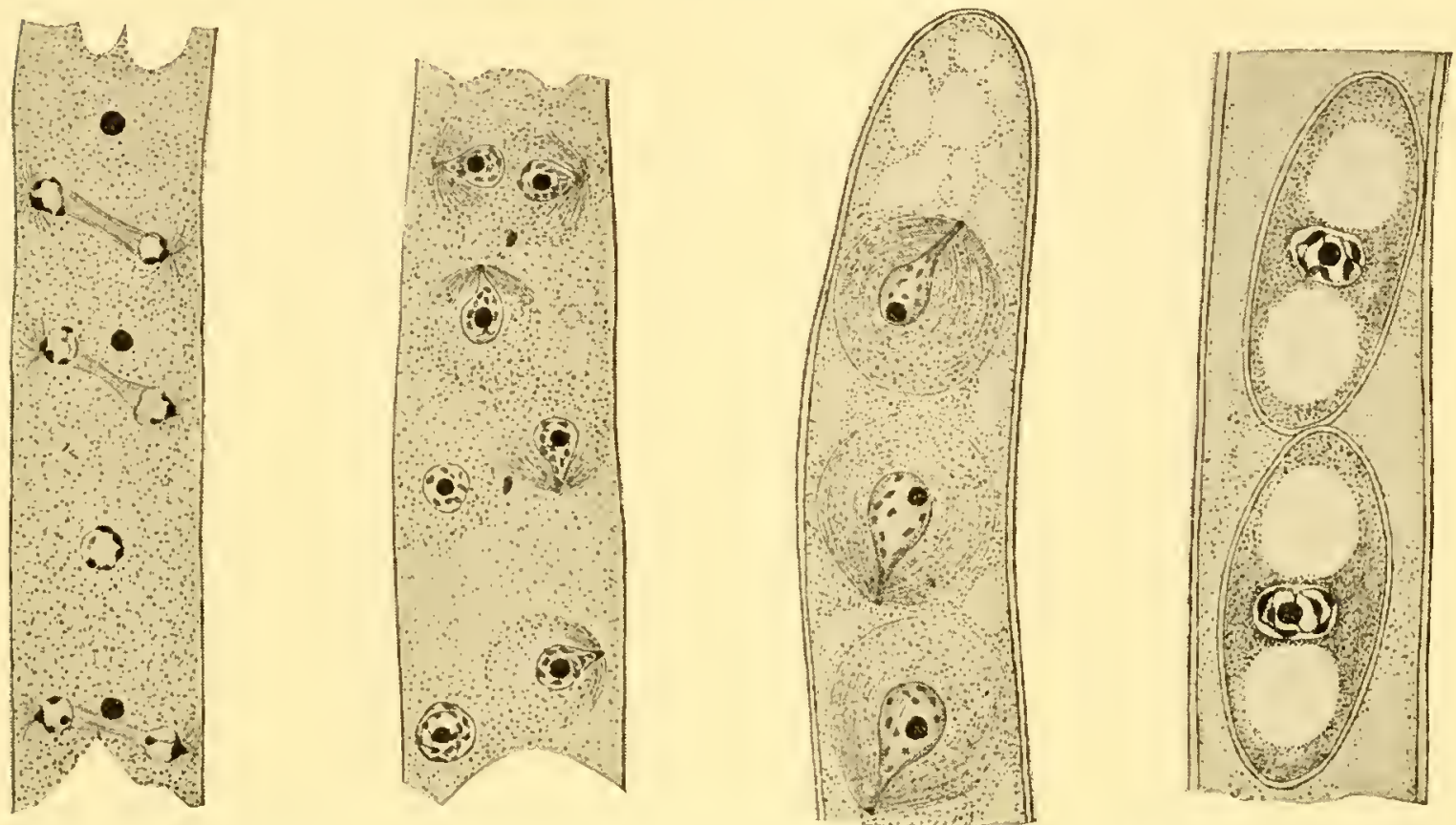

FIG. 85.-Development of spores after last mitosis in ascus of Pustularia bolarioides. (After Bagchee, 1925.)

solid than the rest of the protoplasm, but that their rays, instead of being firm fibers as some had thought, are actually streams of fluid passing inward through the more gel-like granular cytoplasm. This confirmed a widespread view that the aster is primarily an expression of streaming movements in the cytoplasm. The rays merge into a central mass of hyaline fluid which may in some cases begin to accumulate before the cytoplasm becomes sufficiently gelated to reveal the centripetal channels. The aster may sometimes show rather well-marked concentric zones (Fig. 86) as well as one or more concentric series of granules about the centriole. It is questionable how far these are normal appearances, for Chambers (1917b) asserts that some of them may be produced by subjecting eggs to abnormal environmental conditions.

During the anaphase the astral radiations remain conspicuous, but, as the telophase progresses, they gradually fade from view, except in

${ }^{8}$ See the discussion of these cases by Bleier (1931a). 
those forms with more or less permanent asters. The centriole at each pole divides, usually before the anaphase begins and often even earlier. In case the aster about it does not disappear at the close of mitosis, a new amphiaster may differentiate about the daughter centrioles in the midst of the old aster. The latter rarely divides but usually degenerates as the new amphiaster develops within it. ${ }^{9}$

A mass of spindle substance, sometimes surrounded by chondriosomes in the cytoplasm, remains for some time between the chromosome groups and the young nuclei which they form. In animals this substance plays no very conspicuous part in cytokinesis. Granules may be differentiated at the equatorial region, forming the so-called "mid-body," but the division of the cytoplasm is ordinarily brought about by the development of a cleavage furrow, as will be described in the next chapter.

Very characteristic amphiastral figures are developed in the ascomy$\operatorname{cetes}^{10}$ (Figs. 87, 88). The centrosome, which in some ascomycetes is discoid, lies against the nuclear membrane. As mitosis begins, an aster usually develops in the cytoplasm about the centrosome, and the latter divides to form two daughter centrosomes. The central spindle, if formed at all, does not persist. From each of the daughter centrosomes, which begin to move apart along the nuclear membrane, a group of "fibers" extends into the nucleus. This appearance may be due to the fact that the centrosomes here mark regions where there is an active material interchange between nucleus and cytoplasm (Harper, 1919), the result being the orientation of prophasic karyolymph to form the spindle structure. The centrosomes finally reach opposite sides of the nucleus, and the two groups of fibers become arranged in the form of a spindle extending through the nucleus with the chromosomes at the equator. The spindle may occupy nearly all of the nuclear volume or only a small portion of it. The nuclear membrane commonly remains intact until the chromosomes approach the poles at anaphase. It may then disappear, allowing the nucleolus, which has remained visibly unchanged, to escape into the cytoplasm. Between the two densely packed daughter chromosome groups there extends a long strand of spindle substance; this disappears as the daughter nuclei reorganize.

Cytokinesis in fungi is commonly brought about by a cleavage furrow which is independent of the achromatic figure. After the final mitosis in the ascus the astral rays curve around and in some way become involved in the formation of the ascospore membrane. According to Harper, the

${ }^{9}$ See Wilson (1925, p. 680).

${ }^{10}$ Harper (1895, 1897, 1899, 1905), Faull (1905, 1912), Maire (1905a), Guilliermond (1904, 1905, 1911), Fraser (1908), Fraser and Brooks (1909), Fraser and Welsford (1908), Claussen (1912), W. H. Brown (1909, 1910a, 1911b), Bagchee (1925), Carruthers (1911), Schultz (1927), Tandy (1927), Eftimiu (1927, 1929), GwynneVaughan and Williamson $(1930,1931,1932)$. 
rays actually fuse laterally to form the membrane, an interpretation which has been disputed by Faull and others.

Very similar to the amphiastral figures just described are those in certain algæ (Fig. 89). In Dictyota, for example, a curved rod-shaped centrosome lying against the nuclear membrane divides as mitosis begins. The daughter centrosomes, each with an aster, separate and occupy the poles of the intranuclear spindle. In Fucus the two centrosomes are said to arise independently. In Polysiphonia the centriole-like bodies seen in the prophase give way later to larger centrosphere-like masses. In living cells of Sphacelaria, according to W. Zimmermann (1923), the conspicuous rays about the centrioles before the metaphase are made up
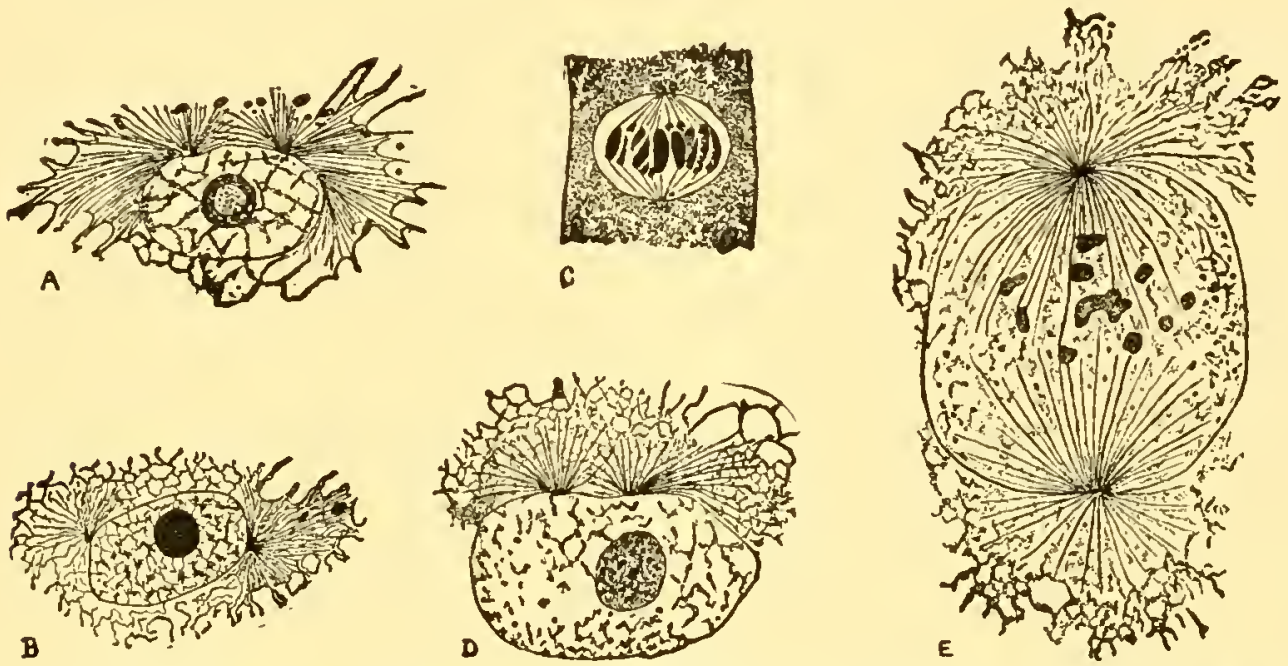

Fig. 89.-Centrosomes in algæ. A, B, Stypocaulon. (After W. T. Swingle, 1897, and Escoyez, 1909.) C, centrosphere-like bodies in Polysiphonia. (After Yamanouchi, 1906.) D, E, Dictyota dichotoma. (After Mottier, 1900.)

of the oriented boundaries of large vacuoles, with fucosan globules and chromatophores lying along them.

Amphiastral figures in the spermatogenous cells of bryophytes and those vascular plants having motile male gametes are described in Chapter XIV (Fig. 122).

The Mechanism of Mitosis.-It has always been tempting to speculate upon the mechanical factors involved in the remarkable process of mitosis. Although the problem is still very far from solution, it is of interest to consider briefly some of the suggestions which have been made. ${ }^{11}$

One of the simplest and most widely accepted theories was that of fibrillar contractility proposed by Klein (1878) and van Beneden (1883, 1887), according to which the chromosomes were supposed to be dragged apart by the contraction of two opposed groups of spindle fibers. Many observations were cited in its favor, and elastic models were made to illus-

${ }^{11}$ See the reviews by Wilson $(1900,1925)$ and Tischler (1921-1922). 
trate the supposed contraction and its results (Heidenhain); but evidence subsequently brought forward ${ }^{12}$ led to the general restriction of the rôle of contractility, until it became apparent that this factor must be one of minor importance.

The striking resemblance between the achromatic figure and the lines of force in an electromagnetic field naturally led to attempts to account for mitosis on the basis of electrical principles. Several investigators, working with various chemical substances, succeeded in modeling fields of force that illustrated graphically the changes supposed to take place in mitosis. In later years the electromagnetic interpretation was again brought into prominence by Gallardo, Hartog, and Prenant. At first Gallardo (1896) believed the two spindle poles to be of unlike sign, but later (1906), as the result of the researches of R. S. Lillie (1903) on the behavior of nucleus and cytoplasm in the electromagnetic field, he concluded that they are of like sign, the centrosomes moving apart because of their similar charge. The movement of the chromosomes to the poles he held to be due to the combined action to two forces: a mutual repulsion of the similarly charged daughter chromosomes and an attraction between the oppositely charged centrosome and chromosome. It has also been thought that the chromosomes may assume their equatorial position because they are repelled by both poles, and that a later weakening of the repelling force relative to the mutual repulsion of the chromosome halves allows the latter to move poleward.

The fact that the two centrosomes and hence the two spindle poles are electrically homopolar (Lillie) at once made it apparent that the mitotic figure does not represent an ordinary electromagnetic field, for in the latter the poles are of unlike sign - the field is heteropolar. It was consequently suggested by Prenant (1910) and Hartog $(1905,1914)$ that the mitotic figure is the seat of a special force peculiar to living organisms. This force they called "mitokinetism." It can scarcely be doubted that electrical forces are in some measure concerned in the mitotic changes, but it is impossible at present to state how they act. ${ }^{13}$ Any theory involving such forces must be applicable not only to ordinary bipolar mitosis but to occasional tripolar, quadripolar, and unipolar (p. 163) mitotic figures as well.

Special significance has been attached to streaming by students of mitosis ever since Bütschli, Fol, and others showed many years ago that currents usually exist in the protoplast. That streams passing poleward within the spindle play a rôle in chromosome movement was suggested by many of the aspects observed, and the subsequent discovery that the astral rays are such streams lent further plausibility to the view. That the "spindle fibers" are such streams was, however, not proved.

${ }^{12}$ Hermann (1891), Drüner (1894, 1895), Calkins (1898), and others.

${ }^{13}$ See Wilson (1925, pp. 184-189). 
All such hypotheses regarding the mechanism of mitosis must be reconsidered in the light of what has recently been learned about the achromatic figure in living cells. As stated above, the aster has been found to be a semisolid region of the cytoplasm with centripetal fluid paths, but, since many achromatic figures have no asters, the causes of mitotic chromosome movement obviously lie primarily in the spindle and the chromosomes.

It is a notable fact that in uninjured living cells the metaphase spindle appears to be optically homogeneous, no "fibers" being visible. ${ }^{14}$ Its density is such that it can be moved bodily through the cytoplasm with the micromanipulator (Chambers) or the centrifuge, ${ }^{15}$ but the microneedles reveal no fibrous structure. In tissue cultures it is observed that when the medium is made acid the fibers appear and the mitotic process ceases; when it is made neutral again the fibers disappear and the process is resumed (Lewis). The fibers can be made to appear by dehydration and by various agents which bring about gelation or coagulation. Hence the coarsely fibrous appearance of the spindle in many fixed preparations is an unnatural one.

It is not to be concluded from these facts that the striations and tractile fibers observed in such preparations have no structural basis whatever in the living cell. We are now forced to view the untreated spindle as a body with a longitudinally oriented finer structure in spite of its optical homogeneity. ${ }^{16}$ In support of this view Bèlar cites the following facts: the distal portions of long chromosomes in the spindle tend to become arranged parallel to its axis; the Brownian movement of occasional included granules is greater in amplitude lengthwise of the spindle than in other directions; mitochondria which sometimes enter an artificially swollen spindle tend to be arranged longitudinally; the spindle has a pronounced axial resistance to contraction; certain agencies cause the spindle to split longitudinally (Fig. 90, c). Hence the spindle may be likened to a crystal which appears homogeneous to the eye but can be shown to have an oriented structure by suitable treatment, or to a block of wood whose splitting and differential swelling indicate the presence of parallel elements.

The exact nature of the invisible local modifications which appear upon fixation in definitely localized positions (notably near the spindleattachment regions of the chromosomes) is not yet known. The fixed images often suggest that they are streams moving in harmony with the cytoplasmic streaming frequently observed during these stages (Fig. 97); but Bělař has shown that the movement of the chromosomes may con-

${ }^{14}$ Chambers (1914, 1915, 1917, 1924), Chambers and Sands (1923), Sands (1923), M. R. Lewis (1923), Lewis and Lewis (1924), Martens (1927c, 1929), Robyns (1929), Bělař $(1927,1929 a b)$.

${ }^{15}$ F. M. Andrews (1915), Němee (1927).

16 Robyns (1926, 1929), Bělař (1927, 1929ab), Martens (1929), Němee (1927, 1929e), Bleier (1930b), Jungers (1931). 
tinue after cytoplasmic streaming has been stopped, and also that the streaming may continue when mitosis is arrested. The appearances in living and fixed cells also suggest a longitudinal tension of some kind in the figure, or even a bundle of tubes through which the chromosomes move (Reuter, Schrader).

As a result of his investigations on the living spermatocytes of Chorthippus (Stenobothrus) and stamen hairs and leaf-cells of Tradescantia, Bèlař advanced the following hypothesis, which is a modification of an earlier one advocated by Drüner (1895) and others, to account for chromo-

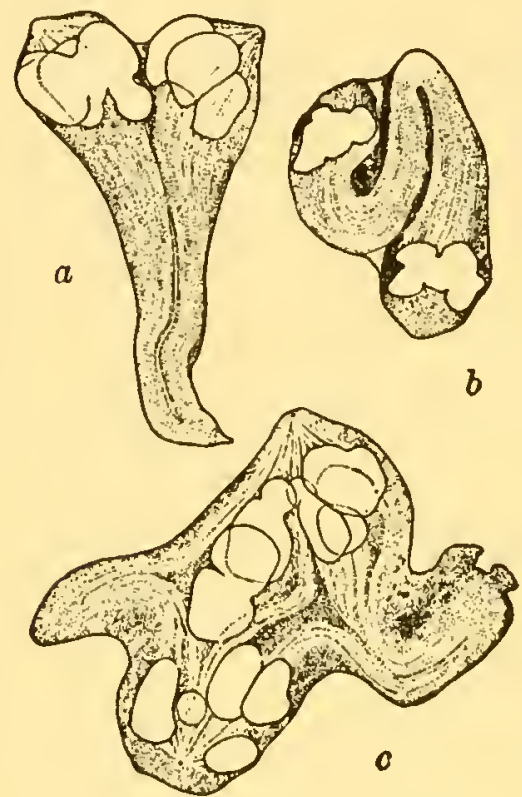

FIG. 90. some movement (Fig. 91). The spindle, which develops progressively from the two poles to the equator, is composed of relatively firm fibers (which are aggregates of micellæ) and a less viscous substance between them.
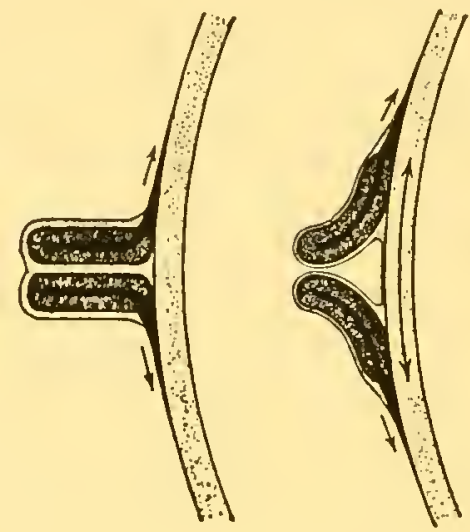

FIG. 91.

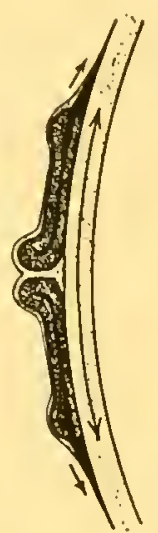

Chorthippus induced by immers-

ing the spermatocytes in hypertonic Ringer's solution. $a, b$, elongation and contortion.
$c$, splitting. The white areas represent the chromosomes. (After Bëlar, 1929a.)

ing the spermatocytes in hypertonic Ringer's solution. $a, b$, elongation and co
$c$, splitting. The white areas represent the chromosomes. (After Bèlarr, 1929a.)

FIg. 91.-Diagram illustrating Bèlař's hypothesis of interaction of chromosomes and spindle. See text for explanation. (After Bèlar̆, 1929a.)

The double chromosomes become fixed in or on the spindle at its equator by their attachment regions. From this region in each halfchromosome there is exuded a fluid droplet which spreads poleward along the spindle; this is the "tractile fiber" of other authors and it does not grow toward the chromosome from outside the nucleus as often supposed. The separation of the daughter chromosomes (or of synaptic mates) is initiated by the chromosomes themselves, the tractile fiber mechanism assisting in some unknown manner, perhaps through forces of adhesion acting between fiber and spindle; but after this the continued separation is due mainly to the elongation of the mass of spindle substance ("Stemmkörper") lying between the diverging groups of chromosomes. In spermatocytes immersed in hypertonic media this elongation may be caused to continue until the mitotic figure is far beyond its natural length and lies folded together within the confining cytoplasm (Fig. 90, $a, b$ ). 
Meanwhile the polar regions of the spindle undergo little change. Long chromosomes like those in Tradescantia undergo a marked shortening during late anaphase.

Although the normal spindle is frequently longer at the end of the anaphase than in the metaphase, a number of workers ${ }^{17}$ object to the emphasis on elongation as a major factor in mitosis. As urged by Bleier, such an interpretation does not apply well to the mitotic phenomena in many hybrids, where double chromosomes of different origin and constitution (bivalents formed by synapsis and univalents which have split) pass poleward at different times in the same spindle (Figs. 207, 208). In Bleier's opinion, all hypotheses postulating only a passive movement of the chromosomes, whether caused by streaming, a contraction of fibers, or a "Stemmkörper," fail in such cases. It is rather to some force resident in the chromosomes themselves that the movement is due primarily; it is provisionally assumed that this is a repulsion force of some kind. It is a suggestive fact that the chromosomes exhibit fully their characteristic reactions in the spindle (attachment at its equator and subsequent separation) only after they have reached a certain stage in the development of their doubleness. The delayed attachment of the univalents may be related to their late splitting, especially at their attachment regions. The repelling force thus comes into play only when the chromosome is properly constituted, and it is accordingly possible to look upon the spindle as a structure which guides the chromosomes instead of actively moving them. The existence of some such force is further rendered plausible by certain other phenomena of synapsis and disjunction to be considered in later chapters, as well as by the successful distribution of daughter chromosomes in narrow root cells and pollen tubes, where limitations of space often prevent the arrangement of the chromosomes in a regular equatorial plane.

Further evidence that the movement of the chromosome is not a passive one is afforded by the remarkable monocentric mitosis discovered in spermatocytes of flies of the genus Sciara by Metz. ${ }^{18}$ In the first meiotic division, although all of the chromosomes show fibers extending from their spindle-attachment regions toward the single pole, four of them move backward away from the pole until they reach the cell boundary, where they move into a compact group and are extruded in a bud from the cell. The behavior of these chromosomes when their extrusion is inhibited shows that they are not merely degenerated material of which the cell is ridding itself. During their backward movement their shapes suggest that a poleward force acting at the spindle-attachment region is overcome by another force acting in the opposite direction. The

${ }_{17}$ Martens (1929), Schaede (1929), Bleier (1930bc). See also Schrader (1932).

${ }^{18}$ See Metz $(1925,1926 c, 1933)$ and Metz, Moses, and Hoppe (1926). See also Huth's (1933) account of Bèlař's work on monasters in Urechis eggs. 
arrangement of materials about the chromosome suggests further that the latter force is not resident in them but in the chromosome itself. A hypothesis involving a progressive alteration at one or both ends of the chromosome is advanced.

Evidence for an active participation by the spindle is afforded by the occasional division of nucleoli in normal bipolar mitosis. ${ }^{19}$ Ordinarily the nucleolus has disappeared by the time the metaphase figure is established in root cells, but frequently a portion of it may remain. In such cases it tends to occupy the equator of the figure with the chromosomes. It may then elongate and divide by constriction, the halves passing toward the spindle poles, sometimes in advance of the chromosomes. A similar behavior is manifested regularly by the "karyosome" in the nuclei of certain lower plants and animals (see Chapter XIII). In the ascus of Pustularia the nucleolus is usually left outside the spindle, but when it lies in the equator it divides. In sea-urchin eggs in hypertonic media Konopacki (1911) observed that a whole nucleus might be separated into two portions if caught between two cytasters.

The foregoing facts seem to show that anaphasic chromosome migration is a process in which more than one of the nuclear constituents play active rôles. As the metaphase approaches, the prophasic karyolymph ${ }^{20}$ becomes organized as a spindle which is the visible expression of a field of forces tending to divide and carry poleward any sufficiently fluid body lying in the equator. The chromosomes, which are less fluid at this stage, are specially adapted to reaction with these forces in being already doubled when they enter the equator and in having specialized attachment regions. Moreover, evidence cited above suggests an active force resident in the chromosomes themselves. To the extent that the spindle substance is actually a constituent of the chromosome (i.e., combined with its matrix) during the metabolic stage, the mitotic division of the nucleus may be regarded as a simultaneous and coöperative division of its several individual chromosomes. In amphiastral figures the process is closely correlated with the multiplication of centrioles and a series of structural alterations in the surrounding cytoplasm, such figures comprising both nuclear and cytoplasmic elements. ${ }^{21}$ The nature and relative importance of the forces involved are still obscure. It seems probable that mitosis involves the action of many factors, including alterations in viscosity, ${ }^{22}$ surface tension, and permeability, as well as streaming, tension, and electrical attractions and repulsions. In no one of these factors alone will the entire solution of the problem of mitosis be found.

${ }^{19}$ Němec (1901) on Alnus, Yamaha and Sinotô (1925) on Glycine and other genera, Zirkle (1928b) on Zea, Frew and Bowen (1929) on Cucurbita and Pustularia, Ghimpu (1930) on Acacia.

${ }^{20}$ The "parachromosomic substance" of Koerperich (1930); the "paragenoplast" of Bleier (1930c). See discussion by Wilson (1932).

${ }^{21}$ See Wilson (1925, pp. 174-178).

${ }^{22}$ Chambers (1917), Heilbrunn (1920a, 1921). 


\section{CHAPTER XII}

\section{CYTOKINESIS AND THE CELL WALL}

The division of the cytosome, or extra-nuclear portion of the protoplast, is known as cytokinesis. It may occur more or less independently of karyokinesis (mitotic nuclear division), or the two processes may be very intimately associated. Cytokinesis is accomplished in a variety of ways in growing and differentiating masses of protoplasm. The principal types are included in the following description. ${ }^{1}$

Furrowing in Plants.-The cleavage of plasmodial masses is most commonly brought about by the formation of furrows, with or without the coopperation of vacuoles. It is well illustrated in the sporangia of myxomycetes and certain fungi. ${ }^{2}$ In Fuligo, cleavage furrows begin to develop at the peripheral membrane of the young sporangium and gradually extend inward, cutting out multinucleate blocks which are subdivided by further furrowing into uninucleate spores. In Didymium the spores are delimited in a similar way by furrows which begin to develop both at the periphery and along the young capillitium filaments in the midst of the protoplasm. In Rhizopus the furrows develop from the peripheral membrane (Fig. 92, A) and from the columella. In the sporangia of Achlya, Saprolegnia, and Olpidiopsis the furrows start from a large central vacuole. In Phycomyces, vacuoles appear in the protoplasm, become stellate in form, and cut out spore masses with from one to about twelve nuclei each (Fig. 92, B). Such vacuoles function together with peripheral furrows in Pilobolus and Circinella. In the sporangia of the phycomycetes the columella is separated from the rest of the sporangium by a dome-shaped layer of vacuoles which coalesce and form a continuous partition between the two regions. Such cleavage is interpreted by Scarth (1927) as an extension and swelling of kinoplasm present at the protoplasmic surfaces.

Similar forms of cytokinesis are found in certain algæ. ${ }^{3}$ In Hydrodictyon, for example, the multinucleate protoplasm, lining the wall of the

${ }^{1}$ A classification of types of cytokinesis, with examples, is given by Yamaha (1926a).

${ }^{2}$ Harper (1899, 1900a, 1914) on Synchytrium, Pilobolus, Sporodinia, Fuligo, and Didymium; D. B. Swingle (1903) and Moreau (1913) on Rhizopus and Phycomyces; Rothert (1892) and Schwarze (1922) on Saprolegnia, Circinella, and Achlya; B. M. Davis (1903) on Saprolegnia; Rytz (1907) on Synchytrium.

${ }^{3}$ Kilebs (1891) and Timberlake (1902) on Hydrodictyon, Yamanouchi (1906) on Polysiphonia, Brand (1908) on Cladophora. For an extensive account of the algæ, see Oltmanns (1922-1923). 
large cell, is divided into uninucleate swarmers by cleavage furrows. These appear to begin their development from the cell and vacuole membranes and from cleft-like vacuoles. Often the furrows thus developing inward from the cell membrane are very narrow. In some

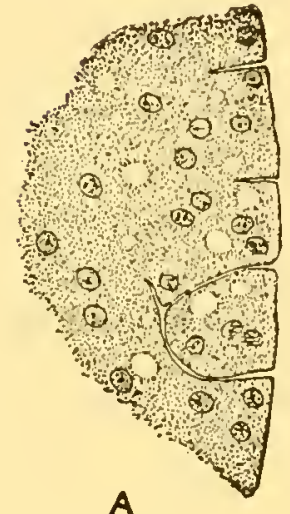

A

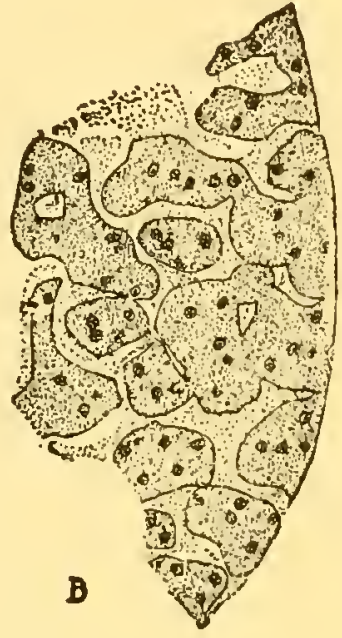

FIG. 92.

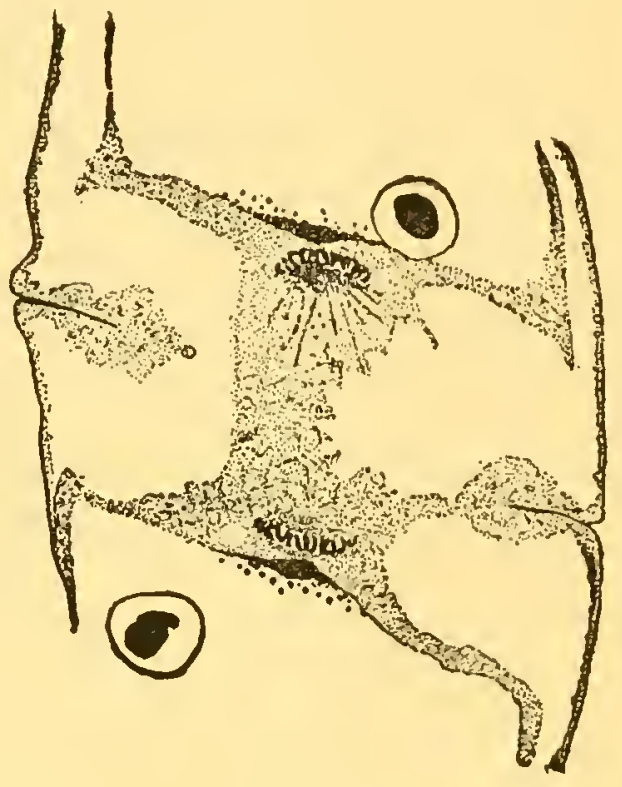

FIG. 93.

F1G. 92.-Cytokinesis by furrowing in sporangia of fungi. A, Rhizopus nigricans. $B$, Phycomyces nitens. (After D. B. Swingle, 1903.)

FIG. 93.-Cytokinesis by furrowing in Closterium. (After Lutman, 1911.)

instances, the wall substance seems to be active in developing the furrow; in such cases a "girdle wall" develops centripetally as a ring-like ingrowth from the sides of the cell (Fig. 94). In Cladophora an accumulation of slimy material, seen against the lateral wall as the process begins, appears to be swollen wall substance. The formation of the girdle wall

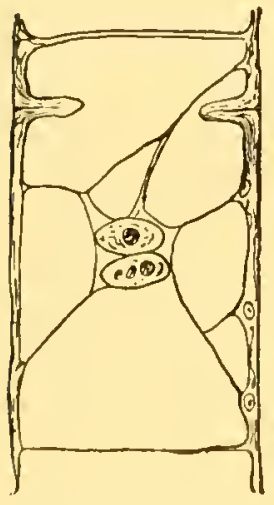

$\boldsymbol{\Lambda}$

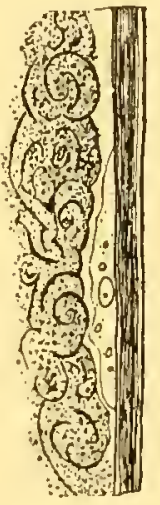

B

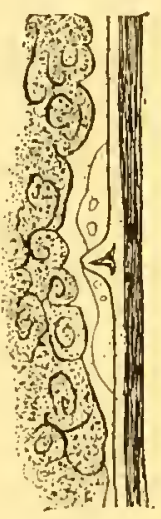

c

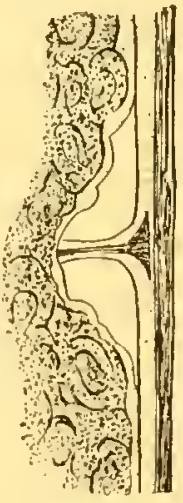

D

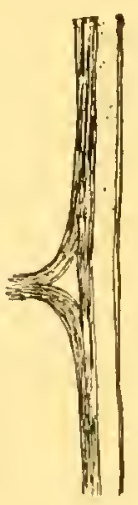

E

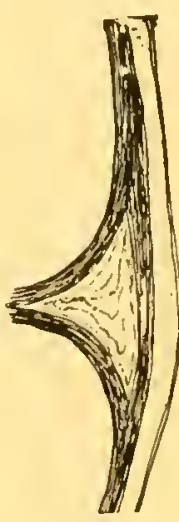

F

Fig. 94.-A, girdle wall developing in Spirogyra. (After Nathansohn, 1900a.) B-F, five stages in the development of the girdle wall in Cladophora. (After Brand, 1908.)

is probably independent of the division of the several nuclei, but in Spirogyra such a wall develops immediately after the single nucleus divides and involves the activity of the achromatic figure (McAllister, 1931). The "division by constriction" seen in unicellular algx, the 
"budding" of yeast cells, and the abstriction of conidia and basidiospores may be regarded as special cases of cytokinesis by furrowing.

The microspore quartets of most vascular plants fall into two classes as regards the shape and arrangement of the spores. If no permanent partition is formed after the first meiotic mitosis, the four spores are delimited by partitions appearing simultaneously after the second mitosis. Such spores, when first formed, commonly, though not always, have the tetrahedral form. If the first mitosis is followed immediately by the formation of a permanent partition through the equator of the sporocyte, each hemisphere being divided by another partition after the second mitosis, the quartet is said to be of the "bilateral" type. ${ }^{4}$

Although the partitions delimiting the four microspores in angiosperms are sometimes formed by the cell-plate method, it has been shown ${ }^{5}$ that furrows developing inward from the periphery are chiefly responsible for cytokinesis here, at least in cases of simultaneous division (quadripartition) to form tetrahedral spores. In Nicotiana, for example, the four microspore nuclei present after the second mitosis all become connected by achromatic "fibers" (Fig. 95). The two sets of connecting fibers of the second mitosis may persist, four new sets being added, or the two may disappear, six sets being developed anew. These fibers have nothing to do with the formation of the partitions; no cell-plates are devel-

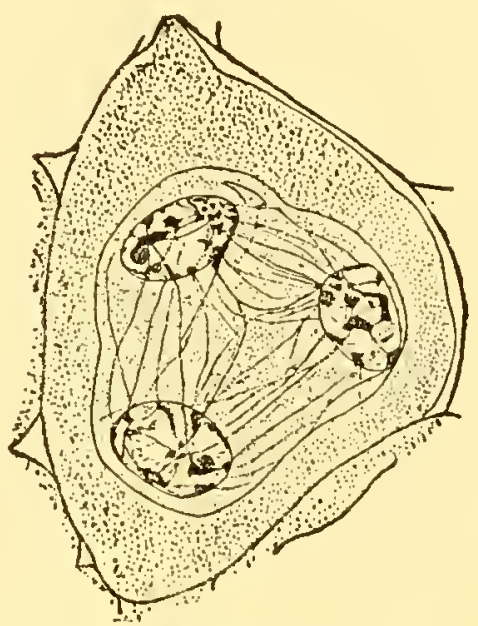

oped. Furrows appear at the periphery and grow inward until they meet at the center, dividing the protoplast simultaneously into four spores. In Nelumbo the furrows are exceedingly narrow, appearing much like cell-plates. As they grow inward they seem simply to cut through any fibers which they may encounter (Farr).

Meanwhile there develops within the original sporocyte wall a mass of callose known as the "special wall." This increases in thickness and follows the furrows inward, forming a sort of matrix in which the young spores lie while their elaborate coats are being differentiated (p. 180).

"Although either the "simultaneous" or the "successive" mode of division may tend strongly to predominate in certain groups of plants, there are so many exceptions to rules and so much variation that the character is of very restricted taxonomic value. See Täckholm and Söderberg (1917, 1918), Söderberg (1919), Palm (1920), Suessenguth (1921), Stenar (1925), and Coulter and Chamberlain (1903).

${ }^{5}$ C. H. Farr $(1916,1918,1922 a b)$, W. K. Farr (1920), Castetter (1925), Gates (1925), Wóycicki (1932). 
By some observers the special wall has been thought to arise through a swelling of the secondary layers of the sporocyte wall, but it now seems evident that it is secreted directly by the protoplast (Gates, Castetter, Wóycicki), which may undergo a distinct contraction at this time. Eventually the sporocyte wall and the material separating the four microspores disappear, leaving the spores free from one another.

Cytokinesis in the microsporocyte of Melilotus alba is of interest in that vacuoles appear to play a conspicuous part in the process (Fig. 96). After the second mitosis small vacuoles develop in the regions between the four nuclei and fuse to form larger ones which nearly separate the protoplasm into four masses. Furrows originating at the surface then
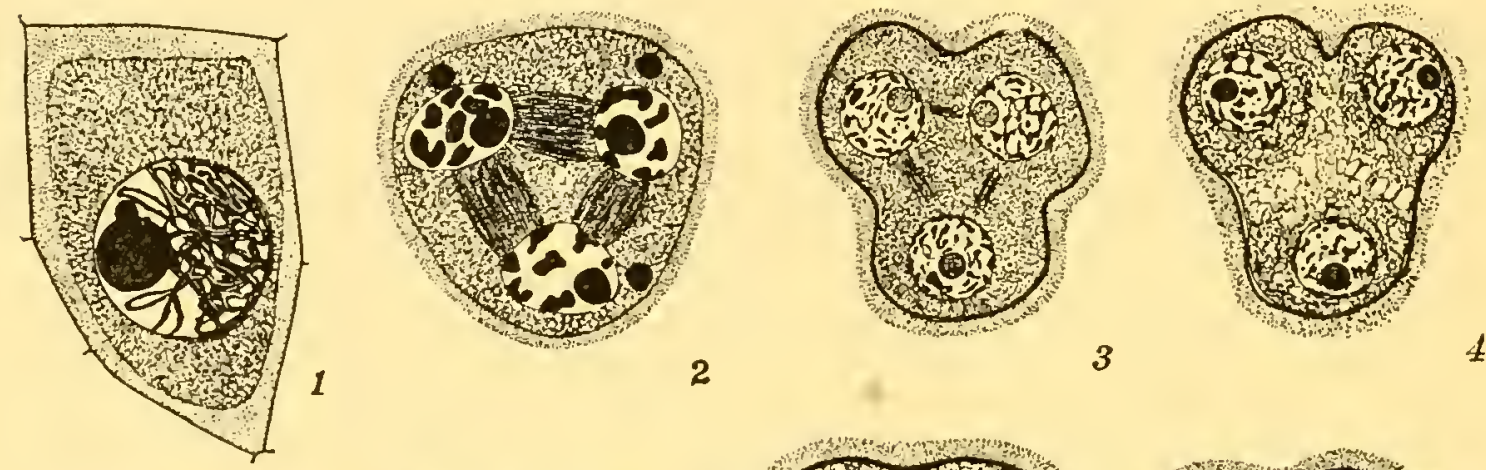

2

3
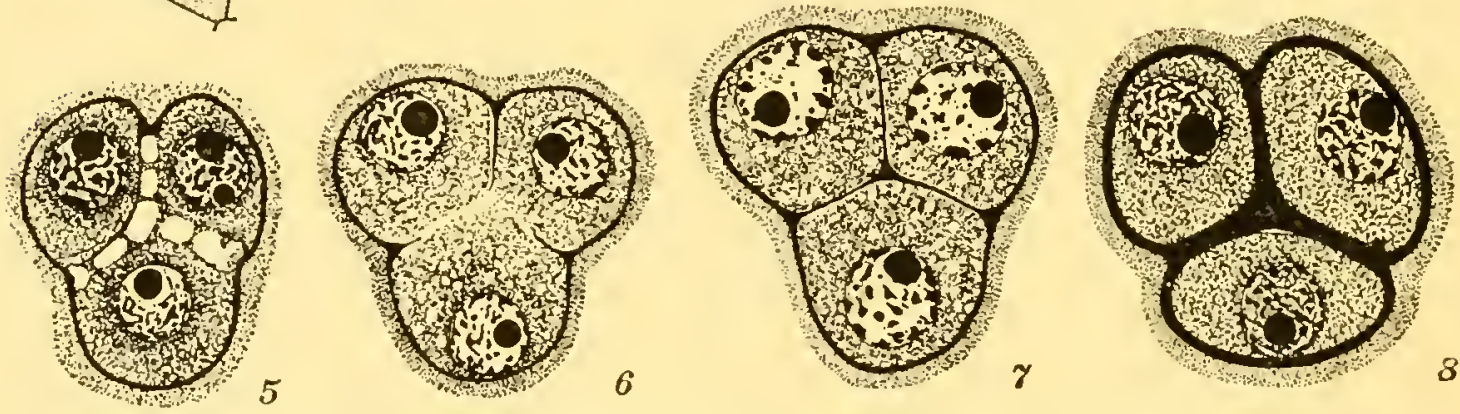

Fig. 96.-Cytokinesis in the microsporocyte of Melilotus. 1, prophase of first meiotic mitosis; eallose (uniformly stippled) being secreted by the protoplast. 2, telophase of second mitosis. 8 , special wall (black) and furrows appearing. 4,5 , vacuoles forming between nuclei. $G$, special wall extending inward. 7 , special wall extensions have met at center. 8, thickened special wall complete. (After Castetter, 1925.)

grow inward, meet the vacuoles, and complete the cleavage of the protoplast (Castetter). A similar process occurs in Gentiana (Wóycicki).

In Magnolia, Farr finds a case in which bilateral quartets are formed by furrowing, rather than by cell-plates as might be expected. Although a transitory cell-plate may be differentiated, it plays no part in cytokinesis. After the first mitosis a cleavage furrow starts to form, but its development is arrested until after the second mitosis, when it resumes its growth and forms a partition through the equator of the sporocyte. At the same time additional furrows subdivide the two hemispheres, thus delimiting the four microspores.

Furrowing in Animals.-Cytokinesis, which in animals is most commonly accomplished by furrowing, has been studied with special care in segmenting eggs. In the case of small eggs, such as those of 
worms, the daughter cells (blastomeres) round up and become more or less spherical, whereas in larger eggs, such as those of frogs, a narrow cleavage furrow appears at one pole and develops through the egg without altering greatly the shape of the latter, so that the first two blastomeres have the form of hemispheres. In many animals, notably birds, the cleavage is superficial, not extending entirely through the yolk-laden egg.

So far as known, no animal cells are divided by cell-plates of the type found so commonly in plants. As noted previously, there is often a slight differentiation (the "mid-body") at the equator of the achromatic figure in the telophase, but it plays no part in cytokinesis. In the spermatogonia of certain insects Janssens (1924) finds a cell-plate-like differentiation which appears to represent a region of protoplasmic continuity rather than a structure concerned in cytokinesis.

Experiments with dividing eggs have led to the identification of some of the factors involved in the development of cleavage furrows. Many
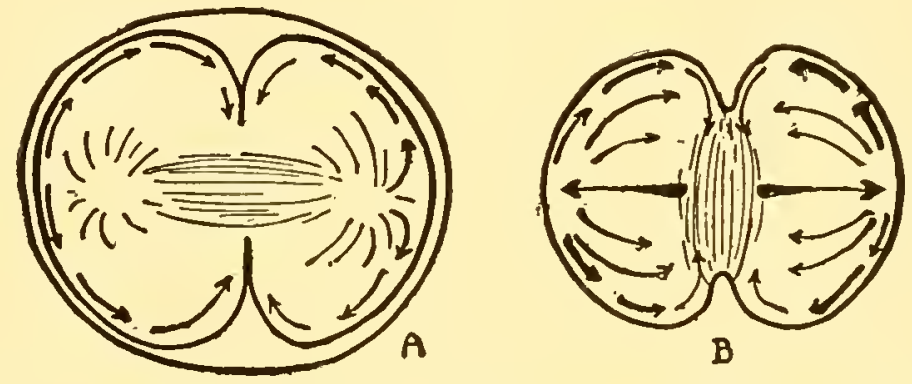

F1G. 97.-Diagram of streaming and furrowing in the egg of Rhabditis pellio $(A)$ and an oil droplet (B). (After Spek, 1918a.)

years ago Bütschli (1876) advanced the view that cytoplasmic currents flowing toward the centrosomes lead to the production of a relatively high surface tension at the equator of the cell, this in turn bringing about furrowing through this region. Evidence favoring this interpretation has been contributed by several later investigators. ${ }^{6}$ Spek imitated furrowing and division with oil and mercury droplets in water and showed that by lowering the surface tension at two poles of the droplet the relatively high surface tension at the equatorial region could be made to bring about the constriction and fission of the droplet. In both droplet and dividing nematode egg he found streamings such as Erlanger (1897) had described in the egg: an inner movement poleward to the region of low surface tension and a superficial streaming toward the equatorial region of higher surface tension, the streams turning inward at the furrow (Fig. 97). Bělař (1927) has observed such cytoplasmic streams in the furrowing spermatocyte of Chorthippus (Fig. 59).

Closely associated with such streaming and surface-tension phenomena are certain periodic alterations in the viscosity of the egg sub-

${ }^{6}$ McClendon (1910, 1913), Spek (1918a, 1920b), Just (1922), Cannon (1923), Bancroft and Gurchot (1927). 
stance. ${ }^{7}$ In the living echinoderm egg it is found that the two asters developed at the first cleavage division are regions in which the protoplasm has become decidedly more viscous, the peripheral and equatorial regions at the same time showing a high degree of fluidity and active streaming like that mentioned above. The growth of the two semisolid masses results in a slight elongation of the egg and a cleavage furrow develops in the more fluid region separating them, after which the asters revert to a less viscous state. In a number of interesting experiments Chambers has shown the dependence of the location of furrows on these local differences in viscosity. When a dividing egg is cut in two obliquely through the two asters, the pieces will continue to cleave along the
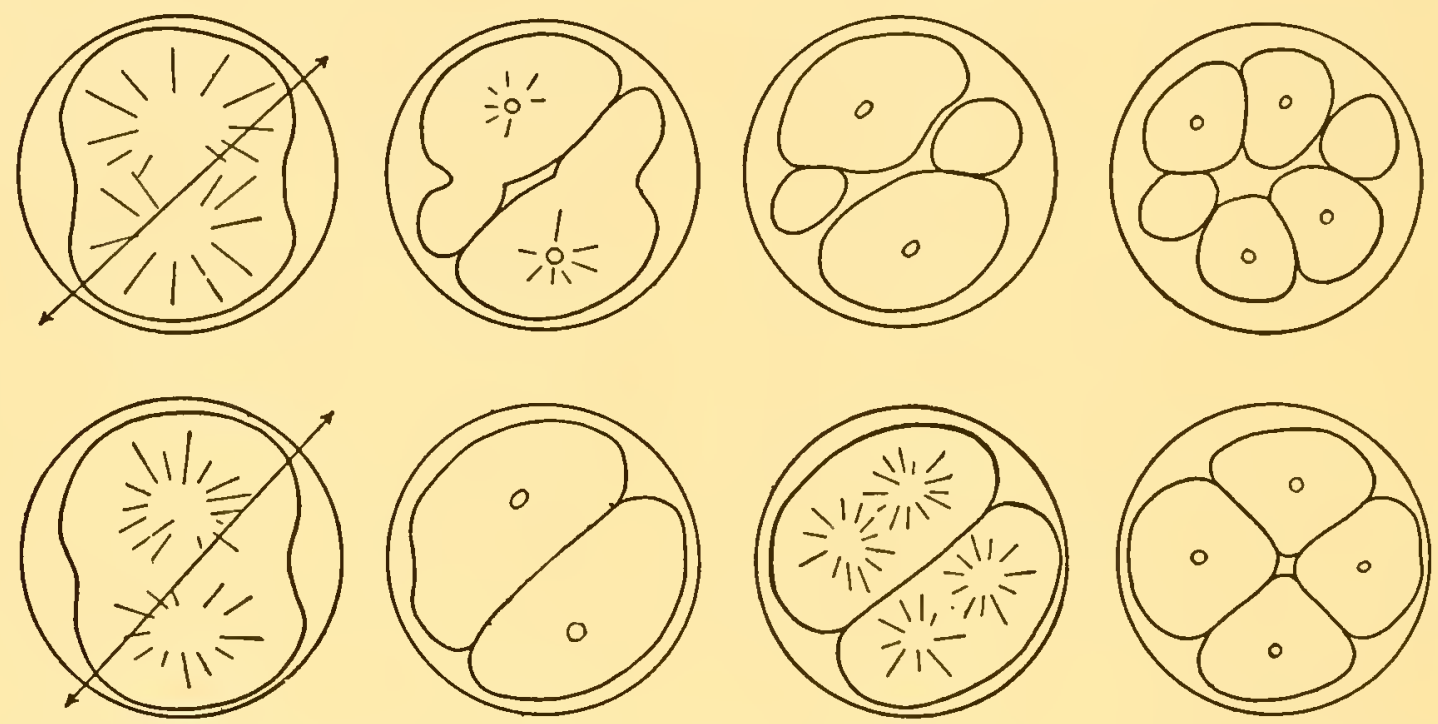

FIg. 98.-Diagrams showing effect of bisecting cleaving echinoderm eggs. First row: without disappearance of asters. Second row: with disappearance of asters. (After Chambers, 1919.)

fluid equatorial plane if the asters persist in the semisolid state; but if the asters revert to the fluid condition, as often happens as the result of rough handling, the cleavage furrow is obliterated, and the pieces produced by the cut divide symmetrically at the next cleavage (Fig. 98). When the formation of a furrow at the first mitosis is prevented by mechanical means, furrows develop between all four asters in the second mitosis, cleaving the egg simultaneously into four blastomeres. According to Heilbrunn, "all agents which stimulate egg cells to segment cause a gelatin or coagulation and . . . all agents which prevent such gelation prevent the division of the egg." In the plant cells mentioned in the preceding section, it is probable that the furrows separate regions of relatively high viscosity even though no asters in the ordinary sense are present.

${ }^{7}$ Heilbrunn $(1915,1920 a, 1921,1925 c)$, Chambers $(1917,1919)$. See Gray (1931). 
Alterations in surface tension and viscosity, together with protoplasmic streaming, are obviously important factors in cytokinesis of certain types, but comparatively little is known about the initial causes of these phenomena. That they are in some way associated with changes in the permeability of protoplasmic membranes seems clear from the work of Spek and others on animal eggs and of Stalfelt (1921) on plant cells. Little more than a promising beginning has been made on the problem of the mechanism of cytokinesis.

Closely allied to the process of furrowing is the type of cytokinesis seen in certain tissues which are more or less plasmodial in their early stages of development. In some forms of cartilage and epithelium, for example, vacuole-like masses of material appear in the cytoplasm and gradually subdivide the plasmodium into cells which, however, remain connected as a syncytium (Fig. 10). In certain cases such masses are metaplasmic in nature (Rohde, see p. 45).

Cytokinesis by Cell-plates.-In the bryophytes and vascular plants cytokinesis in somatic cells commonly begins with the formation of a cell-plate through the equatorial plane of the spindle substance lying between the young sister nuclei. The same process occurs in some sporocytes also. The precise manner in which the cell-plate is formed and becomes involved in the developing cell wall has long been a subject of dispute. According to many of the earlier accounts, ${ }^{8}$ the cell-plate is formed by the fusion of swellings on the middle portions of persisting spindle fibers, the plate then splitting to form the plasma membranes of the two daughter protoplasts, the primary wall layer, or "middle lamella," then being secreted between them.

In a series of more recent papers ${ }^{9}$ it is emphasized that after adequate fixation (Bouin, Benda) the cell-plate first appears as a continuous film in the midst of the oriented "spindle of cytokinesis" and not as a series of swellings or granules. This film proceeds to thicken, of ten unevenly at first. The "splitting of the plate" so often observed is held to be due to improper fixation, for it is said to appear only after the use of certain fluids and not in unfixed cells (Robyns, 1929). Bělař (1929b), in his ingenious experimental studies on living cells of young Tradescantia leaves, finds that the young cell-plate is a fluid layer offering less resistance to shrinking agents than the spindle, so that the halves of the cell can be made to round up and separate along this plane before a primary cell membrane is present. Hence he concludes that the cell-plate is really a transverse cleft in the spindle, the membrane substance being deposited

${ }^{8}$ E.g., Strasburger (1898), Timberlake (1900), and C. E. Allen (1901).

${ }^{9}$ Devisé (1922) on the sporocytes of Larix; Robyns $(1924,1926,1929)$ on the root cells of Hyacinthus, Vicia, and other plants; Martens (1927c, 1929b) on stigma cells of Arrhenatherum and stamen hairs of Tradescantia; Jungers (1931) on the endosperm of Iris. 
later in this cleft. In living stamen hairs of Tradescantia, W. Becker (1932) observes that the cell-plate material appears first in the form of minute droplets which then unite to form a continuous plate (Fig. 99). This may be a more adequate description of what was formerly interpreted as "swellings of the spindle fibers." On the basis of such observations the supposed "splitting of the cell-plate to form the plasma membranes" of the two protoplasts could be regarded as merely the widening of the fluid layer as its substance increases in amount and receives deposits of the primary wall materials. The plasma membranes would be the protoplasmic surfaces lying against the fluid layer. The origin of the fluid which collects thus at the equator to form the cell-plate is unknown, but it has been claimed that a stainable substance migrates to

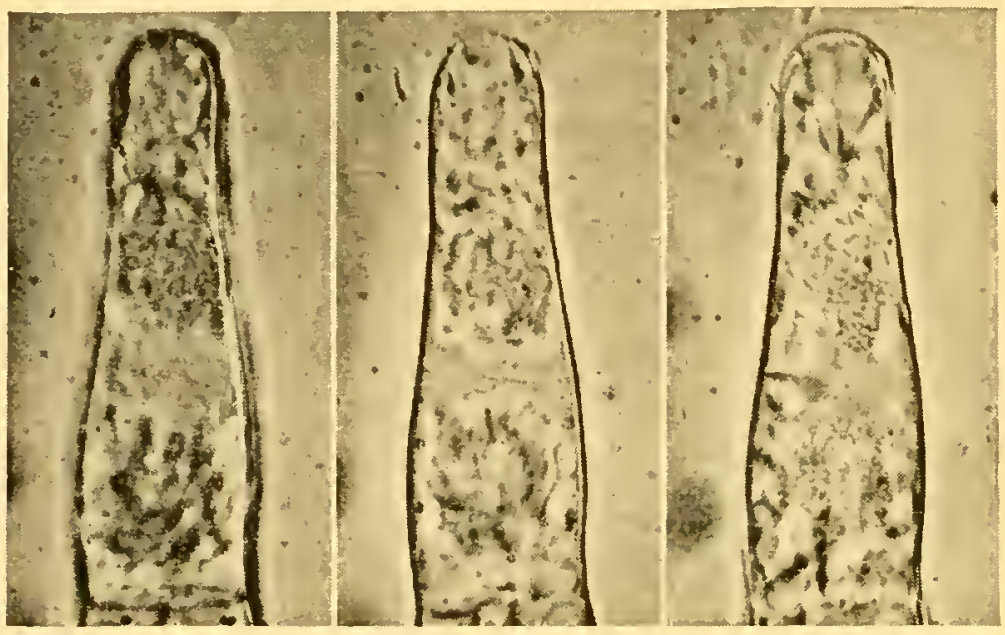

Fig. 99.-Formation of cell-plate by coalescence of droplets in stamen hair of Tradescantia. The ehanges shown occupied 27 minutes. (After W. A. Becker, 1932e.)

this region from the two young nuclei. ${ }^{10}$ The observations of Becker point rather to the conclusion that there is a local dissociation of two protoplasmic phases, one of these forming the cell-plate while the other remains as the plasma membranes. Such a dissociation ("Entmischung"), often reversible, of more and less fluid phases has been induced in the living protoplasm of Didymium and Tradescantia by Bělař (1930).

There are many facts which suggest that the processes of cytokinesis by furrowing, by vacuoles, by membranes, and by cell-plates are not wholly distinct from one another. The formation of the fluid cell-plate from the center outward suggests the cleaving action of vacuoles in the sporangium of Phycomyces; whereas, when spores begin to separate at the periphery after cytokinesis by cell-plates, the appearance is that of furrowing. It may be helpful to think of cytokinesis by cell-plates in vascular plants as a process whose peculiarities are due in large measure to its close association with the mechanism of karyokinesis.

${ }^{10}$ Dembowski and Ziegenspeck (1929), Dannehl and Ziegenspeck (1929). 
The spindle of cytokinesis with its cell-plate commonly extends laterally as a phragmoplast until it reaches the walls of the cell. In wide cells with large vacuoles this may involve the formation of cytoplasmic bridges (p. 151). As multinuclear endosperm becomes cellular, the recently formed pairs of nuclei are connected by spindles on the flanks of which additional spindles arise and so connect all the nuclei. This involves a lateral extension of the spindle substance and apparently also the use of any such substance remaining from the preceding mitosis. Cell-plates appear in all the spindles and so subdivide the multinucleate mass into uninucleate cells (Fig. 100). The development of the cellular
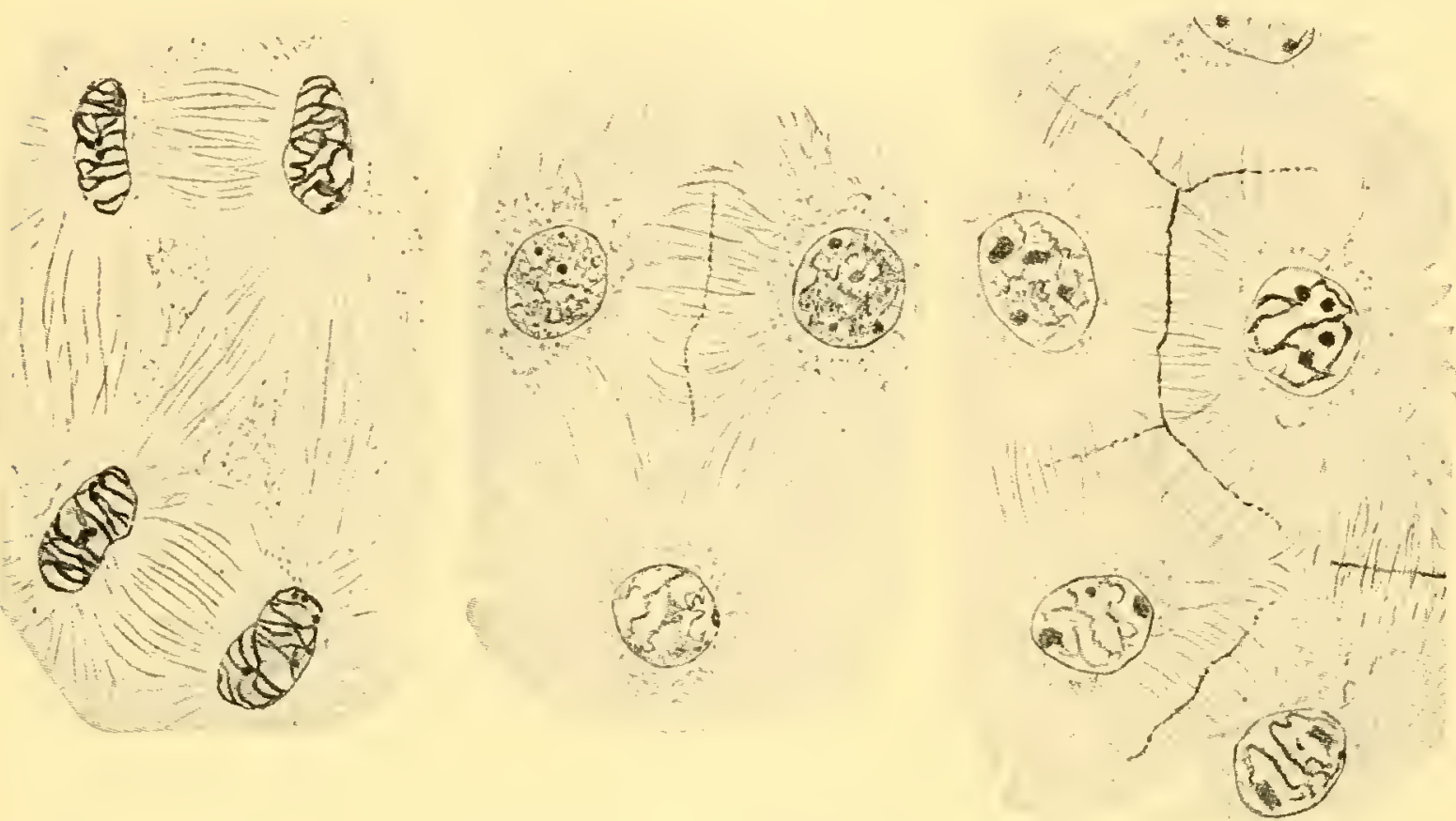

FIG. 100.-Formation of cell partitions in endosperm of Iris. (After Jungers, 1931.)

stage in cycad embryos occurs in a similar manner (Chamberlain, 1910, 1916).

When one of the two telophase nuclei lies near the side of a large cell, as in the angiosperm embryo sac or microspore, the phragmoplast may curve sharply so that the cell-plate and subsequently formed membrane cut off a small cell against the side of the large one (Fig. 125). In extreme cases it may form a complete sphere about one nucleus and so cut out a small cell which lies free in the cytoplasm of the original cell (Fig. 102). Other modes of "free cell formation" are seen in ascospore development (Fig. 88), in the delimitation of the egg in certain phycomycetes and of embryo cells in certain gymnosperms (Fig. 103), and in certain animal tissues (Rohde, 1923). In some cases, notably certain algæ, ${ }^{11}$ partition membranes are reported to form between sister nuclei after the complete disappearance of the achromatic figure.

${ }^{11}$ Strasburger (1892), W. T. Swingle (1897), Mottier (1900). 
The Cell Wall.-Probably the most striking difference which meets the eye in comparing animal and plant tissues is the degree of distinctness with which the limits of the individual cells can be made out. Animal cells, as a rule, are separated only by very thin membranes, which in many tissues are so delicate as to be scarcely discernible; whereas the cells of plants usually possess conspicuous firm walls, which, in the case of woody plants, become greatly thickened and afford mechanical support to large bodies.

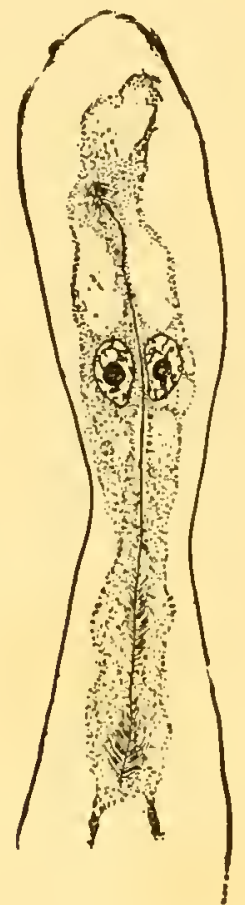

FIG. 101.

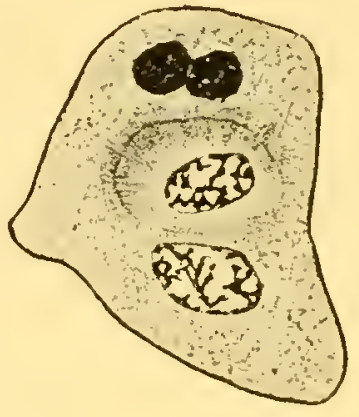

FIG. 102.

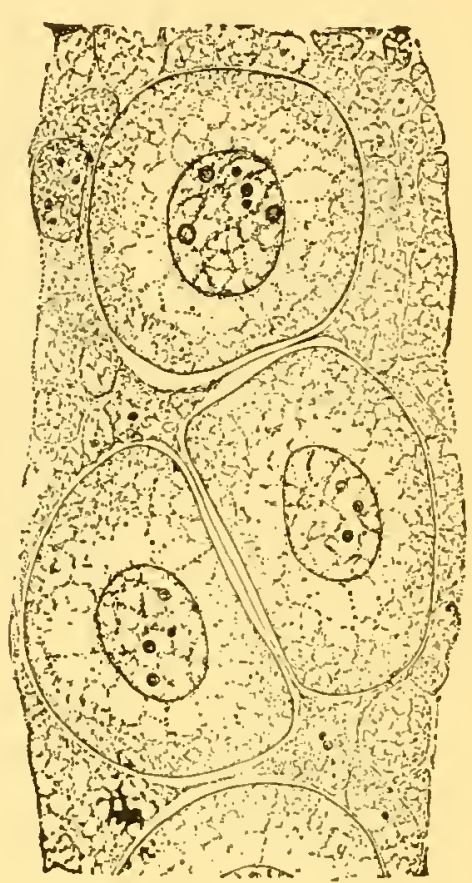

FIG. 103.

Fig. 101.-Continued extension of partition wall after completion of mitosis in endosperm of Physostegia virginiana. (After Sharp, 1911.)

Fig. 102.-Delimitation of generative cell in pollen grain of Scirpus. (The dark bodies are degenerating nuclei of grains which fail to develop.) (After Pieeh, 1928.)

FiG. 103.-Embryonic cells in Ephedra, developed by "free cell-formation" in a common mass of protoplasm. (After Land, 1907.)

As shown in the foregoing section, the development of the cell wall in vascular plants ordinarily begins with the formation of a fluid cell-plate at the close of mitosis. Cytologists are not yet in agreement regarding the exact relation of this layer to the primary wall layer, or middle lamella (the "intercellular substance" and "cement" of early writers). For some time it was thought ${ }^{12}$ that the cell-plate became the middle lamella directly, secondary and tertiary layers being deposited upon it by the protoplasts on either side. Soon it was suggested, ${ }^{13}$ as already stated, that the cell-plate splits, the middle lamella then being deposited between its halves. As we have indicated (p. 171), the young cell-plate has been

12 E.g., by Strasburger $(1875,1882 b, 1884 b)$.

${ }^{13}$ Treub (1878). Strasburger adopted this view in 1898. It was supported by the work of Timberlake (1900), C. E. Allen (1901), and a number of later writers. 
shown to be a fluid film, and it now seems evident that the deposition of salts and other matter in this film transforms it into the middle lamella. Whatever its origin, the middle lamella may increase somewhat in thickness before distinct secondary layers appear. The finer structure of the middle lamella is not adequately known, but there is much to suggest that it consists in reality of two or three differentiated layers, through one of which the lamella splits when intercellular spaces are developed by a rounding up of the cells.

It is probable that the deposition of the secondary layer begins after the cell has reached nearly or quite its full size, though to this there are apparently certain exceptions. The secondary layer, which seems to be formed with considerable rapidity, differs from the primary layer chemically and in structure. There are circular or elongated areas in which no secondary substance is deposited, so that the cells at these places are separated only by the delicate primary membrane. Such a wall is said to be "pitted," the primary layer extending across the pit being termed the closing membrane. The central portion of this membrane (vascular cells of gymnosperms chiefly) has sometimes a more or less conspicuous thickening known as the torus. The portion of the membrane around the torus is pierced by fine pores. In some cases these may become so large and numerous that the torus appears to be suspended on a meshwork, while extreme cases are known in which it is held in place only by a few strands. In bordered pits the secondary wall overarches the margins of the closing membrane. In this type of pit, characteristic chiefly of water-conducting cells of the gymnosperms, the closing membrane is of such a nature that its position in the center of the pit is readily altered. Probably because of changes in pressure it swings to the side of the pit; the torus then lies against the pit opening, or "mouth," and the pit is blocked except for slow diffusion through the rather thick torus.

The secondary wall layer may be even more limited in extent, only a small portion of the primary wall being covered. Such is the case in protoxylem cells, where the secondary layer is deposited in the form of rings and spirals (Fig. 8, $B, C$ ). This form of thickening, together with the peculiarly extensible character of their primary walls, permits the great increase in length of these cells necessitated by the continued growth of the young organs in which they chiefly function. In some cells, notably the tracheids of certain gymnosperms and the vessels of many angiosperms, a tertiary layer is deposited upon the secondary one. This tertiary layer takes the form of slender spirals, rings, and other figures resembling the secondary thickenings of protoxylem cells. ${ }^{14}$ It has been shown in the protoxylem and metaxylem cells of the gourd

${ }^{14}$ For a more complete account of such differentiations, see Eames and MacDaniels (1925). 
Trichosanthes anguina that the position of the spiral and annular thickenings is determined by peripheral bands of cytoplasm separated by more vacuolate regions (Barkley, 1927).

Composition of the Cell Wall.-The principal constituents of the completed cell walls of vascular plants are polysaccharides: cellulose, hemicelluloses, and pectins. With these are often associated other substances, notably lignin, suberin, and cutin. ${ }^{15}$ The distribution of these components in the various layers of the wall has been the subject of many investigations. ${ }^{16}$ The earlier workers, who relied chiefly on staining reactions, concluded that the middle lamella consists of pectic compounds, while the secondary and tertiary layers are composed chiefly of cellulose with or without lignin, pectin, and other additional materials. Subsequent studies with chemical methods have shown that the middle lamella, although it consists chiefly of pectin in one or more of its forms in parenchyma, is principally lignin in woody tissues, and that staining reactions cannot be safely relied upon as criteria of chemical composition. What becomes of the original pectins in the young wall during its lignification is still somewhat uncertain. Harlow finds that the secondary layers of softwoods are appreciably lignified, while those of hardwoods, with few exceptions, have practically no lignin. Tertiary layers are often lignified.

The secondary and tertiary layers are made up of numerous thin lamellæ which appear to differ in the relative amounts of cellulose and pectin present (van Wisselingh) as well as in physical properties. In the wall of the flax fiber the layers have been shown to consist of spirally arranged fibrils, the fibrils in contiguous layers running in opposite directions (D. B. Anderson, 1927). In cotton the direction of coiling changes at intervals along the fiber (Balls, 1923).

Suberin and cutin are varying mixtures of certain organic acids present in part in the form of fats (Priestley, 1921). They are distinguished more by location than by composition, suberin appearing in the walls of periderm cells (cork), while cutin occurs as a cuticle on the epidermis and often in patches in the walls of subepidermal cells. In the resin canals of conifer leaves the suberin is said to be deposited on the primary layer and rarely on the secondary one (Gauba, 1926). Lee and Priestley (1924) have ascribed the formation of plant cuticle to certain

${ }^{15}$ For an extensive account of walls and their constituents, see van Wisselingh (1924). See also Czapek (1913), Molisch (1913), von Wettstein (1921a), Gleisberg (1921), Grafe (1911, 1922), and Gortner (1929). Among the earlier papers on this subject are those of Payen (1842), Frémy (1859), Kabsch (1863b), Wiesner (1864, 1886), Mangin (1888-1893), Schulze (1890-1894), Gilson (1890), van Wisselingh (1888, 1892, 1895), and Allen (1901).

${ }^{16}$ Among recent works, see especially Tupper-Carey and Priestley (1923), Ritter (1925, 1928), Harlow (1927, 1928abc, 1931), D. B. Anderson (1926, 1927, 1928), and Scarth, Gibbs, and Spier (1929). 
alterations in fatty substances which are produced in the protoplasts and then migrate into and along the cell walls to the outer surface of the epidermis. D. B. Anderson (1928) finds the outer wall of the epidermal cell of Clivia to consist of (1) an inner layer of cellulose and pectin; (2) a thin layer consisting mostly or wholly of pectin; (3) a cuticular layer composed of $(a)$ a region of cellulose and pectin lamellæ containing cutin and $(b)$ a region of cutin and cellulose with little or no pectin; and (4) a cuticle of pure cutin. In Aloë new cutin is secreted below the old euticle (Ziegenspeck, 1928).

A variety of mineral substances, such as silica, calcium carbonate, and calcium oxalate, as well as more complex organic compounds, including tannins, oils, and resins, are often deposited in the walls of old cells. The heartwood of trees owes many of its qualities, such as color and density, to the presence of these additional materials in the walls and cell cavities.

Among the thallophytes many species have walls essentially like those of higher plants, cellulose being the chief constituent. Others are characterized by the predominance of other compounds, notably chitin in zygomycetes, ascomycetes, basidiomycetes, and certain alga; pectins in the bacteria; and keratin in myxomycetes. In many specific eases the results of various workers do not agree. ${ }^{17}$

That the cell wall is not wholly an ergastic secretion, but contains protoplasm in some form, is a view which has frequently been maintained. ${ }^{18}$ Wiesner supposed the growing wall to be made up of regularly arranged particles ("dermatosomes") connected by protoplasmic fibrils, growth involving an interealation of new particles between the old ones. Hansteen-Cranner believes the wall to be a colloidal network of celluloses and hemicelluloses with extensions of the plasma membrane in the meshes. In the cellulose walls of a number of meristems Wood (1926) found no more than 0.001 per cent of protein matter, which is less than would be expected if the wall contained significant amounts of protoplasm.

Ultramicroscopic Structure of the Wall.-In connection with this subject, which has recently come into prominence anew, it is of interest to note the prophetic nature of certain early theories. ${ }^{19}$ Von Mohl advanced the view that the cell wall grows by the deposition of material in successive laminæ ("apposition theory"). On the other hand, Nïgeli, as a result of his researches on starch grains and the wall, concluded that the wall is composed of molecular complexes called micellce surrounded by water films and that growth involves the intercalation of new micellæ

${ }^{17}$ See van Wisselingh (1924) and literature there cited; also Mameli (1920), Tiffany (1923), Wurdack (1923), and Thomas (1928).

${ }^{18}$ Wiesner (1886 et seq.), Molisch (1888), Hansteen-Cranner (1919, 1922, 1926).

${ }^{19}$ Von Mohl (1853, 1858), Nägeli (1858, 1863, 1864), Dippel (1879), Ḱrabbe (1887), Strasburger (1882, 1889), Wiesner (1886, 1890, 1892), Correns (1889, 1892, 1893). 
between the old ones ("intussusception theory"). Similar to this was the later view of Wiesner, mentioned in the preceding paragraph. Strasburger held that the units are not micellæ but single molecules linked together in a reticular framework by their chemical affinities. He attributed growth in area to simple stretching without intercalation of particles, and growth in thickness to apposition of small "microsomes." The longitudinal or spiral striations observed in many walls were thought by some to indicate a linear arrangement of particles, while the fine lamination of the wall was attributed either to unlike orientations in contiguous laminæ or to differences in water content.

The recent advances in our knowledge of the finer structure of the cell wall have been made largely through studies of diffraction patterns produced when $\mathrm{X}$-rays ${ }^{20}$ are passed through cellulose and of the effects obtained with polarized light. ${ }^{21}$ These studies, notably those of Sponsler taken together with the chemical researches of Irvine, have shown that the primary structural unit in the wall is an anhydrous glucose residue with the formula $\mathrm{C}_{6} \mathrm{H}_{10} \mathrm{O}_{5}$. Such units are bound together in cellulose chains by primary valencies, while the chains are in turn linked laterally by secondary valencies in such a way that the units form a regular three-dimensional space lattice (Fig. 104). In other words, the wall has a crystalline structure. In the fibers of ramie (Bxhmeria nivea) and the Valonia cell wall, the distance between the centers of adjacent units is calculated to be about $5.15 \AA$. longitudinally, $6.1 \AA$. tangentially, and $5.4 \AA$. radially (Sponsler). There is also evidence that the chains are aggregated into bundles, or colloidal micellce, these probably being

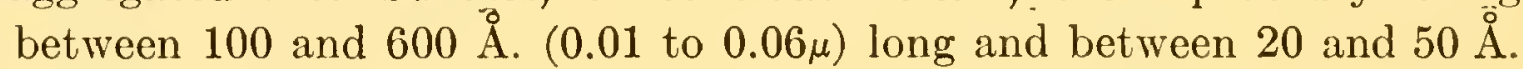
$(0.002$ to $0.005 \mu)$ thick. The nature of the matter between these micellæ, or "crystallites," is unknown.

In a given visible layer of the wall there are many layers of primary units, and the chains they form may be arranged parallel to the longitudinal axis of the cell (ramie), or more or less obliquely (cotton). In Valonia there are two main sets of chains which usually cross each other at an angle of about $80^{\circ}$. This structure extends through the manylayered wall. The chains are found to be parallel to the fine striæ visible on the surface of the wall. There are many facts which indicate that it is not the surface upon which a layer is deposited that determines the arrangement of its elements. It is reported that in the cotton fiber the crystalline pattern becomes more perfect as the fiber matures (Clark et al.). The micellæ evidently come to be much closer together when a

${ }^{20}$ Herzog and Jancke (1919-1920), Sponsler (1925ab, 1926, 1928ab, 1929, 1931; general account, 1933), Sponsler and Dore (1926, 1928), Bragg (1930), Clark, Pickett, and Farr (1930), Astbury, Marwick, and Bernal (1932). Sce Frey (1926a), Meyer and Mark (1928), and Seifriz (1929b).

${ }^{21}$ Balls (1923), Frey (1926b, 1927, 1928), Preston (1931), D. B. Anderson (1927), Mitchell (1930), M. Shaw (1929). 
fiber is dried and farther apart when it is swollen (Frey). Presumably the valencies binding the units into chains offer greater resistance to swelling forces than do those uniting the chains laterally; hence a cellulose fiber swells unequally in different directions. Sponsler and Dore have shown that mercerization involves a lateral shift of the chains in the wall and a partial rotation of their units, together with certain other changes. The precise nature of the structural changes occurring as the
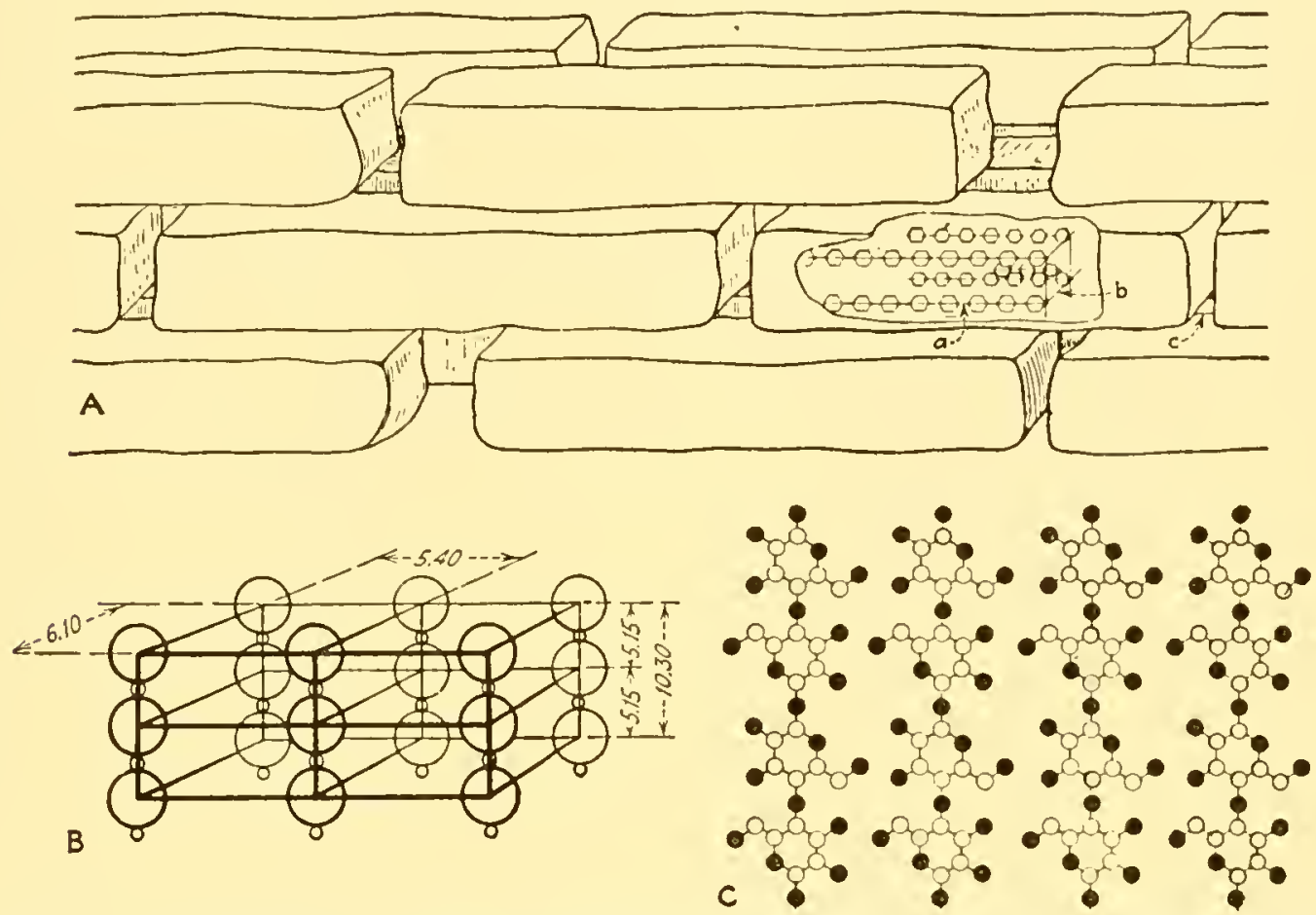

FIG. 104.-Diagrams of cell-wall structure as indicated by X-ray analysis. $A$, large micellæ with chains of glucose residue units showing in one of them. $a$, primary valence forces; $b$, secondary association forces; $c$, tertiary micellar forces. (After Seifriz, 1929b.) $B$, minute portion of cell wall in perspective, showing portions of six chains, each consisting of three glucose residues (large circles) and their connecting oxygen atoms (small circles). (After Sponsler, 1931.) C, inner surface of wall; four chain molecules with four glucose units shown in each. Black circles and white circles represent oxygen atoms and carbon atoms, respectively; hydrogen atoms not shown. (After Sponsler, 1929.)

wall grows in area and becomes impregnated with other substances, such as lignin, remains to be determined.

It is a suggestive fact, as pointed out by Sponsler, that about the only solid materials deposited in large amounts by plant protoplasm, namely, cellulose and starch, consist of $\mathrm{C}_{6} \mathrm{H}_{10} \mathrm{O}_{5}$ units arranged in patterns. There is similar evidence that certain animal products, such as chitin, hair, and silk, have their molecules arranged in regular patterns. ${ }^{22}$ It is possible that this is due not only to the chemical properties of protoplasm and the forces of crystallization but also to certain structural features in protoplasm itself. Seifriz (1929b) has emphasized the idea that the contractility, cohesiveness, elasticity, and tensile strength of protoplasm,

${ }^{22}$ Herzog and Jancke (1921), Brill (1923), Astbury and Street (1931), Astbury and Woods (1932). 
as well as such phenomena as conductance and polarity, are best explained on the assumption that protoplasm itself possesses structural units arranged in linear series, especially since certain other materials exhibiting such properties are known to have such a structure (see Beutner, 1933).

The Walls of Spores.-In bryophytes and vascular plants the walls of spores are developed in two general ways: (1) by the successive formation of layers within the original membrane by the protoplast, often with the addition of material from the anther fluid, and (2) by the deposition of material on the outside of the original membrane by a plasmodium formed by tapetal cells.

In the first method a more or less temporary gelatinous layer is ordinarily developed around each of the young spores, either before or after these have separated from one another (Fig. 96). Upon the inner surface of this special layer the protoplast deposits the exine, or outer wall layer. ${ }^{23}$ In many cases this is at first a homogeneous layer that soon differentiates into an outer lamella and an inner zone with net-like thickenings and spines (the "mesospore"). Finally, there is deposited an inner layer, or intine. This mode of development is widely prevalent, the walls of most spores showing two principal layers, or "coats": an intine, which later becomes greatly extended to form the pollen tube in seed plants, and an exine, which is characteristically thickened and sculptured. In angiosperm pollen the exine has one or more definitely differentiated germ pores through which pollen tubes are to emerge. The characters of the resistant exine are of much value in determining the relationships of living and fossil plants. ${ }^{24}$ The exine, although it begins to differentiate in contact with the protoplast, may continue to thicken and develop its peculiar markings after it has been separated from the protoplast by other layers. ${ }^{25}$ In an extensive study of the composition of pollen-grain walls Biourge (1892) showed that they contain cutin, callose, cellulose, and pectic substances, singly or in various combinations.

The highly specialized coats of the megaspore of Selaginella have been repeatedly studied. In Selaginella rupestris ${ }^{26}$ the coats begin to differentiate in the midst of a thick gelatinous layer developed at the close of the divisions delimiting the spores. The exospore first appears as a double zone, the outer part of which becomes the perinium (Fig. 105) The small protoplast now expands and pushes outward the undifferentiated inner portion of the gelatinous layer, and, as it does so, a second coat, the endospore, is formed at its surface. In S. emiliana the exospore and endospore develop simultaneously. Lyon finds two coats in place

${ }^{23}$ E.g., I pomœa purpurea (Beer, 1911).

${ }^{24}$ E.g., Wodehouse (1928 et seq.) on living plants. For references on fossil pollen, see Erdtman $(1927,1930)$ and Sears (1932).

${ }^{25}$ Beer (1905, 1911), Tischler (1908).

${ }^{26}$ Lyon (1905). The accounts of Fitting $(1900,1906)$ differ from this in certain details. 
of the three reported by Fitting but points out that a portion of the gelatinous layer may remain undifferentiated until a late stage and thus appear like a third coat.

In vascular plants the sporangium (the anther in angiosperms) is lined by a layer of special nutritive cells known as the tapetum. In many known cases, including Equisetum, Botrychium, Marsilia, and a number of angiosperms, the boundaries of these cells break down, allowing the protoplasts to coalesce and form a "tapetal plasmodium," or "periplasmodium." This flows in among the immature spores and contributes to the development of their coats (Fig. 106). In Equisetum the spore has three coats: an endospore, an exospore, and a perispore, the last being formed by the plasmodium and consisting of several layers. Upon the

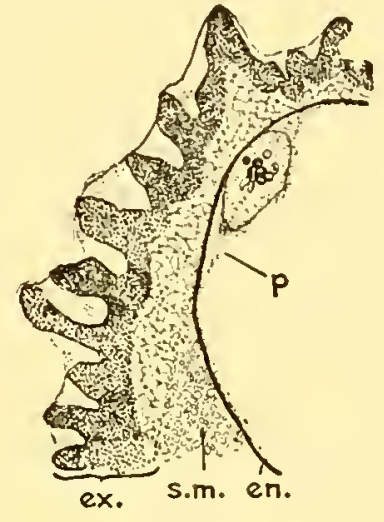

FIG. 105.

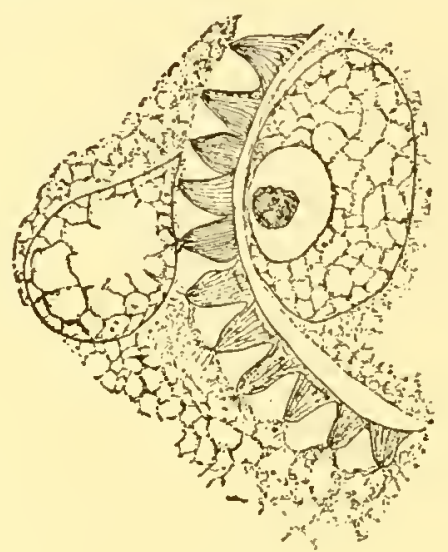

FIG. 106.

FIG. 105.-Developing megaspore coats of Selaginella rupestris. $p$, protoplast with nucleus; en, endospore; s.m., undifferentiated portion of gelatinous layer; $c x$. , exospore, of which the denser portion is the "perinium." (After Lyon, 1905.)

FIG. 106.-Exine of microspore (right) developing in contact with tapetal plasmodium (left) in Commelina. (After Tischler, 1915.)

original membrane the plasmodium deposits successively an inner gelatinous layer, a second layer, an outer gelatinous layer, and finally a layer which later splits up to form the characteristic appendages of the spore. These four layers together constitute the perispore. While they are in the process of formation the original spore membrane becomes transformed into the exospore, and within it the endospore is developed last of all. ${ }^{27}$

In the ascomycete Hydnotrya Tulasnei each spore, after its delimitation from the cytoplasm of the ascus (p. 158), is surrounded by a thin endospore. In the ascus cytoplasm near each spore there appears a rounded mass of fluid, which enlarges, gradually surrounds the spore, and develops into the elaborate exospore. In this case, therefore, the wall material is visible before its deposition on the spore (Nermec, 1929d).

The Cell Membranes of Animals. - The problem of the intimate relation of protoplasm to partitions which subdivide it into cells is encoun-

${ }^{27}$ Beer (1909b), Hannig (1911). 
tered in animals as well as in plants. Zoölogists have differed widely in their interpretations of the "intercellular substance" composing such membranes in animal tissues. Heidenhain and Rohde have emphasized the view that this substance is metaplasm, a special form of living substance which differentiates in protoplasm in connection with special functions and which is capable of growth, response to certain stimuli, and further differentiation. A. Meyer has strongly opposed this conception, holding that the albuminous intercellular substance of animals, like the carbohydrate wall of plants, is ergastic in nature and not to be classed with metaplasmic (alloplasmatic; Meyer) differentiations. The fibrils frequently observed in the intercellular substance, which many have taken as indications of its metaplasmic nature, Meyer regards as modifications of the ergastic material or as substances which have arisen in protoplasmic connections. ${ }^{28}$

The membranes of animal cells differ from those of most plants in consisting largely of such substances as chitin, elastin, keratin, and gelatin rather than cellulose and related carbohydrates. Cellulose has been found only rarely among animals. In the ascidians, as has tong been known, the outer layer of the body wall consists largely of cellulose. A cellulose-like substance has recently been found in the skeletal plates of an infusorian (Dogiel, 1923a).

${ }^{28}$ Heidenhain (1902, 1907), Rohde (1908, 1914, 1923), Meyer (1896, 1920); see also p. 45. See the general account by Studniěka (1925). 


\section{CHAPTER XIII}

\section{ATYPICAL MITOSIS AND OTHER NUCLEAR PHENOMENA}

In the foregoing chapters attention has been restricted largely to typical nuclei and nuclear division as these are observed in the great majority of plant and animal tissues. In this chapter will be reviewed briefly certain additional modes of division and other phenomena which, though in many instances abnormal, have served to broaden our knowledge of the nucleus and its significance.

Amitosis.-In amitotic, or direct, nuclear division the nucleus simply constricts and separates into two portions while in the metabolic condition, no condensed chromosomes or achromatic figure being formed (Fig. 107). When the portions are very unequal in size, the process is called "nuclear budding"; when they are more than two in number it is referred to as "nuclear fragmentation." Such nuclear divisions are not followed by cytokinesis; the cells thus come to have two or more nuclei. Amitosis was at one time looked upon as the prevailing mode of nuclear division, mitosis even being somewhat exceptional, but the true state of affairs, so far as higher organisms are concerned, has turned out to be quite the reverse, mitosis occurring almost universally and true amitosis in comparatively few well-authenticated cases. It is now evident that binucleate cells, constricted or fusing nuclei, and especially the results of aberrant mitosis have often been hastily construed as amitotic division, and it is in

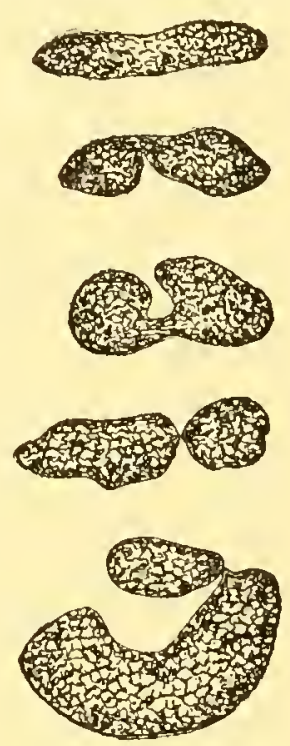

F1G.107.Amitosis in internode of Chara. the light of this fact that many reports of amitosis should be read. ${ }^{1}$

The amitotic phenomena so frequently reported in cells with a distinctly nutritive function, such as certain gland cells of animals and tapetal, antipodal, and endosperm cells of angiosperms, have been thought to substantiate the widely adopted hypothesis that amitosis aids in metabolism by increasing the nuclear surface. The supposed amitosis in tapetal cells has been shown in a number of instances to be aberrant mitosis (Bonnet), but Tischler considers it probable that true

${ }^{1}$ Bonnet (1912), Beer and Arber (1919), Schürhoff (1920), Kater (1927a). For reviews of the literature pertaining to amitosis and irregular mitosis in plants, see Tischler (1921-1922, Chap. VII) and Schürhoff (1926). For the literature on amitosis in animals, see Conklin (1917), Nakahara (1918b), and Wilson (1925, p. 214). 
amitosis as well as deranged mitosis and nuclear fusion may sometimes occur in such cells and the periplasmodia they form.

Another very prevalent opinion regarding the significance of amitosis is that expressed by Flemming (1891), namely, that the process is primarily a degenerative phenomenon, since it is so frequently observed in pathological tissues. In the words of vom Rath (1891), "when once a cell [nucleus] has undergone amitotic division it may indeed continue to divide for a time by amitosis, but inevitably perishes in the end." That this interpretation cannot be of universal application has been contended by those who have found amitosis or amitosis-like appearances in cells which show no other sign of degeneration. ${ }^{2}$ It is probably significant, however, that most if not all of the observed cases of amitosis in living tissues have occurred in cultures which were growing old or showing distinct signs of degeneration, or in which the medium was unfavorable for normal growth. ${ }^{3}$ It is the opinion of Kofoid (1923) that the supposed amitosis in Protozoa is a degenerative or pathological phenomenon, or in some cases a special form of mitosis which has been incorrectly interpreted. Conklin (1917) was able to show that the amitosis-like appearances in Crepidula eggs and embryos were all instances of abnormal mitosis and cytokinesis. He observed that the chromosomes might scatter and fail to unite in a single nucleus; or mitosis might occur without cytokinesis, giving cells with two or more nuclei; or certain chromosomes might fail to separate in the anaphase, leaving a bridge between the sister nuclei; or the nuclear membrane might persist throughout mitosis and finally constrict at the middle. As a result of his many observations and an examination of the evidence offered by others he concluded that there is not a single case of true amitosis known in normally differentiating cells.

The literature contains conflicting opinions on the question of whether or not a portion of a nucleus resulting from amitosis can subsequently divide by mitosis. The observation of amitosis under abnormal conditions followed by mitosis after the tissue has been restored to a normal environment is not conclusive evidence on this point unless it is shown that the mitotically dividing nucleus is actually a product of amitosis. Chambers (1917a) observed that after a nucleus is pinched in two with the micromanipulator the parts may reunite and undergo mitosis. In wound tissue of the stem of Tradescantia, Conard $(1926 a, 1928)$ has shown that markedly lobed nuclei may enter mitosis while in this form, also that they sometimes become constricted into two separate portions while in the metabolic or prophase stage. Two such portions may then

2 Des Cilleuls (1914) on rabbit cells; Saguchi (1917) and Helvestine (1921) on ciliated cells of animals; Bast (1921) on bone; Kisser (1922), Conard (1926a, 1928) and others on vegetative tissues of plants; F. E. V. Smith (1923) on Saprolegnia.

${ }^{3}$ Lewis and Lewis (1924), Drew (1923). 
undergo mitosis side by side, the two spindles often uniting to form only two daughter nuclei. Examination of the chromosomes in such pairs of mitotic figures shows that each nuclear portion after amitosis has only a portion of the full complement, and that certain chromosomes have been fragmented in the amitotic division. The two portions together constitute a complete complement. Since some fragments may pass to the poles along with the unbroken chromosomes, the union of the mitotic figures brings about a partial restoration of the original nuclear organization.

These phenomena suggest that the common failure of amitotic nuclear fragments to undergo mitosis is due not so much to the effects of amitosis itself as to a persistence of the abnormal conditions which originally induced amitotic division. Were such nuclei to continue dividing in a growing and differentiating tissue, derangements in their nuclear organization might well be reflected in abnormalities in the results of differentiation; and were descendants of such nuclei to be transmitted through gametes to the next generation, abnormalities in development would be expected there also. The basis for such an expectation is seen in the known results of chromosome fragmentation and translocation to be described in Chapter XVIII. At present it has not been shown in any case that the descendants of true amitotic nuclei ever become the nuclei of functional gametes.

Induced Aberrations of Mitosis.-Aberrations of mitosis like those so often mistaken for amitosis may be induced by a considerable variety of means. For example, when root tips and other plant tissues are subjected to the action of chloral hydrate and other anesthetizing agents, ${ }^{4}$ the normal course of mitosis is disturbed in various degrees, depending on the strength of the dose. As a rule the achromatic figure develops poorly or not at all, and the chromosomes become irregularly scattered in the cell (Fig. 108). They may form one or more groups and reorganize nuclei which often remain connected by bridges or undergo fusion. Hence it is not uncommon to find in such tissues all gradations between normal mitosis and what looks like amitosis. The latter condition is known as "pseudoamitosis" (Haecker). Němec and Sakamura attribute such scattering of chromosomes to protoplasmic streaming induced by the experimental agency; this is in harmony with G. Ritter's (1911) observation that the nucleus may sometimes be displaced by streaming induced by the wounding of near-by cells. The experiments of Van Regemorter indicate that the failure of the spindle substance to develop a regular figure in chloralized tissue is due to a destruction or impairment of the cell's polarity, the recovery from the effect of the reagent involving a return to the properly polarized condition.

${ }^{4}$ Pfeffer (1899), Nathansohn (1900a), Němec (1904, 1910a, 1929c), Kémp (1910), Sakamura (1920), Van Regemorter (1926). The effects of many other agents are deseribed by Yamaha (1927ab). 
Abnormal temperatures, either high or low, are known to cause aberrations of mitosis in somatic cells and microsporocytes. ${ }^{5}$ Such effects are responsible for the origin of certain races of plants with altered chromosome numbers, as will be shown in Chapter XX.

Wounding, such as that occasioned by grafting or decapitation, of ten disturbs the mitotic process, cells or even branches with altered chromo-

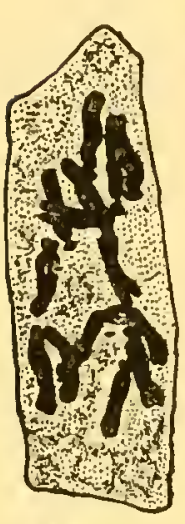

A

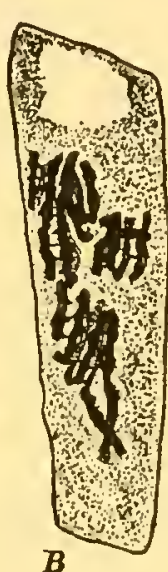

B
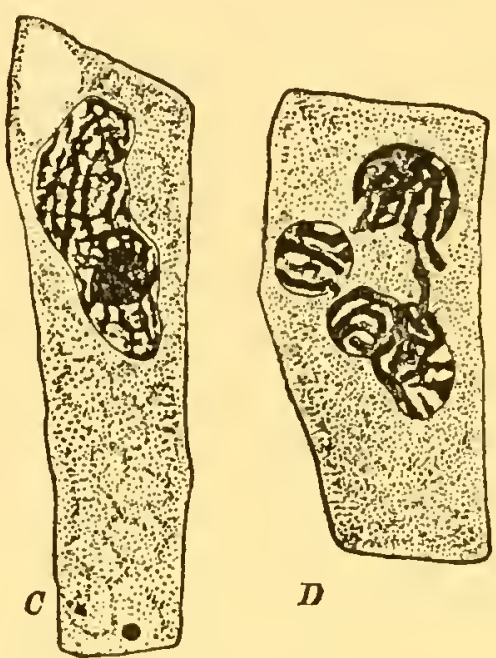

$D$
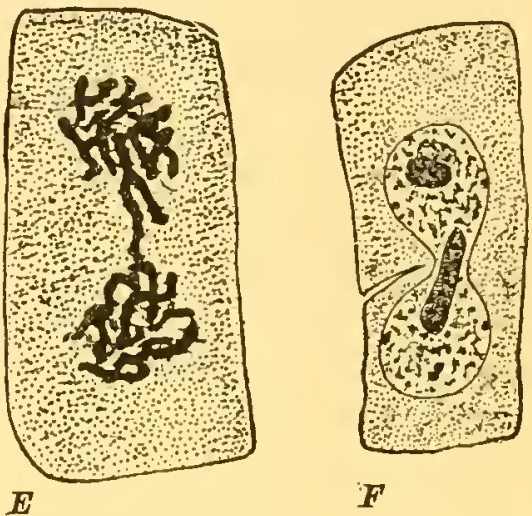

$\boldsymbol{F}$

FIg. 108.-Abnormal mitosis in chloralized root cells of Vicia. A, irregular distribution of chromosomes. $B$, scattered chromosomes beginning to assume nuclear form. $C$, nucleus reconstructed by scattered chromosomes. $D$, chromosomes reconstructing three separate nuclei instead of one. $E$, chromosomes reconstructing two nuclei connected by a bridge. $F$, amitosis-like appearance resulting from condition shown in $E$. (After Sakamura, 1920.)

some numbers sometimes resulting. ${ }^{6}$ Abnormalities also occur in the presence of parasites in gall tissue. ${ }^{7}$

The effects produced by radiations of high frequency ${ }^{8}$ are of special interest in view of their employment in attempts to analyze the functional differentiations of the chromosomes (Chapters XVIII and XX). Among such radiations the $\mathrm{X}$-rays have been studied most. The cytoplasm of cells treated with these rays may show alterations in viscosity ${ }^{9}$ and rate of streaming, ${ }^{10}$ as well as certain degenerative aspects, such as turbidity and

${ }^{5}$ Borgenstam (1922) on Syringa, Belling (1925a) on Uvularia, Sakamura and Stow (1926) on Gagea, Stow $(1926,1927)$ and Fukuda (1927) on Solanum, de Mol $(1927 c$, $1928 a-d)$ on Hyacinthus and Tulipa, Rybin (1927b) on Nicotiana, Shimotomai (1927) on Scilla, Michaelis (1926, 1928) on Epilobium and Enothera, Bleier (1930b) on Triticum, Koshuchow (1928) on Zea and Cucumis. Bleier (1931c) questions the evidence for the rôle of temperature in causing natural sterility in Solanum.

${ }^{6}$ Winkler (1916) and Jørgrensen (1928) on Solanum, Kostoff (1930c, 1931ce) and Kostoff and Kendall (1931a) on Nicotiana, Lindstrom and Koos (1931) on tomato.

${ }^{7}$ Winge $(1927 d)$ on crown gall of Beta; Němec $(1924,1926)$ and Kostoff and Kendall $(1929 a b, 1930 a b)$ on galls of other types.

${ }^{8}$ Certain biological effects of such radiations are reviewed by Packard (1931). Bersa (1926) gives a bibliography. See Popoff (1931) on cell stimulation.

${ }^{9}$ Fairbrother (1928), Wels (1924), Jansson (1927), Nadson (1925).

${ }^{10}$ M. Williams (1923, 1925a), Zuelzer and Philipp (1925). 
vacuolation. ${ }^{11}$ The chromatic matter of the nucleus may contract into a deeply staining mass (pyknosis), diffuse in the karyolymph (karyolysis), or adhere to the membrane. Nuclei about to divide are prevented from doing so, but those in division usually continue or complete the process. Mitosis is often very aberrant, ${ }^{12}$ in many respects resembling that seen in chloralized cells (Fig. 109). The chromosomes are irregularly dis-

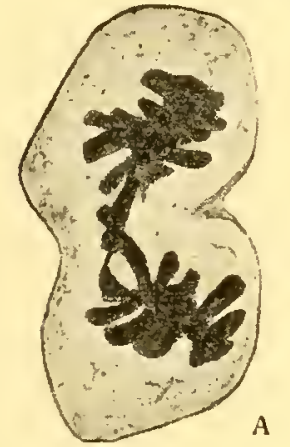

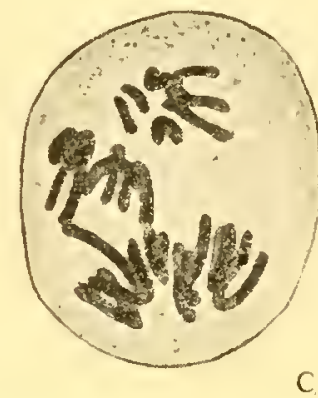

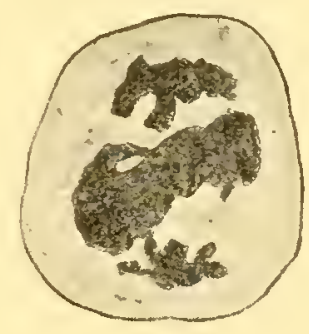

D

FIG. 109. - The effect of Röntgen rays on mitosis in cornea cells of Salamandra. A, chromatic bridge. $B$, "pseudoamitosis." $C$, distribution of chromosomes to three centers. $D$, partial pyknosis; some of the chromosomes have passed to the poles. (After Alberti and Politzer, 1923.)

tributed, hence nuclei and cells with polyploid or otherwise altered chromosome complements are frequently observed in irradiated tissues. The reported occurrence of just such phenomena in ordinary and artificially induced tumors ${ }^{13}$ has excited much interest in connection with the cancer problem, ${ }^{14}$ but at present very little can be said concerning causal relationships.

Of great interest are the more definitely localized effects produced by irradiation with X-rays. ${ }^{15}$ It is found that with proper dosages a

${ }^{11}$ Nadson (1925), Bisceglie and Bucciardi (1929).

${ }^{12}$ E.g., Komuro (1917 et seq.), Pekarek (1927), and Patten and Wigoder (1930) on Vicia roots; Alberti and Politzer (1923, 1924) and Politzer (1925) on cornea cells; Strangeways and Oakley (1923), Strangeways and Hopwood (1926), and Kemp and Juul (1930) on tissue cultures; Pauli and Hartmann (1924) and Pauli and Politzer (1929) on eathode rays; and Bersa (1927) on Zea roots.

${ }^{13}$ In animals: Howard and Schultz (1910), Boveri (1914a), Bichler (1914), Yamagiwa and Iehikawa (1915 et seq.), Lewis and Loekwood (1929), Winge (1930a), Levine (1931, good bibliography), and others.

In plants: Komuro (1922, 1924de, 1925abc, 1928abc, 1930ac), Winge (1927d), Levine (1929), Goldschmidt and Fisher (1929).

${ }^{14}$ See the reviews by Ludford (1925) and Levine (1931).

${ }^{15}$ H. J. Muller (1926, 1927, 1928abc, 1929bc, 1930abc), Muller and Painter (1929), Painter and Muller (1929), Muller and Altenburg (1930), J. T. Patterson (1928, 1929, 1930ab, 1931a), Patterson and Painter (1931), Mavor (1921, 1922, 1923abc, 1925), Hanson (1928), Dobzhansky (1929abcd, 1930ad, 1931ab). The foregoing are on Drosophila. For general accounts, see Muller $(1929 c, 1930 b)$. In plants: Stadler (1928abc, 1929, 1930abc, 1931ab, 1932), Goodspeed and Olson (1928ab), Goodspeed $(1929 a b, 1930 a b c, 1932)$, Goodspeed and Avery (1930), de Mol (1930b, 1931abc), M. Nawasehin (1931c), Delaunay (1930), Randolph (1932), Lervitsky and Araratian (1931). For a general statement, see Stadler (1930b). 
single chromosome only, or even but a small portion of it, may be eliminated or changed. Often a portion of a chromosome becomes detached from the remainder (fragmentation), or attached to another chromosome (translocation), or reversed in position (inversion), or rendered functionless (inactivation), or removed altogether (deletion). These and other alterations, including changes in individual genes, may be brought about not only during certain phases of mitosis but also when the nuclei are in the metabolic condition, as in pollen and dormant seeds. This affords strong support to the view that some characteristic organization is maintained by each chromosome at all stages of the mitotic cycle. Treatments with ultra-violet radiations ${ }^{16}$ and the rays emitted from radium ${ }^{17}$ have also been found to cause alterations of the protoplasm and of nuclear behavior, some of which are comparable to those produced by X-rays. As will appear in later chapters, it is such specific effects of irradiation, particularly those obtained with $\mathrm{X}$-rays, which give the method a high value in modern cytogenetic analysis.

The discovery of "mitogenetic rays" emitted by growing tissues was announced in 1923 by Gurwitsch, ${ }^{18}$ who reported that when a growing onion root (as "sender" of the rays) is pointed directly at the region of cell-division in another root (as "detector") and left for some hours, the detector shows more mitoses in the side toward the sender, the excess over the other side being as much as 50 per cent. Metabolically active tissues in considerable variety have since been reported to emit such rays, and growing yeast cultures have come to be favorite detectors. Attempts to determine the nature of the rays have led to the belief that they are ultra-violet radiations of very low energy content, and that they may possibly be associated with oxidation.

The value of the evidence for the existence of such rays, or for a causal relation between them and the effects observed, has been adversely criticized by a number of investigators. ${ }^{19}$ Reasons for discrepancies in the results of various trials are beginning to be apparent in differences in technique and in the condition of the materials used. At present it appears probable that radiations produced by processes occurring in ${ }^{16}$ Takamine (1923), Sehleip (1923), Addoms (1927), Just (1933), Heilbrunn and Young (1930), Bucholtz (1931).

${ }_{17}$ Mohr (1919), M. Williams (1925a), Gager and Blakeslee (1927), Canti and Spear (1927, 1929), Hanson and Heys $(1928,1929)$, Hanson and Winkleman (1929), Levine (1929), Stein (1929), Stadler (1928b, 1931b), Stoel (1928), Goodspeed (1929b), Goodspeed and Avery (1930).

${ }^{18}$ For general accounts, see Gurwitseh $(1925,1926,1929,1932)$, Reiter and Gábor (1928), and Hollaender and Schoeffel (1931). See also Wagner (1927, 1928), Magrou and Magrou (1927), Baron (1928, 1930), Reiter and Gábor (1929), Frank (1929), Siebert (1928), Borodin (1930), Kisliak-Statkewitsch (1927), Loos (1930), Ferguson and Rahn (1933), Tuthill and Rahn (1933).

${ }^{19}$ Guttenberg (1928), W. Schwarz (1928), Rossmann (1929), Taylor and Harvey (1931), Richards and Taylor (1932), Moissejewa (1931). 
tissues are an important cause of certain phenomena of cell growth and multiplication.

Karyosome Nuclei.-Numerous peculiar types of nuclear organization and behavior are known in the lower organisms, notably among flagellates and Protozoa. ${ }^{20}$ Of these types one of the most prevalent is
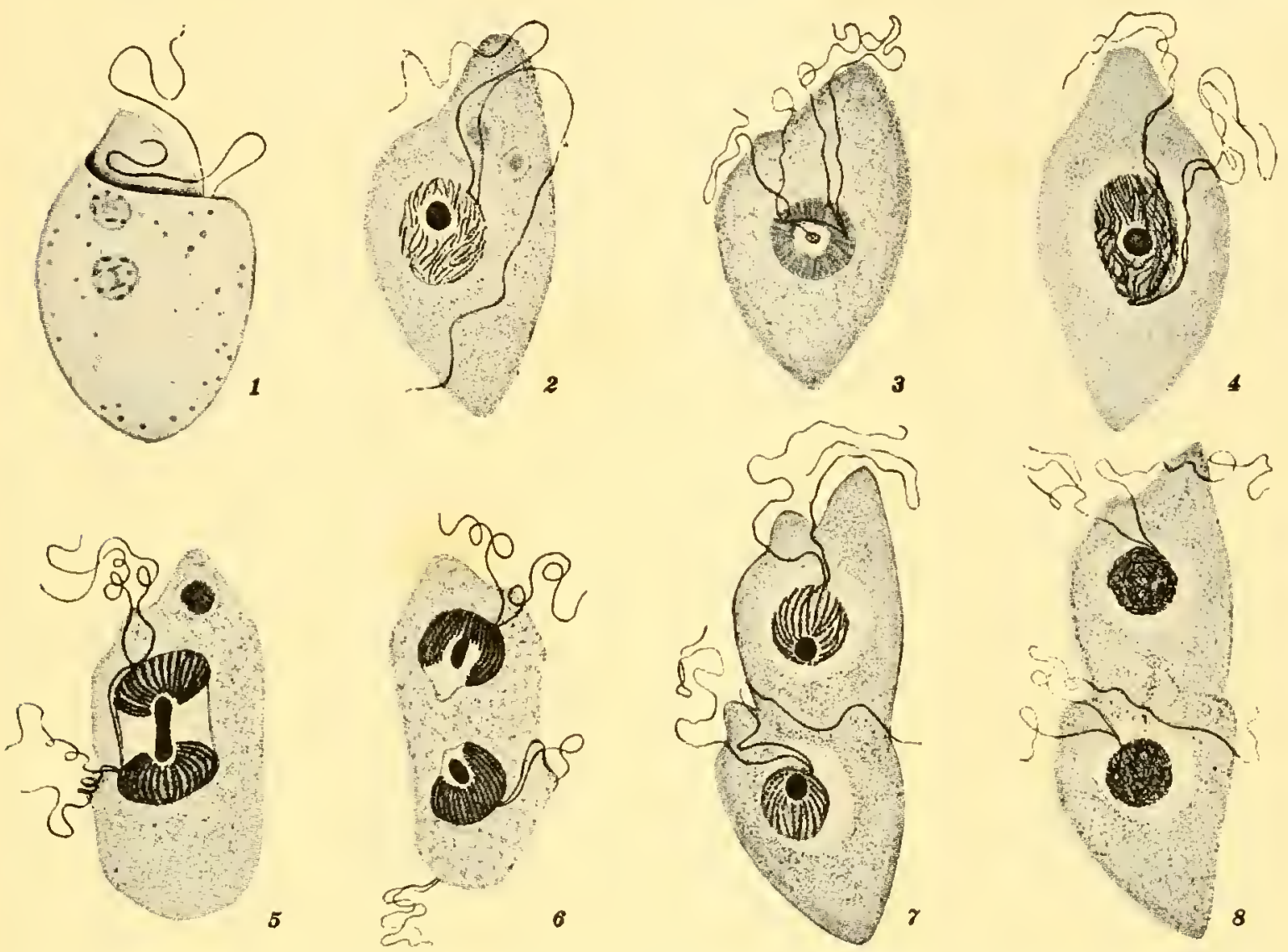

Fig. 110.-Mitosis in Oxyrrhis marina. 1, living individual, showing two flagella and two food bodies. 2,3 , and 4 show the paradesmose between the diverging centrioles. 5 , endosome elongated and about to divide. 6 , early telophase. $\gamma, 8$, fission in progress. (After Hall, 1925a.)

that in which the nucreus contains a large, deeply staining body known as the karyosome, or endosome. ${ }^{21}$ It may contain the only highly chromatic substance in the nucleus at certain stages, but critical studies have shown that the chromosomes, like those in ordinary nuclei, develop in the surrounding region (Figs. 110, 111). The karyosome usually reacts negatively in the Feulgen test, ${ }^{22}$ but it may still be concerned in the changes in chromaticity of the chromosomes (cf. p. 119). Ordinarily

${ }^{20}$ For general accounts of the nuclei of Protista, see M. Hartmann (1911), Minchin (1912), Doflein (1916), Calkins (1926) and Bělař (1926).

${ }^{21}$ Minchin's term endosome is probably used more widely now than karyosome (Hartmann). Other terms are Binnenkörper (Doflein) and nucleolo-centrosome (Keuten). Much of the confusion in the older literature on this subject has been clarified by recent work; see especially Bělař (1926), Hall (1925a) and Hall and Powell (1928).

${ }^{22}$ Reichenow (1928), Roskin and Romanova (1928). 
the karyosome elongates and divides by constriction at the time of nuclear division. Often it forms a dumb-bell-shaped structure, about the middle of which the chromatic granules or chromosomes are grouped and separated into two masses as the nuclear membrane remains intact (Fig. 111). Frequently it does not divide until the elongated chromosomes have reached the poles. The chromosomes lying parallel to the axis of the division figure may appear to break in two at the middle, but it has been shown by Hall and others that in the prophase they are split and opened out from one end, the supposed transverse division
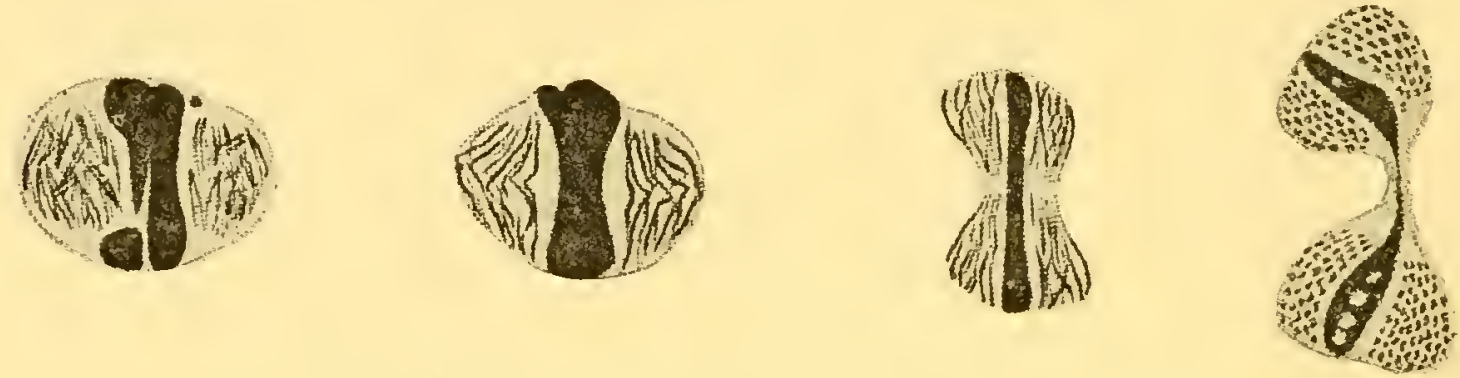

FIG. 111.-Mitosis in Heteronema acus. (After Loefer, 1931.)

being merely the completion of the separation at the other end. ${ }^{23}$ It is claimed that in Euglena the split is present in the preceding telophase. ${ }^{24}$ While these changes are in progress the centrosome and motor elements, if present, become doubled in number in various ways to form the corresponding elements of the daughter cells ${ }^{25}$ (see p. 223).

In the Plasmodiophorales ${ }^{26}$ the chromosomes form a ring at the equator of the mitotic figure; as this ring divides and separates to the two poles, the karyosome it encompasses does likewise. In the green alga Cladophora glomerata, the nucleolus elongates and divides as the split chromosomes separate (Fig. 112). The nuclear membrane remains intact throughout mitosis, and at certain stages the whole figure bears a striking resemblance to those described above. ${ }^{27}$ The behavior of the nucleolus

${ }^{23}$ R. P. Hall on Menoidium (1923), Oxyrrhis (1925a) and Ceratium (1925b); Hall and Powell (1928) on Peranema; Loefer (1931) on Heteronema.

${ }^{24}$ Tschenzoff (1916), Tannreuther (1923), Ratcliffe (1927).

${ }^{25}$ Kofoid and Christiansen (1915), Kofoid and Swezy (1915 et seq.), Swezy (1915, 1916, 1922), Wenrich (1921), Boeck (1917), Hall (1923, 1925ab), Bunting and Wenrieh (1929), Kater (1929).

${ }^{26}$ S. Nawaschin (1899b), Winge (1912) on Sorodiscus, Cook (1928) on Ligniera, Horne (1930) on Spongospora, Milovidov (1931) on Plasmodiophora. For other aecounts of mitosis in myxomycetes, see Strasburger (1884a), Harper (1900a), Jahn (1904, 1908, 1911), Olive (1907), Prowazek (1905), Krränzlin (1907), and Skupieński (1928).

${ }^{27} \mathrm{~T}$ 'Serclaes (1922). For other recent accounts of mitosis in green algæ, see Czurda (1922ab) and Geitler (1930) on Spirogyra, Föyn (1929) on Cladophora and Ulva, Hartmann (1921) on Eudorina, Higgins (1930) and Sehussnig (1930) on Cladophora, Kretschmer (1930) and Ohashi (1930) on Edogonium, Mundie (1929) on Vaucheria, Peterschilka (1922) on Mougeotia, Schussnig (1928ab) on Caulerpa, and M. Williams $(1925 b, 1926)$ on Codium and Vaucheria. 
here tends to be somewhat irregular; in related types it disappears in the prophase, as in higher plants. ${ }^{28}$

Such a range of structural conditions as that reviewed in the foregoing paragraphs suggests that karyosome nuclei differ from the ordinary nuclei of higher organisms chiefly in the amount of nucleolar matter present and in its tendency to be associated with other elements, notably centrosomes. In higher plants the nucleolar matter, which has some structural and functional relationship with the chromosomes (p. 118), ordinarily disappears before the metaphase, but, if any remains, it may sometimes undergo division in the mitotic figure (p. 164). In such forms as Cladophora the latter mode of behavior occurs with some regularity. In still

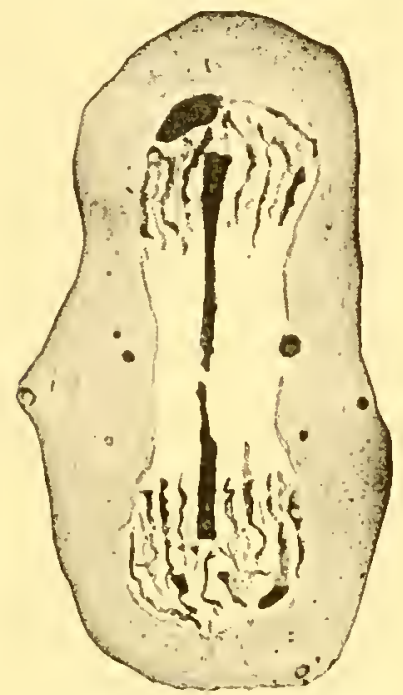

FIG. 112.-Mitosis in Cladophora glomerata. (After T'Serclaes, 1922.)
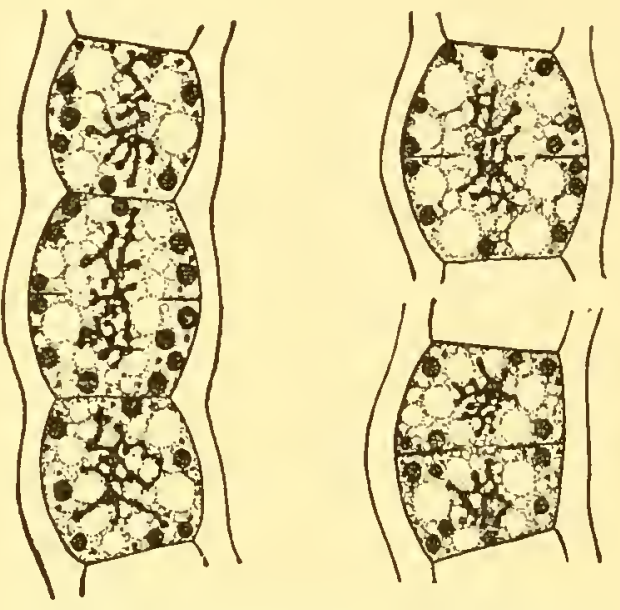

FIg. 113.-Stages of cell-division in Anabeena circinalis. (After Haupt, 1923.)

other forms centrosomal elements are present also. How such conditions have come about is entirely a matter of conjecture. It is not to be assumed, however, that all the cytological differentiations in Protista can be fully explained by reference to those in higher organisins.

With regard to their chromosomal mechanism, it becomes increasingly evident as critical researches multiply that many of the Protista are in certain essential features like higher forms. Both Metcalf (1915) and Kofoid $(1915,1923)$ have emphasized the fundamental similarity of protozoan and metazoan nuclei. In some representatives of all the main groups of Protozoa, elongated chromosomes, which split and show evidence of being made up like those of Metazoa, have been found. Kofoid states that the chromosomes are constant in number and differ in size and shape in certain flagellates. There are genetic data which indicate that, so far as their life cycles show agreement, Protozoa and Metazoa exhibit similar modes of inheritance (see Jennings, 1920). In many Protista, on the contrary, this high degree of nuclear differentiation seems

${ }^{28}$ Němec $(1910 b)$ on the varicty simplicior; Carter (1919c) on Rhizoclonium. 
not to have been reached. To what extent the apparently simpler types of nuclear organization and of division ("promitosis") represent stages in the evolution of more complex types must remain for the future to determine.

Blue-green Algæ.-The structure of the cell in the Cyanophyceæ has long been a subject of controversy. ${ }^{29}$ The chief question at issue has been the nature of the central region, which appears relatively colorless and ill defined in living material. By some observers it has been denied that this in any way corresponds to a nucleus, the contention being that blue-green algæ, like bacteria, have a cell organization fundamentally different from that of other organisms. Others have maintained that the central region, although it is not sharply delimited, may be viewed as a "primitive nucleus," since it is occupied by a material which often stains like "chromatin" and tends to be distributed rather equally to the daughter cells during cell-division ${ }^{30}$ (Fig. 113). It is reported that in some genera it contains a material reacting positively to the Feulgen test, also that in Nostoc no nucleic acid can be demonstrated although its radicles are present. ${ }^{31}$ The degree of distinctness with which such "nuclear" materials are aggregated in the central region varies widely.

At present it is difficult to place a proper evaluation on the conflicting claims made in this field. Many have provisionally concluded with Guilliermond that in at least some blue-green algæ there is a primitive nucleus consisting mainly of chromatic matter often arranged in strands, that such strands are broken as the whole mass divides by a process somewhat intermediate between amitosis and mitosis, and that no distinct membrane appears about the mass because the latter is almost constantly in process of division. Definite chromosomes and longitudinal division of chromatic strands have not been demonstrated.

The peripheral cytoplasm in such cells with a central body contains chlorophyll, together with phycocyanin or phycoerythrin. The exact manner in which these pigments are borne is uncertain, ${ }^{32}$ but it is thought probable that they occur in numerous minute droplets which may some-

${ }^{29}$ Among eytologieal works on the Cyanophyceæ are those of Bütschli (1896), Fischer (1897, 1905), Hegler (1901), Kohl (1903), Olive (1904), Phillips (1904), Gardner (1906), Guilliermond (1906, 1925b, 1926), W. H. Brown (1911a), Acton (1914), Baungärtel (1920), Haupt (1923), S. Lee (1927), Pascher (1929), and Prát (1925). Convenient résumés are given by Olive and Haupt. Lloyd (1924) has given special attention to the pigments.

${ }^{30}$ Kohl (1903) on Tolypothrix; Olive (1904) on Oscillatoria; W. H. Brown (1911) on Lyngbya; Guillicrmond (1906) on Phormidium, Nostoc, and Rivularia; Haupt (1923) on Anabona and Glœocapsa.

${ }^{31}$ Poljansky and Petruschewsky (1929) on Oscillatoria, Tolypothrix, Spirulina, and Glootrichia; Mockeridge (1927) on Nostoc.

${ }^{32}$ See the diseussion of fluoreseent eolors by Lloyd (1924). 
times aggregate to form groups with a plastid-like appearance. Ergastic substances, such as glycogen, fat globules, and "cyanophycin granules," also occur and vary in abundance under different nutritive conditions. Although the cytoplasm is rich in lipides, no chondriosomes are present, according to Guilliermond. This author describes a vacuome consisting of small vacuoles containing metachromatin, the precipitation of this substance giving the granules mistaken for nuclear matter by certain observers.

It is tempting to search among lowly organized plants for light on the origin of structures and modes of behavior seen in more complex types. Although it is improbable that the Cyanophycex had anything to do with the evolution of higher plants, the organization they exhibit affords a hint as to stages which may have occurred in the evolution of the typical nucleus and its elaborate mode of division. The view has long been entertained that certain leading constituents of primitive protoplasm may gradually have aggregated to form a definite organ, the nucleus, thus securing the advantages of more consistent interaction and orderly distribution during growth and differentiation. By arranging various blue-green and other algæ in a suitable series, one can at least illustrate "the conception of cell structure which implies differentiated regions of a colloidal system in which special processes have become localized and tend to remain fixed" (Harper, 1919).

Bacteria.-The bacterial cell presents many obstacles to cytological study. It is extraordinarily small and is often surrounded by a layer of material which interferes with fixation; moreover, it contains ergastic substances which vary in amount and present a great variety of aspects in different preparations. Opinion has ranged all the way from the view that the whole bacterium is a naked nucleus to the idea that it is wholly cytoplasm with inclusions. Most prevalent has been the view that scattered nucleo-proteins are present but not aggregated into a definite nucleus, the cell thus illustrating a primitive stage in the evolution of the nucleus-and-cytoplasm type of organization. ${ }^{33}$

The literature contains many descriptions of small granules supposed to be nuclei or at least granules of "nuclear matter," and the aggregation of such granules to form peculiar spiral masses has frequently been reported. The spiral masses seem to be modifications of the cytoplasm about the vacuoles (Meyer), while the nature of the supposed nuclei has been rendered doubtful in most cases by the presence of ergastic masses which may simulate nuclei. In Bacillus pasteurianus, Meyer regarded as nuclei certain minute bodies which he was able to distinguish from the globules of fat, volutin, and glycogen. The frequently paired arrangement of such small bodies was suggestive of division, and their

\footnotetext{
${ }^{33}$ See the accounts by Meyer (1912), Dobell (1911b), Frost (1917), Löhnis (1922),
} Kirchensteins (1922a), Enderlein (1925), Petit (1927), and Wámoscher (1930). 
position at the center of developing spores recalled the behavior of nuclei elsewhere.

That certain bacteria may contain definite nuclei in spite of the above uncertainties has been rendered more probable by the recent work of Stoughton (1929) on Bacterium (Pseudomonas) malvacearum. By employing the methods of Enderlein (1925) and Nakanishi (1901), in which obscuring nutritive materials are removed and the cells are stained without drying, this investigator has been able to differentiate a central body about $0.5 \mu$ in diameter which shows an affinity for basic dyes and passes through a definite division cycle correlated with that of the cell. In some cases this central body elongates and becomes divided with the cell as the latter constricts, while in others it becomes dumb-bell shaped and completes its division before the cell divides by forming a transverse wall. It can be seen in living cells under dark-field illumination. It is Stoughton's conclusion that this central body is in all probability either a nucleus or possibly (following Enderlein's theory) a nucleus imbedded in a mass of chromatic food-reserve substance. Appearances very suggestive of nuclear fusion and division have been reported for the avian tubercle bacillus (Lindegren and Mellon, 1932).

Chromidia.-In 1902 R. Hertwig described in Actinospharium and certain other rhizopods what appeared to be an emission of chromatic granules from the nucleus into the cytoplasm. The nucleus might even break up completely into such granules. He called these granules chromidia and set forth a "chromidia hypothesis." According to those who developed this hypothesis, ${ }^{34}$ there are in an ordinary nucleus two kinds of "chromatin": idiochromatin, concerned in reproduction; and trophochromatin, concerned in nutrition. The chromidia are granules of trophochromatin which pass into the cytoplasm where they degenerate (Hertwig) or play a rôle in the differentiation of specialized structures (Goldschmidt). In certain rhizopods they may consist of idiochromatin and give rise to gamete nuclei. It was further suggested that in the infusoria the micronucleus contains the idiochromatin and the macronucleus the trophochromatin, and that the ordinary nucleus of higher forms is thus a duplex "amphinucleus." This "binuclearity hypothesis" has now fallen into disfavor.

Chromidia were described in many animal tissues by other investigators, ${ }^{35}$ some of whom did not, however, subscribe to the above views regarding their origin and significance. They were particularly conspicuous in oöcytes and spermatocytes, where a mass of them developed

${ }^{34}$ R. Hertwig (1902, 1904), Goldschmidt (1904 et seq.), Goldschmidt and Popoff (1907), Popoff (1906 et seq.), Schaudinn (1903), Schaxel (1910 et seq.).

35 Marcus (1907), Wassilief (1907), Reichenow (1908), Nowikoff (1909), Buchner (1909), Moroff (1909, 1911), M. Jörgensen (1910, 1913), Nussbaum (1913), Hirschler (1913), van Herwerden (1913). 
near the nucleus during the growth period. When special methods for the study of chondriosomes and Golgi material developed, however, it becaine apparent that chondriosomes, chromidia, secretion droplets, and other ergastic bodies had been hopelessly confused and that many of the elements which had passed as chromidia were, in reality, chondriosomes and other materials for which there was no evidence of nuclear origin. ${ }^{36}$ Cowdry points out that " . . . in practice, under the heading of chromidia, we have therefore to deal with a variety of substances which have been hastily grouped together on account of their general affinity for 'basic' stains and their supposed relation to nuclear chromatin and for which no special methods of fixation are required. It is a branch of cytology which has developed almost wholly apart from methods for the study of living cells."

At present it seems best to reserve the term "chromidia" for the scattered chromatic matter in Protista with no formed nuclei, and in other organisms for the small bodies in the cytoplasm which resemble nuclear matter in composition and chromaticity more closely than do the chondriosomes and other ergastic substances. The view that chromatic granules are transferred bodily through the nuclear membrane has not been well substantiated, but that a nucleic acid compound passes the membrane in solution, takes the form of granular "chromidia" as it reaches the cytoplasm, and is transformed into strands and clumps on fixation is probable (van Herwerden). Such manifestations of nucleocytoplasmic interchange have been clearly shown in echinoderm eggs, ${ }^{37}$ although their full significance and their relation to the general problem of chromidia are not yet apparent. ${ }^{38}$

${ }^{36}$ Duesberg (1911), Fauré-Fremiet (1910), Hirschler (1913), Nussbaum (1913), Jörgensen (1913c), E. V. Cowdry (1924a). The chromidia of Actinospharium are ergastic, according to Rumjantzew and Wermel (1925).

37 Danchakoff (1916), Tennent (1920).

${ }^{38}$ See further on the subject of chromidia Kofoid (1921), Agar (1920a), E. V. Cowdry (1924a), and Wilson (1925, p. 700). 


\section{CHAPTER XIV}

\section{GAMETOGENESIS AND SPOROGENESIS}

In plants there are two principal kinds of specialized reproductive cells: spores, which develop singly, and gametes, which undergo sexual fusion and form a zygote, this in turn developing into a new individual. As already pointed out (p. 122), gametogenesis and sporogenesis are commonly associated with the alternation of two generations in the life cycle of plants, the gametophyte producing gametes and the sporophyte spores. In some of the thallophytes, however, both spores and gametes may be produced rather freely by the same individual, no clear alternation of generations being present. ${ }^{1}$ In the higher animals there are reproductive cells of but one principal kind, namely, the gametes.

The present chapter deals with a few sample cases from among the many diverse types of reproductive phenomena exhibited by different organisms. Discussion of the actual fusion of the gametes and of the behavior of chromosomes in sporogenesis is deferred to the following two chapters.

Algæ. - The two gametes which fuse sexually may be morphologically similar or dissimilar, and motile or non-motile. In Ulothrix and Ectocarpus they are similar and motile, the two cilia of each gamete being equal and terminal in the former but unequal and lateral in the latter (Fig. 138, A, B). In Spirogyra the gametes are but little-modified vegetative cells with no special motile apparatus aside from contractile vacuoles (p. 228).

Examples of unlike male and female gametes are afforded by $E d o-$ gonium, Fucus, Vaucheria, and Polysiphonia. In no two of these genera are the male gametes of the same morphological type. In $E$ dogonium certain small cells of the filament produce two ovoid, green spermatozoids, each with a ring of cilia around one end, while other cells enlarge and become eggs (Fig. 138, C). The egg contains plastids and reserve food materials, while a more or less clear "receptive spot" may often be made out at the point where the spermatozoid is to enter. In Fucus the pri-

${ }^{1}$ For general accounts of the life eyeles of plants, see general textbooks of botany, especially Holman and Robbins (1928), Smith, Overton et al. (1928), Gager (1926), Robbins and Rickett (1929), and Fitting, Sierp, Harder, and Karsten (1930). For algæ, see Oltmanns (1922-1923), Bonnet (1914), West (1927), and G. M. Smith (1933); for fungi, Gäumann-Dodge (1928) and Fitzpatrick (1930); for seed plants, Coulter and Chamberlain $(1903,1910)$. For the eytology of bryophytes, see Motte (1932) and Hoefer (1932). 
mary nucleus of the antheridium initiates a series of mitoses giving rise to a large number of nuclei. About each of these is organized a laterally biciliate spermatozoid. In the oögonium the primary nucleus initiates a series of three mitoses, the protoplasm then subdividing to form eight large eggs. In related genera only four, two, or one egg may be produced, the remaining nuclei degenerating. ${ }^{2}$ In Vaucheria, which is cœnocytic, many colorless, elongated spermatozoids with two cilia near one end are organized about the nuclei in the antheridium. In the maturing oögo-

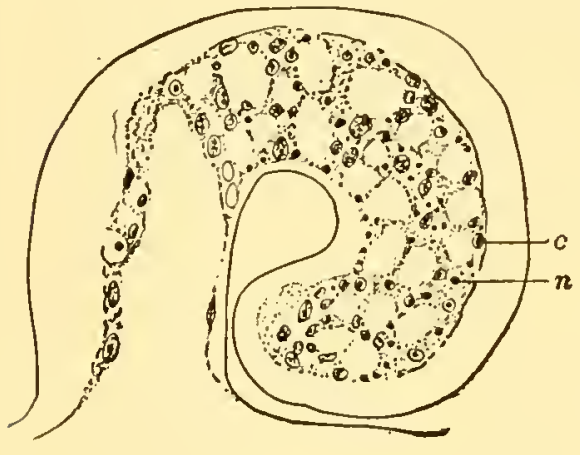

A

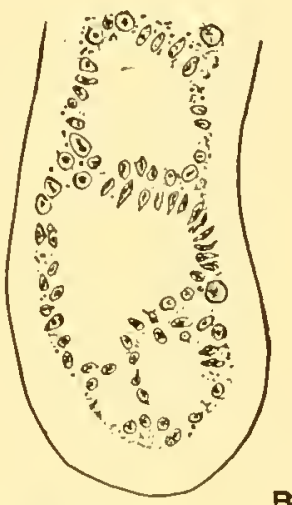

$\mathbf{B}$
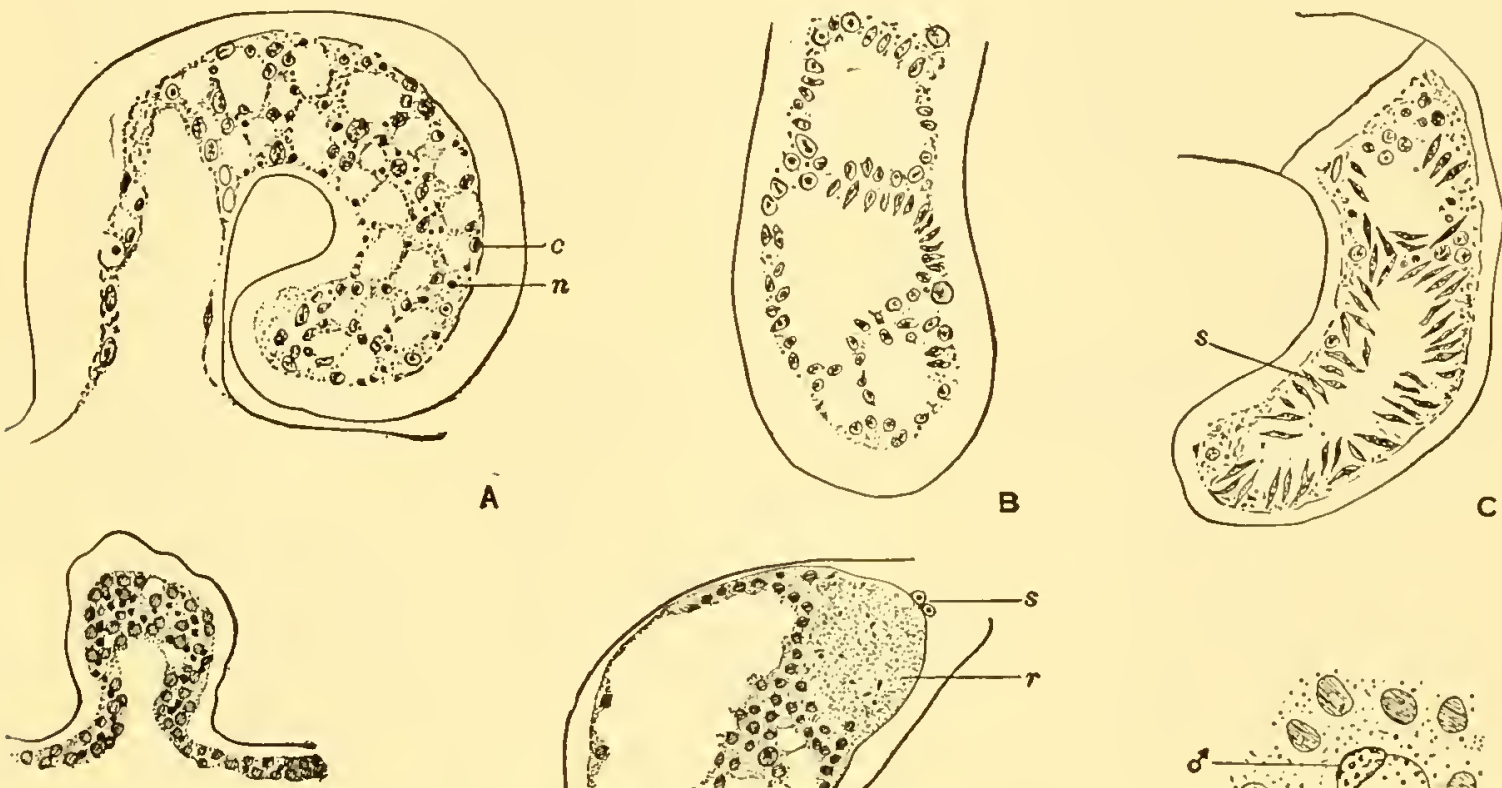

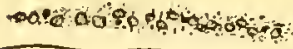
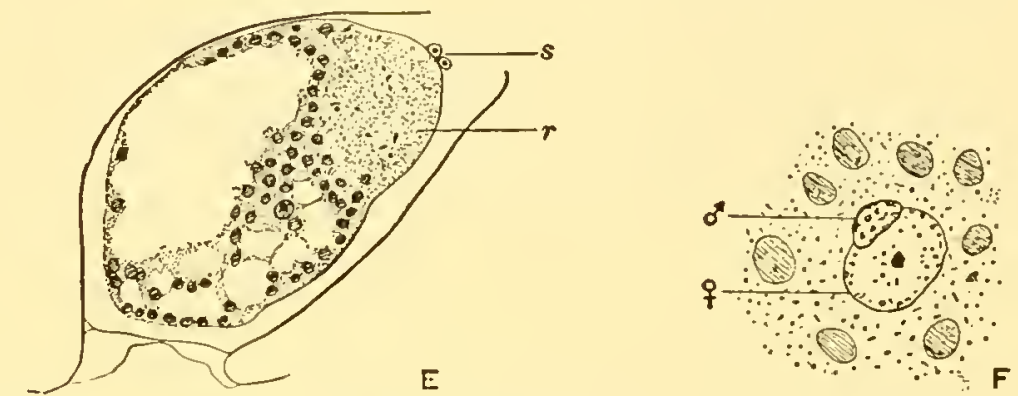

FIG. 114.-Gametogenesis and syngamy in Vaucheria sessilis. $A-C$, differentiation of spermatozoids in cœnocytic antheridium. $D$, early stage in development of oögonium. $E$, mature egg with one nucleus and many plastids. $F$, gametic nuclei uniting in egg $c$, chloroplast; $n$, nucleus; $r$, receptive papilla; $s$, spermatozoids. (After Oltmanns, 1895.)

nium all but one of the many nuclei retreat to the main thread in a mass of "Wanderplasm" or degenerate, that one remaining as the nucleus of the single large $\mathrm{egg}^{3}$ (Fig. 114). In Polysiphonia, non-motile male gametes, or spermatia, are budded off successively from the antheridial cell, each of them being supplied with a nucleus formed by a division of the antheridial nucleus. The female nucleus lies in the enlarged base of the carpogonium, a cell which elongates to form a receptive trichogyne above.

The most characteristic spores in algæ are the zoöspores, which swim about actively by means of cilia. Except for their larger size they resemble the male gametes of their respective genera, being terminally

${ }^{2}$ For recent accounts, see Tahara $(1927,1929)$.

${ }^{3}$ Oltmanns (1895), Heidinger (1908), and Couch (1932b) report retreat; B. M. Davis (1904), M. Williams (1926), and Mundie (1929) report degeneration. 
biciliate in Ulothrix, laterally biciliate in Ectocarpus, and crowned with a ring of many cilia in Edogonium. Non-motile spores (aplanospores) also occur in some genera. In Polysiphonia, carpospores are budded off from the carpogonium and tetraspores are formed in groups of four in the tetrasporangium. In some alga, notably Spirogyra and its relatives, the product of gametic fusion is a resting zygospore.

The cytological phenomena appear to be about the same in the development of motile gametes and spores of a given type. The protoplasm of the antheridium or the sporangium, as the case may be, is often not entirely used up in the formation of the spermatozoids or zoöspores. The chromatophores, if large and continuous, ordinarily break up into numerous smaller ones, while the pyrenoids and starch tend to disappear as the gamete or spore primordia are delimited. In each primordium
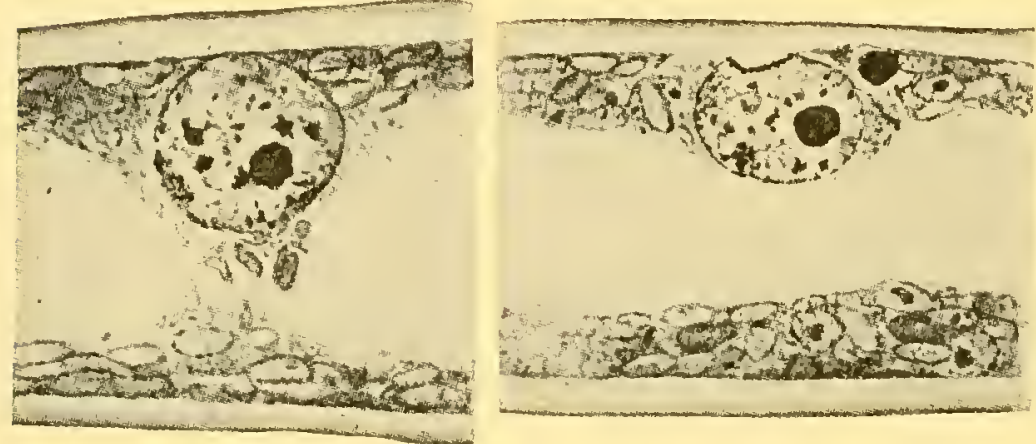

FrG. 115.-Development of blepharoplast in Edogonium.

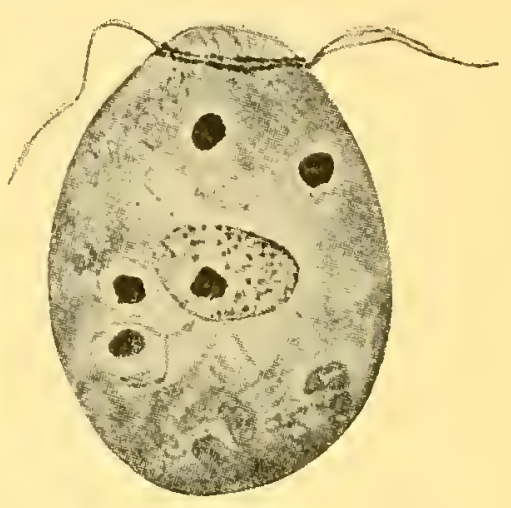

(After Kretschmer, 1930.)

there are differentiated an eyespot and a motor apparatus, the latter consisting of a blepharoplast with its attached cilia.

The development of the blepharoplast, or cilia-bearing organ, has attracted much attention in these and other organisms. In Edogonium (Fig. 115) the nucleus moves against the cell membrane, which there forms a convex thickening. In the plane of contact there appears a ring of granules. As the nucleus loses contact with the thickening the ring becomes double, and from its outer half a crown of cilia grows out. ${ }^{4}$ Each of the many pairs of cilia on the cœnocytic Vaucheria swarm spore is in some way associated with a nucleus lying near the cell membrane. In Eudorina the pointed end of the nucleus, to which a centriole is attached, touches the membrane and then retreats to the center of the cell, leaving behind a double body (centrioles?) from which the cilia grow out (Hartmann, 1921). Such blepharoplasts as that of $E$ dogonium are called "plasmodermal blepharoplasts" to distinguish them from the "centrosomal blepharoplasts" of pteridophytes, bryophytes, and some of the lower forms. 
The mature spermatozoids of the algæ are usually described as having a considerable amount of cytoplasm, but evidently this is not always the case. In Fucus serratus the figures of Guignard (1889) and Kylin (1916) represent the cytoplasm as equaling or exceeding the nucleus in volume, whereas in $F$. Areschougii Kylin (1920) has shown that the main portion of the spermatozoid is nuclear, the cytoplasm being very small in amount (Fig. 116, A). At one side is the eyespot, which is derived from the chromatophore of the antheridial cell through an intermediate color-
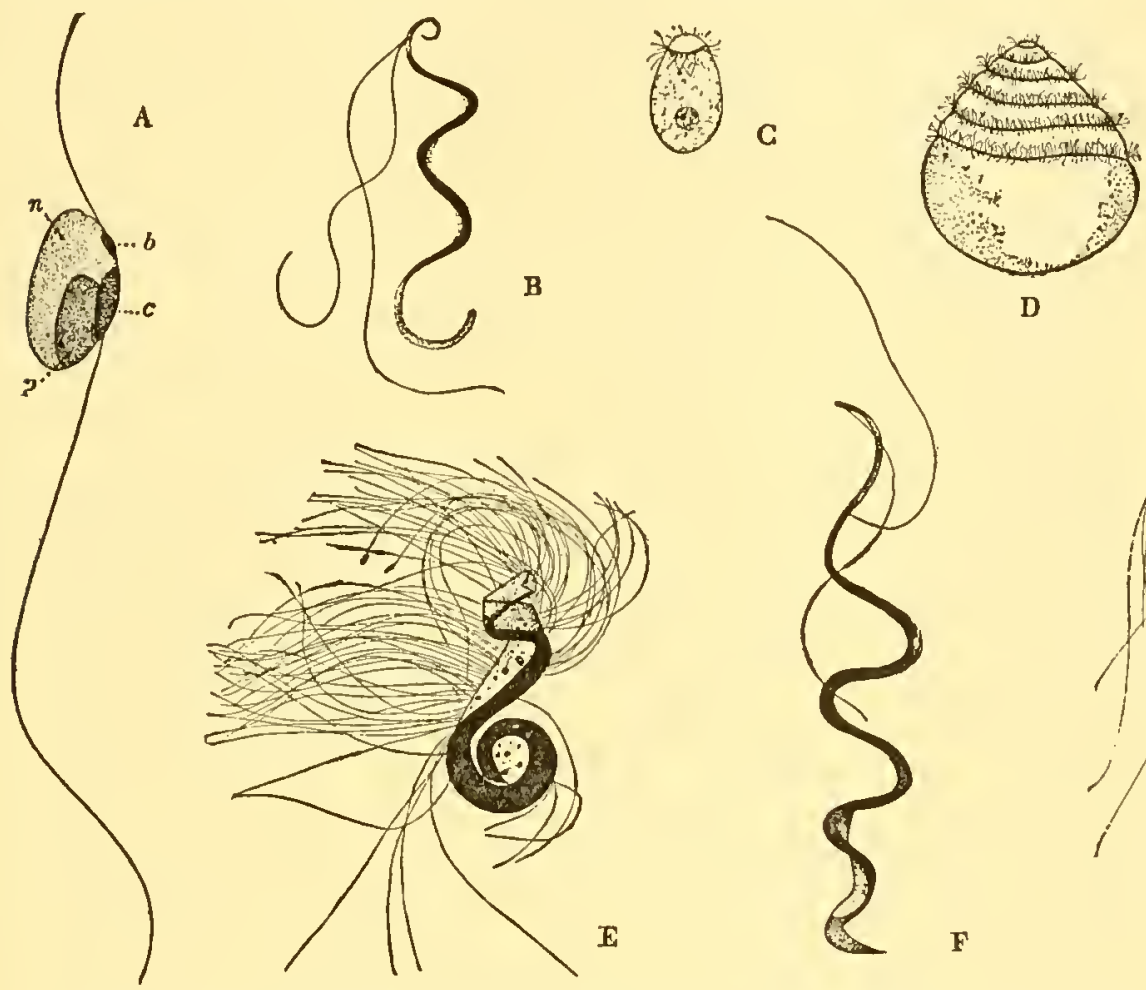

G

F1G. 116.-Spermatozoids of plants. A, Fucus Areschougii; b, blepharoplast; $c$, chromatophore; $n$, nucleus; $p$, plastomere. (After Kylin, 1920.) B, Chara. (After Belajeff.) C, Edogonium. D, Zamia. (After Webber.) E, Onoclea. (After Steil, 1918a.) F, Riccardia. (After Steil, 1923.) $G$, Marsilia; spermatozoid extended as it enters gelatinous material about the archegonium. (After Sharp, 1914.)

less stage. Connected with it is the blepharoplast, which arises from the centrioles, according to Meves $(1918 c)$. Farther back are one or more "plastomeres," which appear to be chondriosomal in nature.

Fungi.--In their reproductive processes the fungi show many interesting parallels with the algæ, at least among the largely conocytic phycomycetes. In Synchytrium the zoöspores and gametes are uniciliate, the gametes being morphologically alike. In Allomyces javanicus the gametes are unlike in size. In Monoblepharis the zoöspores are uniciliate also, while the gametes are differentiated into large eggs borne in oögonia and small uniciliate sperms. In the other oömycetes ${ }^{5}$ the male element is

5 Trow (1895-1904), Wager (1896, 1900b), F. L. Stevens (1901), B. M. Davis (1900, 1903, 1905b), Miyake (1901), P. Claussen (1908), Murphy (1918), P. M. Patterson (1927a), Nishimura (1926), Carlson (1929), G. O. Cooper (1929ab), Couch (1932a). 
represented by the uninucleate or multinucleate contents of the antheridium, some or all of which is discharged into the oögonium through a copulating tube. In Albugo candida and Pythium Debaryanum all but one of the nuclei originally present in the young oögonium retreat to the periplasm, which becomes sharply set off from the central oöplasm with the single functional nucleus (Fig. 117). A dense cytoplasmic mass known as the "cœnocentrum" may appear about the nucleus or in its vicinity. In Saprolegnia the multinucleate protoplasm of the oögonium becomes divided up by one or more vacuoles into several eggs, each of which is at first multinucleate and finally uninucleate. In Mucor and the other zygomycetes the multinucleate contents of two gametangia behave as gametes.
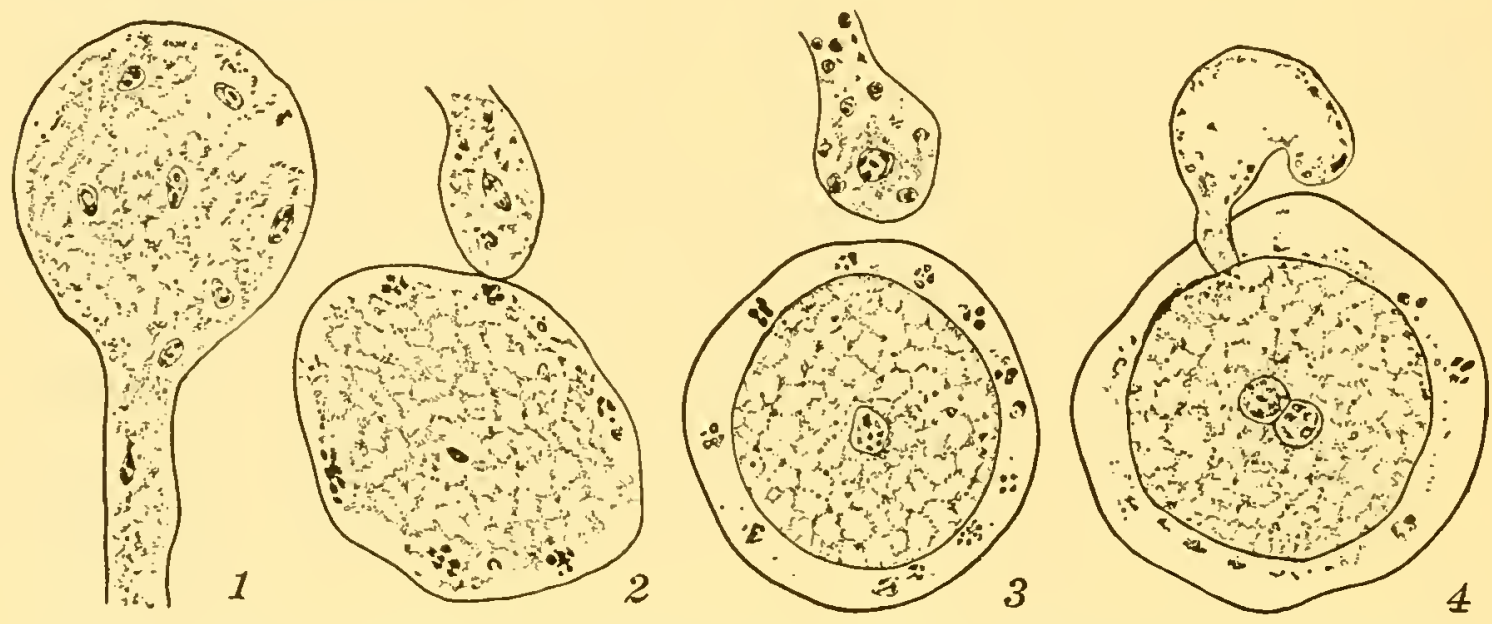

Fig. 117.-Gametogenesis and syngamy in Pythium Debaryanum. 1, formation of öogonium at end of hypha. 2, oögonium and antheridium in contact. 3, egg mature; degenerating nuclei in periplasm; antheridium above, with one functional and several non-functional nuclei. \&, sexual nuclei in contact. (After Miyake, 1901.)

The zoöspores and motile male gametes in the phycomycetes are formed by the subdivision of multinucleate protplasm. In Saprolegnia, for example, the protoplasm in the swollen tip of a hypha rounds up into uninucleate portions by an extension of the vacuole system. These units become terminally biciliate zoöspores. At a later stage of the cycle, Saprolegnia produces also reniform, laterally biciliate zoöspores. Connection of the cilia with a granule (centrosome?) at the apex of a topshaped central body in the nucleus has been described for Leptolegnia (A. C. Mathews, 1932).

In the large sporangia of the zygomycetes the non-motile spores are formed by a progressive furrowing of the multinucleate mass, as already described (p. 165).

The formation of spores exogenously is illustrated by conidia and basidiospores. The former are cut off successively by constriction from the ends of certain hyphæ, each of them receiving one or more nuclei derived by division from the nuclei of these hyphæ. The basidiospores are budded off in quartets from the basidium, whose nucleus by two 
divisions provides them with one nucleus each (Fig. 164). Development of spores by free cell formation occurs in the ascomycetes. After the primary ascus nucleus has divided to eight, a portion of the ascus cytoplasm is cut out about each of these nuclei, the astral rays curving around from the centrosome having some part in the process (p. 158). Finally, resting spores are often formed as a result of a sexual fusion. Examples of this are the zygospore of Mucor and the oöspore of Albugo.

Bryophytes.-In bryophytes, which show a well-marked alternation of generations, the eggs and spermatozoids are borne by the gametophyte in archegonia and antheridia, respectively. The egg is the innermost cell of the axial row in the archegonium (Fig. 118). Commonly it enlarges, rounds up, and lies more or less free in the slimy fluid of the venter, the other axial cells meanwhile breaking down. In some genera (Anthoceros, Sphagnum) the ventral canal cell also is sometimes organized as a functional egg, and there are other facts which suggest that the other cells of the axial row were gametes historically. Besides a large nucleus, the egg may contain plastid primordia and chrondriosomes. In mosses Motte (1928) reports that the egg contains practically no food reserves. In many cases a fairly distinct hyaline receptive spot is present at the side of the egg directed toward the neck of the archegonium.

Spermatogenesis has been studied in both liverworts and mosses. ${ }^{6}$ In Marchantia, according to the

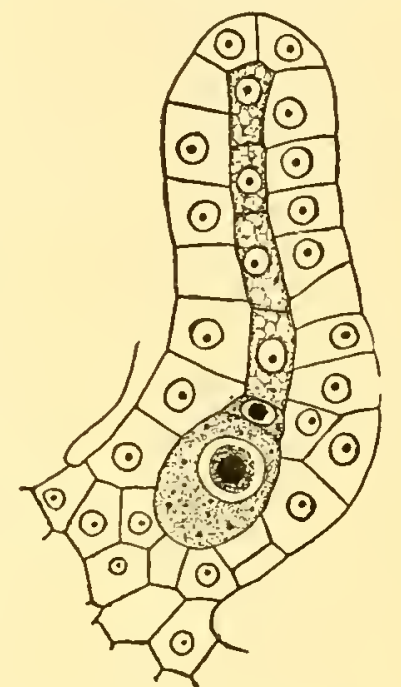

Fig. 118.-Archegonium of Reboulia, showing egg, ventral canal cell, and four neck c a n a l cells. (After Haupt, 1921.) early account of Ikeno, a minute centrosome comes out of the nucleus before each spermatogenous cell-division in the antheridium and divides into two; these pass to opposite sides of the cell and occupy the spindle poles during mitosis. After the last (diagonal) cell-division the centrosome passes to one corner of the cell (spermatid) and there elongates to form the blepharoplast, from which two long cilia grow out (Fig. 119). Meanwhile the nucleus becomes altered in shape and forms the main portion of the body of the spermatozoid. In the cytoplasm of the spermatid was seen another chromatic body of uncertain fate. Later accounts of other liverworts present essentially the same story except that the nuclear origin of the centrosome, which is usually seen only in the last mitosis, has not been substantiated. In Blasia the blepharoplast divides into

${ }^{6}$ On liverworts: Ikeno (1903), Miyake (1905b), C. E. Lewis (1906), Bolleter (1905), Escoyez (1907b), Schaffner (1908), Woodburn (1911, 1913), Sharp (1920b), Motte (1928), Yazawa (1931). On mosses: M. Wilson (1911), Woodburn (1915), C. E. Allen $(1912,1917 a)$, Bowen $(1926 f, 1927 b)$, Motte (1928), Weier (1930, 1931b). See Mühldorf $(1930,1931)$ on the mature spermatozoid. 

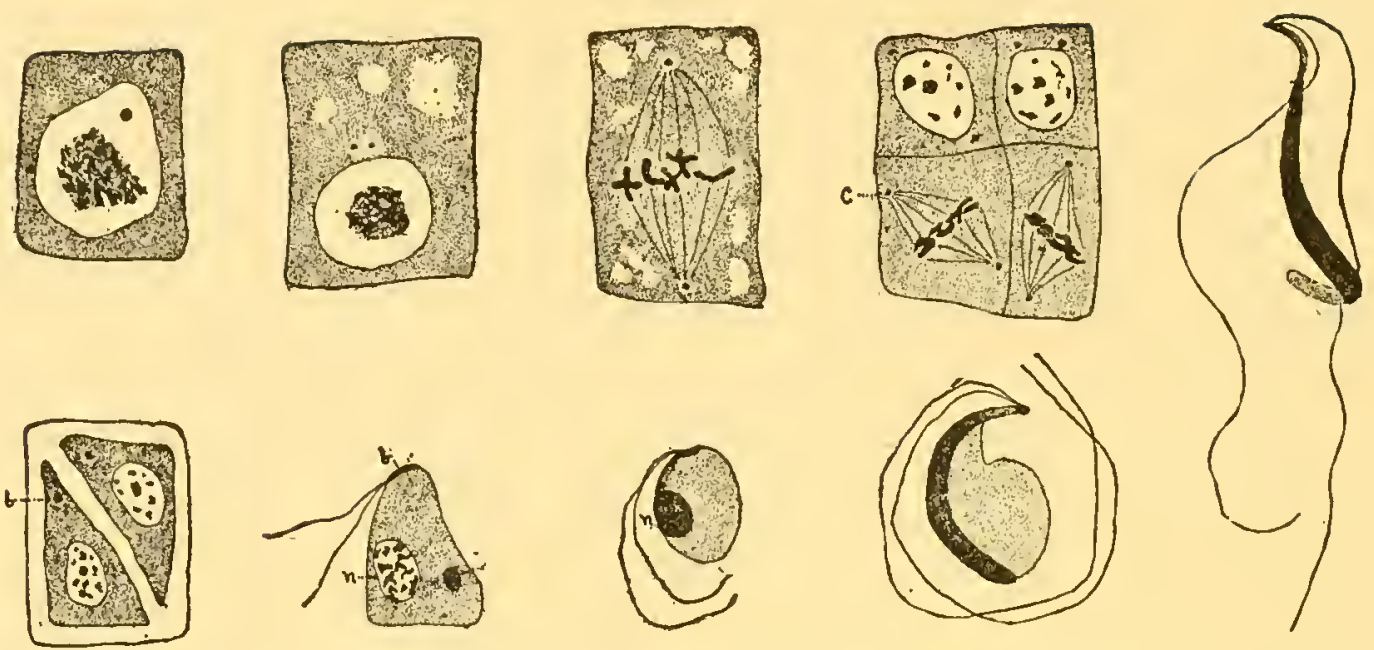

FIG. 119.-Spermatogenesis in Marchantia. b, blepharoplast; $c$, centrosome; $n$, nucleus. (After Ikeno, 1903.)

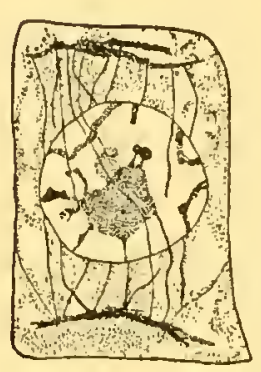

$A$

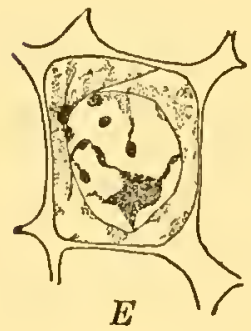

$E$

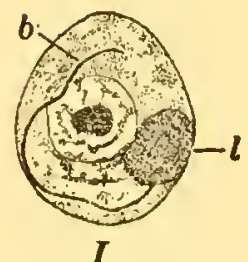

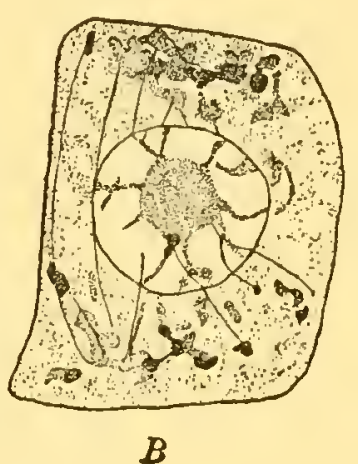
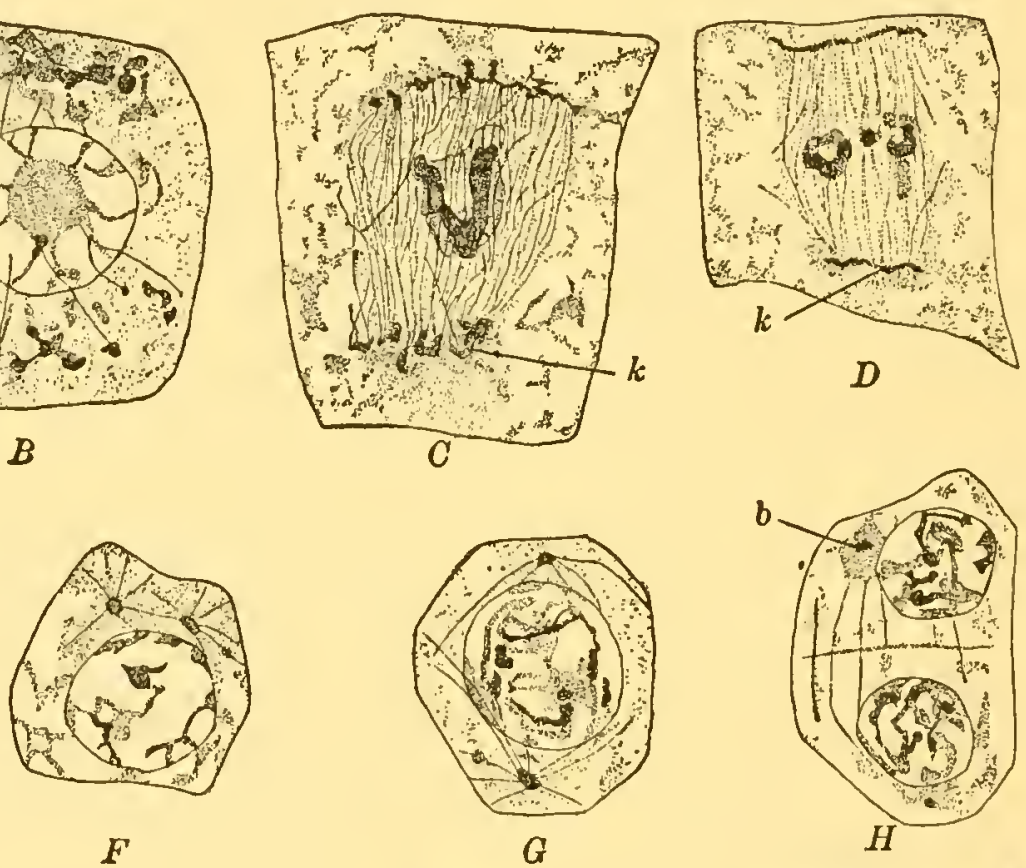

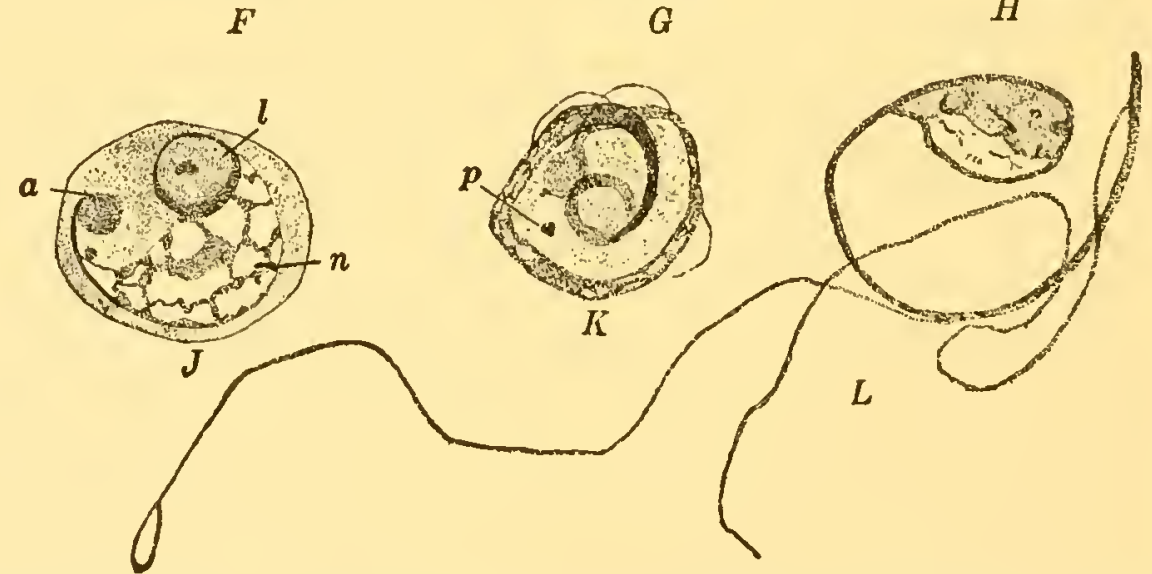

FrG. 120.- Spermatogenesis in Polytrichum. $A-D$, mitosis in androgones, showing appearance of plastid material $(k)$. $E-H$, behavior of centrosome in last division; each androcyte (spermatid) has one centrosome, which acts as a blepharoplast (b). $I-K$, transformation of androcyte into spermatozoid: $a$, apical body; $l$, limosphere; $n$, nucleus; $p$, perenosome. L, mature biciliate spermatozoid. (After C. E. Allen, 1912, 1917a.) 
fragments which together form the cilia-bearing rod, as in certain vascular plants (Sharp, 1920b). In the spermatozoids of Riccardia (Fig. 116, F), Pellia, and certain other genera the two cilia spring from different points, ${ }^{7}$ at which basal granules may be seen.

Recent studies on spermatogenesis in mosses have revealed a remarkable parallelism between the behavior of cell elements here and that in animal spermatogenesis. In the multiplying spermatogenous cells ("androgones") of Polytrichum the plastids form one or two masses,
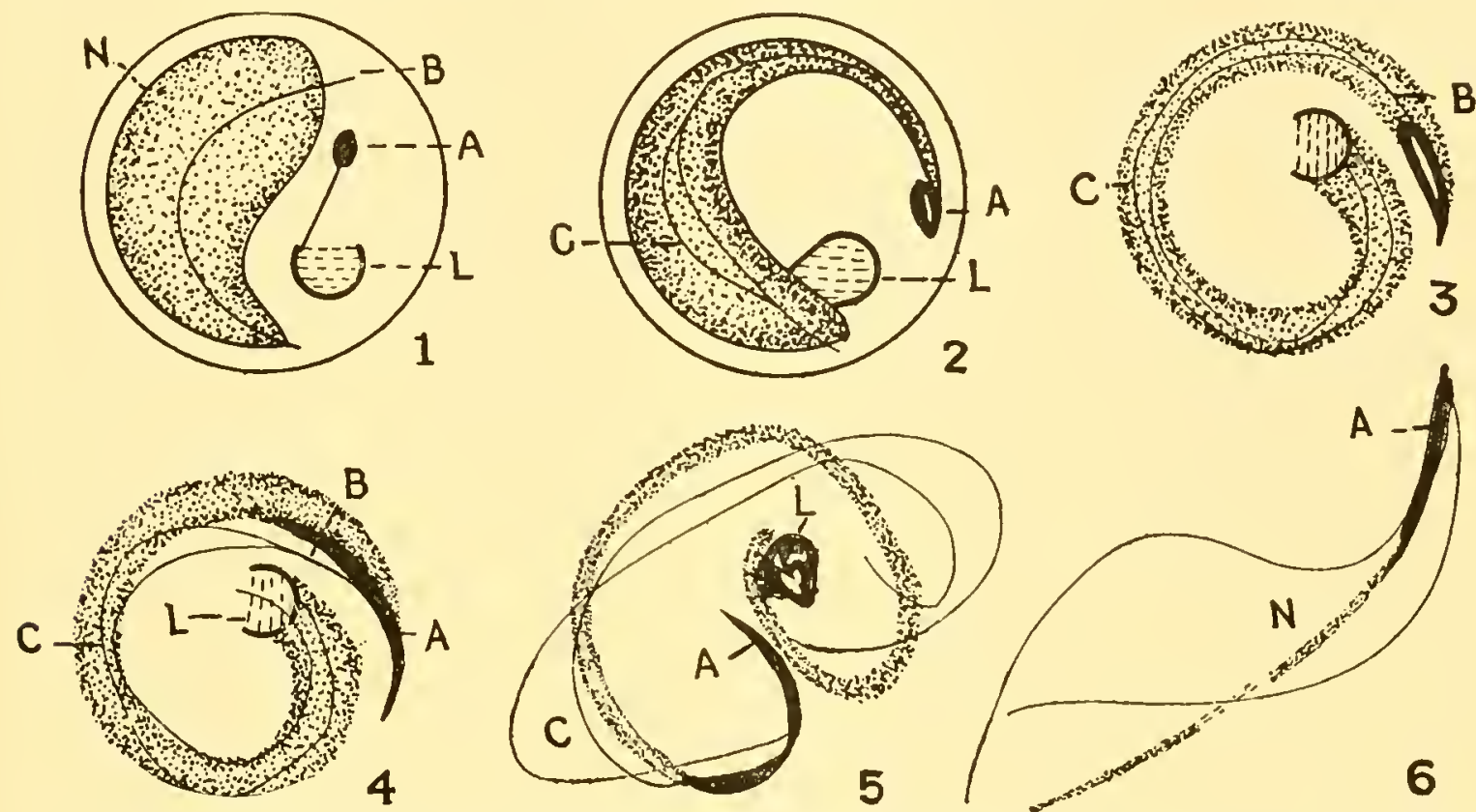

F1G. 121.-Diagram of spermatogenesis in Polytrichum. In 1 the apical body $(A)$ is separating from the limosphere remnant $(L)$. $B$, blepharoplast. $C$, cilia. $N$, nucleus. (After Weier, 1931b.)

which appear after certain fixations like clumps of granules and as large plates, or, after other procedures, like a series of chromophilic plates in a chromophobic substance (see p. 80). In each cell generation these bodies undergo division (Fig. 120). In each cell of the last generation but one, a centrosome with radiations in the cytoplasm divides into two. These move apart and occupy the spindle poles during the last mitosis, which differentiates the spermatids ("androcytes").

In the androcyte the plastid becomes a body known as the limosphere. It becomes somewhat oblong and buds off a small differentiated portion from one end; this is the apical body (Fig. 121). Meanwhile the centro-

${ }^{7}$ Steil (1923), Showalter (1926a), Mühldorf (1931).

${ }^{8}$ The limosphere was named by M. Wilson (1911). Allen (1917a) gave the first adequate deseription of it, diseovered its division to form the apical body, and pointed out evident references to it in the earlier literature. Its derivation from plastids was demonstrated by Weier $(1930,1931 b, 1932)$, see page 81 . The granules and plates in the earlier spermatogenous cells were called "kinetosomes" and "kinoplasmic plates" by Allen (1912). Sapěhin's (1915) claim that they are plastids was confirmed by Bowen (1927b) and Weier. 
some has elongated to form the blepharoplast, and the nucleus has begun to elongate also. The blepharoplast comes against the apical body at one end of the nucleus; this is to be the anterior end of the spermatozoid. The apical body becomes pointed, while the portion of the blepharoplast lying beside it somehow gives rise to the two long cilia. Meanwhile the limosphere remnant takes up a position near the posterior end of the nucleus where it may eventually disappear along with some of the cytoplasm. The mature spermatozoid consists of an elongated body representing chiefly the nucleus, a little cytoplasm, an apical body, and a motor apparatus consisting of blepharoplast and cilia.

It will be noted that the behavior of the limosphere and apical body are almost precisely similar to that of the acroblast and acrosome in animals (p. 220). The acroblast is known to represent the Golgi material of the animal spermatid; hence the demonstration that the limosphere is derived from the plastids of earlier cell generations lends strong support to the view that of all the cell elements in plants the plastids correspond most closely to the Golgi material of animals (see p. 80).

The spores in bryophytes are borne by the sporophyte, which develops from the fertilized egg. In the sporangium certain cells known as sporocytes round up from one another and each of them undergoes two successive divisions, thus giving rise to a quartet of spores. In some liverworts the sporocyte becomes markedly four lobed before it divides. It is in the course of the two mitoses in the sporocyte that the number of chromosomes is reduced one half. Meanwhile the plastids also are divided (at least in some cases) and distributed to the four spores (p. 70). After their walls become fully developed, the spores are freed from the sporangium and later grow into new gametophytes.

Pteridophytes.- In pteridophytes, as in bryophytes, quartets of spores with the reduced, or gametic, chromosome number are produced by the division of sporocytes in the sporangium. Furthermore, the spores germinate to produce gametophytes, which bear eggs and spermatozoids in archegonia and antheridia. In location and general characteristics the egg is much like that of bryophytes. Often it is somewhat flattened on the receptive side at the time of syngamy.

The spermatozoids of all pteridophytes, except Lycopodium, Phylloglossum, and Selaginella, differ from those of bryophytes in being multiciliate. Most of the accounts of spermatogenesis ${ }^{9}$ state that the blepharoplasts appear first either in the spermatids or as centrosomes during the division which differentiates these cells. In Equisetum (Fig. 122) it has been shown that the blepharoplast appears as a functional centrosome

${ }^{9}$ Buchtien (1887), Campbell (1887), Belajeff (1888, 1897, 1898, 1899), Guignard (1889), Schottländer (1893), Shaw (1898a), Thom (1899), Yamanouchi (1908b), R. F. Allen (1911), Sharp $(1912,1914 b)$. See the recent studies on the mature spermatozoid by Dracinschi $(1930,1931,1932)$ and Yuasa $(1932,1933)$. 
in each cell of the penultimate generation; there it divides to two, which separate and occupy the poles of the achromatic figure in the manner of animal centrosomes. At the close of mitosis the blepharoplast in each spermatid fragments into a number of pieces which later join to form a continuous chromatic thread. The spirally coiled body of the mature spermatozoid in pteridophytes consists of the elongated nucleus, a cytoplasmic band lying along the anterior portion of the nucleus and containing the chromatic thread at its margin, a posterior globule of cytoplasm containing starch and other inclusions, and numerous cilia. Students of spermatozoid development have described the cilia as growing out from the chromatic thread ("blepharoplast"), though

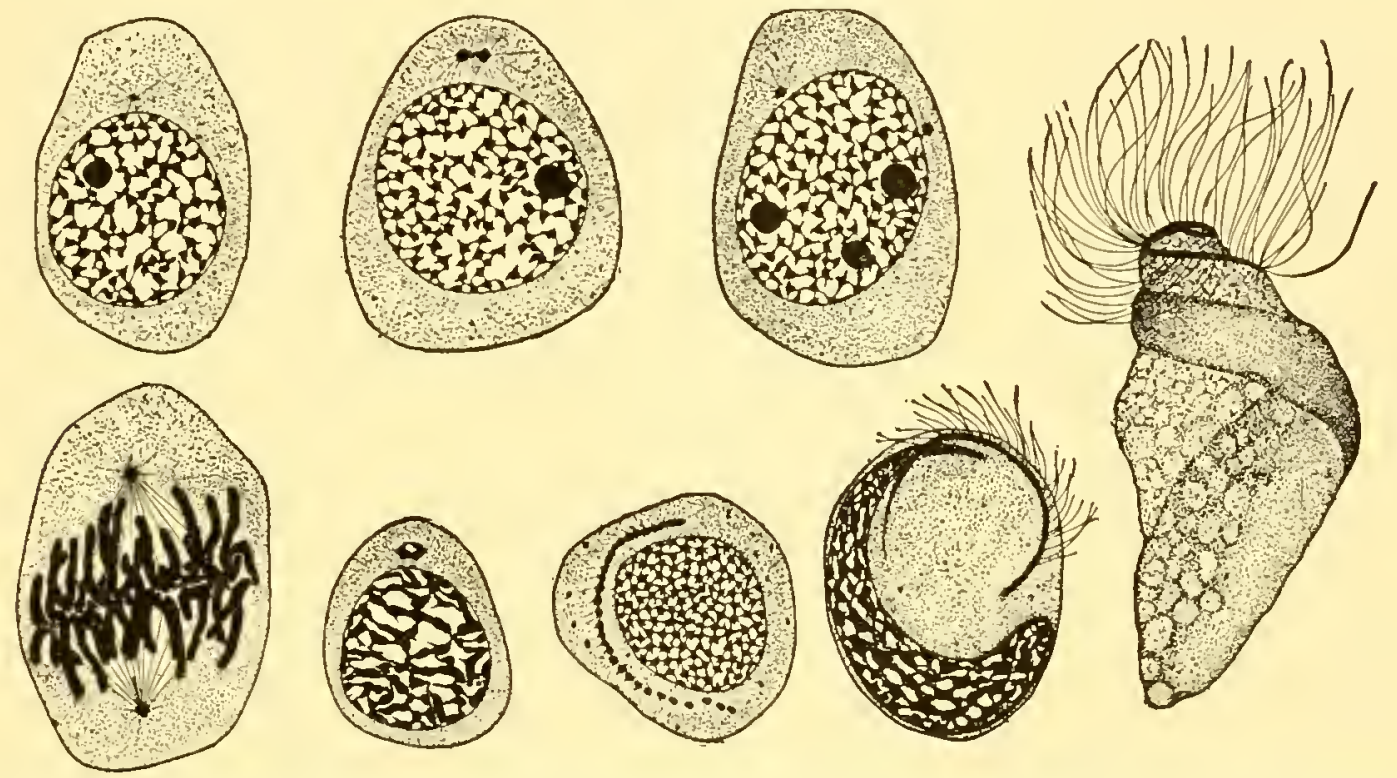

FIG. 122.- Spermatogenesis in Equisetum arvense, showing the behavior of the blepharoplast (centrosome) in the last spermatogenous mitosis and in the transformation of the spermatid into the spermatozoid. (After Sharp, 1912.)

recent observers of mature spermatozoids report that they appear to be attached to basal bodies in the cytoplasmic band whose margin is formed by the thread ("border brim") (Dracinschi, Yuasa).

In Marsilia each of the two primary spermatogenous cells undergoes a series of four divisions which result in 16 spermatids. No distinct centrosomes appear during the first mitosis. During the anaphase of the second mitosis centrosomes develop at the spindle poles. In the telophase they divide but usually then disappear in the cytoplasm. In the third mitosis centrosomes again appear at the spindle poles during the anaphase, and in the telophase they divide. The daughter centrosomes so formed then move apart and occupy the poles of the spindle through the fourth, or final, mitosis. They are at all times accompanied by distinct cytoplasmic radiations, the achromatic figure being strikingly like those in animals and certain thallophytes. Before the last mitosis is completed, the centrosome (blepharoplast) becomes vacuolate, and in the spermatid it breaks 
up into a number of fragments. These later join to form a cilia-bearing band which elongates spirally in close union with the nucleus to form the spermatozoid (Sharp, 1914).

Gymnosperms.- In all seed plants, as well as in certain groups of pteridophytes, spores of two distinct kinds are produced in quartets: microspores and megaspores. ${ }^{10}$ Such plants are said to be heterosporous, while those with only one kind of spore quartet are homosporous. In the gymnosperms the microspore quartets are developed in a manner essentially like that of the quartets of homosporous ferns, i.e., by the subdivision of isodiametric sporocytes. The sporocyte which develops the

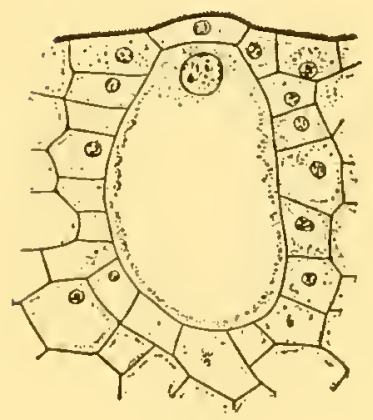

A

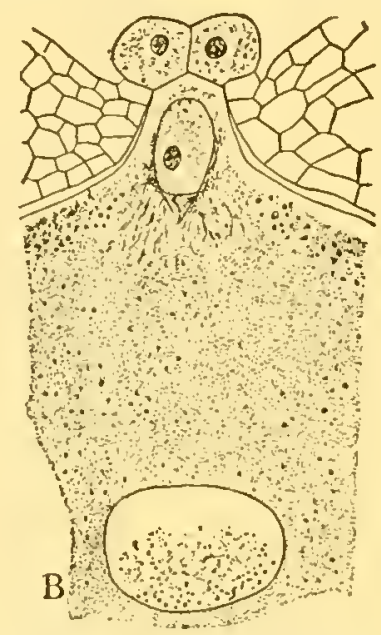

FIG. 123.-Oögenesis in Dioön edule. A, young archegonium. $B$, egg nucleus below

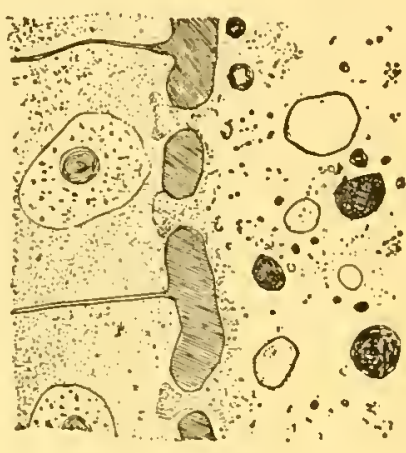

$\mathrm{C}$ and ventral canal nucleus above; two conspicuous neck cells. $C$, protrusions of the egg cytoplasm (at right) extending as haustoria through pores in the egg membrane into the surrounding jacket cells (at left). (After Chamberlain, 1906.)

megaspore quartet in the ovule is commonly elongated, and it divides in such a manner that the spores form a row. The outer three spores ordinarily degenerate, while the innermost one develops into a female gametophyte with archegonia.

The eggs of gymnosperms ${ }^{11}$ are very large cells, the nucleus alone in certain cycads being as much as 0.5 millimeter in diameter. The egg is borne in an imbedded archegonium, which has an evanescent ventral canal cell or nucleus (Fig. 123). The egg cytoplasm is in close communication with surrounding nutritive cells of the gametophyte through pits in the wall. In most cases these pits are said to be closed by a membrane, the only direct connection being through minute plasmodesms. In Dioön, however, Chamberlain has shown that the membrane at first

${ }^{10}$ These standard terms imply a size difference, but the essential difference is one of sexual tendency; androspore and gynospore would be more appropriate. "Megaspores" are sometimes smaller than "microspores."

${ }^{11}$ Goroschankin (1883), Ikeno (1898), Chamberlain (1899, 1906, 1910, 1912, 1916), I. Smith (1904), Ferguson (1904), Stopes and Fujii (1906), Eames (1913), P. Sedgwick (1924), Lawson (1907, 1909, 1926), G. Nichols (1910), Shimamura (1928, 1929), Sinólska (1927), Wakayama (1929), Wóycicki (1923ab), Herzfeld (1927). 
separating the projecting egg haustorium eventually ruptures, the cytoplasm of the egg and nutritive cells then becoming continuous. In the cycads the egg cytoplasm appears relatively free from conspicuous nutritive inclusions, but in conifers, such as Pinus and Agathis, there are often large masses of stainable ergastic material and other substances of uncertain origin and composition. The nucleus of the gymnosperm egg resembles that of certain animal eggs in containing an unusually large amount of material not incorporated in the chromosomes at the time of mitosis.
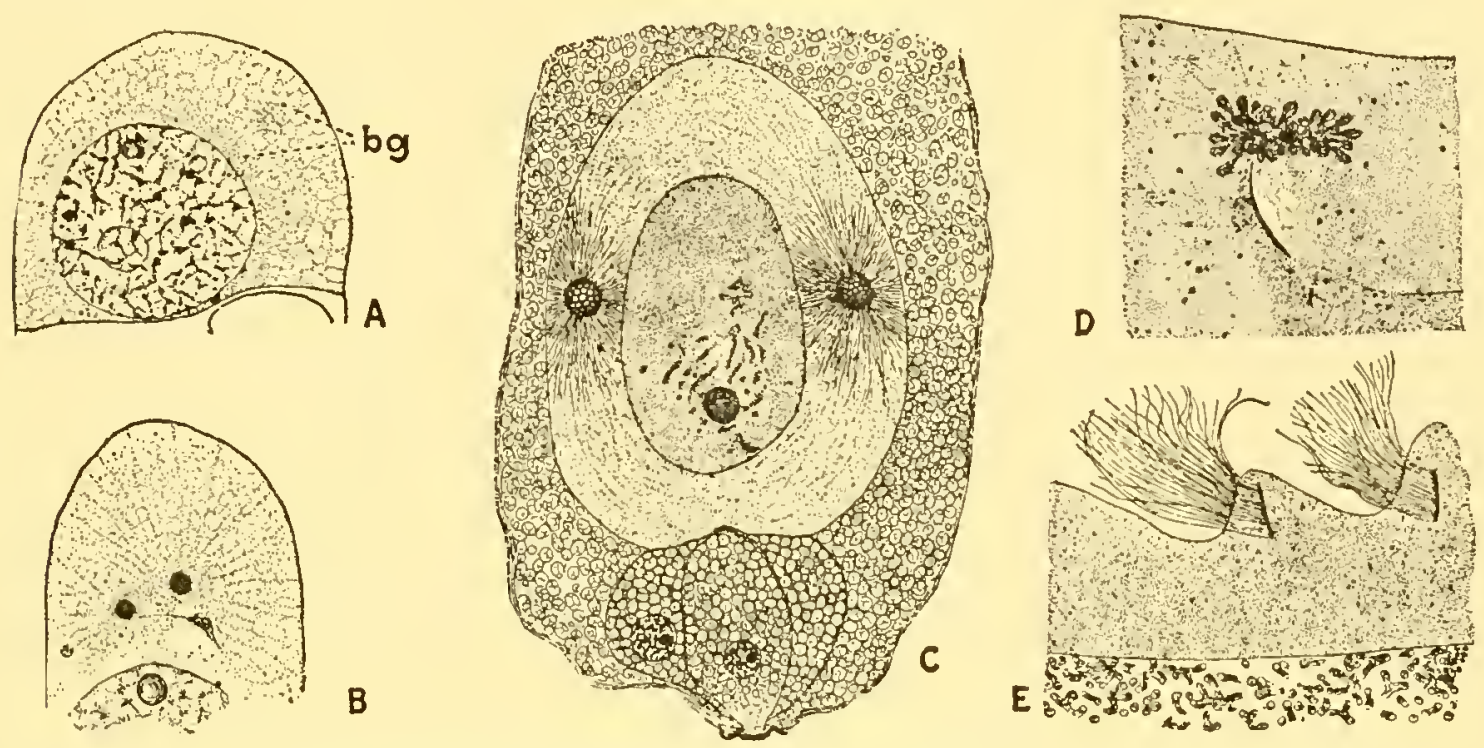

B

FIg. 124.- Stages in spermatogenesis in Dioön edule. A, "body-cell" with black granules in cytoplasm. $B$, two blepharoplasts differentiated. $C$, body-cell with two blepharoplasts; prothallial and stalk cells below; all in end of pollen tube. $D$, fragmentation of blepharoplast in spermatid as spiral band begins to form. $E$, portion of edge of section of spermatozoid ( $c f$. Fig. $116, D$ ) showing spiral blepharoplast eut at two points and cilia growing from it. (After Chamberlain, 1909.)

In the gymnosperms there are two principal types of spermatogenesis, some of the orders (Cycadales, Ginkgoales) having ciliated spermatozoids $^{12}$ and the others (Coniferales, Gnetales) non-motile male cells. In most cycads and Ginkgo a greatly enlarged "body-cell" in the developing pollen tube gives rise to two spermatozoids. In Dioön, for example (Fig. 124), numerous "black granules" appear in the body-cell cytoplasm. Two blepharoplasts now develop, apparently by the enlargement of two of the black granules, and take up positions on opposite sides of the nucleus. Very conspicuous radiations ${ }^{13}$ appear about them as the body-cell divides. The mitotic figure is wholly intranuclear, and, although the blepharoplasts remain opposite its poles, they appear to have no direct connections with it. A wall appears between the two daughter nuclei at the close of mitosis; in each of the resulting cells a

12 Hirasé (1894), Ikeno (1898), Fujii (1898, 1899), Webber (1897, 1901), Caldwell (1907), Miyake (1906), Chamberlain (1909, 1916), Kuwada and Maeda (1929).

${ }^{13}$ Hirasé (1894), Fujii (1899), and Kuwada and Maeda (1929) report that these are invisible in living cells. 
spermatozoid is organized. Even before the division of the body-cell nucleus the enlarging blepharoplasts become vacuolate. After the division is completed, each of them breaks up into a number of small fragments which then coalesce and begin the formation of the spirally coiled band that bears the many cilia. This band-like blepharoplast continues to elongate just within the cell membrane until it has made several turns, a prolongation of the nucleus remaining in contact with its growing end. The mature spermatozoid consists of an enormous nucleus, which in the living condition can be seen to undergo amœboid changes of shape, a cytoplasmic sheath, and the blepharoplast with its cilia.

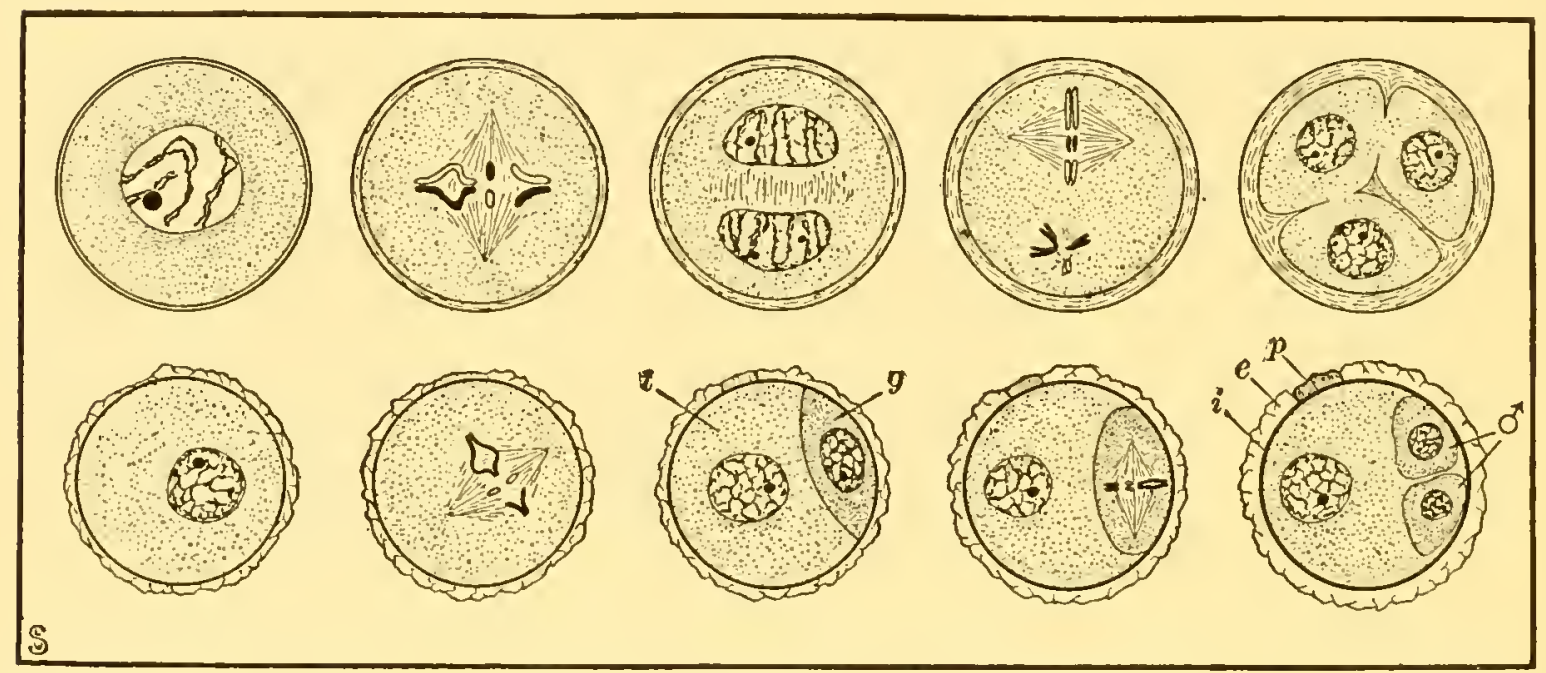

FIG. 125.-Microsporogenesis and development of male gametophyte in an angiosperm. First row: two meiotic mitoses in microsporocyte, followed by cleavage of protoplast into four microspores, three of which are shown. Second row: formation of male gametophyte by one microspore. $t$, tube cell; $g$, generative cell; $\sigma^{7}$, male gametes; $i$, intine; $e$, exine; $p$, germ pore with plug. Semidiagrammatic.

In the conifers, as in the cycads, the body-cell divides to form two male gametes, but here they are non-motile. In some genera (Taxodium) a wall separates the two nuclei after mitosis in the body-cell, but in others (Pinus) these nuclei lie together in the body-cell cytoplasm. As the body-cell moves down the pollen tube its outlines may remain distinct, but in some cases its cytoplasm becomes intermingled with that of the pollen tube. In the latter event the male gametes are simply nuclei, rather than complete cells. In some genera the two male gametes are differentiated in size and apparently in function, a tendency which reaches its fullest expression in the Podocarpineæ.

The theory that the blepharoplasts of bryophytes and vascular plants are homologous with centrosomes ${ }^{14}$ was at one time much disputed, but its validity is now generally conceded. A survey of the known cases suggests that centrosomes originally functioning in the ordinary way through several mitoses have tended to become restricted to one, and

${ }^{14}$ Belajeff (1897), Ikeno (1898 et seq.). See the review by Sharp (1912). 
that their original rôle has grown less evident as they have been modified in connection with their other function, namely, the bearing of cilia. In forms with non-motile gametes they do not appear.

Angiosperms.-In its essential features sporogenesis in angiosperms is like that in gymnosperms. In the anther the microsporocytes, usually as they lie in the anther fluid after rounding up, undergo two divisions to form quartets of microspores (Fig. 125). In some cases a separating wall is formed after the first mitosis, but more commonly cytokinesis does not occur until after the second mitosis, when the cell is divided simultane-
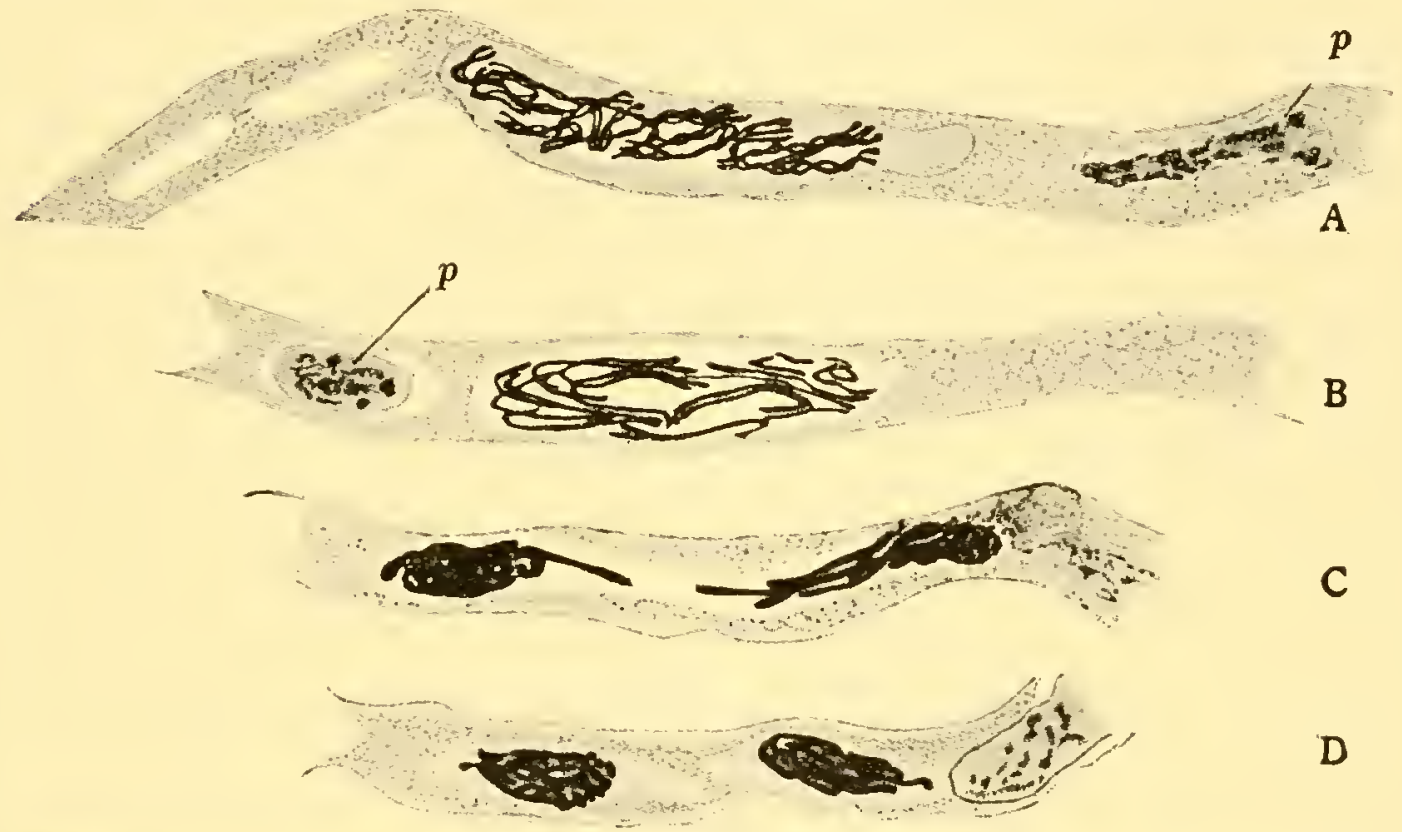

E

FIG. 126.-Development of male gametes in angiosperm pollen tube. $A-D$, division of generative nucleus and delimitation of male cells in Galanthus nivalis. $E$, male cells in Hemerocallis flava. The vegetative pollen-tube nucleus $(p)$ may either precede or follow the generative nucleus through the tube. (After Trankowsky, 1930b.)

ously by furrows or otherwise into the four spores (p. 167). Each microspore develops a thick wall and soon forms a very simple male gametophyte. The microspore nucleus, which has the reduced number of chromosomes, divides to two, around one of which a membrane cuts out a generative cell. The other nucleus and the rest of the microspore cytoplasm constitute the tube cell, which later grows out to form the pollen tube.

The generative cell divides to form two male gametes. This division sometimes occurs in the pollen grain before it is liberated from the anther, but probably more often it takes place in the growing pollen tube after 
pollination ${ }^{15}$ (Fig. 126). It has been shown in many cases ${ }^{16}$ that the male gametes are complete cells, at least while they are in the pollen grain or tube, and sometimes until they reach the embryo sac (p. 236); but it is also reported ${ }^{17}$ that their membranes sometimes disappear early, leaving the male nuclei free in the cytoplasm of the pollen grain or tube. The male nuclei in many species become vermiform, particularly after entering the embryo $\operatorname{sac}^{18}$ (Fig. 144). This change may occur more rapidly in one nucleus than in the other, the two thus being unlike at late stages. It is questionable whether or not such nuclei have any power of movement aside from that occasioned by protoplasmic streaming and surfacetension phenomena.

In the ovule a megasporocyte is developed by the enlargement of a subepidermal cell of the nucellus (Fig. 127). At the close of the first mitosis a wall is formed between the nuclei, and the two resulting cells (secondary megasporocytes) divide again, giving thus four megaspores. (In some cases the outer secondary megasporocyte does not divide.) The outer megaspores disintegrate, while the innermost one enlarges and develops the female gametophyte. Its nucleus, which has the reduced number of chromosomes, initiates a series of three divisions which result in eight nuclei. Ordinarily four of these lie near each end of the elongated embryo sac. In the micropylar end membranes are differentiated in the cytoplasm about three of the nuclei, forming an egg and two synergids, while in the opposite end three antipodal cells are similarly formed. The two remaining free polar nuclei move together and fuse sooner or later. The angiosperm egg usually shows very little special morphological differentiation, and except for its size and the position of its nucleus and

15 This constitutes a systematic character of some value (Schürhoff, 1926, p. 260); see this work for a review of the cytology of angiosperms. Wóyeicki (1926b) attributes the movement of the generative cell into the pollen tube to the action of vacuoles.

${ }^{16}$ E.g., Ishikawa (1918) on Enothera; Wylie (1923) on Vallisneria; Piech (1924b, 1928) and Kostrioukoff (1930) on Scirpus; Trankowsky (1930b) on Hemerocallis; Madge (1929) on Viola; Finn (1925, 1926a, 1928) on Asclepias, Vincetoxicum, and Vinca; and Finn and Rudenko (1930) on Orobanche.

${ }^{17}$ E.g., Dahlgren (1916) on Primula; Trankowsky (1930) on Convallaria and Galanthus; Schürhoff (1919b) on Sambucus; and Poddubnaja (1927) on Echinops.

${ }^{18}$ E.g., Mottier (1898a), S. Nawasehin (1899, 1900, 1909, 1910), Land (1900), Guignard (1900), Blackman and Welsford (1913), Welsford (1914), Sax (1916, 1918), and Heimans (1928).

Fig. 127.-Megasporogenesis, development of female gametophyte, syngamy, and early embryogeny in an angiosperm. 1-5, division of megasporocyte in to linear quartet of megaspores; meiosis oceurs in these two mitoses. 6-12, development of female gametophyte by innermost megaspore, this involving three mitoses. In 12 the largest cell in the upper end of the embryo sae is the egg, and two polar nuclei are in the center. 13, discharge of two male nuclei into embryo sac by pollen tube. 14, double fertilization, one male nueleus fusing with egg nucleus and the other with polar nuclei. 15 , embryo in two-cell stage; endosperm in ccenocytic stage. 16, embryo has developed cotyledons, and endosperm has become cellular. Semidiagrammatic. 


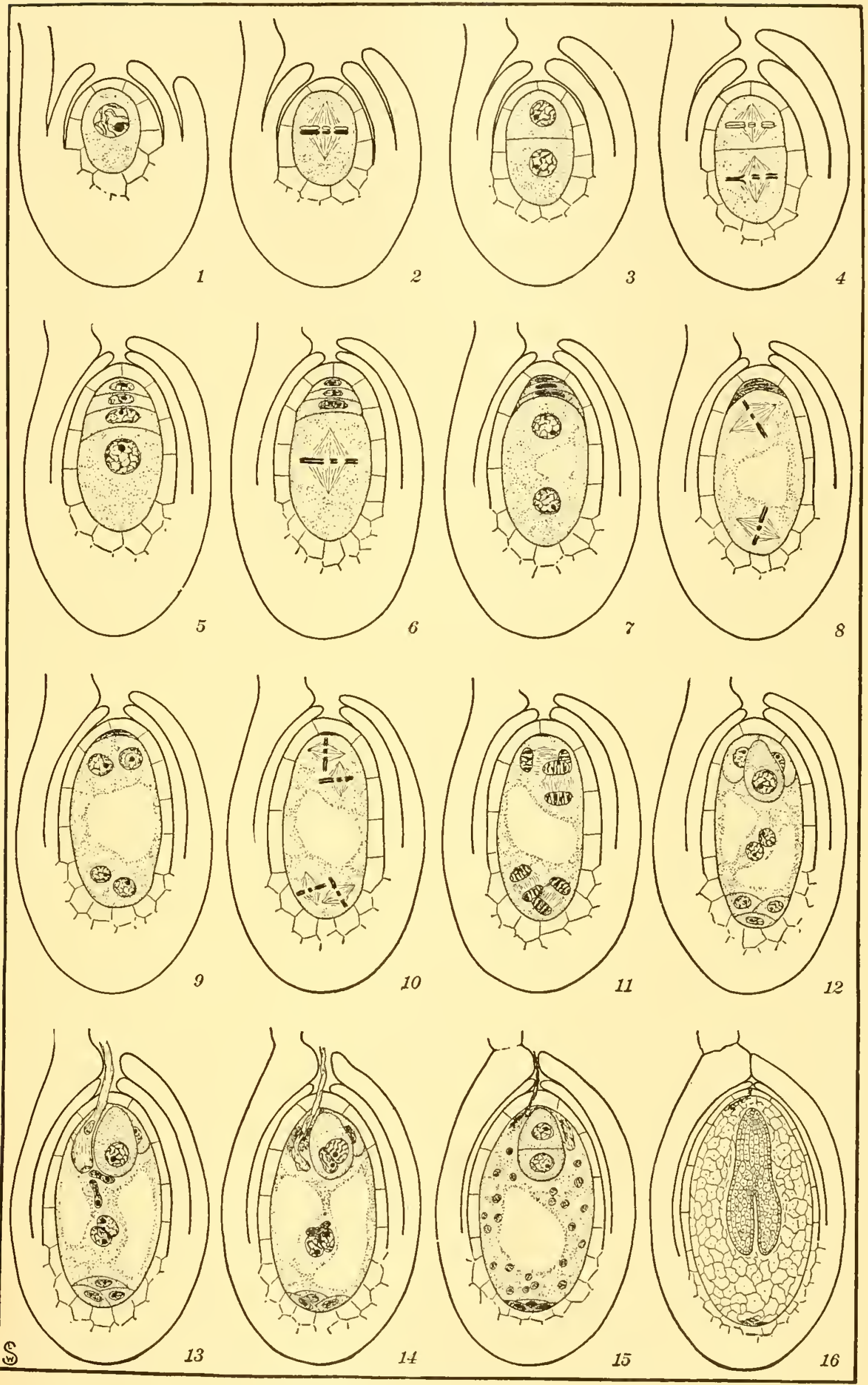

FIG. 127.-See p. 210 for legend. 
racuoles it closely resembles the synergids; in fact, cases are known in which synergids as well as eggs may develop into embryos. Commonly the egg is somewhat larger than the other cells, has its nucleus in the end toward the center of the sac, and has one or more large vacuoles. Visible ergastic materials other than cell sap are usually not conspicuous, but chondriosomes and plastid primordia are probably present regularly.

Many variations of the process just described have been found in the angiosperms. ${ }^{19}$ Probably the commonest of these is that exemplified in Lilium: here no walls are formed after either of the two mitoses in the megasporocyte, the four nuclei lying free in the cytoplasm of the embryo sac where they undergo one further mitosis to form a typical eightnucleate gametophyte (Sargant, 1896). The egg, therefore, is removed from the product of meiosis by a single mitosis, instead of by three mitoses as in the usual type of development.

A condition intermediate between this and the usual one is seen in Smilacina, where the walls form and then disappear, leaving the four nuclei to divide to eight (McAllister, 1909). Of exceptional interest is the situation in Plumbagella micrantha, described by Dalnlgren (1915). Here the four nuclei, formed free as in Litium, divide no further: one of them becomes directly the nucleus of the egg, two of them fuse like polar nuclei, and the fourth disintegrates in the base of the sac. This is the only known case among higher plants in which the gamete nucleus is the immediate product of meiosis, as in animals.

In several known instances the embryo sac is developed neither from a single megaspore as in the usual type, nor directly from the primary megasporocyte as in Lilium and Plumbagella, but from one of the secondary megasporocytes. In Cypripedium, for example, the nucleus of this cell undergoes the second meiotic mitosis, after which another mitosis occurs, giving four nuclei, one of which becomes the nucleus of the egg (Pace, 1907). Embryo sacs with more than eight nuclei have also been found. In Peperomia hispidula no walls are formed after the meiotic mitoses, the four nuclei dividing twice in succession to give 16. An egg and one synergid are organized, and the remaining 14 nuclei fuse (D. S. Johnson, 1907). The attention of the geneticist nray be called to the fact that when all of the embryo-sac nuclei arise from one of the four products of meiosis in a heterozygous plant, they may be assumed to be of the same genetic constitution; but when they are derived from more than one, as in several of the examples cited above, they may differ greatly, so that very unusual correlations between the characters of the sporophyte and those of the endosperm may be expected. In several instances two or more of the described modes of development are known to occur in the same family and even in the same indiridual.

${ }^{19}$ For the types of embryo sac development, see Rutgers (1923), Stenar (1925), Schürhoff (1926), Chiarugi (1927), Schnarf (1929), and Modilewski (1929). 
Oögenesis in Animals. - The development of the animal egg varies considerably in cletail in the different groups. The general features of the process as it occurs in typical cases may be briefly sketched as follows. ${ }^{20}$ In the ovary certain cells known as oögonia enlarge somewhat and become primary oöcytes. When first distinguishable the oöcyte is relatively undifferentiated, but it soon passes through a growth period during which it enlarges greatly and becomes supplied with one or more kinds of yolk (Fig. 128). A rôle in the elaboration of these products has been assigned to the chondriosomes and Golgi material by a number of investigators (pp. 78 to 88). In some animals the changes during the growth period
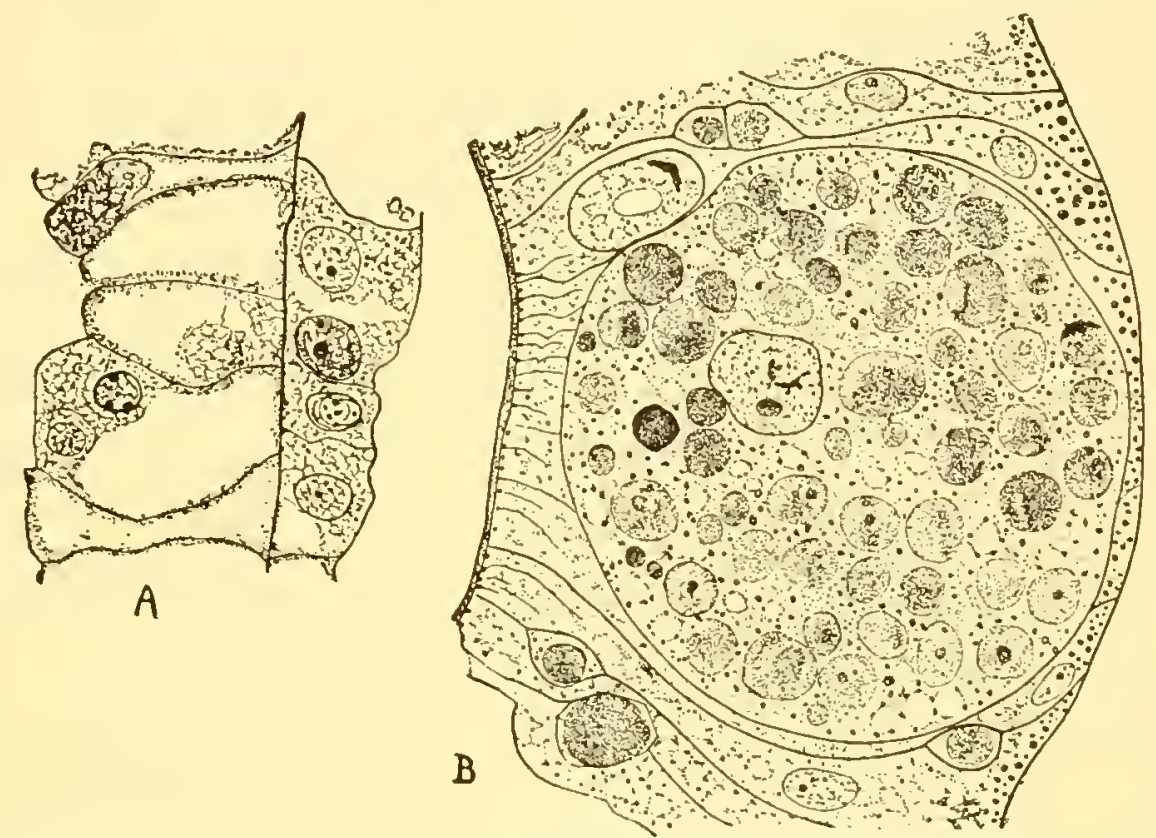

Fig. 128.-Differentiation of oöcyte in Hydra. A, very young oöcyte between ecto. dermal cells at right. $B$, oöcyte after growth period, with yolk globules. (After Downing, 1909.)

involve the activity of a special follicular epithelium organized about the oöcyte, or a group of "nurse cells." The oöcyte at this stage is often called the "ovarian egg" and its large nucleus the "germinal vesicle."

At the close of the growth period the oöcyte nucleus, which is already in an advanced stage of the prophase, undergoes division near the cell membrane. One of the daughter nuclei so formed is included in the first polocyte, or "polar body," a small cell cut off at this point (Fig. 154). The nucleus remaining in the large cell, now called the secondary oöcyte, quickly undergoes another division, one of the resulting nuclei being included in a second polocyte, or "polar body," while the other remains in the now mature ovum, or egg. The first polocyte may divide and thus complete a quartet of cells, but often this does not occur. Normally the polocytes are functionless and soon degenerate, sometimes within the

${ }^{20}$ See Hegner (1914b), Wilson (1925), and Corner (1932). The question of the origin of the germ eells is reviewed by Harms (1926) and Heys (1931). 
egg. The two mitoses in the oöcyte are meiotic in character and result in a reduction from the zygotic to the gametic number of chromosomes. They are frequently called "maturation divisions."

From the foregoing it is evident that the animal ovum develops most of the features characterizing it as a gamete before the meiotic divisions take place. It will be seen later (p. 239) that in some cases the spermatozoön enters the egg before these divisions are completed. It is a further significant fact that in many animals the differentiation of visibly distinct cytoplasmic regions having a relation to parts of the future
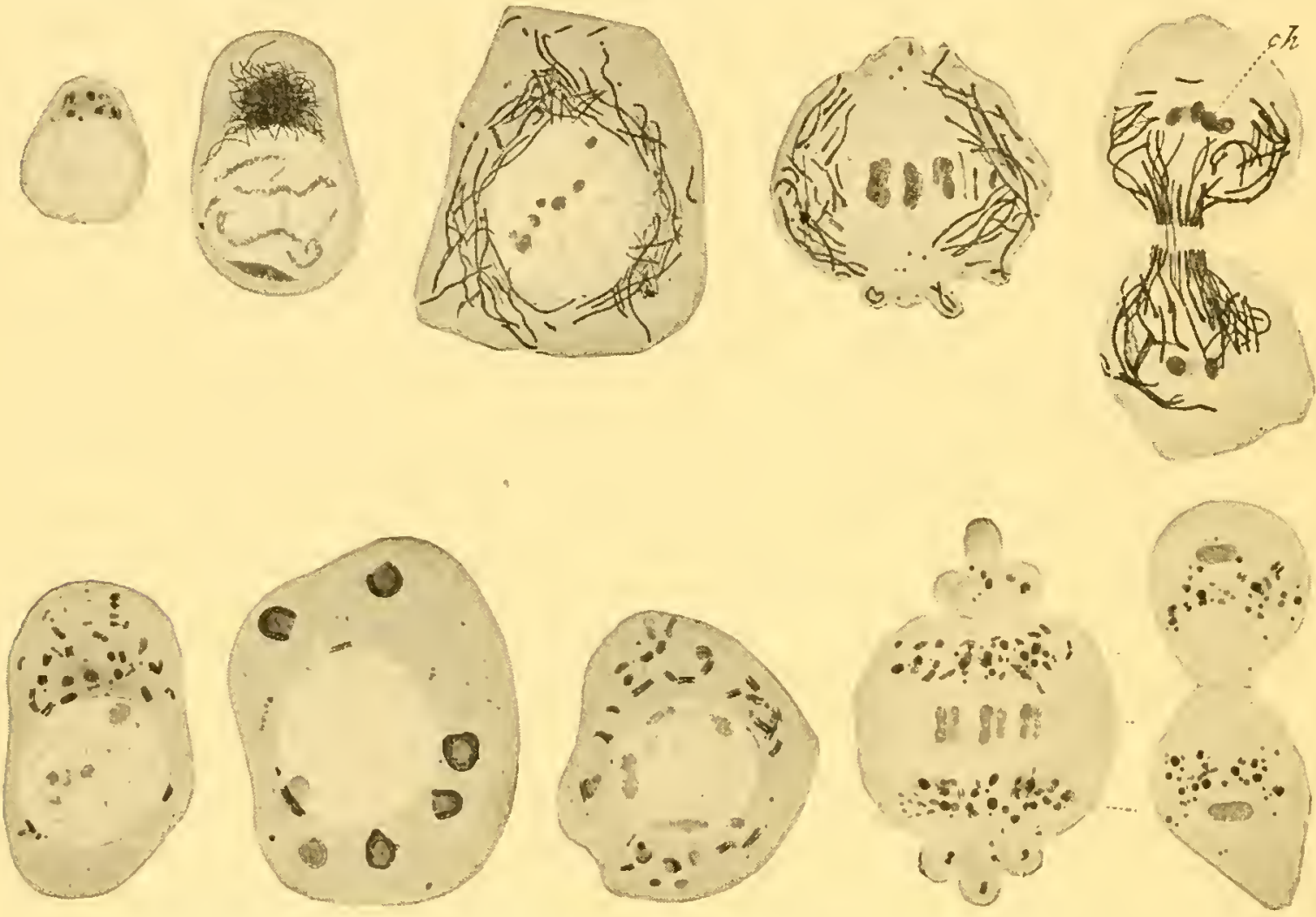

F1G. 129.- First division of spermatocyte of Euschistus, showing behavior of chondriosomes (first row) and Golgi material (second row). ch, chromosomes. (After Bowen, 1920.)

embryo may begin in the oöcyte or ovum before the entrance of the spermatozoön; in other words, embryogeny may actually begin before fertilization (p. 419).

The ovum is surrounded by a delicate vitelline membrane, which becomes conspicuous only after fertilization, a thicker layer of ten showing radial structure (vertebrates) and sometimes a third layer of structureless jelly. The surrounding cells may add certain secondary layers, and later there may be deposited one or more protective envelopes, such as the albumen, shell membrane, and shell of the bird's egg.

Spermatogenesis in Animals. - In the male animal those cells (spermatogonia) in the testis whose ultimate descendants are to become spermatozoa multiply by divisions of the ordinary type until a certain number are prockuced. These cells, now called primary spermatocytes, enlarge and 
undergo two successive divisions: the first division results in two cells called secondary spermatocytes; the second divides the two secondary spermatocytes into four spermatids, each of which then becomes transformed into a spermatozoön. The mitoses in the spermatocyte are meiotic in character and bring about a reduction in the chromosome number. Besides the spermatogenous cells, the testis also contains certain accessory cells, such as the Sertoli cells of mammals and Verson's cells of certain insects.

The transformation of the spermatid into a spermatozoön (spermiogenesis) has long been one of the most fascinating and difficult problems in structural cytology. The several components of the cell undergo an astonishingly complicated series of transformations, and these, together with the types of spermatozoa resulting, differ considerably in detail from group to group. This has naturally led to much confusion in the literature on the subject, although many points were well covered by the early investigations. The outstanding researches of Bowen (1920-1926) have so clarified our conceptions that his descriptions will be used as the principal basis of the following account. Bowen worked chiefly with insects (Hemiptera, Orthoptera, Lepidoptera, Coleoptera, Aptera) and amphibia (Plethodon), but comparisons show that his descriptions, with minor modifications, will apply to the general features of spermatogenesis in various other groups. ${ }^{21}$

The Spermatocyte and Its Divisions (Fig. 129). - In the spermatocyte are the following components: nucleus, cytoplasm, chondriosomes, Golgi material, a pair (usually) of centrioles, and frequently other formed elements of questionable nature. The chondriosomes are present in the form of granules, rods, or threads, and, although these behave variously in different species, they are usually distributed rather equally to the four spermatids in the two divisions. This in some cases involves the passive division of individual chondriosomal threads or an even more precise distribution (p. 86). The centrioles diverge to opposite sides of the cell and occupy the poles of the achromatic figure in each mitosis. At the close of the second mitosis the centriole usually divides, so that the spermatid has a pair of them. Much confusion in terminology and diversity of interpretation have arisen concerning the special differentiations which may occur about the centrioles and the topographic relation which certain other cell components, notably the Golgi material, may bear to them.

Two general modes of arrangement of Golgi material in spermatocytes may be distinguished. In the insects (Bowen) a number of Golgi bodies,

${ }^{21}$ For further gencral accounts of animal spermatogenesis, sec Wilson (1925), Metz (1932), and the résumé by Boweı (1924c). See the extensive researches of Gatenby (1917 et seq.). Other recent works are those of Voïnov $(1925,1927 a b)$, Nath (1925), Chickering (1927), Sokolow (1926, 1929ab), Payne (1927), Hirschler (1928), Pollister (1930), and H. H. Johnson (1931). 
each composed of two substances differing in chromaticity, are scattered throughout the cytoplasm. At each of the meiotic divisions they break up into fragments (dictyosomes) which become arranged around the nucleus at the equator of the cell and pass as equal groups to the two poles in advance of the chromosomes. In Gerris Pollister finds that only the osmiophilic substance forms the dictyosomes, the osmiophobic (and
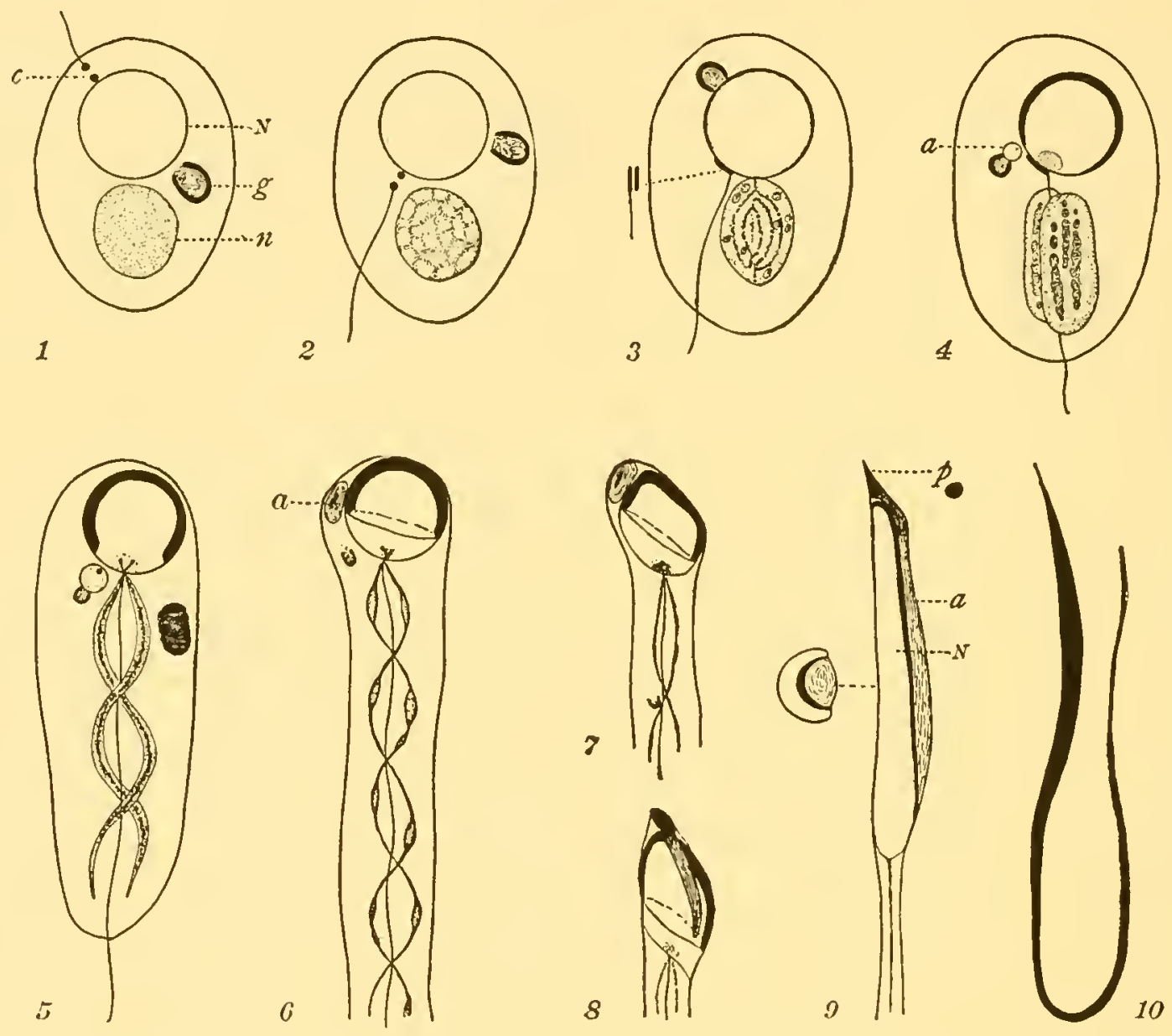

FIG. 130.-Diagram illustrating principal features of spermiogenesis in animals. $a$, acrosome; $c$, centrioles; $g$, Golgi material (acroblast); $N$, nucleus; $n$, nebenkern; $p$, perforatorium. (Based on diagrams and other figures of Bowen.)

neutral-red staining) substance remaining behind. In another series of forms (mollusks, amphibia, other vertebrates) the Golgi bodies are closely aggregated about the centrioles where their lightly staining substance flows together to form the idiosome, to the surface of which their deeply staining constituent adheres as separate chromophilic rodlets. This chromophilic matter often appears as a network or a continuous shell instead of separate rodlets, but it is uncertain how far such appearances are due to the action of fixatives. It was formerly thought that the idiosome substance was related primarily to the centrioles, the Golgi material 
collecting about it at certain stages, but the situation in insects seems to show that the real unit is the Golgi body composed of chromophilic "Golgi matter" and relatively chromophobic "idiosome substance," a close topographic relation to the centrioles being assumed only in certain cases. During the meiotic divisions of the nucleus the Golgi rodlets (or network or shell) form a number of dictyosomes which pass as two groups to the poles; whether each divides or not is a question. The fate of the idiosome substance is difficult to determine, but it is probable that each dictyosome is a minute Golgi body with its two substances, which are thus distributed to the four spermatids.

Each spermatid consists of cytoplasm, a nucleus with the gametic number of chromosomes, some chondriosomes, a pair of centrioles, a number (usually) of Golgi bodies, and often ergastic inclusions. These various elements will now be followed through the metamorphosis of the spermatid into a spermatozoön (Figs. 130 to 134).

Centrioles.-In the Hemiptera the two centrioles are situated near the anterior end of the young spermatid, one of them lying in contact with the nuclear membrane. From the distal member of the pair the delicate axial filament of the flagellum grows out and pierces the cell membrane. In many insects this filament may appear much earlier, before mitosis is complete or

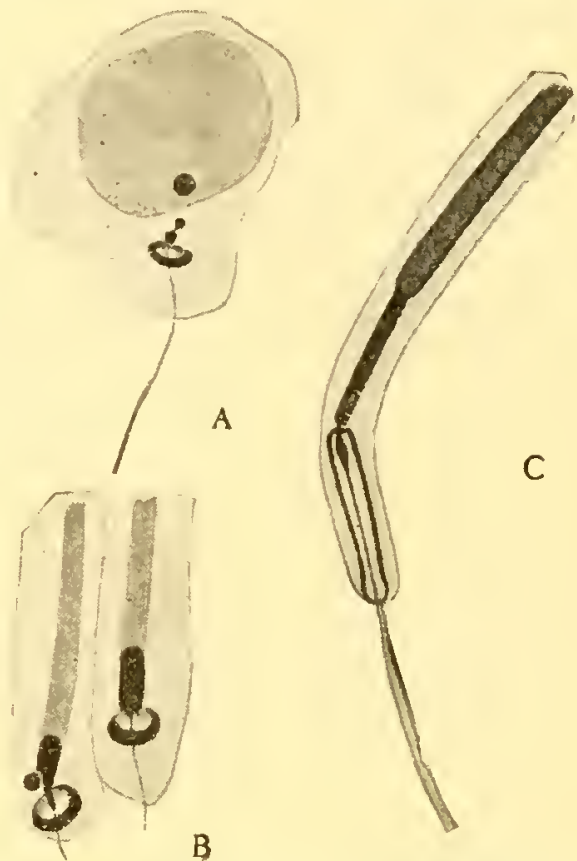

FIG. 131.-Behavior of centrioles in spermiogenesis in Plethodon cinereus. (After Bowen, 1922a.) even in the primary spermatocyte (moths). The centrioles now migrate to a position near the posterior pole of the nucleus, where they take the form of parallel rods lying against the nuclear membrane, and soon form a $\mathrm{V}$, with the elongating axial filament extending from its angle. An intranuclear body ("pseudoblepharoplast") present during these stages has often been confused with the centrioles. The centrioles eventually constitute the so-called "centrosomal middle piece" at the base of the sperm head, but in insects this is not a very distinct structure.

The centrioles in certain other animals show curious modifications into rings and knob-like structures which have been variously interpreted (Fig. 131). In his accounts of spermatogenesis in the salamander Meves $(1896,1897)$ reported that the distal centriole, after the appearance of the axial filament, transforms itself into a ring. The filament then passes through this ring and attaches itself to the proximal centriole, which pushes into the base of the nucleus and enlarges to form the middle piece. Meanwhile the ring divides into two parts, one of which becomes the 
"end-knob" at the posterior end of the middle piece, while the other passes backward along the axial filament. In mammals (rat, man) Meves (1898, 1899) found much the same situation: the proximal centriole remains near the nucleus and does not elongate to form a middle piece. The distal centriole then divides, one part forming the end-knob and the other passing back as a ring to the end of the "connecting piece" (which does not correspond to the middle piece of the salamander). In Ecanthus nigricornis, a cricket, the distal centriole, to which the axial filament is attached, constricts off a portion which encircles the filament and works backward along it to the end of the tail sheath (H. Johnson, 1922, 1931).

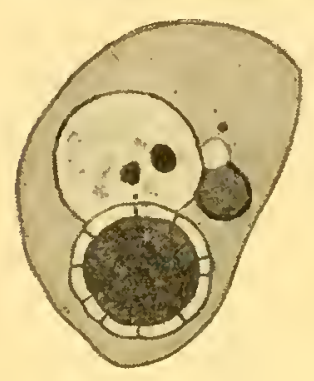

1

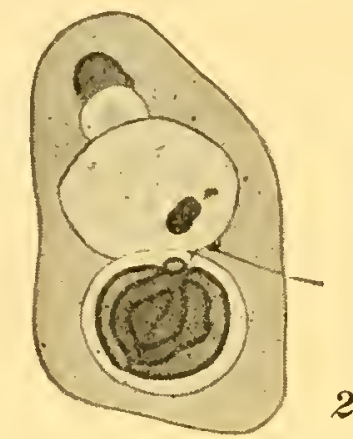

2
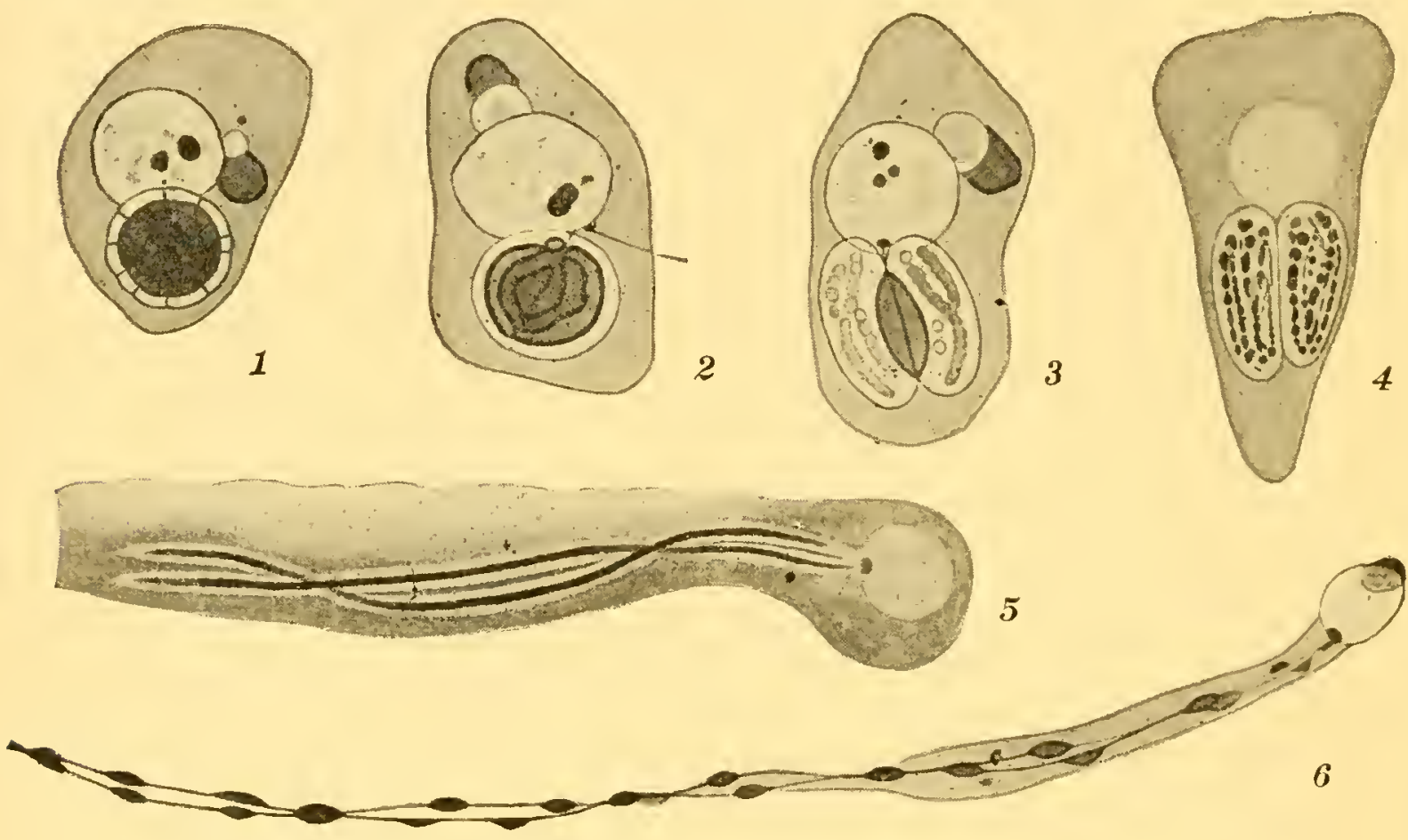

Fig. 132.-Transformation of nebenkern in Brochymena. (After Bowen, 1922cd.)

Chondriosomes.-In insects generally the chondriosomes form a single more or less compact body, the nebenkern (the mitosome of Gatenby) (Fig. 132). Globules of a lightly staining fluid appear in the nebenkern, chiefly near its periphery, giving the "blackberry stage" reported after certain fixatives. Soon the more deeply staining portion takes the form of a series of concentric plates alternating with layers of the lighter substance (the "onion stage"). The platework now becomes a hollow ovoid mass with a median partition. As the nebenkern elongates, the ovoid mass shrinks; as it does so, a furrow follows it inward and eventually cuts the nebenkern in two as the chromatic mass vanishes. Meanwhile globules of an additional "central substance" appear in the light nebenkern material, arrange themselves in rows, and coalesce to form beaded strands. As the nebenkern halves elongate further, these strands unite until each half finally has a single axial core composed of the central substance. 
The nebenkern halves now come to lie on either side of the axial filament and rapidly elongate to form the two "filament sheaths" which become twisted about the axial filament. Their anterior ends appear to attach themselves to the centrioles at the base of the nucleus. As they become longer and thinner they show a series of swellings which may possibly represent accumulations of the central substance. These eventually disappear and the uniform sheaths extend nearly, if not quite, to the tip of the axial filament.

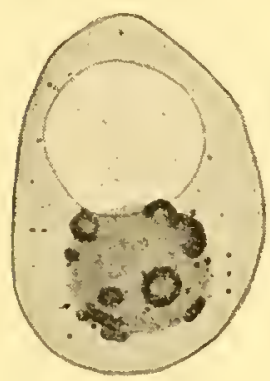

A

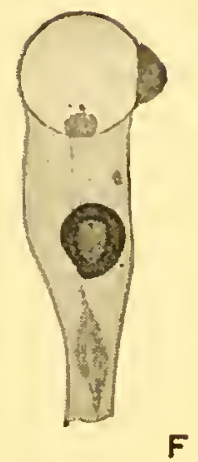

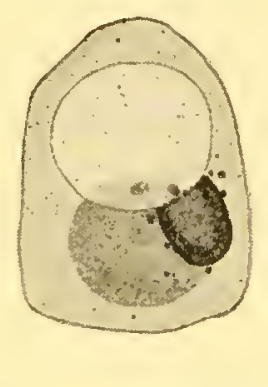

B

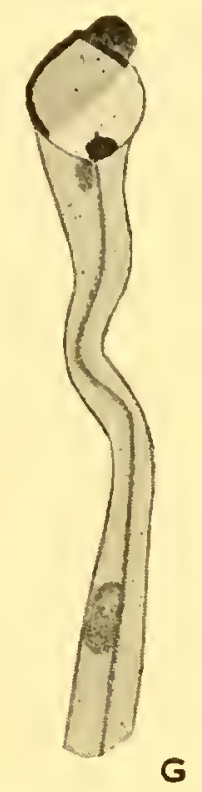

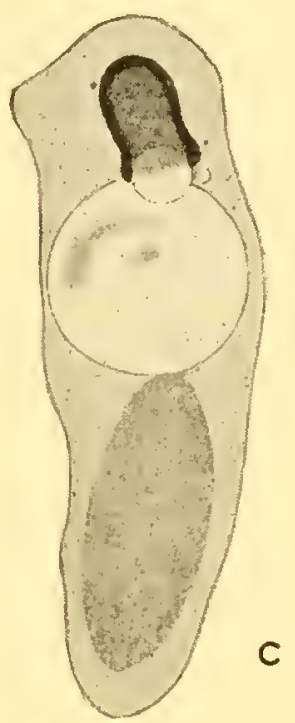

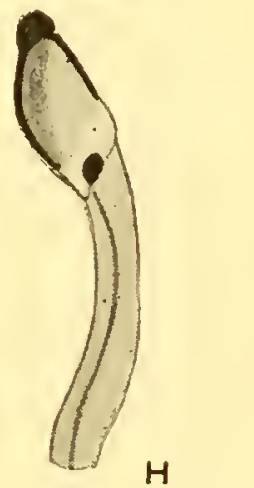

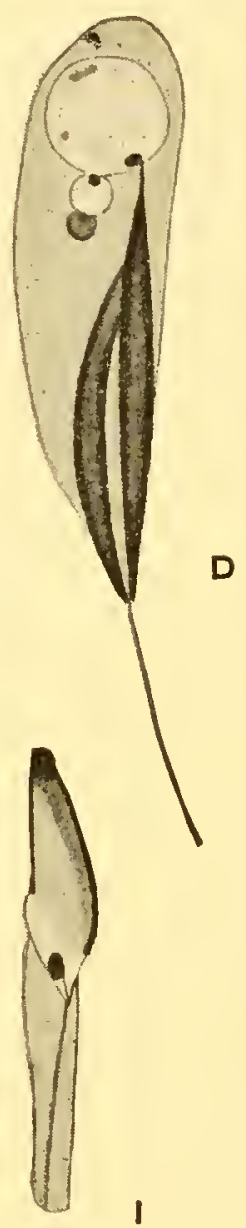

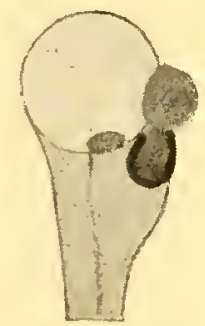

E

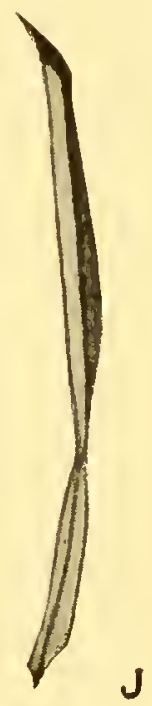

FIG. 133.-Behavior of Golgi material in spermatids of certain insects. A, F, Brochymena; $B-E$, Euschistus; $G-J$, Murgantia. Cf. Fig. 130. (After Bowen, 1920, 1922c.)

Golgi Material (Fig. 133).--In the Hemiptera the Golgi constituents of the spermatid usually behave as follows. At the close of the meiotic divisions the dictyosomes fuse to form several larger Golgi bodies much like those of the spermatocyte. The fusion continues until there is formed a single large acroblast, ${ }^{22}$ which lies near the base of the nucleus on the side opposite that to which the centrioles migrate. Ordinarily it has a heavily staining shell except on the side next to the nucleus. The acroblast soon begins a migration around to the other side of the nucleus, passing first to the anterior end of the cell where it pauses for a time. It

${ }^{22}$ Often referred to in the literature as the "spermatid idiosome," or "sphere." In mollusks this mass has frequently been ealled the "nebenkern." 
then continues along the nuclear membrane on the other side, following the path of the centrioles, and eventually comes to lie near them.

During the migration of the acroblast an acrosome is differentiated from it. This first appears as a delicate vesicle on the side of the acroblast near to the nucleus and seems to consist of a new material, the formation of which is dependent upon the activity of the substance of the acroblast. ${ }^{23}$ Within it is a small granule. Very rarely the acrosome itself has the form of a granule rather than a vesicle (mollusks, Gatenby; grasshopper, Bowen). The acroblast becomes somewhat smaller as the acrosome grows, and together they move again toward the anterior end of the cell. Either before or after reaching the apex they separate, the acrosome taking its final position near the apex while the remainder of the acroblast, known as the "Golgi remnant," passes backward along the tail and is ultimately cast out of the cell in a protoplasmic ball sloughed off at the close of spermiogenesis. ${ }^{24}$

The acrosome, after reaching its definitive position near the anterior end, undergoes a further differentiation. Within it appears a deeply staining mass which may possibly bear some relation to the granule observed in the vesicular acrosome at earlier stages. It rapidly develops into the pointed perforatorium at the tip of the spermatozoön, while the remaining portion of the acrosome grows backward as a band against one side of the asymmetrical and elongating nucleus, with which it becomes very intimately united as the spermatozoön matures. It is the view of Bowen (1924b) that the acrosome or perforatorium is not merely a boring organ, as commonly thought, but a secretion with a special function in fertilization, thus corresponding in origin with the secretion droplets observed to arise in connection with the Golgi material in gland cells (p. 76). Van Herwerden has shown the presence of an oxidase in the acrosome region. Certainly the acrosome cannot be a boring organ in the spermatozoön of Lepisma, in which it lies at the base of the nucleus rather than anterior to it.

Nucleus. - No conspicuous changes occur within the nucleus until the acroblast and its differentiated acrosome have completed part or all of

${ }^{23}$ In Lepidoptera and grasshoppers the Golgi bodies do not fuse but give rise to a eommon acrosome (Bowen, 1922ae). Parat (1928) regards the acrosome as a vacuole, largely because it stains with neutral red. Pollister (1930) finds no neutral-red staining substance in Gerris from the elose of the first spermatocyte division to the formation of the acroblast in the spermatid; it then appears as a secretion within the acroblast. H. Johnson (1932) finds the chondriosomes and the chromophilic rim of the dietyosomes in insect spermatocytes to react similarly to Janus green and certain other vital dyes and questions the complete homology of the dictyosome-acroblast complex with the somatic Golgi apparatus.

${ }^{24}$ Sjövall (1906) on the guinea pig, Terni (1914) on Geotriton, Gatenby (1917) on mollusks and Lepidoptera. In certain mammals a portion of the Golgi remnant is said to remain as a part of the middle piece (Weigl, 1912; Gatenby and Woodger, 1921). 
their migration around it. The chromatic substance is then seen to arrange itself in a layer against the inside of the nuclear membrane, and as spermiogenesis proceeds it abandons the posterior portion and collects as a compact, deeply staining cap at the anterior side. As the acrosome begins to form the perforatorium, this chromatic cap becomes very asymmetrical and the whole nucleus elongates and begins its transformation into the slender sperm head. The "pseudoblepharoplast," which has already been mentioned and which may possibly represent chromosomal material, disappears at about this stage. The head continues to elongate in the form of a narrow spoon with the acrosomic band lying in the hollow; eventually it becomes a slender pointed body in which nucleus and acrosome can no longer be distinguished. During these changes the non-chromatic constituents of the nucleus gradually diminish and disappear, so that the nucleus of the spermatozoön is an extremely concentrated chromatic mass in which structure can be demonstrated only in rare cases.

Cytoplasm.-As the other components of the spermatid undergo the transformation outlined above, the cytoplasmic cell body elongates and remains visible around them (with the possible exception of the tip of the axial filament) until a comparatively late stage. Toward the close of spermiogenesis in the insects it has been ascertained that a cytoplasmic ball, containing the Golgi remnant and other granules of an unknown nature, is sloughed off and ingested by the nutritive epithelial cells lining the spermatic cyst. In other animals a cytoplasmic mass is likewise eliminated, but the amount of "active" protoplasm contained in it is uncertain.

The Mature Spermatozoön (Fig. 134).-The mature spermatozoön has two main parts: the head, which is a dense chromatic mass comprising the nucleus, the acrosome, and sometimes a skeletal fiber of uncertain origin; and a tail, consisting of an axial filament produced as an outgrowth from the distal centriole, the thread-like sheaths formed by the chondriosomal derivatives, and an uncertain amount of residual cytoplasm. It is probable that a thin cytoplasmic membrane exists about the head also, although this is ordinarily invisible. Koltzoff (1909) was able to demonstrate a membrane in this region by using hypotonic solutions. In Arbacia and Nereis, according to Popa (1927), the sperm head con-

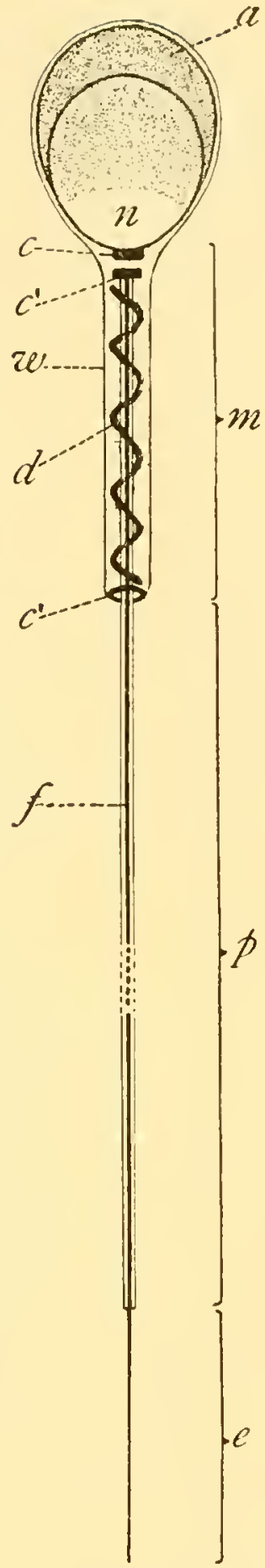

FIG. 134.-Diagram of a typica. manmalian spermatozoön. a, acrosome; $n$, nucleus; $c$, proximal centriole; $c^{\prime}$, distal centriole and derivatives; $w$, cell membrane; $d$, chondriosomes; $f$, axial filament of tail; $m$, $\mathrm{mid} \mathrm{d} \mathrm{le} \mathrm{piece;} p$, principal piece of tail; $e$, end piece of tail. (After Bowen, 1924c.) 
sists of a central hydrophilic substance representing the nucleus, a peripheral lipochromatic layer consisting of cytoplasm with lipides and lipoproteins, and a delicate outer membrane; a fatty layer surrounds the whole cell. In Sepia, W. J. Schmidt (1928) concludes that the head, which is anisotropic in polarized light, is composed largely of submicroscopic rodshaped micellæ arranged parallel to the longitudinal axis.

In many animals, particularly mammals, a "middle piece" is more distinctly differentiated than it is in insects, the spermatozoön therefore appearing to have three main parts. In general it is more convenient to
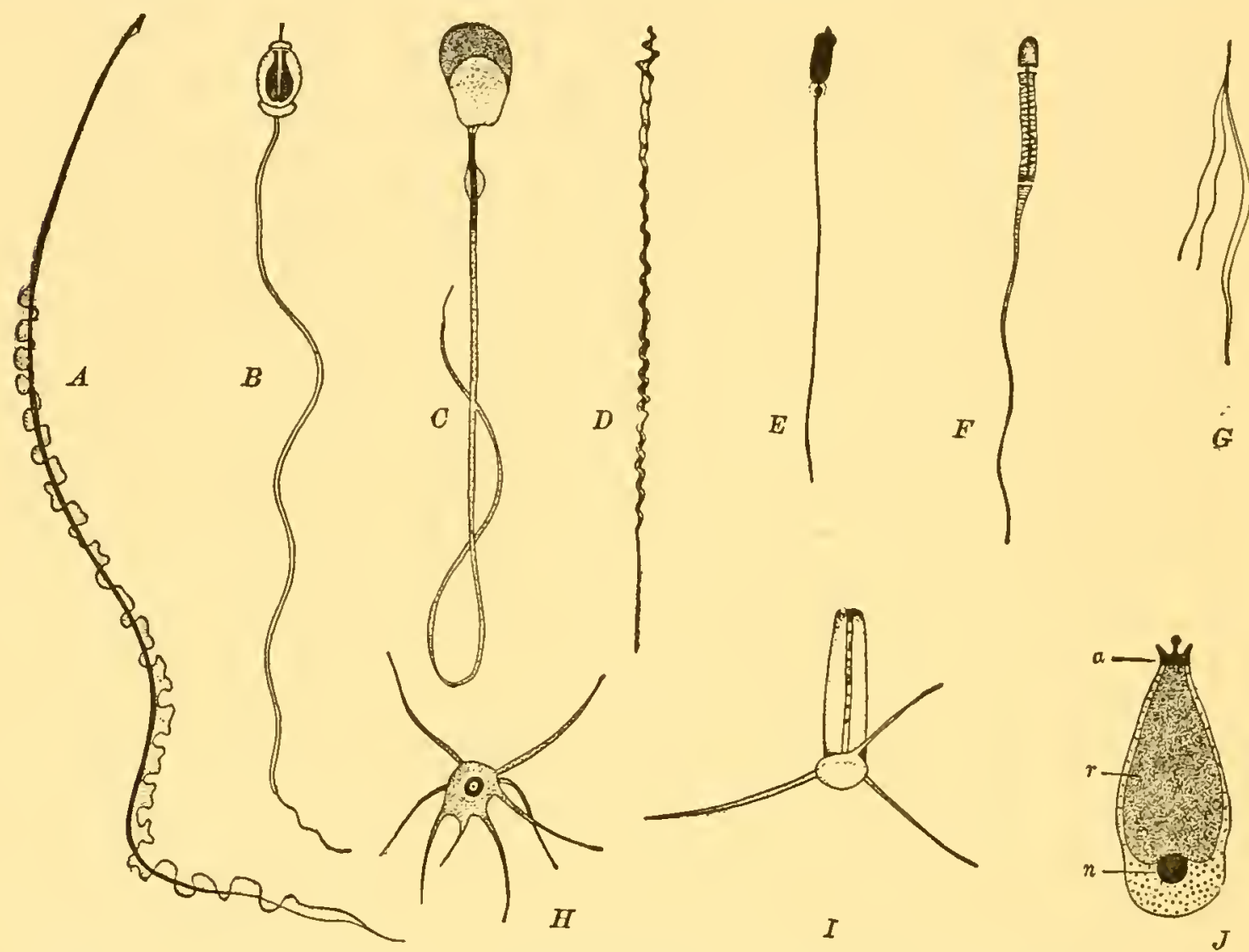

FIG. 135.- Spermatozoa of various animals. A, Triton (salamander). $B$, Nereis (annelid). $C$, guinea pig. $D$, Phyllopneuste (bird). $E$, sturgeon. $F$, Vesperugo (bat). $G$, Castrada (turbellarian). H, Pinnotheres (crustacean). I, Homarus (lobster). J, Ascaris (nematode): $a$, apical body; $n$, nucleus; $r$ " "refringent body." (From the works of Ballowitz, F. R. Lillie, Meves, Luther, Koltzoff, Herrick, and Scheben.)

think of the middle piece as a portion of the tail. The tail of the typical animal spermatozoön as a rule may be said to have three segments, though in certain animals all of them may not be differentiated. These are the "middle piece," in which the axial filament is enclosed in a sheath of undifferentiated cytoplasm carrying the mitochondrial elements and the centrioles; a "principal piece," which has a thin sheath of questionable origin but no undifferentiated cytoplasm; and an "end piece," which is the naked posterior end of the axial filament. Many minor variations in all of these cell elements are known, the spermatozoa of different animals thus differing widely in appearance (Fig. 135). 
In some animals, notably certain arachnids, crustaceans, myriapods, and nematodes, the spermatozoön has no tail. ${ }^{25}$ Although the structures developed in such bizarre types of sperms are very different from those of the ordinary flagellate type, Bowen finds that they are derived from the same components of the spermatid. For example, the "refringent body" in nematodes, the "capsule" in decapods, and the "honeycomb" and "valves" in myriapods are all derived from the Golgi apparatus and hence may be compared to the acrosome of other spermatozoa.

The Structure of Other Ciliated Cells.-In addition to zoöspores and gametes, there are other cells which possess special motor structures. Of particular interest are the motor apparatus of flagellates and the ciliary mechanism of certain epithelial tissues.

Many types of motor apparatus are known in motile organisms. ${ }^{26}$ In Menoidium (Fig. 136) the apparatus consists of a single flagellum, a blepharoplast, a centrosome at the surface of the nucleus, and a rhizoplast connecting the blepharoplast with the centrosome. At the time of cell-division the blepharoplast divides, its halves remaining connected for a time by a slender paradesmose. Similar in many respects is the mechanism in Peranema (Hall and Powell; Brown). In Polytoma and other forms with two flagella (Fig. 110) there are also two blepharoplasts, between which a paradesmose is usually present, at least during division. ${ }^{27}$ In some instances a centronema is said to extend from the centrosome inward to a karyosome. In Trichomonas muris there is a single body which acts as

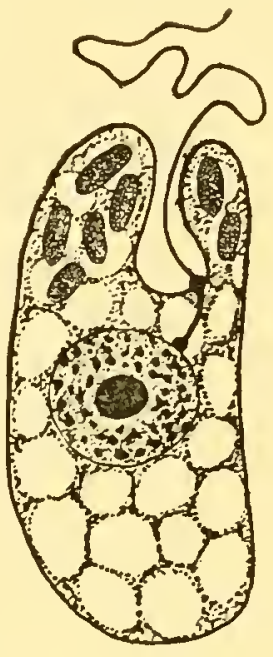

FIG. $136 .-$ Menoidium incurvum, showing motor apparatus. See text. (After $H$ all, 1923.) both blepharoplast and centrosome. To this "centroblepharoplast" are attached three short, free flagella and one long one forming the margin of the undulating membrane. Along the base of the membrane and connected with the blepharoplast is a "chromatic basal rod," and within the cell may be seen certain other peculiar differentiations. As mitosis begins, the blepharoplast buds off a smaller new one and the two diverge like centrosomes to opposite poles of the achromatic

${ }^{25}$ See Bowen (1925b) and literature there cited; also Sokolow (1929ab).

${ }^{26}$ For accounts of the flagellar apparatus of flagellates and Protozoa, see Minchin (1912), M. Hartmann (1911), Wilson (1925), Calkins (1926) and the papers by R. G. Sharp (1914), Yocum (1918), C. V. Taylor (1920), Rees (1921), MeDonald (1922), Hall (1923, 1925a), Hall and Powell (1928), Kofoid and Swezy (1915-1923), Kofoid and Christiansen (1915), Entz (1918), Niesehulz (1922), Bělař (1921), Wenrich (1921), A. S. Campbell (1926, 1927), Bunting and Wenrich (1929), Kater (1929), and V. E. Brown (1930). For accounts of cilia and their action, see Heidenhain (1911), Lundegårdh (1922), Petersen (1929), Gray (1928, 1931), and Lueas (1932).

${ }^{27}$ Polytoma uvella (Entz, 1918), Chlamydomonas nasuta (Kater, 1929), Oxyrrhis marina (Hall, 1925a). 
figure, a paradesmose being drawn out between them. Apparently, one or two of the free flagella aceompany the new blepharoplast, new ones later growing out to restore the full number. It is also aceompanied by a new chromatic basal rod, which begins to differentiate as a row of granules even before the division of the original blepharoplast (Wenrich, 1921).

In the trypanosomes, as in the infusoria, there are two nuclei: the trophonucleus and the kinetonucleus. Motor mechanisms differ considerably within the group, but it seems that the flagellum is usually borne

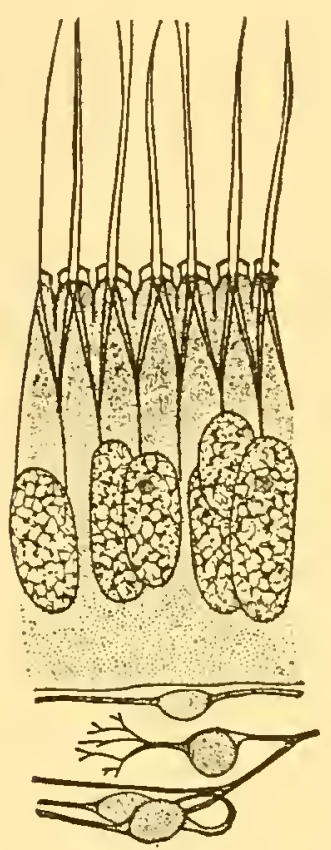

A

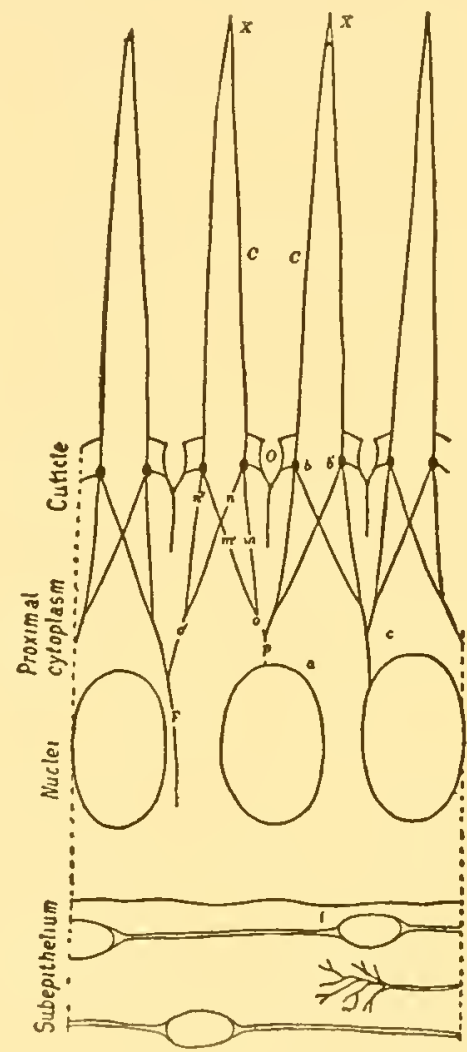

B

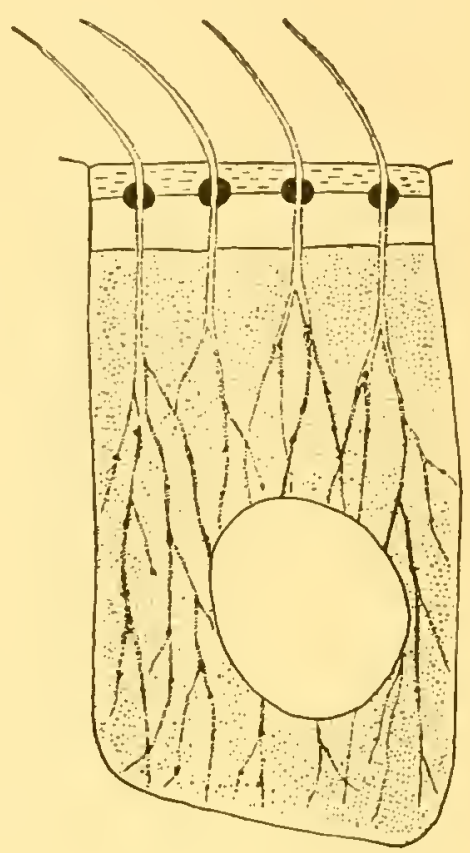

C

Fig. 137.- $A, B$, ciliary mechanism in Lampsilis. The cilia (c) are fusrd in pairs at their tips. Below the basal corpuscles $(b)$ in the cuticle each cilium splits into two strands which connect with strands from other basal corpuscles. (After Grave and Schmitt, 1924.) $C$, diagram of a ciliated epithelial cell based on the figures of Saguchi (1917).

on a basal granule (centrosome?) associated with the kinetonucleus. At the time of cell-division the trophonucleus, kinetonucleus, and basal granule all divide. Although it was formerly thought that the flagellum also split, it now scems clear that it remains attached to one half of the divided basal granule while a new one arises from the other half. ${ }^{28}$

In eiliated epithelial cells each cilium is associated with a basal corpuscle in or near the cell membrane (Fig. 137). The nature of such basal granules has been the subject of a long controversy beginning with the statement of the theory that they, like the granules bearing the axial filament in the spermatozoön, are modified centrioles or centriole deriva-

${ }^{29}$ Rosenbusch (1909), Kuczynski (1917), Hartmann and Nöller (1918). 
tives. ${ }^{29}$ In ciliated epithelial cells, according to Saguchi, there is a basal corpuscle at the base of each cilium. The granules and cilia are in parallel rows. Beneath each row there is a transparent zone in which the rootlets of the cilia are anchored and through which they pass and become continuous with strands of a cytoplasmic reticulum. Saguchi regarded the basal corpuscle as the kinetic center of ciliary movement but not as a centriole derivative.

In the gill cells of certain mollusk $\mathrm{s}^{30}$ the ciliary rootlets below the basal corpuscles form a continuous system which probably represents the structural basis for the coördinated action of the cilia of a given cell. The view of Grave and Schmitt, that the systems of adjacent cells are continuous, is disputed by Bhatia and by Lucas, who believe the coördinating impulses pass from cell to cell through the general cytoplasm and the membranes. In any erent, the eridence shows clearly that the cilium or the flagellum is itself an actively contractile element and not merely a passive element operated by an intracellular mechanism (see Gray, 1931).

${ }^{29}$ Henneguy (1897), Lenhosséli (1898). This theory is favored by Helvestine (1921), Jordan and Helvestine (1923) for certain cells, and Kindred (1927). It was opposed by Saguchi (1917) and by Erhard (1911), who reviewed the evidence then available.

${ }^{30}$ Grave and Schmitt $(1924,1925)$ on Lampsilis and Quadrula, Bhatia (1927) on Mytilus, Lueas (1931) on Mytilus and other genera. 


\section{CHAPTER XV}

\section{SYNGAMY}

The sexual union of two gametes is known as syngamy. In the case of organisms having large female gametes and small, more active male gametes, it is customary to refer to syngamy as the fertilization of the egg by the sperm; but it should be borne in mind that the syngamic reaction is in all cases mutual, and that it is the fusion product of the two gametes which proceeds with development.

Syngamy has two important results: firstly, it has a pronounced effect upon the physiological state of the cells concerned and hence upon. ensuing developmental processes. This may manifest itself in various ways, commonly in the activation of an egg whose development has ceased temporarily, or in the onset of a dormant condition, as in the zygotes of certain lower plants. Secondly, it brings about a significant alteration in the constitution of the nucleus. Syngamy culminates in the fusion of two nuclei each of which has a gametic chromosome complement, typically a single set; hence the zygote nucleus so formed has a zygotic complement composed typically of two sets. This doubling of the chromosome number (diplosis) has far-reaching consequences, as will be emphasized in later chapters.

\section{PLANTS}

The general features of gametic fusion in plants are extraordinarily diversified, notably in algæ and fungi. ${ }^{1}$

Algæ.-Fusions involving gametes of the various types described in the preceding chapter take place as follows. In Ulothrix two morphologically similar, motile gametes meet and fuse to form a resting zygospore (Fig. 138, A). The exact manner of fusion varies greatly in such forms, but commonly it begins at the ciliated end. ${ }^{2}$ In Ectocarpus, also, the gametes are morphologically similar, but some of them become relatively passive and anchor themselves to the substratum by one cilium, while

1 For an extensive account of sexual and asexual cell and nuclear fusions in plants, see Tischler (1921-1922). For angiosperms, see also Schürhoff (1926), Dahlgren (1927a), and Schnarf (1929); for algæ, Oltmanns (1922-1923) and G. M. Smith (1933); for fungi, Guilliermond (1913e), Atkinson (1915), Wager (1920), Gäumann-Dodge (1928), and Fitzpatrick (1930).

2 That such similar gametes are actually unlike sexually, and secrete characteristically different substances into the medium, is shown by the work of M. Hartmann and his associates, chiefly on green algæ. See M. Hartmann (1932). 
more active ones become attached by a cilium to the anchored ones and gradually undergo fusion with them (Fig. 138, B). The fusion culminates in the union of the nuclei. In such gametic fusions the plastids undergo no fusion, so far as is known. In some cases they have been followed through into the new individual. In Fucus the large passive eggs and the small motile spermatozoids are shed from the organs which bear them, their union occurring in the sea water. Many spermatozoids attach themselves to each egg by one cilium, but after one of them enters the egg the others move away. The male nucleus remains relatively small up to the time it fuses with the egg nucleus, and a centrosome with an aster

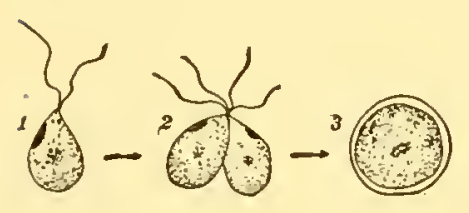

A
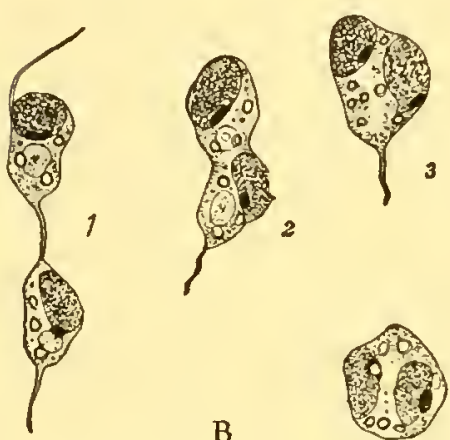

B

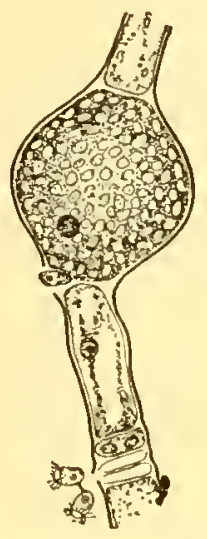

C

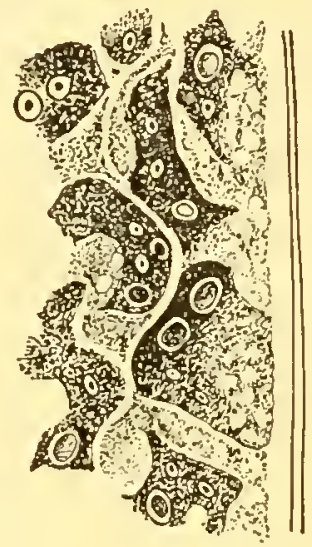

D

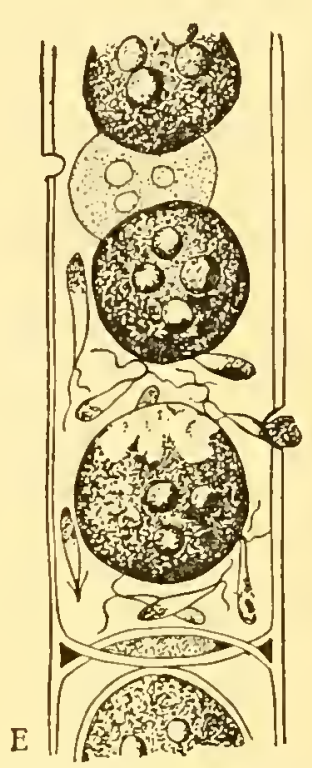

FIG. 138.- Syngamy in various algæ. A, gamete, union of similar gametes (isogamy), and zygote of Ulothrix. $B$, isogamy in Ectocarpus siliculosus. (After Oltmanns, 1897.) $C$, portion of filament of $C$ dogonium; spermatozoids escaping from antheridial cells below; spermatozoid about to enter egg above. (After Coulter.) D, the formation of eggs by subdivision of a coenocytic protoplast in Spharoplea. $E$, portion of filament of Spharoplea showing several cggs; spermatozoids have entered through pores in filament wall. ( $D$ and E after Klebahn.)

appears near the nuclear membrane at the point where the male nucleus enters (Yamanouchi, 1909). This latter phenomenon, which is very suggestive of what occurs in animals, has also been observed in Dictyota (J. Williams, 1904).

In Edogonium and Vaucheria the egg is not liberated from the oögonium but is fertilized in situ by a spermatozoid. The latter passes into the oögonium through a specially differentiated pore and then enters the egg at a more or less distinct receptive spot. In the mature male gametes of Vaucheria, Chara, and Coleochote no plastids have been demonstrated, the new individual apparently deriving these organs wholly from the female gamete. This statement, however, may require revision when male gametes have been more fully studied with methods specially devised for the investigation of cytoplasmic differentiations. In Polysiphonia the non-motile male gamete (spermatium) comes in contact with a prolonga- 
tion (trichogyne) of the female sex organ (carpogonium). Solution of the intervening walls allows the nucleus of the spermatium to pass into the trichogyne and down to the female nucleus in the base of the carpogonium (Fig. 139, A). The male nucleus, when it reaches the female nucleus, appears as a group of 20 chromosomes. In this condition it enters the female nucleus while the latter is yet in the reticular state. Soon the female reticulum condenses into 20 chromosomes, which arrange themselves with the 20 paternal chromosomes in the spindle as the fusion nucleus divides (Yamanouchi, 1906). The spermatium of the Florideæ is said to carry no plastids.

A special condition is found in the Conjugatæ. Here certain vegetative cells, after little morphological alteration, fuse in pairs to produce resting zygospores. In Spirogyra, for example, the entire protoplast passes through a conjugating tube to unite with a similar cell, commonly in another filament. The two nuclei fuse, but the plastids remain distinct and those introduced by the migrating cell are observed in some species to degenerate in the zygospore (Fig. 162). In some species the gametes meet and form the zygospore in the conjugating tube. The movement of these gametes is accomplished through the remarkable action of contractile vacuoles, which withdraw water from the central sap vacuole and discharge it between the protoplast and the wall. ${ }^{3}$

In the diatoms ${ }^{4}$ there are two general modes of sexual behavior, with minor variations in the different species. With rare exceptions these modes characterize the two main groups, Pennatæ and Centrice. In Rhopalodia gibba, a representative of the former group, two individuals meet and form a common mass of jelly between them. In each individual the nucleus undergoes two successive divisions, the protoplast then dividing into two portions, each with a large and a small nucleus. These two portions now fuse with the two produced by the other individual, forming two zygotes which develop into large auxospores. In each case the large nuclei fuse and the small ones degenerate (Klebahn). In some diatoms, for example, certain forms of Cocconeis, the two uniting individuals show distinct differences in size and sexual behavior (Geitler, 1927ab). In the Centricx the auxospores are formed asexually, the sexual act consisting in the union of small biciliate gametes. In Coscinodiscus these are of two types: small colorless ones and larger ones with chromatophores (Pavillard). ${ }^{5}$

${ }^{3}$ Lloyd $(1925,1928 a)$. Conard (1931h) elaims that the eytoplasmic portions of the gametes remain distinct and are separated by the first wall at germination.

${ }^{4}$ Klebahn (1896), Lauterborn (1896), Karsten (1897, 1900, 1904, 1912, 1924), O. Müller (1906), Bergon (1907), Pavillard (1913), Geitler (1927abc, 1928abc, 1929b), von Cholnoky (1927, 1928, 1929), P. Sehmidt (1923, 1927ab). See Pavillard (1910) and Oltmanns (1922).

5 These small cells are termed "microspores" in the literature. The rarely observed "microspores" in Pennatæ seem to be asexual. 
Fungi.-The fusion of the sexual elements described in the preceding chapter (p. 199) proceeds as follows. In Synchytrium the morphologically similar uniciliate gametes unite to form a zygote which penetrates into a host plant. In Monoblepharis the uniciliate male gamete creeps along the surface of the oögonium to its opening, where, after loss of its cilium, it unites with the egg to form a resting oöspore; this matures either inside or outside the oögonium. In Albugo candida and Pythium Debaryanum the egg is entered by a single male nucleus through a conjugating tube from the antheridium (Fig. 139, B). The nuclear fusion in Pythium and Achlya occurs in association with a cytoplasmic "cœnocentrum" (P. Patterson). In Saprolegnia, which has several eggs in the oögonium,

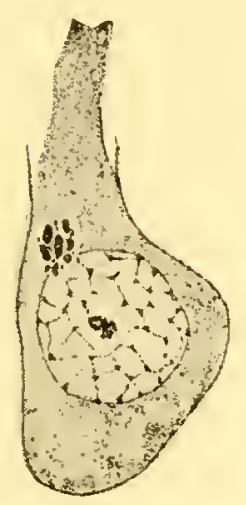

A

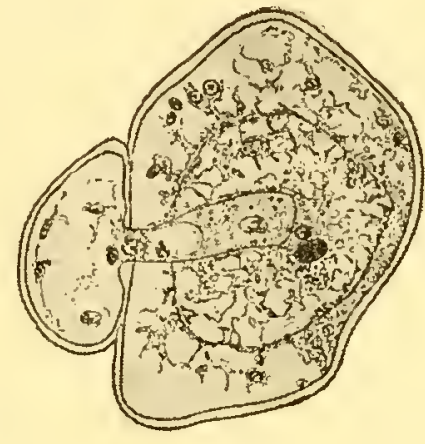

B

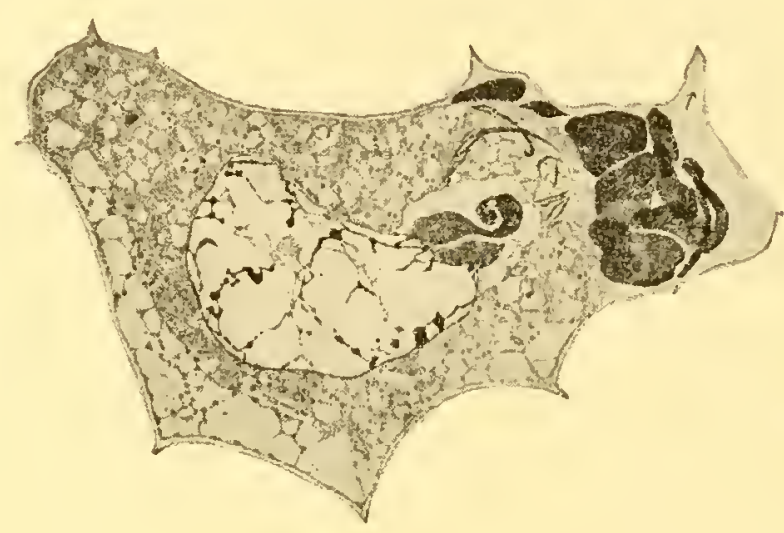

$C$

FIG. 139.-A, Syngamy in Polysiphonia; group of paternal chromosomes about to enter female nucleus. (After Yamanouchi, 1906.) B, Syngamy in Albugo candida; female nucleus in oöplasm near conocentrum (larger dark body); antheridial tube about to discharge male nucleus. (After Davis, 1900.) C, Syngamy in Lygodium palmatum. The coiled nucleus of the functional spermatozoid lies against the right end of the egg nucleus, while its cilia lie in the cytoplasm near by. Outside the egg are other spermatozoids. Arrow indicates neek eanal. (After Rogers, 1926.)

the antheridium sends in a branching tube which delivers a male nucleus to each egg.

In the zygomycetes ${ }^{6}$ the contents of the multirucleate gametangia do not subdivide into small gametes but meet and fuse in their entirety. The product is a heavy-walled zygospore. The extreme minuteness of the nuclei and the abundance of metabolic products in the protoplasm have so far made it impossible to clear up the matter of nuclear behavior in this group.

In the higher ascomycetes the sex organs are typically antheridia and ascogonia. Some species have both organs, some have one or the other, and some have neither. In some cases the organs are uninucleate and in others multinucleate. After an ascogonium has been entered by the contents of an antheridium the nuclei do not fuse (according to one prevalent view) but associate and divide repeatedly in pairs ("conjugate division"

${ }^{6}$ P. A. Dangeard (1906), Moreau (1911, 1913, 1915), Keene (1914), Burgeff (1915), Macormick (1912), Baird (1924). 
of a "dikaryon") in the ascogenous hyphæ which grow out from the ascogonium. In each ascus the two associated nuclei finally fuse. According to this interpretation, ${ }^{7}$ therefore, the nuclear fusion is merely delayed: instead of occurring in the ascogonium, it takes place in the ends of branches produced by the latter, namely, in the asci; and this fusion may be regarded as sexual in nature, as originally stated by Dangeard (1894).

Other observers have claimed that there are two nuclear fusions in the ascomycete life cycle: in addition to the fusion easily observable in the ascus, it is contended that an earlier fusion occurs in the ascogonium after the entrance of the contents of the antheridium, ${ }^{8}$ or in the ascogonium when the antheridium is functionless or absent, ${ }^{9}$ or in vegetative cells when the ascogonium is functionless or absent. ${ }^{10}$ In the ascomycetes generally the fusion nucleus, or "primary ascus nucleus," initiates three successive mitoses to form the eight ascospore nuclei. A reduction in chromosome number occurs in the course of these mitoses. Adherents of the view that there are two nuclear fusions in the life cycle claim that there is a "double reduction" in the ascus (p. 281). In certain yeasts it has been shown that the production of ascospores is preceded by a union of two cells and a fusion of their nuclei, the fusion nucleus dividing to form the spore nuclei (see Guilliermond, 1920n).

In the hymenomycetes it has long been known that a fusion of two nuclei occurs in the basidium, itself the terminal cell of a hypha with binucleate cells, prior to the formation of the four basidiospore nuclei (Fig. 164). The discovery of the origin of the binucleate condition is more recent. ${ }^{11}$

In certain species of several genera, including Coprinus, Aleurodiscus, Pancolus, and Schizophyllum, it has been shown that carpophores, although they will sometimes develop on a mycelium from a single spore, appear freely only when mycelia from different spores are intermingled. Unions then occur between uninucleate hyphæ of different strains and initiate the binucleate dikaryophase. According to Buller, the migrating nucleus divides after entering the adjacent hypha, one of the daughter nuclei then moving on to the next cell to repeat the process. In this way the originally uninucleate mycelium becomes progressively "diplodized." In the resulting binucleate mycelium the paired nuclei

7 P. Claussen (1907, 1912), W. H. Brown (1909, 1910a, 1911b), Faull (1911, 1912), Nienburg (1914), Ramlow (1914), F. Brooks (1910), MeCubbin (1910), H. B. Brown (1913), Fitzpatrick (1918a), Frey (1924), S. G. Jones (1925), Delitsch (1926).

${ }^{8}$ Harper (1895-1905), Blackman and Fraser (1905), Gwynne-Vaughan and Williamson $(1931,1932)$.

${ }_{9}^{9}$ Blackman and Fraser (1906a), Fraser (1907), Welsford (1907), Dale (1909), Gwynne-Vaughan and Williamson (1930).

${ }^{10}$ Fraser (1907, 1908), Carruthers (1911), Blackman and Welsford (1912).

11 Kniep (1915 et seq.), Bensaude (1918), Lehfeldt (1922), Mounce (1921, 1922), Hanna (1925, 1928), Sass (1929), Buller (1930, 1931). 
divide in unison before each cell division, their descendants eventually fusing in the basidium. Here the nuclear fusion ("karyogamy") is delayed until some time after cytoplasmic union ("plasmogamy"), these two events being separated by a binucleate phase in the life cycle.

In the rust fungi there are life cycles of several kinds, these differing in the presence or absence of various spore types and of cell and nuclear fusions. ${ }^{12}$ Some forms have uninucleate cells throughout the cycle, some are entirely binucleate, and some have uninucleate and binucleate, or even short plurinucleate, phases. In a number of forms, including Puccinia graminis, the common wheat rust, each cell of the young teliospore is binucleate. These two nuclei eventually fuse, and as the teliospore germinates to form a promycelium, which is homologous with the basidium of the hymenomycetes, the fusion nucleus divides to four which enter the sporidia. Beginning with the work of Blackman (1904b) and Christman (1905) on Phragmidium and certain other genera, it was thought that the binucleate condition originated by nuclear migration between cells or by cell fusions, particularly in the basal region of the æcial sorus. Evidence recently brought forward ${ }^{13}$ indicates that in certain species of Puccinia a mycelium developing from a single sporidium, or basidiospore, produces as a rule only sterile æcia, and that chains of æciospores develop only when pycniospores of a different strain are added to the infection or when "plus" and "minus" mycelia develop close together. Some of the cytological changes involved here have been ascertained. Andrus (1931) has announced that in Uromyces appendiculatus the sporeforming cells in the base of the xcium are not formed by fusion but are enlarged female cells which send elongated trichogyne-like hyphæ through the opposite side of the leaf, where they meet and fuse with spermatia (pycniospores) from infections of opposite strain. Nuclei apparently pass through the septa of the trichogynes into the female cells, which then proliferate and produce chains of binucleate ceciospores. Similar in many respects is R. F. Allen's (1932a) interpretation of the phenomena in Puccinia triticina. Here the mycelium formed by a single sporidium produces spermatia, receptive hyphæ, and the primordia of æcia. After nuclei from spermatia of the opposite strain enter the receptive hyphæ, the resulting binucleate (or plurinucleate) hyphæ extend into young xcia and develop xciospores.

In the smut fungi, as exemplified by species of Ustilago, the binucleate condition arises as the result of a union of promycelial cells, sporidia, or mycelial cells which they produce, the two nuclei fusing later when chlamydospores are differentiated. ${ }^{14}$ It is known further that the sporidia

12 See Arthur (1929), Gäumann-C. Dodge (1928), B. O. Dodge (1929a), and Jackson (1931).

${ }^{13}$ Craigie (1927ab, 1928, 1931), R. F. Allen (1929, 1930, 1932), Hanna (1929a).

${ }^{14}$ P. A. Dangeard (1893), Lutman (1910), Rawitscher (1912), Kniep (1919, 1920). 
and the mycelia developing from them are of two kinds, only those of unlike strain ("plus" and "minus") uniting when brought together. ${ }^{15}$

The significance of the hyphal and sporidial fusions which lead to the binucleate condition of the cells in the dikaryophase of ascomycetes and basidiomycetes is a matter of debate among mycologists. The view
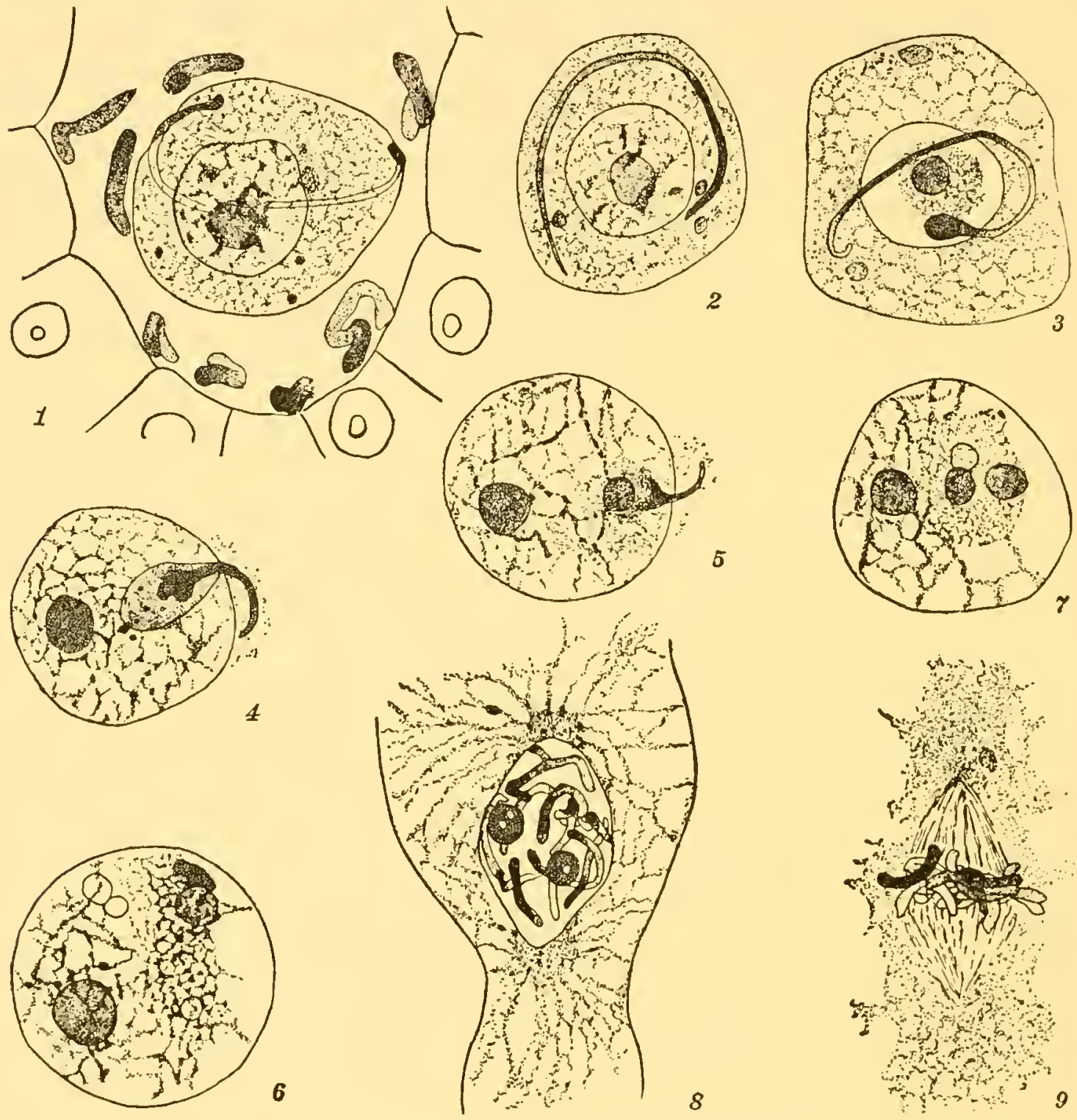

8

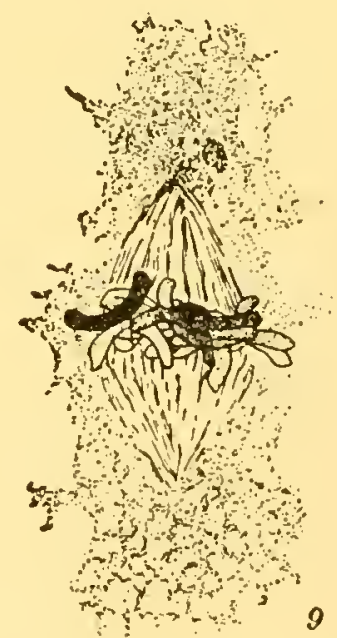

Fig. 140.-Syngamy in Riccardia. 1, sections of spermatozoids in archegonium; one malc nucleus applied to egg. 2, male nucleus in egg cytoplasm. 3-7, passage of male nucleus into egg nucleus and its development of a distinct reticulum. 8,9, first mitosis in zygote; paternal and maternal chromosomes intermingled. (After Showalter, 1926b.)

that they represent a sexual act is disputed by those who hold that they are primarily nutritive in character ("nutritive heterothallism"), although they may be followed by some of the beneficial effects of true sexuality. Comparisons with other plants strongly suggest that sexual differences in fungus nuclei and mycelia, as well as other conditions affecting their interaction, are of ten related to meiotic factor segregation in sporogenesis. This topic is discussed further at page 386.

${ }^{15}$ Kniep (1919, 1920), Stakman and Christensen (1927), Hanna (1929b). 
Bryophytes and Pteridophytes.-The cytological details of the union of the motile spermatozoid with the egg in bryophytes are best known in certain liverworts, owing especially to the researches of Showalter (1926ab, 1927ab, 1929). In Riccardia pinguis (Fig. 140) the elongated body of the spermatozoid applies itself to the surface of the egg, becomes more slender, and gradually sinks through the membrane into the cytoplasm. The chromatic matter of the egg nucleus contracts, and one end of the sperm nucleus extends through the membrane of the egg nucleus, where it becomes considerably swollen. The female nuclear matter then loosens up, and the swollen end of the penetrating male nucleus is seen to lose its definite outline and differentiate a chromatic reticulum and a nucleolus. After penetration is complete the female and male reticula remain distinguishable for some time, but in the late prophase and the metaphase of the first embryonal mitosis the chromosomes of the two parents form a single group. The fate of the cytoplasm, blepharoplast, and cilia of the male gamete is uncertain, but in view of what has been observed in other species it is highly probable that they are absorbed in the egg cytoplasm.

In a number of other liverworts ${ }^{16}$ the male nucleus does not enter the egg nucleus in the compact form but becomes vesicular and reticulate in some degree before the fusion occurs (Fig. 141). In Spharocarpos it ap-

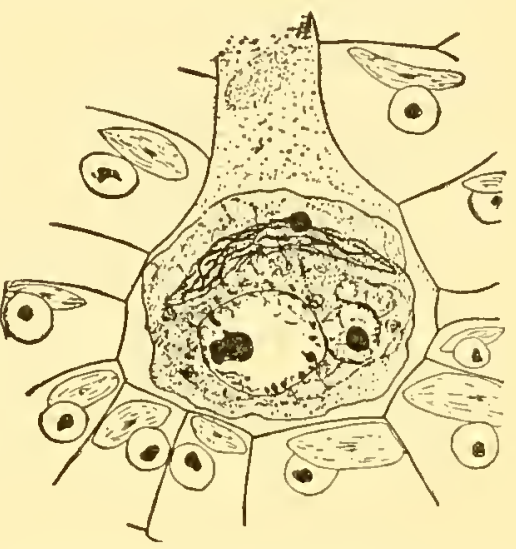

FIG. 141.-Syngamy in Anthoccros. Male and female nuelei about to fuse in lower part of egg in venter of arehegonium; elongated plastid above them. Gametophyte cells show one nucleus and one plastid each. pears that the nuclei do not undergo fusion while in this state but develop separately their chromosomes, which then take their places in a common mitotic spindle, as in many animals. In Preissia, centrosomes are reported in the egg cytoplasm during the fertilization period. This, together with observations on certain algæ (p. 227), suggests that in certain plants, as in animals, the differentiation of division centers in the egg cytoplasm may be induced in some manner by the entrance of the spermatozoid. The fate of the non-nuclear portions of the spermatozoid may prove to be of interest in this connection.

In the pteridophytes ${ }^{17}$ the multiciliate spermatozoid enters the egg cytoplasm. It has been stated that it continues into the egg nucleus in its entirety, but in Lygodium it has been clearly shown that the cilia (and

${ }^{16}$ Pellia Fabbroniana (Showalter, 1927b); Riccia (Garber, 1904; Black, 1913); Corsinia (K. Meyer, 1911); Preissia quadrata (Graham, 1918; Haupt, 1926); Reboulia (Woodburn, 1920); Spherocarpos (Riekett, 1923). In Funaria flavicans (moss) the male nucleus enters rounded up but still compaet (Beardsley, 1931).

${ }^{17}$ Pilularia (Campbell, 1888); Onoclea (Shaw, 1898b); Nephrodium (Yamanouchi, 1908b); Lygodium (Rogers, 1926); Equisetum (Sethi, 1928). 
presumably the blepharoplast and most of the cytoplasm) are left in the cytoplasm as the compact and coiled male nucleus enters the egg nucleus (Fig. 139, C). The male nucleus then gradually becomes reticulate, and eventually the materials contributed by the two gametes become indistinguishable.

The behavior of the plastids during gametogenesis and syngamy in ferns is not well known. Plastids may frequently be seen in the develop-

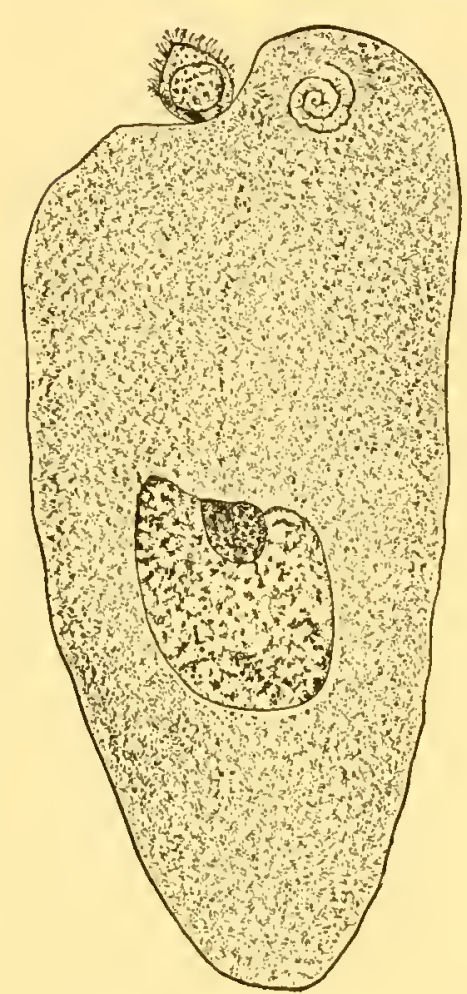

FIg. 142.- Syngamy in Zamia. Male and female nuclei uniting at center; cytoplasmic sheath and spiral blepharoplast of spermatozoid above; another spermatozoid at surface of egg. (After Webber, 1901.) ing egg, and presumably they play a rôle in spermatogenesis somewhat like that in bryophytes (p. 203). At present it seems probable that the plastids of the sporophyte are derived chiefly if not wholly from the female gamete.

Gymnosperms. - In the cycads and Ginkgo the pollen tube discharges two motile spermatozoids near the archegonium, into which one of them passes by squeezing between the neck cells. The nucleus of the spermatozoid then becomes free from its cytoplasmic sheath, blepharoplast, and cilia and advances alone to the egg nucleus, with which it fuses ${ }^{18}$ (Fig. 142). In Ginkgo the cytoplasm and motor apparatus are left in a "receptive spot" formed by vacuoles in the upper part of the egg. In Bowenia the paternal and maternal chromosomes can be distinguished in the first few mitoses preceding wall formation in the embryo.

In the Coniferales and Gnetales the male cells have no motile apparatus. Each consists of a nucleus surrounded by a more or less sharply delimited mass of cytoplasm. In most cases this cytoplasm remains intact until after the male cell has entered the egg, but in other forms it mingles with the cytoplasm of the pollen tube, so that only male nuclei, rather than completely organized male cells, are delivered to the egg. All the nuclei present in the pollen tube-stalk nucleus, tube nucleus, the two male nuclei, and, in certain species, free prothallial nuclei-may be discharged into the egg. All but the functioning male nucleus usually degenerate at once, but in some cases they have been observed to undergo division.

Malc cells of conifers show two general modes of behavior after their entrance into the egg. In some instances the male's cytoplasm is left in the peripheral region of the egg while the nucleus advances alone to

${ }^{18}$ Hirasé (1895, 1898, 1918), Ikeno (1901), Shimamura (1928) and Herzfeld (1927) on Girkigo; Ikeno (1898) and Kuwada (1925a, 1926b) on Cycas; Chamberlain $(1910,1912,1916)$ on Dioön, Ceratozamia, and Stangeria; Lawson (1926) on Bowenia. 
the centrally placed egg nucleus. ${ }^{19}$ In other cases $^{20}$ the male cytoplasm remains intact and surrounds the fusing sexual nuclei. The pollen-tube cytoplasm often plays a conspicuous part in the formation of this "mantle."

The behavior of the chromosomes during the fusion of the gametic nuclei and the first embryonal division has been described in a number of conifers; similar data for the cycads are as yet comparatively few. As a general rule the chromatic elements of the two nuclei, although in the reticular condition when the nuclei unite, do not become very intimately associated in the fusion nucleus but remain distinguishable until the first embryonal mitosis occurs. They then develop two groups of chromosomes which become arranged in a common achromatic figure ${ }^{21}$ (Fig. 143).
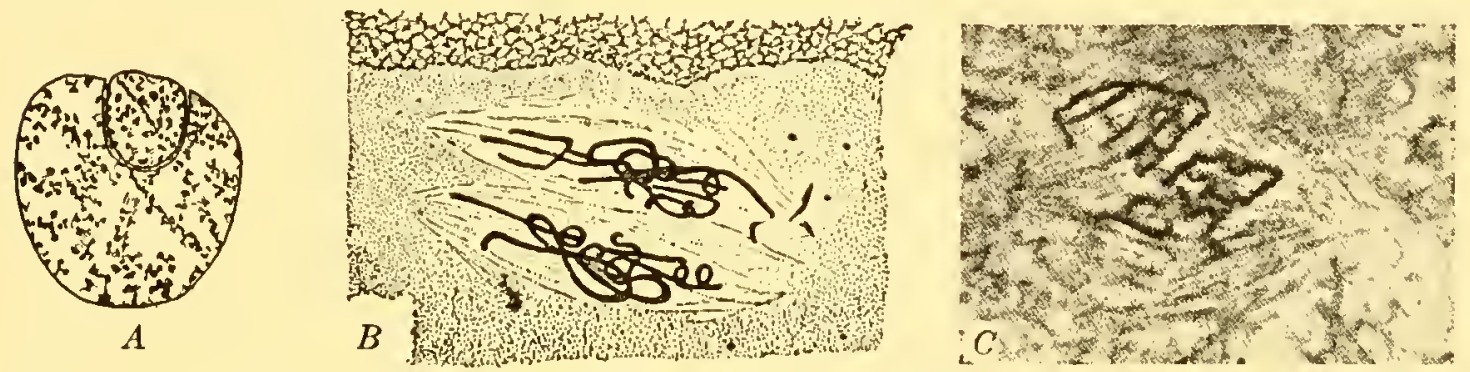

Fig. 143.- Syngamy in conifers. $A$, male nucleus pressing into female nucleus in Pinus. $B$, first mitosis in zygote, showing parental chromosome groups. (After Ferguson, 1904.) C, parental chromosome groups in Larix. (After Wóycicki, 1899.)

In Sequoia the male nuclei escape from their cytoplasm before their discharge from the pollen tube, and after the nuclear fusion in the egg the contributions of the two gametes cannot be distinguished (Lawson, 1904a). In gymnosperms, as in other organisms, all of the chromosomes, paternal and maternal, divide longitudinally in the first embryonal mitosis, the daughter chromosomes being distributed to the daughter nuclei.

Angiosperms.-The pollen tube, which is the elongated tube cell bounded by the greatly extended intine, grows from the pollen grain on the stigma through the style to the ovarian cavity and then by way of the micropyle of the ovule through the nucellus and into the embryo sac (Fig. 127). Usually the tube makes its way between the cells of the style by means of enzymes which dissolve the middle lamellæ of their walls (Paton, 1921). After entering the sac the tube ruptures at its

${ }^{19}$ Pinus (Ferguson, 1901, 1904), Thuja (Land, 1902), Juniperus (Norén, 1904), Cryptomeria (Lawson, 1904b), Libocedrus (Lawson, 1907), Ephedra (Berridge and Sanday, 1907).

${ }^{20}$ Taxodium (Coker, 1903), Torreya (A. Robertson, 1904; Coulter and Land, 1905), Cephalotaxus (Coker, 1907), Phyllocladus (Kildahl, 1908), Juniperus (Nichols, 1910), Agathis (Eames, 1913), Taxus (Dupler, 1917).

${ }^{21}$ Pinus (Blackman, 1898; Chamberlain, 1899; Ferguson, 1901, 1904), Larix (Wóycicki, 1899; Smólska, 1927), Tsuga (Murrill, 1900), Juniperus (Norén, 1907), Cunninghamia (Miyake, 1910), Abies (Hutchinson, 1915), Bowenia (Lawson, 1926). 
end, discharging the two male gametes, together with a certain amount of pollen-tube cytoplasm and sometimes the tube nucleus. Frequently the tube appears to pass directly through a synergid. It has been suggested that the synergids produce enzymes which swell and rupture the membrane of the pollen tube (Goebel; Haberlandt, 1927). As they appear in the embryo sac, the male gametes may be complete cells or only nuclei with no specially delimited cytoplasm (see p. 210). Frequently the nuclei are markedly vermiform. Double fertilization, a process peculiar to angiosperms, now takes place: one male gamete fuses with the egg, while the other unites with the two polar nuclei or their fusion product ${ }^{22}$ (Fig. 144).

The Gametic Fusion.-The nucleus of one of the male gametes enters the egg-through a sort of rift, according to Heimans. In cases where the male gamete is a complete cell in the pollen tube, its nucleus may become free from its cytoplasm before reaching the egg. ${ }^{23}$ In other cases, however, the male cytoplasm remains distinct from that of the pollen tube and embryo sac until the surface of the egg is reached. ${ }^{24}$ It has not yet been demonstrated cytologically in any case that male cytoplasm enters the egg along with the nucleus. Wylie thinks it probable that it does so in Vallisneria, and there is genetic evidence that it may enter in certain other cases (Chapter XXV). It would obviously be difficult to identify male cytoplasm during the passage of the nucleus through the egg membrane, or to show that the nucleus is absolutely freed of all adhering matter previously associated with it. It is expected that refinements in technique will shed light on this question. Occasional differences in chromaticity between male and female nuclei are reported, ${ }^{25}$ so that one may hope for similar evidence for the cytoplasm.

The fusion of the two gamete nuclei probably occurs in most cases very soon after they come in contact, though in certain forms it is known to be considerably delayed. The chromatic matter of the two nuclei at the time they unite may be in the reticular condition, the male and female elements becoming indistinguishable in the fusion nucleus. In other cases ${ }^{26}$ it has already reached the condition characteristic of the prophase, the paternal and maternal elements often being distinguishable in the spindle in the ensuing division of the zygote. That the same species may show considerable variation in this respect is indicated by the situation in

${ }_{22}$ For reviews of the features of fertilization in angiosperms, see Dahlgren (1927a), Schürhoff (1926), Schnarf (1929), and Coulter and Chamberlain (1903).

${ }^{23}$ E.g., Lilium (S. Nawaschin, 1910; Welsford, 1914), Viola (Madge, 1929), and Melandrium (Breslawetz, 1930b). See Tischler (1921-1922, p. 486).

${ }^{24}$ Vallisneria (Wylie, 1922, 1923), Enothera (Ishikawa, 1918), Asclepias (Finn, 1925).

${ }^{25}$ E.g., Heimans (1928; on Lilium) and Kuwada (1925a, 1926b; on Cycas).

${ }^{26}$ E.g., Lilium (Guignard, 1891; Weniger, 1918), Calopogon (Pace, 1909), and Trillium (Nothnagel, 1918). 
Fritillaria, in which Sax $(1916,1918)$ finds that fusion, though it usually occurs in the reticulate stage, sometimes takes place after the thick prophasic threads have been developed. Weniger (1918) reports that in Lilium the egg nucleus is reticulate and the male nucleus in prophase as they unite. Whatever the state of the nuclei at the time of their fusion, all of the chromosomes, paternal and maternal, split longitudinally in the first mitosis in the zygote, the daughter chromosomes so formed
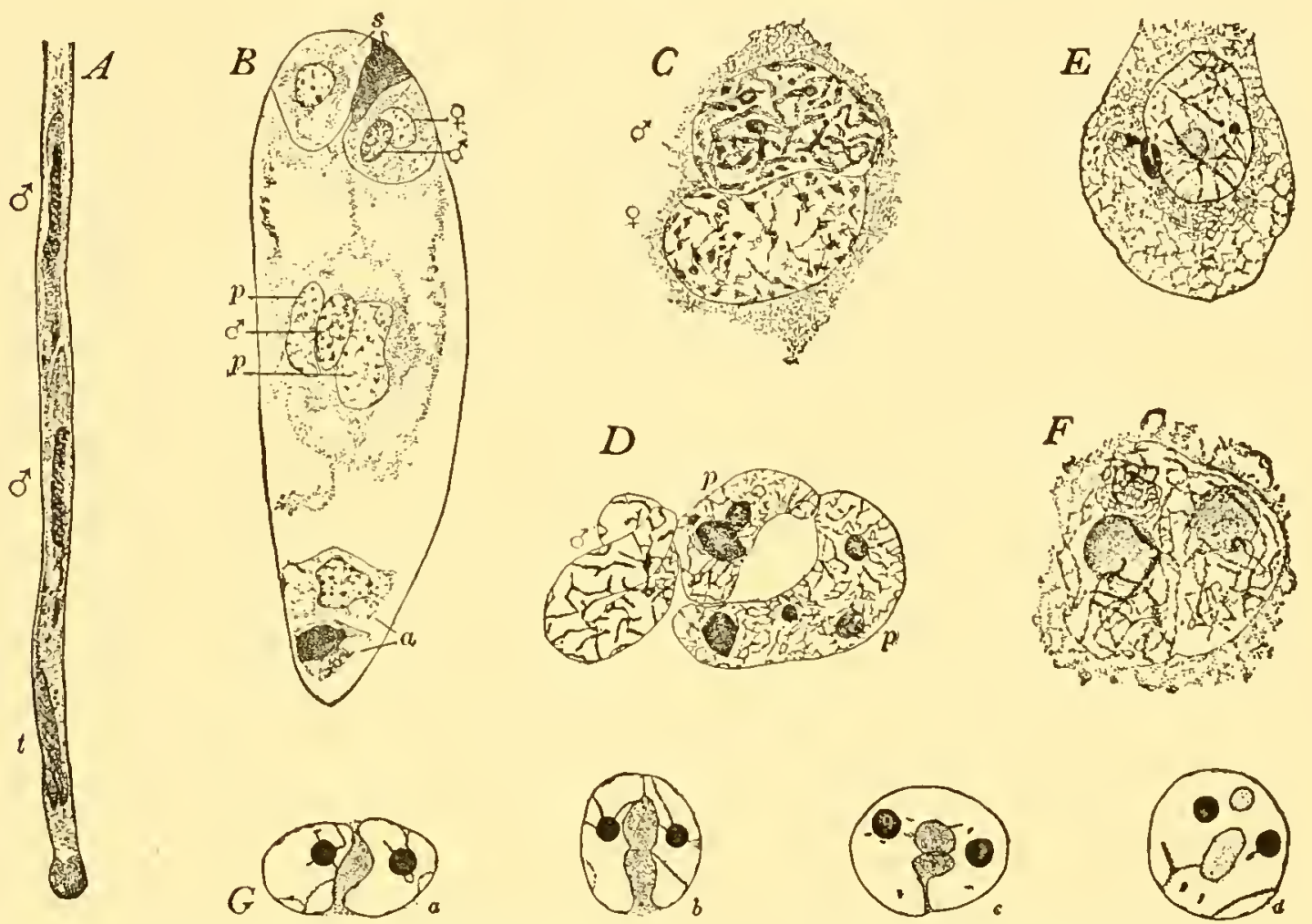

Frg. 144.- Syngamy in angiosperms. A, end of pollen tube from basal portion of style of Lilium auratum, showing two male cells and tube nucleus. (After Welsford, 1914.) $B$, double fertilization in Lilium canadense; sexual nuclei about to fuse in egg; second malc and two polar nuclei fusing at center of embryo sac; $s$, synergids, one degenerated; $a$, antipodal cells. $C$, fusion of sexual nuclei in egg of Lilium philadelphicum. (After Weniger, 1918.) $D$, the second male and two polar nuclei in Lilium Martagon. (After Nothnagel, 1918). E, vermiform male nucleus in contact with egg nucleus in Triticum durum. (After Sax, 1918.) $F$, spireme stage of triple fusion nucleus in Triticum durum, showing distinctness of chromatic elements of the three nuclei. (After Sax.) $G$, inclusion of cytoplasm in fusing sexual nuclei of Peperomia sintenesii; this seems to be an oceasional phenomenon with no special significance. (After W. H. Brown, 1918b.)

being distributed to the two resulting nuclei, just as in all the subsequent divisions in the developing embryo.

The time elapsing between pollination and the union of the gametes varies greatly in different plants, but the variation shows no necessary correlation with the length of their styles. The following examples ${ }^{27}$ may be cited: Zea Mays, 18 to 24 hours; Triticum vulgare, 18 to 20 hours; Lilium philadelphicum, 60 to 72 hours; Phaseolus vulgaris, 8 to 9 hours; Acer negundo, 40 to 72 hours; Monotropa uniflora, 5 days; Secale cereale,

${ }^{27}$ See Schnarf (1929) for an extensive list with citations to literature. 
7 hours; Phajus grandifolius, 2 months; Hicoria pecan, 5 to 7 weeks; Datura stramonium, 25 hours at $20^{\circ} \mathrm{C}$.; Enothera rubrinervis, 36 hours; Quercus rubra, 13 to 14 months. In Beta vulgaris, growing under summer field conditions, the period is 20 hours, and a period of equal length elapses before the fertilized egg divides (Artschwager, unpubl.). It has been shown in certain cases that the rate of pollen-tube growth varies with the temperature and other conditions, which accounts in part for the discrepant reports of different observers.

The Endosperm Fusion. - The second male nucleus (it may be either the first or the second to emerge from the pollen tube) passes to one or both polar nuclei or to the product of their fusion. The fusion of the three nuclei to form the primary endosperm nucleus is carried out in a variety of.ways. The most commonly reported mode is that in which the two polars unite to form a polar-fusion nucleus ("embryo-sac nucleus") before the entrance of the pollen tube, the male nucleus being added later. Less frequently the male nucleus meets and fuses with the polar nucleus of the micropylar end of the sac, the other polar then fusing with the product. This is the method described by S. Nawaschin $(1898,1899 a)$ in his accounts of the discovery of double fertilization in Lilium Martagon and Fritillaria tenella. In Zea the male nucleus and one polar complete their fusion while the two polars are in contact but still unfused (V. Rhoades, unpubl.). The simultaneous fusion of all three nuclei appears to be a common occurrence; it has been described in some detail by Nothnagel (1918) for Trillium and Lilium. Different modes may occur in the same plant under varying conditions, notably of temperature (Shibata, 1902, on Monotropa). Although the three nuclei often appear exactly alike, it is frequently possible to distinguish the male from the polars, not only by its shape and smaller size, but by the condition of its karyotin. In Litium longiflorum (Weniger, 1918), for example, the male nucleus is in the prophase while the polar nuclei are still reticulate. The membranes of the three nuclei may persist for some time after they come into intimate contact, and even after they have disappeared the chromatic elements of the three constituent nuclei may in many cases be distinguished if the section has been made in a favorable plane. When fusion occurs in the metabolic stage this is not so apparent, but when it occurs in the prophase the three chromatic groups are made out with little difficulty.

As the division of the endosperm nucleus approaches, the chromatic elements of the three contributing nuclei become increasingly distinct, even if one or more of the nuclei have fused in the reticulate condition (Fig. 144, F). As the prophase proceeds, all of the chromosomes (the triploid number) are seen to be split longitudinally. Since this is repeated in every subsequent mitosis, the resulting endosperm nuclei are normally all triploid, each of them having one paternal and two maternal chromosome sets. In most angiosperms the developing endosperm passes 
through an early free-nuclear stage, no walls appearing until some time later (p. 173). In other cases walled cells may be formed from the start. ${ }^{28}$

Although the nuclei throughout the endosperm are all derived by repeated division from the triple-fusion nucleus in the great majority of plants studied, cases are known in which at least some of them may be produced by the polar-fusion nucleus alone without the male, or by the fusion product of the male and one polar, or by one polar alone. Combinations of these methods may be found in the same embryo sac. In Petunia the polar-fusion nucleus divides before the pollen tube opens, and four endosperm cells are formed. One male nucleus then fuses with that in the micropylar endosperm cell. That portion of the endosperm which is derived from this cell is triploid (21 chromosomes), while that derived from the three other cells is diploid (14 chromosomes) (Ferguson, 1927). Cases of "mosaic endosperm" frequently reported may have their explanation in such unusual modes of development. Probably more often they are due to aberrant mitotic beharior on the part of endosperm nuclei initially triploid.

The propriety of extending the term "fertilization" to the endosperm fusion may be questioned. Obviously this fusion is not a true syngamic one. It is followed by a resumption of activity on the part of the nuclei in the embryo sac; but since this may occur with no nuclear fusion, it seems evident that it is due to some peculiar condition pervading the sac after the pollen tube enters. Only rarely does the egg develop without a nuclear fusion. An activating, or "fertilizing," effect can be recognized in both fusions, but it now seems proper to regard the endosperm in angiosperms as gametophytic tissue which, unlike that of gymnosperms, is arrested in development until after the pollen tube enters the sac; it then proceeds with development, usually with, but sometimes without, the addition of extra nuclei.

\section{ANIMALS}

The story of syngamy in animals ${ }^{29}$ is complicated by the fact that in many cases the periods of "maturation" and fertilization may overlap. In sea urchins the two meiotic divisions in the oöcyte are completed before the spermatozoön enters. In certain other cases maturation proceeds as far as the metaphase of the first mitosis (certain annelids, nemertines, mollusks, and insects) or the metaphase of the second mitosis (frog, mouse) but goes no further unless penetration occurs. Finally, in Nereis

${ }^{28}$ See Coulter and Chamberlain (1903), Samuelsson (1913), Jacobsson-Stiasny (1914), Dahlgren (1922, 1923), Stenar (1925), Svensson (1925), Schürhoff (1926), and Schnarf (1929).

${ }^{29}$ In the preparation of this portion of the chapter the author has drawn freely upon Prof. F. R. Lillie's Problems of Fertilization, (1919). See also Lillie and Just (1924), Doncaster (1920), Agar (1920a), and Wilson (1925). 
(annelid) and Ascaris (nematode) penetration takes place before the first mitosis ${ }^{30}$ (Fig. 154).

Probably in most animals the whole spermatozoön enters the egg (Fig. 145). In some sea urchins only the head and middle piece enter, while in Nereis the head alone passes in, leaving the middle piece and tail on the egg surface. The details of penetration in two of the best known cases are as follows.

In Nereis the egg has a vitelline membrane, an alveolar cortical layer, many yolk and oil droplets, and a large central germinal vesiele (nucleus). If many spermatozoa are present in the vicinity, a large number attach themselves to the egg, but usually all but one are carried away by an

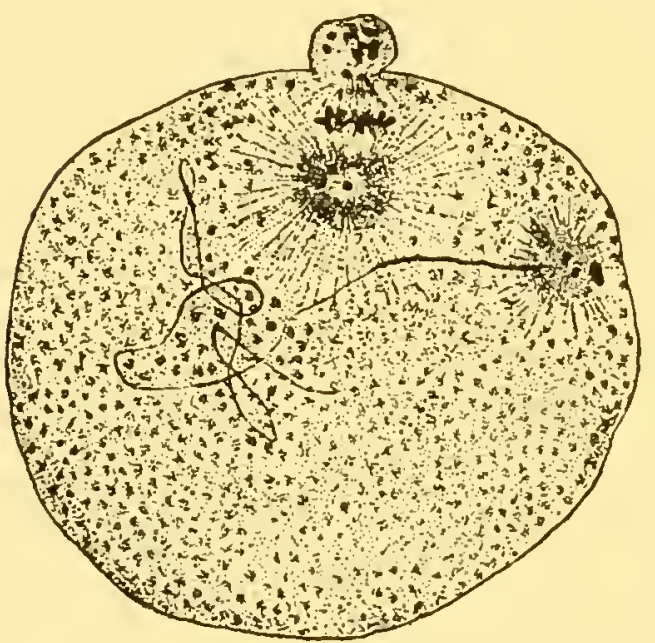

Fig. 145.- Syngamy in Physa (snail). Sperm head and amphiaster at right; long flagellum extending toward left; second meiotic division in progress. (After Kostanecki and Wierzyski, 1896.) outflow of jelly from the alveoles of the cortical layer. This layer now takes the form of a zone traversed by radial protoplasmic plates representing the walls of the alveoles. A transparent "fertilization cone" extends from the inner region of the egg across this zone and touches the membrane at the point where the spermatozoön is beginning to penetrate. The perforatorium goes through the egg membrane and becomes attached to the transparent cone. The latter is now withdrawn, carrying the head of the spermatozoön into the egg with it. Thus it appears that the initiative for the final act of penetration lies with the egg rather than with the spermatozoön. Since only the head enters the egg, it seems that the only necessary portion of the spermatozoön in the actual union is the nucleus; the middle piece and tail are accessory and function only as locomotor organs (F. R. Lillie, 1912, 1919).

In the starfish (Fig. 146) the blunt-headed spermatozoa swim about with spasmodic movements and apparently by chance come in contact with the thick zone of stieky jelly surrounding the egg. The egg then responds to the presence of the spermatozoa by forming one or more hyaline conical projections on its surface. From the summit of each cone a delicate filament grows outward through the jelly until it touches and adheres to any sperm head (now motionless) which happens to lie in its path. The filament then retracts, drawing the spermatozoön inward through the jelly to the summit of the cone. Other filaments are at the same time withdrawn whether they have secured spermatozoa or not.

${ }^{30}$ See Wilson (1925, p. 397) and literature cited; also McKay (1927) and Crabb $(1927 b)$. 
When the spermatozoön arrives at the surface of the cone it is immediately engulfed and passes deeper into the egg. The cytoplasmic granules at the base of the cone disappear, leaving a hyaline pathway along which the sperm head glides. Other spermatozoa being drawn through the jelly are usually dropped by the retracting filaments when the first sperm enters the egg; in case they do reach the egg surface they are stopped by the fertilization membrane (Chambers, 1922c, 1923b, 1930c).

The most striking feature in both of these cases is the relatively passive rôle played by the spermatozoön. Instead of actively forcing its way into the egg by its own power, it is "swallowed" by the egg after its own movements have ceased. In this connection Bowen (1923, 1924ab)
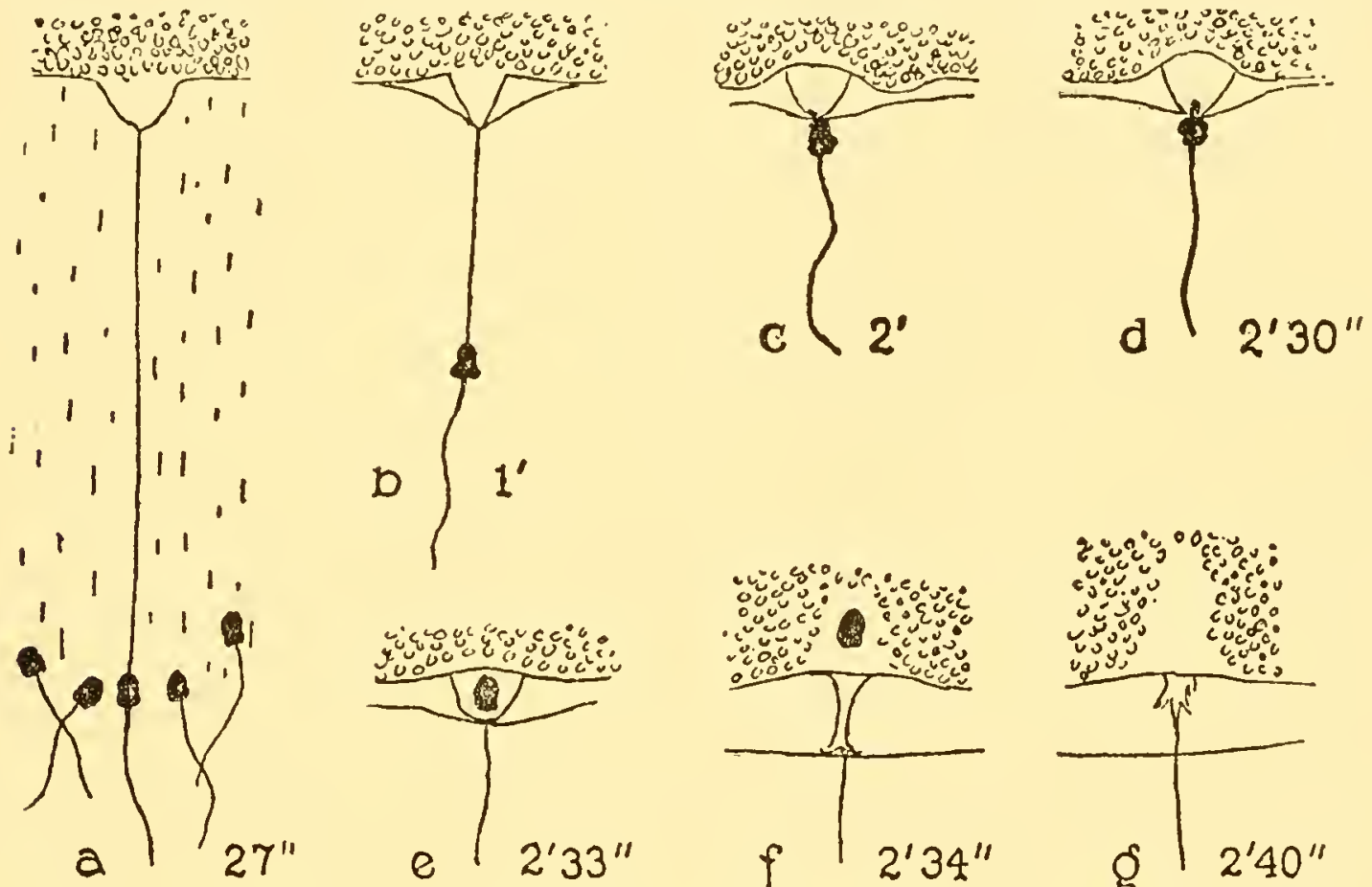

Frg. 146.-The entrance of the spermatozoön into the egg of the starfish. The time occupied by the process is indicated. (After Chambers, 1923b.)

has advanced the view that the acrosome with its perforatorium is not primarily a cutting tool but "essentially a secretory product the principal function of which is to initiate the physico-chemical reactions of fertilization." The spermatozoön may show no such movements as would be necessary for the use of a cutting or boring tool, and in some animals the acrosome is neither of the right shape nor properly located to act in such a capacity. In other animals, however, boring movements do occur. Probably a pointed perforatorium is advantageous in either active or passive movement through the jelly and egg membrane.

A fertilization membrane is commonly formed as a result of fertilization. In the echinoderm it first appears at the point where the spermatozoön is attached and spreads over the egg with great rapidity. This really consists in the elevation of a delicate vitelline membrane already present, 
a process which Heilbrunn has associated with a lowering of surface tension. ${ }^{31}$ These changes do not depend upon the actual entrance of the spermatozoön into the egg; in Nereis they occur before the slow penetration can be completed, or even if the spermatozoön is shaken loose shortly after penetration has begun. In some animals (frog) the amount of fluid in the "perivitelline space" under the membrane is great enough to permit the rotation of the egg within it.

In describing the remarkable transformation undergone by the spermatozoön within the egg it will be convenient to deal with its various parts separately.

The Nucleus.-Immediately after gaining entrance to the egg (Fig. 147), the sperm head begins to enlarge and assume the usual form and

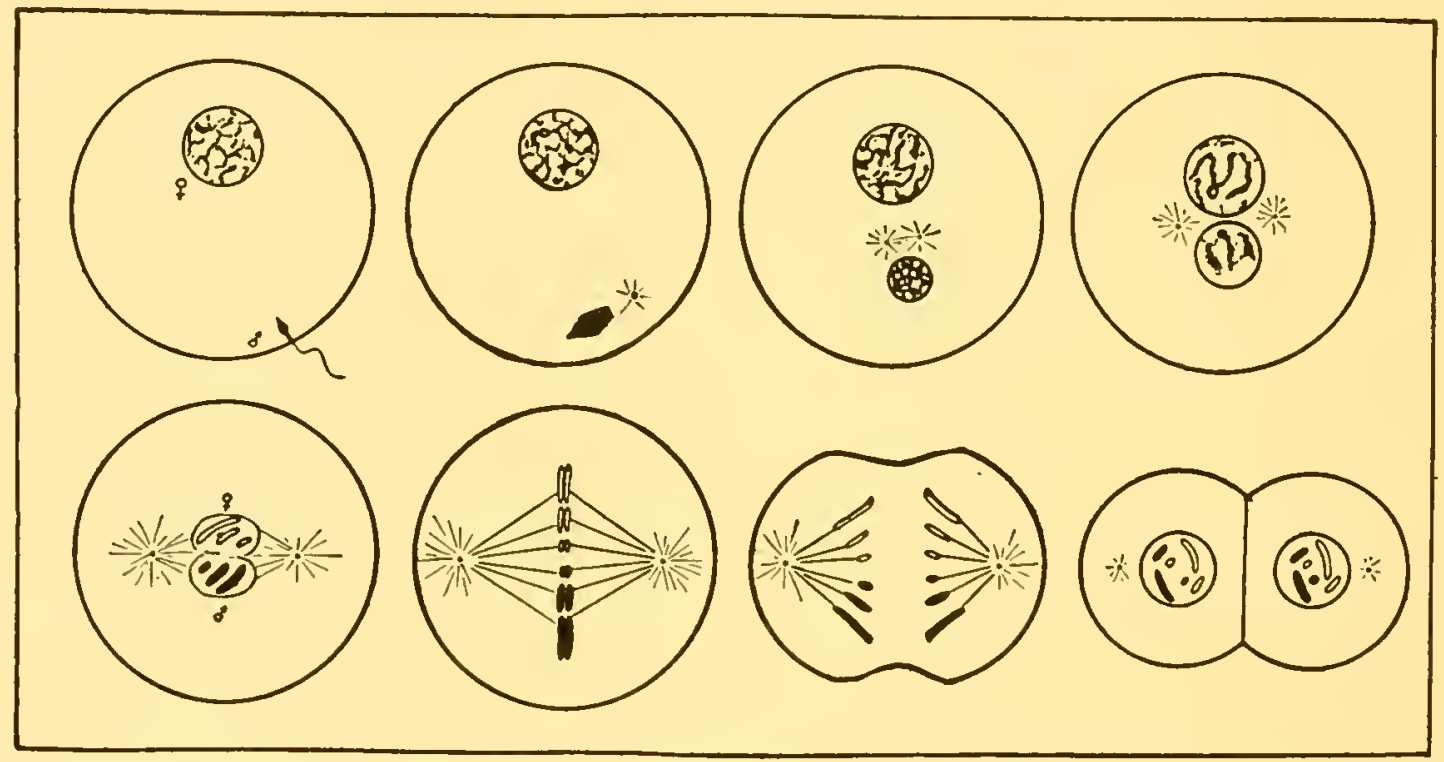

FIG. 147.-Diagram of syngamy and cleavage in an animal. It is assumed in this case that meiosis has been completed before the entrance of the spermatozoön.

structure of a nucleus. Meanwhile it advances toward the egg nucleus. As Lillie points out, both nuclei pass toward a position of equilibrium in a cell preparing to divide and consequently meet; the assumption of an attractive force between them is unnecessary. By the time they meet, the sperm nucleus has usually, but not always, become equal in size and appearance to the egg nucleus. The union of these two "pronuclei" usually occurs at once. In a great many cases there may be no actual fusion of the nuclei as such. As they come close to one another each passes through the prophase stages and gives rise independently to its group of chromosomes, the two groups arranging themselves in a common spindle which organizes as the nuclear membranes disappear. The nuclei may behave variously in this respect within the same species. In Strongylocentrotus lividus, Danchakoff (1916) observed three general

${ }^{31}$ Heilbrunn $(1913,1915,1924 c, 1925 b)$. See also Peterfí (1927), Hobson (1927). and Chambers (1930a) on the fertilization membrane; also the papers of Just (1920 et seq.). 
modes of behavior: (a) the chromatic sperm nucleus may be added to the comparatively achromatic egg nucleus, the two forming a common reticulum from which the chromosomes later condense; $(b)$ the sperm nucleus may become achromatic before uniting with the egg nucleus, the achromatic fusion nucleus forming a reticulum from which the chromosomes develop; (c) the sperm nucleus may condense its chromosomes before uniting with the egg nucleus, no common reticulum being formed and the maternal and paternal chromosomes remaining separate throughout their development.

In the first cleavage mitosis, whether the gamete nuclei have formed a common reticulum or not, each chromosome undergoes a longitudinal division, the halves passing to the two daughter nuclei. This process is repeated in every subsequent somatic mitosis, so that every somatic nucleus has descendants of every parental chromosome originally present in the fertilized egg. In most animals the first cleavage mitosis is followed by the division of the fertilized egg into two cells (blastomeres), but in some cases there are several free nuclear divisions before any cleavage into cells occurs. In some instances it has been shown that the plane of the first cleavage is determined by the chance point of entrance of the spermatozoön, and that the bilaterality of the embryo bears a direct relation to this cleavage. ${ }^{32}$ In other animals, on the contrary, the plane of the first cleavage does not coincide with the plane of bilateral symmetry; moreover, some eggs may show such symmetry before fertilization (e.g., many insects).

The more or less independent formation of the maternal and paternal chromosomes without an intimate nuclear fusion before the first cleavage mitosis is known as gonomery and has been observed in both animals and plants (Fig. 143). It occurs in Drosophila (Huettner, 1924). Furthermore, in several organisms it has been found that the two parental groups of chromosomes can be distinguished not only in the first embryonal mitosis but also in several divisions thereafter. ${ }^{33}$ This is especially evident in hybrids. There is reason to believe that the chromosomes brought together by the two gametes, although they are intermingled in the nuclei of the new individual, never actually fuse in such a way as to lose their essential organization. They are joined with one another in the reticulum and undergo occasional transfers and exchanges of parts, as will be shown in subsequent chapters; but this does not involve the complete mixing of their substance which was once thought to occur. The evident fact that certain nuclear elements maintain their identity through suc-

32 Wilson and Mathews (1895) on the sea urchin; Just (1912) on Nereis. See Morgan (1924b) and Wilson (1925; p. 1012). The plane of cleavage is associated with gravity by Giglio-Tos (1926).

${ }^{33}$ Rückert (1895) and Haecker (1895c) on Cyclops; Conklin (1901a) on Crepidula; B. G. Smith (1919) on Cryptobranchus. 
cessive life cycles in spite of such rearrangements is of the greatest importance in connection with problems of heredity.

The Centrosome.- Shortly after the penetration of the spermatozoön into the egg an aster develops in the cytoplasm near the base of the sperm head, and at the focus of the aster a centriole appears (Figs. 145, 147). The inclusion of a spermatid centriole near the nucleus during spermiogenesis and the appearance of the aster and centriole in this general region after fertilization led to the widely accepted view that the newly appearing centriole is, in reality, that of the spermatid. Whatever its origin, it soon divides into two which function in the first cleavage mitosis. These facts had much to do with the formulation of a theory of fertilization according to which the egg is not able to undergo division because of the lack of any centrosome to initiate the process, while the spermatozoön has a centrosome but not sufficient cytoplasm in which to act. Through the union of the gametes all the organs necessary for division are brought together and cleavage proceeds (Boveri, 1887, 1891).

Various other theories regarding the origin of the cleavage centrioles have been propounded, some workers identifying them with those of the egg and others deriving them from both egg and sperm (see Wilson, 1925, p. 438). In practically all cases there are gaps in the known history of these centrioles. It remains to be clearly proved that the cleavage centrioles are continuous with those of either gamete, although in some cases such proof is very closely approximated. On the other hand, it has been shown that the formation of asters with centrioles can be induced in the cytoplasm by treating the eggs with certain chemicals (see p. 154), and that such structures, provided they originate near the nucleus, may divide and function in the cleavage which follows. ${ }^{34}$ This suggests that the spermatozoön may carry a substance which brings about centriole formation by the cytoplasm in normal fertilization. However this may be, the importance of the centrosome undoubtedly lies in its relation to cleavage rather than to syngamy.

Cytoplasm and Chondriosomes.-In animals, as in plants, the evidence in general indicates that the cytoplasm of the male gamete is of relatively little importance in syngamy. A considerable amount of paternal cytoplasm is introduced in some cases (Ascaris), but in others none at all can be seen to enter. This is particularly true of such a case as Nereis, in which the middle piece and the tail remain outside the egg. These facts are of interest in connection with the suggestion of Meves (1911) that chondriosomes introduced by the sperm are responsible for the transmission of certain paternal hereditary characteristics. The observed behavior of these bodies during cleavage ${ }^{35}$ does not support the theory.

${ }^{34}$ Tharaldsen (1926), Beams (1927).

${ }^{35}$ Van der Stricht (1902), Lams (1913), Meves (1914a). See Wilson (1925; p. 412). 
One should not, however, lose sight of the fact that cytoplasm and chondriosomes are passed from generation to generation through the egg. As will be pointed out later, much that happens in the embryo depends upon the constitution of the egg cytoplasm. That which undergoes development is a complete protoplast; no differentiation occurs without the combination of nucleus and cytoplasm, both of which are derived directly from the preceding generation through one or both of the gametes. The presence of chondriosomes in the egg and sometimes in the sperm probably indicates the occurrence of certain physiological processes as it does in other cells. Such processes may influence the course of differentiation, but there is as yet no adequate evidence that the chondriosomes act as differential factors of inheritance in any way comparable to the action of nuclear factors.

Conjugation and Endomixis.-From among the many kinds of syngamic phenomena in Protozoa ${ }^{36}$ a single peculiar example is selected for description here. Conjugation in Paramœcium, as originally shown by Maupas (1889), involves a complicated series of nuclear changes. Wilson (1900) describes these as follows:

... in Paramocium caudatum, which possesses a single macronucleus and micronucleus, ... eonjugation is temporary and fertilization mutual. The two animals become united by their ventral sides and the macronucleus of each begins to degenerate, while the micronucleus divides twice to form four spindleshaped bodies. Three of these degenerate, forming the "corpuseles de rebut," which play no further part. The fourth divides into two, one of which, the "female pronucleus," remains in the body, while the other, or "male pronucleus," passes into the other animal and fuses with the female pronucleus. Each animal now contains a eleavage-nucleus equally derived from both the eonjugating animals, and the latter soon separate. The cleavage-nucleus in each divides three times successively, and of the eight resulting bodies four become macronuelei and four micronuclei. By two succeeding fissions the four maeronuelei are then distributed, one to each of the four resulting individuals. In some other speeies the mieronuclei are equally distributed in like manner, but in $P$. caudatum the proeess is more eomplieated, since three of them degenerate and the fourth divides twice to produce four new mieronuclei. In either case at the elose of the process each of the conjugating individuals has given rise to four descendants, each containing a maeronucleus and a micronucleus derived from the cleavage-nueleus. From this time forward fission follows fission in the usual manner, both nuclei dividing at each fission, until, after many generations, eonjugation recurs.

It was supposed for a long time that conjugation involving an intermixture of nuclear material from two individuals was necessary for the

${ }^{36}$ Sec M. Hartmann (1909, 1914), Erdmann (1920), G. Hertwig (1921), and especially Jennings (1923), Bělař (1922), Kofoid (1923), E. B. Wilson (1925), and Calkins (1926). For a diagram of conjugation in Paramœcium, see Morgan (1913, p. 6). Conjugation in Dileptus is described by Visscher (1927). 
continued vigor of the race in such animals, and that without a periodic recurrence of the process the race would become increasingly senescent and finally die out. The long-continued experiments of Woodruff, Jennings, and others showed that this is not what happens. Given a properly regulated environment, these organisms will give rise to thousands of successive generations with no conjugation and no decline in vigor. The natural conclusion seemed to be that protoplasm does not necessarily become senescent through continued vegetative activity.

The problem assumed a new aspect with the discovery of endomixis by Woodruff and Erdmann (1914). In conjugation, as shown above, the micronucleus in each individual initiates a series of three mitoses, one of the resulting nuclei passing as a gamete nucleus to the other individual, where a fusion takes place. Since some of the products of the division of the fusion nucleus become macronuclei, conjugation involves a replacement of the old macronucleus by material from the micronucleus, as well as the exchange and fusion of micronuclei. In enciomixis, on the other hand, there is such a replacement but no exchange or fusion. For example, in Paramocium aurelia, which has two micronuclei, it is found that after every 40 or 50 generations (by fission) the macronucleus disintegrates in the cytoplasm, while each micronucleus undergoes two successive mitoses (rather than three). All but one or two of the resulting nuclei degenerate, those which remain later dividing to produce the new macronuclei and micronuclei.

The question of whether the race can continue to live without either conjugation or endomixis has awaited answer for several years. In Paramœcium aurelia and $P$. caudatum the periodic recurrence of this nuclear reorganization is coincident with a rhythmic acceleration in metabolic activity, which at least in part explains the ability of these animals to maintain their vigor without conjugation. Furthermore, if endomixis ceases, the cultures die. But in other species endomixis takes place in the encysted stage or is lacking altogether, yet the metabolic rhythms occur and the race continues. This indicates some factor other than endomixis as the cause of the periodic acceleration of vital activity. The results of several researches ${ }^{37}$ point to the general conclusion that neither conjugation nor endomixis is necessary for the continued existence of the race, although under environmental conditions inducing senile change both processes have rejuvenating effects.

Endomixis thus resembles syngamy in its stimulative effect on metabolism and development, but its possible relation to variation and heredity is not so clear. There is no evidence that in endomixis there is any meiotic disjunction of chromosomes in the formation of the degenerating nuclei in fact, there are certain indications that in the process of conjuga-

${ }_{37}$ Woodruff and Spencer (1924), Spencer (1924), E. I. Moore (1924). See especially Woodruff (1925), Calkins (1926), and Jennings (1920). 
tion disjunction occurs in the third or gamete-producing mitosis, this mitosis being the one which is lacking in endomixis. Molecular rearrangements doubtless occur, but to what extent these may affect the constitution or action of the physical basis of heredity remains for future work to determine.

The Physiology of Fertilization.-The problem of iclentifying the physico-chemical changes involved in the activation of the egg has been attacked through studies of artificial parthenogenesis, as well as through direct analyses of the chemical constitution of the gametes at these stages. ${ }^{38}$

In 1899 Jacques Loeb made the important discovery that the parthenogenetic development of certain animal eggs can be artificially induced. In most cases two successive treatments were found necessary; first, a brief treatment with sea water containing some permeability-increasing substance (certain fatty acids, bases, soaps, alkaloids, glucosides, and foreign blood sera); and, second, a longer treatment with hypertonic sea water, or oxygen-free sea water, or sea water with a trace of $\mathrm{KCN}$, or low temperature. The first treatment caused the formation of a fertilization membrane, which seemed to be a condition necessary for continued development. The critical change was apparently a momentary increase in surface permeability which set in motion the developmental reactions, but in most cases the eggs became sickly and died unless given the second treatment. Loeb concluded that the spermatozoön in normal fertilization may carry two substances, one of which produces the necessary initial surface changes, even when the spermatozoön fails to enter the egg, while the other in some way acts as cloes the second treatment in artificial parthenogenesis. This suggestion seems less plausible in the light of Just's (1922) discovery, that normal sea-urchin larvæ can be produced by treating the eggs with hypertonic solutions alone.

Loeb thought the sickliness of eggs given only his first treatment to be due to a continuance of the cytolytic action begun by this first treatment, but F. R. Lillie pointed out the greater probability of the view that it is due to some internal cause, citing as supporting evidence certain eytological phenomena observed by Herlant in eggs activated by Loeb's method. During the healthy period (12 to 24 hours) immediately following the first treatment, Herlant (1917) observed the following changes. After the formation of the membrane and a hyaline zone, the nucleus becomes the seat of a series of conspicuous alterations. The nuclear membrane disappears, and around the chromosomes there is formed a one-poled achromatic figure but no amphiaster. The chromo-

${ }^{38}$ For general accounts, see Loeb (1913), F. R. Lillie (1919), Lillie and Just (1924), R. S. Lillie (1923), and Gray (1931). For work on plants, see J. B. Overton (1913) on Fucus, Tahara (1927) on Sargassum, and Popoff $(1920,1922,1931)$ on somatic tissues. 
somes divide but do not separate. Although the cytoplasm becomes active, no cytokinesis ensues. The chromosomes then return to the reticulate condition. This process is repeated several times, the nucleus increasing in bulk each time, but it soon becomes very irregular and the egg ultimately breaks down by general cytolysis. The second treatment in some way gives the egg the capacity to divide regularly. Morgan (1899) and Wilson had long before shown that such treatment with hypertonic sea water causes aster formation in the unfertilized sea-urchin egg. Herlant showed that one of these asters and a second aster formed near the egg nucleus together form an amphiaster, normal division then ensuing. Thus the breakdown of the egg after the first treatment alone appears to be related to the absence of a proper coördination of nuclear and cytoplasmic division. The second treatment produces a regulatory effect, partly through aster formation, this resulting in normal development.

Among other agencies inducing activation in certain cases may be mentioned high temperature (R. S. Lillie, 1908, 1915; Heilbrunn, 1925a), carbon dioxide (Herlant, 1920), puncture by needles, allowing gases or other substances to pass the membrane (Bataillon, 1910, and others), and release from the vitelline membrane (Heilbrunn, 1920b). With respect to the effect of heat, Heilbrunn (1925a) finds a measurable increase in viscosity at temperatures inducing artificial parthenogenesis and reiterates his theory (1915) that all such activating methods produce a gelation or coagulation in the egg cytoplasm.

F. R. Lillie (1913 et seq.) advanced the theory that the egg is an "independently activable system," the spermatozoön contributing neither organs nor substances necessary to activation. "The egg possesses all substances needed for activation; the spermatozoön is an inciting cause of those reactions within the egg system upon which development depends." As a result of his direct analysis of the gametes during the fertilization period, Lillie identified a substance in the egg which he called fertilizin. This substance is present in the egg for a short time only. Its formation usually begins at about the time of the breakdown of the germinal vesicle, from which it probably emanates; immediately after fertilization its production ceases. As a rule, it is only during the brief period when fertilizin is present that spermatozoa will enter the egg. Moreover, membraneless egg fragments without fertilizin are not entered. Hence it seems clear that the entrance of more than one spermatozoön is normally prevented, not simply by the mechanical barrier of the fertilization membrane which develops when the first sperm enters, but also by the physiological state of the egg protoplasm itself.

Fertilizin has two effects: it first acts by causing an agglutination of the spermatozoa at the surface of the egg, and later causes the activation of the egg. It may thus be said to stand between the spermatozoön and the activation reactions in the egg. Being present in the egg secretion 
at a certain period, it binds the spermatozoön to the surface of the egg; and the spermatozoön, without necessarily penetrating the egg at all, releases the activity of the fertilizin within the egg, this resulting in development. In brief, the activating substance is already present in the egg and is not brought to it by the spermatozoön. It may be incited to activity by the spermatozoön but by other agencies as well. According to Woodward (1918) and Glaser (1921), the agglutinating material and the parthenogenetic agent are two distinct substances.

Among other immediate physiological consequences usually following fertilization may be mentioned: an increase in permeability to oxygen, $\mathrm{CO}_{2}$, pigments, water, alkalies, vital dyes, and certain other substances; an increase in the rate of oxidation; an increase in viscosity, centering in the sperm aster; a rise in electrical conductivity; the loss of fertilizin (Lillie, 1919). Just (1920) points out that most of these changes are not incident to the fertilization reaction per se but are primarily changes bound up with the egg's division.

In this connection one may be cautioned against the assumption that normal activation is complete when the spermatozoön has gained entrance to the egg. Morgan (1924f) cites the fact that, if an inseminated egg be cut into two pieces in such a way that one fragment contains the egg nucleus and the other the sperm nucleus, only the latter develops; he therefore emphasizes the inadequacy of theories of fertilization which terminate with the entrance of the spermatozoön.

Conclusion.-Syngamy is a mutual process: that which undergoes development following syngamy is a zygote-the fusion product of two gametes, no matter how dissimilar these may have been in their morphological and physiological differentiations. Syngamy has two important results: there is a physiological alteration with profound effects upon the behavior of the protoplasts concerned, and there is a nuclear reorganization which is no less significant. In view of the cardinal importance of the latter change with respect to current theories of heredity, it should be remembered that in the syngamic nuclear union two monoploid sets of chromosomes are brought together and form a diploid complement in the zygote nucleus; and since every chromosome of this complement divides equationally in every somatic mitosis throughout the development of the resulting individual, every nucleus in this individual contains a descendant of every chromosome originally present in the zygote. ${ }^{39}$

As the finer details of syngamy and the significance of its results become better understood, the aptness of Huxley's (1878) often quoted simile, in which he compared the organism to "a web of which the warp is derived from the female and the woof from the male," becomes increasingly striking.

${ }^{39}$ Only typical "diploid" organisms are considered here. The phenomena in "polyploid" organisms are essentially the same except for the number of chromosome sets present (see p. 339). 


\section{CHAPTER XVI}

\section{MEIOSIS}

No subject in the field of cytology is of greater importance than meiosis. This is mainly because the meiotic process affords a key to the explanation of many phenomena of development and inheritance which would otherwise be quite unintelligible; in fact, the modern study of the rôle of chromosomes in heredity has largely centered about changes in nuclear constitution which occur at the meiotic period. Meiosis may therefore be taken as the starting point in our discussion of cytogenetics, which will be begun in the next chapter.

As pointed out at the conclusion of the preceding chapter, each somatic nucleus in ordinary diploid plants and animals contains a complement of chromosomes made up of two intermingled sets descended by division from those brought together in the previous union of gametes. In meiosis there is accomplished what has long been referred to as "the reduction of the chromosomes." This expression has had two meanings. In the first place, it has meant the change from the zygotic to the gametic number which occurs at this time; this is haplosis, or "numerical reduction." In the second place, it has been applied more specifically to the disjunction of the two members of each pair of corresponding ("homologous") chromosomes present in the diploid complement, an event which constitutes the central feature of meiosis.

The Stage in the Life Cycle at Which Meiosis Occurs.-Haplosis and chromosome disjunction are accomplished during the course of two nuclear divisions known as the meiotic divisions. Because of its peculiar appearance the first of these mitoses was termed "heterotypic" by Flemming (1887), while the second, which was seen to resemble a somatic division in certain features, was called "homœotypic." Advances in our knowledge of meiosis have led most cytologists to drop these terms and to refer simply to the "first and second meiotic divisions." They are also called "maturation divisions," especially in the zoölogical literature. Since meiosis involves two divisions, the resulting nuclei or cells are formed in groups of four, or quartets, although it frequently happens that some members of the quartet do not function. For convenience the two meiotic mitoses will be referred to frequently in subsequent pages as $I$ and $I I$.

In the normal life cycle of higher animals meiosis occurs at the time of gametogenesis (Fig. 148). In the male the two divisions result in a 
quartet of spermatids, which then transform into spermatozoa (p. 214). In the female they result in an egg and three (sometimes only two) polocytes (p. 213). Each gamete, male or female, thus contains the reduced, or gametic, number of chromosomes. In bryophytes and vascular plants meiosis occurs normally during sporogenesis, hence the spores are formed in quartets. Each spore carries the reduced number of chromosomes, this number being retained through the development of the gametophyte and its gametes (p. 204). The conditions found in lower plants will be summarized toward the end of the chapter.

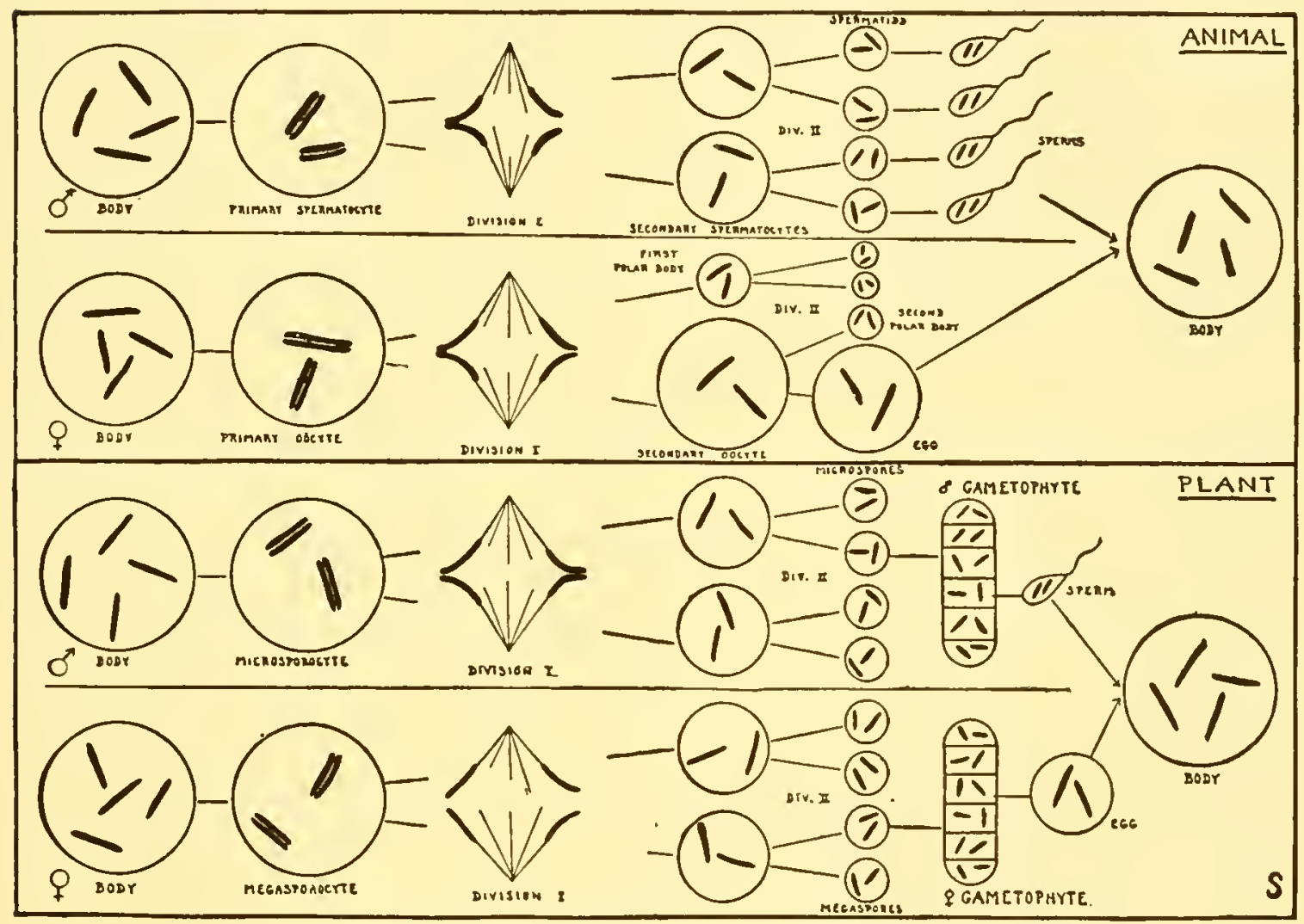

Fig. 148.-Diagram of chromosome cycles of animals and plants.

The term meiocyte may be used to designate any cell in which meiosis is initiated, whatever its origin or position in the life cycle. ${ }^{1}$ In higher animals the meiocytes are therefore the primary spermatocytes in the male and the primary oöcytes in the female. In bryophytes and vascular plants they are the sporocytes-the microsporocytes and megasporocytes in heterosporous forms. The immediate products of meiosis, i.e., the nuclei or cells formed in quartets, may be referred to as gones.

Preliminary Sketch of Meiosis.-Before proceeding with the detailed description of meiosis it will be advantageous to have clearly in mind certain general features of the process without the complications which must be introduced later, and to visualize the more conspicuous differ-

${ }^{1}$ Other terms which have been used in this sense are auxocyte (Lee, 1897) and gonotokont (Lotsy, 1904). 
ences between meiosis and mitosis of the somatic type. These points are illustrated in Fig. 149.

In the division of a somatic nucleus each and every chromosome of the complement divides longitudinally into two halves which come to lie in the two daughter nuclei. Both of these nuclei are therefore like the original nucleus in containing equivalent derivatives of all the chromosomes; hence somatic mitosis is said to be equational.

In the prophase of the first meiotic mitosis the two members of each pair of homologous chromosomes approach each other and come into intimate association; this is known as synapsis. As a result of synapsis the nucleus contains bivalent chromosomes in the reduced number. ${ }^{2}$ At some period during the prophase it becomes evident that each of the members of a synaptic pair is double as the result of a longitudinal splitting. Hence each bivalent appears not simply double, but quadruple: it is a tetrad chromosome. The four members of each tetrad, two of them derived from one synaptic mate and two of them from the other, are known as chromatids. ${ }^{3}$ At the close of the first meiotic prophase, therefore, the meiocyte nucleus contains the reduced number of tetrads.

In the two meiotic mitoses the four chromatids of each and every tetrad are distributed (with or without certain alterations) to the four resulting nuclei. When the tetrads are oriented in the spindle as shown in the diagram, the two paternal chromatids are separated from the two maternal ones ${ }^{4}$ with which they have been in synapsis; this is known as disjunction, or reduction in the stricter sense. Each two associated chromatids passing to each pole constitute a dyad. When disjunction occurs in the first mitosis the second mitosis is obviously equational in character, since dyads which are then separated into single members are sister chromatids formed by longitudinal division.

In some cases it is known that tetrads may be so constituted and oriented in the spindle that they divide (at least in part; see p. 265) along the plane of splitting in the first mitosis and consequently along the synaptic plane (disjunctionally) in the second. Both types of division may occur in the same cell, so that each of the two meiotic mitoses may be both disjunctional (for some elements in the chromosomes) and equational

2 This is often called "pseudoreduction." Bivalent chromosomes are sometimes called gemini (= twins). "Homologous" chromosomes in the complement are those which correspond in function (sce p. 272).

${ }^{3}$ It is customary, though somewhat awkward, to apply the term "chromosome" rather indiscriminately to single chromosomes, split chromosomes, univalents, bivalents, multivalents, and tetrads. When the two halves (chromatids) of a split chromosome or the four chromatids of a tetrad separate from one another, each is then referred to as a "chromosome."

"It is convenient to designate the chromatids in this way because the synaptic mates are ordinarily derived from the male and female gametes. It will be shown, further on, that this is not always the case, and that parental derivation is not the essential feature of "homology." 

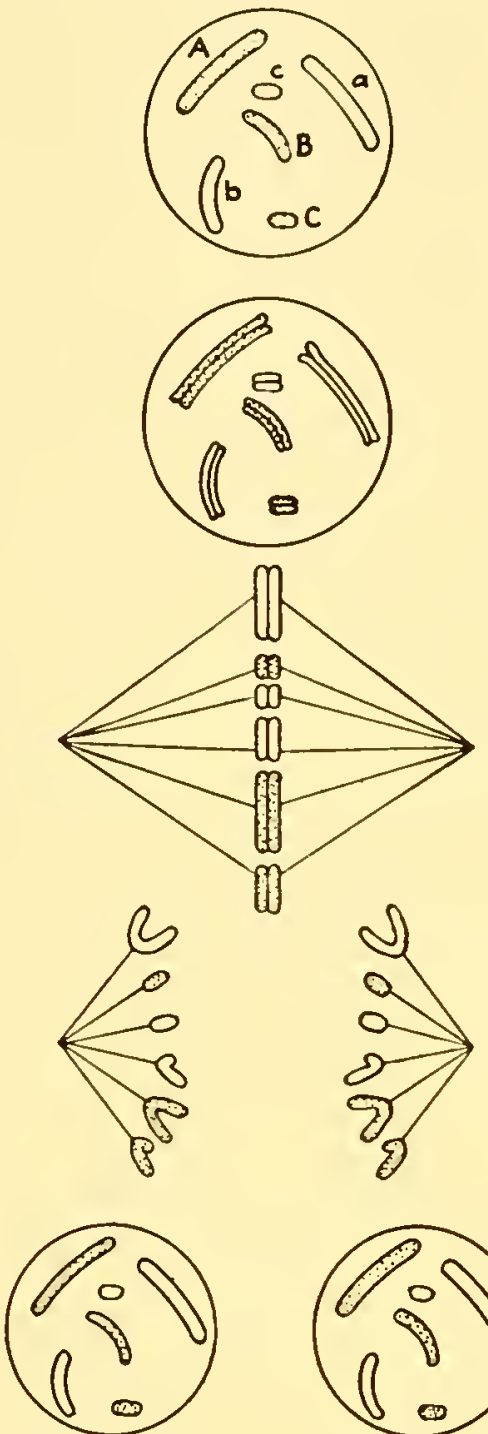

$A B C a b c$

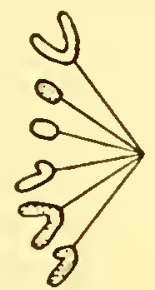

$A B C a b c$
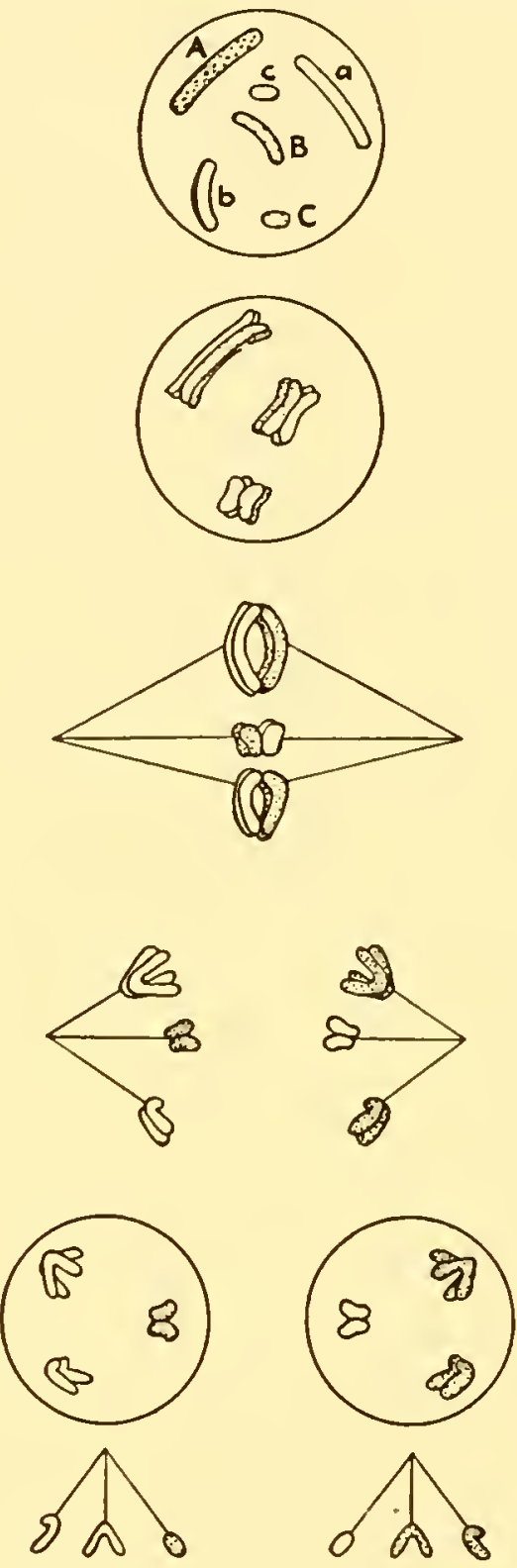

DIAGRAM COMPARING SOMATIC MITOSIS WITH THE MEIOTIC MITOSES

First column: Equational division of a diploid somatic chromosome complement.

Second column: The meiotic divisions, changing the diploid to the monoploid state.
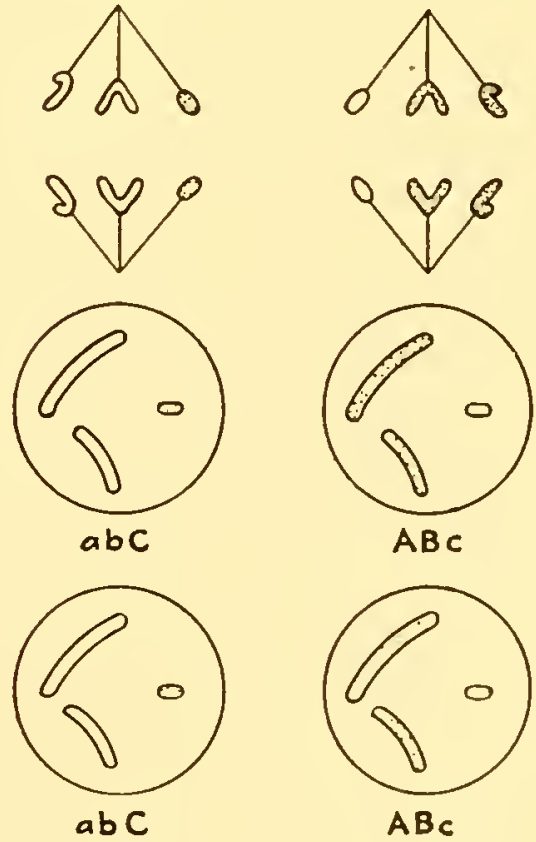
(for other elements). Disjunction in $I$ is called "prereduction"; that in II, "postreduction."

It should be noted that the several tetrads in the first meiotic metaphase are arranged entirely at random with respect to the poles toward which the paternal and maternal members are directed (see Fig. 149). All of the paternal elements may face one way and all of the maternal ones the other, or any other possible arrangement may obtain; apparently this is wholly a matter of chance. ${ }^{5}$ Hence the distribution of chromosomes in meiosis is a random matter so far as their parental derivation is concerned.

At the close of meiosis each of the four resulting nuclei has a monoploid chromosome group (a set) made up of one chromatid of each of the tetrads. In other words, the set comprises a longitudinal half of one member of each of the pairs of homologous chromosomes in the original diploid complement. Thus meiosis involves two nuclear divisions but only one chromosomal division. Representing the three pairs of chromosomes in Fig. 149 by the letters $A a B b C c$, it is found that two of the four nuclei have $A$ and the other two $a$; two have $B$ and two $b$; two have $C$ and two $c$. It should be readily understood that the random assortment of the three pairs of parental elements makes it possible for a nucleus of the quartet to have any one of eight possible combinations: $A B C, A B c$, $A b C, A b c, a B C, a B c, a b C, a b c .^{6}$

A further point should be noted. Two nuclei having $A$ and $a$, respectively, may be considered to differ qualitatively according to the degree of difference between these chromosomes. If, in the meiocyte nucleus, the members of this pair are unlike $(A a)$, two of the quartet nuclei will differ qualitatively from the other two; whereas, if the members are alike $(A A$ or $a a)$ in the meiocyte, all of the quartet nuclei will be alike so far as this pair is concerned. The same holds for all of the pairs. Were the two members exactly alike in every pair, meiosis could occur without producing any qualitative differences whatsoever among the nuclei of the quartet. The essential point to be borne in mind here is that

${ }^{5}$ The same would be true of dyads in the second mitosis in eases of postreduction. Random distribution was first demonstrated with pairs composed of dissimilar members in the orthopteran genera Brachystola and Trimerotropis (Carothers, 1913 1917).

${ }^{6}$ These statements are made on the assumption that the chromosomes remain individually intact throughout meiosis. When homologous chromosomes exchange portions with one another, as will be shown later, the statements will still hold for a given small region of a chromosome.

When all of the chromosomes disjoin in $I$ and divide equationally in $I I$, as shown in the diagram, there are chromosome sets of two types in the nuclei of the quartet, each type being represented twice. When $I$ is equational for portions of tetrads and the dyads are not oriented similarly in the two spindles at $I I$, it is possible to have sets of four types in the quartet. Since the organism usually produces many quartets, there is abundant opportunity for the production of all possible types. 
each nucleus of the quartet resulting from the meiotic divisions contains but one member of each homologous chromosome pair, rather than two as in the meiocyte nucleus. Although qualitative differences among the four nuclei usually obtain as a result of certain dissimilarities in the homologous chromosomes, such differences are not always a necessary consequence of meiosis.

The main points brought out in this section may be summarized provisionally as follows:

In a somatic mitosis each chromosome of the complement is divided longitudinally, the halves passing to the daughter nuclei; hence these nuclei are similar to each other and to the original nucleus: somatic mitosis is equational.

In meiosis each chromosome enters into synapsis with its homologue and also splits longitudinally; this gives a tetrad chromosone composed of four chromatids. The four chromatids of every tetrad are distributed to the four nuclei by the two meiotic mitoses. Each chromosome (or portion of a chromosome) is thus disjoined from its homologue in one mitosis and divided equationally in the other.

Each of the quartet nuclei contains a monoploid chromosome set made up of a longitudinal half of one member of each of the original homologous pairs. The four nuclei differ qualitatively among themselves to the degree in which the various chromosomes differ from their respective homologues, and they furthermore differ from the original meiocyte nucleus in having only half as many chromosomes.

In the next chapter evidence will be presented to show that meiosis involves essentially a change from the condition in which both members of a pair of corresponding genetic units are present in the nucleus to the condition characterized by the presence of but one. This change may be accomplished for all of the units at one of the meiotic mitoses, or for some units at the first mitosis and for others at the second.

Detailed Description of Meiosis.-The literature pertaining to meiosis is characterized by a sharp conflict of opinion regarding the structural alterations which take place, especially during the first prophase. This divergence in interpretation has been both annoying and stimulating, especially because important theoretical considerations have been involved. During the past few years some of the long-debated questions have received generally accepted answers, but other problems have arisen in their places. On the whole, very substantial progress has been made in this important field.

In this section we shall describe the successive appearances presented by the nuclear elements during the process of meiosis. The course of events is naturally not precisely the same in all organisms, but the following account is found to apply very widely, at least so far as essentials are concerned. In order to avoid confusion it will still be necessary to post- 
pone consideration of certain problematic matters until later in the chapter. $^{7}$

As the prophase of the first meiotic mitosis begins, the reticulum of the meiocyte nucleus transforms into the diploid number of long and very slender threads (leptonema stage) (Fig. 150, 1). These thin threads represent essentially the chromonemata of the chromosomes. Owing to their attenuation and the scarcity of enveloping substances they may show clearly their small chromomeres and other structural details. Their doubleness is a matter of debate. Although considerable evidence has been brought forward to show that in some cases at least they are already split at this time, or even in the telophase of the last premeiotic mitosis, ${ }^{8}$ the microscope in other cases reveals no trustworthy evidence of doubleness. The threads may lie without any regular orientation in the nucleus, or, especially in animals, their ends may be directed toward one side, forming what has been called a "bouquet."

The leptonema threads now begin to conjugate in pairs (synapsis). This involves a series of movements which are not well understood but which in some way bring each chromosome where it can enter into close union with its homologue in one or more regions (zygonema stage). Synapsis seems to begin at certain points in the threads, notably at their ends, and then gradually to extend throughout their length. While this is in progress the nucleus may show both paired and unpaired threads; this amphitene condition is well shown in bouquets. During these stages the threads show a notable tendency to shrink and collapse into a more or less tight knot under the influence of fixatives. This shrinking is known as synizesis. In most cases it is obviously an artifact due largely to inadequate fixation of the karyolymph, but in some a certain amount

${ }^{7}$ No attempt can be made in a work of this scope to give a complete summary and classification of all the interpretations that have been put upon the meiotic phenomena. Only enough will be presented to afford a starting point for a study of this complex subject. For a review and criticism of all views expressed up to 1910 , see Grégoire $(1905,1910)$. The subject may be followed further in the more recent general accounts of Agar (1920a), Doncaster (1920a), Tisehler (1921-1922), McClung (1924), Wilson (1925), Bělař (1928), Reuter (1930), and Darlington (1931d, 1932a). Useful lists of works on somatic and meiotic mitosis in angiosperms are given by Picard (1913) and Ruys (1925); see also Schürhoff (1926).

Meiosis as outlined in the following paragraphs was first described by von Winiwarter (1900), A. and K. E. Schreiner (1904 et seq.), and Maréehal (1904 et seq.) for animals; and for plants by Dixon (1895), Grégoire (1904, 1907), Berghs (1904, 1905), Rosenberg (1905, 1907b, 1909a), C. E. Allen (1905bc), and J. B. Overton (1905, 1909). Many of the stages have been observed in living cells: see Chambers (1924, 1925) on Dissosteira, Chodat (1924) on Gymnadenia, Lenoir (1927) on Lilium, and Bělař (1928) on Chorthippus. Recent detailed accounts of meiosis are eited in subsequent footnotes.

${ }^{8}$ W. R. B. Robertson (1919, 1920, 1921, 1931a) and McClung (1924, 1927a, 1928a) on Orthoptera, Kaufmann (1926a, 1931a) and Nebel (1932) on Tradescantia and Rhœo. The significance of these eases will be discussed later in the chapter. 
of shrinkage is claimed to be natural. Eventually the synapsis of homologous threads becomes complete, and the tendency to collapse, which renders many cytological preparations worthless, is no longer so evident.
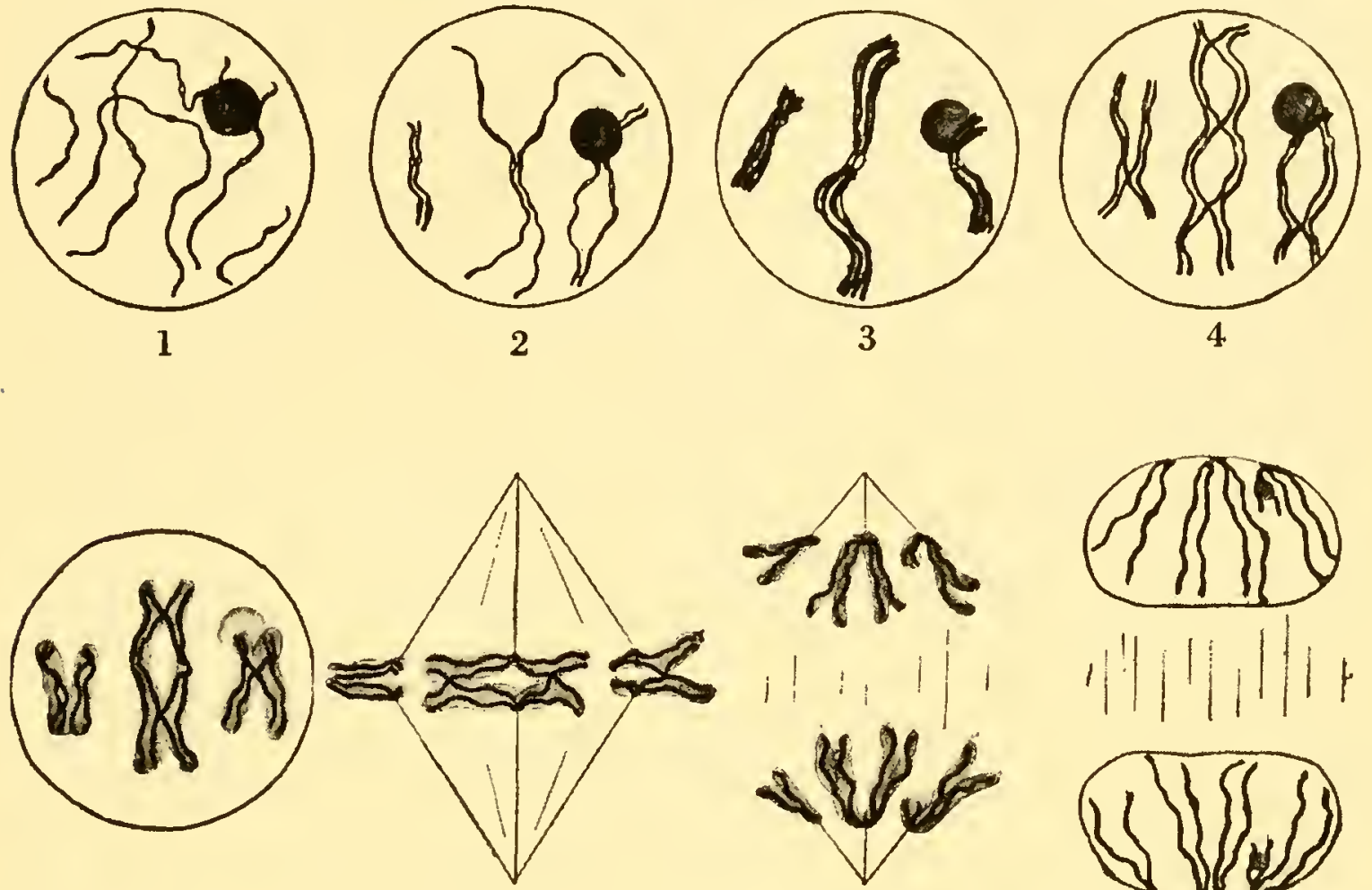

5

6
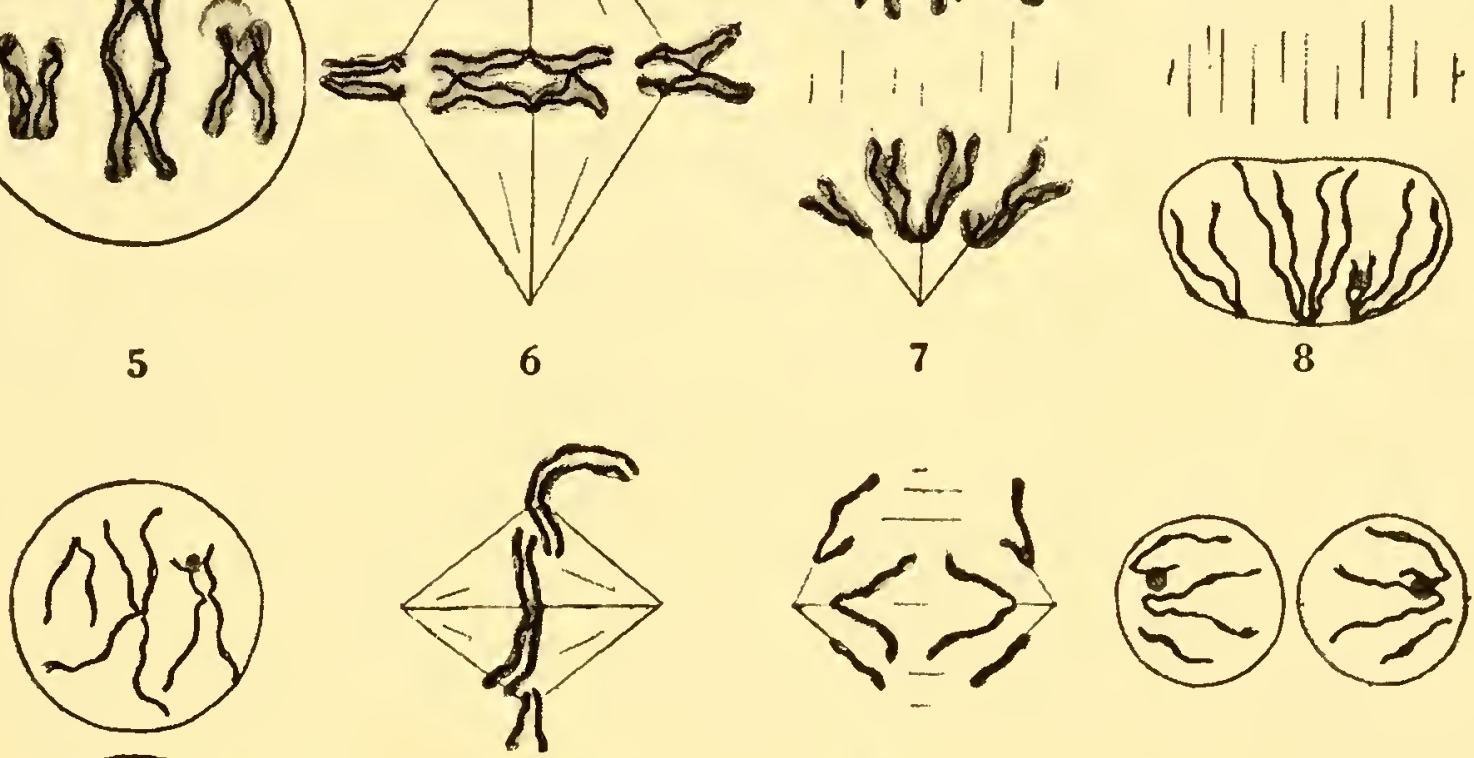

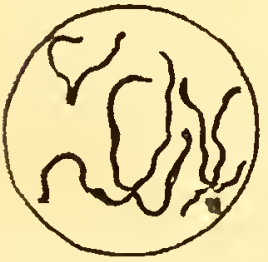

9

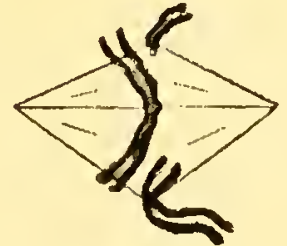

10

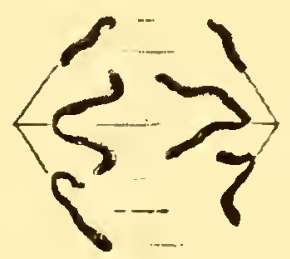

11
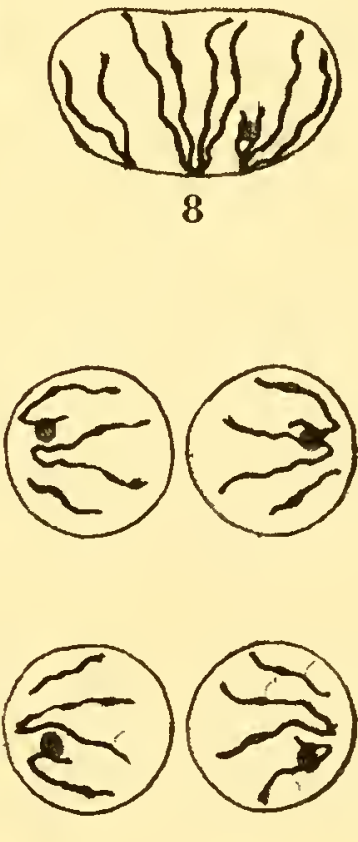

12

Fig. 150.-Semidiagrammatic representation of the principal stages of meiosis. 1 , leptonema; threads present in the diploid number (6). 2, zygonema; synapsis of homologous chromosomes in progress. 3, pachynema. 4, diplonema; note chiasmata. 5 , diakinesis; chiasmata fewer; matrix developed. 6 , metaphase $I . \quad \gamma$, anaphase $I$; tetrads separated into dyads. 8, telophase $I .9$, prophase $I I$; chromatids remain associated at spindle-attachment regions. 10 , metaphase $I I .11$, anaphase $I I .12$, telophase $I I$; each nucleus has the monoploid number (3) of chromatids. The split which is to become effective in the first postmeiotic mitosis is indicated in portions of the chromonemata in 8-12. Cf. Fig. 54 .

The nucleus now contains the reduced number of bivalent threads, each of which represents two homologous chromosomes in synaptic union 
(Fig. 152). These threads soon undergo a marked shortening and commonly become noticeably thicker; hence this is called the pachynema stage. The pachynema threads may lie irregularly in the nucleus, or they may retain the bouquet type of orientation for a time. In suitable preparations their bivalent nature is obvious in their distinct doubleness. Moreover, the chromomeres and other structural features show a close correspondence in the two synapsed threads: the pairing tends to be carried out very precisely, part for part. Closer examination, at least

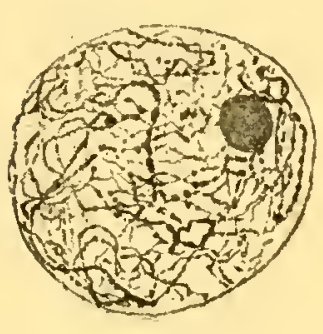

$a$
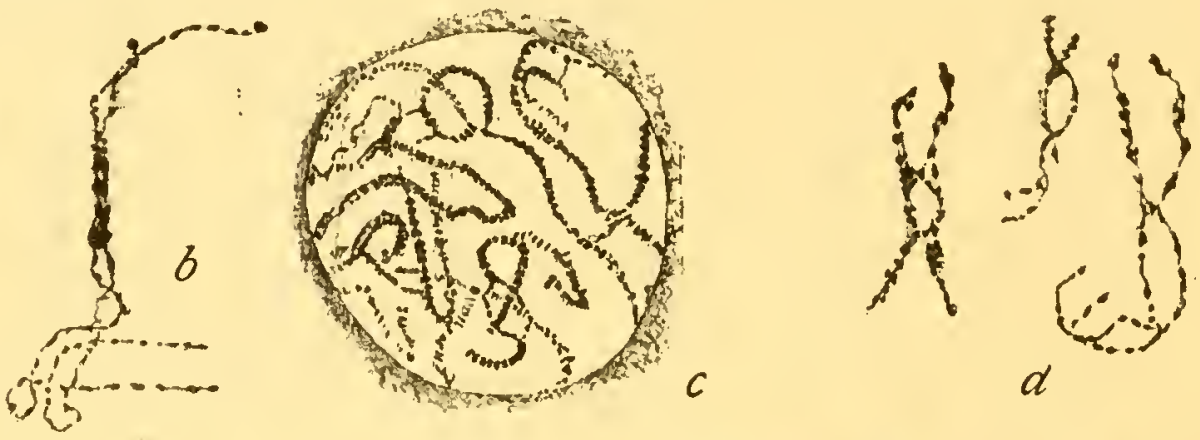

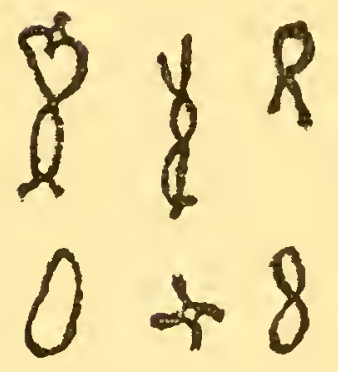

$e$

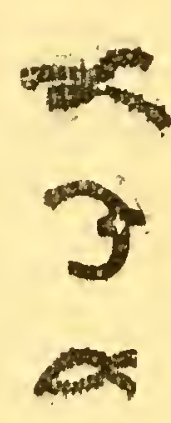

$f$

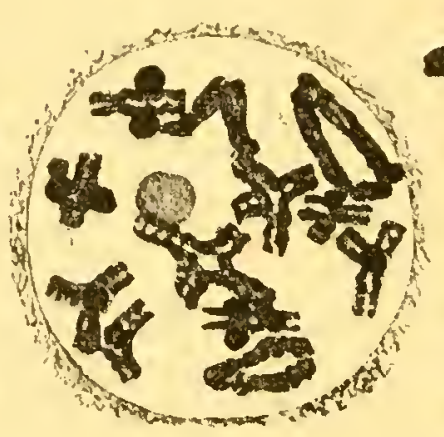

$g$

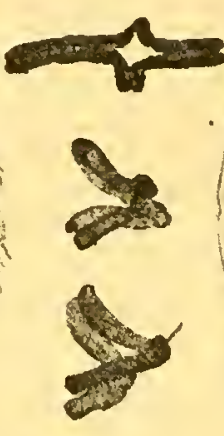

$h$

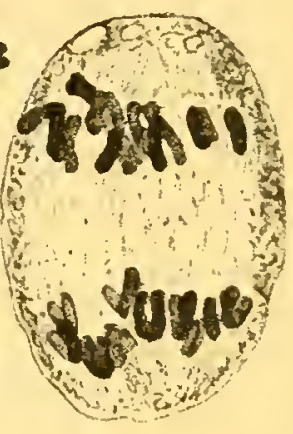

$i$

FIG. 151.--Stages in first meiotic mitosis in Tulipa. a, zygonema (leptonema threads synapsing laterally). $b$, single pair of zygonema threads. $c$, pachynema. $d$, diplonema. $e$, early diakinesis. $f$, late diakinesis, $g$, diakinesis, showing all of the 12 tetrads. $h$, early anaphase, showing tetrads beginning to disjoin into dyads. $i$, later anaphase. (After Newton, 1927.)

late in the pachynema stage, reveals the fact that each of the synapsed threads is double as the result of splitting. Hence each late pachynema thread consists of four chromatids; it is a tetrad. The origin of this split is very difficult to determine. It is said to appear first during late pachynema or in the immediately following stage by several observers. ${ }^{9}$ but others, as already pointed out, find evidence for it at earlier stages. This latter evidence falls in well with that for the presence of two chromonemata in each chromosome in somatic telophases, but it should not be assumed without adequate evidence that the somatic and the last premeiotic mitoses are in all respects alike.

${ }^{9}$ E.g., Newton (1927) and Newton and Darlington (1929) on Tulipa; Darlington (1929c ct seq.) on Hyacirthus, etc.; Belling (1931ab) on Allium and Lilium; Gelei (1921) on Dendrocolum, Janssens (1924) on Stethophyma (Mecostethus); and Huskins (1932) on Trillium. 
The pachynema now passes into the diplonema stage, so called because doubleness in the thick threads, if hitherto obscure, now becomes plainly visible (Figs. 150,$4 ; 151, d ; 155, a$ ). In each tetrad two of the chromatids widen out from the other two through the greater portion of their length, but all four tend to remain together in a characteristic manner in one or more regions. In passing along a tetrad it is often observed that at such a region the paired chromatids appear to exchange partners, thus

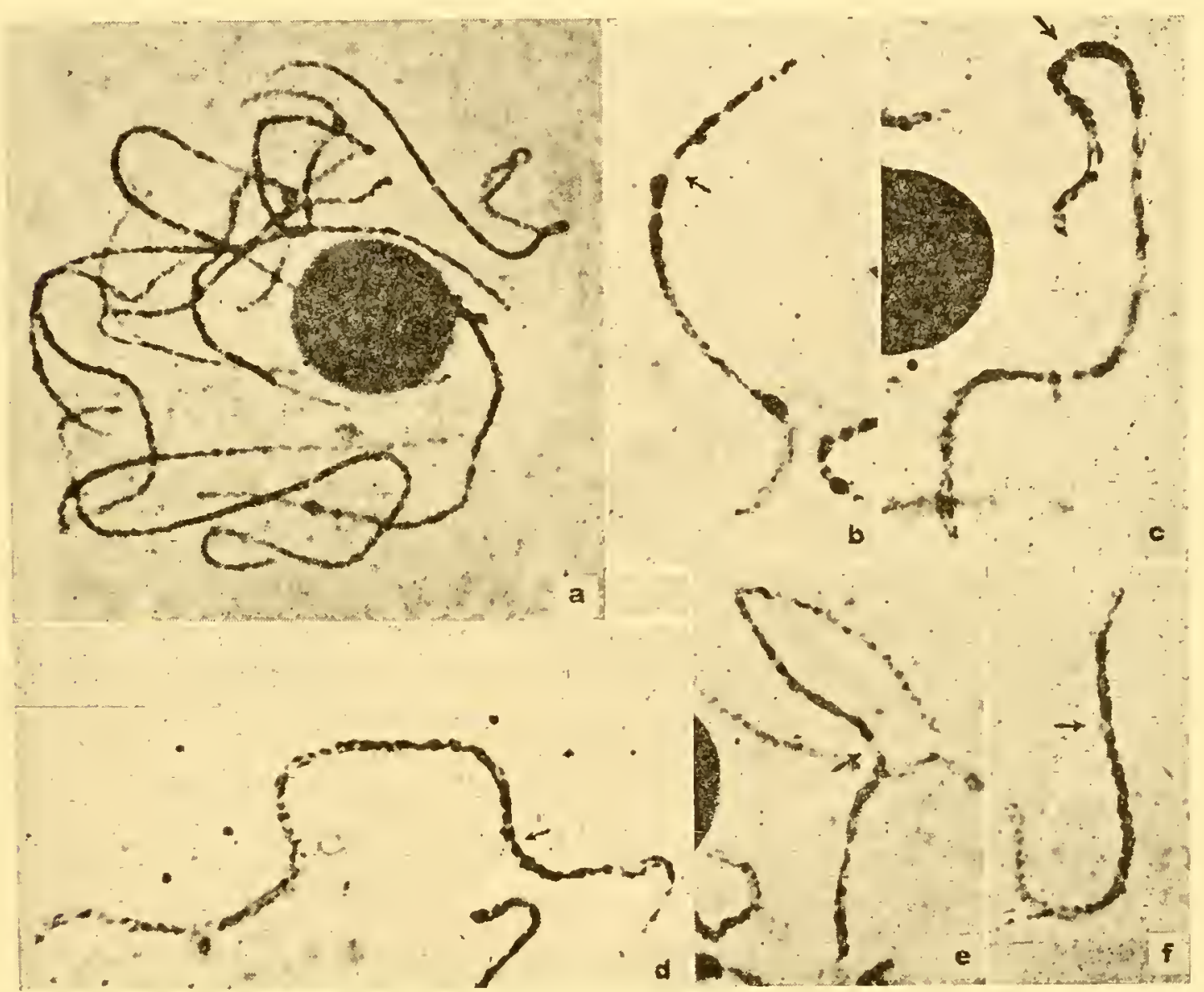

FIG. 152.-Chromosomes of Zea Mays in prophase of first meiotic division in microsporocyte. $a$, whole nucleus, showing all of the chromosomes (in closely synapsed pairs). Chromosome III at upper right; chromosome VI attached to right side of nucleolus; (cf. Fig. 65); looped over VI is chromosome I. b, chromosome VII; note knobs. $c$, chromosome VIII. $d$, chromosome III. e, chromosome IX. $f$, chromosome X. Spindleattachment regions indicated by arrows. Taken from different preparations and therefore not strictly comparable as to size. Cf. Figs. 66 and 170. (Photographs by McClintock.)

constituting a chiasma. The origin and fate of these chiasmata are matters of cardinal importance with respect to the significance of meiosis, hence they will be discussed in detail in a later special section. During this stage the threads often appear markedly twisted (strepsinema condition). It should be noted that a diplonema tetrad which has several chiasmata, and therefore several successive closed or open loops in alternating vertical and horizontal planes, may present a false appearance of twisting when viewed under the microscope. During the 
diplonema stage the threads become progressively shorter ${ }^{10}$ and pass gradually into the stage known as diakinesis, toward the end of which they become compact bodies lying well scattered throughout the nucleus.

At diakinesis the tetrads differ in several respects from those seen earlier in the prophase. Their length has become greatly reduced. In certain cases it has been found that a tetrad may be only one-fifteenth or one-tenth as long as the leptonema and early pachynema threads from which it is derived. This shortening, from the time it begins in the early prophase, involves a number of characteristic alterations which are, however, not precisely comparable in all organisms. As the pachynema threads shorten, their small ("ultimate") chromomeres may group into larger ("secondary") ones ( $c f$. p. 143). Later on, notably as the diplonema stage passes into diakinesis, the matrix becomes conspicuous, giving the tetrad a smoother contour. At the same time the chromonemata become more uniform in thickness and may form rather regular spirals within the matrix. ${ }^{11}$ In rye the chromosome shortens about one-third between diplonema and metaphase, but the chromonemata retain their original length by coiling; during metaphase the chromonemata shorten about one-third while the chromosome length remains unchanged. In other organisms, notably maize and grasshoppers, the chromonemata at diakinesis do not form very conspicuous spirals, the chromosome exhibiting a structure resembling that in the pachynema stage more than would be thought possible after so much shortening. This raises interesting questions regarding the transfer of materials to and from the chromosomes during these stages.

The various peculiar shapes assumed by the tetrads at diakinesis are due mainly to the position of their spindle-attachment regions, which look as if they were repelling each other, and to the number and position of their chiasmata. A single interstitial (non-terminal) chiasma gives the tetrad the form of an $\mathrm{X}$; a terminal chiasma or association gives a V ; two terminal ones give an $\mathrm{O}$; other arrangements of chiasmata give other shapes, as can be readily seen in Figs. 150 to 156 . Since long chromcsomes tend to have more chiasmata than short ones, the small tetrads of a group are in general simpler in form than the large ones. Moreover, the number of chiasmata per chromosome tends in many organisms to be reduced in passing from diplonema to diakinesis, a fact whose theoretical importance will be touched upon in a later section. Although the type of tetrad which develops is thus largely due to the chiasmata formed by the chromonemata, the matrix contributes much to its general appearance. In some tetrads, notably short ones in animal meiocytes, the matrix is

${ }^{10}$ It is here that a "second contraction" stage is often described in accounts of meiosis.

${ }^{11}$ E.g., Babcock and J. Clausen (1929) on Crepis, Sax (1930c) on Secale, Taylor (1931) on Gasteria, and Nebel (1932) on Tradescantia. 
distinct about each of the four chromonemata, so that the whole tetrad is obviously quadruple (Fig. 154). In other cases (Figs. 151, e; 156) it appears to form a common mass about any two chromonemata lying close together and not to divide until the chromonemata are separated later in meiosis. This means that such chromosomes at diakinesis are tetrads with respect only to their chromonemata. ${ }^{12}$ It has now been shown that each of the four chromonemata in the large tetrads of Zea (McClintock), Tradescantia (Nebel), and Trillium (Huskins) is longitudinally double in the late prophase or metaphase. This suggests either that the threads are double before synapsis, or that two splittings must occur in the meiotic prophase (Huskins, 1932a).

Before proceeding further with the history of the tetrads, mention should be made of a remarkable phase through which they pass during the late pachynema and diplonema stages in many meiocytes. During the prophase of the first meiotic mitosis the cell and its nucleus usually become considerably enlarged. In case this growth continues well into the latter part of the prophase, the chromosomes may show a marked tendency to recede from their compact form toward the more finely divided condition of the metabolic stage. In the sporocytes of plants this alteration is ordinarily not noticeable; the diplonema threads may be irregular in outline, but usually they seem to pass into the diakinesis stage with little change other than shortening and thickening. In growing animal spermatocytes this modification of the threads is in some cases carried much further, so that a characteristic "diffuse stage" ensues. It is in the much more extensive "growth period" of the animal oöcyte that it is most pronounced. At this time the cell increases enormously in size and develops most of the differentiation which is to characterize the egg, and as it does so the pachynema or diplonema threads become profoundly altered in appearance. They send out thready processes in all directions, assume an irregular brush-like form, and lose their basichromatic character partially or completely. These changes seem to be associated with the metabolic processes concerned in cell growth. When the growth is completed, the original staining capacity returns while the chromosomes again assume a compact form and pass into the diakinesis stage.

The prophase of the first meiotic mitosis comes to a close with the development of the achromatic figure. If centrosomes are present in the cell (animal spermatocytes and the meiocytes of certain lower plants), asters develop about them in the cytoplasm. With or without an inward shrinking of the nuclear membrane the karyolymph becomes organized as a spindle figure, as already described (p. 151). The tetrads take up

12 The claim is made, however, that in some eases each of the four spiral ehromonemata is aceompanied by its own individual matrix, the several matrices being in contact but not continuous (Nebel, 1932; on Tradescantia and Zebrina). 
their metaphasic positions with their attachment regions in the equator. ${ }^{13}$ Owing to their compactness and their arrangement in a regular plane, the tetrads are easily counted at this stage if viewed from a polar direction.

The anaphasic movement begins very soon. Two chromatids of each tetrad gradually move away from the other two, the movement starting at the attachment regions. As they diverge, it is observed that the chiasmata often appear to offer considerable resistance to the forces of disjunction so that the tetrads may become drawn out into strange shapes which depend upon the relative positions of the attachment regions and the chiasmata as well as upon the number of the latter. Eventually the pairs of chromatids (the dyads) become free and continue their poleward movement.

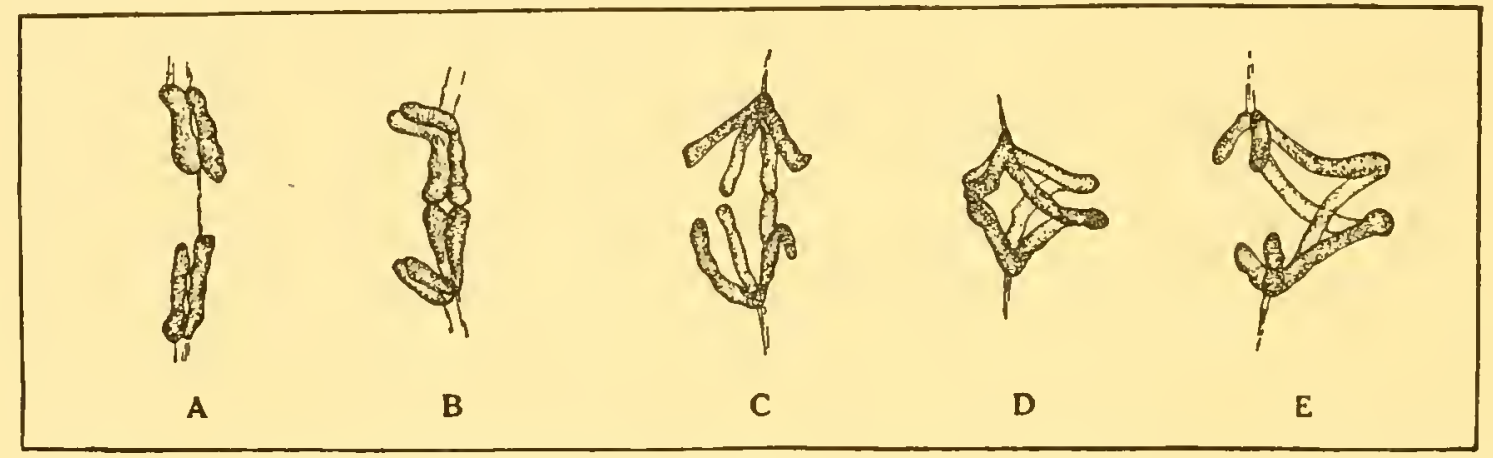

Fig. 153.-Plant chromosomes in anaphase $I$, showing tetrad nature and shapes due to different locations of spindle attachments. (From the works of Allen, Mottier and Strasburger.)

During the anaphase the two chromatids of each dyad tend to spread away from each other except at the spindle-attachment region, even before the dyads have lost their metaphasic contact (Fig. 153). To what extent this involves an actual division of the matrix or only a separation - of two matrices in contact is not altogether clear in many cases. In much of the older literature, particularly that pertaining to plants, it was assumed that this was actually the division of the chromosomes for the second meiotic mitosis; but it is now evident that even in such cases the tetrad chromosomes in the first meiotic metaphase are quadruple with respect to their chromonemata, and that the spreading in the anaphasic dyads represents mercly the divergence of two chromatids developed at a much earlier stage. As a result of this spreading of the free ends a dyad with terminal attachment appears as a single $V$ and one with median attachment as a double $V$. The division of the attachment region is presumably completed in anaphase, telophase, or later.

After the two diverging groups of dyads reach opposite poles, each group usually begins the organization of a telophase nucleus at once. The

${ }^{13}$ The suggestion has been made that if the attachment region in each synapsing chromosome is the last portion to become effectively double, the two chromatids facing the same pole at this region would be regularly sister chromatids. Whether or not this is actually the case is not known. 
extent to which the telophasic transformation is carried varies greatly in different cases. Sometimes what are essentially metabolic nuclei are formed, but probably more often the changes do not proceed far enough to render the individual dyads indistinguishable. In extreme cases, notably certain animal oöcytes (Fig. 154), the second mitosis follows so closely upon the first that the dyads at the close of $I$, immediately and without any structural change, take their places in a spindle newly formed for $I I$. The interval between the two meiotic mitoses is known as interkinesis. Ordinarily the two nuclei at this stage come to be
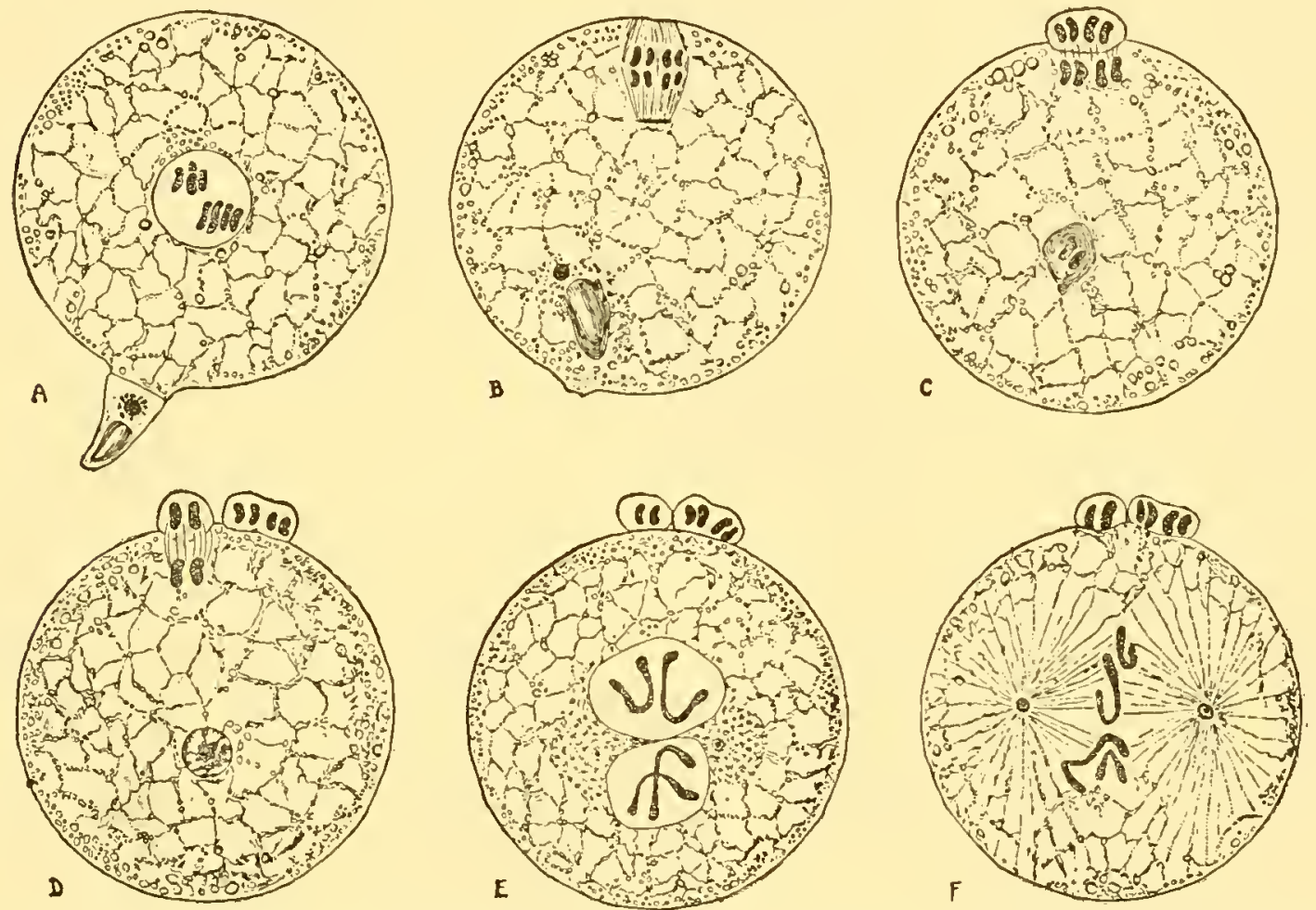

FIG. 154.- Meiosis and syngamy in Ascaris. A, spermatozoön about to enter egg. $B$, spermatozoön inside; first meiotic division in progress. $C$, first mitosis completed; first polar body budded off. $D$, second meiotic division, forming second polar body; nucleus of spermatozoön below. $E$, meeting of male and female pronuclei, each with 2 ehromosomes. $F$, first eleavage mitosis, showing 2 paternal and 2 maternal chromosomes. (After O. Hertwig.)

fairly large, and within them the chromatids appear as somewhat slender, crooked threads associated in dyads at their spindle-attachment regions (Figs. 150, 155). Anastomoses may be more or less evident, depending on the degree of telophasic transformation.

Cytokinesis follows the completion of division $I$ in many meiocytes. In animals the primary oöcyte is thus divided into a secondary oöcyte and a polocyte, and the primary spermatocyte becomes two secondary spermatocytes. In the sporocytes of higher plants cytokinesis may follow division $I$, but in many cases it is delayed until after $I I$, when the sporocyte is divided simultaneously into four spores (p. 167). In the meiocytes of lower plants various conditions are known. 
In the prophase of the second meiotic mitosis the condensing chromosomes appear characteristically in the form of threads or rods, still associated in dyads at their attachment regions but diverging widely elsewhere (Fig. 155). They thus present a marked contrast to the closely parallel halves of split chromosomes seen in a somatic prophase. By the end of prophase $I I$ their matrices have become conspicuous about their chromonemata, and as the spindle develops they take their places with their attachment regions in its equator. Often they appear con-
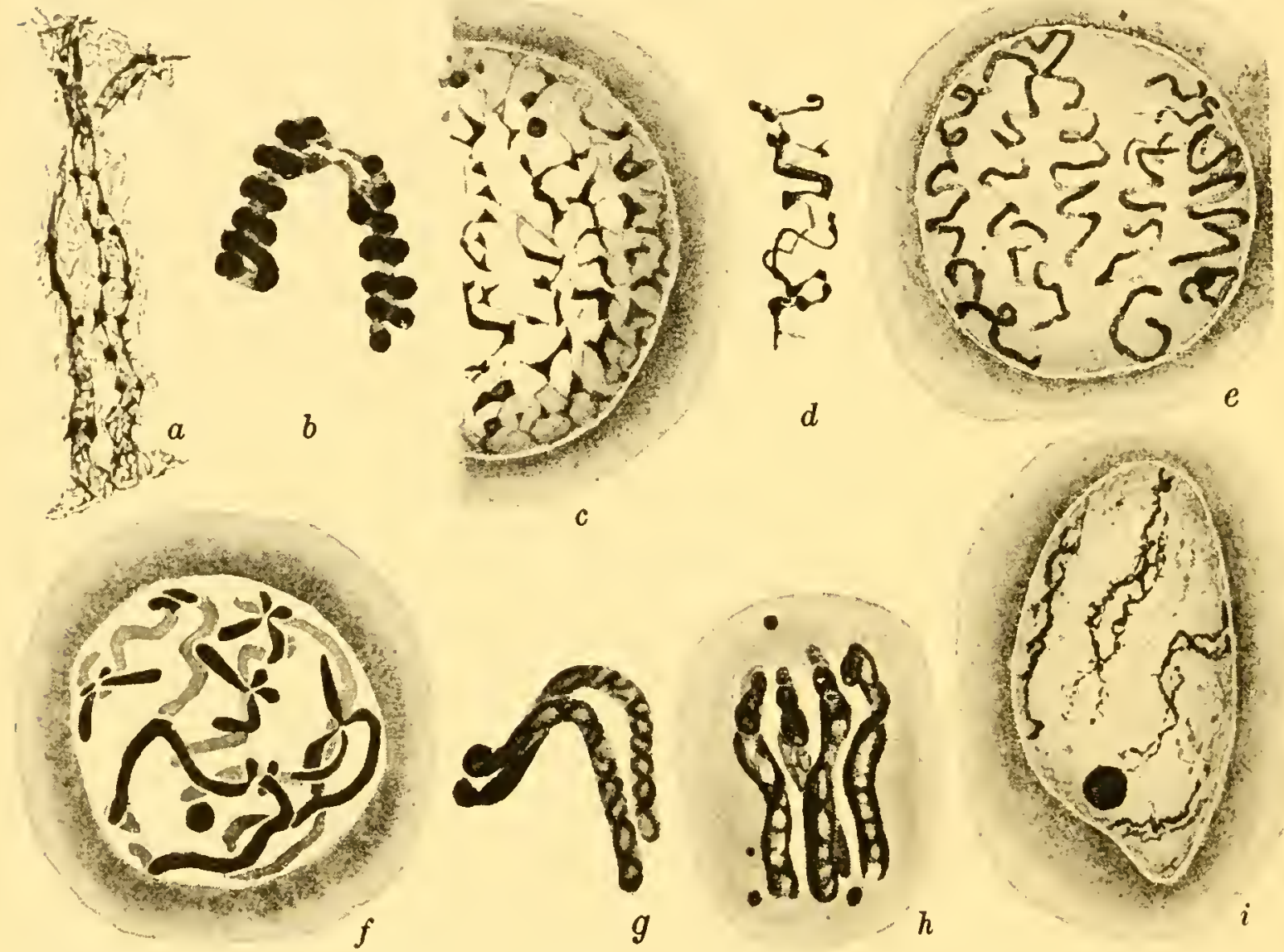

Fig. 155.-Stages in meiosis in Gasteria. a, diplonema. b, dyad in telophase $I . \quad c$, interkinesis. $d$, chromonema in early prophase $I I$, showing doubleness (one ehromatid). $e, f$, prophase $I I$; note association of chromatids by spindle-attachment regions. $g$, metaphase $I I$; chromonema doubled in each chromatid. $h$, late anaphase $I I . \quad i$, telophase II. (After W. R. Taylor, 1931.)

siderably longer and more slender than in the first mitosis and hence tend to resemble somatic chromosomes in this respect. In the anaphase the two chromatids constituting each dyad move toward opposite poles, their shapes at this time depending chiefly upon the position of their attachment regions. After reaching the poles at the end of the anaphase each of the four groups of chromatids begins the telophasic organization of a nucleus which passes into the metabolic condition.

Since each chromatid is now a complete and independent chromosome, it should be evident that each of the four nuclei contains a single monoploid set of chromosomes, the set being composed of one chromatid from 
each of the tetrads seen in the late prophase of the first mitosis. Cytokinesis soon ensues, giving in typical cases four cells (spermatids; egg and polocytes; spores), each with the reduced, or gametic, number of chromosomes in its nucleus.

In certain cases evidence has been brought forward to show that the chromonema in each chromatid at the close of the second meiotic mitosis is already split "in preparation for" the first postmeiotic division. The time of origin of this split is difficult to determine. In Gasteria it is clearly seen in the early prophase of the second meiotic mitosis (Fig. $155, d)$, and its presence in the anaphase of the first mitosis is suggested. In Tradescantia it can be seen at this stage, while in $Z e a$ it has been observed in diakinesis. ${ }^{14}$ This accords with the evidence that in large somatic chromosomes the chromonema is longitudinally doubled at least one mitotic cycle in advance of the anaphase in which separation is to occur along the plane so marked out ( $c f$. p. 137). Whether or not this is true of the last premeiotic mitosis in forms with wholly typical meiosis is still a debated question. We shall revert to this point later in the chapter.

Disjunctional and Equational Division.--It has now been shown that each tetrad chromosome appearing in the late prophase of the first meiotic mitosis is formed by the synaptic union of two homologous chromosomes which in turn are longitudinally split; and, further, that the four chromatids composing the tetrad at late prophase are distributed to four nuclei (and cells) by the two meiotic mitoses. From this it would appear that one of the mitoses must be disjunctional, in that it separates synaptic mates, while the other is equational, since it separates sister chromatids. Which mitosis is to be regarded as disjunctional (reductional in the strict sense) and which as equational?

The four chromatids composing a tetrad are ordinarily similar to one another in appearance when fully condensed, so that it is difficult or impossible to determine by direct observation whether the separation in the first anaphase is along the synaptic or along the equational plane. It has, however, been found in some cases, notably in certain insects, that the synaptic mates are sometimes unlike in size and hence form "heteromorphic" tetrads with two large and two small chromatids. Observations on the behavior of distinguishable chromosomes of this kind have made it possible to show that a given tetrad may divide disjunctionally in $I$ in some cells but equationally in others, at least in the heteromoi pinic region. In certain species there is nevertheless a strong tendency to behave in one way rather than the other. ${ }^{15}$ Furthermore, since the

${ }^{14}$ W. R. Taylor (1931) on Gasteria, Nebel (1932) on Tradescantia, McClintock (unpubl.) on $Z e a$.

${ }_{15}$ Wenrich (1916) on Phrynotettix, MeClung (1928a) on Mecostethus, Carothers (1931) on Trimerotropis, Mecostethus, and Amphitornis. The behavior of heteromorphic sex-chromosome tetrads, summarized by Wilson $(1925$, p. 753$)$, tends to be uniform in a given species. 
several tetrads in one mitotic figure may behave differently in this respect, each of the two mitoses in such cases may be disjunctional for some elements and equational for others. Taking the chromosome complement as a whole, the disjunction of its homologous elements and the equational separation of their halves are complete only at the conclusion of the second mitosis.

As will be shown in subsequent chapters, genetic data yield valuable evidence bearing on the distribution of chromosomes in meiosis. By such methods it can be shown, especially when the four products of meiosis can be followed individually, ${ }^{16}$ that some pairs of genetic units segregate in $I$ while others do so in $I I$. There is evidence which indicates that, although some portions of a tetrad divide equationally in $I$, the division tends strongly to be disjunctional in the neighborhood of the spindle-attachment region. ${ }^{17}$ How such a result could be brought about by an exchange of portions between two non-sister chromatids in the tetrad will be shown in the following section on the chiasma. Certain observations suggest either that sister chromatids in the tetrad are more closely associated at the attachment region than are non-sisters, possibly because this region divides very late, or that for some other reason the non-sisters (i.e., the synaptic mates) tend to separate rather consistently in $I$. To what extent this suggestion can be generalized and applied to the markedly heteromorphic tetrads mentioned above cannot be stated at present.

The Chiasma.- It was once thought by some observers ${ }^{18}$ that the two homologous chromosomes entering into synaptic union fused completely and then split twice along new planes to form the four chromatids of the tetrad. Subsequent investigation has shown this to be erroneous, as was claimed from the first by another group of workers. ${ }^{19}$ Modern cytologists and geneticists are now generally agreed that chromosomes preserve their essential identity through the period of synaptic association, at least so far as concerns those chromonematic elements which are responsible for their finer morphology and genetic functions.

This statement requires one very important qualification. It is now known that frequently two of the chromatids in the tetrad exchange corresponding portions with each other, as originally maintained by Janssens (1909). Beginning with Morgan (1911a) this "crossing-over" has been regarded as the mechanism responsible for the frequent recom-

${ }^{16}$ E.g., the four spores of a single quartet in Sphorocarpos (C. E. Allen, 1924 et seq.; see 1930a).

${ }_{17}$ Morgan (1925), Bridges and Anderson (1925), Anderson (1925, 1929), Redfield (1930).

${ }^{18}$ E.g., Vejdowský (1907), Bonnevie (1906, 1908, 1911), von Winiwarter and Sainmont (1909), and H. Sehneider (1914).

${ }^{19}$ Berghs (1904, 1905), A. and K. E. Schreiner (1905, 1906), Maréchal (1907), Grégoire (1907, 1910), Schleip (1906, 1907), Montgomery (1911), J. B. Overton (1905, 1909), Robertson (1915, 1916), Kornhauser (1914, 1915), Wenrich (1915, 1917). 
bination of characters associated with linked genes (p. 294). That this interpretation is correct has been rendered practically certain by studies on pairing chromosomes in which characteristic structural features were exchanged at the time recombinations were produced (p. 303). That such exchanges somehow involve the chiasmata observed in the tetrads from the diplonema stage onward has been strongly suspected from the first, although much of the evidence has been open to question. It is the prominent rôle played by crossing-over in present-day researches in the field of cytogenetics that now makes it necessary to consider the chiasma in some detail.

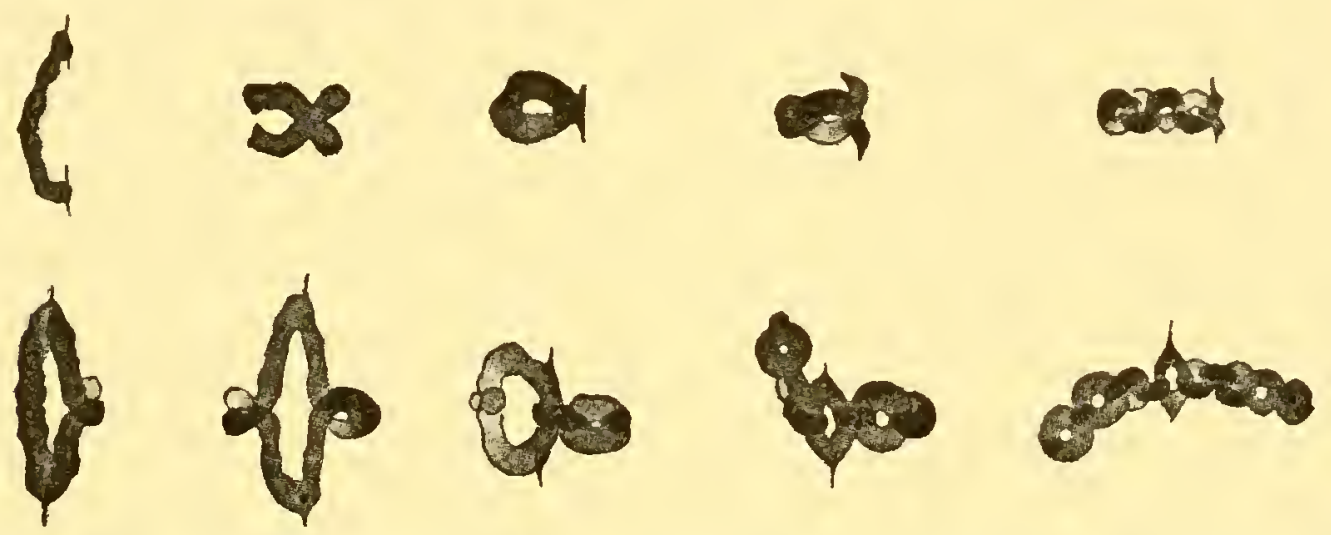

FIG. 156.-Bivalent chromosomes (tetrads) in metaphase and early anaphase of first meiotic mitosis in Vicia faba, showing rarious numbers of chiasmata. First row: chromosomes with subterminal spindle attachment. Second row: longer chromosomes with median attachment. (After Maeda, 1930b.)

As already explained, a chiasma is a region where the four chromatids appear to trade partners when the tetrad opens out in the diplonema stage. The number of chiasmata developing frequently varies with the length of the tetrad. ${ }^{20}$ In Hyacinthus orientalis, for example, there are commonly three in the longest tetrads (which may have as many as six), two in the medium-sized tetrads, and one in the shortest ones. In Vicia faba (Fig. 156) the commonest numbers are three in the short tetrads and eight in the long one. They appear to be located more or less at random in some species, but to be more localized near the spindleattachment region in others. ${ }^{21}$ Of considerable significance is the further fact that in many organisms the number of chiasmata tends to be reduced as the tetrads develop from the diplonema to the diakinesis stage. ${ }^{22}$ In Callisia repens, for example, the six tetrads show a total of about 25

${ }^{20}$ Darlington (1929c) on Hyacinthus, Maeda (1930b) on Vicia. Darlington (1932a) presents an extended discussion of chiasmata and their supposed significance.

${ }^{21}$ At random in Iyacinthus, Fritillaria imperialis, and probably Tradescantia; localized in Fritillaria meleagris (Newton and Darlington 1929, 1930; Darlington, $1929 c, 1930 d)$ and Allium fistulosum (Levan, 1933).

${ }^{22}$ Newton (1927) and Newton and Darlington (1929) on Tulipa, Darlington (1931a) on Primula, Sax (1930cd) on Secale and Callisia, Belling (1928a, 1931b) on Lilium, Philp and Huskins (1931) on Matthiola, Ertanson (1931a) on Rosa. 
chiasmata at diplonema and about 10 at diakinesis. In Rosa the mean number of chiasmata per tetrad is 2.66 at late diplonema and 1.53 at metaphase $I$. As a general rule each tetrad retains at least one chiasma or a terminal association through to the metaphase, indeed, it is believed by some observers that the continued association of the dyads at metaphase is due primarily to the presence of retained chiasmata rather than to any mutual attraction existing between them at this time (Darlington). ${ }^{23}$

A
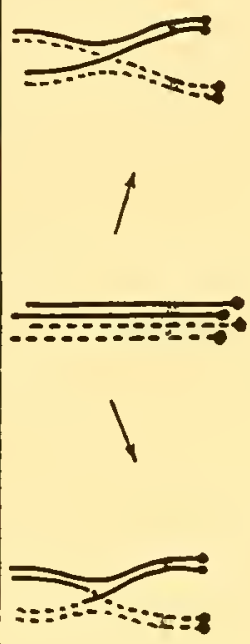

B
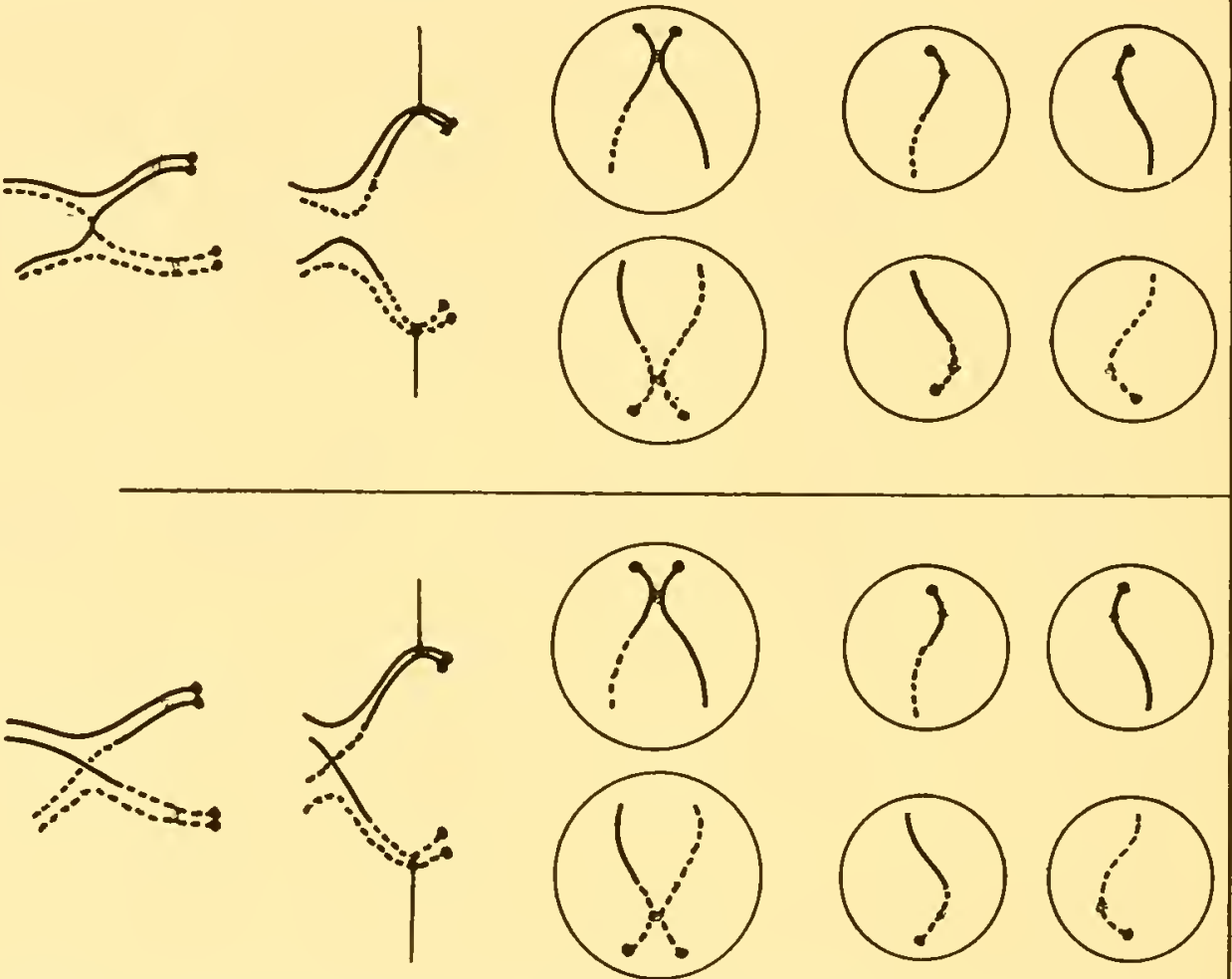

FIG. 157.-Diagram of a tetrad with one ehiasma and chromatid exchange as interpreted on the two-plane theory $(A)$ and the one-plane theory $(B)$. The chromatids of the two eonjugated chromosomes are shown in solid lines and broken lines, respectively, and one end of each is marked with a knob. Spindle-attachment regions indicated by stippling.

With regard to the origin of the chiasma, there are two principal interpretations which for convenience may be called the "two-plane theory" and the "one-plane theory" (Fig. 157).

According to the two-plane theory, ${ }^{24}$ when the four chromatids seen in the pachynema stage open out two by two to give the diplonema condi-

${ }^{23}$ The statistieal statements in this paragraph involve the assumption that the nodes and terminal associations observed in the tetrads actually represent chiasmata. In many instances this interpretation is probably open to question. In view of genetic evidence and the behavior of small chromosomes, particularly in individuals with no crossing-over, it seems especially improbable that strictly terminal associations at metaphase represent chiasmata (Sax, 1930d; Reuter, 1930; Belling, 1931b).

${ }^{24}$ Granata (1910), MeClung (1914, 1924, 1927ab), Wenrich (1916), Robertson (1916), Wilson (1925), Seiler (1926), Bělař (1928), Sax (1930c, 1932), Newton (1927), Newton and Darlington (1929), Darlington (1929c; see, however, our footnote 26), Babcock and J. Clausen (1929). 
tion, the opening is along the synaptic plane in some portions of the tetrad and along the equational plane in other portions. Where two openings in different planes meet, the chromatids must "trade partners." When viewed from a given direction under the microscope two of the chromatids may appear to cross each other at the resulting chiasma while the other two continue more or less straight, and they are usually represented thus in conventional diagrams; but a three-dimensional model makes it clear
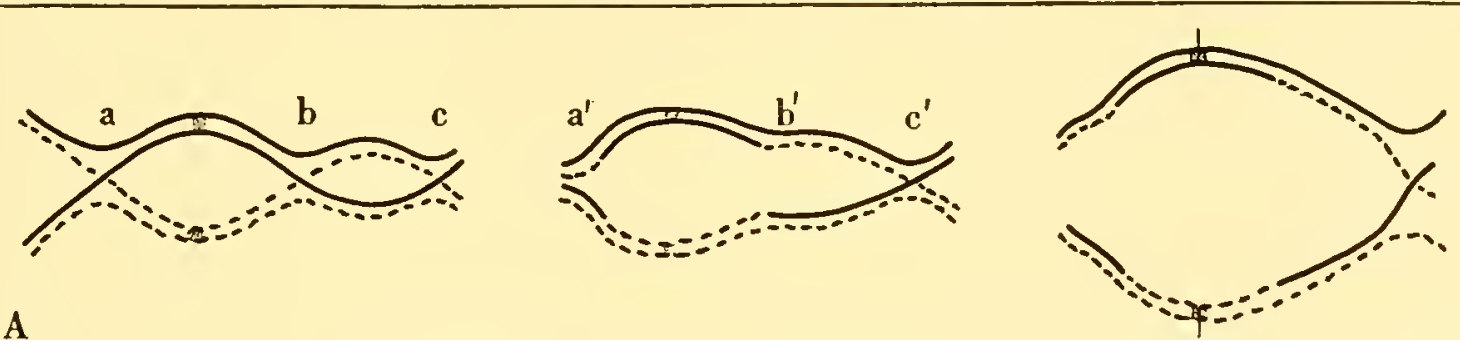

A

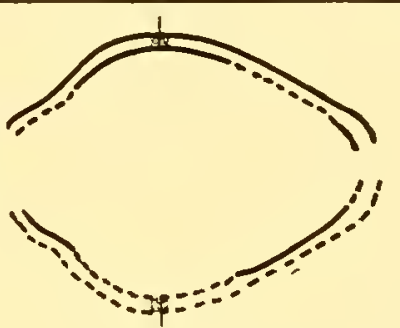

B
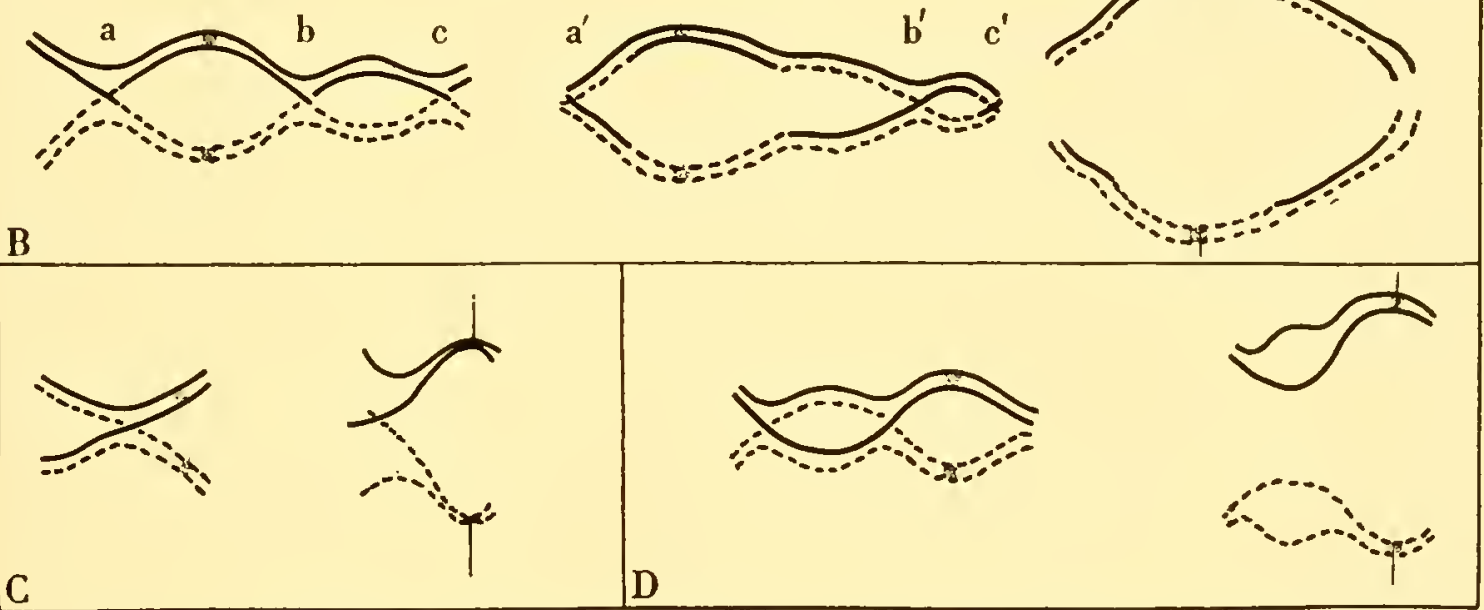

FIG. 158.- $A$, tetrad with three chiasmata $(a b c)$ as interpreted on the two-plane theory. Two of the chiasmata are shown yielding chromatid exchanges $\left(a^{\prime} b^{\prime}\right)$, while the third (c) separates in anaphase (third figure) without exchange. $B$, similar tetrad as interpreted on the one-plane theory, with terminalization: chiasma $a$ has been terminalized to $a^{\prime}, b$ has been moved to $b^{\prime}$, and $c$ has been terminalized to $c^{\prime} . C, D$, tetrads disjoining without chromatid exchange (two-plane theory). Spindle-attachment regions indicated by stippling.

that all of the chromatids may be equally bent, the angle of view determining which two appear to be crossed. If, however, there is a twist of the tetrad at the chiasma, two chromatids may be crossed and in contact while the other two are not. When opening out begins at a number of points, a diplonema tetrad may appear as a series of rings in alternating vertical and horizontal planes (Fig. 158, A).

The fate of the chiasma as meiosis proceeds beyond the diplonema stage may be variously conceived on the basis of the two-plane theory. The four chromatids might contract to the diakinesis condition and move apart in the first anaphase without undergoing any exchange of portions (Fig. 158, C, D). On the contrary, the continued opening out of the tetrad in the diplonema stage and early diakinesis might impose such 
strains upon two of the chromatids, especially those crossed and in contact, that they would find it easier to break at the point of greatest strain than to separate unbroken ( $A$ in Figs. 157 and 158). In this way they would exchange equivalent portions. ${ }^{25}$ Such a process would also result in genetic recombination as well as in a reduction in the number of chiasmata. It has been suggested further that such an exchange between chromatids might be caused by the resistance of chiasmata to the forces which draw the tetrads apart into dyads at anaphase.

The one-plane theory ${ }^{26}$ is essentially the "incomplete chiasmatypy" of Janssens. According to this view, the openings between the chromatids in the diplonema stage are all in the synaptic plane: the portions of two chromatids running closely parallel in any region when the tetrad first opens are always sisters (i.e., two formed by splitting of one) (Figs. $157, B ; 158, B)$. Hence, in order to account for the characteristic arrangement of the four chromatids at the chiasma, it is necessary to assume that an exchange of portions between two of them must have occurred at this point. In other words, chiasmata regularly indicate crossing-over. How such an exchange could occur in the pachynema tetrad before its opening out is an unsolved problem. ${ }^{27}$

To account for the reduction in the number of chiasmata observed between diplonema and late diakinesis, Darlington postulated a process called "terminalization." 28 This is a movement of the chiasmata away from the spindle-attachment region toward the ends of the tetrad, the four threads closing ahead of the moving chiasma and opening in the other plane behind it (Fig. 158, B). The single terminal association remaining in many tetrads at late diakinesis is accordingly regarded as a "terminal chiasma," that is, one which has reached the end of the tetrad.

${ }^{25}$ Aceording to the hypothesis of $\operatorname{Sax}(1930 c)$ a partial twisting of the tetrad brings two of its ehromatids into elose contact, the strains set up by further twisting or unequal contraction causing a break at the point of contact. The same result could be accomplished by the forees opening the tetrad without twisting and contact, provided the paired threads were meanwhile held strongly in synapsis close to the chiasma on either side, for there is evidence that broken ends of ehromosomes have a tendency to reunite (MeClintock, 1932c).

${ }^{26}$ Janssens (1909, 1924), Belling (1927b et scq.), Darlington (1930c et scq.; cf. our footnote 24, p. 268), Maeda (1930ab).

${ }^{27}$ According to the hypothesis of Belling $(1931 b, 1933)$, new longitudinal connecting strands develop between successive daughter chromomeres formed by division in the pachynema stage, sueh strands forming at half-twists in the threads being so arranged that they join portions of different chromatids. If the two strands forming the cross lie in the same plane (i.e., on the same side of the tetrad considered as a regular bundle of four ehromatids), the chiasma is said to be "direct"; whereas, if they lie in different planes (on different sides of the tetrad), it is said to be "oblique." The chromatids "erossed" at the chiasma are unchanged, while those running straight are the exchanged ones.

${ }^{28}$ See especially Darlington (1929b) on Tradescantia and (1931a) on Primula; also $(1931 d, 1932 a)$, where observations of other investigators are interpreted on this basis. 
Comparisons indicate that different species of ten show different characteristic amounts of terminalization, some having almost none while others show only terminal associations at the end of the prophase.

Cytological and genetical evidence is as yet not sufficiently critical to permit a confident decision between the two rival interpretations of the chiasma. Various phenomena have been held to conform better to the one-plane theory ${ }^{29}$ or to the two-plane theory, ${ }^{30}$ but none of them can be regarded as really decisive. What is needed is a series of observations of such a nature that less dependence upon personal judgment will be necessary. Direct evidence has been sought in the behavior of heteromorphic tetrads, in which the two chromatids of one synaptic mate differ visibly from those of the other. In Aloë the opening is regularly in the synaptic plane in the heteromorphic region (Belling, 1931b). Also, in strains of Zea having large terminal knobs on two of the four chromatids the opening appears always to be in the synaptic plane at the ends carrying the knobs. Since such knobs are masses of substance extending beyond the ends of the other chromatids, and since they show a decided tendency to stick together at various stages, it is quite possible that they prevent an opening in the equational plane which might otherwise occur. Tetrads with more minute grades of heteromorphism, particularly in non-terminal regions, may be expected to yield more trustworthy evidence on this point. But even here caution will be necessary in determining along which plane a tetrad first opens in any given region, because terminalization, if this occurs in the cases studied, would separate regions of chromatids originally together and vice versa. Favorable cytogenetic material should yield an answer to the question of whether it is chromatid exchange, or terminalization, or both of these processes, which reduces the number of chiasmata during the later portion of the prophase, and to a considerable extent clarify our notions of the mechanism of genetic recombination.

Before proceeding further it will be well to point out the fact that according to either the two-plane or the one-plane theory a tetrad with one original chiasma and exchange of parts consists of two chromatids altered by the exchange and two unaltered ones. Since these four chromatids are distributed to four nuclei by the two meiotic mitoses, two of these nuclei contain each a chromosome with its original constitution, while the other two contain each a chromosome made up of portions of the two synaptic mates (Fig. 157). This is a point of cardinal importance with respect to the discussions of genetical problems in subsequent chapters.

29. Configurations in trivalent, quadrivalent, and eatenated chromosomes (Belling, 1929; Darlington, 1930cd, 1931e), interloeked tetrads (Belling, 1931b; Catcheside, $1931 b$ ), aspects in pachynema stage (Gelei, 1921; Belling, 1931a).

${ }^{30}$ Genetic data in Drosophila (Sax, 1932; Beadle, 1932). See also S. Emerson and Beadle (1932) and Beadle and Emerson (1932). 
It may be added that when there are several chiasmata in a tetrad the same two chromatids are not necessarily involved at all of them. This creates a more complicated situation, but it should not be allowed to obscure the principle just illustrated with a simpler tetrad. The statement emphasized above still holds for the region about any one chiasma in a more complex tetrad.

It should now be clear why, as a general rule, neither of the two meiotic divisions is wholly disjunctional or wholly equational in character. Even if it be held that the first mitosis is regularly disjunctional for the spindleattachment regions of the chromosomes, it is evident that this mitosis must be equational for certain other regions when chiasmata and chromatid exchange are involved. The conclusions stated at page 255 should now be reread in the light of the foregoing discussion.

The Synaptic Reaction.-The forces bringing about synapsis ordinarily begin to manifest themselves in the early prophase of the first meiotic mitosis and operate for a comparatively brief period. In certain exceptional cases pairing is evident during the closing phases of the last premeiotic mitosis and may become so intimate that it looks much like synapsis. ${ }^{31}$ Pairing has sometimes been reported in the spermatogonia several cell generations before meiosis. ${ }^{32}$ Again, cases are known in which a tendency to associate loosely in pairs is shown in some measure in the somatic cells. In extreme examples the pairing occurs directly after the gametic chromosome sets are brought together in syngamy and persists throughout development. This is notably true of the Diptera, as shown especially by Metz (1916a et seq.) (Figs. 71, 196). A tendency toward such a paired arrangement, but no actual synapsis, has frequently been reported in somatic cells of plants. Reports of a "late synapsis" taking place at diakinesis are frequent in the literature. It has been shown in some such cases $^{33}$ that this is probably "a secondary coupling that has been preceded by a typical synapsis at the usual time and a subsequent deconjugation" (Wilson).

The two chromosomes which undergo synapsis to form each tetrad are the corresponding members of the two monoploid sets composing the diploid complement. They are "homologous" not only because of their ultimate common origin but also in the sense that they perform similar rôles in the life of the organism. More specifically stated, they somehow influence similar groups of functional reactions during the development

${ }^{31}$ Asilus (Metz and Nonidez, 1921), Drosophila (Metz, 1926b). See also Montgomery (1901) on certain Hemiptera, Sutton (1902) on Brachystola, M. Blackman (1903, 1905) on Scolopendra, and Dublin (1905) on Pedicellina.

${ }^{32}$ Certain Hemiptera and Ascaris (Montgomery, 1904, 1905, 1908, 1910), Alytes (Janssens and Willems, 1909), Itelix and Sagitta (Stevens, 1903; Ancel, 1903), certain Diptera (Stevens, 1908, 1911), and Pediculus (Doncaster, 1920b).

${ }^{33}$ Lepidosiren (Agar, 1911), Rhodites (Hogben, 1920a), Cyanotis (Rau, 1930). See Wilson (1925, p. 563). 
of the organism and presumably after maturity. Their effects on the course of development may or may not be precisely the same, but they differ regularly in certain important respects from those exerted by the other chromosomes of the complement. As a consequence of this influence on developmental reactions, the two homologous chromosomes are responsible in some measure for the appearance of morphological and physiological characters which are the results of those reactions. Very little is known concerning the nature of the physical and chemical reactions through which the chromosomes thus influence development, and still less can be said about additional rôles they presumably play in the metabolic activity of the mature organism. It will be seen in the following chapter how researches in the field of cytogenetics have established firmly the theory that a given chromosome influences the development of a certain group of characters because it is composed in part of a certain number of semiindependent elements known as genes. In the terms of genetics, therefore, homologous chromosomes carry similar groups of genes.

Ordinarily the two members of a homologous pair are about alike in size and form, so that in many diploid chromosome complements they can be readily recognized on this basis (Figs. 67, 69). Sometimes they exhibit certain differences, some of which can be shown to bear a relation to differences in function. An extreme case of this is seen in the sexchromosome pair in many animals and plants. Moreover, non-homologous chromosomes are often alike so far as the eye can tell. Hence it becomes unsafe to depend upon size and form alone as criteria of homology.

The most reliable cytological criterion of homology is normal synapsis itself. Caution is nevertheless necessary in using it, for it has been found that a number of agencies may induce a partial or complete failure of synapsis (asynapsis) on the part of chromosomes known to be homologous. ${ }^{34}$ The chromosomes may sometimes synapse in the megasporocytes but not in the microsporocytes. Cases are even known in which asynapsis behaves as a recessive Mendelian character. It should be pointed out that absence of pairing in late prophase and metaphase alone should not be accepted unconditionally as evidence of asynapsis, for the chromosomes may sometimes synapse as usual and then separate before the end of the prophase. Such absence of pairing may therefore indicate desynapsis ("deconjugation") rather than true asynapsis ("non-conjuga-

${ }^{34}$ E.g., Abnormal temperatures (Belling, 1925a, on Uvularia; Sakamura and Stow, 1926, on Gagea; Stow, 1926, 1927, on Solanum; and others); a recessive Mendelian gene (Beadle and McClintock, 1928, and Beadle, 1930a, on Zea; Schwemmle, 1928, on Enothera); X-rays (Goodspeed, 1929b, on Nicotiana); nutritional differences in anthers and ovules (J. Clausen, 1930b, on Viola); genetical conditions affeeting pairing in hybrids (Kihara, 1929c; Karpechenko, 1930). See further footnotes in Chap. XIII. Synapsis is often deficient in plants with more than two homologues (p. 355). 
tion"). This point has been especially neglected in many investigations of meiosis in hybrids.

Recent studies have shown clearly that normal synapsis is due primarily to an interaction of small homologous portions of the two chromosomes rather than of the chromosomes as wholes (Gelei, 1921). Evidence for this is not easy to obtain in normal diploid organisms, but it is inescapable in certain other types to be dealt with in later chapters. A striking case is afforded by a strain of maize in which a small portion

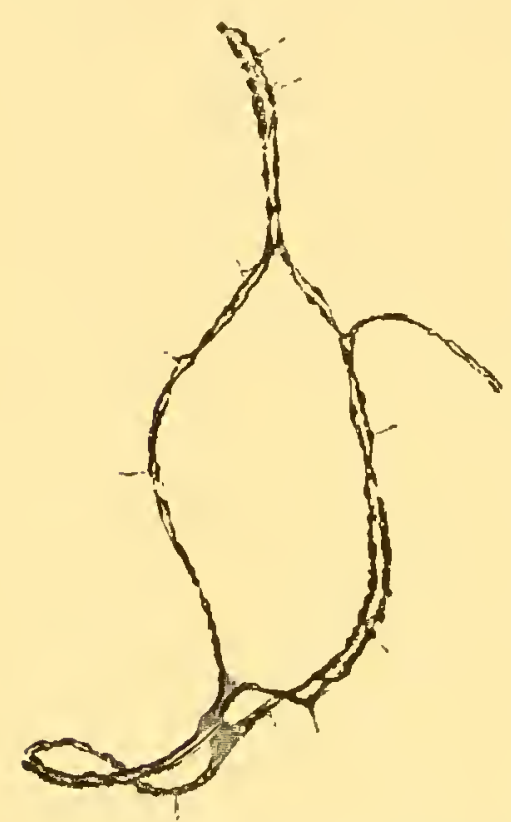

FIG. 159.-Trivalent chromosome in early meiotic prophase in Zea. Note that any one of the three members shows clearly its two chromatids only where it is not synapsed with one of the others. All three are closely assoeiated at the spindleattachment region. (From a preparation by UcClintock.) of one of the homologues has been "deleted." At the time of synapsis the normal chromosome pairs closely with the deleted one along the regions present in both of them, while the region having no counterpart. in the deleted chromosome extends laterally as an unpaired loop (Fig. $182, c)$. Moreover, it sometimes happens that an exchange of segments between non-homologous chromosomes gives rise to a condition in which two regions of one chromosome are homologous respectively with two different chromosomes. Synapsis occurs here between homologous parts and results in a group of three or more chromosomes. Again, a small free fragment of a chromosome tends to synapse at the proper time with the portion of the chromosome with which it would have paired, had it never become free. It is now a workable hypothesis that the synaptic reaction occurs primarily between "homologous" genes, or between substances in their immediate vicinity, the synaptic pairing of whole chromosomes thus being the resultant of the separate synaptic reactions of their many constituent genes.

There is reason to believe further that the synaptic reaction is one which occurs characteristically and fully between two elements only at one time at a given locus in the chromosomes concerned. This is indicated in an interesting way by synaptic behavior in polyploid plants, which have more than two chromosomes of a kind. ${ }^{35}$ In triploid tulips or maize, for example, all three of the homologues come near to one another, but the intimate synaptic union appears characteristically between two only in a given region (except often at the spindle-attachment point) (Fig. 159). The third one may be synapsed with either of them at

${ }^{35}$ M. Lesley (1926) on Lycopersicum, Newton and Darlington (1927b, 1929) on Tulipa, Darlington $(1929 c, 1930 c, 1931 a)$ on Hyacinlhus and Primula, McClintock (unpubl.) on $Z e u$. 
another region; or, if the two are synapsed throughout their length, the third may remain free altogether. The genetic evidence in triploid Drosophila also indicates such behavior. These phenomena suggest that the "synaptic force" operating at any region in the homologues is somehow "exhausted" when two members are intimately synapsed, so that the third member cannot establish synaptic union with them at this region. Chemical and electrical analogies naturally come to mind, but nothing is known about the precise nature of the forces concerned.

That synaptic "attraction" exists primarily between pairs of elements is suggested further by the opening out of ordinary bivalent tetrads at the diplonema stage. After the longitudinal doubleness of each synaptic mate becomes complete and obvious, whatever the time of its inception, the four chromatids "fall apart" into pairs except at chiasmata, as if the forces tending to hold the threads together could now operate between any two of them but not between all four. It is largely upon phenomena of this character that certain current theories of the origin and causes of meiosis have been based.

The suggestive hypothesis advanced by Darlington (see especially 1931d and 1932a) may be stated briefly as follows. During their prophasic contraction, chromosomal threads normally show a strong tendency to be associated in pairs. When the contraction begins in somatic mitosis the threads are already double as a result of splitting, ${ }^{36}$ so that this tendency is already satisfied. In the meiotic prophase, on the contrary, the contraction begins before the splitting; hence the tendency to be double is satisfied by the synapsis of the threads in pairs. After the paired threads become split in the late pachynema stage, this pairing becomes superfluous and the tetrad opens out to give the diplonema condition, the split threads remaining associated only at chiasmata until anaphase. Meiosis is thus conceived to be due to a precocious prophasic contraction of the chromosomes, this precocity being in some way associated with unusual features of the preceding interphase.

An alternative hypothesis is that advanced by Huskins (1932a). This author accepts the evidence that the chromosomal threads may split one mitotic cycle in advance of their separation in somatic divisions and therefore contends that the event which initiates the meiotic process is the suppression or retardation of splitting during the last premeiotic division, rather than the precocious contraction in the meiotic prophase. As a result of such suppression, which is probably due to the peculiar physiological state of the cells at this period, the leptonema threads of the meiotic prophase are single until after the contraction and synapsis begin and must therefore undergo their splitting in the pachynema stage. ${ }^{37}$

${ }^{36}$ Darlington assumes the splitting to take place in the metabolic stage and rejects the evidence for its earlier occurrence.

${ }^{37}$ As already pointed out, Huskins finds the metaphase tetrads of Trillium to have four double chromonemata. Hence the synapsing threads are thought to split twice 
Huskins points out that those organisms in which the chromosomes are reported to be split in the premeiotic telophase (p. 256) are either forms with incomplete synaptic pairing or with no crossing-over, suggesting that such doubleness is actually a hindrance to normal meiosis. This is in accord with his observations on the inverse relation between splitting and pairing in certain speltoid wheats and fatuoid oats.

It is interesting to speculate upon the possible relation between the synaptic "attraction" and the later "repulsion" which is suggested by the behavior of the chromatids in diplonema and anaphase, but such speculations have as yet little of a definite nature to support them. Attempts to identify the immediate causes of meiosis and especially its origin in the evolution of organisms can yield little of value until many other questions have been answered by reliable observation.
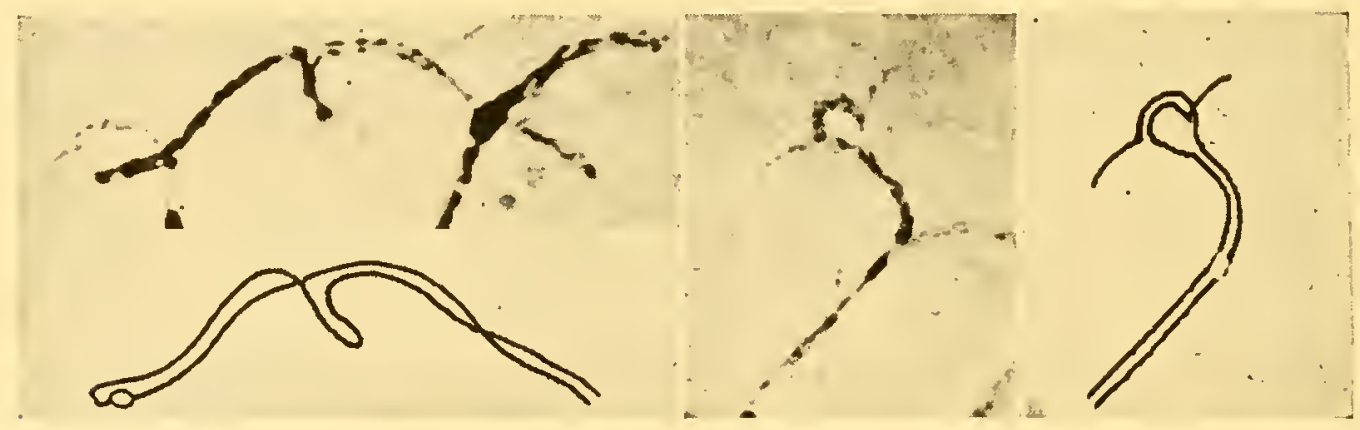

FIG. 160.-At left: Zea chromosome II folded back upon itself in meiotic prophase; spindle-attachment region near one of the folding points. At right: Zea chromosome VIII synapsing with a homologue having a portion of one of its arms inverted; see Fig. 170. (After McClintock, 1933.)

That synapsis is not solely a matter of gene homology, but is influenced by some condition pervading the nucleus or cell generally, is indicated by derangements of synapsis caused by abnormal cultural conditions or by a single Mendelian gene (p. 273). It is also strikingly shown by the fact that non-homologous parts of chromosomes are sometimes observed in a close association to all appearances like that of homologous parts (McClintock, 1932a, 1933; on Zea). For example, when an "interchanged" chromosome synapses with other chromosomes with which it is in part homologous (pp. 329 to 333), the association which begins between homologous parts may sometimes extend along the threads in such a way as to bring non-homologous parts together. Furthermore, a single chromosome normal in other respects may fold back upon itself at various levels, one portion thus entering into close association with the other (Fig. 160). Such association usually disappears before diakinesis. It is rightly emphasized (McClintock, 1932c) that

in the meiotic prophase, the first of these being the split which was delayed from the premeiotic mitosis and taking effect in division $I I$, while the second is that taking effect in the first postmeiotic division. 
synapsis is influenced by a considerable group of factors, including gene homology, a tendency to form pairs, and peculiar conditions at chromosome ends. It is successful and wholly "normal" only when the actions of the various factors are so correlated as to permit homologies to be completely satisfied. When the normal relationship of the several influences is disturbed, as in structurally unbalanced types especially, a variety of unusual results may be expected.

Not only do these phenomena bear upon our hypotheses of synaptic attraction, but they raise fundamental questions regarding the rôle they probably play in the production of translocations, deficiencies, and other modifications of the chromosome complement of significance in the alteration of species. They further serve to emphasize the importance of a knowledge of the finer morphology of the individual chromosomes in the interpretation of the phenomena of meiosis. Much that has been written about meiotic chromosome configurations must be reëvaluated in the light of critical evidence of this kind.

Meiosis in Lower Organisms.-In the foregoing account of meiosis only higher plants and animals have been considered. This section will summarize briefly the conditions in lower groups, with special reference to the stage in the life cycle at which meiosis occurs. The statements should be compared with those made in the preceding two chapters regarding the other reproductive phenomena in these groups.

Among the Protozoa, ${ }^{38}$ the matter of chromosome organization and behavior in many groups is very obseure. In others, however, definite chromosome numbers and meiotic phenomena essentially like those in higher organisms have been demonstrated. In most species with a permanent union of gametes the regetative stages have the zygotic chromosome number, and haplosis occurs in connection with gametogenesis, ${ }^{39}$ as in Metazoa (Fig. 161). In the infusoria, which have a temporary union of cells known as conjugation (see p. 245), meiosis occurs in the first two divisions of the micronucleus just prior to the formation of gamete nuclei. ${ }^{40}$ In a few sporozoa the vegetative stages have nuclei with the gametic number of chromosomes, meiosis taking place in the zygote after the fusion of the sexual nuclei. ${ }^{41}$ In one of these, Aggregata eberthi, the monoploid set is made up of one long, one short and four medium-sized chromosomes. There is also genetic evidence that in the phytoflagellate Chlamydomonas meiosis occurs in the zygote (Pascher,

${ }^{38}$ See Minchin (1912), Jennings (1920, 1929), Calkins (1926), and Bělař (1926).

${ }^{39}$ E.g., Actinosphorium eichhornii (R. Hertwig, 1898b) and Actinophrys sol (Schaudinn, 1896; Bělař, 1922). The paper by Bělař is especially noteworthy.

${ }^{40}$ Opercularia (Enriques, 1907, 1908). Chilodon (Enriques; MacDougall, 1925), Carchesium (Popoff, 1908), Uroleptus (Calkins, 1919), Oxytrichia (Gregory, 1923), Euplotes (Turner, 1930). For further cases, see Calkins (1926, p. 518). 1920).

${ }^{41}$ Aggregata eberthi (Dobell and Jameson, 1915), Diplocystis schneideri (Jameson, 
1916c). Meiosis with spore formation is reported in Paradinium (Chat. ton, 1927).

Turning to the myxomycetes, it seems that meiosis accompanies the formation of the spore nuclei, a fusion of nuclei in the plasmodium having preceded the process. ${ }^{42}$ In Plasmodiophora brassica, according to Prowazek (1905), the plasmodium breaks up into uninucleate masses

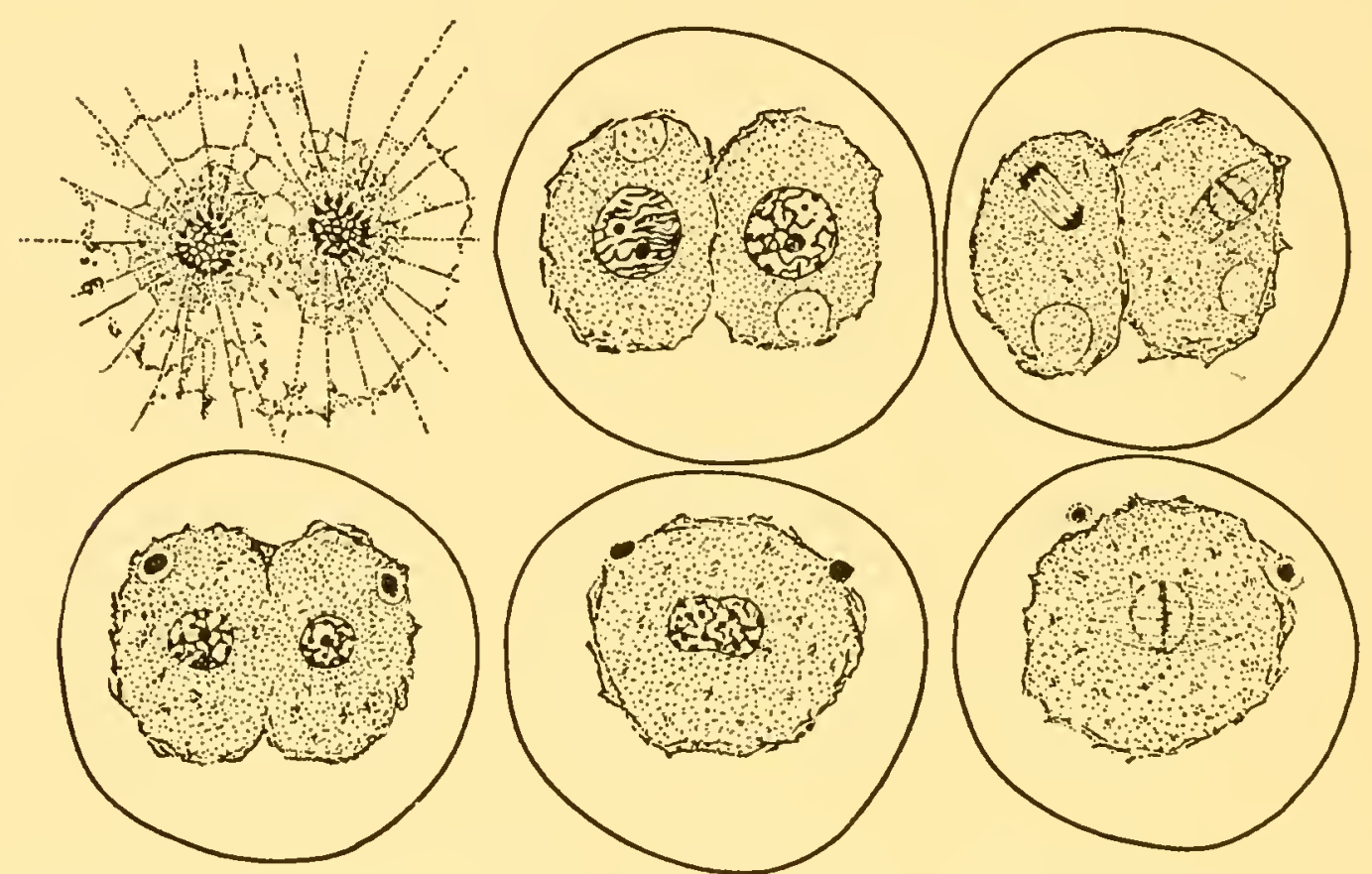

FIG. 161.-Meiosis and syngamy in Actinophrys sol. In each of the copulating individuals meiosis occurs, after which one of the resulting nuclei unites with a similar one from the other individual. (From Minchin, after Schaudinn.)

which fuse in pairs and become spores with two nuclei each. These nuclei in two mitoses give off "reduction nuclei," leaving two sexual nuclei which then fuse. In this species, therefore, the sexual nuclei are the only reduced ones in the cycle.

The green algæ, formerly supposed to show "zygotic" meiosis exclusively, are now known to have other types also. Three general categories may be distinguished: haplonts, diplonts, and diplohaplonts. ${ }^{43}$ As

${ }^{42}$ Ceratiomyxa (Olive, 1907; Jahn, 1908), Trichia and Arcyria (Kränzlin, 1907), Didymium (Skupieński, 1928; genetic evidenec).

${ }^{43}$ Terms introduced by Svedelius $(1915,1931)$. Haplonts are sexual plants in which only the zygote has the zygotic chromosome number. Diplonts are sexual plants in which only the gametes have the gametic number. Diplohaplonts are those with an alternation of two generations having the gametic and zygotic numbers respeetively. For general reviews of reproduction and alternation of generations in the algæ, see Bonnet (1914), Janet (1914), Oltmanns (1922-1923), West (1927), and G. M. Smith (1933). Davis (1916) gives a convenient summary of the life histories of the red alga. Svedelius (1931) and Knight (1931) discuss and diagram the various life cycles in Rhodophyeeæ and Phæoplyyeeæ, respectively. Dodge (1914) compares the cyeles of red alga and ascomycetes. For further diseussions, see Tansley (1912), Buder (1916a), Kylin (1916b), Renner (1916), Fritsch (1916), and Svedelius (1921, 1927b, 1931). 
representatives of the first category may be cited species of Spirogyra (Karsten, 1908; Tröndle, 1911), Zygnema (Kurssanow, 1911), Coleochocte (C. E. Allen, 1905c), Cylindrocystis (H. Kauffmann, 1914), Volvox (W. Zimmermann, 1921), and Ulothrix (I. Gross, 1931; Lind, 1932). These plants have the gametic chromosome number and undergo meiosis in the first two mitoses in the zygote (Fig. 162). In a number of other genera, such as Edogonium, Spharoplea, and Closterium, in which the chromosomes are not so well known, it is probable that the same condition exists, since the zygote upon germination gives rise with considerable regularity to four cells or nuclei; in some cases (Ldogonium) the four cells are zoöspores.

It has recently been discovered that some of the green algæ are diplonts. Here the somatic cells have the zygotic chromosome number, which is reduced at the time of gametogenesis, i.e., meiosis is "gametic."

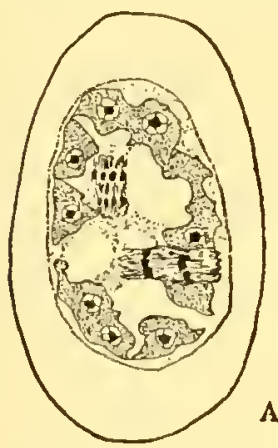

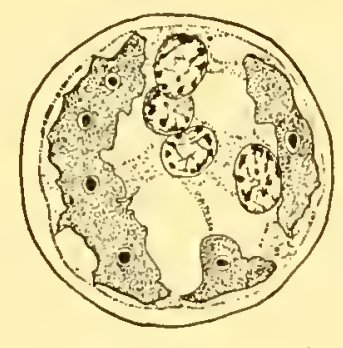

B

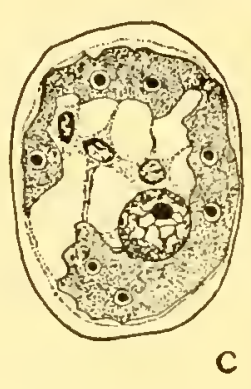

C

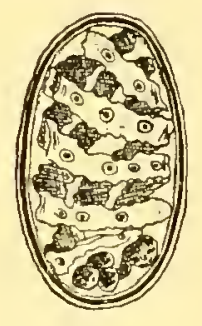

$\mathrm{D}$

FIg. 162.-Behavior of nuclei and plastids in zygospore of Spirogyra. A, second meiotic mitosis in $S$. calospora. $B$, four nuclei resulting from meiotic divisions in $S$. longata. $C$, degeneration of three of the four nuclei after meiosis in $S$. longata. $D$, degeneration of plastids contributed by "male" gamete in S. neglecta. (After Tröndle, 1911.)

In this category are the cœenocyte genera Codium and Acetabularia (M. Williams, 1925b; Schussnig, 1928b, 1930b). There is also some evidence that Vaucheria may have gametic meiosis (Mundie, 1929).

Of even greater interest are the forms with two well-marked generations in the life cycle. Here the gametes are borne on plants with the gametic chromosome number and the spores on other morphologically similar plants with the zygotic number; meiosis occurs at zoösporogenesis. Known examples are Enteromorpha and Chcetomorpha (M. Hartmann, 1921, 1929b, 1930b), Cladophora (Schussnig, 1928b; Föyn, 1929 ; Higgins, 1930) and Ulva (Föyn). Furthermore, it appears that in these cases the sexual plants are dicecious.

Among the Charales, meiosis in the germinating zygote has been described for Chara by Oehlkers (1916). In another form at first thought to be a species of Nitella, Tuttle $(1924,1926)$ reported haplosis in the apical cells of the oögonia and antheridia, which would make the vegetative body a diplont rather than a haplont. In the oögonium the four cells resulting from meiosis were shown to be strikingly like the egg and polocytes of animals. On the contrary, Karling (1926) and Lindenbein 
(1927) find no haplosis in the antheridia of Nitella gracilis, Chara aspera, and other representatives of the group.

In the diatoms ${ }^{44}$ the bilaterally symmetrical Pennatæ and the radially symmetrical Centricæ have been said to be characterized respectively by gametic and zygotic meiosis, but observations in the latter group are scanty and at least one exception to the rule is known. In Surirella saxonica, a pennate form, meiosis occurs at the time of conjugation. In each individual the nucleus divides to four, reducing the chromosome number, after which one of the four unites with one from the other individual as the cells fuse. The fusion product is an auxospore (Karsten). In Corethron valdivice, a centric type, the behavior of the nuclei suggests the occurrence of chromosome reduction in the germination of the zygote, which in this case is formed by the union of motile gametes (Karsten). Biddulphia sinensis, however, resembles pennate forms in having gametic meiosis (Schmidt).

Among the brown algæ, many of which exhibit a well-marked alternation of generations, meiosis occurs in most cases in sporangia on plants with the zygotic chromosome number. In some groups the spores are motile, ${ }^{45}$ whereas in others they are non-motile tetraspores. ${ }^{46}$ In Fucus the vegetative body has the zygotic chromosome number, but it produces gametes instead of spores. Meiosis occurs in the early divisions in the oögonia and antheridia. ${ }^{47}$ Hence Fucus is a diplont with a life cycle closely similar to that of animals. It is the opinion of some algologists (e.g., Svedelius) that spores have been replaced by gametes in Fucus through a progressive abbreviation of the gametophytic phase.

Among the red algx ${ }^{48}$ two principal conditions are known. In Scinaia and Nemalion, meiosis occurs in the zygote. There are then developed gonimoblast filaments and carpospores with the reduced chromosome number, these spores in turn developing into new plants which retain this number. In Polysiphonia and several other forms meiosis does not take place in the zygote, but the carpospores develop into plants with the zygotic number. Meiosis eventually occurs in tetrasporangia on these plants, the resulting tetraspores producing plants with the reduced number.

${ }^{44}$ See especially Klebahn (1896), Karsten (1900, 1904, 1912), P. Schmidt (1923, $1927 a b)$, Geitler (1927abc, 1928abc, 1929b, 1931), and von Cholnoky (1928, 1929).

${ }^{45}$ Cutleria-Aglaozonia (Yamanouchi, 1912), Zanardinia (Yamanouchi, 1913b), Ectocarpus (Kylin, 1918), Pylaiella (Kinght, 1923), Egregia (Myers, 1928), Stypocaulon (Higgins, 1931).

${ }^{46}$ Dictyota (Mottier, 1900; J. Williams, 1904a), Padina (Williams; P. Carter, 1927). Zonaria (Haupt, 1932) has more than four spores.

${ }_{47}$ Yamanouchi (1909) on Fucus; Kunieda (1926) and Okabe (1929) on Sargassum.

${ }^{48}$ Yamanouchi (1906) on Polysiphonia; I. F. Lewis (1909) on Griffithsia; Svedelius (1914abc, 1915) on Nitophyllum, Delesseria, and Scinaia; Cleland (1919) on Nemalion; Yamanouchi (1913c, 1921) on Corallina; Mathias (1928) on Callithamnion. For a summary, see Svedelius (1931). 
Very little is known concerning nuclear behavior in the phycomycetes, largely because of the minuteness of their nuclei. According to Wóycicki (1927), meiosis in Basidiobolus ranarum occurs when the zygote nucleus divides, three of the four nuclei then degenerating. Kniep (1930) reports an alternation of gamete-producing and spore-producing plants in Allomyces javanicus. Here reduction probably occurs in the "resting
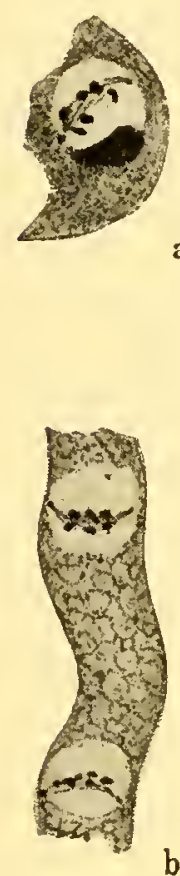

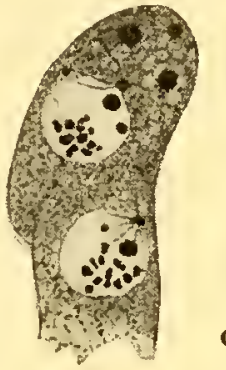

c

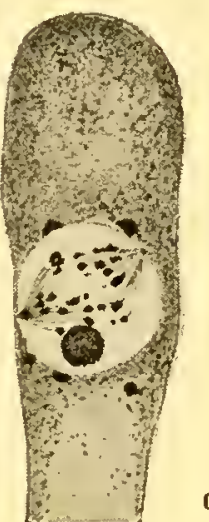

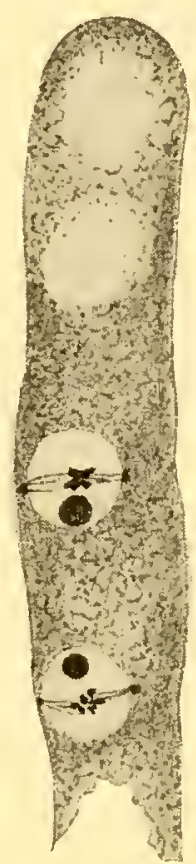

e

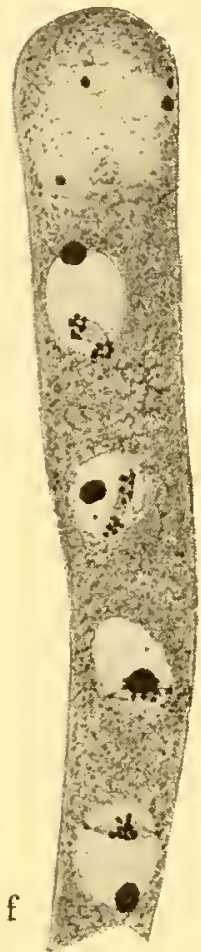

Fig. 163.- "Double reduction" in Pyronema confluens. a, prophase in germinating spore, showing 6 chromosomes. $b$, vegetative hypha. $c$, nuclei in tip of ascogenous hypha, showing 12 chromosomes. $d$, first meiotic mitosis in ascus, showing 24 chromosomes disjoining into groups of 12 . $e$, metaphase of second mitosis. $f$, metaphase (below) and anaphase (above) of third mitosis, showing 6 chromosomes passing to each pole. (After Gwynne-Vaughan and Williamson, 1931.)

cells" producing zoöspores from which the gamete-producing individuals arise.

In the ascomycetes meiosis occurs in the mitoses by which the fusion nucleus in the ascus gives rise to the nuclei of the ascospores. The chief question at issue concerns the nature of the third mitosis, for ordinarily eight spores are formed. Most observers ${ }^{49}$ have contended that meiosis is completed in the first two divisions; but it has also been claimed by some investigators ${ }^{50}$ that a further "brachymeiosis" is accomplished in

${ }^{49}$ Maire (1905a) on Galactinia, Faull $(1905,1912)$ on Hydnobolites and Laboulbenia, P. Claussen (1912) on Pyronema, W. H. Brown (1909, 1911b) on Pyronema confluens and Lachnea, Bagchee (1925) on Pustularia, E. Schultz (1927) on Peziza. Researches on ascomycetes are reviewed by Atkinson (1915).

${ }^{60}$ Fraser $(1907,1908)$ on Humaria; Fraser and Welsford (1908) on Otidea and Peziza; Fraser and Brooks (1909) on Lachnea; Carruthers (1911) on Helvella; GwynneVaughan and Williamson $(1930,1931,1932)$ on Humaria, Pyronema confluens, and Ascobolus; Tandy (1927) for some asci in Pyronema domesticum. 
the third mitosis, such a double reduction being required by the two nuclear fusions supposed to occur in the life cycle (see p. 230) (Fig. 163). A compromise is suggested by Tandy, who reports that only some of the sexual nuclei fuse in the ascogonium, so that the ascogenous hyphæ have both monoploid and diploid nuclei and give rise to asci in which the definitive nucleus is therefore sometimes diploid and sometimes tetraploid. In diploid asci, meiosis is accomplished in the first two mitoses, whereas in tetraploid ones three mitoses are required to produce mono-

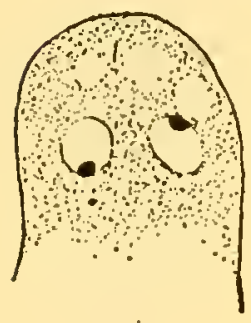

$i$

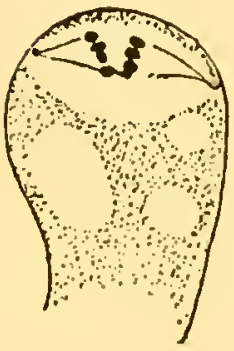

6

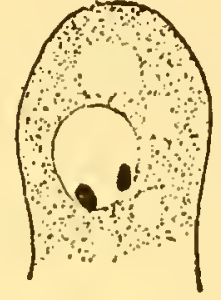

2

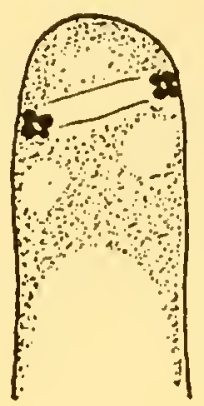

7

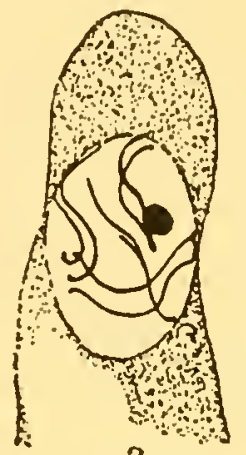

3

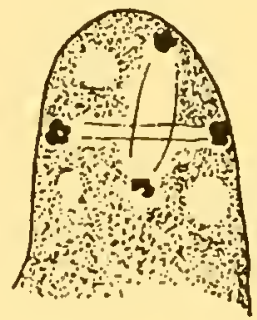

8
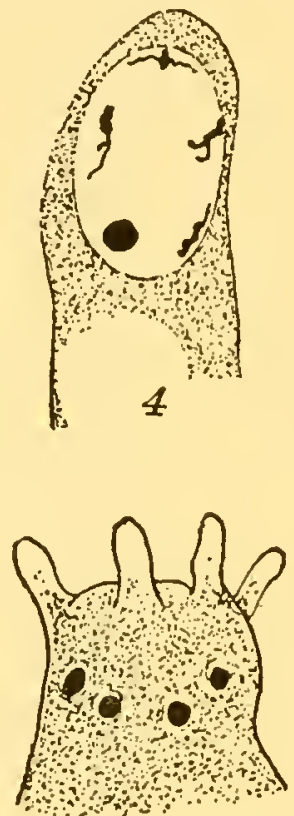

9

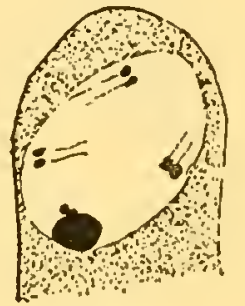

5

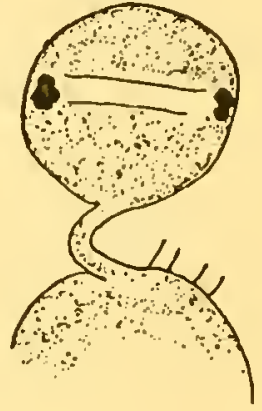

10

FIG. 164.-Meiosis in basidium of Cortinarius cinnamomeus. 1, two nuclei in young basidium. 2, fusion nucleus. 3-5, prophases of division $I$; four bivalents. 6 , early anaphase $I$. 7 , telophase $I$. 8, telophase $I I$; four ehromosomes in each group. 9 , four spore nuclei still in basidium; sterigmata forming. 10 , mitosis in one of the four spores. (After Wakayama, 1930a.)

ploid spore nuclei. An interesting light is thrown upon this problem by the fact that segregation of Mendelian factors (hence presumably of homologous chromosomes) in the asci of Neurospora occurs in either of the first two mitoses but not in the third (B. O. Dodge, 1927 et seq.; Lindegren, 1932, 1933).

In the basidiomycetes, meiosis follows immediately upon the fusion of nuclei, which ordinarily occurs in the basidium or its homologue. In hymenomycetes, the fusion nucleus subdivides twice to form the nuclei of the four basidiospores (Fig. 164), which thus carry the reduced chromosome number. ${ }^{51}$ Cases are known in which no fusion occurs in the basidium, two spore nuclei being formed without meiosis (Bauch). In

${ }^{51}$ Sappin-Trouffy (1896), Juel (1898a), Holden and Harper (1903), Maire (1905b), Guilliermond (1910), Kniep (1911, 1913), Levine (1913), Lindfors (1924), Bauch (1926, 1927), Wakayama (1930a) and others. 
the rusts, meiosis ordinarily occurs as the fusion nucleus in the teliospore divides to form the four nuclei of the sporidia. ${ }^{52}$

Meiosis in bryophytes ${ }^{53}$ and vascular plants takes place in the divisions producing the nuclei of the spore quartets.

Conclusion.-From the foregoing account it may be concluded that meiosis is a process which probably occurs in some form in all nucleated organisms reproducing normally by sexual means. Haplosis in some form is obviously a necessary consequence of syngamic nuclear fusion. The view long current that synapsis is itself a sexual process, the "culmination of fertilization," is, however, rendered less easy of acceptance by what has been ascertained regarding synapsis (1) in polyploids and hybrids, where the synaptic mates are often from the same gamete, (2) in certain parthenogenetic organisms, and (3) between different parts of the same chromosome, exceptional as this may be. Theorizing on this subject will be more profitable when more has been learned about the forces actually concerned in the "attractions" of cells, nuclei, chromosomes, and their constituent elements in the life cycles of different organisms.

The cell in which meiosis is initiated (the meiocyte) may be a sporocyte, a gametocyte, a zygote, an ascus, a basidium, or some other cell; but whatever the relative position of syngamy and meiosis in the life cycle, these two cytological crises are events of the highest significance with respect to the reproduction of the organism. The following chapters will deal with phenomena of heredity exhibited chiefly in successive generations of plants and animals reproducing sexually; hence, if the essential features of chromosome behavior in the two cytological crises are not borne clearly in mind, these chapters will not be intelligible.

${ }^{52}$ W. H. Blackman (1904b), Dietel (1911), Fitzpatriek (1918b), Colley (1918), and others. See Arthur (1929), Gäumann-C. Dodge (1928), B. O. Dodge (1929a), and Jackson (1931) for accounts of the various types of life cycle in rusts.

${ }^{53}$ Farmer $(1894,1895)$ and A. C. Moore $(1903,1905)$ on Pallavicinia; B. M. Davis (1899, 1901) on Anthoceros and Pellia; Walker (1913) and Vandendries (1913) on Polytrichum; Melin (1915) on Sphagnum; C. E. Allen (1917b, 1919) and Schacke (1919) on Sphcrocarpos; Florin (1918a) on Chiloscyphus; Blair (1926) on Reboulia; Heitz (1928ab) on Pellia; Lorbeer $(1924,1927)$ on Anthoceros, Spharocarpos, and Pellia. The cytology of bryophytes is reviewed by Motte (1929). 


\section{CHAPTER XVII}

\section{CHROMOSOMES AND MENDELIAN HEREDITY}

Since the beginning of the present century the study of the rôle of the chromosomes in heredity has been a major activity in biology. The primary stimulus for such investigation came in the discovery that the behavior of the chromosomes through the life cycle, particularly at syngamy and meiosis, afforded an unmistakable clew to an explanation of Mendel's laws of inheritance, which were rediscovered in 1900. Before turning to the special evidence for the chromosome theory of heredity, however, attention should be given to certain more general matters.

Development and Heredity. - The problem of individual development and that of racial heredity can never be wholly divorced. It should be obvious that the course of ontogenetic development depends upon the organization of the protoplast with which it begins, i.e., upon the type of protoplasmic system concerned, and also upon the environmental agencies which influence its action. The problem of development is, therefore, to ascertain in what manner intrinsic and extrinsic factors, together composing a single interacting system, operate to produce the succession of changes which constitute ontogenesis: it is a problem pertaining primarily to the individual. But any inquiry of this nature soon leads beyond the individual life cycle to a consideration of the race and hence to the problem of heredity. The protoplasmic organization upon which the course of development so largely depends is itself an inheritance from the past; indeed, the most fundamental fact which cytology has contributed to the study of heredity is that the protoplasm of successive generations is genetically continuous. These generations pass through the same general series of ontogenetic stages and thus tend to develop similar characters for the reason that the protoplasm with which each ontogenetic cycle begins is of essentially the same constitution by virtue of this continuity. Most characters are not literally "transmitted" but are redeveloped in each generation. Generations may be regarded as periodic developments of a persistent, though not unchangeable, protoplasmic system: the physical basis of heredity is protoplasm.

It is not solely with resemblances that heredity is concerned. The non-appearance of Mendelian characters in certain generations according to definite rules, and hence the frequent unlikeness of parent and offspring, are known to be just as dependent on the operation of intracellular mechanisms as is the regular appearance of such characters in every 
generation. Even wholly new variations permanently affecting the germinal constitution and thus modifying the characters of subsequent generations are supplementary, rather than opposed, to heredity. In the general process of heredity we witness the results of processes occurring in a protoplasmic system which, though it maintains a specific type of organization through successive generations, undergoes minor alterations which modify the characteristics of the race. Hence it may be said that heredity is the occurrence of related but not necessarily identical conditions, events, or characters in successive generations of organisms as a consequence of their protoplasmic organization.

From these considerations it follows that the problem of heredity which confronts the cytologist is that of ascertaining in what respect resemblances and differences in the characters manifested by successive generations are correlated with similarities and dissimilarities in the organization of the protoplasts with which the successive developmental cycles begin, and how such constitutional conditions arise. Search is to be made for a mechanism which remains comparatively stable as it operates through regularly recurring ontogenetic cycles, and yet undergoes orderly alterations of a kind which will help to account for the observed phenomena of heredity and variation, as well as to approach an understanding of evolutionary advance.

The method followed in such studies is mainly that of altering the cytological constitution of the organism and observing the effect of this upon the inherited characters. Such alterations may be induced by various means, but the one which concerns us in this chapter is that of crossing individuals unlike with respect to cytological or external characters and noting the results in following generations. In this way valuable evidence is gained regarding the cytological basis of the inheritance of those characters in which the individuals may differ.

The Rôle of the Nucleus.-The hypothesis that "the nucleus of the cell is the principal organ of inheritance" was suggested by Haeckel in 1866. Cytological evidence in support of this view was brought forward by a number of workers who described the behavior of the nucleus in the various stages of the life cycle, particularly in somatic cell-division, syngamy, and meiosis (p. 441). Of special interest was the discovery by O. Hertwig and Strasburger that in animals and plants the two nuclei which fuse in the process of syngamy are derived from the two gametes and hence from the two parents. Since it was only in their nuclei that the gametes appeared structurally alike in higher organisms, and since inheritance from the two parents in general seemed to be equivalent, Haeckel's hypothesis was advanced to the rank of a theory, and it was not long before the nucleus was assigned a monopoly in hereditary transmission. 
Subsequent researches have shown that the characters in which crossable organisms may differ and which appear in successive generations according to Mendelian rules may be "transmitted" equally by the two parents, and that their development depends in a special way upon the nuclear organization of the gametes and hence of the zygote (or other cell with which development begins). This analysis of the rôle of the nucleus has been possible because the nucleus contains a relatively small number of visible bodies, the chromosomes, whose distribution in successive cycles can be readily traced, so that the effects of their behavior can be stated in simple mathematical terms. The success of the method has led some workers to extend the nuclear theory to all heredity, and to consider the cytoplasm as little more than an adaptive, nutritive medium for the chromosomes.

It is clear that the nucleus does not deserve this monopoly of the responsibility for all hereditary phenomena. Cases are known in which certain characters are obviously due to the constitution of the cytoplasm and may be influenced unequally by the two parents (Chapter XXV). Breeding data indicate clearly a causal connection between chromosomes and Mendelian differences; but since the crosses made must necessarily be narrow, relatively speaking, they yield little evidence as to the basis for the inheritance of those characters which are always the same in the crossed individuals. ${ }^{1}$ It is to be remembered that in all cases the cytoplasm is an essential component of the system which undergoes development and produces the characters; in fact, it is mainly in the extra-nuclear portion of cells that characters are differentiated. The cytoplasm, even though it may be derived from but one parent, obviously is concerned in, and conditions, the reactions which eventuate in inherited characters, and this cytoplasm must not vary in constitution beyond certain limits. Hence the "physical basis of heredity" in a fundamental sense is the whole protoplasmic system concerned in development, although the course of certain developmental reactions and therefore the appearance of certain characters may be correlated with peculiarities in the organization of the nucleus. The nucleus is not an arbitrary determiner of development: it rather contains a set of conditioning or differential factors which somehow influence in particular ways the developmental processes in the protoplast of which they are an integral part. Evidence for this special influence of the nucleus will now be presented. ${ }^{2}$

${ }^{1} C f$. Gates (1915ab), Johanssen (1923), and Winkler (1924).

${ }^{2}$ For accounts of Mendelism and the chromosome theory, see Morgan (1919a, 1925), Morgan et al. (1922, 1925), Walter (1922), Sirks (1922), Castle (1924), Wilson (1925), D. F. Jones (1925), Sinnott and Dumn (1925), Babeock and Clausen (1927), Goldschmidt (1928a), Stern (1928), Bělař (1928a), Baur (1930), Ekman (1930), Punnett (1927), M. Hartmann (1929a), and Sansome and Philp (1932). Matsuura (1929) summarizes researches in plant geneties for 1900-1925. English translations of Mendel's elassic paper may be seen in Jour. Roy. Hort. Soe. 26 (1901), Bateson's 
Examples of Mendelian Heredity.-Mendel crossed plants of a pure-bred race of tall peas ( 6 to 7 feet in height) with plants of a purebred dwarf race ( $3 / 4$ to $1 \frac{1}{2}$ feet in height) (Fig. 165). All the plants of the first filial generation $\left(F_{1}\right)$ were tall like one of their parents. When these tall hybrids were self-pollinated (or bred to one another), it was found that the second generation $\left(F_{2}\right)$ comprised individuals of the two grandparental types, tall and dwarf, in the proportion of $3: 1$. It was further found that the tall individuals of this generation, though alike in visible characters, were unlike in genetic constitution: one-third of them, if bred for another generation, produced nothing but tall offspring, showing that they were "pure" for the character of tallness; whereas the other two-thirds, if similarly bred, produced again in the next generation both tall and dwarf plants in the ratio of $3: 1$, showing that they were hybrids with respect to tallness and dwarfness. The dwarf plants of the second generation $\left(F_{2}\right)$ produced nothing but dwarfs when interbred: they were "pure" for dwarfness. From these facts it was evident that the plants of the $F_{2}$ generation, although they formed only two visibly distinct classes, were in reality of three kinds: pure tall individuals, tall hybrids, and pure dwarfs, these kinds occurring in the ratio of $1: 2: 1$.

It should be understood that the above ratios merely indicate the probability of obtaining the various types through chance combinations of gametes. If the population is sufficiently large, the ratio is approached rather closely; sometimes it is equalled exactly, even in a small population. The ratios, then, represent a statistical result.

The explanation offered by Mendel for these phenomena may be stated briefly as follows. The germ cells produced by the pure tall plant carry something (now termed a factor, or gene, represented in Fig. 165 by $T$ ) which tends to make the resulting plant tall. The germ cells of the dwarf plant carry something $(t)$ causing the dwarf condition. In the first hybrid generation $\left(F_{1}\right)$ both factors are present, $T$ coming from one parent and $t$ from the other, but $T$ "dominates" and prevents the expression of the "recessive" $t$ so that the plants of this generation are all tall. When the hybrid $\left(F_{1}\right)$ produces germ cells, the two factors for tallness and dwarfness segregate, half of the gametes receiving $T$ and the other half $t$. Each gamete therefore carries either one or the other of the two factors in question but never both; it is "pure" either for $T$ or for $t$. This segregation in the germ cells of factors associated throughout the soma is the central feature of the entire series of Mendelian phenomena and is often referred to as Mendel's first law. Since the gametes, both male and female, produced by the hybrid plants of the $F_{1}$ generation are of two kinds (half of them bearing $T$ and half bearing $t$ ), four combinations

Mendel's Principles of Hexedity and the first two editions of Castle's Genetics and Eugenies. 


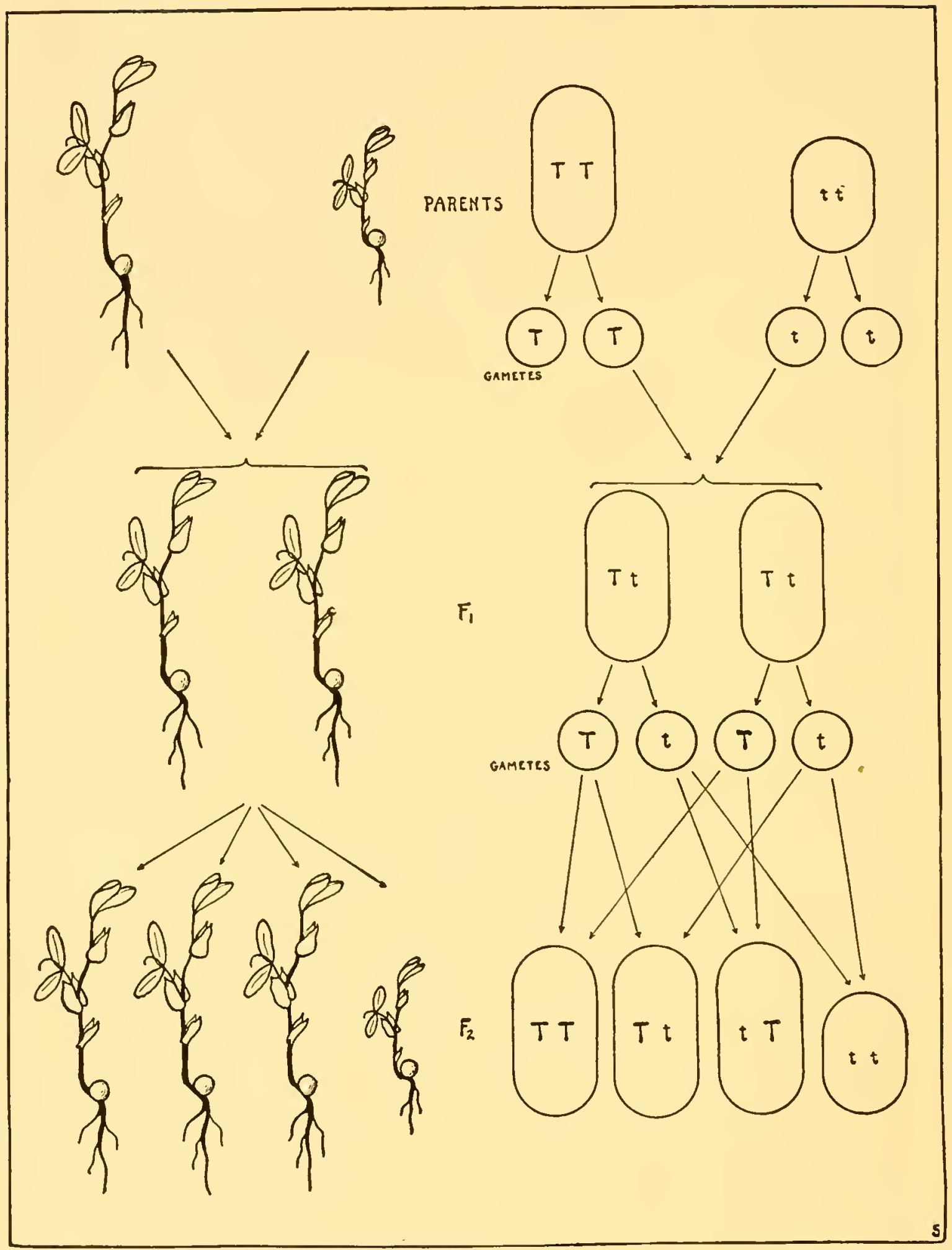

FIG. 165. - A typical example of simple Mendelian heredity. In a cross between pure tall and pure dwarf peas, tallness is dominant over dwarfness in the first filial generation $\left(F_{1}\right)$. In the second filial generation $\left(F_{2}\right)$ tall plants and dwarfs occur in the ratio $3: 1$. At the right is shown the distribution of factors for tallness $(T)$ and dwarfness $(t)$ through these generations. The letters $T$ and $t$ may also be considered as representing a homologous pair of chromosomes. 
are now possible: a $T$-sperm with a $T$-egg, a $T$-sperm with a $t$-egg, a $t$-sperm with a $T$-egg, and a $t$-sperm with a $t$-egg. These four combinations result respectively in a tall plant (pure dominant, $T T$ ), two tall hybrids $(T t)$, and a dwarf plant (pure recessive, $t t$ ). It is obvious that in the long run these three types will tend to occur in the ratio of $1: 2: 1$.

The pure tall individuals and the tall hybrids in $F_{2}$ are ordinarily distinguished from each other by the "back-cross test." It will be readily seen that when a pure tall plant $(T T)$ is crossed with the pure recessive type $(t t)$, all of the offspring will be tall $(T t)$; whereas, when a tall hybrid $(T t)$ is crossed with $t$, half of the offspring will be tall $(T t)$ and half will be dwarf $(t t)$.

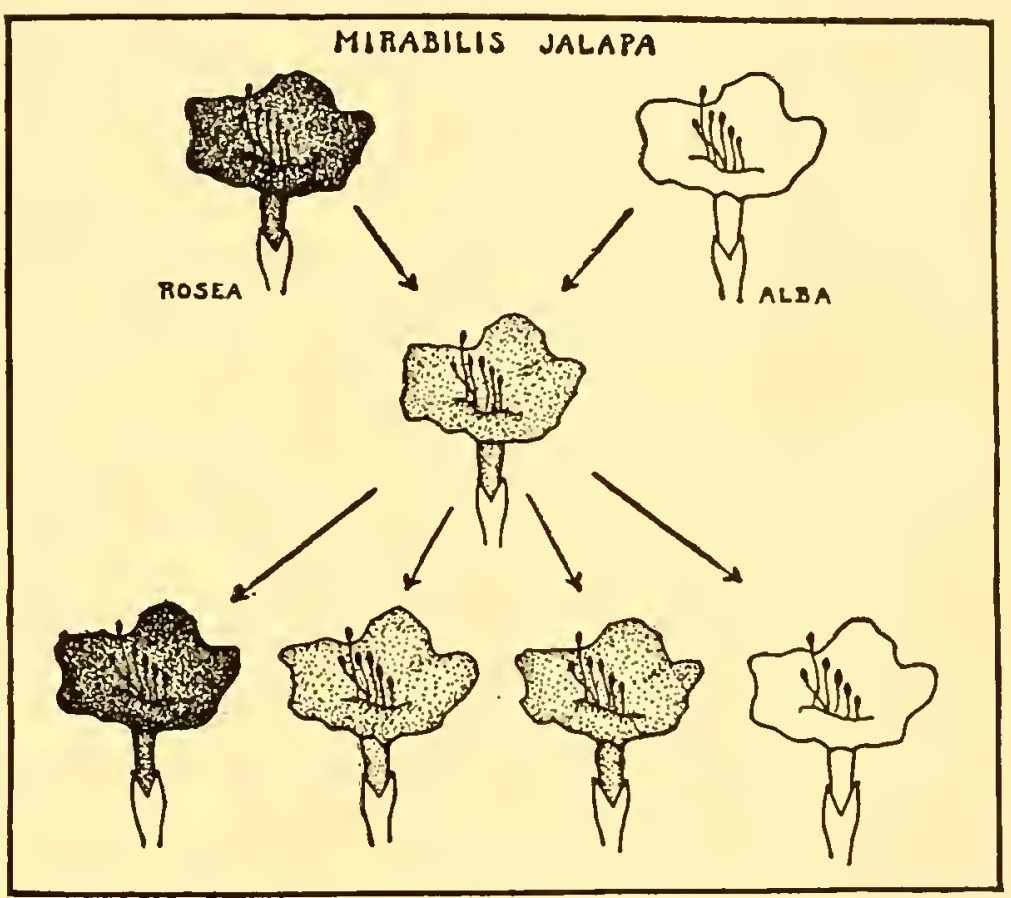

Fig. 166.- Inheritance of flower color in Mirabilis. See text. (Adapted from Correns.)

The Mendelian proportion of hybrids and pure types is perhaps better illustrated by characters in which dominance is imperfect or lacking. In Mirabilis jalapa, for example, the hybrids are more or less intermediate with respect to flower color and are easily distinguishable from the pure parental types (Fig. 166). When plants bearing pure crimson flowers are crossed with those bearing pure white flowers, the hybrid plants of the $F_{1}$ generation have magenta flowers. When these hybrids are bred among themselves, the resulting $F_{2}$ generation comprises plants of three visibly different types: pure dominants with crimson flowers, hybrids with magenta flowers, and pure recessives with white flowers; and these types tend to occur in the ratio of $1: 2: 1 .^{3}$

Mendel's researches on peas included also a study of six other pairs of heritable characters (now known as allelomorphic pairs), the two

${ }^{3}$ See further Kiernan and White (1926) on this case. 
members of each pair behaving toward each other in a manner similar to that described above for tallness and dwarfness. He further observed that the seven pairs were entirely independent of each other in inheritance. For instance, smoothness and wrinkledness of seeds were allelomorphic characters, and either of them was transmitted as often with tallness as with dwarfness in crosses involving both pairs of characters. This independence of the various pairs of factors was set forth in Mendel's second law. The law held for the seven principal pairs studied by Mendel, but it has since been learned that many pairs are not thus independent, as will be pointed out below.

Terminology.-We may here introduce certain terms prominent in the literature of genetics. The genotype is the entire assemblage of genetic factors, or genes, which the organism actually possesses in its constitution, irrespective of how many of these may be expressed in externally visible characters; or, it is a class of individuals with the same genetic constitution. The phenotype is the aggregate of externally visible characters, irrespective of any other factors, unexpressed in characters, which may be present in the organism; or, it is a class of outwardly similar individuals. For illustration: in the case of the tall and dwarf peas there are in the second generation $\left(F_{2}\right)$ three genotypes (with respect to the single character pair discussed): $T T, T t$, and $t t$, represented, respectively, by pure tall plants, tall hybrids, and dwarfs; but there are only two phenotypes: tall and dwarf, because the complete dominance of tallness over dwarfness renders the hybrids externally indistinguishable from the pure tall individuals. Thus one phenotype (tall plants) here includes individuals with two genotypic constitutions, and the two can be distinguished only by a study of their progeny. In Mirabilis, however, there are represented in the $F_{2}$ generation not only three genotypes but also three phenotypes, since incomplete dominance renders the hybrids externally unlike either of the pure forms. Practically, a phenotype is a class of individuals which look alike, and a genotype is a class of individuals which breed alike (Castle).

An individual is said to be homozygous for a given allelomorphic factor pair if it has received the same type of factor from the two parentsa pea, for example, with the constitution $T T$ or $t t$. If it has unlike members in the pair, such as $T t$, it is said to be heterozygous. It may be homozygous for some allelomorphic pairs and heterozygous for others, or it may conceivably be either homozygous or heterozygous for all of its factors. Thus an organism with the genotypic constitution $A A B b c c$ is homozygous for the factors $A A$ and $c c$ and heterozygous for $B b$. It is a pure dominant with respect to $A$ and $a$, a pure recessive with respect to $C$ and $c$, and a hybrid with respect to $B$ and $b$. The phenotypic appearance of the organism is here determined by the dominant factors $A$ and $B$ and the recessive $c$. It is a common practice to represent dominant 
factors by capital letters and their recessive allelomorphs by the corresponding small letters.

The Cytological Basis of Mendelian Heredity.-The history of the chromosomes through the critical stages of the life cycle, as more fully described in the chapters on syngamy and meiosis, must be recalled at this point (see Figs. 148 and 167). Each parent furnishes the offspring with a set of individually different chromosomes, the two sets (represented in Fig. 167 by $A B C D$ and $a b c d$ ) together constituting the diploid complement present in all of the nuclei of the new individual. When gametes (or spores followed later by gametes in the case of higher plants) are to be formed by this individual, descendants of the homologous chromosomes of

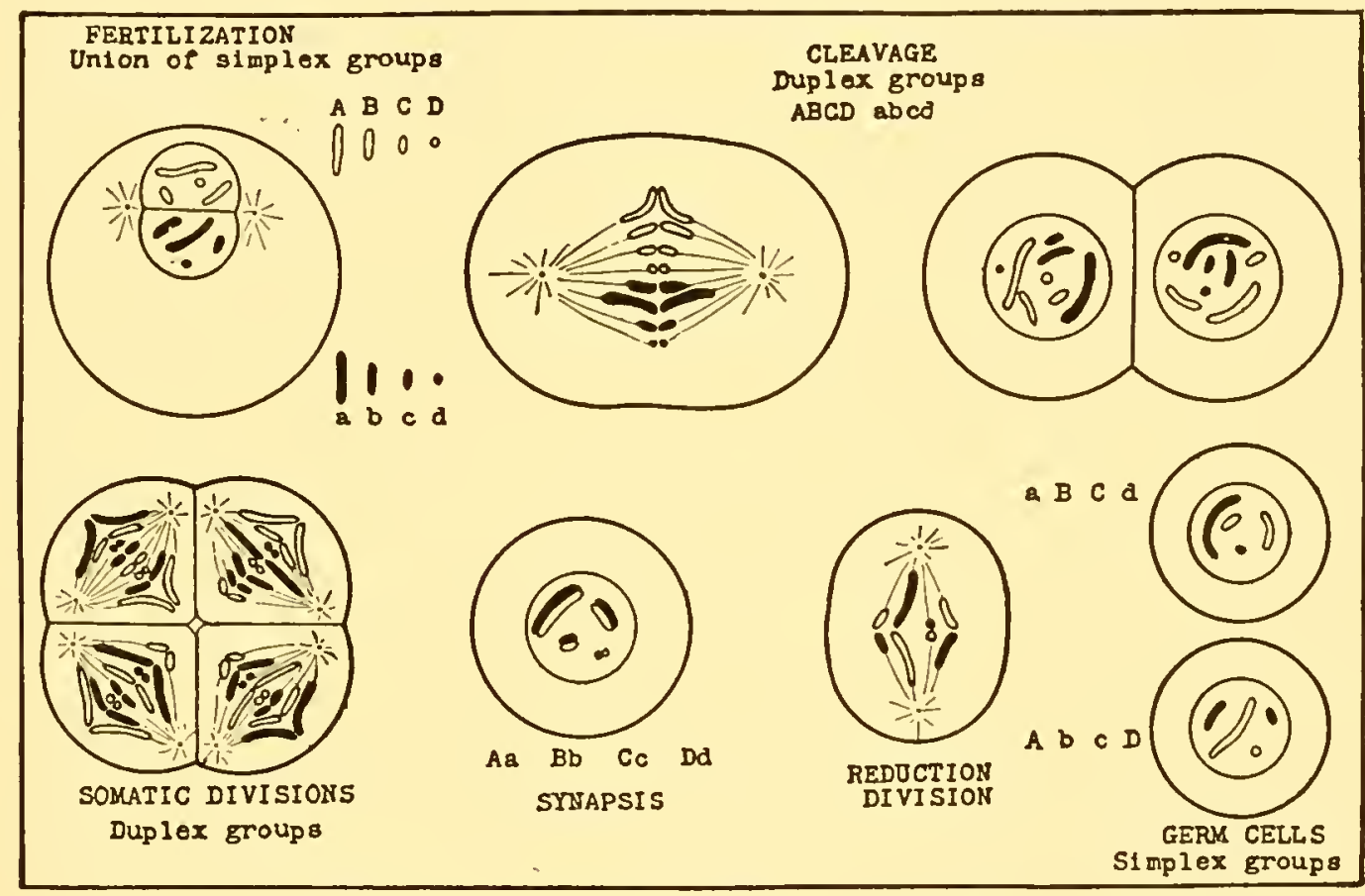

FIG. 167.-Diagram of chromosome cycle. (After E. B. Wilson, 1913.)

the two gametic sets conjugate in pairs (synapsis). In one of the meiotic divisions the two members of each pair disjoin and come to lie in different nuclei. In the other meiotic division the chromosomes divide equationally. The result of the two meiotic divisions, therefore, is a group of four gametes (or spores), two of which differ from the other two with respect to any given chromosome pair: two of them have derivatives of $A$ while the others have derivatives of $a$, and so on for all the other pairs. The chromosomes of the diploid complement are in this way assorted into monoploid sets, each gamete (or spore) having a set made up of one member of each of the pairs. This set represents the contribution made to the following generation.

It is of importance to recall alsö that the various pairs of chromosomes are independent of each other as regards their orientation in the mitotic figures and hence in their distribution to the daughter nuclei (p. 254). 
As a result a given gamete (or spore) in an organism with four pairs of chromosomes may have any one of 16 possible monoploid combinations (Fig. 168). ${ }^{4}$

It will be observed at once that the distribution of the chromosomes through the life cycle and successive generations is precisely like that of the Mendelian factors. Two groups of factors are brought together in syngamy and are associated in the body of the offspring. In the germ cells the factors of each allelomorphic pair segregate and pass to different

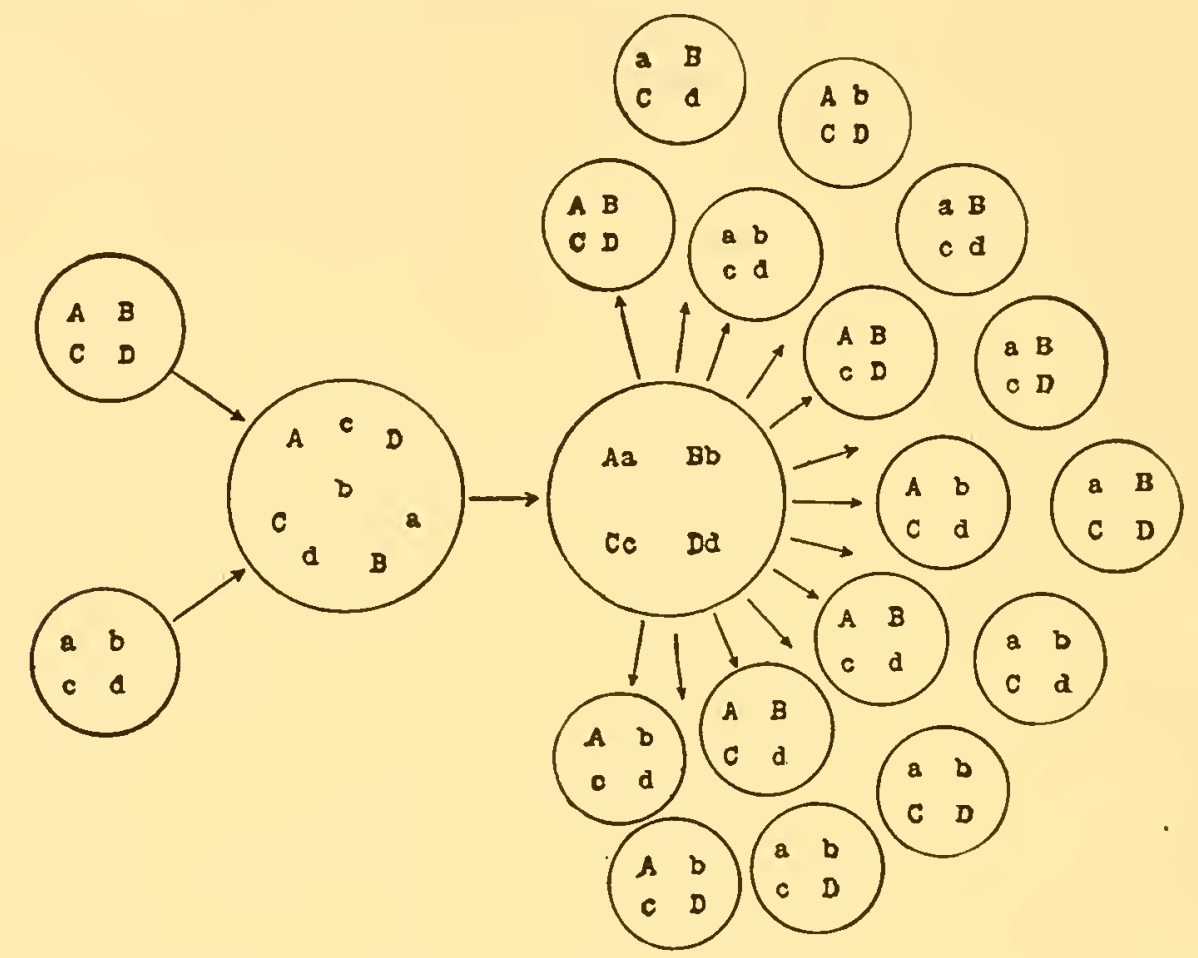

FIG. 168.-Diagram showing the 16 gametic combinations formed by four independent heterozygous pairs of factors. (After $E, B$. Wilson, 1913.)

gametes (or spores). The factors and the chromosomes alike form a duplex group in each somatic nucleus and a simplex group in each gamete (or spore): both chromosomes and factors are aggregated in syngamy and segregated in meiosis. The precise nature of the parallelism is seen not only in the factor and chromosome groups as wholes but also in the individual pairs. When a single pair of chromosomes, for example $A a$ in Fig. 167, is followed through successive cycles, it is seen that there is an exact parallelism between the distribution of a given homologous pair of chromosomes and that of a single allelomorphic pair of Mendelian factors. This is just the condition that should result if the two factors are present

${ }^{4}$ The possible number of different monoploid gametic combinations and of diploid zygotic combinations after self-fertilization (or crossing with an exactly similar individual), when each ehromosome pair shows some degree of heterozygosity, may be readily calculated. Where $n=$ the number of pairs, the number of gametic combinations is $2^{n}$ and the number of zygotic combinations is $3^{n}$. If two individuals differing in all their ehromosomes are erossed, there will be $4^{n}$ possible kinds of combinations in $F_{1}$ and $10^{n}$ in $\mathrm{F}_{2}$ (see Winge, 1919b). 
as units of some kind in the two members of the pair of chromosomes. In the disjunction of these members in meiosis is recognized a cytological mechanism adequate to accomplish the factorial segregation underlying Mendel's first law. In Fig. 165 the symbols $T$ and $t$ may be taken to represent either a pair of factors or a pair of chromosomes. The same is true of the letters in Fig. 167.

The cytological basis for Mendel's second law is seen in the independent distribution of the various chromosome pairs. Different factor pairs are independently distributed, as Mendel held, if they are associated with different chromosome pairs; but this will not be the case if they lie in the same pair. The discovery of "linkage," to be described below, has accordingly made necessary an important qualification of Mendel's second law, but this has served to show even more strikingly the significant relation between chromosome behavior and Mendelian heredity.

Mendelian studies are often rendered more difficult by a number of phenomena which tend to alter the characteristic ratios obtained. It frequently happens that among sister individuals with unlike genic combinations some do not proceed far with development, so that an expected class may be partially or wholly absent from the progeny. The germination of pollen grains with certain combinations is also sometimes deficient; even when all the grains germinate it may be found that the pollen tubes carrying certain combinations outgrow the others and so reach all or most of the ovules before the slower tubes arrive. Furthermore, there are known a number of "lethal factors" which in certain combinations retard or prevent development at certain stages, notably in the gametes and zygotes. As would be expected, occasional abnormal chromosome behavior results in abnormal ratios also. Because of these phenomena there is often a marked "developmental sclection" which favors the multiplication of certain genetic types at the expense of others. In such cases the analysis of the genetic data becomes a more complicated problem, but in this analysis the fundamental assumptions regarding the association of chromosomes and factors are found to be adequate. ${ }^{5}$

Linkage and Crossing-over.-When two pairs of Mendelian characters are due to differential factors located in different chromosome pairs they are inherited independently, that is, either character of one pair has an equal chance of appearing with either character of the other pair because of the independent distribution of the two chromosome pairs. On the contrary, when their differential genes are located in the same chromosome pair, certain character combinations tend to appear much

${ }^{5}$ Buchholz and Blakeslee $(1922,1927 a, 1929,1930 a c, 1932)$ on Datura pollen tubes; Buchholz (1922) on developmental selection in general; Brink and MacGillivray (1924), Brink (1925b, 1927a), Brink and Burnham (1927), Mangelsdorf (1929), Brieger (1926), Nishiyama (1928) and D. F. Jones (1928) on selection among pollen and male gametes. For lethal factors, see Muller (1917, 1918), Li (1927), B. M. Davis (1923), and Morgan et al. (1922). 
more frequently than others. Such linkage of genes and of characters is well illustrated in the following case in Drosophila (Fig. 169).

Two well-known characters in Drosophila cultures are black body and vestigial wings. Each of these is a recessive character, appearing in a fly only when the gene has been received from both parents in the recessive condition $b$ or $v$. In the dominant conditions $B$ and $V$ these genes produce, respectively, normal gray body and normal long wings. When a fly, homozygous for both dominant factors, is mated to one with all of the corresponding factors recessive, the offspring all have normal body and

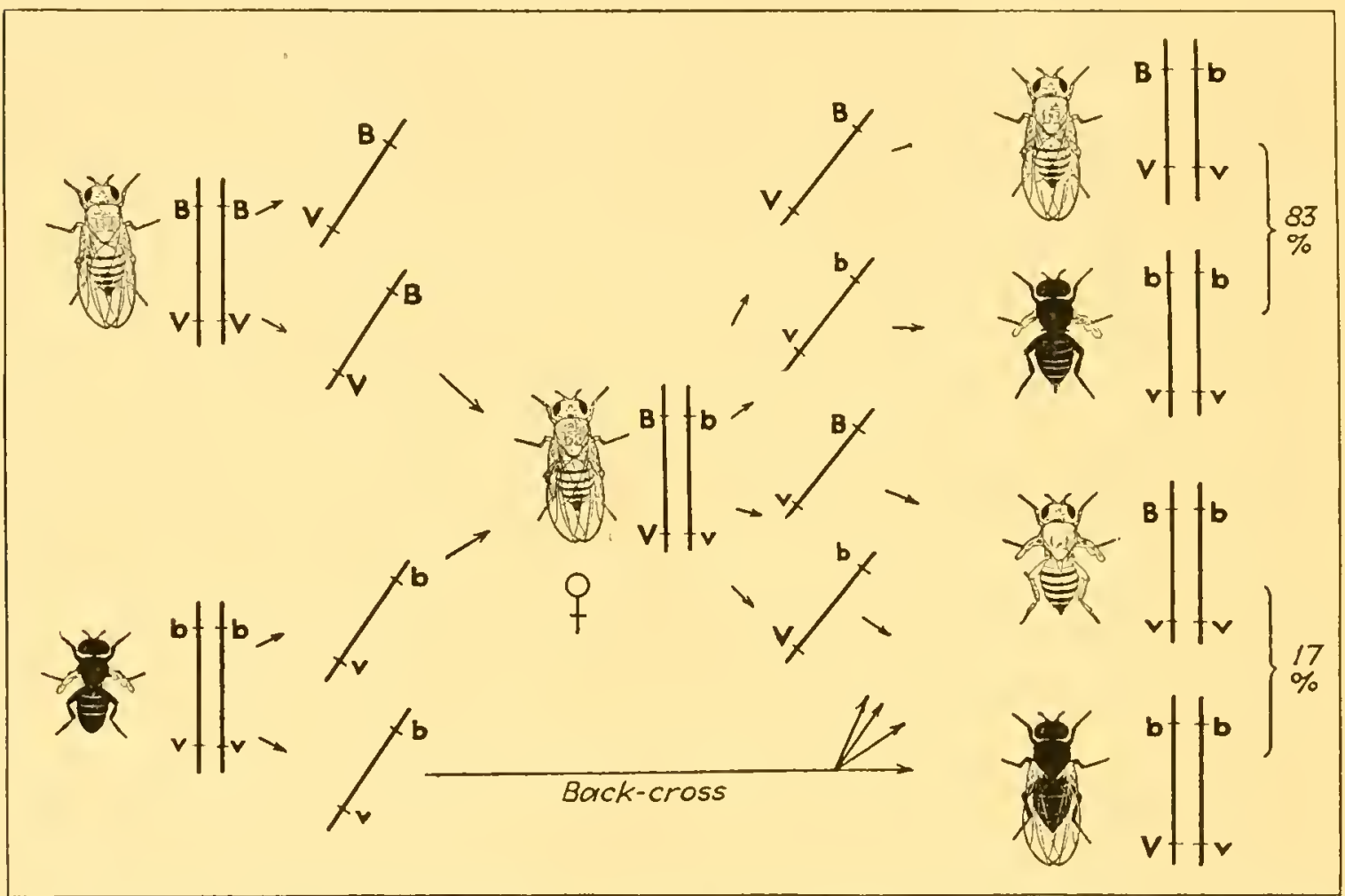

FIG. 169.-Linkage in Drosophila. One pair of somatic chromosomes in each fly is represented by parallel lines; chromosomes in gametes represented by diagonal lines. For explanation, see text. (Adaptèd from Morgan et al., 1922.)

wings, because of the dominance of $B$ and $V$ over $b$ and $v$, respectively. If the females of this $F_{1}$ generation are back-crossed to the homozygous recessive, flies of four types appear in the next generation: gray-long, black-vestigial, gray-vestigial, and black-long. Those flies with the original combinations (gray-long and black-vestigial) together comprise 83 per cent of the total number; only 17 per cent are of the new types (gray-vestigial and black-long). It thus appears that if the two characters, gray body and long wings, are "contributed" to the offspring by the same parent, they tend to appear together in the majority of the individuals resulting from the back-cross; in other words, they are "linked." This is explained by the fact that the differential genes concerned are located in the same chromosome. The same is obviously true of the allelomorphic characters, black body and vestigial wings: their genes are 
also carried in the same chromosome. Hence in the $F_{1}$ fly one chromosome of a certain homologous pair carries $B V$, while the other carries $b v$, and they tend strongly to continue into the next generation in these conditions. Were the two pairs of genes in question, $B b$ and $V v$, carried by different pairs of chromosomes instead of in the same pair, there would be no linkage: the two characters, gray and long, and likewise the two characters, black and vestigial, would then be exhibited together in the next generation by about 50 per cent of the flies, the chance frequency, rather than 83 per cent.

We have next to inquire into the origin of the new combinations appearing in 17 per cent of the flies after the back-cross. In the original female both chromosomes carry $B V$; hence every egg has this combination. The male has $b v$ in both chromosomes of the pair; hence every sperm has $b v$. All flies in $F_{1}$ will therefore have $B V$ in one chromosome of the pair and $b v$ in the other; they are heterozygous for both pairs of genes. When the females of the $F_{1}$ generation mature their eggs, the two chromosomes disjoin in meiosis so that half of the eggs carry one and half the other. If the chromosomes are passed along unaltered, no new combinations appear in the next generation.

Now let it be supposed that in some of the oöcytes ${ }^{6}$ two non-sister chromatids exchange portions at some point between the two pairs of genes in question. This will mean that some eggs will carry unaltered chromosomes $(B V)(b v)$ while others will carry altered ones $(B v)(b V)$. Fertilization of these four classes of eggs by sperms carrying $b v$ will obviously result in flies of four classes, two of which are of new kinds. This mutual exchange of corresponding portions of homologous chromosomes, which may result in such recombinations of linked characters, is known as crossing-over. The percentage of recombinations appearing depends upon the proportion of the oöcytes in which chromatid exchange occurs between the two pairs of genes. If it occurs in every oöcyte, 50 per cent of the resulting flies should show the recombination (other things being equal), since two normal and two altered chromatids result in any one cell. From this it can readily be seen that the frequency (17 per cent) of recombination in the above example is due to the fact that the proper chromatid exchange occurred in but 34 per cent of the oöcytes. Such crossing-over between pairs of genes linked in various degrees is a phenomenon occurring generally in plants and animals, although in some cases, notably in the males in Drosophila, it is absent. ${ }^{7}$

${ }^{6}$ That crossing-over occurs in the primary oöcytes rather than in earlier or later cells is shown by the work of Plough (1917) and Gowen $(1929 a, 1933)$.

${ }^{7}$ Huettner (1930) reports that in Drosophila males synapsis is incomplete and of brief duration, but that the meiotic mitoses are otherwise normal. Other works on meiosis in Drosophila are those of Huettner (1924), Metz (1926b), League (1929), Guyénot and Naville (1929), and Woskressensky and Scheremetzewa (1930). 
It should be pointed out that if the original cross in the foregoing example had been made between a fly homozygous for black-long and one homozygous for gray-vestigial, these combinations would have appeared in about 83 per cent of the $F_{2}$ individuals after back-crossing to a pure recessive, while gray-long and black-vestigial would have formed about 17 per cent of recombinations because of crossing-over. In other words, one combination is as likely to appear as another in a long series of generations, barring detrimental effects which may attend one of them. The condition rather arbitrarily called "normal" is usually the one which is most prevalent in healthy flies; sometimes it is the one which happens to be observed first.

Because the Mendelian factor pairs so far outnumber the chromosome pairs, it is evident that each of the chromosomes must carry a considerable number of genes. Extensive researches on linkage relations in various plants and animals have brought out the fact that the genes and hence the Mendelian characters fall into linkage groups, the members of each group being linked to one another in various degrees but showing independent assortment with the members of other groups. Moreover, when the linkage relations of enough genes are known, it is found that the number of such linkage groups in an organism is the same as the number of its chromosome pairs. This is well illustrated by Drosophila melanogaster and Zea Mays, the two organisms concerning whose genetic behavior our knowledge is most advanced. In Drosophila melanogaster there are four pairs of chromosomes; pair I is rod-shaped, pairs II and III are longer and bent, and pair IV is very small (Fig. 196). The genes in this species fall into four linkage groups, and it is noteworthy that three of the groups are large while the fourth comprises only a very few known genes (Morgan, Muller, Sturtevant et al.). In Drosophila Willistoni there are three chromosome pairs and three linkage groups (Metz) and in $D$. obscura five pairs and five groups (Lancefield). In Zea Mays, where there are normally 10 pairs of chromosomes, 10 linked groups have been identified (Emerson et al.). In Pharbitis Nil, which has 15 pairs of chromosomes, there have been identified 10 groups and several independent factors probably representing the five other groups (Imai, Hagiwara). It is an interesting fact that Mendel, in his classic researches on Pisum, which has only seven chromosome pairs, happened to select for special study seven pairs of characters evidently belonging to as many different linkage groups and so did not detect the phenomenon of linkage. In view of such facts, it is possible to look upon a chrcmosome as a body containing a definite group of genes which influence the development of a definite group of characters; but it is not to be concluded from this that the chromosome in question is solely responsible for these characters.

Assignment of Linkage Groups to Chromosomes.-The first character found to be definitely associated with a distinguishable chromosome was 
that of sex (McClung). In certain insects it was observed that one pair of chromosomes was markedly different from the others in appearance and behavior; moreover, the two members were unlike in size, so that they could be followed through successive generations. Furthermore, males and females were observed to differ with respect to this chromosome pair, females commonly having two large ones $(X X)$ while the males had one large and one small $(X Y)$. In some species the $Y$ was absent altogether, so that the two sexes were characterized by different chromosome numbers. The logical conclusion that this chromosome pair exerts a special influence upon the sex of the organism has since been borne out in a large number of unisexual animals and plants. In typical cases this "sexchromosome" mechanism tends to produce equal numbers of males and females in each generation. A number of characters other than sex were seen to be linked with sex in inheritance, so that their genes were assigned to the sex-chromosomes. Such genes are said to be "sex-linked." These topics will be discussed further in Chapter XXIII.

The studies of Morgan and his associates on Drosophila melanogaster have shown that pair I, which is heteromorphic in the male, has a special influence on sex determination and carries a considerable group of genes for sex-linked characters. Pair IV, the smallest of the group, carries the very small linkage group, as was shown particularly well by occasional flies which had one too many or too few of these small chromosomes. The two large linkage groups were accordingly assigned to the two large chromosome pairs, and it has since been demonstrated that the slightly longer pair carries the genes of the "third" linkage group (Dobzhansky, $1929 a b)$.

In Zea Mays the 10 chromosomes of the monoploid set (and hence the 10 pairs) can all be distinguished on the basis of their size and structure (McClintock, 1929b, 1932c). It has now been possible to identify each linkage group worked out by Emerson and his associates with one of these 10 chromosomes (Fig. 170). This has been accomplished largely through a study of occasional "trisomic" plants. In such plants one of the members of the set is present in triplicate in the somatic cells while the nine others are in duplicate as usual; hence at the time of meiosis some spores, and therefore gametes, receive one member of the "trisome" while others receive two. From this it follows that any characters due to genes in the triplicate chromosome should appear in abnormal proportions in successive generations, while other characters should at the same time appear in normal Mendelian ratios. The method is, therefore, to determine genetically which characters give "trisomic ratios" when the plants are bred, and to observe cytologically which chromosome of the set is present in triplicate in the somatic cells and in duplicate in some of the microspores. In this way it was found that in a strain of maize with an extra chromosome, number $\mathrm{X}$ (the smallest of the set), an aleurone color 
due to the gene $R$ showed trisomic ratios while characters in other linkage groups showed ordinary ("disomic") ratios. Hence this gene and other genes of the same linkage group are carried by the smallest chromosome of the normal set (McClintock and Hill, 1929, 1931) (Fig. 66, d).

In Datura stramonium, which ordinarily has 12 pairs of chromosomes, similar studies on trisomic strains have led to the assignment of certain genes to certain distinguishable chromosomes. In this species each of the 12 chromosomes produces, when present in triplicate, a characteristic

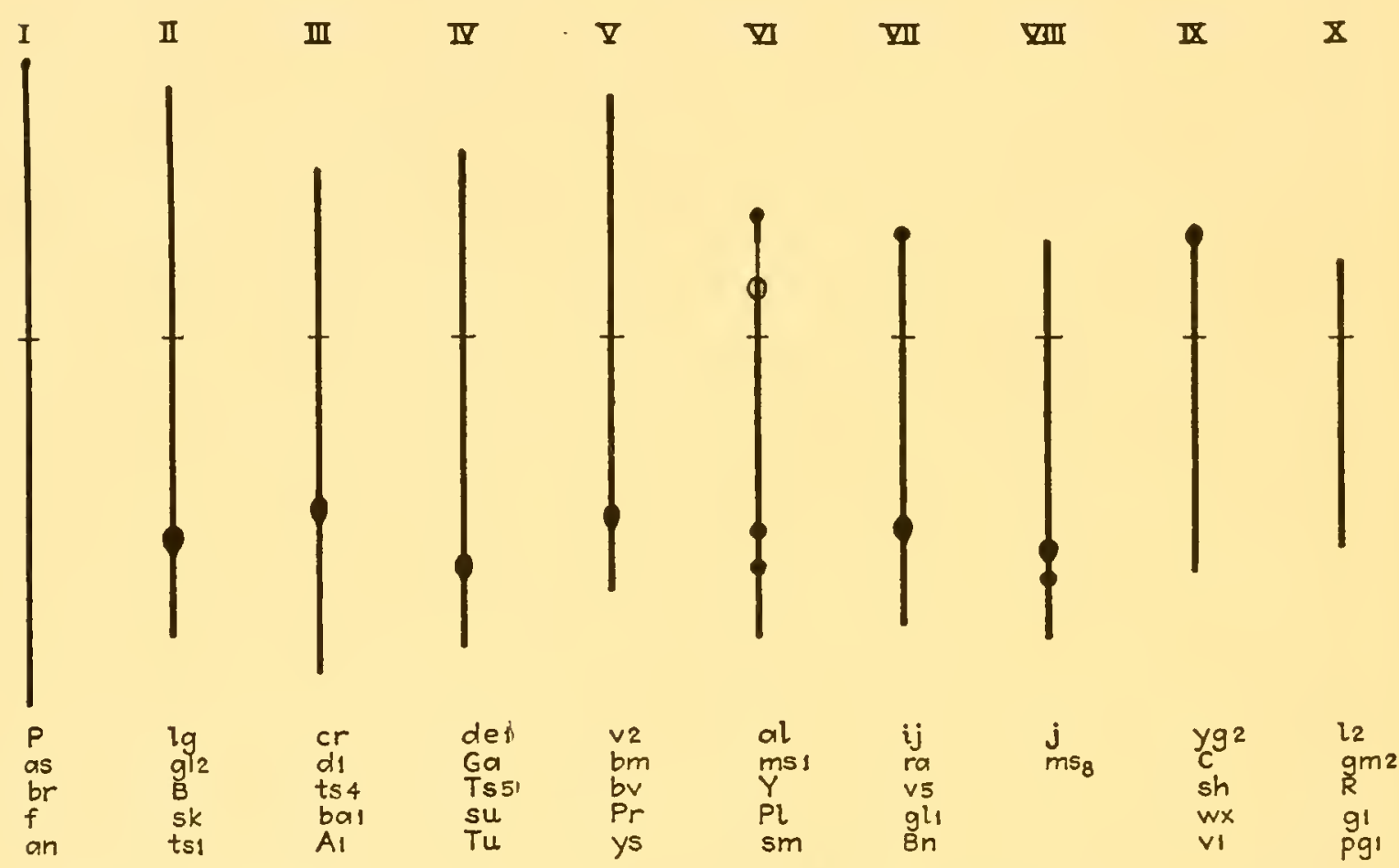

Fig. 170.-Diagram of chromosome set of Zea Mays, showing the relative lengths and the location of the spindle-attachment regions (cross lines) in the 10 members. Inversions and translocations sometimes cause variations in these features. Chromatic knobs appearing in one or more of the chromosomes of certain strains are also indicated. Below the chromosomes are some of the genes which they carry. $C f$. Figs. 171, 66, and 152 . (Based on diagrams and data of McClintock, Emerson, and others.)

appearance in the plant which may be readily recognized in the breeding plot (Belling, Blakeslee).

Such results as these serve to emphasize the fact that the chromosome set is a definitely differentiated system, each member containing a group of elements with specific effects upon the course of development and therefore upon the characters which appear in the organism.

Location of Genes in the Chromosome.-That the genes of a given linkage group are arranged in a definite linear order in the chromosome was first clearly shown by the extensive researches on linkage relations in Drosophila. It was observed that some factor pairs were closely linked, i.e., crossing-over occurred between them very rarely; whereas, other pairs were more loosely linked, as shown by the consistently high frequency of crossing-over between them. The hypothesis was advanced 
that the frequency with which crossing-over occurs is a function of the actual distance apart of the factor pairs in question (Sturtevant, 1913). In other words, the farther apart two pairs of genes lie, the greater the chance for a crossover to occur between them. It was assumed that when flies showed 1 per cent of recombinations after a back-cross, the two pairs of genes concerned were one "unit" apart in the chromosome, and so on for other percentages. Certain refinements have since been made in this hypothesis, but linkage studies in many organisms, together with

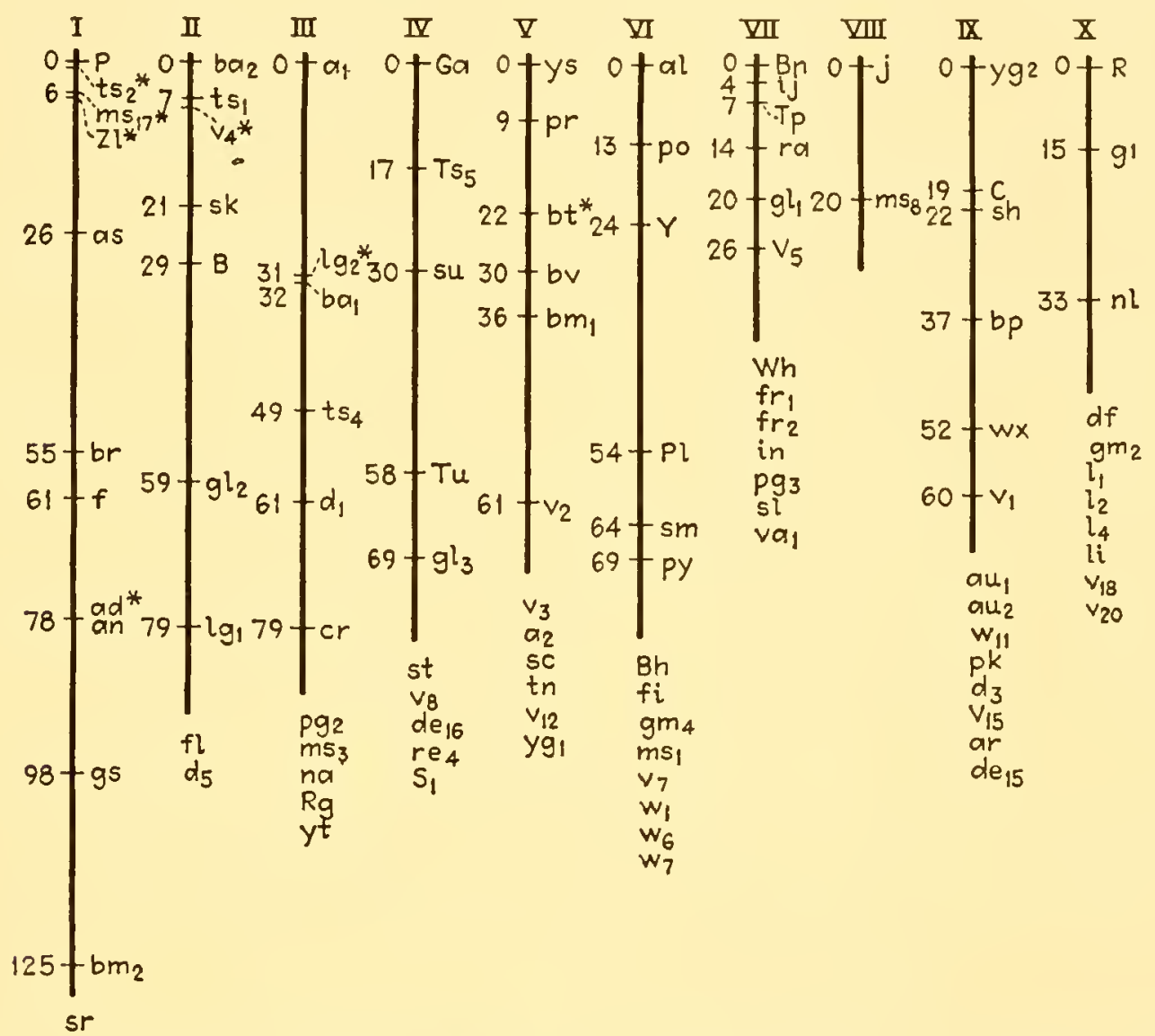

FIG. 171.-Linkage map for Zea Mays, showing the arrangement of the genes as determined genetically. The length of the lines does not indicate actual chromosome length; cf. Fig. 170. The position of genes marked with asterisks is only approximate. The genes below the lines are known to belong to the linkage groups indicated, but they have not yet been assigned to definite loei. (After R. A. Emerson, 1933.)

evidence from translocations of portions of chromosomes (see next chapter), have given it striking confirmation.

The method of determining the relative position of genes by studying their linkage relations may now be illustrated with a case in Zea (Fig. 172). In chromosome pair II there are, among others, the following pairs of genes $=L g l g$, for liguled $v s$. liguleless leaves; $B \quad b$, for more intense $v s$. less intense plant color; $S k s k$, for normal vs. silkless ears. Between $L g l g$ and $B b$ (region $\alpha$ ) the percentage of recombination was found to be 28.6 , so that these pairs of genes were assigned to loci 28.6 units apart in the "map" of the chromosomes. Between $B b$ and $S k$ sk (region $\beta$ ) the 
percentage was 6.9 , so that $S k s k$ had to be placed this number of units from $B b$. Either of two positions was possible for $S k$ sk: to the right or to the left of $B \quad b$. Obviously a choice between these positions might be made if the percentage of recombination between $L g l g$ and $S k$ sk were known. This turned out to be 34 ; hence $S k$ sk was assigned a position farther away from $\mathrm{Lg} l g$ than was $B b$. The relative position of the three pairs of genes was thus established.

It will be noted that the percentage of recombination between $\mathrm{Lg} l g$ and $S k s k$ is somewhat less than the sum of the percentages shown in regions $\alpha$ and $\beta$. This is due to the fact that two crossovers sometimes occur between widely separated genes in the same chromosome pair. Thus two exchanges between $L g l g$ and $S k s k$ would leave the same genes linked, one crossover neutralizing the effect of the other. Since all three gene pairs were studied simultaneously in the above experiment, it was

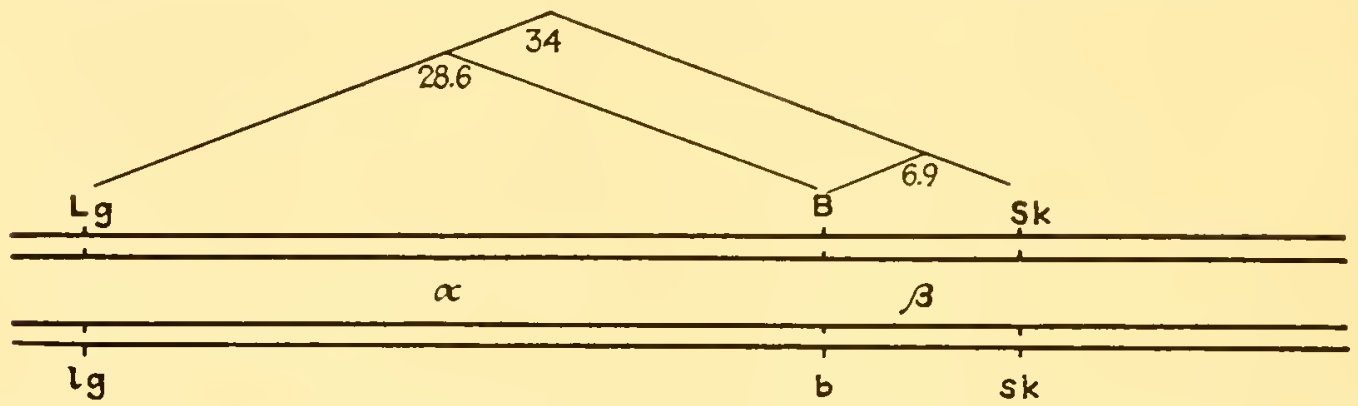

FIg. 172.-Diagram of linkage relations of certain genes in chromosome II of Zea Mays. The four lines represent portions of the chromatids of a synapsed pair. See text. (After data from M. M. Rhoades.)

possible to show that crossing-over had actually occurred simultaneously in regions $\alpha$ and $\beta$ in 11 out of the 1,392 cases. Because of such "double crossing-over," the map distances often exceed the percentages of recombination for widely separated genes.

The observed percentages of recombination due to random crossingover between the four chromatids of a tetrad approach 50 as a limit since only two of the four chromatids are altered by any one exchange (Emerson and Rhoades, 1933). In well-developed chromosome maps like those of Zea and Drosophila (Figs. 171, 173) it will, however, be noted that map distances may exceed 100 units. This is because the map is built up by adding the distances between closely linked genes and not by observing directly the recombination percentages for widely separated genes. It does not take account of such modifying influences as double crossingover. Hence the map distances represent actual recombination percentages only for rather closely linked genes (not more than about 10 units apart in Drosophila). It will accordingly be observed that the map distance between "black" and "vestigial" in the second chromosome is 18.5 units, whereas the percentage of recombination is 17 , as shown in Fig. 169. 


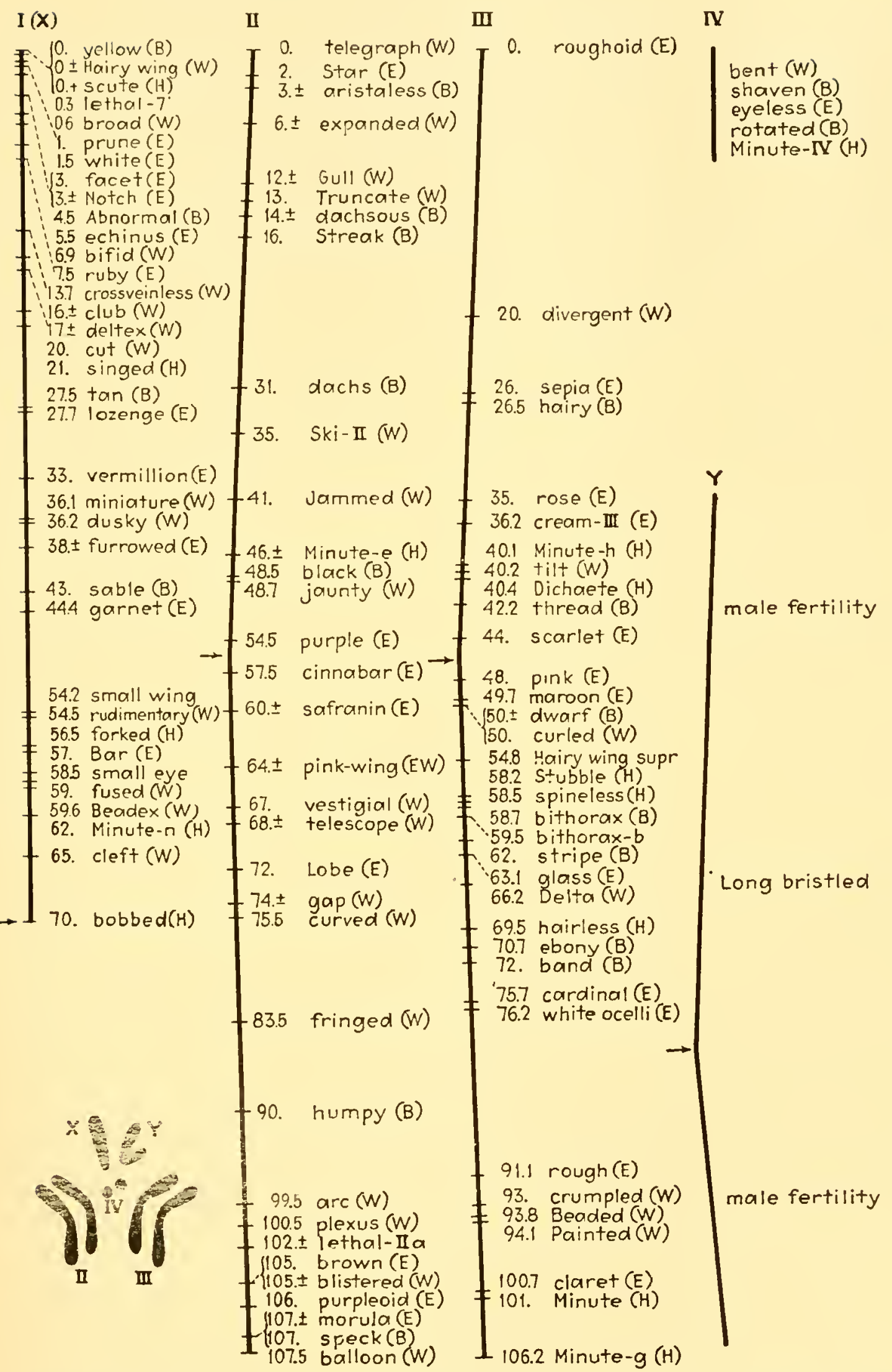

FIG. 173.-Linkage map for Drosophila melanogaster, showing relative positions of many of the known genes in the ehromosomes as determined genetically. The letters in parentheses indicate the portion of the fly in which the characters appear: $B$, body; $E$, eye; $H$, hairs; $W$, wings. The arrows indicate positions of spindle-attaehment regions. In the $Y$-chromosome, "Long bristled," which is the normal allelomorph of "bobbed," and the two faetors for male sterility have not been precisely located. In chromosome IV the genes are all very closely linked. (Adapted from Morgan, Sturtevant, and Bridges (1925) and Stern (1929)). 
It is important to note further that crossovers may not occur with equal frequency in all portions of the chromosome. For instance, it has been shown that crossing-over in Drosophila occurs less frequently near the spindle-attachment region than elsewhere. Of interest in this connection is the observation in Zea that the chromatids, as they open out in the diplonema stage, tend to remain together and therefore to form fewer chiasmata near the attachment region (McClintock). From such facts it follows that the map, although it shows correctly the serial order and linkage relations of the genes, may not give a true picture of their actual spacing in the chromosome. We shall revert to this topic in the next chapter.

In interpreting recombination data it is often necessary to consider a phenomenon known as interference. If the genes are arranged in a close linear series in the chromatid, and especially if chiasmata account for crossing-over, it might be expected that when crossing-over occurs between any two pairs of genes, the near-by pairs would show no crossing-over, since the physical conditions might prevent the formation of two chiasmata or of two breaks very near each other. It has been found that the data often conform with these expectations. The recombination percentage characteristic for genes in a given region of the chromosome is noticeably lowered when crossing-over occurs at a near-by point. This interference with crossing-over varies from a high value for closely linked genes to zero for very loosely linked ones (Muller, Sturtevant, Weinstein). $\Lambda$ further interesting fact is that interference in Drosophila and Zea is weak across the spindle-attachment region; each arm of the chromosome behaves more or less independently in this respect. 'The frequency of crossing-over has been shown to be affected in certain cases by age, temperature, sex, and irradiation. ${ }^{8}$

Chromatid Exchange as the Mechanism of Recombination.Many years ago it was suggested that an exchange of some sort occurs between homologous chromosomes during the synaptic period. ${ }^{9}$ Cytological evidence for an exchange of portions between chromosomes in certain insects was brought forward by Janssens in 1909. Although this evidence was not considered conclusive by many cytologists, chromosome exchange was provisionally assumed to represent the physical basis of recombination by Morgan and his associates in their successful development of the chromosome theory of Mendelian heredity. Many bits of evidence have pointed toward the correctness of the exchange hypothesis, but a complete demonstration of the fact that two chromosomes actually exchange corresponding portions as the genes in these regions are recom-

${ }^{8}$ E.g., Bridges $(1915,1927,1929)$ on age; Plough $(1917,1921)$ and Stern $(1926 c)$ on temperature; Mavor (1923b), Mavor and Svenson (1923), E. G. Anderson (1925b), and Muller (1926) on X-radiation.

${ }^{9}$ Correns (1902), DeVries (1903), Strasburger (1905a), C. E. Allen (1905a). 
bined has proved difficult to obtain. In 1931 evidence of great cogency was yielded by both Zea and Drosophila. In each of these cases the chromosome pair involved was heteromorphic in two regions and had two pairs of genes between these regions which could be followed genetically as the history of the chromosomes was being traced.

The situation in Zea Mays is as follows (Creighton and McClintock, 1931) (Fig. 174). The plant selected for the test had a heteromorphic

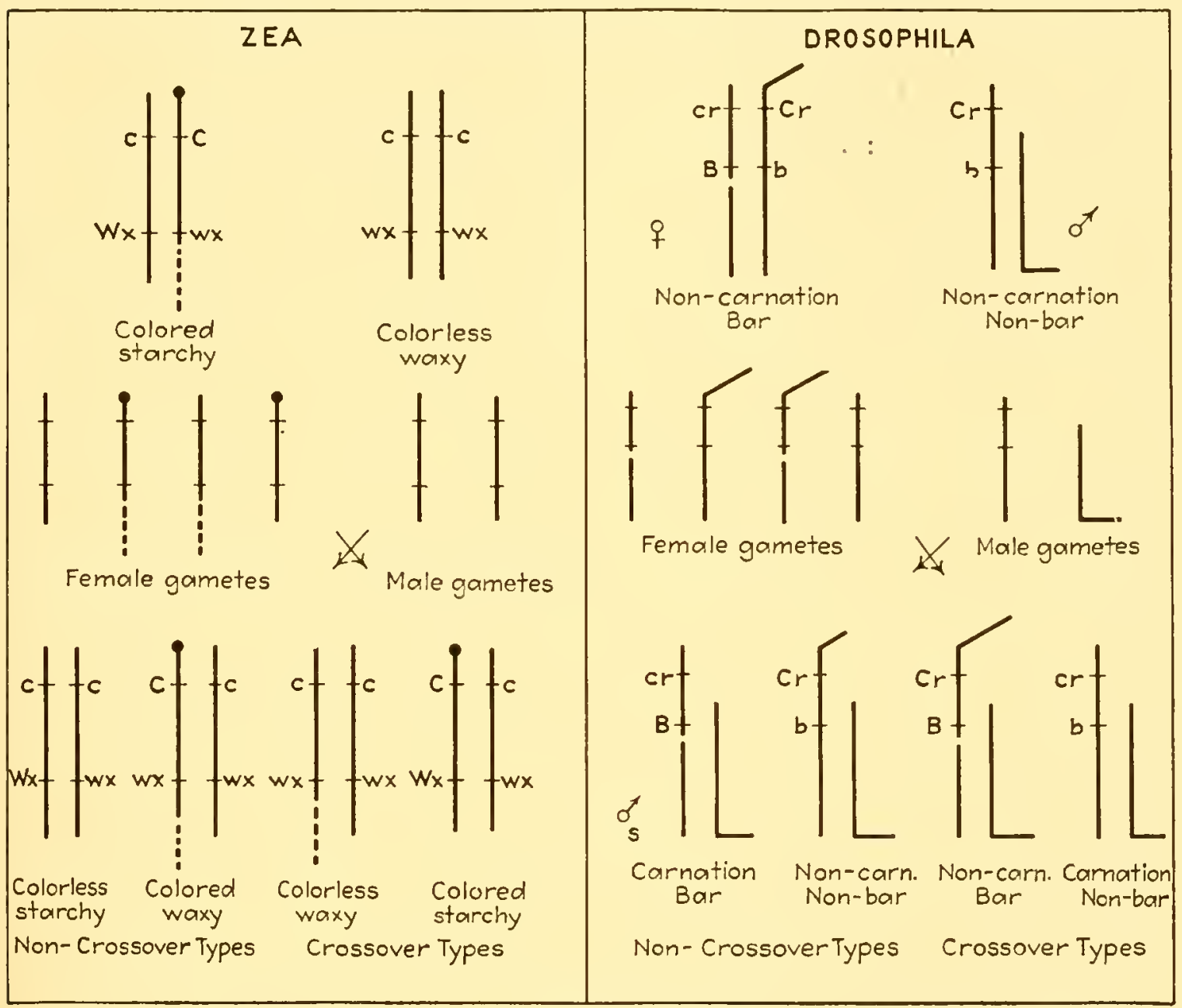

FIG. 174.-Diagram of evidence for the view that genetie recombination is due to an exehange of portions of homologous chromosomes. In Drosophila, only the males among the progeny are represented; the females also showed some crossover types. The L-shaped chromosome is the $Y$-chromosome. Further explanation in text. (Based on diagrams of Creighton and McClintock for Zea, and of Stern for Drosophila.)

chromosome pair. One member of the pair had a large knob at one end and a portion of a non-homologous chromosome attached to the other end; it also carried the gene $C$ for colored endosperm and the gene $w x$ for waxy endosperm. The other member of the pair had no knob and no translocated piece, and it carried the gene $c$ for colorless endosperm and the gene $W x$ for starchy endosperm. The approximate positions of these genes in the chromosomes were known. This plant was crossed with one having two knobless, normal chromosomes carrying $c$ wx..$^{10}$

${ }^{10} c w x, c W x$ in the original experiment; $c w x, c w x$ in later crosses. 
Among the offspring were four types. In the first type the maternal member of the chromosome pair in question had neither knob nor translocated piece, and the characters of the plants showed that it carried $c$ and $W x$. In the second type the chromosome had both knob and translocated piece, and it carried $C$ and $w x$. The two remaining types had morphologically altered maternal chromosomes, one of them showing the piece but no knob while the other had a knob but no piece. Hence the female parent had furnished gametes of four kinds with respect to the chromosome in question, crossing-over having resulted in the two altered chromosomes. The characters of the plants showed that the genes concerned as well as portions of the chromosomes had been exchanged in the crossover.

Almost at the same time there appeared Stern's (1931a) report of an essentially similar situation in Drosophila (Fig. 174). In this case two special strains were used, one having a portion of the $Y$-chromosome attached to an $X$-chromosome, and the other having one of its $X$-chromosomes broken in two. By suitable crossing there was produced a female with one $X$-chromosome fragmented and the other carrying the attached piece of a $Y$; hence this chromosome pair was heteromorphic at two points: the attachment and the break. The fragmented $\dot{X}$ carried the genes $\mathrm{cr}$ (for recessive carnation eye-color) and $B$ (for dominant bar eye), while the other $X$ carried their respective normal allelomorphs. When this fly was crossed to a normal male, it was observed that among the offspring some of the male individuals (which receive their $X$-chromosome from their mother; see p. 378) carried unaltered $X$-chromosomes and the maternal factor combinations, whereas others indicated in their chromosome morphology and genetic behavior that an exchange somewhere between $\mathrm{Cr} \mathrm{cr}$ and $\mathrm{B} b$ had taken place in the maternal $X$-chromosome pair. The females also gave similar cvidence that an exchange of corresponding regions by homologous chromosomes is associated with a recombination of linked characters.

Among other observations suggesting an exchange of portions of homologous chromosomes may be mentioned the following. In the diplonema tetrads of Stethophyma grossum, Janssens (1924) described breaks or thin regions in two of the four chromatids at the chiasma, and in some cases two of the four chromatids in anaphase $I$ appeared to be composite in structure. Darlington (1930c) reported configurations in the multivalent chromosomes of polyploid Hyacinthus which, on the assumption that more than two homologous chromosomes never associate closely enough for chromatid exchange in one small region, he could only interpret on the basis of chromatid exchange. Further evidence was seen in the peculiar "figure-of- 8 " configurations and the interlocking bivalent rings in Enothera (Darlington, 1931e; Catcheside, 1931b). In 
none of these cases was the distribution of genes followed as in Drosophila and $Z e a$.

The behavior of knobbed chromosomes in Zea has served further to show directly that in a diploid meiocyte an exchange may occur between only two of the four chromatids in a given level (Creighton and McClintock, 1932). In the plant in question there was a chromosome having

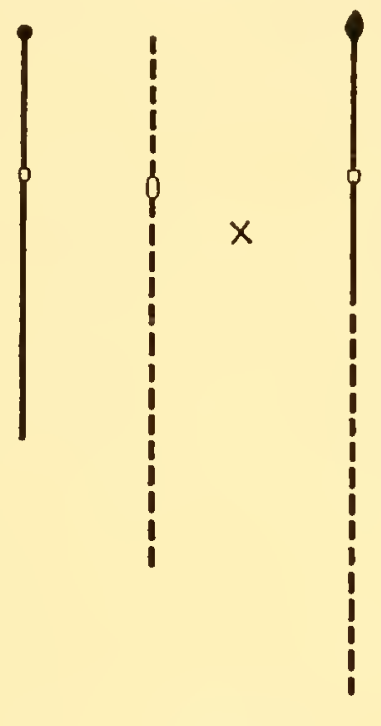

Gametes

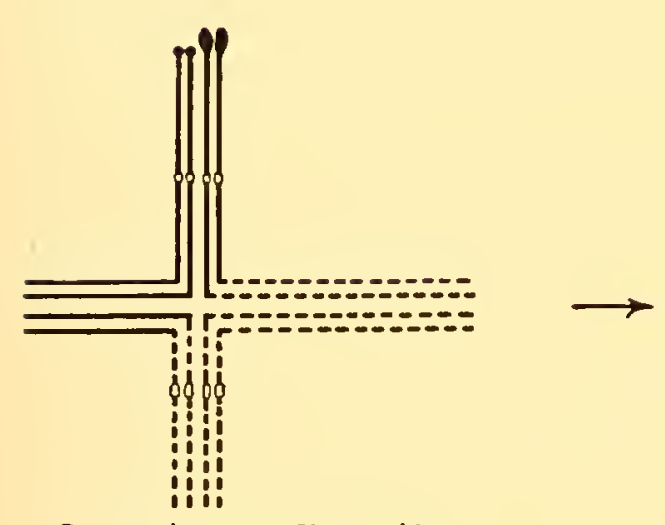

Synaptic Configuration
Zygote

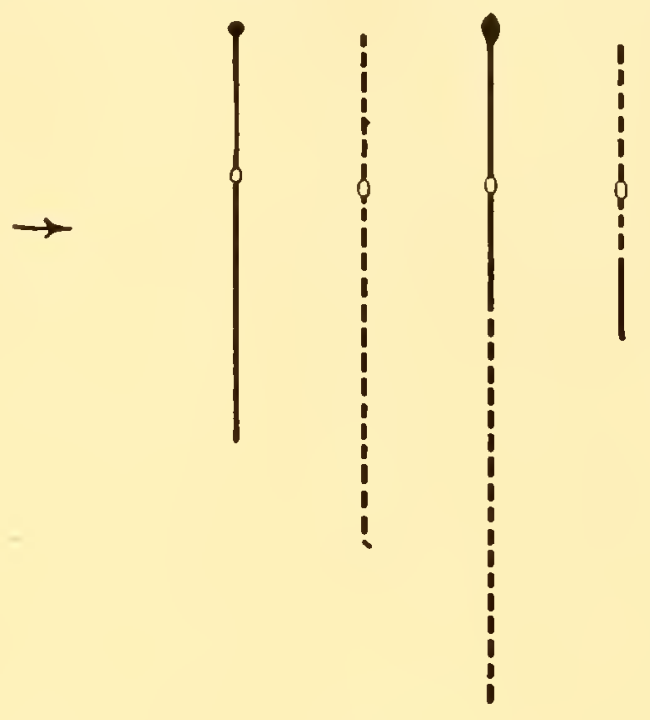

\section{.}


there has been at hand genetic evidence that crossing-over usually, if not always, involves only two of the four chromatids at a given locus, and it has also been shown that the same two chromatids are not always involved in all the crossovers in one tetrad. ${ }^{12}$ Recent data from Drosophila indicate further that crossing-over occurs only rarely, if at all, between sister chromatids. ${ }^{13}$

Mendelism in Monoploid Plants.-The principles enunciated by Mendel were based on phenomena observed in organisms with diploid

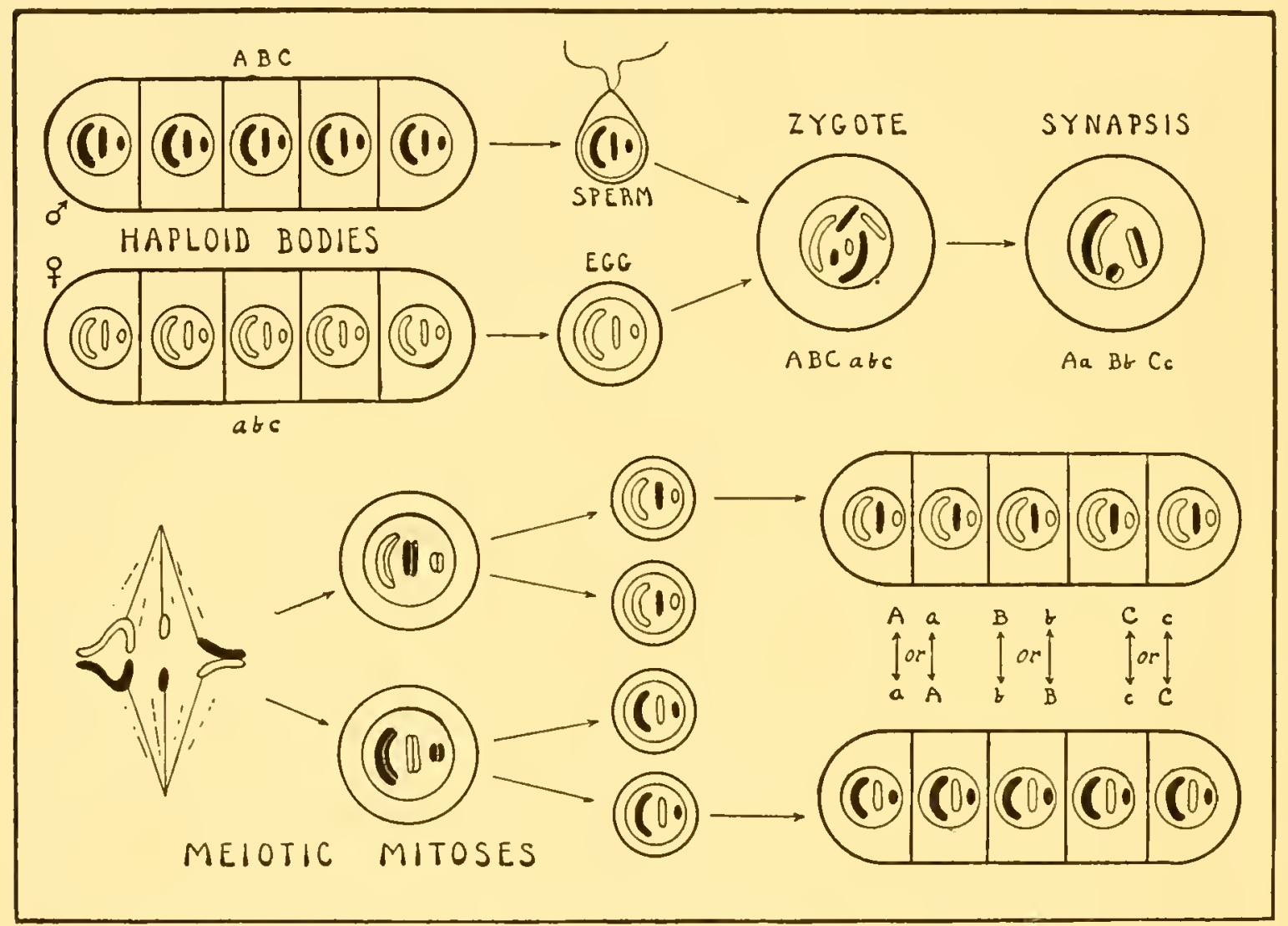

Fig. 176.-Diagram illustrating chromosome cycle of plant with monoploid (haploid) body. Owing to independent orientation of the several chromosome pairs in the meiotic mitoses, a given monoploid individual may have any one of eight possible chromosome assortments when there are three pairs of chromosomes.

somatic nuclei and monoploid gametes. Since that time most genetic investigations have been carried on with such organisms. It will now be of interest to see how the same principles hold in the case of organisms having the monoploid number of chromosomes in their somatic nuclei. Genetic work of this nature has been done with haplonts (in which only the zygote is diploid; p. 278) and with the gametophytes of diplohaplonts, especially among bryophytes.

The chromosome cycle of a haplont (e.g., certain green algæ) is shown in Fig. 176. The monoploid plants produce monoploid gametes which

12 Bridges (1916), Bridges and Anderson (1925), Anderson (1925c, 1929), L. V. Morgan (1925), Rhoades (1932), Redfield (1930), Anderson and Rhoades (1931).

${ }^{13}$ Weinstein (1932), L. V. Morgan (1933). 
fuse to form a diploid zygote (oöspore or zygospore). When the zygote germinates, the chromosome number is at once reduced, the monoploid products of meiosis (here represented as spores) giving rise to the individuals of the next generation. The cycle is the same in the bryophytes, except for the important fact that the zygote germinates to form a diploid sporophyte in which meiosis is delayed until sporogenesis (Fig. 217). In the body of a haplont or of a moss gametophyte, therefore, only one member of each chromosome pair is present in each nucleus rather than both members as in the diploid bodies of animals and plant sporophytes.

The expectations based on the chromosome theory of Mendelism are as follows. Two contrasting "allelomorphic" characters of the monoploid individuals introduced into a zygote by crossing should segregate at once in the next generation in the ratio of $1: 1$. In these individuals, therefore, there should be no "dominance," since only one factor of a given pair is present. Every factor should have an opportunity to produce its full effect upon the characters. With respect to a given pair of characters, diploid individuals show one genotype in $F_{1}$ (after pure types are crossed) and three in $\mathrm{F}_{2}$, whereas monoploid ones show two in every generation.

In the moss genera Funaria and Physcomitrium, von Wettstein ${ }^{14}$ studied the progeny of crosses between types differing in various pairs of contrasting gametophytic characters and found that such characters segregate 1:1 in the next generation. For example, when two types differing in leaf form were crossed, the spores from the hybrid capsule produced gametophytes of the two parental types in equal numbers. The sporophytic characters (form and color of capsule) were inherited as would be expected in diploid individuals. Von Wettstein was able to show that the segregation of the factors for the gametophytic characters takes place in each sporocyte: two spores of a quartet produce one type of gametophyte and two produce the other. This clearly indicates a segregation in one of the meiotic divisions.

In the liverwort, Sphcerocarpos Donnellii, C. E. Allen ${ }^{15}$ has shown that the gametophytic character "polycladous" segregates from its allelomorph "typical" nearly $1: 1$ in the first gametophyte generation after a cross, and that two spores of a quartet produce one type and two the other. The same holds for the character "tufted." Both characters are independent of sex, which had formerly been shown to be inherited in the same manner (p. 382). Hence the spores produced by a single hybrid sporophyte give rise, for example, to tufted males, tufted females, typical males, and typical females. Ordinarily the spores of a single quartet are of only two of these types, but Allen found a few exceptional cases in

${ }^{14}$ Von Wettstein $(1921 b, 1923,1924 a b)$. Literature on heredity in bryophytes is reviewed in 1924c. Later papers $(1926,1927,1928,1930)$ deal largely with heredity in polyploid mosses and the hereditary rôle of the cytoplasm.

${ }_{15}$ C. E. Allen $(1924 a b, 1925 a, 1926 a b c, 1930 a b)$. For a general account, see $1930 a$. 
which all four were present. Whether this should be attributed to an occasional disjunction of two chromosome pairs in different meiotic mitoses or to some other cause is not yet clear.

In Spirogyra, a typical haplont, three of the four nuclei formed at meiosis in the zygospore degenerate. Hybrids between species are of the two parental types in equal numbers, so far as any one pair of characters is concerned. When two pairs of characters are considered, the hybrids are of four types; when three are considered, they are of eight types. These results are in accord with expectations based on the independent assortment of factors and the equal chance of survival of the four meiotic nuclei (Transeau, 1919).

The pollen grains of angiosperms, since they normally belong to the reduced phase of the life cycle, may be expected to show a 1:1 segregation of parental pollen characters in each generation if such characters are due primarily to the constitution of the monoploid nuclei in the grains. Such a segregation occurs in a number of known instances. ${ }^{16}$ Contrasted with these are certain other cases ${ }^{17}$ in which pollen characters (chiefly color and shape) show dominance and behave as sporophytic characters, which is not surprising in view of the fact that the grains are developed in the midst of diploid sporophytic cells (tapetum, etc.) and are known to make use of materials derived from them. Color, for example, may be due to substances produced in the sporophytic cells and deposited in all the spore coats irrespective of the genetic constitution of the protoplasts they enclose. Pollen may therefore exhibit "sporophytic" as well as "gametophytic" characters, owing to the intimate association of the two phases of the life cycle.

The above results of researches on monoploid plants afford definite confirmation of the chromosome theory of Mendelian heredity as originally developed through studies on diploid organisms. Very striking additional confirmation will be brought out in later chapters dealing with unusual types of chromosome behavior.

The Gene Theory.-It is only for the sake of simplicity that Mendelian characters have been treated as if they might be due to single genes, for this is far from what is actually assumed on the gene theory. It is a well-established fact that the development of a given character involves the complemental action of two or more pairs of genes; indeed, it is doubtless due to the interaction of a very large number of genes, known and unknown. When a single pair of genes is singled out for study in a cross, it is done with the understanding that other genes affecting the results are similar in the organisms used; in other words, the one pair is

${ }^{16}$ Enothera (Renner, 1919ab), Oryza (Parnell, 1921), Zea (Demeree, 1924; Brink and MacGillivray, 1924; Longley, 1925).

${ }^{17}$ Epilobium, Papaver, and Geranium (Correns, 1900h, 1902), Lathyrus (Bateson, Saunders, and Punnett, 1905), Nicotiana (Thomas, 1913). See Correns (1928b). 
treated as the differential element in a large system of factors. "The factorial hypothesis does not assume that any one factor produces a character directly and by itself, but only that a character in one organism may differ from a character in another because the sets of factors in the two organisms have one difference" (Morgan et al., 1915).

Bridges (1923) has described the supposed activity of genes and the principle of "genic balance" as follows:

... the genes of the entire complement act together, since all are liberating their chemical products into the common cytoplasm. But since the products of the different genes are different, they take effect in different ways upon the developing organism. Thus some of the genes will have much effect upon one character but little effect upon another. Each character will be determined by all the genes, but in each case most of the effect will be produced by a much more limited number. Each particular character shows a grade of development corresponding to the number and effectiveness of the genes that are producing it. In the case of many of the characters there are genes tending to make the character less pronounced, and the grade realized is that corresponding to the equilibrium between these plus and minus modifiers. The directions of modification for each character are in general quite diverse, and it is somewhat for the sake of simplicity that they are lumped together as plus and minus modifiers.

It is thus thought that the genes affect the characters mainly by influencing the course of metabolism and the processes of differentiation during ontogeny. Very little is known on this subject. It is thought that a few hundred genes can produce a much larger number of distinct effects upon characters because they act in different combinations and probably in different ways at different stages of development. Their actions are in part responses to changing conditions in the cytoplasm and external environment, i.e., in the other portions of the system of which they are a part. Still less is known about the functions of genes in the mature organism, although a beginning has been made on this problem.

How the influences of genes are transmitted to the cell or tissue in which they lie is at present wholly a matter of conjecture. It has been held that certain products of genic activity are liberated into the cytoplasm at the time of mitosis; this is suggested by the time of appearance of differentiated regions in certain animal eggs (p. 419). It has also been thought that nucleolar matter so liberated may possibly carry such products (p. 120). In this connection it should be remembered that many tissues undergo a considerable amount of growth and differentiation and perform their characteristic functions long after all the nuclear and cell-divisions are completed, which strongly suggests that genes exert influences upon development and behavior while the nucleus is in the active metabolic stage. 
It is usually assumed as a workable hypothesis that a gene may exist in two or more allelomorphic states. ${ }^{18}$ It is only when at least two states are available that the function of the gene can be conveniently studied in crosses. Hence the genes used in genetic studies and appearing in chromosome maps are "mutant genes," i.e., genes which in some cases have become "mutated," or altered from a former condition, so that the effects of two allelomorphic states can be compared. A large share of the responsibility for the production of a given character may lie with uniform and unknown genes.

The term mutation may be used in a general sense for all types of alteration in the genotype aside from that accomplished by normal meiosis and syngamy, the types being classified on the basis of the amount of the substance involved (gene; group of genes or section of chromosome; chromosome; chromosome set) and the nature of the process causing the alteration (Bridges, 1923). There is a tendency among some geneticists, however, to restrict the term to gene mutations. The hypothesis that a comparatively stable gene may under certain occasional circumstances (perhaps as it reproduces) undergo a qualitative change with an effect upon one or more characters underlies much of the genetic investigation now in progress. It is supposed that such an alteration may involve a single locus or gene ("point mutation") or a group of linked genes occupying a certain region of a chromosome ("regional mutation," or "complex mutation"); but it is not clear in many cases whether the change is strictly a qualitative alteration in the gene, and therefore a true gene mutation, or a mere derangement of a very small region of the chromosome.

There is genetic evidence to show that gene mutations may occur at any place in the organism or stage of the life cycle, but they are most frequent in the germ cells at or near the meiotic period. ${ }^{19}$ Ordinarily, therefore, only a few of the many reproductive cells transmit the altered gene. When, as seems to be true of the majority of known cases, the mutation is from a dominant to a recessive condition, the effect on the characters of the diploid organism is not fully manifested until two such recessive allelomorphic genes are brought together. Mutations occurring in body cells are known as "somatic mutations." Two of the most conspicuous results of somatic mutation in plants are bud sports and chimeras. If the mutation occurs in the meristematic tissue of a very young bud, the product of the bud-branch, flower, or flower cluster

${ }_{18}$ When more than two are known, they are ealled "multiple allelomorphs." In Phytodecta, for example, the color of the beetle is affected by such a series of four allelomorphs: $N, R, A, L$. Each of these is dominant to any one on its right in the order stated (Zulueta, 1925).

${ }_{19}$ Emerson (1914, 1917, 1922), Eyster (1924), Bridges (1919), Muller (1920), Blakeslee (1921), Anderson and Eyster (1928), MacArthur (1928), Gowen (1929), Demerec $(1929 d)$. 
- may have a new genotypic constitution and exhibit an appearance often strikingly different from that of the other branches or flowers of the plant; hence the term "bud sport." Such a mutation may result in a chimera, in which a distinct portion of the mature structure, commonly a sharply defined sector, differs constitutionally and in appearance from the other portions. Analogous changes occur in animals. Such mutational changes are inherited only if they take place in the lineage of the reproductive cells.

Many "mutations" in both somatic and germ cells are known to be due to relatively gross alterations in the chromosome complement. On the other hand, the nature of the alteration in a true gene mutation is unknown, since the nature of the gene itself is almost wholly a matter of conjecture. The literature of genetics contains many interesting speculations on this subject, various comparisons with molecules, radicals, colloidal aggregates, and enzymes being suggested. Whatever their nature, most genes appear to have a high degree of stability and are not easily influenced. So far almost nothing is known about the causes of natural gene mutation. Many environmental factors appear to have little or no effect upon the process. Its frequency, which in nature is very low, can be markedly increased by X-rays and radium and possibly also by certain other agencies, such as abnormal temperature. ${ }^{20}$ Often the mutations so obtained are qualitatively the same as those occurring in non-treated material. In certain cases it is apparent that the alteration is a structural deficiency rather than an actual change of the gene, which raises an interesting question regarding the real relation of minute deficiencies to what have been regarded as mutations in the gene itself. Whatever may be the ultimate nature of the change whose effects are observed, it is probable that many variations which constitute initial steps in species alteration and evolution originate in this manner. ${ }^{21}$

Assuming, as many do, that genes are discrete material particles, tentative calculations place them well below the limit of visibility with the ordinary microscope and suggest that they are of the same order of magnitude as certain large protein molecules. ${ }^{22}$ No worker should claim, however, that any accurate determination of their size is at present

${ }^{20} \mathrm{X}$-rays: Muller (1927, 1928ab, 1929c, 1930ab), Weinstein (192S), Whiting (1928), Stadler (192Sabc, 1929, 1936abc, 1931b, 1932), Hanson (1928), Patterson (192S, 1929), Gowen (1929b), Goodspeed and Olson (1928ab), Goodspeed (1930abc), Oliver (1929), Patterson and Muller (1930), Whiting (1932b) and Tim oféeff-Ressovsky (1930). Earth radiation: Olson and Lewis (192S) and Hanson and Heys (1929). Radium: Gager and Blakeslee (1927), Stadler (1928b), Goodspeed (1929), Hanson and Heys (1928), Hanson and Winkleman (1929), and Buchholz and Blakeslee (1930b). Temperature: Muller and Altenburg (1919), Muller (1928a), Goldschmidt (1929a), Jollos (1930), Rokizky (1930), and Plough and Ires (1932).

${ }^{21}$ See Conklin (1919-1920), Muller (1922, 1923), Muller and Altenburg (1919), Morgan (1922), Castle (1923), Hagedoorn et al. (1921), and Gates (1915, 1920b).

${ }^{22}$ See Morgan (1922) and Gowen and Gay (1933). 
possible. As pointed out in an earlier chapter (p. 141) the chromonema often exhibits a series of chromomeres arranged in a characteristic pattern, and it is expected by many observers that these bodies may be shown sooner or later to have some definite and significant relation to the genetic units known as genes, as has long been conjectured. Whether or not improved optical methods will bring visual confirmation of the view that the gene is a discrete material unit, it will still remain true that it is a distinct constitutional feature which is definitely localized in the nucleus and which produces certain specific effects during the life of the organism. Hence the geneticist can treat it as a unit, whatever its ultimate physicochemical nature may be. He may thus make effective use of it, no matter how he may regard certain speculative hypotheses ${ }^{23}$ suggesting that genes are ultimate living particles which have somehow aggregated and differentiated to form chromosomes, nuclei, and organisms.

The expression "factorial theory" usually has reference to the conception of Mendelian factors or genes, but it should be obvious that any comprehensive interpretation of organic phenomena in terms of contributory causes must include the action of other factors as well. It is to be strongly emphasized that the system in which the phenomena of heredity and development occur is the organism-environment. There is probably no character which can be attributed to the organism or to the environment alone; these two components of the system cannot be separated in any real sense. What an organism inherits is not a set of characters but a protoplasmic organization giving it the capacity to react in certain ways under certain conditions, the developed characters being the result of the action of the entire system of which the organism and environment are integral parts. The relative differential effects of genetic and nongenetic factors may be expected to vary under appropriately varying circumstances. ${ }^{24}$

Summary.-According to the chromosome theory of heredity, each chromosome carries a linear series of genes, which are units having specific effects upon the course of development and hence upon the morphological and physiological characters of the organism. Characters dependent on genes in the same chromosome tend to be inherited together as a linked group. A gene may be constant in its composition and effects, or it may occasionally mutate and therefore exist in two or more allelomorphic states. In the latter case its rôle may be studied in crosses.

Two sets of chromosomes and hence two lots of genes are brought into association in syngamy. When the resulting diploid organism forms gametes (in most animals) or spores followed later by gametes (in most plants), the homologous chromosomes and therefore the corresponding

${ }^{23}$ See Alexander and Bridges (1928) and Darlington (1932a, Chap. XVI).

${ }^{24}$ For discussions of this point, see Emerson (1924), Jennings (1924, 1930), and Sharp (1925). For a full account of the modern gene theory, see Morgan (1928). 
groups of linked genes are segregated, each gamete (or spore) carrying one chromosome of each homologous pair and hence one linked group of each kind. Investigation of the individuals of the next generations shows that two corresponding gene groups may have exchanged some of their members, this recombination being accomplished by an exchange of portions between homologous chromosomes. It was this phenomenon which first made it possible to determine the relative order of the genes in the linear series in the chromosome.

Because of the distribution of multiplying generations of chromosomes through successive life cycles and the activity of genes carried by them, the characters appear in successive generations according to definite rules first discovered by Mendel, who was not, however, aware of the cytological reasons for the phenomena he observed. Such recombinations of genes and Mendelian characters with occasional mutation are plainly responsible for much of the diversity of type observed among species in nature. It is accordingly inferred that the prevalence and high specialization of that form of reproduction involving gametic union and meiosis, with its random assortment and crossing-over of chromosomes, are evidences of its important evolutionary rôle in producing frequent and diverse recombinations of hereditary elements. Its full value in this respect has not been measured. 


\section{CHAPTER XVIII}

\section{FRAGMENTATION AND TRANSLOCATION}

In the foregoing chapters we have described the chromosomes and their normal behavior through the life cycles of representative organisms, together with the application of such nuclear phenomena to the problems of Mendelian heredity. Several chapters will now be devoted to less common types of chromosome behavior and their genetic consequences. Such phenomena afford valuable natural checks upon current theories. Moreover, it has been found that the frequency of their occurrence can in
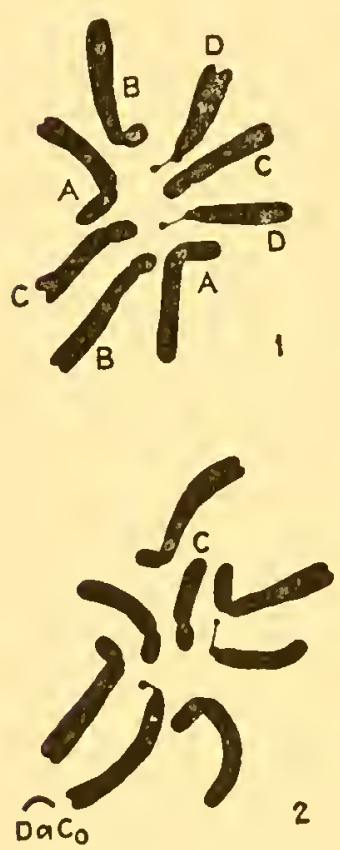

FIG. 177. some instances be markedly increased by artificial means, enabling the investigator to acquire an abundance of material for cytogenetic studies. They also constitute a basis for alterations of character beyond those attributable to gene mutation and Mendelian recombination. ${ }^{1}$

This is a relatively new field in cytogenetics, and data as well as interpretations are multiplying very rapidly. In the following pages will be given examples

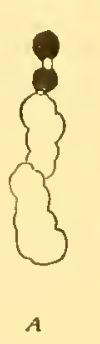

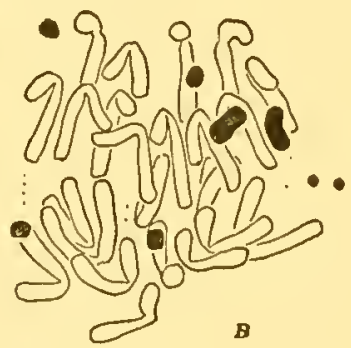

Fig. 178.

FIG. 177.- Translocation indueed by X-rays in Crepis tectorum. 1, normal somatic complement. 2, a portion of a $C$-ehromosome has been transloeated to a $D$-chromosome. (After M. Nawaschin, 1931e.)

Fig. 178.-Chromosome fragments in Tradescantia. A, bivalent fragment associated with normal pair in meiosis. $B$, anaphase of mitosis in microspore, with some of the fragments failing to divide. (Aftcr Darlington, 19296.)

of the better known types of aberrant chromosome behavior, the object being to furnish little more than an introduction to the subject. It should be remembered that opinions in such a field are subject to frequent change, and that further refinements in method will doubtless make necessary a redefinition of the categories into which the observed phenomena now appear to fall.

1 For references to the literature on the effects of various agencies on chromosome behavior and gene activity, see footnotes in Chap. XIII and footnote $20 \mathrm{on} \mathrm{p.} 311$. 
Fragmentation of Chromosomes.-A chromosome may sometimes be "broken" transversely into one or more pieces. In the literature there are numerous reports of small bodies interpreted as such "fragments" of "normal" chromosomes, and in some of these cases the morphology of the normal chromosome set is known well enough to render the interpreta-

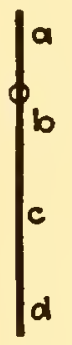

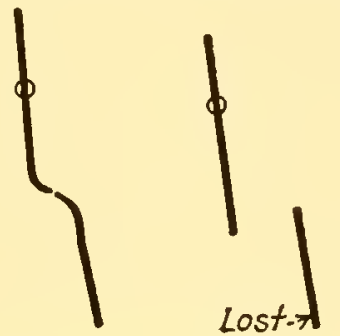

1 Terminal deletion
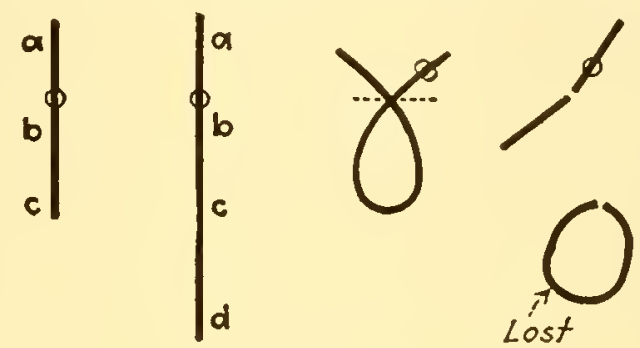

2 Intercalary deletion
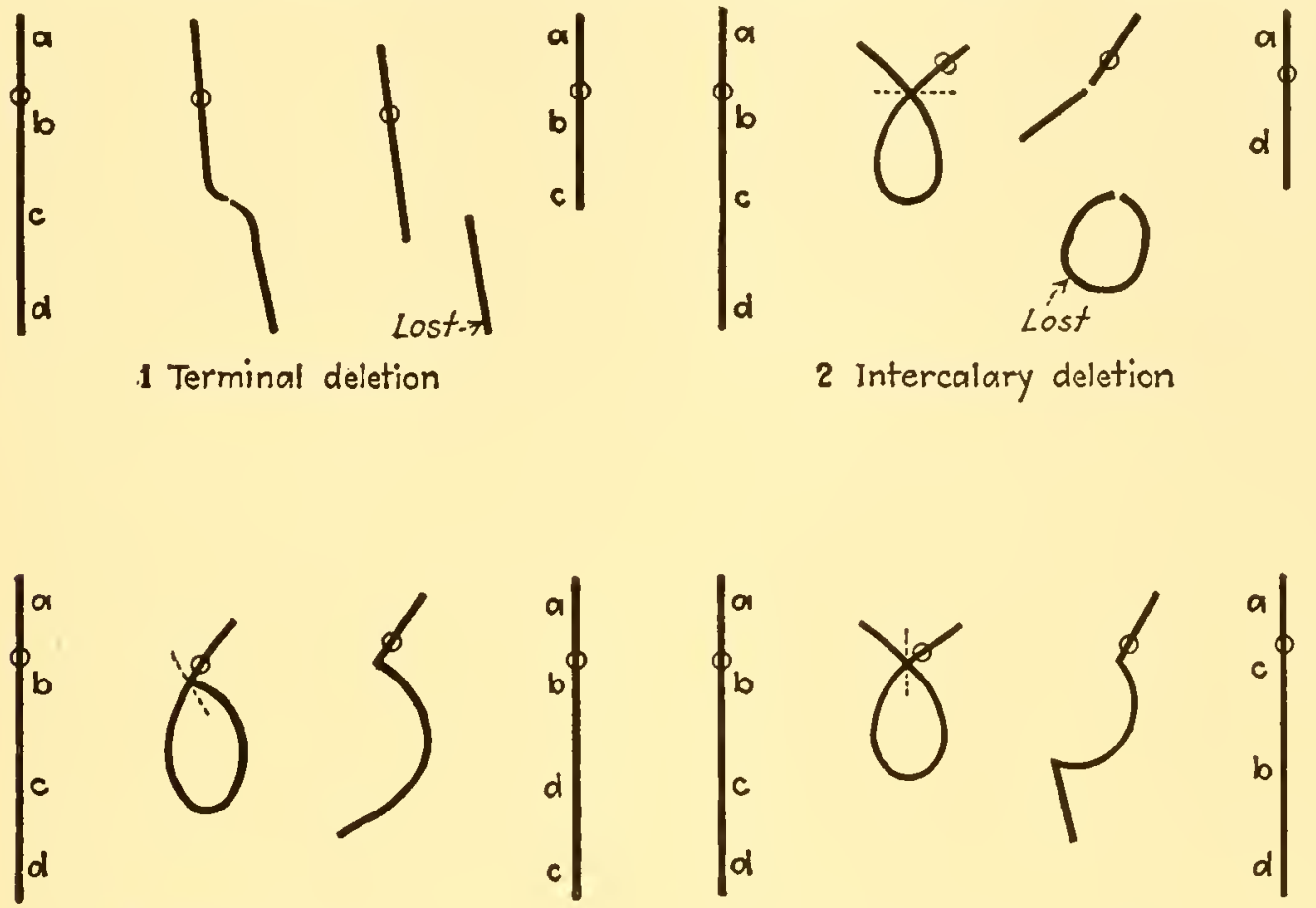

3 Terminal inversion

4 Intercalary inversion
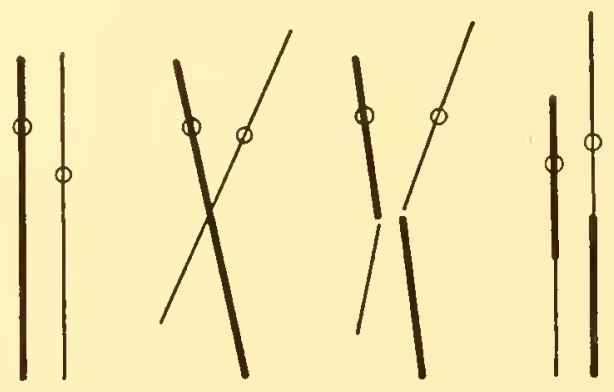

6 Reciprocal translocation

Fig. 179.-Diagram illustrating possible modes of origin of various chromosome abnormalities. Spindle-attachment region indicated. (In part after Serebrovsky, 1929.)

tion valid. The effects of such fragmentation on the appearance of the chromosome complement are various. Sometimes the chromosome number is apparently raised by one, since both pieces of the broken chromosome are present (Fig. 198, F). Or, one of the pieces may be lost (deleted) or inverted, so that the complement is still normal with regard to number but abnormal in the morphology of one member. Again, a fragment may be attached to another chromosome (translocation), so that the number remains normal while the morphology of two members is 
altered (Fig. 177). Finally, one or more fragments additional to a normal complement may be present after a number of generations (Fig. 178).

Our knowledge of the actual mechanism of fragmentation is rather meager. Available evidence, including the fact that portions of chromosomes are much more often translocated or inverted than left as free fragments, suggests that contacts or entanglements between chromosomal threads are in part responsible (Fig. 179). It is not uncommon to observe prophasic threads interlocked and stretched as if a rupture or exchange were soon to follow at the point of contact. The frequency of translocation and related alterations can be notably increased by the use of X-rays, even when the nuclei treated are known to be in the metabolic condition. For example, kernels of barley and maize, after being irradiated when in the dormant state, give rise to individuals showing a relatively high
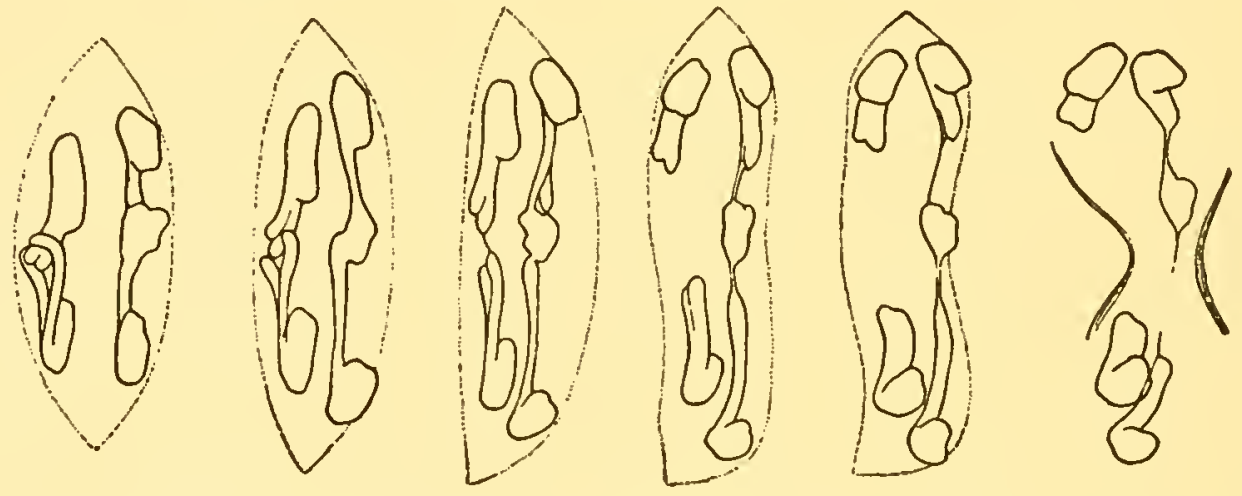

Fig. 180.-Breaking of chromosome after failure of distal ends to disjoin in living spermatocyte of Chorthippus. The changes shown occupied 40 minutes. (After Bèlaŕ, 1929a.)

percentage of translocations in the multiplying cells (Stadler, Randolph). Similar results follow the irradiation of mature pollen. In Drosophila such aberrations can be induced by irradiating the mature spermatozoa. ${ }^{2}$ The natural inference from such data is that the chromosomal threads persist in some form throughout the entire nuclear cycle, and that X-radiation increases the frequency of fragmentation and the like by inducing localized alterations in the position, consistency, and tension of these threads. In Zea certain translocations have been shown to be due to the occasional association of non-homologous elements in the meiotic prophase (p. 276).

Another mode of fragmentation is that in which a small portion of one chromosome is torn away by its synaptic mate in the first meiotic anaphase. This has been observed in a living spermatocyte (Fig. 180), and a similar process is strongly suggested by a $Z e a$ sporocyte observed by Randolph; but it is probable that mitotic derangements of this sort play only a minor rôle in the production of alterations in the chromosome complement.

${ }^{2}$ Muller (1927), Muller and Altenburg (1930). 
In certain tissue cultures it appears that the cells are especially susceptible to the disturbing effect of $\mathrm{X}$-rays just before the visible prophasic changes begin. ${ }^{3}$ The results of such derangements are sometimes not evident until the succeeding anaphase, when the lack or abnormal position of spindle-attachment regions, together with other agencies, interferes with the regular movement of the affected chromosomes. Marked chromosomal alterations are obtained in Zea by irradiating the young ear during fertilization or soon afterward, when the embryos are beginning their development. ${ }^{4}$ Of special interest is the induction of aberrations by subjecting the young ears to treatment with heat, since the temperatures used need not be higher than those occasionally occurring under natural conditions in the field.

Behavior of Free Fragments.-In the behavior of fragments much depends upon the spindle-attachment region. Researches on Drosophila and Zea indicate that a fragment not including the attachment region tends to be lost in mitosis unless it is translocated to another chromosome with such a region. For example, when a deletion occurs in Zea as shown in Fig. 179, 2, the rod-shaped fragment with the attachment region survives and the ring is lost; but when the ring includes the attachment region, the ring remains and the rod is eliminated. ${ }^{5}$ One case is known in which the break passed through the elongated attachment region, both ring and rod then possessing functional portions of it. In one strain of Zea a minute chromosome consisting of an attachment region and a single chromomere has been observed.

Ordinarily deletions tend to affect the viability of the cells or organisms concerned, but this is found to vary with the amount and nature of the material eliminated; it also varies at different stages of the life cycle. When viability is not too seriously impaired, it can be shown by genetical tests that the cells actually lack certain genes; in other words, the deletion of a portion of a chromosome has created a genetic deficiency. The first known case of this kind was one in which a normal gene, dominating one for the waltzing character in certain mice, was lost along with a portion of a chromosome (Painter, 1927). Some genetic deficiencies have been interpreted as being due to an inactivation of certain genes rather than to an actual chromosomal deletion.

The method of determining the location of genes in the chromosome by means of induced deletions may be illustrated by a case in $Z e a$. It had been shown previously that the satellited chromosome (number VI) carries a gene $P l$, causing a dominant purple color in the plant. Pollen from homozygous recessive plants $(p l p l)$, and therefore all carrying $p l$, was

${ }^{3}$ Strangeways and Oakley (1923), Strangeways and Hopwood (1926).

${ }^{4}$ Stadler (1928a, 1930b, 1931a), Randolph (1932).

s The peculiar behavior of such ring-shaped ehromosomes has been correlated with a certain type of variegation (McClintock, 1932b). 
placed on the silks of homozygous dominant plants $(P l P l)$. Normally all of the immediate offspring $(P l p l)$ of such a cross are purple, because of the dominant $P l$ derived from the pistillate parent. When, however, the young embryos were $\mathrm{X}$-rayed (20 hours after pollination), some of them developed into non-purple plants. When such non-purple plants were examined cytologically, it was found that one of the satellited chromosomes lacked a portion of its longer arm (Fig. 181, E). From this it could be concluded that the irradiation had somehow caused the loss
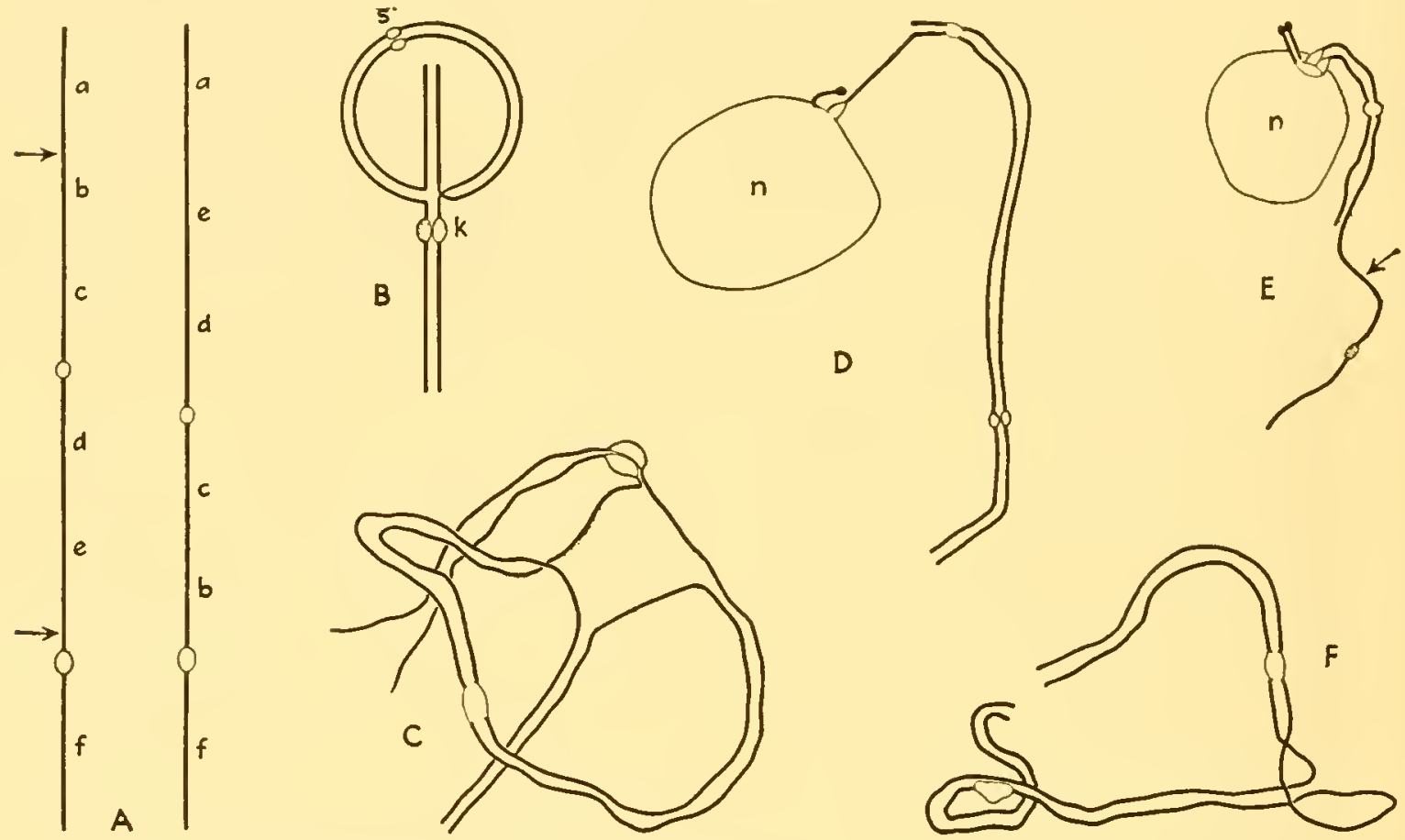

FIG, 181.-Diagrams of inversion and deletion in $Z e a$. A, the region between the arrows in a normal chromosome (left) became inverted (right). Synapsis of homologous regions of an inverted with a normal chromosome gave configuration shown diagrammatically in $B$ and semidiagrammatically in $C$; $f$. Fig. 182, $a$; also Fig. 160 . $D$, normal satellited chromosome synapsed with one lacking most of its shorter arm; $c f$. Fig. $182, b$. $E$, normal satellited chromosome synapsed with one lacking a large portion of its longer arm. Arrow indicates suspected position of gene $P l$. F, synapsed number VII chromosomes, one of which lacks a median portion, leaving the corresponding portion of the other extending as a loop; $c f$. Fig. 182, c. The pair in this plant is heteromorphic for the chromatic knob. $k$, chromatic knob. $n$, nucleolus. $s$, spindle-attachment region. (After McClintock, 1931b.)

of this portion and that the gene $P l$ had been located in it. Loss of the dominant $P l$ left the plants with only the recessive $p l$ derived from the pollen parent; hence the plants were non-purple. Similarly, it has been shown in another such test that the gene $\lg$ (liguleless) probably is near the end of the shorter arm of chromosome II (McClintock, 1931b).

Especially instructive are certain cases in Drosophila reported by Painter and Muller (1929). By the use of X-rays there were obtained several flies in which one chromosome had undergone "intercalary deletion," i.e., the middle region had been lost, leaving the two ends as a single small chromosome (Fig. 179, 2). This small chromosome remnant 
was of three sizes in three flies, and genetic data showed that in each successively larger deletion one more known gene had been eliminated. Moreover, the order of disappearance was in exact accordance with the order previously assigned to these genes in the chromosome map built up from crossover data. This amounts to a direct proof of the essential correctness of the map of this region of the chromosome, so far as the serial order of the genes is concerned.

Another mode of behavior on the part of free fragments is that in which they act regularly in the somatic divisions but manifest irregularities at the time of meiosis, in part because of deficient synaptic reaction. When fragments are present in addition to a fully normal chromosome complement, they may be eliminated altogether or pass to some of the
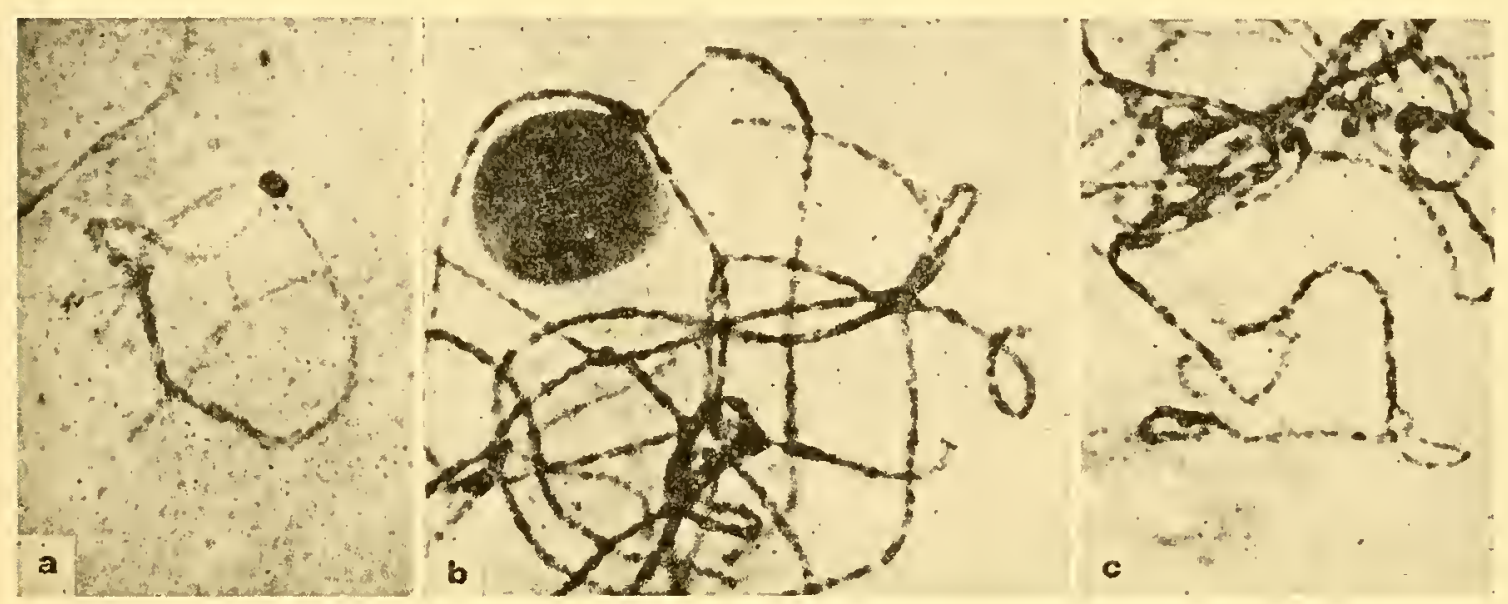

FIG. 182.- Synaptic configurations after inversion and deletion in Zea. a, after inversion of median portion of one member; $c f$. Fig. 181, $A-C$. $b$, after deletion of portion of shorter arm of satellited chromosome; $c f$. Fig. 181, D. c c, after deletion of small median region; cf. Fig. 181, F. (After McClintock, 1931b.)

spores (or gametes) only. In this way gametes with or without extra fragments may arise. When there are several fragments in the sporocyte they may sometimes synapse among themselves, as in certain strains of Tradescantia (Darlington, 1929b). In other cells they seem to be attached to the normal bivalents in various ways. They may be distributed unequally to the spores, or, because of lagging, they may be partly or completely lost, especially when unsynapsed. Not infrequently, however, one or more are transmitted through the gametes to the next generation.

Finally, fragments may sometimes pass regularly through the entire life cycle, behaving as extra independent chromosomes. This may be expected when, for example, there are in the somatic cells two fragments which are able to react properly in equational nuclear division because they have spindle-attachment regions and to undergo synapsis regularly because of their homologies.

The genetic effect of the presence of extra chromosomes or fragments is known as duplication, i.e., certain genes are present in excess. Con- 
sequently the organisms carrying transmissible duplications may be expected to show unusual ratios for some of their Mendelian characters if the additional genes concerned are such as to affect the conditions of dominance. Another general effect is sometimes observed in the size or habit of the plant. In Matthiola, for example, it has been observed that the presence of fragments is correlated with small size, the amount of dwarfing being proportional to the degree of unbalance caused in the chromosome complement by the fragments (Lesley and Frost, 1928). ${ }^{6}$

Inversion.--The reversal in position of a portion of a chromosome is called inversion. The portion inverted may include one end, or it may involve an interstitial region only (Fig. 179, 3, 4). Several such inversions have been detected in the altered linkage relations of the genes without being visible in the chromosomes themselves. In one of the linkage groups of Drosophila, for example, the genetic data for certain genes indicated that a considerable portion of the "right" end of one chromosome carrying them had been reversed in position. They indicated further that when crossing-over occurred in the left end, none took place in the right end, presumably because inverted and non-inverted chromosome sections did not have homologous loci opposite each other. In one case it appeared that the two chromosomes must have arranged themselves so as to allow crossing-over in the right end; here none occurred in the left end. ${ }^{7}$

In $Z e a$ it has been possible to observe the effect of an inversion on the synaptic behavior of the chromosomes. In one case, for example (Figs. 181, $A$ to $C$ and $182, a$ ), a long section of a chromosome was inverted as a result of X-ray treatment. When this modified chromosome met its normal synaptic mate, their homologous elements moved together in spite of the abnormal arrangement in one chromosome and so produced the peculiar configuration shown. Similarly, the inversion of a terminal portion resulted in the formation of a loop at synapsis (Fig. 160). Almost nothing is known about the mechanism of such inversions. It is possible, as Serebrovsky (1929) has suggested, that at some stage the chromosomal threads may undergo bending, fusion, and breakage as shown in Fig. 179. It is probable that some other mechanism accounts for inversions induced in metabolic nuclei by X-radiation.

It will be readily understood that inversions and other aberrations involving portions of chromosomes may often alter the position of the spindle-attachment region. Helwig (1929) believes that in Circotettix

${ }^{6}$ For further aecounts of chromosome fragments, see Lutz (1916) and Hanee (1918a) on Enothera; M. Nawaschin (1926, 1931a) on Crepis; Belling (1925a) on Uvularia; Morgan, Sturtevant, and Bridges (1928) on Drosophila; Goodspeed (1929ab, 1930c) and Goodspeed and Avery (1930) on Nicotiana; and Lesley and Lesley (1929) on Lycopersicum.

${ }^{7}$ Sturtevant (1926a), Sturtevant and Dobzhansky (1931). 
inversion is responsible for a change to the atelomitic from the telomitic position, which is regarded as primitive for Acrididæ.

Translocation.-The transfer of a piece of one chromosome to another, aside from that involved in normal crossing-over, is called translocation (Fig. 179, 5). It seems probable that as a rule the transfer is accomplished at one step and does not represent the attachment of a fragment previously free. Commonly the translocated piece is borne terminally by the receiving member; rarely it extends laterally from some intermediate point (Fig. 67, D). The transfer may be to either a homologous or a nonhomologous member of the complement. It has been known to occur naturally in many instances, but its frequency can be markedly increased by the use of X-rays and heat. The first known case was discovered genetically in non-irradiated Drosophila (Bridges, 1919, 1923). The transfer of a single piece, or "simple translocation," is to be distinguished from the mutual exchange of pieces known as "reciprocal translocation" (Fig. 179,6). The latter type will be dealt with in the following chapter.

Apparently any portion of one chromosome may be translocated to any other chromosome. Because of the rôle of the spindle-attachment region, many translocations must result in mitotic derangements serious enough to prevent development. Hence in translocations induced by $\mathrm{X}$-rays it is commonly the "distal" portion without the attachment region which appears to have been transferred, the "proximal" portion with the attachment region remaining as an altered independent chromosome. ${ }^{8}$

Among the most favorable plant species for the study of translocations are Crepis capillaris and C. tectorum since they normally have respectively but three and four pairs of easily distinguishable chromosomes. The results of many modifications are thus readily detected in the metaphase ${ }^{9}$ (Figs. 69, 198). For the investigation of alterations involving only very minute portions of the chromosomes, reliance must be placed on such forms as Zea Mays with larger chromosomes exhibiting individually their characteristic aspects with great clearness in the meiotic prophase.

Among simple translocations involving only homologous chromosomes the first to be worked out both cytologically and genetically was that in which a portion of the $Y$-chromosome was found to be attached to the $X$-chromosome in Drosophila (Stern, 1926b, 1927a). Since that time a number of other examples involving various pairs have been discovered. The translocations can often be detected in the altered length of the chromosomes concerned and, when their morphology is sufficiently well known, in their altered form as well (Fig. 183).

${ }^{8}$ E.g., Dobzhansky (1931a) on Drosophila, M. Nawaschin (1931c) and Lewitsky and Araratian (1931) on Crepis.

${ }^{9}$ M. Nawasehin (1926, 1930, 1931ac), Lewitsky and Araratian (1931). 
Among simple translocations involving non-homologous chromosomes the first case known both cytologically and genetically was one in which a portion of the $X$-chromosome was transferred to one of the long chromosomes (III) in Drosophila. ${ }^{10}$ As an example of this general class of translocations may be taken one in which the "right" end of chromosome III was transferred to chromosome II (Fig. 184). By crossing flies having this translocation with normal ones, "hyperdiploid" flies were obtained with a normal complement plus the translocated piece. Studies

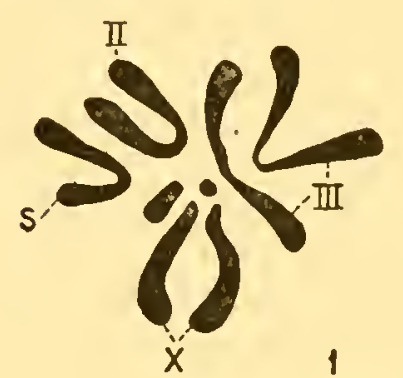

1

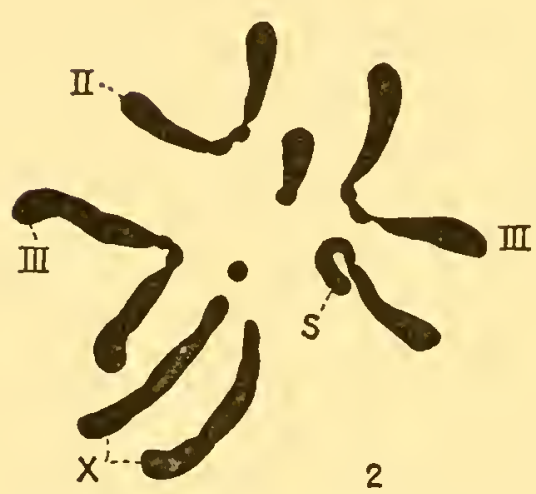

FIg. 183.-Translocation of portion of chromosome II to chromosome IV in Drosophila. Shortened chromosome II marked $S$. 1, metaphase in oögonium; 2, metaphase in neurocyte. (After Dobzhansky, 1931b.) microscope directly, as well as genetically in altered linkage relations. Thus in one case it has been shown that a translocation which at first appeared to be simple was in reality reciprocal, one of the exchanged pieces carrying only a single characteristic chromomere (or at most two) and apparently being too small to complete the synaptic configuration ordinarily distinguishing reciprocal translocations (Burnham, 1932). This naturally' raises a question regarding other supposed simple translocations.

Certain translocations throw an interesting light upon the problem of the forces concerned in meiotic disjunction. In Drosophila it has been reported that a pair of chromosomes, one of which carried a translocated piece, did not assort at random with the pair from which the piece was

${ }^{10}$ Muller (1926). See Painter and Muller (1929b). 
derived (Muller, 1930c). This indicates that in such cases the disjunctive force may not be limited to the spindle-attachment region of the chromosome, homologous distal portions tending to pass toward opposite poles even when one of these portions has been translocated (Blakeslee, Darlington, Muller). Many more observations must be made before safe generalizations can be made regarding these matters.

Bearing on the Chromosome Map.-In some of the foregoing examples of translocation the abnormalities were detected first in altered genetic behavior and then confirmed cytologically, while in others they were first seen cytologically and then confirmed genetically. This is a striking corroboration of the cytogenetic theory that certain genes are definitely

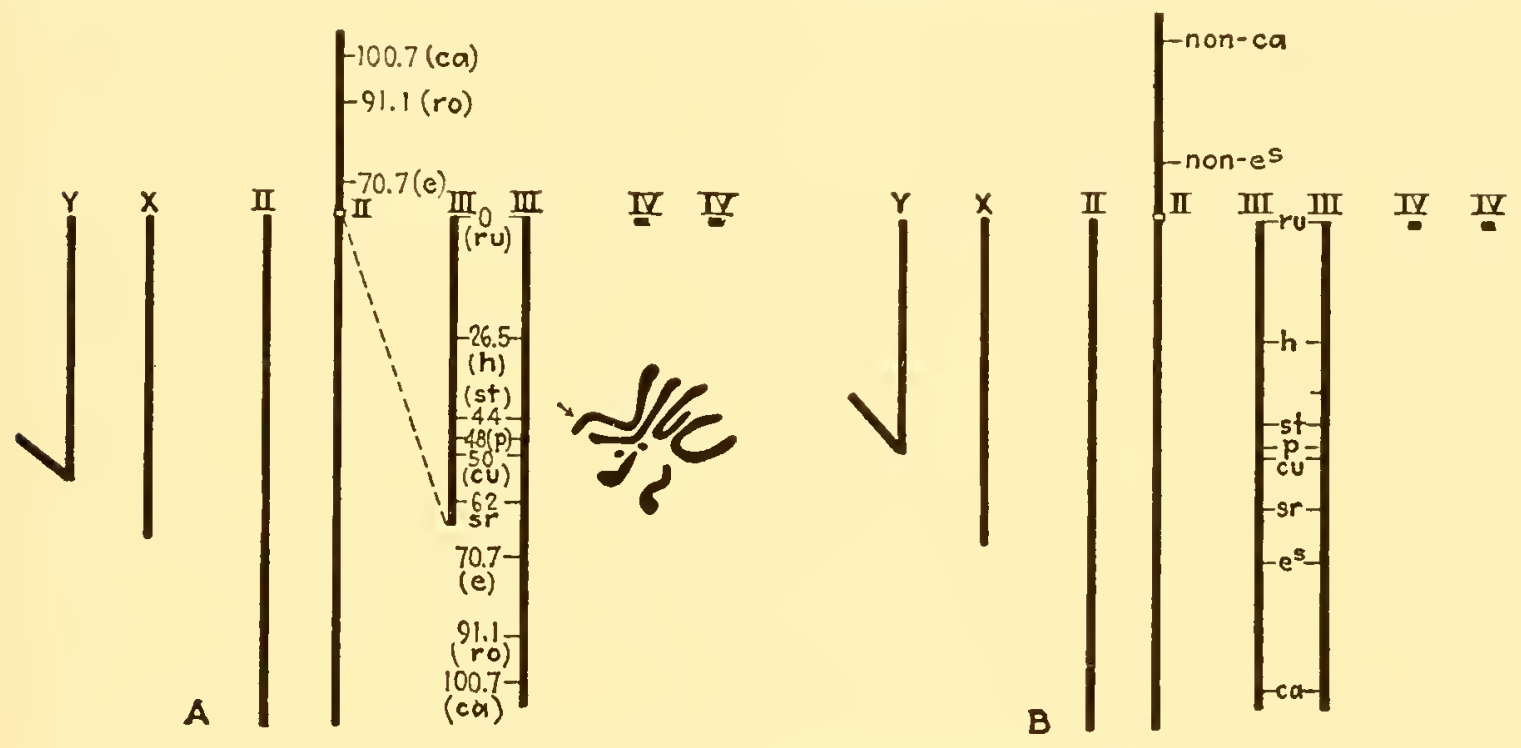

Fig. 184.-Chromosome aberrations induced by X-rays in Drosophila. A, a "III to II" translocation. In the drawing of the chromosome complement at the right the translocated piece (indicated by arrow) came from the V-shaped chromosome at upper right. $B$, hyperdiploidy produced by crossing a fly having the translocation with a normal fly. (After Painter and Nuller, 1929.)

located in certain chromosomes. In each case where the data were adequate the genetic results of translocation and inversion showed that the genes must have been arranged in the order previously assigned to them in the "genetic map" of the chromosome on the basis of crossover values. On the other hand, they showed that these genes did not necessarily have the spacing shown in the map. For example, the translocated piece in Fig. 184, A was actually smaller than would be expected from the large portion of the genetic map altered. This and other cases in Drosophila have shown that the genes in the middle portion of the "genetic map" of chromosomes II and III are represented closer together than they are in the "cytological map" of the actual metaphase chromosome (Fig. 185). The reason for this seems to lie in the fact that in these chromosomes crossing-over is less frequent in the middle region near the spindle-attachment point than it is toward the ends. The genetic map was originally built up on the hypothesis that crossing- 
over is of about equal frequency in all regions; hence the genes in the middle region were assigned to loci too close together. In a similar manner it has been shown that several genes shown very near the "right" end of chromosome $I$ in the genetic map must lie much farther to the
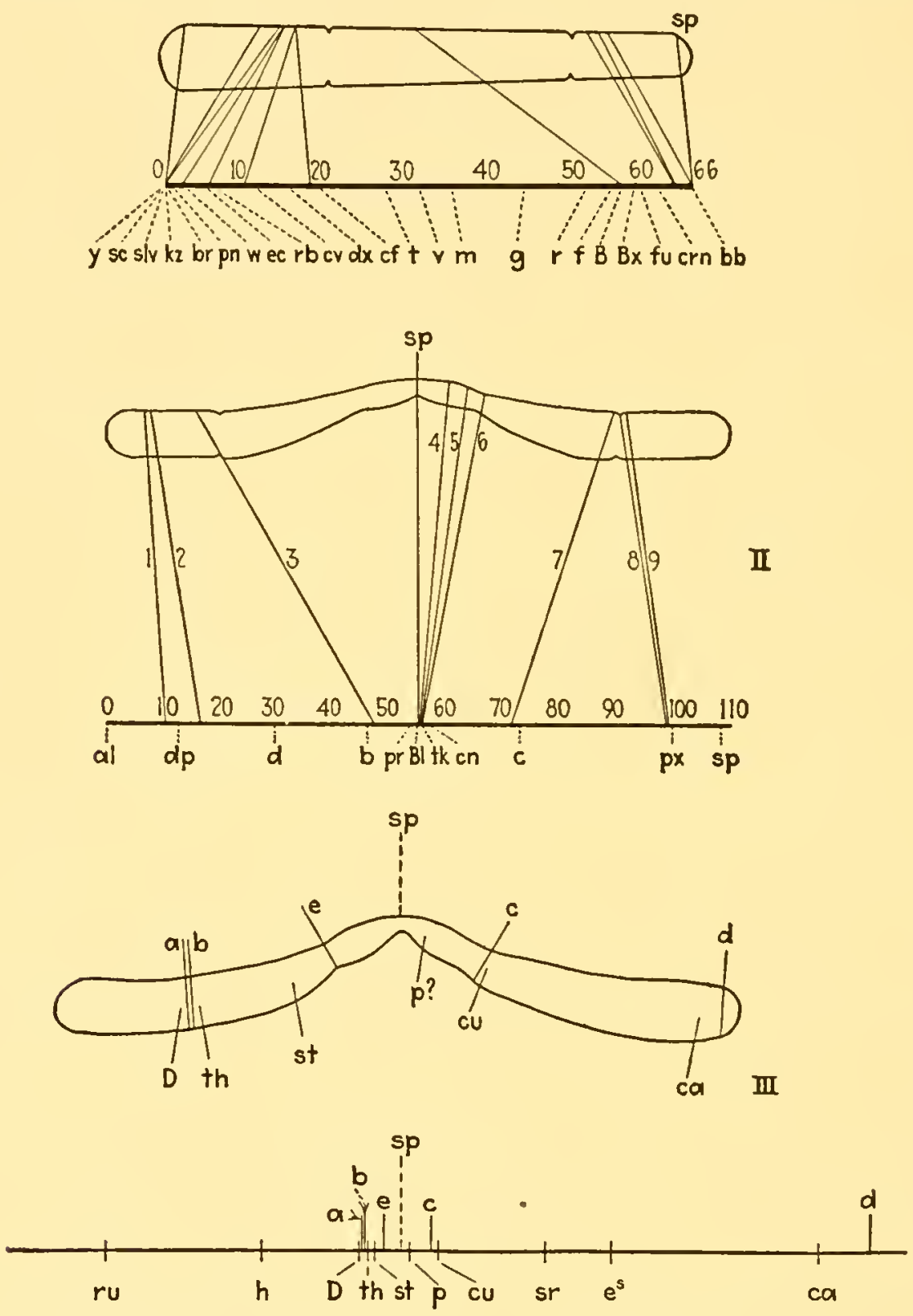

FIG. 185.-Comparison of "genetical maps" of chromosomes I, II, and III of Drosophila melanogaster with "cytological maps" of these chromosomes in metaphase as indicated by translocation data. $s p$, spindle-attachment region. Cf. Fig. 173. (After Dobzhansky, $1932 a, 1930 d, 1929 a$.)

"left," the right end with the spindle-attachment point being for the most part devoid of known mutant genes. ${ }^{11}$ The older genetic maps still serve as diagrams of the order of the genes and their crossover values, but they may not show accurately the true spacing. It is to be remem-

${ }^{11}$ For chromosomes II and III, see especially Muller and Painter (1929) and Dobzhansky (1929ab, 1930ad, 1931b). For chromosome I (X-chromosome), see Painter (1931) and Dobzhansky (1932a). 
bered that the metaphase chromosomes studied in making these cytological maps have chromonemata within them, so that a map of a chromonema might show a still different spacing. For chromosomes appearing clearly in the prophase as long threads with characteristic chromomere patterns it should be possible to build up more accurate maps than can be expected for chromosomes which are "good cytological material" only at the metaphase.

Discussion.-From the facts set forth above may be drawn a number of interesting inferences, some of which are the following. A chromosome contains a group of elements (genes) with special individual functions, and the group may often be enlarged or diminished through translocation without impairing the ability of the chromosome to behave regularly as a whole. Apparently one of the chief requirements is the capacity to react consistently with the spindle mechanism.

The monoploid chromosome set, or genom, is a group of chromosomes differing among themselves in the number and kind of their component elements. Ordinarily all or nearly all of the genetic elements (genes) are probably necessary to the normal activity of the nucleus; in other words, the genom is a harmonious, differentiated system of elements, the majority of which are essential parts of the system. Spores or gametes with extra elements (duplications) are often fully able to function, although they may not compete successfully with those having normal sets. Those lacking certain elements (deficiencies) usually tend to be non-viable unless the deficiency is very small and involves no essential genes. A spore or gamete carrying a set with a translocation, i.e., a set in which all of the elements are present but arranged in a new grouping in the chromosomes, is ordinarily viable. Hence it appears that the set functions best when there are present all of its naturally evolved group of elements and no more, but that the arrangement of the elements within the group is of somewhat secondary importance.

In the diploid chromosome complements of somatic cells also duplications are usually less detrimental than deficiencies. Moreover, a translocation involving only one of the sets (the individual therefore being "heterozygous for the translocation") does not interfere greatly with somatic development, ${ }^{12}$ although there are exceptions to this, notably in Drosophila. ${ }^{13}$ In diploid somatic tissues the important consideration is evidently "balance," i.e., there should be present two elements of each kind and no more. The complement may be balanced when a deficiency in one set is offset by a duplication in the other set. The diploid complement must meet stricter requirements at the time of meiosis when homologous elements undergo synapsis. A complement in which the two sets have the same elements arranged in different ways, or in which

${ }^{12}$ E.g., Crepis (M. Nawaschin, 1931c).

${ }^{13}$ Muller and Altenburg (1930), Dobzhansky (1930a, 1931a). 
extra elements are present, forms abnormal synaptic configurations. These often lead to marked irregularities in chromosome distribution in the meiotic mitoses and hence to sterility among the spores or gametes. Thus a complement ordinarily requires a certain structural "symmetry" as well as numerical "balance" if the organism is to retain its full fertility.

Since it is known that translocations take place from time to time under natural conditions, and also that some of these, when homozygous, do not impair the developmental and reproductive capacities of the organism, one of the reasons why related organisms have chromosome sets differing in minor details of their morphology should be evident. Such differences are very common among related species (p. 126). Even within the same species one may expect to find races which differ less in the outfit of genes they carry, i.e., in genotype, than they do in the arrangement of these genes and in chromosome morphology. The individuals of two such races might look alike, yet show different linkage relations among their characters when bred. Furthermore, it seems altogether probable that losses, duplications, and translocations have played a considerable rôle in the production of widely different chromosome complements and genotypes and therefore in the origin of stable and fertile types to which specific rank has been assigned. Hence such cytogenetic phenomena are of great importance to students of phylogeny and evolution.

Finally, the discovery that certain agencies, such as X-rays and heat, can be used to increase the frequency with which such chromosomal changes occur has placed in the hands of the investigator a tool with which he will be able not only to analyze the functions of chromosomes in greater detail but also to bring about the production of new types of plants and animals more rapidly than they would otherwise appear in nature. 


\section{CHAPTER XIX}

\section{RECIPROCAL TRANSLOCATION}

The mutual exchange of portions of chromosomes known as reciprocal translocation, or often as "segmental interchange," differs in a number of respects from that occurring in ordinary crossing-over. In crossingover, two homologous chromatids of a tetrad exchange portions which are, with rare exceptions, exactly equivalent; and this is done with characteristic frequencies in most of the diploid organisms which have been adequately studied. On the other hand, in reciprocal translocation, which is of relatively rare occurrence, the chromosomes involved may be either homologous or non-homologous, and the exchanged pieces may be of any relative size. When the exchange is between homologues and involves approximately equivalent pieces, the effects may resemble those produced by crossing-over; but ordinarily, especially when non-homologues are involved, the cytological and genetical consequences of reciprocal translocation are highly characteristic and not to be confused with those of normal crossing-over. Just how such translocations are accomplished is not yet known, but processes like those supposed to bring about simple translocation are suggested. Moreover, it is not improbable that many supposedly simple translocations are actually reciprocal, one of the exchanged pieces being missed because of its minuteness and the consequent failure to form the chromosome rings characteristic of translocations of the latter type. Just such a case has been described in Zea (Burnham, 1932).

Origin of the Theory.-In Datura stramonium, Blakeslee and his associates $^{1}$ found that any one of the 12 chromosomes might be present in triplicate in the somatic cells, giving 12 visibly different "primary mutants" with 25 chromosomes instead of the normal 24. The primary type due to the presence of an extra chromosome I (the longest of the set) was called "Rolled." When Rolled plants were bred there appeared among the offspring four types: (1) normal plants without the extra chromosome, due to the fact that half of the spores of Rolled do not contain it; (2) Rolled plants carrying the extra chromosome; (3) plants of a "secondary" 25-chromosome type called "Sugarloaf"; and (4) another secondary type called "Polycarpic." Other primary types also were found to have their corresponding secondaries. The secondaries in

${ }^{1}$ For general accounts of the phenomena treated here, see Blakeslee $(1928,1929 a$, 1931b). See also Belling (1927bcd, 1928f) and Bergner et al. (1933). 
turn gave rise to (1) their corresponding primary type, (2) normals, and (3) plants like themselves.

In studying the chromosomes of these 25-chromosome ("simple trisomic") mutants, Belling observed that the three members of the trisome tended to form characteristically different trivalent configurations at diakinesis and metaphase $I$ in the primary and two secondaries (Fig. 186). In the primary, Rolled, they most frequently formed either a V-shaped chain of three or a ring of two with the third one projecting

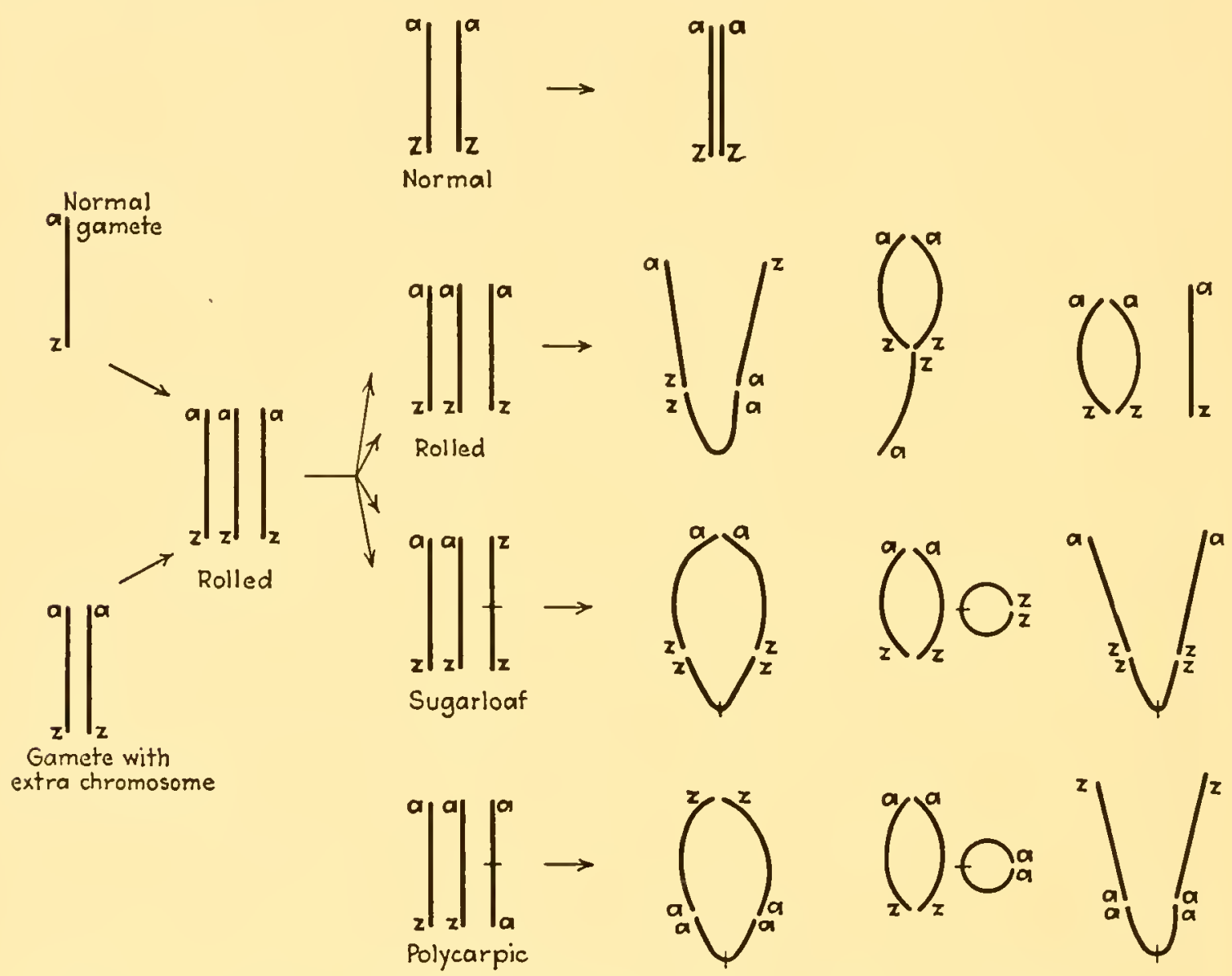

FIG. 186.-Diagram showing chromosome constitutions of the primary Datura mutant "Rolled" and its two secondaries, "Sugarloaf" and "Polycarpic," as postulated by Belling. See text.

from one point of union; and when the third chromosome was free from the others it was straight. In the corresponding secondaries, on the contrary, the commonest configuration was a closed ring of three, usually with one of the members sharply bent; and when the third chromosome was free from the others it formed a small ring by itself. To account for these configurations and for the characteristic outward differences between the primary plant and its corresponding secondaries, Belling and Blakeslee (1924b) announced the hypothesis that in some manner an interchange of unlike terminal segments had occurred between two chromosomes I, giving a chromosome with two "right" ends and another with two "left" ends. When the extra chromosome in a 25 -chromosome 
plant was of the former interchange type, the plant was a secondary; when it was of the latter interchange type, the plant was the other secondary; when it was of the normal type, the plant was a primary. The trivalent configurations were explained on the assumption that only homologous ends are paired in the late prophase and metaphase. As would be expected, these abnormalities led to a certain amount of sterility in trisomic plants because of the abnormal chromosome distribution in meiosis.

In addition to the primaries and secondaries certain "tertiary" types were discovered. The breeding behavior of the tertiary known as "Wiry" suggested that both chromosome I (giving "Rolled" as a primary) and chromosome IX (giving "Poinsettia" as a primary) were somehow involved. Cytological observations showed that these two chromosomes actually were connected with each other in meiosis. This led to the definite formulation of the theory that non-homologous chromosomes occasionally undergo reciprocal translocation (Belling and Blakeslee, 1926). On the theory that only homologous portions undergo synapsis, it was found possible to explain the curious configurations seen at meiosis after various crosses and to account for the unusual genetic behavior of the plants concerned. The continuation of these investigations led to results of great importance in the field of cytogenetics. Reciprocal translocation has since been reported for other organisms also. ${ }^{2}$ It has received definite cytological proof and promises to explain many hitherto puzzling phenomena in both plants and animals.

Reciprocal Translocation in Zea.-For a fuller description of reciprocal translocation in a diploid plant we may select a particularly clear case from among several known in Zea (Figs. 187 to 189). This case ${ }^{3}$ moreover, is the one in which the peculiar morphology of the chromosomes concerned permitted the first complete cytological proof of the correctness of Belling's hypothesis of reciprocal translocation.

In a certain strain of maize known as "semisterile-2" it was observed that approximately one half of the pollen grains and one half of the ovules were functionless. Cytological examination of the sporocytes of semisterile plants revealed the presence of eight bivalents and a ring of four instead of the 10 bivalents seen in normal plants. It followed that certain nonviable chromosome combinations produced by the disjunction of the four

${ }^{2}$ E.g., Zea (Burnham, 1930; McClintock, 1930; Cooper and Brink, 1931; Brink and Cooper, 1931); Pisum (Håkansson, 1929a, 1931a, 1932; Hammarlund and Håkansson, 1930; E. Richardson, 1929; Pellew and E. Sansome, 1931; E. Sansome, 1932); Campanula (Gairdner and Darlington, 1930); Rhœo, Tradescantia, and Zebrina (Darlington, 1929ab; Sax, 1931e; Nebel, 1932b); Aucuba (Meurman, 1929a); Drosophila (Sturtevant and Dobzhansky, 1930; Muller, 1930c; Dobzhansky, 1933); Enothera (see later in chapter); Godetia and Clarkia (Håkansson, 1925, 1931b); Hypericum (Hoar, 1931); and Circotettix (Helwig, 1932).

${ }^{3}$ Burnham (1930), McClintock (1930ab). 


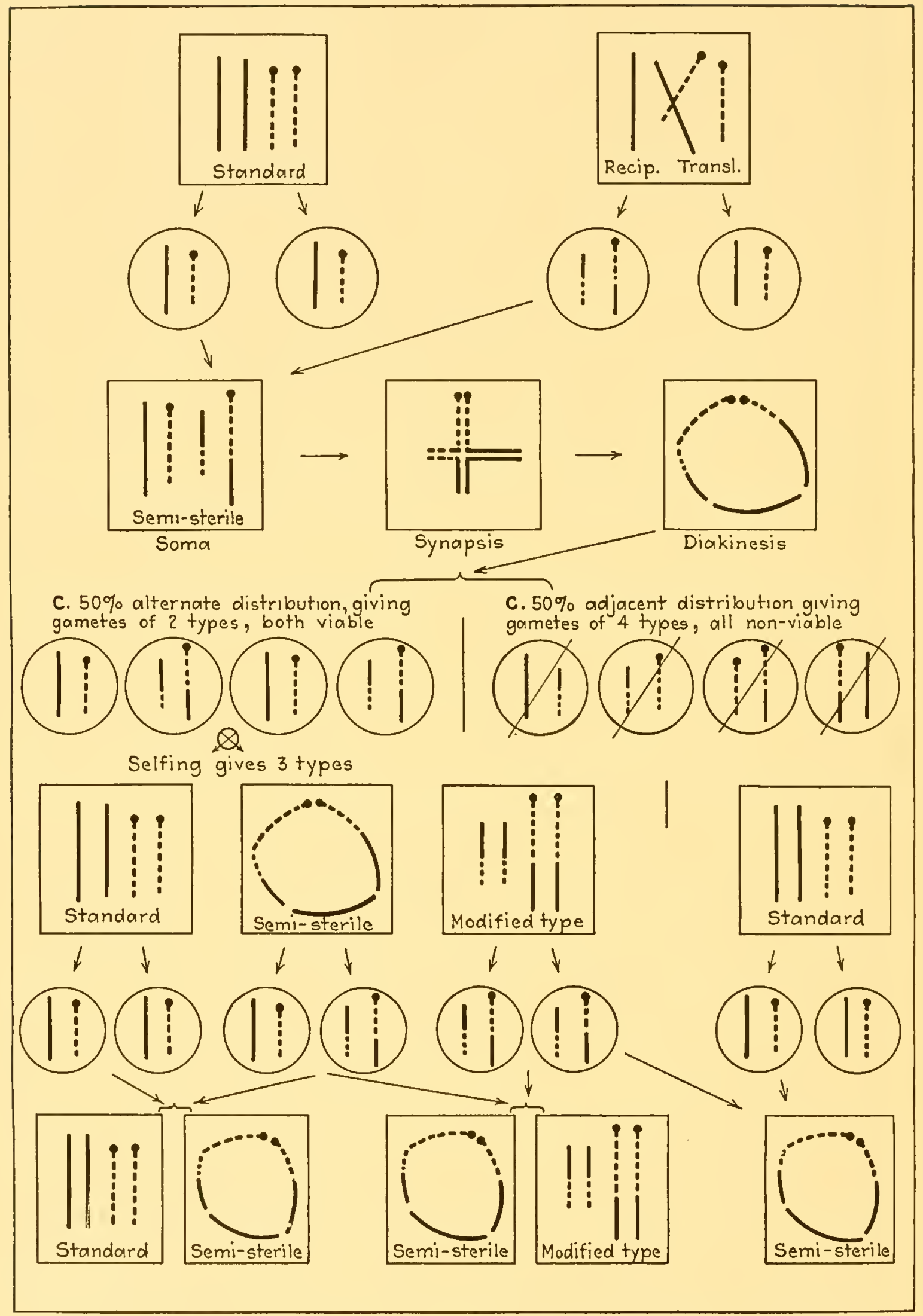

FIG. 187.-Diagram of reciprocal translocation and its results in the "semisterile-2" strain of Zea Mays, based on data of Burnham (1930) and MeClintock (1930ab). Chromosome VIII represented by solid line and chromosome IX by broken line. For simplicity the two chromatids of each chromosome are not shown. The rectangles represent the plants, and the circles their (spores and) gametes. Further explanation in text. 
members of the ring were responsible for the sterility and that the ring itself was a result of reciprocal translocation. ${ }^{4}$ The chromosomes concerned were number VIII (carrying the $j$ - $m s_{s}$ linkage group) and number IX (carrying the $C$-sh-wx group), and these could be distinguished on the basis of length, position and size of attachment region, and a terminal knob on IX. The semisterile plant contained (in addition to eight normal chromosome pairs) one normal VIII, one normal IX, one interchange chromosome composed of portions of VIII and IX, and one interchange chromosome composed of the remaining portions of IX and VIII. The portions interchanged were unequal in length and were such that each of the modified chromosomes had an attachment region.

In the meiotic prophase the synapsis of homologous portions in such semisterile plants gives rise to a cross-shaped group of four, the center of the cross marking the points of interchange in two of the members. Later in the prophase the four open out, remain attached at their ends, and shorten to form the conspicuous ring seen at diakinesis and metaphase $I$. In anaphase $I$ the four members pass two by two to opposite poles. In about half of the sporocytes adjacent members go to one pole, while in the other sporocytes alternate ones go together. When distribution is "adjacent," all of the chromosome sets resulting are abnormal, since they have a certain portion of one chromosome in excess and lack a portion of the other chromosome concerned. The pollen grains containing such sets abort: hence the sterility observed. On the other hand, when distribution is "alternate," all of the resulting pollen grains contain complete sets and are viable, although in one-half of them the chromosomal materials have a new arrangement.

When a semisterile-2 plant is selfed or crossed to another of its kind the progeny includes plants of three different chromosomal types in the ratio of $1: 2: 1$ : plants with the standard chromosome complement, semisterile plants again forming a ring of four in meiosis, and plants with two modified chromosome pairs but fully fertile because the complement is symmetrical and balanced. Had the "modified" type been discovered first, it might have been regarded as the "standard." Thus in Datura stramonium it appears that the standard line $1 A$, which has been so extensively studied, is actually less prevalent in nature than the $B$-race, these two types evidently having been differentiated by reciprocal translocation. The discovery of such related chromosomal types may be expected in other genera also, especially among self-pollinated species.

The diagram for Zea (Fig. 187) shows further that in this case standards give about 50 per cent semisteriles when crossed with semisteriles, that modifieds give about 50 per cent semisteriles when crossed with

${ }^{4}$ This explanation of semisterility was first suggested by Belling (1925c) for Stizolobium. It also applies to certain forms of Pisum sativum (Pellew and E. Sansome, 1931). 
semisteriles, and that when standards and modifieds are crossed only semisteriles result. When selfed, standards and modifieds give only their own kind. All of these findings are consistent with the conclusion that sterility here is due to abnormal assortments of chromosomal materials.

In addition to semisterile-2, described above, there are several other instructive semisterile races in Zea. Semisterile- 4 has a reciprocal translocation involving chromosome II ( $B$-lg linkage group) and chromosome V $\left(\mathrm{Pr}_{-} \mathrm{v}_{2}\right)$ (Rhoades, 1931b). Semisterile-1 involves chromosomes I and II and accordingly the $P$-br and $B$-lg linkage groups (Brink and Cooper, 1931), while semisterile-3 involves I and VII. When two such
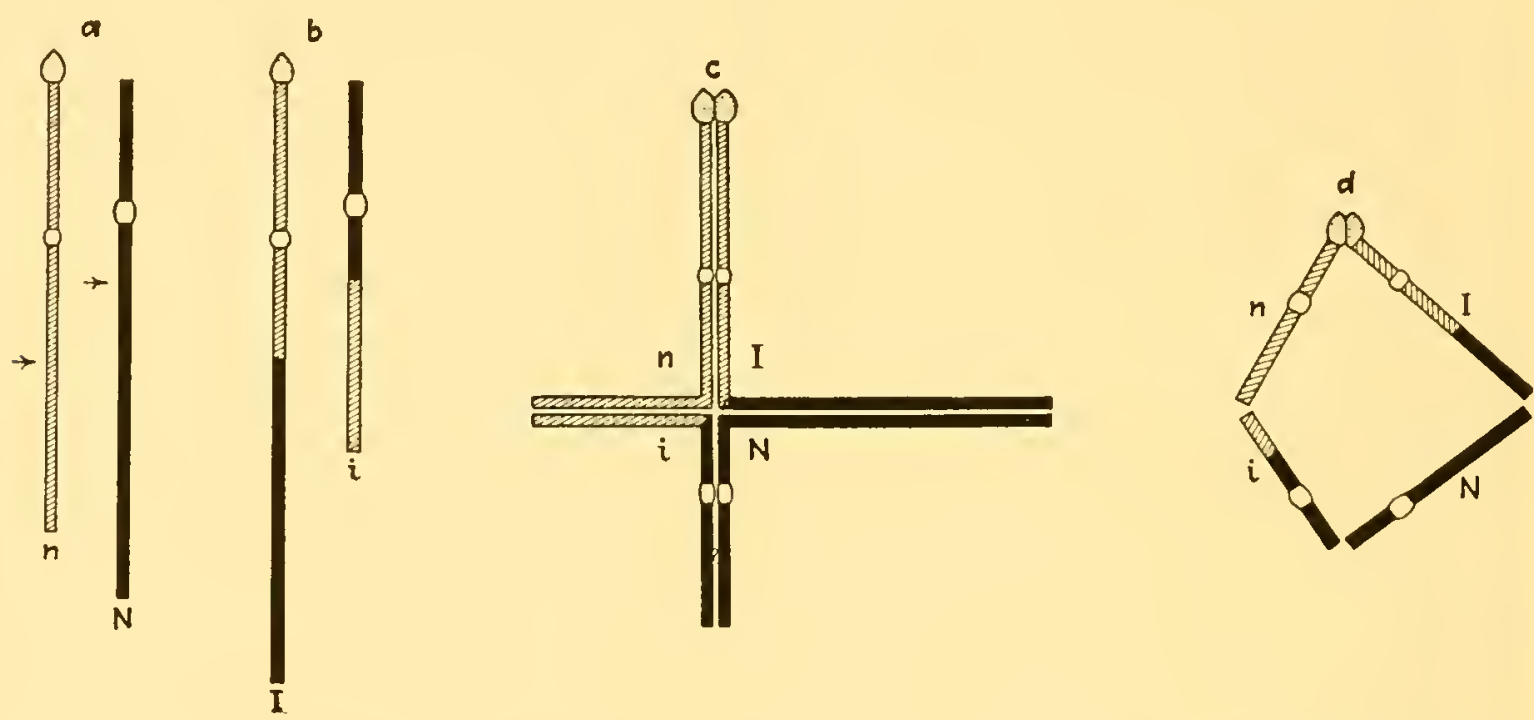

F1G. 188.-Diagram of reciprocal translocation in "semisterile-2" strain of Zea. a, normal chromosomes IX (diagonal shading) and VIII (solid black). The clear portions represent spindle-attachment regions. Arrows indicate location of interchange to produce condition in $b . \quad c$, synaptic complex in mid-prophase of meiosis after crossing a normal plant with one carrying the interchange. $d$, the ring at diakinesis formed by opening out of synaptic complex. $N$, larger normal chromosome (VIII); $n$, smaller normal chromosome (IX); $I$, larger interchange chromosome (IX-VIII); $i$, smaller interchange chromosome (VIII-IX). Cf. Figs. 187 and 189. (After McClintock, 1931a.)

races having interchanges involving one chromosome in common are crossed, a race showing a ring of six at meiosis may result (Fig. 190). This occurs in crosses between semisteriles- 1 and -5 (Brink and Cooper, 1932) and between semisteriles- 1 and -3 . The members of the ring of six are variously distributed in the meiotic divisions, with the result that about 75 per cent of the pollen aborts. It may be calculated further that with a ring of eight or a ring of ten the sterility would be still greater. The useful term catenation has been suggested for such chain formation by chromosomes (Gates, 1931b).

It is an interesting fact that in some cases in Datura and in Enothera the distribution of the catenated chromosomes is rather regularly alternate, giving a high degree of fertility, and not so often adjacent as in the semisterile strains of $Z e a$ mentioned above. In semisterile- 2 the 

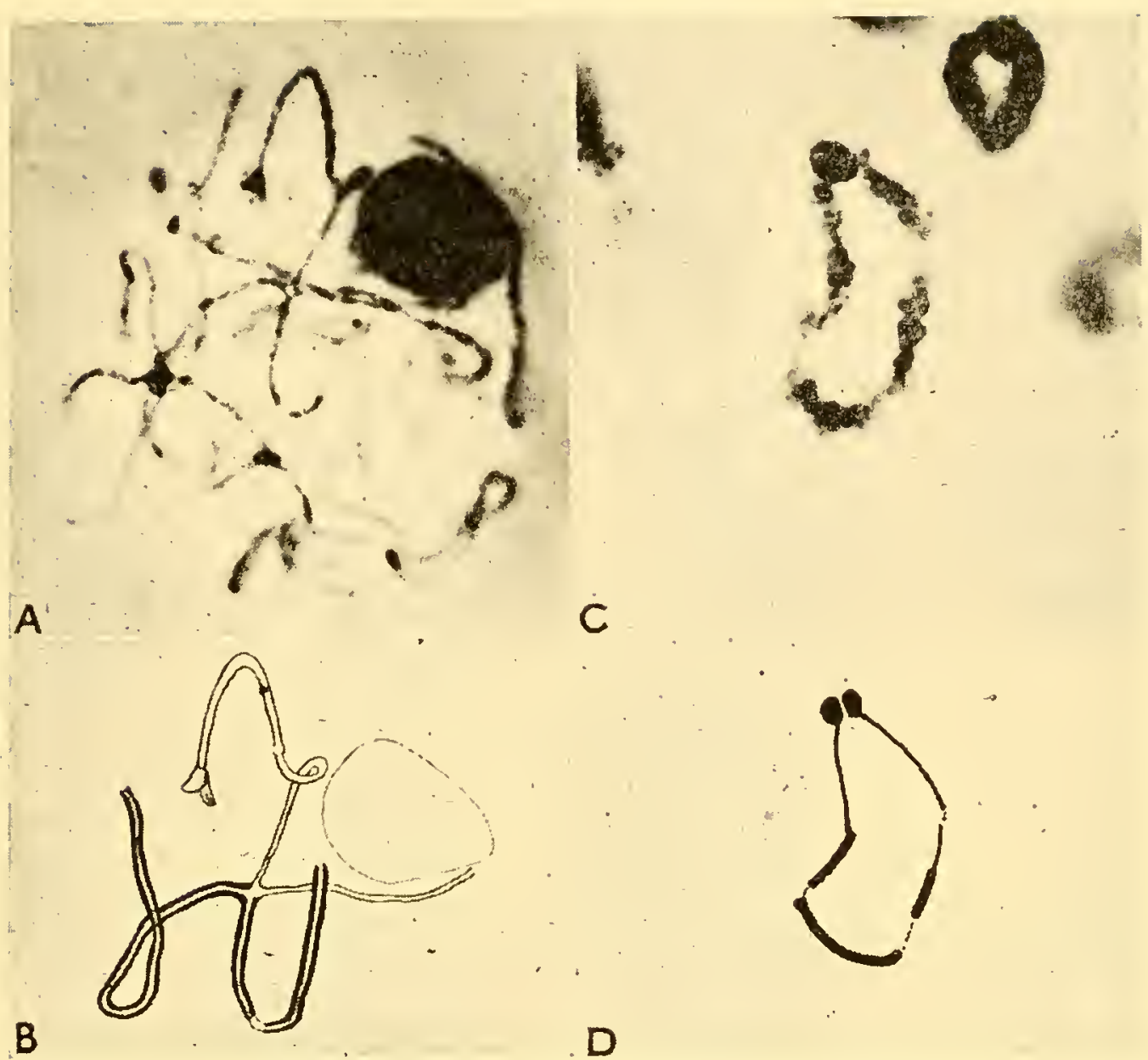

Fig. 189.-Chromosome complexes produced by reciprocal translocation. A, synaptic complex formed by two normal and two reciprocally translocated chromosomes; $c f$. key diagram $(B)$ and Fig. 188. $C$, ring of four in diakinesis formed by opening out of synaptic complex; $c f$. key diagram $(D)$ and Fig. 188. A, $\times 900 ; C, \times 1800 . \quad$ (From McClintock.)
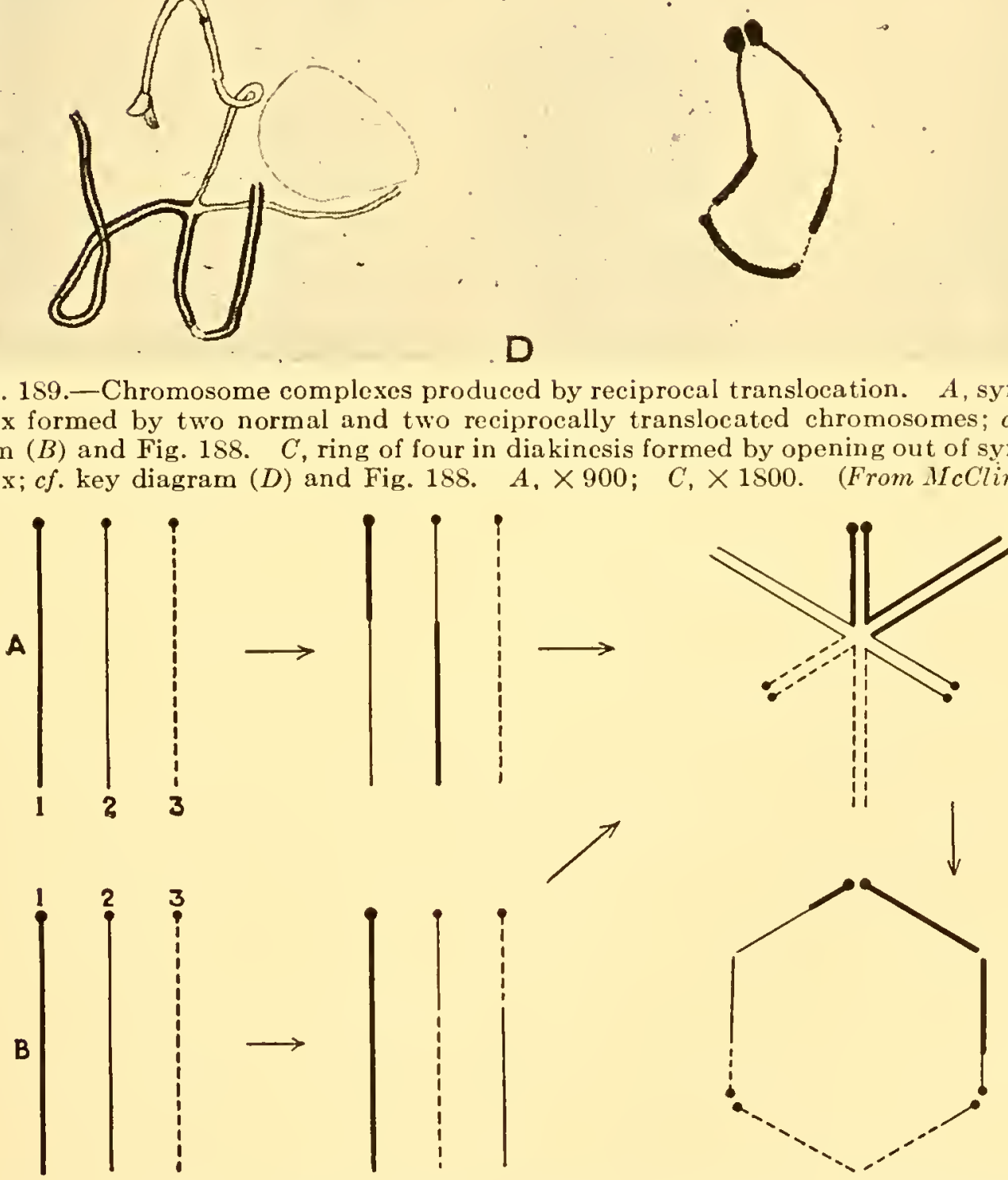

FIG. 190.-Formation of ring of six by reciprocal translocation. The translocation involves chromosomes 1 and $\mathscr{Q}$ in race $A$, and chromosomes 2 and $B$ in race $B$. In the hybrid between $A$ and $B$ the six chromosomes tend to form a six-rayed synaptic complex in early meiotic prophase and a ring of six at diakinesis. For easy interpretation one end of each chromosome is marked with a knob. 
distribution differs significantly from a random one, which indicates some influence favoring one type of orientation in the spindle rather than the other. What it is that thus affects the distribution is not yet known. As observations multiply it will doubtless be found that the ratio of the various types of distribution varies widely in different cases.

Enothera.-The genus Enothera has occupied a prominent position in genetics ever since de Vries formulated his mutation theory largely
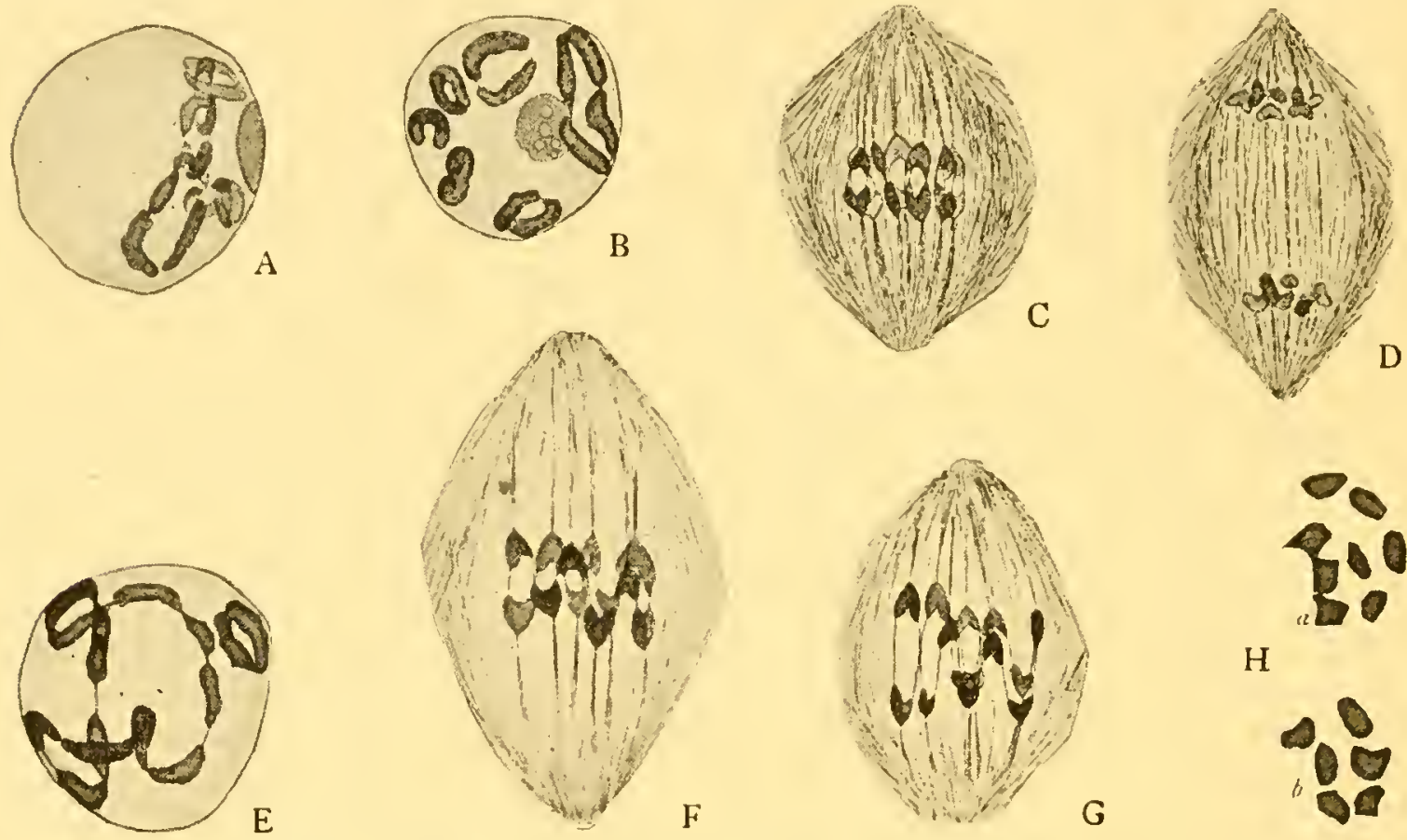

Fig. 191.- Stages in meiosis in the microsporoeytes of Enothera. $A-D, E$. franciscana: $A$, prophase showing a ring of 4 , and 5 pairs. $B$, diakinesis, after separation of pairs from the ring. $C$, metaphase of $I$. $D$, anaphase, showing regular distribution. $E-H$, E. franciscana sulphurea: $E$, prophase showing ring of 12 and one pair. $F$, alternate chromosomes in the ring of 12 passing to the same pole in anaphase; single pair not shown. $G$, later stage; all chromosomes shown. $H$, anaphase of division $I I$, with 8 chromosomes in one figure and 6 in the other; this is due to an occasional irregularity of distribution in $I$. (After Cleland, 1922, 1924.)

on the basis of the peculiar genetic behavior of $E$. Lamarckiana. Not only did this species give rise to occasional "mutants," but it displayed a number of other genetical peculiarities for which little by way of explanation was available. It was soon found that the Onagra section of the genus was peculiar cytologically, the chromosomes in meiosis forming rings or chains with various numbers of members in the different species. ${ }^{5}$

${ }^{5}$ Gates (1907 et seq.), Lutz (1907 et seq.), Geerts (1907-1911), B. M. Davis (19091911), Cleland (1922 et seq.). Later works: S. H. Emerson (1924a, 192sb, 1931ab), Boedijn (1924, 1926), Ochlkers (1926), Håkansson (1926a, 1928a, 1930be, 1931e), Valkanover (1926), Kihara (1927a), Sinotô (1927), Sheffield (1927, 1929), Schwemmle (1924 et seq.), Gerhard (1929), Kulkarni (1929abc), Gates and Sheffield (1929ab), Illick (1929, 1932), Leliveld (1928, 1931), Weier (1930b), Cleland and Oehlkers (1929, 1930), Catcheside-(1930, et seq.), Gates and Goodwin (1931), Darlington $(1929 a, 1931 e)$, and Hedayetullah (1932). For general aceounts of research in Enothera, see Lehmann (1922), Oehlkers (1924), Remner (1925), and Gates (1928). 
For example, E. Hookeri had seven normal bivalents, $E$. franciscana a ring of four and five bivalent pairs, $E$. rubrinervis a ring of six and four pairs, $Q$. rubricalyx a ring of eight and three pairs, $\mathscr{E}$. biennis (from Munich) a ring of eight and a ring of six, $E$. strigosa $\times$ Lamarckiana cruciata a ring of ten and two pairs, E. Lamarckiana a ring of 12 and one pair, and $E$. muricata a ring of 14 with no free pairs. Genetical investigations showed that the more chromosomes there are in the ring or chain, the larger the number of linked genes (Cleland and Oehlkers). Of special importance were the discovery by Cleland of the fact that alternate
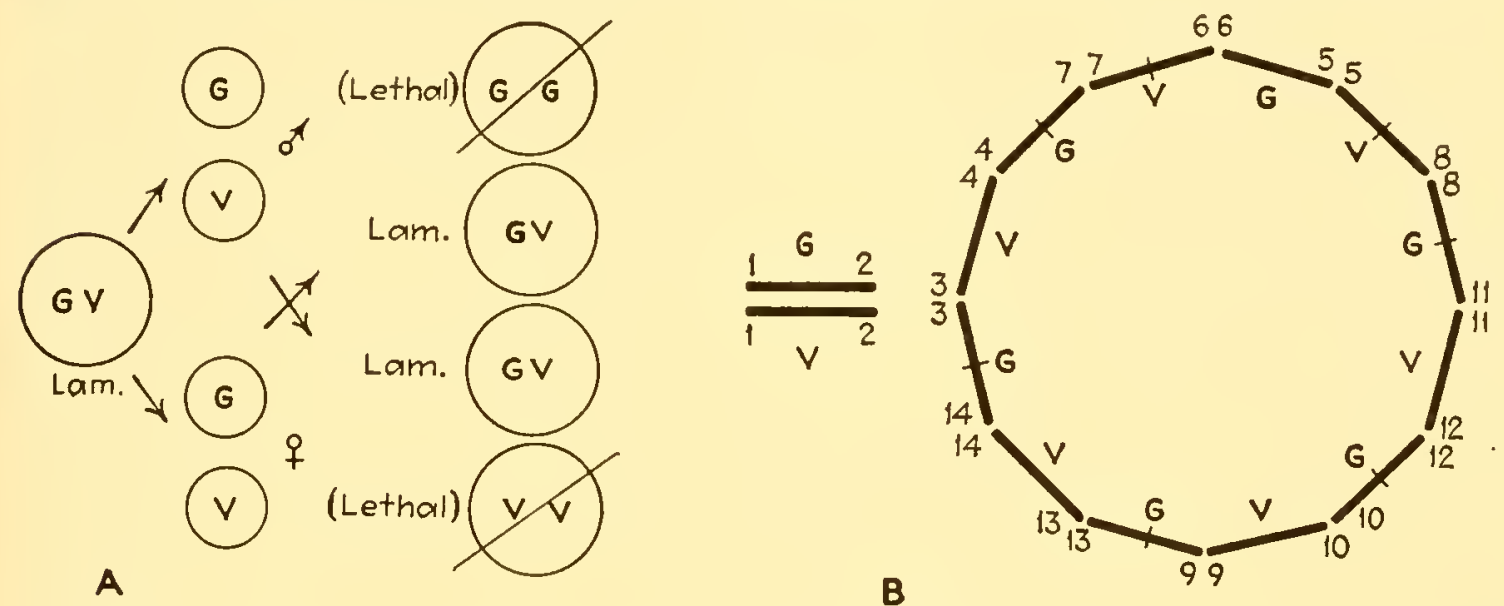

FIG. 192.-The cytogenetic composition of Enothera Lamarckiana. A, distribution of the Renner complexes gaudens $(G)$ and velans $(V)$ in reproduction. Only the heterozygotes survive. $B$, the chromosomes at diakinesis. Those carrying the gaudens and velans complexes are marked $G$ and $V$, respectively. Interchanged chromosomes indicated by transverse marks. (Based on data of Renner, Cleland, and Blakeslee.)

chromosomes in the ring as a rule pass to the same pole in anaphase $I$ (Fig. 191) and his suggestion that each chromosome has a constant position in the ring. ${ }^{6} \quad$ This results in the formation of spores and gametes with only two assortments of chromosomes, so far as those in the ring are concerned. Since certain chromosomes thus tend to remain together, they function like a single large chromosome as carriers of linked genes.

As soon as the theory of reciprocal translocation had been formulated, it was evident to those ${ }^{7}$ who employed it in their investigations that here was probably one of the principal keys to the Enothera problem. Its

For lists of chromosome configurations in the various species, see Cleland (1925), Cleland and Oehlkers (1929, 1930), and Illick (1932b).

${ }^{6}$ It has since been shown directly in Rhœo that the 12 chromosomes in the ring do have constant positions (Sax, 1931e), and furthermore that the direction in which the chromonemata coil tends to be constant for the given distinguishable members (Nebel, 1932b).

${ }^{7}$ Belling, Sturtevant, S. H. Emerson, Meurman. The first discussions of the question in the literature were those of Hakansson (1928a) and Darlington (1929a, 1931e). That $E$ nothera Lamarckiana combines unlike chromosome sets was suggested by Lotsy $(1917,1919)$. 
adequacy was demonstrated in an extensive series of crosses which gave the chromosome configurations predicted on the basis of the theory. ${ }^{8}$ Accordingly, the forms of Enothera with catenated chromosomes are now regarded as "structural hybrids," i.e., the two sets of chromosomes in the diploid complement are unlike in the arrangement of the elements composing them. This, together with the action of factors which are lethal in certain combinations, goes far to explain the peculiar genetical and cytological behavior observed in the genus.

The general situation may be illustrated by the case of $E$. Lamarckiana. Some years ago Renner (1915 et seq.) advanced the hypothesis that in Enothera there are several different genetic factor complexes which are combined in pairs in the various species and that these complexes segregate as wholes in meiosis, each gamete carrying one or the other. Moreover, certain complexes are lethal in gametes, while some combinations are lethal in zygotes. The two complexes in $E$. Lamarckiana are called gaudens and velans. Hence gaudens- and velans-carrying (spores and) gametes are formed; and by their unions three different combinations are possible in the offspring (Fig. 192). But both gaudens and velans are lethal when in the homozygous condition in the zygote, hence the only surviving plants are Lamarckiana. In other words, these plants breed true, not because they represent a pure species as supposed by de Vries, but rather because they are "complex heterozygotes" whose homozygous offspring do not survive.

It was suggested at once that the two genetic complexes must be carried by different gametes produced after meiosis, ${ }^{9}$ but only after the theory of reciprocal translocation had been developed was it fully apparent why the sets should segregate as wholes. The make-up of the gametic sets of seven carrying the gaudens and velans complexes, respectively, is such that, in meiosis, one free pair is formed while the remaining chromosomes form a ring of 12 with the members of the two sets alternate in fixed positions (Fig. 192). Since alternate chromosomes of the ring usually pass to the same pole in anaphase $I$, the members of each set remain together when reproductive cells are formed. The single free pair disjoins at random; but its two members are similar as to their shares of the complexes concerned, so that their freedom does not alter the complexes. Hence the different sets of seven chromosomes, like the genetic complexes, may be treated as characteristically stable units.

The way in which the various genetic complexes are combined in the species of Enothera occurring in nature has been extensively studied genetically, particularly by Renner. In addition, several combinations not before known in nature have been produced by appropriate crosses.

${ }^{8}$ Blakeslee and Cleland (1930), Cleland and Blakeslee (1930, 1931), Emerson and Sturterant (1931), Cleland (1932, 1933).

${ }^{9}$ Bartlett (1915, 1916), Cobb and Bartlett (1919), Renner (1917). 
In this way it has been possible to determine the degree of similarity, or relationship, between the various complexes. In a parallel series of cytological researches by Cleland and Oehlkers it has been shown that the chromosome sets carrying the genetic complexes show clearly their degree of relationship in the configurations produced at diakinesis after two such sets have been combined in a cross. Complexes which are closely related genetically combine to form configurations with most or all of the chromosomes in separate pairs at diakinesis, whereas complexes which are more distantly related give configurations with more of the chromosomes involved in circles. The more distant the relationship, the larger the chains or circles. Plants with identical genetic constitutions tend to show similar configurations at diakinesis. ${ }^{10}$ The genetical and cytological data are in striking harmony, which confirms the theory that in these plants several genetic complexes differentiated by rearrangement and gene mutation are carried by chromosome sets differentiated largely by reciprocal translocation.

The development of the chromosome ring in the meiotic prophase of Enothera is presumably like that in Zea, where it has been shown that homologous portions synapse laterally and later open to form the ring (p. 331). Enothera is ill adapted to a study of these stages, but aside from reports of parallelism of prophasic threads ${ }^{11}$ there are other indications of lateral synapsis at some stage earlier than diakinesis. Among these are interlocking of ring-shaped bivalents and larger rings and the presence of chiasmata. ${ }^{12}$ Moreover, crossing-over has been demonstrated genetically for chromosomes in the ring, ${ }^{13}$ which indicates a lateral association. In view of the striking way in which parasynapsis with reciprocal translocation accounts for the phenomena in Enothera, as it does for those in organisms (e.g., Zea) whose prophasic changes can be clearly followed, this is now generally accepted as the proper interpretation of meiosis in this genus. For many years cytology witnessed a battle between "parasynapsis" (lateral synapsis) and "telosynapsis" (synapsis primarily end to end ${ }^{14}$ ), but the battle has now practically ended with the retirement of telosynapsis from the field as a general interpretation of the meiotic prophase.

${ }^{10}$ See Hoeppener and Renner (1928) and Cleland (1931a) for diagrams of relationship of complexes and of chromosome configurations in crosses.

${ }^{11}$ Gates and Goodwin (1931), Weier (1930b), S. Emerson (1931b).

12 Catcheside (1931b), Darlington (1931e).

${ }^{13}$ See S. H. Emerson (1931a). Crossing-over between catenated chromosomes has also been demonstrated in Zea, where the parasynaptic condition is particularly clear (Creighton and MeClintock, 1931), and in Datura (Blakeslee and Bergner, 1932).

${ }^{14} \mathrm{Mid}$-prophasic doubleness was attributed to splitting rather than synapsis. This interpretation will apply to unsynapsed portions of threads in translocation complexes, multivalents (Fig. 159) and elsewhere, but not to normal meiosis. "Telosynapsis" of catenated chromosomes is but the result of a restriction of parasynapsis to terminal regions. 
Enothera exhibits other genetic peculiarities, such as the production of twin hybrids, unlike reciprocals, ${ }^{15}$ and a rarity of typical Mendelian ratios. These find their explanation in the phenomena outlined above. In contrast to the "superlinkage" displayed by forms with catenated chromosomes, those with freely assorting pairs (Hookeri; grandiflora of Davis) show ordinary linkage and typical Mendelian ratios. One of the most important conclusions to be drawn from these researches is that $E$. Lamarckiana, which de Vries regarded as a pure species giving rise to mutants by some unknown process, is a "permanent hybrid" whose constancy is maintained by lethal factors and whose mutants are due to occasional non-disjunction of some of the catenated chromosomes, to translocations, to doubling of the chromosome number, and to disturbances of the lethal mechanism by crossing-over. Had modern cytogenetical work on Enothera begun earlier, the mutation theory would have been formulated in a very different manner. Moreover, the solution of the Enothera puzzle would not have been possible without the theory of reciprocal translocation, which has now become more than a theory.

${ }^{15}$ Lehmann (1922) describes these phenomena with the aid of diagrams. 


\section{CHAPTER XX}

\section{HETEROPLOIDY}

The course of modern cytology was markedly influenced by two discoveries made in the early years of the century. Firstly, it was observed that when Drosera rotundifolia (20 chromosomes) was crossed with D. longifolia (40 chromosomes), there resulted a hybrid with 30 chromosomes, of which 10 were contributed by rotundifolia and 20 by longifolia. When synapsis occurred in the microsporocytes in this hybrid only 10 bivalents were formed, 10 chromosomes remaining unpaired. Irregular distribution of these unpaired chromosomes in the meiotic mitoses then followed (Rosenberg, 1904a, 1909b). Secondly, it was found that while Enothera Lamarckiana had 14 chromosomes (diploid), its mutant gigas had 28 (tetraploid) (Lutz, 1907; Gates, 1908a). Since that time the number and the behavior of chromosomes in related types and their hybrids have been very extensively studied, especially in plants, where the phenomena are best displayed. The results of these studies will now be summarized in three chapters.

Terminology.-A nucleus with some number other than the true monoploid or diploid number of chromosomes is said to be heteroploid. This term and others given below are also applied to cells, tissues, individuals, races, or species with such nuclei. When the number is an exact multiple of the monoploid, the nucleus (or tissue, etc.) is euploid. The terms designating the multiples up to 10 , beginning with the triple number, are as follows: triploid, tetraploid, pentaploid, hexaploid, heptaploid, octoploid, enneaploid, decaploid. The higher multiples, which are of rarer occurrence, are usually designated as 11-ploid, 12-ploid, and so on. Euploid types are often said to be polyploid. In such species the zygotic and gametic chromosome numbers are, for example, hexaploid and triploid, or tetraploid and diploid, rather than diploid and monoploid as in the types selected for discussion in foregoing chapters. ${ }^{1}$ Non-heteroploid groups may be called homoploid.

A nucleus (or tissue, etc.) with some number other than an exact multiple of the monoploid number is aneuploid. When the number is a little lower than some multiple it is hypoploid; when it is a little higher it is hyperploid. Obviously, a number falling between the diploid and triploid numbers, for example, may be called either hyperdiploid or hypotriploid.

${ }^{1}$ It was formerly customary to refer to the zygotic and gametic numbers as "diploid" and "haploid," regardless of the number of chromosome sets actually present. 
A chromosome complement in which the heteroploidy is due to the multiplication of a single kind of monoploid set (or of some of its members) is said to be autoheteroploid; whereas, one in which specifically different sets or members are combined, as in a hybrid, is alloheteroploid. Although this distinction cannot always be sharply drawn, it is of considerable importance, as will appear later. ${ }^{2}$

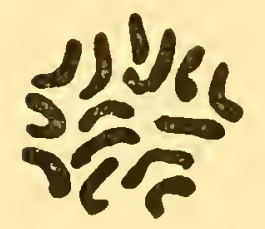

A
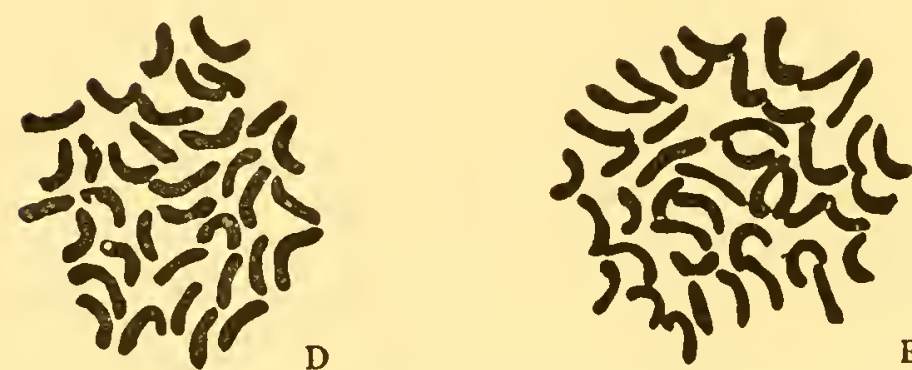

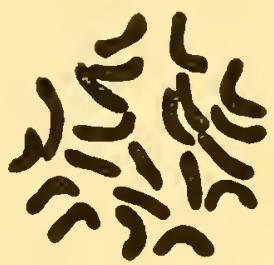

B

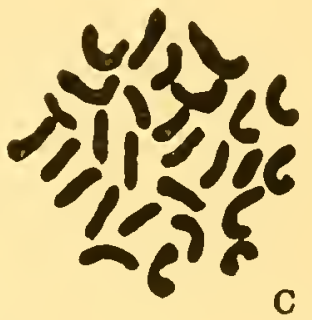

C

FIG. 193.- Somatic chromosome complements of various species of Rosa, illustrating euploidy. A, R. webbiana, 14 chromosomes. $B, R$. chinensis, 21. C, "Konrad Ferdinand Meyer," 2S. D, R. tomentosa cuspidatoides, 35. E, R. nutkana, 42. F, an octoploid hybrid, 56. (After Täckholm, 1922.)

Heteroploidy in Related Species.-Very often the chromosome numbers in the various species of a given genus form a more or less complete euploid series (Fig. 193). For example, in Chrysanthemum the various species have $9,18,27,36$, and 45 pairs of chromosomes; hence they range from diploid to decaploid, with 9 as the "basic" number (Tahara, 1915b, 1921). In Potentilla, with 7 as a basic number, the species range from diploid to $16-$ ploid. ${ }^{3}$ The species of Thalictrum, also with 7 as a basic number, range from diploid to 12-ploid. ${ }^{4}$ Such long

${ }^{2}$ In many reported eases it is not known which type of heteroploidy is represented. It is largely for this reason that "heteroploidy" is here used singly and in combination to indicate number only, without special reference to the kinds of sets concerned. In this we follow Winkler $(1916,1920)$. Winkler refers to complements with similar sets (genoms) as "isogenomatie" and to those with unlike sets as "anisogenomatic." The terms "autopolyploidy" and "allopolyploidy" were suggested by Kihara and Ono (1926). Hurst (1927b) uses the parallel terms "duplicational polyploidy" and "differential polyploidy." Täcklolm (1922) introduced "euploid" and "aneuploid." For further discussions of terms, see Langlet (1927a), Brieger (1927b), and von Wettstein (1927).

${ }^{3}$ Shimotomai (1929, 1930), Müntzing (1931).

${ }^{4}$ Langlet (1927a), Kuln (1928a, 1930c). 
unbroken series may be somewhat exceptional, but series with two or three members are very common, especially in the angiosperms. Extremely high multiples are found occasionally, as in Prunus laurocerasus with about 176 somatic chromosomes (ca. $22 \times 8)^{5}$ and Senecio Roberti-Friesii with about $180($ ca. $36 \times 5) .{ }^{6}$ Euchlona mexicana has 10 pairs of chromosomes and is annual, while $E$. perennis has 20 and is perennial; the same is true of Sorghum sudanensis and S. halepensis. ${ }^{7}$
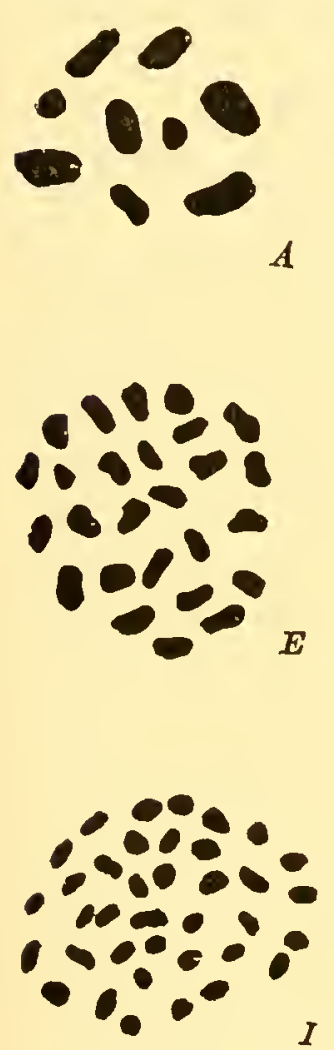

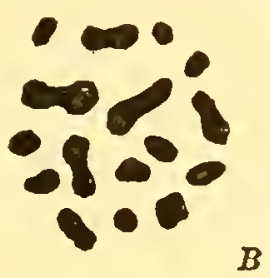

$\boldsymbol{B}$

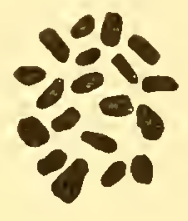

C
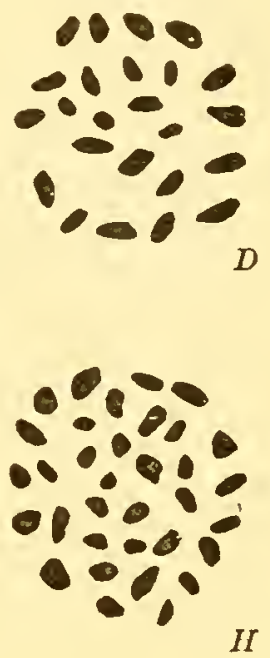

$D$
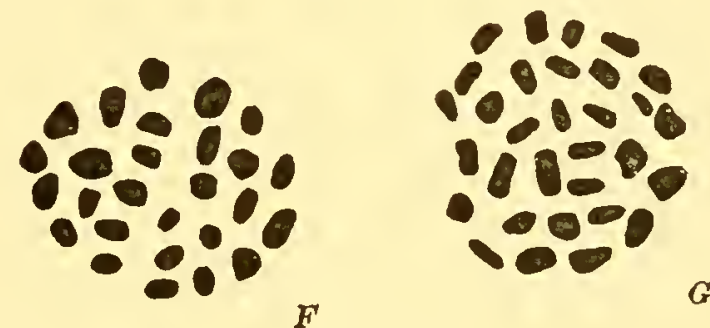

FIG. 194.-The chromosomes in the metaphase of the first meiotic mitosis in the microsporocytes of various species of Carex, illustrating aneuploidy. A, pilulifera, 9 chromosomes. $B$, sparsiflora, 16. $C$, montana, 19. D, tomentosa, 24. $E$, digitata, $26 . F$, loliacea, 27. $G$, caryophyllea, 29. $H$, punetata, 34. I, distans, 37. J, vesicaria, 41. $K$, Goodenoughii, 42. L, hirta, 56. (After Heilborn, 1924.)

A striking example of aneuploidy within a genus is afforded by Carex, in which the species examined show 22 gametic numbers, all but one of which lie between 9 and 42 (Fig. 194). Taxonomic relationship is roughly indicated here by the number and size of the chromosomes. ${ }^{8}$ Another example of such marked aneuploidy is Scirpus. ${ }^{9}$ Heilborn thinks it probable that this exceptional condition is connected with the peculiar mode of pollen formation in Cyperaceæ. Frequently a genus shows two or more euploid series, or it may have euploid combined with aneuploid

${ }^{5}$ Meurman (1929b).

${ }^{6}$ Afzelius (1924).

${ }^{7}$ Longley (1924c, 1932); see also Huskins and Smith (1932).

${ }^{8}$ Heilborn (1922, 1924, 1925, 1932).

${ }^{9}$ Hảkansson (1928b), Hicks (1928). 
numbers. Thus in Brassica the gametic numbers are 9, 18, 10, 20, and $19 .{ }^{10}$ In Rumex the species of the Eulapathum section show gametic numbers of $10,20,30,40,50,60$, and 100, while those of the Acetosa section show 7, 8, 9, 10, 20, 21, and 24. ${ }^{11}$ Another such genus is Viola. ${ }^{12}$

Of special interest is the relation sometimes exhibited between chromosome number and taxonomic division into subgenera. An outstanding example is Triticum, in which the gametic numbers are 7 in the einkorn group of species, 14 in the emmer group, and 21 in the spelta group. ${ }^{13}$ Similar correlations are shown in a number of other genera. ${ }^{14}$

In contrast with the above are certain homoploid genera, such as Pinus, with 12 pairs of chromosomes in every species yet studied: Quercus, also with 12; Lilium, with 12 ; Philadelphus, with 13; Epilobium, with 18; and Mecostethus, with $12 .{ }^{15}$

Numbers in Related Genera.-Related genera often show "related" chromosome numbers. In the large systematic group Bicornes (Ericales) the gametic numbers found in the various genera are $6,12,18,24,36,48$; also $8,16,13,23$, and 26 . In the Antirrhineæ the numbers are 6, 7, 8, 9, and 12; here there appear to be related pairs of genera which differ by one pair of chromosomes. The Orchidaceæ have $8,11,12,14,16,17,20$, and 28, with 16 and 20 most frequent. ${ }^{16}$ Zea Mays and certain species of Euchlcna and Sorghum have 10 pairs. Perennial species of the latter two genera are tetraploid, and it is of interest to find that an induced tetraploid from a diploid annual Euchlana is perennial (Randolph, 1932).

Heteroploidy within a Species.--Individuals or races within a species often differ characteristically in chromosome number; in many cases such differences have been seen to arise in the breeding plot. In Zea Mays, whose normal diploid number is 20 , individuals have also been found with

${ }^{10}$ Karpechenko (1924a, 1928), Shimotomai (1925), Morinaga (1929), and others.

11 Roth (1906), Kihara and Ono (1926), Ono (1926 et seq.), Jaretzky (1927ab, 1928b).

${ }^{12}$ J. Clausen (1927, 1929a), Gershoy (1928).

${ }^{13}$ Sakamura (1918), Kihara (1919 et seq.), Sax (1918 et seq.), Nikolaewa (1920a, 1924), de Mol (1924), Watkins (1924, 1925, 1927, 1930), Bleier (1926 et seq.), Percival (1926), Aase and Powers (1926), Aase (1930), Kagawa (1927b), W. P. Thompson (1925 et seq.), and others. Cf. Schulz (1913), Zade (1914, 1918), and Vavilov and Jakushkina (1925) on relationships. See also footnote 8, p. 366.

${ }^{14}$ E.g., Valeriana (Senjaninova, 1927a), Gossipium (Denham, 1924ab), Bursa (S. E. Hill, 1927), Polygonum (Jaretzky, 1928b).

${ }_{15}$ Chamberlain (1899), Ferguson (1901, 1904), and others on Pinus; Ghimpu (1929bf, 1930), Jaretzky (1930), Friesner (1930), and others on Quercus; Bangham (1929) on Philadelphus; Håkansson (1924b), Schwemmle (1924), Michaelis (1905), and Johansen (1929) on Epilobium; McClung (1923, 1924) on Mecostethus. For Lilium and other cases, see the lists of Tischler $(1927 a, 1931)$.

${ }_{16}$ Hagerup (1928b) on Bicornes, Heitz (1927a) on Antirrhineæ, Hoffman (1929) on Orchidaceæ. See also Langlet $(1927 a, 1932)$ on Ranunculaceæ, Jaretzky (1928a, 1932) and Manton (1932b) on Cruciferæ, Fernandes (1931) on Liliacex, Wanscher (1931, 1932) on Umbelliferæ, and Tscheehow (1932) on Trifolieæ. 
10, 30, 40, 80, and certain aneuploid numbers. Euploid individuals and races are now known in a large number of species in many groups of plants. In Enothera Lamarckiana, where the diploid $(2 n)$ number is 14, some of the well-known mutants have $4 n, 3 n$, and $2 n+1$ chromosomes. The primary and secondary mutants of Datura stramonium are $2 n+1$ forms. In Thalictrum minus there are three races (species ?) with gametic numbers of 7,14 , and 21 . Vicia græca has races with 6,7 , and 14 . In Matthiola incana have been seen individuals with $14,14+$ two fragments, and $7+$ one fragment. In Pellia epiphylla and $P$. Neesiana races with diploid gametophytes are known. In Funaria hygrometrica there are races with 14, 28, and 56. A few such cases are known among the algæ. Among grains certain speltoid wheats, dwarf wheats, and fatuoid oats may have one or two chromosomes more or less than the normal number. In Artemia salina, a crustacean, there are diploid, tetraploid, and octoploid races. ${ }^{17}$

Heteroploidy within the Individual.Occasionally one region of an individual differs from the remainder in chromosome number. Diploid Datura plants, for example, have been seen to bear $2 n+1,2 n-1$, and $4 n$ branches. Sometimes the modified region is a well-defined sector of the plant (Figs. $195,204) .^{18}$ The differing portions may also be arranged concentrically: in Spinacia the plerome and dermatogen may be $2 n$ and the periblem $4 n$ or $8 n .{ }^{19}$ Such sectorial and

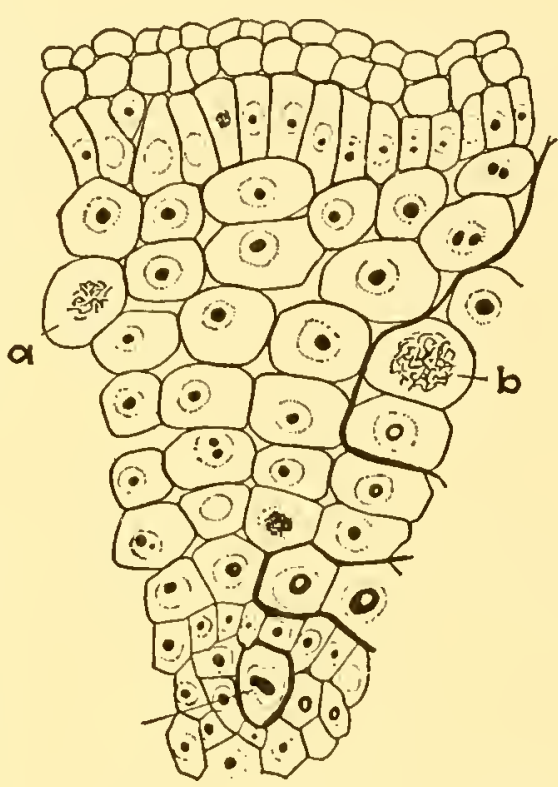

FIG. 195.-Portion of transverse section of root of chromosomal chimera in Nicotiana. Cells at left of heavy line have 24 chromosomes, as at $a$; cells at right have 48 , as at $b$. (After Ruttle, 1928.)

periclinal types are known as "chromosomal chimeras." Of very frequent occurrence are scattered cells with aberrant numbers, especially in roots or anthers subjected to abnormal cultural conditions. The formation of tetraploid shoots is sometimes induced from callus tissue

${ }^{17}$ Randolph and McClintock (1926) and Randolph (1932) on Zea; Blakeslee, Belling, and Farnham (1920) on Datura; Kuhn (1928a) on Thalictrum; Sweschnikova (1927a) on Vicia; Lesley and Frost (1928) on Matthiola; Heitz (1927b) and Showalter (1927c) on Pellia; von Wettstein (1924b) on Funaria; Winge (1924), Huskins (1928ab, 1931a), Müntzing (1930a) and Håkansson (1933a) on Triticum; Huskins (1927, 1928a) and Nishiyama (1931) on Avena; Artom (1912) and F. Gross (1932) on Artemia. For Enothera, see footnote 5, p. 334.

${ }^{18}$ Nawaschin $(1926,1930 a)$ and Hollingshead (1930c) on Crepis; Lesley (1925) on Lycopersicum; Ruttle (1928) on Nicotiana; Jaretzky (1927ab) on Thalictrum. Langlet (1927b) lists known cases.

${ }^{19}$ De Litardière (1923c) and Langlet (1927b) on Spinacia and Cannabis. 
after grafting or decapitation, notably in Solanaceæ. ${ }^{20}$ Another interesting condition is seen in certain gynandromorphs, where portions of the body differing in chromosome number differ also in sex (p. 381). In certain Diptera it has been observed that tracheal cells, intestinal cells, or rectal gland cells may become polyploid while the germ cells retain the normal number. ${ }^{21}$

How Differences in Number Arise.-Sudden alterations in chromosome number are brought about by aberrations of mitosis, by the union of unusual or unlike complements in syngamy, and by a combination of these two processes. In a number of instances such aberrations have been directly observed in progress, while in certain others it can reasonably be inferred that they are responsible for differences in number found in the field or breeding plot. Moreover, some of them may be induced experimentally, as by means of heat or X-rays. ${ }^{22}$ Some of the more common phenomena leading to numerical changes are listed below.

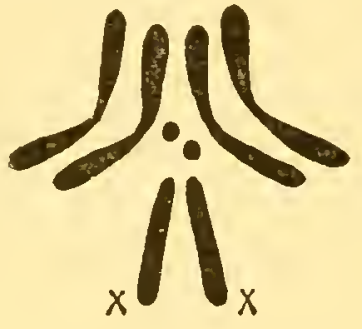

운

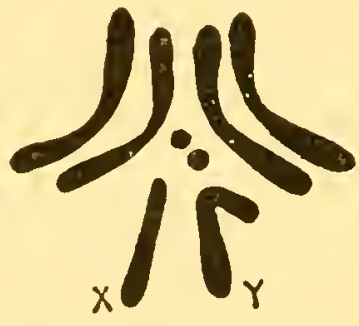

ð

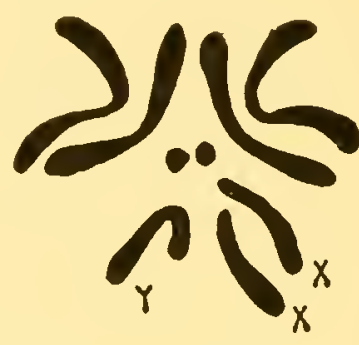

q

FIG. 196.-Chromosome complements of Drosophila melanogaster as they appear during mitosis in a female, a male, and a non-disjunctional female with an extra $X$-chromosome. (After Morgan.)

Two chromosomes may fail to separate in the anaphase of mitosis and pass together to one pole. In meiosis this non-disjunction results in the formation of some spores or gametes lacking one chromosome $(n-1)$ and others with both members of the non-disjoined pair $(n+1)$. Unions of gametes after this aberration may give rise to new individuals with $2 n-2,2 n-1,2 n, 2 n+1$, or $2 n+2$ chromosomes (Fig. 196). Gametes and zygotes with extra chromosomes are viable more often than those lacking chromosomes, so that hyperploid types arise more frequently than hypoploid ones. Sometimes more than one chromosome pair may fail to disjoin, giving still greater departures from the diploid number in the offspring. When the halves of a split chromosome in somatic tissue pass to the same pole instead of separating, hyperploid and hypoploid nuclei result. Such an aberration may have an immediate visible effect upon

${ }_{20}$ Winkler (1916), Jørgensen (1928), F. Sansome (1929), Lindstrom and Koos (1931).

${ }^{21}$ Holt (1917; on Culex), Frolowa (1926, 1929; on Drosophila and other genera).

22 For literature, see footnotes in Chapter XIII. 
the tissue concerned, giving one type of "somatic mutation," but it affects the next generation only if it occurs in the lineage of the reproductive cells. ${ }^{23}$

Very often, especially in meiocytes, one or more of the chromosomes may fail to pass far enough toward either pole to be included in one of the new nuclei. This is known as lagging, and it may play a very important rôle in determining the make-up of the chromosome complements of spores, gametes, and offspring, particularly in hybricls. The extra chromosome in $2 n+1$ types is very often lost through lagging, clecreasing the expected number of $n+1$ gametes.

Sometimes the entire complement of chromosomes behaves in an aberrant manner. In the meiocytes the synaptic reaction may be deficient, some of the chromosomes failing to conjugate (asynapsis) or losing their synaptic association prematurely (desynapsis). Irregularities in chromosome distribution naturally follow. In case the whole complement is involved, complete ameiosis may result; the chromosomes may all divide equationally in the single mitosis which occurs, so that diploid spores or gametes are formed. ${ }^{24}$ A union of such a gamete with a normal monoploid gamete results in a triploid individual. ${ }^{25}$ When ameiosis occurs in both the microsporocytes and the megasporocytes, two diploid gametes may unite to form a tetraploid indiridual.

Similar results may follow from non-division in somatic cells. Here the chromosomes split, thus doubling their number, but there is no division of the nucleus as a whole. It is probable that such an aberration occurs frequently in the zygote or young embryo, giving a tetraploid individual directly. ${ }^{26}$ The formation of a tetraploid plant in this way has been artificially induced by heating young ears of $Z e a$ soon after fertiliza-

${ }^{23}$ Non-disjunetion, originally discovered by R. Gates (1908) in Enothera. is responsible for some of the $2 n+1$ chromosome mutants of Enothera and Datura. In Datura, Belling and Blakeslee (1924b) saw eight cases of it in 1,137 sporoeytes; in Uvularia Belling (1925a) saw 10 cases in three buds. Other examples are Nicotiana (Goodspeed, 1923; Ruttle, 1927) and Zea. In Nicotiana tabacum the forms ealled "enlarged" and "fluted" have $2 n+1$ and $2 n-1$ elromosomes, respeetively (Clausen and Goodspeed, 1924, 1926a).

${ }^{24}$ This may be the reason why in Zea, for example, tetraploid plants so rarely arise directly from diploids with aberrant sporogenesis. Aberrations at this stage more often lead to triploidy.

${ }^{25}$ Triploidy was first discovered in Enothera by Stomps (1912) and Lutz (1912) and was explained by them in the above manner. Triploids have since been produeed frequently by crossing diploids with forms producing occasional diploid pollen grains, e.g., in Hyacinthus (de Mol, 1923).

${ }^{26}$ Gates (1909b, 1913, 1924a) and Davis (1911) for Enothera and other genera; Strasburger (1910b) for Enothera and Wikstromia; Tisehler (1910) for Musa; Sax (1921, 1922) for Triticum; Tahara (1921) for Chrysanthemum; Afzelius (1924) for Senecio; Clausen and Goodspeed (1925) for Nicotiana. Winge (1917) attributes such doubling in hybrid zygotes to chromosomal incompatibility (see p. 369). 
tion (Randolph, 1932). It has been observed in roots of Spinacia that the chromosome halves may separate during the prophase and undergo a second splitting so that each telophase nucleus receives the tetraploid number (24) rather than the diploid (12). Repetition of the process may give octoploid nuclei. ${ }^{27}$ Sometimes diploid plants show tetraploid sporocytes undergoing meiosis along with the normal diploid ones, which suggests the occurrence of non-division in the development of the anther. A similar condition has been seen in the spermatocytes of Euschistus. ${ }^{28}$ In $Z e a$ the occasional appearance of triploid plants strongly suggests that functional diploid spores and gametes may thus arise and lead to heteroploidy. The origin of tetraploidy through a fusion of two such gametes would depend on the occurrence of the aberration in the lineage of both sperms and eggs. In a monœcious plant a single aberration of this type

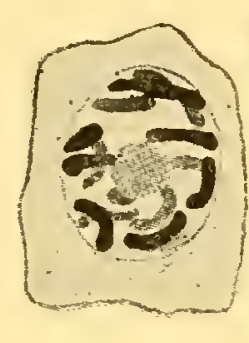

$A$

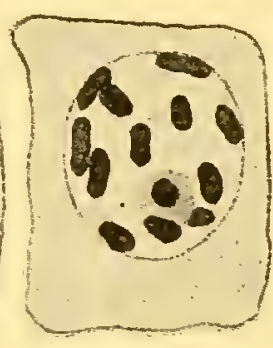

$B$

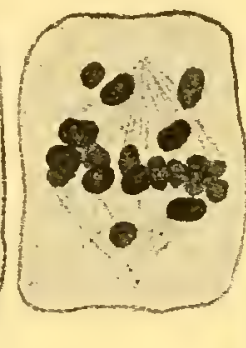

$C$

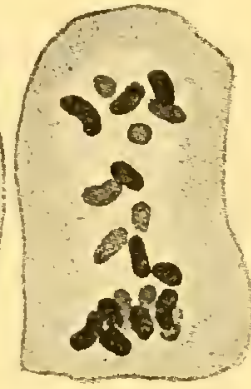

$D$

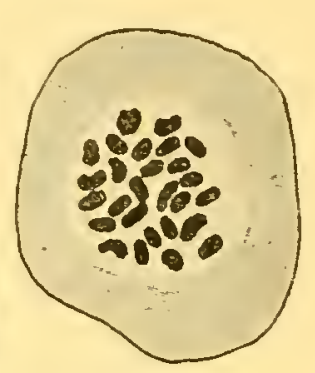

$E$

FIg. 197.- "Semiheterotypic" mitosis, with formation of "restitution nucleus" in Hieracium. $A, B$, prophase; $C$, irregular metaphase; $D$, irregular anaphase. $E$, polar view of metaphase of division of restitution nucleus formed when all of the chromosomes in the preceding anaphase (not all shown in $D$ ) reorganize as one nucleus instead of two. (After Rosenberg, 1927a.)

occurring before the differentiation of the carpellate and staminate portions might result in the production of diploid gametes of both sexes, whereas in a diœcious plant or in a monœcious one after the differentiation of the carpels and stamens, two similar aberrations would be required for the production of tetraploidy in the immediate offspring.

Another aberration in sporogenesis is the formation of a "restitution nucleus" (Fig. 197). Usually after deficient synapsis the chromosomes are scattered along the first meiotic spindle, and before they reach the poles a single large nucleus is formed, enclosing all of them. This nucleus and the sporocyte then divide once, giving two large microspores, each with the unreduced number of chromosomes. ${ }^{29}$ In several instances nuclei formed by the meiotic mitoses have been seen fusing in the sporo-

${ }^{27}$ De Litardière $(1923 c)$. Other probable cases of non-division: Cannabis (de Litardière, 1924; Langlet, 1927b) and Lycopersicum (Lesley, 1925).

${ }^{28}$ Randolph (1928) and McClintock (1929a) on Zea; Bowen (1922f) on Euschistus; Fukushima (1921) on Brassica; Geerts (1909) on megasporocyte in Enothera.

${ }^{29}$ Rosenberg $(1917,1926 b, 1927 a b)$ on Hieracium, Kuwada (1928) on Balanophora, Tischler (1928) on Ribes, Kagawa (1929b) on Triticum, Buxton and Newton (1928) on Digitalis. 
cyte; occasionally it is the mitotic figures themselves which unite. ${ }^{30}$ The result in either case would be unreduced spore nuclei. The rôle of the last mentioned aberrations, as well as that of abnormal nuclear fusions occasionally seen at other stages, in the production of heteroploidy is problematical. The fusion of two male gametes with the egg (dispermy) may possibly lead to triploidy in some cases. ${ }^{31}$

Although there are many ways in which the chromosome number may be altered, it is probable that tetraploidy arises most often through a doubling of the number in a diploid zygote or young embryo, and that triploidy is usually the result of a union between a monoploid and a diploid gamete, the latter being formed either after meiosis in a tetraploid plant or after ameiosis in a diploid one. Further increases in number may follow. Doubling in a triploid gives a hexaploid; crossing a hexaploid with a tetraploid may give a pentaploid; doubling in a tetraploid, or crossing a hexaploid with a diploid followed by doubling, may give an octoploid, and so on. Thus it appears that the origin of tetraploidy is of ten the first step in the development of higher euploid types. Aneuploid plants with one or two chromosomes lacking or in excess are probably due chiefly to non-disjunction; and since such plants produce spores and gametes carrying various chromosome numbers, further unions may extend the aneuploid series. As would be expected, many of the altered types fail to meet the test of viability; or, if viable, they may fail to compete successfully with normal types because of their reduced vigor or fertility. Nevertheless, the fact that the monoploid chromosome numbers of so few flowering plants, relatively speaking, are prime numbers indicates the important rôle of polyploidy in the evolution of this great group (Winge, 1917).

With regard to the evolution of new "basic" chromosome numbers, from which new polyploid series may in turn be developed, it seems improbable that simple additions or losses of whole chromosomes are very significant in this connection, because of the physiological unbalance which such changes introduce. If, however, they are accompanied by compensatory alterations, they may become very important. Thus M. Nawaschin (1932) cites evidence suggesting the following "dislocation hypothesis": An extra chromosome is first added in one of the ways mentioned above, so that the somatic cells of an individual contain three

${ }^{30}$ Gates (1915) on Enothera, Rosenberg (1917) on Hieracium, Blackburn and Harrison (1921) on Rosa, Ljungdahl (1922) on Papaver, McClintock (1929a) on triploid Zea, Prywer (1931) on Beta. In Nereis oöcytes treated with ultra-violet light both meiotic mitoses occur near the center of the cell; the four resulting nuclei may then unite with the sperm nucleus and a pentaploid embryo may begin development (Just, 1933).

${ }^{31}$ Dispermy has been seen in Gagea (Němcě, 1912), Enothera (Ishikawa, 1918), and other plants. It can easily be brought about in echinoderm eggs (Boveri). 
chromosomes of one kind (a "trisome"); through elimination and translocation the materials of one member of the trisome, except for the kinetic body of the spindle-attachment region, are then replaced by some of the materials of a non-homologous chromosome (or of more than one of these), so that the materials of the latter chromosome come to be associated with two kinetic bodies instead of one; through disjunction at meiosis the normal homologue of the contributing chromosome is eliminated, and gametes with two chromosomes in its place are formed; union of two such gametes gives an individual having one more chromosome pair than the original type but with the same assemblage of chromosomal materials. The basic number may also be changed in the opposite direction if translocations and eliminations leave a kinetic body with little or no chromosomal matter, and this body is then segregated out.

Some of the irregularities described in the foregoing pages, notably asynapsis, lagging, and zygotic doubling, have been observed repeatedly in interspecific hybrids. The hasty conclusion that all such aberrations indicate hybridity has been proved erroneous by what has been learned about the effects of cultural conditions; but it remains true that heteroploidy has one of its chief causes in hybridization, ${ }^{32}$ as will be made clear in a later chapter. In the study of such phenomena and their significance it therefore becomes necessary to distinguish two kinds of heteroploidy: autoheteroploidy and alloheteroploidy.

Autoheteroploidy and Alloheteroploidy.-In autoheteroploid types the chromosomes of only one kind of set are involved. For example, a plant with three sets which are alike (within the usual specific limits) is an autotriploid, whereas a plant with two sets from one species and one set from another is an allotriploid. Similarly, a plant with a single one of its chromosomes in triplicate is an "autohyperploid," whereas one in which the extra chromosome is one from the set of another species is an "allohyperploid." In other words, the autoheteroploid types are specifically "pure" as regards their chromosome complements, while the alloheteroploid types are "hybrid." It is obvious that such a distinction cannot always be sharply drawn, since hybridity may exist in so many gradations; moreover, both conditions may be present together, as, for example, in a hexaploid having three sets from one species and three from another. Nevertheless, failure to distinguish the conditions as far as possible has brought much confusion into the literature on this subject.

When the origin of the heteroploid condition is not directly known, an examination of the morphology of the chromosomes composing the complement often yields decisive evidence as to the kind of heteroploidy represented. For example, in euploid forms of Crepis, whose chromosome

32 Rosenberg (1917), Ernst (1917, 1918), Winge (1917), I. Holmgren (1919), Täckholm (1920, 1922), Jeffrey et al. (1922), Jeffrey (1925a), Afzelius (1924), Gates $(1924 a, 1925,1929 a)$. 
morphology is well known, inspection of the complement in the root tips enables one to tell whether a single set has been multiplied to produce an autoheteroploid type or unlike sets have been combined to make an alloheteroploid one. Similarly, one may of ten tell whether an apparently $2 n+1$ type has arisen through the duplication of a chromosome by non-disjunction (Fig. 198, $E$ ), or through the fragmentation of a chromosome (Fig. 198, F), or through hybridization (Fig. 206, 3). Furthermore,

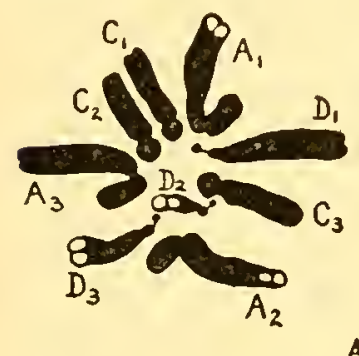

A

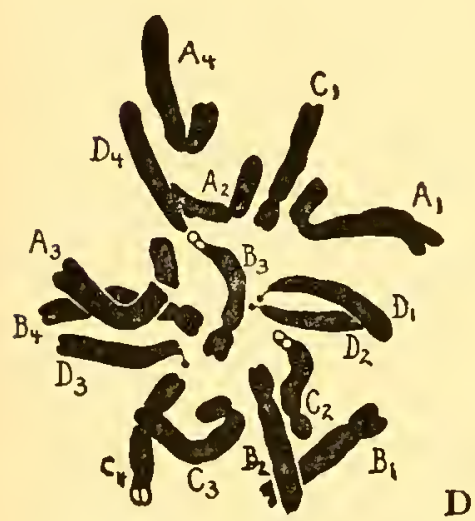

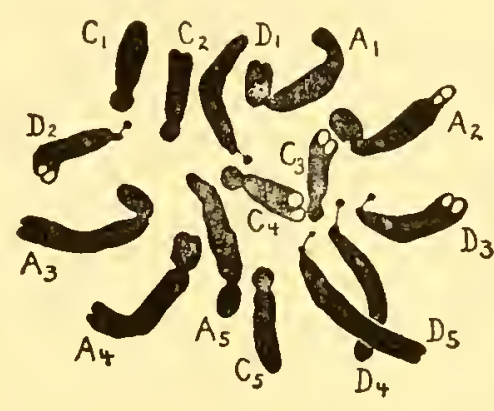

B

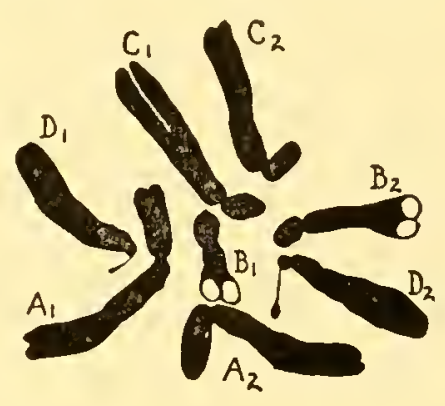

C

FIG. 198.- Somatic chromosome complements in Crepis, showing autopolyploidy and other conditions. $A$, triploid complement in $C$. capillaris. $B$, pentaploid complement in C. capullaris. $C$, diploid complement in an individual "asymmetrical" for size of satellites. $D$, tetraploid complement in $C$. tectorum. $E$, hyperdiploid complement in tectorum plant with extra $B$-chromosome. $F$, diploid complement in tectorum plant with one $D$-chromosome fragmented into two portions: $D_{o}$ and $d$. (Aftcr M. Nawaschin, 1926.)

a sufficient knowledge of the chromosome morphology in an organism under investigation makes it possible to detect alterations, such as deletions and translocations, not affecting the number of chromosomes present. The occurrence of such alterations makes it necessary to use the criterion of chromosome morphology with considerable caution.

Other criteria of value in the analysis of heteroploid complements are the meiotic behavior of the chromosomes and their functions as indicated by genetic data. Autoheteroploids and alloheteroploids frequently differ characteristically in their meiotic and genetic behavior. It is the purpose of the next two chapters to describe some of the most important of such phenomena. 


\section{CHAPTER XXI}

\section{THE CYTOGENETICS OF AUTOHETEROPLOID PLANTS}

A somatic nucleus in a plant sporophyte or higher animal is autoheteroploid when one or more of the chromosomes of the normal set are present in some number other than two. ${ }^{1}$ A gamete or gametophyte nucleus is autoheteroploid when one or more of the members of the normal set are present in some number other than one. In other words, only one kind of set is represented, but some or all of its members are present in unusual numbers. The cytological and genetical behavior shown by autoheteroploid organisms may be expected to differ from that in monoploid-diploid forms. It is the purpose of this chapter to review some of the more characteristic types of such behavior.

Tetraploids and Tetrasomics.-A plant with four similar complete chromosome sets is "autotetraploid." One with a single chromosome in quadruplicate but all of the others in duplicate is "simple tetrasomic." It is found that the four homologous chromosomes in the latter type behave in general like any one of the groups of four in the autotetraploid. Hence the simple tetrasomic type, when bred, may be expected to show "tetrasomic" genetic ratios in characters due to mutant factors in one chromosome and ordinary "disomic" ratios for those due to factors in all the other chromosomes, whereas in an autotetraploid type the ratios will be tetrasomic for all characters. To illustrate such ratios, one may therefore select either a tetraploid or a tetrasomic type.

In autotetraploids and tetrasomics any four homologous chromosomes ordinarily tend to form a quadrivalent group in meiosis (Figs. 199, $g$ to $i$; 200). As synapsis begins, it is seen that any one of the four may associate with any other one in a given region, or with more than one in different regions. If all four form and retain such synaptic associations a quadrivalent chromosome is present at the end of the prophase, hence in a tetraploid plant there may be the monoploid number of quadrivalents. Often the synaptic association is such as to group the four members into two bivalents, or into a trivalent and a free univalent. Thus tetraploid sporocytes may sometimes exhibit the diploid number of bivalents (the "double-diploid" condition) or varying numbers of quadrivalents, trivalents, bivalents, and univalents. The reasons for this variation are not fully known, but it seems that the manner in which the four members

${ }^{1}$ This does not apply to normally unpaired or multiple sex-chromosomes. $C f$. definitions on pp. 340 and 348 . 
happen to associate as synapsis begins, together with the number and persistence of chiasmata, is an important factor in this connection. The behavior is apparently influenced in some cases by the relative positions of the chromosomes in the nucleus before synapsis begins; also, it tends to differ characteristically in different species or genera. ${ }^{2}$ In addition to the "primary association" determined by the ordinary synapsis in the early prophase, there is frequently a "secondary association" at the end of the prophase when homologous bivalents previously separate move together to form loose quadrivalent groups. ${ }^{3}$
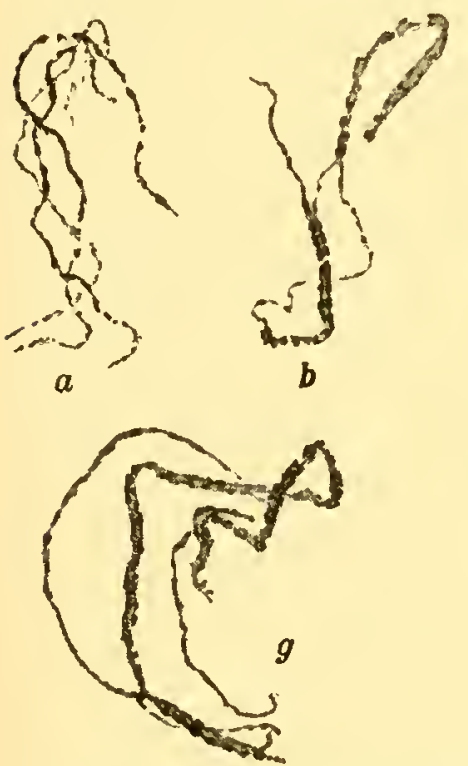

FIG. 199.- Synaptic configurations in heteroploid plants. $a-f$, trivalent chromosomes in Hyacinthus. $g, h$, quadrivalent chromosomes in Hyacinthus. $i$, quadrivalent in Tulipa. $e, f$, and $i$ are postdiplonema stages. (After Newton and Darlington, 1929.)

The phenotypic ratio shown by a pair of Mendelian characters in breeding tetraploids obviously depends upon the degree of dominance exhibited, and upon the manner in which the four chromosomes carrying the four differential factors are distributed in the meiotic divisions. In tetraploid Primula sinensis ${ }^{4}$ the several combinations, $D D D D, D D D d$, $D D d d, D d d d$, and $d d d d$, give a series in depth of flower color, the first three being white or faintly tinged, the fourth weakly colored, and the fifth more deeply colored. Hence various types are to be expected

2 There are sometimes $n$ quadrivalents in Datura tetraploids (Belling and Blakeslee 1923, 1924a; Belling, 1927d). The number in most cases is smaller, as in Prunus, Hyacinthus, and Primula sinensis (Darlington, 1927b, 1929c, 1931a; Dark, 1931); also in Euchlona perennis (Randolph, unpubl.) and Dahlia (Lawrence, 1929). In Campanula most of the chromosomes are in pairs (Gairdner, 1926). The "double-diploid" condition occurs sometimes in Hyacinthus (Belling, 1925b) and rarely in Datura. In $Z e a$ there are quadrivalents and bivalents, and the segregation is at random (Randolph, Rhoades).

${ }^{3}$ Darlington (1928) on Prunus, Darlington and Moffett (1930) on Pyrus, Lawrence (1931c) on Dahlia.

${ }^{4}$ Gregory (1914), Bateson, Sverdrup, Haldane (1929). 
among the offspring of plants bearing both $D$ an $d$. On the other hand, a single $G$-factor (for green stigma) is able to dominate three $g$-factors (for red stigma); hence only two types appear among the progeny, so far as this character is concerned.

The ratios in which the two types may be expected to appear in tetraploid plants with such strong dominance may be readily computed. ${ }^{5}$ In Primula, for example, only green stigmas are to be expected in the progeny of a $G G G G$ plant after selfing or back-crossing to gggg, because every individual has at least one dominant $G$; whereas in the progeny of a

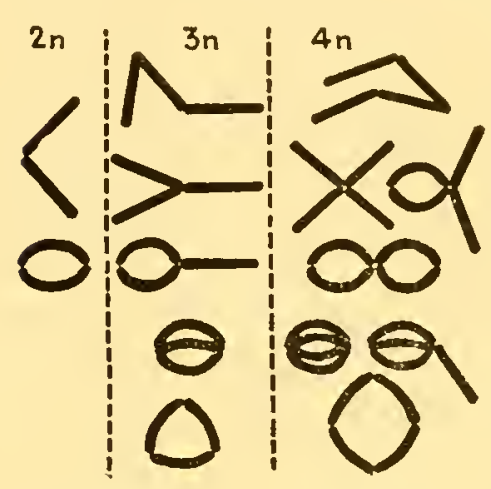

Fig. 200.-Arrangements assumed by the chromosomes in the first meiotic division in diploid, triploid, and tetraploid sporocytes of Datura. The lowest two configurations in the $3 n$ column are rare. In the $4 n$ column the commonest types are the figure of eight (middle) and the one below and to the left of it. (After Betling and Blakestee, 1923.) gggg plant after selfing or back-crossing to gggg, only red stigmas appear, since all of the factors present are recessive. In the progeny of plants with $G G G g, G G g g$, or $G g g g$, characteristic ratios are to be expected, these depending largely upon the manner in which the four homologous chromosomes carrying the factors in question behave during meiosis. Thus when the four form a quadrivalent with the constitution $G G g g$ and are disjoined two from two at random, the ratios will not be the same as when they form two bivalents arranged $G g$ and $G g$.

In tetraploids and tetrasomics a certain amount of sterility may be expected to result from occasional irregularities in chromosome distribution, but, generally speaking, such plants are much more stable and fertile than those having an odd number of certain chromosomes or chromosome sets as in trisomics, triploids, pentaploids, etc. This is because elements present in even numbers are more regularly distributed in the meiotic mitoses than are those present in odd numbers.

Triploids and Trisomics.-In autotriploid plants the chromosomes most commonly form trivalent groups in the meiotic prophase, although the number of these is often rather variable. ${ }^{6}$ As described in an earlier chapter (p. 274), the synaptic association in a given region ordinarily

${ }^{5}$ Muller (1914); Blakeslee, Belling, and Farnham (1923); Haldane (1929, 1930b). Such computations have ordinarily been made on the basis of the distribution of four chromosomes. Calculations based on the distribution of eight ehromatids should be more accurate; see Haldane.

6 Among researches on the cytology of triploid plants are those of Belling and Blakeslee (1922, 1923, 1924a, 1926) and Belling (1931b) on Dalura; Lesley (1926) on Solanum; Ono (1927b) and Tomowo (1927) on Primula; Newton and Darlington (1927, 1929) and Darlington (1929c) on Tulipa, Belling (1929) and Darlington (1929c) on Hyacinthus; Nagao (1929ab) on Narcissus; MeClintock (1929a) on Zea; Håkansson (1926a), Capinpin (1930), and Cateheside (1931a, 1933a) on Enothera; and Steere (1932) on Peturia. 
involves a close union of only two of the three homologues, although one of them may be synapsed with both of the others in different regions (Figs. $159 ; 199, a$ to $f$ ). As chiasmata develop, the trivalent may assume a rather complicated structure. If the three members remain associated until the metaphase, the trivalent usually appears as a compact but asymmetrical triple body in the equator of the spindle; but if only two of the three chromosomes synapse or remain together, one of them may appear at metaphase as a univalent while the two others constitute a bivalent (Fig. 201, a). The chiasmata or points of contact between the members of the trivalent at diakinesis and metaphase may be situated in various positions along their length in some plants (Hyacinthus, Fig. 199), while in others the association is practically always terminal (Datura,

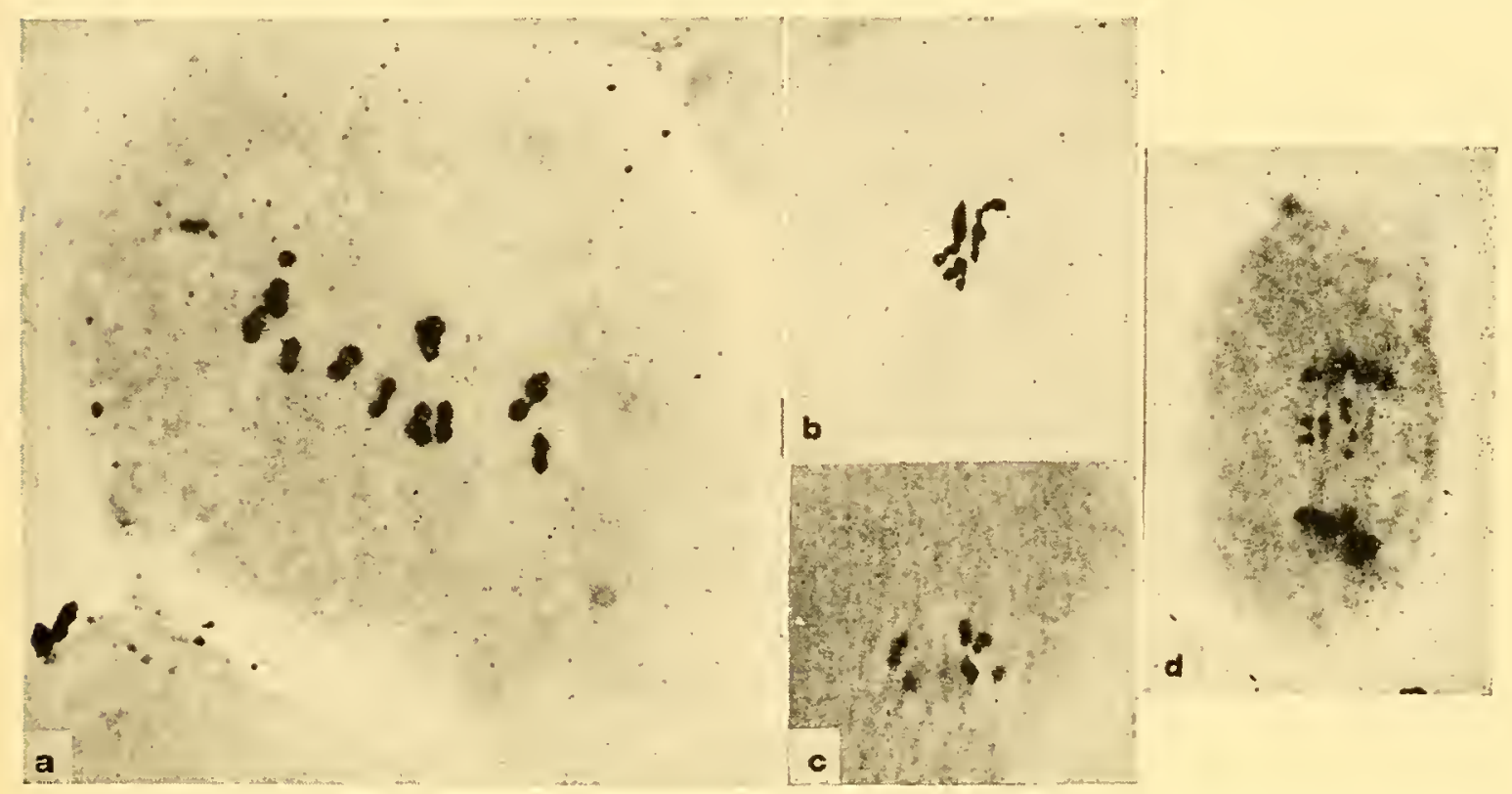

FIG. 201.-Chromosomes in heteroploid maize. $a$, metaphase $I$, showing three trivalents, seven bivalents, and one univalent. (Spot at upper left is not a chromosome.) $b$, three trivalents in metaphase. $c$, trivalent disjoining two from one. $d$, tardily split univalents ready to separate at equator of spindle. (After McClintoch, 1929a.)

Fig. 200). The genetic data indicate that crossing-over may occur between all three chromosomes (in different regions), that it takes place when six chromatids are present, and that crossover values between certain genes differ somewhat in diploids and triploids. ${ }^{7}$

In the first meiotic mitosis the three members of a trivalent tend to disjoin at random, except in those regions near the spindle-attachment point where crossing-over may affect the type of disjunction. In the second mitosis the halves of all of the chromosomes pass to opposite poles. Often one chromosome of the group lags and is lost. Typically, as in triploid $Z e a$, the different trivalents are variously distributed in mitosis $I$ (Fig. 201), so that spores are formed with chromosome numbers ranging from the monoploid to the diploid. Although many of the microspores

${ }^{7}$ Bridges and Anderson (1925) and Redfield (1930, 1932) on Drosophila. 
and male gametes with unusual numbers may not function, especially in competition with normal ones, the megaspores and female gametes are more successful, so that plants with various chromosome numbers appear among the progeny of a triploid. Meiotic irregularity may result in almost complete sexual sterility in some triploid plants; these are often propagated regularly by vegetative means. ${ }^{8}$

The behavior of a pair of Mendelian characters when three homologous chromosomes are present is well illustrated in trisomic maize. ${ }^{9}$ In Zea the smallest chromosome (number $\mathrm{X}$ ) of the set carries a factor $R$ for colored aleurone; when the factor is recessive $(r)$, it tends to make the aleurone colorless. In the sporocytes of plants trisomic for this chromosome there are usually nine bivalents and one trivalent. The distribution of the three members of the trivalent is such that somewhat more than half of the pollen grains carry the normal set of 10 chromosomes, while the remainder (somewhat less than half because of lagging on the part of the extra chromosome) carry 11, including two number X's. However, the 11-chromosome grains do not germinate and develop pollen tubes as rapidly as the competing normal ones, so that the 11-chromosome male gametes tend to be eliminated. From this it follows that the extra chromosome is practically always transmitted to the next generation through the female gamete alone. ${ }^{10}$

On the basis of these facts it is possible to calculate the characteristic ratios $^{11}$ of types expected after selfing and back-crossing plants with $R R R$ ("triplex"), RRr ("duplex"), Rrr ("simplex"), and $r r r$ ("nulliplex"). It is found that the results conform satisfactorily with the expectations. Moreover, since the elimination of male gametes with an extra chromosome is much more complete than that of the corresponding female gametes, the ratios yielded by reciprocal crosses differ. In actual tests the following results were obtained. When pollen from an $\mathrm{Rrr}$

${ }^{8}$ E.g., Hyacinthus, Narcissus, Tulipa, Canna, Hemerocallis, and Rosa.

${ }^{9} \mathrm{H}$. Hill (1930), MeClintock and Hill (1931).

10 The same is true in strains trisomic for chromosome II, which carries the $B-l g$ linkage group (Besley, 1930).

11 Blakeslee and Farnham (1923) calculated the ratios for Datura on the basis of a random distribution, two from one, of the three members of the trisome concerned. So calculated, the back-cross ratios for an $R r r$ maize plant would be $1 R: 1 r$ when the trisomic plant is used as the female parent, and $1 R: 2 r$ when it is used as the male parent. The corresponding ratios for an $R R r$ plant would be 5:1 and 2:1. However, if ealculations are made on the basis of the random distribution of six chromatids, the ratios tend to be $7: 8$ and $1: 2$ for an $R r r$ plant, and $4: 1$ and $2: 1$ for an $R R r$ plant. The degree of approximation to these latter ratios may be expected to vary with the distance of the genes concerned from the spindle-attachment point, genes near this point tending rather to give the ratios based on the distribution of three whole chromosomes. This is because the chromatids in this region do not segregate at random, and show a relatively low frequency of crossing-over (pp. 302,323); hence only those genes located far enough away from the spindle-attachment point to escape these restrictions actually give the ratios based on random chromatid distribution. 
plant was placed on the silks of an $r r$ plant, the ear produced 13 colored and 27 colorless kernels (expectation, 1:2). Pollen from an $R R r$ plant on silks of an $\mathrm{rr}$ plant gave 646 colored and 355 colorless kernels (expectation, 2:1). Pollen from a normal $r r$ plant on the silks of an $R R r$ plant gave 819 colored and 213 colorless kernels (expectation, about $4: 1$ ). A certain amount of deviation from expected ratios in such cases is due to the lagging and loss of the extra chromosome in meiosis, so that the spores and gametes with $n$ chromosomes actually outnumber those with $n+1$. This results in a deficiency of dominant plants among the offspring.

In the foregoing example one dominant factor $(R)$ is sufficient to overcome the effect of two recessives $(r r)$. Other genes are known of which this is not true, the appearance of the triploid tissue depending rather upon which type of factor outnumbers the other. ${ }^{12}$ In such cases, of course, a different set of ratios is expected.

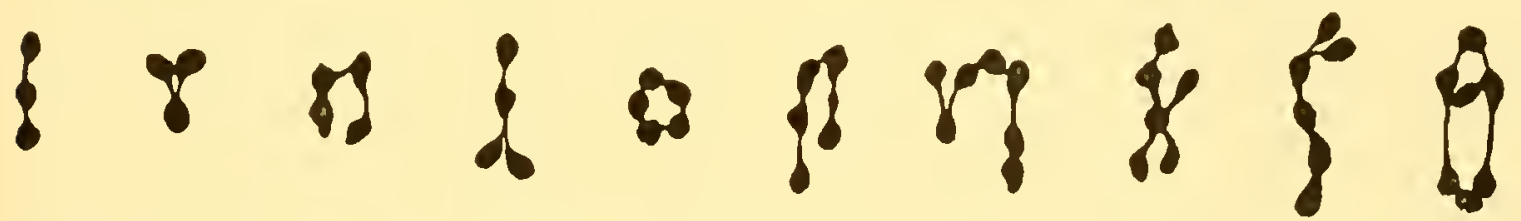

Fig. 202.-Multivalent chromosomes in Prunus laurocerasus. (After Meurman, 1929b.)

Other Heteroploids. - The cytological and genetical features of plants with five or more homologous chromosomes or chromosome sets are not so well known as those of triploids and tetraploids. The chromosome configurations in meiosis are very variable. In the pentaploid Tulipa Clusiana (60 chromosomes) there are univalents, bivalents, trivalents, quadrivalents, and quinquevalents; but among these the quinquevalents are least frequent and the bivalents most frequent. In the octoploid Dahlia variabilis (64 chromosomes) there are bivalents, quadrivalents, sexavalents, and octovalents, with the last named the least frequent. In Dahlia Merckii with 36 chromosomes $(4 n+4)$ there are quadrivalents and two sexavalents, suggesting that the plant is a tetraploid with two of the eight chromosomes of the set still further reduplicated. In the $22 n$ Prumus laurocerasus there are many multivalent associations, with trivalents most, common (Fig. 202). Such variations in plants not known to be truly autoheteroploid may be due in part to incomplete homology. The genetic ratios observed in such plants will obviously depend upon the strength of dominance, the manner in which the multivalents are distributed in meiosis, and the viability and fertility of the various heteroploid gametes and zygotes. ${ }^{13}$

12 East and Hayes (1915) on Zea endosperm, von Wettstein (1923) on triploid moss gametophytes.

${ }^{13}$ Newton and Darlington (1929) on Tulipa, Lawrence (1929) on Dahlia, Meurman (1929b) on Prunus. Haldane (1930b) gives expected genetie ratios for $4 n, 6 n, 8 n$, $10 n, 12 n$, and $16 n$ autopolyploid types. 
Monoploid Sporophytes.-In a number of angiosperm genera, individual plants have occasionally appeared with the gametic rather than the zygotic number of somatic chromosomes. ${ }^{14}$ In several of these, including Datura stramonium, Zea Mays, Crepis capillaris, Enothera Hookeri, and Triticum monococcum, it has been shown that the complement is truly monoploid (Fig. 203). The evidence at hand indicates that such monoploid sporophytes, which are relatively rare, usually arise as the result of the parthenogenetic development of the egg. Since only one chromosome of each kind is present in the sporocyte, there is no normal
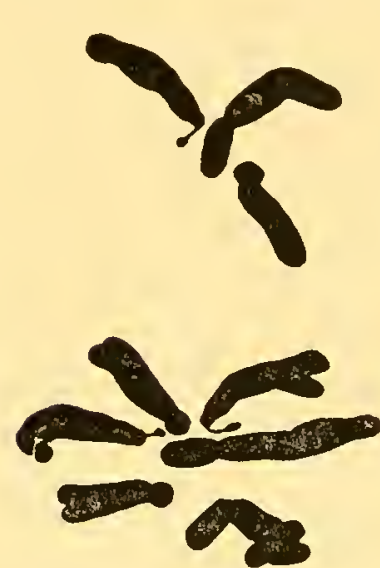

FIG. 203.-Somatic chromosome complements from roots of monoploid and diploid plants of Crepis capitlaris. (After Holtingshead, 1930c.)

rearite synapsis, all of the chromosomes appearing as univalents at the end of the meiotic prophase. The distribution of these chromosomes is not the same in all cases. In Datura they are distributed at random to the two poles in $I$ and divide in $I I$. Although the plants are highly sterile as a result of this irregularity, occasional spores with all 12 chromosomes are developed, so that selfing yields a few diploid offspring. In Enothera Hookeri a random distribution of the seven chromosomes occurs in about one-quarter of the sporocytes; in the remainder there is a splitting and separation of some or even all of the chromosomes and a second splitting may follow in $I I$ (Bleier). In the practically monoploid Matthiola ( $7+$ a fragment) some of the sporocytes show irregular distribution of the chromosomes, while the majority show a splitting of all of them in the single mitosis occurring. The dependence of fertility upon diploidy is well illustrated by a monoploid and sterile Crepis plant which produced a diploid and fertile branch. Even in this branch, however, synapsis was somewhat deficient, indicating that diploidy and homozygosity do not always insure fully normal meiotic regularity.

Other Features of Autoheteroploids.-Tetraploid mutants are frequently somewhat larger than the corresponding diploids; this is true

${ }^{14}$ E.g., Datura (Blakeslee et al., 1922; Blakeslee and Cartledge, 1926, 1927; Belling and Blakeslee, 1923, 1927); Crepis capillaris (Hollingshead, 192Sa, 1930c); Matthiola (Lesley and Frost, 1928); Nicotiana glutinosa (Goodspeed and Avery, 1929b); Nicotiana tabacum (Clausen and Mann, 1924; Chipman and Goodspeed, 1927; Ruttle, 1928; see our p. 365); Solanum (Jørgensen, 1928); Triticum (Gaines and Aase, 1926); Lycopersicum (Lindstrom, 1929; Lindstrom and Koos, 1931); Enothera (S. H. Emerson, 1929; Gates, 1929b; Gates and Goodwin, 1930; Davis and Kulkarni, 1930; Bleier, 1933a; Catcheside, 1932); Zea (Stadler and Randolph, 1929; Randolph, 1932); Oryza (Morinaga and Fukushima, 1931b).

In his paper on Enothera (1933a; see also 1933b) Bleicr classifies known sporophytes with the gametic chromosome number (these he calls "haplodiplonts") according to whether this number is truly monoploid or not, and discusses the bearing of their chromosome behavior on ecrtain problems of meiosis. 
of the "gigas" forms of Enothera. Often diploids, triploids, and tetraploids are of about the same size, but monoploids are almost always noticeably smaller. Tetraploid species may even be smaller than the related diploid species, as in Plantago and Alyssum. Silene ciliata has $4 n$ and $16 n$ races which are practically alike in appearance. In a chromosomal chimera the portions with different chromosome numbers may differ in size (Fig. 204). In some cases gigantism is associated with an increase in the size of the chromosomes but not in their number; this is true of Primula sinensis and Phragmites communis. In moss gametophytes, diploids tend to be larger than monoploids, and tetraploids larger than diploids. Here the increase in plant size is a compromise between a decrease in the number of cells and an increase in their volume, which tends to be proportional to the number of chromosomes. In polyploid tulips the surface area of the pollen grain and of its nucleus is found to vary proportionally with the number of chromosome sets. The monoploid, diploid, and tetraploid pollen grains show a volume ratio of $8: 27$ : 64 (Fig. 205)..$^{15}$

The histological structure of heteroploids usually

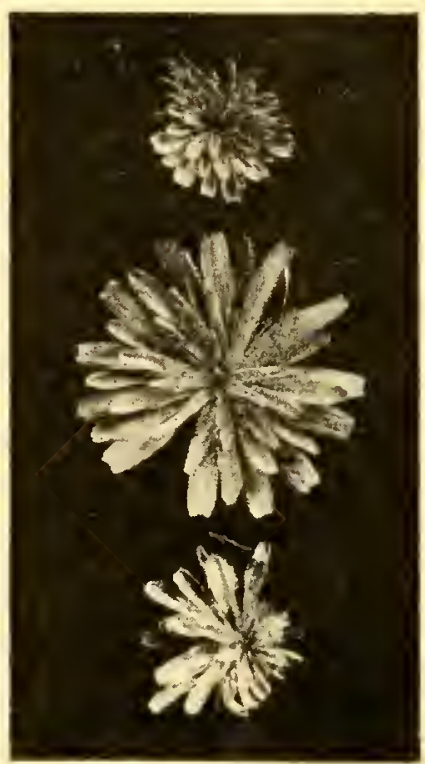

FIG. 204.-Flower heads borne on monoploid individuals of Crepis capillaris. Top: monoploid head. Middle: diploid head. Bottom: chimeral head, with monoploid and diploid sectors. (After Hottingshead, 1930c.) differs in certain respects from that of the corresponding diploids. In
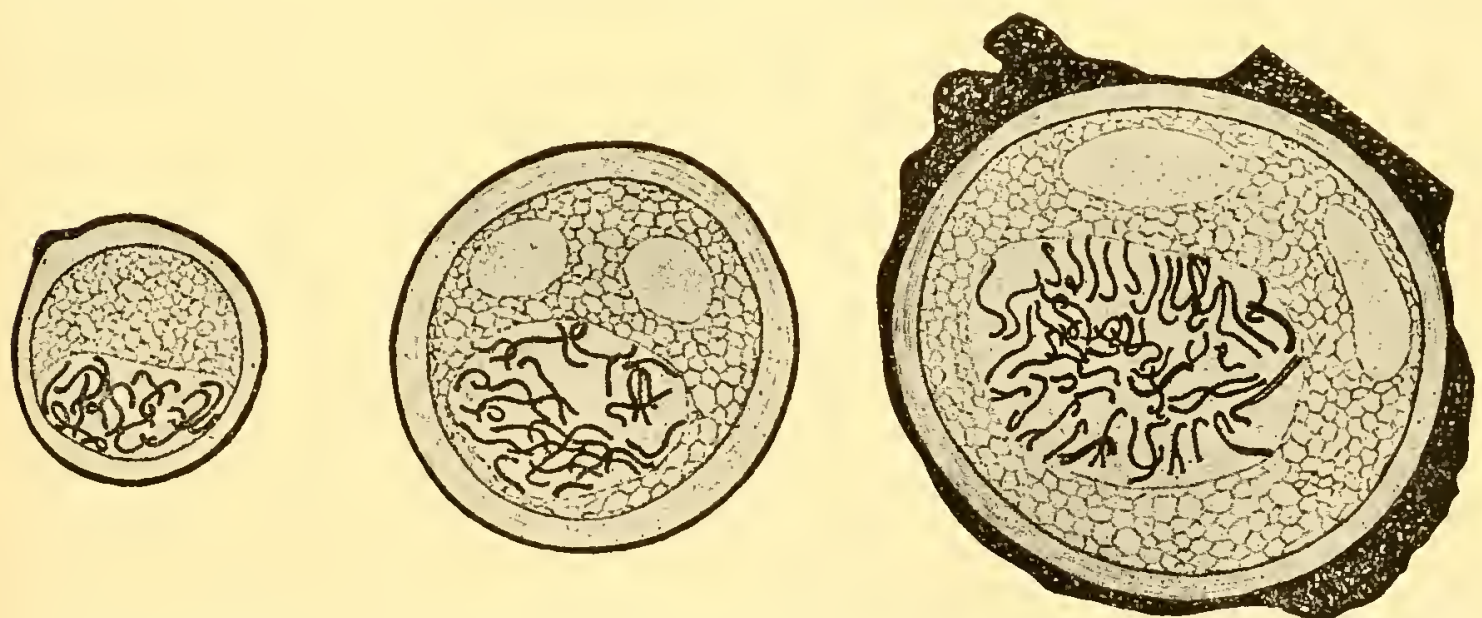

Fig. 205.-Microspores of Tulipa suaveolens with 12 (monoploid), 24 (diploid), and 48 (tetraploid) chromosomes. (After de Mol, 1928d.)

Solanum the cells of the mesophyll, stomates, hairs, and vascular bundles are all larger in the tetraploids, and the leaves are greener because of the

${ }^{15}$ Sinotô (1925) on Plantago, Jaretzky (1928a) on Alyssum, Blackburn (1927a) on Silene, Gregory $(1909,1914)$ on Primula, Tischler (1918) on Phragmites, von Wettstein (1923 et seq.) and Schratz (1924) on mosses, de Mol (1928d) on Tulipa, de Mol (1932b) on Narcissus. 
presence of the larger and more numerous chloroplasts. In Zea the monoploid, diploid, tetraploid, and octoploid plants can be distinguished by examining the size and distribution of the stomates. In Datura each of the 12 primary $2 n+1$ mutants differs characteristically from all of the others in its histological structure. ${ }^{16}$ Aneuploid plants usually differ in appearance from the normal diploid much more than do the tetraploids, triploids, or other euploid forms. There is a "balance" within the complement which is maintained even when three, four, five, or more sets are present, but which is disturbed when any set lacks one or more of its members.

The effect of heteroploidy upon sexual fertility has already been mentioned. Autotetraploids often show comparatively little sterility because the chromosomes, although present in double the usual number, are all able to form synaptic groups of even number (bivalents or quadrivalents), enabling the meiotic mitoses to be carried out without great irregularity. In triploids, on the contrary, the odd number of elements in the synaptic groups (trivalents) and the frequently unassociated univalents interfere with proper meiotic distribution, so that a large proportion of the spores or gametes are non-functional. In the higher euploid types such as pentaploids, hexaploids, etc., the meiotic irregularity and sterility tend to be rather great, especially in the ones with odd numbers of sets. Thus in Rubus the pollen sterility is very low in $4 n, 6 n$, and $8 n$ forms, but very high in $3 n$ and $5 n$ forms (Longley, 1927a). For similar reasons aneuploid types tend to show considerable sterility. Frequently a change to the heteroploid condition causes greater sterility in one sex than in the other. Thus in a triploid Enothera the eggs are apparently able to function with any chromosome number from 7 to 14 , whereas the only male gametes functioning are those with either 7 or 14, because the pollen grains with intermediate numbers abort (van Overeem, 1921, 1922). In some heteroploids the sterility is so complete in one or both sexes that the type cannot persist except through asexual propagation. This is notably the case in heteroploids of hybrid constitution, as will be shown in the next chapter. Asexual reproduction, however, is not necessarily an indication of hybridity. ${ }^{17}$ The fact that organisms reproducing asexually without effective meiosis show no Mendelian segregation affords strong support to the chromosome theory of heredity. The effect of heteroploidy upon the sexual development of the organism will be discussed further in Chapter XXIII.

${ }^{16}$ Winkler (1916) on Solanum, Randolph (1932) on Zea, Sinnott and Blakeslee (1922) on Datura. See also Tupper and Bartlett (1916) on Enothera.

${ }_{17}$ Tischler $(1928 c)$ reviews many cases of spore sterility in angiosperms. 


\section{CHAPTER XXII}

\section{THE CYTOGENETICS OF HYBRIDS}

In modern genetics the principal criterion of hybridity is heterozygosity rather than the relative taxonomic standing of the parents known or supposed to have been crossed. The union of any two protoplasts differing in hereditary potency, no matter how slight the difference, constitutes an act of hybridization in the fundamental sense. Accordingly, a hybrid is defined as an individual arising from the union of genetically dissimilar gametes ${ }^{1}$ and maintaining the resulting heterozygosity in its somatic nuclei. Moreover, its spores and gametes are commonly of more than one kind.

The degree of heterozygosity differs widely in different cases. It may involve only one or relatively few genes, as in the ordinary intraspecific hybrids so widely studied by geneticists; here there is usually no disturbance of the normal pairing and distribution of the chromosomes in meiosis. As will be shown below, the chromosomes in many interspecific and even in some intergeneric hybrids may show a high degree of compatibility in both somatic development and meiosis, fertility not being impaired in any considerable measure. In other instances, where the chromosome sets brought together differ widely in genic composition, the hybrid may undergo successful vegetative development and then prove to be sexually sterile as a result of a partial or complete failure of meiosis. This difficulty at the meiotic period commonly has its chief visible expression in asynapsis or desynapsis (see p. 345), this being followed by other irregularities which prevent the formation of functional spores and gametes. Irregularities of this kind are even more pronounced when the parental chromosome groups differ in number of members as well as in genic composition. Deficient synapsis is not, however, wholly responsible for the sterility observed in hybrids, since degenerative changes often begin well in advance of the synaptic period.

As a general rule, one may expect the difficulty of obtaining a hybrid to increase with numerical and qualitative dissimilarities in the chromosome complements of the parental types. Failure may be due to the inability of the pollen tube to grow properly in a "foreign" style, to a lack of gametic interaction, to a developmental incapacity on the part

1 This holds for diploid and most other hybrids but not without qualification for those of the amphidiploid type (see p. 369). It is understood that a hybrid individual may also arise from a hybrid parent by vegetative means. 
of the zygote at some stage, or to unfavorable relations existing between the embryo and the surrounding tissues. Even when a hybrid is obtained it may prove to be sexually sterile, as pointed out above, and so fail to maintain its type.

In earlier chapters we have dealt with ordinary Mendelian hybrids and with those special cases in which heterozygosity is automatically maintained by virtue of peculiar meiotic mechanisms and lethal factors (p. 336). The present chapter is mainly a series of examples of the more prevalent modes of chromosome behavior which have been observed in hybrid plants derived from fairly wide crosses. The chief interest of these phenomena is in the light they throw upon the causes of normal chromosome behavior and upon the problem of the origin of new stable and fertile types through hybridization. Most of the researches so far carried out in this field have dealt with meiotic phenomena and their genetic results. ${ }^{2}$ Somatic chromosome complements have also been studied, but relatively little attention has been devoted to syngamy in such plants.

Diploid Hybrids with Good Synapsis and Fertility.-When Zea Mays $(n=10)$ is crossed with the Durango or the Chalco race of Euchlcena mexicana $(n=10)$, the fertile $F_{1}$ hybrid shows 10 bivalents which behave regularly in meiosis. In subsequent generations the parental types are recovered among others, indicating a random distribution of the chromosomes and the occasional reconstitution of essentially normal Zea and Euchlona sets. Furthermore, some crossing-over occurs between the Zea and Euchlcena chromosomes. This high degree of chromosome compatibility, which is somewhat exceptional in interspecific or intergeneric hybrids, doubtless indicates a close natural relationship between the two parents. When a Florida race of Euchlcena mexicana is crossed with $Z e a$, the hybrid shows some unequal chromosome pairs and considerable sterility. Triploid hybrids formed by crossing Zea with the tetraploid Euchlona perennis show much meiotic irregularity and a rather high degree of sterility. ${ }^{3}$

In the Lepidoptera fertile interspecific and intergeneric hybrids are frequently obtained. For example, a hybrid between Chorocampa elpenor $(n=29)$ and Metopsilus porcellus $(n=29)$ shows regular synapsis and fertility, as well as the combined characters of the parents. In moth hybrids the amount of synapsis varies with the closeness of relationship of the parents (Federley, 1923, 1928).

That good synapsis alone does not insure complete fertility is shown by hybrids between Pisum humile $(n=7)$ and $P$. sativum $(n=7)$.

${ }^{2}$ For a more extensive account of chromosome behavior in polyploids and hybrids, see Darlington (1932a).

${ }^{3}$ Data in this paragraph are from Kuwada (1919), Longley (1924c), Beadle (1932c), and Randolph. 
Here meiosis is regular in $F_{1}, F_{2}$, and $F_{3}$, but the high sterility and the appearance of abnormal types in $F_{2}$ and $F_{3}$ indicate that many of the combinations of humile and sativum chromosomes formed by random assortment function poorly or fail completely in development (Lutkov, 1930). Certain Digitalis hybrids are also sterile in spite of good synapsis (Haase-Bessell, 1932a).

Partial Asynapsis and Its Results.-Some varietal crosses in Viola arvensis show regular Mendelian segregation, but others exhibit faulty synapsis coupled with genetic irregularities. When $V$. arvensis $(n=17)$ is crossed with $V$. tricolor $(n=13)$, the $F_{1}$ hybrid shows various numbers of bivalents and univalents, and some sterility. Later generations include

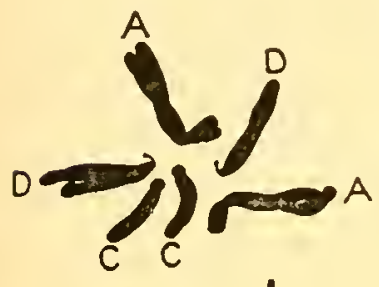

1
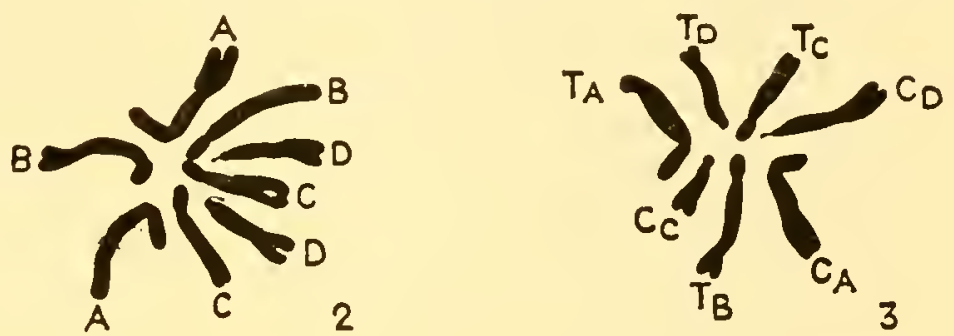

2
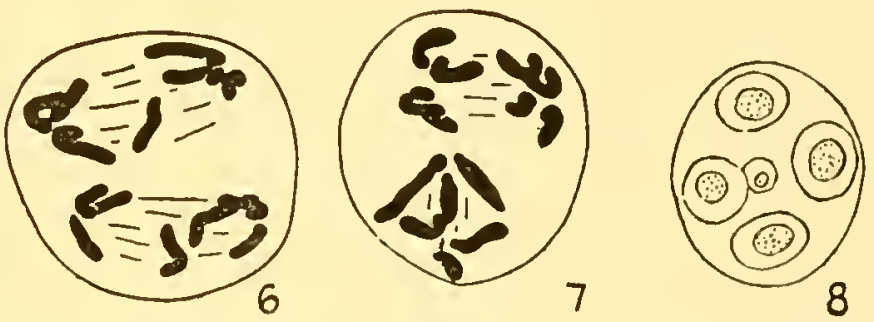

FIg. 206.-Chromosome behavior in $F_{1}$ hybrid, Crepis capillaris $\times$ Crepis tectorum. $1,2,3$, somatic complements of $C$. capillaris, C. tectorum, and $C$. capillaris $\times$ tectorum. 4,5 , first meiotic mitosis in hybrid. 6,7 , second meiotic mitosis. 8 , group of microspores, including an extra one formed after chromosome lagging. The satellite on the tcctorum $D$-chromosome does not show in the hybrid. (After Hollingshead, 1930a.)

the parental types and a number of new ones which are constant and fertile (J. Clausen, 1924, 1926, 1931c).

Crepis tectorum $(n=4) \times C$. capillaris $(n=3)$ gives an $F_{1}$ hybrid in which all of the seven parental chromosomes can be distinguished on the basis of their morphology (Fig. 206). For some reason, however, the tectorum satellite is not evident in the hybrid. When one strain of tectorum is used in this cross, three bivalents and one univalent appear most often in meiosis, but when another strain is used this configuration is least common. The univalents divide in either meiotic mitosis, and the hybrid is almost completely sterile (Hollingshead, 1930a; M. Nawaschin, 1927b).

Synapsis in Relation to Polyploidy in Hybrids.-Highly characteristic of many hybrids is the type of meiosis first observed in Drosera by Rosenberg (1904a, 1909b). When Drosera rotundifolia (10 gametic chromosomes) was crossed with $D$. longifolia (20 gametic chromosomes), 
the 30-chromosome hybrid (D. obovata) showed 10 bivalents and 10 univalents in meiosis. Two interpretations of such synaptic behavior were possible: (1) the rotundifolia chromosomes might have paired with 10 of those from longifolia (allosynapsis), leaving the other 10 of longifolia unpaired; (2) the longifolia chromosomes might have paired among themselves (autosynapsis) because of diploidy in the longifolia gametic complement, leaving the rotundifolia chromosomes unpaired. Subsequent researches on a number of genera have shown that in some auto-

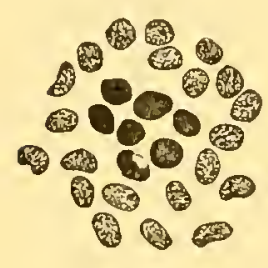

A

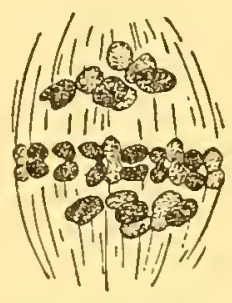

B

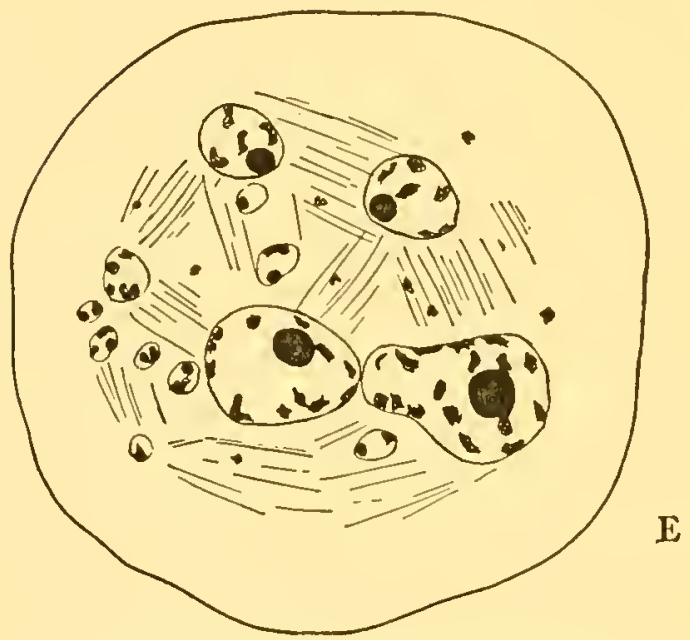

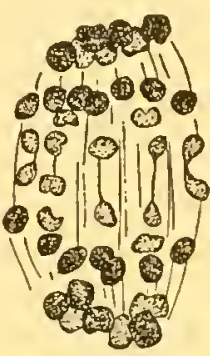

C
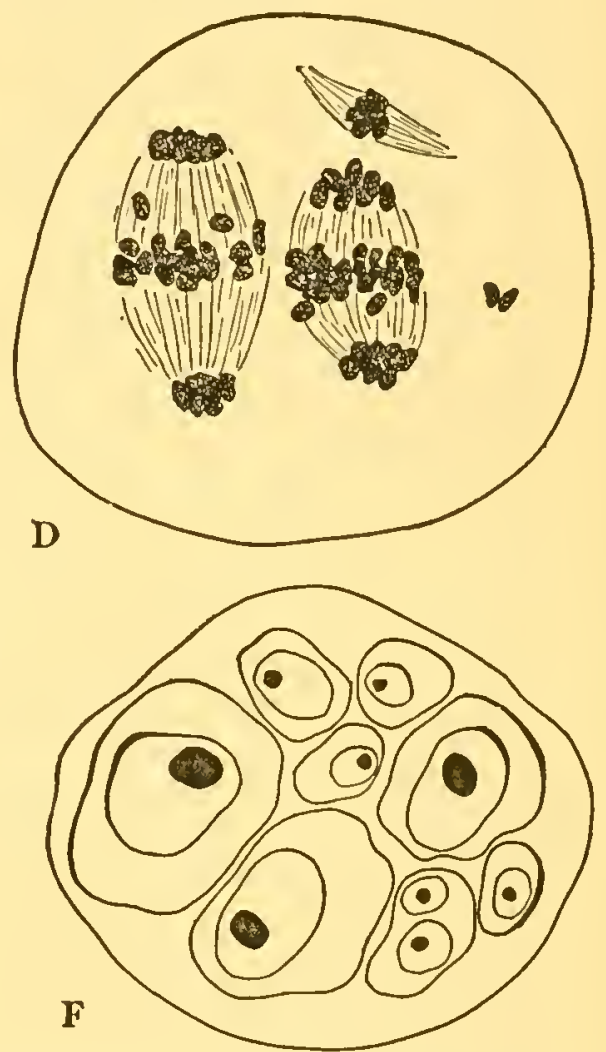

Fig. 207.-Chromosome behavior in Rosa hybrids. A, polar view of the chromosomes in the metaphase of the first meiotic mitosis in the microsporocyte of $R$. glauca dilatans, showing 7 bivalents surrounded by 21 univalents. $B$, anaphase of mitosis $I$ in $R$. glauca plebeia; the bivalents have disjoined and the split univalents lie at the equator. $C$, late anaphase in $R$. pomifera Grenieri. $D$, division $I I$ in $R$. Junzillii; ehromosomes which failed to reach poles in $I$ have formed an extra mitotic figure. $E$, telophase of $I I$ in the same, showing the formation of supernumerary nuclei. $F$, polyspory (more than 4 spores) resulting from mitotic irregularities in $R$. pomifera. (After Täckholm, 1922.)

synapsis, in others allosynapsis, and in still others both types of synapsis may be the rule. Synapsis is generally regarded as an indication of homology in the chromosomes involved, and when used judiciously this criterion is a useful one in the analysis of hybrid chromosome complements.

An example of autosynapsis is afforded by 24-chromosome Crepis hybrids formed by crossing C. setosa (4 in gamete) with C. biennis (20 in gamete). In the microsporocytes of this hybrid there are 10 bivalents and four univalents; this indicates autosynapsis among the chromosomes of biennis, a polyploid species. Allosynapsis seems to obtain in the asexually reproducing hybrids in the section Canince of the genus Rosa (Fig. 207). In these hybrids, which have 28 (tetraploid), 35 (pentaploid), 
and 42 chromosomes (hexaploid), there are regularly seven bivalents in the microsporocytes, the other chromosomes $(14,21$, and 28 in the three types) remaining unpaired. Evidently in each case a set of seven descended from one gamete pairs with a set of seven from the other gamete, leaving the extra sets unpaired. Both types of synapsis must occur in hexaploid Papaver hybrids with 42 chromosomes formed by crossing $P$. nudicaule ( 7 in gamete) with $P$. striatocarpum ( 35 in gamete). These hybrids show 21 bivalents in meiosis, indicating a synapsis of all six sets irrespective of gametic derivation. On the theory that synapsis is determined mainly by homology, the nudicaule set of seven must be

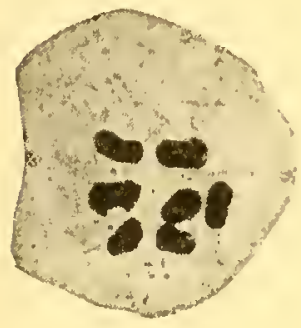

A

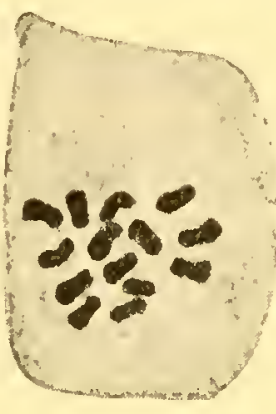

$\boldsymbol{B}$

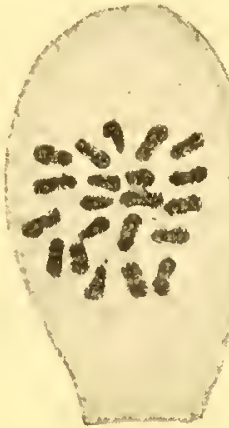

$C$

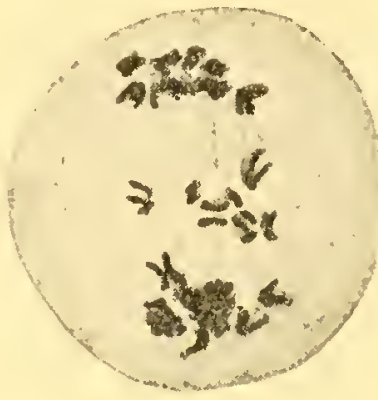

$D$

FIG. 208.-The chromosomes of Triticum. A, metaphase of division $I$ in $T$. monococcum; 7 chromosomes. $B, T$. durum; 14 chromosomes. $C, T$. vulgare; 21 chromosomes. $D$, division $I$ in hybrid between members of emmer group $(n=14)$ and vulgare group $(n=21)$; the 14 bivalents have disjoined and the 7 univalents are separating equationally. (After Sax, 1922a.)

homologous with at least one set in striatocarpum, and the four other sets of the latter species must be of but one or two kinds. In certain Digitalis hybrids with 72 chromosomes (48 lutea +24 micrantha), 36 bivalents appear at diakinesis, indicating the occurrence of autosynapsis. ${ }^{4}$

The behavior of the chromosomes in meiosis when both bivalents and univalents are present varies considerably in different cases. The bivalents rather regularly disjoin in $I$ and divide in $I I$, but the univalents behave more irregularly. In mitosis $I$ they may wander at random as wholes to the two poles (Drosera and some Triticum hybrids); or they may split and occupy the equator, the daughter halves then separating poleward (some Triticum hybrids and microsporocytes of Canince roses; Figs. 207, 208); or some of them may distribute as wholes while the others split and separate. This variable behavior seems to be associated with the time at which the division of the univalent chromosome, particularly its spindle-attachment region, takes place. If the division is sufficiently early, the univalent reacts by entering the equator and separating; whereas, if the division is delayed, it fails to behave in such a manner and tends to be carried toward the pole nearer which it happens

${ }^{4}$ Collins and Mann (1923) on Crepis; Täckholm (1920, 1922) on Rosa; Ljungdahl (1924) on Papaver; Haase-Bessell $(1921,1932 a)$ on Digitalis. 
to lie. Rarely all of the univalents may pass to one pole (megasporocytes of Canince roses). In mitosis $I I$ the chromosomes which disjoined as bivalents in $I$ split and separate, as in normal meiosis. The former univalents may be distributed at random as wholes, or they may divide and separate, sometimes even after having divided in $I .{ }^{5}$ Very often one or more of the univalents may lag behind and fail to be included in either daughter nucleus. They may then degenerate, but often they form small nuclei about which small supernumerary spores ("microcytes") are differentiated (Figs. 206, 207). Such cells are functionless. Moreover, many of the spores and gametes which are viable carry very abnormal chromosome complements. Hence plants with such meiotic irregularities are characterized by considerable sterility and the production of abnormal progeny.

The union of gametes with altered complements naturally leads to diversity in the following generations. Many combinations do not result in viable forms, but others give rise to types which develop normally until meiosis, when chromosomal aberrations may again appear. In successive hybrid generations meiotic irregularity and genetic instability are observed as long as extra unpaired chromosomes are present. These tend to be eliminated gradually through irregular mitoses, until relatively stable types with one or more numbers of regular chromosome pairs are established. Frequently, in the course of such adjustment through elimination of extra chromosomes, some members of the complement become lost while others are duplicated, so that, although the chromosome number of one of the original parents may be restored, the assortment is a new one and the plants are correspondingly altered in character. This again emphasizes the fact that it is the qualitative constitution of the complement, rather than the mere number of chromosomes, that is of primary significance in species alteration.

Analysis of Alloheteroploid Types.-The formation of bivalents rather than multivalents in the euploid plants mentioned above is attributed to dissimilarities in the chromosome sets, that is, some of the sets are homologous enough to undergo synapsis, while others are not. The plants in question are alloheteroploid, at least in part. This condition is generally ascribed to hybridization. It is probable, however, that in autopolyploid forms differences sometimes develop in the associated sets through mutation, translocation, and the like, the differentiation finally being great enough to prevent synapsis between the members of certain sets. At the same time, there is evidence which points to the

${ }^{5}$ Two suecessive splittings are reported for Hieracium (Rosenberg, 1917), Raphanus $\times$ Brassica (Karpechenko, 1927b), and Rosa (Tackholm, 1922; Hurst, 1931). The types of behavior on the part of unpaired elements in $I$ are tabulated by Täckholm. 
union of gametes carrying dissimilar sets as a more frequent cause of allopolyploidy.

Although the degree of validity of this basis for the analysis of chromosome complements is a subject of debate, certain cases may be cited to illustrate the method. One of the best known is that of Nicotiana tabacum, which has 48 somatic chromosomes. When $N$. sylvestris (12 in gamete) is crossed with $N$. tomentosa (12), the resulting hybrid shows almost complete asynapsis among its 24 chromosomes. This is taken to mean that the sylvestris set $S$ and the tomentosa set $T$ are not closely homologous. Similarly, the hybrid $N$. sylvestris $(12) \times N$. rusbyi (12) shows no synapsis. When $N$. tabacum (24) is crossed with $N$. sylvestris (12), the 36-chromosome hybrid shows 12 bivalents and 12 univalents in meiosis. Also, when $N$. labacum (24) is crossed with $N$. tomentosa or $N$. rusbyi (12), the hybrid shows 12 bivalents and 12 univalents. Finally, in the triple hybrid [tabacum $(24) \times$ rusbyi $(12)] \times$ sylvestris (12), there are 24 bivalents. From these data on synapsis it is inferred that the gametic complement of $N$. tabacum actually consists of two sets of 12 , one of them being homologous with the tomentosa set $(T)$ and the other with the sylvestris (S) (or rusbyi) set. Hence $N$. tabacum is an allotetraploid hybrid with the somatic composition TTSS, its gametes carrying TS. It is believed to be a descendant of tomentosa (or tomentosiformis) and sylvestris or closely related progenitors, the cross being followed by chromosome doubling and the attainment of stability and fertility. ${ }^{6}$ Other cases of this kind will be described in the next section.

On the basis of genetic data and synaptic behavior, Hurst has advanced the theory that in the genus Rosa there are five fundamental diploid species, each with its characteristic set of seven chromosomes. The diploid somatic complements of these species may be represented as $A A, B B, C C, D D$, and $E E$. Multiplication of any one of the sets gives autopolyploid "varieties," such as $A A A A$ or $D D D$. Hybridization leads to the establishment of allopolyploid "species." These may be "regular," with bivalents only (e.g., $A A B B$, or $A A D D E E$ ); or "irregular," with bivalents and univalents (e.g., $A A B D E$, or $A A C C E$ ). Although the limits of the applicability of this theory are not agreed upon by all workers, the responsibility of hybridization for the production of many known rose types is unmistakable. ${ }^{7}$

On similar grounds Morinaga $(1928,1929)$ postulates the occurrence of three kinds of basic set in Brassica: $A$, with 10 chromosomes; $B$, with 8 ; $\mathrm{C}^{\prime}$, with 9 . Synaptic behavior in hybrids and the numbers present

${ }^{6}$ R. E. Clausen and Goodspeed (1925), Goodspeed and Clausen (1927b, 1928), Brieger (1930), Clausen (1932). See the eriticism by East (1933).

${ }^{7}$ Hurst (1925 et seq.), Erlanson (1929, 1931ab), Harrison and Blackburn (1927), Blackburn and Harrison (1924b), Blackburn (1925). 
suggest that the gametic complements of certain species are made up as follows: cernua, $A B$; chinensis, $A$; Rapa, $A$; Napella, $A C^{\prime}$. The hybrids tend to resemble the parent with the higher chromosome number.

Very extensive studies of this kind have been made upon Triticum and the closely related genus Egilops. ${ }^{8}$ In Triticum the three main groups of species are characterized by different chromosome numbers. In the einkorn wheats (monococcum, villosum, agilopoides) the gametic number is 7 ; in the emmer wheats (dicoccum, polonicum, turgidum, durum) the gametic number is 14 ; in the spelt wheats (vulgare, compactum, spelta) the gametic number is 21 . In many of the hybrids between members of these groups, as well as in those between Triticum and Egilops, the chromosomes tend very strongly to form a characteristic number of bivalents and univalents, as in Drosera and other cases mentioned on foregoing pages. There is, however, some variation in behavior under different conditions.

On the basis of the synaptic relations exhibited, the theory has been developed ${ }^{9}$ that the chromosome complements of Triticum and Egilops combine in various ways as many as five different but related sets of 7 $(A, B, C, D, E)$. In Thompson's recent tabulation these sets are present as follows in the gametic complements: einkorns, $A$; emmers, $A B$; spelts, $A B C$; Egilops cylindrica, CD; E. ovata, DE; E. crassa, C. Other species of Egilops have less certain combinations, and Secale (rye) has $D$.

Studies on the morphology of Triticum chromosomes suggest that the species with the larger numbers of sets are not simply autopolyploid derivatives of some ancestor with two similar somatic sets, for in four species about 17 different types of chromosome are found (Kagawa). Some types appear in only one of the species, while others appear in two, three, or four. This suggests hybridization in the differentiation of such species. On the other hand, the chromosomes in certain crosses may undergo synapsis in spite of their differences in type, which points to translocation and deletions as probable causes of some of the cytological diversities in the members of this genus. That synaptic behavior is a reliable measure of relationship in grains is questioned by Bleier, who lays emphasis on the probable rôle of the spindle substance in determining irregular meiosis in hybrids $(1930 c, 1931 a)$. Furthermore, it is to be borne in mind that failure to pair may often be due not so much to lack

${ }^{8}$ Sax (1922 et seq.), Watkins (1924 et seq.), Kihara (1919 et seq.), Gaines and Aase (1926), Kagawa $(1927 a b, 1929 a c)$, Bleier $(1928 a b, 1930 a c d, 1931 d)$, W. P. Thompson (1926 et seq.), Melburn and Thompson (1927), Huskins (1928ab), Jenkins (1929), Percival (1930), Tschermak and Bleier (1926), Tschermak (1929), Kihara and Nishiyama $(1928,1930 a)$, Kihara and Katayama (1931). For convenient general accounts, see Aase (1930), Watkins (1930), and Thompson (1931b). See also footnote 13, p. 342.

${ }^{9}$ Gaines and Aase (1926), Sax (1928), Kihara (1928), Thompson (1931b), Kihara and Lilienfeld (1932). 
of homology as to conditions affecting the relative rate of development of the various chromosomes in the prophase or to one of the modifying influences mentioned at page 273 .

Amphidiploid Hybrids.-Primula floribunda $(n=9)$ was crossed with $P$. verticillata $(n=9)$, producing a sterile $F_{1}$ hybrid with 18 somatic chromosomes. From this plant there arose a bud sport known as $P$. Kewensis; this was found to be tetraploid (36 chromosomes) and fertile. Since the tetraploid condition arose through some form of somatic chromosome doubling in the $F_{1}, P$. Kewensis has two floribunda and two verticillata chromosome sets. It is said to be amphidiploid, since it is diploid for each kind of set present. It is also said to be allotetraploid, because it has four sets of nine which are in part unlike (see p. 340). Such forms are called "sexual nuclear chimeras" by Tschermak. In some such cases the original parental sets do not have the same number of chromosomes (Fig. 209). For example, Nicotiana glutinosa $(n=12) \times N$. tabacum $(n=24)$ gave in $F_{2}$ the fertile amphidiploid $N$. digluta with 72 chromosomes. This was the first such hybrid whose cytological constitution was understood. Amphidiploidy is one of the most significant of

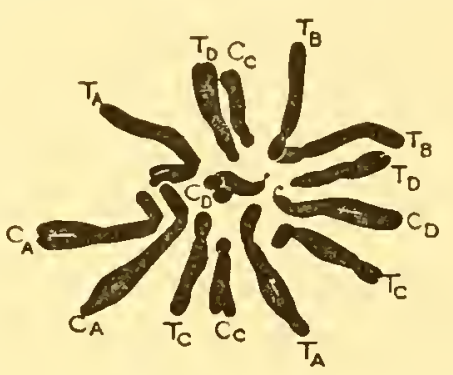

FIG. 209.- Somatic chromosome complement of an amphidiploid Crepis capillaris $\times$ C. tectorum hybrid. It comprises two sets from each parental species. Satellites on tectorum D-chromosomes do not show at metaphase in this hybrid. (After Hollingshead, 1930a.) all cytological phenomena in hybrids because of its evident relation to the origin of new fertile types. Several such fertile amphidiploids are now known, ${ }^{10}$ among which the following are of much interest.

Raphanus sativus (radish; $n=9$ ) was crossed with Brassica oleracea (cabbage; $n=9$ ). In some of the sporocytes of the $F_{1}$ hybrids the 18 chromosomes showed deficient synapsis and other irregularities, giving various numbers in the spores (Fig. 210). In some of these were all 18 chromosomes, and from the union of such gametes there were obtained $F_{2}$ plants with 36 . These amphidiploid plants, with two radish and two cabbage sets, were fertile, and they differed in character from their

${ }^{10}$ Primula Kewensis (Digby, 1912; Newton and Pellew, 1929); Raphanus $\times$ Brassica (Karpechenko, 1924ab, 1927ab; Karpechenko and Shehavinskaia, 1930); Triticum $\times$ Egilops (Tsehermak and Bleier, 1926; Kragawa, 1928, 1929; Tsehermak, 1929, 1930; 'Taylor and Leighty, 1931); Fragaria (Ichijima, 1926); Nicotiana (Clausen and Goodspeed, 1925; Rybin, 1927, 1929); Rosa Wilsoni (Blackburn and Harrison, 1924b); Brassica (Frandsen and Winge, 1932); Digitalis (Buxton and Newton, 1928; Buxton and Darlington, 1931); Aquilegia (Skalinska, 1932); Spartina (Huskins, 1931c). Amphidiploids which are largely or completely sterile are known in Nicotiana (Lammerts, 1931, 1932), Solanum (Jørgensen, 1928), Triticum $\times$ Secale (Lewitsky and Benetzkaja, 1930, 1931), Crepis (Babeock and Nawasehin, 1930; Hollingshead, 1930a; Poole, 1931, 1932), and Saxifraga (Marsden-Jones and Turrill, 1930). Winge (1932) ruviaws most of the known cases. 
parents. Moreover, only with difficulty could they be crossed with the parents, and the few offspring obtained were highly sterile; hence they had the sexual isolation which aids in the establishment of new independent types. The type was given the new generic name Raphanobrassica. Other heteroploid types were obtained also; these will be discussed later in the chapter.

After crossing Triticum durum $(n=14)$ with Egilops ovata $(n=14)$, it was found that the $F_{6}$ plants were fertile, genetically stable, and

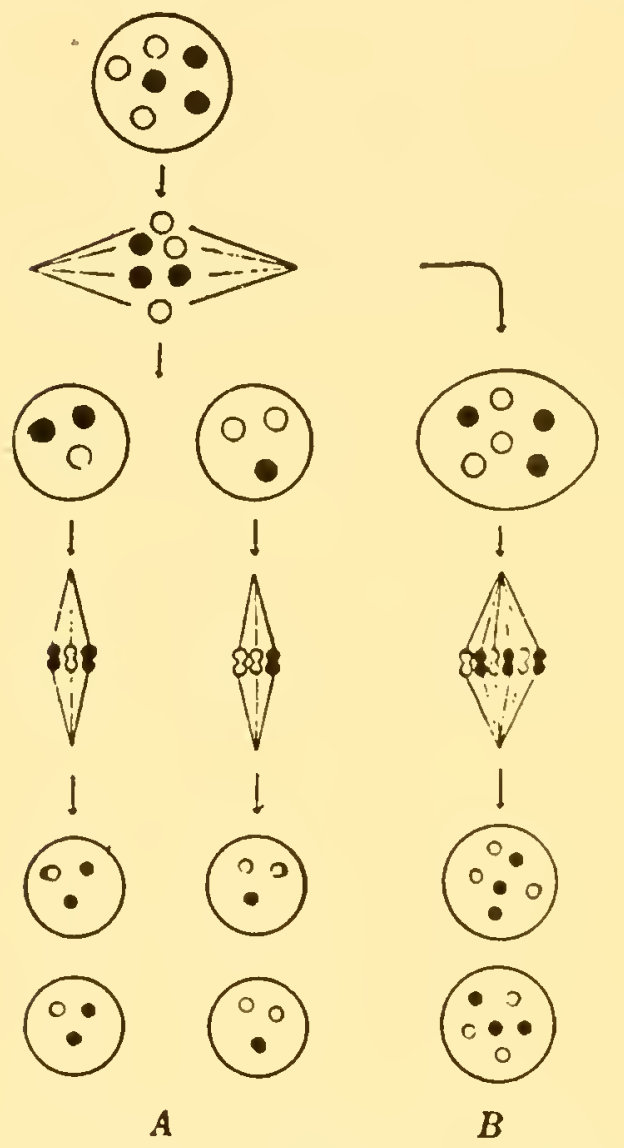

Fig. 210.-Diagram of mitoses in mierosporoeytes of $F_{1}$ hybrids of Raphanus sativus $\times$ Brassica oleracea. $A$, usual course of divisions, forming monoploid spores. $B$, formation of diploid spores following "asynapsis." (After Karpechenko, 1927a.)

seed, which grew into a fertile plant with 72 chromosomes. Probably the chloroform treatment induced ameiosis, leading to amphidiploidy (Rybin).

The formation of unreduced gametes is also known in moth hybrids. Pygcera anachoreta $(n=30) \times P$. curtula $(n=29)$ gave an $F_{1}$ hybrid with

${ }^{11}$ If, as suggested by the theory mentioned on p. 366. Triticum durum and Egilops ovata may be regarded as amphidiploids with the somatic formulæ $A A B B$ and $D D E E$, respectively, the 56-chromosome hybrid formed by crossing them would be amphidiploid in the second degree: it would be an allo-octoploid with the formula $A A B B D D E E$. type was named Egilotricum (Tschermak and Bleier). Since the $F_{1}$ of such a cross was later found to be diploid (28), it is probable that it produced diploid spores through some form of ameiosis (Kagawa). Certain other Triticum-Egilops hybrids are more variable in later generations (Taylor and Leighty). Triticum turgidum $(n=14) \times$ Triticum villosum $(n=7)$ gave a constant and fertile hybrid with 42 chromosomes (Tschermak). By crossing this hybrid with Egilotricum there was obtained a plant combining the gametic complements of four species (Berg, 1931b). In the notation given on page 366 , the somatic formula of this plant would be $A A A B B D E$. A fertile, balanced hybrid between Triticum and Secale is reported by Lebedeff (1932).

Nicotiana tabacum $(n=24)$ crossed $F_{1}$ hybrid with 36 chromosomes. A branch of this plant was treated with chloroform vapor 8 days before the opening of the flower. Self-pollination then resulted in the production of a single amphidiploid (56 chromosomes). ${ }^{11}$ The 
little or no synapsis in its spermatocytes. The chromosomes divided in both meiotic mitoses, giving gametes with all of the 59 chromosomes. No allotetraploid type was obtained, but allotriploids resulted from backcrossing the hybrid to one parent. ${ }^{12}$

From the foregoing examples it can be concluded that amphidiploidy may arise if the two unlike chromosome sets brought together in a cross are compatible enough to exist together in the zygote and if the chromosomes then become doubled in some manner. The rôle of interaction of homologous elements in the origin of such alloheteroploid types is conceived by Winge (1917) as follows. When two specifically different chromosome sets of like number are brought together, they may sometimes act in complete harmony at all stages of somatic development and meiosis so that a new sexually reproducing hybrid type results. If the compatibility of the chromosomes is less, the chromosomes, after having coöperated throughout somatic development, synapse poorly or not at all in the sporocytes so that diploid spores and gametes are formed. Finally, if the compatibility is insufficient for the "action in pairs" favoring the development of the diploid soma, all of the chromosomes in the zygote or early embryo may split, the resulting halves then acting as homologous pairs in the development of the resulting allotetraploid individual. Such an individual may reproduce sexually as a "permanent hybrid" if it produces diploid spores and gametes after meiosis. ${ }^{13}$ If meiosis and sporogenesis fail, the tetraploid type may reproduce by asexual means alone or disappear altogether. These several conditions are observed in nature, and the interpretation outlined above harmonizes well with certain phenomena in apomictic diploid plants (p. 407).

In some cases there is a doubling of the chromosomes of one parent only. Thus Saccharum officinarum of $(n=40) \times S$. spontaneum or $(n=56)$ gave a fertile hybrid with 136 chromosomes, the set of 40 from officinarum evidently having doubled in the zygote. When this hybrid was back-crossed as pollen parent to S. officinarum, the progeny showed about 148 chromosomes, indicating that the officinarum chromosomes had again doubled (Bremer, 1928a).

Because of their cytological and genetical constitution, alloheteroploid plants may be expected to differ in many ways from autoheteroploid ones. An autotetraploid, for example, may differ from its corresponding diploid mainly in a quantitative way, whereas an allotetraploid usually shows qualitative peculiarities of character due to the combination of unlike elements. Instead of forming quadrivalents as in typical autotetraploids, the allotetraploid types ordinarily form bivalents like a diploid, although

12 Federley (1913 et seq.). See his general account (1928).

${ }^{13}$ Thus an individual of hybrid constitution may arise from the union of gametes which are genetically similar (cf. p. 359), but here the gametes themselves are hybrid in character. 
quadruple groupings sometimes occur, particularly when the species crossed are nearly related. The fertile allotetraploid tends to breed
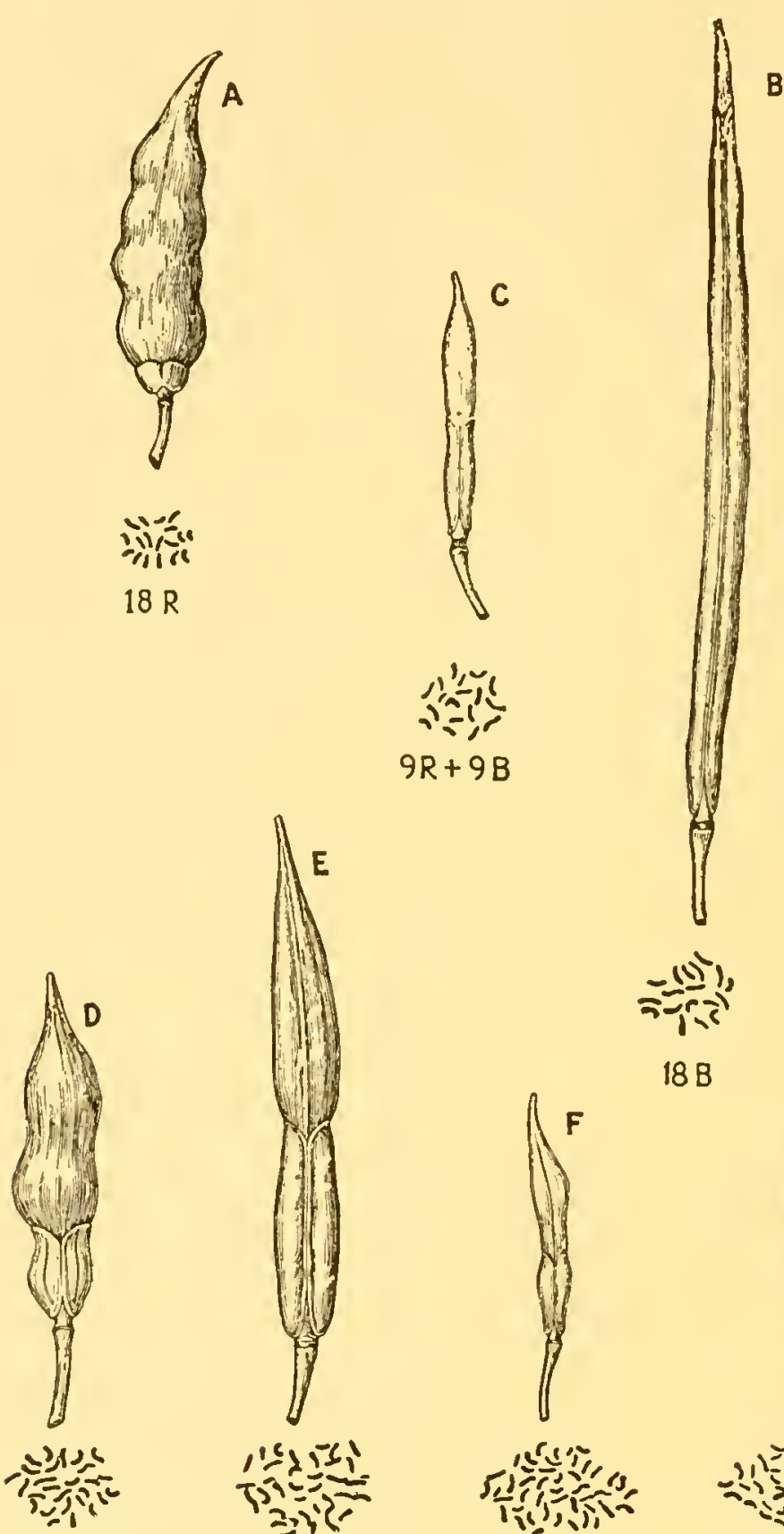

$18 R+9 B$

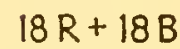

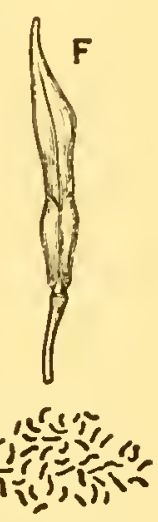

$27 R+18 B$
B
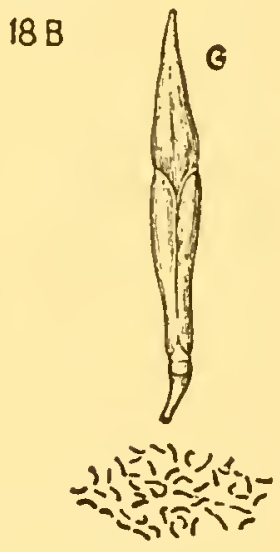

$24 R+27 B$

FIG. 211.-Corrclation of seed pod characters with chromosome constitution in Raphanus $\times$ Brassica. A, Raphanus sativus: upper, non-dehiscent secd-bearing part large; lower part small and mostly seedless. B, Brassica oleracea: upper part nearly seedless; lower part much elongated, with seeds. $C$, diploid $F_{1}$ hybrid between $A$ and $B$ : upper and lower parts about equal in size. $D, F$, triploid and pentaploid types derived from cross of $A$ and $C$ : each has more Raphanus than Brassica chromosomes, and upper part of pod is larger. $E$, fertile tetraploid type (Raphanobrassica) obtained by selfing $C$ : two sets of chromosomes from each grandparent; capsule parts about equal in size. $G$, hypohexaploid type somewhat deficient in Raphanus chromosomes. (After Karpechenko, 1927b.)

true, apparently because of the regular pairing between chromosomes from similar sets (autosynapsis) and their regular distribution. The occasional segregation of differing types, as in Primula and Aquilegia, is 
attributed to the synapsis of chromosomes from dissimilar sets in the complement (allosynapsis) or to secondary pairing and atypical distribution of autosynaptic pairs. Studies on such alloheteroploid plants, taken together with comparisons of the conditions observed in many related species in nature, have strengthened the theory that amphidiploidy has played an important rôle in the differentiation of species.

Parental Characters in Polyploid Hybrids.--There is a marked tendency on the part of polyploid plants of hybrid origin to show a greater resemblance to the parent contributing the larger number of chromosome sets. In crosses between Raphanus and Brassica the sterile $F_{1}$ hybrid with one set from each parent $(R B)$ is intermediate in capsule characters (except size) (Fig. 211). The fertile $F_{2}$ Raphanobrassica with two sets of each kind $(R R B B)$ and a hexaploid with three of each kind $(R \dot{R} R B B B)$ are intermediate also. A pentaploid form with $R R R B B$ is somewhat more like Raphanus, while the resemblance is still greater in a triploid $(R R B)$. Thus is the ratio of parental chromosome sets reflected in external characters (Karpechenko, 1927b).

When Rubus rustica inermis, a diploid species, was crossed with $R$. thyrsiger, a tetraploid, three $F_{1}$ hybrids were obtained. Two of these were triploid $(T T R)$ and resembled $R$. thyrsiger, while the third was tetraploid (TTRR) and was more like $R$. rustica inermis. The tetraploid probably arose from a diploid egg produced by rustica after some meiotic aberration (Darlington, 1929f).

Euchlena mexicana $(n=10)$ crossed with Zea Mays $(n=10)$ gave an intermediate hybrid with one set from each parent $(E Z)$. The tetraploid $E$. perennis (gametic number $=20$ ) when similarly crossed to Zea gave a triploid hybrid $(E E Z)$ which was more like Euchlona. One plant in the progeny of this cross was considerably more like $Z e a$, and cytological examination showed it to be tetraploid. Presumably the fourth set was formed from the Zea set by doubling, giving the formula $E E Z Z$ and a more even balance between the characters of the two parents ( $R$. Emerson and Beadle, 1930).

The same phenomenon is manifested by polyploid moss hybrids. In hybrids between Physcomitrella patens and Funaria hygrometrica the peristome of the capsule becomes less like that of the former genus $\left(P^{2}\right)$ and more like that of the latter $\left(F^{2}\right)$ in passing through the series $P^{2}, P^{1} F^{1}, P^{8} F^{9}, P^{4} F^{5}, P^{2} F^{3}, P^{1} F^{2}, P^{2} F^{5}, P^{1} F^{3}, F^{2}$ (von Wettstein, 1926). In narrow crosses in mosses the degree of dominance is roughly proportional to the relative number of dominant genes, whereas in wide crosses (specific or generic) it may increase up to a certain ratio and then decrease. This is attributed to differences in the parental cytoplasms (von Wettstein, 1927).

In hybrids between Crepis capillaris $(n=3)$ and C. aspera $\left(n^{\prime}=4\right)$ where a doubling of one chromosome set has occurred, it is found that 
10-chromosome plants with one aspera and two capillaris sets $(A C C)$ are more like capillaris, while 11-chromosome plants with the formula $A A C$ are more like aspera. A triple hybrid involving these two species and $C$. setosa (ACS) showed certain setosa characters (M. Nawaschin, 1927b). Similarly, in hybrids between $C$. tectorum and $C$. alpina the characters of the achenes vary according to the numbers of parental chromosome sets involved (M. Nawaschin, 1930b).

Segregation of Parental Chromosome Sets.-It frequently happens that when a hybrid forms spores and gametes, only those which contain a complete chromosome set from one parental species or the other are functional. Or, if the gametes carry various combinations, possibly only those zygotes survive which have two complete sets, be they of one or of both species. In a Crepis tectorum and $C$. alpina cross like that just mentioned, the only three plants obtained in $F_{2}$ contained, respectively, two tectorum sets ( $T T$ ), two alpina sets $(A A)$, and one of each $(T A)$. Of further interest is the fact that the $F_{2}$ plant with two alpina sets was precisely like the original alpina plant, notwithstanding the fact that its cytoplasm had been derived from tectorum (M. Nawaschin, 1927a).

In a Crepis hybrid containing two capillaris sets and one tectorum set, the six capillaris chromosomes formed three bivalents at meiosis, leaving the four of tectorum as univalents. Although most or all of the female gametes formed by this hybrid were able to function, only those male gametes with none or all of the tectorum chromosomes were functional. $\quad F_{2}$ plants with one or more tectorum chromosomes were less viable than those with capillaris chromosomes only (Hollingshead, 1930a). These facts all emphasize the importance of "balance" in the chromosome complement, particularly in the pollen.

A Fertile Tetraploid Obtained through a Sterile Triploid.-In the genus Galeopsis the gametic chromosome numbers of four species are as follows: pubescens, 8 ; speciosa, 8; tetrahit, 16; bifida, 16 . In the progeny of a cross between pubescens and speciosa was an almost completely sterile triploid plant with 24 somatic chromosomes. When this plant was pollinated with pubescens pollen, a single seed was obtained; this grew into a fertile plant with 32 somatic chromosomes, of which 24 were probably contributed by a triploid gamete from the triploid plant and 8 by the gamete from pubescens. The plant would not cross with either pubescens or speciosa, although it crossed readily with either tetrahit or bifida. It was morphologically and genetically indistinguishable from $G$. tetrahit, and it had the same chromosome number. It was therefore called "artificial tetrahit"; and Müntzing (1930b) described it as "the first case where a Linnxan species has been synthesized by means of species hybridization and chromosome summation."

Conclusions. - The observational and experimental evidence reviewed in these chapters indicates that distinct heritable changes in the char- 
acters of organisms may involve Mendelian recombinations of genetic factors, or genes, actual mutations of these genes, alterations in the number and assortment of chromosomes as results of aberrations in mitotic behavior, and hybridization, by which many new combinations are produced. Any stable sexual type is characterized by a chromosome complement consisting of a certain number and assortment of elements showing individual functional and morphological differences; all of them constitute a balanced, harmonious system. Any change in the constitution of this system, whether quantitative or qualitative, large or small, is accompanied by a corresponding alteration of the type, although this alteration is not always immediately visible.

With regard to the rôle of genes and their mutation in the origin of species, Morgan (1923c) points out that the inheritance of the results of gene mutations as Mendelian characters suggests the origin of Mendelian differences in general in such mutations. "It is ... now clear that these smaller mutant variations must be those small heritable variations that Darwin himself appealed to as furnishing the materials for organic evolution." These must not be confused with non-inherited environmental variations. In reply to the objection that changes due to gene mutations are too trivial to be of significance in evolution, Morgan further points out that the minor superficial characters used by the geneticist and the taxonomist may of ten be secondary results of changes which have some invisible but more fundamental physiological effects with an actual survival value. Moreover, "useless" characters may be closely linked with "useful" ones. The systematist of necessity distinguishes many species on the basis of characters which have played no part in the actual production of those species.

Change of type is effected not only by such mutations of genes and their Mendelian recombination through normal chromosome distribution but also by more extensive and less orderly alterations in the grouping and interaction of inheritance materials through abnormalities in chromosome behavior. Non-division, non-disjunction, translocation, and other aberrations all play rôles in the production of new chromosome complements which differ from the original complements in the number, size, and assortment of their constituent elements. The range of variation is greatly increased by crossing among the altered types. It seems abundantly evident that hybridization involving types with chromosomes that are in some measure incompatible is one of the chief causes of chromosomal aberrations leading to the establishment of qualitatively new gene systems. The irregularities in chromosome behavior immediately following such crossing gradually disappear in subsequent inbred generations through the elimination of unpaired chromosomes, until there may remain a chromosome complement whose members act in harmony, although they may constitute a very new type of interacting system. 
In more general terms, hybridization, or outcrossing, plays its chief rôle by inducing variability in immediately subsequent generations, the amount of variability depending on the degree of constitutional difference between the types involved. If free intercrossing with the parental and other types continues, the variability is kept up. In order for stable types to differentiate from the series of variants, crossing must be restricted by some form of isolation, such as natural or artificial selection, geographic isolation, intersterility, and the like, whereupon some of the inbreeding types rapidly become stable. The origin of species is thus frequently conceived as a fixation of stable independent types through the inbreeding of variable types originally resulting from hybridization. We have also seen that hybridization, if accompanied by chromosome doubling, may yield new and stable types almost at once.

Since hybridization has long been recognized as an important cause of diversity in organisms, it may be well to summarize the contributions which cytology has made in this connection. Cytology has shown the close relation between hybridization and heteroploidy, between heteroploidy and certain classes of mutations, and hence between hybridization and such mutations. In its elucidation of chromosome aberrations in hybrids it has thrown much light on the curious behavior of inherited characters in hybrid lines; the apparently non-Mendelian inheritance in crosses between species, varieties, and mutants, the genetic constancy of certain hybrids, the unexpected gametic ratios frequently obtained, the unlikeness of reciprocal hybrids, sterility, and a variety of otherwise unintelligible phenomena. It has heightened the precision with which certain changes in character can be attributed to crossing, often affording real proof in the case of suspected hybrids. It has shown the value of cytological data in determining taxonomic relationships within narrow circles of affinity and the prime necessity of utilizing such data in many problematic cases. It has shown the importance of apomixis and somatic mutation as factors in heredity and hence their importance to the geneticist and field taxonomist. In short, cytology has afforded a common rationale for a great variety of phenomena hitherto studied by distinct groups of biologists. 


\section{CHAPTER XXIII}

\section{CHROMOSOMES AND SEX}

During the closing decades of the nineteenth century many researches were carried out in the hope of identifying the controlling agency in sex determination with one or more of the environmental factors. The effects of light, temperature, moisture, and nutrition were examined, and although a number of workers believed their methods to be in a certain measure successful, the results were on the whole inconclusive. The period during which sex was thus looked upon as a character more or less under the control of the environmental factors was succeeded by one in which it came to be regarded as something regulated mainly by some internal mechanism or condition, and as relatively unalterable by external agencies. This conclusion, definitely reached by Cuénot (1899) for animals and by Strasburger $(1900 c)$ for plants, received the support of a number of experimental researches on animals and diœcious plants. It was further strengthened by the discovery that in many organisms males and females differ visibly in their chromosome complements.

The modern interpretation of sex determination combines the elements of truth in both of these theories. The course of development in an organism is dependent on a series of interactions between a protoplasmic system of a certain kind and a particular group of environmental conditions. It is found that the differentiation of sex, like that of any other character, involves the action of both internal and external factors. In some cases the tendency to develop one sex or the other is so strongly set by the internal factors that it can be modified only with difficulty, if at all, by altering the external factors; whereas in other cases the internal factors are so balanced that the course of differentiation can be thrown toward either sex with comparative ease by altering the environment in which the individual develops.

Sex-chromosomes in Animals. - In a great many animals it has been found that the approximate numerical equality of males and females is related to the fact that the gametes of one sex (more commonly the male) are of two kinds, the sex of the offspring depending upon which kind happens to function in syngamy. The difference in the two kinds of gamete is often due to the meiotic disjunction of the unlike members of a distinguishable pair of chromosomes which evidently play a special rôle in sex determination. Such chromosomes are known as sex-chromosomes; 
the other members of the complement are called autosomes. Typical examples of sex-chromosomes in animals are given below. ${ }^{1}$

In 1891 Henking noticed that half of the spermatids of Pyrrhocoris apterus, an insect, contain a body which he thought might be a nucleolus. It was subsequently shown" that this supposed nucleolus is an "accessory chromosome" which remains compact while the other chromosomes become diffused or reticulate in form. That this accessory chromosome exerts a special influence in sexual differentiation was suggested by McClung $(1901,1902)$ and proved by him and many other observers.

When some of the sperms have one sex-chromosome $(X)$ and the others none, the animal is said to show the "XO type" of sex-chromosome differentiation. A good example is Protenor belfragei, a bug ${ }^{3}$ (Fig. 212). In the somatic nuclei and spermatogonia of the male there are six pairs of autosomes and a single unpaired $X$-chromosome. The autosomes undergo disjunction in $I$ as usual and divide equationally in $I I$, but the $X$-chromosome divides in $I$ and passes undivided to one pole in $I I$. Two sperms of the quartet receive one $X$ each, while the other two receive none. In the female somatic cells and the oöcytes there are two $X$-chromosomes besides the autosomes; every egg receives one $X$. Fertilization by a spermatozoön with an $X$ results in a female with two $X \mathrm{~s}$, whereas that by one with no $X$ results in a male with only one. In a number of insects, domesticated mammals, and lizards, the $X$-chromosome passes undivided to one pole in $I$ and divides in $I I$, the end result being the same as in Protenor. ${ }^{4}$ The plotted measurements of the spermatozoa of Canis form a bimodal curve, the indicated size dimorphism probably being associated with the chromosomal difference. ${ }^{5}$

Two variants of the $X O$ type are found in the nematode genus Ascaris. In A. megalocephala bivalens (Fig. 212) there are in the spermatocyte four autosomes and a single small $X$, which is usually attached to the end of one of the autosomes. The $X$ passes undivided to one pole in $I$ and divides in $I I$; hence it is present in half of the spermatozoa. In the oöcyte there are two such $X$-chromosomes, which disjoin in $I$ and divide in $I I$, every egg thus receiving one. Fertilizations of eggs by sperms with and without an $X$ result in females and males, respectively.

${ }^{1}$ For extensive reviews, see Crew (1927c), F. Sehrader (1928), Wilson (1925), and Witsehi (1929). See M. Hartmann $(1929 b, 1930)$ on Protista.

${ }^{2}$ MeClung (1899), Paulmier (1899), de Sinéty (1901), Montgomery (1901).

${ }^{3}$ Montgomery (1901), Wilson (1906), Morrill (1910).

${ }^{4}$ The behavior of sex-chromosomes in $I$ and $I I$ is tabulated by Wilson (1925). A tendency on the part of the $X^{r}$ to lag may result in both modes of distribution in the same species (Edwards, 1910).

${ }^{5}$ Malone (1918) on the dog. Other reports of XO type: Wodsedalek (1913, 1914, 1920, 1922) on horses, sheep, pigs, and cattle; Masui (1919ab) on horses and eattle; Painter (1920, 1921b) on lizards; Gulick (1911) and Mulsow (1912) on nematodes; Brauer (1928) on Bruchus; and Mols (1928) on guinea pig. See also next footnote. 


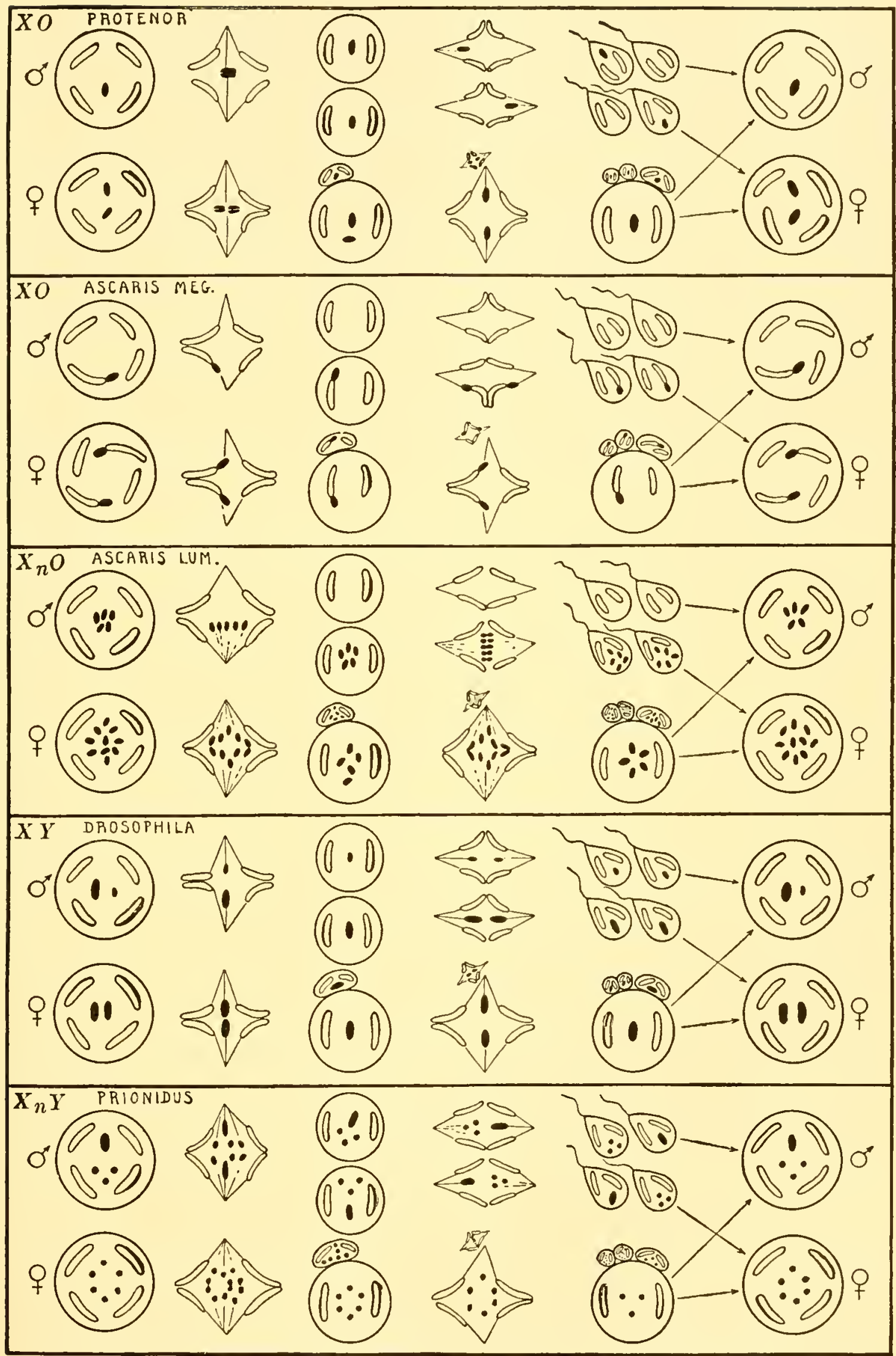

FIG. 212.- Several types of sex-chromosome behavior in animals with heterogametic males. For the sake of clearness all are shown with two pairs of autosomes. Sex-chromosomes in the female are shown disjoining in the same mitosis as in the male, though this is not known to occur in all five cases. 
A. lumbricoides has in the male an $X$-complex made up of five elements (Fig. 212). All five pass to one pole in $I$, and two classes of spermatozoa are formed, with 19 and 24 chromosomes respectively. This is sometimes referred to as the " $X_{n} O$ type."

The "XY type" is well exemplified by Drosophila. In D. melanogaster the somatic complement comprises four chromosome pairs, including a pair of sex-chromosomes (Figs. 196, 212). In the female the two members of this pair are alike $(X X)$, while in the male they differ slightly in size and greatly in function $(X Y)$. Owing to disjunction in meiosis, half of the sperms carry $X$ and half carry $Y$, while every egg has $X$. Fertilization of an egg by an $X$-sperm results in a female with $X X$, while that by a $Y$-sperm results in a male with $X Y{ }^{7}$ In some genera the $X$ and $Y$ divide equationally in $I$ and disjoin in $I I$. It will be shown later in the chapter that sex is correlated with the ratio of $X$-chromosomes to autosome pairs, rather than with the simple presence of $X$ or $Y$ or the number of $X$ 's.

The $X Y$ type is the most prevalent form of sex-chromosome differentiation in animals. Like the $X O$ type, it is best known in insects and mammals ${ }^{8}$ including man. In a number of cases the genetic evidence indicates the presence of an $X Y$ pair, ${ }^{9}$ although it has not been possible to identify it with the microscope. Apparently the sex-chromosomes are often not visibly differentiated from the other members of the complement. A variant of the $X Y$ type is seen in Prionidus, in which the $X$ is represented by three small elements (Fig. 212). In the female there are six of these $X$-elements, while in the male there are three $X$-elements and a $Y$. In spermatogenesis the $X$-elements disjoin from the $Y$ in $I I .{ }^{10}$

${ }^{6}$ Works on sex-ehromosomes in Ascaris: Boveri (1909b), Boring (1909), Edwards (1910, 1911), Frolowa (1912), Walton (1918, 1921, 1924). Walton concludes from his extensive researehes on nematodes that each autosome in $A$. megalocephala is equivalent to 22 somatic chromosomes, and that the $X$ is equivalent to 8 . Sex-ehromosomes attached to autosomes are also reported for Choaborus plumicornis (Frolowa, 1929b).

'Stevens (1907, 1908a), Morgan (1911), Metz (1914). In Drosophila Willistoni it has been shown by Lancefield and Metz $(1921,1922)$ that the sex-chromosomes are represented by one of the large bent pairs rather than the shorter rod-shaped pair; and there is evidence in the linkage of genetie factors that the rod-shaped chromosome of melanogaster corresponds to one arm of the large bent sex-chromosome in Willistoni and obscura.

8 Works on mammals: Painter $(1921 a, 1922 b)$ on opposum, $(1922 a, 1923 b)$ on monkeys, (1926) on rabbit; Agar (1923), Greenwood (1923) and Saez (1930c) on marsupials; Cox (1926) and Minouchi (1928e) on the mouse; Minouchi (1928c) and Pincus (1928) on the rat; Starks (1928) on the seal; Minouchi (1928a) on the eat. Winiwarter and Sainmont (1909) reported XO in the cat.

${ }^{9}$ E.g., in the fish genera Lebistes (J. Schmidt, 1920; Winge, 1922, 1923a, 1927b, 1930b; Vaupel, 1929; Iriki, 1932) and Aplocheilus (Aida, 1921, 1930; Iriki, 1932).

${ }^{10}$ Payne (1909). Several other cases of this $X_{n} Y$ type of differentiation are known. In the Mantidæ the $\mathrm{X}$-complex consists of two elements, the male having $24+X_{a} Y X_{b}$ and the female $24+X_{a} X_{a} X_{b} X_{b}$ (King, 1931; Oguma, 1921). 
The number of chromosomes in man and the make-up of the complement in the two sexes have long been subjects of controversy. ${ }^{11}$ It has now been established that there are 24 pairs represented in the complement. According to most recent workers (Painter, Evans and Swezy, Kemp), these include an $X Y$ pair in the male and $X X$ in the female; but others (de Winiwarter, Oguma) contend that there is no $Y$, the sexchromosomes being of the $X O$ type. In Painter's material there can be little doubt that a large, unequal pair resembling $X Y$ pairs in other mammals is present and disjoins in $I$ (Fig. 213).
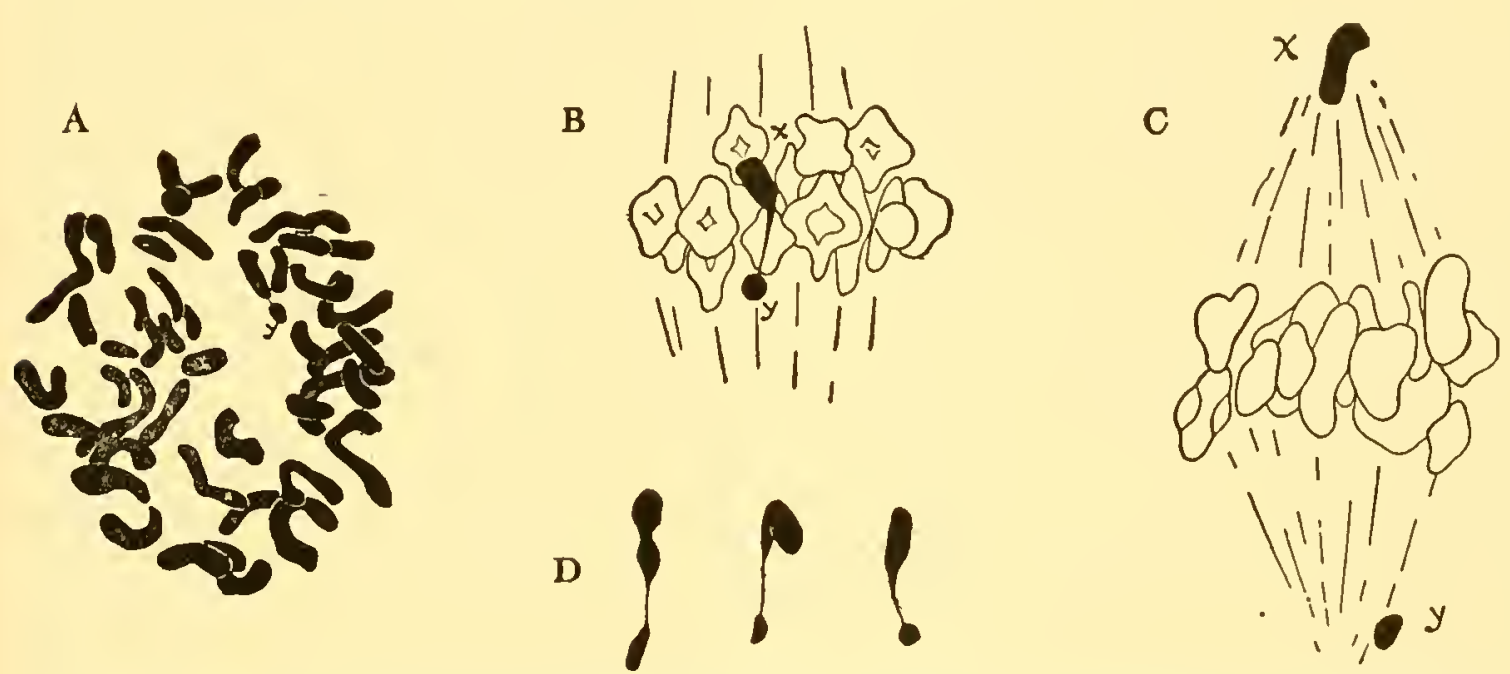

Fig. 213.- $A$, diploid chromosome complement in man. $B$, metaphase $I$ in spermatocyte, showing $X Y$ pair about to disjoin. $C$, the $X$ and $Y$ have disjoined and passed to poles in advance of autosomes. $D, X Y$ pairs in opossum, monkey, and man. (After Painter, 1923a, 1922a.)

There is considerable evidence for the view that the $X O$ type has been derived from the $X Y$ type through loss of the $Y$. In different organisms many stages in the supposed decrease in size and restriction of function on the part of the $Y$ are known. Moreover, the $Y$ may be either present or absent in Metapodius, and such an explanation has been suggested for the divergence of opinion regarding man (Wilson, 1909b, 1925).

In all of the foregoing cases it is the male which is "digametic," or "heterogametic," sperms of two kinds fertilizing eggs of one kind. In some groups, notably birds, moths, and butterflies, the female is digametic. Here the sperms are all alike, while the eggs are of two sorts. (In such cases the sex-chromosomes are often referred to as $Z$ and $W$ instead of $X$ and $Y$.)

11 Reports on the chromosomes of man have been made by many investigators, among whom may be mentioned Flemming (1882b, 1898), Moore and Arnold (1906), Duesberg (1906), Guyer (1910), Gutherz (1912), Montgomery (1912), de Winiwarter (1912, 1921ab), Wieman (1917), Evans (1918), Painter (1921a, 1922a, 1923a, 1930), Grosser (1921), Rappeport (1922), Oguma and Kihara (1922, 1923), de Winiwarter and Oguma (1925, 1926), Oguma (1930), Kemp (1929), Evans and Swezy (1928, 1929). 
The $X O$ type (Fig. 214) is exemplified by the moth Taloporia tubulosa. In the female there are 59 chromosomes, including an unpaired $X$. In some oöcytes the $X$ passes to the first polar body at $I$, while in others it remains in the secondary oöcyte nucleus. All of the chromosomes appear to divide in $I I$. Hence there are two classes of eggs, with 29 and 30 chromosomes respectively. In the male there are 60 chromosomes, including an $X X$ pair, and all of the spermatozoa are alike in having one $X$. Fertilization of the two kinds of eggs would therefore be expected to give $X O\left(\right.$ o ) and $X X\left(\sigma^{\top}\right)$ (Seiler, 1917).

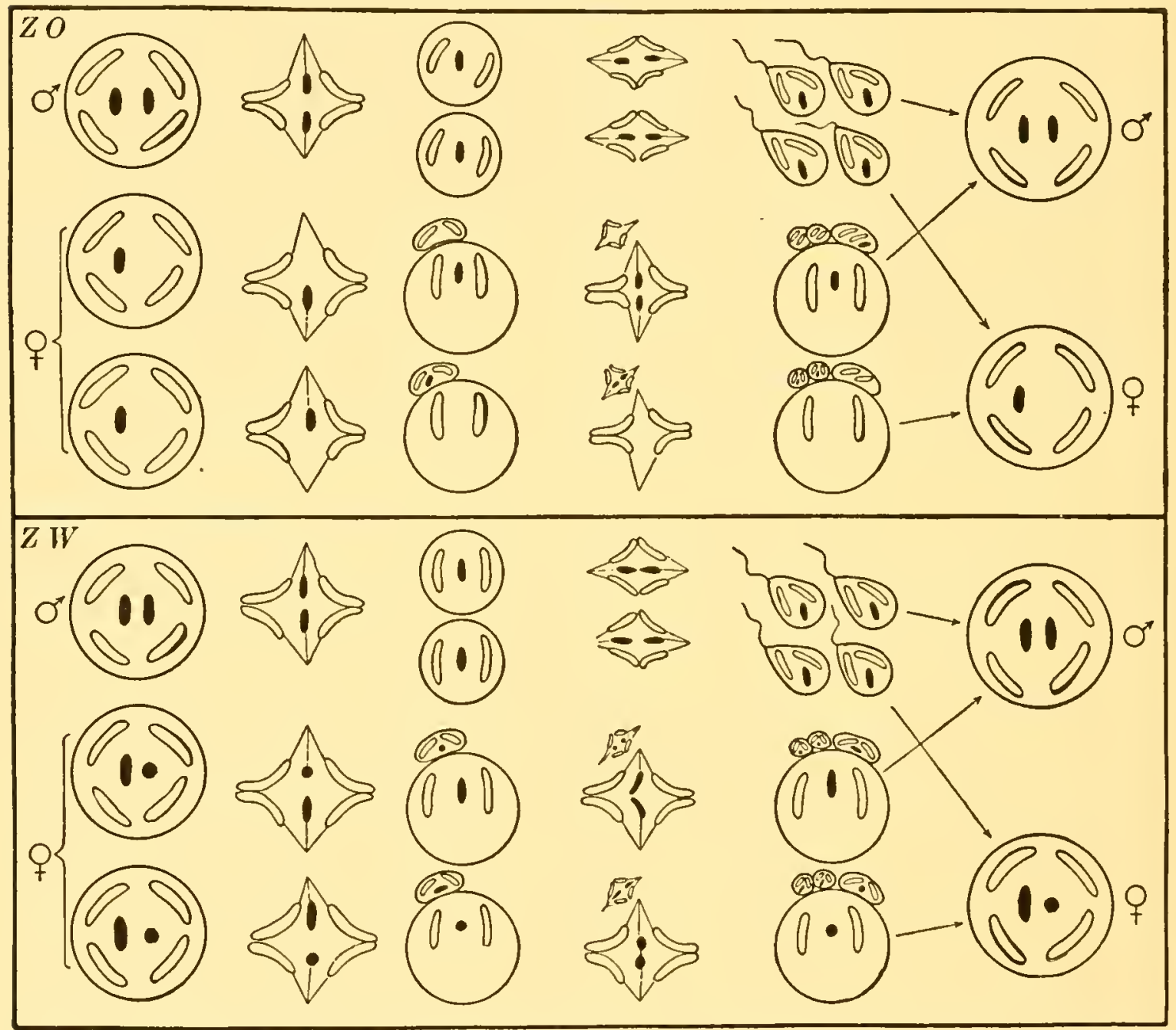

Fig. 214.-Sex-chromosome behavior in animals with heterogametic females. Division $I$ arbitrarily shown as disjunctional in all cases. $Z O=X O ; Z W=X Y$.

Another moth, Phragmatobia fuliginosa, exhibits a modified $X Y$ type of sex-chromosome behavior (Seiler, 1913). In Abraxas grossulariata the somatic nuclei of both sexes usually have 56 chromosomes, but the females of some strains have only 55 and produce two classes of eggs with 27 and 28, respectively (Doncaster, 1914). It is not unreasonable to suppose that in the 56-chromosome strains the $X$ is paired with an inert $Y$ and that the latter has been lost in the 55-chromosome strains (Agar, 1920). 
The $X O$ type is found in the chicken, the duck, and the pigeon. ${ }^{12}$ In the chicken the number of small autosomes is uncertain, but the largest chromosome of the complement is represented once in the female and twice in the male (Fig. 215). In the pigeon the male has 62 somatic chromosomes, including two large $X$-chromosomes; every spermatid retains one $X$. Some embryos have 62 , including $X X$, while others have 61 , including $X$. The latter are in all probability females.

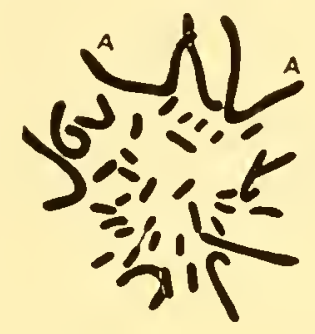

A

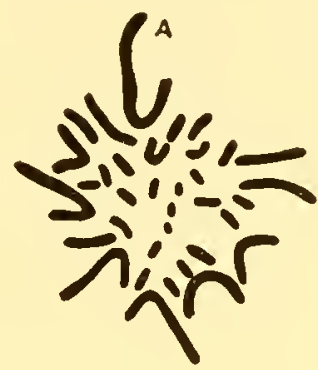

$B$

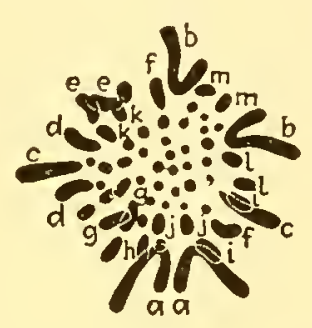

$c$

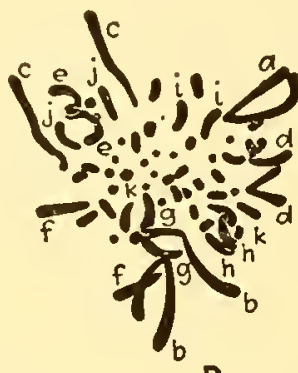

D

FIG. 215.-Chromosomes in birds. $A$, diploid complement in male chicken; two $X$-chromosomes (marked $A$ ). $B$, diploid complement in female; one $X$-chromosome. (After Hance, 1924.) $C$, diploid complement in male pigeon, showing a $X X$ pair (marked aa). $\quad D$, from a presumably female embryo, showing one $X(a)$. (After Oguma, 1927.)

In a genus of fishes, Platypocilus, the genetic data indicate the presence of an $X Y$ pair in the female. ${ }^{13}$ The fact that the nearly related genus Lebistes appears on similar evidence to have the $X Y$ in the male suggests that one condition may be derived from the other, possibly by crossingover or translocation. This is further suggested by the occasional appearance of $X X$ males in Lebistes (Winge, 1930b).

Gynandromorphs afford further evidence of the rôle of chromosomes in sex determination. Such individuals, with male characters in certain parts of the body and female characters in other parts, are known in several groups of animals which are normally unisexual, but most of them occur among insects. Such a condition may arise in several ways. In Drosophila the genetic evidence, including that in X-rayed flies, shows that it is mainly due to the elimination of one $X$-chromosome during an early mitosis in a female embryo (Fig. 216). Thus one part of the body develops with $X$ and the other portion with $X X$, the autosomes in both portions being the same. ${ }^{14}$ In the bee it is probable that gynandromorphism results from the entrance of a sperm into one blastomere of a divided egg. ${ }^{15}$ In the Lepidoptera the morphological and genetical data favor the theory that an egg may sometimes be formed with two nuclei, with $X$ and $Y$ respectively, and that a sperm nucleus may then

12 Guyer (1909, 1916), Stevens (Boring, 1923), Hance (1924, 1926), and Akkeringa (1927) on the chicken; Werner (1927) on the duck; Oguma (1927) on the pigeon. In the turkey, Shiwago (1929) reports the $X Y$ type, the $Y$ being very small.

${ }^{13}$ Bellamy (1922, 1924), Gordon (1926, 1927).

14 T. H. Morgan (1914), L. V. Morgan (1929), Patterson (1931a).

${ }^{15}$ Boveri (1888), Goldschmidt (1923a). 
fuse with each of them. ${ }^{16}$ Binucleate eggs also appear to be responsible for gynandromorphs in Habrobracon. ${ }^{17}$ Gynandromorphism in the katydid (Amblycorypha) may possibly be due to chromosomal elimination or to the entrance of two sperms, with $X$ and $Y$, respectively, into a binucleate egg. Polyspermy is known to occur in Drosophila, but its relation to gynandromorphism has not been proved. ${ }^{18}$

Certain further facts are also in harmony with the view that the sex of the individual is dependent upon the genetical constitution of the protoplast with which development begins. In a number of animals exhibiting parthenogenesis, sex is correlated with chromosome number (p. 410). Human twins, if "identical" (produced by the same zygote), are invariably of the same sex; if "fraternal" (produced by different
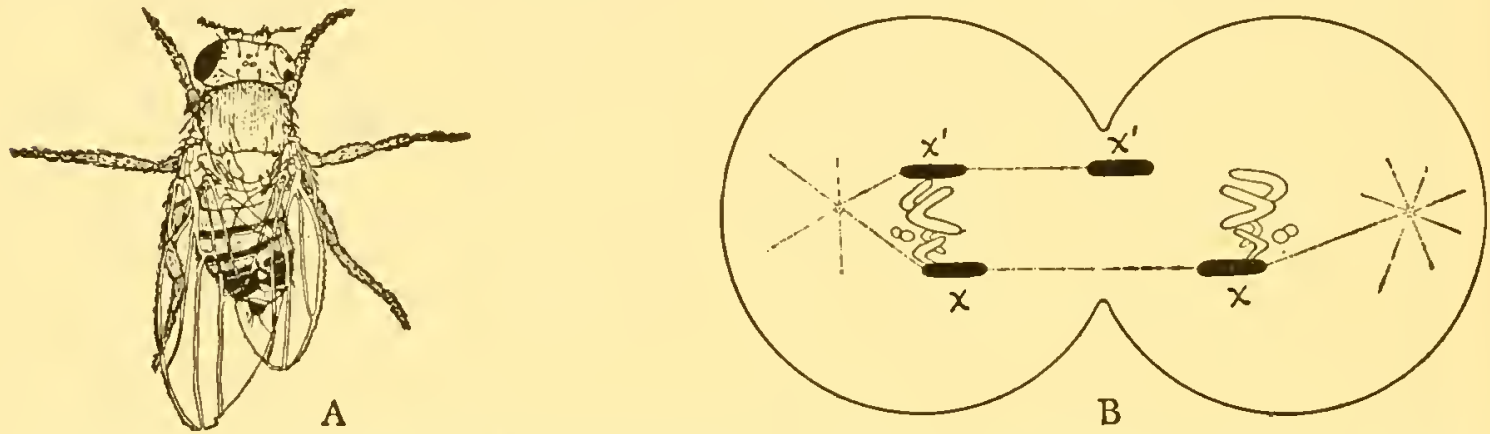

Fig. 216.-A, a Drosophila gynandromorph, female on left side and male on right. $B$, explanation suggested by Morgan for this condition: in an early eleavage division one $X$-chromosome fails to be included in either daughter nucleus, so that the nuclei in a portion of the fly have $X X$ while those in the remaining portion have $X$. (After MLorgan et al., 1922.)

zygotes) they may or may not be of the same sex. In the nine-banded armadillo one zygote commonly gives rise to four individuals, which are invariably all male or all female. Similar examples of polyembryony are known in insects. In the light of what has been set forth in the foregoing paragraphs it appears probable that the constitutional factors affecting sexual differentiation are largely in the chromosomes.

Sex-chromosomes in Bryophytes.-In Sphwrocarpos, a genus of heterothallic liverworts, two spores of a single quartet develop into male gametophytes while the other two give rise to females. This is associated with the behavior of a pair of sex-chromosomes, the first to be discovered in plants ${ }^{19}$ (Figs. 218, 219). The sporophyte has eight pairs of chromosomes, including a very unequal $X Y$ pair. At the time of sporogenesis the $X$ and $Y$ disjoin in the first mitosis and divide in the second. The two

${ }^{16}$ Doncaster (1914), Goldschmidt and Fiseher (1927), Goldschmidt and Katsuki (1927).

17 Whiting (1931), Whiting and Stancati (1931).

${ }_{18}$ Pearson $(1927,1929)$ on Amblycorypha, Huettner $(1924,1927)$ on Drosophila.

${ }^{19}$ C. E. Allen $(1917,1919)$. See also Schacke (1919), Wolfson (1927), and Lorbeer (1930). The sexual tendeneies of the four spores were discovered by Douin (1909) and Strasburger (19096). 


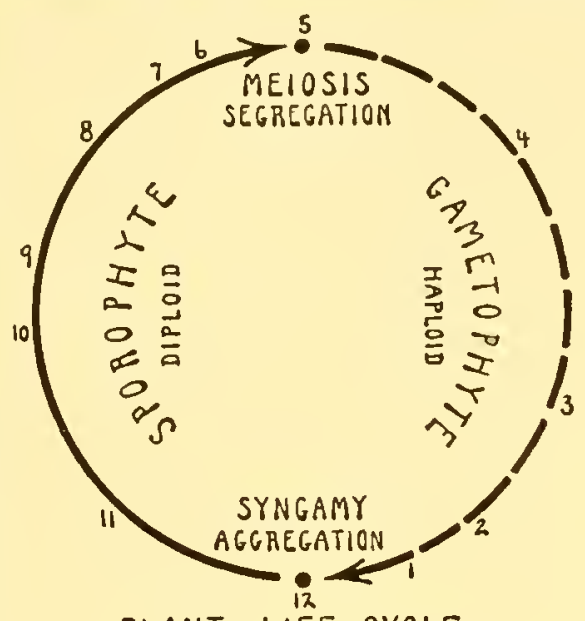

PLANT LIFE CYCLE

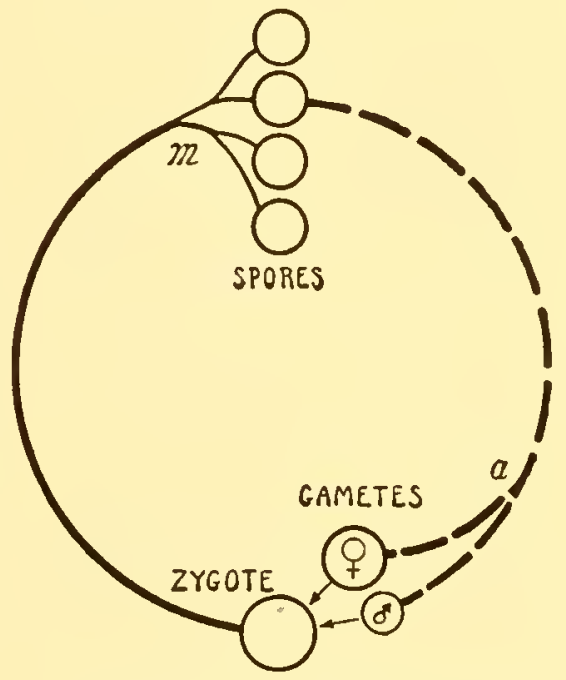

MOMOTHALLIC BRYOPHYTE

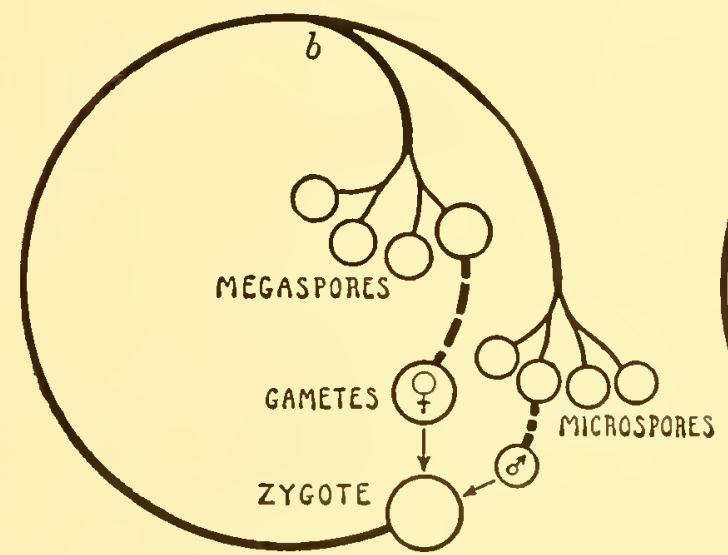

\& HOMOPHYTIC SEED PLANT

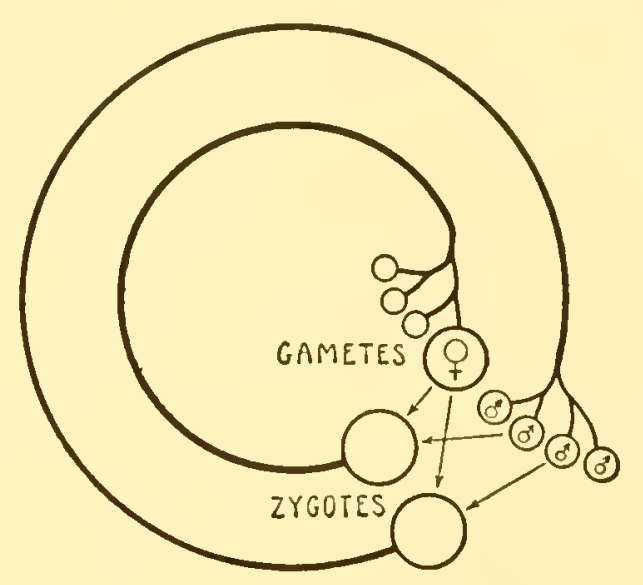

ANIMAL LIFE CYCLE

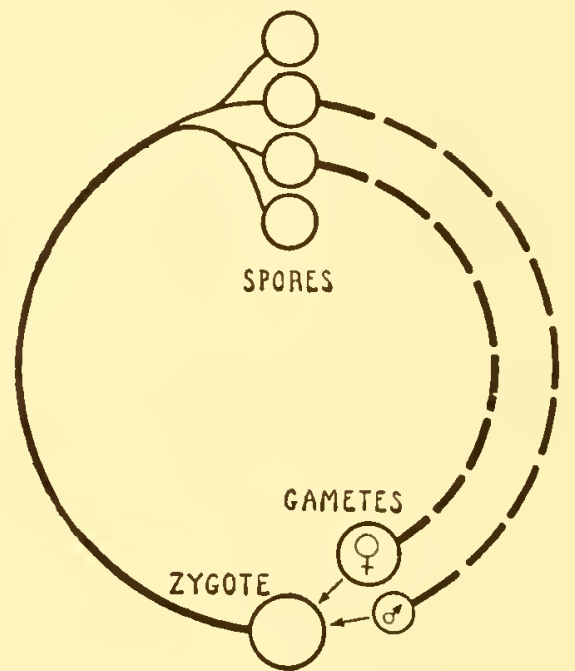

HETEROTHALLIC BRYOPHYTE

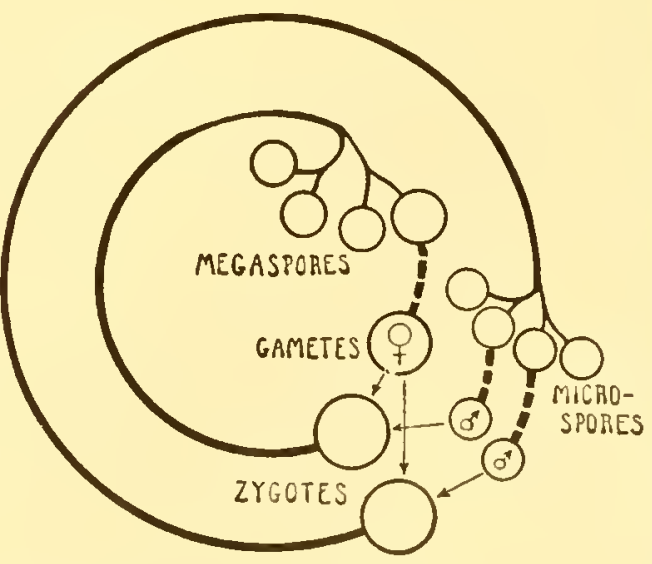

HETEROPHYTIC SEED PLANT

FIG. 217.-Diagrams of life cycles of several types. The first diagram is a modification of one by Schaffiner (1923c). (After Sharp, 1925). 
spores with $7+X$ develop into female gametophytes, while those with $7+Y$ develop into males. The total chromosome volume in a female gametophyte nucleus is about 1.7 times that in a male, and the volumes of the cells differ by about the same amount (Lorbeer).

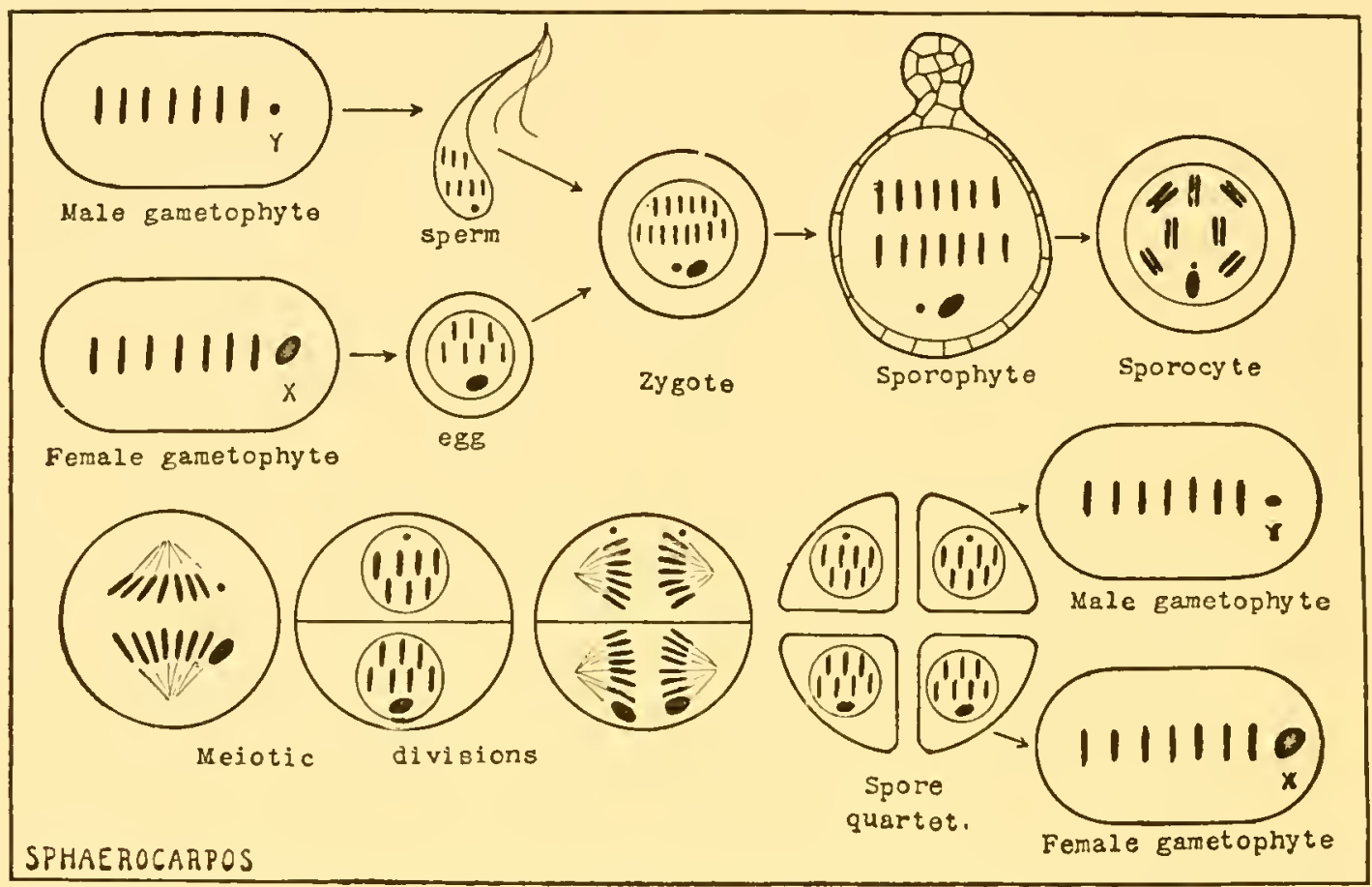

FIG. 218.-Chromosome cycle in Spharocarpos. (Based on data of C. E. Allen, 1917, 1919.)

The genus Pellia also shows well-differentiated sex-chromosomes ${ }^{20}$ (Figs. 219, 220). In P. Neesiana the male gametophyte has nine chromosomes, including a $Y$ with unequal arms. In the female there is an equal-armed $X$ in place of the $Y$. Considerable portions of the $X$ and $Y$

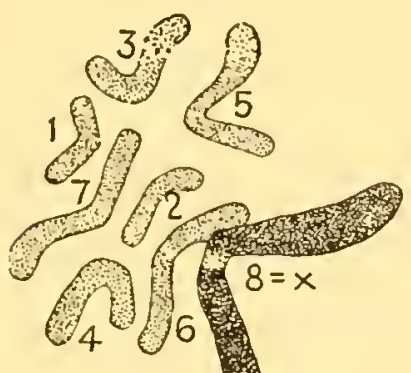

A

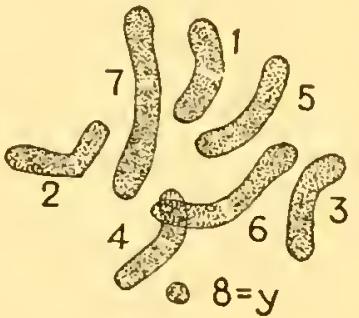

B

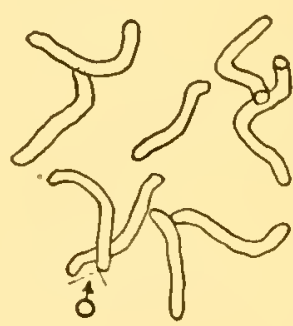

c

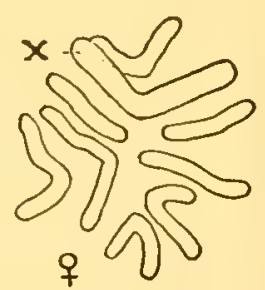

D

Fig. 219.- Sex-chromosomes in liverworts. $A, B$, Sphorocarpos Donnellii: $A$, from apical region of female gametophyte; $B$, from spermatogenous tissue of male. (After Lorbeer, 1930.) $\quad C, D$, Pcllia Neesiana: sets from male and female gametophytes. Which of the nine chromosomes in the male is the $Y$ has not been determined. (After Showalter, 1928.)

remain condensed (heteropyknotic) through the telophase and interphase. A comparison of the chromosome complement with that of $P$. epiphylla brings out the interesting fact that in homothallic species also a specialized heteropyknotic " $M$-chromosome" may be present and suggests that the

${ }^{20}$ Heitz (1927ho. 1928ab), Showalter (1927c, 1928). 
heterothallic condition may have arisen through the loss of a portion of this chromosome in some plants. This would give sex-chromosomes of two kinds: an unmodified $X$ and a shortened $Y$. It would also give two

Pellia epiphylla.

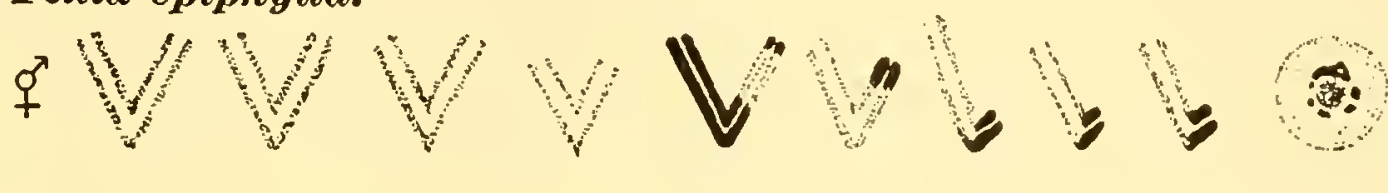

\section{Pellia Neesiana.}
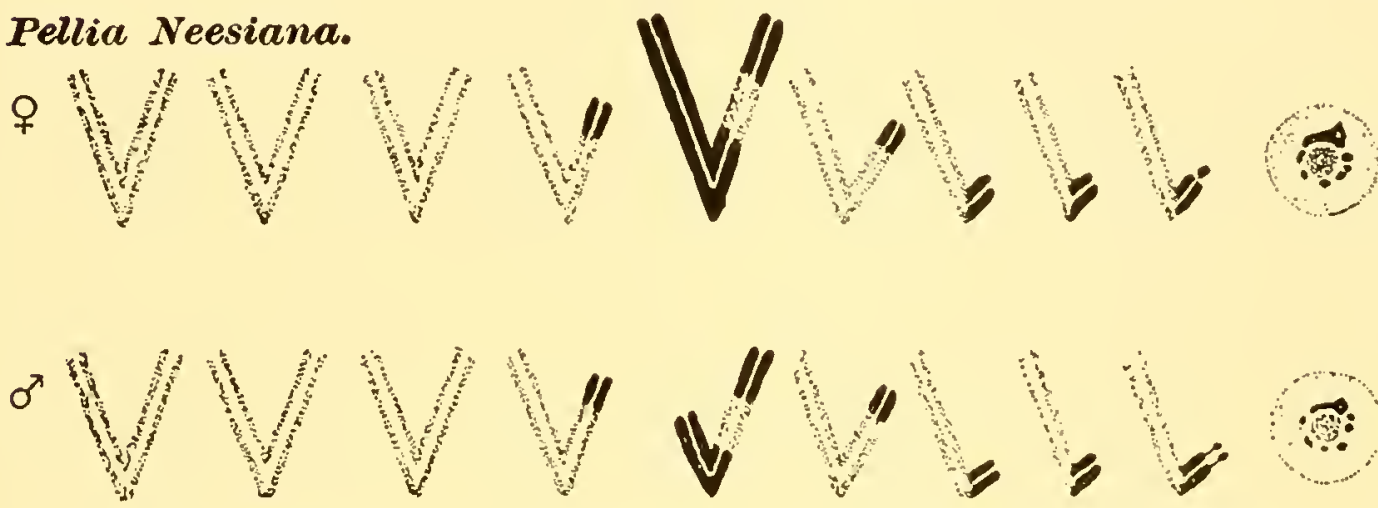

Fig. 220.-Diagram of chromosome sets of homothallic and heterothallic species of Pellia. The fifth chromosome in each row is the sex-chromosome. At right: metabolic nuclei, showing heteropyknotic chromosomal regions. Cf. Fig. 80. (After Heitz, 192Sb.)

sexual manifestations, assuming that the $M$-chromosome carries factors specially influencing sexual development.

Sex-chromosomes essentially like those of Pellia Neesiana have been described in the heterothallic moss Pogonatum inflexum (Fig. 221).
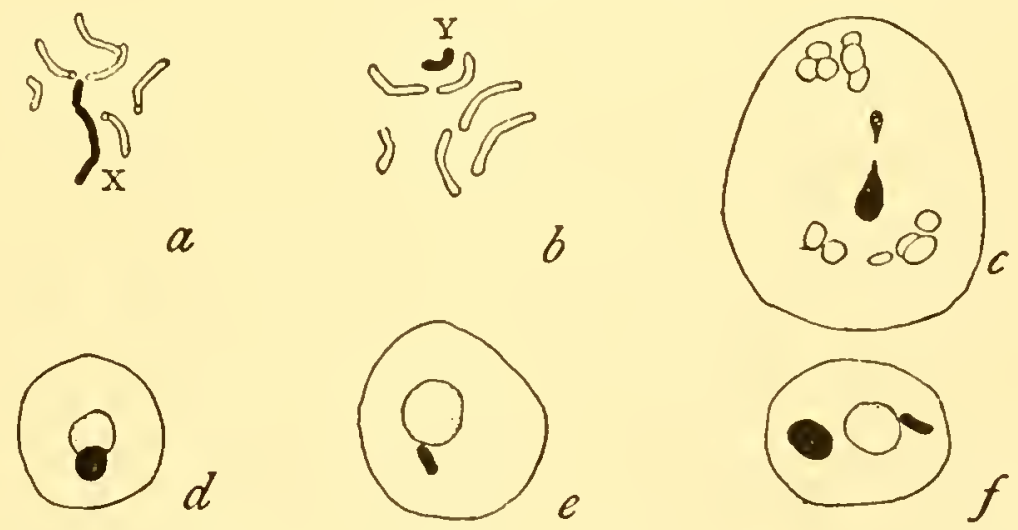

Fig. 221.-Chromosomes of Pogonatum inflexum. a, from female gametophyte $(6+x)$. $b$, from male gametophyte $(6+y)$. $e$, disjunction of $Y$ and $X$ in first mitosis in sporocyte. $d, e, f$, metabolic nuclei from female gametophyte, male gametophyte, and sporophyte, showing heteropyknotic sex-chromosomes (also nucleolus). (After Shimotomai and Koyama, 1932.)

In other heterothallic bryophytes, whose chromosomes are not well known, there is evidence for the meiotic segregation of sex factors. In certain mosses there are produced in the capsule spores of two kinds which 
develop into male and female plants, respectively. ${ }^{21}$ In these gametophytes, which may be reproduced by regeneration, the sexes are very firmly fixed, but gametophytes produced by regeneration from diploid tissue of the sporophyte are bisexual. In pteridophytes the sexual tendencies of ordinarily heterothallic gametophytes are not so firmly fixed and can often be readily altered by varying the cultural conditions. ${ }^{22}$ In these forms very little is known about the chromosomes or factor segregation.

Algæ and Fungi.-A series of experiments performed by Klebs and others many years ago showed that in certain green algæ the environmental conditions very largely determine whether the plants shall grow vegetatively, produce zoöspores, or reproduce sexually. The factors influencing the kind of sex developed have, however, been very little investigated in the algæ. A great many species are homothallic. In Vaucheria it has been found that bisexual plants may be formed by regeneration from the contents of either the antheridium or the oögonium; this indicates that the differentiation of the sex organs can hardly involve a segregation of genetical sex factors. In the heterothallic Cladophora Suhriana it has been reported that the diploid sporangium-bearing plant has 13 chromosomes, including an unpaired $X$. In meiosis both halves of the split $X$ pass to the same pole in the first mitosis and separate in the second, so that half of the spores and diœcious gametophytes carry six chromosomes while the other half carry $6+X$. In certain red algæ, for example Polysiphonia violacea, the development of distinct male and female plants from different tetraspores suggests a segregation of sex factors in meiosis. Studies on diatoms have yielded evidence that such factors segregate at $I$ in Nitzschia subtilis and at $I I$ in Anomœoneis sculpta. ${ }^{23}$

In the fungi comparatively little is known about the chromosomes aside from their number and the occurrence of meiosis at sporogenesis in certain groups (p. 281). In many forms there is, however, strong evidence for a meiotic segregation of factors influencing the course of sexual reproduction. In Coprinus, Aleurodiscus, Ustilago, Puccinia, and other genera of basidiomycetes the spores produced by a single basidium may be of two "strains," as indicated by the behavior of the mycelia developing from them. Hyphal unions initiating the binucleate phase of the life cycle take place only when mycelia of opposite strain are brought together. ${ }^{24}$ In some cases the mycelia from the spores of a

21 El. and Em. Marchal (1906, 1907, 1909), Schweizer (1923), Fleischer (1920).

22 Wuist (1913), Czaja (1921), Mottier (1927, 1931), Nagai (1914, 1915).

${ }^{23}$ Von Wettstein (1920) on Vaucheria; Schussnig (1930ab) on Cladophora; Geitler (1928b, 1929b) and von Cholnoky (1928) on diatoms.

${ }^{24}$ Kniep (1919 et seq.), Vandendries (1923 et seq.), Brunswik (1924, 1926), Hanna (1925, 1928, 1929b), D. Newton (1926), Sass (1928, 1929), Zattler (1924), Craigie 
quartet are of four types, each one reacting with only one of the other three.

A parallel situation is found in ascomycetes. For example, in Neurospora tetrasperma the four spores usually developed in a single ascus are binucleate and produce "homothallic" mycelia, while occasional uninucleate spores produce "heterothallic" ones. In the ascus of $N$. sitophila there are regularly eight spores, of which four are "plus" and four "minus," as indicated by the interactions of the mycelia they form. By studying the relative positions in which the spores with the various tendencies are borne in the ascus, it is possible to show that the factors concerned in the differentiation of these tendencies may be segregated at either the first or the second meiotic mitosis and that this segregation is independent of that of certain other factors. ${ }^{25}$

In certain heterothallic phycomycetes, notably in Phycomyces nitens, the spores in a germ sporangium develop two kinds of mycelia, zygospores being formed only when gametangia borne on the plus and minus strains are brought together. ${ }^{26}$

It has been generally assumed that the plus and minus strains in the fungi represent the two sexes, the factors segregated at meiosis being "sex factors" analogous to those in bryophytes. It was to this sexually diceious condition that the term heterothallism was originally applied (Blakeslee, 1906). When it was found that the mycelia in certain basidiomycetes were of four types rather than two, some observers concluded that there must be four grades of sex, this "multipolar" condition being determined by the independent segregation of two or more pairs of sex factors in meiosis.

A number of recent researches in this field tend to support another interpretation of such phenomena. In certain species, representing both ascomycetes and basidiomycetes, it has been shown that the plus and minus strains are not unisexual: the mycelium from a single spore bears functional organs of both sexes, ${ }^{27}$ but sexual unions occur only between those of opposite strain. From this it is inferred that the meiotic segregation in such cases is not one of sex factors but rather one of factors inducing self-sterility and cross-fertility between certain classes of

(1927, 1928), R. F. Allen (1929, 1930, 1932). Kniep (1928) and M. Hartmann (1929bc, 1930) review the subject of sexuality in lower plants. See also the experiments of Harder (1927) (p. 419).

${ }^{25}$ B. O. Dodge (1927, 1928ab, 1929b), Wilcox (1928), Lindegren (1932). See also Dowding (1931) and Ames (1932).

${ }^{26}$ Blakeslee $(1906,1915,1920 b)$, Burgeff $(1913,1914,1915)$, Couch (1926). See, further, footnote 28.

${ }^{27}$ E.g.: Antheridia and aseogonia in Ascobolus (Gwynne-Vaughan and Williamson, 1932); spermatia (mieroconidia) and ascogonia in Pleurage (Ames, 1932) and Sclerotinia (Drayton, 1932); and spermatia and receptive hyphæ in Puccinia (R. F. Allen, 1932). 
individuals. Unfortunately such species have been said to be "heterothallic" along with those showing true sexual diœeism, thus creating a confusion which should be clarified.

It is evident, therefore, that the problem of sex in fungi is complicated, as it is in higher plants, by factors which render certain bisexual individuals or strains intersterile. While the differentiation into plus and minus strains is plainly of this nature in the instances cited above, there is evidence ${ }^{28}$ that in others it may be truly sexual. A full understanding of the situation can scarcely be reached until much more has been learned about the physico-chemical nature of the sexual states themselves and about the factors by which they are controlled.

Sex-chromosomes in Angiosperms.-There has long been reason to believe that the heterophytic (diœcious) condition in flowering plants is related to a qualitative difference among the pollen grains. Correns (1907) concluded from his work on Bryonia that the eggs all have a female sex "tendency"; that there are two kinds of microspores, and hence male gametes, with male and female tendencies respectively; and that the male tendency dominates the female, so that half of the sporophytes are staminate and half of them carpellate. Strasburger (1910a), who worked with Elodea, Melandrium, and Mercurialis, assumed that the tendency of all the pollen grains, and hence male gametes, is toward maleness: some have a "strong" male tendency which dominates the female tendency of the egg, staminate plants being so produced, while others have a "weak" male tendency which is dominated by the female tendency of the egg, carpellate plants so resulting.

The view that the pollen grains are of two kinds with a differential effect upon the sex of the offspring received cytological confirmation in 1923, when several species of heterophytic angiosperms were shown to have heteromorphic sex-chromosome pairs. ${ }^{29}$ Not all heteromorphic pairs in higher plants, however, are sex-chromosomes.

Sex-chromosomes in heterophytic angiosperms are most commonly of the $X Y$ type. In Elodea canadensis (Fig. 222), the first known case in vascular plants, the microsporocytes show 24 pairs, including an unequal $X Y$ pair. All of the pairs disjoin in the first division, and the second

${ }^{28}$ Chemical researches on strains of phycomycetes (Satina and Demerec, 1925; Satina and Blakeslee, 1925 et seq.). Vandendries and Brodie (1933) report evidence for the view that repulsion between certain strains of a basidiomycete, Lenzites betulina, is due to a radiation under the influence of a Mendelian factor pair and not to chemical interaction.

${ }^{29}$ For lists of known cases, see Kihara (1929b) and Sinotô (1929b). Correns (1928a) reviews the subject of sex in higher plants. Yampolsky (1922) lists the types of sexual manifestation in angiosperms. Lindsay (1930) gives a list of heterophytic plants whose chromosomes have been studied. Lindsay (1929) and Cooper (1929) describe a heteromorphic pair in the homophytic Bougainvillea. For a discussion of the application of terms implying sexuality to sporophytes, see Sharp (1925). 
division is equational, so that half of the microspores (and hence male gametes) receive a $Y$-chromosome and 23 autosomes while the other half receive 23 autosomes and an $X$-chromosome. In the nuclei of the

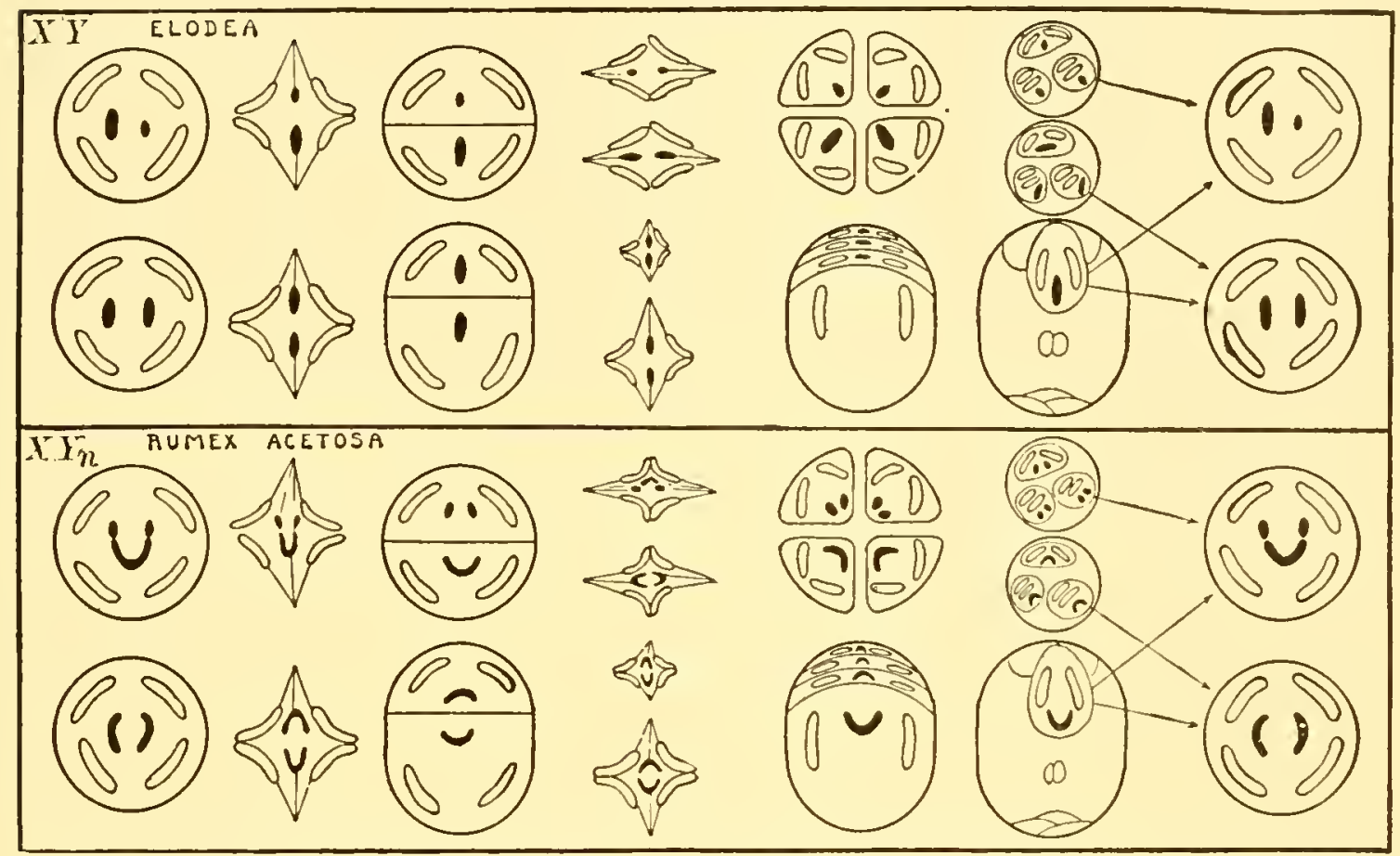

FIG. 222.-Diagrams of two types of sex-chromosome behavior in angiosperms. In each case the upper row represents the staminate plant and the lower row the carpellate plant. Number of autosomes arbitrarily shown as four.

carpellate plant there are two $X$-chromosomes, every egg receiving one of them. It is therefore evident that the union of an egg with a male gamete carrying a $Y$-chromosome results in the production of a staminate

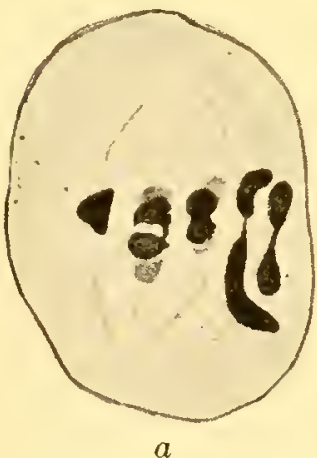

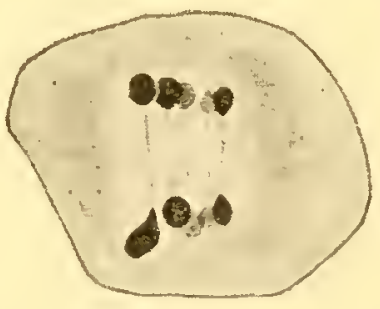

c

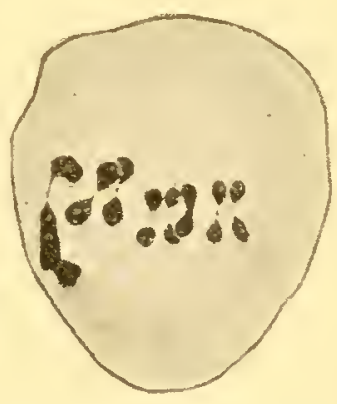

$d$

FIG. 223.-Sex-chromosomes in heterophytic angiosperms. a, division $I$ in microsporocyte of Melandrium album, showing unequal XY pair about to disjoin. b, Populus balsamifera. c, Valeriana dioica. $d$, Rumex acetoselia, showing $M m$ disjoining from $m$. (c after Winge, 1923b; b-d after Meurman, 1925.)

plant $(46+X Y)$, while a union with a male gamete with an $X$-chromosome leads to the production of a carpellate plant $(46+X X)$ (Santos, 1923, 1924). 
In Melandrium album (Lychnis alba) ${ }^{30}$ an $X Y$ pair behaves essentially as in Elodea (Fig. 223). This holds also for Lychnis dioica, in which the staminate plants have long been known to be heterozygous for sex (Shull). In seeking an explanation for the preponderance of carpellate plants in this genus, Correns found that the pollen tubes formed by "female-producing" grains grow more rapidly than those of the "maleproducing" grains and thus reach a larger proportion of the ovules. When relatively small amounts of pollen are used, thus lessening the competition unfavorable to the male-producing pollen, the ratio of

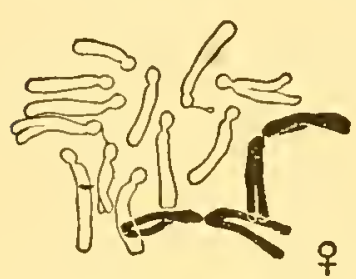
staminate and pistillate plants among the offspring is changed decidedly toward equality. ${ }^{31}$

A peculiar form of sex-chromosome behavior is manifested by Rumex acetosa and certain related species. $^{32}$ In $R$. acetosa (Figs. 222, 224) the staminate plant has 15 chromosomes in the somatic nuclei. In

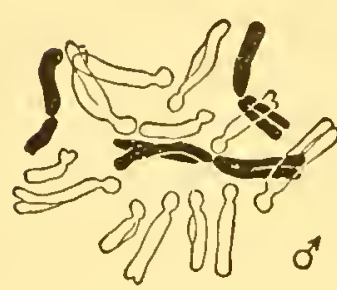

FIG. 224.-Somatic chromosome complements of carpellate $(X X)$ and staminate $(Y X Y)$ plants of Rumex acetosa. Sex-chromosomes in black. (After Ono, 1930b.) the microsporocytes there are six bivalents and a triple group composed of a large $X$ with two smaller elements $\left(Y_{1}\right.$ and $\left.Y_{2}\right)$ attached to its ends. In the first meiotic mitosis $X$ disjoins from $Y_{1}$ and $Y_{2}$, and in the second mitosis all of the chromosomes divide; hence two microspores of the quartet receive 7 chromosomes $(6+X)$ while the other two receive $8\left(6+Y_{1} Y_{2}\right)$. The somatic nuclei of the carpellate plant contain 14 chromosomes $(12+X X)$. Thus it appears that fertilization by a male gamete with $X$ results in a carpellate plant and that by a gamete with $Y_{1} Y_{2}$ in a staminate one. This may be called the $X Y_{n}$ or $Y X Y$ type of sex-chromosome behavior. It is reported that in $R$. acetosella the large chromosome and one small one disjoin from the other small one (Fig. $223, d)$.

A similar triple chromosome group is found in Humulus japonicus. ${ }^{33}$ The distribution of the three members differs in the various sporocytes, giving different chromosomal races (Tuschnjakova). The full sig-

${ }^{30}$ Blackburn (1923, 1924), Winge (1923b), Heitz (1925b). Other cases are Populus and Valeriana (Meurman, 1925), Morus, Cannabis, and Salix (Sinotô, 1928), and Silene (Blackburn, 1928, 1929).

${ }^{31}$ G. H. Shull (1910, 1911), Correns (1918b, 1920b, 1921, 1922b).

32 Kihara and Ono (1923ab, 1925, 1926), Ono (1926, 1927a, 1928, 1930ab), Ono and Shimotomai (1928), Meurman (1925), Kihara and Yamamoto (1931). The presence of two kinds of pollen was demonstrated by Correns (1922b).

${ }^{33}$ Kihara (1928, 1929a), Winge (1923b, 1929a), Sinotô (1929b), Tuschnjakova $(1929,1930)$. It seems probable that more complex configurations observed here (Kihara, 1929d) and in H. lupulus (Sinotô, 1929ab) are due to more extensive chromosome association (Winge, 1929b), possibly through translocation. 
nificance of this with respect to sex and other features has not been determined.

The first known case of $X Y$-chromosomes in digametic females in plants was that of Fragaria elatior. The genetic evidence that the carpellate plants in Fragaria produce two sorts of megaspores, and therefore female gametes, is supported by cytological studies on $F$. elatior. In the megasporocyte there are 21 pairs, including an unequal $X Y$ pair which disjoins in the first meiotic mitosis, each member then dividing in the second. Fertilization of $X$-eggs and $Y$-eggs by $X$-sperms thus gives staminate plants $(X X)$ and carpellate plants $(X Y)$, respectively. The breeding behavior of Thalictrum Fendleri shows that this species also is heterozygous for sex in the female. ${ }^{34}$

Sex-linkage.-As explained in Chapter XVII, characters which are dependent upon differential genes located in the same chromosome are "linked" in inheritance. Since the sex-chromosomes carry genes affecting non-sexual characters also, such characters exhibit a peculiar relation to sex in successive generations: they are sex-linked characters. ${ }^{35}$ The following example will make this clear.

When a red-eyed male Drosophila is mated to a white-eyed female, the $F_{1}$ individuals are white-eyed males and red-eyed females; each eye-color is, as it were, transferred to the opposite sex (Fig. 225). When the $F_{1}$ flies are interbred, the resulting $F_{2}$ generation comprises four types: red-eyed males and females, and white-eyed males and females. It is observed that red eye-color appears in every fly, male or female, which possesses a derivative of the $X$-chromosome of the original red-eyed male; whereas, white eyes characterize every male deriving the $X$-chromosome from the original white-eyed female, and in every female having two such chromosomes. This is because the original male's $X$-chromosome carries a dominant factor for red eyes, while each of the original female's $X$-chromosomes carries a recessive factor for white eyes. This explanation also applies to the reciprocal cross (white-eyed male $\times$ red-eyed female), in which, however, the relative proportions of red-eyed and white-eyed flies in $F_{1}$ and $F_{2}$ are different: in $F_{1}$ all flies of both sexes have red eyes, while in $F_{2}$ all of the females and one half of the males have red eyes, white eyes appearing only in one half of the males.

${ }^{34}$ Genetic evidence in Fragaria: C. Richardson (1914), Valleau (1923), Correns (1928a). Cytological: Kihara $(1926,1930 b)$. Thalictrum: Kuhn (1930d).

${ }^{35}$ Sex-linked characters are not to be confused with sex-limited characters. The latter are those found exclusively in individuals of one sex and are usually called "secondary sexual characters." In several cases it has been shown that their genes are located in the autosomes. It is suggested by Winge (1922a) that inheritance of secondary sexual characters through autosomes be referred to as "sex-limited" inheritance, that of characters through the sex-chromosome common to both sexes $(X)$ as "sex-linked" inheritance, and that of characters through the sex-chromosome occurring in only one sex $(Y)$ as "one-sided" inheritance. 
The form of human colorblindness known as Daltonism, in which green and red are not properly distinguishable, is a sex-linked character which is inherited precisely like white eyes in Drosophila. The presence of this defect more commonly in men than in women, and its appearance in so few individuals in affected lines, are due to the fact that it is a recessive and sex-linked character. It occurs in a woman only if both of her $X$-chromosomes bear factors for it, which means that one such factor must have been received from each parent; whereas one factor is sufficient

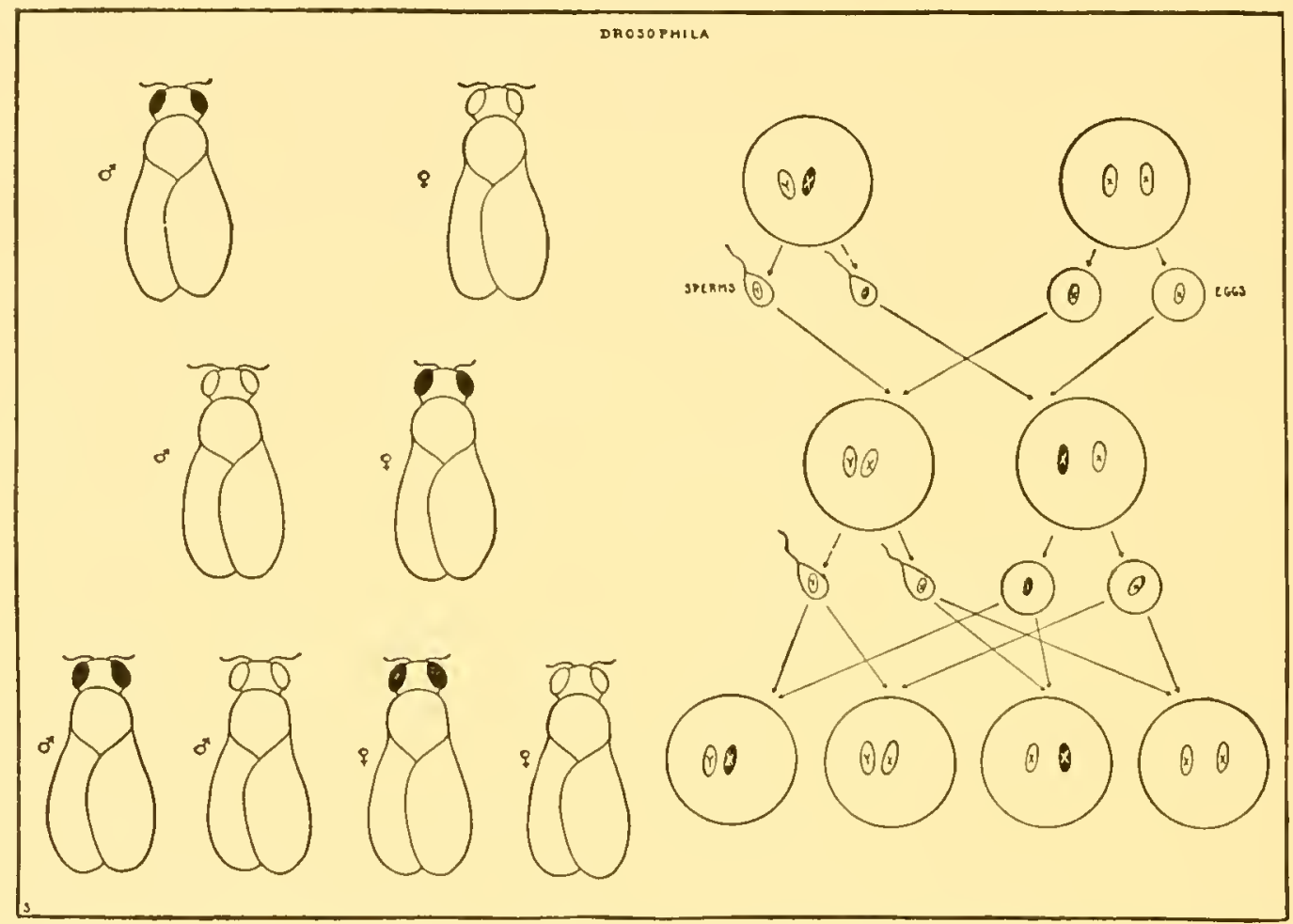

F1G. 225.- Sex-linkage in Drosophila. Three suecessive generations at left; red eyes shown in black. History of sex-chromosomes through these generations shown at right, the $X$-chromosome of the original male in black. (Adapted from Morgan.)

to produce it in a man, because his $Y$-chromosome carries no factors which might dominate it. Furthermore, since the $X$-chromosome of the male is always derived from the mother, a man can inherit colorblindness from his mother but not from his father. He might appear to do so, however, if his father were colorblind and his mother apparently normal but carrying one affected $X$-chromosome: the defect in this case would actually be transmitted by the mother, though apparently by the father. In general, therefore, a colorblind woman transmits the defect to all of her sons and to half of her grandsons and granddaughters, whereas a colorblind man transmits it to none of his children and only to one half of his grandsons. Hæmophilia is inherited in a similar manner. ${ }^{36}$

Sex-linkage in plants was first observed in Melandrium (Lychnis). ${ }^{37}$ The genetic data indicate that the genes for "angustifolia" and "aurea"

${ }^{36}$ For literature on sex-linkage in man, see Davenport (1930).

${ }^{37}$ Baur (1912), G. H. Sluull (1914), Winge (1927c, 1930c, 1931). 
are located in the $X$-chromosome, but certain lethal effects of these genes result in atypical ratios. A gene for "abnormal" may be present in either the $X$ or the $Y$ (Winge).

Crossing-over is often rare or absent altogether in individuals heterozygous for the sex factors. In Drosophila it is normally only in females $(X X)$ that crossing-over takes place: new combinations resulting from this process occur in the eggs but not in the spermatozoa, a fact which greatly simplifies genetic analysis in this organism. The lack of crossingover between $X$ and $Y$ is doubtless associated in some way with their wide difference in constitution and function, particularly with the large amount of genetically "inert" material in them. ${ }^{38}$ Lack of crossing-over in the accompanying autosomes as well indicates the action of general crossover suppressors. The common fowl, in which the female is heterozygous for sex $(X Y)$, shows crossing-over only in the male $(X X)$. On the other hand, the genetic evidence indicates that in the fish genera Aplocheilus and Lebistes crossing-over occurs frequently between the $X$ and $Y$. Thus it was observed that a certain character showing "onesided masculine inheritance," presumably because of a gene in the $Y$-chromosome, suddenly began to behave as though the gene had been transferred to the $X$. In a later generation it was apparently returned to the $Y$ by a second crossover (Winge, 1923a). In the related genus Platypxcilus, which is heterogametic in the female, there is evidence of crossing-over between the $X$ and $Y$ (Gordon, 1927). In many organisms, crossing-over occurs regularly in both sexes. It seems probable that these are forms with less highly differentiated sex mechanisms and that lack of crossing-over in forms like Drosophila is of value in preserving the state of sexual heterozygosity.

In Drosophila the normal allelomorph for the mutant character "bobbed" is carried in the $Y$-chromosome. In Phytodecta any one of a group of four allelomorphic genes (p. 310) may be carried by either the $X$ or the $Y .{ }^{39}$

The general conclusions regarding the function of sex-chromosomes are amply supported by the results of chromosome aberrations, particularly non-disjunction. ${ }^{40}$ In all such cases "an abnormal distribution of the sex-chromosomes goes hand in hand with an abnormal distribution of all sex-linked factors" (Morgan).

${ }^{38}$ Muller (1914b, 1918), Muller and Painter (1932).

${ }^{39}$ Drosophila: Stern (1926a). Phytodecta: Zulueta (1925; see Morgan, 1926a); Galán (1931) on chromosomes.

${ }^{40}$ Non-disjunction of the two $X$-chromosomes in a normal female is called "primary" non-disjunction; the separation of $X X$ from $Y$, or $X Y$ from $X$, in an $X Y Y$ female is called "secondary" non-disjunction. Non-disjunction is also termed "reductional" or "equational" according as it occurs in a disjunctional or an equational meiotic division. The frequency of non-disjunction in Drosophila can be markedly increased by X-rays (Mavor, 1921 et seq.; E. G. Anderson, 1924c). 
Heteroploidy and Sex.-A valuable clew to the probable mode of action of chromosomes in the determination of sex is afforded by heteroploid organisms. It was long supposed that sex in Drosophila, for example, was a matter of the $X$-chromosomes alone, one of these causing maleness and two, femaleness. In his researches on heteroploid flies, however, Bridges ${ }^{41}$ was able to show that the two sexes are correlated not simply with the number of $X$-chromosomes present but rather with the numerical ratio between these and the autosomes. Individuals in which the ratio is $1: 2$ (one $X$ to two autosome sets) are male; those with $2: 2$ or $3: 3$ are females (diploid and triploid). An intermediate ratio $2: 3$ is correlated with the intersexual state, while flies with the extreme ratios $3: 2$ and 1:3 are "superfemales" and "supermales," respectively. In certain mosaic individuals the monoploid regions with a ratio of $1: 1$ are female in character, although other known monoploid insects are male.

The study of sexual and other characters in such heteroploid flies led Bridges to adopt the hypothesis of "genic balance," according to which "each character of an individual is the index of the point of balance in effectiveness of a large but unknown number of genes, some of which have a tendency to change development in one direction and others in the opposite." 42 With respect to sex itself, it had been suggested by Goldschmidt (1911) that the sex grade is a sort of balance between determiners of opposite tendency. As stated by Bridges, both maleness and femaleness are due to the simultaneous action of two opposed sets of genes, whose influences on the organism are toward maleness and femaleness, respectively. These genes, which may affect other characters also and are regarded as distinct from other genes, must lie in most if not all of the chromosomes of the complement; the $X$-chromosome is peculiar merely in having a preponderance of genes tending to produce female characters. The balance is such that, if the number of $X$-chromosomes equals the number of autosome sets, the female-producing genes in the complement as a whole outweigh in effect the genes tending toward maleness and the individual is consequently female; whereas, if there are only half as many $X$-chromosomes as autosome sets, the femaleproducing genes present are insufficient to accomplish this result and the individual is male. Intermediate and extreme ratios would be expected to produce the results actually observed. Since this is precisely what is found in the case of characters other than sex, Bridges concludes that "the conception of the nature and action of genes as gained from the study of non-sexual characters is valid in interpreting sex phenomena." This conclusion is borne out by the fact that in strains showing additional fragments of $X$-chromosomes, the sexual expression varies with the size

${ }^{41}$ Briclges (1921a, 1922, 1925, 1930, 1932).

${ }^{42}$ Genic balance is also correlated with metabolic rate and duration of life by Gowen (1931ab). 
of the fragments, indicating the presence of many sex factors located in various portions of the $X .{ }^{43}$

Comparable with the foregoing conception is the "algebraic-sum" hypothesis of Schrader and Sturtevant (1923), who hold that, although the determination of sex depends upon a preponderance of the genetic effect of the factors for one sex over those of the other sex, a certain threshold degree of preponderance is necessary before there can be clearcut determination. ${ }^{44}$ The case of Habrobracon, in which the monoploid parthenogenetically produced males and the occasional diploid biparental males are similar sexually, appears to require a somewhat different explanation. ${ }^{45}$

The effect of heteroploidy on sex in plants is well illustrated by Rumex and Pellia. In Rumex acetosa, as pointed out in an earlier section, the diploid somatic complement of the female consists of 12 autosomes + $X X$ while that of the male consists of $12+Y X Y$; hence the $X$ :autosome set ratio is $2: 2$ in the female and $1: 2$ in the male. In a series of heteroploid forms the following conditions were observed:46 a triploid plant with $18+X X X$ (ratio $=3: 3$ ) was female; a triploid with $18+X X Y Y$ $($ ratio $=2: 3)$ was bisexual; a tetraploid with $24+X X X Y Y$ (ratio $=$ $3: 4$ ) was bisexual; a triploid with $18+X Y Y Y$ (ratio $=1: 3$ ) was male; a tetraploid with $24+X X Y Y Y$ (ratio $=2: 4$ ) was male.

In Pellia Neesiana, a heterothallic liverwort, the female gametophyte ordinarily has 8 autosomes $+X$ (ratio $=1: 1$ ), while the male has $8+Y$ (ratio $=0: 1)$. A race has been discovered in which the gametophyte is diploid (18 chromosomes) and the sporophyte tetraploid (36); moreover, this gametophyte is bisexual. ${ }^{47}$ Although the mode of origin and cytological constitution of the original bisexual gametophyte are not certainly known, it is thought probable that it arose from a diploid spore formed after ameiosis in a normal sporophyte $(16+X Y)$. If this is true its $X$ :autosome ratio $(1: 2)$ is intermediate between those of monoploid males and females. In Spharocarpos Donnellii, diploid gametophytes arising from such unreduced spores $(14+X Y)$ are functionally female but frequently show a male tendency in the structure of their sex organs. ${ }^{48}$ Simple doubling of the chromosome set in the monoploid

${ }^{43}$ Dobzhansky and Schultz (1931). Other portions appear to be without such genes. See Muller and Altenburg (1928), Muller and Stone (1930), Patterson (1931c), and Muller and Painter (1932). For a discussion of the supposed origin of the sexchromosome mechanism, see Muller (1932).

${ }^{44}$ See the discussion by Schrader and Schrader (1931).

${ }^{45}$ P. W. Whiting (1921), A. R. Whiting (1927, 1928), Torvik (1931).

${ }^{46}$ Ono $(1928,1930 a b)$, Ono and Shimotomai (1928), Kihara and Yamamoto (1931), Ono (1932).

${ }^{47}$ Showalter (1927c). Also in P. epiphylla (Heitz, 1927b).

${ }^{48}$ Lorbeer, 1927; C. E. Allen, 1932. 
gametophytes would be expected to have no effect upon the sex expression, since the ratio would not be altered.

Data such as the foregoing are still rather few, and generalizations should therefore be tentative. At the same time they show clearly that the development of the sexual states is a resultant of the activities of both sex-chromosomes and autosomes. They also suggest one reason for the rarity of polyploidy among animals as compared with plants (Muller, 1925). In animals, which are so commonly unisexual with welldifferentiated sex-chromosomes, the addition of a set of chromosomes seriously disturbs the normal $X$ :autosome ratio, and hence development and fertility, in the heterozygous sex. This offers a serious obstacle to the establishment of triploid and other races with odd numbers of chromosome sets. On the other hand, in bisexual plants there is no such critical ratio to be disturbed, so that triploid and pentaploid races are not uncommon. The origin of tetraploid races directly from diploids in animals may be expected occasionally through a duplication of all the somatic chromosomes, since such duplication does not alter the $X$ :autosome ratio. Noteworthy in this connection is the fact that heterophytic plants tend strongly to remain diploid, or, when polyploid, to show only even numbers of chromosome sets; whereas homophytic plants exhibit very freely all types of polyploidy, with odd as well as even numbers of sets. There is also evidence for the view that polyploid races in the angiosperms tend to maintain their distinctness because of disturbed relations between embryo, endosperm, and surrounding tissues in backcrosses to the original parents. ${ }^{49}$

Modification of Sex.-Field taxonomists and experimental workers are well acquainted with the frequent intergrades between the purely staminate and purely carpellate conditions in normally heterophytic flowering plants, and with the changes in sex expression which may be induced in both heterophytic and homophytic forms by appropriate alterations in the environmental conditions. ${ }^{50}$ The effects of age, nutrition, length of day, and other factors have been investigated and shown to be very marked in a number of plants, including Cannabis sativa, Morus alba, Salix amygdaloides, Humulus japonica, Thalictrum dasycarpum, and Arisama Dracontium.

In only a few such cases are the cytological conditions known. In Cannabis, which is normally heterophytic and is reported to have $X Y$ sex-chromosomes, ${ }^{51}$ the sex ratio is ordinarily $1 \%: 1 \sigma^{7}$. When pollen from anthers appearing on a carpellate plant was transferred to the pistils of a normal carpellate plant, all of the offspring were carpellate. This

${ }^{49}$ See Müntzing (1930, 1933), W. P. Thompson (1930ab), and Watkins (1932).

${ }^{50}$ See Schaffner (1919 et seq.); also Correns (1908), Bartlett (1911), Davey and Gibson (1917), Stout (1919), and Yampolsky (1919, 1920).

${ }^{51}$ Hirata $(1924,1927 b)$, Sinotô (1928). 
indicates that all of the pollen grains carried an $X$, instead of half of them $X$ and half of them $Y$. There was also some evidence that a staminate plant bearing carpels produced some eggs with $X$ and some with $Y$. In Melandrium album an exceptional bisexual individual was shown to have $X Y$ in both micro- and megasporocytes. The same is true of a bisexual Populus tremuloides. ${ }^{52}$ Thus under certain conditions it is possible for reproductive elements of one sex to develop with the chromosome complement ordinarily characterizing the opposite sex.

Such cases of sex modification in plants and animals ${ }^{53}$ serve to emphasize the fact that it is not simply the sex-chromosomes but a complicated system of which the chromosomes are a part that is responsible for the development of the sexes. The development of sex, like that of any other character, depends upon the constitution of the protoplasm concerned as well as upon the conditions to which this protoplasm is compelled to react. Although the protoplasmic constitution is directly inherited, its reactions in the course of ontogeny can be influenced within certain limits through external agencies. Hence the determination of sex, like that of other heritable characters, involves the interaction of two sets of factors: (1) genetic factors (inherited protoplasmic constitution; intrinsic factors; genes); and (2) non-genetic factors (extrinsic factors; environmental agencies, external and internal).

The state of balance between those genetic factors whose influence is toward maleness and those tending toward femaleness varies greatly in different organisms. In bisexual forms it is so delicate that the two sexes develop in response to different conditions prevailing in different regions of the same individual, just as other distinct characters develop near one another. In unisexual forms, especially in those with welldifferentiated sex-chromosomes, two unlike complexes of genetic factors are established through meiosis and syngamy. The balance of maleproducing and female-producing factors in these two complexes is such that, under average conditions, one of them predisposes development toward maleness and the other toward femaleness. In some cases, notably the bryophytes, this predisposition is very strong and has not been overcome by environmental alterations so far tried; whereas, in other cases, such as the angiosperms mentioned above, the natural predisposition can be overcome more or less easily. In no case can the action of either genetic or non-genetic factors be denied. It now seems

${ }^{52}$ McPhee (1925) on Cannabis, Bèlař (1925) on Melandrium, Erlanson and Hermann (1928) on Populus.

${ }^{53}$ Among the many accounts of sex modification and its physiological significance in animals may be cited those of Riddle (1912 et seq.; see 1927), Crew (1923, 1926, 1927abc), Banta (1916 et seq.), Baltzer (1914), F. R. Lillie (1917), Minoura (1921), Goldschmidt (1911 et seq.; see 1923a, 1927ab, 1928b, 1929b, 1931), Witschi (1922 et seq.; see 1929 and 1932), Seiler (1920), and Brambell (1930b). 
evident that all nuclei, monoploid and otherwise, however greatly they may differ in the proportions of their "male-producing" and "femaleproducing" genes, regularly carry both kinds; and it is probable that the tissues in which they lie might be made to develop either sex if we but knew how to alter the other factors concerned.

In the evolution of sex-chromosomes organisms have established a mechanism insuring a degree of sex separation which is sufficient to confer upon them the benefits of such separation, but the condition has apparently become rigid and unalterable only in a minority of the cases, at least among plants. As emphasized by Correns (1926a), the essence of diœcism is the presence of two genotypes correlated with sex and not the uniformly sharp separation of the sexes. The rôle of genetic factors in sex differentiation is strikingly illustrated in the production of a diœeious strain of maize by the genetic manipulation of genes previously known to be associated with male or female sterility in the ordinary monœecious strains. ${ }^{54}$

Only a beginning has been made on the problem of the ontogenetic development of the sexual states. The influences of genetic and environmental factors are exerted through a long and complicated series of intracellular and intercellular reactions. The correlation of sexual differences with metabolic rate (Riddle et al.), chemical composition (Manoilov et al.), ${ }^{55}$ and endocrine secretions (F. R. Lillie, Witschi, et al.) are of special interest in this connection. Goldschmidt, as a result of his extensive researches on intersexuality in diploid moths, ${ }^{56}$ has suggested that the final expression of sexual characters is dependent upon the relative velocities of the male-producing and female-producing reactions in the early stages of ontogenetic differentiation. Moreover, the velocities vary with the amounts of gene substance present. As an embryo with the male genetic complex begins development, the male-producing reactions proceed at a faster rate than the female-producing ones, and this continues throughout development, giving a normal male moth. When, however, the quantity of the male-producing gene substance is less, the rate of the male-producing reactions may sooner or later fall below that of the female-producing ones; if this occurs before the critical stage of differentiation is reached, female characters develop and the moth is intersexual. The earlier the turning point with reference to

${ }^{54}$ D. F. Jones (1931). See Emerson $(1924,1932)$. For discussions of the factorial interpretation of sex determination in the life cycles of plant groups, see Correns (1928a) and Sharp (1925).

${ }^{55}$ Manoilov (1922 et seq.), Grünberg (1922, 1923), Minenkov (1924), Satina and Demerec (1925), Satina and Blakeslee (1925, 1926ab, 1927), Joyet-Lavergne (1927 et seq.), Camp (1929), Dahlgren (1929). For general accounts, see Sehratz (1928), Joyet-Lavergne (1928c, 1932), and Abromavich and Lynn (1930).

${ }^{56}$ See Goldschmidt's recent review (1931). For a brief summary of work on amphibians, see Witschi (1932). 
the critical stage, the greater the amount of sex reversal. The same interpretation applies, mutatis mutandis, to intersexes beginning development as females. In triploid Drosophila, also, the degree of intersexuality varies with the time of sex reversal, which is influenced by both genetic factors and temperature (Dobzhansky, 1930c).

These suggestions are of special importance in that they direct attention to the quantitative and ontogenetic aspects of genic action, a fuller knowledge of which is prerequisite to a true understanding of the physical basis of heredity.

The direct control of sex in a given individual is not to be confused with the control of sex in a population. Nutritive conditions sometimes influence the form of reproduction and therefore the sex of the animals resulting; but here the sex of no individual, once decided, is altered. ${ }^{57}$ Abnormal temperatures may prevent the laying of the eggs from which one sex develops, without interfering with those giving rise to the other sex. ${ }^{58}$ In such cases the environmental influences operate "by affecting the production and survival of sexually predestined germ-cells" (Thomson, 1913).

Conclusion. - In bisexual organisms the gene complexes in all individuals are nearly enough alike (with respect to genes affecting sex) for these to show the same sex manifestation under similar environmental conditions, and the constitution of this gene complex is such as to result in the production of both sexes as responses to the different special conditions in tissues in different regions or at different stages of development. Artificial modifiability here depends on the extent to which those particular special conditions, which are in this case the differential factors, can be controlled or altered.

In unisexual (diœcious) organisms the gene complexes of some individuals differ from those of others in such a way that some develop one sex, and others the other sex, under the same general environmental conditions and in the presence of the range of special internal conditions normally prevailing. Under ordinary conditions, therefore, it is the genetic factors that are differential. In the heterothallic bryophytes the segregation of genes at meiosis results directly in spores and gametophyte individuals of the two sexes; in heterophytic seed plants it results in two sorts of microspores and hence male gametophytes whose respective gametes cause a difference in the sex expression of the succeeding sporophytes; in animals it results directly in two kinds of gametes of one sex. Artificial modifiability here depends on the kind and degree of difference between the male and female gene complexes, and on the extent to which their differential effect can be overcome by altering controllable extrinsic

${ }^{57}$ Whitney $(1914,1916,1919)$ on Hydatina senta.

${ }^{58}$ Malsen (1906; on Dinophilus). 
factors of the system, i.e., the extent to which the latter factors can be made to assume or to share in the differential function.

In many plants and animals the genic sexual differences have been correlated with visible and constant differences in the chromosomes. This serves to emphasize the necessity of considering cytological and genetical data in any attempt to understand sex and its control. 


\section{CHAPTER XXIV}

\section{APOMIXIS AND RELATED PHENOMENA}

The many known kinds of reproduction in organisms are ordinarily grouped in two main categories, sexual and asexual. In the latter category are several modes which seem clearly to represent aberrations or derivatives of the sexual process in that they involve the structures commonly concerned in sexual reproduction. Often there is no sexual fusion whatsoever, in which case the reproductive process is referred to as apomixis. This is in contradistinction to amphimixis, in which the new individual arises from the fusion product of two gametes. In many organisms apomixis recurs regularly in successive life cycles owing to other compensatory processes. In other instances it may occur occasionally without leading to the establishment of apomictic races.

In this chapter attention will be devoted chiefly to apomixis in its two principal forms, parthenogenesis and apogamy. The first of these occurs in both plants and animals, while the second, as well as a related condition known as apospory, occurs in plants only. ${ }^{1}$

\section{PLANTS}

The life cycle in vascular plants, bryophytes, and certain thallophytes is characterized by the alternation of two generations differing in chromosome number. This is not, however, true of all cases. Many plants are now known in which the life cycle shows the two generations as usual but without any changes in the number of chromosomes. This must be borne in mind in reading the following pages.

Apomixis.- No sexual fusion occurs.

A. Parthenogenesis. - The development of a sporophyte from a female gamete (or sometimes a male gamete?) without syngamy.

1 The classification employed here for apomixis in plants follows, in the main, that drawn up by Winkler $(1908,1920)$ with certain modifications. The elassification for animals resembles that of Prell (1923b). To aid the student in interpreting the confused terminology in the literature, the terms of other authors are included in brackets under each heading. It is because of complications introduced by heteroploidy that we substitute the words "reduced" and "unreduced" for the conventional terms "haploid" and "diploid" ordinarily used in such classifieations. For discussions of the phenomena treated in this chapter, some of them with classifications of cases, see Winkler (1908, 1920), Strasburger (1909b), Ernst (1918), Renner (1916), M. Hartmann (1909), Prell (1923b), Vines (1911), P. Hertwig (1920a), Tischler (19211922), Ankel (1927), Rosenberg (1930), and Darlington (1932a). 
1. Reduced Parthenogenesis.-The dereloping gamete has the reduced (gametic) number of chromosomes.

[Called haploid parthenogenesis by Hartmann (1909) and Renner (1916), generative parthenogenesis by Winkler (1904, 1908, 1920), and true parthenogenesis by Strasburger $(1907 a)$.]

For many years no angiosperm sporophytes with the reduced chromosome number were known, but during the last decade several cases have been discovered. They occur in Datura, Nicotiana, Triticum, Crepis, $Z e a$, and other genera (see p. 356). In some instances it is clear that the chromosome set is truly monoploid and not merely the reduced complement of a polyploid race. The origin of such monoploid sporophytes has not been traced in detail, but the genetic evidence leaves little doubt that reduced eggs have developed. Mitosis in such eggs has been observed. ${ }^{2}$ In none of these cases has a regularly apomictic race been established.

This mode of development is known to occur also in thallophytes. In Fucus the development of the unfertilized egg has been induced by artificial means (J. B. Overton, 1913), but the cytological features here are unknown. Motile gametes of certain other alga have been observed to develop without conjugation, as in Ectocarpus (Kylin, 1918). In Vaucheria (von Wettstein, 1920) the contents of either the oögonium or the antheridium may be made to regenerate a new individual; what is virtually "male parthenogenesis" occurs in the latter case. An example of reduced parthenogenesis in the fungi is afforded by Saprolegnia (Mäckel, 1928; Schlösser, 1929).

2. Unreduced Parthenogenesis.-The developing gamete has the unreduced (zygotic) number of chromosomes.

[Called diploid parthenogenesis by Hartmann and Renner, somatic parthenogenesis by Winkler, oöapogany by Strasburger, Juel, and Ernst, and parthenapogamy by Farmer and Digby (1907).]

This form of parthenogenesis occurs very frequently in vascular plants as a regularly recurring reproductive phenomenon. A few representative cases are listed in the footnote. ${ }^{3}$ The unreduced state of the egg is due to a previous failure of meiosis, the sporophytic number of chromosomes being carried through to the gametophyte. The two sporocyte divisions

${ }^{2}$ Kusano (1915) on Gastrodia, Haberlandt (1921, 1922) on Enothera.

${ }^{3}$ Marsilia Drummondii (Strasburger, 1907a); Athyrium filix-fœmina, var. clarissima and Scolopendrium vulgare (Farmer and Digby, 1907); Alchomilla (Murbeck, 1901; Strasburger, 1904c; Böös, 1917, 1920); Antennaria alpina (Juel, 1898b, 1900); Archieracium (Rosenberg, 1906, 1917; Ostenfeld, 1910, 1912); Atamosco texana (Pace, 1913); Erigeron ammuns (Tahara, 1915a, 1921; I. Holmgren, 1919); Eupatorium glandulosum (Holmgren, 1919); Calycanthus (Schürhoff, 1923); Taraxacum (Murbeck, 1904; Juel, 1904, 1905; Osawa, 1913; Sears, 1917, 1922; Stork, 1920a); Chondrilla (Rosenberg, 1912); Wikstræmia (Winkler, 1906; Strasburger, 1909a); Allium odorum (Haberlandt, 1923); Humulus lupulus (von Wettstein, 1925); Ochna scrrulata (Chiarugi and Francini, 1930). An example among lower plants is Chara crinita (Ernst, 1918, $1921 a)$. 
may occur, giving unreduced megaspores (Marsilia), or only one division may take place (Taraxacum), or the gametophyte may arise from the megasporocyte itself without haplosis (Antennaria).

B. Apogamy.-The apomictic development of a sporophyte from a cell or cells of the gametophyte instead of from a gamete.

1. Reduced Apogamy.-The developing cells have nuclei with the reduced (gametic) chromosome number.

[Called haploid apogamy by Hartmann, generative apogamy by Winkler, haploid apogamety by Renner, and meiotic euapogamy by Farmer and Digby.]

The development of sporophytes from vegetative tissue of gametophytes with the reduced chromosome number and without nuclear fusion

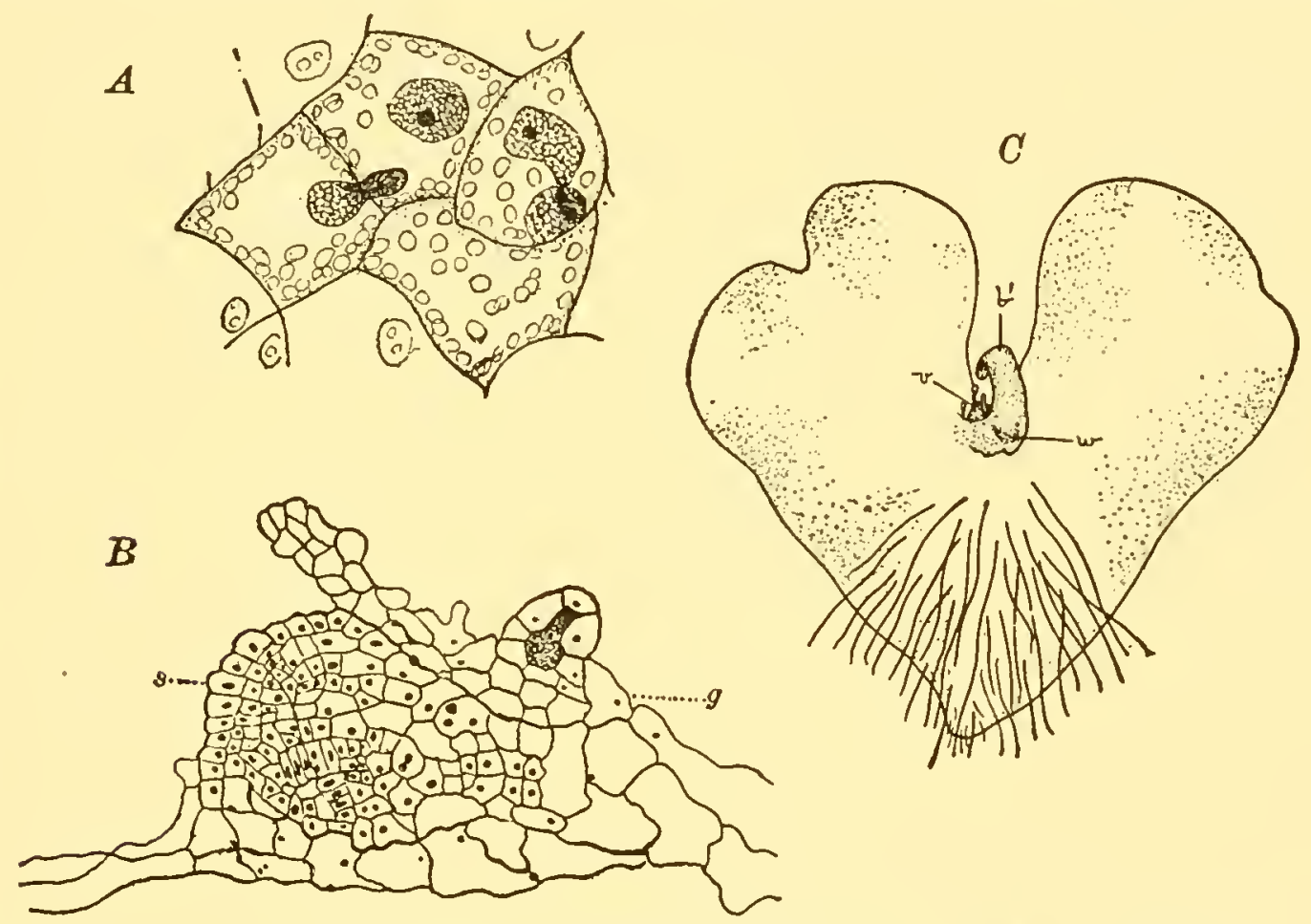

FIG. 226.-A, muclear migration in gametophyte cells of Lastraca. (After Farmer and Digby, 1907.) $B$, section through gametophyte, showing young sporophytic tissue (s) developing from gametophytic tissue $(g)$. (After Farmer and Digby.) $C$, sporophyte arising apogamously from gametophyte in Pteris cretica; $b^{\prime}$, first leaf; $v$, stem apex; $w$, root. (After de Bary.)

has frequently been observed among ferns. ${ }^{4}$ The process commonly begins with the division of the gametophytic cells to form a mass of "engrafted tissue" which then grows into a new sporophyte (Fig. 226, B). Ordinarily, apogamy is offset in the life cycle by apospory. In Nephrodium hirtipes the spores are formed, though in an abnormal manner: when there are eight sporogenous cells in the sporangium there is an incomplete nuclear division, each nucleus coming to have the unreduced number of chromosomes. These eight cells then function as sporocytes

${ }^{4}$ E.g.: Lastroa pseudo-mas, var. cristata apospora (Farmer and Digby, 1907); Nephrodium molle (Yamanouchi, 1908c); and Nephrodium hirtipes (Steil, 1919a). 
and produce 32 reduced spores (Fig. 227, B). The reduced number here $(60+)$ is doubtless a polyploid one.

2. Unreduced Apogamy.-The developing cells have nuclei with the unreduced (zygotic) chromosome number.

[Called diploid apogamy by Hartmann, somatic apogamy by Winkler and Ernst, diploid apogamety by Renner, and euapogamy by Farmer and Digby.]

This type of apogamy appears to occur in certain ferns. In one reported case ${ }^{5}$ the sporophyte arises from unreduced "engrafted tissue"
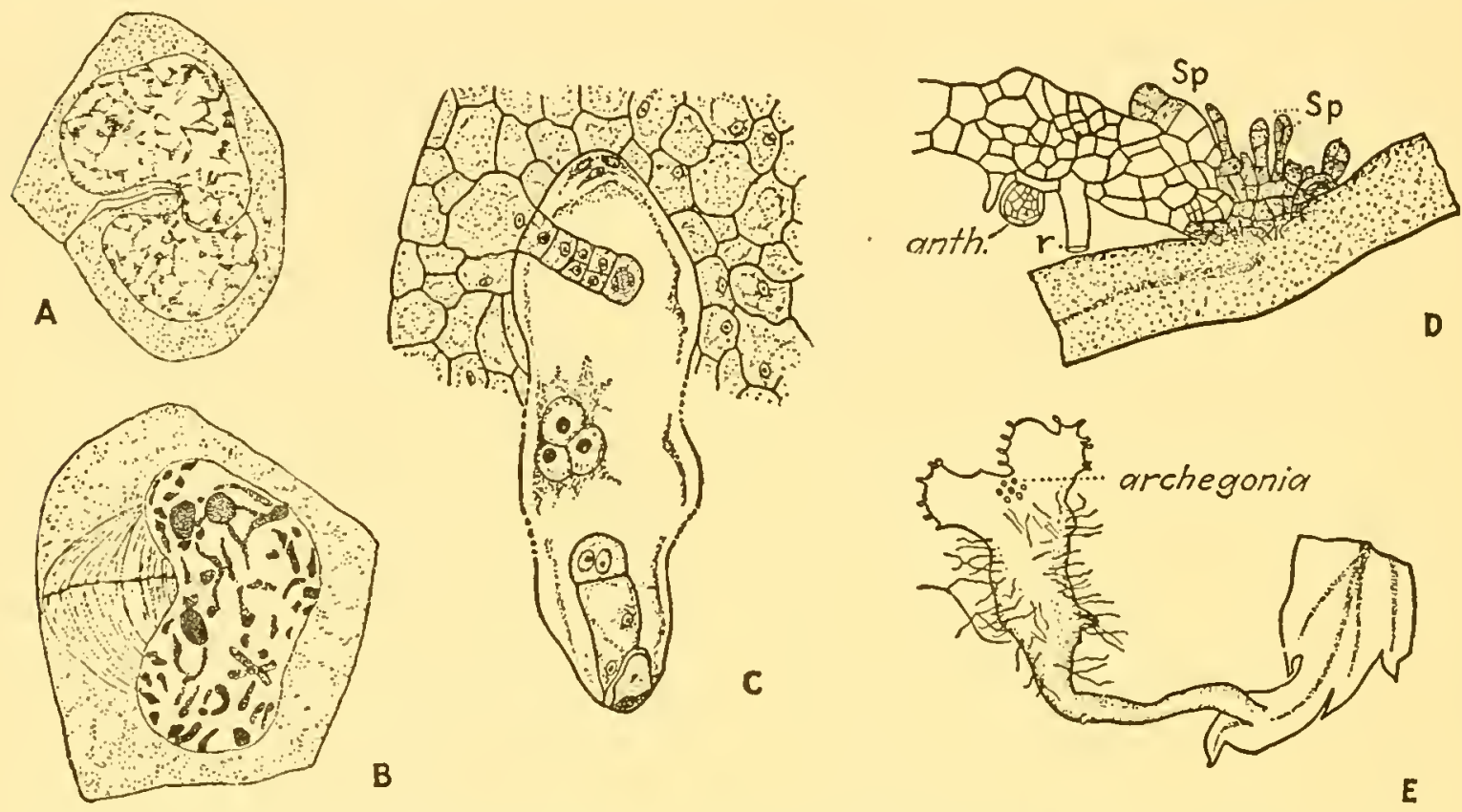

FIG. 227.- $A$, nuclear abnormality in sporangium of Aspidium falcatum. (Afier R. F. Allen, 1911.) $B$, restitution nucleus in Nephrodium hirtipes. (After Steil, 1919a.) $C$, unreduced parthenogenesis and sporophytic budding in embryo sac of Alchemilla pastoralis. The egg is developing one embryo below and a nucellar cell is forming another above; two polar nuclei and one synergid nucleus at middle. (After Murbeck, 1901.) $D$, gametophyte with antheridium and rhizoids arising aposporously from tissue of sorus in Polystichum; sp., sporangia. $E$, gametophyte with archegonia arising from tip of pinnule in Polystichum. ( $D$ and $E$ after Bower.)

which develops after a nuclear migration and fusion in certain cells of the reduced gametophyte (Fig. 226, A). Such a fusion in cells not specifically organized as gametes has been called pseudomixis. In angiosperms the formation of embryos by unreduced synergids or antipodal cells has been reported, ${ }^{6}$ but the cytological data in most such reports are rather scanty.

C. Sporophytic Budding. - The formation of new sporophytes by sporophytic cells surrounding the embryo sac, such cells projecting into the sac and developing into embryos. Commonly the cells in question

${ }^{5}$ Lastróa pseudo-mas, var. polydactyla (Farmer, Moore, and Digby, 1903; Farmer and Digby, 1907).

${ }^{6}$ E.g., Alchemilla scricata (Murbeck, 1902), Burmannia colestis (Ernst and Bernard, 1912), and Allium odorum. (Haberlandt, 1923). 
belong to the nucellus ("nucellar embryony"), but sometimes they are integument cells.

This process occurs in a number of angiosperms, ${ }^{7}$ commonly in species exhibiting parthenogenesis or apogamy also (Fig. $227, C$ ). The saes into which the embryos project may have either reduced or unreduced nuclei. Embryos may arise from the egg, other gametophytic cells, and sporophytic cells in the same plant, the condition known as polyembryony often resulting. ${ }^{8}$ In genetic researches the exact mode of origin of such embryos is a matter of considerable moment.

Apospory.-The development of a gametophyte from a cell (or cells) other than a spore (in plant groups normally developing gametophytes from spores).

As a rule both the sporophyte and the aposporous gametophyte arising from it have the unreduced chromosome number. In several genera of ferns ${ }^{9}$ gametophytes may develop as buds on the sporophyte, commonly from the leaf margin or the meristematic tissue at the base of the sorus (Fig. 227, D, E). Such aposporous development of diploid gametophytes can be induced by cultural means, and this may be followed by the production of tetraploid sporophytes. ${ }^{10}$ Ordinarily, apospory is balanced by a pogamy, the chromosome number remaining the same throughout the life cycle. In mosses ${ }^{11}$ also the development of diploid ganretophytes from portions of the sporophyte can be induced in this way. In some cases these diploid gametophytes produce diploid gametes which fuse and give rise to tetraploid sporophytes bearing diploid spores. In Amblystegium a tetraploid gametophyte has been obtained. Von Wettstein $(1924 b)$ reports the production of octoploid gametophytes in certain intergeneric hybrids.

Apospory may also occur in angiosperms. In such cases ${ }^{12}$ an unreduced female gametophyte develops from a cell of the nucellus or integument, often in addition to the gametophyte arising normally from the megaspore (Fig. 228, $D, E$ ).

False Hybrids.-After cross-pollination individuals sometimes develop which are not true hybrids because they carry nuclear factors from only one of the parents. Such "false hybrids" are metromorphic

${ }^{7}$ E.g., Alchemilla (Murbeck, 1901), Citrus (Osawa, 1912), Rosa (Täckholm, 1922), Spathiphyllum (Schürhoff and Jüssen, 1925), Nigritella (Afzelius, 1928), Artcmisia (Chiarugi, 1926), and Ochna (Francini, 1928).

${ }^{8}$ For cases of polyembryony in angiosperms, sce Ernst (1918, p. 436).

${ }^{9}$ Pteris, Asplenium, Athyrium, Polystichum, Scolopendrium, Lastrca.

${ }^{10}$ Lang (1924) and Manton (1932a) on Osmunda; Lawton (1932) on Aspidium and Woodwardia; Andersson-Kottö (1931, 1932) on Scolopendrium.

${ }_{11}$ Mnium, Bryum, Phascum, Amblystegium (Ë. and Ëm. Marchal, 1909, 1912; Schweizer, 1923).

12 Hieracium flagellare and H. excellens (Rosenberg, 1906, 1907a; see 1930), Artemisia (Chiarugi, 1927), Oxyria (Edman, 1929). 
(like the mother) or patromorphic (like the father), according to the derivation of their genetic factors; ${ }^{13}$ hence two main processes may be distinguished: pseudogamy and androgenesis.

A. Pseudogamy.-The development of metromorphic offspring induced by pollination, but without complete syngamy. The possible explanations are several:

1. Offspring with the reduced chromosome number may arise by (a) reduced parthenogenesis, the male gamete not entering the egg; or (b) gynogenesis, the male gamete entering the egg, but playing no further rôle; or $(c)$ reduced apogamy.
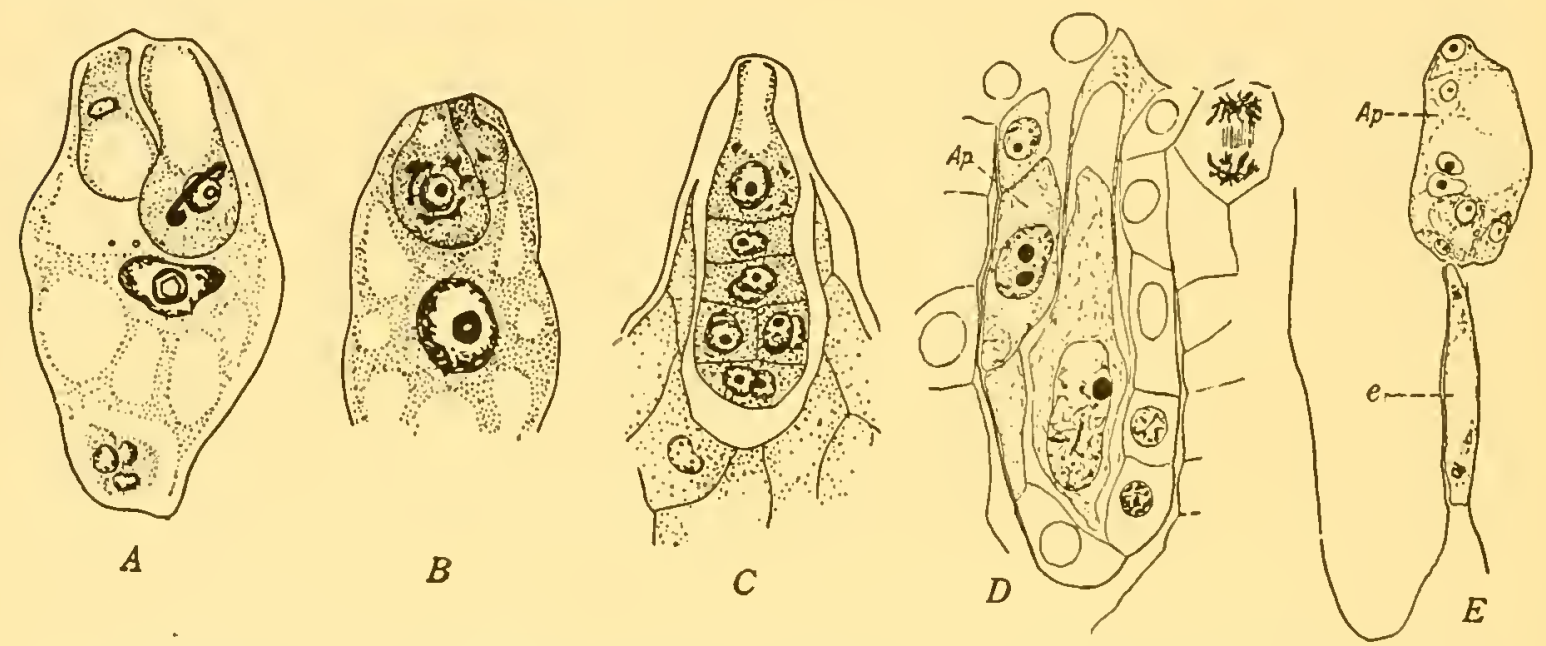

FIG. 228.- $A-C$, gynogenesis in Solanum nigrum. $A$, embryo sac, with curved male nucleus near egg nucleus. $B$, male nuclear matter degenerating in egg. $C$, young embryo, with male matter still evident in upper cell. (After C. Jórgensen, 192S.) $D, E$, apospory in angiosperms. $D$, nucellar cell $(A p)$ beginning development of aposporous embryo sac in Hieracium flagellare. $E$, aposporous embryo sac $(A p)$ outgrowing a normal one $(e)$ in $H$. excellens. (After Rosenberg, 1930.)

2. Offspring with the unreduced chromosome number may arise by $(a)$ reduced parthenogenesis followed by chromosome doubling; or (b) unreduced parthenogenesis; or $(c)$ unreduced apogamy; or $(d)$ sporophytic budding.

Examples of some of the above modes of behavior are the following: Datura stramonium of $\times D$. ferox $\sigma^{7}$ yielded a metromorphic monoploid plant, in all probability as a result of reduced parthenogenesis (explanation, 1a) (Belling and Blakeslee, 1927). Solanum nigrum $q \times S$. luteum or yielded a few plants with the maternal gametic chromosome number, and cytological study showed that the male nucleus degenerates after entering the egg (explanation, 1b) (Fig. 228, $A$ to $C$ ) (Jørgensen, 1928). Fragaria vesca $\& \times F$. chiloensis $\sigma^{7}$ gave metromorphic offspring with the maternal zygotic chromosome number (probable explanation,

${ }^{13}$ Millardet used the term "false hybrids" for actual hybrids which were strongly metroclinous or patroclinous, but Renner (1929) and Kulin (1930b) employ it only for individuals arising apomictically. We follow Kuhn in using the terms pseudogamy (Focke, 1881) and androgenesis (Kuhn). 
2a) (Longley, 1926a; East, 1930). In Atamosco the male nucleus entered a diploid egg and later degenerated, the embryo continuing development with maternal chromosomes only (explanation, 2b) (Pace, 1913). In certain species and biotypes of Potentilla pollination is necessary for the setting of seeds, but the offspring of crosses are purely maternal in characters and chromosome number (Müntzing, 1928).

B. Androgenesis.-Development of offspring with the paternal chromosomes only. How the condition arises is not yet known.

Examples: Tripsacum dactyloides $\& \times$ Euchlcena mexicana $\sigma^{7}$ yielded a plant which was morphologically and genetically like the male parent, although the seed from which it arose was outwardly like that of the other parent (Collins and Kempton, 1916). Nicotiana digluta $\circ \times N$. tabacum or yielded, among other types, one plant which was morphologically and cytologically like other tabacum plants with the reduced chromosome number (Clausen and Lammerts, 1929). Similarly, N. tabacum macrophylla of $\times N$. Langsdorffii $\sigma^{7}$ gave a typical monoploid Langsdorffii individual (Kostoff, 1929).

C. Merogony.-Similar in some respects to androgenesis is merogony, in which an egg fragment without a nucleus develops after the entrance of a sperm. This has been induced in the brown alga Cystosira barbata by Winkler (1901) and in several animals (p. 412).

Causes of Apomixis.-It is a noteworthy fact that apomictic plants are usually characterized by various irregularities in sporogenesis. The megasporocyte, for example, may form a quartet of unreduced spores, a pair rather than a quartet, or no spores at all. Similar irregularities in microsporogenesis lead to the production of varying numbers of functionless pollen grains; in extreme cases no pollen is formed.

It is evident that abnormal chromosome behavior is largely responsible for such defective sporogenesis. In the megasporocyte of Marsilia Drummondii the chromosomes undergo synaptic pairing but then dissociate and split longitudinally as in a somatic mitosis. In Antennaria the meiotic phenomena are fewer, and the mitosis passes sooner into the vegetative form. In Wikstrœmia there is no trace of synaptic association, the mitosis being purely vegetative in character; here only two cells are formed. Finally, in Elatostema sessile there are no meiotic phenomena and no cell-division; the sporocyte forms the female gametophyte directly. These cases ${ }^{14}$ illustrate a series of transitional conditions between normal meiosis associated with sexuality on the one hand and the failure of meiosis associated with apomixis on the other.

The situation is better known in microsporogenesis, where a series of increasingly aberrant modes of chromosome behavior may be seen within a single genus, notably in Hieracium (Rosenberg, 1917). In Hieracium

${ }^{14}$ Strasburger (1907a, 1904c, 1909a), Modilewski (1908). 
pilosella, which is not apomictic, normal synapsis and disjunction are constant in both megasporocytes and microsporocytes. In the species boreale, lavigatum, and pseudoillyricum, all of which belong to the subgenus Archieracium, are seen three stages in the transition from normal meiosis with sexual reproduction, such as occurs in $H$. pilosella, to ameiotic sporocyte divisions with apomixis. These cases show clearly that there is a connection of some sort between apomixis and a weakness or absence of the synaptic attraction between homologous chromosomes.

Another notable fact is the common association of apomixis with polyploidy. When the chromosome number of an apomictic species is compared with that of a nearly related sexual species, it is commonly found that the former is larger. In about one-half of the known cases in angiosperms the apomictic species have twice the numbers found in the related sexual species, and in some cases the multiple is still larger. Furthermore, polyploid forms, like apomictic forms, are often characterized by conspicuous irregularities in chromosome behavior at the time of meiosis. A common cause underlying meiotic irregularity, polyploidy, and apomixis has consequently been sought.

That a primary cause of these chromosomal aberrations, together with the associated substitution of apomictic for sexual reproduction, is hybridization, is a theory which has been supported by a number of students of such phenomena. ${ }^{15}$ The phenomena in question are so exactly paralleled in hybrids of known origin that little doubt can be entertained regarding the essential correctness of the theory, though the limits of its application cannot be stated at present. The importance of deficient synapsis in bringing about polyploidy and apomixis has been emphasized by Winge in a special hypothesis involving the synaptic reaction (see p. 369). The sexual sterility so common in hybrids and polyploid forms is largely a result of the incompatibility of chromosomes of unlike origin; indeed, it is highly probable that many apomictic forms have arisen through the crossing of normally sexed forms, as Ernst maintained. In certain moss hybrids apomixis is believed to be due to the action of genes which are freed for action by hybridization or polyploidy. ${ }^{16}$

The advocates of the hybridization theory do not hold hybridization to be the sole cause of apomixis. There is evidence that sexual sterility with apomictic development may also be the outcome of nutritive disturbances set up by other causes, such as abnormal cultural conditions, parasitism, and wounding. The cells involved in sexual reproduction seem to be especially sensitive to unfavorable environmental influences, so that they can easily be made to undergo abnormal development or

15 Ernst (1917, 1918), Rosenberg (1917 et seq.), Winge (1917), I. Holmgren (1919), Täckholm (1920, 1922), Ostenfeld (1921), Turesson (1930).

${ }^{16}$ Von Wettstein (1927). See Zattler (1924). 
degeneration. In Enothera, Haberlandt (1921, 1922) finds that wounding brings about the formation of adventitious embryos and he attributes this to the action of a wound hormone originating in the injured tissue.

The importance of apomixis to the geneticist and field taxonomist is well illustrated by the condition in the Canina section of the genus Rosa (Täckholm, 1920, 1922). Among these roses there are types which had long been regarded as distinct species because of their remarkable constancy in external characters and the lack of intermediate types. Täckholm found that the section includes tetraploid, pentaploid, and hexaploid forms, and that they all show most strikingly the Drosera type of chromosome behavior in meiosis. As a result the microspores and male gametes in the pentaploid forms, for example, contain from 7 to 22 chromosomes, while the eggs usually have 28 . These facts clearly indicate the hybrid nature of the Caninc roses. They are stable genetically because they are apomictic: the embryo arises not from sexual cells but by budding from the nucellus. They are therefore not pure species but asexually reproducing chones which maintain their hybrid chromosome complements and the same external characters from generation to generation because the reassorting of chromosomes and genes at meiosis plays no part in determining the make-up of the next generation. Apomixis obviously retards the process of forming new combinations, but it preserves new types once they are formed, just as in other vegetatively propagated plants. The importance of such facts for students of the origin of species and varieties can scarcely be overestimated.

\section{ANIMALS}

The best known examples of natural parthenogenesis in animals are found among the rotifers, crustaceans, and insects, this being the regular mode of reproduction in some species. Other modes may also occur in such organisms under certain conditions or after a certain number of generations. Some species produce parthenogenetic and sexual eggs which show conspicuous structural differences. Moreover, parthenogenesis may be artificially induced in the eggs of other animal groups, notably echinoderms, mollusks, and amphibians. Around this fact centers much significant work in modern experimental biology. ${ }^{17}$

Parthenogenesis.-In true parthenogenesis the egg develops without syngamy or other nuclear fusion.

1. Reduced Parthenogenesis.-The developing gamete has the reduced (gametic) chromosome number, two maturation divisions having effected haplosis as usual.

${ }_{17}$ Robertson (1931b) introduces the useful term parthenote for an individual derived from an egg with only one gametic nucleus. 
The type case of this category has long been the honey bee Apis mellifica, in which the eggs may develop parthenogenetically into drones or, after syngamy, into females. ${ }^{18}$ Such a development of males with the reduced chromosome number has also been demonstrated in a number of other insects $^{19}$ (Fig. 229). In such males the divisions differentiating the spermatids are purely equational in character; often there is but one mitosis. It is probable that in certain rotifers also the male parthenotes have the reduced chromosome number. ${ }^{20}$

In addition to the foregoing cases in which the reduced chromosome number is retained to sexual maturity, there are others in which the number somehow becomes doubled during ontogenesis. Investigators have repeatedly found that the larvæ of echinoderms, insects, amphibians,
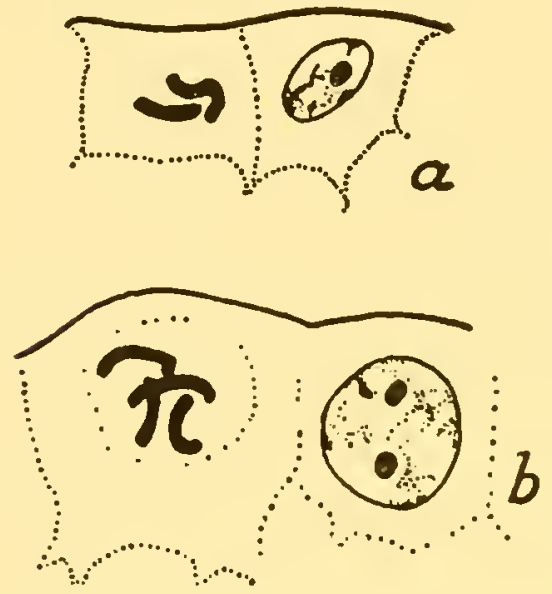

FIG. 229.-Chromosomes of Icerya littoralis. $a$, in parthenogenetically produced male (monoploid). $b$, in sexually produced female (diploid.) (After HughesSchrader, 1930b.) and other animals, whether produced by inducing the parthenogenetic development of reduced eggs or by causing the insemination of enucleate egg fragments, soon show a characteristic inability to continue very far with their metamorphosis. For example, when frogs' eggs are induced to develop by artificial means, mitoses with both the gametic and zygotic chromosome numbers can be seen in the cleavage stages and in the tadpoles. The adults all have the zygotic number. ${ }^{21}$ As in plants, there may be animals in which the somatic number is gametic though not truly monoploid; but in Tetranychus bimaculatus, with three chromosomes, and in Icerya purchasi, with two, differing in size, true monoploidy cannot be doubted. ${ }^{22}$ Nevertheless, viable monoploid offspring are not, so far as known, produced by animals normally diploid in both sexes.

2. Unreduced Parthenogenesis.-The unfertilized egg develops with the unreduced (zygotic) chromosome number.

${ }_{18}$ Petrunkewitsch (1901), Meves (1904b, 1907c), Doneaster (1906, 1907), Mark and Copeland (1906), Nachtsheim (1913).

${ }^{19}$ E.g.: Tetranychus, Trialeurodes, Icerya, Paracopidosomopsis (Sehrader, 1920, 1923c; Thomsen, 1927; Hughes-Schrader, 1925, 1926, 1927, 1930ab; Patterson, 1917, 1921; and others). In their useful review of haploidy in Metazoa Schrader and Hughes-Sehrader (1931) list the eases in which the reduced chromosome number in the male has been demonstrated, as well as those in which the evidence is not conclusive. See also the diseussion by Metz, Moses, and Hoppe (1926).

${ }^{20}$ Whitney (1909, 1924, 1929), A. F. Shull (1921), Storeh (1924).

${ }^{21}$ Parmenter (1920, 1925, 1926). See G. Hertwig (1918), Goldschmidt (1920b), and the discussions by P. Hertwig (1920a), Morgan (1924f), and Bosteus (1926). Doubling also occurs in Apotettix and Paratettix (W. Robertson, 1925, 1930).

22 Schrader (1923c), Schrader and Hughes-Schrader (1926). 
In some cases $^{23}$ it is reported that two maturation divisions occur without accomplishing haplosis. More commonly there is but one division, a single polar body being formed with no haplosis. ${ }^{24}$ In Rhabditis monohystera, a nematode worm, the 20 chromosomes in the oöcyte form 10 synaptic pairs and separate in the single meiotic mitosis which occurs. During the telophase the chromosomes appear double as in ordinary meiosis, but since there is no second mitosis to separate them the egg retains the diploid number, 20. This egg develops without syngamy into a diploid animal. Apparently in rare cases there may be no maturation divisions. In Neuroterus lenticularis, a gall-fly, the nucleus in certain eggs approaches the surface as if to produce a polar body but then returns to the middle of the egg and divides equationally. Development then proceeds with the unreduced chromosome number, 20 (Doncaster, 1910, 1911, 1916).

Parthenogamy.- Under this heading may be placed those comparatively rare cases in which an egg develops without union with a male gamete but after a fusion of two nuclei. For example, it has been reported that a polar body nucleus may sometimes fuse with the egg nucleus, after which development proceeds. ${ }^{25}$ In Mactra, after the formation of the two polar bodies has been induced by treatment with salt solutions, the nucleus of the egg divides into two which then fuse, and development proceeds (Kostanecki, 1911). The fusion of cleavage nuclei at later stages has been observed in certain species of Solenobia (Seiler, 1923).

False Hybrids.-As in plants, so in animals there are rare instances of the development of an embryo which, though supposedly the offspring of two parents of the same or different species, contains the functional nuclear factors of only one of them. Cases like that of Nereis (p. 242), in which the egg can be activated by a sperm without the latter's entrance, may be included here. Those in which entrance occurs may be grouped under the following heads.

A. Gynogenesis.-Here the male nucleus degenerates in the cytoplasm of the egg, development proceeding with maternal nuclei alone. This appears to occur normally in Rhabditis, a genus of nematodes. The eggs form only one polar body and retain the unreduced chromosome number. They are then penetrated by the sperm, which degenerates

${ }^{23}$ Rhodites rosæ (Henking, 1892; Schleip, 1909b; Hogben, 1920a), Nematus lacteus (Doncaster, 1907b).

${ }^{24}$ Phylloxera (Morgan, 1906), Aphis (de Baehr, 1908-1912, 1920a), Daphnia (Kühn, 1908), Miastor (Kahle, 1908), Simocephalus (Chambers, 1913), Lecanium (Thomsen, 1927), Rhabditis (Bělař, 1923), Aspidiotus (Schrader, 1929), probably Tettigidæ (Robertson, 1930).

${ }^{25}$ Artemia (Brauer, 1894; F. Gross, 1932), Asterias (O. Hertwig, 1890; Buchner, 1911). 
without fusing with the egg nucleus. In one species the sperm is necessary for activation, although it does not participate further in development. ${ }^{26}$

Similar phenomena are frequently encountered in the course of hybridization experiments and in pathological material. The degeneration of the sperm nucleus in the egg cytoplasm has been observed in a cross between a sea urchin and a mollusk. ${ }^{27}$ In certain other crosses the paternal nuclear matter is extruded from the fusion nucleus or during the early cleavage mitoses. ${ }^{28}$ Such elimination during cleavage results largely from the lagging of the paternal chromosomes in mitosis; in fish hybrids this appears to be related to the viscosity of the egg cytoplasm (Pinney). Embryos formed by gynogenesis are maternal in character. Gynogenesis has been induced in amphibians and echinoderms by treating the sperm with radium. ${ }^{29}$

B. Androgenesis.- The development of embryos with the paternal chromosomes alone has been induced by incapacitating the egg nucleus with radium before syngamy. ${ }^{30}$ In Chatopterus it has been shown that such larvæ have the monoploid chromosome number, 9 . Whether such larvæ may develop into adults or not is unknown.

C. Merogony.-The development of an enucleate egg fragment which has been entered by a normal sperm may be induced more or less readily in a number of animals. Such enucleate fragments are obtained by shaking the eggs ${ }^{31}$ or by cutting them in two with a knife or hair noose. ${ }^{32}$ The development of merogonic individuals usually does not continue beyond the larral stage.

Many such experiments have been made with the idea of testing the nuclear theory of heredity. The results have varied with the species used, the methods, and the direction of the crosses, but, when all things are considered, they have tended to support the nuclear theory. The evidence in such cases is often inconclusive because larval characters, which must be chiefly depended upon, are not widely different in the species crossed and may begin their differentiation in the egg cytoplasm before syngamy (p. 419). ${ }^{33}$

${ }^{26}$ Krüger (1913), P. Hertwig (1920a). See Wilson (1925, p. 460).

${ }^{27}$ Echinus $\& \times$ Mytilus ơ (Kupelwieser, 1912).

${ }^{23}$ Godlewski (1911), Baltzer (1910), Kupelwieser (1912), Tennent (1912), Pinney $(1918,1922)$.

${ }^{29}$ O. Hertwig (1910 et seq.), G. Hertwig (1911 et seq.), P. Hertwig (1911 et seq.).

${ }^{30}$ G. Hertwig (1911, 1913; on amphibians), Packard (1918; on Chætopterus).

${ }^{31} \mathrm{O}$. and R. Hertwig (1887), Boveri (1889 et seq.), and Godlewski (1906) on echinoderms.

${ }^{32}$ Wilson (1903), Yatsu (1904, 1910), Zeleny (1904), Spemann (1914, 1919; hair noose) and Baltzer (1921) on nemertines, mollusks, and amphibians.

${ }^{33}$ For more extensive aecounts of experiments of the kind eited in this seetion, see Morgan (1924a-g), Conklin (1924), G. Hertwig (1920ab), P. Hertwig (1923), Stomps (1923), Wilson (1925), and Brachet (1927). 
Parthenogenesis and Heteroploidy.-Although heteroploidy occurs much less commonly in animals than in plants, there is some evidence that here also a causal relationship often exists between apomixis, heteroploidy, and hybridization. In Tephrosia the crossing of two nonparthenogenetic species resulted in intermediate hybrids which produced parthenogenetic eggs. A race of Daphnia, in which the egg forms only one polar body without haplosis and develops parthenogenetically, is evidently hexaploid. In Trichoniscus provisorius there is a diploid race with normal sexual reproduction and a triploid race showing unreduced parthenogenesis. ${ }^{34}$

As in the case of plants (p. 407), one may arrange a series of instances in animals illustrating a transition from normal meiosis with sexuality to the absence of meiosis with parthenogenesis, one of the characteristic features of the abnormal cases being a weakness or absence of synaptic interaction of chromosomes in the meiotic prophase. ${ }^{35}$ To what extent this abnormal synaptic behavior is due to hybridity in the animals concerned, as it often appears to be in plants, remains to be determined.

${ }^{34}$ J. Harrison (1920) and Harrison and Peacock (1926) on Tephrosia; Schrader (1925) on Daphnia; Vandel (1928) on Trichoniscus.

${ }^{35}$ See de Baehr (1920a) on Aphis and Fries (1909) on Artemia. 


\section{CHAPTER XXV \\ CYTOPLASMIC HEREDITY}

In the foregoing discussions of heredity attention has been limited to those characters which develop anew in successive generations under the differential influence of nuclear factors. Since these factors are carried by chromosomes, which are distributed in a definite manner through successive life cycles, the characters dependent upon them are inherited according to Mendelian rules. The characters in which crossable organisms differ appear to be mainly of this kind (cf. p. 286). In addition, there are some characters whose inheritance is non-Mendelian and depends rather directly upon peculiarities of the cytoplasm or something it contains. Among these the best known are certain chlorophyll characters of plants.

Chlorophyll Inheritance.-It is obvious that two successive generations of cells reproducing by division resemble each other partly because the organs of a given cell may actually become the corresponding organs of its daughter cells. Thus, in the case of a unicellular green alga the daughter individuals are like the mother individual in being green because the chloroplast of the mother cell is divided and passed on directly to them. In those algae in which a swarm spore germinates to produce a multicellular individual or associates with others of its kind to form a colony, the color of the successive colonies or multicellular individuals is a "metidentical" character transmitted directly by the repeated division of chloroplasts. ${ }^{1}$

A similar interpretation has been placed upon the inheritance of chlorophyll characters in the higher plants, the supposition being that plastids, multiplying only by division, are responsible for the distribution, in the individual plant and through successive generations, of those characters which manifest themselves in these organs. Abnormalities in chlorophyll coloring, such as pale greenness, whiteness, and variegation, are accordingly attributed to an abnormal condition or behavior of the chloroplasts. Since the color itself is not present in the plastids of angiosperm gametes, this character may resemble ordinary Mendelian characters in being developed anew in each generation, but it differs from them in depending upon the reproduction and distribution of differentiated cytoplasmic organs, the plastids. Indeed, it has been shown that the various known chlorophyll characters, even those appearing much

${ }^{1}$ See Harper $(1906,1918 a b)$ on Hydrodictyon and Pediastrum. 
alike, fall into two categories: (1) those inherited according to ordinary Mendelian rules, which is taken to mean that in such cases the processes concerned in their development are under the influence of differential nuclear factors; and (2) those not so inherited and therefore seeming to have their differential basis in the cytoplasm. Both types may appear in the same genus or species, e.g., in Zea Mays. It is to be emphasized that the characters in both categories are developed under the influence of both nucleus and cytoplasm, but that they differ with regard to the location and nature of the factors acting differentially in the developing system. Only the second category-the "non-Mendelian" chlorophyll characters-will be considered in the following classification of the best known cases. ${ }^{2}$

1. The Inheritance Is Maternal- - $a$. The variegated plants when selfed or crossed produce variegated, green, pale-green, and white offspring.

The classic example of this type is Mirabilis jalapa albomaculata, described by Correns (1909a). Plants of this race have some branches with normal green leaves, some with white leaves, and some with variegated leaves. Flowers are borne on branches of all three types. Crosses between unlikes result in seedlings with the color of the maternal parent. For instance, if a flower on a green branch is pollinated with pollen from a flower on a white branch, the offspring are all green. In the reciprocal cross the offspring are all white and soon die because of the lack of chlorophyll. If flowers on variegated branches are pollinated, offspring of all types may result. In no case does the pollen affect the color of the progeny. ${ }^{3}$

$b$. The variegated plants produce only variegated progeny, irrespective of the type of pollen used: Humulus japonicus albomaculata (Winge, $1919 a)$.

c. The affected plants produce only self-colored (green or pale-green) progeny: Glycine hispida (Terao, 1918).

2. The Inheritance Is Biparental.-Crosses between flowers on the green and the white parts of the variegated plant may result in green, white and variegated offspring; selfing variegateds results only in white offspring. The type case in this class is Pelargonium zonale albomarginata

2 This classification was drawn up by Demerec (unpubl.). See also Winge (1919a) and the recent reviews by Correns (1928b) and Chittenden (1927a). For ferns, see Andersson-Kottö (1931). Küster (1927) deseribes the anatomy of variegated leaves. The relation of chlorophyll inheritance and other genetic phenomena to pathology is discussed by Link (1932).

${ }^{3}$ Other cases: Stellaria, Senecio, Taraxacum, Hieracium, Arabis, and Mesembryanthemum (Correns, 1919, 1922, 1928b, 1931); Antirrhinum (Baur, 1907, 1910); Melantrium (G. H. Shull, 1913); Primula (Gregory, 1915); Zea Mays (E. G. Anderson, 1923; Demerec, 1927); Viola (J. Clausen, 1927), Oryza (Kondo, Takeda, and Fujimoto, 1927); and Sorghum (Karper and Conner, 1931). 
(Baur, 1909, 1919). This form, which is characterized by white-margined leaves, often has pure green and pure white branches, as does Mirabilis. Crosses either way between flowers on these two kinds of branches may result in variegated offspring; inheritance is here not purely maternal as in Mirabilis. ${ }^{4}$

Theories of Chlorophyll Inheritance.--It has been suggested that there are two types of non-Mendelian chlorophyll inheritance, biparental and maternal, because the male gamete in some cases introduces cytoplasm into the egg while in others it does not. Maternal inheritance would also occur if the male cytoplasmic elements were functionless after entering the egg. Such explanations do not seem improbable in view of the available descriptions of syngamy in angiosperms (p. 236), but nothing can be stated with assurance until much more has been learned concerning the history of cytoplasm and cytoplasmic inclusions throughout the life cycle.

With regard to the "albomaculata type" of variegation (1a), it is the hypothesis of Correns that the condition is due to a cytoplasmic disease which prevents the normal development of the chloroplasts. It is therefore delivered directly to the next generation through the egg cytoplasm and is not transmitted by the male parent because the male gamete introduces no cytoplasm into the egg. If it were due solely to nuclear factors, it would be transmitted equally by both parents, since the nuclear contributions of the two are equivalent. The variegation is the result of different reactions in the unstable affected cytoplasm during the development of the tissues in various portions of the leaf. Winge contends that in types giving greens, whites, and variegateds $(1 a)$ the defect is in the plastids themselves, whereas in those giving rise to variegateds only (1b) it is in the cytoplasm in which the plastids lie. For the "albomarginata type" (2) Baur holds that the appearance of green and white tissues is due to a sorting out of two distinct kinds of plastids or their primordia, green and white, as the cells multiply by division. ${ }^{5}$

That variegation may be due to nuclear genes is suggested in the hypothesis of Demerec (1927), who assumes the presence of a gene which is highly mutable when associated with the cytoplasm of another plant, and also in the hypothesis of Eyster (1928), who postulates a gene composed of a number of "genomeres" assorting at random as the gene

${ }^{4}$ Other cases: probably Enothera (Stomps, 1920; see Renner, 1924b, on hybrids); probably Viola (J. Clausen, 1930a); Hypericum perforatum (Correns, 1931). A blotehing of the leaves in Avena is inherited in a similar manner (Ferdinandsen and Winge, 1930).

5 This hypothesis is adopted by Gregory (1915) for Primula, E. G. Anderson (1923) for Zea, Clausen (1927b) for Viola, and Yasui (1929) for Hosta. These are examples of class $1 a$. 
divides in somatic mitosis. When the genomeres of a gene are unlike with respect to their effect upon chlorophyll development, their segregation results in variegation.

The cytological evidence bearing on the foregoing theories is as yet rather meager. Green, yellowish, and colorless plastids have frequently been observed in variegated plants, but too little is known about their origin and behavior to warrant generalizations. In normal plants and in several Mendelian and non-Mendelian abnormal chlorophyll types of Zea Mays, Randolph (1922) found the same kind of visible structure in the meristematic cells: all contain very minute proplastids (plastid primordia) which, so far as the microscope shows, are of one kind. In normal plants the proplastids develop into large green chloroplasts, whereas in pale-green, yellowish, or white plants, or in the various regions of variegated plants, they fail to attain the normal color, the normal size, or both. No evidence was found that green and colorless plastids represent fundamentally distinct types; they appear rather to be end results of different modes of development of one type of initial body. Moreover, they are connected by a series of all conceivable intermediate conditions, particularly in cells on the boundary between green and white areas in variegated leaves. Hence no support is found for the hypothesis that plastids of two initially distinct types undergo a sorting out during the development of the tissues. Why it is that the primordia develop so differently in different cells can only be conjectured, but it appears probable that their peculiar behavior is an indication of some invisible differentiation being carried out in the cytoplasm. In Abutilon, Tsinen $(1923,1924)$ attributes variegations to alterations occurring in the plastids before, during, or after their development from primordia. Although the green, yellow, and colorless plastids in rariegated individuals of IIosta japonica are indistinguishable in the primordial stage, Yasui (1929) is inclined to regard them as initially unlike.

The full evaluation of the various theories of non-Mendelian chlorophyll inheritance must await a fuller knowledge of the cytoplasm and its many differentiations. When certain variegation patterns are compared with the patterns assumed by the leaf-cells as a result of their lineage (Noack, 1922), it is difficult to regard a simple sorting out of plastids or of genomeres in successive cell-divisions as an adequate explanation of the variegation. It seems more likely, in some cases at least, that the peculiar behavior of the plastids is associated causally with less evident conditions in the cytoplasm. Such conditions arising during the course of tissue differentiation might well affect the growth and greening of plastids, as they do other developmental processes, and so result in the appearance of "chlorophyll characters." The development of all such characters doubtless involves the interaction of both nuclear and cytoplasmic elements, but in the non-Mendelian category the latter elements exert a more 
pronounced differential influence than is usually the case. It remains for future studies to reveal the nature of these extra-nuclear elements or conditions and to demonstrate their location with respect to the plastids manifesting the characters for which they are responsible.

Mosses.- In bryophytes and vascular plants most if not all of the cytoplasm of a given individual is derived from the mother, since the male gamete brings little if any cytoplasm into the egg at the time of syngamy. Hence it should be possible to detect any differential effect of the cytoplasm upon inherited characters by comparing the results of reciprocal crosses between unlike individuals. When the cross is made in one direction the cytoplasm is contributed by one parent, while in the reciprocal cross it is derived from the other; the nuclear constitution after both crosses is the same. In general, it is found that reciprocal crosses between races or Mendelian forms within the same species give similar progeny, which is taken to mean that the cytoplasm is alike in the parents, or that any slight differences are neutralized or dominated by the nucleus in the offspring and therefore exert no differential influence upon characters. ${ }^{6}$ In interspecific and intergeneric crosses, on the other hand, it is often found that cytoplasmic differences are not thus neutralized, so that it becomes possible to relate these to certain differences in character exhibited by the reciprocal offspring.

These principles are well illustrated by extensive researches on mosses carried out by F. von Wettstein $(1926,1928,1930)$. When two races of Funaria hygrometrica are crossed, the reciprocals ${ }^{7}$ are alike; hence the cytoplasms of these races are similar so far as genetic effects are concerned. When $F$. hygrometrica is crossed with $F$. mediterranea, and especially when it is crossed with the more distantly related Physcomitrium piriforme, the reciprocals differ and tend to be almost wholly like their respective mothers in certain characters. Furthermore, such differences persist through repeated back-crossing to the paternal species, indicating that they are related to some stable element in the cytoplasm which does not become altered by the paternal nuclear elements.

This genetic element always present in the cytoplasm is called by von Wettstein the plasmon. ${ }^{8}$ Hence the entire genetic complex consists of factors in the chromosome set (the genom) and the plasmon. In the interspecific crosses it is found that the relative effects of these two elements are not the same for all character differences. Thus paraphysis form appears to be controlled wholly by the genom, the shape of the leaf

${ }^{6}$ Chlorophyll eharacters treated in the preceding section are not to be included here.

${ }^{7}$ I.e., the gametophytes produced by spores from the reciprocal capsules. Certain sporophytic eharacters have also been studied.

${ }^{8}$ Correns (1928b) has employed Strasburger's term "eyto-idioplasm." It does not include the results of previous nuelear influence nor ergastic materials. 
tip by both genom and plasmon, and the length of the mid-rib almost wholly by the plasmon. ${ }^{9}$

Other Cases.- In a few higher plants it has been shown that certain characters, notably sterility, are determined in part by some element or condition in the cytoplasm. For example, certain reciprocal hybrids in Epilobium show differences in fertility which indicate an interaction of genes and unlike maternal cytoplasmic elements. ${ }^{10}$ A somewhat similar situation is found in Linum. In crosses between two varieties of Vicia Faba, major and minor, certain genes are found to produce different effects in the cytoplasms of the two varieties. This is also observed in reciprocal crosses of Nicotiana Langsdorffi $i$ and $N$. Sanderc. ${ }^{11}$ In a certain race of Zea Mays with pollen which degenerates, usually after the division forming the generative cell, it has been shown by an extensive series of crosses that the peculiarity is due directly to something in the cytoplasm and is not affected differentially by known genes in the various linkage groups. Moreover, the sterility is not transmitted by pollen from partially sterile plants. The cytoplasm in the sporocytes differs visibly in sterile and non-sterile individuals, but the real nature of the difference is not known. ${ }^{12}$ In a fungus, Pholiota mutabilis, mycelia may be obtained from a cell having a nucleus of one strain in a mixture of cytoplasms from two strains, and these mycelia may produce fruit bodies. The form of these fruit bodies is affected by the cytoplasm, the various gradations between the forms of the parental strains varying apparently with the proportions in which the parental cytoplasms are mixed (Harder, 1927).

The larval characters of certain animals should be mentioned here. The mature egg, notably in echinoderms, ascidians, and certain other groups, exhibits a visible cytoplasmic differentiation, and by virtue of this fact develops for a time in a definite manner irrespective of the type of sperm causing its activation. Among the characters appearing in the early stages of embryogeny are some which are inherited in each generation from the mother only, since their differentiation is actually under way in the cytoplasm before syngamy occurs. If this "promorphology" of the egg and therefore such embryonic characters are under the differen-

9 When the effect is produced chiefly by the genom, the genom is said to be antecedent and the plasmon recedent; when it is due mainly to the plasmon, the latter is antecedent and the genom recedent (von Wettstein, 1926; p. 260).

${ }^{10}$ Lehmann (1918 et seq.), Sehwemmle (1924), Lehmann and Sehwemmle (1927), Renner and Kupper (1921), Michaelis (1929).

${ }^{11}$ Chittenden and Pellew (1927) and Chittenden (1927b) on Linum, Sirks (1931) on Vicia, East (1932) on Nicotiana.

12 Rhoades $(1931 a, 1933)$. The distribution on the cob of kernels producing sterile and non-sterile plants rules out the hypothesis of a somatic segregation of a small number of elements ( $c f$. Anderson, 1923, and Demerec, 1927, on chlorophyll characters). 
tial control of genes, these characters, although inherited "maternally" in any particular first-generation hybrid because of the late addition of the male nucleus, should show biparental inheritance through subsequent generations.

Certain evidence obtained on this point is in accord with these expectations. For example, in cross-fertilized sea urchin or fish eggs the rate and the type of cleavage are the same as in the mother, no matter what the direction of the cross; they are characters impressed upon the egg during its ovarian history, and the male nucleus fails to change the condition already induced in the egg by maternal nuclear factors. When the hybrid matures and produces eggs, however, it is found that these all show the dominant rate and type of cleavage, no matter which parent contributes the dominant factor. This shows that the male does affect the character in the second generation. ${ }^{13}$ Breeding experiments with moths and butterflies have given similar results with respect to some embryonic characters. ${ }^{14}$ The conclusion is that many embryonic characters whose differentiation is initiated in the egg cytoplasm before syngamy, although fundamentally Mendelian in their inheritance, may be peculiar in that the visible effect of the male gamete is delayed for one generation.

Conclusions.-The phenomena described in this chapter serve to emphasize the fact that the differential factors responsible for those differences in heritable characters which constitute the materials for genetic study are not all similarly located in the protoplasmic system. In crosses between nearly related types it appears that the differences in character are due mainly if not entirely to differences in the genetic factors in the chromosomes, i.e., to the genes. Such characters show Mendelian inheritance because the genes are distributed in a characteristic manner by the chromosomes through successive life cycles. In crosses between more distantly related organisms there may be, in addition to genic differences, cytoplasmic differences which are sufficiently great and persistent to reveal their influence on the development of hereditary characters. When the cytoplasmic differences are the only ones, as may be the case in reciprocal hybrids, the development of the persistent diversities in character may be said to be "controlled" by these differential cytoplasmic conditions, just as the genes "control" the development of Mendelian characters in narrower crosses.

It should be recognized that in all cases the development of the inherited characters involves the activity of both nucleus and cytoplasm. The characters are brought to expression through the action of the entire (1927).

${ }^{13}$ See Conklin (1915, 1917, 1924), Morgan (1924afg), Wilson (1925), and Brachet

$$
{ }_{14} \text { Toyama (1913), Tanaka (1916), Uda (1923). See Morgan et al. (1922) and }
$$
Morgan $(1924 \mathrm{ag})$. 
protoplasmic system, this including its interaction with the environment. If we define heredity as "the occurrence of related but not necessarily identical conditions, events, or characters in successive generations of organisms as a consequence of their protoplasmic organization" (p. 285), we must regard the entire protoplasmic system as the "physical basis of heredity." The nuclear theory of heredity, properly conceived, does not attribute the existence of a given character wholly to nuclear action, although it rightly explains many of the differences between related individuals (probably all of such differences in many cases) as results of diversities in nuclear constitution. It is such differences which have been studied most in modern cytogenetics, but the striking success attending these studies should not obscure the fact that any complete explanation of the phenomena of heredity must include much that lies outside of the nucleus.

It should now be more plainly evident why we have stated ( $p .284$ ) that the problem of development cannot be entirely divorced from that of heredity. Much has been learned about the transmission of hereditary elements through successive generations, but the manner in which these elements function in the actual development of the characters remains to be discovered. This is one of the major biological problems of the future, and not before it has been solved can we be satisfied with our conceptions of the mechanism of either development or heredity. 


\section{CHAPTER XXVI \\ HISTORICAL SKETCH}

Cytology is about a century old. Before protoplasm had come to be recognized as the physical basis of life, and the cell as essentially a protoplasmic unit, cytology as a distinct branch of biology scarcely existed, although important investigations of the structure and development of plants and animals had been in progress for many years. The course followed later by the growing science was so profoundly affected by some of these early investigations that they will be set forth below as a part of the background of our subject. It will be seen that the discovery of the cellular organization of most organisms, and the formulation of an influential theory based on the cell as a unit of cardinal importance in development, long antedated a proper conception of the true significance of protoplasm. As a result, cytology has not only derived its name from the cell, but it has been dominated by the cell concept from the first, notwithstanding the fact that many of the fundamental problems confronting the cytologist are presented by organisms which do not have what is ordinarily regarded as cellular organization.

The investigation of such protoplasmic structures as nuclei and plastids was begun a century ago, long before their constitutional relationship was suspected, but most of our knowledge of these and other kindred elements has been acquired during the past 50 years. The last quarter of the nineteenth century witnessed an intensive study of the behavior of cells, nuclei, and chromosomes in the various processes involved in the growth and differentiation of the organism. Among the noteworthy results of this study was the theory that the phenomena of inheritance are in some way closely dependent on the activities of the nucleus, a theory which received striking support after the principles of Mendelian heredity were rediscovered in 1900. This rediscovery has very largely determined the character of cytological research so far in the present century. It has brought cytology into intimate association with genetics and taxonomy, while a renewed study of living protoplasm has resulted in similar alliances with physiology and biochemistry. One of the most conspicuous and encouraging tendencies of the present day is this closer correlation of the various subdivisions of biology.

The Discovery of the Cellular Organization of Plants and Animals.A perusal of Aristotle's De Partibus Animalium and the Historia Plantarum of Theophrastus serves to show that the ancients had considerable 
knowledge of the organs and tissues of which organisms are composed. The cells, however, escaped observation until many centuries later, when suitable lenses became available. The first compound microscope ${ }^{1}$ appears to have been produced in about 1590 by Jans and Zacharias Janssen, spectacle makers of Middleburg in the Dutch province of Zeeland, and during the first part of the seventeenth century other improved models were designed by other workers. These instruments in the hands of men with scientific curiosity soon led to many significant discoveries. A new world was opened to the eye of science, and the compound microscope has since remained an indispensable instrument in many branches of biological research.

The first description of the cellular organization of plants was given in 1665 by Robert Hooke (1635-1703), a resident of London. Hooke's interest in optics led him to examine all sorts of objects with the compound microscope. In charcoal, and later in cork and other plant tissues, he found small cavities like those in a honeycomb; these cavities he called "cells." He had no distinct notion of the cell contents but spoke of a "nourishing juice," which he inferred must pass through pores from one cell to another. His many observations were embodied in his Micrographia (1665). The chapter containing his remarks on cells is entitled, "Of the schematisme or texture of cork and the cells and pores of some other such frothy bodies." Quaint and crude as it now appears to us, the Micrographia will always be of special interest because it was the earliest work to deal with cells, which were to become the subject matter of a new science.

Nehemiah Grew (1641-1712), an English physician and botanist, began a careful study of plant structure in 1664; in 1670 he read his first important paper before the Royal Society. Further contributions followed at intervals until 1682, when all of them were published under the title The Anatomy of Plants. Like Malpighi, an abstract of whose first work on plants was presented to the Royal Society in 1671, Grew was interested in tissues and gave particular attention to the combinations of these tissues in different plant organs. He was strongly impressed by the manner in which the cells, which he also called "vesicles" and "bladders," appeared to make up the bulk of certain tissues:" . . . the parenchyma of the Barque," he said, "is much the same thing, as to its conformation, which the froth of beer or eggs is, as a fluid, or a piece of fine Manchet, as a fixed body." He further believed the walls of the cells to be composed of numerous extremely fine fibrils; in the vessels or longitudinal elements these fibrils were wound in the form of a close spiral, while the vessels themselves were bound together by a transverse series of interwoven threads. He accordingly compared the structure of the

${ }^{1}$ For the early history of the microscope, see Disney (1928). 
plant with that of a basket, also with "fine bone-lace, when the women are working it upon the cushion."

Marcello Malpighi (1628-1694), an Italian physiologist and professor of medicine, is best known for his important pioneer work in anatomy and embryology. Most of his observations on plants were included in his Anatome Plantarum (1675) and had to do largely with the various kinds of elements making up the body of the vascular plant. A foreshadowing of the cell theory is seen in his remarks concerning the importance of the "utriculi" in the structure of the body. At Pisa, Malpighi was associated with G. A. Borelli, who was one of the first to use the microscope on the tissues of higher animals.

Antoni van Leeuwenhoek (1632-1723) of Delft is remembered for his pioneer researches in the field of microscopy. He constructed a number of simple lenses of high power, and with these he was able to see for the first time certain Protozoa, bacteria, and other minute forms of life. In the course of his investigations he observed the cells ("globules") in the tissues of higher organisms.

Preformation and Epigenesis.-After the death of the above named observers there ensued a period during which the actual investigation of the structure of organisms remained practically at a standstill. There was, however, considerable indulgence in speculation; this should be recorded here, not because it can be regarded as scientific cytology, but because of the influence it exerted upon the formulation of many cytological problems in later years. Such speculation resulted in the division of the biologists of the day into two schools, the main controversy being over the manner in which the embryo develops from the egg. The two theories formulated in answer to this question were called the preformation theory and the theory of epigenesis. ${ }^{2}$

According to the preformation theory, the basis for which was laid in the seventeenth century works of Swammerdam, Malpighi, and van Leeuwenhoek, the egg contains a fully formed miniature individual, which simply unfolds and enlarges as development proceeds. Because of this unfolding, the theory was also known as the "theory of evolution," an expression which has a quite different connotation today. In the eighteenth century the preformation idea was carried to an absurd extreme by Bonnet (1720-1793) and others, who argued that if the egg contains the complete new individual, the latter must in turn contain the eggs and individuals of all future generations successively encased within it, like an infinite series of boxes one within another. The preformationists soon became separated into two groups: the spermists, or animalculists, and the ovists. By the former the new individual was supposed to be encased in the spermatozoön, and figures were actually

${ }^{2}$ See the account by Cole (1930). 
published showing a small human figure, or "homunculus," within the sperm head. The ovists, on the contrary, held that the individual is encased in the egg. A bitter strife was carried on over this question by the two groups and various interesting compromises were made, but all extreme forms of preformationism were to disappear in the light of more critical investigations, which went far to support the opposing theory of epigenesis.

Two of the early champions of the theory of epigenesis were William Harvey (1578-1667; Exercitationes de Generatione Animalium, 1651), and Caspar Friedrich Wolff (1733-1794; Theoria Generationis, 1759). As the result of many careful observations on the embryogeny of the chick, Wolff was able to show beyond question that development is epigenetic: neither egg nor spermatozoön contains a formed embryo; development consists not in a process of unfolding, but in "the continual formation of new parts previously non-existent as such" (Wilson). Here was room for the principle of true generation, or "the production of heterogeneity out of homogeneity." Wolff also discovered the vegetative growingpoint of plants and described the new formation of the successive lateral members. The Theoria Generationis is to be regarded as one of the really great contributions to biological science, for the theory of epigenesis, to which it furnished substantial support, later became established with modifications as a fundamental principle of embryology, particularly through the work of Karl Ernst von Baer (1792-1876) in the nineteenth century.

In commenting on preformation and epigenesis Whitman (1894) emphasizes the fact that the tendency of modern biology has not been to show the entire falsity of either of these views, but to seek out the germs of truth possessed by each, and to relate them to modern biological conceptions. Our present position, although it excludes both views in their crude original form, involves in a new sense both conceptions. When we say that the egg is organized, possessing an architecture or mechanism in its cytoplasm or nucleus which largely predetermines the course of development, we are making a modernized statement of the preformation idea. When we say that the parts of the individual are in no way delineated in the egg but are mainly determined by external conditions during the course of development, we are speaking in terms of modern epigenesis. "The question is no longer whether all is preformation or all postformation; it is rather this: How far is postformation to be explained as the result of preformation, and how far as the result of external influences?" (Whitman). When, therefore, it is borne in mind that one of the outstanding problems of modern cytology is that of identifying the factors involved in the development of an organized and highly differentiated individual from an organized but relatively undifferentiated egg, it is evident that any sketch of cytological history would be incomplete 
without some reference to the early theories of preformation and epigenesis.

The Renewal of the Study of Organic Structure.-The researches of Hooke, Grew, and Malpighi in the seventeenth century had made it apparent that "cells," or "globules," are important structural elements in organisms. When attention was again directed to such matters torard the end of the eighteenth century, a number of interesting suggestions were offered regarding the origin and significance of these elements.

One of the earliest theories of cell-formation was that which had been put forward by Wolff in his Theoria Generationis (1759). According to Wolff, every organ is at first a clear, viscous fluid with no definite structural organization. In this fluid, cavities ("Bläschen," "Zellen") arise and become cells or, by elongation, vessels. These may later be thickened by deposits.from the "solidescible" nutritive fluid. The cavities, or cells, are not to be regarded as independent entities: organization is not effected by them, but they are rather the passive results of an organizing force (vis essentialis) inherent in the living mass.

K. Sprengel (1766-1833) stated that cells originate in the contents of other cells as granules or vesicles which absorb water and enlarge. This theory, in spite of its poor observational basis, was upheld by L. C. Treviranus (1779-1864) in a work appearing in 1806, and both men fought many years for its support. Kieser (1812) further developed the idea that granules in the latex are "cell germs" which later hatch in the intercellular spaces to form new cells. With a much clearer understanding of the nature of the problems involved, a number of excellent observations were made by J. J. Bernhardi in 1805 , by H. F. Link and K. A. Rudolphi in 1807, and by J. J. P. Moldenhawer in 1812 . It is to be regretted that the deserved attention was not given to their results, for they promised to lead in the right direction.

Because of their relation to the cell theory, which is soon to be discussed, special consideration should be given the views of J. B. P. Lamarck (1744-1829), C. F. Mirbel (1776-1854), and R. J. H. Dutrochet (17761847). As emphasized by Gerould (1922), certain aspects of the cell theory were taught in Paris at the opening of the nineteenth century, 40 years before the publication of the works which brought it into prominence.

The famous French biologist Lamarck, in his Philosophie Zoologique (1809), strongly emphasized the fundamental importance of "cellular tissue" in the structure and development of organisms. In his own words, ". . cellular tissue is the matrix in which all the organs of living bodies have been successively formed, and . . . the movement of fluids through it is nature's method of gradually creating and developing those organs out of this tissue" (Elliott's translation, p. 230). He adds in a footnote that he had been teaching this doctrine since 1796 . By 
"fluids" he evidently means subtle influences of undetermined nature. With regard to the internal structure of plants, he says: " . . . all that we can find is, among the simplest, a cellular tissue without vessels but variously modified and stretched or compressed according to the special shape of the plant; and in the more complex, an assemblage of cells and vascular tubes of various sizes, mostly with lateral pores, and a varying number of fibers, resulting from the compression and hardening that a portion of the vascular tube has undergone" (p. 235).

As Gerould points out, Lamarck's cellular-tissue theory, like his theory of evolution, was not supported by a body of well-authenticated published facts. It was rather Mirbel (1808) who attempted to furnish such observational data. In a work on Marchantia (1831-1833) Mirbel distinguished three modes of cell-formation: the formation of cells on the surface of other cells, the formation of cells within older cells, and the formation of cells between older cells. The first mode apparently represented the budding of the germ tube arising from the spore, while the second and third modes were formulated as the result of a misinterpretation of the process of cell multiplication in growing gemmæ. Special notice should be taken of the fact that by both men it was cellular tissue, and not the individual cell, that was regarded as fundamental. Both looked upon the organism as a cellular whole rather than an association of elementary unicellular organisms.

It was Dutrochet (1824) who first insisted upon the primary importance of the cells as individuals. This was largely because he was able to resolve plant tissues by maceration into distinct cell units. Of the cell he writes, "this astounding organ is truly the fundamental element of organization; everything, indeed, in the organic tissues of plants is evidently derived from the cell, and observation has just proved to us that it is the same with animals." This is the essential point of the cell theory; but before proceeding to a discussion of this theory we should call attention to the discovery of the nucleus.

Although nuclei were occasionally seen by earlier observers, the major share of the credit for its discovery goes to Robert Brown (17731858), for it was he who was first impressed by its probable importance and emphasized it as a normal constituent of cells. Brown, a British botanist celebrated chiefly for his taxonomic monographs and morphological researches, announced his discovery in a paper read before the Linnæan Society in 1831. It was in leaf-cells of orchids that he saw the nuclei most clearly. Concerning these observations he wrote as follows:

In each cell of the epidermis of a great part of this family, especially of those with membranaceous leaves, a single circular areola, generally somewhat more opaque than the membrane of the cell, is observable. This areola, which is more or less distinctly granular, is slightly convex, and although it seems to be on the surface is in reality covered by the outer lamina of the cell; it is not unfrequently 
however central or nearly so. . . . This areola, or nucleus of the cell as perhaps it might be termed, is not confined to the epidermis, being also found not only in the pubescence of the surface, particularly when jointed, as in Cypripedium, but in many cases in the parenchyma or internal cells of the tissue, especially when these are free from the deposition of granular matter.

After Brown's announcement, observations on nuclei in various tissues multiplied rapidly, and it was not many years before nuclei came to be recognized as important components of practically all organisms.

The Cell Theory.-The generalization that the bodies of organisms are regularly composed of cells and their products, and that the cell units are of prime importance in determining growth and differentiation, was made by R. J. H. Dutrochet (1776-1847) in France and by M. J. Schleiden (1804-1881) and T. Schwann (1810-1882) in Germany. In addition to the statement quoted in an earlier paragraph, Dutrochet says in his Recherches Anatomiques et Physiologiques sur la Structure Intime des Animaux et des Végétaux et sur leur Motilité (1824):

... The globular corpuscles which make up all the tissues of animals are really globular cells of an extreme smallness, which are united only by cohesion. ... This uniformity of ultimate structure proves that organs really differ one from the other only in the nature of the substances which are contained in the vesicular cells of which they are composed... Growth results from the increase in the volume of the cells, and from the addition of new little cells.

Dutrochet noted that certain structures, notably feathers, are made up of dead cells, and that others represent cell secretions. Moreover, he recognized the cell as a unit of physiological function. He did not, however, make a clear distinction between true cells and other globules visible in tissues (see Rich, 1926).

Schleiden and Schwann made their formulation of the cell theory more or less jointly after comparing their observations on plants and animals. In his Beiträge zur Phytogenesis (1838) Schleiden says,

... every plant developed in any higher degree is an aggregate of fully individualized, independent, separate beings, even the cells themselves. Each cell leads a double life: an independent one, pertaining to its own development alone; and another incidental, in so far as it has become an integral part of a plant. It is, however, easy to pereeive that the vital process of the individual cell must form the first, absolutely indispensable fundamental basis, both as regards vegetable physiology and comparative physiology in general. . . .

Schwann stated the theory in concise form in 1838, and 1839 he published a full account under the title, Mikroskopische Untersuchungen über die Uebereinstimmung in der Struktur und dem Wachsthum der Thiere und Pflanzen. In this classic work a great variety of animal cells are carefully described and figured, and the cell stands forth as an indi- 
vidual unit with a clearness unapproached in any earlier treatise. This was due in part to Brown's discovery of the nucleus, which enabled Schwann to surpass his predecessors in distinguishing true cells from other elements with which they had long been confused. With regard to the general significance of cells Schwann says:

The elementary parts of all tissues are formed of cells in an analogous, though very diversified manner, so that it may be asserted that there is one universal prineiple of development for the elementary parts of organisms, however different, and that this prineiple is the formation of cells. . . All organized bodies are composed of essentially similar parts, namely, of cells. . . . The whole organism subsists only by means of the reciprocal aetion of the single elementary parts. [And further:] The development of the proposition that there exists one general prineiple for the formation of all organie production, and that this prineiple is the formation of cells, as well as the conclusions which may be drawn from this proposition, may be comprised under the term Cell Theory. . . .

It should be carefully noted that the essential point in this theory was that cells, no matter how diverse they may be in appearance, are all morphologically equiralent and are elementary living units whose action determines the development of the organism; the cell is the primary agent of organization. It is an observable fact that most bodies are composed of cells and their products, and that the life cycles of such organisms may be described as cell successions, but the theory lies rather in the conception of the cell individual as the leader in the development of organic structure and in function. With Lamarck and Mirbel, even as with Wolff, cells were not very definitely individualized and were more or less passive in the formation of organs in the fundamental cellular matrix; theirs was a tissue theory rather than a cell theory. With Schleiden and Schwann, on the other hand, cells were definite elementary organisms primarily responsible for the development and activity of the body. Dutrochet seems to have been the only one to approach this conception previously.

Interesting consequences followed from the extension of the cell theory to the Protozoa. The protozoön was found usually to be a uninucleate individual not greatly unlike one of the cells of a larger animal or plant. It was therefore concluded that Protozoa are primitive one-celled organisms that in the course of evolution somehow aggregated to form cell republics, or multicellular organisms; and this was further taken to mean that the protozoön was to be homologized with a single cell of the multicellular body. This conception of the Protozoa and of the phylogeny of multicellular organisms has been justly criticized by many biologists, who hold that if multicellular animals have evolved from Protozoa it has been by developing internal cellular structure rather than by colonial aggregation, and that the protozoön is therefore homologous with the entire multicellular individual (see Dobell, 1911a). 
None of the observers named above knew how the cells multiplied. In his Beiträge zur Phytogenesis, which dealt largely with the origin of cells, Schleiden set forth his theory of "free cell-formation," which was essentially as follows. In the general cell contents or mother liquor ("cytoblastema") there are formed, by a process of condensation, certain small granules (later called "nucleoli" by Schwann). Around these many other granules accumulate, thus forming nuclei ("cytoblasts"). Then, "as soon as the cytoblasts have attained their full size, a delicate transparent vesicle appears upon their surface." This vesicle in each case enlarges and forms a new cell, and, since it arises upon the surface of the cytoblast (nucleus), "the cytoblast can never lie free in the interior of the cell, but is always enclosed [i.e., imbedded] in the cell wall. . . ." Cell-formation was thus regarded as endogenous ("cells within cells") rather than the result of cell-division. This erroneous idea was adopted by Schwann, and although it detracted little from the value of his histological work, it required correction before cytology could develop further along progressive lines.

Cell-multiplication.-The cell theory as outlined above was at once adopted as a fundamental proposition in biological research, though in certain of its aspects it underwent considerable modification as knowledge increased. It was especially desirable to clear up the question of cell origin, and to this task a number of able observers addressed themselves. Among these should be mentioned Hugo von Mohl (1805-1872), Wilhelm Hofmeister (1824-1877), F. J. F. Meyen (1804-1840), Franz Unger (1800-1870), and Carl von Nägeli (1807-1891). The multiplication of cells by division was observed by several investigators between 1830 and 1840, von Mohl being the first to describe the process in some detail.

As Sachs has pointed out, Schleiden's strange theory of cell-formation could hardly have been developed if the true relation of cell-division to what various workers had called free cell-formation had been recognized earlier. Von Mohl $(1835,1844)$ maintained that there are two modes of cell-formation: by division and by the formation of cells within cells; but considerable uncertainty remained regarding the behavior of the vacuolated protoplast ("primordial utricle") during these processes. At the same time Nägeli (1844) produced an exhaustive treatise on the nucleus, cell-formation, and growth. In algæ and the microsporocytes of angiosperms he clearly showed that cells multiply by division, and Schleiden was forced to admit that this might be "a second kind of cellformation." The continuation of Nägeli's researches in 1846 completely overthrew Schleiden's conception of free cell-formation, establishing the significant fact that practically all vegetative cell-formation is by celldivision. Many similar observations had been made by Unger and von Mohl, but Nägeli elaborated a broad theory which took into account 
all of the data at hand. He distinctly defined cell-division and free cell-formation, and showed that what had been taken for the latter might be regarded as a special case of the former. Nägeli's conclusions were supported by new evidence furnished by other investigators, who further held that not only vegetative cells, but also those reproductive cells (in thallophytes) which Nägeli thought in some cases might be formed freely, originate by a modified process of cell-division. It now seemed clear to these men that cells arose only from preëxisting cells, a conception which had been emphasized by Remak (1841), and which Virchow (1855) expressed in the dictum, omnis cellula e cellula. This dictum is frequently employed today, but it is obvious that some qualification should be made for the origin of cells in plasmodial masses.
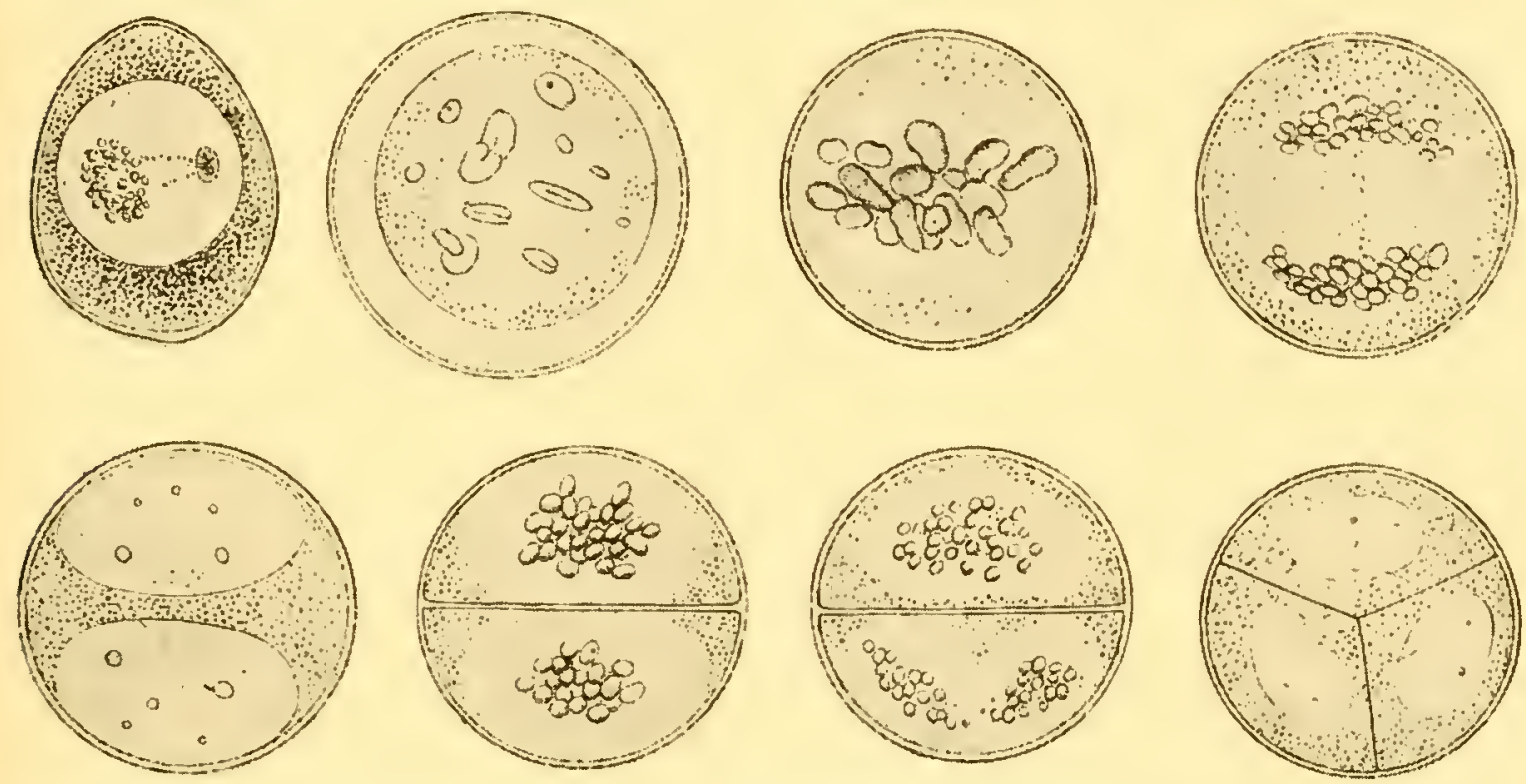

FIG. 230.-Figures of division of Tradescantia microsporocyte drawn by Wilhelm Hofmeister (1S48) many years before chromosomes were recognized as definite nuclear units.

Opinions concerning the origin of the nucleus and its rôle in cell-division varied greatly among these workers, reliable observations being insufficient to allow any definite conclusion. In 1841 Henle believed with Schleiden that the nucleus was formed by the aggregation of "elementary granules," and that it was not constantly present. Better observations soon showed the falsity of this view. Von Kölliker (1845) asserted that nuclear division precedes the division of the cell. Hofmeister, studying embryo development in angiosperms, reported "that the nucleus of the mother-cell divides into two; that one half of the contents of the cell collects around each of the daughter-nuclei . .." (1847). It was commonly believed at this time that the nucleus actually dissolved just before cell-division, new nuclei being formed in the daughter cells; but Hofmeister, although he described the dissolution of the nuclear membrane and the nucleoli (1848), held that the main bulk of the nuclear material actually separated into two masses. At this early date he 
clearly observed the poleward migration of what we now call chromosomes ${ }^{3}$ in fresh microsporocytes of Tradescantia (Fig. 230). Remak, as a result of his observations on blood cells in the chick embryo, formulated a definite theory of cell-division $(1841,1858)$. He believed cell-division to be a "centrifugal" process: the nucleolus, nucleus, cytoplasm, and cell membrane were supposed to divide in turn by simple constriction. Von Mohl, who in the main agreed with Hofmeister, wrote as follows (1851):

The second mode of origin of a nucleus, by division of a nucleus already existing in the parent-cell, seems to be much rarer than the new production of them.... [And again] ... it is possible that this process [nuclear division] prevails very widely, since . . . we know very little yet respecting the origin of nuclei. Nägeli thinks that the process is quite similar to that in cell-division, the membrane of the nucleus forming a partition, and the two portions separating in the form of two distinet cells.

It was not until many years later, in connection with researches upon syngamy and embryogeny, that the behavior of the nucleus in celldivision became known in detail and its probable significance pointed out (p. 441). In 1879 Eduard Strasburger (1844-1912) announced definitely that nuclei arise only from preexisting nuclei. W. Flemming was led to the same conclusion by his studies on animal cells and expressed it in the dictum, omnis nucleus e nucleo (1882a).

The Protoplasm Doctrine.-The cell theory and all of its corollaries were placed in a new light with the development of a more adequate conception of the significance of protoplasm. A number of early investigators had seen protoplasm and had been impressed by certain of its activities. As early as 1772 Corti, and a few years later Fontana (1781), saw the rotation of the "sap" in the Characes and other plants. After being long forgotten, this phenomenon was rediscovered by $\mathrm{L}$. C. Treviranus (1811) and G. B. Amici (1819). The cell to its discoverers meant nothing more than a wall surrounding a cavity; they spoke only in the vaguest terms of the "juices" present in cellular structures. The founders of the cell theory held a position but little in adrance of this; they observed the cell contents but regarded them as of relatively slight importance.

Felix Dujardin (1801-1860) in 1835 described the "sarcode" of the lower animals as a substance having the properties of life. Von Mohl had

${ }^{3}$ Concerning the behavior of these bodies in the formation of daughter nuelei, Hofmeister says: "Ich kann die Bildung der Toehterkerne in keiner andern Weise mir denken, als dass die in Centrum der Mutterzelle angesammelten eyweissartigen Stoffe, aus zur Zeit umbekannten Ursaehen in zwei Gruppen sich sondern, die in Form abgeplatteter elliptischer Sphäroïde sieh individualisieren; später naeh Aussen sieh mit einer Membran bekleiden und im Inmern Bildungen aus diehteren Stoffen erzeugen können." Sinjilar statements accompany his figures of Psilotum in Die Lehre von der Pflanzenzelle (1867). 
seen a similar substance in plant-cells; in 1846 he called it "Schleim," or "Protoplasma," the latter term having been used shortly before by Purkinje in a somewhat different sense. Nägeli and A. Payen (1795$1871)$ in 1846 recognized the importance of protoplasm as the vehicle of the vital activity of the cell, and Alexander Braun (1805-1877) in 1850 pointed out that, swarm spores, which are cells, consist of "naked" protoplasm. An important point was reached when Payen (1846) and Ferdinand Cohn (1850) concluded that the "sarcode" of the animal and the "protoplasm" of the plant are essentially similar substances. In the words of Cohn:

The protoplasm of the botanist, and the contractile substance and sarcode of the zoölogist, must be, if not identical, yet in a high degree analogous substances. Hence, from this point of view, the difference between animals and plants consists in this; that, in the latter, the contractile substance, as a primordial utricle, is enclosed within an inert cellulose membrane, which permits it only to exhibit an internal motion, expressed by the phenomena of rotation and circulation, while, in the former, it is not so enclosed. The protoplasm in the form of the primordial utricle is, as it were, the animal element in the plant, but which is imprisoned, and only becomes free in the animal; or, to strip off the metaphor which obscures simple thought, the energy of organic vitality which is manifest in movement is especially exhibited by a nitrogenous contractile substance, which in plants is limited and fettered by an inert membrane, in animals not so.

Protoplasm was now studied more intensively than ever. H. A. de Bary (1831-1888), working on myxomycetes and other plant forms, and Max Schultze (1825-1874), investigating animal cells, demonstrated the correctness of Cohn's view. The work of Schultze (1861) was especially important in that it firmly established the protoplasm doctrine, namely, that the units of organization are masses of protoplasm, and that this substance is, in general, similar in all living organisms. Schultze described the cell as a mass of protoplasm containing a nucleus, both nucleus and protoplasm arising through the division of the corresponding elements of a preëxisting cell. The cell wall, upon which the early workers had focused their attention, turned out to be of secondary importance. The cell was thus seen to be primarily the organized protoplasmic mass, to which Hanstein in 1880 applied the convenient term protoplast.

Extensive studies on the physical nature of protoplasm were soon undertaken. Brücke (1861), who was one of the first to lay emphasis on the fact that protoplasm is an organized substance, looked upon it as a contractile, semisolid material through which there streams a fluid carrying granules. Similar to this was the idea of Cienkowski (1863), who believed he saw in the protoplasm of myxomycetes two fluids, one of them hyaline and only semifluid (the "ground substance"), and the 
other a more limpid fluid with granules suspended in it. De Bary $(1859,1864)$, on the other hand, regarded protoplasm as a single semifluid substance, contractile throughout, but showing many local differences due to varying water content. Probably the first man to recognize the importance of the colloidal state of matter, newly distinguished by Graham, in the study of protoplasm was W. Hofmeister $(1862,1867)$, but many years were to elapse before such studies could be effectively pursued.

More influential for a time were the structural theories associated with the names of Klein, Flemming, Altmann, and Bütschli, and known respectively as the "reticular," "fibrillar," "granular," and "alveolar" theories. The reticular theory, which was formulated by Frommann $(1865,1875,1884)$, was developed especially by Klein $(1878,1879)$ and supported by van Beneden, Carnoy, Leydig, and others. These workers saw in protoplasm a reticulum or fine network of a rather solid substance (spongioplasm) which held a fluid and granules in its meshes.

The fibrillar, or filar, theory announced by Velten $(1873,1876)$, as a result of his observations on Tradescantia and other forms, stated that protoplasm is composed of fine fibrils, which, though often branched, do not form a continuous network. This idea was developed mainly by Flemming (1882), who called the substance of the fibrils mitome and the fluid bathing it paramitome. Some observers asserted that the fibrils were in reality minute canals filled with a liquid, the granules seen by others being merely sections of these canals.

According to the granular theory, protoplasm was a compound of innumerable minute granules which alone form the essential active basis for the phenomena exhibited; the observed fibrillar and alveolar structures were of secondary importance. For Altmann (1886 et seq.), who was the most prominent exponent of the theory, the granules were actual elementary living units, or "bioblasts," the liquid containing them being a non-living hyaloplasm. The cell was therefore looked upon not as a unit but as an assemblage of bioblasts, "like bacteria in a zoöglœa," and the bioblasts were believed to arise only by division of others of their kind (omne granulum e granulo!).

The alveolar theory, also known as the emulsion, or foam, theory, was elaborated principally by Bütschli (1892 et seq.), and is of special interest in view of certain present-day notions of protoplasmic structure. According to Bütschli, protoplasm consists of minute droplets (averaging $1 \mu$ in diameter) of a liquid "alveolar substance" (enchylema) suspended in another continuous liquid "interalveolar substance." The structure is, therefore, that of an extremely fine emulsion, and the appearances described by other workers are due to optical effects encountered in examining the minute alveolar structure. Bütschli supported his theory 
by making artificial emulsions with soaps and oils which showed amœboid movement and other striking resemblances to living protoplasm.

The modern studies discussed in Chapter II have placed a new evaluation on these early structural theories. What their proponents saw was not the fundamental structure of protoplasm but secondary structural modifications and differentiation products. Thus Bütschli's alveoles were innumerable minute masses of various vacuolar and other substances. Any distinction between large vacuoles, alveoles, and ultramicroscopic colloidal masses of the same material is more or less arbitrary, though the physico-chemical properties of the system may be expected to vary with the degree of subdivision. It is now evident that it is mainly in the hyaline "ground substance" that fundamental structure is to be sought, and that the appearances observed by the above pioneers do not have the significance originally attributed to them.

It would be difficult to overestimate the value, both practical and theoretical, of the protoplasm doctrine, for its establishment has not only led to knowledge by which the conditions of life have been materially improved but has also been an important factor in assisting man to a modern, rational outlook on organic nature. It is not too much to say that the identification of protoplasm as the material substratum of the life processes was one of the most significant events of the nineteenth century. The doctrine was furnished with a popular expression by Huxley in his well-known essay, The Physical Basis of Life (1868).

The Organismal Theory.-The conception of the cell had by this time developed into something quite different from what it had been in the minds of the founders of the cell theory. The cell was now recognized as a protoplasmic unit, and the ideas of these men concerning the origin of cells had been overthrown. Future researches were to show more clearly the rôle of cells in connection with development and inheritance, and certain limits were to be set to the conception of the cell as a unit of function and organization. To Dutrochet and Schwann the multicellular plant or animal appeared as little more than a cell aggregate, the cells being the primary individualities; the organism was looked upon as something completely dependent upon their varied activities for all its phenomena. "The cause of nutrition and growth," said Schwann, "resides not in the organism as a whole, but in the separate elementary parts-the cells." This elementalistic conception of the organism as an aggregate of independent vital units governing the activities of the whole dominated biology for many years, nowithstanding its severe criticism by Sachs, de Bary, and many other later writers who pointed out that, owing to the high degree of physiological differentiation among the various tissues and organs, the cell cannot be regarded merely as an independent unit but as an integral part of a higher individual organization, and that 
as such the exercise of its functions must be governed to a considerable extent by the organism as a whole.

That it is thus the living system as a whole, and not the individual cell, that is the "primary agent of organization" was definitely maintained by a number of biologists, who, unable to accept the orthodox cell theory, developed and supported the "organismal theory." 4 According to this theory, evidence for which was cited in Chapter I, the multicellular plant or animal is not a colony or republic of elementary cell individuals but rather a more or less continuous mass of protoplasm which has become incompletely subdivided into subordinate centers of action, the cells, during the course of ontogenesis; the many cells are an accompaniment or a result, rather than the cause, of development and differentiation. In the words of de Bary, "die Pflanze bildet Zellen, nicht die Zelle bildet Pflanzen." The phylogenetic corollary of this theory is that multicellular organisms have evolved not by an aggregation of many individuals but rather by the growth, differentiation, and septation of one, i.e., by just such a process as is observable in ontogeny. This general theory has made slow progress in competition with the descriptively convenient cell theory, but the tendency at the present time is to give it increasing recognition in discussions of the organism and its activity.

Syngamy and Embryogeny. Plants.-Although it was known to the ancients that there is in plants something corresponding to the sexual reproduction seen in animals, their ideas of the organs and processes involved were very vague. Like Grew and others in the seventeenth century, the botanists of antiquity were aware of the fact that the pollen in some way influences the development of the ovary into a fruit with seeds. That this involves a sexual act was clearly shown by the observations and experiments of R. J. Camerarius (1694), C. Mather (1716, 1721), T. Fairchild (1717, 1724), P. Miller (1721), J. Logan (1735), and others, ${ }^{5}$ but in spite of these and the subsequent researches of $\mathrm{J}$. G. Koelreuter (1761), C. K. Sprengel (1793), and K. F. Gaertner (1849) the idea of sexuality in plants was vigorously opposed in certain quarters for many years.

An important step in advance was made when G. B. Amici (1830) followed the growth of the pollen tube from the pollen grain on the

${ }^{4}$ Here may be mentioned the names of de Bary (1862), Hofmeister (1863, 1867), Sachs (1882), Rauber (1883), Heitzmann (1883), O. Hertwig (1884), Whitman (1893), A. Serlgwick (1894), Heidenhain (1902, 1907), Schlater (1911), Dobell (1911a), Gurwitsch (1913), Ritter (1919), and Rohde (1885 et seq.; see 1923). See the recent discussions by Ritter and Bailey (1928) and Russell (1930). The theory has also been known as the plasma theory, tissue theory, and plasmodial theory.

${ }^{5}$ For an account of these early investigations, see Zirkle (1932b). See also Roberts (1929). 
stigma to the ovule. Schleiden (1837) and Schacht $(1850,1858)$ took up the study and made a curious misinterpretation: they regarded the ovule as merely a place of incubation for the end of the pollen tube, which they thought entered the ovule and enlarged to form the embryo directly. The work of Amici (1842), Tulasne (1849), and others showed the falsity of this notion, but an acrimonious discussion raged about the subject for a number of years. After W. Hofmeister (1849) and Radlkofer (1856) had followed the process with their characteristic thoroughness, there could remain no doubt concerning the error of Schleiden and Schacht. Hofmeister clearly demonstrated that the embryo arises, as Amici contended, not from the end of the pollen tube, but from an egg contained in the ovule, the egg being stimulated to development by the pollen tube. He was wrong, however, in supposing that the tube aid not open and that a fertilizing substance diffused through its wall.

It was in the algæ that the union of the sperm cell with the egg cell (syngamy) was first seen in the case of plants. In 1853 Thuret saw spermatozoids attach themselves to the egg of Fucus, and in 1854 he showed that they are necessary to its development. The actual entrance of the spermatozoid into the egg was first observed in 1856 by Nathanael Pringsheim (1824-1894) in Edogonium. The fusion of the parental nuclei was seen by Strasburger (1877) in Spirogyra, but he thought they thereupon dissolved. This error was corrected shortly afterward by Schmitz (1879b), who was thus the first to show clearly that the central feature of the sexual process in plants is the union of two parental nuclei to form the primary nucleus of the new individual. The demonstration of such a nuclear fusion in a number of algæ and fungi soon followed (see Tischler, 1921-1922, p. 462).

The fusion of the gametic nuclei in bryophytes and pteridophytes was first seen by Kruch (1890) in Riella, and by Campbell (1888) in Pilularia. That the same process occurs in syngamy in the seed plants was demonstrated by Strasburger, who in 1884 described the union of the egg nucleus with a nucleus brought in by the pollen tube ( $c f .1877, \mathrm{pp} .56,76)$. In 1898 and 1899 S. Nawaschin and L. Guignard completed the story by describing "double fertilization," wherein one male nucleus unites with that of the egg while a second male nucleus unites with the two polar nuclei to form the primary endosperm nucleus. The subsequent work of Strasburger and others on the gymnosperms and angiosperms greatly cleared up the whole matter of syngamy and embryogeny in these plants.

Animals.-It is probable that the spermatozoön was first seen in 1677 by Ludwig Hamm, a pupil of Leeuwenhoek. The credit for the discovery is, however, usually given to Leeuwenhoek, since it was he who brought the matter to the attention of the Royal Society and pursued such studies further. He asserted that the spermatozoa must penetrate 
into the egg, but it was thought at the time and for many years afterward that they were parasitic animalcules in the spermatic liquid; hence the name "spermatozoa."

Although L. Spallanzani (1786) is usually said to have shown by a filtration experiment that the spermatozoön is the fertilizing element, it is pointed out by Lillie (1916) that Spallanzani did not draw the correct conclusion; he even denied that the spermatozoön is the active element, holding rather that the fertilizing power lies in the spermatic liquid. It was Prevost and Dumas who corrected this mistake and demonstrated the true rôle of the spermatozoön (1824). The spermatozoön was later shown by Schweigger-Seidel (1865) and La Valette St. George (1865) to be a complete cell with its nucleus and cytoplasm, as von Kölliker had maintained. That Schwann (1839) had been right in regarding the egg as a cell was shown by Gegenbaur in 1861. The polar bodies formed at the time the egg matures are said to have been first seen by Carus (1824). Bütschli (1875) showed them to be formed in connection with the division of the egg nucleus, and Giard (1877) and Mark (1881) interpreted them as abortive eggs.

The penetration of the spermatozoön into the egg was not actually seen until Newport (1854) observed it in the case of the frog. In 1875 O. Hertwig (1849-1922) announced the important discovery that the two nuclei which fuse in the fertilized egg are furnished by the egg and the spermatozoön. The rôle of the nucleus in syngamy was thus demonstrated in animals only shortly before it was in plants, and it is interesting to note that the first complete description of the union of the germ cells in animals was given by H. Fol in the same year (1879) that Schmitz described clearly the process in plants. It was now evident that syngamy in both kingdoms consists in the union of two gametes which are ordinarily single cells, one from each parent (in diœcious forms), and that the central feature of the process is the union of two gamete nuclei, the new individual therefore deriving a portion of its nuclear substance from each parent.

Although the cleavage of the fertilized animal egg in the development of the embryo had been seen many years previously, it was first definitely described by Prevost and Dumas in 1824 for the frog. At that time neither the egg nor the products of its division were clearly recognized as cells. The true meaning of cleavage was elucidated by M. Barry, who held that the blastomeres are cells and that their division is preceded by the division of their nuclei; also by a number of later writers, including A. von Kölliker, Whitman and Rabl, who traced in detail the long series of changes undergone by the multiplying embryonic cells as the various tissues and organs are differentiated. Embryogeny was thus shown to involve the division and differentiation of cells, the fertilized egg initiating a series of divisions giving rise to all the cells of the body and to the germ- 
cells. It was now possible to describe the life cycle in terms of cell successions; and since the egg was seen to be a descendant of the egg of the previous generation, it became evident that there has been an uninterrupted series of cell-divisions from the remote past down to the organisms in existence today. The statement of this conception, without, however, any necessary emphasis on cells rather than protoplasm itself, is known as the law of genetic continuity. In the words of Locy (1915):

The conception that there is unbroken continuity of germinal substance between all living organisms, and that the egg and the sperm are endowed with an inherited organization of great complexity, has become the basis for all current theories of heredity and development. So much is involved in this conception that . . . it has been designated (Whitman) "the central fact of modern biology." The first clear expression of it is found in Virchow's Cellular Pathology, published in 1558. It was not, however, until the period of Balfour, and tbrough the work of Fol, Tan Beneden (chromosomes, 1853) Boveri, Hertwig, and others, that the great importance of this conception began to be appreciated, and came to be woven into the fundamental ideas of development.

Mitosis and Meiosis. - We have pointed out that cells were once believed to arise de novo from a mother liquor, or "cytoblastema." This misconception was removed by later investigations in which it was shown beyond question that cells arise in general by the division of preëxisting cells. By several early observers the nucleus was seen to have a more or less prominent part in the process, its division preceding that of the cell; but, in the words of Wilson (1900),

. . . it was not until 1873 that the way was opened for a better understanding of the matter. In this year the discoveries of Anton Schneider, quickly followed by others in the same direction by Bütschli, Fol, Strasburger, Van Beneden, Flemming, and Hertwig, showed cell-division to be a far more elaborate process than had been supposed, and to involve a complicated transformation of the nucleus to which Schleicher (1S7S) afterward gave the name karyokinesis. It soon appeared, however, that this mode of division was not of universal occurrence; and that cell-division is of two widely different types, which Van Beneden (1876) distinguished as fragmentation, corresponding nearly to the simple process described by Remak, and division, involving the more complicated process of karyokinesis. Three years later Flemming (1879) proposed to substitute for these terms direct and indirect division, which are still used. Still later (1S82a) the same author suggested the terms mitosis (indirect or karyokinetic division) and amitosis (direct or akinetic division), which have rapidly made their way into general use, though the earlier terms are often employed. Modern research has demonstrated the fact that amitosis, or direct division, regarded by Remak and his followers as of universal occurrence, is in reality a rare and exceptional process; . . . it is certain that in all the higher and in many of the lower forms of life, indirect division or mitosis is the typical mode of cell-division.

The chromosomes appeared in published figures long before their significance was appreciated. Thus in 1848 Wilhelm Hofmeister exam- 
ined Tradescantia sporocytes in water and described "sphericaldroplets of a strongly refractive substance" formed by a "slow coagulation of albuminoids in the cell contents" (see p. 431). They were more adequately described a quarter of a century later by the investigators named in the preceding paragraph. Waldeyer (1888) gave them their name. The longitudinal splitting of the prophasic chromosomal threads was discovered by Flemming in 1879; shortly thereafter (1884) van Beneden and Heuser showed in animals and plants, respectively, that the daughter halves pass to opposite poles in the anaphase. That they maintain their individuality through the nuclear cycle was maintained by van Beneden (1883), Rabl (1885), and Boveri (1887 et seq.). Drawings of the achromatic figure were published by Kowalevsky (1871) and Fol (1873), but Bütschli (1875) was the first to describe it in detail.

The reduction of the number of chromosomes was discovered by van Beneden (1883), who announced that the nuclei of the egg and spermatozoön of Ascaris each contain one half the number found in somatic cells. Strasburger (1888) showed that in angiosperms the number of chromosomes in the egg and male nuclei is fixed by a reduction occurring in the megasporocyte and microsporocyte, respectively. This was at once confirmed by Guignard $(1889,1891)$. E. Overton (1893) found that the female gametophyte cells in a cycad, Ceratozamia, have half the number present in the cells of the sporophyte. He further suggested that reduction probably occurs in the sporocytes in mosses and ferns. In a liverwort, Pallavicinia, Farmer (1894) found the gametophyte cells to have four chromosomes and the sporophyte cells eight. That Overton's theory of reduction in the sporocytes of bryophytes and pteridophytes was correct was demonstrated by Strasburger (1894), who postulated the occurrence of a periodic reduction in the number of chromosomes in all organisms reproducing sexually.

How the reduction is accomplished was not at first apparent. The formation of functionless polar bodies by animal oöcytes suggested that the change in number is brought about by the simple casting out of half the chromosomes during the development of the reproductive cells. This view proved to be incorrect when it was shown (1890-1893) by Henking, Rückert, Haecker, vom Rath, and others that the double chromosomes appearing in the reduced number in the first meiotic mitosis are really pairs of chromosomes. These bivalent pairs were seen to arise by a synapsis of chromosomes two by two, the members of each pair then disjoining in one of the meiotic divisions. Thus it became evident that reduction is effected by a redistribution of the chromosomes to different nuclei and cells and that the degeneration of the polar bodies is a phenomenon without any general significance in meiosis. Studies on meiosis in many groups of organisms were soon undertaken, and no department of cytology has witnessed the development of a larger or more contro- 
versial literature. Interest in the subject was greatly accentuated by the suggestion that the mode of chromosome reduction affords a key to certain phenomena of heredity.

Cytology and Heredity.-As Wilson (1900) points out, the many facts brought to light in the early days of cytology were of the greatest significance in connection with the theory of evolution and the problem of heredity, though for many years this was only vaguely perceived. Darwin, aside from his hypothesis of pangenesis, scarcely mentioned the theories of the cell, and not until many years later was the cell investigated with reference to these matters. Researches on the origin of the germ-cells, nuclear division, and fertilization, which brought cytological research and the study of evolution and heredity into intimate association, began shortly after 1870 with the works of Schneider, Auerbach, Fol, Bütschli, O. Hertwig, C. Bernard, van Beneden, Strasburger, and Flemming. These were followed by the noteworthy achievements of Boveri, Driesch, Herbst, Morgan, Loeb, and others. These men laid the foundations for the work which has followed, and their researches, greatly aided by the development of new refinements in microtechnique, ${ }^{6}$ ushered in modern cytology.

A powerful stimulus to investigation was given when the zoölogists Hertwig, von Kölliker, and Weismann, and the botanist Strasburger, concluded independently and almost simultaneously (1884-1885) that the nucleus is the "vehicle of heredity," an idea which Haeckel had put forward as a speculation in 1866 . The announcement of this conception led to an even more intensive study of the nucleus and of its rôle in heredity, a study which is now in progress and which, more than any other one thing, can be said to characterize the work of our modern period.

Special mention should be made of the theory developed by August Weismann (1834-1914), because the modern nuclear theory of heredity, although resting on a substantial foundation of observational and experimental evidence, is largely an outgrowth of his speculations. It may be noted that Weismann incorporated in his theory several points of earlier theories, particularly those of Darwin, de Vries, and Nägeli. ${ }^{7}$ His various hypotheses were set forth in his Das Keimplasma (1892) and in more elaborated form in his Vorträge über Deszendenztheorie (1902).

Weismann identified the supposedly distinct inheritance material, or germ-plasm (idioplasm), about which there had been much speculation, with the chromatic substance of the nucleus. His conception of its constitution was essentially as follows. The ultimate living unit is the

${ }^{6}$ For the history of staining, see Conn (1928ab, 1929, 1930ab, 1933), Conn et al. (1929), Conn and Kornhauser (1928), Kornhauser (1930), and Conn and Cunningham (1932).

${ }^{7}$ For fuller treatments of this subject, see Kellogg (1907), Delage and Goldsmith (1913), Thomson (1899, 1913), Conklin (1915), and the second edition of this book. 
biophore, a minute particle capable of growth and reproduction. The many kinds of biophores in a given cell are the elements upon whose presence the development of the cell's characters depends. The biophores are grouped to form units of a higher order, known as determinants. The determinant, since it is composed of the many kinds of biophores, has the power of determining the development of a certain type of cell or group of cells. In general, therefore, there are as many sorts of determinants in the organism as there are types of cells, or "independently variable parts."

The determinants are, in turn, grouped into $i d s$. A single id contains all the kinds of determinants and so stands for the sum of all the characters of the organism. The ids in a given species differ only slightly among themselves, the differences corresponding to the variations observed within the species: they are the "ancestral germ-plasms" contributed by past generations. The ids are identified with the visible chromatic granules in the nuclear reticulum or in the chromatic thread during mitosis. In most cases the ids are grouped to form idants, or chromosomes. The id, rather than the chromosome, is the unit of primary importance. The several ids in a chromosome are arranged in a linear series, as suggested for the hereditary "qualities" by Roux (1883).

Quoting Wilson (1900) again,

The end of fertilization is to produce new combinations of variations by the mixture of different ids. Since, however, their number, like that of the chromosomes which they form, is doubled by the union of two germ-nuclei, an infinite complexity of the chromatin would soon arise did not a periodic reduction occur. Assuming, then, that the "ancestral germ-plasms" (ids) are arranged in a linear series in the spireme thread or the chromosomes derived from it, Weismann ventured the prediction (1887) that two kinds of mitosis would be found to occur. The first of these is characterized by a longitudinal splitting of the thread, as in ordinary cell-division, "by means of which all the ancestral germ-plasms are equally distributed in each of the daughter-nuclei after hav ng been divided into halves." This form of division, which he called equal division (सquationstheilung), was then a known fact. The second form, at that time a purely theoretical postulate, he assumed to be of such a character that each daughternucleus should receive only half the number of ancestral germ-plasms possessed by the mother-nucleus. This he termed a reduction division (Reduktionstheilung) and suggested that this might be effected either by a transverse division of the chromosomes or by the elimination of entire chromosomes without division. By either method the number of "ids" would be reduced; and Weismann argued that such reducing divisions must be involved in the formation of the polar bodies and in the parallel phenomena of spermatogenesis.

With the aid of this elaborate mechanism Weismann explained ontogenetic development in the following manner. In the zygote from which the individual is to develop, all the kinds of determinants are present: those of the female parent were contained in the egg nucleus and those 
of the male parent were brought in by the nucleus of the spermatozoön. During the long series of nuclear divisions beginning with that in the zygote and ending with the completion of the mature organism, the many kinds of determinants are sorted out through a progressive disintegration of the ids and are distributed in a definite and orderly manner to the different parts of the body. Many somatic mitoses are therefore regarded not as equational ("erbgleich") but in reality qualitative ("erbungleich"). When a given determinant finally reaches the proper cell, i.e., when that cell is finally formed, the determinant splits up into its constituent biophores, and these, through their action on the protoplasm, give to the cell its specific characters. The general character of a cell is accordingly due to the type or types of determinant which it receives.

Weismann accounted for the presence of a complete outfit of determinants in the gametes and zygote, and hence for the phenomenon of hereditary resemblance, by assuming that a certain portion of the complete germ-plasm is carried along unchanged during ontogenesis and is delivered intact to the germ-cells. He thus rejected Darwin's suggestion that representative particles (gemmules) were transmitted from all the body-cells to the germ-cells. It had been shown that in certain animals the primitive germ-cells are set aside at once when development begins, and Weismann pointed out that they are therefore differentiated before any sorting out of the hereditary units could have taken place. Hence the germ-cells are really produced by the germ-cells of the previous generation and not by the individual's own soma (body); they are present from the beginning of development with the full hereditary outfit, and by a few equational divisions give rise to the gametocytes. In the case of those animals and plants whose germ-cells appear later in the ontogeny, Weismann held that, although a sorting out of the units occurs in the majority of the cells during ontogenesis, those meristematic cells which constitute the chain connecting the zygote with the germ-cells-the germ-track (Keimbahn)-maintain the undiminished germ-plasm. Hence in all cases there is a continuity of the germ-plasm, if not a continuity of the germ-cells (unless meristematic cells also are germ-cells).

Weismann argued that since there is no contribution of hereditary elements from the soma to the germ-cells, somatic changes being in no way impressed upon the germ-cells from which the next generation is to arise, there can be no inheritance of acquired somatic modifications. In multicellular animals the only inherited variations are those originating in the germ-plasm of the germ-cells or germ-track as responses to internal (nutritive, etc.) or external environmental stimuli and those due to recombinations of hereditary units at the time of syngamy (amphimixis). He admitted that the germ-plasm, though remarkably stable, might be altered directly by the environment (parallel modification of 
germ and soma), or even by modifications in the surrounding soma; but he denied that in the latter case the alteration would be of such a nature as to cause the reappearance of the same somatic modifications in the next generation. He accounted for the internally induced heritable variations on the basis of his theory of germinal selection. He supposed that the determinants, while multiplying in the germ-cells, are subject to selection like all other organic units. Some determinants, being better placed with respect to nutritive conditions, are favored thereby and grow stronger and more influential, while others undergo changes in the opposite direction. The parts of the organism receiving the determinants which have had the advantage in the struggle become better developed than those receiving the weaker determinants. As this process continues from generation to generation, the new variation gradually increases until it becomes pronounced enough to be laid hold of by natural selection.

A review of the points brought out in earlier chapters will show that modern genetics, although it has shown the inadequacy of certain elements in the hypotheses sketched above, owes many of its leading ideas to Weismann. These ideas were put to the test in the brilliant experimental researches on development carried out by Roux, Boveri, R. and O. Hertwig, and others, and later in the researches of modern students of cytogenetics. Weismann's view that heredity is conditioned primarily by the genetic continuity of germ-plasm has its modern expression in the statement that a specific type of nuclear composition, upon which the development of characters largely depends, is maintained by growth and division from generation to generation. That this composition or organization is preserved only in germ-cells sharply distinct from somatic cells is, however, a conception which no longer squares with the results of experiment. It now appears that the complex of nuclear genetic units is complete in both regetative and reproductive cells, the differentiation of cell types not being due to any sorting out of the units during ontogenesis. Our knowledge in this field is little more than fragmentary, but it seems likely that differentiation is largely a response to the diverse conditions inevitably arising in a growing mass, such conditions stimulating different groups of genes to action at different stages and in different regions. The results of such action are, in turn, a part of the cause of what follows. The gene, moreover, is now regarded as a constitutional factor somehow involved with others in the metabolic processes and thus influencing the development of many characters, rather than something standing for the characters of a complete organism or a structural part thereof as did Weismann's id and determinant.

The "germ-plasm," if this term is to be retained for the genes or chromosomes, thus represents an integral part of a developing system rather than an arbitrary determiner of development. For this reason 
many prefer to apply the term, if at all, to the entire protoplasmic system capable of differentiation and reproduction ( $c f$. p. 421). With regard to the origin of heritable variations, geneticists are still in accord with Weismann in attributing them chiefly to hybridization and alterations in the inheritance units themselves. They are not, however, inclined to see in any process like "germinal selection" the underlying cause of such gene mutations. Weismann's prediction that there would be found a reduction in the number of genetic units at gametogenesis (in animals) by a special form of nuclear division has been fulfilled, though the precise manner in which it is accomplished differs from that postulated by him.

Notwithstanding the abandonment of Weismann's theory of ontogenesis and the changes made in his theory of heredity, his influence on both cytology and genetics can never be forgotten, chiefly because of his emphasis on the need for careful studies of the nuclear mechanism at the critical stages of the life history, and upon the idea that this mechanism is in some way bound up with the phenomena of heredity. "It has been Weismann's service to place the keystone between the work of the evolutionists and that of the cytologists" (Wilson, 1900).

The Twentieth Century.--The year 1900 marks the beginning of a new era in cytology, for reasons which may be stated in the words of Wilson (1924):

This era of cell research coincides with the new era in genetics that opened in 1900 with the rediscovery of the Mendelian phenomena of heredity by de Vries, Correns, and Tschermak. This discovery was the outcome of purely genetic experiments on hybrids; but almost at the moment of its announcement, by a remarkable coincidence, cytologists had independently arrived at a point where the cytological basis of the phenomena could be clearly recognized. Rückert eight years earlier (1892) had briefly suggested a conjugation and disjunction of corresponding paternal and maternal chromosomes in meiosis and an exchange of material between them ("amphimixis of the chromosomes"), thus to a certain extent foreshadowing the modern explanation of the Mendelian segregation and of recombination by "crossing-over." Montgomery (1901), without knowledge of Mendel's fundamental law of segregation, brought together almost all of the essential data for its explanation, though he did not bring them into specific relation with the genetic phenomena. He pointed out the constant size differences of the chromosomes, emphasized the presence in the diploid groups of paternal and maternal homologues in pairs, and accepted the conjugation of these homologues in synapsis and their disjunction in the reduction division. Boveri, in his remarkable paper on multipolar mitosis (1902), demonstrated experimentally the determinative action of the chromosomes in development and proved their qualitative differences in this respect. A possible connection between the Mendelian disjunction and the reduction division was suggested nearly at the same time by several observers, including Strasburger, Correns, Guyer, and Cannon. It was, however, Sutton $(1902,1903)$ who first clearly set 
forth in all its significance the cytological explanation of the Mendelian phenomena that is offered by the behavior of the chromosomes, and thus initiated the remarkable movement in this direction that followed.

It was in such a manner that cytology was brought into an alliance with genetics. As in other instances in the history of science, the frontier of one field of research turned out to be the border of a neighboring field, and the gratifying yield of significant results along the line of meeting has been due in no small measure to the fact that those who formerly worked independently on the two sides can now employ their combined methods and speak the same language. The field of physics has contributed a valuable tool in the form of X-radiation. By means of this tool workers in modern cytogenetics are now able to obtain desired data far more rapidly than would be possible without it, and they are entitled to hope that they may some day exercise a fuller measure of control over the course of evolution by some such means.

Closely associated with the movement outlined above has been another line of investigation characteristic of the present century, namely, the study of the morphology of the chromosomes and the comparison of the chromosome complements of related organisms. Research in this field was stimulated by the discovery of heteroploidy and observations on chromosome behavior in hybrids early in the century (p. 339 et seq.), while its problems were placed in a much clearer light by the work on the morphology of individual chromosomes begun about 1910 by S. Nawaschin (1857-1930) and his associates. Such researches have already yielded important evidence with regard to systematic relationships, particularly among plants, and they promise to contribute largely to the solution of many outstanding problems of this nature. During the years which lie ahead, one of the most fruitful regions in the field of biology with its disappearing lines of subdivision should be this cytotaxonomy.

Of special importance also is the modern renewal of the investigation of protoplasm, its inclusions, its differentiations, and its reactions in the living state. This type of study has been greatly promoted not only by the development of methods of tissue culture and micromanipulation but also by the conception of protoplasm as a colloidal system. Investigations in this field are removing many misconceptions growing out of the too exclusive use of fixed material and are yielding partial explanations for a variety of puzzling cytological phenomena. Moreover, they are bringing cytology into ever more intimate associations with physical chemistry, physiology, and experimental medicine, just as the study of chromosomes has allied it with genetics and systematics. To such fields cytology has made invaluable contributions; in return it has received incentives to deal more directly and experimentally with major problems 
as well as with objects. Every field concerned has thus gained a wider appreciation of the significance of its own data. No tendency of the present is more encouraging to the investigator and promising for the future of biology than the development of such alliances, for they afford our only strong hope that we shall come nearer to a solution of the peculiar problems presented by living organisms. 



\section{BIBLIOGRAPHY}

Aase, H. C. 1930. Res. Studies State Coll Wash. 2: 5-60. illus. [Grain hybrids.]

Aase, H. C., and L. Powers. 1926. Amer. Jour. Bot. 13:367-372. illus. [Chromosome numbers.]

Abderhalden, E. (ed.). 1923. Handbuch der Biologischen Arbeitsmethoden. Berlin.

Abromavich, C. E., and W. G. Lrnn. 1930. Quar. Rev. Biol. 5: 68-78. [Sex.]

ACQUA, C. 1891. Malpighia 5: 3-39. pls. 1, 2. [Rôle of nucleus.]

1910. Ann. di Botanica 8: 43-50. [Rôle of nucleus.]

Acton, E. 1914. Ann. Botany 28: 433-454. pls. 23, 24. [Cyanophyceæ.]

Apdous, R. M. 1927. Amer. Jour. Bot. 14: 147-165. illus. [Coagulation.]

Afzelius, K. 1924. Acta Horti Bergiani 8:123-219. figs. 33. [Senecio.] 1928. Svensk Bot. Tidskr. 22: 82-91. illus. [Nigritella.]

Agar, W. E. 1911. Quar. Jour. Mier. Sci. 67: 1-44. illus. [Lepidosiren.] 1912. Quar. Jour. Micr. Sci. 58: 285-298. pls. 12, 13.

1920a. Cytology, with Special Reference to the Metazoan Nucleus. London.

1920b. Jour. Geneties 10:303-330. figs. 3. [Daphnia hybrid.]

1923. Quar. Jour. Micr. Sci. 67: II 183-202. pls. 12-14. [Marsupials.]

AIdA, T. 1921. Genetics $6: 554-573.1 \mathrm{pl}$. [Aplocheilus.]

1930. Genetics 15:1-6. [Aplocheilus.]

Akneringa, L. J. 1927. Zeitschr. Mikr. Anat. Forsch. 8: 325-342.

Albach, W. 1928. Protoplasma 5 : 412-443. [Vital staining.]

1929. Protoplasma $7: 395-422$.

Alberti, W., und G. Politzer. 1923. Arch. Mikr. Anat. u. Entw. 100: 83-109.

1924. Arch. Mikr. Anat. u. Entw. 103: 284-307. [X-rays and mitosis.]

Alexander, J., and C. B. Bridges. 1928. Chap. I of Colloid Chemistry, Theoretical and Applied. New York.

Alexieff, A. 1917a. Compt. Rend. Soc. Biol. Paris 69: 358-361.

1917b. Compt. Rend. Soc. Biol. Paris 69: 361-363.

1917c. Compt. Rend. Soc. Biol. Paris 69: 499-502.

1917d. Compt. Rend. Soc. Biol. Paris 69: 510-512.

1923. Compt. Rend. Soc. Biol. Paris 75: 728-730. [Bacteria.]

1925. Arch. Protist. 49:396-432. illus. [Bacteria.]

1928. Arch. Protist. $60: 269-286$. illus.

Allen, C. E. 1901. Bot. Gazette 32:1-34. [Middle lamella.]

1905a. Ann. Botany 19: 189-258. pls. 6-9. [Meiosis.]

1905b. Jahrb. Wiss. Bot. 42: 72-82. pl. 2. [Meiosis.]

1905c. Ber. Deu. Bot. Gesell. $23: 285-292$. pl. 13. [Coleochæte.]

1912. Arch. Zellf. 8: 121-188. pls. 6-9. [Polytrichum.]

1916. Amer. Jour. Bot. 3:456-460. figs. 2. [Catherinea.]

1917a. Ann. Botany 31:269-292. pls. 15, 16. [Polytrichum.]

1917b. Science $46: 466-467$. [Spherocarpos.]

1919. Proc. Amer. Phil. Soc. 58: 289-316. figs. 28. [Sphterocarpos.]

1924a. Proc. Amer. Phil. Soc. 63: 222-235. [Spharocarpos.] 
1924b. Genetics 9:530-587. figs. 60. [Sphœrocarpos.]

1925a. Geneties 10:1-16. figs. 11. [Spharocarpos.]

1925b. Genetics 10: 72-79. [Sphorocarpos.]

1926a. Genetics 11: 83-87. [Spharocarpos.]

1926b. Proc. Nat. Acad. Sci. 12: 2-7. [Sphærocarpos.]

1926c. Proc. Nat. Acad. Sci. 65 : 83-89. [Sphcrocarpos.]

1930a. Science 71: 197-204. [Sphorocarpos.]

1930b. Genetics 15: 150-188. [Spharocarpos.]

1932a. Amer. Naturalist 66:97-107. [Sex inheritance.]

1932b. Proc. 6 Internat. Congr. Genetics 2: 1-2. [Polyploidy.]

Allen, R. F. 1911. Trans. Wis. Acad. Sci. 17: 1-56. pls. 1-6.

1929. Science 70: 308-309. [Puccinia.]

1930. Jour. Agr. Res. $40: 585-614$. illus. [Puccinia.]

1932a. Jour. Agr. Res. 44: 734-754. illus. [Puccinia.]

1932b. Jour. Agr. Res. $45: 513-541$. illus. [Puccinia.]

Altannn, R. 1886. Studien über die Zelle. I. Leipzig.

1887. Die Genese der Zellen. Leipzig.

1889. Arch Anat. u. Physiol. 409-411.

1890, 1894. Die Elementarorganismen, usw. Leipzig.

Alvarado, S. 1923a. Trab. Mus. Nac. Cien. Nat., Madrid, Ser. Bot., No. 17.

1923b. Ber. Deu. Bot. Gesell. 41: 85-96. pl. 1. [Chondriosomes, plastids.]

Alverdes, F. 1912. Arch. Zellf. 9: 168-204. pls. 15, 16. [Chironomus.]

Anes, L. M. 1932. Bull. Torr. Bot. Club 59:341-345. illus. [Pleurage.]

Amici, G. B. 1830. Ann. Sci. Nat. Bot. I 21: 329-332.

ANCEL, P. Arch. de Biol. 19: 389-652. pls. 12-18. [Helix pomatia.]

Anderson, D. B. 1926. Science 64:64-65. [Flax fibers.]

1927. Amer. Jour. Bot. 14: 187-211. illus. [Flax fibers.]

1928. Jahrb. Wiss. Bot. 69:501-515. illus. [Clivia wall.]

Anderson, E. G. 1923. Bot. Gazette 76:411-418. figs. 2. [Variegation.] 1924c. Papers Mich. Acad. Sci., Arts, Letters $4: 523-525 . \quad$ [X-rays.]

1925b. Papers Mich. Acad. Sc1., Arts, Letters 5: 355-366. [X-ray treatments.]

1925c. Genetics 10: 403-417. [X-ray treatments.]

1929. Zeitschr. Ind. Abst. Vererb. 51:397-441. [Non-disjunction.]

Anderson, E. G., and W. H. Eyster. 1928. Genetics 13: 111-120. [Variegation.]

Anderson, E. G., and M. M. Rhondes. 1931. Papers Mich. Acad. Sci. 13 : 227-239.

[Interference.]

Andelison, R. J., and W. L. Kulp. 1922. Jour. Biol. Chem. 50: 433.

1923. N. Y. Agr. Exp. Sta. Techn. Bull. 92. [Pollen analysis.]

Andelisson, I. 1923. Jour. Genetics 13:1-12. 2 pls. [Variegation in fern.] 1924. Jour. Geneties $14: 185-195$. [Variegation in Barbarea.]

Andersson-Kотtö, I. 1931. Bibliog. Genet. 8: 269-294. [Ferns.] 1932. Svensk Bot. Tidskr. $26: 99-106$. [Ferns.]

Andrews, E. A. 1898. Roux's Arch. Entw. 6:228-248. 24 figs. [Plasma bridges.]

Andrews, F. M. 1901. Beih. Bot. Centralbl. 11: 134-142. 1 pl. [Mitosis.] 1902. Jahrb. Wiss. Bot. 38: 1-40. pl. 1. [Centrifuged cells.]

1915. Jahrb. Wiss. Bot. 56:221-253. pl. 1. 2 figs. [Centrifuged cells.]

Andrews, G. F. 1897. Jour. Morph. 12:367-389. [Plasma bridges.]

Andrus, C. F. 1931. Jour. Agr. Res. 42: 559-587. illus. [Uromyces.]

Anitschkow, N. 1923. Arch. Mikr. Anat. 97. pl. 1. [Chondriosomes.]

ANkel, W. E. 1927. Zeitschr. Ind. Abst. Vererb. $45: 232-278$.

Arber, A. 1920. Jour. Roy. Mier. Soe. London 1920; 1-21. pl. 1. 2 figs.

Alnold, G. 1912a. Quar. Jour. Mier. Sci. 57:283-299. pl. 25. [Chondriosomes.] 1912b. Arch. Zellf. 8: 252-271. pl. 12. [Chondriosomes.] 
Arthor, J. C. 1929. The Plant Rusts. New York.

Arton, C. 1912. Arch. Zellf. 9:87-113. illus. [Artemia.]

Astbury, W. T., T. C. Marwick, and J. D. Bernal. 1932. Proc. Roy. Soc. B 109 : 443-450. illus. [Valonia wall.]

Astbury, W. T., and A. Street. 1931. Phil. Trans. Roy. Soc. London A 230 : $75-101$.

Astbury, W. T., and H. J. Woons. 1932. Jour. Textile Inst. 23: T17-T34.

Atkinson, G. F. 1915. Ann. Missouri Bot. Garden 2:315-376. 10 figs. [Ascomycetes.]

Avdulow, N. P. 1931. Bull. Appl. Bot., Genet., Plant Breed., suppl., 43: 1-428. illus. [Graminece.]

Avel, M. 1925. Compt. Rend. Soc. Biol. Paris 90: 794.

Avery, P. 1929. Univ. Calif. Publ. Bot. 11: 265-284. illus. [Nicotiana.]

B

BaAche Wilg, S. 1925. Compt. Rend. Acad. Sci. Paris 180:309-311. [Erisiphe.] ВАвсоск, E. B. 1924a. Amer. Naturalist 58: 296-310. [Crepis hybrids.]

1924b. Science 59:327-328. [Genetics and taxonomy.]

Babcock, E. B., and J. Clausen. 1929. Univ. Calif. Publ. Agr. Sci. 2 : 410-432. illus. [Crepis.]

Babcock, E. B., and R. E. Clausen. 1927. Genetics in Relation to Agriculture. New York.

Babcock, E. B., and M. Nawaschin. Bibliog. Genet. 6:1-90. illus. [Crepis.]

BacCarini, J. 1908. Nuovo Giom. Bot. Ital. N. S. $15:$ 189-204. pl. 7.

von BAER, K. E. 1828, 1837 . Ueber Entwickelungsgeschichte der Thiere.

DE BAEHr, V. B. 1920a. La Cellule 30:315-353. 1 pl. [A phis palma.]

von Baenr, W. B. 1908. Zool. Anzeiger 33: 507-517. 14 figs. [Aphidæ.]

1909. Arch Zellf. 3: 269-334. pls. 12-15. [Aphis saliceti.]

1912. La Cellule $27: 385-450.2$ pls. [A phis saliceti.]

Bagchee, K. 1925. Ann. Botany 39:217-266. pls. 5-8. [Pustularia.]

Bailey, I. W. 1919. Proc. Nat. Acad. Sci. 5: 283-285. 1 fig. [Cambium.]

1920a. Amer. Jour. Bot. $7: 355-367.3$ figs. [Cambium.]

1920b. Amer. Jour. Bot. $7: 417-434$. pls. 26-29. [Cambium.]

1930. Zeitschr. Zellf. Mikr. Anat. 10 : 651-682. illus. [Vacuome.]

Bailey, I. W., and C. Zirkle. Jour. Gen. Physiol. 14:363-383. [Vacuome.]

Baird, E. A. 1924. Trans. Wis. Acad. Sci. 21: 357-380. 2 pls. [Zygomycetes.]

Balbiani, E. G. 1876. Compt. Rend. Acad. Aci. Paris 83: 831-834.

1881. Zool. Anz. 4: 637-641. 7 figs.

Ballowitz, K. Anat. Anz. 1, 1886; 27, 1905; 28, 1906; Arch. Mikr. Anat. 32, 1888

(birds); 36, 1890 (fishes, amphibia); 65, 1904; 70, 1907; Zeitschr. Wiss Zool. 50,

1890 (insects); 52, 1891 (mammals); 91, 1908; Arch. Gosammt. Physiol. 46, 1890.

Balls, W. L. 1923. Proc. Roy. Soc. London B 95: 72-89. illus. [Cotton wall.]

Baltzer, F. 1920. Verhl. Schwciz. Naturf. Gesell.

Bancroft, W. D. 1921. Applied Colloid Chemistry. New York.

Bancrowt, W. D., and C. Gurchot. 1927. Jour. Phys. Chem. 31: 430-438.

Banghan, W. 1929. Jour. Arnold Arbor. 10:167-169. [Philadelphus.]

Banta, A. M. 1916. Proc. Nat. Acad. Sci. $2: 578-583 . \quad$ [Sex in crustacea.]

1918. Proc. Nat. Acad. Sci. 4:373-379. [Sex in crustacea.]

1919. Anat. Record $15: 355-356$. [Sex in crustacea.]

Baranetzky, J. 1880. Bot. Zeitung 38: 241-247, 265-274, 281-296. pl. 5.

Baranov, P. 1926. Zeitschr. Zellf. Mikr. Anat. 3: 130-148. illus.

BArber, M. A. 1911b. Jour. Infect. Diseases 9:117-129. [Microdiss. app.] 
BAron, M. A. 1928. Centralbl. Bakt. 73 : II 373-379. illus.

1930. Planta 10:28-83. [Mitogenetic rays.]

Bartlett, H. H. 1911. Rhodora 13: 199-206. 3 figs. [Sex in Plantago.]

1915. Amer. Jour. Bot. 2 : 132-155. [Experimental genetics.]

1916. Amer. Naturalist $50: 513-529$. [Enothera and mutation.]

DE BARY, H. A. 1S5S. Untersuchungen über die Familien der Conjugaten. Leipzig.

1859. Zeitschr. Wiss. Zool. 10: 88-175. 5 pls. [Myxomycetes.]

1862. Flora 20. [Protoplasm.]

1864. Die Mycetozoen. 2d ed. Leipzig. [Protoplasm.]

Bast, T. H. 1921a. Amer. Jour. Anat. 29: 139-157. 1 pl. [Amitosis.]

1921b. Amer. Jour. Anat. $29: 321-339.1$ pl. [Amitosis.]

Bataillon, E. 1910. Compt. Rend. Acad. Sci. Paris 150: 996-998. [Parthenogenesis.]

1929. Roux's Arch. Entw. Org. 115 : 707-778. illus.

Bateson, Sadnders, and Ponrett. 1905. Rept. II to Evol. Comm. Roy. Soc. London.

Bateson, W., and R. C. Punnett. 1906. Rept. to Evol. Comm. Roy. Soc. London.

BAUCH, R. 1926. Zeitschr. Bot. 18: 337-387. illus. [Camarophyllus.] 1927. Arch. Protist. 58: 285-299. illus. [Clavaria.]

BaUmgärtel, O. 1920. Areh. Protist. 41: 50-148. 1 pl. [Cyanophyceæ.]

BAUR, E. 1907. Ber. Deu. Bot. Gesell. 25: 442-454. [Antirrhinum.]

1909. Zeitschr. Ind. Abst. Vererb. 1: 330-351. 20 figs. [Pelargonium.]

1910. Zeitschr. Ind. Abst. Vererb. 4: 81-101. [Melandrium, etc.]

1912. Zeitschr. Ind. Abst. Vererb. 8: 335-336.

1930. Einführung in die Experimentelle Vererbungslehre. Berlin.

BAyliss, W. M. 1915. Principles of General Physiology. London.

1920. Proc. Roy. Soc. London B 91: 196-201. [Reversible gelation.]

1921. Rept. II on Colloid Chem., British Assoc. Adv. Sci. [Permeability.]

1923. Interfacial Forces and Phenomena in Physiology. New York.

Beadle, G. W. 1930a. Cornell Univ. Agr. Exp. Sta. Memoir 129. [Asynapsis.]

1932a. Proc. Nat. Acad. Sci. 18: 160-165. [Crossing-over.]

1932c. Zeitschr. Ind. Abst. Vererb. 62: 291-304. illus. [Euchlana $\times$ Zea.]

Beadle, G. W., and S. Enierson. 1932. Proc. 6 Internat. Congr. Genet. 2 : 7.

Beadle, G. W., and B. McClintock. 1928. Science 58: 433. [Asynapsis.]

BEAMS, H. W. 1927. Biol. Bull. 53: 155-172. illus. [Activation.]

1929. Anat. Record $42: 323-332$. illus. [Golgi material.]

1930a. Anat. Record 45: 137-161. illus. [Vacuome and Golgi.]

1930b. Anat. Record 46: 305-327. illus. [Vacuome, Golgi, chondriome.]

Beans, H. W., and J. B. Goldsmith. 1930. Jour. Morph. Physiol. 50: 497-515.

Beans, H. W., and R. L. King. 1932. Jour. Morph. Physiol. 53: 59-95. illus.

[Ganglion cells.]

Beams, H. W., and C. F. Wu. 1929. Jour. Morph. Physiol. 47: 261-281. illus.

[Secretion.]

Beardsley, M. L. 1931. Ann. Missouri Bot. Garden. 18: 509-540. illus.

Beauverie. 1919. Sci. Amer. Suppl. 87 : No. 2244, pp. 2-3, 7. [Pigments.]

Bechimol, H. 1912. Die Kolloide in Biologie und Medizin. Dresden. (Engl.

transl. by Bullowa, New York, 1919.)

Becker, E. R. 1926. Biol. Bull. $50: 382-392$. illus.

Becker, W. A. 1932a. Protoplasma 15: 478-481. illus. [Cell-plate.]

1932b. Compt. Rend. Acad. Sci. Paris 194: 1850-1852.

1932c. Acta Soc. Bot. Polon. 9: 381-419. illus.

BEER, R. 1905. Beil. Bot. Centralbl. I 19: 2S6-313. pls. 3-5. [Pollen wall.] 1909a. Ann. Botany 23: 63-72. pl. 4. [Elaioplasts.] 
1909b. New Phytol. 8: 261-266. [Equisetum.]

1911. Ann. Botany 25: 199-214. pl. 13. [Spore wall.]

Beer, R., and A. Arber. 1919. Proc. Roy. Soc. London B 91:1-17. pl. 1.

1920. Jour. Roy. Micr. Soc. 23-31. [Multinucleate cells.]

Berkirch, H. 1925. Bot. Arch. 12:389-445. illus. [Streaming.]

BelajefF, W. 1888. Ber. Deu. Bot. Gesell. 7 : 122-125. [Spermatozoids.]

1894a. Flora, Ergänzb. 79: 1-48. pl. 1. [Spermatozoids.]

1894b. Flora 79: 430-441. pls. 12, 13. [Spermatozoids.]

1897a. Ber. Deu. Bot. Gesell. 15 : 337-339. [Spermatozoids.]

1897b. Ber. Deu. Bot. Gesell. $15: 339-342$. [Spermatozoids.]

1897c. Ber. Deu. Bot. Gesell. 15 : 342-345. [Spermatozoids.]

1898. Ber. Deu. Bot. Gesell. 16: 140-144. pl. 7. [Blepharoplast.]

1899. Ber. Deu. Bot. Gesell. 17: 199-205. pl. 15. [Blepharoplast.]

BĚLA Ř, K. 1921. Biol. Zentralbl. 41:385-394. illus. [Actinophrys.]

1922. Areh. Protist. 46:1-96. illus. [Actinophrys.]

1923. Biol. Zentralbl. 43 : 513-518. figs. 3. [Rhabditis.]

1924. Zeitschr. Zellen- u. Gewebelehre 1: 1-21. figs. 13. [Rhabditis.]

1925. Zeitsehr. Ind. Abst. Vererb. 39: 184-190. illus. [Melandrium.]

1926. Ergebn. u. Fortschr. Zool. 6: 235-654. illus. [Nucleus.]

1927. Naturwissenschaften 15: 725-734. illus. [Mechanism of mitosis.]

1928a. Die Cytologischen Grundlagen der Vererbung. Berlin.

1928b. Zeitschr. Ind. Abst. Vererb., suppl., 1: 402-407.

1929a. Roux's Areh. Entw. 118: 359-484. illus. [Mech. of mitosis.]

1929b. Zeitschr. Zellf. Mikr. Anat. 10: 73-134. [Meeh. of mitosis.]

1929c. Collecting Net, Woods Hole, 4. [Centriole.]

1930. Protoplasma 9: 209-244. illus. [Protoplasm.]

Bellamy, A. W. 1922. Anat. Record 24:419-420. [Platypcecilus.]

1924. Genetics 9: 513-529. [Platypocilus.]

Belling, J. 1923. Second Internat. Eugenies Congr. 1: 84-95. Baltimore.

1925a. Jour. Geneties $15: 245-266.11$ figs. [Uvularia.]

1925b. Genetics 10: 59-71. illus. [Triploidy.]

1925c. Zeit. Ind. Abst. Vererb. 39 : 286-2S8. [Stizolobium.]

1927b. Proe. Nat. Acad. Sci. 13: 717-718. [Reciprocal translocation.]

1927c. Biol. Bull. 52: 480-487. illus. [Reciprocal translocation.]

1927d. Jour. Genetics 18: 177-205. illus. [Spindle attachment.]

1928a. Biol. Bull. 64: 465-470. illus. [Chiasmata.]

1928b. Univ. Calif. Publ. Bot. 14: 335-343. illus. [Chromomeres.]

1928c. Univ. Calif. Publ. Bot. 14: 307-31s. illus. [Chromomeres.]

1928f. Univ. Calif. Publ. Bot. 14 : 283-291. illus. [Reciprocal translocation.]

1928g. Nature (London), 122: 882 . [Chromomeres.]

1929. Univ. Calif. Publ. Bot. 14: 379-388. illus. [Trivalents.]

1931a. Univ. Calif. Publ. Bot. 16:153-170. illus. [Chromomeres.]

1931b. Univ. Calif. Publ. Bot. 16:311-338. illus. [Chiasmata.]

1933 (posthumous). Genetics 18: 388-413. [Crossing-over.]

Belling, J., and A. F. Blakeslee. 1922. Amer. Naturalist 66:339-346. S figs.

1923. Proe. Nat. Acad. Sci. 9: 106-111. [Meiosis in polyploids.]

1924a. Amer. Naturalist 58: 60-70. 4 figs. [Meiosis in polyploids.]

1924b. Proc. Nat. Acad. Sci. 10: 116-120. 2 figs. [Datura, reciprocal trans-

location.]

1926. Proe. Nat. Acad. Sci. 12: 7-11. illus. [Reciprocal translocation.]

1927. La Cellule $37: 355-365$. illus. [Monoploid Datura.]

Belzung, E. 1887. Ann. Sci. Nat. Bot. VII 5 : 179-310. pls. 5-8. [Starch.] 
Benda, C. 1897. 1898. Verh. Physiol. Gesell. Berlin.

1899ab. Verh. Physiol. Gesell. Berlin. [Chondriosomes.]

1902. Die Mitochondria. Ergeb. Anat. 11. Entw. 12: 743-781.

van Beneden, E. 1876. Bull. Acad. Roy. Belg. 41:1160-1205; 42: 35-97. 3 pls. 1883. Arch. de Biol. 4.

van Beneden, E., and Julin. 1884. Bull. Acad. Roy. Belg. III 7 : 312-342.

van Beneden, E., and Neyt. 1887. Bull. Acad. Roy. Belg. III $14: 215-295$. pl. 1.

Benoist, H., V. Golblin, and W. Kopaczewski. 1929. Protoplasma 5 : 481-510.

Bensaude, M. 1918. Diss. Nemours. 156 pp., 13 pls. (See Bot. Gazette 68:

67-68, or Bot. Absts. 3 : 49.)

Bensley, R. R. 1910. Biol. Bull. 19: 174-194. 3 figs. [Canaliculi.] 1911. Amer. Jour. Anat. 12: 297-388. 15 figs. [Pancreas.]

1916. Amer. Jour. Anat. $19: 37-54$. [Thyroid.]

Berg, K. H. 1931ab. Akad. Wiss. Wien, Akad. Anz. No. 22.

von Bergen, F. 1904. Arch. Mikr. Anat. 64: 498-574. pls. 29-31.

Berghs, J. 1904. La Cellule 21 : 383-394. 1 pl. [Meiosis.]

1905. La Cellule 22 : 141-160. 2 pls. [Meiosis.]

1906. La Cellule 23: 53-86. 3 pls. [Spirogyra.]

1909. La Cellule 25: 73-84. 1 pl. [Marsilia.]

Bergner, A. D., S. Satina, and A. F. Brakeslee. 1933. Proc. Nat. Acad. Sci. 19 : $103-115$. illus.

Bergon, P. 1907. Bull. Soc. Bot. France 54:327-358. illus. [Diatoms.]

Bernard, C. 1878. Leçons sur les Phénomènes de la Vie. Paris.

Bernhardi, J. J. 1805. Beobachtungen über Pflanzengefässe.

Berridge, E. M., and F. Sanday. 1907. New Phytol. 6: 128-134, 167-174.

Bersa, E. 1926. Protoplasma 1: 159-166. [Irradiation: bibliography.]

Besley, H. 1930. Cornell Univ. thesis.

Besta, C. 1910. Anat. Anz. 36: 476-486. 1 pl. [Golgi material.]

Beutner, R. 1933. Physical Chemistry of Living Tissues and Life Processes. Baltimore.

Beyer, W. 1921. Jenaische Zeitschr. 57: 491-512. pl. 25. [Blood cells.]

Bhatia, D. 1927. Quar. Jour. Micr. Sci. 70:681-691. illus. [Ciliated cells.]

Bhatia, D., and V. NAth. 1931. Quar. Jour. Micr. Sci. 74: 669-699. illus.

Bhattacharya, D. A., and K. B. LAl. 1929. Allahabad Univ. Stud. 6: 1-20.

Bhattacharya, D. A., and C. B. Mathur. 1930. Allahabad Univ. Stud. 6 : 29-40. [Yolk.]

Bichler. 1914. Wiener Klin. Wochenschr. No. 26. [X-ray tumors.]

Binder, S. 1927. Zeitschr. Zellf. Mikr. Anat. 5 : 293-346. illus.

Binz, A. 1892. Flora 76: 34-91. pls. 5-7. [Starch grain.]

Biodrge, P. 1892. La Cellule 8: 47-76. 2 pls. [Pollen walls.]

Bisceglie, V., and G. Bucciardi. 1929. Arch. Exper. Zellf. $7: 444$.

Black, C. A. 1913. Ann. Botany 27: 511-532. pls. 37, 38.

Blackidns, K. B. 1923. Nature, Nov. 10, 1923. 1 fig. [Lychnis chromosomes.] 1924. Brit. Jour. Exp. Biol. 1:413-430. 2 pls. [Lychnis chromosomes.]

1925. Amer. Naturalist $59:$ 200-205. [Rosa chromosomes.]

1927a. Nature (London) 120:157-158. [Silene.]

1928. Zeitschr. Ind. Abst. Vererb., suppl. 1: 439-446. illus. [Silene.]

1929. Proc. Internat. Cong. Plant Sci., Ithaca 1: 299-306. illus.

Brackburn, K. B., and J. W. H. Harrison. 1921. Ann. Botany 35 : 159-188.

1924a. Ann. Botany 38 : 361-378. 11 figs. [Salicacex: chromosomes.]

1924b. Brit. Jour. Exp. Biol. 1 : 557-570. 2 pls. [Hybrid roses.]

Blackman, M. W. 1903. Biol. Bull. 5: 187-217. 22 figs. [Scolopendra or.] 1905. Bull. Mus. Comp. Zoöl. Harvard 48:1-137. 9 pls. [Scolopendra ơ.] 
Blackman, V. H. 1898. Phil. Trans. Roy. Soc. London B $190:$ 395-426.

1904a. New Phytol. 3: 149-158. [Apogamy and parthenogenesis.]

1904b. Ann. Botany 18: 323-369. pls. 21-24. [Uredineæ.]

Blackman, V. H., and H. C. I. Fraser. 1905. Ann. Botany 19 : 567-569.

1906a. Proc. Roy. Soc. London B 77:354-368. pls. 13-15.

1906b. Ann. Botany $20: 35-48$. pls. 3, 4. [Uredineæ.]

Blackman, V. H., and E. J. Welsford. 1912. Ann. Botany 26: 761-767. 2 pls. 1913. Ann. Botany 27:111-114. pl. 12. [Syngamy in Lilium.]

Blair, M. C. 1926. Bot. Gazette $81: 377-400$. illus. [Reboulia.]

Blakeslee, A. F. 1904. Proc. Amer. Acad. Arts Sci. 40: 205-319.

1906. Bot. Gazette $42: 161-178$. pl. 6.3 figs. [Sex.]

1915. Biol. Bull. $29: 87-89.3$ pls. [Mucors.]

1920a. Genetics 5: 419-433. 1 fig. [Portulacca.]

1920b. Science 51:375-382, 403-409. 4 figs. [Mucors.]

1921a. Amer. Naturalist $55: 254-267$. [Datura.]

1921b. Genetics 6: 241-264. 1 fig. [Datura.]

1921c. Proc. Nat. Acad. Sci. $7:$ 148-152. [Datura.]

1922. Amer. Naturalist 56:16-31. 7 figs. [Datura.]

1928. Zeitschr. Ind. Abst. Vererb., suppl. 1:117-130. illus.

1929a. Jour. Heredity 20: 177-190. illus.

1931b. Smithsonian Rept. 1930, 431-450. illus.

Blakesiee, A. F., and J. Belling. 1924a. Jour. Heredity 15: 195-206. 8 figs. Blakeslee, A. F., J. Belling, and M. E. Farnham. 1920 . Science 52: 388-390. 1923. Bot. Gazette 76: 329-373.

Blakeslee, Belling, Farnham, and Bergner. 1922. Science б5: 646-647.

Blakeslee, A. F., and D. Bergner. 1932. Proc. Nat. Acad. Sci. 18: 151-159.

Blakeslee, A. F., and J. L. Cartledge. 1926. Proc. Nat. Acad. Sci. $12:$ 315-323. 1927. Memoir Hort. Soc. N. Y. 3: 305-312. illus.

Blakeslee, A. F., and R. E. Cleland. 1930. Proc. Nat. Acad. Sci. 16: 177-183.

Bleier, H. 1925. Jahrb. Wiss. Bot. 64, 604-636. 6 figs.

1926. Zeitschr. Pflanzenzücht. $11: 302-310$.

1928a. Verh. 5 Internat. Kongr. Vererb., Berlin.

1928b. Bibliogr. Genet. 4:321-398. [Grain hybrids.]

1928c. Genetica 11:111-118. [Vicia.]

1930a. Der Züchter 2:12-22. illus. [Egilops $\times$ Triticum.]

1930b. Zeitschr. Zellf. Mikr. Anat. 11:218-236. illus. [Temperature.]

1930c. La Cellule $40: 85-144$. illus. [Spindle.]

1930d. (Paper Cambridge Cong. 1930.)

1931a. Genetica 13:27-76. illus. [Mechanism of mitosis.]

1931b. Zeitschr. Züchtung A $17: 70-79$. illus. [Grain hybrids.]

1931c. Arch. Pflanzenbau 5: 545-560. illus. [Potato.]

1933a. Genetica 15:129-176. illus. [Monoploid Enothera.]

1933b. Der Züchter 5 : 159-168. illus. [Haplodiplonts.]

Blunt, G. M. 1932. Science $76: 372$.

Bobilioff-Preisser, W. 1917. Beil. Bot. Centralbl. I $34: 459-492.18$ figs.

Bock, F. 1926. Arch. Protist. 56:321-356. illus. [Plasmodesms.]

Воеск, W. C. 1917. Univ. Calif. Publ. Zoöl. 18: 1-26. pl. 1. [Giardia.]

Boedyn, K. 1924. Zeitschr. Zellen- u. Gewebelehre 1:265-277. 17 figs.

1926. Zeitschr. Bot. 18: 161-171. [Enothera.]

Вокке, J. 1926. Zeitschr. Zellf. Mikr. Anat. 7: 95-120. [Neurofibrils.]

Bokorny, T. 1888. Jahrb. Wiss. Bot. 19: 206-220. [Vacuoles.]

Bord, H. C. 1931. Bull. Torr. Bot. Club 57:577-604. illus. [Chlorococcum.]

Bolieter, E. 1905. Beih. Bot. Centralbl. 18: 327-408. illus. 
Bonnet, J. 1912. Arch. Zellf. 7: 604-722. pls. 39-45. 17 figs.

1914. Prog. Rei Bot. 5: 1-126. 65 figs. [Algæ: life cycles.]

Bonnevie, K. 1906. Jenaische Zeitschr. 41: 229-428. pls. 16-23. 10 figs.

1908. Arch. Zellf. $2: 201-278$. pls. 13-19. 23 figs.

1911. Arch. Zellf. 6: 190-253. pls. 10-13. [Meiosis in Allium.]

Böös, G. 1917. Lunds Univ. Årsskr., N. F., Avd. 2, $13: 31$ pp. 17 figs.

1920. Bot. Notiser, Lund. [Parthenogenesis in Alchemilla.]

Borgenstam, E. 1922. Arch. Bot. 17:1-27. pl. 1. [Syringa.]

Boring, A. M. 1909. Arch. Zellf. 4:120-131. pl. 10. [Ascaris: chromosomes.]

1923. (Stevens.) Science 58: 73-74. 6 figs. [Chick chromosomes.]

Borodin, O. N. 1930. Plant Physiol. 5: 119-129. [Mitogenetic rays.]

Borzì, A. 1886. Malpighia 1: 74, 97, 145, 197. pl. 3. [Plasina bridges.]

Bosaeus, W. 1926. Upsala Läkarefören. Förhandl. 31: 415-462.

Bose, J. C. 1918. Proc. Roy. Soc. London B 90: 364-400. 17 figs.

Bose, S. R. 1927. Nature 120: 805-806. [Golgi material.]

1931. Ann. Botany $45: 303-314$. ilhus. [Golgi material.]

Bourquin, H. 1917. Bot. Gazette 64: 426-434. pl. 27. [Starch formation.]

Bouygues, H. 1924. Actes Soc. Linn. Bordeaux 76:5-166. illus. [Chondriome.]

Boveri, T. 1887ab. Sitzber. Gesell. Morph. Physiol. München 3.

1887c. Anat. Anz. 2: 688-693. [Nuclei in cleavage.]

1887d. Zellen-Studien I. Jenaische Zeitschr. 21: 423-515. pls. 25-28.

1888a. Zellen-Studien II. Jenaische Zeitschr. 22 : 685-882. pls. 19-23.

1888b. Sitzber. Gesell. Morph. Physiol. München 4.

1889. Sitzungsber. Phys.-Med. Gesell. Würzburg.

1890. Zellen-Studien III. Jenaische Zeitschr. 24: 314-401. pls. 11-13.

1891. Befruchtung. Ergeb. Anat. Entw. 1:386-485. 15 figs.

1892. Sitzber. Gesell. Morph. Physiol. München 8.

1895a. Roux's Arch. Entw. 2 : 394-443. pls. 24, 25. [Enucleate cells.]

1895b. Verh. Phys.-Med. Gesell. Würzburg, N. F., 29. [Centrosome.]

1899. Festsehr. f. C. von Kupffer, 383-430. pls. 40-45.

1901. Zellen-Studien IV. Jenaische Zeitschr. 35: 1-220. 8 pls.

1902a. Verh. Phys.-Med. Gesell. Würzburg 35. [Multipolar mitosis.]

1902b. Das Problem der Befruchtung. Jena. [Fertilization.]

1903a. Ueber die Konstitution der chromatischen Kernsubstanz. Leipzig.

1903b. Roux's Arch. Entw. 16: 340-363. pl. 15. 3 figs.

1904a. Zool. Anz. 27 : 406-417. (Boveri und Stevens.)

1904b. Ergebnisse über die Konstitution der chromatisehen Kernsubstanz. Jena.

1905. Zellen-Studien V. Ueber die Abhängigkeit der Kerngrösse und Zellenzahl

der Seeigel-Larven von der Chromosomenzahl der Ausgangszellen. Jena.

1909a. Sitzber. Physs.-Med. Gesell. Würzburg. [Chromosomes and sex.]

1909b. Arch. Zellf. 4: 132-141. figs. 2. [Chromosomes and sex.]

1909c. Arch. Zellf. 3: 181-268. pls. 7-11. 7 figs.

1910a. Festschr. f. R. Hertwig III 131-214. pls. 11-16.

1910b. Roux's Arch. Entw. 30: II 101-125. 32 figs. [Centrifuged eggs.]

1911. Verh. Phys.-Med. Gesell. Würzburg 41. [Hermaphroditism.]

1914a. Zur Frage der Entstehung maligner 'Tumoren. Jena.

1914b. Verh. Phys.-Med. Gesell. Würzburg 43. [Echinoderm hỵbids.]

1918 (posthumous). Areh. f. Entw. 44: 417-471. 3 pls.

Bowen, R. H. 1920. Biol. Bull. 39: 316-362. 2 pls. [Spermatogenesis.]

1922a. Amer. Jour. Anat. 30: 1-23. 2 pls. [Spermatogenesis.]

1922b. Anat. Record 24: 159-180. 3 figs. [Spermatogenesis.]

1922c. Jour. Morph. 37: 79-193. 10 pls. 5 figs. [Spermatogenesis.]

1922d. Biol. Bull. 41: 53-85. 2 pls. [Nebenkern.] 
1922e. Quar. Jour. Micr. Sci. 66: 595-626. pls. 24-26. [Spermatogenesis.]

1922f. Biol. Bull. 43 : 184-202. 2 pls. [Abnormal mitoses.]

1923a. Anat. Record 26:153-160. [Chondriosomes.]

1923b. Proc. Nat. Acad. Sci. 9: 349-352. [Secretory granules.]

1924a. Amer. Jour. Anat. 33: 197-217. 1 pl. [Secretory products.]

1924b. Anat. Record $28: 1-13.2$ figs. [Acrosome; secretion.].

1924c. Jour. Morph. 39: 351-413. 4 pls. [Insect spermatogenesis.]

1925a. Science $61: 545-546$. [Golgi material in gland cells.]

1925b. Anat. Record 31:201-231. illus. [Non-flagellate sperm.]

1926c. Anat. Record 32: 151-193. [Golgi apparatus: review.]

1926f. Science 44:188-190. [Polytrichum sperm.]

1926h. Anat. Record 34: 143. [Plant cells.]

1926j. Quar. Jour. Micr. Sci. 70: 419-449. illus. [Gland cells.]

1927a. Anat. Record $35: 309-335$. illus. [Golgi and vacuome.]

1927b. Biol. Bull. 53: 179-196. illus. [Plant cells.]

1928a. Zeitschr. Zellf. Mikr. Anat. 6: 689-725. illus. [Osmiophilic platelets.]

1928b-g. Anat. Record 38, 39, 40. [Golgi methods.]

1929a. Zeitschr. Zellf. Mikr. Anat. 9:1-65. illus. [Plastidome and chondriome.] 1929b. Ann. Botany 43: 309-327. illus. [Plastidome and chondriome.]

1929c. Bull. Torr. Bot. Club $56: 33-52$. illus. [Osmic methods.]

1929d. Quar. Rev. Biol. 4: 299-324, 484-519. illus. [Sccretion.]

Bowen, R. H., and L. H. Buck. 1930. Ann. Botany 44:565-586. illus.

Bozler, E. 1927. Zeitschr. Vergl. Physiol. 6: 255-263.

Brachet, Q. 1927. Quar. Rev. Biol. 2: 204-229. [Developmental factors.]

BragG, William. 1930. Nature. London. suppl. 125:315-322. illus.

Brambell, F. W. R. 1930a. The Development of Sex in Vertebrates. N. Y. 1930b. Science Progress $24: 643-664$. illus.

Brambell, F. W. R., and N. S. Rau. 1925. Proc. Roy. Soc. London B 97. [Golgi.] Brand, F. 1908. Ber. Deu. Bot. Gesell. 26: 114-143. pl. 5. [Wall formation.]

Brauer, A. 1894. Arch. Mikr. Anat. 43:162-222. pls. 8-11. [Artemia.] 1928. Jour. Morph. Physiol. 46:217-239. illus.

Braun, Alex. 1850. Betrachtungen über die Erscheinung der Verjüngung, usw.

Bremer, G. 1922. Arch. Suikerind. Nederl.-Indië, 1-111, 92 figs.

1924a. Arch. Suikerind. Nederl.-Indië, 151-180.

1924b. Arch. Suikerind. Nederl.-Indië, 477-508. illus. [Sugar cane.]

1924c. Genetica 6:497-525. illus. [Sugar cane.]

1925. Genetica $7: 293-322$. illus.

1928a. Arch. Suikerind. Nederl.-Indië No. 11.

1928b. Rec. Trav. Bot. Néerl. 25a: 82-91. illus.

1929a. Proc. 3 Cong. Internat. Soc. Sugar Cane Tech. 408-415.

1929b. Proc. 3 Cong. Internat. Soc. Sugar Cane Tech., Bull. 15.

1931a. Arch. Suikerind. Nederl.-Indië, 583-607. illis.

1931b. Arch. Suikerind. Nederl.-Indië, 1349-1391.

1932. Paper 4 Cong. Internat. Soc. Sugar Cane Tech.

Breslawetz, L. 1930. Proc. U. S. S. R. Congr. Genetics, etc. 2 : 181-186.

Bresslad, E., and Harnisch. 1927. Tabulæ Biol. 4: 83-113. [Animal chromosome numbers.]

Bridges, C. B. 1913. Jour. Exp. Zoöl. 15: 587-606. [Non-disjunction.]

1915. Jour. Exp. Zoöl. 19: 1-21. [Age and crossing-over.]

1916. Genetics 1:1-52, 107-163. [Non-disjunction.]

1919. Proc. Soc. Exp. Biol. Med. 17: 1-2. [Mutations.]

1921 $a$. Science 54: 252-254. [Triploid intersexes.]

1922. Amer. Naturalist 56:51-63. 7 figs. [Origin of variations.] 
1923. Genetics, Eugenics and The Family 1: 76-81.

1925. Amer. Naturalist 59: 127-137. [Sex and chromosomes.]

1927. Jour. Gen. Physiol. 8: 689-700. [Age and crossing-over.]

1929. Carnegie Inst. Wash. Publ. 399:63-89. [Age and crossing-over.]

1930. Science 72: 405-406. [Monoploidy and genic balance.]

1932. Sex and Internal Secretions 55-93. [Genetics of sex.]

Bridges, C. B., and E. G. Anderson. 1925. Genetics 10:418-441.

Brieger, F. G. 1926. Amer. Naturalist 60:183-191. [Selective fertilization.] 1927b. Naturwissenschaften 15: 734-740. illus.

1930. Ber. Deu. Bot. Gesell. 48: 95-98. [Chromosome doubling.]

Brill, R. 1923. Liebig's Ann., 434. [Silk fiber.]

Brink, R. A. 1925b. Genetics 10: 359-394. [Gametophytic ratios.]

1927a. Zeitschr. Ind. Abst. Vererb. 44:129-14S. [Linaria.]

1927c. Amer. Naturalist 61: 280-283. [Development.]

1927e. Geneties 12: 461-491. [Sugary gene.]

1928. Biochem. Jour. 22: 1349-1361. [Waxy starch.]

1929a. Genetics 14:569-590. [Waxy gene.]

1929b. Quar. Rev. Biol. 4: 520-543. [Waxy gene.]

Brink, R. A., and F. A. Abegg. 1926. Genetics 11:163-199. illus.

Brink, R. A., and C. R. Burnham. 1927a. Proc. Nat. Acad. Sci. 13: 238-242. 1927b. Geneties 12:348-378. [Genes and male gametophytes.]

Brink, R. A., and D. C. Cooper. 1931. Genetics 16:595-628. illus. 1932. Proc. 6 Internat. Cong. Genetics 2 : 16-17.

Brink, R. A., and J. H. MacGillivray. 1924. Amer. Jour. Bot. 11: 465-469.

Brooks, F. T. 1910. Ann. Botany 24:585-605. pls. 48, 49. [Gnomonia.]

Brooks, W. K. 1899. The Foundations of Zoölogy. New York.

Brown, E. D. W. 1919. Bull. Torr. Bot. Club 46:27-30. pl. 2. [Apogamy.]

Brown, H. B. 1913. Ann. Mycol. 11: 1-13. pls. 1, 2. [Xylaria.]

Brown, R. 1833. Trans. Linn. Soc. 16:685-745. illus. Also privately printed, 1831. [Nucleus.]

1866. Miscellaneous Botanical Works. (Ray Society.) (Observations of 1827.)

Brown, V. E. 1930. Quar. Jour. Micr. Sci. $73: 403-419$. illus. [Peranema.]

Brow, W. H. 1909. Johns Hopkins Univ. Circ. 6: 42-45. [Pyronema.]

1910a. Bot. Gazette 50:443-459. 47 figs. [Leotia.]

1910b. Bot. Gazette 49: 1S9-194. pl. 13. [Peperomia.]

1911a. Bot. Gazette 51:390-391. [Lyngbya.]

1911b. Bot. Gazette 52:275-305. pl. 9. 51 figs. [Lachnea.]

Brôzek, A. 1923. Stud. Plant Phys. Lab. Charles Univ. Prague 1: 45-79.

BRǗcke, C. 1S61. Sitzber. Akad. Wiss. Wien. II $44: 381-406$.

Brunelli, G. 1911. Mem. Acc. d. Lincei, Ser. 5a, 8. [Tryxalis ơ.] See also Ser. $33,16$.

Bruns, E. 1894. Flora 79: Ergänzungsb., 159-178. pl. 6.

Brunswik, H. 1924. Zeitschr. Ind. Abst. Vererb. 33: 257-258. [Coprinus.] 1926. Zeitschr. Bot. 18: 481-498. [Coprinus.]

Buchrolz, J. T. 1922. Bot. Gazette 73: 249-286. illus. [Developmental selection.]

Buchiolz, J. T., and A. F. Blakestee. 1922. Science 55:597-599. [Pollen tube.] 1927a. Proc. Nat. Acad. Sci. 13: 242-249.

1929. Genetics 14:538-568. illus. [Pollen-tube growth.]

1930a. Proc. Nat. Acad. Sci. 16: 190-195. [Pollen-tube growth.]

19306. Jour. Heredity $21: 119-129$. illus. [Radium.]

1930c. Bot. Gazette 90: 366-383. [Pollen-tube growth.]

1932. Aner. Jour. Bot. 19: 604-626. illus. [Pollen-tube growth.] 
Bdchner, P. 1909. Arch. Zellf. 3: 335-430. pls. 16-21. [Chromidia.]

1911. Arch. Zellf. 6: 577-612. pls. 31-34. 7 figs.

Bucholtz, A. F. 1931. Ann. Missouri Bot. Garden 18: 489-508. [Ultra-violet light.]

Buchtien, O. 1887. Bibl. Botaniea 8: 1-49. pls. 1-6. [Equisetum.]

BUdEr, J. 1911. Zeitsehr. Ind. Abst. Vererb. 5: 209-284. 21 figs.

1916a. Ber. Deu. Bot. Gesell. 34:559-576. [Alternation of generations.]

Buller, A. H. R. 1930. Nature. London, 126:686-689. illus.

1931. Researches on Fungi, 4. London.

Bunting, M., and D. H. Wenrich. 1929. Jour. Morph Physiol. 47 : 37-87. illus.

Burgeff, H. 1913. Ber. Deu. Bot. Gesell. 30:679-685. [Phycomyces.]

1914. Flora 107: 259-316. pls. 16, 17. 20 figs. [Phycomyces.]

1915. Flora 108: 353-448. 13 figs. [Phycomyces.]

Burnham, C. R. 1930. Proc. Nat. Acad. Sei. 16: 269-277. illus.

1932. Proe. Nat. Aead. Sei. 18: 434-440. illus. [Reeiprocal translocation.]

Bütschli, O. 1875ab. Zeitsehr. f. Wiss. Zool. $25: 201,426$.

1876. Abhandl. Senckenb. Naturf. Gesell. 10.

1890. Ueber den Bau der Bakterien, usw. Leipzig.

1892. Untersuchungen über mikroskopische Sehäume und das Protoplasma.

1896. Weitere Ausführungeu über den Bau der Cyanophyceen und Bakterien.

1898. Untersuchungen über Strukturen. Leipzig.

1901. Roux's Arch. Entw. 11: 499-584. pl. 20.

1902. Areh. Protist. 1: 41-58. illus. [Cyanophyceæ and bacteria.]

Buxton, B. H., and C. D. Darlington. Nature, Jan. 7, 1931. [Digitalis.]

Buxton, B. H., and W. C. F. Newton. 1928. Jour. Geneties 19:269-279. illus.

C

Cajal, S. R. 1903. Trab. Lab. Invest. Biol. Univ. Madrid 2: 129-221. 38 figs.

1914. Trab. Lab. Invest. Biol. Univ. Madrid 12: 127-227.

Caldwell, O. W. 1907. Bot. Gazette 44:118-141. pls. 10-13. [Microcycas.]

Calkins, G. N. 1897. Bull. Torr. Bot. Club 24: 101-115. pls. 295, 296.

1898. Jour. Morph. 15 : 711-770. pls. 40-42. [Mitosis in Protozoa.]

1919. Jour. Exp. Zoöl. 27 : 293-357. illus. [Uroleptus.]

1926. The Biology of the Protozoa. Philadelphia and New York.

Camerarios, R. J. De Sexu Plantarum. 1694.

Camp, W. H. 1929. Amer. Jour. Bot. 16: 221-224. [Catalase.]

Campbell, A. S. 1926. Univ. Calif. Publ. Bot. 29 : 179-236. illus.

1927. Univ. Calif. Publ. Bot. 29 : 429-452. illus. [Neuromotor apparatus.]

Camprelit, D. H. 1887. Ber. Deu. Bot. Gesell. 6:120-127. pl. 6. [Spermatozoid.] 1888. Ann. Botany 2 : 233-264. pls. 13-15. [Spermatozoid.]

Cannon, H. G. 1923. Jour. Geneties 13:47-78. 4 figs.

Canti, R. G., and F. G. Spear. 1927, 1929. Proc. Roy. Soc. London B 102 : 92-101.

illus; 105 : 93-98.

Capinpin, J. M. 1930. Amer. Naturalist 64:566-570. [Triploid Enothera.]

Carleton, H. M. 1920. Quar. Jour. Mier. Sei. 64:329-344. pl. 17.

Carlson, M. C. 1929. Ann. Botany 43:111-117. illus. [Achlya.]

Carothers, E. E. 1913. Jour. Morph. 24: 447-506.

1917. Jour. Morph. 28: 445-521. 14 pls. 5 figs. [Orthoptera.]

1921. Jour. Morph. 35: 457-483. 5 pls. [Circotettix.]

1931. Biol. Bull. 61:324-349. illus. [Disjunetion.]

Carrel, A. 1924. Physiol. Rev. 4: 1-20. [Tissue cultures.]

1931. Science $73: 297-303$. 
Carrothers, D. 1911. Ann. Botany 25:243-252. pls. 18, 19. [Helvella.] 1921. Arch. Zellf. 15: 370-376. pl. 20. [Hyacinthus: mitosis.]

Carter, G. S. 1926. Brit. Jour. Exper. Biol. 4: 1-26. [Cilia.]

Carter, N. 1919ab. Ann. Botany 33:215-254. pls. 14-18; 295-304. pls. 19, 20. 1919c. Ann. Botany 33: 467-478. pl. 27. [Cladophora.]

1920ab. Ann. Botany 34: 265-285; 303-320. pls. 10-16.

1926. Ann. Botany $40: 665-689$. illus. [Ulva.]

1927. Ann. Botany 41:139-159. illus. [Padina.]

Carus, C. 1824. Von den äusseren Lebensbedingungen der Thiere, usw. Leipzig.

Cassaigne, Y. 1931. Rev. Gén. Bot. 43: 140-167. illus. [Vacuome.]

Castetter, E. F. 1925. Amer. Jour. Bot. 12: 270-286. pls. 15-17.

Castle, IV. E. 1923. Amer. Naturalist 57: 559-561. 1924. Genetics and Eugenics. 3d ed. Cambridge, Mass.

Catcheside, D. G. 1930. Trans. Roy. Soc. Edinb. 56: 467-484. illus.

1931a. Jour. Genetics $24: 145-163$. illus. [Triploid Enothera.]

1931b. Proc. Roy. Soc. London B 109: 165-184. illus. [Parasynapsis.]

1932. Cytologia 4:68-113. ilhs. [Monoploid Enothera.]

1933a. Genetica 15:177-201. illus. [Trisomic Enothera.]

1933b. Jour. Geneties 27 : 45-69. illus. [Enothera hybrids.]

Cattaneo, D. 1914. Arch. Ital. d'Anat. Embriol. 12.

Cavers, F. 1914. New Phytol. 13: 96-106. [Chondriosomes: review.]

Chalaud, G. 1929-1930. Rev. Gén. Bot. 41, 42. [Fossombronia.]

Chamberlain, C. J. 1898. Bot. Gazette 26:431-435. [Blepharoplast.]

1899. Bot. Gazette 27 : 268-280. pls. 4-6. [Pinus.]

1906. Bot. Gazette $42: 321-358$. pls. 13, 14. [Diön.]

1909. Spermatogenesis in Dioön edule. Bot. Gaz. 47:215-236. pls. 15-18.

1910. Bot. Gazette 50:415-429. pls. 14-17. [Dioön: fertilization, embryo.]

1912. Bot. Gazette 53 : 1-19. pl. 1. 7 figs. [Ceratozamia.]

1916. Bot. Gazette 61:353-372. pls. 24-26. [Stangeria.]

Chanbers, R. 1913. Biol. Bull. $25: 134-140 . \quad$ [Simocephalus spermatogenesis.]

1914. Science $40: 824-827$. [Physical properties of nucleus.]

1915. Science $41: 290-293$. [Microdissection of germ cells.]

1917a. Amer. Jour. Physiol. 43: 1-12. 2 figs. [Protoplasmic structure.]

1917b. Jour. Exp. Zoöl. 23 : 483-504. [Aster.]

1918. Biol. Bull. 34: 121-136. [Microvivisection method.]

1919. Jour. Gen. Physiol. 2 : 49-68. [Viscosity and division.]

1921a. Science 54:411-413. 2 figs. [Apparatus.]

1921b. Jour. Gen. Physiol. 4: 33-39. See also 41-44. [Aster.]

1921c. Biol. Bull. 41:318-350. [Structure of echinoderm egg.]

1922a. Anat. Rec. 24: 1-19. 5 figs. [Apparatus.]

1922b. Jour. Gen. Physiol. 5:189-193. [Permeability.]

1922c. Proc. Soc. Exp. Biol. Med. 20:137-138. [Fertilization.]

1923a. Proc. Soc. Exp. Biol. Med. 20:367-368. [Death changes.]

1923b. Jour. Gen. Physiol. 5: 821-829. 7 figs. [Fertilization.]

1924. Chapter $\mathrm{V}$ in General Cytology, ed. by Cowdry.

1925. La Cellule 35: 107-124. 2 pls. [Dissection of spermatocyte.]

1928. Biol. Bull. 55: 369-376. [pH of protoplasm.]

1930a. Science 71: 340. [Egg membrane.]

1930c. Biol. Bull. 58 : 344-369. illus. [Insemination.]

1930d. [In] The Laws of Life. Prag. 1930.

1932. Jour. Cellul. Compar. Physiol. 1: 65-70.

Chambers, R., and G. Cameron. 1932. Jour. Cellul. Compar. Physiol. 2: 99-103.

Chambers, R., and J. A. Dawson. 1925. Biol. Bull. 48: 240-242. illus. 
Chambers, R., and K. Höfler. 1931. Protoplasma 12: 338-355. illus.

Chambers, R., and T. Kerr. 1932. Jour. Cellul. Compar. Physiol. 2 : 105-119.

Chambers, R., and H. Pollack. 1927. Jour. Gen. Physiol. 10: 739-755. illus.

Chambers, R., H. Pollack, and S. Hiller. 1927. Proc. Soc. Exp. Med. 24: 760-

761. [Protoplasmic $p \mathrm{H}$.]

Chambers, R., H. Pollack, and B. Cohen. 1929. Brit. Jour. Exp. Biol. 6: 229

247. [Reduction potentials.]

Chambers, R., and G. S. Rényi. 1925. Amer. Jour. Anat. 35: 385-402.

Chambers, R., and H. C. Sands. 1923. Jour. Gen. Physiol. 5: 815-819. 1 pl.

Champy, C., and H. M. Carleton. 1921. Quar. Jour. Mier. Sei. 65: 589-610.

Chatton, É. 1927. Compt. Rend. Acad. Sci. Paris 185: 553-555. [Paradinium.]

Chevalier, M. 1927. Elementos de Citologia y de Embriologia Fisiologica del

Hombre y de los Vertebrados. 1. Citologia Animal. Barcelona.

Chinrugi, A. 1926. Atti R. Accad. Naz. Lincei, Rend. Cl. Fis. Mat. e Nat. 3 :

281-284. [Apospory and apogamy in Artemisia.]

1927. Nuov. Giorn. Bot. Ital. 34: 5-133. illus. [Embryo sac.]

Chiarugi, A., and E. Francini. 1930. Niov. Giorn. Bot. Ital. 37: 1-250. illus.

Chickering, A. M. 1927. Jour. Morph. Physiol. 44: 541-607. illus.

Child, C. M. 1911a. Biol. Bull. 21: 280-296. 16 figs.

1911b. Jour. Exp. Zoöl. 10: 265-320. 7 figs. [Axial gradient.]

1912. Jour. Exp. Zoöl. 13 : 103-152. 46 figs. [Axial gradient.]

1913. Arch. Entw. 37: 108-158. 13 figs. [Axial gradient.]

1915. Senescence and Rejuvenescence. Chicago.

1916. Bot. Gazette 62: 89-114. [Axial gradients in algæ.]

1921a. Jour. Exp. Zoöl. 33: 409-433. 33 figs. [Morphogenesis.]

1921b. Amer. Jour. Bot. 8: 286-295. [Axial gradients.]

1923. Genetics 8: 336-354. 14 figs. [Symmetry and polarity.]

Chipman, R. H., and T. H. Goodspeed. 1927. Univ. Calif. Publ. Bot. 11: 141-

158. illus. [Haploid Nicotiana.]

Chittenden, R. J. 1927a. Bibliog. Genet. 3: 355-439. [Veg. Segregation.] 1927b. Jour. Heredity 18: 337-343. illus. [Flax.]

Chittenden, R. J., and C. Pellew. 1927. Nature 119: 10-11.

Chodat, R. 1924. Compt. Rend. Soc. Phys. et Hist. Nat. Genève 41: 96-99. 1925. Bull. Soc. Bot. Genève, 17 : 1-30. 17 figs. [Meiosis.]

Cholnoky, B. von. 1927. Areh. Protist. 60:8-33. illus. [Rhoicosphenia.] 1928. Arch. Protist. 63:23-57. illus. [Anomøoneis.]

1929. Areh. Protist. 68: 471-502. illus. [Auxospores.]

Christian, A. H. 1905. Bot. Gazette 39: 267-274. pl. 8. [Uredinea.] 1907. Bot. Gazette 44: 81-101. pl. 7. [Uredinew.]

Cienkowski, L. 1863. Jahrb. Wiss. Bot. 3:325-337. [Protoplasm.]

Clark, G. L., L. W. Pickett, and W. K. FArr. 1930. Science 71: 293-294.

Clausen, J. 1922. Bot. Tidskr. (Dansk.) 37:363-416. 9 figs. [Viola.]

1924. Hereditas 5 : 29-32. 5 figs. [Viola.]

1926. Hereditas 8: 1-156. illus. [Viola.]

1927a. Ann. Botany 41:677-714. illus. [Viola.]

1927b. Hereditas $9: 245-256$. illus. [Plastid segregation.]

1929a. Ann. Botany $43: 741-764$. illus. [Viola.]

1930a. Hereditas 13:342-356. illus. [Variegation.]

1930b. Hereditas 14: 53-72. illus. [Male sterility.]

1931c. Hereditas 15: 219-308. illus. [Viola.]

Clausen, R. E. 1932. Svensk Bot. Tidskr. 26: 123-136. [Nicotiana hybrid.]

Clauden, R. E., and T. H. Goodspeed. 1924. Genetics 9: 181-197.

1925. Geneties 10:278-284. illus. [Amphidiploid Nicotiana.]

1926ab. Univ. Calif. Publ. Bot. 11 : 61-82, illus.; 83-101. 
Cla usen, R. E., and M. C. Mann. 1924. Proc. Nat. Acad. Sci. 10: 121-124.

Claussen, P. 1905. Bot. Zeitung 68:1-27. [Boudiera.]

1907. Ber. Deu. Bot. Gesell. $25: 586-590$. [Pyronema.]

1908. Ber. Deu. Bot. Gesell. 26: 144-161. [Saprolegnia.]

1912. Zeitschr. Bot. 4:1-64. pls. 1-6. [Pyronema.]

Cleland, R. E. 1919. Ann. Botany 33:323-352. pls. 22-24. [Nemalion.]

1922. Amer. Jour. Bot. 9: 391-413. pls. 25-27. [Enothera.]

1923. Amer. Naturalist 57:562-566. 14 figs. [Enothera.]

1924. Bot. Gazette $77:$ 149-170. pls. 14, 15. [Enothera.]

1925. Amer. Naturalist 59: 475-479. 8 figs. [Enothera.]

1926a. Genetics 11: 127-162. illus. [Meiosis in Enothera.]

1926b. Bot. Gazette 82 : 55-70. illus. [Meiosis in Enothera.]

1929a. Tübinger Naturwiss. Abh. 12: 50-55. illus.

1929b. Proc. Internat. Cong. Plant Sci., Ithaca $1:$ 317-331.

1929c. Zeitschr. Ind. Abst. Vererb. 51: 126-145. illus.

1931a. Amer. Jour. Bot. 18: 629-640. illus.

1931b. Proc. Nat. Acad. Sci. 17 : 437-440.

1932. Genetics $17: 572-602$.

1933. Amer. Naturalist $67: 407-418$.

Cleland, R. E., and A. F. Blakeslee. 1930. Proc. Nat. Acad. Sci. 16: 183-189. 1931. Cytologia 2: 175-233. [Reciprocal translocation.]

Cleland, R. E., and F. Oenlkers. 1929. Amer. Naturalist 63 : 497-510.

1930. Jahrb. Wiss. Bot. 73: 1-124. illus.

Clowes, G. H. A. 1916ab. Jour. Phys. Chem. $20: 407-451$. Science 43: 750-757.

Сoвв, F., and H. H. Bartlett. 1919. Jour. Wash. Acad. Sci. 9: 462-483.

Conn, F. 1850. Nova Acta Acad. Cres. Leop. Carol. Nat. Cur. Bonn 22: 605-764.

Coker, W. C. 1903. Bot. Gazette 36:1-27, 114-140. pls. 1-11. [Taxodium.]

1907. Bot. Gazette $43: 1-10$. pl. 1. 5 figs. [Cephalotaxus.]

Cole, F. J. 1930. Early Theories of Sexual Generation. Oxford University Press.

Colley, R. H. 1918. Jour. Agr. Res. 15: 619-659. illus. [Cronartium.]

Collins, G. N., and J. H. Kempton. 1916. Jour. Heredity $7:$ 106-118. illus.

Collins, J. L., and M. C. Mann. 1923. Genetics 8:212-232. 9 figs.

Conard, A. 1926a. Bull. Acad. Roy. Belg., Cl. d. Sci., 1925, 731-739.

1928. Mém. Acad. Roy. Belg., Cl. d. Sci., IX, 1-66. illus.

1931h. Compt. Rend. Soc. Biol. Paris 108: 1172-1174.

Conklin, E. G. 1897. Jour. Morph. 13:1-226. pls. 1-9. [Embryo of Crepidula.]

1901a. Biol. Bull. 2: 257-265. [Cleavage nuclei in Crepidula.]

1912a. Jour. Acad. Nat. Sci. Phila. 15: 503-591. pls. 43-49.

1912b. Jour. Exp. Zoöl. 12: 1-98. 37 figs. [Cell and nuclear size.]

1912c. Jour. Morph. 23: 159-188. 12 figs. [Cell and body size.]

1915. Heredity and Environment. Princeton.

1917. Biol. Bull. 33 : 396-436. 10 pls. [Amitosis.]

1919-1920. Sci. Monthly 9, 10. [Mechanism of evolution.]

1924. Chapter IX in General Cytology, ed. by Cowdry.

1931. Jour. Exper. Zoöl. 60: 1-119. illus.

Conn, H. J. 1928a. Stain Tech. 3:1-11. [History of staining.]

1928b. Stain Tech. 3:110-121. [History of staining.]

1929. Stain Tech. $4: 37-48$. [History of staining.]

1930ab. Stain Tech. 5: 3-12; 39-48. [History of staining.]

1933. Stain Tech. 8:4-8. [History of staining.]

Conn, H. J., and R. S. Cunningham. 1932. Stain Tech. $7:$ 81-90.

Conn, H. J., and S. I. Kornhauser. 1928. Stain Tech. 3: 110-121.

Conn, H. J. et al. 1929. Biological Stains. Geneva, N. Y. 
Соoк, W. R. I. 1926. Trans. Brit. Mycol. Soc. 11: 196-213. illus.

Cooper, D. C. 1929. Proc. Nat. Acad. Sei. 15 : 885-887. illus.

Cooper, D. C., and R. A. Brink. 1931. Proc. Nat. Acad. Sci. 17 : 334-338. illus.

Cooper, G. O. 1929a. Trans. Wis. Aead. Sei. $24: 303-308$. illus. [Achlya.]

1929b. Trans. Wis. Acad. Sci. $24: 309-322$. illus. [Brevilegnia.]

Corner, G. W. 1932. Special Cytology (Cowdry, ed.). See. XXXIX.

Correns. 1889. Flora, 1889, 298-347. illus. [Cell membrane.]

1892. Jahrb. Wiss. Bot. 23: 254-338. illus. [Cell membrane.]

1893. Ber. Deu. Bot. Gesell. 11: 410-425. illus. [Cell membrane.]

1900a. Ber. Deu. Bot. Gesell. 18: 158-168.

1900b. Bot. Zeitung 58: II. 229-235.

1902. Bot. Zeitung 60: II. 65-82.

1906. Ber. Deu. Bot. Gesell. 24 : 459-474. [Sex.]

1907a. Die Bestimmung und Vererbung des Geschlechts, usw. Berlin.

1907b. Jahrb. Wiss. Bot. $44: 124-176.4$ figs. [Sex.]

1908. Ber. Deu. Bot. Gesell. 26a : 686-701. [Sex.]

1909a. Zeitschr. Ind. Abst. Vererb. 1: 291-329. 2 figs. [Chlorophyll types.]

1918a. Naturwissenschaften 6:277-280. [Melandrium.]

1918b. Sitzber. Preuss. Akad. Wiss. Berlin 1175-1200. 3 figs.

1919a. Sitzber. Preuss. Akad. Wiss. Berlin 585-610. [Capsella.]

1919b. Sitzber. Preuss. Akad. Wiss. Berlin 820-857. [Arabis, ete.]

1920b. Natur u. Technik $2: 65-71.2$ figs. [Sex.]

1920c. Zeitschr. Bot. $12: 49-60.2$ figs. [Funaria.]

1921. Hereditas $2: 1-24.5$ figs. [Melandrium.]

1922a. Sitzber. Preuss. Akad. Wiss. Berlin 460-486. [Stellaria, Urtica, ete.]

1922b. Biol. Zentralbl. 42: 465-480. [Rumex.]

1926a. Zeitschr. Ind. Abst. Vererb. 41:5-40. [Sex.]

1928a. Bestimmung, Vererbung und Verteilung des Geschlechtes bei den höheren

Pflanzen. Berlin.

1928b. Zeitschr. Ind. Abst. Vererb. Suppl. Bd. I: 131-168.

1931. Sitzungsber. Preuss. Akad. Wiss., Phys.-Math- Kl., 203-231. illus.

Corti, B. 1772. Observationi misc. sulla Tremella, etc. Lucca, 1774.

Couch, J. N. Ann. Botany 40:849-881. illus. [Dictyuchus.]

1931. Jour. Elisha Mitehell Sci. Soc. $46: 231-239$. illus.

1932a. Amer. Jour. Bot. 19: 584-599. illus. [Leptolegnia.]

1932b. Bot. Gazette 94:272-296. illus. [Vaucheria.]

Cotlter, J. M., and C. J. Chamiberlain. 1903. Morphology of Angiosperms.

1910. Morphology of Gymnosperms. Chieago.

Coulter, J. M., and W. J. G. Land. 1905. Bot. Gazette $39: 161-178$. pls. A, 1-3.

Coulter, M. C. 1923. Outline of Geneties. Chicago.

Cowdry, E. V. 1912. Internat. Monatschr. Anat. Physiol. 29 : 473-504.

1914a. Internat. Monatsehr. Anat. Physiol. 31: 267-286.

1914b. Amer. Jour. Anat. 17: 1-29. 3 pls. [Ganglion cells.]

1914c. Anat. Rec. 8 : 102-103. [Chondriosomes, mitosis, and amitosis.]

1914d. Amer. Jour. Anat. 15: 389-429. 5 pls. [Neurofibrils.]

1916a. Amer. Jour. Anat. 19: 423-446. [Chondriosomes; review.]

1916b. Carnegie Inst. Wash. Publ. 224: 27-43. 1 pl.

1918. Carnegie Inst. Wash. Publ. 271: 39-160. 1 pl. [Chondriosomes; review.]

1921a. Jour. Exp. Med. 33: 1-11. pl. 1. [Blood cells.]

1921b. Anat. Rec. $22: 239-250$. [Nomenclature.]

1922. Amer. Jour. Anat. 30: 25-37. 2 pls. ['Thyroid cells.]

1923a. Amer. Jour. Anat. 31: 339-343. 1 pl. [Root nodules.]

1923b. Seience 58: 1-7. [Golgi material.] 
1923c. Jour. Exp. Med. 37 : 431-456. [Rickettsia.]

1924a. Chapter VI in General Cytology, ed. by E. V. Cowdry.

1924b. Amer. Naturalist 58: 97-114. [Chondriosomes and pathology.]

1926b. Amer. Naturalist 60: 157-165. [Mitochondria.]

1928. Special Cytology, Section XXVII.

1932. (Editor) Special Cytology. New York (Hoeber). 1st ed., 1928.

Cowdry, E. V., and P. K. Olitsky. 1922. Jour. Exp. Med. 36: 521-533.

Cowdry, N. H. 1917. Biol. Bull. 33 : 196-228. 26 figs. [Chondriosomes.]

1918. Biol. Bull. 35: 71-94. 1 pl. [Chondriosomes in myxomycetes.]

1920. Biol. Bull. 39 : 18S-206. 3 pls. [Experimental studies on chondriosomes.]

Cox, E. K. 1926. Jour. Morphol. Physiol. 43: 45-54. illus. [Mouse.]

CrabB, E. D. 1927b. Biol. Bull. 53:67-108. illus. [Lymnaa.]

Craigie, J. H. 1927a. Nature (London) 120:116-117. [Sex in rusts.]

1927b. Nature (London) 120: 765-767. illus. [Pycnia.]

1928. Phytopath. 18: 1005-1015. illus. [Pyenia and cecia.]

1931. Phytopath. 21: 1001-1040. illus. [Sex in rusts.]

Creighton, H., and B. McClintock. 1931. Proc. Nat. Acad. Sci. 17: 492-497. 1932. Proc. 6 Internat. Cong. Genet. $2: 392$.

Crew, F. A. E. 1921. Jour. Genetics 11: 141-181. 23 figs. [Sex in frogs.]

1923. Jour. Heredity 14:361-362. 5 figs. [Sex-reversal in fowl.]

1926. Quar. Rev. Biol. 1: 315-359. [Abnormal sexuality.]

1927a. Quar. Rev. Biol. 2 : 249-266. illus. [Abnormal sexuality.]

1927b. Quar. Rev. Biol. 2: 427-441. [Sex reversal.]

1927c. The Genetics of Sexuality in Animals. New York.

Cú́not, L. 1899. Bull. Sci. France et Belg. 32. [Sex determination.]

Cunha, A. G. DA. 1929. Arch. Portug. Sci. Biol. 2 : 242-285. illus.

CzaJA, A. T. 1921. Zeitschr. Bot. $13: 545-589$. [Sex in ferns.]

Czapeк, F. 1910a. Ber. Deu. Bot. Gesell. 28: 147-169. [Plasma membrane.] 1910b. Ber. Deu. Bot. Gesell. 28: 480-487. [Plasma nembrane.]

1911b. Chemical Phenomena of Life. New York.

1913, 1920. Biochemie der Pflanzen. 2d ed., Jena.

Czurda, V. 1922ab. Arch. Protist. 44:346-374. pls. 14, 15. 45: 163-199.

1928. Beih. Bot. Centralbl. 45 : I 97-270. illus. [Starch.]

1929. Ber. Deu. Bot. Gesell. 47 : 181-185. [Pyrenoid.]

Dahlgren, K. V. O. 1915. Arkiv. Bot. 14:1-10. 5 figs. [Plumbagella.]

1916. Kgl. Svensk Vet. Akad. Handl. 56 : $\$ 0$ pp. 3 pls. 137 figs.

1922. Svensk Bot. Tidskr: 16: 77-87. [Spigelia.]

1923. Botaniska Notiser 1923, 1-24. 5 figs. [Endosperm.]

1927a. Hereditas 10:169-229. [Syngamy in angiosperms.]

1929. Bot. Notiser, 341-353. [Catalase.]

Dale, E. 1909. Ann. Mycol. $7: 215-225 . \quad$ pls. 2, 3. [Aspergillus.]

Danciп коFF, V. 1916. Jour. Morphol. $27: 559-603.5$ pls. [Nuclcar extrusion.]

Dangeard, P. 1920. Compt. Rend. Acad. Sci. Paris 170:474-477. 8 figs.

1921abc. Compt. Rend. Acad. Sci. Paris 172: 857-859, 995-998, 1401-1403.

1922a. Compt. Rend. Acad. Sci. Paris 174: 319-321. [This and 1921abc on aleurone.]

1922b. Bull. Soc. Bot. France 69: 112-118. 3 figs. [Anthocyanin.]

1923a. Recherche sur l'Appareil Vacuolaire, etc. Thesis, Paris.

1923b. Compt. Rend. Acad. Sci. Paris 177: 67-69. [Oil.] 
1924. Arch. d'Anat. Micr. 20 : 461-464. 6 figs. [Ricinus.]

1927c. Le Botaniste 18: 245-264. illus. [Vacuoles.]

Dangeard, P. A. 1S92. Le Botaniste 3:28-31. pl. 2. [Cyanophyceæ.]

1893. Le Botaniste $3: 240-281$. [Sex in fungi.]

1894a. Le Botaniste 4: 119-181. 24 figs. [Basidiomycetes.]

1894b. Le Botaniste 4:21-58. 10 figs. [Ascomycetes.]

1895. Le Botaniste 5 : 245-284. 17 figs. [Ascomycetes.]

1906. Compt. Rend. Aead. Sci. Paris 142 : 645-646. [Mueorinex.]

1916. Bull. Soc. Bot. France $63:$ 179-187. [Vacuoles.]

1918. Compt. Rend. Aead. Sci. Paris 166: 439. [Chondriome.]

1919. Compt. Rend. Acad. Sci. Paris 169: 1005-1010.

1920ab. Compt. Rend. Acad. Sci. Paris 170: 301-306; 709-714.

1920cd. Compt. Rend. Acad. Sci. Paris 171: 69-74; 1016-1019.

1925. La Cellule $35: 239-250.1$ pl.

1929. Le Botaniste, Sér. XXI, 281-344. illus. [Vacuome.]

1931. Le Botaniste, $22: 325-389$. illus. [Cell elements in fungi.]

Dannehl, H., and H. Ziegenspeck. 1929. Bot. Areh. 25 : 243-251.

Darbishire, O. V. 1896. Wissenschaftliche Meersuntersuchungen, N. F., 1.

Dark, S. O. S. 1931. Jour. Genetics 25: 91-95. illus. [Triploid Primula.]

Darlington, C. D. 1926. Jour. Genetics 16:237-251. illus. [Scillece.]

1927b. Nature 119: 390-391. [Prunus.]

1928. Jour. Genetics 19: 213-256. illus. [Prunus.]

1929a. Genetics $20: 345-363$. illus. [Enothera, Tradescantia.]

1929b. Jour. Genetics 21: 207-286. illus. [Tradescantia.]

1929c. Jour. Genetics 21:21-56. illus. [Aneuploid Hyacinthus.]

1929f. Nature (London), July 13 and 20. [Polyploidy.]

1930c. Proc. Roy. Soc. London B 107: 50-59. illus. [Crossing-over.]

1930d. Cytologia 2:37-55. illus. [Fritillaria.]

1931a. Jour. Genetics 24: 65-96. illus. [Primula, meiosis.]

1931d. Biol. Rev. 6: 221-264. illus. [Meiosis.]

1931e. Jour. Genetics 24: 405-474. illus. [Enothera.]

1932a. Recent Advances in Cytology. New York.

Darlington, C. D., and A. A. Moffett. 1930. Jour. Genetics 22: 129-151. illus.

Darwin, C. 1868. Animals and Plants under Domestication.

Davenport, C. B. 1930. Geneties 15: 401-444. illus. [Sex-linkage in man.]

Davey, A. J., and C. M. Gibson. 1917. New Phytol. 16: 147-151.

Davis, B. M. 1896. Ann. Botany 10:49-76. pls. 6, 7. [Batrachospermum.]

1898. Ber. Deu. Bot. Gesell. 16: 266-272. pls. 16, 17. [Corallina.]

1899. Bot. Gazette 28: 89-109. pls. 9, 10. [Anthoceros.]

1900. Bot. Gazette $29: 297-311$. pl. 22. [Albugo.]

1901. Ann. Botany 15: 147-180. pls. 10, 11. [Pellia.]

1903. Bot. Gazette 35: 233-249, 320-349. pls. 9, 10. [Saprolegnia.]

1904. Bot. Gazette 38:81-98. illus. [Vaucheria.]

1905a. Amer. Naturalist 38, 39. [Review of plant cytology.]

1905b. Bot. Gazette 39:61-64. [Saprolegniales.]

1908. Ann. Botany 22:1-20. pls. 1, 2. [Derbesia.]

1909. Ann. Botany $23: 551-571$. pls. 41, 42. [Enothera.]

1910. Ann. Botany 24:631-651. pls. 52, 53. [Enothera.]

1911. Ann. Botany 25: 941-974. pls. 71-73. [Enothera.]

1916. Amer. Naturalist 50: 502-512. [Red algæ.]

1923. Amer. Jour. Bot. 10:462-467. [Sterility in hybrids.]

Davis, B. M., and C. G. Kúlkarni. 1930. Geneties 15: 55-79. [Monoploid Enothera.] 
DAY, H. C. 1927. Jour. Morphol. Physiol. 44:363-372. [Contractile vacuole.] 1930. Physiol. Zool. $3: 56-71$. illus.

Dehorne, A. 1911. Arch. Zellf. 6: 613-639. pls. 35, 36. 2 figs.

DeinekA, D. 1912. Anat. Anz. 41: 289-309. [Golgi material in cell-division.]

1914. Anat. Anz. 46:97-126. 16 figs. [Golgi material; chondriosomes.]

Delage, Y. 1903. Structure du Protoplasma et les Théories sur l'Hérédité, etc. Paris.

Delage, Y., and M. Goldsuith. 1913. The Theories of Evolution. New York.

Delaunay, L. N. 1925. Science 62:15-16. 1 fig. [Ornithogalum chromosomes.] 1926a. Zeitschr. Zellf. Mikr. Anat. 4: 338-364. illus.

1929. Planta 6: 100-112. illus. [Chromosome morphology.]

1930a. Wissensch.-Selek.-Inst., Kiew 6. [X-rays.]

1930b. Zeitschr. Ind. Abst. Vererb. 55: 352-355. illus. [X-rays.]

Delitsch, H. 1926. Zur Entwicklung der coprophilen Ascomyceten, usw. Leipzig.

Dellinger, O. P. 1909. Jour. Morphol. $20: 171-209.4$ pls. 12 figs. [Cilium.]

Dembowski, J., and H. Ziegenspeck. 1928. Bot. Arch. 22. [Nucleoli.] 1929. Bot. Arch. $24: 492-504$.

Demerec, M. 1923. Genetics 8: 561-593. [White maize seedlings.] 1924. Amer. Jour. Bot. 11: 461-464. 1 fig. [Maize pollen.]

1927. Bot. Gazette 84: 139-154.

1929d. Proc. Nat. Acad. Sci. 15: 870-876. [Mutation.]

Denuam, H. J. 1924ab. Ann. Botany 38:407-432; 433-438. [Cotton: meiosis.]

Des Cilleuls, J. 1914. Arch. d'Anat. Micr. 16: 132-148. pls. 7, 8.

Dessiatoff, N. 1911. Ber. Deu. Bot. Gesell. 29:33-39. 17 figs.

Devisé, R. 1914. Compt. Rend. Acad. Sci. Paris 158: 1028-1030.

1922. La Cellule $32: 249-309.4 \mathrm{pls}$. [Achromatic figure.]

Dietel, P. 1911. Centralbl. Bakt. II 31: 95-106. [Uredineæ.]

DigBy, L. 1910. Ann. Botany $24: 727-757$. pls. 59-63. [Gallonia; mitosis.]

1912. Ann. Botany $26: 357-388.4$ pls. [Primula.]

1914. Arch. Zellf. 12: 97-146. pls. 8-10. [Crepis.]

1919. Ann. Botany 33: 135- 172. pls. 8-12. [Osmunda.]

Dippel, L. 1879. Abh. Senckenb. Gesell. 11: 154.

Disney, A. N. 1928. Origin and Development of the Microscope. London.

Dixon, H. H. 1895. Proc. Roy. Irish Acad. III 3: 707-720. [Meiosis.]

Dobell, C. 1911a. Arch. Protist. $23: 269-310$.

1911b. Quar. Jour. Micr. Sci. 56: 395-506. pls. 16-19. [Bacteria.]

1914. Arch. Protist. 34: 137-189. illus. [Amœba.]

Dobell, C., and A. P. Jameson. 1915. Proc. Roy. Soc. London B 89: 83-94.

Dobzhansky, T. 1929a. Biol. Zentralbl. 49: 408-419. illus. [Translocation.]

1929b. Proc. Nat. Acad. Sci. 15: 633-638. [Translocation.]

1929d. Arch. Entw. Org. 115: 363-379. illus. [Cell size.]

1930a. Genetics 15:349-399. illus. [Translocation.]

1930d. Biol. Zentralbl. 50:671-655. illus. [Translocation.]

1931a. Amer. Naturalist 65: 214-232. illus. [Crossover frequeney.]

1931b. Genetics 16:629-658. illus. [Translocation.]

1932a. Biol. Zentralbl. 52 : 493-509. illus. [X-chromosome map.]

1933. Zeitschr. Ind. Abst. Vererb. 44: 269-309.

Dobzhansky, T., and J. Schultz. 1931. Proc. Nat. Acad. Sci. 17: 513-578.

DODEl, A. 1892. Flora 75: 267-280. pls. 5, 6. [Starch grain.]

Donge, B. O. 1914. Bull. Torr. Bot. Club 41: 157-202. 13 figs.

1927. Jour. Agr. Res. 35: 289-305. illus. [Neurospora.]

1928a. Mycologia 20:18-21. [Neurospora.]

19286. Jour. Agr. Res. 36:1-14. illus. [Neurospora.] 
1929a. Proc. Internat. Cong. Plant Sci., Ithaca, 2: 1751-1766.

1929b. Science 70:222. [Segregations in Neurospora.]

Doflein, F. 1928. Lehrbuch der Protozoenkunde. Jena.

1918. Zool. Jahrb., Anat. Abt. 41: 1-112. 9 pls. 32 figs. [Polytomella.]

Dogiel, V. 1923a. Biol. Zentralbl. 43:289-291. [Cellulose in infusoria.]

Dolley, D. H. 1913. Arch. Zellf. 9: 485-551. pls. 24-26. 5 figs.

1925. Amer. Jour. Anat. $35: 153-195.1$ pl. 3 figs. [Nucleoplasmic ratio.]

Doncaster, L. 1907a. Anat. Anz. 31 : 168-169. [A pis ơ'-genesis.]

1907b. Quar. Jour. Micr. Sci. 51: 101-114. pl. 8. [Nematus.]

1910-1911. Proc. Roy. Soc. London B 82: 88-113. pls. 1-3. 83: 476-489.

1914a. Quar. Jour. Micr. Sci. 59: 487-522. 4 figs.

1914b. The Determination of Sex. Cambridge, Eng.

1916. Proc. Roy. Soc. London B $89:$ 183-200. pls. 6, 7. [Neuroterus.]

1920a. An Introduction to the Study of Cytology. London.

1920b. Quar. Jour. Mier. Sei. 64:303-328. illus. [Pediculus.]

Dorsey, E. 1924. Cornell Univ., Thesis [Triticum; Avena.]

Douвт, D. G. 1928. Bot. Gazette 86: 330-344. illus. [Halidrys.]

Douin, C. 1909. Rev. Bryologique 36:37-41. 10 figs. [Sphærocarpos.]

Dowding, E. S. 1931. Ann. Botany 45: 1-14. illus. [Pleurage.]

Downing, E. R. 1909. Zool. Jahrb., Anat. Abt. 28: 295-324. pls. 11, 12.

Dracinschi, M. 1930. Ber. Deu. Bot. Gesell. 48: 295-311. illus.

1931. Bul. Facul. Stiinte Cernäuti 5: 84-95. [Spermatozoid.]

1932. Bul. Facul. Stiinte Cernäuti 6:63-134. illus. [Spermatozoid.]

Drayton, F. L. 1932. Cornell Univ. Thesis. [Sclerotinia.]

Drew, A. H. 1920. Jour. Roy. Micr. Soc. April, 1920: 295-297. 4 figs. 1923. The Lancet, 785-787, 833-835. [Tumor cultures.]

DrüNer, L. 1894. Jenaische Zeitschr. 28:469-474. [Mechanism of mitosis.] 1895. Jenaische Zeitschr. 29: 271-344. pls. 4-8. [Mechanism of mitosis.]

Dublin, L. I. 1905. Ann. N. Y. Acad. Sci. 16:1-64. pls. 1-3. [Meiosis.]

Dubredil, G. 1913. Arch. d'Anat. Micr. 15: 53-151. [Chondriosomes.]

Duesberg, J. 1906. Anat. Anz. 28: 475-479. 3 figs. [Human chromosomes.]

1909a. Arch. Zellf. 4:602-671. pls. 28-30. 10 figs. [Myofibrils.]

1909b. Verh. Anat. Gesell. 24: 123-126.

1911. Ergeb. Anat. Entw. 20: II 567-916. [Review.]

1914a. Anat. Anz. 46: Ergänzungsheft, 11-80. [Golgi material.]

1914b. Verh. Anat. Gesell. Innsbruck, 11-80. [Golgi material.]

1919. Biol. Bull. 36: 71-81. [Chondriosome problem.]

Dufrenoy, J. 1927. Rev. Path. Vég. Entom. Agr. 14: 54-61. illus.

1928b. Ann. Epiphyt. 14: 227-268. illus. [Vacuoles.]

1928c. Ann. Epiphyt. 14: 199-210. illus. [Sugar-cane mosaic.]

1929b. Boll. R. Staz. Patol. Veg. Roma 9. [Wheat mosaic.]

1929c. Publ. Cinquantenaire de l'Inst. Nat. Agron. Paris.

1930c. Ann. Inst. Nat. Agr. II $23: 1-104$. illus.

1931a. Compt. Rend. Soc. Biol. Paris 107: 868-870. illus.

1931c. Rev. Pathol. Vég. Entom. Agr. 18: 74-80. illus.

1931e. 2 Cong. Internat. Pathol. Comp. 309-319. illus.

Dufrenor, J. et al. 1929. Rev. Pathol. Vég. Entom. Agr.

Dujardin, F. 1835. Ann. Sci. Nat. Zool. II $4:$ 364-377.

Dupler, A. W. 1917. Bot. Gazette 64:115-136. pls. 11-14. [Taxus.]

Dutrochet, R. J. H. 1824. Recherches Anatomiques et Physiologiques sur la

Structure Intime des Animaux et des Végétaux et sur leur Motilité. Paris.

1826. L'Agent Immédiat du Mouvement Vital, Dévoilé dans sa Nature et dans

sa Mode d'Action chez les Végétaux et chez les Animaux. Paris.

1837. Mémoires pour Servir a l'Histoire Anatomique, etc. 
$\mathrm{E}$

EAmes, A. J. 1913. Ann. Botany 27: 1-38. pls. 1-4. 92 figs. [Agathis.]

Eanes, A. J., and L. H. MacDaniels. 1925. An Introduction to Plant Anatomy. New York.

East, E. M. 1929. Bibliog. Genetica 5 : 331-370. [Self-sterility.]

1930. Science 72: 148-149. [Fragaria.]

1932. Geneties 17: 175-202. [Nicoliana hybrids.]

1933. Amer. Jour. Bot. 20: 269-289. illus. [Triploid Nicotiana.]

East, E. M., and H. K. Hayes. 1915. Conn. Agr. Exp. Sta. Bull. 188. [Inheritance in maize.]

Eberdt, O. 1891. Jahrb. Wiss. Bot. 22: 293-348. pls. 11, 12.

Eduan, G. 1929. Acta Horti Bergiani 9. [Oxyria.]

Edwards, C. L. 1910. Arch. Zellf. 5: 422-429. illus. [Ascaris.] 1911. Areh. Zellf. $7:$ 309-313. pl. 28. [Ascaris felis.]

Eнтімiu, P. 1927a. Rev. Pathol. Vég. Entom. Agr. 14: 166-175. [Exoaseaceæ.] 1927b. Le Botaniste 18: 1-154. illus. [Exoaseacex.]

1929a. Compt. Rend. Aead. Sei. Paris 188: 266-269. [Spathularia.] 1929b. Le Botaniste 20:228-237. [Spathularia.]

Eichrorn, A. 1927. Bull. d'Hist. Appl. 4. [pH.]

Ersen, G. 1899. Bot. Centralb]. 19: 130-136. 5 figs. [Chromioles.]

Ekman, G. 1930. Ann. Soc. Zool. Bot. Fenn. Vanamo. 10:1-141. illus.

Emberger, L. 1920ab. C. R. Acad. Sci. Paris 170: 282-284, 469-471. 12 figs. 1920cd. C. R. Acad. Sci. Paris 171: 263-266. 6 figs.; 735-737. 1922a. Aich. de Morph. Gén. et Exp. 1: 1-190. [Origin of plastids.] 1927. Rev. Gén. Bot. 39 : 341-363, 420-448. illus.

Euerson, R. A. 1914. Amer. Naturalist 48: 87-115. [Variegation in maize.] 1917. Genetics $2: 1-35$. [Trriegated pericarp in maize.]

1922. Amer. Naturalist 56:64-79. [Bud variations.]

1924. Seience 59: 176-182. [Sex inheritance.]

1932. Seience $75: 546$. [Sex in maize.]

1933. Proe. 6 Internat. Cong. Genet. 1. [Geneties of maize.]

Emerson, R. A., and G. IT. Beadle. 1930. Amer. Naturalist 64: 190-192. illus.

Emerson, R. A., and M. M. Rhoddes. 1933. Amer. Naturalist 67: 374-377.

Exierson, S. H. 1924a. Papers Mich. Acad. Sei. ete. 4: 111-114. pls. 12-14.

1924b. Jour. Wash. Acad. Sei. 14 : 277-284.

1928a. Univ. Michigan. Thesis.

1928b. Papers Mieh. Acad. Sci. 9: 117-120. illus. [Enothera.]

1929. Ia Cellule 39: 159-165. illus. [Monoploid Enothera.]

1931a. Geneties 16:325-348. illus. [Enothera hybrids.]

1931b. Aner. Naturalist $65: 551-555$. illus. [Parasynapsis.]

Enerson, S., and G. W. Beadle. 1932. Proe. 6 Internat. Cong. Genet. 2: 48-49.

Emerson, S. H., and A. H. Sturtevant. 1931. Zeitschr. Ind. Abst. Vererb. 59: 395-419. [OEnothera.]

Emmel, V. E. 1924. Amer. Jour. Anat. 33: 347-405. 7 pls. [Blood cells.]

Endelztein, G. 1925. Bacterien Cyelogenie. Berlin.

Enrigues, P. 1907. Areh. Protist. 9: 195-296. illus.

1908. Arch. Protist. 12: 213-276. illus.

Entz, G. 1918. Arch. Protist. 38: 324-354. pls. 12, 13. 5 figs.

Erduan, R. 1920. Roux's Areh. Entw. 46: 85-148. 12 figs. [Endomixis.]

Erituan, C. 1927, 1930. Geol. Fören. Stoekh. Förh. 49, 52.

Erhard, H. 1911. Ergeb. Anat. Entw. 19: 893-929. 
von Erlanger, R. 1897. Biol. Centralbl. 17: 152-160, 339-346. 25 figs.

Erlanson, E. W. 1929. Bot. Gazette 87:443-506. illus. [Rosa.] 1931a. Cytologia $2: 256-282$. illus. [Rosa.]

1931b. Geneties 16: 75-96. illus. [Rosa.]

Ertanson, E. W., and F. J. Hermann. 1928. Papers Mich. Acad. Sci. 8: 97-110.

Enns', A. 1917. Vierteljahrsehr. Naturf. Gesell. Zürich 62: 336-348.

1918. Bastardierung als Ursache der Apogamie im Pflanzenreich. Jena.

1921a. Zeitschr. Ind. Abst. Vererb. $25: 185-197$. [Chara crinita.]

Ernst, A., and C. Bernard. 1912. Ann. Jard. Bot. Buit. II 11: 234-257. illus.

Escoyez, E. 1907a. La Cellule 24 : 355-366. 1 pl. [Zygnema.]

1907b. La Cellule 24:247-256. [Marchantia.]

1909. La Cellule 25:181-201. 1 pl. [Stypocaulon.]

Evans, H. M., and O. Swezr. 1928. Geneties $13: 532-543$. illus. [Man.] 1929. Mem. Univ. Calif. 9. [Chromosomes in man.]

Eyster, W. H. 1924. Genetics 9: 372-404.

1928. Zeitschr. Ind. Abst. Vererb., suppl. 1: 666-686.

1929. Science 69: 48. [Maize plastids.]

Fairbrother, J. A. V. 1928. Brit. Jour. Radiol. 1: 125.

Fairchild, T. 1717. See R. Bradley: New Improvements of Planting and Gardening, ete. London. Also: Phil. Trans. Roy. Soe. (London) 33:127-129. 1724.

Fankhauser, G. 1929. Rev. Suisse Zool. 36: 179-187. illus. [Cytaster.]

Farmer, J. B. 1894. Ann. Botany 8:35-52. pls. 6, 7. [Pallavicinia.] 1895. Ann. Botany 9:469-523. pls. 16-18. [Pallavicinia.]

1907. Proc. Roy. Soc. London B. $79: 446-464$.

FAruer, J. B., and L. Digby. 1907. Ann. Botany 21: 161-199. pls. 16-20. 1914. Phil. Trans. Roy. Soc. London B 205: 1-26. 2 pls.

Farmer, J. B., J. E. S. Moore, and L. Digby. 1903. Proc. Roy. Soc. London 71: $453-457$.

FArr, C. H. 1916. Mem. N. Y. Bot. Gard. 6:253-317. pls. 27-29. [Cytokinesis.] 1918. Amer. Jour. Bot. 5: 379-395. pls. 30-32. [Magnolia: cytokimesis.] 1922a. Bull Torr. Bot. Club 49:51-61. pl. 2. [Sisyrinchium.] 1922b. Amer. Jour. Bot. 9: 296-306. pl. 15. [Nelumbo.]

Farr, W. K. 1920. Bull. Torr. Bot. Club 47:325-337. pl. 14. [Cobaa.]

Fadlu, J. H. 1905. Proc. Boston Soc. Nat. Hist. 32: 77-113. pls. 7-11. 1911. Ann. Botany 25: 649-654. [Laboulbeniales.] 1912. Ann. Botany 26:325-355. pls. 37-49. [Laboulbenia.]

Fauré-Fremiet, M. E. 1910a. Areh. d'Anat. Mier. 11: 457-648. pls. 19-22. 1913. Arch. d'Anat. Micr. 15: 435-757. pls. 12-14. 136 figs.

1921. Compt. Rend. Acad. Sci. Paris 173: 1023-1026.

Faussek, W. 1913. Arch. Mikr. Anat. $82: 39-60$. pls. 3, 4.

Federley, H. 1913. Zeitschr. Ind. Abst. Vererb. 9: 1-110. pls. 1-4. 5 figs. 1923. Hereditas $4: 161-170$. [Species hybrids.]

1928. Zeitschr. Ind. Abst. Vererb., suppl., 1: 194-222. illus.

Feld. 1926. Sce Kiesel, 1930.

Feudinandsen, C., and $\varnothing$. Winge. 1930. Hereditas $13: 164-176$. illus.

Ferguson, A. J., and O. Ralin. 1933. Areh. Mikrobiol. [Mitogenetie rays.]

Fergusox, M. C. 1901. Ann. Botany 15: 193-223. pl s. 12-14. [Pinus.] 1904. Proc. Wash. Acad. Sci. 6: 1-202. pls. 1-24. [Pinus.]

1927. Bull. Torr. Bot. Club 54:657-664. illus. [Petunia.]

Fernandes, A. 1931. Bol. Soc. Broter. (Coimbra) 7:1-122. 
Fetter, D. 1926. Jour. Exp. Zoöl. 44: 279-283. [Viscosity.]

Feulgen, R. 1926. Abderhalden's Handbuch Biologischer Arbeitsmethoden 213. Feulgen, R., and H. Rossenbeck. 1924. Zeitschr. Physiol. Chem. 135: 203-248.

Feulgen-Bradns, F. 1924. Arch. Gesammt. Physiol. 203: 415-435.

F1KRY, M. A. 1930. Jour. Roy. Micr. Soc. III 50:387-419. illus.

FINDLAY, G. M. 1927. Jour. Roy. Micr. Soc. 47:258-265. illus.

Finv, W. W. 1925. Bot. Gazette $80: 1-25$. pls. 1-3. 2 figs. [ơ cells.]

1928. Ber. Deu. Bot. Gesell. $46: 235-246$. illus. [Vinca.]

FinN, W. W., and T. RUdenko. 1930. Bull. Jard. Bot. Kieff 11:69-82. illus.

Fischer, A. 1897. Untersuchungen über den Bau der Cyanophyceen und Bakterien. 1899. Fixicrung, Färbung und Bau des Protoplasmas. Jena.

1905. Bot. Zeitung 63: 51-129. pls. 4, 5. [Cyanophyceæ.]

Fischer, M. H. 1923. Science 57: 724-727.

FISK, E. L. 1925. Proc. Nat. Acad. Sci. 11:352-356. illus. [Zea.] 1927. Amer. Jour. Bot. 14: 53-75. illus. [Zea.]

Fitting, H. 1900, 1906. Bot. Zeitung 58: I 107-164. pls. 5, 6; 64: 42-43.

1909. Zeitschr. Bot. 1: 145-147.

1927. Jahrb. Wiss. Bot. $67: 427-596$. illus.

Fitting, Sierp, Harder, and Karsten. 1930. A Textbook of Botany. 6th Eng. ed. transl. from 17th German ed. New York.

Fitzpatrick, H. M. 1918a. Bot. Gazette 65:201-226. pls. 3, 4. [Rhizina.] 1918b. Amer. Jour. Bot. 5: 397-419. pls. 30-32. [Eocronartium.]

1930. The Lower Fungi. Phycomycetes. New York.

Fleischer, M. 1920. Ber. Deu. Bot. Gesell. 38: 84-92. pl. 2. [Sex in mosses.]

Flemming, W. 1875. Sitzber. Wiener Akad. Wiss. 71.

1879a. Arch. Mikr. Anat. 16: 302-436. illus.

1879b. Virchow's Archiv 77.

1880. Arch. Mikr. Anat. 18: 151-259. illus.

1882a. Zellsubstanz, Kern und Zelltheilung. Leipzig.

1882b. Arch. Mikr. Anat. 20: 1-86. pls. 1-4.

1898. Anat. Anz. 14: 171-174. 1 fig.

FLorin, R. 1918a. Ark. Bot. 15: 1-10. 1 pl. [Chiloscyphus.]

Fоске, W. O. 1881. Die Pflanzen-Mischlinge. Berlin.

FoL, H. 1873. Jenaische Zeitschr. 7.

1875. Arch. de Zool. 4.

1879. Mém. Soc. Phys. et Nat. Genève $26:$ 89-397.

Fontana. 1781. Traité sur le Venin de la Vipère. Florence.

Foot, K., and E. C. Strobell. 1905. Amer. Jour. Anat. 4: 199-243. pls. 1-9.

Forenbacher, A. 1911. Ber. Deu. Bot. Gesell. $29: 648-660$. pl. 25.

Fortuin, C. E. D. 1926. Proc. Koninkl. Akad. Wetensch. Amsterdam 29 : 979-988.

Föyn, B. 1929. Ber. Deu. Bot. Gesell. 47: 495-506. illus. [Cladophora, Ulva.]

Francini, E. 1928. Rend. Acc. Naz. dei Lincei 7: 92. [Ochna.]

Frandsen, H. N., and $\varnothing$. Winge. 1932. Hereditas 16: 212-219.

Frank, G. 1929. Biol. Zentralbl. 149:129-141. illus.

Fraser, H. C. I. 1907. Ann. Botany 21: 349-360. pls. 29, 30. [Lachnea.]

1908. Ann. Botany 22:35-55. pls. 4, 5. [Humaria.]

Fraser, H. C. I., and W. E. Brooks. 1909. Ann. Botany 23 : 538-549. [Ascus.]

Fraser, H. C. I., and E. J. Welsford. 1908. Ann. Botany 22: 465-477.

Franzé, R. 1893. Zeitschr. Wiss. Zool. 56: 138-164. pl. 8. [Eyespot.]

Free, E. E. 1918. Plant World 21: 141-150. [Permeability.]

Frémy, E. 1859. Compt. Rend. Acad. Sci. Paris 48: 202-212, 667-673, 862-868.

Freundici, H. 1926. Colloid and Capillary Chemistry. London.

Freundilch, H., und W. Seifriz. 1923. Zeitschr. Phys. Chem. 104: 233-261. 
Frew, P. E., and R. H. Bowen. 1929. Quar. Jour. Micr. Sci. 73: 197-214. illus.

Frey, A. 1926a. Ber. Deu. Bot. Gesell. 44: 564-570. [Micellar theory.]

1926b. Jahrb. Wiss. Bot. 65: 195-223. illus. [Cell membrane.]

1927. Naturwissenschaften $15: 760-765$. illus. [Cell membrane.]

1928. Ber. Deu. Bot. Gesell. $46: 444-456$. [Cell membrane.]

Frey, C. N. 1924. Trans. Wis. Acad. Sci. $21: 303-343.2$ pls. [Venturia.]

Friedrichs, G. 1922. Jahrb. Wiss. Bot. 61:430-458. pl. 1. [Plastids.]

Fries, R. E. 1911. Svensk Bot. Tidskr. 5:241-251. pl. 1. [Hygrophorus.]

Fries, W. 1909. Arch. Zellf. 4: 44-80. pls. 3-5. [Meiosis and parthenogenesis.]

Friesner, R. C. 1919. Mich. Acad. Sci. Report 21 : 233-234.

1920. Amer. Jour. Bot. $7: 380-407$. pls. 24, 25. [Division rhythm.]

1930. Butler Univ. Bot. Studies 1: 77-103. illus.

Frisendahl, A. 1912. Kgl. Svensk Vet. Handl. 48. [Myricaria.]

Fritsch, F. E. 1916. New Phytol. 15: 233-250. [Alternation of generations.]

Frolowa, S. 1912. Arch. Zellf. 9: 149-167. pls. 13, 14. [Ascaris.]

1926. Zeitschr. Zellf. Mikr. Anat. 3: 682-694. illus.

1929a. Zeitschr. Zellf. Mikr. Anat. 8: 542-565. illus. [Polyploid Diptera.]

1929b. Zeitschr. Zellf. Mikir. Anat. 9: 66-82. illus. [Choaborus.]

Frolowa, S. L., and B. L. Astatrow. 1929. Zeitschr. Zellf. Mikr. Anat. 10: 201213. illus.

Frommann, C. 1865. Centralbl. Med. Sci. 3. [Structure of protoplasm.] 1875. Jenaische Zeitschr. 9 (Other papers listed). [Structure of protoplasm.] 1884. Jenaische Zeitschr. 17: 1-349. 3 pls. [Structure of protoplasm.]

Frost, W. D. 1917. The Cytology of Bacteria. In Marshall's Microbiology.

Fry, H. J. 1928, 1929, 1932. Biol. Bull. 54: 363; 56: 101; 57: 131, 151; 63: 149.

FoJ11, K. 1898, 1899. Bot. Mag. Tokyo $12: 287-290$. 13:260-266. pl. 7.

FukUdA, Y. 1927. Bot. Mag. Tokyo 41:459-474. illus. [Solanum.]

Fondshima, E. 1931. Jap. Jour. Bot. 5: 273-283. illus. [Brassica.]

Folton, J. F. 1922. Quar. Jour. Micr. Sci. 66: 339-396. [Pigments.]

Gaertner, K. F. 1849. Versuche und Beobachtungen über die Bastardzeugung.

GaGer, C. S. 1926. General Botany. Philadelphia.

Gager, C. S., and A. F. Blakeslee. 1927. Proc. Nat. Acad. Sci. 13: 75-79.

Gaines, E. F., and H. C. Aase. 1926. Amer. Jour. Bot. 13: 373-385. illus.

[Monoploid wheat.]

Gairdner, A. E. 1926. Jour. Genetics 16:341-351. illus. [Campanula.]

Gairdner, A. E., and C. D. Darlington. 1930. Nature. London, Jan. 18.

1931. Genetica $13: 113-150$.

Ga1ser, L. O. 1923. Bull. Torr. Bot. Club 60:389-398. pl. 20. [Crystals.] 1926. Genetica $8: 401-484$.

1930a. Bibliog. Genetica 6: 171-466. [Chromosome numbers.]

1930b. Genetica 12:162-260. [Chromosome numbers.]

Galán, F. Eos 7:461-501. illus. [Phytodecta.]

Gallardo, A. 1896. Ann. Soc. Cien. Argent. 42. Ann. Mus. Nac. Buenos Aires.

1906. Ann. Mus. Nac. Buenos Aires 13: 259.

Garber, J. F. 1904. Bot. Gazette 37:161-177. pls. 9, 10. [Ricciocarpus.]

Gardiner, M. S. 1927. Jour. Morphol. and Physiol. 44:217-262. illus.

Gardiner, W. 1884. Arb. Bot. Inst. Würzburg 3 : 52-87. [Plasmodesms.]

1885. Proc. Roy. Soc. London 39 : 229-234.

1897. Proc. Roy. Soc. London $62: 100-112.8$ figs. [Plasmodesms.]

1900. Proc. Roy. Soc. London 66: 186-188. [Plasmodesns.] 
Gardiner, W. and Hill, A. W. 1901. Phil. Trans. Roy. Soc. London B 194: 83-125. 5 pls. [Plasmodesms.]

Gardner, N. L. 1906. Univ. Calif. Publ. Bot. 2: 237-296. pls. 21-26.

Gargeanne, A. J. M. 1903. Flora 92: 457-482. 18 figs. [Oil bodies.]

Gatenbi, J. B. 1917ab. Quar. Jour. Mier. Sei. 62:407-463; 555-611.

1918. Quar. Jour. Mier. Sei. 63: 197-258. pls. 16-18. 3 figs.

1919a. Quar. Jour. Micr. Sei. 63: 445-492. pls. 27, 28.

1919b. Jour. Roy. Mier. Soc. London 93-119. 14 figs.

1920. Quar. Jour. Mier. Sei. 64: 267-302.

1922. Quar. Jour. Mier. Sei. 66: 1-48. pls. 1-4.

1925. Quar. Jour. Mier. Sci. 69: 629-642. illus.

1928. Nature. London 121: 11-12. [Golgi material in plants.]

1929. Proe. Roy. Soe. London B 104: 302-321. illus. [Golgi.]

1930. Jour. Roy. Mier. Soc. III 50: 20-29. [Cell nomenclature.]

Gatenby, J. B., and J. H. Woodger. 1920. Jour. Roy. Mier. Soe., 129-156. pl. 2. 1921. Quar. Jour. Mier. Sei. 65:265-291. pls. 11, 12.

Gatenby, J. B., and S. B. Woodger. 1929. Proc. Roy. Soc. London B 104: 471480 . illus.

Gates, R. R. 1907a. Bot. Gazette 43: 81-115. illus. [Enothera.]

1907b. Bot. Gazette 44:1-21. [Enothera.]

1908a. Science 27: 193-195. [Enothera.]

1908b. Bot. Gazette 46:1-34. pls. 1-3. [Enothera.]

1909a. Bot. Gazette 48: 179-199. pls. 12-14. [Enothera.]

1909b. Areh. Zellf. $3: 525-552$. [Enothera.]

1911a. Ann. Botany 25: 909-940. pls. 67-70. [Enothera.]

1911b. Bot. Gazette 51: 321-344. [Mode of meiosis.]

1912. Ann. Botany 26: 993-1010. pl. 86. [Enothera: somatie mitosis.]

1913. Biol. Centralbl. 33: 93-99, 113-150. 7 figs. [Tetraploidy.]

1915a. Amer. Jour. Bot. 2 : 519-52S. [Mutations.]

1915b. The Mutation Factor in Evolution. London.

1920a. Proc. Roy. Soe. London B 91: 216-223. 2 figs. [Lactuca.]

1920b. New Phytol. 19: 26, 64, 132, 172, 213. [Mutations.]

1923. Nature $111: 635-636$. [Chloroplasts.]

1924a. Brit. Jour. Exp. Biol. 1: 153-182. [Polyploidy.]

1924b. Nature 114: 353-356. [Species and ehromosomes.]

1925. Amer. Naturalist 59: 193-200. [Species and chromosomes.]

1928. Bibliog. Genetiea 4:401-492. illus. [Enothera.]

1929a. Conference on Polyploidy, J. Innes Hort. Inst., Jan. 19.

1929b. Nature. London 124: 948. [Monoploid Enothera.]

1931b. Amer. Naturalist 65: 97-120. [Mutation.]

1932a. Jour. Roy. Mier. Soe. 52 : 1-19. [Nuelear strueture.]

Gates, R. R., and K. M. Goodwr. 1930. Jour. Geneties 23:123-156. illus.

1931. Proe. Roy. Soc. London B 109: 149-164. illus. [Enothera.]

Gates, R. R., and J. Latter. 1927. Jour. Roy. Mier. Soc. 47: 209-225. illus.

Gates, R. R., and E. M. Rees. 1921. Ann. Botany 35: 365-398. pl. 20.

Gates, R. R., and F. M. L. Sheffield. 1929a. Proc. Roy. Soc. London B 105: 499-517. illus.

1929b. Phil. 'Trans. Roy. Soc. London B 217 : 367-394. [Enothera.]

Gates, R. R., and N. Thonis. 1914. Quar. Jour. Micr. Sci. $59: 523-571$.

GaUBA, E. 1926. Biologia Generalis 2: 301-337. illus. [Cutinization.]

Gaudissart, P. 1913. La Cellule 30:29-43. pls. 1, 2. [Myofibrils.]

Gäumann, E. A. (Transl. by Dodge) 1933. Comparative Morphology of Fungi.

Ed. 2. (Ed. 1, 1928.) New York. 
Geerts, J. M. 1907. Ber. Deu. Bot. Gesell. 25: 191-195. pl. 6. [Enothera.]

1908. Ber. Deu. Bot. Gesell. 26a: 608-614. [Enothera.]

1909. Ree. Trav. Bot. Néerl. 5: 93-206. [Enothera.]

1911. Ber. Deu. Bot. Gesell. 29: 160-166. 1 pl. [Enothera.]

Gegenbaur, K. 1861. Areh. Anat. Physiol. 451-529.

Geitler, L. 1926. Areh. Protist. 56: 128-144. illus. [Pyrenoid.]

1927a. Areh. Protist. 59: 506-549. illus. [Cocconeis.]

1927b. Biol. Zentralbl. 47: 307-318. ilhus. [Cocconeis.]

1927c. Areh. Protist. 58: 465-507. illus. [Cymbella.]

1925a. Oesterreich. Bot. Zeitsehr. 77: 81-91. illus. [Amphora.]

1928b. Biol. Zentralbl. 48:648-663. illus. [Cymbella.]

1928c. Areh. Protist. 61: 419-442. illus. [Nitzschia.]

1929b. Areh. Botanique 3 : 105-112. illus. [Sex in diatoms.]

1930. Areh. Protist. 71: 79-100. illus. [Spirogyra.]

1931. Beilı. Bot. Centralbl. I $48: 1-14$. illus. [Diatoms.]

Gelei, J. 1921. Areh. Zellf. 16: 88-169. 6 pls. 6 figs. [Parasynapsis.]

1922. Arch. Zellf. 16: 299-370. 1 fig. [Parasynapsis.]

Gellhorn, E. 1929. Das Permeabilitïtsproblem. Berlin.

Gerassinow, J. J. 1890-1901. Bull. Soc. Sei. Moseow: 1890, 548-554; 1892,

$109-131 ; 1896,477-480 ; 1899,220-267 ; 1901,185-220$.

1904. Beih. Bot. Centralbl. 18: 45-118. pls. 2.

1905a. Hedwigia 44:50-56. [Enucleated cells, ete.]

1905b. Flora 94: 79-98. 7 pls. [Etherized Spirogyra.]

Gerhard, K. 1929. Jenaisehe Zeitsehr. Naturwiss. 64: 283-338. [Enothera.]

Gerould, J. H. 1922ab. Sci. Mo. 14: 267-276. Seience 55 : 421-422.

Gershor, A. 1928. Vermont Agr. Exp. Sta. Bull. 279. illus. [Viola.]

Ghinipu, V. 1929b. 14 Cong. Internat. Agr., Bucharest.

1929f. Rev. Bot. Appl. Agrie. Coloniale 9: 176-179. illus.

1930. Areh. d'Anat. Mier. 26: 135-249. illus.

Giard, A. 1877. Sur la Signifieation Morphologique des Globules Polaires.

Gickliorn, J. 1927. Protoplasma 2:1-16. illus. [Vital staining.]

Gicklhorn, J., and L. Möschl. 1930. Protoplasma 9: 521-535. illus.

Gigulo-Tos, E. 1926. Areh. Entw. Org. 107: 186-212. illus.

Gilson, E. 1890. La Cellule 6: 63-114. 1 pl. [Suberin.]

Giroud, A. 1925a. Areh. d'Anat. Mier. 21: 145-252. 17 figs. [Chondriome.] 1925b. Compt. Rend. Soe. Biol. Paris 93: 158-160. [Chondriosomes.]

1928. Compt. Rend. Acad. Sei. Paris 186: 794-795. [Chondriosomes.]

1929. Protoplasma $7: 72-98$. illus. [Chondriosomes.]

Glaser, O. 1921a. Anat. Ree. 20: 227-228. [Egg secretions.]

1921b. Biol. Bull. 41: 63-72. [Egg secretions.]

Gleisberg, W. 1921. Beih. Bot. Centralbl. 38:217-265. [Cell membrane.]

Godlewsk1, E. 1906. Roux's Arch. Entw. 20:579-643. pls. 22, 23. [Echinoderm hybrids.]

1911. Roux's Areh. Entw. 33: 196-254. pls. 11-13.

Goldschindt, R. 1904a. Biol. Centralbl. 24: 241-251. 4 figs. [Chromidia.]

1904b. Zool. Jahrb., Ontog. Abt. 21: 49-140. pls. 3-8. [Chromidia.]

1908. Arch. Zellf. 1: 232-24t. pl. 7. [Dicroculium.]

1909. Areh. Zellf. 4: 81-119. pls. 6-9. 3 figs. [Chromidia.]

1910. Festsehr. f. R. Hertwig. Jena.

1911. Einführung in die Vererbungswissensehaft. Leipzig.

1912. Zeitsehr. Ind. Abst. Vererb. 8: 79-8S. [Sex.]

1916a. Amer. Naturalist 50: 705-718. [Sex.]

1916b. Proe. Nat. Acad. Sci. 2 : 53-58. [Sex.] 
1917a. Jour. Exp. Zoöl. 22 : 593-611. 53 figs. [Sex.]

1917b. Geneties $2: 82-95$. [Crossing-over.]

1919. Biol. Zentralbl. 39 : 498-512. [Intersexuality.]

1920a. Zeitschr. Ind. Abst. Vererb. 23: 1-199. [Intersexuality.]

1920b. Arch. Zellf. $15: 283-290$. [Sex of parthenogenetic frog.]

1920c. Mechanismus und Physiologie der Geschlechtsbestimmung. Berlin.

1921ab. Nature 107: 780-784. Naturwissensehaften 9:315-316. 2 figs. [Sex.]

1922ab. Biol. Zentralbl. 42 : 301-302; 481-487.

1923a. The Mechanism and Physiology of Sex Determination. New York.

1923b. Zeitschr. Ind. Abst. Vererb. 31: 100-133. 10 figs.

1925b. Biol. Zentralbl. $45: 536-541$. [Intersexes.]

1926a. Biol. Zentralbl. 46: 193-200. [Intersexes.]

1926b. Biol. Zentralbl. 46:441-452. [Bonellia.]

1927a. Physiologische Theorie der Vererbung. Berlin.

1927b. Ergeb. Biol. 2: 554-684. illus. [Sex determination.]

1928a. Einführung in die Vererbungswissensehaft. 5th ed. Leipzig.

1928b. Quar. Rev. Biol. 3: 307-324. [The Gene.]

1929a. Biol. Zentralbl. 49: 437-448. [Mutation.]

1929b. Biol. Zentralbl. 49:641-648. [Sex determination.]

1931a. Quar. Rev. Biol. 6: 125-142. [Intersexuality.]

1931b. Die Sexuellen Zwischenstufen. Berlin. [Intersexuality.]

Goldschmidt, R., and E. Fischer. 1927. Arch. Entw. Org. 109:1-13. illus. 1929. Zeitschr. Krebsf. 30:281-285. illus.

Goldschmidt, R., and K. Katsuki. 1927. Biol. Zentralbl. 47:45-54. illus. Goldschmidt, R., and M. Popoff. 1907. Areh. Protist. 8:321-343. 6 figs. Goldstein, B. 1928. Bot. Gazette 86: 365-383. illus. [Nuclear form.]

Golgi, C. 1898ab. Arch. Ital. Biol. 30:60-71; 278-286. 1 pl. 1900. Arch. Ital. Biol. 31: 273-280. 1 pl. [See also Opera Omnia.]

Goodspeed, T. H. 1923. Svensk Bot. Tidskr. $17: 472-478$. illus.

1929a. Bot. Gazette $87: 563-582$. illus. [X-rays.]

1929b. Jour. Heredity $20: 243-259$. illus. [X-rays and radium.]

1930a. Univ. Calif. Publ. Bot. 11: 285-298. illus. [X-rays and radium.]

1930b. Univ. Calif. Publ. Bot. 11: 299-308. illus. [X-rays and polyploidy.]

1930c. Univ. Calif. Publ. Bot. 11: 309-318. illus. [X-rays and meiosis.]

1932. Svensk Bot. Tidskr. 26: 147-162. illus. [X-rays; radium.]

Goodspeed, T. H., and P. Avery. 1929b. Proe. Nat. Acad. Sei. 15: 502-504.

1930. Cytologia 1:308-327. illus. [Meiosis after X-rays.]

Goodspeed, T. H., and R. E. Cluausen. 1927b. Univ. Calif. Publ. Bot. 11:127-140.

1928. Univ. Calif. Publ. Bot. 11: 245-256. illus. [Nicotiana hybrids.]

Goopspeed, T. H., and A. R. Olson. 1928a. Science 47:46. [X-rays.]

1928b. Proc. Nat. Acad. Sci. 14: 66-69. [X-rays.]

Gordon, M. 1926. Anat. Rec. 34: 172. [Platypœcilus.]

1927. Genetics $12: 253-283$. illus. [Platypœcilus.]

Goroschankin, J. 1883. Bot. Zeitung 41: 825-831. pl. 7A.

Gortwer, R. A. 1929. Outlines of Biochemistry. New York.

Gотон, K. 1924. Bot. Mag. Tokyo 38: 135-152. 13 figs. [Sccale.]

Gowen, J. W. 1929a. Proe. Nat. Acad. Sci. 15: 266-268. [Crossing-over.]

1929b. Science 70:358-360. [Gene action.]

1931a. Jour. Gen. Physiol. 14: 447-461. [Chromosome balance.]

1931b. Jour. Gen. Physiol. 14: 463-472. [Chromosome balance.]

1933. Jour. Exper. Zoöl. 65: 83-106.

Gowen, J. W., and E. H. GaY. 1933. Genetics 18: 1-31. illus. 
Grafe, V. 1911. In Abderhalden's Biochemischen Handlexikon. Abt. II. 1922. Chemie der Pflanzenzelle. Berlin.

1925. Biochem. Zeitschr. 159.

Grafe, V., and H. Magistris. 1926. Planta 2.

Graham, M. 1918. Ann. Botany 32:415-420. pl. 10. [Preissia.]

Granata, L. 1910. Arch. Zellf. 5 : 182-214. pls. 14-16.

Grave, C., and F. O. Schmitt. 1924. Science 60:246-248. 2 figs. [Cilia.] 1925. Jour. Morphol. and Physiol. 40:479-515. 2 pl. 1 fig.

Gray, J. 1925. Proc. Cambridge Phil. Soc. Biol. Series 1, 225. [Oxidation.] 1927b. Brit. Jour. Exp. Biol. 5: 102-111. illus. [Effect of gravity.] 1928. Ciliary Movement. Cambridge University Press.

1931. A Text-book of Experimental Cytology. Cambridge University Press.

Greenwood, A. W. 1923. Quar. Jour. Micr. Sci. 67: II 203-218. pls. 15, 16.

Grégoire, V. 1899. La Cellule 16:235-297. 2 pls. [Meiosis in Lilium.]

1904. La Cellule 21: 297-314. [Meiosis.]

1905. La Cellule 22: 221-376. [Meiosis: review.]

1906. La Cellule 23: 311-353. 2 pls. [Chromosome structure.]

1907. La Cellule 24:369-420. 2 pls. [Meiotic prophase.]

1909a. La Cellule $25: 245-285.2$ pls. [Meiosis in Zoögonus.]

1909b. La Cellule 25: 87-99. [Meiosis.]

1910. La Cellule 26 : 223-422. 145 figs. [Meiosis: review.]

1911. Rev. Quest. Sci., October, 1911; April, 1912. [Mendelism.]

1912. Ann. Soc. Sci. Bruxelles 34. [Somatic mitosis.]

1925. Rev. Quest. Sci., July, 1925. [Chromosomes and Mendelism.]

1928. Bull. Acad. Roy. Belg., Cl. d. Sci., V 13. [Genetics and cytology.]

1932. Bull. Acad. Roy. Belg., Cl. d. Sci., 17: 1435-1448. illus. [Euchromocenters.]

Grégoire, V., and J. Berghs. 1904. La Cellule 21: 193-238. 2 pls. [Pellia.]

Grégoire, V., and A. Wygaerts. 1903. La Cellule 21: 7-67. 2 pls. [Mitosis.] 1907. La Cellule 24:369-420. 2 pls. [Meiotic prophase.]

Gregory, R. P. 1909. Proc. Cambridge Phil. Soc. 15:239-246. 1 pl. [Primula.] 1914. Proc. Roy. Soc. London B 87 : 484-492. [Primula.]

1915. Jour. Genetics $4: 305-321$. [Primula.]

Grew, N. 1682. The Anatomy of Plants. London.

Griggs, R. F. 1912. Bot. Gazette 53:127-173. pls. 11-16. [Rhodochytrium.]

Gris, A. 1864. Ann. Sci. Nat. Bot. V 2:5-123. 14 pls. [Germination.]

Gross, F. 1932. Naturwissenschaften 20:962-967. illus. [Artemia.]

Gross, I. 1931. Arch. Protist. 73: 206-234. illus. [Ulothrix.]

Gross, R. 1916. Arch. Zellf. 14:279-354. pls. 15, 16. 13 figs. [Nucleus.]

Grosser, O. 1921. Anat. Anz., Erg. 54: 181-185. 3 figs.

Guignard, L. 1889. Rev. Gén. Bot. 1: 11, 63, 136, 175. pls. 2-6. [Sperms.]

1891a. Compt. Rend. Acad. Sci. Paris 112: 1074-1076.

1891b. Ann. Sci. Nat. Bot. 14: 163-296. pls. 9-18.

1899. Compt. Rend. Acad. Sci. Paris 128: 864-871. 19 figs.

1900. Ann. Sei. Nat. Bot. VIII 11: 365-387. pls. 9-11.

Guilliermond, A. 1904. Rev. Gén. Bot. 16:129-143. pls. 14, 15. [Ascomycetes.]

1905. Ann. Mycol. 3 : 344-361. pls. 10-12. [Ascomycetes.]

1906. Rev. Gén. Bot. 18: 392-408, 447-465. pls. 9-13. [Cyanophycea.]

1907a. Compt. Rend. Soc. Biol. Paris 59: II 216-218. [Aleurone.]

1910. Bull. Sei. France et Belg. 44: 109-196. [Fungi.]

1911a. Rev. Gén. Bot. $23: 89-121$. pls. 4, 5. [Ascus.]

1911b. Compt. Rend. Acad. Sci. Paris 153: 199-201. 4 figs.

1912a. Arch. d'Anat. Micr. 14: 309-428. pls. 13-18. [Starch.] 
1913e. Prog. Rei Bot. 4 : 389-542. 82 figs. [Fungus cytology.]

1915a. Compt. Rend. Soc. Biol. Paris 67: 241-249.

1915b. Rev. Gén. Bot. 27 : 193, 236, 271, 297, 315. 12 pls.

1918. Compt. Rend. Acad. Sci. Paris 167: 430-433.

1919a. Rev. Gén. Bot. 31: 372, 446, 532, 635. 60 pls. 35 figs.

1919c. Compt. Rend. Soc. Biol. Paris 82: 309-312.

1920abde. Compt. Rend. Acad. Sci. Paris 170: 194, 612, 1329, 1003.

1920n. The Yeasts. (Engl. transl. by F. W. Turner.) New York.

1921i. Titres et Travaux Scientifiques de M. Alex. Guilliermond. Lyon.

1922ab. Compt. Rend. Soc. Biol. Paris 86:434; 437.10 figs.

1922e. La Cellule $32: 429-451.3$ pls.

1923a. Compt. Rend. Soc. Biol. Paris 89.

1923b. Arch. d'Anat. Micr. 20: 1-210. 15 pls.

1924. Ann. Sci. Nat. Bot. X $6: 1-52.2$ pls.

1925a. Compt. Rend. Acad. Sci. Paris 180:221-223. illus.

1925b. Compt. Rend. Acad. Sci. Paris 180: 951-954. [Cyanophyceæ.]

1926a. Compt. Rend. Soc. Biol. Paris 94: 993-996. illus. [Vacuome.]

1926b. Rev. Gén. Bot. 38: 129-145, 177-190. [Cyanophyceæ.]

1927. Arch. d'Anat. Micr. 23: 1-98. illus. [Golgi and vacuome.]

1928. Compt. Rend. Soc. Biol. Paris 98: 368-371.

1929a. Amer. Jour. Bot. 16: 1-22. illus. [Vacuonre.]

19296. Compt. Rend. Soc. Biol. Paris 101: 567-572. illus.

1929c. Compt. Rend. Soc. Biol. Paris 101: 619-622. [Dark-field observations.]

1929e. Compt. Rend. Acad. Sci. Paris 188: 1003-1006. illus.

1930a. Rev. Gén. Bot. 42: 327-347. illus.

Gullliermond, A., and G. Mangenot. 1922. C. R. Acad. Sci. Paris 174: 692-694.

1923. Compt. Rend. Acad. Sci. Paris 177: 600-603. 8 figs.

1928. Rev. Gén. Bot. 38: 251-262. [Nucleolus.]

Gullleriond, A., G. Mangenot, and L. Plantefol. 1932. Traité de Cýtologic

Végétale. Paris.

Gulick, A. 1911. Areh. Zellf. 6: 339-382. pls. 18-20. 5 figs.

Gurwitsch, A. 1904. Morphologie und Biologie der Zelle. Jena.

1913. Vorlesungen über Allgemeine Histologie. Jena.

1923. Arch. Mikr. Anat. 100: 11-40.

1924. Arch. Mikr. Anat. 103: 490-498. [Mitogenetic rays.]

1925. Bot. Gazette $80: 224-226$. illus. [Mitogenetic rays.]

1926. Das Problem der Zellteilung Physiologisch Betrachtet. Berlin.

1929. Protoplasma 6 : 449-493. illus. [Mitogenetic rays.]

1932. Die Mitogenetische Strahlung. Berlin (Springer).

Gurwitscil, L., and H. Perepelinina. 1927. Arch. Entw. Org. 109: 362-379.

Guthenz, S. 1912. Arch. Mikr. Anat. 79: 79-95. pl. 6.

Gutstein, M. 1926. Virchows Arch. 261.

Guttenberg, H. von. 1928. Biol. Zentralbl. 48: 31-39.

Guyénot, E., and A. Naville. 1929. La Cellule 39:27-82. illus. [Meiosis in Drosophila.]

Guyer, M. F. 1909. Anat. Anz. 34:573-580. 2 pls.

1910. Biol. Bull. 19: 219-234. pl. 1. [Human chromosomes.]

1914. Science $39: 721-722$. [Human ehromosomes.]

1916. Biol. Bull. 31: 221-268. 7 pls. [Chromosomes of fowl.]

1931. Science 74: 159-166. [Internal secretions.]

Grynne-Vaugman, H. C. I. 1928. Sex and Nutrition in Fungi. Brit. Assoc. Adv.

Sci. Glasgow. 
Gwynne-Vaughan, H. C. I., and H. S. Whlliamson. 1930. Ann. Botany 44 : 127-145. illus. [Humaria.]

1931. Ann. Botany $45: 355-371$. illus. [Pyronema.]

1932. Ann. Botany $46: 653-670$. illus. [Ascobolus.]

1933. Ann. Botany 47:375-383. illus. [Lachnca.]

$\mathrm{H}$

HaAs, P., and T. G. Hind. 1928. An Introduction to the Chemistry of Plant Products. 4th ed. London and New York.

Hanse-Bessell, G. 1921. Zeitsehr. Ind. Abst. Vererb. 27: 1-26. [Digitalis.] 1928b. Planta 6: 767-789. illus. [Anthurium.]

1932a. Beih. Bot. Centralhl. $49: 129-155$. illus. [Digitalis.]

1932b. Ber. Deu. Bot. Gesell. 50a: 61-91. illus. [Rosa.]

Haberlandt, G. 1857. Ueber die Bezielnung zwisehen Funktion und Lage des Zellkerns bei den Pflanzen. Jena.

1890. Das Reizleitende Gewebesystem der Simpflanzen. Leipzig.

1918. Physiologisehe Pflanzenanatomie. 5th ed.

1919ab. Sitzber. Akad. Wiss. Berlin 322-348; 721-733. 12 figs.

1921ab. Sitzber. Preuss. Akad. Wiss. 1921, VIII 221-234; XL 695-725.

1921c. Beitr. Allg. Bot. 2 : 1-53. 12 figs. [Wound hormones.]

1922. Biol. Zentralbl. $42: 145-172.9$ figs. [Wound hormones.]

1923. Ber. Deu. Bot. Gesell. 41: 174-179. 3 figs. [Allium.]

1929. Biol. Zentralb)1. 49: 226-230.

Haecker, E. 1866. Generelle Morphologie der Organismen. Jena.

Haecker, V. 1890. Zool. Anz. 13:551-558. 1 fig. [Cyclops.]

1892. Zool. Juhrb. 5:211-248. pl. 19. [Cyclops.]

1895a. Ann. Botany 9: 95-102. [Meiosis.]

1895c. Arch. Mikr. Anat. 46: 579-617. pls. 28-30.

Hagedoorn, A., and A. C. Hagedoorn-Vorstheuvel La Brand. 1921. The

Relative Value of the Processes Causing Evolution. The Hague.

Hagerul, O. 1928b. Dansk Bot. Arehiv. 6: 1-26. illus. [Bicornes.]

HÅnansson, A. 1924a. Hereditas 5: 93-96. 5 figs. [Enothera.] 1924b. Botan. Notiser 1924, 269-278. 23 figs. [Epilobium.]

1925. Hereditas $6: 257-274.64$ figs. [Godetia.]

1926a. Hereditas 8: 255-304. illus. [Enothera.]

1928a. Hereditas 11:129-282. illus. [Enothera.]

1928b. Hereditas 10:277-292. illus. [Scirpus.]

1929a. Hereditas 12:1-10. illus. [Pisum.]

1930b. Jahrb. Wiss. Bot. 72:385-402. illus. [Enothera.]

1930c. Hereditas 14:1-32. illus. [Enothera.]

1931a. Hereditas 15:17-61. illus. [Pisum.]

1931b. Ber. Deu. Bot. Gesell. 49: 22S-234. illus. [Godetia, Clarkia.]

1931c. Bot. Notiser 1931, 339-342. illus. [Enothera.]

1932. Hereditas 16:155-159. illus. [Pisum.]

1933. Hereditas $17: 155-196$. illus. [Triticum.]

Haldane, J. B. S. 1929. Conference on Polyploidy, J. Innes Hort. Inst.

1930b. Jour. Geneties $22:$ 359-372. [Polyploid ratios.]

Hatu, R. P. 1923. Univ. Calif. Publ. Zool. $20: 447-476$. pls. 40, 41.

1925a. Univ. Calif. Publ. Kool. 26: 281-324. illus. [Oxyrrhis.]

1925b. Univ. Calif. Publ. Zool. 28: 29-64. illus. [Ceratium.]

1929. Jour. Morphol. Physiol. 48: 105-121. illus. [Pcranema.]

1930a. Arch. Protist. 69:7-22. illus. [Flagellates.] 
1930b. Jour. Morphol. Physiol. 49:139-151. illus. [Amœba.]

1931 a. Ann. de Protistol. 3: 57-68. illus. [Vacuome.]

1931b. Zeitschr. Zellf. Mikr. Anat. 13: 770-782. illus. [Stylonychia.]

Hall, R. P., and F. W. Duninue. 1931. Trans. Amer. Mier. Soc. 50: 196-205. illus. [Vorticella.]

HALL, R. P., and T. L. JAHN. 1929. Seience 69: 522 . [Eyespot.]

Hall, R. P., and R. F. Nigrelit. 1931. Jour. Morphol. Physiol. 51: 527-543. illus. [Chlamydomonas.]

Hall, R. P., and W. N. Powell. 1928. Biol. Bull. 54:36-64. illus. [Peranema.] Hammarlund, C., and A. HÁkansson. 1930. Hereditas 14: 97-98. [Pisum.]

Hammarsten, O. 1909. A Textbook of Physical Chemistry. 5th ed. New York. HaNCE, R. T. 1918a. Geneties 3: 225-275. 7 pls. [QEnothera.]

1924. Seience $59: 424-425.3$ figs. [Chromosomes of ehick.]

1926a. Biol. Bull. 51: 443-447. illus. [Chiek.]

1926b. Jour. Morphol. Physiol. 43: 119-145. illus. [Chick.]

Hanna, W. F. 1925. Ann. Botany 39:431-457. [Coprinus.]

1928. Ann. Botany $42: 379-389$. [Coprinus.]

1929a. Nature. London 124: 267. [Puccinia.]

1929b. Phytopathol. 19:415-442. illus. [Ustilago.]

HANNIG, E. 1911. Flora $102: 209-278$. pls. 13, 14.17 figs. [Spore coat.]

Hanson, F. B. 1928. Seience $67: 562-563$. [X-ray mutations.]

Hanson, F. B., and F. M. Heys. 1928. Seienee 68: 115-116. [Radium.]

1929. Science $71: 43-44$. [Earth radiation.]

Hanson, F. B., and E. Winkleman. 1929. Jour. Heredity $20: 277-286$. illus.

Hansteen-Cranner, B. 1919. Ber. Deu. Bot. Gesell. 37 : 380-391. pls. 3, 4.

[Cell wall.]

1922. Meld. fra Norges Landbrukshoiskole $2: 1-160$. pls. 1-17.

1926. Planta 2: 438-445. [Cell membrane.]

Hanstern, J. 1864. Die Milchsaftgefässe, usw. Berlin.

1868. Bot. Zeitung 26: 697, 721, 745, 769.

1880a. Die Protoplasma als Träger der Pflanzlichen und Thierischen Lebensver-

richtungen. Heidelberg.

1880b. Hansteins Bot. Abhl. 4: 1-56.

Harder, R. 1927. Zeitschr. Bot. 19: 337-407. illus. [Rôle of nucleus.]

HARDY, W. B. 1899. Jour. Physiol. 24: 158-210. illus.

1913. Jour. Physiol. 47 : 108-111.

Harlow, W. M. 1927. N. Y. State Coll. Forestry Tech. Publ. 21. [Cell wall.] 1928a. N. Y. State Coll. Forestry Teeh. Publ. 24. [Lignifieation.]

1928bc. N. Y. State Coll. Forestry Teeh. Publ. 26. [Reactions of cell walls.] 1931. Indust. Eng. Chemistry 23: 419-423. illus. [Cell wall.]

Harman, M. T. 1915. Seience $41: 440$. 1920. Biol. Bull. 38: 213-230. [Paratettix.]

Harms, J. W. 1926. Körper und Keimzellen. Berlin.

Harper, R. A. 1895. Ber. Deu. Bot. Ges. 13: (67)-(68). pl. 27. [Ascus.]

1896. Jahrb. Wiss. Bot. $29: 655-685$. pls. 11, 12. [Aseus.]

1897. Jahrb Wiss. Bot. 30: 249-284. pls. 11, 12. [Ascus.]

1899. Ann. Botany 13: 467-525. pls. 24-26. [Aseus.]

1900a. Bot. Gazette 30:217-251. pl. 14. [Fuligo.]

1900b. Ann. Botany 14: 321-396. pls. 19-21. [Pyronema.]

1905. Carnegie Inst. Wash. Publ. 37. [Mildews.]

1906. Bot. Soe. Amer. Publ. 36: 281-334. 4 pls. 1908. [Cœnobic plants.]

1914. Amer. Jour. Bot. 1: 127-144. pls. 11, 12. [Didymium.]

1918a. Proc. Amer. Phil. Soe. $57: 375-439.2$ pls. 35 figs. [Pediastrum.] 
1918b. Mem. Torr. Bot. Club 17: 210-240. 27 figs. [Pediastrum.]

1919. Amer. Jour. Bot. 6: 273-300. [Structure of Protoplasm.]

1929. Proc. 4 Internat. Cong. Plant Sci. 1: 311-316. [Elaioplasts.]

Harrison, J. W. H. 1920. Jour. Genetics 10:61-85. [Tephrosia.]

Harrison, J. W. H., and K. B. Blackburn. 1927. Mem. Hort. Soc. N. Y. 3 : 23-32. [Rosa.]

Harrison, J. W. H., and A. D. Peacock. 1926. Trans. Nat. Hist. Soc. Northumberland, etc. II $6: 201-217$. [Tephrosia.]

Harrison, W. 1911. Zeitschr. Chem. Ind. Koll. 9: 5-9. [Starch.]

Hartig. 1856. Bot. Zeitung, p. 257. Sec also 1855, p. 881.

Hartuann, M. 1909. Arch. Protist. 14: 264-334.

1911. Die Konstitution der Protistenkerne, usw. Jena.

1914. Verh. Deu. Zool. Gesell., 24 Versamml., 15-50.

1921. Arch. Protist. 43: 223-286. illus. [Eudorina.]

1929a. Fortpflanzung und Befruchtung als Grundlage der Vererbung. Berlin.

1929b. Ber. Deu. Bot. Gesell. 47 : 485-494. illus. [Chatomorpha, ctc.]

1929c. Handbuch der Vererbungswissenschaft (BAUR und HARTManN) II, E.

1930a. Zeitschr. Ind. Abst. Vererb. $54: 76-126$. illus. [Sex.]

1930b. CEsterreich. Bot. Zeitschr. 79: 269-272.

1932. Naturwissenschaften $20: 567-573$. illus. [Isogamy.]

Hartiann, M., und W. Nöller. 1918. Arch. Protist. 38: 355-374. illus.

HartmanN, O. 1919a. Arch. Zellf. 15:160-176. illus. [Chromatophores.]

Harvey, L. A. 1925. Quar. Jour. Micr. Sci. 69: 291-316. illus.

Harvey, Wm. 1651. Exercitationes de Generatione Animalium.

Haupt, A. W. 1921a. Bot. Gazette 71:61-74. illus. [Reboulia.]

1923. Bot. Gazette 75:170-190. pl. 7. [Cyanophyceæ.]

1926. Bot. Gazette 82:30-54. illus. [Preissia.]

1932. Amer. Jour. Bot. 19: 239-254. illus. [Zonaria.]

Hazen, T. E. 1922. Bull. Torr. Bot. Club 49: 123-137. pls. 5, 6.

Hedayetullah, S. 1931. Jour. Roy. Micr. Soc. 51:347-386. illus. [Narcissus.] 1932. Jour. Genetics $26: 179-197$. illus. [CEnothera.]

Hegler, R. 1901. Jahrb. Wiss. Bot. 36:229-354. pls. 5, 6. [Cyanophyceæ.]

Hegner, R. W. 1914a. Jour. Morphol. 25: 375-509. 74 figs. [Keimbahn determinants.]

1914b. The Germ-cell Cycle in Animals. New York.

1919. Proc. Nat. Acad. Sci. 5: 19-22. [Nucleoplasmic ratio.]

Hegner, R. W. and H. Wu. Amer. Naturalist 65 : 335-346. 20 figs.

Heidenhain, M. 1897. Würzburger Sitzungsber.

1902. Anat. Anz. 21: 609-640.

1907, 1911. Plasma und Zelle. Jena.

Heidinger, W. 1908. Ber. Deu. Bot. Gesell. 26:313-364. [Vaucheria.]

Heilborn, O. 1921a. Ark. Bot. 17: 7 pp. 7 figs. [Ananas.] 1921b. Ark. Bot. 17: 16 pp. 1 pl. 17 figs. [Carica.]

1922. Svensk Bot. Tidskr. 16: 271-274. 1 fig. [Carex.]

1924. Hereditas $5: 129-216.1$ pl. 22 figs. [Carex.]

1925. Bibliog. Genetica 1: 459-461. [Carex.]

1932. Svensk Bot. Tidskr. $26: 137-146$. illus. [Carex.]

Heilbrons, A. 1922. Jahrb. Wiss. Bot. 61: 284-338. [Viscosity.]

Heilbrunn, L. V. 1913. Biol. Bull. 24: 345-361. [Activation.]

1915. Biol. Bull. 29 : 149-203. [Artificial parthenogenesis.]

1920a. Jour. Exp. Zoöl. 30: 211-238. [Artificial parthenogenesis.]

1920h. Biol. Bull. 38: 317-339. [Artificial parthenogenesis.]

1920c. Biol. Bull. 39: 307-315. [Anesthesia and viscosity.] 
1921. Jour. Exp. Zoöl. 34: 417-447. [Viscosity and mitosis.]

1923. Amer. Jour. Physiol. 64: 481-498. [Electrical phenomena.]

1924a. Amer. Jour. Physiol. 68: 645-648. [Temperature effects.]

1924b. Amer. Jour. Physiol. 69: 190-199. [Heat coagulation.]

1924c. Biol. Bull. 46: 277-280. [Egg membrane elevation.]

1925a. Joır. Exp. Zoöl. 41: 243-260.

1925b. Biol. Bull. $49: 241-249$.

1925c. Arch. Mikr. Anat. 104:313-316. [Gelation and mitosis.]

1925f. Biol. Bull. 49 : 461-476. [Viscosity.]

1926c. Jour. Exp. Zoöl. 44: 255-278. [Viscosity.]

1927. Quar. Rev. Biol. 2 : 230-248. [Viscosity.]

1928. The Colloid Chemistry of Protoplasm. Berlin.

1929a. Protoplasma 8: 58-64. [Viseosity.]

1929b. Protoplasma 8: 65-69. [Viscosity.]

1930a. Proc. Amer. Phil. Soc. 69 : 295-301. [Surface precipitation.]

Heilbrunn, L. V., and R. A. Young. 1930. Physiol. Zoöl. $3:$ 330-341.

Heinans, J. 1928. Rec. Trav. Bot. Néerl. 25A:138-167. illus. [Lilium.]

Heıtz, E. 1922. Untersuchungen über die Teilung der Chloroplasten, usw. Strassburg.

1925a. Biol. Zentralbl. 45: 179-186. 2 figs. [Chloroplasts.]

1925b. Arch. Wiss. Bot. 1: 241-259. pl. 1. 6 figs. [Mclandrium.]

1927a. Planta 4:392-410. illus. [Antirrhinum.]

1927b. Abh. Naturwiss. Ver. Hamburg 21: 48-58. illus.

1927c. Ber. Deu. Bot. Gesell. $45: 607-610$. illus. [Pcllia.]

1928a. Planta 5: 725-768. illus. [Pellia.]

1928b. Jahrb. Wiss. Bot. 69: 762-818. illus. [Pellia.]

1929a. Ber. Den. Bot. Gesell. 47: 274-284. illus. [Heterochromatin.]

1931a. Planta 12: 775-844. illus. [Nucleoli.]

1931b. Planta 15:495-505. illus. [Nucleoli in Vicia.]

Heitzuann, C. 1883. Microscopical Morphology, etc. New York.

Helvestine, F. 1921. Jour. Morphol. Physiol. 36: 103-117. 2 pls. [Amitosis.]

Helwig, E. R. 1929. Jour. Morphol. Physiol. $47: 1-36$. illus.

1932. Science $76: 352$. illus.

Henckel. 1901. Nyt Mag. Naturvid. 39: 355. [Cystoclonium.]

Henderson, L. J. 1913. Thie Fitness of the Environment. New York.

von Henking, H. 1891. Zeitschr. Wiss. Zool. 51: 685-736. pls. 35-37.

1892. Zeitschr. Wiss. Zool. 54: 1-274. pls. 1-12. 12 figs.

Hennegur, L. F. 1897. Arch. d'Anat. Mier. 1: 481-496. 5 figs.

Hering, G. 1888. Lotos 9. See Brain 20: 232-258. 1897.

Herla, V. 1893. Arch. de Biol. 13: 423-520. pls. 15-19. [Ascaris.]

Herlant, M. 1917. Arch. de Biol. 28: 505-60s. [Artificial parthenogenesis.] 1920. Compt. Rend. Soe. Biol. Paris 83: 188-190. [Artificial parthenogenesis.]

Hermann, J. 1891. Arch. Mikr. Anat. 37:569-586. pl. 31.

Hertwig, G. 1911. Areh. Mikr. Anat. 77: II 165-209. illus. [Radium.]

1912. Arch. Mikr. Anat. 79: II 201-241. illus. [Radium.]

1913. Arch. Mikr. Anat. 81: II 87-127. illus. [Radium.]

1918. Areh. Mikr. Anat. 91: II 1-271. 10 pls.

1920a. Arch. Mikr. Anat. 94: 2SS-302. 1 fig.

1920b. Zeitschr. Strahlentherapie 11. [Radium experimentation.]

1921. Biol. Zentralbl. $41: 49-87$. [Sex.]

Hertwig, G., und P. Hertwig. 1922. Zeitschr. Ind. Abst. Vererb. 28: 259-294.

Hertwig, G., F. K. Stunnicka, and E. Tschope. 1929. Allgemeine Mikroskopische

Anatomie der Lebenden Masse. Berlin. 
Hertwig, O. 1875. Morph. Jahrb. 1. See also 2-4. [Fertilization.]

1884. Jenaische Zeitschr. 18: 276-318. [Fertilization.]

1890. Areh. Mikr. Anat. 36: 1-138. pls. 1-4.

1893. Die Zelle und die Gewebe. Jena.

1900. Die Entwicklung der Biologie im 19 Jahrhundert. Jena.

1909. Allgemeine Biologie. 3d ed. Jena.

1910. Sitzungsber. Kigl. Preuss Akad. Wiss. 11.

1911, 1913. Areh. Mikr. Anat. 77: II 1-164; 82. [Radium.]

Hertwig, O., and R. Hertwig. 1887. Jenaisehe Zeitsehr. 20 : 120-241. illus.

Hertwig, P. 1911. Areh. Mikr. Anat. 77: II 301-312. illus. Also 81: II.

1920a. Biol. Zentralbl. 40: 145-174. [Parthenogenesis.]

1920b. Areh. Mikr. Anat. 94:303-337. pl. 21. [Parthenogenesis.]

1923. Areh. Mikr. Anat. 100: 41-60. 1 pl. 3 figs. [Enucleation.]

Hertwig, R. 1889. Abh. Bayer. Akad. Wiss. II 17.

189Sa. Sitzber. Gesell. Morph. u. Physiol. Münehen 14.

189\$b. Abh. Bayer. Akad. Wiss. II 19: III.

1899. Festschr. f. C. von Kuppfer. Jena. [Chromidia.]

1902. Areh. Protist. 1.

1903. Biol. Centralbl. 23 : 49-62, 108-119. [Kernplasma relation.]

1904. Festschr. f. E. Haeckel, 301-354. pls. 9-12. [Chromidia.]

1908. Areh. Zellf. 1: 1-32. figs. 9.

van Hertwerden, M. A. 1910, 1911. Anat. Anz. 36: 193-207; 38:387-393.

1913. Arch. Zellf. 10: 431-449. 14 figs. [Chromidia.]

1917. Proc. See. Sei., Koninkl. Akad. Wetensch. Amsterdam 20. [Volutin.]

Herzfeld, S. 1927. Jahrb. Wiss. Bot. 66: 814-862. illus. [Ginkgo.]

Herzog, R. O., and W. JANCKe. 1921. Festsehr. Kiaiser Wilhelm. Gesellschaft.

Heuser, F. 1884. Bot. Centralbl. 17: 27, 57, 117, 154. pls. 1, 2.

Hers, F. 1931. Quar. Rer. Biol. 6:1-45. [Germ-cells.]

Hibbard, H. 192S. Jour. Morphol. Physiol. 45: 233-257. illus.

Н1ск, T. 1883. Nature 28: 581. [Plasmodesms.]

1855. Jour. Botany 23 : 97-102, 354-357. pl. 255. [Plasmodesms.]

Hicks, G. C. 1928. Bot. Gazette 86: 295-317. illus. [Scirpus.]

Higgins, E. M. 1930. Ann. Botany $44: 587-592$. illus. [Cladophora.] 1931. Ann. Botany $45: 345-353$. illus. [Stypocaulon.]

Hill, A. W. 1900. Proe. Roy. Soe. London 67: 437-439. [Plasmodesms.] 1901. Ann. Botany $15: 575-611$. pls. 31-33. [Plasmodesms.]

Hill, H. E. 1930. Cornell Univ. Thesis. [Trisomie Zea.]

Hult, S. E. 1927. Biol. Bull. 53: 413-415. illus. [Bursa.]

Hırasé, S. 1895: Jour. Imp. Coll. Sei. Tokỵo 8:307-322. illus. 189S. Jour. Imp. Coll. Sei. Tokyo 12: 103-149. illus. [Ginkgo.]

1918. Bot. Mag. Tokyo 32. [Ginkigo.]

Hirata, K. 1924. Jour. Soc. Agr. Forestry 16:37-60. [Cannabis.] 1927b. Jour. Geneties 19 : 65-79. [Cannabis.]

Hinschler, J. 1913. Arch. Zellf. 9: 351-398. pls. 20, 21. [Chromidia.] 1928. Zeitsehr. Wiss. Biol. B $7: 62-82$. illus.

HoAr, C. S. 1931. Bot. Gazette $92: 396-406$. [Hypericum.]

HöвER, R. 1909 . Biochem. Zeitsehr. $20: 56$.

1922. Physikalisehe Chemie der Zelle und der Gewebe. Leipzig.

Hobson, A. D. 1927. Proe. Roy. Soe. Edinb. 47: I 94-117. [Fertilization membrane.]

Hoefer, K. 1932. Verdoorn's Manual of Bryology, Chap. VII. The Hague.

Hoeppener, E., and O. Renner. 1928. Zeitschr. Ind. Abst. Vererb. 49: 1-25. illus. 
Hof, A. C. 1898. Bot. Centralbl. 76:65-69, 113-118, 166-171, 221-226. pls. 3, 4. Hoffmann, K. 1929. Ber. Deu. Bot. Gesell. 47 : 321-326. [Orchids.]

Hofmeister, W. 1847. Bot. Zeitung 5: 785-782. illus. [Cell-division.]

1848. Bot. Zeitung 6:425-434, 671-674. illus. [Cell-division.]

1849. Die Entstehung des Embryos der Phanerogamen. Leipzig.

1858. Jahrb. Wiss. Bot. 1: 82-188. illus.

1862. Flora 45: 497-503, 513-517. [Protoplasm.]

1865. Flora 48: 7-12. [Protoplasm.]

1867. Die Lehre von der Pflanzenzelle. Leipzig.

Hogben, L. T. 1920a. Proc. Roy. Soc. London B 91:305-329. illus.

Holden, R. J., and R. A. Harper. 1903. Trans. Wis. Acad. Sei. 14: 63-82. illus.

Hollaender, A., and E. Schoeffel. 1931. Quar. Rev. Biol. 6: 215-222. illus.

Hollingshead, L. 1928a. Amer. Naturalist 62: 283-284. [Monoploid Crepis.] 1930a. Univ. Calif. Publ. Agr. Sci. 6: 55-94. illus.

1930c. Univ. Calif. Publ. Agr. Sci. 6: 107-134. illus. [Monoploid Crepis.] 1932. Cytologia 3:119-141. illus. [Triticum.]

Hollingshead, L., and E. B. Babcock. 1930. Univ. Calif. Publ. Agr. Sci. 6: 1-53. illus.

Holman, R. M., and W. W. Robbins. 1928. A 'Textbook of General Botany. New York.

Holmgren, I. 1916. Svensk Bot. Tidskr. 10: 263-268. 10 figs. [Eupatorium.] 1919. Kgl. Svensk Vet. Handl. 59: 118 pp. 24 figs. [Eupatorium, Erigeron.]

Holt, C. M. 1917. Jour. Morphol. 29: 609-627. 4 pls. [Culex.]

Номès, M. 1929. Acad. Roy. Belg., Cl. d. Sci., Mém. in 8vo. 10: 5-54.

Нооке, R. 1665. Micrographia. London.

Horne, A. S. 1930. Ann. Botany 44:199-230. illus. [Plasmodiophorales.]

Horning, E. S. 1926. Austral. Jour. Exp. Biol. Med. Sci. 3: 89-95. illus.

1927a. Austral. Jour. Exp. Biol. Med. Sci. 4: 69-73. illus. [Chondriosomes.]

1927b. Austral. Jour. Exp. Biol. Med. Sci. 3 : 149-159. illus. [Chondriosomes.]

1928a. Austral. Jour. Exp. Biol. Med. Sei. 5: 143-148. illus. [Chondriosomes.]

Horning, E. S., and A. H. K. Petrie. 1927. Proc. Roy. Soc. London B 102: 188-206. illus.

Hoven, H. 1910a. Arch. de Biol. 25: 427-494. pls. 15, 16. [Neurofibrils.] 1910b. Anat. Anz. 37:343-351. 7 figs. [Gland cells.]

Howard, G. E. 1925. Ann. Missouri Bot. Garden 12: 145-212. [Carotinoids.]

Howard, W. T. 1910. Festschr. f. R. Hertwig, pp. 1-18. pls. 1-3. ['Tumors.]

Howard, W. T. and Schultz, O. T. 1910. Jour. Exp. Med. [Tumors.]

Huettner, A. F. 1923. Jour. Morphol. 37: 385-423. 2 pls. [Germ cells.]

1924. Jour. Morphol. $39: 249-265.3$ pls. [Meiosis and syngamy in Drosophila.]

1927. Zeitschr. Zellf. Mikr. Anat. 4: 599-610. illus. [Polyspermy.]

1930. Zeitschr. Zellf. Mikr. Anat. 11:615-637. illus. [Meiosis.]

Hughes-Schraner, S. 1925. Zeitschr. Zellf. Mikr. Anat. 2: 264-292. illus. 1926. Science 68: 500-501. [Icerya.]

1927. Zeitschr. Zellf. Mikr. Anat. 6:509-540. illus. [Icerya.]

1930a. Ann. Entomol. Soc. Amer. 23: 359-380. [Icerya.]

1930b. Jour. Morphol. Physiol. 50: 475-495. illus. [Icerya.]

Hume, M. 1913. New Phytol. $12: 216-221.1$ fig. [Plasmodesms.]

Hurst, C. C. 1925. Experiments in Genetics. Cambridge University Press.

1927a. Science 65:271-273. [Rosa species.]

1927b. Zeitschr. Ind. Abst. Vererb. 46:46-47. [Polyploidy in Rosa.]

1927c. Eugenies Review 19: 19-31. [Polyploidy in Rosa.]

1927d. Cambridge Meeting of Genetical Society, July 5.

1928. Zeitschr. Ind. Abst. Vererb., suppl., 2 : 866-906. 
1929a. Rose Annual, 1929, 37-64. illus. [Genetics of Rosa.]

1929b. Conference on Polyploidy, J. Innes Hort. Inst., Jan. 19.

1930. Gardener's Chronicle, Oct. 18. [Species concept.]

1931. Proc. Roy. Soc. London B 109: 126-148. illus. [Rosa: ‥]

Huskins, C. L. 1927. Jour. Geneties $18: 315-364$. illus. [Fatuoid oats.]

1928a. Zeitschr. Ind. Abst. Vererb., suppl., 2: 907-916. illus.

1928b. Jour. Genetics $20: 103-122$. illus. [Speltoid wheats.]

1931a. Jour. Genetics 25: 113-124. illus. [Dwarf wheat.]

1931c. Genetica $12: 531-538$. [Spartina.]

1932a. Trans. Roy. Soc. Canada, Sec. V, 1-12. [Meiosis.]

1932b. Proc. 6 Internat. Cong. Genet. 2: 95-96. [Meiosis.]

1933. Nature 132:62-63. illus. [Meiosis.]

Huskins, C. L., and S. G. Smith. 1932. Jour. Genetics $25: 241-249$. illus.

Hutchinson, A. H. 1915. Bot. Gazette 60:457-472. pls. 16-20. 1 fig. [Abies.] Huth, W. 1933. Zeitschr. Zellf. Mikr. Anat. 17: 51-66. [Bělař on Urechis.]

Huxley, J. S. 1912. The Individual in the Animal Kingdom. Cambridge.

Huxley, T. H. 1868. The Physical Basis of Life. (Collected essays.)

1878. Evolution in Biology. (Collected essays.)

HYMAN, L. H. 1916. Jour. Exp. Zoöl. 20:99-163. 24 figs. [Axial gradient.]

1921. Biol. Bull. $40: 32-72.3$ pls. [Axial gradient.]

Hyman, L. H., and A. W. Bellamy. 1922. Biol. Bull. 23: 313-348. [Axial gradient.]

Iснiлina, K. 1926. Genetics 11:590-604. illus. [Fragaria.]

IKARI, J. 1923. Bot. Mag. Tokyo 37: 96-108. illus. [Coscinodiscus.]

IKEDA, T. 1928. Folia Anat. Japon. 6: 389-423. illus. [Golgi and chondriosomes.]

Ikeno, S. 1898. Jahrb. Wiss. Bot. 32:557-602. pls. 8-10. [Cycas.]

1901. Ann. Sci. Nat. Bot. VIII 13:305-318. pls. 2, 3. [Ginkgo.]

1903. Beih. Bot. Centralbl. 15: 65-88. pl. 3. [Marchantia: $\left.\sigma^{7}.\right]$

1904. Beih. Bot. Centralbl. 24 : 211-221. 3 figs. [Blepharoplasts.]

1906. Flora $96: 538-542$. [Homology of blepharoplasts.]

Illick, J. T. 1929. Genetics 14: 591-633. illus. [Enothera.]

1932a. Bot. Gazette 93:313-327. illus. [Enothera.]

1932b. Bot. Gazette $94: 1-50$. [Enothera.]

InAriyama, S. 1928. Bot. Mag. Tokyo 42:486-489. illus. [Hosta.]

Ingvar, S. 1920. Proc. Soc. Exp. Biol. Med. 17 : 198-199. [Polarity.]

Iriki, S. 1932a. Proc. Imp. Acad. (Japan) 8: 262-263. [Fish chromosomes.]

1932b. Sci. Rep. Tohoku Bunrika Daigaku B 1:127-131. illus. [Fish chromosomes.]

Irvine, J. C. 1927. Carbohydrates. In Contemporary Developments in Chemistry. Columbia University Press.

Ishikawa, M. 1916. Bot. Mag. Tokyo 30: 404-448. [Plant chromosome numbers.]

1918. Ann. Botany 32:279-317. pl. 7. 14 figs. [Enothera.]

1921a. Bot. Mag. Tokyo 35: 206-218. pl. 4. [Porphyra.]

1921b. Bot. Mag. Tokyo 35: 153-159. [Lactuca.]

JAckson, H. S. 1931. Bull. Torr. Bot. Club 18.: 1-108. [Uredinales.]

JAcobs, M. H. 1924. Chapter III in General Cytology, ed. by Cowdry. [Permeability.] 
JACOBS, W. 1927. Ergeb. Biol. 2: 357-415. illus. [Golgi; review.]

1929. Zeitsehr. Wiss. Biol. B 8: 1-62. illus. [Golgi.]

Jacobsson-Stiasny, E. 1914. Sitzber. K. Akad. Wiss. Wien Math.-Nat. Kl. I 123 : $1-137$.

Jahn, E. 1904. Ber. Deu. Bot. Gesell. 22 : 84-92. pl. 6. [Stemonitis.] 1908. Ber. Deu. Bot. Gesell. 26a: 342-352. [Ceratiomyxa.]

1911. Ber. Deu. Bot. Gesell. 29 : 231-247. pl. 11.

Jameson, A. P. 1920. Quar. Rev. Mier. Sci. 64: 207-266. illus. [Diplocystis.]

JANet, C. 1914. L'Alternanee Sporophyto-Gamétophytique de Générations chez les Algues. Limoges.

Janssens, F. A. 1901. La Cellule 19:7-116. 3 pls. [Triton: spermatogenesis.] 1905. La Cellule $22: 379-425.7$ pls. [Batracoseps: spermatogenesis.]

1909. La Cellule 25: 389-411. 2 pls. [Chiasmatypy.]

1924. La Cellule 34: 135-359. 21 pls. [Chiasmatypy.]

Janssens, F. A., and J. Willems. 1909. La Cellule 25: 151-178. 2 pls.

JANsson, G. 1927. Acta Radiol. 8: 427.

Jaretzky, R. 1927a. Ber. Deu. Bot. Gesell. 45: 48-54. illus. [Polygonaceæ.] 1927b. Jahrb. Wiss. Bot. 66: 301-320. illus. [Rumex.]

1928a. Jahrb. Wiss. Bot. 68: 1-45. illus. [Crueiferæ.]

1928b. Jabrb. Wiss. Bot. 69: 357-490. illus. [Polygonaceæ.]

1930. Planta 10:120-137. illus. [Fagales.]

1932. Jahrb. Wiss. Bot. 76:485-527. illus. [Crueifere.]

JAretzky, R., and T. Triebel. 1932. Areh. Pharm. u. Ber. Deu. Pharmazeut. Gesell., 94-97.

Jasswoin, G. 1925. Zeitschr. Zellf. Mikr. Anat. 2: 741-765. illus.

Jeffrer, E. C. 1922. Science 55: 566-567. [Druses.] 1925a. Amer. Nat. 59: 209-217. [Polyploidy.]

Jeffrey, E. C., A. E. Longley, and C. W. T. Penland. 1922. Science 55:517-518. Jenkins, J. A. 1929. Amer. Jour. Bot. 16:238-245. illus. [Triticum $\times$ Egilops.] Jenkins, J. A., and W. P. Thompson. 1930. Canad. Jour. Res. 2: 162-170.

Jennings, H. S. 1920. Life and Death, Heredity and Evolution in Unicellular Organisms. Boston.

1923. Proc. Nat. Acad. Sci. 9: 141-147. See also Geneties $8: 393-457$.

1924. Sei. Mo. 19: 225-238. [Heredity and environment.]

1927. Science $65: 19-25$.

1929. Bibliog. Genetica 5 : 105-330. illus. [Geneties of Protozoa.]

1930. The Biological Basis of Human Nature. New York.

Johansen, D. A. 1929. Amer. Jour. Bot. 16: 595-597. [Onagraceæ.]

Johanssen, W. 1923. Hereditas 4: 133-141. [Units of heredity.]

Johnson, D. S. 1907. Johns Hopkins Univ. Circ. 195: 19-21. pls. 5, 6.

1914. Science 39: 299-319. [Sex in plants; historical.]

Johnson, H. H. 1922. Seience 56: 759-760. [Geanthus.]

1931. Zeitsehr. Wiss. Zool. 140: 115-166. illus. [Gryllidæ.]

1932. Proc. Soc. Exp. Biol. Med. 29: 818-821.

Johnson, L. N. 1893. Bot. Gazette 18: 294-298. pl. 32. [Draparnaldia.] Jollos, V. 1930. Biol. Zentralbl. 50:5+1-554. [Mutation.]

Jones, D. F. 1925. Geneties in Plant and Animal Improvement. New York.

1928. Selective Fertilization. University of Chicago Press.

1931. Science 73: 432. [Dioceious maize.]

Jones, S. G. 1925. Ann. Botany 39: 41-75. pl. 4. 23 figs. [Rhytisma.]

Jönsson, B. 1892. Ber. Deu. Bot. Gesell. 10:494-513. pl. 27.

Jolidan, H. E., and F. Helvestine. 1923. Anat. Rec. 25:7-17. 1 pl. [Cilia.] 
Jórgensen, C. A. 1923. Dansk. Bot. Tidskr. 38: 81-126. 19 figs. [Callitriche.] 1928. Jour. Genetics 19:133-211. illus. [Solanum.]

Jörgensen, I., and W. Stiles. 1917. New Phytologist. Reprint No. 10.

Jörgensen, M. 1910ab. Areh. Zellf. 4:163-242. pls. 11-15. Fest. f. R. Hertwig. 1913a. Arch. Zellf. 10: 1-126. pls. 1-12. 15 figs. [Egg growth.]

1913b. Arch. Zellf. 10:125-160. pls. 13-18. [Pisciola.]

1913c. Areh. Zellf. 10: 161-202. pls. 19, 20. 11 figs. [Chromidia.]

Joret-Lavergne, P. 1927a. Compt. Rend. Soc. Biol., Paris 96 : 25-26.

1927b. Compt. Rend. Acad. Sci. Paris 184: 293-295. [Sex.]

1928a. Compt. Rend. Acad. Sci. Paris 186: 595-597. [Chondriome in Saprolegnia.]

1928b. Compt. Rend. Soc. Biol., Paris 98: 567-569. [Nucleolus.]

192Sc. Protoplasma 3: 357-390. illus. [Sex.]

1929. Compt. Rend. Acad. Sci. Paris 189: 409-412. [Sex.]

1932. La 'Théorie Physico-chimique de la Sexualité. Paris (Doin).

Juel, H. O. 1S9Sa. Jahrb. Wiss. Bot. 32:361-38S. pl. 4. [Basidium.]

1898b. Bot. Centralbl. 74:369-372. [Antennaria: parthenogenesis.]

1900. Kógl. Svensk Vet. Akad. Handl. $33: 59$ pp. 6 pls. [Antennaria.]

1904. Ark. Bot. 2: 1-9. [T'araxacum.]

1905. Kgl. Svensk Vet. Akad. Handl. 39:1-20. pls. 1-3. [Taraxacum, etc.]

Jungers, V. 1930. La Cellule 40:7-82. illus. [Plasmodesms.]

1931. La Cellule $40: 293-353$. illus. [Cell plate.]

1933. La Cellule $42: 7-28$. illus. [Comnections in algae.]

Just, E. E. 1912. Biol. Bull. 22 : 239-251. [Cleavage plane.]

1920. Biol. Bull. 39: 280-305. [Activation of egg.]

1922abc. Biol. Bull. 43: 384-400; 401-410; 411-422. [Activation.]

1923. Biol. Bull. 44:1-21. [Fertilization reaction.]

1933. Zeitschr. Zellf. Mikr. Anat. 17: 25-50. illus.

II

KABsch, W. 1863a. Zeitschr. Anal Chem. 2:216-217. [Starch.]

1863b. Jahrb. Wiss. Bot. $3: 357-399$. [Chemistry of tissues.]

Kachidze, N. 1929. Planta $7: 482-502$. illus. [Dipsacaceæ.]

Kagawa, F. 1927a. La Cellule 37: 231-322. illus. [Triticum chromosomes.]

1927b. Proc. Inp. Acad. Japan 3: 304-306. [Triticum chromosomes.]

1928. Jap. Jour. Bot. 4:1-26. illus. [Triticum $\times$ Egilops.]

1929a. Jour. Coll. Agr. Imp. Univ. Tokyo 10: 273-228. illus.

1929b. Jap. Jour. Bot. $4: 345-361$. illus.

1929c. Jap. Jour. Bot. $4: 363-383$. illus. [Chromosomes in cereals.]

Kahle, W. 1908. Zoologica 21: [Miastor.]

Karling, J. S. 1926. Bull. Torr. Bot. Club 53: 319-379. illus. [Nitella.]

Karpechenko, G. D. 1924a. Bull. Appl. Bot. Plant Breed. 13:1-14. [Cruciferce.] 1924b. Jour. Geneties 14:375-396. pls. 21, 22. [Brassica.]

1927a. Hereditas 9:249-268. illus. [Raphanus $\times$ Brassica.]

1927b. Bull. Appl. Bot. Plant Breed. 17: (3) 305-408. illus. [Raphanus $\times$ Brassica.]

1927c. Bull. Appl. Bot. Plant Breed. 17: (4) 343-350. [Triticum $\times$ Egilops.]

1928. Zeitsehr. Ind. Abst. Vererb. 48:1-85. illus. [Raphanus $\times$ Brassica.]

1930. Proe. U. S. S. R. Cong. Genet., etc. 2: 277-294. illus. [Raphanus $\times$ Brassica.]

Karpechenko, G. D., and S. A. Shchavinskala. 1930. Proc. U. S. S. R. Cong. Genet, etc. $2: 267-276$. illus. 
Karper, R. E., and A. B. Conner. 1931. Genetics 16:291-308. [Sorghum.] Karpova, L. 1925. Zeitschr. Zellf. Mikr. Anat. 2 : 495-514. illus.

Karsten, G. 1897. Flora 83:33-53. pls. 1, 2. [Diatoms.]

1900. Flora $87: 253-283$. pls. 8-10. [Diatoms.]

1904. Ber. Deu. Bot. Gesell. 22 : 544-554.

1907. Wiss. Ergeb. Deu. Tiefsee Exped. 2.

1908. Flora 99:1-11. pl. 1. [Spirogyra zygote.]

1912. Zeitschr. Bot. 4: 417-426. pl. 7. [Surirella: meiosis.]

1915. Zeitschr. Bot. 7:1-34. [Growth periodicity.]

1918. Zeitschr. Bot. 10:1-20. pl. 1. [Mitosis: periodicity.]

1924. Internat. Rev. Gesammt. Hydrobiol. Hydrograph. 12 : 116-120.

Kassuann, F. 1926. Planta 1:624-656. illus. [Chondriosomes.]

Kater, J. McA. 1927a. Biol. Bull. 62: 436-449. illus. [Yeast.]

1928c. Zeitschr. Zellf. Mikr. Anat. 8:186-221. illus. [Yolk.]

1929a. Univ. Calif. Publ. Zool. 33 : 125-168. illus. [Chlamydomonas.]

1929b. Northwest Sci. Assoc. [Amitosis.]

Kater, J. McA., and D. M. Smith. 1932. Anat. Rec. 52: 55-68. illus.

Kauffiann, H. 1914. Zeitschr. Bot. 6: 721-784. [Cylindrocystis.]

Kaufmann, B. P. 1925. Amer. Naturalist 59: 190. [Tradescantia.]

1926a. Amer. Jour. Bot. 13: 59-80. illus. [Tradescantia.]

1926b. Aner. Jour. Bot. 13: 355-363. illus. [Podophyllum.]

1931a. Amer. Naturalist 65: 280-282. [Chromonemata.]

1931b. Amer. Naturalist 65: 555-558. illus. [Drosophila.]

Kawakami, J. 1930. Bot. Mag. Tokyo 44:319-328. illus. [Leguminosæ.]

Keene, M. L. 1914. Ann. Botany 28: 455-470. pls. 35, 36. [Sporodinia.]

Kellicott, W. E. 1904. Bull. 'Torr. Bot. Club 31: 529-550. [Periodicity.]

KellogG, V. L. 1907. Darwinism Today. New York.

Kenp, H. P. 1910. Ann. Botany $24: 775-803$. pls. 66, 67.

Kемг, T. 1929. Zeitschr. Mikr. Anat. Forsch. 15:1-20. illus.

Kemp, T., and J. JuUl. 1930. Acta Path. Microbiol. Scand. 7: 279-308. illus.

Keuten, J. 1895. Zeitschr. Wiss. Zool. 60:215-235. pl. 11. [Euglena.]

Kienitz-Gerloff, F. 1891. Bot. Zeitung 49: 1, 17, 33, 49, 65. 2 pls.

Kinernan, F. P., and O. E. White. 1926. Jour. Heredity 17: 383-386. [Mirabilis.]

Kiesel, A. 1925. Zeitschr. Physiol. Chem. 150: 149. [Protoplasm: analysis.]

1927. Zeitschr. Physiol. Chem. 164: 103.

1930. Chemie des Protoplasmas. Berlin.

Kieser, 1812. Mćmoire sur l'Organisation des Plantes.

Kinara, H. 1919ab. Bot. Mag. Tokyo 32: 17-38. figs. 21. 33: 95-98. 1921. Bot. Mag. Tokyo $35: 19-44$. pl. 1. [Triticum hybrids.]

1924. Mem. Coll. Sci. Kyoto Imp. Univ. B 1: 1-200. 5 pls.

1925. Jap. Jour. Bot. 2 : 299-304. pl. 14. [Triticum.]

1926. Zeitschr. Ind. Abst. Vererb 41: 41-42. [Fragaria.]

1927a. Jahrb. Wiss. Bot. 66: 429-460. illus. [Rumex, Enothera.]

1928. Bot. Mag. Tokyo $42: 237-238$. illus. [Humulus.]

1929a. Jap. Jour. Genetics $4: 55-63$. illus. [Humulus.]

1929b. Jap. Jour. Genetics $4: 90-101$. [Sex-chromosomes in plants.]

1929c. Cytologia 1: 1-15. illus. [Triticum $\times$ Egilops.]

1929d. Jap. Jour. Genetics 5 : 73-80. illus. [Humulus.]

1930a. (Kinara and Nishiyama). Cytologia 1: 263-284. illus. [Triticum;

Egilops.]

1930b. Cytologia 1:345-367. illus. [Fragaria.] 
1931. (Kinara and Katayama). Cytologia 2: 234-255. illus. [Triticum, Egilops.]

1932. Jap. Jour. Bot. 6:35-62. illus. [Triticum.]

Kihara, H., and F. Lilienfeld. 1932. Cytologia 3: 384-456.

Kinara, H., and I. Nishiyama. 1928. Bot. Mag. Tokyo 42:221-231. illus.

Kihara, H., and T. Ono. 1923ab. Bot. Mag. Tokyo 37: (84)-(90). [Rumex.]

1925. Zeitschr. Ind. Abst. Vererb. 39: 1-7. illus. [Rumex.]

1926. Zeitschr. Zellf. Mikr. Anat. 4:475-481. illus. [Rumex.]

Kinara, H., and Y. Yamanoto. 1931. Cytologia 3: 84-118. illus. [Rumex.]

Kinara, H., Y. Yamamoto, and S. Hosono. 1931. A List of Chromosome Numbers of Plants Cultivated in Japan. Tokyo.

Kildahl, N. J. 1908. Bot. Gazette 46:339-348. pls. 20-22. [Phyllocladus.]

Kindred, J. E. 1927. Jour. Morphol. Physiol. 43: 267-297. illus.

KING, H. D. 1912. Jour. Exp. Zoöl. 12:319-336. [Sex-determination in amphibia.]

KInG, R. L. 1923. Jour. Morphol. 38: 19-63. 8 pls. [Pseudotrimerotropis.]

1931. Jour. Morphol. Physiol. 52 : 525-533. illus. [Mantidæ.]

King, S. D. 1926. Proc. Roy. Soc. London B 100:1-10. illus. [Oniscus.]

1927. Jour. Roy. Micr. Soc. $47: 342-355$. illus. [Golgi in Protozoa.]

King, S. D., and J. B. Gatenby. 1923. Quar. Jour. Mier. Sci. 67: III 381-390.

Kingery, H. M. 1917. Jour. Morphol. 30:261-316. 2 pls.

K1ngsbury, B. F. 1912. Anat. Rec. 6: 39-52. [Cytoplasmic fixation.]

Kinney, E. 1926. Biol. Bull. 51: 405-434. illus. [Secretion.]

Kirby, K. S. N. 1928. Jour. Roy. Micr. Soc. 48: 10-35. illus. [Plastids.]

Kirchensteins, A. 1922a. Structure Intérieure et Mode de Développement des Bactéries. $90 \mathrm{pp} .7 \mathrm{pls}$. Riga, University de France.

Kisliak-Statkewitsci, M. 1927. Zeitschr. Wiss. Biol. D $109: 283-286$.

Kisser, J. 1922. Sitzber. Akad. Wiss. Wien, Math.-Naturw. Kl. I 131: 105-128.

Kıte, G. L. 1913. Amer. Jour. Physiol. 32: 146-164. [Microdissection.]

Kryohara, K. 1927. Bot. Mag. Tokyo 41: 211-218. illus. [Plastids.]

1930. Cytologia 1:328-334. illus. [Plastids.]

Klebahn, H. 1892. Jahrb. Wiss. Bot. 24:235-267. pl. 3. [Edogonium.]

1896. Jahrb. Wiss. Bot. $29: 595-654$. pl. $10 . \quad$ [Rhopalodia.]

KLeBs, G. 1883. Untersuch. Bot. Inst. Tübingen 1:233-262. pls. 2, 3.

1887. Biol. Centralbl. $7: 161-168$. [Influence of nucleus.]

1888. Untersuch. Bot. Inst. Tübingen $2: 489-568$. pls. 5, 6.

1891. Bot. Zeitung 49: 789, 805, 821, 837, 853. pl. 9.

1892. Zeitschr. Wiss. Zool. 55 : 265-445. pls. 13-18.

KLein, E. 1878. Quar. Jour. Micr. Sci. 18: 315-339. pl. 16.

1879. Quar. Jour. Micr. Sci. 19: 125-175. pl. 7. Also Centralbl. Med. Wiss.

Klingstedt, H. 1928. Mém. Soc. Fauna et Flora Fennica 4:32-37.

KnıeP, H. 1911. Zeitschr. Bot. 3: 529-553. pls. 3, 4. [Hymenomycetes.]

1913. Zeitschr. Bot. 5: 593-637. pls. 2-5. [Hymenomycetes.]

1915, 1916, 1917. Zeitschr. Bot. $7: 369 ; 8: 353 ; 9: 81$. [Hymenomycetes.]

1919. Zeitschr. Bot. 11:257-284. [Ustilago.]

1920. Verhandl. Phys.-Med. Gesell. Würzburg 46:1-18. [Ustilago: sex.]

1922. Verhandl. Phys.-Med. Gesell Würzburg 47:1-29. figs. 6. [Aleurodiscus.]

1928. Die Sexualität der Niederen Pflanzen. Jena.

1930. Zeitschr. Bot. $22: 433-441$. illus. [Allomyces.]

Knight, M. 1923. Trans. Roy. Soc. Edinb. 53: II 343-360. 6 pls. [Pylaiella.]

1931. Beih. Bot. Centralbl. I 48: 15-37. illus.

Koch, A. 1925. Zeitschr. Zellf. Mikr. Anat. 2 : 293-346. illus.

Koelredter, J. G. 1761-1766. Vorläufige Nachricht von einigen das Geschlecht der Pflanzen betreffenden Versuchungen und Beobachtungen. 
Koenperich, J. 1930. La Cellule 39: 309-397. illus. [Mitosis.]

lífold, C. A. 1915. Seience 42 : 658. [Protozoan nueleus.] 1923. Seience $57: 397-408$. [Life eycle of Protozoa.]

Kiofoid, C. A., and E. B. Christensen. 1915a. Proc. Nat. Acad. Sci. 1: 547-552. 1915b. Univ. Calif. Publ. Zoöl. 16: 30-54. pls. 5-8. [Giardia.]

Kofold, C. A., and O. Swezy. 1915a. Proc. Nat. Acad. Sci. 1:315-321. 9 figs. 1915b. Proc. Amer. Acad. Arts and Sei. Boston $2: 289-378.8$ pls.

1920abcd. Univ. Calif. Publ. Zoöl. 20 : 21-40, pls. 3, 4; 41-98, pls. 2-12; 99-116. pls. $13-14 ; 117-144$, pls. $15-17$.

1922. Univ. Calif. Publ. Zoöl. 20 : 199-234. pls. 23-26. 11 figs.

1923. Univ. Calif. Publ. Zoöl. $20: 373-390$. pl. 37. 1 fig.

Koнl, F. G. 1891. Ber. Deu. Bot. Gesell. 9:9-17. pl. 1. [Plasmodesnis.]

1897. Bot. Centralbl. 72:257-265. pl. 4. [Plasmodesms.]

1900. Ber. Deu. Bot. Gesell. 18: 364-372. pl. 12. [Plasmodesms.]

1902. Beih. Bot. Centralbl. 12: 343-350. pls. 10, 11. [Plasmodesms.]

1903. Ueber die Organisation und Physiologie der Cyanophyceenzelle, usw. Jena.

Kojima, H. 1928. Jour. Dept. Agr. Kyushu Imp. Univ. 2: 75-91.

von Kölliker, A. 1845. Zeitschr. Wiss. Bot. 2.

1885. Zeitschr. Wiss. Zool. 42.

Koltzoff, N. K. 1905. Arch. Mikr. Anat. 67: 364-572. pls. 25-29. 37 figs. 1909. Arch. Zellf. 2: 1-65. pls. 1-5. 18 figs.

Konuro, H. 1917. Irigaku Ryôhô Zassi (Jour. Phys. Therapy) No. 6. [X-rays.] 1922. Bot. Mag. Tokyo 36:41-45. [Effect of X-rays on root cells.]

1924a. Bot. Gazette $77: 446-452.1$ fig. [Effect of X-rays on root cells.]

1924d. Jap. Jour. Bot. 2 : 133-156. pls. 7, 8. 12 figs. [X-rays.]

1924e. Gaun 18: 317-327. [X-ray tumors.]

1925a. Zeitschr. Tirebsf. 22: 199-209. illus.

1925b. Bot. Mag. Tokyo 39: 220-224.

1928a. Gann 22:4-14. illus.

1928b. Proc. Imp. Acad. 4: 404-406. illus.

1928c. Zeitsehr. Krebsf. 28: 371-373. illus.

1930a. Zeitschr. Krebsf. 31:490-494. illus.

1930b. Fortschr. Gebiete Röntgenstrahlen 41.

1930c. Gamn 24: 1-16. illus.

Kíndo, M., M. Takeda, and S. Fujnoto. 1927. Ber. Ohara Inst. Landw. Forseh. $3: 291-317$. illus.

Konopack, M. 1927. Bull. Histol. Appl. Physiol. Pathol. 4: 40-51. illus.

Kopsch, F. 1926. Jahrb. Morphol. u. Mikr. Anat. II 5: 221-284. illus.

Kornhauser, S. I. 1914. Arch. Zellf. 12:241-298. pls. 18-22. [Enchenopa.] 1915. Arch. Zellf. 13: 399-445. pls. 17-19. 9 figs. [Hersilia.]

1930. Stain Tech. 5: 117-125. [History of staining.]

Korotneff, A. 1909. Areh. Mikr. Anat. 74: 1000-1016. 2 pls.

Korschelt, E. 1896. Arch. Mikr. Anat. 47: 500-549. pls. 26-28.

Kosiucirow, Z. A. 1928. Angew. Bot. 10: 140-148. illus.

Kosser, A. 1880. Zeitschr. Physiol. Chem. 3, 4. [Nuclein.]

1886. Zeitschr. Physiol. Chem. 10. [Nuelein.]

1896. Zeitschr. Physiol. Chem. 22. [Nuclein.]

1911. Münch. Med. Wochenschr. 58: 65-69. [Chemistry of nucleus.]

Kostaneck1, K. 1911. Arch. Mikr. Anat. 78: II 1-62. pls. 1-4. [Mactra.]

Kostanecki, K., und A. Wierzesski. 1896. Areh. Mikr. Anat. 47: 309-385.

Kostoff, D. 1929. Zeitschr. Zellf. Mikr. Anat. 9: 640-642. illus. 1930c. Jour. Geneties 22 : 399-418. illus. [Grafting.] 
1931c. Svedenia po Zemledelieto Godina 12. [Grafting.]

1931e. Bulgar. Agr. Soc. Sei. Publ. 2S. [Sterility.]

Kostoff, D., and J. Kendall. 1929a. Biol. Bull. 56:402-459. illus. [Galls.]

1929b. Jour. Geneties 21 : 113-115. [Alnormal meiosis.]

1930a. Genetica 12:140-144. illus. [Abnormal meiosis.]

1930b. Zentralbl. Bakt. II 81: 86-91. illus. [Mitosis in galls.]

1931a. Biol. Generalis 7: 271-282. illus.

Kostrioukoff, X. 1930. Bull. Jard. Bot. Kieff 11: 10-17. illus.

Kowalevsky, A. 1871. Mém. Acad. Imp. Sei. St. Petersburg VII 16: 13. pl. 4.

Kowalsk1, J. 1915 (1919). La Cellule 30:83-119. 2 pls. [Pyrrhochoris.]

Krabie, G. 1887. Jahrb. Wiss. Bot. 18: 346-423. illus. [Cell wall.]

Kramer, H. 1902. Bot. Gazette 34: 341-354. pl. 11. [Stareh grain.]

KrR̈̈nzurN, H. 1907. Areh. Protist. 9: 170-194. pl. 4. [Myxomycetes.]

Kretschmer, H. 1930. Arch. Protist. 71: 101-13S. illus. [QEdogonium.]

Kropp, B., and R. M. MAY. 1924. Anat. Ree. 27: 289-292. [Blood cells.]

Ḱrdch, O. 1890. Malpighia 4:403-423. pls. 17, 18. [Riella.]

KrÜGER, E. 1913. Zeitsehr. Wiss. Zool. 105: 87-124. illus. [Rhabditis.]

Krupko, S. 1926. Aeta Soe. Bot. Polon. 4: 77-86. illus. [Plastids.]

Kuczynski, M. H. 1917. Areh. Protist. 38:94-112. pls. 3, 4.

Konla, F. 1900. Bot. Zeitung 58: 29-5S. pl. 3. [Plasmodesms.]

Küнn, A. 1908. Areh. Zellf. 1: 538-586. pls. 18-21. 6 figs.

Kuнn, E. 1928a. Jahrb. Wiss. Bot. 68: 382-430. illus. [Thalictrum.]

1929a. Ber. Deu. Bot. Gesell. $47: 420-430$. illus. [Chromocenters.]

1930b. Der Züchter 2: 124-136. illus. [Pseudogamy.]

1930c. Biol. Zentralbl. 50: 79-102. illus. [Thalictrum.]

1930d. Absts. 5 Internat. Bot. Cong., 161-162.

Kulkarni, C. G. 1929a. Bot. Gazette 87:218-259. illus. [Enothera.] 1929b. Papers Mich. Acad. Sei. 9: 223-225. illus. [Enothera.]

1929c. Amer. Jour. Bot. 16: 606-620. illus. [Enothera.]

Kunieda, H. 1926. Bot. Mag. Tokyo 40:545-550. illus. [Sargassum.]

Kupelwieser, H. 1912. Arch. Zellf. 8: 352-395. pls. 13-15. 4 figs.

Kunssanow, L. 1911. Flora 104:65-84. pls. 1-4. [Zygnema.]

Kusano, S. 1915. Jour. Coll. Agr. Tokyo 6: 7-120. pls. 5-9. 28 figs.

Kǘster, W. 1894. Die Oelkörper der Lebermoose, usw. Inaug. Diss., Basel.

1911. Ber. Deu. Bot. Gesell. 29:362-369. 4 figs. [Plastids.]

1918a. Ber. Deu. Bot. Gesell. 36 : 283-292. 3 figs. [Vacuole.]

1918b. Zeitsehr. Wiss. Mikr. 35. [Vital staining.]

1926. Zeitsehr. Wiss. Mikr. 43. [Vital staining.]

1927. Anatomie des Panaschierten Blattes. Berlin.

1929. Pathologie des Protoplasmas. Berlin.

Kuwada, Y. 1919. Jour. Coll. Sei. Tokyo 39:1-148. 2 pls. [Zea ehromosomes.] 1921. Bot. Mag. Tokyo 35: 99-105. 1 pl. [Vicia: mitosis.]

1925a. Bot. Mag. Tokyo $39: 128-132$. [Cyeas.]

1926a. Mem. Coll. Sci. Kyoto Imp. Univ. B 2:1-13. [Chromosomes.]

1926b. Bot. Mag. Tokyo 40:198-201. illus. [Cycas.]

1927. Bot. Mag. Tokyo 41: 100-109. illus. [Chromosome strueture.]

1928. Bot. Mag. Tokyo 42: 117-129. illus. [Balanophora.]

1929. Mem. Coll. Sei. Kíroto Imp. Univ. B 4: 199-264. illus.

1932a. Bot. Mag. Tokyo 46: 257-258, 307-310. illus.

1932b. Seience (Japanese) 4:180-183. [Chromosome structure.]

Kuwada, Y., and T. Maeda. 1929. Mem. Coll. Sei. Kyoto Imp. Univ. B 4:

165-174. illus. [Cyeas.] 
Kuwada, Y., and T. Sakamura. 1926. Protoplasma 1:239-254. illus. [Chromonemata.]

Kuwada, Y., and T. Suginoto. 1926. Bot. Mag. Tokyo 40:19-20. illus. [Chromonemata.]

KYlin, H. 1913. Zeitschr. Physiol. Chem. 23: 171.

1916a. Zeitschr. Bot. 8: 97-123. 1 pl. 11 figs. [Griffithsia.]

1916b. Zeitschr. Bot. 8: 545-586. 11 figs. [Bonnemaisonia.]

1918. Svensk Bot. Tidskr. 12: 1-64. [Phæophyceæ.]

1920. Ber. Deu. Bot. Gesell. 38: 74-78. 2 figs. [Fucus sperm.]

$\mathrm{L}$

Laburu, R. P. J. A. 1916. Bull. Soc. Espan. Biol. 33: 104-107. [Golgi material.]

Lagerberg, T. 1909. Kgl. Svensk Vet. Handl. 44. [Adoxa.]

Laguesse, E. 1926. Arch. d'Anat. Micr. 22: 129-175. illus.

Laibach, F. 1907. Beih. Bot. Centralbl. 22:191-210. [Prochromosomes.]

LAmarck, J. B. P. 1809. Philosophie Zoologique. Paris. (Elliott's translation.)

Lambin, P. 1923. Revue d. Questions Sei., January and April. [Blood cells.]

Lannerts, W. E. 1931. Genetics 16: 191-211. illus. [Amphidiploid Nicotiana.] 1932. Cytologia 4:46-51. illus. [Amphidiploid Nicotiana.]

LAMPE, L. 1931. Bot. Gazette 91: 337-376. illus. [Carbohydrate reserves.]

Lampe, L., and M. T. Meyers. 1925. Science 290-291. [Storage in Zea.]

Lass, H. 1913. Arch. de Biol. 28: 229-324. pls. 11-14.

LANCEFIELD, D. E. 1922. Geneties $7: 335-384.3$ figs. [Sex-linkage.]

Lancefield, R. C., and C. W. Metz. 1921. Proc. Nat. Acad. Sei. 7: 225-229.

1922. Amer. Naturalist 56: 211-241. 18 figs. [Sex-linkage.]

LaNd, W. J. G. 1900. Bot. Gazette 30:252-260. pls. 15, 16 . [Double fertilization.]

1902. Bot. Gazette 34: 249-259. pls. 6-8. [Thuja.]

1907. Bot. Gazette $44: 273-292$. pls. 20-22. [Ephedra.]

Landis, E. M. 1920. Amer. Naturalist 54: 453-457. [Amicronucleate Paramocium.]

Lang, W. H. 1924. Mem. and Proc. Manchester Lit. and Phil. Soc. 68 : 53.

Langlet, O. F. I. 1927a. Svensk Bot. Tidskr. 21:1-17. illus. [Ranunculaceæ.] 1927b. Svensk Bot. Tidskr. 21: 397-422. illus. [Chromosome doubling.]

1928. Svensk Bot. Tidskr. 22 : 169-184. illus. [Berberidaceæ.]

1932. Svensk Bot. Tidskr. $26: 381-400$. illus. [Ranunculaceæ.]

Latter, J. 1926. Ann. Botany 40:277-313. illus. [Lathyrus.]

Ladghlin, H. H. 1919. Carnegie Inst. Wash. Publ. 265, pp. 488.7 pls.

Lauterborn, R. 1896. Untersuchungen über Bau ... der Diatomen. Leipzig.

LaValette St. George. 1865. Arch. Mikr. Anat. 1. See also 2 and 3.

Lawrence, W. J. C. 1929. Jour. Genetics $21: 125-158$. illus. [Dahlia.]

1931ab. Jour. Geneties $24: 257-306 ; 307-324$. illus. [Dahlia.]

1931c. Cytologia $2: 352-384$. illus. [Secondary association.]

Lawson, A. A. 1904a. Ann. Botany 18: 1-28. pls. 1-4. [Sequoia.]

1904b. Ann. Botany 18: 417-444. pls. 27-30. [Cryptomeria.]

1907. Ann. Botany 21: 1-23. pls. 1-4. [Cephalotaxus.]

1909. Ann. Botany $23: 163-180$. illus. [Pseudotsuga.]

1910. Ann. Botany $24: 403-421$. illus. [Sciadopitys.]

1911a. Trans. Roy. Soc. Edinb. 47 : III 591-604. pls. 1, 2. [Synizesis.]

1911b. Trans. Roy. Soc. Edinb. 48: 137-161. pls. 1-4. [Nuclear osmosis in mitosis.]

1926. Trans. Roy. Soc. Edinb. 54: 357-394. illus. [Bowenia.] 
LAwton, E. 1932. Amer. Jour. Bot. 19: 303-333. illus. [Apospory.]

League, B. B. 1929. Science 71: 99. [Drosophila, meiosis.]

LebedefF, V. N. 1932. Ukrainian Inst. Sei. Res.; Unis'a, Kieff.

LEe, A. B. 1897. La Cellule 13: 199-278. illus. [Helix.]

1921. The Microtomist's Vade-Mecum. 8th ed. Philadelphia.

1924. Quar. Jour. Micr. Sci. 69: 1-25. pl. 1. [Paris chromosomes.]

Lee, B., and J. H. Priestley. 1924. Ann. Botany $38: 525-545.12$ figs.

LeE, S. 1927. Bot. Gazette 83: 420-424. illus. [Stigonema.]

Leeman, A. 1928. Planta 6:216-233. illus. [Secretion.]

van Leeuwenhoek, A. Letter No. 29 to Royal Society, 1680.

Lehfeldt, W. 1922. Hedwigia 64: 30-51. [Hymenomycetes.]

Lehman, E. 1918. Zeitsehr. Bot. 10: 497-511.

1919. Ber. Deu. Bot. Gesell. 37 : 347-357. illus.

1922. Die Theorie der CEnotheraforsehung. Jena.

1924. Biol. Zentralbl. 44 : 243-254. illus.

1925. Zeitsehr. Ind. Abst. Vererb. $37: 1-27$. illus.

1929. Proc. Internat. Congr. Plant Sci. (Ithaea) 1: 787-801.

1932. Zeitschr. Zücht., Reihe A, 17: 157-172. illus.

Lehmann, E., and J. Schwemile. 1927. Biblioth. Bot. 95: 1-156. illus.

Lelivetd, J. A. 1928. Ree. Trav. Bot. Néerl. 25a: 237-243. illus. [Enothera.] 1931. La Cellule 40:195-256. illus. [Enothera.]

von Lenhossék, M. 1898. Verhandl. Anat. Gesell. Kiel. 12: 106.

Lenorr, M. 1923. Compt. Rend. Acad. Sci. Paris 176: 1648-1651. 1927. Compt. Rend. Acad. Sei. Paris 184: 1664-1666. [Lilium, meiosis.]

Lepeschin, W. 1923. Ber. Deu. Bot. Gesell. 41: 179-187.

1924. Kolloidehemie des Protoplasmas.

Lesley, J. W., and M. M. Lesley. 1929. Geneties 14:321-336. illus.

Lestey, M. M. 1925. Amer. Naturalist 59: 570-574. illus. [Chimera.] 1926. Genetics 11:267-279. illus. [Meiosis in triploid.]

Lesley, M. M., and H. B. Frost. 1928. Amer. Naturalist $62: 22-33$. illus.

Levan, A. 1931. Hereditas 15:347-356. [Allium.]

1932. Hereditas $16: 257-294$. illus. [Allium.]

1933. Svensk Bot. Tidskr. 27: 211-232. illus. [Allium.]

Levi, G. 1911. Arch. Ital. Anat. Embr. 10: 168-195.

1916. Monit. Zoöl. Ital. 77-84.

Levine, M. 1913. Bull. Torr. Bot. Club 40:137-181. pls. 4-8. [Boletus.] 1929. Proc. Internat. Cong. Plant Sci. Ithaca 1: 271-297. illus.

1931. Amer. Jour. Cancer 15: 144-211, 788-834, 1410-1494. illus.

LEwis, C. E. 1906. Bot. Gazette 41: 109-138. pls. 5-9. [Riccia.]

LEwis, I. F. 1909. Ann. Botany 23: 639-689. pls. 49-53. [Griffithsia.]

Lewis, M. R. 1917. Carnegie Inst. Wash., Contrib. to Embryol. 17. 1923. Johns Hopkins Hosp. Bull. 34 : 373-379.

Lewis, M. R., and W. H. Lewis. 1915. Amer. Jour. Anat. $17: 339-401.26$ figs.

Lewis, M. R., and J. Lockwood. 1929. Johns Hopkins Hosp. Bull. 44: 187-199.

Lewis, W. H. 1922a. Anat. Rec. 23: 177-184. 4 figs. [Mesenchyme.] 1922b. Amer. Jour. Anat. 30: 39-59. 5 pls. [Endothelium.]

1923. Anat. Rec. 26: 15-29. [Tissue cultures; dark-field.]

Lewis, W. H., and M. R. Lewis. 1917. Anat. Ree. 13: 359-368. [Mitosis.] 1924. Chapter VII in General Cytology, ed. by Cowdry.

Lewitsky, G. A. 1910. Ber. Deu. Bot. Gesell. 28:538-546. pl. 17. [Chondriosomes.]

1911. Ber. Deu. Bot. Gesell. 29:697-703. pl. 28. [Plastid origin.]

1914. Ber. Deu. Bot. Gesell. 31:517-528. 1 pl. [Chondriosomes in fungi.] 
1924. Zeitschr. Bot. $16: 65-89.2$ pls. [Chondriosomes in myxomycetes.] 1925. Arch. Wiss. Bot. 1:310-316. pl. 2. [Equisetum.]

1930. Proc. U. S. S. R. Cong. Genet. Plant Animal Breed. 2 : 87-105. illus. 1931a. Bull. Appl. Bot. Genet. Plant Breed. 27 (1): 19-173. illus.

1931є. Bull. Appl. Bot. Genet. Plant Breed. 27 (1):187-240. illus. [Karyotype in systematics.]

Lewitsky, G. A., et al. 1931d. Amer. Naturalist 65:564-567. illus.

Lewitsky, G. A., and G. A. Araratian. 1931. Bull. Appl. Bot. Genet. Plant

Breed. 27 (1): 265-304. illus.

Lewitsky, G. A., and G. К. Benetzkaja. 1927. Bull. Appl. Bot. Genet. Plant Breed. 17 (3): 289-303. illus.

1930. Proc. U. S. S. R. Cong. Genet. Plant Animal Breed. 2: 345-352. illus. 1931abe. Bull. Appl. Bot. Genet. Plant Breed. 27 (1): 19-173; 175-185; 187-240.

Lewitsky, G. A., and E. J. Tron. Planta 9: 760-775. illus. [Muscari.]

LI, J. C. 1927. Genetics 12:1-58. [Aberrations in Drosophila.]

Lillie, F. R. 1896. Jour. Morphol. 12: 239-249. [Regeneration in Stentor.]

1902. Arch. Entw. 14:477-499. [Differentiation in Chatopterus.]

1906. Jour. Exp. Zoöl. 3 : 153-268. [Chatopterus.]

1912. Jour. Exp. Zoöl. 12: 413-476. pls. 1-11. [Nereis: fertilization.]

1913. Jour. Exp. Zoöl. 14: 515-574. [Fertilization.]

1914. Jour. Exp. Zoöl. 16:523-590. [Fertilization.]

1916. Science 43: 39-53. [Fertilization; historical.]

1917a. Proc. Nat. Acad. Sci. 3: 464-470. [Sex determination.]

1917b. Jour. Exp. Zoöl. 23 : 371-452. [Sex determination.]

1917c. Science 45:354. [Sex determination.]

1919. Problems of Fertilization. Chicago.

1921ab. Biol. Bull. 40:1-22; 23-31. [Fertilization.]

Lillie, F. R., and E. E. Just. 1924. Chapter VIII in General Cytology (Cowdry).

Lillie, R. S. 1902. Amer. Jour. Physiol. 7:412-421. 1 fig. [Oxidation.]

1903. Amer. Jour. Physiol. 8 : 273-283. [Elcctrical charge of nucleus.]

1905. Biol. Bull. 8 : 193-204. illus. [Mitosis.]

1908. Jour. Exp. Zoöl. 5 : 375-428. [Artificial parthenogenesis.]

1913. Jour. Biol. Chem. 15:237-248. pl. 1. [Indophenol.]

1915. Biol. Bull. $28: 260-303$. [Artificial parthenogenesis.]

1919. Sci. Monthly 8: 456-474, 552-567. [Protoplasmic transmission.]

1922. Sci. Monthly 14:113-130. [Growth.]

1923. Protoplasmic Action and Nervous Action. Chicago.

1924a. Chapter IV in General Cytology, ed. by Cowdry.

1924b. Amer. Naturalist 58: 219-236. [Morphogenesis.]

Lind, E. M. 1932. Ann. Botany $46: 711-725$. illus. [Ulothrix.]

Lindegren, C. C. 1932. Bull. Torr. Bot. Club 59:119-138. [Neurospora.] 1933. Bull. Torr. Bot. Club 60:133-154. illus. [Neurospora.]

Lindegren, C. C., and R. R. Mellon. 1932. Proc. Soc. Exp. Biol. Med. 30 : 110-112. illus.

Lindenbein, W. 1927. Planta 4:437-466. illus. [Nitella etc.]

Lindfors, T. 1924. Svensk Bot. Tidskr. 18: 1-84. illus.

Lindsay, R. H. 1929. Proc. Nat. Acad. Sei. 15: 611-613.

1930. Amer. Jour. Bot. $17:$ 152-174. illus.

Lindstron, E. W. 1929. Jour. Heredity $20: 23-30$. illus.

Lindstrom, E. W., and K. Koos. 1931. Amer. Jour. Bot. 18: 398-410. illus.

LinK, G. K. K. 1932. Quar. Rev. Biol. $7: 127-171$. illus. [Genetics in pathology.] Link, H. F. 1807. Grundlehren der Anatomie und Physiologie der Pflanzen.

Linsbauer, K. 1921. Ber. Deu. Bot. Gesell. 39:41-49. [Cystoliths.] 
de Litardière, R. 1921b. La Cellule 31:255-473. 9 pls. [Mitosis in ferns.] 1923b. Bull. Soc. Bot. France 70: 193-197. 3 figs. [Crepis.]

1923c. Rev. Gén. Bot. 35: 369-381. pls. S, 9. [Spinacia.]

1924. La Cellule $35: 21-25$. illus. [Didiploidy.]

1925a. La Cellule $35: 21-25.2$ figs. [Didiploid figures.]

1925b. Compt. Rend. Soc. Biol. Paris. 92: 796-797. [Heat experimentation.]

Ljungdahl, H. 1922. Svensk Bot. Tidskr. 16: 103-114. 6 figs. [Papaver.] 1924. Svensk Bot. Tidskr. 18: 279-291. 4 figs. [Papaver.]

Lloyd, F. E. 1915. Yearbook Carnegie Inst. Wash. 14: 66-69. [Protoplasm.] 1923. Science $57: 273-274$. [Druse.]

1924. Science 59: 241-248. [Fluorescent colors of plants.]

1925. Trans. Roy. Can. Inst. 'Toronto 15: 129-134. pl. 10. [Spirogyra.]

1928a. Protoplasma 4:45-66. illus. [Spirogyra gametes.]

1928b. Biol. Rev. and Biol. Proc. Cambridge Phil. Soc. 3 : 329-358. illus.

Llooyd, F. E., and G. W. Scarti. 1926. Science 63: 459-460. [Vacuoles.]

Locy, W. A. 1901. Pop. Sci. Monthly 58: 561-584. [Early microscopists.]

1905. Pop. Sei. Monthly 67: 97-126. [von Baer and embryology.]

1915. Biology and Its Makers. 3d ed. New York.

Loeb, J. 1899. Roux's Areh. Entw. 8: 689-693. [Nucleus and regeneration.]

1900. Amer. Jour. Physiol. 3: 434-471. [Artificial parthenogenesis.]

1911. Science $34: 653-655$. [Rôle of silts.]

1913. Artificial Parthenogenesis and Fertilization. Chicago.

1918. Proc. Nat. Acad. Sei. 4: 60-62. [Sex of parthenogenetic frog.]

1921a. Jour. Gen. Physiol. 3: 539-545. 3 figs. [Sex of parthenogenetic frog.]

Loefer, J. B. 1931. Areh. Protist. 74: 449-470. illus. [Heteronema.]

Logan, J. 1735. See Phil. Trans. Roy. Soc. (London) 39: 192-195. 1736.

Löhnıs, F. 1922a. Mem. Nat. Acad. Sci. 16: 335 pp. [Cycles in baeteria.] 1922b. Centralbl. Bakt. II 56:528-544. illus. [Cycles in bacteria.]

Longley, A. E. 1923. Amer. Naturalist. 57: 568-569. [Rubus; Cratogus.]

1924a. Amer. Jour. Bot. 11: 249-282. pls. 11-15. 13 figs. [Rubus.]

1924b. Amer. Jour. Bot. 11: 295-317. pls. 16-18. [Cratægus.]

1924c. Jour. Agr. Res. 28: 673-682. 3 pls. [Euchlena; Zea; Coix.]

1925. Science 61:542-543. [Carbohychates in Zea pollen.]

1926a. Jour. Agr. Res. $32: 559-568$. illus. [Fragaria.]

1926b. Jour. Wash. Acad. Sci. 16:543-545. illus. [Triploidy.]

1927a. Mem. Hort. Soc. N. Y. 3: 15-17. [Rubus, Fragaria.]

1927c. Jour. Agr. Res. 35: 769-784. illus. [Zea.]

1932. Jour. Agr. Res. 44: 317-321. illus. [Sorghum.]

Longley, A. E., and C. F. Clark. 1930. Jour. Agr. Res. 41: 867-888. illus.

[Solanum.]

Loos, W. 1930. Jahrb. Wiss. Bot. 72:611-664. [Mitogenetic rays.]

Lorbeer, G. 1924. Ber. Deu. Bot. Gesell. 4.2:231-237. illus. [Anthoceros.]

1927. Zeitschr. Ind. Abst. VererJ. 44:1-109. illus.

1930. Zeitschr. Bot. 23 : 932-956. illus. [Sphcerocarpos.]

Lotsy, J. P. 1904. Flora 93: 65-86. 19 figs. [Meiosis.]

1916. Evolution by Means of Hybridization. The Haguc.

1917. Arch. Néerl. Sci. Exact. et Nat. III B 3:324-350.

1919. Genetica 1: 7-63, 113-129.

Loul, J. von. 1930. Planta 12: 191-23s: illus. [Plastids.]

Lowe, C. W., and F. E. Lloyd. 1928. Trans. Roy. Soe. Canada III 21 (V): 279287. illus.

Löwschin, A. M. 1913. Ber. Deu. Bot. Gesell. 31: 203-209. [Chondriosomes.] 1914. Ber. Deu. Bot. Gesell. 32: 266-270. pl. 5. [Chondriosomes.] 
Loyez, M. 1909. Compt. Rend. Assoc. Anat. 189-195.

Lubluenko, V. 1921. Compt. Rend. Acad. Sci. Paris 173:365-367. [Chlorophyll]. Lubosch, W. 1902a. Jenaische Zeitschr. 37: 217-296. pls. 12-16. [Nucleolus]. 1902b. Ergeb. Anat. u. Entw. 11: 709-783. 2 figs. [Nucleolus.]

Lucas, A. M. 1931. Jour. Morphol. Physiol. 51: 147-193. illus.

1932. In Special Cytology (Cowdry, ed.), Sec. XI.

Lucas, F. F., and M. B. Stark. 1931. Jour. Morphol. Physiol. 52: 91-113. illus. Ludford. R. J. 1921. Jour. Roy. Micr. Soc. 121-133. pls. 3, 4. [Nucleolus.]

1922a. Jour. Roy. Micr. Soc. II 113-150. pls. 3-5. [Nucleolus.]

1922b. Quar. Jour. Micr. Sci. 66:151-158. 4 figs. [Golgi bodies.]

1925. Proc. Roy. Soc. London B 98: 557-577. illus.

1927. Proc. Roy. Soc. London B 101:409-429. illus. [Golgi in tissue cultures.] 1928. Proc. Roy. Soc. London B 103: 288-301. illus. [Vital staining.]

LÜDTke, F. 1890. Jahrb. Wiss. Bot. 21:62-127. pls. 2-4. [Aleurone.]

LUND, E. J. 1921. Jour. Exp. Zoöl. 34: 471-493. [Electrical polarity.]

LUNDEGARDH, H. 1910a. Jahrb. Wiss. Bot. 48: 285-378. pls. 6-8. [Vicia root.] 1910b. Svensk Bot. Tidskr. 4: 174-196. 11 figs. [Mitosis.]

1912a. Jahrb. Wiss. Bot. 51: 236-282. pl. 2. [Mitosis: living material.]

1912b. Arch. Zellf. 9: 205-330. pls. 17-19. 9 figs. [Nucleus.]

1912c. Beitr. Biol. Pflanzen 11: 373-542. pls. 11-14. [Mitosis.]

1914a. Svensk Bot. Tidskr. 8 : 161-180. 18 figs. [Mitosis.]

1922. Zelle und Cytoplasma. Berlin. (Linsbauer's Handbuch.)

Lutkov, A. N. 1930. Proc. U.S.S.R. Cong. Genet. Plant Animal Breed. 2 : 354-367.

Lutuan, B. F. 1910. Trans. Wis. Acad. Sci. 16: 1191-1244.

1911. Bot. Gazette 51: 401-430. pls. 22, 23. [Closterium.]

Lotz, A. M. 1907. Science 26: 151-152. [Tetraploid Enothera.]

1912. Biol. Centralbl. $32: 385-435.7$ figs. [Enothera.]

1916. Amer. Jour. Bot. 3: 502-526. pl. 24. 7 figs. [Enothera.]

1917. Amer. Jour. Bot. $4: 53-111.9$ figs. [Enothera.]

LynCH, V. 1919. Amer. Jour. Physiol. 48: 258-283. [Nucleus and synthesis.]

Lyon, E. P. 1907. Arch. Entw. Org. 23: 151-173. [Centrifuged eggs.]

Lyon, F. 1905. Bot. Gazette 40: 285-295. pls. 10, 11. [Spore coat.]

MA, R. M. 1930. Bull. Torr. Bot. Club $57: 277-284$. illus. [Chloroplast.] MA, W. C. 1928b. Chinese Jour. Physiol. 2: 247-254. illus. [Secretion.]

MA, W. C., and H. C. Chang. 1928. Chinese Jour. Physiol. 2: 381-388. illus. Ma, W. C., H. C. Chang, and A. C. Liu. 1929. Chinese Jour. Physiol. 3 : 29-40.

M acallum, A. B. 1891. Trans. Canad. Inst. 1: 247-278. 2 pls. [Nucleolus.]

MacArthur, J. W. 1928. Geneties 13:410-420. [Linkage in tomato.]

MacDodgal, D. T. 1920. Carnegie Inst. Wash. Publ. 297. [Hydration.]

MacDodgall, M. S. 1925. Quar. Jour. Micr. Sci. 69: 361-384. illus.

Macfarlane, J. M. 1892. Contrib. Bot. Lab. Univ. Pa. 1:7-44. pl. 4.

MacKay, M. E. 1927. Proc. and Trans. Nova Scotian Inst. Sci. 17: 46-60. illus.

M ̈̈скеi, H. G. 1928. Jahrb. Wiss. Bot. 69:517-548. illus. [Saprolegnia.]

Macormick, F. A. 1912. Bot. Gazette 53:67-68. [Rhizopus zygospore.]

MAdGe, M. A. P. 1929. Ann. Botany. 43: 545-577. illus. [Viola.]

MaEda, T. 1928. Bot. Mag. Tokyo 42:191-195. illus. [Chromonemata.]

1930a. Mem. Coll. Sci. Kyoto Imp. Univ. B 5 : 89-123. illus. [Chiasma.]

1930b. Mem. Coll. Sci. Kyoto Imp. Univ. B 6: 125-137. illus. [Chiasmata.]

Magrou, T., and M. Magrou. 1927. Rev. Path. Végét. et Entom. Agr. 14: 244246. 
Maige, A. 1923ab. Compt. Rend. Soc. Biol. Paris 88: 97-99; 530-533.

Maire, R. 1905a. Ann. Mycol. 3: 123-154. pls. 3-5. [Ascomycetes.] 1905b. Compt. Rend. Soc. Biol. Paris 57 : 726-728. [Basidiomycetes.] 1911. Progr. Rei Bot. 4:110-162. [Uredinales.]

Malone, J. Y. 1918. Trans. Amer. Micr. Soc. $37: 97-110$, pls. 9, 10. [Dog.] Malpighi, M. 1675. Anatome Plantarum.

vov Malsen, H. 1906. Arch. Mikr. Anat. 69:63-99. pl. 2. [Dinophilus.] Malte, M. O. 1910. Embryologiska och cytologiska undersökningar öf ver Mercurialis annua L. Lund. 96 pp. 3 pls.

Maneli, E. 1920. Atti Inst. Bot. Univ. Pavia II 17: 257-264. [Cell wall.]

Mangelsdorf, P. C. 1929. Amer. Naturalist $63: 139-149$. [Style length.]

Mangelsdorf, P. C., and R. G. Reeves. 1931. Jour. Heredity 22: 329-343.

Mangenot, G. 1920abcd. Compt. Rend. Acad. Sci. Paris 170:63-65; 200-201;

1458-1459; 1595-1598. [Fucus, Vaucheria, Florideæ; plastids.]

1920e. Compt. Rend. Soc. Biol. Paris 83: 275-276. [Fucus: §.]

1921. Compt. Rend. Acad. Sci., Paris 172: 1198-1200. [Fucus: oౌ.]

1923a. Compt. Rend. Acad. Sci., Paris 176: 183-185. [Floridean starch.]

1923b. Compt. Rend. Soc. Biol. Paris 75: 522-523. [Phæoplast.]

1925. Compt. Rend. Acad. Sci., Paris 180: 157-160. illus.

1926. Bull. Hist. Appl. Physiol. et Path. 3: 142-160. illus.

1927. Compt. Rend. Soc. Biol., Paris $97: 342-345$. illus.

1929a. Compt. Rend. Acad. Sci., Paris 188: 1431-1434.

1929b. Arch. d'Anat. Micr. 25: 507-518. illus.

Mangenot, G., and L. Emberger. 1920. Compt. Rend. Soc. Biol. 83 : 418-420.

Mangin, L. 1888-1893. Compt. Rend. Acad. Sci., Paris 107: 144; 109: 579; 110 :

$295 ; 111: 120 ; 113: 1069 ; 116: 653$. [Chemistry of cell wall.]

Mann, M. C. 1924. Univ, Calif. Publ. Agr. Sci. 2: 243-248. pl. 44. [Ginkgo.] 1925. Univ. Calif. Publ. Agr. Sci. $2: 297-314$. illus.

Manollov, E. O. 1922-1923. Bull. Appl. Bot. Plant Breed. 13 (2): 503-505.

Manton, I. 1932a. Jour. Genetics 25: 423-430. illus. [Apospory.] 1932b. Ann. Botany $46: 509-556$. illus. [Cruciferæ.]

Marchal, Êl. and Êx. 1906. Mém. Cour., Cl. d. Sci., Dec. 15, 1905. 50 pp.

1907. Bull. Acad. Roy. Belg., Cl. d. Sci., 765-789. [Apospory; sex.]

1909. Bull. Acad. Roy. Belg., Cl. d. Sci., 1249-1288. [Apospory; sex.]

1911. Bull. Acad. Roy. Belg., Cl. d. Sci., 750-778. 1 pl. [Apospory; sex.]

1912. Bull. Acad. Roy. Belg. 51: 189-203. 1 pl. [Amblystegium.]

Marchal, Ém. 1920 (1914). Mém. Acad. Roy. Belg. II 4: 108 pp. 4 pls.

Marcos, H. 1907. Verhandl. Anat. Gesell. 21. [Thymus.]

1908. Arch. Mikr. Anat. 71: 734-767. [Thymus.]

Maréchal, J. 1904. Anat. Anz. $25: 383-398.25$ figs. [Meiosis; oöcyte.]

1905. Anat. Anz. 26: 641-652. 27 figs. [Meiosis; oöcyte.]

1907. La Cellule $24: 1-239.10$ pls. [Meiosis; oöcyte.]

Margolena, L. A. 1932. Stain Tech. 7:9-16. [Feulgen reaction.]

MArk, E. L. 1881. Bull. Mus. Comp. Zoöl. Harvard Coll. 6: 173-625. 5 pls.

MArk, E. I., and M. Copeland. 1906. Proc. Amer. Acad. Arts Sci. 42: 101-112.

Mark, E. L., and L. C. Wyman. 1922. Science 55: 488-489. [Chondriosomes.]

Marsden-Jones, E. M., and W. B. Turrill. 1928. Nature (London) $122: 58$.

Marshak, A. G. 1931. Cytologia 2:318-339. illus. [Pisum chromosomes.]

Marston, H. R. 1923. Biochem. Jour. 17 : 851-859. [Enzymes.]

1926. Austral. Jour. Exp. Biol. Med. Sci. 3 : 233-236.

Martens, P. 1922a. Bull. Acad. Roy. Belg., Cl. d. Sci., 124-130. 1 pl.

1922b. La Cellule $32: 333-428.4$ pls. [Mitosis in Paris.]

1924. Compt. Rend. Acad. Sci., Paris 179: 1280-1282. [Mitosis in Listera.] 
1925. La Cellule $36: 125-214.2$ pls. [Mitosis in Listera.] 1927ab. Compt. Rend. Acad. Sei. Paris 184:615-617; 758-760.

1927c. La Cellule 38:69-174. illus. [Mitosis in vivo.] 1928. Bull. d'Histol. Appl. 5 : 229-252. illus. [Mitosis in vivo.] 1929a. Bull. Aead. Roy. Belg., Cl. d. Sci., V 15: 160-169. illus. [Mitosis in vivo.] 1929b. La Cellule 39:169-215. illus. [Mitosis in vivo.]

Martens, P., and R. Chambers. 1932. La Cellule $41: 131-144$. illus.

Martins-Mano, T. 1904. La Cellule $22: 57-76.4$ pls. [Nueleolus.]

Maschke, O. 1859. Bot. Zeitung, 409, 417, 429, 437. [Aleurone.]

Massee, G. 1884. Jour. Roy. Mier. Soe. II 4: 198-200. pl. 6.

MAST, S. O. 1916. Jour. Exp. Zoöl. 20:1-17. 6 figs. [Gonium.] 1926. Jour. Morphol. Physiol. 41:347-425. illus. [Amœba.]

Masvi, K. 1919ab. Jour. Coll. Agr. Tokyo 3 : 357-376; 377-403. 6 pls.

Mather, C. 1716. See Religio Philosophica. London, 1721.

Mathews, A. C. 1932. Jour. El. Mitchell Sei. Soc. 47:281-292. illus.

Mathews, A. P. 1903. Amer. Jour. Physiol. 8: 294-299. [Eleetrical polarity.] 1916. Physiologieal Chemistry.

1924. Chapter II in General Cytology, ed. by Cowdry.

Mathias, W. T. 1928. Publ. Hartley Bot. Lab. (Liverpool), No. 5.

Matsumoto, T. 1920. Johns Hopkins Hosp. Bull. 31:91-93. [Nerve fibers.]

Matsuura, H. 1929. A Bibliographical Monograph on Plant Geneties. Tokyo.

Maupas, M. 1889. Arch. de Zool. II 7. [Rejuvenescence in eiliates.]

Madrer, F. 1915. Grundzüge der Vergleichenden Gewebelehre. Leipzig.

Mavor, J. W. 1921. Seience 54:277-279. [X-rays and chromosome behavior.] 1922. Seience 55 : 295-297. [X-rays and ehromosome behavior.]

1923a. Seience $57: 503-504$. [X-rays and chromosome behavior.] 1923b. Geneties 8: 355-366. 2 figs. [X-rays and crossing-over.] 1923c. Amer. Jour. Roentg. Radium. Therapy 10: 968-974. illus. 1925. Sei. Monthly 21: 355-363. illus.

Mavor, J. W., and H. K. Svenson. 1923. Seience 58: 124-126. [X-rays.]

Maziarski, S. 1910. Areh. Zellf. 4: 443-601. pls. 24-27. [Secretion.] 1911. Areh. Zellf. 6:397-442. pls. 22, 23. [Secretion.]

McAllister, F. 1909. Bot. Gazette 48:200-215. pl. 15. [Smilacina: ᄋ.] 1913a. Trans. Wis. Acad. Sci. $17: 599-660$. pls. 56-5S. [Smilacina: ㅇ.] 1913b. Anu. Botany $27: 681-696$. pl. 56. [Tetraspora.] 1914. Amer. Jour. Bot. $1: 79-95$. pl. 8. [Anthoceros pyrenoid.] 1927. Amer. Jour. Bot. 14: 246-257. illus. [Pyrenoid.] 1931. Amer. Jour. Bot. 18: 838-853. illus. [Spirogyra spindle.]

McClendon, J. F. 1910a. Roux's Areh. Entw. 31: 80-90. pl. 3.2 figs. 1910b. Amer. Jour. Physiol. $27: 240-275 . \quad$ [Permeability.]

1913. Roux's Arch. Entw. $37: 233-247.10$ figs. [Surface tension.]

McClintock, B. 1929a. Geneties 14:180-222. illus. [Triploid Zea.] 1929b. Seienee 69: 629. illus. [Zea chromosome set.]

1930a. Anat. Ree. 47:380. [Reciprocal transloeation.]

1930b. Proc. Nat. Acad. Sei. 16: 791-796. illus. [Reeiprocal transloeation.]

1931a. Proc. Nat. Acad. Sci. $17: 485-491$. illus. [Order of genes.]

1931b. Univ. Missouri Aga. Res. Sta. Res. Bull. 163. illus. [Deficiencies ete.] 1932a. Proe. 6 Internat. Cong. Genet. $2: 126-128$. [Non-homologous association.] 1932b. Proe. Nat. Acad. Sei. 18: 677-681. [Variegation.]

1933. Zeitsehr. Zellforseh. Mikr. Anat. 19: 191-237. illus. [Non-homologous association.]

1934. Zeitschr. Zellforsch. Mikr. Anat. 21:291-32S. illus. [Nucleolus.]

McClintock, B., and H. E. Hill. 1929. Anat. Rec. 44:291.

1931. Geneties 16:175-190. illus. [Trisomic Zca.] 
MCClung, C. E. 1899. Zoöl. Bull. 2 : 187-197. 14 figs. [Sex-chromosome.] 1900. Kansas Univ. Quar. A 9: 73-100. pls. 15, 16. [Meiosis.]

1901. Anat. Anz. 20:220-226. [Accessory chromosome.]

1902a. Kansas Univ. Sei. Bull. 11. [Meiosis in Locustidæ.]

1902b. Biol. Bull. 3:43-84. [Sex-chromosome.]

1905. Biol. Bull. 9 : 304-340. 21 figs. [Chromosomes in orthopterans.]

1908. Kansas Univ. Bull. 4. [Cytology and taxonomy.]

1914. Jour. Morphol. 25: 651-749. pls. 1-10. [Orthopteran chromosomes.]

1917. Jour. Morphol. 29: 519-604. 8 pls. [Multiple chromosomes.]

1923. Eugenies, Genetics and Family 1: 65-75.

1924. Chapter X in General Cytology, ed. by Cowdry.

1927a. Jour. Morphol. Physiol. 43:181-265. illus. [Meiosis.]

1927b. Quar. Rev. Biol. 2: 344-366. illus. [Chiasmatype theory.]

1928a. Zeitschr. Zellf. Mikr. Anat. 7: 756-778. illus. [Meiosis.]

1928b. Arch. de Biol. 38: 503-528. illus.

McCubbin, W. A. 1910. Bot. Gazette 49: 195-206. pls. 14-16. [Helvella.]

McDonald, J. D. 1922. Univ. Calif. Publ. Zoöl. 20 : 243-300. pls. 27, 28.

McPhee, H. C. 1925. Jour. Agr. Res. 31: 935-943. [Sex in hemp.]

MeAD, A. D. 1898. Jour. Morphol. 14: 181-218. [Centrosomes in egg.]

Meek, C. F. U. 1920. Proc. Roy. Soc. London 91: 157-165. [Chromosome dimensions.]

Melburn, M. C., and W. P. Thompson. 1927. Amer. Jour. Bot. 14: 327-333.

Meuin, E. 1915. Svensk Bot. Tidskr. 9: 261-293. [Sphagnum.]

Mendel, G. 1865 (1866). Versuche über Pflanzenhybriden. Verh. Nat. Ver. Brünn. Reprinted in Flora $89: 364-403.1901$.

Metcalf, M. M. 1915. Science 42:658. [Chromosomes in Protozoa.]

Metz, C. W. 1914. Jour. Exp. Zoöl. 17: 45-56. 1 pl. [Chromosomes of Diptera.] 1916a. Jour. Exp. Zoöl. 21:213-280. pls. 1-8. [Chromosomes of Diptera.]

1916b. Amer. Naturalist 50:587-599. pl. 1. [Chromosomes of Diptera.]

1918. Geneties $3: 107-134$. [Linkage in Drosophila viritis.]

1922a. Anat. Rec. 23: 95-96. [Incomplete synapsis.]

1922b. Biol. Bull. 43 : 253-266. 2 pls. [Incomplete synapsis.]

1922c. Biol. Bull. 43: 369-373. 6 figs. [Association of homologues.]

1923. Amer. Naturalist 57:381-384. [Drosophila Willistoni.]

1925. Science 61: 212-214. illus. [Sciara.]

1926b. Zeitsehr. Zellf. Mikr. Anat. 4: 1-28. illus. [Drosophila, meiosis.]

1926c. Science 63:190-191. illus. [Sciara.]

1932. In Special Cytology (Cowdry, ed.), Sec. XLIII.

1933. Biol. Bull. 64:333-347. illus. [Sciara.]

Metz, C. W., and M. S. Moses. 1923. Jour. Heredity 14: 195-204.

Metz, C. IV., M. S. Moses, and E. N. Hople. 1926. Zeitsehr. Ind. Abst. Vererb. $42: 237-270$. illus.

Metz, Moses, and E. D. Mason. 1923. Carnegie Inst. Wash. Publ. 328. 94 pp.

Metz, C. W., and J. F. Nonidez. 1921. Jour. Exp. Zoöl. 32: 165-185. [Asilus.] 1923. Arch. Zellf. 17: 438-449. pls. 21-23. [Asilus: meiosis.]

1924. Biol. Bull. 46: 153-164. 1 pl. [Lasiopogon: meiosis.]

Metzner, P. 1920. Biol. Zentralb1. 40: 49-87. 18 figs. [Flagellum.]

Medrman, O. 1925. Soc. Sci. Fennica, Comm. Biol. 2 : No. 3. [Sex-chromosomes.] 1929a. Hereditas 12:179-209. illus. [Aucuba.]

1929b. Jour. Geneties 21: 85-94. illus. [Prunus.]

Meves, F. 1896. Arch. Mikr. Anat. 48: 1-83. pls. 1-5. [Salamandra: ơ.]

1897. Arch. Mikr. Anat. 50: 110-141. pls. 7, 8. [Salamandra: o7.]

1898. Verh. Anat. Gesell. Jena. 14. [Man and rat: spermatogenesis.] 
1899. Arch. Mikr. Anat. 54: 329-402. pls. 19-21. 16 figs.

1904a. Ber. Deu. Bot. Gesell. 22 : 284-286. pl. 16. [Chondriosomes.]

1904b. Anat. Anz. 24:29-32. illus. [Apis: ơ.]

1907ab. Anat. Anz. 31:399-407; 561-569. [Chondriosomes.]

1907c. Arch. Mikr. Anat. 70:414-491. illus. [Apis: $\left.\sigma^{7}.\right]$

1908. Arch. Mikr. Anat. 72: 816-867. pls. 39-42. [Chondriosomes.]

1911. Areh. Mikr. Anat. 76: 683-714. pls. 27-29. [Chondriosomes.]

1914a. Arch. Mikr. Anat. 85: II 1-8. pls. 1, 2. [Cleavage.]

1918a. Areh. Mikr. Anat. 92: II 41-136. 18 figs. [Chondriosomes.]

Meyen, F. J. F. 1826. De Primis Vitæ Phenomenis in Fluidis.

1830. Lehrbuch der Phytotomie. Berlin.

1837-1839. Neues System der Pflanzenphysiologie.

Meyer, A. 1881. Bot. Zeitung 39: 841-846, 857-864. pl. 9. [Starch grain.]

1883a. Bot. Zeitung $41: 489-498,505-514,525-531$. [Plastids.]

1883b. Das Chlorophyllkorn. 91 pp. 3 pls. Leipzig.

1895. Untersuchungen über die Stärkekörner. Jena.

1896. Bot. Zeitung 54:187-217. [Protoplasmic connections in Volvox.]

1911. Ber. Deu. Bot. Gesell. 29: 158-160. [Chondriosomes.]

1912. Die Zelle der Bakterien. Jena.

1913. Kolloid-Chem. Beihefte 5. [Jellies.]

1916. Ber. Deu. Bot. Gesell. $34: 168-173$. [Plant chondriosomes.]

1917a. Ber. Deu. Bot. Gesell. $35: 333-338$. [Nucleolus.]

1920-1921, 1926. Morphologisehe und Physiologische Analyse der Zelle der

Pflanzen und Tiere. Jena.

1922. Ber. Deu. Bot. Gesell. 40:161-167. 1 fig. [Plastid boundary.]

Meyer, K. 1911. Bull. Soc. Imp. Moseow 236-286. [Corsinia.]

Meyer, K. H., and H. Mark. 1928. Ber. Deu. Chem. Gesell. 61: 593.

1930. Der Aufbau der Hochpolymeren Organischen Naturstoffe. Leipzig.

Michaelis, P. 1925. Ber. Deu. Bot. Gesell. 43:61-67. illus. [Epilobium.]

1926. Planta 1:569-582. illus. [Temperature effects.]

1928. Biol. Zentralbl. $48: 370-374$. [Temperature effects.]

1929. Biol. Zentralbl. $49: 302-316$. illus. [Rôle of eytoplasm.]

Miescher, F. 1871. Med.-Chem. Untersuch. Lab. Chem. Tübingen 4. Berlin.

Miller, P. 1721. See Bradley: Treatise of Husbandry and Gardening 1: 330-332. London, 1726.

Milovidov, P. F. 1928b. Arch. d'Anat. Mier. 24 : 19-31. illus.

1928c. Compt. Rend. Acad. Sei. Paris $187:$ 140-142. [Chondriosomes.]

1929. Rev. Gén. Bot. 41: 193-208. illus. [Chondriosomes.]

1931. Areh. Protist. 73:1-46. illus. [Plasmodiophora.]

Minchin, E. A. 1912. An Introduction to the Study of the Protozoa. London.

Minenkov, A. 1924. Nautschno Agron. Zurnal 1.

Minot, C.S. 1891. Jour. Physiol. $12: 97-153 . \quad$ [Senescence.]

1908. The Problem of Age, Growth, and Death. New York.

1913. Moderne Probleme der Biologie. Jena.

Minouchi, O. 1928a. Proc. Imp. Acad. Japan 14: 128-130. illus. [Cat.] 1928c. Jap. Jour. Zoöl. 1 : 235-254. illus. [Rat.]

1928e. Jap. Jour. Zoöl. 1: 269-273. illus. [Mouse.]

Minours, T. 1921. Jour. Exp. Zoöl. 33: 1-41. pls. 1-10. [Tissue grafts.]

Mirande, M. 1919. Compt. Rend. Acad. Sci. Paris 168: 283-286. 7 figs.

1923. Compt. Rend. Acad. Sci. Paris 176: 327-330. 21 figs. Also 596-598, $769-771$. 
Miriele, C. F. 1801. Traité d'Anatomie et de Physiologie Végétale.

1808. Exposition et Defense de ma Théorie de l'Organisation Végétale.

1833. Mém. French. Inst. 1835. [Marchantia.]

Mitchell, E. M. 1930. Ainer. Jour. Bot. 17: 117-138. illus. [Hemicelluloses.]

MIYAKE, K. 1901. Ann. Botany 15:653-667. pl. 36. [Pythium.]

1905a. Jahrb. Wiss. Bot. $42: 83-120$. [Meiosis.]

1905b. Bot. Mag. Tokyo 19 : 98-101. [Centrosome.]

1906. Jour. Appl. Micr. Lab. Meth. 5: 1773-1780. [Ginligo: o'.]

1910. Beih. Bot. Centralbl. $27: 1-25.5$ pls. [Cunninghamia.]

Möвıus, M. 1920. Ber. Deu. Bot. Gesell. 38: 224-232. [Chloroplast size.]

1927. Die Farbstoffe der Pflanzen. Berlin.

Mockeridge, F. A. 1927. Brit. Jour. Exp. Biol. 4: 301-304. [Nostoc.]

Modilewsk1, J. 1908. Flora 98: 423-470. illus. [Apomixis.] 1929. Ukrainian Bot. Rev. 5: 5-40. [Embryo sacs.]

Moenkhaus, W. J. 1904. Jour. Anat. 3: 29-65. [Fish hybrids.]

Moffett, A. A. 1931. Proe. Roy. Soc. London B 108: 423-446. illus.

voN Mont, H. 1835, 1837. Flora $45: 1837$. Diss. Tübingen, 1835. [Plastids.]

1844. Bot. Zeitung 2 : 273-277, 289-294, 305-310, 321-326, 337-342. pl. 2.

1846. Bot. Zeitung 4: 73-78, 89-94. [Protoplasmic streaming.]

1851. Grundzüge der Anatomie und Physiologie der Vegetabilisehen Zelle.

1853. Bot. Zeitung 11:753-762, 769-775. [Cell membrane.]

1858. Bot. Zeitung 16:373-375. 1 pl. [Use of polarized light.]

Монr, O. H. 1919. Arch. Mikr. Anat. 92:298-368. pls. 11-16. [Radium, ete.]

Moissejewa, M. 1931. Biochem. Zeitschr. 241: 1-13.

Mol, W. E. DE. 1923. Genetica 5: 225-272. 6 pls. [Hyacinthus.]

1924. Genetiea 6: 289-336. 57 figs. [Meiosis in wheat.]

1927c. Genetica 9: 116. [Polyploidy in tulips.]

1928a. Genetica 11:119-212. illus. [Polyploidy in tulips.]

1928b. Zeitschr. Ind. Abst. Vererb. 48: 145-148. [Hyacinths, tulips.]

1928c. Weekblad v. Bloembolleneultuur, July 20.

1928d. Vierteljahrsehr. Naturf. Gesell. Zürieh 73: 73-97. illus.

1930b. Zeitsehr. Ind. Abst. Vererb. 54:363-367. [X-rays.]

1931a. Nederl. Tijdschr. Geneesk. 75 : 1086-1088. [X-rays.]

1931b. Handel. 23 Nederl. Nat. en Geneesk. Cong. Delft.

1931c. Zeitsehr. Ind. Abst. Vererb. 59: 280-283. [X-rays.]

1932b. Der Züchter 4: 61-66. illus.

Moldenifater, J. J. P. 1812. Beiträge zur Anatomie der Pflanzen.

Molisch, H. 1888. Sitzungsber. K. Akad. Wiss. Wien, Math.-Naturwiss. Cl., I

97 : 264-299. illus. [Tyloses.]

1913. Mikrochemie der Pflanze. Jena.

Mous, G. 1928. Arch. de Biol. $38: 31-86$. illus.

Montgonery, T. H. 1899. Jour. Morphol. 15: 265-582. [Nucleolus; review.]

1901a. Trans. Amer. Phil. Soe. $20: 154-236$. pls. 4-8. [Meiosis.]

1901b. Proc. Acad. Nat. Sci. Phila. 53: 261-271. pl. 10. [Meiosis.]

1903. Biol. Bull. $4: 259-269.8$ figs. [Meiosis.]

1904. Biol. Bull. 6 : 137-158. 3 pls. [Meiosis.]

1905. Proc. Acad. Nat. Sci. Phila. 57: 162-205. pls. 9, 10. [Meiosis.]

1906. Trans. Amer. Phil. Soe. $21: 97-174$. pls. 9-13. [Meiosis.]

1908. Arch. Zellf. $2: 66-75$. pls. 6, 7. [Ascaris ehromosomes.]

1910. Arch. Zellf. 5: 121-145. pls. 9, 10. [Euschistus sperm.]

1911. Jour. Morphol. 22 : 731-798. pls. 1-5. [Euschistus spermatogenesis.]

1912. Jour. Acad. Nat. Sei. Phila. 15: 1-22. pls. 1-4. [Human ehromosomes.] 
Moore, A. C. 1903. Bot. Gazette 36:384-388. illus. [Pallavicinia.] 1905. Bot. Gazette 40: 81-96. pls. 3, 4. [Pallavicinia.]

Moore, B. 1921. Bioehemistry. London.

Moore, E. L. 1924. Jour. Exp. Zoöl. 39: 317-337. 3 figs. [Endomixis.]

Moore, J. E. S., and G. Arnold. 1906. Proc. Roy. Soc. London B 77 : 563-570.

Moreau, F. 1911a. Bull. Soe. Myeol. France $27: 334-341.4$ figs. [Mucorinex.] 1911b. Bull. Soc. Bot. France 58:618-623. 4 figs. [Mucorinex.]

1913a. Recherehes sur la Reproduction des Mucorinées. Thèse, Paris.

1913b. Bull. Soc. Bot. France 60: 121-123. [Rhizopus zygospore.]

1914. Bull. Soe. Bot. France 61: 139-142. [Vaucheria; chondriosomes.]

1915ab. Compt. Rend. Soc. Biol., Paris 78: 171-172; 143-144.

Morelle, J. 1923. Bull. Acad. Roy. Belg., Cl. d. Sci., 139-157. 1 pl. [Pancreas.] 1926. La Cellule 37: 77-141. illus. [Seeretion.]

1927. Ann. Soe. Sei. Bruxelles 47.

Morgan, C. L. 1923. Emergent Evolution. New York.

Morgan, L. V. 1925. Geneties 10: 148-178.

1929. Carnegie Inst. Wash. Publ. 399: 223-296.

1933 Genetics 18: 250-283. illus.

Morgan, T. H. 1896. Roux's Arch. Entw. 3: 339-361. pl. 19. [Cytasters.]

1899. Roux's Arch. Entw. 8: 448-539. pls. 7-10. 21 figs.

1906. Biol. Bull. $10: 201-206.4$ figs. [Phylloxerans.]

1908. Proc. Soe. Exp. Biol. Med. 5. [Phylloxerans.]

1909a. Science 29: [Sex and parthenogenesis in phylloxerans and aphids.]

1909b. Jour. Exp. Zoöl. 7 : 239-352. 1 pl. [Sex and parthenogenesis.]

1910a. Proc. Soc. Exp. Biol. Med. 7. [Sex and parthenogenesis.]

1910b. Proc. Soc. Exp. Biol. Med. 8. [Sex-limited inheritance.]

1910c. Science 32:120-122. [Sex-limited inheritance.]

1910d. Amer. Naturalist 44:449-496. [Chromosomes and heredity.]

1910e. Jour. Exp. Zoöl. 9: 593-655. 8 pls. [Centrifuged eggs.]

1911a. Science 34: 384. [Crossing-over.]

1911b. Jour. Exp. Zoöl. 11: 365-413. 1 pl. [Chromosome analysis.]

1912. Jour. Exp. Zoöl. 12: 479-498. 29 figs. [Phylloxerans.]

1913. Heredity and Sex. New York.

1914. Amer. Naturalist 48: 577-5S3. [Sex-linkage; sex-limited characters.]

1919a. The Physical Basis of Heredity. Philadelphia.

1919b. Anat. Rec. 15: 357. [Gynandromorphism.]

1919c. Carnegie Inst. Wash. Publ. 285. [Secondary sexual characters.]

1920a. Science 61: 73-80. [Whitman's work on pigeons.]

1920b. Endoerinology 4:381-385. [Hen-feathered fowls.]

1922. Proc. Roy. Soc. London B 94: 162-197. pls. 7, 8.

1923a. Amer. Naturalist 57: 476-477. [Lutear cells.]

1923b. Physiol. Rev. 3: 603-627. 8 figs. [Geneties and embryology.]

1923c. Sei. Monthly 16:237-246. [Mendelism and origin of speeies.]

1924a. Sei. Monthly 18:1-17. [Heredity of embryonic charaeters.]

1924b. Sci. Montbly 18: 205-215. 8 figs. [Median plane of embryo.]

1924c. Sei. Monthly 18: 273-290. [Asymmetry.]

1924d. Sei. Monthly 18: 354-371. 16 figs. [One embryo from two eggs.]

1924e. Sei. Monthly 18:529-546. 14 figs. [Two embryos from one egg.]

1924f. Sci. Monthly 18 : 561-579. 15 figs. [Development of egg fragments.]

1924g. Chapter XI in General Cytology, ed. by Cowdry. [Mendelism.]

1925. La Cellule 36: 111-123. illus. [Crossing-over.]

1928. The Theory of the Gene. Yale University Press, New Haven.

Morgan, T. H., and C. B. Bridges. 1919. Carnegie Inst. Wash. Publ. 278. 
Morgan, T. H., C. B. Bridges, and A. H. Sturtevant. 1925. Bibliog. Genet. $2: 1-262$. illus.

Morgan, T. H., A. H. Sturtevant, and C. B. Bridges. 192S. Carnegie Inst. Yearbook No. 27. 330-335.

Morgan, Sturtevant, H. J. Muller, and Bridges. 1922. The Mechanism of Mendelian Heredity. 2d ed. New York. (1st ed., 1915.)

Morinag., T. 1928. Proc. Imp. Acad. Japan 4:620-622. illus. [Brassica.] 1929a. Cytologia 1: 16-27. illus. [Brassica.] 1929b. Jap. Jour. Bot. 4: 277-289. illus. [Brassica.]

1929c. Jour. Dept. Agr. Kýushu Imp. Univ. 2: 199-206. illus. [Brassica.]

Morinaga, T., and E. Fukushina. 1931b. Proe. Imp. Acad. Japan $7:$ 383-384.

Morita, Y., and R. Chambers. 1929. Biol. Bull. $56: 64-67$.

Moroff, T. 1909a. Areh. Zellf. 2: 432-493. pls. 34-36. 11 figs. 1909bc. Areh. Zellf. 4: 142-161. 57 figs. Centr. f. Physiol. 22.

1911. Biol. Centralbl. $31: 144-150.3$ figs.

Morrill, C. V. 1910. Biol. Bull. 19: 79-127. 2 pls. [Protenor.]

Morrıs, M. 1914. Jour. Exp. Zoöl. 16: 501-522. 5 pls. [Fish hỵrids.]

Motтe, J. 1928. Ann. Sei. Nat. Bot. X 10:293-543. illus. [Mosses.] 1929. Rev. Bryologique 56: II 167-206. [Mosses.]

1932. Verdoorn's Manual of Bryology, Chap. VI. The Hague.

Mottier, D. M. 1898b. Ber. Deu. Bot. Gesell. 16: 123-128. 5 figs. [Dictyota.] 1899. Ann. Botany 13: 325-361. pl. 18. [Centrifuged cells.]

1900. Ann. Botany 14: 166-192. pl. 11. [Dictyota.]

1904a. Ann. Botany 18: 245-254. pl. 17. [Chara spermatozoid.]

1905. Bot. Gazette $40: 171-177$. [Meiosis.]

1907. Ann. Botany $21: 309-347$. pls. 27, 28. [Meiosis.]

1909. Ann. Botany $23: 343-352$. pl. 23. [Meiosis.]

1914. Ann. Botany 28: 115-133. pls. 9, 10. [Meiosis.]

1915. Jahrb. Wiss. Bot. 56: 65-84. [Apogamy.]

1918. Ann. Botany 32: 91-114. pl. 1. [Chondriosomes and plastids.]

1921. Ann. Botany 35: 349-364. pl. 15. [Plastids; protein; oil.]

1927. Bot. Gazette $83: 244-266$. illus. [Ferm prothallia.]

1931. Bot. Gazette 92: 218-223. illus. [Fern prothallia.]

DE Moulin, F. 1923. Areh. Zellf. 17 : 389-396. [Ganglion cell.]

Mounce, I. 1921, 1922. Trans. Brit. Mycol. Soe. 7 : 198-217; 8: 256-269.

MÜHLdorf, A. 1930a. Bot. Areh. 30: 167-194. illus.

1930b. Beih. Bot. Centralbl. I $47: 169-191$.

1930c. Biolog. Generalis 6: 457-482. [Spermatozoids.]

1931. Ber. Deu. Bot. Gesell. 49: 21-28. illus.

Müller, H. A. C. 1912. Arch. Zellf. 8: 1-51. pls. 1, 2. [Najas.]

Muller, H. J. 1914a. Amer. Naturalist 48: 508-512. [Tetraploid ratios.]

1914b. Jour. Exp. Zoöl. 17: 326-328.

1918. Geneties 3 : 422-499. [Balanced lethals.]

1920. Jour. Exp. Zoöl. 31: 443-473. 3 figs. [Mutation.]

1922. Amer. Naturalist 56: 32-50. [Mutation.]

1923. 2 Internat. Cong. Eugenies 1: 106-112. [Mutation.]

1925. Amer. Naturalist 59: 346-353. [Polyploicly.]

1926. Amer. Naturalist 60:192-195. [X-rays and crossing-over.]

1927. Science $66: 84-87$. [Gene mutation.]

1928a. Zeitschr. Ind. Abst. Vererb., suppl. 1: 234-260.

1928b. Proc. Nat. Acad. Sci. 14: 714-726. [X-ray mutations.]

1928c. Anat. Rec. 41: 100. [X-ray translocations.]

1929b. Amer. Naturalist 63: 481-486. [Translocation.] 
1929c. Sci. Monthly 29:481-505. [Mcthod of cvolution.]

1930a. Amer. Naturalist 64:28-59. [Radiation and genetics.]

1930b. Jour. Genetics $22: 299-334$. illus. [X-ray modifications.]

1930c. Jour. Genetics 22 : 335-357. [Chromosome linkage.]

1932. Amer. Naturalist 66:118-138. [Geneties of sex.]

Muller, H. J., and E. Altenburg. 1919. Proc. Soc. Exp. Biol. Med. 17 : 10-14. 1930. Genetics $15: 283-311$. [X-rays.]

Muller, H. J., and T. S. Painter. 1929. Amer. Naturalist 63: 193-200. illus. 1932. Zcitschr. Ind. Abst. Vererb. 62: 316-365.

Muller, H. J., and W. S. Stone. 1930. Anat. Ree. 47 : 393-394.

Müller, O. 1906. Jahrb. Wiss. Bot. 43:49-88. illus.

Mulsow, K. 1912. Areh. Zellf. 9:63-72. pls. 5, 6. 5 figs. [Ancyracanthus.]

Mundie, J. R. 1929. Bot. Gazette $87: 397-410$. illus. [Vaucheria.]

Müntzing, A. 1928. Hereditas 11: 267-283. illus. [Pseudogamy.]

1930a. Botaniska Notiser 35-47. illus. [Speltoid mutants.]

1930b. Hereditas $14: 153-172$. illus. [Galeopsis.]

1931. Hereditas 15:166-178. illus. [Potentilla.]

1932. Hereditas 16:105-154. illus. [Galeopsis.]

1933. Hereditas 18: 33-55. [Poly poidy]

Murbeck, S. 1901. Lunds Univ. Årsskr. $36: 41$ pp. 6 pls. [Alchemilla.] 1904. Botaniska Notiser, Lund, 285-296. [Taraxacum; Hieracium.]

Murphy, P. A. 1918. Ann. Botany 32: 115-153. illus. [Phytophthora.]

Murray, J. A. 1898. Zool. Jahrb., Anat. Abt., 11: 427-440. illus.

Murrill, W. A. 1900. Ann. Botany 14:583-607. pls. 31, 32. [Tsuga.]

Myers, M. E. 1928. Univ. Calif. Publ. Bot. 14:225-246. illus. [Egregia.]

\section{$\mathrm{N}$}

Nachtsheim, H. 1913. Arch. Zellf. 11: 169-241. pls. 7-10. [Apis.] 1921. Biol. Zentralbl. 41 : 459-479. 1 fig. [Haploid Metazoa.]

Nadson, G. A. 1925. Biochem. Zeitschr. 155: 381.

NAGaI, I. 1914. Flora N. F. 60:281-330. illus. [Sex in ferns.] 1915. Jour. Coll. Agr. Univ. Tokyo 6:127-164. pl. 10. [Sex.]

NAGAO, S. 1929a. Mem. Coll. Sei. Kyoto Imp. Univ. B 4: 175-198. illus. 1929b. Mem. Coll. Sei. Kyoto Imp. Univ. B 4: 347-352. illus.

voN NäGeLI, C. 1844, 1846. Zeitschr. Wiss. Bot. 1, 3. [Cell-division, ete.] 1846. On the Utricular Structures in the Contents of Cells. Ray Society, 1849. 1858. Die Stärkekörner. Zïrich.

1863a. Nägeli's Bot. Mitt. 1, 2. [Stareh grain and cell wall.]

1863b. Beitr. Wiss. Bot. 3: 1-126. pls. 1-7. [Use of polarized light.]

1864. Sitzber. Ḱgl. Bayer. Akad. Wiss. München. [Cell membrane.]

1881. Bot. Zeitung. 39: 633-651, 657-678. [Growth of starch grain.]

Nakahar,a, W. 1917. Jour. Morphol. 29: 55-74. 2 pls. [Nucleolus.]

1918a. Anat. Ree. 15: 203-216. 9 figs. [Nucleolus.]

1918b. Jour.. Morphol. 30: 483-526. 6 pls. 36 figs. [Amitosis.]

Nakanishi, K. 1901. Centralbl. Bakt. I 30:97, 145, 193, 225. [Bacteria.]

Nassonow, D. 1918. Arch. Russe d'Anat. Histol. et Embr. 2: 95-127, pls. 3, 4. 1923. Arch. Mikr. Anat. u. Entw. 97: 136-186. pls. 10-12. [Golgi; secretion.] 1924a. Arch. Mikr. Anat. u. Entw. 100: 433-472. pls. 2-4. [Golgi: seeretion.] 1924b. Areh. Mikr. Anat. u. Entw. 103: 437-482. [Contractile vacuole.] 1926. Zeitsehr. Zellf. Mikr. Anat. 3: 472-502. illus.

Nath, V. 1925. Quar. Jour. Micr. Sei. 69:643-659. illus. [Tail sheath.] 1926. Biol. Rev. 2 : 52-79. [Chondriome, Golgi.] 1928a. Quar. Jour. Micr. Sci. 72: 277-300. illus. [Yolk.] 
1929. Zeitschr. Zellf. Mikr. Anat. 8: 655-670. illus. [Yolk.]

1930. Quar. Jour. Micr. Sci. 73:477-506. illus. [Golgi.]

Nath, V., and M. T. Husain. 1928b. Quart. Jour. Micr. Sci. 72 : 403-418. illus.

Nath, V., and D. R. Metha. 1929. Quart. Jour. Micr. Sci. 73: 7-24. illus.

Nath, V., and P. Mohan. 1929. Jour. Morphol. Physiol. 48: 253-279. [Yolk.]

NAth, V., and M. D. Nangia. 1931. Jour. Morphol. Physiol. 52: 277-307. illus.

Nathansohn, A. 1900a. Jahrb. Wiss. Bot. 35: 48-78. pls. 2, 3. [Amitosis.] 1900b. Ber. Deu. Bot. Gesell. 18: 99-109. 2 figs. [Parthenogenesis.]

Nawaschin, M. 1925a. Zeitschr. Zellf. Mikr. Anat. 2: 98-110. 1 pl. [Crepis.] 1925b. Geneties 10: 583-592. illus. [Polyploid Crepis.]

1926. Zeitschr. Zellf. Mikr. Anat. 4: 171-215. [Crepis.]

1927a. Ber. Deu. Bot. Gesell. 45: 115-126. illus.

1927b. Zeitschr. Zellf. Mikr. Anat. 6: 195-233. illus. [Crepis hybrids.]

1930a. Univ. Calif. Publ. Agr. Sci. 6: 95-106. illus. [Chimera.]

1930b. Zeitschr. Zellf. Mikr. Anat. 55: 348-352. illus. [Crepis hybrids.]

1931a. Univ. Calif. Publ. Agr. Sci. 6 : 201-206. [Translocation.]

1931c. Amer. Naturalist $65: 243-252$. illus. [X-rays.]

1932. Zeitschr. Ind. Abst. Vererb. $63: 224-231$.

Nawaschin, S. 1898. Bull. Acad. Imp. Sei. St. Petersburg V 9: 377-382.

1899a. Bot. Centralbl. 77:62. [Double fertilization.]

1899b. Flora 86:404-427. illus. [Plasmodiophora.]

1900. Ber. Deu. Bot. Gesell. 18: 224-230. illus. [Syngamy.]

1909. Ocsterreich. Bot. Zeitschr. 59: 457. [Male nucleus.]

1910. Ann. Jard. Bot. Buit. 12 : Suppl. III, 871-904. pls. 33, 34.

1912. Bull. Acad. Imp. Sci. St. Petersburg 6: 373-385. [Galtonia.]

1915. Bull. Acad. Imp. Sci. St. Petersburg 9: 1821-1834. [Galtonia.]

1916. Rec. d'Art. Sci. dédić à C. Timiriaseff. Moscow.

1927 (1913). Ber. Deu. Bot. Gesell. 45: 415-428. illus. [Galtonia.]

Nebel, B. R. 1932ab. Zeitschr. Zellf. Mikr. Anat. 16: 251-284; 285-304. illus.

NĚmec, B. 1901. Beitr. Wiss. Bot. 4. [Alnus nucleoli.]

1904. Jahrb. Wiss. Bot. $39: 645-730.157$ figs. [Chloralized cells.]

1909. Ber. Deu. Bot. Gesell. 27 : 43-47.

1910. Das Problem der Befruchtungsvorgänge, usw. Berlin.

1912. Bull. Intern. Aead. Sei. Bohême, 1-17. 19 figs. [Gagea.]

1924. Stud. Plant Physiol. Lab. Ch. Univ., Prague, 2: 47-94. 3 pls.

1926. Biol. Generalis 2 : 96-103. illus. [Multipolar figures.]

1927. Arch. Exper. Zellf. 5: 77-82. illus. [Spindle.]

1929c. Z Vestniku Král. Ces. Spol. Nauk 2. [Multipolar mitosis.]

1929d. Z Vestniku Král. Ces. Spol. Nauk 2. [Ascospore wall.]

1929e. Protoplasma $7: 99-105$. illus. [Influence of nicotine.]

1929f. Protoplasma $7: 423-443$. illus. [Structure of nucleus.]

von Neuenstein, H. 1914. Arch. Zellf. 13: 1-91. 20 figs. [Alga nuclei.]

Newport, G. 1851, 1853, 1854. Phil. Trans. Roy. Soc. London. [Fertilization.]

Newton, D. E. 1926. Ann. Botany 40: 891-917. [Sex in Coprinus.]

Newton, W. C. F. 1924. Ann. Botany 38: 197-206. pl. 9. 3 figs. [Galtonia.] 1927. Jour. Linn. Soe. (Bot.) $47: 339-354$. illus. [Meiosis.]

Newton, W. C. F., and C. D. Darlington. 1927. Nature (London), July 2.

1929. Jour. Geneties 21:1-15. illus. [Meiosis in polyploids.]

1930. Jour. Genetics $22: 1-14$. illus. [Chiasmata in Fritillaria.]

Newton, W. C. F., and C. Pellew. 1929. Jour. Geneties $20: 405-469$. illus.

Nichols, G. E. 1910. Beih. Bot. Centralbl. $25: 201-241 . \quad$ pls. 8-17. [Juniperus.]

Nichols, S. P. 1922. Amer. Jour. Bot. 9: 18-27. pl. 3. [Healing of cells.]

1925. Bull. Torr. Bot. Club 52: 351-363. illus. [Nitella.] 
Nicholson, F. M. 1923. Jour. Exp. Med. 37: 221-230. [Rickettsia.] Nicolosi-Roncati, F. 1912. Boll. Soc. Bot. Ital. 59-62. [Red algæ.] Nienburg, W. 1914. Zeitschr. Bot. 6:369-400. 17 figs. [Polystigma.] Nieschulz, O. 1922. Arch. Protist. 45:241-263. illus. [Trypanosome.] Nikolaewa, A. 1920a. Verh. Kong. Pflanzenzücht. Saratov. [Triticum.] 1924. Sci. Agron. Jour. 1:570-576. illus. [Rye $\times$ wheat.]

Nishimura, M. 1926. Jour. Coll. Agr. Hokkaido Imp. Univ. 17: 1-61. illus. 1928. Bot. Mag. Tokyo 42: 154-177. illus. [Triticum hybrids.]

Nishiyama, I. 1931. Jap. Jour. Genetics 7:49-102. illus. [Fatuoid oats.]

NOACK, K. 1921. Zeitschr. Bot. 13:1-35. pls. 1, 2. [Origin of plastids.] 1922. Jahrb. Wiss. Bot. 61: 459-534. 56 figs. [Pelargonium.] 1924. Verh. Phys.-Med. Gesell. Würzburg 49:45-93. [Pelargonium.] 1925. Verh. Phys.-Med. Gesell. Würzburg 60:47-97. [Varicgation.]

Noul, F. 1903. Biol. Centralbl. 23: 281-297, 321-337, 401-427. [Bryopsis.]

Norén, C. O. 1904. Ark. Bot. Svensk Vet.-Akad. 3: pp. 11. [Juniperus.] 1907. Uppsala Univ. ̊̊rsskr., 64 pp. 4 pls. [Juniperus.]

Nothnagel, M. 1916. Bot. Gazette 61: 453-476. pls. 28-30. [Meiosis.] 1918. Bot. Gazette 66:143-161. pls. 3-5. [Double fertilization.]

Nowikoff, M. 1909. Anat. Anz. 34: 168-173. pl. 2. [Chromidia.] Nussbaum, J. 1913. Arch. Zellf. 10: 359-367. [Golgi matcrial, chromidia, etc.]

Oenlkers, F. 1916. Ber. Deu. Bot. Gesell. 34: 223-227. [Chara.] 1924. Zeitschr. Ind. Abst. Vererb. 34: 259-283. [Enothera: review.] 1926. Jahrb. Wiss. Bot. 65: 401-446. [Enothera.]

Oes, A. 1908. Bot. Zeitung 66:89-120. pl. 5. [Action of nuclease.] 1910. Zeitschr. Bot. 2: 39-49. 6 figs. [Action of nucleasc.]

Ogata, M. 1883. Arch. Anat. Physiol., Physiol. Abt., 405-437. pl. 6.

Oguma, I. 1921. Jour. Coll. Agr. Hokkaido Imp. Univ. 10. [Mantis.] 1927. Jour. Coll. Agr. Hokkaido Imp. Univ. 16: 203-227. illus. [Pigeon.] 1930. Arch, de Biol. 40 : 205-226. illus. [Man.]

Ogdia, K., and H. Kinara. 1922. Dobutsu-gaku Zasshi 34: 424-435. 4 figs. 1923. Arch. de Biol. 33 : 493-514. 3 pls. [Human chromosomes.]

OGUma, K., and S. MaKino. 1932. Jour. Genetics $26: 239-254$.

Онashi, H. 1930. Bot. Gazette 90:177-197. illus. [Edogonium.]

Orabe, S. 1929. Sci. Rep. Tohoku Imp. Univ. 4:661-669. illus. [Sargassum.] Ouve, E. W. 1904. Beih. Bot. Centralbl. 18: 9-44. pls. 1, 2. [Cyanophyceæ.] 1907. Trans. Wis. Acad. Sci. 15: 754-773. pl. 47. [Ceratiomyxa.]

Oliver, C. P. 1929. Science 71: 44-46. [X-rays and mutation.]

Oliver, F. W. 1888. Ann. Botany 1: 237-253. pl. 12. [Plasmodesmis.]

Otson, A. R., and G. N. L.ewis. 1928. Nature. London 121:673-674.

Ottmanns, F. 1889. Bibl. Bot. 14:1-94. 15 pls. [Fucacex.] 1895. Flora 80: 388-420. pls. 6-10. [Vaucheria.] 1898. Flora 85: 1-18. pls. 1, 2. [Coleochate.] 1922-1923. Morphologie und Biologic der Algen. 2d ed. Jena.

O'vara, J. 1931. Cytologia 3:66-76. illus. [Yucca.]

Ono, T. 1926. Sci. Rep. Tohoku Imp. Univ. IV 2: 159-160. illus. [Rumex.]

1927a. Bot. Mag. Tokyo 41:632-633. illus. [Rumcx.]

1927b. Bot. Mag. Tokyo 41: 601-604. [Triploid Primula.] 1928. Bot. Mag. Tokyo 42: 524-533. illus. [Polyploid Rumex.] 1930a. Bot. Mag. Tokyo 44: 168-176. illus. [Intersexes in Rumex.] 
1930b. Sci. Rep. Tohoku Imp. Univ. IV 5: 415-422. illus. [Rumex.]

1932. Bot. Mag. Tokyo 46:321-327. illus. [Polyploidy in Rumex.]

Ono, T., and N. Shimotomai. 1928. Bot. Mag. Tokyo 42: 266-270. illus.

[Polyploidy in Rumex.]

Onslow, M. W. 1923. Practical Plant Biochemistry. Cambridge, Eng.

Orman, E. 1913. La Cellule 28: 365-441. 4 pls. [Cytoplasmie differentiations.]

Osawa, J. 1912. Jour. Coll. Agr. Imp. Univ. Tokyo 4: 83-116. illus.

1913. Areh. Zellf. 10: 450-469. pls. 37, 38. [Taraxacum.]

Ostenfeld, C. H. 1910. Zeitschr. Ind. Abst. Vererb. 3: 241-285. pl. 4. 1912. New Phytol. 11:347-354. [Hieracium: apogamy and hybridization.]

1921. Jour. Geneties 11: 117-122. 2 pls. [Hieracium: apogamy, ete.]

Osterhout, IV. J. V. 1900. Flora 87: 109-115. pl. 5. [Batrachospermum.] 1911. Science $34: 187-189$. [Permeability.]

1917. Seience $46: 367-369$. [Nucleus and oxidation.]

Ostwald, Wolfgang. 1917. Kolloidehemie. I. Dresden und Leipzig.

van Overeem, C. 1921. Beih. Bot. Centralbl. 38: 73-113. 6 pls. [Enothera.] 1922. Beil. Bot. Centralbl. 39:1-80. 15 pls. [Enothera; bibliography.]

Overton, E. 1889. Bot. Centralbl. 39: 65, 113, 145, 177, 209, 241, 273.4 pls.

1893. Ann. Botany $7: 139-143$. [Chromosome reduction in plants.]

1895. Vierteljahrssehr. Nat. Gesell. Zürich. 40 : 159-184. [Permeability.]

1899. Vierteljahrssehr. Nat. Gesell. Zürich. 44: 85-135. [Permeability.]

1900. Jahrb. Wiss. Bot. 34: 669-701. Also Pflïger's Arehiv 92.

Overton, J. B. 1902. Bot. Gazette 33:363-375. pls. 12, 13. [Thalictrum.]

1905. Jahrb. Wiss. Bot. 42: 121-153. pls. 6, 7. [Meiosis.]

1909. Ann. Botany 23: 19-61. pls. 1-3. [Meiosis; chromosome individuality.]

1911. Science 33: 193-194. [Somatie mitosis.]

1913. Seience 37: 841-844. [Artificial parthenogenesis in Fucus.]

1922. Trans. Wis. Acad. Sci. $20: 275-322$. pl. 7. [Somatic mitosis.]

Owens, H. B., and R. R. Bensley. 1929. Amer. Jour. Anat. 44 : 79-109. illus.

Ozawa, Y. 1927. Rev. Gén. Bot. 39 : 218-233. illus. [Chondriome.]

\section{$\mathrm{P}$}

Pace, L. 1907. Bot. Gazette 44:353-374. pls. 24-27. [Cypripedium.]

1909. Bot. Gazette 48: 126-137. pls. 7-9. [Calopogon.]

1913. Bot. Gazette 66 : 376-394. pls. 13, 14. [Atamosco.]

PACKARD, C. 1918. Biol. Bull. 35: 50-70. illus. [Radium.] 1931. Quar. Rev. Biol. 6: 253-280. illus. [Irradiation.]

Painter, T. S. 1920. Anat. Ree. 17: 328. [Anolis: spermatogenesis.] 1921a. Seience 53: 503-504. [Sex-chromosomes in opossum and man.] 1921b. Jour. Exp. Zoöl. 34: 281-327. 4 pls. [Lizards: spermatogenesis.]

1922a. Seience 56:286-287. 4 figs. [Sex-ehromosomes of monkey.]

1922b. Jour. Exp. Zoöl. 35: 13-45. 3 pls. [Didelphys: spermatogenesis.]

1923a. Jour. Exp. Zoöl. $37: 291-335.6$ pls. 4 figs. [Man: spermatogenesis.]

1923b. Science 58: 247-248. [Sex-chromosomes of monkey.]

1925. Amer. Naturalist 59 : 385-409. 21 figs. [Mammal ehromosomes.]

1926. Seience 64:336. illus. [Rodents.]

1927. Geneties $12: 379-392$. illus. [v-o mice.]

1930. Jour. Heredity 21:61-64. illus. [Man.]

1931. Seience 73:647-648. illus. [X-chromosome map.]

Painter, T. S., and H. J. Muller. 1929. Jour. Heredity $20: 287-298$. illus. 
Palla, E. 1889. Ber. Deu. Bot. Gesell. $7: 330-331 . \quad$ [Enucleated cells.] 1890. Flora 73: 314-331. pl. 13. [Enucleated cells.]

1906. Ber. Deu. Bot. Gesell. 24: 408-414. pl. 19. [Enucleated cells.]

Palladin, V. I. 1923. Plant Physiology. (Engl. transl. ed. by Livingston.)

Palm, D. 1920. Svensk Bot. Tidskr. 14: 261-266. [Pollen development.]

Palmer, L. S. 1922. Carotinoids and Related Pigments. New York.

Paltauf, A. 1928. Sitzungsber. Akad. Wiss. Wien, Math.-Naturw. Kl., I 137 : 691-716.

Pappenheimer, A. M. 1916. Anat. Rec. 11: 107-148. [Golgi material; review.] Parat, Marguerite, 1927. Compt. Rend. Soc. Biol. Paris 96:1360-1363. illus.

Parat, Marguerite, and Maurice Parat. 1927. Compt. Rend. Soc. Biol. Paris

$96: 1363-1364$. [Vacuome.]

1930. Arch. d'Anat. Micr. 26: 447-474. illus. [Golgi zone.]

Parat, Maurice. 1926. Compt. Rend. Assoc. Nat. 21: (1-8). [Golgi zone.]

1928. Arch. d'Anat. Micr. 24: 73-357. illus. [Vacuome, Golgi, chondriome.]

Parat, Maurice, and E. Gambier. 1926. Compt. Rend. Soc. Biol. 94: 748-749.

Parat, Maurice, and J. Painlevé. 1924ab. Compt. Rend. Acad. Sci. Paris 179:

$543-544 ; 612-614$. illus.

1924c. Compt. Rend. Acad. Sci. Paris 179: 844-846. [Golgi and vacuome.]

1925a. Bull. Histol. Appl. [Vacuome and chondriome.]

1925b. Compt. Rend. Soc. Biol. Paris 77 I: 65-67, 767-768. [Secretion.]

1925c. Compt. Rend. Acad. Sci. Paris 180: 1134-1137. illus. [Vacuome and

Golgi.]

1925dc. Compt. Rend. Soc. Biol. Paris 92: 65-67. [Secretion.]

Paravicini, E. 1918. Centralbl. Bakt. II 48:337-340. 12 figs. [Bacteria.]

Parker, G. H. 1929a. Amer. Naturalist 63: 97-117. illus. [Neurofibrils.]

1929b. Quar. Rev. Biol. 4: 155-178. illus. [Neurofibrils.]

Parmenter, C. L. 1920. Jour. Gen. Physiol. 2: 205-206. [Chromosomes of parthenogenetic frogs.]

1925. Jour. Gen. Physiol. 8: 1-20. illus. [Frog.]

1926. Anat. Record $34: 150$. [Frog.]

Parnell, F. R. 1921. Jour. Geneties 11: 209-212. pl. 21 . [Rice pollen.]

Pascher, A. 1916c. Ber. Deu. Bot. Gesell. 34: 228-242. 5 figs.

1918a. Ber. Deu. Bot. Gesell. 36: 163-168. [Meiosis and segregation.]

1918b. Ber. Deu. Bot. Gesell. 36:168-172. [Edogonium.]

1929a. Jahrb. Wiss. Bot. 70:329-347. illus. [Endonema.]

1929b. Jahrb. Wiss. Bot. 71: 386-462. [Endosymbiosis.]

Paton, J. B. 1921. Amer. Jour. Bot. 8: 471-501. [Pollen tube growth.]

Patten, R. E. P., and S. B. Wigoder. 1930. Quar. Jour. Micr. Sci. 73: 633-650.

Patterson, J. T. 1917. Biol. Bull. 33: 57-66. illus. See also $38-50$.

1921. Jour. Morphol. Physiol. 36: 1-69. illus.

1928. Science 68: 41-43. [X-rays and mutation.]

1929. Jour. Heredity $20: 261-267$. illus. [X-rays and mutation.]

1930a. Genetics 15: 141-149. [X-rays and mutation.]

1930b. Proc. Nat. Acad. Sci. 16: 109-111. [X-rays and inutation.]

1931a. Jour. Exp. Zoöl. 60: 173-211. illus. [Gynandromorphs.]

1931c. Anat. Rec. $51: 111$. [ $X$-chromosome.]

Patterson, J. T., and H. J. Muller. 1930. Geneties 15: 495-578. [X-ray mutations.]

Patterson, J. T., and T. S. Painter. 1931. Science 73: 530-531. illus.

Patterson, P. M. 1927a. Jour. El. Mitchell Sci. Soc. 43: 108-123. illus. [Achlya.]

1927b. Jour. El. Mitchell Sci. Soc. 43: 124-128. illus. [Pythium.] 
Pauli, W. E., and A. Hartiann. 1924. Arch. Mikr. Anat. 103: 95-167. illus. Paull, W. E., and G. Politzer. 1929. Zeitschr. Zellf. Mikr. Anat. 8: 404-424. Paulmier, F. C. 1899. Jour. Morph. 15: suppl., 223-272. pls. 13, 14. [Anasa.] Pavillard, J. 1910. Prog. Rei Bot. 3 : 474-544. [Veg. protistology.]

Payen, A. 1842. Mémoires sur les développements des végétaux. Paris. 1846. Mém. Acad. Paris 9.

PAYNe, F. 1909. Biol. Bull. 16: 119-166. pl. 1. 12 figs. [Sex-chromosomes.] 1916. Jour. Morphol. $28: 287-327.4$ pls. 5 figs. [Gryllotalpa.] 1927. Jour. Morphol. Physiol. 43 : 299-345. illus. [Gelastocoris: ơ'-genesis.] 1932. Jour. Morph. 53 : 523-591. illus. [Golgi bodies.]

Pearson, N. E. 1927. Amer. Naturalist 61: 283-285. [Gynandromorphs.] 1929. Jour. Morphol. Physiol. $47: 531-553$. ilhus. [Gynandromorphs.]

Pekarek, J. 1927. Planta $4: 299-357$. illus. [X-rays and initosis.] Pellew, C., and E. R. Sansone. 1931. Jour. Genetics 25:25-54. illus. [Pisum.] Pensa, A. 1910. Anat. Anz. 37: 325-333. [Chondriosomes.] 1912. Arch. Zellf. 8: 612-662. pls. 25-28. [Chondriosomes.] 1913a. Anat. Anz. 45: 81-90. [Chondriosomes.] 1913b. Arch. Zellf. 11: 557-582. pls. 27, 28. [Chondriosomes.] 1914. Anat. Anz. 46:13-22. [Chondriosomes.] 1925. Compt. Rend. Assoc. Anat. [Proplastids.]

Percival, J. 1926. Jour. Geneties 17:49-68. illus. [Egilops $\times$ Triticum.] 1930. Jour. Genetics 22: 201-278. [Triticum.]

Perroncito, A. 1909. C. Rend. Ist Lombard. 41: Ser. II. [Golgi material, etc.] 1910. Areh. Ital. de Biol. 54:307-345. 3 pls. [Golgi material, etc.]

Perry, K. M. 1932. Jour. Roy. Micr. Soc. 52: 344-356. illus. [Galanthus.]

PÉterfi, T. 1923. Abderhaldens Handbuch Biologischer Arbeitsmethoden. 5: $479-516$.

1927. Roux's Arch. Entw. Org. 112: 660-695. illus.

Peterschilka, F. 1922. Arch. Protist. 45: 153-162. pl. 2. [Mougeotia.] 1923. Arch. Protist. 46: 153-165. pls. 9, 10. [Spirogyra.]

Petersen, J. B. 1929. Dansk Bot. Tidskr. 40: 373-389. illus. [Flagella.]

Petit, A. 1929. Ann. Serv. Bot., Tunis $4: v i+120$. ilhis. [Bacteria.]

Petrunkewitsch, A. 1901. Zool. Jahrb., Anat. Abt., 14:573-608. illus.

Pfeffer, W. 1872. Jahrb. Wiss. Bot. 8: 429-574. pls. 36-38. [Aleurone.] 1874. Flora $57: 2-6,17-27,33-43$. pl. 1. [Oil bodies.]

1889. Flora $72: 46-54$. [Vacuoles.]

1890. Abh. Sächs. Gesell. Wiss. Math.-Phys. Kl. 16. [Vacuoles.]

1899. Ber. Sächs. Gesell. Wiss. Math.-Phys. Kil. [Amitosis.]

1900. The Physiology of Plants. I. Oxford.

Pfeiffer, H. 1930, 1931, 1932. Protoplasma 11, 12, 14, 15, 16.

Pfitzner, W. 1881. Morph. Jahrb. $7: 289-311.2$ figs. [Nuclear structure.] Phelps, L. A. 1926. Trans. Amer. Micr. Soe. 45: 133-145. [Enucleated amoba.] Phillips, O. P. 1904. Contrib. Bot. Lab. Univ. Pa. 2: No. 3. [Cyanophyceæ.] Philp, J., and C. L. Huskins. 1931. Jour. Geneties 24 : 359-404. illus. Picard, M. 1913. Bull. Torr. Bot. Club 40:575-590. [Bibliography of mitosis.] Piech, K. 1924b. Bull. Acad. Polon. Sci. Lett. B, 605-621. illus. 1928. Bull. Acad. Polon. Sci. Lett. B, 1-43. illus. [Scirpus: ơ cells.]

Pincus, G. 1928. Jour. Morphol. Physiol. 44: 515-538. illus. [Rat.]

Pinney, E. 1908. Kansas Univ. Sei. Bull. 4. [Phrynotettix.]

Pinney, E. 1918. Jour. Morphol. 31: 225-292. 14 pls. [Fish hybrids.] 1922. Jour. Morphol. 36 : 401-419. 2 pls. [Fish hybrids.]

Plotnikowa, T. W. 1931. Zeitsehr. Zücht. A 16:662-668. illus. 
Plovgh, H. H. 1917. Jour. Exp. Zoöl. 24: 147-210. 9 figs. 1921. Jour. Exp. Zoöl. 32: 197-202. 3 figs. [Temperature and crossing-over.]

Plough, H. H., and P. T. Ives. 1932. Proc. 6 Internat. Cong. Genet. 2 : 156-158.

Plowe, J. Q. 1931a. Protoplasma 12:196-220. illus. [Membranes.] 1931b. Protoplasma 12: 221-240. [Membranes.]

Poddubnaja, V. 1927. Planta $4: 284-298$. illus. [Compositæ.]

Poirault, G. 1893. Ann. Sci. Nat. Bot. VII 18: 113-256. 43 figs.

Policard, A. 1912. Compt. Rend. Soc. Biol. Paris 72:228-230. [Chondriosomes.]

Policard, A., and G. Mangenot. 1922. Compt. Rend. Acad. Sci. Paris 174:

645-647.

1923ab. Compt. Rend. Acad. Sci. Paris 176: 1841-1844; 177: 346-348. [Oil.]

Politis, I. 1914a. Atti Inst. Bot. Univ. Pavia II 14: 335-361. pls. 13-15.

Poljansky, G., and G. Petruschewsky. 1929. Arch. Protist. 67: 11-45. illis.

Pollack, H. 1928. Biol. Bull. 55 : 383-385. [ $p \mathrm{H}$ in amoba.]

Pollister, A. W. 1930. Jour. Morphol. Physiol. 49: 455-507. illus. [Gerris.] 1932. Science $75: 390-391$. [Centriole.]

Poole, C. F. 1931. Univ. Calif. Publ. Agr. Sci. 6: 169-200. illus. [Crepis.] 1932. Univ. Calif. Publ. Agr. Sci. 6:231-255. illus. [Crepis hybrid.]

Popa, G. T. 1927. Biol. Bull. 52: 238-257. illus. [Spermatozoön.]

Popoff, M. 1906. Anat. Anz. 29 : 249-25s. 4 figs. [Chromidia.]

1907a. Arch. Protist. 1: suppl. 44-82. [Chromidia.]

1907b. Arch. Mikr. Anat. 70: 43-129. pls. 4-8. [Chromidia.]

1908. Zeitschr. Wiss. Zool. $89: 487-524$. illus.

1910. Festschr. f. R. Hertwig. Jena. 19-48. pls. 4-6. [Chromidia.]

1916. Biol. Centralbl. 36: 175-191. illus. [Stimulation of somatic cells.]

1922. Jahrb. Univ. Sofia 19 : II. [Stimulation of somatic cells.]

1931. Die Zellstimulation. Berlin.

Popovici, H. 1925. Compt. Rend. Acad. Sci., Paris 181: 126-128. [Oils.]

Portier, P. 1917. Compt. Rend. Acad. Sci. Paris 165: 267-269.

1918. Les Symbiotes. Paris.

PrÁt, S. 1925. Arch. Protist. 52:142-165. illus. [Cyanophyceæ.]

Pratje, A. 1920. Biol. Zentralbl. 40: 88-112. [Chemistry of nucleus.]

Prell, H. 1923a. Genetica 5: 177-190. 4 figs. [Rhegmatypy.]

1923b. Genetica 5: 191-208. 8 figs. [Apomixis.]

Preston, R. D. 1931. Proc. Leeds Phil. Soc. 2: 185-195. illus. [Valonia wall.]

Prevost et Dumas. 1824. Ann. Sci. Nat. Bot. 1: 1, 167; 2: 100, 129.

Priestley, J. H. 1921. New Phytol. 20:17-29. [Suberin and cutin.]

Pringshem, N. 1854. Untersunchungen über Bau und Bildung der Pflanzenzelle.

Berlin.

1855, 1856. Monatsber. Kgl. Akad. Wiss. Berlin. [Syngamy in algæ.]

Prowazek, S. 1905. Arb. Kais. Gesundheitsamt 21. [Plasmodiophora.]

Prywer, C. 1931. Acta Soc. Bot. Polon. 8: 19-46. illus. [Beta.]

Punnett, R. C. 1927. Mendelism. 7th ed. London.

Py, G. 1929. Compt. Rend. Soc. Biol. Paris 101: 919-921. illus.

Quisumbing, E. 1925. Bot. Gazette 80:439-449. illus. [Plasmodesms.]

$\mathrm{R}$

RABL, C. 1885. Morphol. Jahrb. 10: 214-330. pls. 7-13. 5 figs. RACiborski, M. 1893. Anz. Akad. Wiss. Krakau, p. 259. [Elaioplasts.] 
Radlkofer, L. 1856. Ann. Sci. Nat. IV Bot. 5: 220-250. illus.

Ranlow, G. 1914. Mycol. Centralbl. 5: 177-198. pls. 1, 2. 20 figs. [A scobolus.] Ramsden, W. 1904. Proc. Roy. Soc. London 72:156-164. [Colloids.]

Randolph, L. F. 1922. Bot. Gazette 73: 337-375. pls. 11-16. [Plastids.] 1928. Cornell Univ. Agr. Exp. Sta. Mem. 117. [Zea.]

1932. Proe. Nat. Acad. Sei. 18: 222-229. [Temperature effects.]

Randolph, L. F., and B. McClintock. 1926. Amer. Naturalist 60:99-102. illus. Rappeport, T. 1922. Arch. Zellf. 16: 371-382. pl. 18. [Human ehromosomes.] Ratcliffe, H. L. 1927. Biol. Bull. 53: 109-123. illus. [Euglena.] vom Rath, O. 1891. Zool. Anz. 14:331, 342, 355. 3 figs. [Amitosis.] 1892. Areh. Mikr. Anat. 40: 102-132. pl. 5. [Gryllotalpa: ơ-genesis.] 1893. Zeitsehr. Wiss. Zoöl. 67: 97-185. pls. 7-9. [Salamandra.] 1895. Areh. Mikr. Anat. 46:168-238. pls. 6-8. [Meiosis in $\left.\sigma^{7}.\right]$ RAU, N. S. 1930. Jour. Indian Bot. Soe. 9: 79-113. illus. [Cyanotis.] Rauber, A. 1883. Morphol. Jahrb. 8: 233-338. pls. 11-14. Rawin, W. 1924. Areh. Mikr. Anat. 101:53-61. Rawitscher, F. 1912. Zeitschr. Bot. 4:673-706. [Ustilago.] Redfield, H. 1930. Geneties $15: 205-252$. [Triploid Drosophila.] 1932. Geneties 17: 137-152. illus. [Triploid Drosophila.]

Reed, G. 1923. Amer. Jour. Bot. $10: 234-238$. [Species coneept.]

REED, G. B. 1915. Jour. Biol. Chem. 22: 99-111. [Oxidases.]

Reed, T. 1914. Ann. Botany 28: 271-281. pls. 18, 19. [Meiosis in Allium.] Rees, C. W. 1921. Amer. Naturalist 55: 464-469. figs. 2. [Neuromotor apparatus.]

1922b. Univ. Calif. Publ. Zoöl. 20 : 333-364. pls. 32-36. [Neuromotor app.] 1931. Jour. Morphol. Physiol. 52 : 195-213. illus. [Diplodinium.]

Reeves, R. G. 1928. Amer. Jour. Bot. 16: 114-122. illus. [Cell plate.]

Regaud, C. 1908. Compt. Rend. Soc. Biol. Paris 65:660-662. [Chondriosomes.] 1911. Rev. de Méd. [Chondriosomes.]

1919. Compt. Rend. Soe. Biol. Paris $82: 244-247$. [Symbiotes.]

Reichenow, E. 1908. Areh. Mikr. Anat. 72:671-718. pl. 33. [Chromidia.] 1909. Arb. Kais. Gesundheitsant 33. [Hæmatococcus.]

1928. Arch. Protist. 61: 144-166. illus. [Endosome.]

Reichert, E. T. 1913. Carnegie Inst. Wash. Publ. 173.900 pp. 102 pls.

1914. Science 40:649-661. [Germ-plasm as a stereochemic system.]

1919. Carnegie Inst. Wash. Publ. 270. 834 pp. 34 pls. [Starch; h:emoglobin.] Reichent, E. T., and A. P. Brown. 1909. Carnegie Inst. Publ. 116.338 pp. Reinke, F. 1894. Arch. Mikr. Anat. 43: 377-422. pls. 22-24.

Reinke, F., und H. Rodewald. 1881. Untersuch. Bot. Lab. Göttingen, Heft $2,1-75$.

Reiss, P. 1922. Compt. Rend. Soe. Biol. Paris 87: 255-256. [Golgi material.] Reiter, T., and D. Gábor. 1928. Zellteilung und Strahlung. Berlin. 1929. Strahlentherapie 28: 125-131. [Ultra-violet rays.]

Remak, R. 1841. Areh. Anat. Physiol, 177-188. pl. 8. [Cell-division.]

Renaut, J. 1907, 1911. Areh. Mikr. Anat. 9. Rev. de Méd. 31.

Renner, O. 1913. Ber. Deu. Bot. Gesell. 31:334-335. [Gnothera: syngamy.]

1914. Flora 107: 115-150. pls. 12, 13. [Enothera: syngamy.]

1916a. Ber. Deu. Bot. Gesell. 34: 858-869. [Enothera.]

1916b. Biol. Centralbl. 36:337-374. [Alternation of generations.]

1917a. Zeitsehr. Ind. Abst. Vererb. 18: 121-394. [QEnothera.]

1919a. Ber. Deu. Bot. Gesell. $37: 129-135.2$ figs. [Enothera pollen.]

1919b. Zeitschr. Bot. 11: 305-380. 39 figs. 1 pl. [Enothera pollen.]

1920. Biol. Zentralbl. 40:268-277. [Nature of gene.] 
1924a. Zeitschr. Ind. Abst. Vererb. 33: 317-347. [Species hybrids.]

1924b. Biol. Zentralbl. 44: 309-336. illus. [Variegation.]

1925a. Untersuchungen über die faktorielle Konstitution einiger Komplex-hetero-

zygotischer Enotheren. Leipzig.

1925b. Bibliotheca Genetica 9. $168 \mathrm{pp}$. illus.

1929. Artbastarde bei Pflanzen. Berlin.

Renner, O., and W. Kupper. 1921. Ber. Deu. Bot. Ges. 39 : 201-206.

Reuter, E. 1909. Acta Soc. Sci. Fennicae $37:$ 1-52. [Merokinesis.]

1930. Acta Zool. Fennica 9:1-487. illus.

Reznikoff, P., and H. Pollack. 1928. Biol. Bull. 55: 377-382. [pH.]

Rhondes, M. M. 1931a. Science 73:340-341. [Cytoplasm in sterile Zea.]

1931b. Proc. Nat. Acad. Sci. $17: 694-698 . \quad$ [Zea, linkage.]

1931c. Genetics 16: 490-504. [Translocation.]

1932. Proc. Nat. Acad. Sci. 18: 481-484. [Crossing-over.]

1933. Jour. Geneties $27: 71-93$. [Cytoplasm in sterile Zea.]

Rhombler, L. 1896-1903. Roux's Arch. Entw. 3 : 527-623, pl. 26; 4:659-730, pl. 38;

$7: 535-554$, pl. $12 ; 8: 187-238,28$ figs; $16: 476-535,36$ figs.

Rich, A. R. 1926. Johns Hopkins Hosp. Bull. 39: 330-365. [Dutrochet.]

Richards, A. 1916, 1917. Science 43: 178. Biol. Bull. $32: 249-290.4$ pls.

Richards, O. W. and G. W. 'Taylor. 1932. Biol. Bull. 63: 113-128.

Richardson, C. W. 1914. Jour. Genetics 3 : 171-178. See also 13 : 147-152.

Richardson, E. 1929. Nature. London. Oet. 12. [Pisum.]

Rickett, H. W. 1923. Ann. Botany 37: 225-260. pls. 3, 4. [Spharocarpos.]

Riddle, O. 1914. Bull. Amer. Acad. Med. 15. [Sex control.]

1916. Amer. Naturalist $50: 385-410$. [Sex control.]

1917a. Jour. Wash. Acad. Sei. $7: 319-356 . \quad$ [Sex Control.]

1917b. Science $46: 19-24$. [Sex control.]

1920. Proe. Soc. Exp. Biol. Med. 18: 88-91. [Sex control.]

1924. Amer. Naturalist 58: 167-181. 1 fig. [Sex control.]

1927. Zeitschr. Sexualwiss. 1: 193-218. [Sex reversal.]

Riddle, O., and A. A. Spohn. 1912. Science 35: 462-463. [Sex control.]

Riddle, O., and E. H. Behre. 1921. Amer. Jour. Physiol. $57: 228-249$. [Sex.]

RigG, G. B. 1924. The Pharmacist's Botany. New York. [Pigments.]

Riker, A. J. 1921. Bull. Torr. Bot. Club 48: 141-148. pl. 3. [Chondriosomes.]

Ritter, G. 1911. Zeitschr. f. Bot. $3: 1-42$. [Streaming.]

Ritter, G. J. 1925. Indus. Eng. Chem. 17: 1194. [Lignin.]

1928. Indus. Eng. Chem. $20: 941-945$. illus. [Cell wall structure.]

Ritter, W. E. 1919. The Unity of the Organism. Boston.

Ritter, W. E., and E. W. Bailey. 1928. Univ. Calif. Publ. Zoöl. 31 : 307-358.

Rivett, M. F. 1918. Änn. Botany 32:207-214. pl. 6. 3 figs. [Oil bodies.]

Robrins, W. J., and H. W. Ricketт. 1929. Botany. New York.

Roberts, H. F. 1929. Plant Hybridization before Mendel. Princeton.

Robertson, A. 1904. New Phytol. 3: 205-216. pls. 7-9. [Torreya.]

Robertson, T. B. 1908. Jour. Biol. Chem. 4: 1-.

1920. Prineiples of Biochemistry. Philadelphia and New York.

1923. The Chemical Basis of Growth and Senescence. Philadelphia.

Robertson, W. R. B. 1915. Jour. Morphol. 26: 109-141. pls. 1-3. [Synapsis.]

1916. Jour. Morphol. $27:$ 179-332. pls. 1-26. [Meiosis.]

1919. Proc. Amer. Soc. Zoöl., Dec. 1919.

1920. Anat. Rec. 17: 329. [Presynaptic split.]

1921. Anat. Ree. 20 : 199. [Chromatid arrangement.]

1925. Anat. Rec. 31: 307-308. [Parthenogenetic Tettigidæ.] 
1930. Jour. Morphol. Physiol. 50 : 209-257. illus. [Tettigidæ.]

1931a. Jour. Morphol. Physiol. 51:119-145. illus. [Presynaptic split.]

1931c. Genetics 16:349-352. illus. [Split in anaphase $1 I$.]

Robyns, W. 1924. La Cellule $34: 367-454.5$ pls. [Achromatic figure.]

1926. La Cellule $37:$ 144-177. [Spindle of cytokinesis.]

1928. La Cellule 38: 177-191. illus. [Raphides.]

1929. La Cellule 39: 85-118. illus. [Spindle in vivo.]

Rogers, L. M. 1926. La Cellule 37:327-352. illus. [Lygodium, syngamy.]

Roнde, E. 1903. Zeitschr. Wiss. Zool. 73:497-682. pls. 32-40. [Nucleolus.]

1908. Histogenetische Untersuchungen. I. Breslau. [Plasmodial tissues.]

1923. Zeitschr. Wiss. Zool. 120: 325-535. 111 figs. [Plasmodial theory.]

Roкizky, F. T. 1930. Biol. Zentralbl. 50:554-566. [Temperature and mutation.]

Rosen, F. 1892. Beitr. Biol. Pflanzen (Cohn) 5:443-459. pl. 16.

Rosenberg, O. 1904a. Ber. Deu. Bot. Gesell. 22: 47-53. illus. [Drosera.]

1905. Botan. Notiser, 1-24. 14 figs. [Meiosis.]

1906. Ber. Deu. Bot. Gesell. 24: 157-161. pl. 11. [Apomixis.]

1907a. Dansk Bot. Tidskr. 28: 143-170. 2 pls. 13 figs. [Apomixis.]

1907b. Svensk Bot. Tidskr. 1:398-410. pl. 7. [Meiosis.]

1909a. Svensk Bot. Tidskr. 3: 64-77. pl. 1. [Meiosis.]

1909b. Kgl. Svensk Vet. Handl. 43: 3-64. 4 pls. 33 figs. [Drosera.]

1909c. Svensk Bot. Tidskr. 3: 163-173. pl. 5. [Prochromosomes.]

1909d. Svensk Bot. Tidskr. 3: 150-162. 7 figs. [Taraxacum; Rosa.]

1912. Svensk Bot. Tidskr. 6: 915-919. 11 figs. [Chondrilla.]

1917. Svensk Bot. Tidskr. 11: 145-206. 26 figs. [Hieracium: meiosis.]

1918. Ark. Bot. 15: 1-16. 6 figs. [Crepis chromosomes.]

1920. Svensk Bot. Tidskr. 14: 320-326. [Crepis chromosomes.]

1926b. Ber. Deu. Bot. Gesell. 44: 455-460. [Chromosome doubling.]

1927a. Hereditas 8: 305-338. illus. [Semiheterotypic mitosis.]

1927b. Hereditas 9: 285-288. illus. [Semiheterotypic mitosis.]

1930. Apogamie und Parthenogenesis bei Pflanzen. Berlin.

Rosenbusch, T. 1909. Arch. Protist. 15: 263-296. pls. 25-27. [Trypanosomes.]

Rosenvinge, L. K. 1888. Dansk Bot. Tidskr. $17:$ 10-19.

Roskin, G., and K. Romanowa. 1928. Arch. Protist. 60:482-491. illus.

Rossmann, B. 1929. Arch. Entw. Org. 114: 583-586.

Roтн, F. 1906. Disst., Bonn, Verh. Naturw. Ver. Rheinl.-Westf. 63: 327-360.

Rotilert, W. 1892. Beitr. Biol. Pflanzen 5:291-349. pl. 10. [Saprolegnia.]

1912. Bull. Acad. Sci. Cracovie, pp. 91, 192, 210. [Chromoplasts.]

Roux, W. 1883. Ueber die Bedeutung der Kernteilungsfiguren. Leipzig.

Rǘckert, J. 1891. Anat. Anz. 6: 308-322. [Meiosis.]

1892. Anat. Anz. $7: 107-158.6$ figs. [Meiosis.]

1893. Ergeb. Anat. Entw. 3: 517-583. [Meiosis.]

1894. Ergeb. Anat. Entw. Anat. Hefte 4:261-352. pls. 21-25. [Meiosis.]

1895. Arch. Mikr. Anat. 45: 339-369. pls. 21, 22. [Cleavage mitosis.]

Rudolph, K. 1912. Ber. Deu. Bot. Gesell. 30: 605-629. pl. 18. [Plastid.]

Ruhland, W. 1908. Jahrb. Wiss. Bot. 46: 1-54. [Permeability.]

1912. Jahrb. Wiss. Bot. 51: 376-431. [Permeability.]

1915. Biochem. Zeitschr. 54:59-77. [Permeability.]

Ruhland, W., and K. Wetzel. 1924. Ber. Deu. Bot. Gesell. 42:3-14. illus.

Runijantzew, A. 1926. Arch. Exper. Zellf. 3: 115-165. illus.

Rumjantzew, A., and E. Wermel. 1925. Arch. Protist. 52:217-264. illus.

Russeld, E. S. 1930. The Interpretation of Development and Heredity. Oxford. Russow, A. 1883. Sitzber. Nat. Gesell. Univ. Dorpat 6: 562. [Plasmodesms.] 
Rutgers, F. L. 1923. Embryosac ... of Moringa. Thesis, Utrecht. Leiden.

Ruttle, M. L. 1927. Univ. Calif. Publ. Bot. 11: 159-176. illus.

1928. Univ. Calif. Publ. Bot. 11: 213-232. illus. [Haploidy. Chimera.]

RuYs, J. D. 1925. Énumération des Plantes Phanérogames Angiospermes Examinées au Point de Vue de la Karyologie. Leiden. [1200 titles.]

Rybin, V. A. 1927b. Bull. Appl. Bot. Genet. Plant Breed. 17(3): 191-240. illus. 1929. Ber. Deu. Bot. Gesell. $47: 385-394$. illus. [Nicotiana.]

Rytz, W. 1907. Centralbl. Bakt. II $18: 635-655,799-825$. illus.

\section{S}

von SAchs, J. 1875. Geschichte der Botanik. (Engl. transl. 1889).

1882. Vorlesungen über Pflanzenphysiologie.

1892. Flora 75: 57-67. 1893. Flora $77: 49-81$.

1895. Flora (Ergänzungsband) $81: 405-434$.

Safz, F. A. 1928. Conf. y Res. Cien., R. Soc. Españ. Hist. Nat. 2. illus. 1930a. Rev. Museo La Plata 32: 317-361. illus.

1930c. Aetas Cong. Internat. Biol. Montevideo. [Didelphis.]

SAGUCHI, S. 1917. Jour. Morphol. $29: 217-279$. pls. 1-4. [Ciliated cells.] 1920ab. Amer. Jour. Anat. $26: 1-57,6$ pls.; 347-421, 5 pls. [Panereas.]

SAKAliura, T. 1914. Bot. Mag. Tokyo 28:131-147. pl. 2. [Mitosis in Vicia.] 1915. Bot. Mag. Tokyo 29: 287-300. pl. 13. [Chromosome constrictions.]

1918. Bot. Mag. Tokyo $32: 151-154$. [Triticum ehromosomes.]

1920. Jour. Coll. Sei. Imp. Univ. Tokyo 39: $221 \mathrm{pp} .7 \mathrm{pls}$.

1927a. Protoplasma 1:537-565. illus. [Chromonemata.]

1927b. Bot. Mag. Tokyo 41:59-63. illus. [Chromonemata.]

Sakamura, T., and I. Stow. 1926. Jap. Jour. Bot. 3:111-137. illus.

Salter, J. H. 1898. Jahrb. Wiss. Bot. 32: 117-166. pls. 1, 2. [Starch.]

Samuelsson, G. 1913. Svensk Bot. Tidskr. $7:$ 97-188. 17 figs. [Endosperm.]

Sanchez, M. 1922bc. Bol. R. Soc. Españ. Hist. Nat. 22 : 37S-381; 456-462.

Sande-Bakhuyzen, H. L. van de. 1926. Proc. Soc. Exp. Biol. Med. 23: 302-305.

Sands, H. C. 1923. Amer. Jour. Bot. 10:343-360. pls. 29, 30.

Sansome, E. R. 1932. Cytologia $3: 200-219$. illus. [Pisum.]

Sansome, F. W. 1929. Conference on Polyploidy, J. Innes Hort. Inst., Jan. 19. 1930. Proc. 2 Internat. Cong. Sex Res., 248-257.

Sansome, F. W., and J. Philp. 1932. Recent Advances in Plant Geneties. Philadelphia.

Santos, J. K. 1923. Bot. Gazette 75: 42-59. pl. 3. [Sex in Elodea.] 1924. Bot. Gazette $77: 353-376$. pls. 23-27. [Sex in Elodea.]

SAp̌̌Hin, A. A. 1911. Ber. Deu. Bot. Gesell. 29:491-496. 5 figs. [Plastids.] 1913a. Investigations on the individuality of plastids. (Russian.) Odessa. 1913b. Ber. Deu. Bot. Gesell. 31: 14-16. 1 fig. [Individuality of plastid.] 1915. Arch. Zellf. 13 : 319-398. pls. 10-26. [Individuality of plastid.]

Sappin-'Trodffy, P. 1896. Le Botaniste 5: 59-244. illus.

SARGANt, E. 1896. Ann. Botany 10:445-477. pls. 22, 23. [Lilium: q-genesis.] 1897. Ann. Botany 11: 187-224. pls. 12, 13 . [Lilium: o'-genesis.]

SAss, J. E. 1928a. Science $68: 548$. [Coprinus.] 1928b. Papers Mich. Acad. Sei. 9: 287-298. illus. [Psalliota.] 1929. Amer. Jour. Bot. 16:663-701. illus. [Coprinus etc.]

Satina, S., and A. F. Blakeslef. 1925. Proc. Nat. Acad. Sci. $11: 528-534$.

1926a. Proc. Nat. Acad. Sei. 12: 191-196. [Sex in Mucor.] 1926b. Proc. Nat. Aead. Sci. 12: 197-202. [Sex in green plants.] 1927. Proc. Nat. Acad. Sci. 13: 115-122. [Sex in plants.] 
1928a. Proc. Nat. Acad. Sci. 14: 229-235. [Sex in Mucor.]

1928b. Proc. Nat. Acad. Sci. 14: 308-316. [Sex in Mucor.]

1929. Proc. Nat. Acad. Sci. 15: 735-740. [Sex in Mucor.]

1930. Bot. Gazette 90:299-311. illus. [Sex in Mucor.]

Satina, S., and M. Denerec. 1925. Science, 42:225-226. [Sex.]

SAx, K. 1916. Bull. Torr. Bot. Club 43: 505-522. pls. 27-29. [Syngamy.]

1918. Genetics 3: 309-327. pls. 1, 2. [Syngamy.]

1921. Science $54: 413-415$. [Triticum chromosomes.]

1922a. Geneties $7: 513-552.3$ pls. [Chromosomes in Triticum liybrids.]

1922b. Genetics 7:553-558. [Triticum: endosperm, sterility.]

1923. Genetics 8: 301-321. [Triticum: chromosome number and characters.]

1928. Zeitschr. Ind. Abst. Vererb., suppl. 2: 1267-1284. [Triticum.]

1929a. Proc. 4 Internat. Cong. Plant Sci. 1: 345-350.

1930c. Jour. Arnold Arbor. 11: 193-220. illus. [Crossing-over.]

1931a. Proc. Nat. Acad. Sci. 17:601-603. illus. [Crossing-over.]

1931e. Cytologia 3: 36-53. illus. [Rhœo.]

1932. Jour. Arnold Arbor. 13: 180-212. jllus. [Crossing-over.]

Sax, K., and E. F. Garnes. 1924. Jour. Agr. Res. 28: 1017-1032. 2 pls.

SAX, K., and H. J. SAX. 1924. Geneties 9:454-464. pl. 2. [Egilops X Triticum.] Scarth, G. W. 1924. Quar. Jour. Exp. Physiol. 14: 99-113, 115-122.

1927. Protoplasma 2: 189-205. illus. [Living cells.]

Scarth, G. W., R. D. Gibbs, and J. D. Spier. 1929. Trans. Roy. Soc. Canada

III 23 (V): 269-279, 281-287. illus. [Cell wall.]

Scarth, G. W., and F. E. Lloyd. 1927. Science 65: 599-600. [Kinoplasm.]

Schacht, H. 1850. Ann. Sci. Nat. Bot. III 15: 80-109. 1851. [Pollen tube.]

1858. Jahrb. Wiss. Bot. 1: 193-232. pls. 11-15. [Pollen tube.]

1860. Jahrb. Wiss. Bot. 2: 107-168. pls. 14-18. [Pollen walls.]

Schacke, M. A. 1919. Science 44: 218-219. [Sex-chromosomes in Spherocarpos.]

Schatde, R. 1925. Beitr. Biol. Pflanzen (Cohn) 14:231-260. illus.

1929. Planta 8: 383-397. illus. [Mechanism of mitosis.]

1930. Ber. Deu. Bot. Gesell. 48 : 342-348. illus. [Nuelear structure.]

Schaeffer, A. A. 1920. Ameboid Movement. Princeton.

Schaffner, J. H. 1908. Ohio Naturalist 9: 383-388. [Centrosomes.]

1919b. Ohio Jour. Sci. 19:409-416. [Sex in Morus and Salix.]

1919c. Ohio Jour. Sei. 20:25-34. [Sex in Thalictrum.]

1921. Bot. Gazette 71: 197-219. pl. 11. [Sex in hemp.]

1922a. Amer. Jour. Bot. 9: 72-78. [Sex in Arisœma.]

1922b. Ohio Jour. Sei. $22: 149-154$. [Scx in Arisæma.]

1923a. Bull. 'Torr. Bot. Club 60: 73-79. pl. 2. [Sex in IHumulus.]

1923b. Ohio Jour. Sci. 33: 149-159. [Sex in several genera.]

1923c. Ohio Jour. Sci. 33 : 225-240. [Time of sex-determination.]

1923d. Ecology 4 : 323-334. 3 figs. [Sex and length of day.]

1925a. Bull. Torr. Bot. Club 52: 35-47.

1925b. Amer. Naturalist 69: 115-127. [Sex modification.]

1926ab. Ohio Jour. Sci. 26. Bull. Torr. Bot. Club 63.

1927ab. Ohio Jour. Sci. 27. Amer. Naturalist 61.

1927cd. Bull. Torr. Bot. Club 54. Bot. Gazette 84.

1928. Amer. Jour. Bot. 15: 77-85. illus.

1929. Bot. Gazette 87 : 653-659.

1931. Amer. Jour. Bot. 18: 424-430.

Schaudinn, F. 1896. Sitzungsher. Akad. Wiss. Berlin. 1896. I, 83-89. illus. 1903. Arb. Kais. Gesundheitsamt 19. [Rhizopoda.] 
Schaxel, J. 1910a. Areh. Zellf. 4: 265-308. pls. 19-21. [Chromidia.] 1910b. Zool. Anz. 35: 407-414. 3 figs. [Chromidia.] 1910c. Festsehr. f. R. Hertwig. Jena. [Chromidia.] 1910d. Zool. Anz. $36: 33-42.7$ figs. [Chromidia.]

1911a. Zool. Jahrb., Anat. Abt., 31:613-656. pls. 31-33. [Chromidia.]

1911b. Arch. Mikr. Anat. 76: 543-607. pls. 19-23. [Chromidia.]

1911c. Anat. Anz. 39: 337-353. 16 figs. [Chromidia.]

1912. Zool. Jahrb., Anat. Abt., 34: 381-472. pls. 16-28. [Chromidia.]

Scheben, L. 1905. Zeitschr. Wiss. Zool. 79 : 396-431. pls. $20,21$.

Scherrer, A. 1914. Flora $107: 1-56$. pls. 1-3. [Chondriosomes and plastids.]

Schilling, A. J. 1891. Flora 74: 220-299. pls. 8-10. [Eyespot.]

Schimper, A. F. W. 18s0-1S81. Bot. Zeitung 38: 881; $39: 185,201,217$.

1883. Bot. Zeitung $41: 105,121,137,153$. pl. 1. [Plastids.]

1885. Untersuchungen über die Chlorophyllkörper und die ihnen homologen Gebilde.

Sсніӧтz, I. 1920. Brit. Jour. Ophth. 4: 349-359, 393-403. [Colorblindness.]

Schlater, G. 1911. Die Cellularpathologie, usw. Jena.

Schleiden, M. J. 1837. Wiegmann's Archiv 1:289. [Origin of embryo.]

1838. Beitrage zur Phytogenesis. Arch. Anat. Physiol. Wiss. Med. 137-176. illus. [Cell theory.]

1842. Grundzüge zur wissensehaftlichen Botanik. $2 \mathrm{~d}$ ed.

1844. Flora 27: 787-789. [Origin of embryo.]

Schleip, W. 1906. Zool. Jahrb., Anat. Abt., $23: 357-380$. pls. 23, 24. [Planaria.] 1907. Zool. Jahrb., Anat. Abt., 24:129-174. pls. 14, 15. [Planaria or.] 1909b. Zool. Anz. 35: 203-213. 10 figs. [ -genesis; Rhodites.] 1923. Arch. Zellf. 17: 289-367. pl. 15. 24 figs. [Ultra-violet light.]

Schlösser, L. A. 1929. Planta 8: 529-570. illus. [Saprolegnia.]

Schmidt, E. W. 1912. Progr. Rei Bot. 4: 163-181. 6 figs. [Chondriosomes.] 1914. Ber. Deu. Bot. Gesell. 32: 35-47. 7 figs. [Centrifuged cells.]

Schmid, J. 1920. Compt. Rend. Trav. Lab. Carlsberg 14: 1-12. 5 pls.

Schmid, P. 1923. Internat. Rev. Gesammt. Hydrobiol. Hydrograph. 11: 114-147. 1927a. Internat. Rev. Gesammt. Hydrobiol. Hydrograph. 18: 400-414. illus. 1927b. Internat. Rev. Gesammt. Hydrobiol. Hydrograph. 17: 274-288. illus.

Schmid, W. J. 192S. Zool. Jahrb., Allg. Abt., 45: 177-216. illus.

Schmitz, F. 1879a. Festschr. Naturf. Gesell. Halle, 275-320. pl. 12.

1879b. Verh. Naturhist. Ver. Preuss. Rheinl. u. Westf., p. 346.

1852. Verh. Naturhist. Ver. Preuss. Rheinl. u. Westf., 40. (See also Sitzber.

Med. Ges. Nat. u. Heilk. Bonn. 1880.)

1884. Jahrb. Wiss. Bot. 15: 1-177. pl. 1.

Schnarf, K. 1929. Embryologie der Angiospermen. Berlin.

1931. Vergleichende Embryologie der Angiospermen. Berlin.

Schneider, A. 1873. Jahrb. Oberhess. Gesell. Natur-Heilk. 14. Giessen.

Schneider, H. 1914. Areh. Zellf. $12: 359-371$. pl. 28. [Meiosis.]

Schneider, K. C. 1910. Festsehr. f. R. Hertwig. [Chromosome structure.]

Schottl Änder, P. 1593. Beitr. Biol. Pflanzen 6: 267-304. pls. 4, 5.

Schrader, F. 1920. Jour. Morphol. 34: 267-306. 4 pls. [Trialeurodes.] 1921. Biol. Bull. 40: 259-270. 2 pls. [Pseudococcus.]

1923a. Arch. Zellf. $17: 45-62$. pls. 5, 6. [Pscudococcus.]

1923b. Zeitschr. Ind. Abst. Vererb. 30: 163-182. pls. 2-5. [Pseudococcus.]

1923c. Arch. Mikr. Anat. 97:610-622. pl. 26. [Tetranychus.]

1925. Zeitsehr. Ind. Abst. Vererb. 40:1-27. illus. [Daphnia.]

1928. Die Geschlechtsehromosomen. Berlin. 
1929. Psyche 36: 232-236. illus. [Aspidiotus.]

1932. Zeitschr. Wiss. Zool. 142: 520-539. illus.

Schrader, F., and S. Hughes-Schrader. 1924. Jour. Morphol. 39: 157-205.

4 pls. [Acroschismus.]

1926. Zeitschr. Wiss. Zool. 128: 182-200. illus.

1931. Quar. Rev. Biol. 6: 411-438. [Haploidy in Metazoa.]

Schrader, F., and A. H. Sturtevant. 1923. Amer. Naturalist 67: 379-381.

Schratz, E. 1924. Biol. Zentralbl. 44:593-623. illus. [Plastids.]

1928. Ergeb. d. Biol. 3: 228-264. [Manoilov reaction.]

Schreiner, A. and K. E. 1904. Anat. Anz. 24: 561-578. 24 figs. [Meiosis.]

1905. Arch. de Biol. 21 : 183-355. pls. 5-14. [Myxine: meiosis.]

1906a. Arch. de Biol. 22 : 1-70. pls. 1-3. [Tomopteris: spermatogenesis.]

1906b. Arch. de Biol. 22 : 419-492. pls. 23-26. [Salamandra, Spinax, Myxine.]

1906c. Anat. Anz. $29: 465-479.17$ figs. [Ophryotrocha.]

1907. Vid.-Selsk. Skrift, 1-31. [Enteroxenos: meiosis.]

1908a. Vid.-Selsk. Skrift. [Parasynapsis.]

1908b. Vid.-Selsk. Skrift. [Zoögonus: meiosis.]

Scr ÜEPp, O. 1926. Meristeme. Berlin.

Schultz, E. S. 1927. Amer. Jour. Bot. 14:307-322. illus. [Peziza.]

Schultze, M. 1861. Arch. Anat. Physiol. Wiss. Med. 1-27. [Protoplasm doctrine.]

Schultze, W. H. 1913. Verh. Deir. Path. Gesell. 16 : 161-168.

Schulz, A. 1913. Die Geschichte der Kultivierten Getreide. [Halle.]

Schulze, E. 1890, 1892, 1894, 1909. Zeitschr. Physiol. Chem. 14, 16, 19, 61.

Sch Ürhoff, P. N. 1918. Flora 110: 52-66. 3 figs. [Nucleolus.]

1919b. Arch. Zellf. 15: 145-159. 1 pl. [o nuclei.]

1920. Beih. Bot. Centralbl. 37 : I 381-389. 6 figs. [Amitosis.]

1923. Flora N. F. 16:73-84. pl. 1. [Apomixis in Calycanthus.]

1924. Die Plastiden. Berlin.

1926. Die Zytologie der Blütenpflanzen. Stuttgart.

Sch ürhoff, P. N., and F. J. Jǘssen. 1925. Ber. Deu. Bot. Ges. 43: 454-456.

Schussnig, B. 1928a. Oesterreich. Bot. Zeitschr. 77:62-67. illus. [Cladophora.]

1928b. Ber. Deu. Bot. Ges. 46:481-490. illus. [Cladophora.]

1930a. Oesterreich. Bot. Zeitschr. $79: 273-278$. illus. [Cladophora.]

1930b. Absts. 5 Internat. Bot. Cong., 189. Cambridge.

Schwann, TH. 1839. Mikroskopische Untersuchungen über die Uebereinstimmung in der Struktur und dem Wachsthum der Thiere und Pflanzen. Berlin. Prelim.

statement: Froriep's Notizen No. 91: 103, 112. 1838.

Schwarz, W. 1928. Biol. Zentralbl. $48: 302-308$. [Mitogenetic rays.]

Schwarze, C. A. 1922. Mycologia 14: 143-172. pls. 15, 16. [Cytokinesis.]

Schweigger-Seidel, O. 1865. Arch. Mikr. Anat. 1:309-335. pl. 19.

Schweizer, J. 1923. Flora N. F. 16:1-72. 56 figs. [Polyploidy in moss.]

Schwemmle, J. 1924a. Ber. Deu. Bot. Gesell. 42:238-243. 1 pl. [Meiosis.]

1924b. Zeitsehr. Ind. Abst. Vererb. 34: 145-185.

1926. Jahrb. Wiss. Bot. 65: 778-818. illus. [Eucharidium.]

1927. Jahrb. Wiss. Bot. 66:579-595. illus. [Enothera.]

1928. Jahrb. Wiss. Bot. 67: 849-876. illus. [Enothera.]

1929. Tübinger Naturwiss. Abh. $12: 42-49$. illus.

Scott, F. M. 1929. Amer. Jour. Bot. 16: 598-605. illus. [Golgi.]

Sears, P. B. 1917. Ohio Jour. Sci. 27: 97-100. [Taraxacum: apomixis.]

1922. Bot. Gazette $73:$ 308-325, 425-446. pls. 9, 10. [Taraxacum: meiosis.]

1932. Ecology $13: 1-6$. [Fossil pollen.] 
Sedgwick, A. 1894. Quar. Jour. Micr. Sci. 37:87-101. [Organismal Theory.] 1895. Quar. Jour. Micr. Sci. 38: 331-337. [Organismal Theory.] Sedgwick, P. J. 1924. Bot. Gazette 77:300-310. illus. [Encephalartos.] SEIFriz, W. 1918. Biol. Bull. 34: 307-424. 3 figs. [Structure of protoplasm.]

1920. Bot. Gazette 70:360-386. [Viseosity of protoplasm.]

1921. Ann. Botany 35: 269-296. 1 fig. [Membranes; miscibility.]

1923a. Ann. Botany 37: 489-510. 4 figs. [Protoplasm.]

1923bc. Science 57 : 694-696. Amer. Jour. Physiol. 66:124-139. [Phase reversal.]

1924a. Brit. Jour. Exp. Biol. 1: 431-443. [Protoplasm and gels.]

1924b. Brit. Jour. Exp. Biol. 2: 1-11. [Elasticity; viscosity.]

1926a. Amer. Naturalist $60: 124-132$. [Elasticity.]

1926b. Protoplasma 1:1-14. illus. [Echinarachnius oöcytes.]

1926c. Protoplasma 1:345-365. illus. [Erythrocytes.]

1929a. Proc. 4 Internat. Cong. Plant Sci. Ithaca 1: 251-258.

1929b. Amer. Naturalist 63: 410-434. illus. [Contractility.]

1930a. Protoplasma 9: 177-208. illus. [Alveolar structure.]

1931a. Science 73:64S-649. illus. [Structure of protoplasm.]

1931b. Jour. Phys. Chem. 35: 118-129. illus. [Structure of protoplasm.]

Seiler, J. 1913. Zool. Anz. 41 : 246-251. 4 figs. [Lepidoptera.]

1917. Zeitschr. Ind. Abst. Vererb. 18: 81-92. [Lepidoptera.]

1920. Arch. Zellf. $15: 249-268$. pl. 13. [Effect of heat on sex-chromosomes.]

1923. Zeitschr. Ind. Abst. Vererb. 31: 1-99. 3 pls. [Lepidoptera.]

1926. Zeitschr. Ind. Abst. Vererb. 41: 259-284. [Chiasmatypy.]

Selim, A. G. 1930. Cytologia 2: 1-26. illus. [Oryza.]

Senjaninova, M. 1926. Zeitschr. Zellf. Mikr. Anat. 3: 417-430. illus.

1927a. Zeitschr. Zellf. Mikr. Anat. 5: 675-679. illus. [Valeriana.]

1927b. Zeitsehr. Zellf. Mikr. Anat. 6: 464-492. illus. [Moss Plastids.]

1927c. Zeitschr. Zellf. Mikr. Anat. 6: 493-508. illus. [Nephrodium.]

Senjaninova-Korczagina, M. V. 1930. Proc. U.S.S.R. Cong. Genet. Plant

Animal Breed. $2: 453-466$. illus. [Egilops.]

1932. Bull. Appl. Bot. Genet. Plant Breed. 28: 91-118. illus. [Vicia.]

Senn, G. 1908. Die Gestalts- u. Lageveränderung der Pflanzen-Chromatophoren.

Serebrovsky, A. S. 1929. Amer. Naturalist $63: 374-378$. illus.

Sethi, M. L. 1928. Ann. Botany 42:729-738. illus. [Equisetum.]

Shaffer, E. L. 1920. Biol. Bull. 38: 83-103. [Lachnosterna.]

Sharp, L. W. 1911. Bot. Gazette 52:218-225. pls. 6, 7. [Physostegia.]

1912. Bot. Gazette 54:89-119. pls. 7, 8. [Equisetum: spermatogenesis.]

1913. La Cellule $29: 297-331.2$ pls. [Vicia: somatic mitosis.]

1914a. Bot. Gazette 57:531. [Vicia: meiosis.]

1914b. Bot. Gazette 58: 419-431. pls. 33, 34. [Marsilia: spermatogenesis.]

1920a. Amer. Jour. Bot. $7: 341-354$. pls. 22, 23. [Tradescantia: mitosis.]

1920b. Bot. Gazette 69:258-268. pl. 15. [Blasia: spermatogenesis.]

1925. La Cellule 35: 193-235. 1 fig. [Sex determination.]

1929. Bot. Gazette $88: 349-382$. illus. [Chromosome structure.]

Sharp, R. G. 1914. Univ. Calif. Publ. Zoöl. 13: 43-122. [Neuromotor apparatus.]

Shaw, M. 1929. Amer. Jour. Bot. 16: 259-276. [Nelumbo fruit.]

Shaw, W. R. 1898a. Ber. Deu. Bot. Gesell. 16: 177-184. pl. 11. [Blepharoplast.]

1898b. Ann. Botany $12: 261-285$. pl. 19. [Syngamy in Onoclea.]

Silearer, C. 1906. Proc. Roy. Soc. London B 77:498-504. pl. 19.

Sheffield, F. M. L. 1927. Ann. Botany 41: 779-816. illus. [Enothera.]

1929. Proc. Roy. Soc. London B 105: 207-230. illus. [Enothera.]

Sheppard, E. J. 1909. Jour. Roy. Mier. Soc. 551-554. pl. $19 . \quad$ [Nucleolus.]

Shibata, K. 1902. Flora 90:61-66. illus. [Monotropa, syngamy.] 
Shigenaga, M. 1933. Mem. Coll. Sci. Kyoto Imp. Univ. B 8: 217-231. illus.

Shimamora, T. 1928. Bot. Mag. Tokyo 42: 71-82. illus. [Ginkgo.] 1929. Cytologia 1: 59-67. illus. [Pinus egg.]

Sнimotomai, N. 1925. Bot. Mag. Tokyo 39: 122-127. illus. [Brassica.] 1927. Bot. Mag. Tokyo 41: 149-160. illus. [Temperature and meiosis.]

1929. Sci. Rep. Tohoku Imp. Univ. IV 4:369-372. [Potentilla.] 1930. Jour. Sei. Hiroshima Univ. B, 2, 1:1-11. illus. [Potentilla.] 1932. Jour. Sci. Hiroshima Univ. 1: 117-120. illus. [Chrysanthemum.]

Shimotomai, N., and Y. Koyama. 1932. Jour. Sci. Hiroshima Univ. 1: 95-101. illus. [Pogonatum.]

Shinke, N. 1930. Mem. Coll. Sei. Kyoto Imp. Univ. B 5: 239-245. illus. Shinke, N., and M. Shigenaga. 1933. Cytologia 4:189-221. illus.

Shiwago, P. I. 1929. Zeitschr. Zellf. Mikr. Anat. 9: 106-115. illus.

Showalter, A. M. 1921. Bot. Gazette 72: 245-249. pls. 4, 5. [Conocephalum.] 1923. Amer. Jour. Bot. 10: 170-172. 1 pl. [Riccardia ehromosomes.]

1926a. Ann. Botany 40:691-707. illus. [Antherozoids.]

1926b. Ann. Botany 40:713-726. illus. [Syngamy, Riccardia.]

1927a. Ann. Botany 41:37-46. illus. [Syngamy, Fossombronia.]

1927b. Ann. Botany 41: 409-417. illus. [Syngamy, Pellia.]

1927c. Proe. Nat. Acad. Sci. 13: 369-372. [Hermaphroditism.]

1928. Proe. Nat. Acad. Sci. 14:63-66. illus. [Pellia chromosomes.]

1929. La Cellule 38: 295-348. illus. [Syngamy, Riccardia.]

Shold, A. F. 1910. Jour. Exp. Zoöl. 8: 311-354. 1 fig. [Hydatina cycle.]

1921. Biol. Bull. $41: 55-61.6$ figs. [Hydatina: meiosis.]

Shull, G. H. 1910. Bot. Gazette 49: 110-125. 2 figs. [Sex in Lychnis.]

1911. Bot. Gazette $52: 329-368.15$ figs. [Sex in Lychnis.]

1913. Ber. Deu. Bot. Gesell. 31: 40-80. [Melandrium: color.]

1914. Zeitschr. Ind. Abst. Vererb. $12: 265-302$. pls. 1, 2. [Lychnis.]

Siebert, W. W. 1928. Zeitschr. Kilin. Med., 109-260.

De Sinéty, R. 1901. La Cellule $19: 119-278.5$ pls. [Sex-chromosome.]

Sinigaglia, G. 1910. Arch. Ital. de Biol. 53: 392-400. 1 pl. [Golgi material.]

Sinnott, E. W. 1930. Bull. Torr. Bot. Club 57: 1-20. [Cell and organ size.]

Sinnott, E. W. and A. F. Blakeslee. 1922. Proc. Nat. Acad. Sci. 8: 17-19.

Sinnott, E. W., and L. C. Dunn. 1932. Prineiples of Geneties. New York.

Sinotô, Y. 1925. Bot. Mag. Tokyo 39: 159-166. 2 figs. [Plantago.]

1927. Bot. Mag. Tokyo 41: 225-234. illus. [Enothera.]

1928. Proc. Imp. Acad. Japan 4: 175-177. illus. [Sex-chromosomes.]

1929a. Proc. Imp. Acad. Japan 5: 46-47. illus. [Humulus ehromosomes.]

1929b. Cytologia 1: 109-191. illus. [Sex-chromosomes.]

Sirks, M. J. 1922. Handboek der Algemeene Erfelijkheidsleer. The Hague.

1931. Proe. KoninkI. Akad. Wetensch. Amsterdam 34. [Vicia.]

SJövall, E. 1906a. Anat. Hefte 30:259-392. pls. 13-17. [Golgi material.]

1906b. Anat. Anz. 28:561-579. 5 figs. [Golgi material.]

Skalinska, M. 1932a. Proc. 6 Internat. Cong. Genet. 2: 185-186.

1932b. Compt. Rend. Soe. Biol. (Varsovie) 111: 97-99.

Skupieński, F. X. 1928. Acta Soc. Bot. Polon. 5: 255-336. illus. [Didymium.]

Small, J. 1929. Protopl. Monographien 2. [pH.]

De Smet, E. 1914. La Cellule $29: 335-377.3$ pls. [Nucleolus, mitosis.]

Sмiтн, B. G. 1919. Biol. Bull. $37: 246-287.9$ pls. [Cleavage nuclei.]

1929. Jour. Morphol. Physiol. 47: 89-133. illus. [Chromosomal vesicles.]

Sмитн, D. M. 1931. Jour. Morphol. Physiol. 62 : 485-511. illus.

Sirtu, E. L. 1923. Bull. Torr. Bot. Club 50:1-16. pl. 1.

Smith, F. E. V. 1923. Ann. Botany 37:63-73. 12 figs. [Amitosis.] 
Sмıтн, F. H. 1932. La Cellule $41: 243-263$. illus. [Galtonia.]

Sмnth, G. M. 1913. Bull. Torr. Bot. Club 40: 75-87. pl. 1. [Tetradesmus.] 1914. Arch. Protist. 32: 278-297. pls. 16, 17. [Scenedesmus.]

1933. The Fresh-water Algæ of The United States. McGraw-Hill. New York.

Simth, G. M., J. B. Overton, E. M. Gilbert, R. H. Denniston, G. S. Bryan, and

C. E. Allen. 1928. A Textbook of General Botany. New York.

Sмiтh I. 1904. Bot. Gazette $37: 346-352.6$ figs. [Zamia egg.]

Smb́lska, A. 1927. Bull. Acad. Polon. Aci. Lett., Cl. d. Sci. Math. Nat., B, 9931038. illus.

Söderberg, E. 1919. Svensk Bot. Tidskr. 13: 204-211. [Pollen development.]

Sokolow, I. 1913. Arch. Zellf. 9: 399-432. pls. 22, 23. [Scorpion.] 1926. Zeitschr. Zellf. Mikr. Anat. 3:615-681. illus. [Obiscum, Chelanops.] 1929a. Zeitschr. Zellf. Mikr. Anat. 8: 617-654. illus. [Nemastoma.] 1929b. Zeitschr. Zellf. Mikr. Anat. 10:164-194. illus. [Opiliones.]

Sorokin, H. 1924. Publ. Fac. Sci. Charles Univ. Prague 13:15 pp. 2 pls. 1929. Amer. Jour. Bot. 16: 407-420. illus. [Ranunculaceæ.]

Spallanzani, L. 1786. Expériences pour Servir à l'Histoire de la Génération des Animaux et des Plantes. Genève.

Spek, J. 1918a. Roux's Arch. Entw. 44: 1-113. 25 figs. [Surface tension.] 1918b. Roux's Arch. Entw., 44: 217-255. 15 figs. [Streaming.]

1920b. Kolloidchem. Beih. 12:1-91. [Surface tension.]

1926. Protoplasma 1:302-303. [Protoplasmic movement: bibliography.]

Spemann, H. 1914. Verh. Deu. Zool. Gesell., 216-221. illus.

1918. Roux's Arch. Entw. 43: 448-555. [Differentiation.]

1919. Naturwissenschaften $7: 32$. [Differentiation.]

1923. Zur Theorie der Tierischen Entwicklung. Freiburg.

1924. Zeitschr. Ind. Abst. Vererb. 33: 272-294.

Spencer, Herbert. 1864. Principles of Biology.

Spencer, Hope. 1924. Jour. Morphol. Physiol. 39 : 543-551.

Spitzer, W. 1897. Arch. Ges. Physiol. 67:615-656. [Nucleoproteins.]

Spoenr, H. A. 1917. Plant World 20:365-379. [Nucleic acid; pentoses.] 1919. Carnegie Inst. Wash. Publ. 287, 79 pp. [Pentosans.]

Sponsler, O. L. 1922. Amer. Jour. Bot. 9: 471-492. 9 figs. [Starch grain.] 1923. Jour. Gen. Physiol. 5: 757-776. [Starch structure.]

1925a. Science 62: 547-548. [Wall structure.]

1925b. Jour. Gen. Physiol. 9: 221-223. [Wall structure.]

1926. Jour. Gen. Physiol. 9: 677-695. [Wall structure.]

1928a. Amer. Jour. Bot. 15: 525-536. illus. [Wall structure; summary.]

1928b. Indus. Eng. Chem. 20: 1-7. [X-ray methods.]

1929. Plant Physiol. $4: 329-336$. illus. [Wall formation.]

1931. Protoplasma 12:241-254. illus. [Wall of Valonia.]

1933. Quar. Rev. Biol. 8: 1-30. illus.

Sponsler, O. L., and W. H. Dore. 1926. Colloid Sympos. Monogr. 4: 174-202.

1928. Jour. Amer. Chem. Soc. 50: 1940-1950. illus. [Ramie cellulose.]

Sprengel, C. K. 1793. Das neu Entdeckte Geheimniss der Natur in Bau und Befruchtung der Blumen. Berlin.

Sprengel, K. 1802. Anleitung zur Kenntniss der Gewächse.

Stadler, L. J. 1928a. Proc. Nat. Acad. Sci. 14: 69-75. [X-ray mutation.]

1928b. Science 68: 186-187. [X-ray mutation.]

1928c. Anat. Record 41: 97. [X-ray mutation.]

1929. Proc. Nat. Acad. Sci. 15: 876-881. [X-ray mutation.]

1930a. Anat. Rec. 47: 381. [X-ray mutation.]

1930b. Jour. Heredity 21:3-19. illus. [X-ray mutation.] 
1931a. Sci. Agric. 11: 557-572. illus. [Induced chromosome irregularities.] 1931b. Sci. Agric. 11:645-661. illus. [Induced gene mutation.] 1932. Proc. 6 Internat. Congr. Genetics 1: 274-294. [Nature of mutations.] Stadler, L. J., and L. F. Randolph. 1929. Paper read before Sec. O, Amer. Assoc. Adv. Science.

Stakman, E. C., and J. J. Christensen. 1927. Phytopath. 17: 827-834. STÅlfelt, M. G. 1919. Svensk Bot. Tidskr. 13:61-70. [Periodicity.] 1921. K. Svensk Vet. Akad. Handl. $62: 1-114$. [Periodicity in cell-division.] Starks, D. J. 1928. Amer. Jour. Anat. 40:471-499. illus. [Callorhinus.] Steere, W. C. 1932. Amer. Jour. Bot. 19:340-357. illus. [Triploid Petunia.] Sterl, W. N. 1918a. Bot. Gazette 65: 562-563. 1 fig. [Fern spermatozoid.] 1918b. Bull. Torr. Bot. Club 45: 93-108. pls. 4, 5. [Apogamy in ferns.] 1919a. Ann. Botany 33: 109-132. pls. 5-7. [Apogamy in Nephrodium.] 1919b. Bot. Gazette 67: 469-482. pls. 16, 17. [Apogamy in Pteris.] 1923. Bull. Torr. Bot. Club 50: 197-201. 5 figs. [Riccardia sperm.]

Stein, E. 1929. Biol. Zentralbl. 49:112-126. illus. [Radium.] Stenar, A. H. S. 1925. Embryologische Studien I, II. Uppsala.

Stern, C. 1926a. Biol. Zentralbl. 46:344-348. [Y-chromosome gene.] 1926b. Biol. Zentralbl. 46: 505-508. illus. [Attached $X$ and $Y$.] 1926c. Proc. Nat. Acad. Sci. 12: 530-532. illus. [Crossing-over.] 1927a. Naturwissenschaften 15:465-473. illus. [Rôle of $Y$-chromosome.] 1928. Ergeb. d. Biol. 4: 205-359. illus. [Chromosome theory.] 1929. Der Züchter 1: 237-243. [Drosophila chromosome map.] 1931a. Biol. Zentralbl. 51:547-587. illus. [Proof of chromatid exchange.] Stern, K. 1920. Ber. Deu. Bot. Gesell. 38: 28-34. [Chlorophyll.] 1921. Zeitschr. Bot. 13 : 193-239. [Chlorophyll.]

Stevens, F. L. 1901. Bot. Gazette 32 : 77-98. pls. 1-4. [Albugo.] Stevens, N. M. 1903. Zool. Jahrb. 18:227-240. pls. 20, 21. [Sagitta \&-genesis.] 1905. Carnegie Inst. Wash. Publ. 36. [Accessory chromosome.] 1907. Proc. 7 Internat. Zool. Cong., Boston. [Drosophila chromosomes.] 1908a. Jour. Exp. Zoöl. 5 : 453-470. 3 pls. [Diabrotica chromosomes.] 1908b. Jour. Exp. Zoöl. 5 : 359-374. 4 pls. [Sex-chromosomes; synapsis.] 1911ab. Biol. Bull. 20:121-122. 21: 155-167. 35 figs. [Guinea pig.] 1911c. Biol. Bull. 20 : 109-120. 38 figs. [Culex chromosomes.] Steward, F. C. 1928a. Biochem. Jour. 22 : 268-275. [Cell membrane.] 1928b. Proc. Leeds Phil. Soc. 1: 258-270. [Cell membrane.] 1929. Protoplasma $7: 602-621$. [Cell membrane.]

Stieve, H. 1921. Arch. Mikr. Anat. 95: II 1-202. pls. 1-7. [Proleus oöcyte.] Stiles, W. 1924. Permeability. London. Stoel, G. 1928. Zeitschr. Krebsf. $26: 386-403$. illus. [Radium.] Śtolc, A. 1910. Roux's Arch. Entw. 29 : 152-168. pls. 4, 5. [Enucleated cells.] Stolte, H. A. 1922. Arch. Protist. $45: 344-389.30$ figs. [Macronucleus.] Stomps, T. J. 1910. Kerndeeling en synapsis bij Spinacia oleracea. (See Biol. Centralbl. $31: 257-320$. 3 pls.)

1912. Biol. Centralbl. 32: 521-535. 1 pl. [Enothera mutation.] .

1916. Biol. Centralbl. 36 : 129-160. [Chromosome number: plant size.]

1919. Zeitschr. Ind. Abst. Vererb. 21: 65-90. 3 pls. [Gigantism.]

1920. Zeitschr. Ind. Abst. Vererb. 22: 261-274. [Variegation.]

1923. Erblichkeit und Chromosomen. Jena.

Stopes, M., and K. Fujir. 1906. Beih. Bot. Centralbl. $20: 1-24.1$ pl.

Storch, O. 1924. Zool. Jahrb., Anat. Abt., 45: 309-404. illus. [Asplanchna.] Stork, H. E. 1920a. Bull. Torr. Bot. Club 47: 199-210. pls. 6, 7. [Taraxacum.] 1920b. Amer. Jour. Bot. 7:445-457. pls. 31-33. [Aleurodiscus.] 
Stoughton R. H. 1929. Proe. Roy. Soe. London B 105: 469-484. illus.

Sтобт, A. B. 1919. Bot. Gazette 68: 109-133. pls. 12, 13. [Intersexes.]

1923. Amer. Jour. Bot. $10: 57-66$. pl. 6. [Sex in Cleome.]

Stow, I. 1926. Proc. Imp. Acad. Japan 2: 426-430. illus. [Solanum.]

1927. Jap. Jour. Bot. 3 : 217-238. illus. [Solanum.]

1930. Cytologia 1: 417-439. illus.

Strangeways, T. S. P. 1922. Proc. Roy. Soc. London B 94: 137-141. pls. 2-5.

Strangeways, T. S. P., and R. G. Canti. Quar. Jour. Mier. Sci. 71: 1-14. illus.

Strangeways, T. S. P., and F. L. Hopwood. 1926. Proc. Roy. Soc. London B 100.

Strangeways, T. S. P., and H. E. H. Oakley. 1923. Proc. Roy. Soc. London B

95 : 373-381. illus.

Strasburger, E. 1875. Ueber Zellbildung und Zelltheilung. Jena.

1877. Ueber Befruchtung und Zelltheilung. 108 pp. Jena. See also Jenaische Zeitsehr. 11.

1879. Die Angiospermen und die Gymnospermen. Jena.

1880. Ueber Zellbildung und Zelltheilung. 3d ed. Jena.

1882a. Arch. Mikr. Anat. 21: 476-588. pls. 25-27. [Mitosis.]

1882b. Ueber den Bau und das Wachsthum der Zellhäute. Jena.

1884a. Neue Untersuchungen über den Befruchtungsvorgang, usw. Jena.

1884b. Arch. Mikr. Anat. 23 : 246-304. pls. 13, 14. [Mitosis.]

1888. Histol. Beitr. 1. [Nuclear and cell division; fertilization.]

1889. Histol. Beitr. 2. Ueber das Wachstum vegetabilischer Zellhäute.

1892. Histol. Beitr. 4. [Swarm-spores; gametes; syngamy.]

1893. Histol. Beitr. 5. [Nuclear and cell size.]

1894. Ann. Botany 8: 281-316. [Periodic eliromosome reduction.]

1895. Jahrb. Wiss. Bot. 28 : 151-204. pls. 2, 3. [Mitosis.]

1897a. Jahrb. Wiss. Bot. 30: 375-405. 2 figs. [Mitosis.]

1897b. Jahrb. Wiss. Bot. $30: 351-374 . \quad$ pls. 27, 28. [Fucus.]

1898. Jahrb. Wiss. Bot. $31: 534-598$. pls. 15, 16 . [Cell wall.]

1900a. Histol. Beitr. 6. [Meiosis; spindle; centrosomes; blepharoplasts.]

1900b. Bot. Zeitung 58: 293-316. [Double fertilization.]

1900c. Biol. Centralbl. 20: 657-665, 689-698, 721-731, 753-785. [Sex.]

1901a. Jahrb. Wiss. Bot. 36: 493-610. pls. 14, 15. [Plasmodesms.]

1901b. Bot. Zeitung 59: II 353-368. [Fertilization.]

1904a. Festschr. f. E. Haeckel. [Gymnosperm gametophyte.]

1904b. Sitzber. Akad. Wiss. Berlin 18: 587-614. 9 figs. [Meiosis.]

1904c. Jahrb. Wiss. Bot. 41: 88-164. pls. 1-4. [Apomixis.]

1905a. Jahrb. Wiss. Bot. $42: 1-71.1$ pl. [Mitosis; meiosis.]

1907a. Flora $97: 123-191$. pls. 3-8. [Apomixis in Marsilia.]

1907b. Jahrb. Wiss. Bot. 44: 482-555. pls. 5-7. [Chromosome individuality.]

1907c. Progr. Rei Bot. 1. Die Ontogenie der Zelle seit 1875.

1908. Jahrb. Wiss. Bot. 45: 479-568. pls. 1-3. [Chromosome number etc.]

1909a. Ann. Jard. Bot. Buit. II 3 : Suppl. 13-18. 3 figs. [Wikstroemia.]

1909b. Histol. Beitr. 7. [Sex; apomixis; meiosis.]

1910a. Jahrb. Wiss. Bot. 48: 427-520. pls. 9, 10. [Sex.]

1910b. Flora 100: 398-446. pl. 6. [Chromosome number.]

1910c. Jahrb. Wiss. Bot. 47: 245-288. 4 pls. [Apomixis.]

$1910 d$. The minute structure of cells in relation to inheritance. In "Darwin and

Modern Science" (Seward, editor).

1911. Flora 102: 1-23. pl. 1. [Individuality of chromosome.]

Strugger, S. 1930. Protoplasma $10: 363-378$. illus. [Nucleus.]

Stubbe, H. 1930. Zeitschr. Ind. Abst. Vererb. 56: 1-38. illus.

Studnıčk A, F. K. 1925. Zeitsehr. Zellf. Mikr. Anat. 2: 408-479. illus. 
Sturtevant, A. H. 1913. Jour. Exp. Zoöl. 14: 43-59. [Sex-linkage.]

1915. Zeitschr. Ind. Abst. Vererb. 13 : 234-266. [Linkage.]

1920a. Science 51:325-327. [Intersexes in Drosophila.]

1920b. Proc. Soc. Exp. Biol. Med. 17: 70-71. [Gynandromorphism.]

1921ab. Geneties 6 : 43-64. [Sex-linkage]; 179-207 [Linkage.]

1921c. Proe. Nat. Acad. Sei. $7: 181-183$. [Linkage maps.]

1926a. Biol. Zentralbl. 46: 697-702. [Inversion.]

Sturtevant, A. H., and T. Dobzhansky. 1930. Proc. Nat. Aead. Sei. $16: 533-536$. 1931. Carnegie Inst. Wash. Publ. 421. [Chromosome anomalies.]

Suessenguth, K. 1921. Beih. Bot. Centralbl. 38:1-79. 18 figs.

Somner, J. B. 1926. Jour. Biol. Chem. 69:435-441. See also 70:97-98. [Urease.]

Sonner, J. B., and D. B. Hand. 1928. Jour. Biol. Chem. $76: 149-162$.

Sotton, W. S. 1902. Biol. Bull. 4:24-39. 11 figs. [Brachystola ehromosomes.] 1903. Biol. Bull. 4:231-251. The ehromosomes in heredity.

Svedberg, T. 1921. Rept. Faraday Soc. and Phys. Soc. London on Colloids, 55-56. 1928. Colloid Chemistry. New York.

Svedelius, N. 1911. Svensk Bot. Tidskr. 5: 260-324. pls. 2, 3. [Delesseria.] 1912. Svensk Bot. Tidskr. 6: 239-265. pls. 5, 6. 11 figs. [Dclesseria ơ.] 1914a. Ber. Deu. Bot. Gesell. 32: 48-57. 1 pl. [Nitophyllum.] 1914b. Ber. Deu. Bot. Gesell. 32 : 106-116. pl. 2. [Nitophyllum.]

1914c. Svensk Bot. Tidskr. 8: 1-32. 2 pls. [Delesseria.]

1915. Nova Acta Reg. Soc. Sei. Uppsala IV 4: No. 4. [Scinaia.]

1921. Ber. Deu. Bot. Gesell. 39: 178-187. [Alternation of generations.]

1927b. Bot. Gazette $83: 362-384$. [Alternation of generations.]

1931. Beih. Bot. Centralbl. 48: 38-59. illus.

Svensson, H. G. 1925. Uppsala Univ. Årsskr., Mat. o. Nat., 2. [Endosperm.]

Sweschnikowa, I. 1927a. Zeitsehr. Ind. Abst. Vererb. 46:54-55. [Vicia.] 1927b. Bull. Appl. Bot. Genet. Plant Breed. 17(3):37-72. illus. [Vicia.] 1928. Zeitsehr. Ind. Abst. Vererb., suppl. 2: 1415-1421. illus. [Vicia.]

1929. Ann. Timir. Agr. Acad. (Moseow), 1-22. illus.

Swezy, O. 1915ab. Univ. Calif. Publ. Zoöl. 16: 71-88; 89-94. pls. 9-12. 1916. Univ. Calif. Publ. Zoöl. 16: 127-138. pls. 16, 17.

1922. Univ. Calif. Publ. Zoöl. 20 : 313-332. pls. 29-31.

Swingle, D. B. 1903. U. S. Dept. Agr. Pl. Ind. Bull. 37. [Phycomycetes.]

Swingle, W. T. 1897. Jahrb. Wiss. Bot. $30: 296-350$. pls. 15, 16. [Sphacelaria.]

Swingle, W. W. 1920. Amer. Naturalist 64:349-357. [Sex in frogs.]

1921. Jour. Exp. Zoöl. $32: 235-331.15$ pls. [Sex in frogs.]

Syкes, M. G. 1908. Arch. Zellf. 1:381-398. pls. 8, 9. [Meiosis.]

Sypkens, B. 1904. Proe. Konink1. Akad. Wetensch. Amsterdam, Dec. 1904. $412-419$.

Täскноцм, G. 1920. Svensk Bot. Tidskr. 14: 300-311. 3 figs. [Rosa.] 1922. Acta Horti Bergiani 7 : 97-381. 56 figs. [Rosa.]

Täскholm, G., und E. Sönerberg. 1917. Ark. Bot. 15: 14 pp. [Pollen.] 1918. Svensk Bot. Tidskr. 12 : 189-201. 7 figs. [Pollen development.]

Tahara, M. 1910. Bot. Mag. Tokyo 24:281-289. pl. 9. [Morus.]

1915a. Bot. Mag. Tokyo 29: (245)-(254). 5 figs. [Erigeron.]

1915b. Bot. Mag. Tokyo 29 : 48-50. 1 pl. [Chrysanthemum.]

1921. Jour. Coll. Sei. Imp. Univ. Tokyo 43 : 53 pp. 4 pls.

1927. Bot. Mag. Tokyo 41: 142-148. illus. [Sargassum.]

1929. Sci. Rept. Tohoku Imp. Univ. 4:551-556. illus. [Coccophora.] 
Takamine, N. 1923. Bot. Mag. Tokyo $37: 109-112$. pl. 5. [Ultra-violet light.]

1927. Bot. Mag. Tokyo 41:118-122. illus. [Najas.]

Tammes, T. 1900. Flora 87: 205-247. pl. 7. [Carotin.]

TanakA, Y. 1916. Jour. Coll. Agr. Sapporo 7: 129-255. [Silkworm.]

Tandy, G. 1927. Ann. Botany 41:320-325. illus. [Pyronema.]

TANGL, E. 1879. Jahrb. Wiss. Bot. 12: 170-190. pls. 4-6. [Plasmodesms.]

Tannreuther, G. W. 1923. Roux's Arch. Entw. 52:367-383. 52 figs. [Euglena.] TANsley, A. G. 1912. New Phytol. 11: 213-216. [Alternation of generations.] Tänzer, E. 1921. Zeitschr. Wiss. Zoöl. 111: 114-153. pl. 6.19 figs.

Taylor, C. V. 1920. Univ. Calif. Publ. Zoöl. 19: 403-470. pls. 29-33. 1923a. Jour. Exp. Zoöl. $37: 259-287.3$ pls. [Contractile vacuole.] 1923b. Science 68:308. [Removal of micronucleus.] 1929. Amer. Naturalist 63: 328-345. illus. [Neuromotor system.]

Taylor, G. W., and E. N. Harvey. 1931. Biol. Bull. 61: 280-293.

Taylor, J. W., and C. E. Leighty, 1931. Jour. Agric. Res. 43: 661-679. illus.

Taylor, W. R. 1924. Amer. Jour. Bot. 11: 51-59. 25 figs. [Gasteria.] 1925a. Amer. Jour. Bot. 12: 104-115. 35 figs. [Velthemia, Allium, Cyrtanthus.] 1925b. Amer. Jour. Bot. 12: 219-223. 13 figs. [Gasteria, Aloë, Haworthia.] 1925c. Amer. Jour. Bot. 12: 238-244. pl. 22. [Chromosome constrictions.] 1925c. Amer. Jour. Bot. 12: 238-244, illus. [Chromosome morphology.] 1931. Amer. Jour. Bot. 18:367-386. illus. [Gasteria, meiosis.]

Telezynski, H. 1930a. Compt. Rend. Soc. Biol., Paris 104: 167-169. 1930b. Acta Soc. Bot. Polon. $7: 381-433$. illus. [Chromonemata.] 1931a. Compt. Rend. Soc. Sci. Lett. Varsovie 23 : IV 116-118.

1931b. Acta Soc. Bot. Polon. 8: 109-132. illus. [Chromonemata.]

Tennent, D. H. 1910. Roux's Arch. Entw. 29: 1-14. 2 figs. [Echinoderm hybrids.]

1912a. Jour. Morphol. 23: 17-30. 20 figs. [Echinoderm hybrids.] 1912b. Jour. Exp. Zoöl. 12: 391-411. 21 figs. [Echinoderm hybrids.] 1920. Proc. Nat. Acad. Sci. 6: 217-221. [Nuclear extrusion.]

Terao, H. 1918. Amer. Naturalist 62:51-56. [Maternal inheritance.]

Terletzki, P. 1884. Jahrb. Wiss. Bot. 15: 452-501. pls. 24-26. [Plasmodesms.] Terni, T. 1914. Arch. Zellf. 12:1-96. pls. 1-7. [Chondriosomes.]

Tharaldsen, C. E. 1926. Jour. Exp. Zoöl. 44: 159-217. illus. [Cytasters.]

Thatcher, R. W. 1921. The Chemistry of Plant Life. New York.

Thom, C. 1899. Trans. Acad. Sci. St. Louis 9: 285-314. pls. 36-38. [Syngamy.] Thomas, R. C. 1928. Amer. Jour. Bot. 15: 537-547. [Hypha wall.]

Thomas, R. H. 1913. Rapp. 4 Conf. Internat. Génétique, Paris, 450-461.

'Thompson, D.'A. W. 1917. On Growth and Form. Cambridge.

Thompson, W. P. 1925. Genetics 10:285-304. [Triticum hybrids.]

1926a. Jour. Genetics $17: 43-48$. illus. [Triticum hybrids.]

1926b. Genetics $11: 317-332$. illus. [Wheat $\times$ rye.]

1927. Sci. Agric. 8: 56-62. illus. [Triticum hybrids.]

1928. Trans. Roy. Soc. Canada, Biol. Sci. 22 : 335-348. illus.

1930a. Genetics 15: 99-113. [Shrivelled endosperm.]

1930b. Amer. Naturalist 64: 407-421. [Reciprocal crosses.]

1931a. Genetics 16:309-324. illus. [Triticum hybrids.]

1931b. Canadian Jour. Res. 4: 624-634. [Triticum, Secale, Egilops.]

Thompson, W. P., and D. R. Cameron. 1928. Genetics 13:456-469. [T'riticum.]

Thompson, W. P., and L. Hollingshead. 1927. Jour. Genetics 17: 283-307.

[Triticum hybrids.]

Thompson, W. P., and H. T. Robertson. 1930. Cytologia 1:252-262. illus.

Thomsen, M. 1927. Zeitschr. Zellf. Gewebelehre 5: 1-116. illus. 
Thomson, J. A. 1899. The Science of Life. London. 1913. Heredity. 2d ed. London and New York. 1920. The System of Animate Nature. New York.

Thuret, G. 1854-5. Ann. Sei. Nat. Bot. IV 2, 3. [Algæ.]

Thurlow, M. DeG. 1917. Carnegie Inst. Wash. Contrib. Embryol. 16, 37-44. Tiffany, L. H. 1923. Dissert., Ohio State Univ.

Timberlake, H. G. 1900. Bot. Gazette $30: 73-99,154-170$. [Cell-plate.] 1901. Ann. Botany 15:619-635. [Stareh formation in Hydrodictyon.] 1902. Trans. Wis. Aead. Sei. 13: 486-522. pls. 29, 30. [Zoöspores.]

1903. Seience 17: 460. [Py renoid.]

Timoféeff-Ressovsky，N. W. 1930. Naturwissensehaften 18: 434-437. [X-ray mutations.]

Tischler, G. 1906. Jahrb. Wiss. Bot. 42: 545-578. 1 pl. [Ribes pollen.] 1908. Areh. Zellf. 1: 33-151. 120 figs. [Mitosis; eell wall.] 1910. Areh. Zellf. 5: 622-670. pls. 30, 31. [Musa.] 1915. Jahrb. Wiss. Bot. 55: 53-90. illus. [Periplasmodium.] 1916. Prog. Rei Bot. 5 : 164-284. [Review: ehromosome number, form, etc.] 1918. Ber. Deu. Bot. Gesell. $36: 549-558$. pl. 17. [Phragmites.] 1921-1922. Allgemeine Karyologie. Berlin.

1924. Jahrb. Wiss. Bot. 64:121-168. 5 figs. [Nueleoplasmic ratio.] 1925a. Bibliog. Genetica 1: 39-68. [Cytology of hybrids.] 1925b. Jahrb. Wiss. Bot. 64: 121-168. [Nueleoplasmie ratio.] 1927a. Tabulæ Biol. 4: 1-83. [Plant ehromosome numbers.] 1928c. CEsterreich. Bot. Zeitschr. 77: 292-306. [Sterility.] 1931. Tabulæ Biol. Periodieæ. 1: 109-226. (Tab. Biol. 7.) Tomowo, O. 1927. Bot. Mag. Tokyo 41:600-604. illus. [Triploid Primula.] Torvik, M. M. 1931. Biol. Bull. 61: 139-156. [Hadrobracon.] Townsend, C. O. 1897. Jahrb. Wiss. Bot. 30: 484-510. pls. 20, 21. Toyama, K. 1913. Jour. Geneties 2: 351-404. [Silkworm.]

Trankowsky, D. A. 1930a. Zeitschr. Zellf. Mikr. Anat. 10:736-743. illus. 1930b. 'Planta 12:1-19. illus. [Division of generative cell.]

Transeau, E. N. 1919. Amer. Naturalist 53:109-119. 7 figs. [Spirogyra.] Treub, M. 1878. Quelques Recherehes sur le Rôle du Noyau, ete. Amsterdam. Treviranus, L. C. 1806. Vom inwendigen Bau der Gewächse.

1811. Beiträge zur Pflanzenphysiologie.

Trier, G. 1924. Chemie der Pflanzenstoffe. Berlin.

Tröndle, A. 1911. Zeitsehr. Bot. 3: 593-619. 1 pl. 20 figs. [Spirogyra.] 1912. Zeitsehr. Bot. 4:721-747. pl. 9. [Spirogyra.]

Trow, A. H. 1895. Ann. Botany 9:609-652. pls. 24, 25. [Saprolegnia.] 1899. Ann. Botany 13:131-179. pls. 8-10. [Achlya.] 1901. Ann. Botany 15: 269-312. pls. 15, 16. [Pythium.] 1904. Ann. Botany 18: 541-569. pls. 34-36. [Syngamy in Saprolegniaceæ.] Tschechow, W. 1930. Planta 9: 673-680. illus. [Galegee.] 1932. Bull. Appl. Bot. Genet. Plant Breed. 11 ser., No. 1, 119-146. Tschechow, W. P., and N. N. Kartaschowa. 1932. Bull. Tomsk State Univ. $85: 1-22$. illus.

Tschenzoff, B. 1916. Areh. Protist. $36: 137-173$. pls. 11, 12. [Euglena.] Tschermak, E. 1929. Ber. Deu. Bot. Gesell. 47: 253-261. [Nuclear chimeras.] 1930. Absts. 5 Internat. Bot. Cong., 152.

Tschermak, E., and H. Bleier. 1926. Ber. Deu. Bot. Gesell. 44: 110-132. illus. Tschernoyarow, M. 1914. Ber. Deu. Bot. Gesell. 32:411-416. pl. 10. 
Tschirch, A. 1887. Sitzungsber. Gesell. Naturforsch. Freunde, Berlin, 19 April, p. 51.

1889. Angewandte Pflanzenanatomie. Wien und Leipzig.

de T'Serclaes, J. 1922. La Cellule $32: 313-326.2$ pls. [Cladophora.]

Tsinen, S. J. 1923. Bull. Soe. Sci. Nancy IV 2: 25-26. [Variegation.]

1924. Thesis, Univ. Naney. 104 pp. illus. [Variegation.]

'Tswett, M. 1906ab. Ber. Deu. Bot. Gesell. 24:316-323; 384-392. [Chlorophyll.] Tuan, H. C. 1931. Bot. Gazette 92: 45-65. illus. [Gasteria.]

Tulasne, L. R. 1849. Ann. Sei. Nat. Bot. III $12: 21-137$. pls. 3-7.

Tupper, W. W., and H. H. Bartlett. 1916. Science 43: 292.

Tupper-Carey, R. M., and J. H. Priestley. 1923. Proc. Roy. Soc. London B 95. Turesson, G. 1930. Hereditas 13:177-184. illus. [Festuca.]

Turner, J. P. 1930. Univ. Calif. Publ. Zoöl. 33: 193-258. illus. [Euplotes.]

Tuschnjakowa, M. 1929. Planta 7:427-443. [Hydrocharis, Spinacea, Humulus.] 1930. Planta $10: 597-610$. illus. [Humulus.]

Tuthill, J. B., and O. Rahn. 1933. Arch. Mikrobiol. [Mitogenetic rays.]

Tutrle, A. H. 1924. Science 60:412-413. [Nitella.]

1926. Univ. Calif. Publ. Bot. 13 : 227-234. illus.

Twiss, W. C. 1919. Amer. Jour. Bot. 6: 217-234. pls. 23, 24. [Plastid origin.]

UdA, H. 1923. Genetics 8: 322-335. [Maternal inheritance.]

Umrath, K. 1930. Protoplasma $9: 576-597$. illus. [Streaming.]

Unger, F. 1844. Bot. Zeitung. 2: 489-494, 506-511, 521-526. [Internodes; meristems.]

Unva, P. G. 1921. Abderhalden's Handbuch der Biologischen Arbeitsmethoden, Abt. 5, T. 2, Heft 1.

Unna, P. G., und H. Fein. 1921. Biol. Zentralbl. 41: 495-507. [Chromolysis.] Unna, P. G., and E. T. Tielenann. 1918. Centralbl. Bakt. I 80:66-89. illus.

Valkanover, R. 1926. La Cellule 37:203-222. illus. [Enothera: meiosis.] Vallead, D. W. 1923. Amer. Jour. Bot. 10:259-274. [Sex in Fragaria.] VAN Camp, G. M. 1924. La Cellule 34: 7-48. 2 pls. [Nucleolus in mitosis.] Van Cleave, H. J. 1932. Quar. Rev. Biol. 7 : 59-67. [Cell number.]

VANdel, A. 1928. Bull. Biol. France et Belg. 62: 164-281. illus.

Vandendries, R. 1913. La Cellule 28: 257-261. 11 figs. [Polytrichum.] 1923a. Mém. Cour. Acad. Roy. Belg. 5 : 63 . [Sex in basidiomycetes.] 1923b. Bull. Soc. Roy. Bot. Belg. 16. [Sex in basidiomycetes.] 1924. Bull. Soc. Roy. Bot. Belg. 57, fase. 1. [Sex in basidiomyeetes.] 1925a. La Cellule 35: 129-155. 2 pls. [Sex in basidiomycetes.] 1925b. Bull. Soc. Roy. Bot. Belg. 57, fasc. 2. [Sex in basidiomycetes.] 1925c. Bull. Trim. Soc. Mycol. France 41: 358-374.

1926. Bull. Soc. Roy. Bot. Belg. 58. [Sex in Coprinus.]

1927. Bull. Soe. Roy. Bot. Belg. 60:62-65.

1929. Bull. Soc. Roy. Bot. Belg. 61: 123-135.

1930. Bull. Soe. Roy. Bot. Belg. 62 : 133-136; 63 : $26-35$.

1931. Rec. Trav. Cryptog. dédié à L. Mangin.

Vandendries, R., and H. J. Brodie. 1933. Bull. Acad. Roy. Belg. V 19 : 3-8.

Vandendries, R., and G. Robyn. 1929. Mém. Acad. Roy. Belg., Cl. d. Sci., II 9 : $3-117$. 
Van Der Stricht, O. 1902. Verh. Anat. Gesell. 163-168. [Fate of sperm.] Van Durme, M. 1914. Arch. de Biol. 29 : 71-200. pls. 4-8.

VAN Regenorter, D. 1926. La Cellule $37: 43-73$. illus. [Chloralized cells.] Van Tieghem, P. 1888. Jour. Bot. Paris 2:429-432. [Vacuoles.]

VAuper, J. 1929. Jour. Morphol. Physiol. 47:555-573. illus. [Lebistes.]

Vavilov, N. I., and O. V. Jakushinina. 1925. Bull. Appl. Bot. Plant Breed. 15 : 3-159. illus.

Vejdowsk Ý, F. 1907. Kgl. Böhm. Gesell. Wiss. Prag. [Meiosis; syngamy.] 1912. Zum Problem der Vererbungsträger. pp. 184. pls. 12. Prag.

Velten, W. 1876. Sitzber. Akad. Wiss. Wien, Math.-Nat. Kl., 73: I 131-151.

Vines, S. H. 1911. Article on Reproduction in Plants. Encyel. Brit., 11th. ed.

Virchow, R. 1855. Arch. Path. Anat. Physiol. 8. [Cellular Pathology.] 1858. Die Cellularpathologie, usw. (Transl. by Chance, 1860.)

Visscher, J. P. 1927. Jour. Morphol. Physiol. $44: 383-415$. illus.

Voïnov, D. 1916. Compt. Rend. Soc. Biol. Paris 68: 451-454. 4 figs. 1925. Arch. de Zool. Exp. et Gén. 63: 437-523. 7 pls. [Gryllotalpa.] 1927a. Compt. Rend. Soc. Biol. Paris 96: 1017-1018. illus. 1927b. Arch. d. Zool. Exp. Gén. 67: 1-22. illus. [Notonecta.]

Volkonsky, M. 1930. Compt. Rend. Soc. Biol. Paris 105: 619-623. illus. Vouk, V. 1925. Acta Botanica (Zagreb) 1:37-43. illus. [Aleurone.] De Vries, H. 1885. Jahrb. Wiss. Bot. 16 : 465-598. [Vacuole.] 1889. Intracelluläre Pangenesis. Jena. 1903. Die Mutationstheorie. II. Leipzig.

W

WADA, B. 1930. Cytologia 1:404-416. illus. [Coagulation.] W Ager, H. 1896. Ann. Botany $10: 295-342$. pls. 15, 16 . See also 90-91. 1900a. Jour. Linn. Soc. 27:463-481. pl. 32. [Eyespot; flagellum.] 1900b. Ann. Botany $14: 263-279$. pl. 16. [Peronospora: syngamy.] 1903. Proc. Roy. Soc. London 72: 401-408. 3 figs. [Cyanophyceæ.] 1904. Ann. Botany 18: 29-55. pl. 5. [Nucleolus.] 1911. Article on Plants: Cytology. Encycl. Brit., 11th ed. 1920. Trans. Brit. Mycol. Soc. 6:305-317. [Sexuality in fungi.]

Wagner, N. 1915. Mem. Soc. Nat. Kiew 25. [Chondriosomes, plastids.] 1927a. Biol. Generalis 3: 15-66. illus. [Chondriosomes, plastids.] 1927b. Biol. Generalis $3: 329-346$. illus. [Chondriosomes, plastids.] 1927c. Biol. Zentralbl. 47:670-678. [Mitogenetic rays.] 1928. Planta 5 : 70-88. illus. [Mitogenetic rays.]

Wakayama, K. 1929. Cytologia 1:68-75. illus. [Pinus embryo.] 1930a. Cytologia 1:369-388. illus. [Agaricacex.] 1930b. Cytologia 2: 27-36. illus. [Morchella.]

WAKKer, J. H. 1888. Jahrb. Wiss. Bot. $19: 423-496$. pls. 12-15.

Waldeyer, W. 1888. Arch. Mikr. Anat. 32:1-122. [Early cytol. literature.] Walker, C. E., and F. M. Tozer. 1909. Quar. Jour. Exp. Physiol. 2: 187-200 Walker, N. 1913. Ann. Botany 27:115-132. pls. 13, 14. [Polytrichum ơ.] Wallin, I. E. 1922ab. Amer. Jour. Anat. 30 : 203-229, 1 pl.; 451-471, 2 pls. 1923. Anat. Record 25: 1-6. [Portier's symbiotes.] 1924. Amer. Jour. Anat. 33: 147-173. 2 pls. [Cultures of mitochondria.] 1925a. Amer. Jour. Anat. 35: 403-419. 2 pls. [Cultures of mitochondria.] 1925b. Amer. Jour. Anat. 36: 131-149. illus.

1926. Science $44: 173-175$.

1927. Symbionticism and the Origin of Species. Baltimore. 
Walter, H. 1921. Biochem. Zeitschr. 122: 86-99. [Analysis of protoplasm.]

Walter, H. E. 1922. Genetics. 2d ed. New York.

Walton, A. C. 1918. Jour. Morphol. 30:527-604. 9 pls. [Ascaris: $q$-genesis.] 1921. Jour. Exp. Zoöl. $34: 189-201.2$ pls. [Ascaris felis.]

1924. Zeitschr. Zellen- und Gewebelehre 1: 167-239. pls. 8-11.

WÁmoscher, L. 1930. Zeitsehr. Hyg. Infekt. 111: 422-460. illus. [Baeteria.]

Wanscher, J. H. 1931. Hereditas 15: 179-184. illus. [Umbelliferæ.] 1932. Dansk Bot. Tidskr. 42: 49-58. illus. [Umbelliferx.]

WarbUrG, O. 1923. Pflüger's Archiv 200: 203.

1930. Biochem. Zeitschr. $227:$ 177, 184.

1931. Biochem. Zeitschr. $235: 240$.

1932. Zeitschr. Angew. Chemie 45.

Wassillef, A. 1907. Arch. Mikr. Anat. 70:1-42. pls. 1-3. [Blatta: o'-genesis.]

Watkıns, A. E. 1924. Jour. Genetics 14:129-171. 77 figs. [Triticum.] 1925. Jour. Genetics $15: 323-366.27$ figs. [Triticum.]

1927a. Jour. Genetics 18:375-396. illus. [Triticum hybrids.]

1927b. Genetics $19: 81-96$. illus.

1930. Jour. Genetics 23: 173-263. illus. [Wheat species.]

1932. Jour. Genetics $25: 125-162$.

Weatherby, J. H. 1927. Biol. Bull. 52:208-218. [Contractile vacuole.]

Weatherwax, P. 1923. The Story of the Maize Plant. Chicago.

Webber, H. J. 1897a. Bot. Gazette 23: 453-459. pl. 40. [Zamia pollen tube.] 1897b. Bot. Gazette 24:16-22. 5 figs. [Zamia spermatozoid.]

1897c. Bot. Gazette 24:225-235. pl. 10. [Zamia sygnamy; Ginkgo pollen tube.] 1901. U. S. Dept. Agr. P. Ind. Bull. 2. 100 pp. 7 pls. [Zamia.]

Weber, F. 1922a. Naturwiss. Wochensehr. N. F. 21: 113-125. [Viscosity.] 1922b. Ber. Deu. Bot. Gesell. 40:212-216. [Viscosity of protoplasm.] 1923. Arch. Ges. Physiol. 198: 644-647. [Viscosity of protoplasm.] 1925a. Planta 1:374-378. illus. [Stomates.]

1926a. Protoplasma 1: 167-176. [Elasticity, viscosity: bibliography.]

1926b. Protoplasma 1: 486-496. [Permeability: bibliography.]

1927. Protoplasma $2: 305-311$. [Stomates.]

Weier, T. E. 1930a. Proc. Nat. Acad. Sci. 16: 536-543. [Moss plastids.] 1930b. La Cellule 39:271-305. illus. [Enothera, meiosis.]

1931 $a$. La Cellule 40: 261-290. illus. [Moss plastid; sporogenesis.]

1931b. La Celhule $41: 51-84$. illus. [Moss Plastid; spermatogenesis.]

1932a. Biol. Bull. 62: 126-139. illus. [Plastid and Golgi.]

1932b. Amer. Jour. Bot. 19: 659-672. illus. [Plastid and Golgi.]

Weigl, R. 1910. Bull. Acad. Sci. Cracovie. [Golgi material.]

1912. Bull. Acad. Sci. Cracovie, 417-448. [Golgi material.]

Weinstein, A. 1918. Genetics 3: 135-173. [Coincidence of crossing-over.]

1928. Science $67: 376-377$. [X-ray mutations.]

1932. Proc. 6 Internat. Cong. Genetics 2 : 206-208.

Weis, A. 1925. Arch. Wiss. Bot. 1: 145-186. 9 figs. [Plasma membrane.]

Weismann, A. 1885. Die Kontinuitït des Keimplasmas, usw. Jena.

1887. Ueber die Zahl der Richtungskörper, usw. Jena.

1891-1892. Essays upon Heredity. Oxford.

1892. Das Keimplasma. (Engl. transl., The Germ-Plasm, 1893.)

1902, 1913. Vortrïge über Deszendenztheoric. Jena. (The Evolution Theory, 1904.)

Welrs, H. G. 1914. Chemical Pathology. 2d ed. Philadelphia. Chap. I.

WeLs, P. 1924. Fortschr. Roentgenforsch. 15: 112. 
Welsford, E. J. 1907. New Phytol. 6: 156-161. pl. 4. [Ascobolus.]

1914. Ann. Botany 28 : 265-270. pls. 16, 17. [Lilium: or nuclei.]

Wendel, E. 1918. Beitr. Allg. Bot. 1: 45-80. 11 figs. [Nuclear function.]

Weniger, W. 1918. Bot. Gazette 66:259-268. pls. 11-13. [Lilium: syngamy.]

WENRICH, D. H. 1915. Science 41: 440. [Synapsis; individuality of chromosome.]

1916. Bull. Mus. Comp. Zoöl. Harvard Coll. 60 : 55-136. 10 pls.

1917. Jour. Morphol. 29: 471-516. pls. 1-3. [Chorthippus; Trimerotropis.]

1921. Jour. Morphol. 36: 119-155. 4 pls. [T'richomonas.]

1926. Jour. Morphol. Physiol. 43 : 81-103. illus.

1928a. Trans. Amer. Micr. Soc. 47: 256-261. illus. [Paramœcium.]

Went, F. A. F. C. 1888. Jahrb. Wiss. Bot. $19: 295-356$. pls. 7-9. [Vacuole.]

1890. Jahrb. Wiss. Bot. $21: 299-366$. [Vacuoles.]

1926. Proc. Konink1. Akad. Wetensch. Amsterdam 29. [Latex.]

1931. In Kostytsehew und Went: Lehrbuch der Pflanzen-Physiologie. II. Berlin.

Wermel, E. 1927. Zeitsehr. Zellf. Mikr. Anat. 5: 400-414. [Feulgen reaction.]

Werner, O. S. 1927. Biol. Bull. 52:330-372. illus. [Duck.]

West, G. S. 1927. A Treatise on the British Freshwater Algæ. Cambridge.

Westвrook, M. A. 1930a. Ann. Botany 44:1011-1015. illus. [Callithamnion.]

Wester, D. H. 1909. Arch. d. Pharm. 247: 303. [Chitin.]

Wettstein, F. von. 1920. Ber. Deu. Bot. Gesell. 38: 260-266. [Vaucheria.]

1921a. Sitzber. Akad. Wiss. Wien. Math.-Nat. Kl., 130:3-20. [Chitin.]

1921b. Ber. Deu. Bot. Gesell. 39: 274. [Funaria hybrids.]

1923. Biol. Zentralbl. 43: 71-83. 1 fig. [Funaria hybrids.]

1924a. Zeitschr. Ind. Abst. Vererb. 33:1-236. 12 pls. [Moss hybrids.]

1924b. Zeitschr. Ind. Abst. Vererb. $33: 253-257$. [Generic crosses in mosses.]

1924c. Bibliog. Genetica. 1: 1-30. [Bryophyte genetics.]

1926. Nachr. Gesell. Wiss. Göttingen, Math.-Phys. K1., 250-281. illus. [Plasmon.] 1927. Ergeb. d. Biol. 2:311-356. illus. [Heteroploidy.]

1928. Ber. Deu. Bot. Gesell. 46 : (32)-(49). [Plasmon.]

1930. Nachr. Gesell. Wiss. Göttingen, Math.-Phys. Kl., VI 109-118.

Wettstein, R. von. 1925. Flora 118-119: 600-604. illus. [Humulus.]

Wherry, E. T. 1913. Seience $37: 494-496$. [Vahlkampfia.]

Whiting, A. R. 1927. Biol. Bull. 53: 438-449.

1928. Amer. Naturalist $62: 55-58$.

Whiting, P. W. 1917. Jour. Morphol. $28: 523-577.7$ pls. [Culex chromosomes.] 1921. Biol. Bull. $41: 42-54$.

1928. Science 68: 59. [X-ray mutations.]

1931. Biol. Bull. 61: 478-480. [Gynandromorphs.]

1932b. Genetics 17: 1-30. illus. [X-ray mutation.]

Whiting, P. W., and M. F. Stancati. 1931. Biol. Bull. 61: 481-484. illus.

Whiting, P. W. and A. R. 1927. Biol. Bull. 62: 89-121. illus. [Gynandromorphs.]

Wintman, C. O. 1893. Woods Hole Biol. Leetures 2: 105-124. [Organismal Theory.]

1894. Woods Hole Biol. Lectures. [Evolution and epigenesis.]

Whitney, D. D. 1909. Jour. Exp. Zoöl. 6: 137-146. 5 figs. [Hydatina.]

1914. Jour. Exp. Zoöl. $17: 545-558$. [Sex control in Hydatina.]

1916. Jour. Exp. Zoöl. 20:263-296. 6 figs. [Sex control in Hydatina.]

1917. Jour. Exp. Zoöl. 24: 101-138. 4 figs. [Sex control in Hydatina.]

1919. Jour. Exp. Zoöl. 28: 469-492. [Sex control in Hydatina.]

1924. Anat. Rec. 29: 107. [Asplanchna.]

1929. Jour. Morphol. Physiol. 47: 415-434. illus. [Asplanchna.] 
Whong, S. H. 1931. Protoplasma 12 : 123-128. illus. [Cell bridges.]

Wieman, H. L. 1917. Amer. Jour. Anat. 21:1-22. 4 pls. [Human chromosomes.] Wiesner, J. von. 1864. Sitzber. Akad. Wiss. Wien, Math.-Naturw. K1., II 50 : $442-453$.

1886. Sitzber. Akad. Wiss. Wien, Math.-Naturw. Kl., I 93: 17-80. 5 figs. [Cell wall.]

1890. Sitzungsber. Akad. Wiss. Wien 99.

1892. Die Elementarstruetur und das Wachstum der Lebenden Substanz. Wien.

Wiesner, J. von, et al. 1932. Die Rohstoffe des Pflanzenreichs. Leipzig.

Wilcox, M.S. 1928. Mycologia 20:3-17. illus. [Neurospora.]

Wildeman, E. De. 1891. Ann. Soc. Belg. Micr. 15. [Temperature and mitosis.]

Wille, N. 1883. Ber. Deu. Bot. Gesell. 1: 243-246. [Wall pores.]

Willials, J. L. 1904a. Ann. Botany 18: 183-204. pls. 12-14. [Dictyota.]

Williams, M. 1923. Ann. Botany 37:217-224. [X-rays and plant cells.]

1925. Ann. Botany $39: 547-562$. [Radium and plant cells.]

Willians, M. M. 1925. Proc. Linn. Soc. N.S.W. 50:II 98-111. illus. [Codium.] 1926. Proc. Linn. Soc. N.S.W. 51: II 283-295. illus. [Vaucheria.]

Willstätter, R., und A. Stoll. 1913. Untersuchungen über Chlorophyll. Berlin. Wilson, E. B. 1892. Jour. Morphol. 6:361-480. pls. 13-20. [Cell lineage.]

1893. Woods Hole Biol. Leetures, 1-14. [Development.]

1899. Jour. Morphol. 15 : Suppl., 1-23. [Echinoderm eggs.]

1900. The Cell in Development and Inheritance. $2 \mathrm{~d}$ ed.

1901. Roux's Arch. Entw. 12: 529-596. pls. 11-17. [Parthenogenetic eggs.]

1903. Roux's Arch. Entw. 16: 411-460. illus.

1905a. Jour. Exp. Zoöl. 2 : 371-406. 7 figs. [Idiochromosomes.]

1905b. Jour. Exp. Zoöl. $2: 507-546$. [Idiochromosomes.]

1905c. Seience 20:564. [Chromosomes and sex.]

1906. Jour. Exp. Zoöl. $3: 1-40.6$ figs. [Sex-chromosomes.]

1907. Seience N. S. 25:376-379. [Sex and parthenogenesis.]

1909a. Jour. Exp. Zoöl. 6: 69-100. 2 pls. [Syromastes; Pyrrochoris.]

1909b. Jour. Exp. Zoöl. 6: 147-206. 1 pl. [Metapodius chromosomes.]

1909c. The eell in relation to heredity and evolution. In Fifty Years of Darwinism, New York.

1910. Seience Progress $16: 570-592$. [Chromosomes and sex.]

1911a. Areh. Mikr. Anat. 77: II 249-271. 5 figs. [Sex-ehromosomes.]

1911b. Jour. Morphol. 22 : 71-110. 1 pl. [Nezara chromosomes, ete.]

1912a. Amer. Naturalist 46:57-67. [Cytology and geneties.]

1912b. Jour. Exp. Zoöl. 13 : 345-449. 9 pls. [Meiosis.]

1913. Science 37: 814-826. 3 figs. [Heredity and microscopical researeh.]

1914. Proc. Roy. Soc. London B 88: 333-352. [Heredity and mieroseopical researeh.]

1916ab. Science 43 : 539. Proc. Nat. Aead. Sei. 2: 321-324. [Seorpions.]

1923. Seience $57: 277-286$. The Plyysical Basis of Life.

1924. General Cytology (Cowdry, ed.). Introduetion.

1925. The Cell in Development and Heredity. 3d ed. New York.

1926. Amer. Naturalist $60: 105-120$. illus. [Strueture of protoplasm.]

1930b. Science 71: 661-662. [Centrioles.]

1932. Jour. Morph. 53: 443-471. illus. [Metaphase patterns.]

Wilson, E. B., and A. F. Huettner. 1931. Science 73:447-448. [Centrioles.]

Wilson, E. B., and A. P. Matilews. 1895. Jour. Morphol. 10 : 319-342.

Wilson, M. 1911. Ann. Botany 25: 415-457. pls. 37, 38. [Moss: ơ-genesis.] 
Winge, $\varnothing$. 1912. Ark. Bot. 12: 1-39. 3 pls. [Plasmodiophoraceæ.]

1917. Compt. Rend. Trav. Lab. Carlsberg 13:131-275. 1 pl. 46 figs. [Chromosomes: number, etc.]

1919a. Compt. Rend. Trav. Lab. Carlsberg 34: 1-20. 4 figs. [Variegation.]

1919b. Jour. Genetics 8: 133-138. 5 pls. [Lathyrus chromosomes.]

1922a. Jour. Geneties 12: 137-144. pl. 1. [Lebistes.]

1922b. Jour. Genetics $12: 145-162$. pls. 12, 13. [Lebistes.]

1923a. Compt. Rend. Trav. Lab. Carlsberg. $14: 18$ pp. [ $X-Y$ erossing-over.]

1923b. Compt. Rend. Trav. Lab. Carlsberg. 15: 1-26. 4 pls. 35 figs. [Sexchromosomes in angiosperms.]

1924. Hereditas 5 : 241-286. 30 figs. [Triticum chromosomes.]

1925. La Cellule $35: 305-324$. 2 pls. [Chromosome numbers.]

1927a. Jour. Genetics 18: 99-107. illus. [Vallisneria.]

1927b. Jour. Genetics 18: 1-43. illus.

1927c. Hereditas 9:274-284. [Y-linked gene.]

1927d. Zeitschr. Zellf. Mikr. Anat. 6: 397-423. illus. [Crown gall.]

1929a. Hereditas 12: 53-63. illus. [Humulus.]

1929b. Hereditas $12: 269-270$. [Humulus.]

1930a. Zeitschr. Zellf. Mikr. Anat. 10:683-735. illus. [Tar tumors.]

1930b. Jour. Genetics $23: 69-76$. [Lebistes; XX o.]

1930c. Absts. 5 Internat. Bot. Cong., 121-122. [ $X$ - and $Y$-linkage.]

1931. Hereditas 15: 127-165. illus. [ $Y$ - and $Y$-linkage.]

1932. Svensk Bot. Tidskr. $26: 107-122$. [Amphidiploids.]

Winiwarter, H. DE. 1900. Arch. de Biol. 17: 33-199. pls. 3-8. [Man; rabbit.]

1912. Arch. de Biol. 27: 91-189. pls. 6, 7. [Human chromosomes.]

1921a. Revue Anthropol., xxv-xxxii. [Human chromosomes.]

1921b. Compt. Rend. Soc. Biol. Paris 85: 266-267. [Human chromosonies.]

1925. Compt. Rend. Assoc. Anat. Turin. Paris.

Winiwarter, H. DE, and K. Oguma. 1925. Compt. Rend. Assoc. Anat. Turin.

[Human chromosomes.]

1926. Arch. de Biol. 36: 99-166. illus. [Human chromosomes.]

Winiwarter, H. De, and G. Sainmont. 1909. Arch. de Biol. 24: 1, 165, 373, 627.

Winkler, H. 1898. Jahrb. Wiss. Bot. 32:525-556. [Plastids and starch.]

1901. Jahrb. Wiss. Bot. 36: 753-775. illus. [Cystosira.]

1904. Ber. Deu. Bot. Gesell. 22:573-580. [Wikstramia: parthenogenesis.]

1906. Ann. Jard. Bot. Buit. 5: 208-276. pls. 20-23. [Wikstrœmia.]

1908. Prog. Rei. Bot. 2 : 293-454. [Apomixis; review.]

1916. Zeitschr. Bot. 8:417-531. pls. 4-6. 17 figs. [Solanum.]

1920. Verbreitung und Ursache der Parthenogenesis im Pflanzen- und Tierreiche.

1924. Zeitschr. Ind. Abst. Vererb. 33: 238-253. [Rôle of nucleus.]

1930. Die Konversion der Gene. Jena.

1932. Biol. Zentralbl. 52: 163-189. illus. [Conversion of gene.]

van Wisselingh, C. 1888, 1892, 1895. Arch. Néerl. Sci. Ex. et Nat. 22 : 253; 26 :

$305 ; 28: 373$. [Cell wall.]

1898. Bot. Zeitung 56:195-226. pl. 10. [Spirogyra nucleolus.]

1900. Flora $87: 355-386$. pl. 15. [Mitosis in Spirogyra.]

1909. Beih. Bot. Centralbl. 24 : I 133-210. pls. 4-6. [Spirogyra.]

1914. Rec. Trav. Bot. Néerl. 11: 1-13. [Zygnema: mitosis, nucleolus.]

1921. Beih. Bot. Centralbl. 38: 273-354. 103 figs. [Spirogyra.]

1924. Die Zellmembran. Berlin.

Wiтschi, E. 1922. Zeitschr. Ind. Abst. Vererb. 29:31-68. 1 pl. [Sex in frog.] 1923. Zeitschr. Ind. Abst. Vererb. 31: 287-312. [Sex in frog.]

1924. Zeitschr. Zellen- und Gewebelehre 1:523-561. 3 pls. [XY chromosomes.] 
1929. Bestimmung und Vererbung des Geschlechtes bei Tieren. Berlin.

1932. Amer. Naturalist 66: 108-117. [Physiology of sex differentiation.]

Wodehouse, R. P. 1928. Ann. Botany 42: 891-934. illus. [Pollen.]

1929a. Bull. Torr. Bot. Club 56:123-138. illus. [Pollen.]

1929b. Amer. Jour. Bot. 16: 297-313. illus. [Pollen.]

1929c. Bull. Torr. Bot. Club 56:339-350. illus. [Pollen.]

1930. Bull. Torr. Bot. Club $57: 21-46$. illus. [Pollen.]

1932. Bull. Torr. Bot. Club 59: 313-340. illus.

Wodsedalek, J. E. 1913. Biol. Bull. 25: 8-44. 6 pls. [Pig spermatogenesis.]

1914. Biol. Bull. $27: 295-324.6$ pls. [Horse spermatogenesis.]

1920. Biol. Bull. 38 : 290-316. 5 pls. [Cattle spermatogenesis, etc.]

1922. Anat. Rec. 23: 103. [Sheep spermatogenesis, etc.]

Wolfe, J. J. 1904. Ann. Botany 18:607-630. pls. 40, 41. [Nemalion.]

1918. Jour. Eli. Mitchell Soc. 34: 78-109. [Padina.]

Wolff, C. F. 1759. Theoria Generationis.

Wolfson, A. M. 1927. Amer. Jour. Bot. 14:516-519. illus. [Spharocarpos.]

Wollenweber, W. 1907. Ber. Deu. Bot. Gesell. $25: 316-320$. pl. 11. [Eyespot.] 1908. Ber. Deu. Bot. Gesell. 26 : 238-298. pls. 12-16. 12 figs. [Eyespot.]

Wood, F. M. 1924. Ann. Botany 38: 273-398. [Cellulose membrane.]

1926. Ann. Botany $40: 547-570$. illus. [Cell wall.]

Woodburn, W. L. 1911. Ann. Botany $25: 299-313$. pl. 25. [Spermatogenesis.] 1913. Ann. Botany 27 : 93-101. pl. 11. [Spermatogenesis in Blasia.]

1915. Ann. Botany 29:441-456. pl. 21. [Spermatogenesis in Mnium.]

1920. Bull. Torr. Bot. Club $46: 461-464$. pl. 19. [Reboulia embryo.]

WOODRUFF, L. L. 1921. Jour. Exp. Zoöl. 34:329-337. [Amicronucleate infusoria.] 1925. Amer. Naturalist 59: 225-249. [Conjugation and endomixis.]

Woodruff, L. L., and R. Erdians. 1914. Jour. Exp. Zoöl. 17 : 425-516. 4 pls.

Woodroff, L. L., and Hope Spencer. 1924. Jour. Exp. Zoöl. 39 : 133-196.

Woodward, A. E. 1918. Jour. Exp. Zoöl. 26: 459-501. [Egg secretions.]

Wortmann, J. 1887. Bot. Zeitung 45: 785, 801, 817, 833. [Plasmodesms.]

1889. Bot. Zeitung 47: 453, 469, 485. [Plasmodesms.]

Woskressensky, N. M., and E. A. Scheremetzewa. 1930. Zeitschr. Zellf. Mikr.

Anat. 10: 411-426. illus. [Drosophila, meiosis.]

Wórcicki, Z. 1899. Fertilization in Coniferæ. (Russian.) 57 pp. 2 pls. Warsaw. 1912. Sitzungsher. Warschaner Gesell. Wiss. 5. [Malva.]

1923a. Acta Soc. Bot. Polon. 1: 149-164. illus. [Larix.]

1923b. Bull. Acad. Polon. Sci. Lett. B, 79-107.

1926b. Bull. Acad. Polon. Sci. Lett. B, 177-188. illus. [Hæmanthus.]

1927. Flora 122: 159-166. illus. [Basidiobolus.]

1929b. Bull. Acad. Polon. Sci. Lett. B, 25-39. illus. [Oleosomes.]

1932 Acta Soc. Bot. Polon. 9: 457-472. illus. [Gentiana.]

Wuist, E. D. 1913. Physiol. Res., Prelim. Abst., 1: 93-94. [Sex in fern.]

WURDACK, M. E. 1923. Ohio Jour. Sci. 23: 181-191. [Cell walls of algæ.]

Wylie, R B. 1922. Science 66:422-423. [Vallisneria: or cells.]

1923. Bot. Gazette 75: 191-202. pls. 8-10. [Vallisneria: ơ cells.]

Yamagiwa K. and K. Ichikawa. 1915-1919. Mitt. Med. Fak. Univ. Tokyo 15,

17, 19, 22. Amer. Jour. Cancer Res. 11. 1917.

YamahA, G. 1926a. Jap. Jour. Bot 3: 139-162. illus. [Cytokinesis.]

1926b. Bot. Mag. Tokyo 40: 172-197. illus. [Artefacts.]

1927ab. Jour. Fac. Sci. Imp. Univ. Tokyo 2: 1-214; 215-296. illus. 
1927c. Bot. Mag. Tokyo 41: 181-210. illus.

1932. Sei. Rept. Tokyo Bunrika Daigaku, B 1: 1-21. [Staining.]

Yamaha, G., and Y. Sinotô. 1925. Bot. Mag. Tokyo 39: 205-219. illus.

Yamanouchi, S. 1906. Bot. Gazette 42:401-449. pls. 19-28. [Polysiphonia.]

1908a. Bot. Gazette 45: 1-20. pls. 1-4. [Nephrodium: meiosis.]

1908b. Bot. Gazette 45: 145-175. pls. 6-8. [Nephrodium: gametogenesis, fertilization.]

1908c. Bot. Gazette $45: 289-318$. pls. 9, 10. [Nephrodium: apogamy.]

1909. Bot. Gazette $47: 173-197$. pls. 8-11. [Fucus.]

1910. Bot. Gazette 49:1-12. pl. 1. [Osmunda.]

1912. Bot. Gazette 54:441-502. pls. 26-35. 15 figs. [Cutleria.]

1913a. Bot. Gazette 55: 74-79. [Hydrodictyon.]

1913b. Bot. Gazette 56: 1-35. pls. 1-4. 24 figs. [Zanardinia.]

1913c. Bot. Mag. Tokyo 27: (279)-(285). (Japanese) [Corallina.]

1921. Bot. Gazette 72:90-96. (Transl. of 1913c) [Corallina.]

Yampolsky, C. 1919. Amer. Jour. Bot. 6:410-442. pls. 37-40. [Sex.]

1920ab. Amer. Jour. Bot. 7:21-38; 95-100. pl. 5. [Sex intergrades.]

YAMPOLSKY, C. and H. 1922. Bibliog. Genetica 3:1-162. illus. [Sex.]

YASUI, K. 1929. Cytologia 1: 192-215. illus. [Hosta plastids.]

Yats , N. 1904. Biol. Bull. 6: 123-136. illus. [Egg fragments.]

1905. Jour. Exp. Zoöl. 2 : 287-312. illus. [Egg fragnents.]

1910. Jour. Coll. Sci. Tokyo 27.

YAZAWA, H. 1931. Cytologia 2:157-173. illus. [Makinoa: $\left.\sigma^{7}.\right]$

Yocom, H. B. 1918. Univ. Calif. Publ. Zoöl. 18: 337-396. pls. 14-16.

Yuasa, A. 1932. Bot. Mag. Tokyo 46:4-12. [Pteris sperm.]

1933. Cytologia 4:305-337. illus. [Spermatozoids.]

\section{Z}

Zacharias, E. 1881. Bot. Zeitung 39: 169-176. [Nucleus.]

1882. Bot. Zeitung 40: 611-616, 627-649, 651-653. [Nucleus.]

1883. Bot. Zeitung 41: 209-215. [Plastin, etc.]

1885. Bot. Zeitung 43: 257-265, 273-283, 289-296. [Nueleolus.]

1887. Bot. Zeitung 45: 297-304. pl. 4. [Nucleus; sexual cells.]

1888. Bot. Zeitung 46: 33-40, 51-62. pl. 2. [Nucleus; cell-division ]

1890. Bot. Zeitung $48: 1-10,17-26,33-43$, etc. [Cyanophyceæ.]

1892. Bot. Zeitung 60:617-624. [Cyanophyceæ.]

1895. Flora $81: 217-266$. illus. [Nucleus.]

1898. Ber. Deu. Bot. Gesell. 16: 185-198. [Nucleus.]

1902. Ber. Deu. Bot. Gesell. 20 : 298-320. illus. [Nucleus.]

1903. Jahrb. Hamburg. Wiss. Anst. 21: 47-89. 1 pl. [Cyanophyceæ.]

1907. Bot. Zeitung 65: II 264-287. [Cyanophyceæ.]

1910. Prog. Rei Bot. 3: 67-258. [Chemistry of protoplasm.]

ZADE, A. 1914. Zeitschr. Pflanzenzücht. 2:101-151. [Serological studies.]

1918. Der Hafer. 355 pp. 32 figs. Jena.

Zattler, F. 1924. Zeitschr. Bot. 16:433-499. illus.

Zeleny, C. 1904. Jour. Exp. Zoöl. 1: 293-329. illus.

Ziegenspeck, H. 1928. Bot. Archiv 21: 1-8. illus. [Cutin.]

Zimmermann, A. 1893. Beitr. Morph. Physiol. Zelle 1: 185-197. [Elaioplasts.] 1893-1894. Sammel-Referate. Beih. Bot. Centralbl. 3-4.

1896. Morphologie und Physiologie des Pflanzlichen Zellkerns. Jena.

ZimmermanN, W. 1921. Jahrb. Wiss. Bot. 60: 256-294. 1 pl. [Volvox.]

1923. Zeitschr. Bot. 15: 113-175. 7 figs. [Duration of initosis.] 
ZirkLE, C. 1926. Amer. Jour. Bot. $13: 301-320,321-341$. illus. [Plastid.] 1927a. Amer. Jour. Bot. 14: 429-445. illus. [Plastids.]

1928a. Protoplasma 4: 201-227. illus. [Fixation.]

1928b. Bot. Gazette 86: 402-418. illus. [Nucleolus.]

1929a. Protoplasma 5: 511-534. illus. [Fixation.]

1929c. Bot. Gazette 88: 186-203. illus. [Plastid development.]

1931. Cytologia 2: 85-105. illus. [Nucleolus.]

1932a. Zeitschr. Zellf. Mikr. Anat. 16: 26-47. illus. [Vacuoles.]

1932b. Jour Heredity $23: 433-448$. [Early hybridizers.]

1933. Protoplasma 18: 90-111. See also $19: 565-577 ; 20: 169-179$.

Zuelzer, M., and F. Philipp. 1925. Strahlentherapie 20: 787.

Zulueta, A de. 1925. Eos 1. [ $Y$-chromosome genes.]

Zweibaum, J., and A. Elkner. 1926. Bull. Histol. Appl. Phys. Path. 3: 218-221.

illus. [Golgi.] 


\section{INDEX}

Numbers in bold-face indicate pages bearing illustrations.

A

Aase, 121, 342, 356, 366

Abderhalden, 100

Abegg, 99, 100

Aberrant mitosis, 184-188

Abies (fir), 95, 235

Abnormal mitosis, 184, 186

Abraxas (moth), 380

Abromavich, 398

Abutilon (angiosperm), 417

Acacia (angiosperm), 164

Acer (maple), 237

Acetabularia (green alga), 279

Achlya (fungus), 165, 229

Achromatic figure, 149-164, 440

Aconitum (angiosperm), 129

Acqua, 58

Acquired somatic modification, 443

Acrididæ (see also Grasshopper), 130, 136

Acroblast, 7S, 204, 216, 219, 220

Acrosome, 78, 204, 216, 219, 220, 221, 241

Actinophrys (protozoan), 277, 278

Actinospharium (protozoan), 194, 195, 277

Activation of egg, 247

Acton, 192

Addoms, 188

Adsorption, 38, 89

Egilops (angiosperm), 128, 129, 366

Egilotricum (angiosperm), 368

Ethalium (slime mold), 26

Afzelius, 341, 345, 348, 405

Agar, chromidia, 195

chromosome, 116, 142

meiosis, 256, 272

nucleus, 48

sex-chromosome, 378,380

syngamy, 239

Agathis (conifer), 23, 24, 207, 235

Agave (century plant), 103

Agglutination, 248

Aggregata (protozoan), 277

Aglaozonia (brown alga), 280

Agropyrum (angiosperm), 128

Aida, 378

Akkeringa, 381

Albach, 53

Alberti, 187

Albugo (fungus), 10, 200, 201, 229

Albumin, 84, 96

Alchemilla (angiosperm), 402, 404, 405

Aleurodiscus (fungus), 230, 386

Aleurone, 95, 98, 101, 103

Alexander, 144, 312
Alexieff, 82

Algæ, apomixis, 402

cell wall, 177

gametogenesis, 196

heredity, 306

meiosis, 278-280

mitosis, 159, 191

plastids, 63,64

sex, 386

sporogenesis, 196

syngamy, 226-228

Alkaloids, 96

Allelomorph, 2\$9, 310

Allen, C. E., cell wall, 176

chromomeres, 142

cytokinesis, 171, 174

heredity in bryophyte, 307

meiosis, 256, 262, 266, 279, 283

recombination, 302

sex determination, 382, 384, 395

spermatogenesis, 201, 202, 203

Allen, R. F., 204, 231, 387

Allium (onion), canaliculi, 79

chondriosomes, 83

meiosis, 258, 267

parthenogenesis, 402, 404

root tip, 5,79

somatic chromosomes, 124, 130, 132, 133, 134

tonoplast, 42

Alloheteroploidy, 340, 348, 369

Allomyces (fungus), 199, 281

Alloplasm, 45

Allosynapsis, 362

Alnus (angiosperm), 164

Alö̈ (angiosperm), 129, 130, 177, 271

Altenburg, $187,311,316,325,395$

Alternation of generations, 122, 196, 279-281, 401

Altmann, 26, 82, 89, 434

Alvarado, 90

Alveolar spheres, 32

Alveolar theory of protoplasm, 434

Alveolation theory of chromosomes, 132, 140

Alverdes, 51

Alyssum (angiosperm), 357

Alytes (toad), 272

A mblycorypha (katydid), 382

A mblystegium (moss), 405

A meiosis, 345

Ames, 387

A mici, 432, 436, 437

A mides, 96

A mitosis, 183-185, 439

Amaba (protozoan), 39, 43, 58, 59

Amphiaster, 156 
Amphiastral figure, 153-159

A mphibia, 114

A mphidiploid hybrids, 367-371

Amphimixis, 401

Amphitène, 256

Amphitornis (insect), 265

A mygdalus (angiosperm), 103

Amyloplast, 62, 65, 98

Amylose, 99

Anabcna (blue-green alga), 191, 192

Anaphase, meiotic, 257, 262

somatic, 106, 107, 109, 134, 135

Anastomoses, 54, 106, 136, 137

Anastral figures, 149-153

Ancel, 272

Anderson, D. B., 176-178

Anderson, E. G., 30, 266, 302, 306, 310, 353, 393, $415,416,419$

Anderson, R. J., 30

Andersson-Kottö, 405, 415

Andrews, E. A., 18

Andrews, F. M., 18, 57, 58, 161

Androcyte, 202, 203

Androgenesis, 407, 412

Androgone, 202, 203

Androspore, 206

Andrus, 231

Anesthetizing agents, 185

Aneuploidy, 339, 341

Anisogenomatic, 340

Anitschkow, 83

Ankel, 401

Anomaoneis (diatom), 386

Antecedent genom, 419

Antennaria (angiosperm), 402, 403, 407

Antheridium, 197, 229, 230

Anthoceros (liverwort), meiosis, 283 plastid, $61,63,70$

pyrenoid, 68,69

syngamy, 233

zygospore, 201

Anthocyanin pigments, 96,97

Anthoxanthum (angiosperm), 133

Anthurium (angiosperm), 103

Antipodal cells, 183, 210, 211, 237

Antirrhinum (angiosperm), 97, 342, 415

A phis (aphid), 411, 413

A pical body, 71, 81, 202, 203, 204, 222

Apical cell, 12, 93

Apis (bee), 410

Aplanospores, $19 \mathrm{~S}$

Aplocheilus (fish), 378, 393

A pogamy, 403

Apomixis, 401-413

Apospory, 404, 405

A potettix (grasshopper), 410

Apposition, 177

Aquilegin (angiosperm), 367, 370

Arabis (angiosperm), 415

Araratian, 121, 124, 187, 321

Arbacia (sea urchin), 32, 40, 154, 221

Arber, 49, 183

Archegonium, 201, 206, 233

Archieracium (angiosperm), 402

Archoplasm, 44
Arctostaphylos (angiosperm), 103

Arcyria (myxomycete), 278

A risama (angiosperm), 396

Aristotle, 422

Arnold, 85, 87, 88, 379

Arrangement of chromosomes, 125

Arrhenatherum (angiosperm), 54, 111, 171

Artemia (crustacean), 343, 411,413

Artemisic (angiosperm), 405

Arthur, 231, 283

Artichoke, 103

Artom, 343

Artschwager, 238

Ascaris (nematode), centrosome, 153

chromosomes, 132

fertilization, 240,244

meiosis, 263, 272, 440

sex-chromosomes, $376,377,378$

spermatozoön, 222

Ascidian, 182

Asclepias (angiosperm), 210, 236

Ascobolus (fungus), 281, 387

Ascogonium, 229

Ascomycetes, achromatic figure, 156, 157, 158 meiosis, 281

sex, 387

spore coat, 181

spore formation, 157, 173, 201, 230

syngamy, 229,230

A silus (insect), 272

Asparagin, 94, 96

Asparagus (angiosperm), 90

Aspidiotus (insect), 411

Aspidium (fern), 404, 405

Asplenium (fern), 405

Astaurow, 129

Astbury, 178, 179

Aster, 3, 111, 149, 155, 156, 157, 170, 244

Asterias (starfish), 154, 411

Astral rays, 157

Asynapsis, 273, 345, 359

Atamosco (lily), 402, 407

Atelomitic, 116

Athyrium (fern) 402, 405

A tkinson, 226, 281

Attachment constriction, 116

A ucuba (angiosperm), 329

Auerbach, 441

Autoheteroploidy, 340, 348, 350-35s

Autosome, 376

Autosynapsis, 362

Auxin, 19

Auxocyte, 251

Auxospore, 228

Avdulow, 121, 128

Avena (oats), 343, 416

Avery, 124, 187, 188, 320, 356

Axial filament, 217, 219, 221

Axial gradient, 19

Axon, 13, 14

Azolla (water fern), 138

B

Baache-Wiig, 96

Babcock, 128, 139, 142, 260, 268, 286, 367 
Baccarini, 56

Bacillus, 89, 193

Back-cross test, 289, 295

Bacteria, 82, 89, 100, 177, 193, 194

Bacterium, 194

de Baehr, 411, 413

von Baer, 425

Bagchee, 156, 157, 158, 281

Bailey, E. W., 21, 436

Bailey, 1. W., 40, 51, 94, 96, 97

Baird, 229

Balanced emulsions, 36

Balanophora (angiosperm), 346

Balbiani, 51, 141

Balfour, 439

Ballowitz, 222

Balls, 176,178

Baltzer, 397, 412

Bancroft, 33, 169

Bangham, 342

Banta, 397

Baranetzky, 132

Baranov, 118

Barkley, 176

Baron, 188

Barry, 438

Bartlett, 336, 358, 396

de Bary, 23, 403, 433-436

Basal granules or corpuscles, 203, 205, 224

Basic number of chromosomes, 340,347

Basichromatin, 54, 55

Basidiobolus (f ungus), 281

Basidiomycetes, gametogenesis, 200 meiosis, 282

sex, 386

syngamy, 230

Basidiospores, 200

Basidium, 200, 230, 282

Bast, 184

Bataillon, 154, 248

Bateson, 286, 308, 351

Bauch, 282

Baumgärtel, 192

Baur, 286, 392, 415, 416

Bayliss, 25, 33, 38, 40, 43

Beadle, 271, 273, 360, 371

Beams, 58, 78, 244

Beardsley, 233

Beauverie, 64

Bechhold, 33

Becker, 58, 172

Beckwith, 85

Bee, 410

Beer, 49, 66, 180, 181, 183

Beikirch, 31

Belajeff, 199, 204, 208

Bèlař, achromatic figure, 155, 161, 162, 163

cell-plate, 171, 172

chiasma, 268

fixation, 7

flagella, 223

fragmentation, 316

furrowing, 169

meiosis, 256, 277

Mendelism and chromosomes, 286
Bëlař, nucleus, 189

parthenogenesis, 411

reticulum, 54

sex, 397

somatic mitosis, 108, 109, 112

spindle attachment, 116

syngamy, 245

Bellamy, 381

Bellevalia (angiosperm), 128

Belling, chromomeres, 142

chromonema, 139

chromosome fragments, 320

chromosome morphology, 124

genes, 144

meiosis, 258, 267, 268, 270, 271, 273

meiosis and heteroploidy, 351, 352

monoploid Datura, 356, 406

non-disjunction, 345

reciprocal translocation, $327-329,331,335$

reticulum, 54

spindle attachment, 116

temperature effect, 186

trisomic mutants, 298, 327, 343

Belzung, 100

Benda, 82, 87

van Beneden, achromatic figure, 159

centrosome, 153

cytology and heredity, 439, 441

individuality of chromosome, 141, 145

mitosis, 159, 440

protoplasmic structure, 434

Benetzkaja, 367

Benoist, 53

Bensaude, 230

Bensley, 79, 80, 84, 95

Berberidacew, 129

Berberis (angiosperm), 18

Berg, 368

von Bergen, 73

Rerghs, 256, 266

Bergner, 124, 327, 337

Bergon, 228

Bernal, 178

Bernard, 58, 441

Bernhardi, 426

Berridge, 235

Bersa, 186, 187

Bertholletia (Brazil nut), 101, 103

Besley, 354

Beta (angiosperm), 186, 238, 347

Beutner, 20, 38, 40, 42, 180

Beyer, 49

Bhatia, 88, 225

Bhattacharya, 78

Bichler, 187

Bicornes (angiosperms), 342

Biddulphia (diatom), 280

Binder, 139

Binnenkörper, 189

Binuclearity hypothesis, 194

Binz, 100

Bioblast, 82, 89, 434

Biogen, 46

Biophore, 442

Biourge, 180 
Bisceglie, 187

Bivalent chromosomes, 252, 351, 440

Black, 233

Blackburn, 347, 357, 365, 367, 390

Blackman, M1. W., 272

Blackman V. H., 210, 230, 231, 235, 283

Blair, 283

Blakeslee, Datura chromosomes, 124

gene mutation, 310

heteroploidy, 343, 352, 358

monoploid sporophyte, 356

non-disjunction, 345

pollen tube growth, 293

primary mutants, 327

radium treatment, 188,311

sex, 387, 388, 398

tetraploidy, 351

translocation, 323, 329, 335-337

triploidy, 354

trisomic mutants, 298

Blasia (liverwort), 201

Blastomere, 243, 438

Bleier, $149,157,161,163,164,186,342,356,366$, 367

Blepharisma (protozoan), 44

Blepharoplast, 198, 199, 201-208, 202, 205, 207, 223,234

Blood, 14, 49, 73, 89

Blue-green algæ, 191-193

Blunt, 133

Bobilioff-Preisser, 58

Bock, 22

Boeck, 190

Boedijn, 334

Bæhmeria (angiosperm), 178

Boeke, 13

Bokorny, 79

Bold, 68

Bolleter, 201

Bone, 14

Bonnet, C., 424

Bonnet, J., 183, 196, 278

Bonnevie, 132, 133, 142, 266

Böös, 402

Border brim, 205

Bordered pit, 175

Borelli, 424

Borgenstam, 186

Boring, 378, 381

Borodin, 188

Borzl, 16

Bosaeus, 410

Bose, 20, 79

Botrychium (fern), 181

Bouin, 93

Bougainvillea (angiosperm), $3 \subseteq 3$

Bouquet stage, 256

Bourquin, 65

Bouygues, 83, 90

Boveri, amitosis, 187

centrosome, 153

cytology and heredity, 439, 444

dispermy, 347

fertilization, 244

individuality of chromosome, 145, 146, 440
Boveri, nucleus size, 51

parthenogenesis, 412

rôle of chromosomes, 445

sex-chromosomes, 378,381

Bowen, chondriosome, $86,89,90,91$

Golgi material, 73, 75, 76, 79, 80

heteroploidy, 346

leucoplasts, 62

plastids, 69, 70, 91

secretion, $75 \notin$.

spermatogenesis, 201, 203, 214

spermiogenesis, 215-223, 216, 217, 218, 219, 221

spindle, 164

syngamy, 241

Bowenia (cycad), 234, 235

Bower, 404

Bozler, 13

Brachet, 412, 420

Brachiomonas (green alga), 68

Brachymeiosis, 281

Brachystola (grasshopper), 122, 254, 272

Bragg, 178

Brambell, 74, 397

Brand, 165, 166

Brassica (angiosperm), 342, 346, 364, 365, 367, 370

Brauer, 376,411

Braun, 433

Bremer, 369

Breslawetz, 236

Bresslau, 121

Bridges, crossing-over, 306

factorial hypothesis, 312

fragmentation, 320

gene theory, 144, 309, 312

homologous chromosomes, 123

linkage, 301

meiosis, 266

mutation, 310

non-disjunction, 345

sex, 394

translocation, 321

triploidy, 353

Brieger, 293, 340, 365

Brill, 179

Brink, 99, 100, 293, 305, 329, 332

Brochymena (insect), 218, 219

Brodie, 388

Brooks, F. T., 230

Brooks, W. E., 2 S1

Brooks, W. K., 47, 158

Brown, A. P., 30

Brown, H. B., 230

Brown, R., 427

Brown, V., 223

Brown, W. H., 158, 192, 230, 237, 281

Brownian movement, 6, 31, 43, 53, 161

Bruchus (insect), 376

Brücke, 433

Bruns, 100

Brunswik, 386

Bryonia (angiosperm), 388

Bryophytes, cytoplasmic inheritance, 418

gametogenesis, 201-204

heredity in, 307 
Bryophytes, meiosis, 283 plastid, 63, 71, 80 sex-chromosomes, 382 sporogenesis, 204 syngamy, 233

Bryopsis (green alga), S, 22

Bryum (moss), 405

13ucciardi, 187

Buchholz, J., 293, 311

Buchner, 194, 411

Bucholtz, 188

Buchtien, 204

13uck, 90

Bud sport, 311

Buder, 17, 278

Bufo (toad), 80

Buller, 230

Bunting, 190, 223

Burgeff, 229, 387

Burmannia (angiosperm), 404

Burnham, 100, 293, 322, 327, 329, 320

Burrows, 6

Bursa (angiosperm), 342

Bütschli, achromatic figure, 160, 440

blue-green algæ, 192

cytology and heredity, 441

furrowing, 169

polar bodies, 438

protoplasm, 434

Buxton, 346, 367

C

Cabomba (angiosperm), 69, 85

Cajal, 73

Cald well, 207

Calkins, 43, 160, 189, 223, 245, 246, 277

Callisia (angiosperm), 267

Callithamnion (red alga), 280

Callose, 167, 180

Calopogon (orchid), 236

Calycanthus (angiosperm), 402

Cambium, 12, 51, 94, 97

Camerarius, 436

Cameron, 29

Camp, 398

Campanula (angiosperm), 329, 351

Campbell, A. S., 223

Campbell, D. H., 204, 233, 437

Canaliculi, 79

Cancer problem, 187

Canis (dog), 376

Canna (angiosperm), 354

Cannabis (hemp), 343, 346, 390, 396, 397

Cannon, 125, 169, 445

Canti, 3, 188

Caoutchouc, 97

Capinpin, 352

Carbohydrate, 27, 96, 98-100, 99

Carbohydrate-protein ratio, 30

Carchasium (infusorian), 277

Carex (sedge), 341

Carleton, 50, 57

Carlson, 199

Carnoy, 434
Carothers, 115, 116, 123, 141, 265

Carotin, 64

Carpogonium, 228

Carrel, 6, 47

Carruthers, 158, 230, 281

Carter, G., 41

Carter, N., 63, 68, 191, 280

Cartilage, 14, 15, 171

Cartledge, 356

Carus, 435

Cassaigne, 96

Castetter, 167, 168

Castle, 256, 290, 311

Castrada (flatworm), 222

Cat, 378

Catalysis, 38

Catcheside, 271, 304, 334, 337, 352, 356

Catenation, 332

Catherinea (moss), 71

Cathode ray, 187

Cattaneo, 78

Cattle, 376

Caulerpa (green alga), 8, 10, 11, 190

Cavers, 82, 90

Cell, discovery, 2, 423

enucleated, 58

number, 9,357

size, 23,357

Cell-division, 110, 430

duration and periodicity, 111

Cell membrane of animals, 181

Cell-plate, 109, 110, 150, 171-174, 172

Cell sap, 2, 29, 96

Cell theory, 20 ff., 428

Cell wall, 4, 12, 100, 165-168, 174-181

Cells and tissues, 1-24

Cellulose, 100, 176, 1S0, 192 in animals, 182

Central spindle, 111, 155

Centrifuged cells, $31,32,57,58,84,161$

Centriole, 3, 153, 155, 215, 217, 221

Centroblepharoplast, 223

Centronema, 223

Centrosome, 3, 153, 154, 156, 159

in mitosis, 110

in syngamy, 244

Centrosphere, 3,153

Centrurus (scorpion), 86

Cephalobrachial chromosomes, 116

Cephalotaxus (conifer), 95, 235

Ceratiomyxa (slime mold), 278

Cетаtium (dinoflagellate), 190

Ceratopteris (fern), 138

Ceratozamia (cycad), 234, 440

Cerebratulus (worm), 154

Chania (protozoan), $\mathbf{5 1}$

Charocampa (insect), 360

Chatomorpha (green alga), 279

Chetopterus (annelid worm), 23, 412

Chalaud, 70

Chamberlain, cell-plate, 173

cytokinesis, 167

heteroploidy, 342

life cycle, 196

oögenesis, 206 
Chamberlain, spermatogenesis, 207

syngamy, 234-236, 239

Chambers, achromatic figure, 157, 161, 164, 170

amitosis, 184

Brownian movement, 32

chondriosomes, $\$ 3,85,86$

chromosome, 142

ectoplasm, 44

Golgi material, 74

hydrogen-ion concentration, 29

meiosis, 256

microdissection, 6, 142

nucleus, $52-54,59$

parthenogenesis, 411

plasma bridges, 18

plasma membrane, 39,40

syngamy, 241, 242

tonoplast, 42

viscosity, 31

Champy, 50

Chang, 74

Chara (stonewort) and other Charales, 183, 199, $227,279,402$

Chatton, 278

Chiarugi, 212, 402, 405

Chiasma, 257, 259, 260, 268, 269, 271, 337

Chicken, 381, 393

Chickering, 215

Child, 19, 20, 51

Chilodon (infusorian), 277

Chiloscyphus (liverwort), 283

Chimera, 311, 343, 357, 367

Chipman, 356

Chironomus (insect), 51

Chitin, 177, 179, 182

Chittenden, 415, 419

Chlamydomonas (green alga), 68, 223, 277

Chloralized cells, 115, 185, 186

Chlorococcum (green alga), $6 \mathrm{~S}$

Chlorophyll, 64, 67, 192

inheritance, 414-418

Chloroplast, 6367,68

Chouborus (insect), 378

Chodat, 256

Cholesterol, 27

von Cholnoky, 228, 280, 386

Chondrilla (angiosperm), 402

Chondriocont, $\$ 3$

Chondriome, 83

Chondriomite, 83

Chondriosomal mantle, 151, 152

Chondriosome, 3, \$2-92, 214

function, 87

relation to plastid, $90 \mathrm{fJ}$.

rôle in secretion, 77

in spermatogenesis, 86

in spermiogenesis, $215,218,221$

in sporogenesis, 85,86

in syngamy, 244

Chorthippus (grasshopper), 7, 112, 162, 169, 256, 316

Christiansen, 190, 223, 232

Christman, 231

Chromatid, 252, 257, 264

Chromatin, 27, 52, 55
Chromatium (bacterium), 43

Chromatophore, 62

Chromidia, 194

Chromioles, 55, 141, 143

Chromocenter, 2, 56

Chromomere, 142-144, 312

in meiosis, 259, 260

in reticulum, 56

theory, 132, 141

Chromonema, 54, 105, 106, 114, 133, 134, 135, $139,140,142-144,260,264,312,335$

theory, 132

Chromoplasts, $62 \mathrm{ff}$.

Chromosomal vesicle, 136

Chromosome cycle, 251, 291

Chromosomes, $105 \mathrm{ff}$.

aberrations, $185 \not f ., 359 \not f$.

alveolation, 132, 140

arm, 116

arrangement, 125

balance among, 325

basic number, 340,347

complement, $121 \mathrm{fJ}$.

constrictions, $115 f$.

continuity, 145

fragmentation, $117,185,314 f f ., 349$

in heredity, $284 f f$.

heteromorphic, 265,272

historical, 439

homologous pairs, 252, 272

human, 379

in hybrids, $359 \mathrm{ff}$.

individuality, 145

knob, 119, 123, 271, 298, 305, 318, 332, 333

in life cycle, 122, 251, 291, 383, 440

map, 299, 301, 323, 324

matrix, 106, 133-144

in Mendelian heredity, $291 \mathrm{f}$.

morphology, 114-131, 349, 446

multivalent, 304, 355

non-homologous association, 276

nucleolus-forming region, 118-121

number, $121,339 \mathrm{f}$.

alterations, 344

and taxonomy, $126,340 f f ., 446$

pairing in somatic cells, $123,126,272$

satellites, $118 \mathrm{f}$.

set, $121,254,325$

sex, 297, 375-400

size and shape, $114 f f$.

sinall, 138

splitting of, $105,113,133,137,145,190,265$, 363,440

splitting in meiosis, 252, 255, 25S, 261, 265, 275

structure, 132-148

tetrads, $252 \not f$.

trivalent in synapsis, 274

$\mathrm{X}$-chromosome, $304,376 \mathrm{ff}$.

$Y$-chromosome, 304, $378 \mathrm{ff}$.

Chrysanthemum (angiosperm), 340, 345

Cienkowski, 433

Cilia, 43, 197, 203, 207, 208, 223, 229, 233

Ciliary mechanism, 224, 225

Ciliated cells, 184, 223, 224, 225

des Cilleuls, 184 
Circinella (fungus), 165

Circotettix (grasshopper), 320, 329

Cirri, 43

Citrus (angiosperm), 405

Cladophora (green alga), alternation of generations, 279

body structure, $8,23,49$

cytokinesis, 165, 166

eyespot, 67

mitosis, 190, 191

nuclei, number, 9

sex, 386

Clark, 178

Clarkia (angiosperm), 329

Clausen, J., 139, 142, 260, 268, 273, 342, 361, 415,416

Clausen, R. E., 286, 345, 356, 365, 367, 407

Claussen, P., 158, 199, 230, 281

Cleavage of egg, 168-171, 242-244

furrowing, $165-171$

historical, 438

and promorphology of ovum, 23 rate, 420

Cleland, meiosis, 280, 334

nucleol us, 58

Enothera, 334, 335, 336

translocation, 336,337

Clivia (angiosperm), 177

Closing membrane, 175

Closterium (desmid), 166, 279

Clowes, 36, 37, 41

Coagulation, 37,53

Cobb, 336

Cocconeis (diatom), 228

Codium (green alga), 190, 279

Conocentrum, 200, 229

Cœnocytes, 8, 10, 21-23, 48, 197

Cœnocytic embryo, 23, 24

Ccenogametes, 10

Cohen, 29

Cohn, 433

Coker, 235

Cole, 424

Coleochcete (green alga), 227, 279

Colley, 283

Collins, G. N., 407

Collins, J. L., 124, 363

Colloidal state, $33 f f ., 434$

Colorblindness, 392

Commelina (angiosperm), 181

Complement of chromosomes, $121 \not f ., 340 f f$.

Components (of colloids), 34

Conard, 184, 228

Conduction of stimuli, 18-20, 39

Conductivity of protoplasm, 180

Conidia, 167, 200

Conjugate division of nuclei, 229

Conjugation, in algæ, 228

in Paramoecium, 245

Conklin, amitosis, 183, 184 cellular differentiation, 9 cleavage, 23 cytoplasmic inheritance, 420

fertilization, 243

fibrils, 87
Conklin, individuality of chromosomes, 136, 146 mutation, 311

nucleoplasmic ratio, 51

parthenogenesis, 412

promorphology of ovum, 23

Weismannism, 441

Conn, 441

Connecting piece, 218

Connective tissue, $14,15,18$

Conner, 415

Constrictions in chromosomes, 115, 116

Continuity of protoplasm, 16-18

Contractile vacuole, A moba, 39

Diplodinium, 10

discharge, 42

origin, 44

Spirogyra, 45, 228

Convallaria (angiosperm), 210

Cook, 190

Cooper, D., 329, 332, 388

Cooper, G., 199

Copeland, 410

Coprinus (mushroom), 230, 356

Corallina (red alga), 280

Corethron (diatom), 280

Cork, 176

Cornea, 75, 154, 187

Corner, 213

Correlation, 9, 15-20

Correns, cytoplasmic inheritance, 418

Mendelism, 289, 445

pollen characters, 308

recombination, 302

sex, 388, 390, 391, 396, 398

variegation, 415,416

wall structure, 177

Corsinia (liverwort), 233

Corti, 432

Coscinodiscus (diatom), 228

Cotton fiber, 176, 178

Couch, 64, 197, 199, 387

Coulter, 167, 196, 227, 235, 236, 239

Cowdry, E. V., chondriosomes, $82,83,84,87,89$, 90

chromidia, 195

Golgi material, 73-75, 78

respiration, 89

Cowdry, N. H., 82-84

Cox, 378

Crabb, 240

Craigie, 231, 386

Creighton, 121, 303, 305, 337

Crepidula (mollusk), 136, 184, 243

Crepis (angiosperm), chromomere, 142

chromosome complement, 124, 127, 349

chromosome duplication, 325

fragmentation, 320

heteroploidy, 343,348

hybrid, 361, 362, 363, 367, 371, 372

kinetic bodies, 117

meiosis, 260

monoploid sporophyte, 356,357

parthenogenesis, 402

somatic chromosomes, 124, 127, 130, 139

species and chromosomes, 127, 128 
Crepis (angiosperm), translocations, 314, 321

Crew, 376, 397

Crossing-over, 266-272, 293-295, 298-306, 320, $323,327,337,393$

cytological evidence, 302-304

in triploid, 353

between two chromatids only, 305

Crown gall, 186

Cruciferæ, 342

Cryptobranchus (amphibian), 76, 136, 243

Cryptomeria (conifer), 235

Cryptoplasm, 33

Crystallites, 178

Crystalloid, 101

Crystals, 101-103

Cucumis (angiosperm), 186

Cucurbita (angiosperm), 164

Cuénot, 375

Culex (mosquito), 344

Cumingia (mollusk), 32

Cunha, 90

Cunningham, 441

Cuscuta (angiosperm), 17

Cuticle, 176

Cutin, 176, 180

Cutleria-Aglaozonia (brown alga), 280

Cyanophycex, 191-193

Cyanophycin granules, 193

Cyanotis (angiosperm), 272

Cycad, 206, 234

Cycas (cycad), 234, 236

Cyclops (copepod), 136, 243

Cylindrocystis (green alga), 279

Cypripedium (orchid), 212

Cyrtanthus (angiosperm), 130

Cystolith, 103

Cystosira (brown alga), 407

Cytaster, 154, 248

Cytoblast, 430

Cytoblastema, 430

Cyto-idioplasm, 418

Cytokinesis, 85, 86, 105 ff., 111, 116, 152, 165-173

by furrowing, 109, 155, 165-171

in meiocyte, 263

Cytome, 83

Cytomicrosome, 82

Cytoplasm, cell constituent, 2

in heredity, 286

mantle, 235

in syngamy, 234, 236

Cytoplasmic heredity, 414-419

Cytosome, 2, 83, 91

Cytotaxonomy, 126, $340 \not f ., 446$

Czaja, 386

Czapek, 25, 33, 93, 97, 100, 176

Czurda, 68, 190

\section{D}

Dahlgren, 210, 212, 226, 236, 239, 398

Dahlia (angiosperm), 351, 355

Da Fano, 74

Dale, 230

Daltonism, 392

Danchakoff, 195, 242
Dangeard, aleurone, 101

ascomycetes, 231

chondriosomes, 61, 69, 83, 90, 91

elements of protoplast, 104

ergastome, 102

nucleus, 53

syngamy, 229, 230

vacuole, $93-97$

vital staining, 6

Dannehl, 172

Daphnia (copepod), 411, 413

Darbishire, 100

Dark, 351

Dark field, 3, 6, 43, 84

Darlington, amphidiploidy, 367

chiasma, 267, 268, 270, 271

chromosome complement, 129

disjunction, 323

fragments, 314, 319

gene theory, 312

meiosis, 256, 258, 275

Enothera, 304, 334, 335, 337

reciprocal translocation, 329

synapsis in heteroploid, $274,351,352$, 355

Darwin, 373,441

Datura (angiosperm), aleurone, 103

chromosome complement, 124

heteroploidy, 343, 352, 359

monoploid sporophyte, 356

non-disjunction, 345

parthenogenesis, 402, 406

primary mutants, $327, \mathbf{3 2 8}$

reciprocal translocation, $331,332,337$

syngamy, 238

tetraploidy, 351

triploidy, 353, 354

trisome, 298

Davenport, 392

Davey, 396

Davis, B. M., achromatic figure, 151

a]gæ, 197, 278

cytokinesis, 165

heteroploidy, 345

meiosis, 283

monoploid sporophyte, 356

Enothera, 334

oömycetes, 199

plastid, 70

protoplasmic connections, 16

syngamy, 229

Dawson, 44

Day, 43

Decaploidy, 339

Deconjugation, 272, 273

Decticus (insect), 142

Deficiency, 317,325

Dehorne, 133

Deineka, 73, 76

Delage, 46, 441

Delaunay, 116, 124, 126, 128, 157

Delesseria (red alga), 280

Deletion, 188, 274, 315, 317, 318

Delitsch, 230

Delphinium (angiosperm), 129 
Dembowski, 172

Demerec, 308, 310, 38s, 398, 415, 416, 419

Dendrite, 13, 14

Dendrocœlum (flatworm), 258

Denham, 342

Dermatosome, 177

Desynapsis, 273,345

Determinant, 442

Deutoplasm, 100

Development, 7

and cells, $20 \mathrm{ff}$.

chromosome infuence, 273

gene theory, 309

and heredity, 284 ff.

Weismann's theory of, 442

Developmental selection, 293

Devisé, $71,86,151,152,171$

Dextrin, 99

Diakinesis, 257, 258, 260, 334

Diatoms, 228, 2s0, 356

Dictyokinesis, 75

Dictyosomes, 75, 216, 220

Dictyota (brown alga), 159, 227, 280

Didelphys (opossum), 378, 379

Didymium (slime mold), 165, 172, 278

Dietel, 283

Differential polyploidy, 340

Differentiation, 9 ff., 443, 444

Diffuse stage, 261

Digby, apomixis, 402, 403, 404

chromosome number and taxonomy, 126 hybrids, 367

nucleolus, 57,58

Digestion, 89

Digitalis (angiosperm), 346, 361, 363, 367

Dikaryon, 230

Dikaryophase, 230, 232

Dileptus (protozoan), 245

Dinophilus (annelid worm), 399

Dicecism, 385, 398

Dioncea (anglosperm), 18

Diospyros (angiosperm), 17

Dioön (cycad), 50, 206, 207, 234

Diplocystis (protozoan), 277

Diplodinium (protozoan), 10

Diplodization, 230

Diplohaplont, 278

Diplonema, 257, 258, 259, 264

Diplont, 278

Diplosis, 226

Dippel, 177

Dipsacaceæ, 129

Diptera, 122, 125, 272

Disjunction, 250, 252, 255, 265, 272, 291, 322

Dislocation hypothesis, 347

Disney, 423

Dispermy, 347

Disperse phase, 33

Dispersions medium, 33

Dissosteira (grasshopper), 86, 256

Dixon, 256

Dobell, 10, 21, 193, 277, 429, 436

Dobzhansky, 123, 187, 297, 320-325, 322, 324, $329,395,399$

Dodel, 100
Dodge, B. O., 231, 278, 282, 283, 387

Dodge, C. W., 196, 226, 231, 283

Doflein, 27, 189

Dogiel, 182

Dolley, 51

Dominance, 287

Doncaster, 239, 256, 272, 380, 382, 410, 411

Dore, 178, 179

Double erossing-over, 300

Double fertilization, 211, 236, 237, 238, 437

Double reduction, 230, 281

Doubt, 16

Douin, 382

Dowding, 387

Downing, 213

Dracinschi, 204, 205

Drayton, 387

Drew, 79, 184

Driesch, 441

Drosera (angiosperm), 339, 361

Drosophila (fruit fly), bar eye, 304

black body, 294, 300

carnation eye, 304

chromonema, 139

chromosome complement, 122, 129, 344

chromosome map, 324

crossing-over, 302, 303, 353, 393

embryo, 24

fragmentation, 316,318

gynandromorph, 382

inversion, 320

linkage, 294

linkage groups, 296-29s

linkage map, 301

meiosis, 295

non-disjunction, 345

polyploidy, 275

reciprocal translocation, 329

recombination, 304

sex-chromosomes, $377,378,381$

sex-linkage, 391, 392

synapsis, 272

syngamy, 243

translocation, $121,317,318,321, \mathbf{3 2 2}, \mathbf{3 2 3}, 325$

triploidy, 353

vestigial wings, 294, 300

X-ray treated, 187

Drüner, 160,162

Druse, 102, 103

Dublin, 272

Dubreuil, 88

Duck, 351

Duesberg, 73, 75, \$2, 85, 87, 195, 379

Dufrenoy, 91,98

Dujardin, 432

Dumas, 438

Dunn, 286

Dupler, 235

Duplex genes, 354

Duplex group of chromosomes, 292, 325

Duplication of genes, 319,322

Duplicational polyploidy, 340

Dutrochet, 20, 426-428, 435

Dyad, 252, 262, 264 
E

Eames, 12, 24, 175, 206, 235

East, 355, 365, 407, 419

Eberdt, 100

Echinops (angiosperm), 210

Echinus (sea urchin), 57, 412

Ectocarpus (brown alga), 196, 226, 227, 280, 402

Ectoplasm, 39, 43

Ectoplast, 3, 39

Edman, 405

Edwards, 378

Eftimiu, 158

Egg, of animals, 213

of plants, 197, 206, 210, 211, 232

Egregia (brown alga), 280

Eichhorn, 29

Eisen, 55

Eisentraut, 136

Ekman, 286

Elais (palm), 103

Elaioplast, 66, 102

Elasticity of protoplasm, 31, 37

Elastin, 182

Elatostema (angiosperm), 407

Electosome theory, 88

Electrical phenomena, 39, 52

Electrical theories of mitosis, 160

Elkner, 74

Elodea (angiosperm), 31, 44, 68, 388, 389

Emberger, 69, 83, 90

Embryo sac, 210, 212, 235, 237

Embryogeny, 211, 436-439

Emerson, R. A., 296, 298, 299, 300, 310, 312, 371 , 398

Emerson, S. H., 271, 334-337, 356

Emmel, 49

Emulsifying agent, 35

Emulsion, 35

Enchylema, 434

End piece, 221, 222

Enderlein, 193, 194

Endocrine secretions, 398

Endomixis, 246

Endoplasm, 39

Endosome, 189, 190

Endosperm, amitosis, 183

cytokinesis, 171,173

development of, 211, 212, 238

effect on embryo, 396

genetics of, 303,355

plasmodesms, 17, 18

storage, 95, 96, 100, 101

Endospore, 180, 181

Engrafted tissue, 403, 404

Enneaploid, 339

Enriques, 277

Enteromorpha (green alga), 279

Entz, 223

Enucleated cells, 58

Enucleated egg fragments, 410, 412

Environment, 47

Enzyme, 28, 38, 89, 96, 235, 236

Ephedra (gymnosperm), 174, 235

Epigenesis. 424
Epilobium (angiosperm), 186, 308, 342, 419

Epithelium, 15, 18, 171, 224

Equational division, 113, 252, 253, 255, 265, 272

Erdmann, 245, 246

Erdtman, 180

Equisetum (pteridophyte), chondriosomes, 86, 91 spermatogenesis, 204, 205

spore coat, 181

stem, 103

syngamy, 233

Ergastic materials, 4, 46, 93

Ergastome, 102

Ergastoplasm, 44, 93

Ergosterol, 27

Erhard, 225

Ericales, 342

Erigeron (angiosperm), 402

von Erlanger, 169

Erlanson, 267, 365, 397

Ernst, 348, 401, 402, 408

Erysiphe (fungus), 96

Erythroblast, 73

Erythrocyte, 49

Erythroplastid, 49

Escoyez, 159, 201

Euastrum (green alga), 63

Euchromocenters, 56, 57

Euchlana (angiosperm), 341, 342, 351, 360, 407

Euchlana-Zea hybrids, 371

Eudorina (green alga), 22, 67, 68, 190, 198

Euglena (flagellate), 66, 67

Eupatorium (angiosperm), 402

Euploidy, 339, 340

Euplotes (protozoan), 19, 42, 49, 277

Euschistus (bug), 75, 86, 214, 219, 346

Evans, 379

Evolution, 441

Exine, 180, 181, 208

Exospore, 180, 181

Eyespot, 44, 66

Eyster, 61, 310, 416

F

$F_{1}$ and $F_{2}, 287$

Factor (see also Gene), 287, 290

Factorial theory, 308-312

of sex, 397

Fairbrother, 186

Fairchild, 436

False hybrids, 405, 406, 411

Fankhauser, 154

Farmer, achromatic figure, 151

apogamy, 404

chromosomes and species, 126

meiosis, 283, 440

nucleolus, 58

parthenogenesis, 402, 403

Farnham, 124, 343, 352, 354

Farr, C. H., 167

Farr, W. K., 167, 178

Fats, 27, 88, 102

Fatuoid oats, 276

Faull, 158, 159, 230, 281

Fauré-Fremiet, 30, 51, 82, 84, 86, 93, 195 
Faussek, 51

Federley, 360, 369

Fein, 57

Fels, 57

Ferdinandsen, 416

Ferguson, A. J., 188

Ferguson, M., 206, 235, 239, 342

Fernandes, 342

Fertilization (see also Syngamy), animal, 239-249 cone, 240, 241

historical, 436-439

membrane, 241,247

physiology of, 247

plants, 226-239

Fertilizin, 248

Fetter, 32

Feulgen, 52

Feulgen reaction, 52, 120, 192

Fiber-attachment point (see Spindle-attachment region)

Fibrillar theory of protoplasm, 434

Ficus (rubber plant), 103

Fikry, 120

Fila, 82

Filament sheath, 219

Findlay, 82

Finn, 210, 236

Fischer, A., 55, 192

Fischer, E., 382

Fischer, M., 37

Fish, 378, 381, 393

Fisk, 123

Fission, 189

Fitting, 31, 67, 180, 181, 196

Fitzpatrick, 196, 226, 230, 283

Fixation, achromatic figure, 151, 152

of cell, 5,7

cell-plate, 171

chromosomes, 114, 115, 139, 140, 142

Golgi material, 73

nucleus, 54

plastids, 69

and chondriosomes, 91

and Golgi inaterial, 79-81

protoplasm, 37

Flagellates, 189, 190, 191, 223

Flagellum, 43, 44, 189, 223

Flavon and flavonol pigments, 97

Flax fibers, 176

Fleischer, 386

Flemming, amitosis, 184

cell-division, 439

chondriosomes, 82

chromosome division, 440

chromosomes in man, 379

meiosis, 250

net knots, 56

nucleolus, 58

nucleus, by division only, 432

in heredity, 441

protoplasm, 434

Floridean starch, 62, 65, 100

Florin, 283

Fluorescence, 68, 91

Focke, 406
Fæniculum (angiosperm), 103

Fol, 160, 438-441

Fold-back, 276

Fontana, 432

Forenbacher, 85, 90

Fortuin, 112

Fossil pollen, 180

Fowl, 381, 393

Föyn, 190, 279

Fragaria (angiosperm), 367, 391, 406

Fragmentation of chromosomes, 188, 314-326, 349

Francini, 402, 405

Frandsen, 367

Frank, 188

Franzé, 66, 67

Fraser, 158, 230, 281

Free, 41

Free cell-formation, 173, 174, 201, 430

Frémy, 176

Freundlich, 31, 33

Frew, 164

Frey, 178, 230

Friedrichs, 69, 85, 90

Fries, W., 413

Friesner, 112, 342

Fritillaria (angiosperm), 237, 238, 267

Fritsch, 278

Frog, 23, 169, 239, 242, 410, 438

Frolowa, 129, 344, 378

Frommann, 434

Frost, 193, 320, 343, 356

Fry, 155

Fucoxanthin, 64

Fucus (brown alga), achromatic figure, 159

egg, 35

eyespot, 66

gametes, 196, 199

meiosis, 280

parthenogenesis, 247,402

syngamy, 227,437

Fujimoto, 415

Fukuda, 186

Fukushima, 346, 356

Fuligo (slime mold), 26, 28, 165

Fulton, 64

Funaria (moss), 65, 233, 307, 343, 371, 418

Fundulus (fish), 136

Fungi, cell wall, 177

gametogenesis, 199

meiosis, 281-283

mitosis, 156-158

sex, 386-388

sporogenesis, 199

syngamy, 229-232

Furrowing (cytokinesis), 109, 155

G

Gábor, 188

Gartner, 436

Gagea (angiosperm), 186, 273, 347

Gager, 188, 196, 311

Gaines, 356, 366

Gairdner, 329, 351 
Gaiser, 103, 121

Galactinia (f ungus), 281

Galín, 393

Galanthus (angiosperm), 133, 209, 210

Galeopsis (angiosperm), 372

Gallardo, 160

Galls, 186

Galtonin (angiosperm), 117-119, 133, 139

Gametangia, 229

Gametes, 226, 227, 287

Gametic meiosis, 279

Gametic number, 122

Gametogenesis, 196-223

Gametophyte, 122, 208, 211, 251, 306-308, 343, $382-356,401 £$.

Gamophase, 122

Ganglion cell, 73

Garber, 233

Gardiner, M1., 58

Gardiner, W., 16, 18, 19, 98

Gardner, 192

Gargeanne, 102

Garnier, 93

Gasteria (angiosperm), 129, 130, 139, 260, 264, 265

Gastrodia (orchid), 402

Gatenby, 74, 78, 79, $88,215,218,220$

Gates, catenation, 332

chromosomes and taxonomy, 126, 334

cytokinesis, 167, 168

heredity, 286

heteroploidy, $339,345,347,348$

monoploid sporophyte, 356

mutation, 311

nuclear membrane, 136

nucleoplasmic ratio, 52

plastids, 61

translocation, 337

Gauba, 176

Gaudissart, 87

Gäumann, 196, 226, 231, 283

Gay, 144, 311

Geerts, 334, 346

Gegenbaur, 438

Geitler, $68,190,228,280,386$

Gel, 34

Gelatin, 34, 182

Gelei, 258, 271, 274

Gellhorn, 40

Gemini, 252

Gene string, 144

Gene theory, 144 ff., 308-312

Generative cell, 208, 209

Generative nucleus, 209

Genes, 144, 287, 290

in chromonema, 144

in crosses, 287, 290

in development, 273,398

mutations, 310

nature of, 311,312

rôle in origin of species, 373

Genetic continuity, 439

Genic balance, 309, 394

Genom, 121, 325, 340, 419

Genomeres, 416

Genotype, 290
Gentiana (angiosperm), 168

Geotriton (salamander), 87, 220

Geranium (angiosperm), 308

Gerassimow, 50, 58

Gerhard, 334

Germ cell, 213, 441, 444

Germ-plasm, 441, 442, 444

Germ pore, 150, 208

Germ-track, 443

Germinal selection, 444

Germinal vesicle, 213

Gerould, 426

Gerris (insect), 216, 220

Gershoy, 342

Ghimpu, 164, 342

Giard, 438

Gibbs, 176

Gibson, 396

Gicklhorn, 6, 53

Giglio-Tos, 243

Gill cells, 225

Gilson, 176

Ginkgo (gymnosperm), 71, 207, 234

Girdle wall, 166

Giroud, 82, 84

Gland cell, 75, 84, 154, 1 S3

Glaser, 249

Gleisberg, 176

Globoid, 101

Globulin, 101

Glœocapsa (blue-green alga), 192

Glœotrichia (blue-green alga), 192

Glucose residue units, 178,179

Glucoside, 96

Glutamin, 96

Glutathione, 89

Glycine (angiosperm), 164, 415

Glycogen, 27, 62, 100, 193

Goblet cell, 76, 78

Godetia (anglosperm), 329

Godlewski, 15, 412

Goebel, 236

Goldschmidt, chromidia, 194

gynandromorph, 381

Mendelism, 286

parthenogenesis, 410

sex-determination, $382,394,397,398$

temperature and mutation, 311

Goldsmith, 441

Goldsteın, 50, 51

Golgi, 73

Golgi material, 3, 73-\$1

fixation, 73

remnant, 220

rôle in secretion, $75-78$

in spermiogenesis, 214-217, 219

zone, 77

Gones, 251

Gonium (green alga), 22, 67

Gonomery, 235, 243

Gonotokont, 251

Goodspeed, 1S7, 1S8, 273, 311, 320, 345, 356, 365. 367

Goodwin, 334, 337, 356

Gordon, 381, 393 
Goroschankin, 16, 206

Gortner, 25, 27-29, 33, 41, 64, 93, 97, 176

Gossipium (angiosperm), 342

Gowen, 144, 295, 310, 311, 394

Grafe, 25, 52, 176

Grafting, 17, 156, 344

Graham, 233

Gramineæ, 121

Granata, 268

Granular theory of protoplasm, 434

Grasshopper, 86, 114, 126, 130, 136, 139, 141, $220,254,256,258,265,304,320,329,410$

Grave, 67, 224, 225

Gray, cell form, 9

cleavage, 170

colloids, 33

fertilization, 247

flagella, cilia, 43, 223, 225

nucleolus, 57

plasma membrane, 40,41

Greenwood, 378

Grégoire, chromocenters, 57

chromomeres, 142

chromosomes, 136, 140

meiosis, 256, 266

nuclear reticulum, 55,56

Gregory, 277, 351, 357, 415, 416

Grew, 423

Griffithsia (red alga), 280

Gris, 101

Gross, 53, 279, 343, 411

Grosser, 379

Growth, 7

Growth period in oöcyte, 147, 213, 261

Gruber, 51

Grünberg, 397

Gryllotalpa (mole cricket), $86, \mathbf{8 7}$

Guignard, 66, 199, 204, 210, 236, 437, 440

Guilliermond, ascomycetes, 158

canaliculi, 79

chondriosomes, S0, S2-85, 83, 89, 91, 102

Cyanophyceæ, 192

elaioplast, 66

elements of protoplast, 104

meiosis, 282

nucleolus, 57

plastids, $69,71,80,90$

reticulum, 54

syngamy, 226, 230

vacuole, $93,96,101$

Guinea pig, 220, 222, 376

Gulick, 376

Gums, 97, 100

Gurchot, 169

Gurwitsch, 87, 188, 436

Gutherz, 379

Gutstein, 52

Guttenberg, 158

Guyénot, 295

Guyer, 19, 379, 381, 445

Gwynne-Vaughan, 158, 230, 281, 387

Gymnadenia (angiosperm), 256

Gymnosperms, 206, 234

Gynandromorph, 344, 381, 382
Gynogenesis, 406, 411

Gynospore, 206

II

Haas, 64, 93

Haase-Bessell, 120, 361, 363

Haberlandt, apogamy, 404

apomixis, 409

nucleus, 50,58

parthenogenesis, 402

plasmodesms, 16

plastid, 61

syngamy, 236

wounds, 19

Habrobracon (insect), 382, 395

Haeckel, 285, 441

Haecker, 146, 185, 243, 440

Hamanthus (angiosperm), 133, 135

Hæmoglobin, 30

Homatococcus (green alga), 44

Hagedoorn, 311

Hagerup, 342

Hegiwara, 296

Hair, 179

Håkansson, 329, 334, 335, 341-343, 352

Haldane, 351, 352, 355

Hall, 66, 73, 74, 81, 189, 190, 223

Hamm, 437

Hammarlund, 329

Hammarsten, 25-27

Hance, 320, 381

Hand, 28

Hanna, 230-232, 386

Hannig, 181

Hanson, 187, 188, 311

Hansteen-Cranner, 52, 177

Hanstein, 2, 45, 46, 93, 433

Haplodiplonts, 356

Haploid (see also Monoploid), 122, 339, 410

Haplont, 278, 306

Haplosis, 250, 253, 440

Harder, 196, 387, 419

Hardy, 52, 55

Harlow, 176

Harman, 123

Harms, 213

Harnisch, 121

Harper, algæ, 193, 414

cell constitution, 4

centrosome, 158

cleavage furrow, 165

meiosis, 282

mitosis, 158, 190

plastids, $66,72,90$

protoplasm, 31

syngamy, 230

Harrison, J. W. H., 347, 365, 367, 413

Harrison, R. G., 6

Harrison, W., 99

Hartig, 101

Hartmann, A., 187

Hartmann, M., apomixis, 401

heredity, 286 
Hartmann, M., karyosome, 189

meiosis in algæ, 279

mitosis, 190

motile apparatus, 198, 223, 224

on Protista, 376

pyrenoid, 68

sex in lower plants, 226,387

syngamy in Protozoa, 245

Hartmann, O., 61

Hartog, 160

Harvey, E. N., 188

Harvey, L., 88, 121, 425

Hatschek, 33

Haupt, 191, 192, 201, 233, 280

Haustoria, 206, 207

II aworthia (angiosperm), 129, 130

Hayes, 355

Hazen, 68

Headed chromosome, 116

Heartwood, 177

Heberer, 136

Hedayetullah, 133, 137, 334

Hegler, 192

Hegner, 51, 213

Heidenhain, achromatic figure, 153

animal cells, 13

cell theory, 20

chondriosomes, 87

cilia, 43,223

connective tissue, 16

intercellular substance, 182

living cells, 3

metaplasm, 45

mitosis, mechanism, 160

nuclear structure, 55

organismal theory, 24,436

protoplasmic structure, 46

Heidinger, 197

Heilborn, 126, 341

Heilbronn, 31

Heilbrunn, 25, 31, 32, 40, 164, 170, 188, 242, 248

Heimans, 125, 210, 236

Heitz, chromosome morphology, 116, 118, 129

function of nucleus, 58

heteroploidy, $342,343,395$

heteropyknosis, 146, 386

meiosis, 283

nucleolus, 57, 120, 121

plastids, 69

sex-chromosomes, $\mathbf{3 8 4}, \mathbf{3 8 6}, 390$

Heitzmann, 436

Hlelix (snail), 80, 272

Helleborex, 128

Helleborus (angiosperm), 86

Helvella (fungus), 281

Helvestine, 184, 225

Helwig, 115, 320, 329

Hlemerocallis (angiosperm), 209, 210, 354

Hemicellulose, 100, 176

Henckel, 65, 100

Henderson, 28

Henking, 376, 411, 440

Henle, 431

Henneguy, 225

Heptaploid, 339
Herbst, 441

Heredity, algæ, 306

cytology and, $284 f f ., 441$

defined, 285

fungi, 387

heteroploids, $350 \not f$.

Mendelian, $284 f f$.

nucleus in, 285

physical basis of, $286 f$.

Weismann's theory of, 441

Hering, 20

Herla, 146

Herlant, 247, 248

Hermann, 160, 397

Herrick, 222

Hertwig, G., 245, 410, 412

Hertwig, O., heredity, 444

meiosis, 263

nucleus in heredity, 2S5, 439, 441

organismal theory, 436

parthenogenesis, 411,412

syngamy, 438

Hertwig, P., 401, 410, 412

Hertwig, R., 51, 58, 194, 277

van Herwerden, 27, 51, 194, 195, 220

Herzfeld, 206, 234

Herzog, 178, 179

Heterobrachial chromosomes, 116

Heteromorphic pairs of chromosomes, 265, 271, 303,388

Ifeteronema (flagellate), 190

Heterophytic angiosperms, 388

Heteroploidy, 339 ff. and sex, 394

Heteropyknosis, 56, 146, 385

Heterospory, 206

Heterothallism, 232, 387

Heterotypic mitosis, 250

Heterozygous, 290

Heuser, 440

Hexaploidy, 339

Hexose, 27, 30

Heys, 188, 213, 311

Hibbard, 78

Hick, 16

Hicks, 341

Hicoria (angiosperm), 238

Hieracium (angiosperm), 346, 347, 364, 405, 406, 407,415

Higgins, 190, 279, 280

Hill, H. E., 298, 354

Hill, S. E., 342

Hill, T. G., 14, 16, 64, 93

Hiller, 29

Hilum, 98

Hirase, 207, 234

Hirata, 346

Hirschler, 78, 194, 195, 215

Histon, 27

History of cytology, 422-447

Hoar, 329

Höber, 40

Hobson, 242

Hoefer, 196

Hoeppener, 337 
Hof, 12

Hoff man, 342

IIöfler, 39

II of meister, $23,430-\mathbf{4 3 9}, \mathbf{4 3 1}$

Hogben, 272, 411

Holden, 282

Hollaender, 188

Hollingshead, 124, 128, 343, 356, 357, 361, 367, 372

Holman, 196

Holmgren, I., 348, 402, 408

Holt, 344

Homarus (lobster), 222

HIomès, 98

Homœetypic mitosis, 250

Homology of chromosomes, 252, 272

Homoploid, 339

Homospory, 206

Homozygous, 290

Hlooke, 2, 423

Hoppe, 163, 410

Hoppe-Seyler, 26

Hopwood, 187, 317

Hormones, 19

Horne, 190

Horning, 85, 89, 90

IIorse, 376

Hosono, 121

Hosta (angiosperm), 139, 416, 417

Hoven, 87, 88

Howard, 51, 97, 187

Huates, 133

II uettner, 24, 155, 243, 295, 382

Hughes-Schrader, 410

II umaria (fungus), 281

Hume, 16

Itumulus (angiosperm), 390, 396, 402, 415

Hurst, 340, 364, 365

Huskins, 133, 139, 258, 261, 267, 275, 341, 343, 366,367

Hutchinson, 235

IIuth, 163

Huxley, J. S., 22

Huxley, T. H., 45, 249, 435

Ilyacinthus (angiosperm), aberrant mitosis, 186 achromatic figure, 150

cell-plate, 17I

chondriosomes, 91

heteroploidy, 345

meiosis, 258, 267, 274, 304

in heteroploids, 351-354

plastids, 62

Hyaloplasm, 31, 32

Hyalotheca (green alga), 63

Hybridization, $361 f f ., 408$

Hybrids, 287, 348, 359-374

amphidiploid, 367-37I

false, $405,406,411$

permanent, 338

structural, 336

twin, 338

Hydatina (rotifer), 399

IIydnobolites (fungus), 281

Hydnotrya (ascomycete), 181

Hydra (coelenterate), 9, 213
Hydrodictyon (green alga), 63, 165, 414

Hydrogen-ion concentration, 5, 29, 84, 91, 97, 139

Hyperdiploidy, 322, 323

Hypericum (angiosperm), 329, 416

Hyperploidy, 339

Hypoploidy, 339

\section{I}

Icerya (insect), $\mathbf{4 1 0}$

Ichijima, 367

Ichikawa, 187

Id, 442

Idiochromatin, 194

Idiogram, 128

Idioplasm, 441

Idiosome, 216, 219

Ikari, 68

Ikeda, 78

Ikeno, 201, 202, 207, 208, 234

Illick, 334

Imai, 296

Impatiens (angiosperm), 117

Inactivation, 188,317

Inariyama, 139

Independence of factor pairs, 290

Individuality, of chromosome, $145 \mathrm{ft}$.

of plastid, $70 \mathrm{ft}$.

of vacuole, $94 \not 5$.

Ingvar, 20

Inheritance, $284 \mathrm{ff}$.

Insectivorous plants, 98

Insertion of spindle fibers, $115 \mathrm{ff}$.

Intercellular substance, 4, 182

Interkinesis, 263, 264

Interlocked tetrads, 271

Interphase, 109, 134

Intersex, 394, 398

Intine, 180, 208

Intussusception, 178

Inulin, 103

Inversion of chromosomes, 188, 276, 315, 318 , 319,320

I pomøa (angiosperm), 180

Iriki, 378

Iris (angiosperm), 79, 133, 171, 173

Irvine, 178

Ishikawa, 68, 210, 236, 347

Isobrachial chromosomes, 116

Isoelectric point, 29

Isoga my, 227

Isogenomatic, 340

Isomerism, 30

Ives, 311

Jackson, 231, 283

Jacobs, 40, 73, 77

Jacobsson-Stiasny, 239

Jahn, 66, 190, 278

Jakushkina, 342

Jameson, 277

Jancke, 178, 179

Janet, 278 
Janssen, 423

Janssens, cell-plate, 169

chiasmatypy, 266,270

chromosomes, 132, 141

meiosis, 258, 272

recombination, 302, 304

Jansson, 186

Janus green, 84, 89, 90

Jaretsky, 88, 111, 342, 343, 357

Jasswoin, 77

Jeffrey, 103, 348

Jelly, 34

Jenkins, 366

Jennings, 47, 191, 245, 246, 277, 312

Johansen, 342

Johanssen, 286

Johnson, D. S., 212

Johnson, H. H., 155, 215, 218, 220

Johnson, L. N., 66

Jollos, 311

Jones, D. F., 286, 293, 398

Jones, S. G., 230

Jönsson, 16

Jordan, 225

Jorgensen, C., 186, 344, 356, 367, 406

Jörgensen, I., 64

Jörgensen, M., 57, 58, 156, 194, 195

Joyet-Lavergne, 89,398

Juel, 282, 402

Juglans (walnut, butternut), 101

Jungers, 16, 17, 161, 171, 173

Juniperus (juniper), 235

Jüssen, 405

J ust, 169, 18s, 239, 242, 243, 247, 249, 347

Juul, 187

K

Kabsch, 100, 176

Kachidze, 129

Kagawa, 129, 342, 346, 366, 367

Kahle, 411

liangaroo, 139

Karling, 279

Karpechenko, 273, 342, 364, 367, 368, 370, 371

Karper, 415

Karpova, 74

Karsten, 112, 196, 228, 279, 280

Kartaschowa, 129

Karyogamy, 231

Karyokinesis, $105 \not f ., 439$

Karyolymph, 2, 53, 135, 136, 149

Karyolysis, 187

Karyomeres, 136, 146

Karyoplasm, 2

Karyoplasmic ratio, 51

Karyosome, 56, 164, 189, 190, 223

Karyotin, 2, 55

Karyotype, 126

Kassmann, 69, \$5, 90

Katayama, 366

Kater, 68, 78, 88, 183, 190, 223

Katsuki, 382

Kauff mann, H., 279

Kaufmann, B. P., 133, 136, 137, 139, 140, 256
Kawakami, 121

Keene, 229

Keimbahn, 443

Kellicott, 112

Kellogg, 46, 441

Kemp, 185, 187, 379

Kempton, 407

Kendall, 186

Keratin, 177, 182

Kerr, 29

Keuten, 189

Kidney, 77

Kienitz-Gerloff, 16

Kiernan, 289

Kiesel, 25, 26

Kieser, 426

Kihara, asynapsis, 273

chromosome complement, 121, 129

grains, 366

heteroploidy, $340,342,395$

human chromosomes, 379

Enothera, 334

sex-chromosomes, $388,390,391,395$

Kildahl, 235

Kindred, 225

Kinetic bodies, 116, 117

Kinetic constriction, 116

Kinetochore, 116

Kinetonucleus, 224

Kinetosomes, 203

King, R. L., 78, 115, 378

King, S. D., 73, 78

Kingery, 84

Kingsbury, 82, 88

Kinney, 58

Kinoplasm, 44, 165

Krinoplasmic plates, 203

Kirby, 90

Kirchensteins, 193

Kisliak-Statkewitsch, 188

Irisser, 53, 184

Kite, 6, 39, 52-54

Kriyohara, 69, 80

Klebahn, 227, 228, 280

Kilebs, 58, 66, 165, 386

Klein, 159, 434

Klingstedt, 53

Kniep, 230-232, 281, 282, 386, 387

Knight, 278, 280

Knob on chromosome, 119, 123, 143, 271, 298, $303,318,332$

Koch, 58

Koelreuter, 436

Koerperich, 136, 140, 149, 164

Kofoid, 10, 183, 190, 191, 195, 223, 245

KohI, 16, 192

Kojima, 112

Kolatchev, 74

von Kölliker, 431, 438, 441

Koltzoff, 221, 222

Komuro, 187

Kondo, 415

Konopacki, 88, 164

Koos, 186, 344, 356

Kopsch, 73, 74 
Kornhauser, 266, 441

Korotneff, 86

Iiorschelt, 51

Koshuchow, 186

Kossel, 26, 30, 58

Kostanecki, 240, 411

Kostoff, 186, 407

Kostrioukoff, 210

Kowalevsky, 440

Kowalski, 14

Loyama, 385

Krabbe, 177

Kramer, 100

Ǩränzlin, 190, 278

Kretschmer, 190, 193

Iŕropp, 89

Kruch, 437

Krüger, 412

Kripko, 90

Kuckuck, 63

Kuczynski, 224

Kuhla, 16

Kühn, A., 411

Kuhn, E., 56, 57, 118, 340, 343, 391, 406

líulkarni, 334, 356

liulp, 30

Kunieda, 280

liupelwieser, 412

Kupper, 419

Liurssanow, 72, 279

Kusano, 402

Kiüster, $6,53,62,66,98,415$

Kuwada, chromomeres, 142

chromonemata, 139,140

chromosome arrangement, 125

hybrids, 360

nucleolus, 57

restitution nucleus, 340

spermatogenesis, 207

syngamy, 234, 236

Zea chromosomes, 123

Kylin, apomixis, 402

eyespot, 66

Floridean starch, 65, 100

gametes, 199

meiosis, 278, 280

L

Laboulbenia (f ungus), 281

Laburu, 79

Lachnea (fungus), 281

Lagging chromosomes, 345, 355, 361-363, 364

Laguesse, 87

Laibach, 57

Lamarck, 426

Lammerts, 367, 407

Lampe, 99, 100

Lampsilis (mollusk), 224, 225

Lams, 244

Lancefield, 296, 378

Land, 174, 210, 235

Landis, 49

Lang, 405

Langlet, $129,340,342,343,346$
Larix (conifer), 86, 151, 162, 171, 236

Larval characters, 419

Lastrca (fern), 403,405

Latex, 97

Lathyrus (angiosperm), 308

Latter, 58

Laughlin, 111

Lauterborn, 228

La Valette St. George, 438

Lawrence, 351, 355

Lawson, 206, 234, 235

Lawton, 405

League, 295

Lebedeff, 368

Lebistes (fish), 378, 381, 393

Lecanium (insect), 411

Lecithin, 27

Lee, A. B., 73, 251

Lee, B., 176

Lee, S., 192

Leeman, 102

van Leeuwenhoek, 424, 437

Leguminosæ, 121, 129

Lehfeldt, 230

Lehmann, 334, 338, 419

Leighty, 367

Leliveld, 334

Lenhossék, 14, 225

Lenoir, 58, 256

Lenzites (fungus), 388

Lepeschkin, 26, 33, 40

Lepidosiren (fish), 272

Lepisma (nematode), 220

Leptolegnia (fungus), 200

Leptonema (brown alga), 63

Leptonema, 256, 257

Lesley, J. W., 320

Lesley, M. M., 274, 320, 343, 346, 352, 356

Lethal factors, 293, 336, 338

Leucoplast, 62, 64, 65, 69, 90

Levan, 124, 267

Levi, 87

Levine, 187, 188, 282

Lewis, C. E., 201

Lewis, G. N., 311

Lewis, I. F., 280

Lewis, M. R., 187

Lewis, M. and W., achromatic figure, 161 chondriosomes, 84, 85, 87

neurofibrils, 13

nuclear fragmentation, 184

nucleus, 53

tissue cultures, 6,111

Lewitsky, amphidiploidy, 367

chondriosomes, $82,84,86,90$

chromosomes, 114-116, 121, 124

and taxonomy, 126, 128, 129

irradiation, 187

plastids, $69,71,90$

translocation, 321

Leydig, 434

Libocedrus (conifer), 235

Life cycles, 383

Ligniera (myxomycete), 190

Lignin, 100, 176 
Liliaceæ, 342

Lilienfeld, 26, 366

Lilium (lily), canaliculi, 79 chondriosomes, 71, 83 chromonema, 139, 142 homoploidy, 342

megaspore nuclei, 212 meiosis, 256, 258, 267 nucleus, 50 syngamy, 236, 237, 238

Lillie, F. R., fertilization, 239, 240, 242, 247-249, 438

sex-determination, 397,328

spermatozoön, 222

Lillie, R. S., activation of egg, 247, 248

chromosome arrangement, 125

differentiation, 11

electrical phenomena, 52, 160

oxidation, 59

plasma membrane, 40

polarity, 20

protoplasmic structure, $31,37,38$

specificity of protoplasm, 30

Limosphere, 71, S1, 202-204

Limosphere remnant, 203, 204

Lind, 279

Lindegren, 194, 282, 387

Lindenbein, 279

Lindfors, 282

Lindsay, 388

Lindstrom, $186,344,356$

Linin, 55

Link, G. K. K., 415

Link, H. F., 426

Linkage, 293-296, 298-306

groups, 296

map, 299, 301

Linum (angiosperm), 419

Lipide, 140

Lipide theory, 40

de Litardière, alveolation theory, 140

chromomeres, 142

heteroploidy, 343,346

matrix, 136

mitosis, 138

nucleolus, 58

reticulum, 54,55

Liu, 74

Liver, 77, 88

Liverworts, 352

Lizard, 376

Ljungdahl, 347, 363

Jloyd, 41, 43-45, 63, 64, 68, 103, 192, 228

Lockwood, 187

Locy, 439

Loeb, J., 59, 247, 441

Loeb, L., 6

Loefer, 190

Logan, 436

Liihnis, 90, 193

Longley, 100, 123, 308, 341, 358, 360, 407

Loos, 188

Lophius (fish), 15

Lorbeer, 283, 382, 384, 395

Lotsy, 251, 335
Loui, 90,91

Lowe, 63

Löwschin, 84, 85, 90

Loyez, 88

Lubimenko, 64

Lubosch, $5 \mathrm{~S}$

Lucas, 139, 223, 225

Ludford, 57, 58, 74, 77, 187

Lüdtke, 101

Lund, 20

Lundegårdh, chondriosomes, 82, 90

chromomeres, 142

chromosomes, 140

cilia, 43, 223

colloids, 33

karyotin, 55

plasma membrane, 40

plasmodesms, 16

protoplasm, 25, 31

reticulum, 54, 55

vacuoles, 93

Lupinus (angiosperm), 71

Luther, 222

Lut kov, 361

Lutman, 166, 231

Lutz, 320, 334, 339, 345

Lychnis (angiosperm), 390, 392

Lycogala (myxomycete), 26

Lycopersicum (angiosperm), 186, 274, 343, 346, 356

Lycopodium (pteridophyte), 204

Lygodium (fern), 229, 233

Lynch, 58, 59

Lyngbya (blue-green alga), 192

Jynn, 398

Lyon, 31, 180, 181

Lyophilic, 34

Lyophobic, 34

M

Ma, R., 69

Ma, W., 74

Macallum, 58

MacArthur, 310

MacDaniels, 12, 175

MacDougal, 27

MacDougalI, 49, 277

Macfarlane, 18

MacGillivray, 100, 293, 308

Mäckel, 402

Macormick, 229

Macronucleus, 10, 49, 194, 245, 246

Macrosomes, 32

Mactra (mollusk), 411

Madge, 210, 236

Maeda, 124, 139, 207, 267, 270

Magistris, 52

Magnets, 125

Magnolia (angiosperm), 168

Magrou, 188

Maige, 51

Maire, 158, 281, 282

Maize (see Zea)

Makino, 121 
Male cells and nuclei, 208, 209, 210, 234, 235-237 Malone, 376

Malpighi, 423, 424

Malsen, 399

Malte, 55

Mameli, 177

Mammals, 376,378

Man, centrosome, 154 chromosomes, 379

colorblindness, 392

Mangelsdorf, 293

Mangenot, canaliculi, 79

chondriosomes, 82, 83, 90, 91

eyespot, 66

Floridean starch, 100

nucleolus, 57

oil, 102

plasmodesms, 16

plastids, 65

vacuoles, 97, 98

Mangin, 176

Mann, 71, 124, 356, 363

Manoilov, 398

Mantidæe, 378

Mantle of cytoplasm, 235

Manton, 342, 405

Marchal, 386, 405

Marchand, 15

Marchantia (liverwort), 201, 202, 427

Marcus, 51, 194

Maréchal, 142, 256, 266

Margolena, 52

Mark, E. L., 87, 410, 438

Mark, H., 178

Marsden-Jones, 367

Marshak, 124

Marsilia (water fern), apomixis, 402, 403, 407 spermatogenesis, 205

spermatozoid, 199

spore coat, 181

Marsupials, 378

Martens, cell-plate, 171

chromonema, 139

chromosomes, 119

fixation, 7,54

matrix, 136

micromanipulation, 6

nucleolus, 58

somatic mitosis, 111

spindle, 161, 163

Martins-Mano, 55, 142

Marwick, 178

Maschka, 10I

Masdevallia (orchid), is

Mason, 129

Massee, 16

Mast, 43,67

Masui, 376

Maternal inheritance, $415 \mathrm{ff}$.

Mather, 436

Mathews, A. C., 200

Mathews, A. H., 20, 25, 243

Mathias, 280

Matrix, 106, 119, 133, 134-137, 140, 143, 144, 260

Matsumoto, 13
Matsuura, 286

Matthiola (angiosperm), 267, 320, 343, 356

Maturation divisions, 214, 239, 250

Maupas, 245

Naurer, 17

Mavor, 187, 302, 393

May, 89

Mayer, 89

Mayer, Rathery, and Schaeffer, 89

Maziarski, 58

McAllister, 68, 166, 212

McClendon, 169

MfcClintock, anastomoses, 120, I35

asynapsis, 273

chromosome matrix, 120

chromosome morphology, 115, 118, 119, 123, 298

crossing-over, $303,305,337$

deletion, 318, 319

fragmentation, 317

heteroploidy, $343,346,347$

inversion, 318, 319

linkage groups, 298

meiosis, 259, 261, 265, 270, 302

non-homologous association, 276

nucleolus, 118, 119, 120

reciprocal translocation, $329,330,332,333$

synapsis, 274

triploidy, 352, 353, 354

MeClung, chiasma, 268

chromosome individuality, 115, 141

chromosomes and taxonomy, 123, 126, 129, 130,342

linkage and chromosomes, 297

meiosis, 256, 265

reticulum, 55

sex-chromosomes, 376

McCubbin, 230

AlcDonald, 10, 223

MeKay, 240

McPhee, 397

Mead, 154

Mecostethus (insect), 129, 258, 265, 342

Meek, 126

Megaspore, 180, 206, 210, 211

Megasporocyte, 210

Mehta, 58

Meiocyte, 251, 282

Meiosis, 250-283

algæ, 278

and apomixis, 407

gametic, 279

historical, 440

in hybrids, $360 \not f$.

in living cells, 256

and Mendelian segregation, 291-293

in monoploid sporophyte, 356

outline of, 252, 253

in plant groups, $278 \mathrm{ff}$.

in polyploids, $350 \mathrm{ff}$.

in Protozoa, 277

summarized, 255

theory of, 275

Melandrium (angiosperm), 236, 388, 389, 390, $392,397,415$ 
Míelburn, 366

Melilotus (angiosperm), 168

Melin, 283

Mellon, 194

Membranellæ, 43

Mendelism, 284-313

in algæ, 306

and chromosomes, $284 \not f ., 445$

rediscovery, $\mathbf{4 4 5}$

Menoidium (flagellate), 190, 223

Mercerization, 179

Mercurialis (angiosperm), 388

Meristem, 11, 12, 94, 96

Merogony, 407, 412

Merokinesis, 146

Mesembryanthemum (angiosperm), 415

Mesoderm, 17

Mesospore, 180

Metabolic gradient, 19

Metabolic nucleus, 105, 106, 134, 136, 147

Metabolic rate, 398

Metabolism and genes, 309

and sex, 398

Metachromatic corpuscles, 101

Metachromatin, 97, 101, 193

Metachrome, 95

Metaphase, 106, 107, 108, 134, 137

Metaplasm, $45 \not f ., 93$

Metapodius (insect), 379

Metaxylem, 175

Metcalf, 191

Metopsilus (insect), 360

Metz, chromosome morphology, 123

chromosomes and species, 129

haploidy, 410

linkage, 296

meiosis, 272, 295

monocentric mitosis, 163

sex-chromosomes, 378

somatic pairing, 126

spermatogenesis, 215

Metzner, 43

Meurman, 329, 335, 341, 355, 389, 390

Meves, centrioles in sperm and oöcyte, 155, 217, 218

chondriosomes, $\$ 2,83,90$

chondriosomes and fertilization, 244

differentiation theory, 87

parthenogenesis, 410

spermatozoid, 199

spermatozoön, 222

Meyen, 430

Meyer, A., bacteria, 193

chondriosomes, $82,85,88$

colloidal state, 33

elements of protoplast, 104

ergastic substances, $30,93,94,96,101$

intercellular substance, 182

nucleolus, 57,58

pigment, 97

plasmodesms, 16

plastid, $61,63,65,67,70,90,91$

protoplasm, $25 f f ., 31,45,46$

starch, 98-100

Meyer, K., 233
Meycr, K. H., 178

Mleyers, 100

Miastor (fly), 411

Micellæ, 33, 99, 162, 177-179

Michaelis, 186, 342, 419

Microcentrum, 153

Micromanipulation, $6,52,53,142,161,184,446$

Micromeric theories of protoplasm, 46

Micronucleus, 10, 49, 194, 245, 246

Microscope, 423

Microsome, 92, 178

Microspore, 206, 208, 209

of diatoms, 228

Microspore quartet, 167

Microsporocyte, achromatic figure, 151, 152

cytokinesis, 167

meiosis, 209, 364

Microsporogenesis, 85

Mid-body, 155, 158, 169

Middle lamella, 171, 174

Middle piece, 217, 218, 221, 222, 240

Miescher, 26, 30

Millardet, 406

Miller, 436

Milovidov, 82, 84, 89, 90, 190

Mimosa (angiosperm), 18

Minchin, 43, 44, 189, 223, 277, 278

Minenkov, 398

Minot, 51

Minouchi, 378

Minoura, 397

Mirabilis (angiosperm), 289, 415, 416

Mirande, 82, 103

Mirbel, 426, 427

Mitchell, 178

Mitochondria (see also Chondriosomes), 82

Mitogenetic rays, 188

Mitokinetism, 160

Mitome and paramitome, 434

Mitosis, aberrant, 184-1S8, 186

duration and periodicity, 111, 112

historical, 439

mechanism of, 159-164

monocentric, 163

premeiotic, 272

in Protozoa, 189

semiheteroty pic, 346

somatic, 105-113, 110, 112

Mitosome (Gatenby), 218

Miyake, 57, 199, 200, 201, 207, 235

Mnium (moss), 405

Möbius, 61, 64, 97

Mockeridge, 192

Modilewski, 212, 407

Moenkhaus, 146

Moffett, 351

von Mohl, 94, 177, 430, 432

Mohr, 188

Moissejewa, 188

de $\mathbf{M o l}, 186,187,342,345,357$

Moldenhawer, 426

Molge (amphibian), 76

Molisch, 17, 93, 176, 177

Mols, 376

Monkey, 378, 379 
Monoblepharis (fungus), 199, 229

Monocentric mitosis, 163

Monoploid, 121

Monoploid plants, Mendelism, 306

Monoploid sporophytes, 356, 402

Monotropa (angiosperm), 59, 237, 238

Montgomery, chondriosomes, 86

chromosome individuality, $\mathbf{4 4 5}$

meiosis, 266, 272

sucleolus, 57

sex-chromosomes, 376,379

Moore, A. C., 151, 283

Moore, B., 25

Moore, E. L., 246

Moore, J. A., 116

Moore, J. E. S., 379

Moreau, 85, 90, 165, 229

Morelle, 77, 85

Mlorgan, C. L., 47

Morgan, L. V., 306, 381

Morgan, T. H., activation, 249

chromosome complement, $\mathbf{3 4 4}$

chromosome set, 123

crossing-over, 266

cytasters, 154, 248

cytoplasmic inheritance, 420

factorial hypothesis, 309

fragmentation, 320

gene theory, 312

genes, 144

lethal factors, 293

linkage, 294, 296, 301, 392

Mendelism, 256, 373

mutations, 311

parthenogenesis, 410-412

sex-chromosomes, 378,393

sex determination, 381,382

syngamy, 243, 245

Morinaga, 342, 356, 365

Moroff, 194

Morphallaxis, 9

Morrill, 376

Morris, 146

Morus (mulberгy), 390, 396

Mosaic endosperm, 239

Moses, 129, 163, 410

Mosses, cytoplasmic inheritance, 418

heredity in, 307

heteroploid, 357

hybrids, 371,418

Moths, 368, 398

Motile structures, 14

Motte, 69, 70, 90, 196, 201, 283

Mottier, achromatic figure, $\mathbf{1 5 9}$

aleurone, 101

centrosomes, 159

chromomeres, 142

eytokinesis, 173

male cells, 210

meiosis, 262, 280

nucleolus, 57

plastids, 69, 90, 91

sex, 386

Mougeotia (green alga), 68, 190

de Moulin, 13
Mounce, 230

Mouse, 378

Mucilage, 100, 102

Mucor (fungus), 8, 10, 50, 200, 201

Mühldorf, 201, 203

Müller, H. A. C., 124, 142

Muller, H. J., lethal factors, 293

linkage, 296

mutation, 310,311

reciprocal translocation, 329

sex-chromosomes, 393,395

somatic pairing, 123

tetraploid ratios, 352

translocation, 316, 318, 322-325

X-radiation, 187, 302, 311, 316

Müller, O., 228

Mulsow, 376

Multiple allelomorphs, 310

Multivalent chromosomes, 304, 355

Mundie, 190, 197, 279

Müntzing, $340,343,372,396,407$

Murbeck, 402, 404, 405

Murgantia (insect), 219

Murphy, 199

Murray, 74

Murrill, 235

Musa (angiosperm), 345

MI uscari (angiosperm), 128

Muscle, 14, 18

Mutation, 188, 310, 338

Myers, 280

Myofibril, 15, 87, 88

Myoneme, 44

Myristica (angiosperm), 103

Mytilus (mollusk), 225, 412

Myxomycete, chondriosomes, 86

cytokinesis, 165

glycogen, 100

meiosis, 278

mitosis, 190

protoplasm, 26, 32, 39

surface-volume ratio, 8

N

Nachtsheim, 410

Nadson, 186, 187

Nagai, 386

Nagao, 352

von Nägeli, cell-division, 430,431

cell wall, 177

idioplasm theory, 441

importance of protoplasm, 433

starch grain, 99, 100

vacuole, 94

Najas (angiosperm), 117, 124

Nakahara, 5s, 183

Nakanishi, 194

Nangia, 74, 78

Narcissus (angiosperm), 133, 139, 352, 354, 357

Nassonow, 76, 77, 90

Nasturtium (angiosperm), 64

Nath, 58, 73, 74, 78, 82, 88, 215

Nathansohn, 166, 185

Naville, 295 
Nawaschin, M., amphidiploidy, 367

chromosome morphology, 115, 121, 124, 127, 128

dislocation hypothesis, 347

fragmentation, 320,349

heteroploidy, 343,349

hybrids, 361,372

translocation, 314, 321, 325

$\mathrm{X}$-radiation, 187

Nawaschin, S., chromosome morphology, 114$119,128,446$

fertilization, $236,238,437$

male nuclei, 210

Sorodiscus, 190

Nebel, 133, 137, 139, 256, 260, 261, 265, 329, 335

Nebenkern, 88, 90, 216, 218, 219

Nebennucleoli, 56

Neck canal cells, 201

Nelumbo (angiosperm), 167

Nemalion (red alga), 280

Nematodes, 376

Nematus (saw-fly), 411

Nèmec, altered chromosomes, 186

chloralized cells, 185

dispermy, 347

mitosis, 191

nucleolus, 57

nucleus, $52,53,55$

spindle, 161, 164

spore wall, 181

Nephrodium (fern), 86, 233, 403, 404

Nereis (annelid worm), 221, 222, 238, 240, $242-244,247$

Nerve cells, $13,14,83$

von Neuenstein, 48

Neurofibrils, 13, 87

Neuromotor apparatus, 10, 19

Neurospora (fungus), 282, 387

Neuroterus (gall-fly), 411

Newport, 438

Newton, D., 386

Newton, W. C. F., 258, 267, 268, 274, 346, $351,352,355,367$

Nichols, G. E., 206, 235

Nichols, S., 31

Nicholson, 89

Nicolosi-Roncati, 71,82

Nicotiana (angiosperm), amphidiploidy, 367 androgenesis, 407

chimera, $\mathbf{3 4 3}$

chromosome morphology, 124

cytokinesis, $\mathbf{1 6 7}$

cytoplasmic heredity, 419

digluta, 367

fragmentation, 320

haploidy, 356, 402

non-disjunction, 345

pollen characters, 308

tabacum, 365, 368

temperature effect, 186

$\mathrm{X}$-radiation, 273

Nienburg, 230

Nieschulz, 223

Nigella (angiosperm), 129

Nigritella (angiosperm), 405
Nikolaewa, 342

Nishimura, 199

Nishiyama, 293, 343, 366

Nissl substance, 13

Nitella (stonewort), 97, 279

Nitophyllum (red alga), 280

Nitzschia (diatom), 356

Noack, 69, 90, 417

Nodules of legumes, 90

Noll, 22

Nöller, 224

Non-conjugation, 273

Non-disjunction, $338,344,345,393$

Non-division, 345,346

Non-homologous association of chromosomes, 276, 316

Nonidez, 272

Non-Mendelian inheritance, $415 f f$.

Norén, 235

Nostoc (blue-green alga), 192

Nothnagel, 153, 236, 237, 238

Nowikoff, 194

Nucellar embryo, 404, 405

Nuclear budding, 183

Nuclear fragmentation, 183

Nuclear membrane, 2, 136

Nuclear reticulum, 53, 105, 134, 136

Nucleic acid, 26, 27, 29

Nuclein, 26, 27

Nucleolo-centrosome, 189

Nucleolus, 2-59 ff., 118-121, 119, 164

Nucleome, 49

Nucleoplasm, 2

Nucleoplasmic ratio, 8,51

Nucleo-proteins, 26, 140

Nucleus, 48-60

of bacteria, 193, 194

cell constituent, 2

chemical nature, 52

discovery, 427

division, $105 \mathrm{ff}$

evolution of, 193

fusion and heteroploidy, 346

in heredity, 2s5, 441

metabolic or resting stage, 59

migration of, 403, 404

origin by division, 432

oxidation, 59

in Protista, 189 ff.

restitution, 346

size and chromosome number, 51

structure, $53 \mathrm{ff}$.

synthesis, $58 f f$.

Nulliplex, 354

Nurse cells, 213

Nussbaum, 74, 194, 195

Nymphøa (water lily), 83

$\mathrm{O}$

Oakley, 187,317

Obersteiner, 14

Ochna (angiosperm), 402, 405

Octoploidy, 339

Ecanthus (cricket), 218 
Edogonium (green alga), blepharoplast, 198 gametes, 196, 199

meiosis, 279

mitosis, 190

pyrenoids, 63

syngamy, 227, 437

zoöspores, 198

(Ehlkers, 279, 334, 337

Einothera (angiosperm), 334-338

abnormal mitosis, 186

apomixis, 409

cytogenetic composition, 335

fragmentation of chromosomes, 320

gigas mutant, 339

heteroploidy, $339,343,346,347$

meiosis, 273, 334

monoploid sporophyte, 356

non-disjunction, 345

parthenogenesis, 402

pollen characters, 210,308

recombination, 304

Renner complexes, 335, 336

semisterility, 332

structural hybridity, 336

syngamy, 236, 238

tetraploid, 357

triploid, 352, 358

twin hybrids, 338

variegation, 416

Oes, 55

Ogata, 56

Oguma, 121, 378, 379, 381

Ohashi, 190

Oil, 102

body, 102

droplet division, 169

Okabe, 280

Oleosome, 66

Olitsky, 89

Olive, 190, 192, 278

Oliver, 18, 311

Olpidiopsis (f ungus), 165

Olson, 187, 311

Oltmanns, 11, 64, 165, 196, 197, 226, 227, 278

One-plane theory of chiasma, 268, 269, 271

One-sided inheritance, 391

Ono, 340, 342, 390, 395

Onoclea (fern), 199, 233

Onslow, 25, 64, 93, 97, 100

Ontogenesis (see Development)

Oöcytes or ovocytes, 213, 238

Oögenesis, in animals, 213, 214

in plants, 196, 197, 200, 201, 206, 210, 211

Oögonia or ovogonia, 213

Oögonium (of plants), 197

Oömycetes, 199, 229, 251

Opercularia (infusoria), 277

Ophiocephalus (fish), 78

Opisthacanthus (scorpion), 86

Opossum, 378, 379

Orchidaceæ, 342

Orchis (orchid), 62

Organelle, 10

Organic acids, 96

Organismal theory, 20 ff., 435
Orman, 85

Ornithogalum (angiosperm), 66

Orobanche (angiosperm), 210

Oryza (angiosperm), 30s, 356, 415

Osawa, 402, 405

Oscillatoria (blue-green alga), 192

Osmiophilic platelets, 71,81

Osmosis, 42

Osmunda (fern), 93, 405

Ostenfeld, 402, 408

Osterhout, 40, 59

Ostwald, 33

Otidea (f ungus), 281

van Overeem, 358

Overton, E., $16,40,66,440$

Overton, J. B., chromocenters, 57

life cycle, 196

meiosis, 256, 266

parthenogenesis, 247,402

somatic mitosis, 140

Ovule, 210, 211

Ovum, 213

Owens, 80

Oxidase, 220

Oxidation, and chondriosomes, 88,89

and nucleus, 59

in respiration, 64

Oxychromatin, 54, 55

Oxyria (angiosperm), 405

Oxyrrhis (flagellate), 189, 190, 223

Oxytrichia (infusorian), 277

Ozawa, 82

\section{$\mathrm{P}$}

Pace, 212, 236, 402, 407

Pachynema, 257, 258

Packard, 186, 412

Padina (brown alga), 280

Painter, deletion, 317,318

sex-chromosomes, $376,378, \mathbf{3 7 9}, 393,395$

somatic pairing, 123

translocation, 322, 323, 324

I-radiation, 187

Palla, 5s

Palladin, 25, 64

Pallavicinia (liverwort), 283, 440

Palm, 167

Palmer, 64

Paltauf, 6, 53

Paludina (gastropod), 74

Pancoolus (fungus), 230

Pancreas, 77, 85

Pandorina (green alga), 22

Pangenesis hypothesis, 441

Papaver (angiosperm), 308, 347, 363

Pappenheimer, 73

Parachromosomic, substance, 136, 149, 164

Paracopidosomopsis (insect), 410

Paradesinose, 189, 223

Paradinium (Hagellate), 278

Paragenoplast, 149, 164

Paramitome, 434

Paramœcium (infusorian), 32, 42, 49, 245, 246

Paramylum, 66 
Parasynapsis, 337

Parat, 76-80, 220

Parasettix (grasshopper), 126, 410

Parenchyma, 12

Parker, 13

Parmenter, 410

Parnell, 308

Parthenogamy, 411

Parthenogenesis, in animals, 409 artificial, 247

and heteroploidy, 413

and hybridity, 408

and meiosis, 407

in plants, 401

and sex, 410

Parthenote, 409

Pascher, 89, 192, 277

Pathological tissue, 98, 184

Pathology and genetics, 415

Paton, 235

Patten, 187

Patterson, J. T., 187, 311, 381, 395, 410

Patterson, P. M., 189

Pauli, 187

Paulmier, 376

Pavillard, 228

Payen, 176, 433

Payne, 78, 86, 215, 378

Pea, Mendelism, 257-289, 296

Peacock, 413

Pearson, 382

Pectin, 100, 176

Pediastrum (green alga), 414

Pedicellina (flatworm), 272

Pediculopsis (insect), 136

Pediculus (insect), 272

Pekarek, 187

Pelargonium (angiosperm), 62, 415

Pellew, 329, 331, 367, 419

Pellia (liverwort), chromosome morphology, 129 heteroploidy, 343, 395

heteropyknosis, 146

meiosis, 283

sex-chromosomes, 384, 385, 395

spermatozoid, 203

syngamy, 233

Pcncillium (fungus), 96

Pensa, 69, 72, 91

Pentaploidy, 339, 409

Pentose and pentosan, 27, 30, 62

Peperomia (angiosperm), 212, 237

Peptization, 34

Peranema (flagellate), 190, 223

Percival, 342, 366

Percnosome, 202

Perforatorium, 78, 216, 220, 240, 241

Periderm, 176

Perinium, 180, 181

Periplasm, 200

Periplasmodium, 181

Periplast, 43

Perisperm, 101

Perispore, 181

Perivitelline space, 242

Permanent hybrid, 338
Permeability, 37, 40

Perroncito, 74

Perry, 133

Péterfi, 6, 242

Peterschilka, 68, 190

Petersen, J. B., 43, 44, 223

Petit, 193

Petrunkewitsch, 410

Petruschewsky, 192

Petunia (angiosperm), 239, 352

Peziza (fungus), 281

Pfeffer, 40, 94, 101, 102, 185

Pfeiffer, 29

Pfitener, 141

$p H, 5,29,84,91,97,139$

Phacus (flagellate), 44

Phæoplast, 102

Phajus (orchid), 65, 238

Phaneroplasm, 33

Pharbitis (angiosperm), 296

Phascum (moss), 405

Phase reversal, 36, 41

Phaseolus (angiosperm), 56, 237

Phases in colloids, 34

Phelps, 5s

Phenotype, 290

Philadelphus (angiosperm), 342

Philipp, 186

Phillips, 192

Philp, 267, 286

Phloem fiber, 12

Pholiota (fungus), 419

Phormidium (blue-green alga), 192

Phospholipide, 27, 84

Photosynthesis, 64, 65

Phragmatobia (moth), 380

Phragmidium (fungus), 231

Phragmites (angiosperm), 357

Phragmoplast, 150, 173

Phrynotettix (grasshopper), 118, 136, 141, 265

Phycocyanin, 64, 192

Phycoerythrin, 64, 192

Phycomyces (fungus), 165, 166, 172, 387

Phycomycetes, 199, 229, 281

Phycophæin, 64

Phyllocladus (gymnosperm), 235

Phylloglossum (pteridophyte), 204

Phyllopneuste (bird), 222

Phylloxera (aphid), 411

Physa (snail), 240

Physcomitrella (moss), 371

Physcomitrium (moss), 307, 418

Physical basis of heredity, 286, 421

Physiological gradient, 19

Physostegia (angiosperm), 174

Phytodecta (beetle), 310, 393

Phytosterol, 103

Picard, 165, 256

Pickett, 178

Piech, 174, 210

Pieris (butterfly), 50

Piersol, 14

Pig, chromosomes, 376

Pigeon, 381

Pigments, 96, 97, 192 
Pilayella (brown alga), 63, 280

Pilobolus (fungus), 165

Pilularia (pteridophyte), 233, 437

Pincus, 378

Pinney, 136, 141, 412

Pinnotheres (crustacean), 222

Pinus (gymnosperm), 57, 207, 208, 235, 342

Pisciola (leech), 156

Pisum (pea), aleurone, 101

chondriosomes, $\mathbf{8 3}, 90$

chromosome complement, 124

hybrids, 360

leucoplast, 62

Mendel's work 287-289, 296

reciprocal translocation, 329,331

root hairs, 50

Pits, 175

Plantago (angiosperm), 357

Plantef ol, 90

Plasma membrane, 2, 39, 172

Plasma theory, 436

Plasmodermal blepharoplast, 67, 198

Plasmodesms, 16, 17, 22

Plasmodial theory, 436

Plasmodial tissues, 10, 22

Plasmodiophora (myomycete), 190, 278

Plasmodium, 171

Plasmogamy, 231

Plasinolemma, 39

Plasmon, 418, 419

Plasmosome, 2, 57

Plastid, 3, 61-72, 80, 279

and chondriosomes, 90-92

development, 417

and Golgi material, 80, 81

individuality, $70 \mathrm{ff}$.

origin, $69 \mathrm{fJ}$.

primordia, 69, 70, 101

in syngamy, 233, 234

in zygospore, 279

Plastidome, 61

Plastomere, 199

Plastonema, 70

Plastosome, 70

Platypæcilus (fish), 381, 393

Plethodon (amphibian), 215, 217

Pleurage (f ungus), 387

Plough, 295, 302, 311

Plowe, 39, 40, 42

Plumbagella (angiosperm), 212

Plus and minus strains, 231, 232

Poddubnaja, 210

Podophyllum (angiosperm), 133, 134, 139

Pogonatum (angiosperm), 385

Poirault, 16

Polar bodies, 213, 263

Polar caps, 149, 150

Polar-fusion nucleus, 238

Polar nuclei, 210, 211, 237

Polarity, 20, 75, 180, 185

Polarized light, 84, 98, 99, 178

Pole field, 108

Polianthes (angiosperm), 66

Policard, 84, 91

Politis, 66
Politzer, 187

Poljansky, 192

Pollack, 29

Pollen, characters, 308

grain, $\mathbf{1 7 4}$

tube, 208, 209, 235, 237, 293, 436, 437

wall, 180

Pollination, 237

Pollister, 78, 155, 215, 216, 220

Polocyte, 213

Polyembryony, 382, 405

Polygonum (angiosperm), 342

Polyploidy (see also Heteroploidy), 125, 130, 339

in a nimals, 396

and apomixis, 408

in gametophyte, $343,357,395$

and heredity, $350 \mathrm{ff}$

and meiosis, 274

and size, 356,357

Polypodium (fern), 138

Polysiphonia (red alga), centrospheres, 189

furrowing, 165

gametes, 196, 197

meiosis, 280

sex, 386

syngamy, 227, 229

zoöspores, 198

Polyspory, 361, 362

Polystichum (fern), 404, 405

Polytoma (green alga), 62, 223

Polytrichum (moss), 70, 71, 80, 202, 203, 283

Poole, 367

Popa, 221

Popoff, 51, 1S6, 194, 247, 277

Popovici, 102

Populus (angiosperm), 389, 390, 397

Porphyra (red alga), 68

Portier, 89

Postmeiotic division, 265

Postreduction, 254

Potentilla (angiosperm), 340, 407

Powell, 190, 223

Powers, 121, 342

Prát, 192

Pratje, 25

Preformation, 424

Preissia (liverwort), 233

Prell, 401

Premeiotic mitosis, 272

Prenant, 93, 160

Prereduction, 254

Preston, 178

Prevost and Dumas, 438

Priestley, 176

Primary constriction, 116

Primary mutant, 327

Primordial utricle, 430

Primula (angiosperm), chiasma, 270

gametes, 210

heteroploidy, $351,352,357$

hybrids, 367

meiosis, 267, 274

variegation, 415,416

Principal piece, 221, 222

Pringsheim, 437 
Prionidus (bug), 377, 378

Prochromosomes, 57

Promitosis, 192

Promorphology of ovum, 419

Pronucleus, 242, 263

Prophase, meiotic, 256 second meiotic, 264 somatic, 105, 106, 134, 137

Proplastid, 69, 91, 417

Protamine, 27

Protein, $100 \mathrm{fl}$.

Protenor (bug), 376, 377

Prothallial cell or nucleus, 207

Protista, ectoplast, 39, 43 Jf.

mitosis, 191

nucleus, 48,189

organismal theory, 22

sex, 376

Protoplasm, 25-47

chemical nature of, $25-31$

colloidal nature of, 36-39

continuity, 16, 17, 18

doctrine, 432

early observations, 433

physical nature, 31-33

streaming, $31,160,161,169,185,186$

structural theories, 433-435

term, 433

Protoplast, 2, 433

outline of structure, 103-104

Protoxylem, 175

Protozoa, amitosis, 184, 191

cell theory, 429

chondriosomes, 89

differentiation, 10

ergastic substance, 101

flagellar apparatus, 223

Golgi material, 74, 81

meiosis, 277

nucleus, 59, 189

relation to Metazoa, 21

syngamy, 245

Prowazek, 190, 278

Prozymogen, 77

Prunus (angiosperm), 341, 351, 355

Prywer, 347

Pseudoamitosis, 185, 187

Pseudoblepharoplast, 217, 221

Pseudochondriosomes, 91

Pseudogamy, 406

Pseudomixis, 404

Pseudomonas (bacterium), 194

Pseudonucleoli, 56

Pseud opodium, 32, 43

Pseudoreduction, 252

Psilotum (pteridophyte), 432

Pteridophytes, 204, 233, 386

Pteris (fern), 12, 138, 403, 405

Puccinia (fungus), 231, 386, 387

Punnett, 286, 308

Purkinje, 433

Purkinje cells, 73

Pustularia (fungus), 83, 156, 157, 164, 281

Py, 71

Pycniospore, 231
Pygara (butterfly), 368

Pyknosis, 187

Pylaiella (brown alga), 63, 280

Pyrenoid, 64, 68, 198

Pyronema (fungus), 281

Pyrrhocoris (insect), 376

Pyrus (angiosperm), 351

Pythium (fungus), 200, 229

\section{Q}

Quadripartition, 167

Quadripolar spindle, 151

Quadrivalent chromosomes, 350, 351

Quadrula (mollusk), 225

Quartets, 250

Quercus (angiosperm), 238, 342

Quisumbing, 16, 17

$\mathrm{R}$

Rabbit, 184, 378

Rabl, 145, 438, 440

Raciborski, 66

Radiations, 186, 189, 273, 316, 388

Radium, 188, 311, 412

Radlkofer, 437

Rahn, 188

Ramie, 178

Ramsden, 40

Ramslow, 230

Randolph, heat treatment, 345

heteroploidy, $342,343,346,351,356,358$

hybrids, 360

irradiation, 187

plastid, $69,72,417$

Zea chromosomes, $123,316,317$

Random assortment of chromosomes, 254, 291293

Ranunculacex, 342

Raphanobrassica (angiosperm), 367, 368, 370, 371

Raphanus (angiosperm), 364, 367, 370

Raphides, 102, 103

Rappeport, 379

Rat, 378

Rateliffe, 190

vom Rath, 184,440

Rathery, 89

Rau, 74, 272

Rauber, 436

Rawitscher, 231

Reboulia (liverwort), 201, 233, 283

Recedent genom, 419

Receptive spot, 196, 197, 234

Recessive, 287

Reciprocal crosses, 418

Reciprocal translocation, 315, 322, 327-338

Recombination of factors, 294-304

Redfield, 266, 306, 353

Reduction, 89

of chromosomes (see also Meiosis), 250-283

Reed, G., 30, 57, 58, 59

Rees, C. W., 10, 223

Refringent body, 222

Regaud, 82, 84, 89 
Reichenow, 27, 189, 194

Reichert, 30, 100

Reinke, 26, 63

Reiss, 75

Reiter, 188

Rejuvenescence, 246

Remak, 431, 432

Renaut, 76

Renner, alternation of generations, 278 apomixis, 401

false hybrid, 406

Enothera, 334, 335, 337

pollen characters, 308

reciprocal hybrid, 419

variegation, 416

Renner complex, 335, 336

Renyi, 18

Repulsion in mitosis, 163

Resin, 97

Respiration, 64, 65, 88

Restitution nucleus, 346, 404

Reticular theory of protoplasm, 434

Reticularia (myxomycete), 26

Reticulum of nucleus, $2,53,106$

Reuter, 105, 136, 144, 146, 162, 256

Reznikoff, 29

Rhabditis (nematode), 169, 411

Rheum (angiosperm), 103

Rhizoclonium (green alga), 191

Rhizoplast, 189, 223

Rhizopus (fungus), 83, 165, 166

Rhoades, M. M1., 300, 306, 322, 332, 351, 419

Rhoades, V., 238

Rhodites (gall-fly), 272, 411

Rhodochorton (red alga), 63

Rhæo (angiosperm), 256, 329, 335

Rhopalodia (diatom), 228

Rhumbler, 58

Ribes (gooseberry), 346

Riccardia (liverwort), 199, 203, 232, 233

Riccia (liverwort), 233

Rich, 428

Richards, A., 136, 146

Richards, O., 188

Richardson, C., 391

Richardson, E., 329

Ricinus (castor bean), 95, 96, 101, 103

Rickett, 196, 233

Riddle, 397, 398

Riella (liverwort), 437

Rigg, 93

Rings, due to deletion, 315, 317

due to reciprocal translocation, $328 \mathrm{ff}$.

Rita (fish), 78

Ritter, G., 185

Ritter, G. J., 176, 185

Ritter, W. E., 21, 436

Rivett, 102

Rivularia (blue-green alga), 192

Robbins, W. J., 196

Robbins, W. W., 196

Roberts, 436

Robertson, A., 235

Robertson, T. B., 25, 33, 40
Robertson, W. R. B., chromomeres, 141 chromosome arrangement, 126 chromosome morphology, 123, 130 meiosis, 256, 266, 268

parthenogenesis, 409-411

Robinia (angiosperm), 94

Robyns, 102, 149, 150, 161, 171

Rodewald, 26

Rogers, 229, 233

Rohde, classification of cell elements, 45 cytokinesis, 171,173

intercellular substance, 182

nucleolus, 58

organismal theory, 24, 436

Rokizky, 311

Romanova, 189

Root hairs, 50

Rosa (angiosperm), apomixis, 405, 409

chiasma, 267, 268

chromosome sets, 365

heteroploidy, 340,347, 354

hybrids, 362-364

meiosis, 267

Wilsoni, 367

Rosen, 56

Rosenberg, apomixis, 401-408, 406 chromocenters, 57

chromosome morphology, 124

chromosomes in hybrids, 339,361

heteroploidy, 346-348

hybrids, 364

meiosis, 256

Rosenbusch, 224

Rosenvinge, 16

Roskin, 189

Rossenbeck, 52

Rossmann, 188

Roth, 342

Rothert, 63,165

Roux, 141, 442, 444

Rubus (angiosperm), 358, 371

Rückert, 146, 243, 440

Rudenko, 210

Rudolph, 90

Rudolphi, 426

Ruhland, 40, 71, 91

Rumex (angiosperm), 129, 342, 389, 390, 395

Rumjantzew, 84, 195

Russell, 21, 47, 436

Russow, 16

Rusts, 283

Rutgers, 212

Ruttle, 343, 345, 356

Ruys, 105, 256

Rybin, 186, 367, 368

Rytz, 165

S

Sabellaria (annelid), 30

Saccharum (angiosperm), 369

Sachs, 23, 46, 50, 430, 435, 436

Saez, 129, 130, 378

Sagitta (round worm), 272

Saguchi, 57, 58, 77, 184, 224, 225 
Sainmont, 266, $37 \mathrm{~s}$

Sakamura, abnormal mitosis, 185, 186

chromonema, 139, 140

chromosome morphology, 115

chromosome size, 115

chromosomes in Triticum, 342

homologous chromosomes, 124, 273

Salamandra (amphibian), 133, 155, 187

Salix (willow), 390,396

Salter, 100

Salvia (angiosperm), 88

Sambucus (angiosperm), 210

Samuelsson, 239

Sanchez, 79

Sanday, 235

Sande-Bakhuyzen, 98

Sands, 161

Sansome, E., 329, 331

Sansome, F., 286, 344

Santos, 389

Sapěhin, 70, 90, 203

Sappin-Trouffy, 282

Saprolegnia (f ungus), 96, 165, 184, 200, 229, 402

Sargant, 212

Sargassum (brown alga), 247, 280

Sass, 230, 386

Satellites, 118, 119, 124, 127, 128, 130, 314, 349, 361, 367

Satina, 388, 398

Saunders, 308

Sax, chiasma, 268, 270, 271

chromonema, 139

heteroploidy, 342,345

hybrids, 363, 366

male nuclei, 210

meiosis, 260, 267, 268

reciprocal translocation, 329,335

syngamy, 237

Saxifraga (angiosperm), 367

Scarth, 32, 44, 53, 54, 165, 176

Scenedesmus (green alga), 68

Schacht, 437

Schacke, 283, 382

Schaede, 53, 163

Schaeffer, 31, 39, 40, 89

Schaffner, 201, 383, 396

Schaudinn, 194, 277, 278

Schaxel, 84, 194

Scheben, 222

Scheremetzewa, 295

Scherrer, 63, 70, 85, 90

Schilling, 66

Schimper, 61, 65, 68, 70, 99, 100

Schizophyllum (f ungus), 230

Schlater, 436

Schleicher, 439

Schleiden, 20, 428, 437

Schleip, 188, 266, 411

Schlösser, 402

Schmidt, E., 57, 82

Schmidt, J., 378

Schmidt, P., 228, 280

Schmidt, W. J., 222

Schmitt, 224, 225

Schmitz, 63, 68, 100, 437, 438
Schnarf, 212, 226, 237, 239

Schneider, A., 439, 441

Schneider, H., 266

Schneider, K., 133

Schoeffel, 188

Schottländer, 204

Schrader, achromatic figure, 162, 163 apomixis, $410,411,413$ sex, 376,395

Schratz, 357, 398

Schreiner, 256, 266

Schüepp, 11

Schultz, E., 51, 158, 187, 281, 395

Schultze, M., 433

Schultze, W., 59

Schulz, 342

Schulze, 176

Schürhoff, amitosis, 183

apomixis, 402,405

embryo sac, 212

male cells, 210

meiosis, 256

mitosis, 105

nucleolus, 58

plastids, 61

syngamy, 226, 236

Schussnig, 190, 279, 386

Schwann, 20, 428, 435, 438

Schwarz, 188

Schwarze, 165

Schweigger-Seidel, 438

Schweizer, 386,405

Schwemmle, 273, 334, 342, 419

Sciara (fly), 163

Scillex, 129, 186

Scinaia (red alga), 280

Scirpus (angiosperm), 174, 210, 341

Sclerotina (f ungus), 387

Scolopendra (centipede), 272

Scolopendrium (fern), 402, 405

Scopolia (angiosperm), 50

Scorpion, 86

Scott, 79

Seal, 378

Sears, 180, 402

Sebaceous gland, 77

Secale (angiosperm), 143, 237, 260, 267, 366

Second contraction, 260

Secondary association of bivalents, 351

Secondary constriction, 115, 118

Secondary mutant, 327,328

Secondary wall layer, $12,175,176$

Secretion, 14, 98

chondriosomes in, 77,88

Golgi material in, 75-78

and nucleolus, 58

Sedgwick, 21, 206, 436

Seeds, storage, 101, 102

Segmental interchange, 327-338

Segregation, 287

Seifriz, cryptoplasm and phaneroplasm, 45 emulsion, 35

hyaloplasm, 33

micromanipulation, 6

nucleus, 52 
Seifriz, protoplasm, 31, 32, 37, 39

X-ray analysis, 178,179

Seiler, 268, 350, 397, 411

Selaginella (pteridophyte), 61, 69, 180, 181, 204

Selim, 119

Semiheterotypic mitosis, 346

Semipermeability, 37, 40, 41

Semisterile plants, 329-334

Senecio (angiosperm), 341, 345, 415

Senescence, 246

Senjaninova, 70, 71, S6, 90, 11S, 342

Senjaninova-Korczagina, 128, 129

Senn, 61

Sepia (mollusk), 222

Serebrovsky, 315, 320

Sertoli cells, 215

set of chromosomes, $121,254,325$

Sethi, 233

Sex, chemical reactions, 226,398

chromosomes, 297, 375-400

genes, 394

and heteroploidy, 394

historical, $\mathbf{4 3 6}$

intergrades, 394

in life cycles, $\mathbf{3 8 3}$

-limited characters, 391

linkage, 297, 391-393

metabolic theory, 398

modification, 396-399

and parthenogenesis, 410

Shaffer, 88

Sharp, L. W., blepharoplast, 208

chromosomes, form and structure, 116, 124, 133,136

cytokinesis, $\mathbf{1 7 4}$

life eycles, $\mathbf{3 8 3}$

mitosis, 133, 134, 137, 140, 142

reticulum, 55

organism-environment, 47,312

sex, 3Ss, $39 S$

spermatogenesis, 199, 201, 203, 204, 205

Sharp, R. G., 10, 223

Shaw, 178, 204, 233

Shchavinskaia, 367

Shearer, 18

Sheep, 376

Sheffield, 334

Sheppard, 58

Shibata, 238

Shigenaga, 52, 57, 120, 140

Shimamura, 57, 206, 234

Shimotomai, 1S6, 340, 342, 385, 390, 395

Shinke, 52, 57, 120, 139, 140

Shiwago, 381

Showalter, 203, 232, 233, 343, 383, 395

Shull, A. F., 410

Shull, G. H., 390, 392, 415

Siebert, 188

Sierp, 196

Sieve tube, 12,13

Silene (angiosperm), 357, 390

Silk, 179

Simocephalus (crustacean), 411

Simplex, 354

Simplex group of chromosomes, 292
Simultaneous division, 167

Sinapis (angiosperm), 50

de Sinéty, 376

Sinigaglia, 73

Sinnott, 23, 286, $35 \mathrm{~S}$

Sinotô, 164, 334, 357, 38s, 390, 396

Sirks, 286, 419

Sisa, 121

Sjövall, 220

Skalinska, 367

Skupieński, 190, 278

Slime, 96,100

Small, 29

de Smet, 124

Smilacina (angiosperm), 212

Smith, B. G., 136, 146, 243

Smith, D., 88

Smith, E. L., 100, 102

Smith, F. E., 154

Smith, F. H., 133, 139

Smith, G. M1., 68, 196, 226, 278

Smith, I., 206

Smith, S. G., 139, 341

Smólska, 206, 235

Söderberg, 167

Sokolow, S7, 215, 223

Sol, 34

Solanum (angiosperm), 186, 273, 352, 356-358, 367,406

Solenobia (moth), 411

Solvation, 34

Somatic mitosis, 105-113, 106, 112, 133-139

duration and periodicity, 111

outline, 105-109

Somatic mutation, 310,345

Somatic pairing of chromosomes, 123, 126, 272

Sorghum (angiosperm), 341, 342, 415

Sorodiscus (myxomycete), 190

Sorokin, 116, $11 \mathrm{~S}$

Space lattice, 178

Spallanzani, 435

Spartina (angiosperm), 367

Spathiphyllum (angiosperm), 405

Special wall, 167, 168

Specificity of organisms, 30

Spek, 31, 169, 171

Speltoid wheats, 276

Spemann, 23, 412

Spencer, Herbert, 47

Spencer, Hope, 246

Spermatia, 197, 227, 231

Spermatid, 201, 215

Spermatocyte, 214, 215

Spermatogenesis, in animals, $81,214-223$

in plants, 70, 196-211, 202, 205

Spermatogonia, 214

Spermatozoids, 197, 199, 202, 227, 232, 239

Spermatozoön, 26, 215, 221, 222, 240, 241, 437

Spermiogenesis, 215-217

Sphacelaria (brown alga), 111, 159

Sphorocarpos (liverwort), 233, 266, 283, 307, 382, 384, 395

Sphcroplea (green alga), 227, 279

Sphagnum (moss), 201, 283

Spier, 176 
Spierer objective, 33

Spinacia (angiosperm), 343, 346

Spindle, 106, 107, 149, 152, 161, 162, 259, 274 of cytokinesis, 171,173

Spindle-attachment region, 106-109, 116-119, 123, 148, 332

altered position, 320

effect on crossing-over, 302

fragments without, 317

in translocations, 321

Spindle fiber, 150

Spindle substance, $149,151,152$

Spirillum (bacterium), 43

Spirogyra (green alga), conjugation, 228

cytokinesis, 111, 166

gametes, 43, 45, 196

hybrids, 308

kinoplasm, 44

meiosis, 279

mitosis, 190

nucleus, early observations, 437

plastid, 63, 64, 72, 279

pyrenoid, 68

syngamy, 228, 437

tonoplast, 42

vacuoles, 228

zygospores, 279

Spirostomum (protozoan), 50, 51

Spirulina (blue-green alga), 192

Spitzer, 59

Splitting of chromosomes, in meiosis, 252, 255, $258,261,265,275$

somatic, 105, 113,133,137, 145, 190

Spoehr, 27

Spongioplasm, 434

Spongospora (myxomycete), 190

Sponsler, 99, 100, 178, 179

Spore coat, 180, 181

Sporidia, 231

Sporocyte, 204

Sporodinia (f ungus), 165

Sporogenesis, 70, 196-198, 200, 204, 206, 209-211

Sporophyte, 122, 204, $383 f f ., 401 f f$.

Sporophytic budding, 404

Sprengel, C. K., 436

Sprengel, K., 426

Stabilizer, 35

Stadler, 187, 188, 311, 316, 317, 356

Stains, 5, 7, 84, 139, 142

Stakman, 232

Stålfelt, 112, 171

Stalk cell, 207

Stemmkörper, 162, 163

Stancati, 382

Stangeria (cycad), 234

Starch, 65, 80, 97, 98, 99

Starfish, 240, 241

Stark, 139

Starks, 378

Steere, 352

Steil, 199, 203, 403, 404

Stein, 61, 188

Stellaria (angiosperm), 415

Stenar, 167, 212, 239

Stenobothrus (grasshopper), 7, 162
Stentor (protozoan), 50

Sterility, 352, 354, 356, 358, 359 ff., 387, 419

Stern, 68, 286, 301, 302, 303, 304, 321, 393

Stethophyma (grasshopper), 258, 304

Stevens, F. L., 199

Stevens, N. M., 272, 378, 381

Steward, 40

Stieve, $\mathbf{5 5}$

Stigeoclonium (green alga), 23

Stigma (eyespot), 66

Stiles, 40, 64

Stizolobium (angiosperm), 331

Stoel, 188

Śtolc, 58

Stoll, 64, 68

Stolte, 49

Stolze, 121

Stomates in polyploid Zea, 358

Stomps, 142, 345, 412, 416

Stone, 395

Stopes, 206

Storch, 410

Stork, 402

Stoughton, 194

Stout, 396

Stow, 186, 273

Strangeways, 3, 111, 187, 317

Strasburger, apomixis, 401, 402, 407

blepharoplast, 198

cell-division, 439

cell wall, $173,174,177,178$

chondriosomes, 82

chromocenters, 57

chromomeres, 142

chromosome number, 126

cytokinesis, 171,173

eyespot, 67

kinoplasm, 44

meiosis, 262, 440

Mendelism and chromosomes, 445

mitosis, 190

nuclei, origin of, 432

nucleolus, 58

nucleoplasm, 2

nucleoplasmic ratio, 51

nucleus in heredity, 285, 441

protoplasmic continuity, 16

recombination, 302

sex determination, $375,382,388$

starch, 65

syngamy, 437

tetrad chromosome, 262

tetraploidy, 345

vacuoles, 94

Streaming, 31, 185, 186

in cell-division, $160,161,169$

Street, 179

Strepsinema, 259

Stroma, 67, 68

Strongylocentrotus (sea urchin), 242

Structural hybrids, 336

Strugger, 53

Studnicka, 15, 182

Sturgeon, 222

Sturtevant, crossing-over, 299, 302, 320 
Sturtevant, linkage groups, 296, 301 Enothera, 335, 336

reciprocal translocation, 329

sex determination, 395

Stypocaulon (brown alga), 159, 280

Suberin, 176

Successive division, 167

Suessenguth, 71, 167

Sugars, 100

Sugimoto, 139

Sumner, 28

Superlinkage, 338

Supersexes, 394

Surface tension, 169

Surirella (diatom), 280

Sutton, 122, 272, 445

Svedberg, 33

Svedelius, $122,278,280$

Svenson, 302

Svensson, 239

Sverdrup, 351

Swammerdam, 424

Swarm spores, 197

Sweschnikowa, 124, 129, 343

Swezy, 10, 190, 223, 379

Swingle, D. B., 165,166

Swingle, W. T., 159, 173

Symbiosis, 89

Symphoricarpos (angiosperm), 44

Symplasm, 90

Synapsis, 252, 256, 257, 272-277

and apomixis, 407

and deletion, 319

in heteroploids, 350, 351, 352

in hybrids, $359 \mathrm{ff}$.

after inversions, $\mathbf{3 1 9}, 320$

in polyploid hybrids, $361 \mathrm{ff}$.

after reciprocal translocation, $331, \mathbf{3 3 2}, 333$

in tetraploid, 350

in triploid, 352

Synchytrium (fungus), 50, 165, 199, 229

Syncytium, 15, 24, 171

Synergids, 210, 211, 237

Syngamy, in algæ, 226

in animals, 239-249, 263

in fungi, 229

historical, 436-439

physiology of, 247

in plants, 197, 200, 211, 226-239

Synizesis, 256

Sypkens, 55

Syringa (angiosperm), 186

$\mathrm{T}$

Täckholm, apomixis, 405, 408, 409 cytokinesis, 167

heteroploidy, 340, 348

hybrids, 362-364

Tahara, 197, 247, 340, 345, 402

Takamine, 124, 188

Takeda, 415

Talceporia (moth), 380

Tammes, 64

Tanaka, 420
Tandy, 158, 281, 282

Tangl, 16, 18

Tannin, 96, 97

Tannreuther, 190

Tansley, 278

Tänzer, 51

Tapetal plasmodium, 181

Tapetum, 79, 83, 181, 183, 308

Taraxacum (angiosperm), 402, 403, 415

Tassement polaire, 135

Taxodium (gymnosperm), 208, 235

Taxus (gymnosperm), 235

Taylor, C. V., 6, 10, 19, 31, 49, 53, 223

Taylor, G., 188

Taylor, J. W., 367

Taylor, W. R., 114, 124, 129, 130, 139, 142, 260, 264,265

Telezynski, 54, 133, 135

Teliospore, 231

Telomitic, 116

Telophase, meiotic, 262

somatic, 106, 108, 134, 135

Teosinte (see Euchlona)

Telosynapsis, 337

Temperature effects, activation, 248

chromosome behavior, 273

crossing-over, 302

mutation, 311,345

rate of mitosis, 111,186

translocation, 317,321

Tennent, 146, 195, 412

Tephrosia (moth), 413

Terao, 415

Terletzki, 16

Terminalization, 268-271

Terni, 85, 86, 87, 220

Tertiary mutants, 329

Tertiary wall layer, 175,176

Tetrad chromosome, 252, 258, 262, 263, 271

T'ctradesmus (green alga), 68

Tetranychus (mite), 410

Tetraploidy, 339, 342, 347, 350, $365 \not J ., 395$

Tetrasomic plants, 350

Tetrasomic ratios, 352

Tetraspores, 280

Thalictrum (angiosperm), 340, 343, 391, 396

Tharaldsen, 154, 244

Thatcher, 25, 64, 93

Theophrastus, 422

Thom, 204

Thomas, 177, 308

Thompson, D'A. W., 19, 23

Thompson, W. P., 342, 366, 396

Thomsen, 410, 411

Thomson, 47, 399, 441

Thuja (gymnosperm), 235

Thuret, 437

Tiffany, 177

Timberlake, 165, 171, 174

Timoféeff-Ressovsky, 311

Tischler, achromatic figure, 153

amitosis, 183

apomixis, 401

cambial initial, 51

chromomeres, 142 
Tischler, chromosome number, 121, 126, 342 exine, 180,181

heteroploidy, 345, 357, $35 \mathrm{~s}$

meiosis, 256

mitosis, $105,111,159$

nuclear crystals, 101

nuclear function, 58

nuclear membrane, 136

nucleolus, 57

nucleoplasmic ratio, 52

nucleus, 48-50

protoplasm, 25

restitution nucleus, $346^{\circ}$

syngamy, 226, 236

Tissue cultures, $6,85,187$

Tissue theory, 436

Tissues, 1-24

Tolypothrix (blue-green alga), 192

Tomowo, 352

Tonoplast, 2, 42, 94

Torreya (gymnosperm), 235

Torus, 175

Torvik, 395

Townsend, 58

Toyama, 420

Tozer, 57

Tracheid, 12, 13, 175

Trachelomonas (protozoan), 67

Tractile fibers, $150,151,162$

Tradescantia (angiosperm), achromatic figure, 162 amitosis, 184

cell-plate, 171, 172

chromosomes, 132, 133, 139, 143, 163

fragmentation, 314, 319

kinoplasm, 44

meiosis, 256, 260, 261, 265, 267, 270, 431, 432, 440

micromanipulation, 6,54

protoplasm, 31, 434

reciprocal translocation, 329

somatic mitosis, 111, 139

Trankowsky, 116, 117, 209, 210

Transeau, 308

Translocation, 121, 188, 305, 314-326

reciprocal, $\mathbf{3 1 5}, 322,327-338$

Trennungstelle, 116

Treub, 174

Treviranus, 426, 432

Trialeurodes (fly), 410

Trichia (myxomycete), 278

Trichites, 99

Trichocyst, 44

Trichogyne, 228, 231

Trichomonas (flagellate), 223

Trichoniscus (crustacean), 413

Trichosanthes (gourd), 176

Triebel, 88

Trier, 25, 93

Trifolieæ, 342

Trillium (angiosperm), 117, 134, 139, 236, 238, 258,261

Trimerotropis (grasshopper), 254, 265

Triple fusion, 237, 238

Triplex, 354

Triploidy, 339, 345, 346, 352
Tripsacum (angiosperm), 407

Trisome, 297, 327

Trisomic plants, 352

Trisomic ratios, 297,354

Triticum (wheat), abnormal mitosis, 186 chromosomes in hybrids, 129, 363

heteroploidy, 342, 343, 345, 356

parthenogenesis, 402

restitution nucleus, 346

syngamy, 237

vacuole, 102

Triticum $\times$ Égilops, 366, 367, 368

Triticum $\times$ Secale, 367, 368

Triton (amphibian), 222

Trivalent chromosome, 274, 351, 353

Tron, 128

Tröndle, 72, 279

Trophochromatin, 194

Trophonucleus, 224

Trophoplasm, 44

Trow, 199

Trypan blue, 76

Trypanosoma (flagellate), 44

Trypanosomes, 224

Tschechow, 129, 342

Tschenzoff, 190

Tschermak, 366, 367, 445

Tschernoyarow, 124

Tschirch, 101, 103

T'Serclaes, 190, 191

Tsinen, 417

Tsuga (gymnosperm), 235

Tswett, 68

Tuan, 139

Tube cell or nucleus, 208, 209, 237

Tulasne, 437

Tulipa (angiosperm), 186, 258, 267, 274, 351, 352, $354,355,357$

Tumor, 187

Tupper, 358

Tupper-Carey, 176

Turesson, 408

Turgor, 42

Turkey, 381

Turner, 277

Turrill, 367

Tusehnjakova, 390

Tuthill, 188

Tuttle, 279

Twin hybrids, 338

Twins, 382

Twiss, 85, 382

Two-plane theory of chiasma, 268, 269, 271

Tyloses, 17

Tyndall phenomenon, 33

U

Uda, 420

Ulothrix (green alga), 68, 196, 198, 226, 227, 279

Ultramicroscopic structure, 177

Ultra-violet light, 139, 188

Ulva (green alga), 68, 190, 279

Umbelliferre, 342

Undulating membrane, 44, 223 
Unger, 430

Unna, 26, 57

Urease, 28

Urechis (echinoderm), 163

Urediner, 283

Uroglena (yellow-green alga), 44

Uroleptus (infusorian), 277

Uromyces (fungus), 231

Ustilago (f ungus), 231, 386

Uvularia (angiosperm), 186, 273, 320, 345

\section{V}

Vacuole, 2, 93-96, 210

contractile, 42, 228

in cytokinesis, $165,168,171$

ergastic contents, 96-98, 101, 102

membrane, 42 ff.

origin of, 94

Vacuome, 94, 193

Valkanover, 334

Valeriana (angiosperm), 342, 389, 390

Valleau, 391

Vallisneria (angiosperm), 210, 236

Valonia (green alga), 97, 178

Van Camp, 58

Van Cleave, 9

Vandel, 413

Vandendries, 283, 386, 388

Van der Stricht, 244

Van Durme, ss

Vanessa (butterfly), 50, 51

Van Regemorter, 185

Van't Hoff's law, 112

Van Teighem, 95

Variegation, 317,415

Vaucheria (green alga), body structure, 8, 10, 23 gametes, 196

gametic meiosis, 279

mitosis, 190

parthenogenesis, 402

sex, 386

syngamy, 197, 227

zoöspore, 198

Vaupel, 378

Vavilov, 342

Vegetative propagation, 354

Vejdovský, 132, 133, 142, 266

Velten, 434

Verson's cells, 215

Verworn, 46

Vesperugo (bat), 222

Vessel, 12

Vicia (angiosperm), abnormal mitosis, 186, 187

cell-plate, 171

chondriosomes, 91

chromosome complement, 115, 118, 124, 128, 129

cytoplasmic heredity, 419

heteroploidy, 343

leucoplast, 62

meiosis, 267

nucleolus, 120, 121

plastids, 69

somatic mitosis, 133,134
Vinca (angiosperm), 210

Vincetoxicum (angiosperm), 210

Vines, 401

Viola (angiosperm), 210, 236, 273, 342, 361, 415, 416

Virchow, 431, 439

Viscosity, 31, 169, 186, 248, 249

Viscum (angiosperm), 17

Visscher, 245

Vital dyes, 6

Vitamin D, 27

Vitelline membrane, 214, 240, 241

Voinov, 78, 86, 87, 215

Volkonsky, 62

Volutin, 27, 193

Volvocales (green algæ), 16, 68, 279

Vouk, 101

de Vries, Enothera species, 336, 338

recombination, 302

rediscovery of Mendelism, 445

theory of heredity, 441

tonoplast, 42

vacuoles, 79,94

W

W-chromosome, 379, 380

Wager, 57, 66, 67, 199, 226

Wagner, 71, 85, 188

Wakayama, 57, 206, 282

Wakker, 66, 101

Waldeyer, 440

Walker, 57, 283

Wall thickening, 50

Wallin, 89,90

Walter, H., 25

Walter, H. E., 286

Walton, 378

Waltzing mice, 317

Wámoscher, 193

Wanderplasm, 197

Wanscher, 342

Warburg, 38, 89

Wassilief, 194

Watkins, 342, 366, 396

Wax, 102

Weatherby, 43

Webber, 199, 207, 234

Weber, 31, 32, 40, 61

Weier, Enothera, 334, 337

plastids, $68-71$ and Golgi material, $\mathbf{8 0}, 81$ spermatogenesis, 201, 203

Weigl, 74, 220

Weinstein, 302, 306, 311

Weismann, 141, 441-445

Wells, 25, 26

Wels, 186

Welsford, 210, 230, 236, 237, 2 S1

Wendel, 50

Weniger, 236, 237, 238

Wenrich, amitosis, 190

chiasma, 268

chromomeres, 136, 141

flagella, 223, 224 
Wenrich, meiosis, 265, 266

nucleolus, 118

nucleus, 49

vacuoles, 42

Went, 19, 95, 97

Wermel, 52, 120, 195

Werner, 381

West, 196, 278

von Wettstein, apomixis, 402

cell size, 357

cell wall, 176

cytoplasmic heredity, 418, 419

gametophytic characters, 307,355

heteroploidy, $340,343,405$

polyploid moss hybrids, 371, 405, 408

sex in algæ, 386

Wetzel, 71,91

Wherry, 59

White, 289

Whiting, A. R., 395

Whiting, P., 311, 382, 395

Whitman, 21, 425, 436, 438, 439

Whitney, 399, 410

Whong, 18

Wieman, 379

Wierzyski, 240

Wiesner, 93, 176, 177, 178

Wigoder, 187

Wikstrcemia (angiosperm), 345, 402, 407

Wilcox, 387

Wildeman, 111

Wille, 16

Willems, 272

Williams, J. L., 227, 280

Williams, M., 186, 188, 190, 197, 279

Williamson, 158, 230, 281, 387

Willstätter, 64, 68

Wilson, E. B., amitosis, 183

aster, 154

cell-division, historical, 439

centrosome, 153,155

chondriosomes, 86,88

chromatin, 55

chromidia, 195

chromosomes, and heredity, 286, 291, 292 somatic, 121,125

cytology and genetics, 445

cytoplasmic heredity, 420

epigenesis, 425

flagellum, 223

individuality of chromosomes, 145

karyosome, 189

meiosis, 256, 265, 268, 272

mitosis, $159,160,164$

nucleoplasmic ratio, 51

organismal theory, 22

parthenogenesis, 412

protoplasm, 31, 32, 93

sex-chromosomes, 376,379

spermatogenesis, 213,215

syngamy, 239-245, 248

Weismannism, 442

Wilson, H. V., 6

Wilson, M1., 201, 203
Winge, alloheteroploidy, 369

amphidiploidy, 367

apomixis, 408

chromosome number, 124

factor combinations, 292

galls and tumors, 186,187

heteroploidy, $343,347,348$

linkage, 391,392

myxomycete, 190

sex-chromosomes, 378, 381, 389, 390, 393

variegation, 415,416

de Winiwater, 58, 256, 266, 378, 379

Winkleman, 188, 311

Winkler, apomixis, 100, 401, 402, 407

altered chromosomes, 186

heredity, 286

heteroploidy, 340,358

grafting, 344

nuclear phases, 122

nucleoplasmic ratio, 51

van Wisselingh, $58,176,177$

Witschi, 376, 397, 398

Wodehouse, 180

Wodsedalek, 376

Wolff, 425

Wolfson, 382

Wollenweber, 66

Wood, F. M., 177, 179

Wood fiber, 12, 13

Woodburn, 201, 233

Woodger, 78, 88, 220

Woodruff, 49, 246

Woodward, 249

Woodwardia (fern), 405

Wortmann, 16

Woskressensky, 295

Wound hormone, 409

Wounding, 185, 186

Woycicki, crystals, 101 cytokinesis, 167, 168

elaioplast, 66

gonomery, 235

gymnosperm egg, 206

meiosis in fungus, 281

plastids, 71

pollen tube, 42,210

Wu, 51, 58

Wuist, 386

Wurdack, 177

Wygaerts, 55, 140, 142

Wylie, 210, 236

Wyman, 87

$\mathrm{x}$

$\mathrm{X}$-chromosome, 304, $376 f f$.

$\mathrm{X}$-ray analysis, of cell wall, 178 of chromosome, $317 \mathrm{ft}$. of starch granules, 99

$\mathrm{X}$-ray effects, on chromosomes, $186,187,273$ on crossing-over, 302

and inversion, 320

on mutation, 311

and translocation, $317,318,321$

Xanthophyll, 64 
Y

$Y$-chromosome, 304, 378 f.

Yamagiwa, 187

Yamaha, 5, 27, 120, 164, 165, 185

Yamamoto, 121, 129, 390, 395

Yamanouchi, achromatic figure, 159, 165

apogamy, 403

chondriosomes, 86

eyespot, 66

meiosis, 280

spermatogenesis, 204

syngamy, 227, 228, 229, 233

Yampolsky, 388, 396

Yasui, 416, 417

Yatsu, 154, 412

Yazawa, 201

Yeast, 96,167

Yocum, 10, 223

Yolk, 78, S8, 100, 213

Young, R. A., 32, 188

Yuasa, 204, 205

\section{Z}

Z-chromosome, 379, 380

Zacharias, 25, 56, 57

Zade, 342

Zamia (cycad), 199, 234

Zanardinia (brown alga), 66, 280

Zattler, 386, 408

Zea (maize), abnormal mitosis, 186, 187 chiasma, 271

chromomeres, 142, 143, 259, 274, 276

chromosome set, 298

chromosomes, $115,118,119,121,123,135,140$, $259,274,276$

crossing-over, 302, 303, 305

cytoplasmic heredity, 419

endosperm, 355

heteroploidy, $342,343,345,346$

inversion and deletion, 318, 319, 320

liguleless leaves, 299

linkage, 296, 300

linkage groups, 296, 297, 298

linkage map, 299

matrix, 120, 136

meiosis, 259, 261, 265, 273, 274, 276
Zea (maize), monoploid sporophyte, 356 non-disjunction, 345

non-homologous association, 276

nucleolus, 119, 120

plastid development, 69

pollen characters, 30,308

root tip, 5

reciprocal translocation, 327, 329-334, 330, 332

silkless ears, 299

spindle, 164

stomates in polyploids, 358

syngamy, 237, 238

temperature effects, 317,345

tetraploidy, 345

translocation, $316,317,321,322$

triploidy, 346, 347, 352, 354

trisomic inheritance, 297, 354

variegation, $415-417$

Zea-Euchlcena hybrid, 360, 371

Zebrina (angiosperm), 261, 329

Zeleny, 412

Ziegenspeck, 172, 177

Zimmermann, A., 25, 66, 154

Zimmermann, W., 31, 68, 111, 159, 279

Zirkle, chondriosome, 90, 91

chromosome, 133

early hybridizers, 436

fixation, 5

nucleolus, 58, 118, 120

permeability, 40

plastids, $67, \mathbf{6 8}, 69$

spindle, 164

vacuole, $79,93,94,96,97$

Zonaria (brown alga), 280

Zoöspores, 197, 198, 199

Zuelzer, 186

Zulueta, 310, 393

Zweibaum, 74

Zygnema (green alga), 61, 65, 72, 279

Zygomycetes, 200

Zygonema, 256, 257, 258

Zygophase, 122

Zygospore, 198, 22S, 229, 279, 307

Zygote, 122, 249

Zygotic meiosis, 278

Zygotic number, 122

Zymogen, 88 




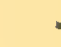





